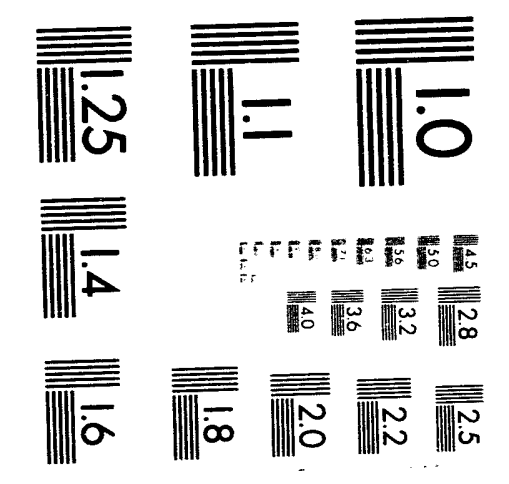



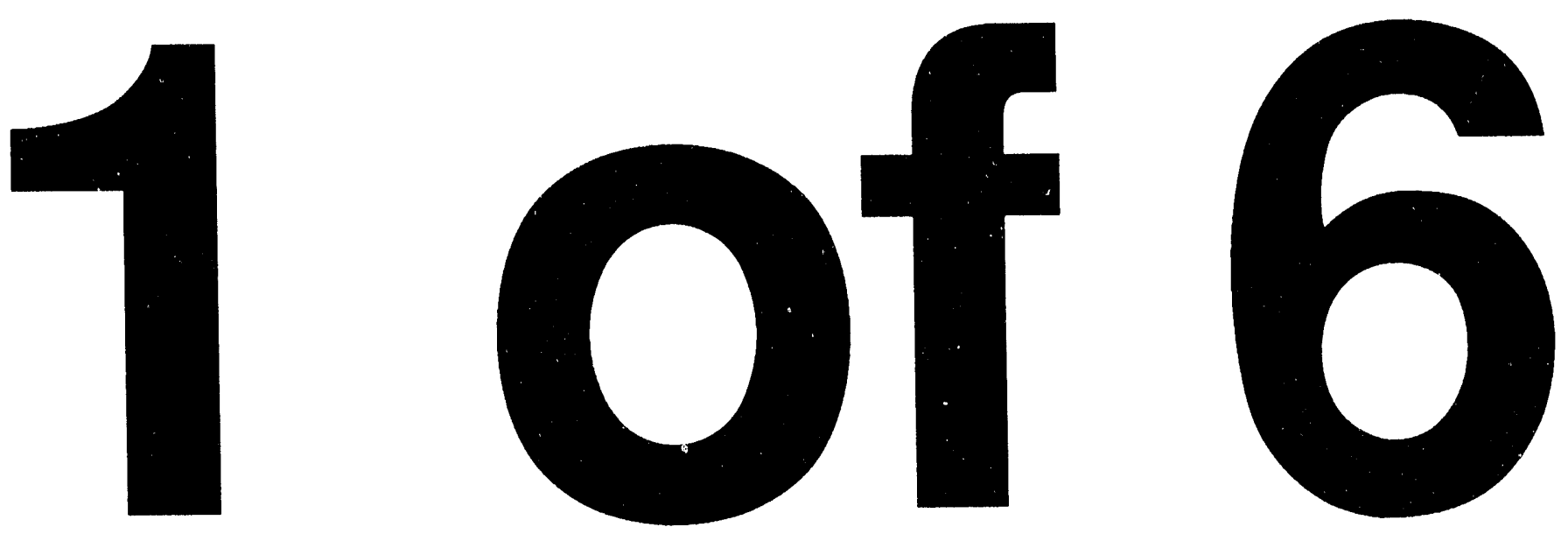


\title{
FINAL REPORT ON DECOMMISSIONING BOREHOLES AND WELLSITE RESTORATION, GULF COAST INTERIOR SALT DOMES OF MISSISSIPPI
}

\author{
REVISION 0
}

APRIL 1989

Prepared by: Stone \& Webster Engineering Corporation

Prepared for: U.S. Department of Energy

Salt Repository Project Office 
1.0 INTRODUCTION 1

2.0 PREREQUISITES TO FIELD ACTIVITIES

3.0 FIELD ACTIVITIES

3.1 Well Reconnaissance $\quad 8$

3.2 Well Plugging 9

3.2.1 Deep Wells 9

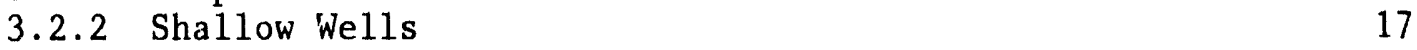

3.2.2.1 PVC Monitoring Wells 17

3.2.2.2 Water Supply Wells 18

$\begin{array}{ll}3.2 .2 .3 \text { Conductor Pipes } & 19\end{array}$

3.2.3 Testing of Retained Water Wells 19

3.2.4 Site Restoration 21

4.0 MODIFICATIONS TO ACTIVITY PLAN 22

5.0 SUMMARY 23

Appendices

A Weekly Reports

B Site Reconnaissance Form

C Schematic of Preabandonment Site Status

D Plats of Well Locations

E Site Restoration Form

F Waste Hauler's Manifests

G Plugging Reports

H Borehole Completion Sketches

I Damage Releases and Transfer Agreements

\section{DISCLAIMER}

This report was prepared as an account of work sponsored by an agency of the United States This report was prepared as an account of work sponsored by an agency thereof, nor any of their Government. Neither the United States Government nor any agency thereof, liability or responsiemployees, makes any warranty, express or implied, or assumes any lion, apparatus, product, or bility for the accuracy, completeness, or usefulness of any information, apparatus, product, process disclosed, or represents that its use would not infringe private trade name, trademark, ence herein to any specific commercial product, process, or service by trade ndorsement, recommanufacturer, or otherwise does not necessarily constitute or imply its endorsement, The views mendation, or favoring by the United States Government or any agency thereof The ve and opinions of authors expressed herein do not

United States Government or any agency thereof. 
Table

1 Well Summary Data Sheet

2 Water Quality Testing Results
Page

10

20

\section{LIST OF FIGURES}




\subsection{INTRODUCTION}

In 1978, eight salt domes in Texas, Louisiana, and Mississippi were identified for study as potential locations for a nuclear waste repository as part of the National Waste Terminal Storage (NWTS) program funded by the Department of Energy (DOE). Three domes were selected in Mississippi for "area characterization" phase study as follows: Lampton Dome near Columbia, Cypress Creek Dome near New Augusta, and Richton Dome near Richton. The purpose of the studies was to acquire geologic and geohydrologic information from shallow and deep drilling investigations to enable selection of sites suitable for more intensive study.

Eleven deep well sites were selected for multiple-well installations to acquire information on the lithologic and hydraulic properties of regional aquifers (Figure 1). In addition, the Cypress Creek and Richton Domes were further investigated by the drilling of 50 and 35 shallow borings, respectively (Figures 2, 3, 4, and 5). Information from these borings was used to delineate the near surface stratigraphy and structural geology in the vicinity of the domes. This phase of the work was performed between 1979 and 1981 by Law Engineering and Testing Company (LETCO) under subcontract to Battelle Memorial Institute, the lead contractor for the DOE.

In 1982, the Earth Technology Corporation (TETC), as the Southern Region Geologic Program Manager, assumed responsibility for the work. Field activities at this time consisted of quarterly water level monitoring of the existing wells. No new test wells were drilled by TETC.

In 1986, the Gulf Coast salt domes were eliminated from further consideration for repository development by the selection of three candidate sites in other regions of the country. In 1987, well plugging and restoration of these deferred sites became a closeout activity under the direction of Stone \& Webster Engineering Corporation (SWEC), the Technical and Field Services Contractor (TFSC) for the Salt Repository Project office (SRPO) of the DOE. The primary objectives of this activity are to plug and abandon all wells and boreholes in accordance with state regulations, restore all drilling sites to as near original condition as feasible, and convey to landowners any wells on their property that they choose to maintain.

This report describes the activities undertaken to accomplish these objectives, as outlined in Activity Plan 1-2, "Activity Plan for Well Plugging and Site Restoration of Test Hole Sites in Mississippi."

\subsection{PREREQUISITES TO FIELD ACTIVITIES}

Considerable effort was required before plugging activities could commence. Some of the more notable activities are briefly described below:

\section{Change of Operatorship and Application for Permits}

Formal application for a change of operatorship of 30 deep wells from TETC to SWEC was made with the Oil and Gas Board. "Notice of Intention to Plug and Abandon" forms were also filed with the board for these wells. 


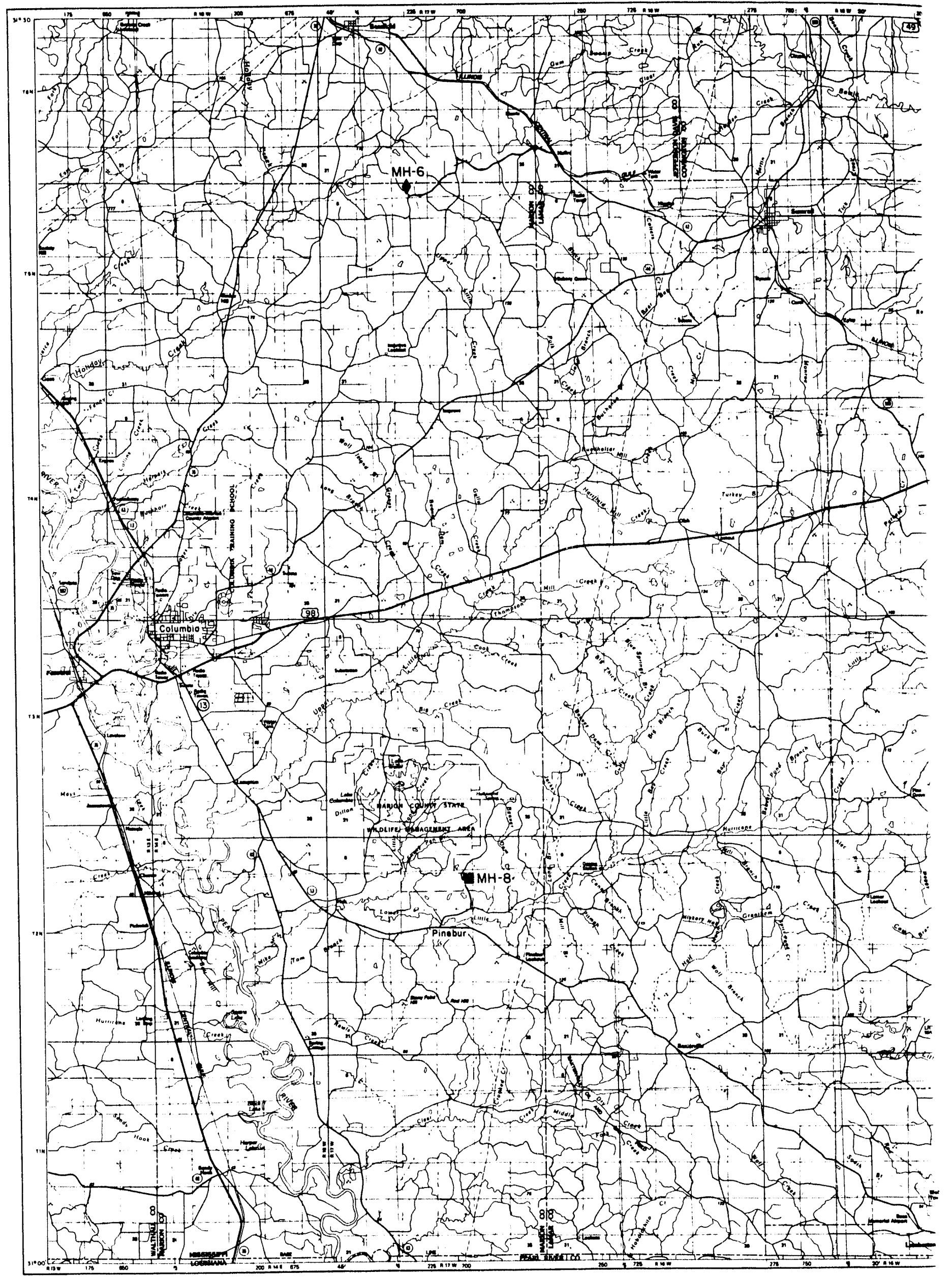




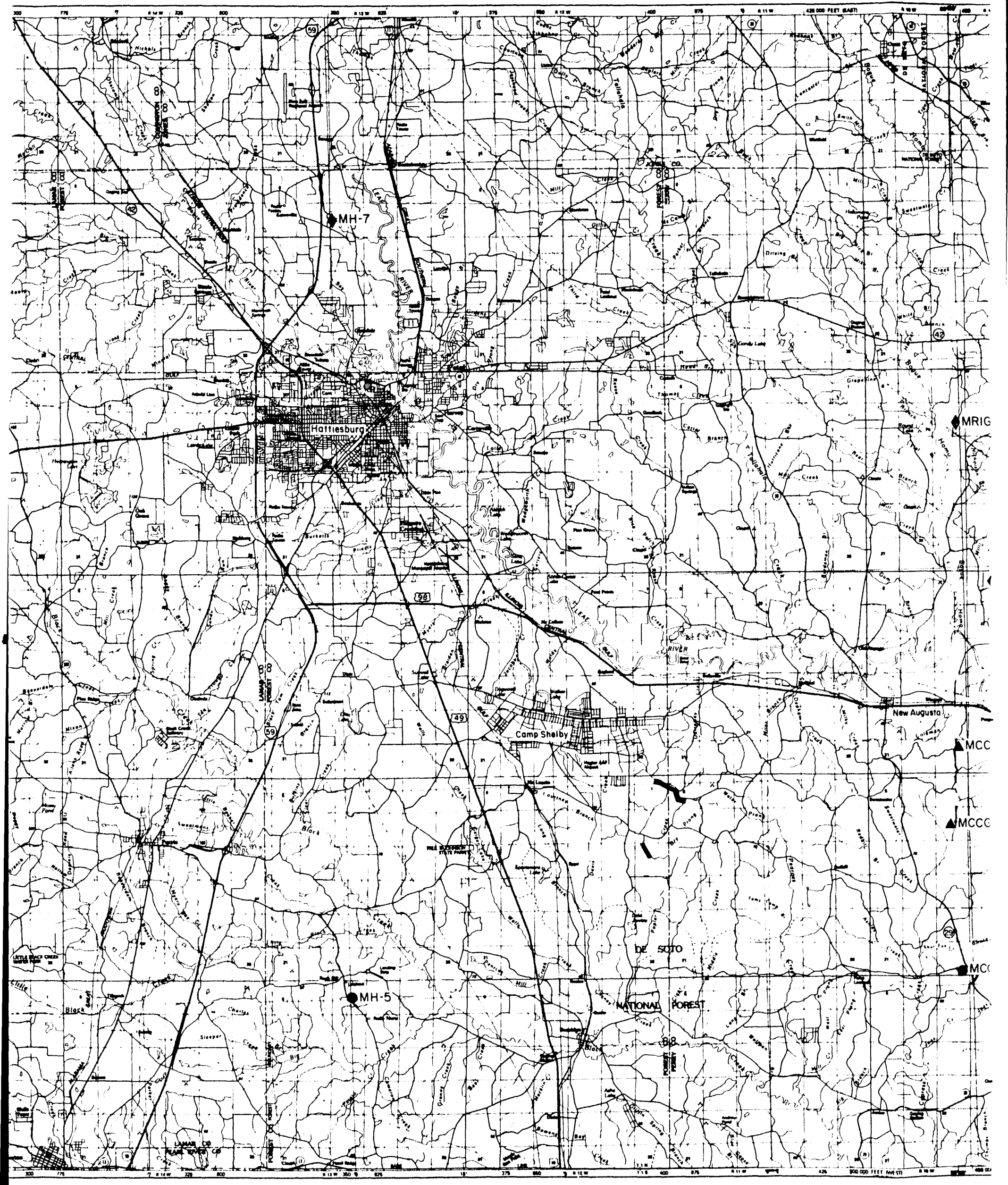




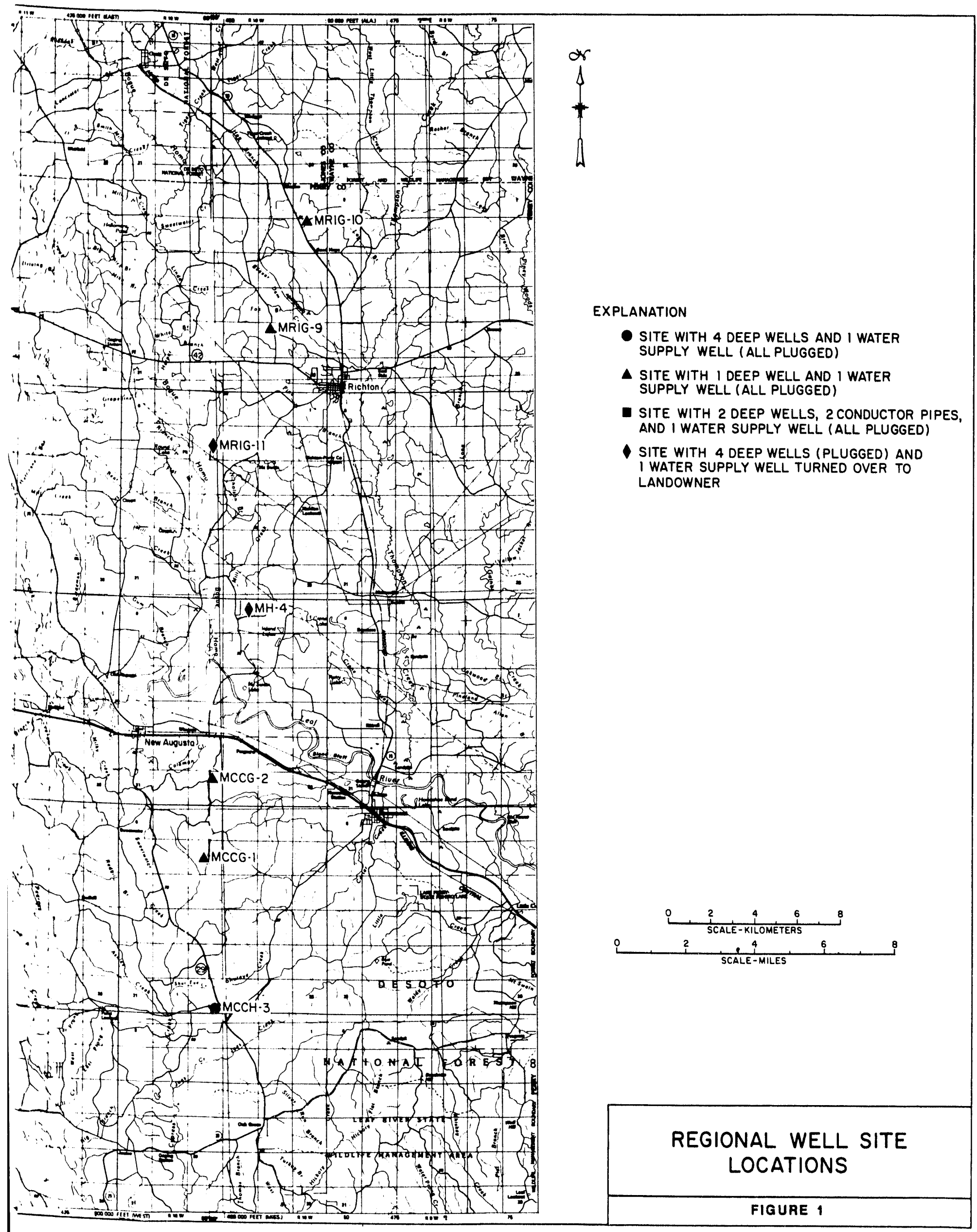




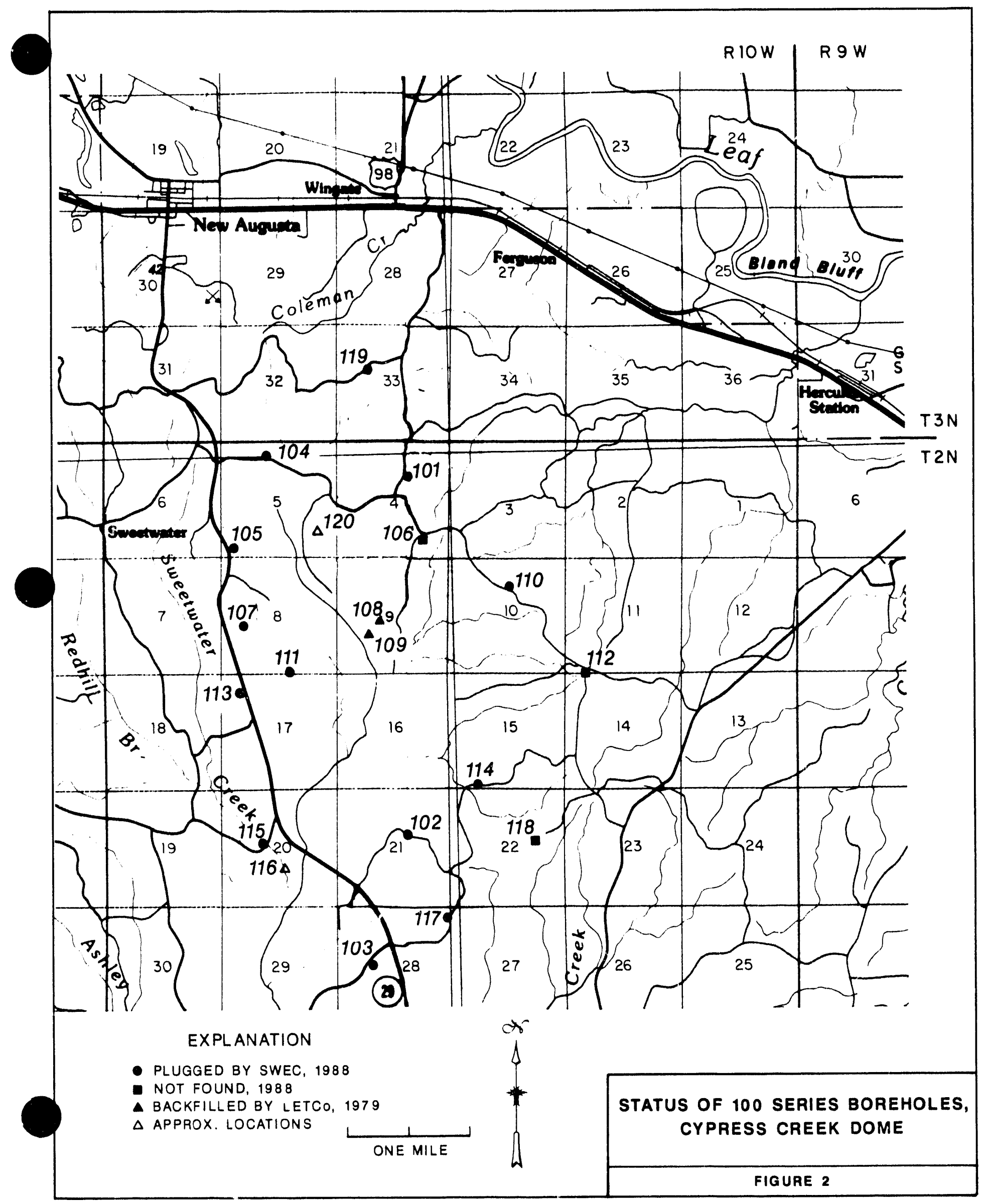




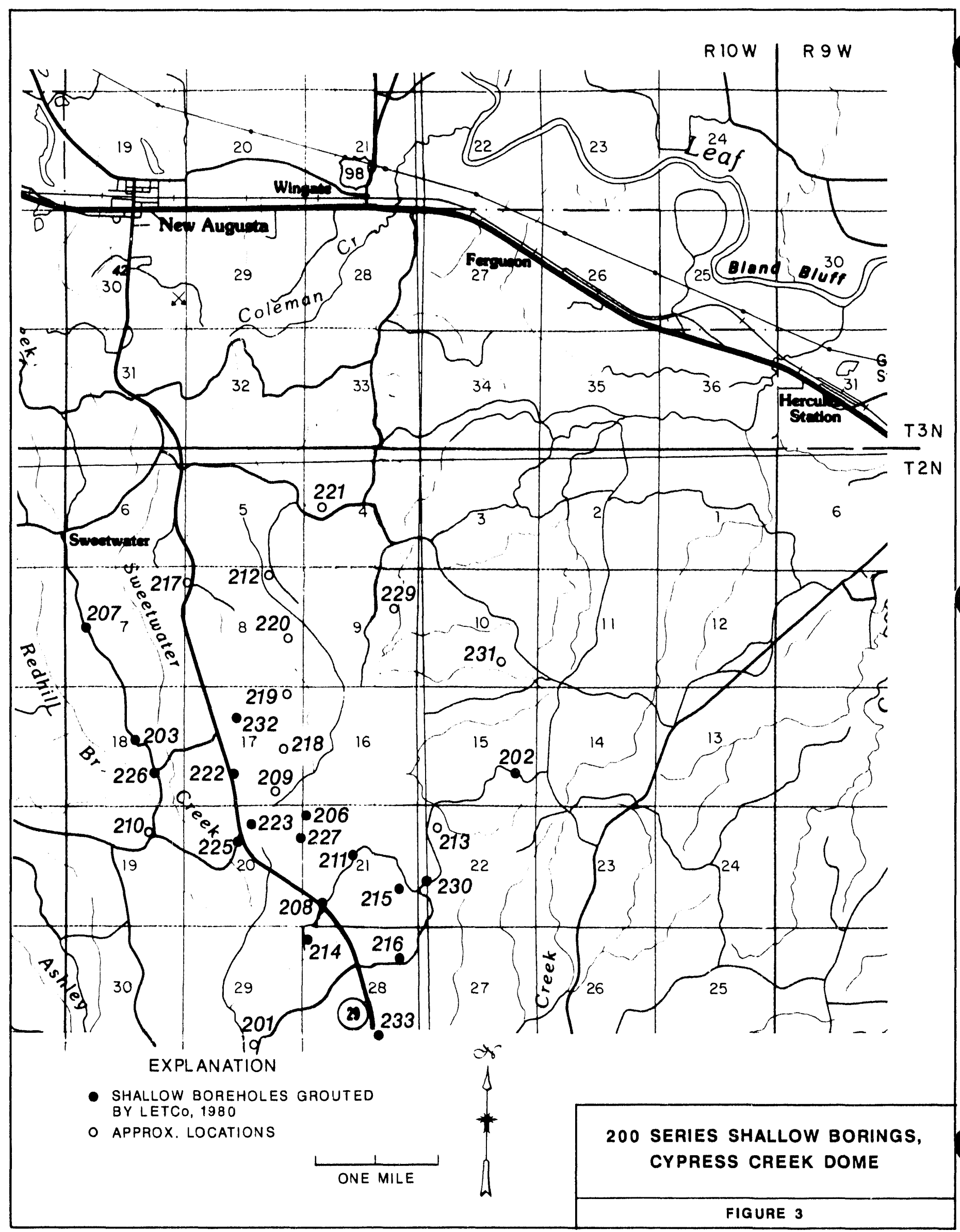




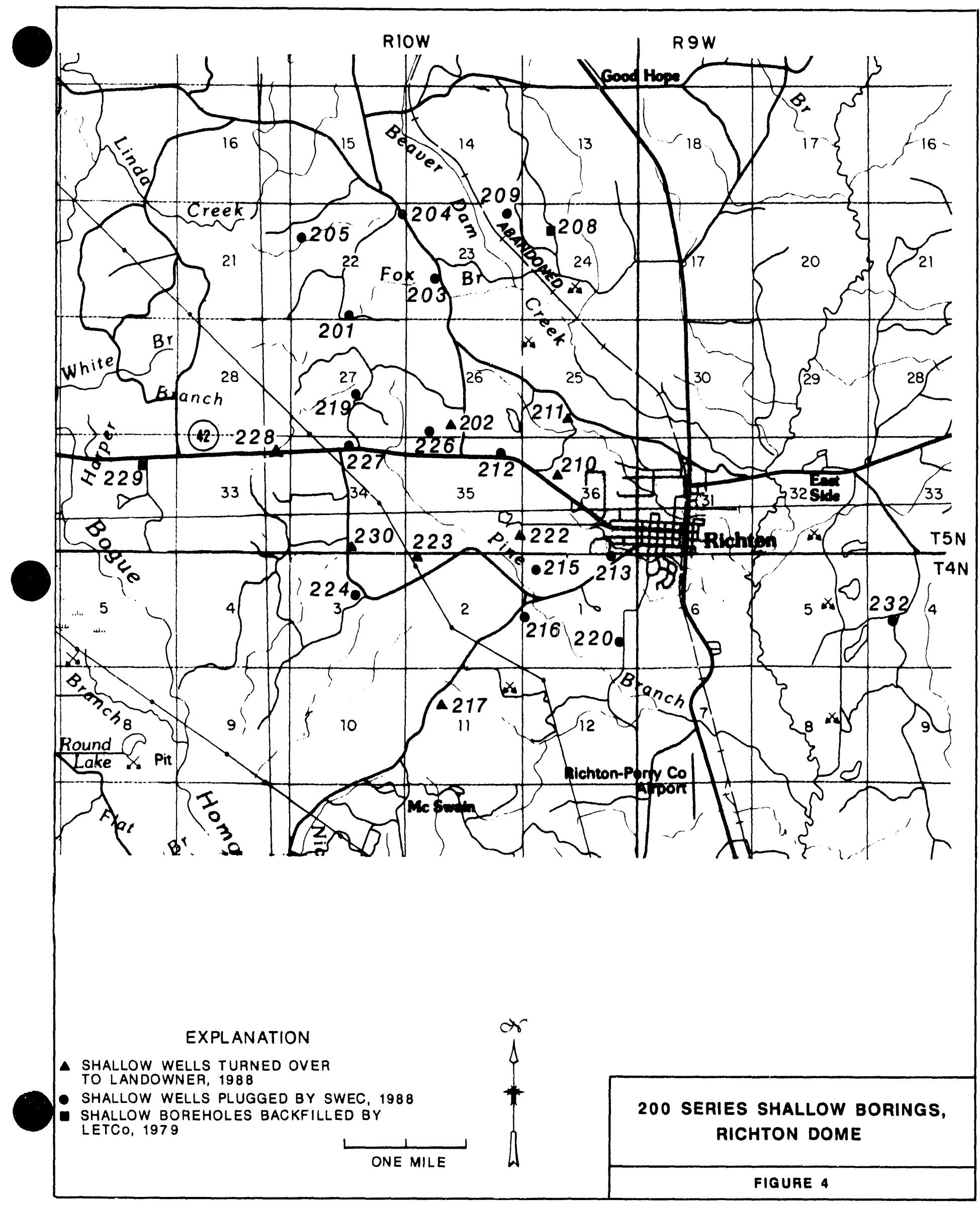




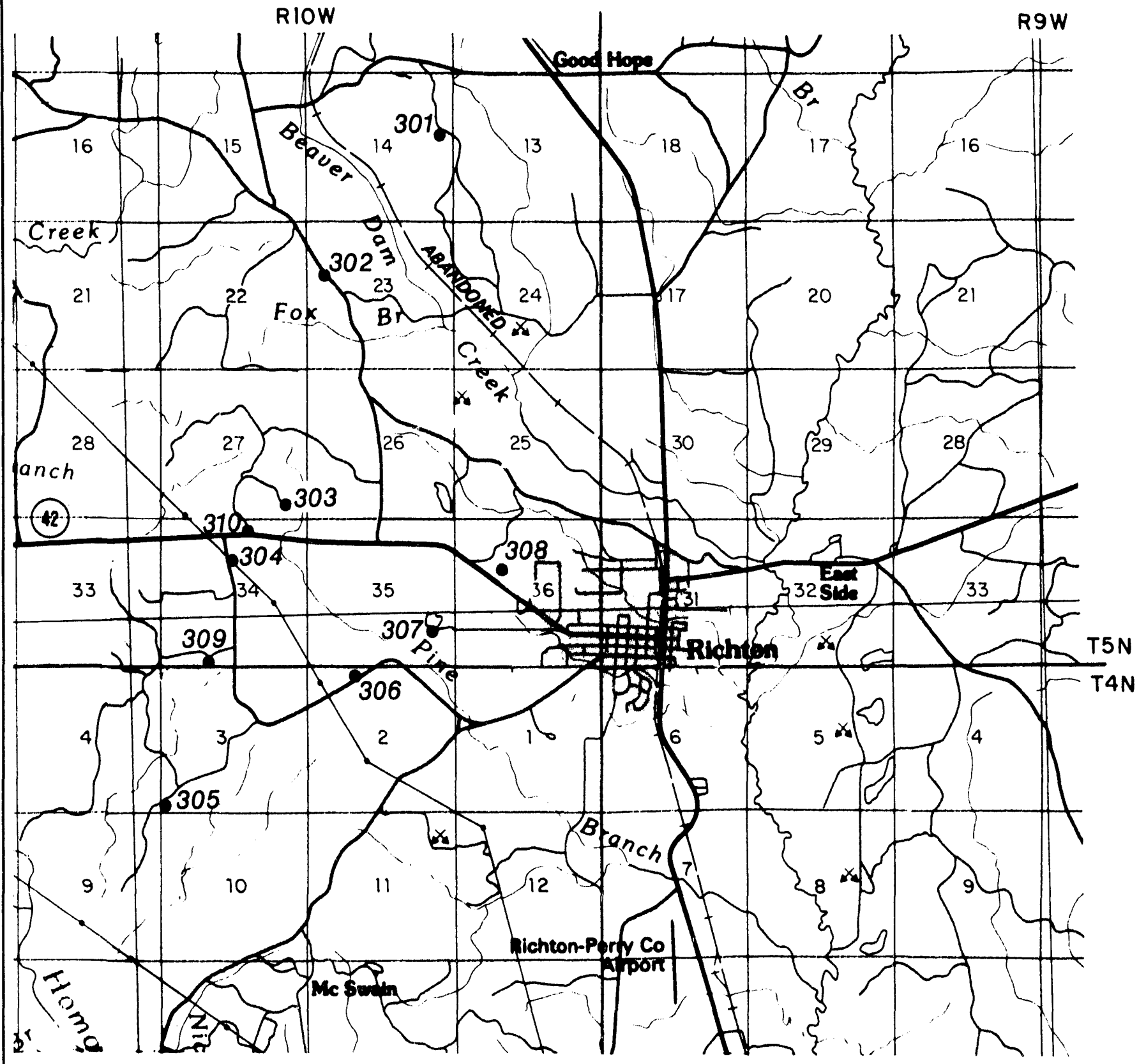




\section{Changes to Lease and Access Agreements}

The lessor was changed from TETC to SWEC on leases for all deep and shallow drill sites. Landowners were contacted and made aware of the changes and plans to plug the wells. In some instances, new leases were negotiated for expired agreements.

\section{Preparation of Plans and Procedures}

SWEC prepared an Activity Plan and Project Technical Procedure which defined the scope of the activities, the methods to be utilized, the sequence of events, and the documentation required during the performance of the work.

\section{Meetings with State and Federal Agencies}

Meetings were held with the following agencies:

Bureau of Land and Water Resources, Bureau of Geology, Bureau of Pollution Control, Board of Health, Department of Energy and Transportation, Oil and Gas Board, Mississippi National Guard, and the U.S. Forest Service.

Discussions resulted in several riodifications of the methods to be used in plugging as discussed in the Activity Plan and clarification of the jurisdictions of the various agencies.

5. Preparation of Subcontracts to Perform the Field Work

Scopes of work, bid documents, and purchase orders were prepared for subcontracts to support the field activities. These resulted in contracts for the following work:

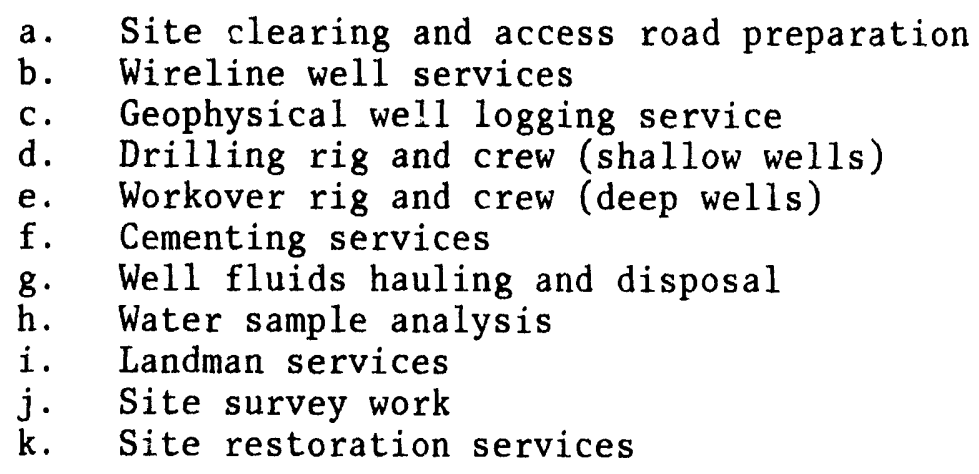

\subsection{FIELD ACTIVITIES}

As a result of SWEC's prerequisite activities, the scope of the field work was determined as follows:

During the initial drilling program (1979-1981), a total of 128 boreholes and wells were drilled. Of these, 40 were previously backfilled by LETCO and required no additional work. The remaining 88 are divided into several groups: 30 deep wells, ranging between 940 to $6,041 \mathrm{ft}$, permitted by the 0il 
and Gas Board at 11 regional sites; 11 water-supply wells ranging between 398 and $514 \mathrm{ft}$, at each deep well site; two 60-ft deep conductor pipe installations; 25 shallow wells at the Richton Dome, and 20 shallow wells at Cypress Creek Dome, genera1ly less than $100 \mathrm{ft}$ deep.

Two state agencies regulate the plugging of wells in Mississippi. The 0il and Gas Board governs all wells that penetrate below freshwater-bearing strata. The 30 wells at the 11 regional sites are in this category. Guidelines for plugging these wells are part of the "State 0il and Gas Board Statutes, Rules of Procedure, and Statewide Rules and Regulations" as revised (August 10,1987). The Bureau of Land and Water Resources regulates all ground-water observation wells, water wells and geotechnical borings. Plugging of the 45 shallow wells, 11 water wells, and 2 conductor pipes at Richton and Cypress Creek Domes was accomplished with close coordination of the Bureau and satisfies its "Surface Water and Ground Water Use and Protection Regulations" published in June 1988.

\subsection{Well Reconnaissance}

Actual work in the field started in early July 1988. A week-by-week summary of all field activities is included as Appendix A.

The first task to be undertaken involved the location and documentation of well and site conditions at all the sites. These conditions were recorded on the Site Reconnaissance Form and are found in Appendix B. Appendix C, Schematic of Preabandonment Site Status, contains maps to locate each well and a sketch of the wellheads before plugging activities. Surveyed plats of each well location are found in Appendix D. Appendix E, Site Restoration Form, contains a photograph of the well sites before plugging. As part of the reconnaissance activity, fluid samples were taken from each well and analyzed for conductivity, $\mathrm{pH}$, and total dissolved solids (TDS). This analysis enabled planning for proper disposal of fluids displaced during plugging. The fluid analyses are found on the Site Reconnaissance Form in Appendix B. A TDS level of $1000 \mathrm{ppm}$ was specified in the Activity Plan as the point at which the fluids would require disposal at a licensed facility. In practice, all fluids with a TDS content greater than about $500 \mathrm{ppm}$ were hauled to the disposal facility. Copies of the waste hauler's manifests are found in Appendix F. The internal configuration of the wells was also verified at this time, using a ring gauge and bailer on a wireline, to enable preparation of well-specific plugging plans and procedures. Casing installation records and cement bond logs were missing for three of the deep wells. These wells, MH-3A, $-3 \mathrm{~B}$, and $-4 \mathrm{D}$, were logged by Inland Well Surveys and subsequently were determined to be adequately cemented by the $0 i 1$ and Gas Board. No perforating and squeeze cementing was required in any of the deep wells prior to plugging. Site clearing and upgrading of access roads was necessary in many cases to enable equipment, such as drilling rigs and cement trucks, to gain access to the wells. The extent of the work varied from essutially none to complete clearing and leveling of 1 to 2 acres of 10-year-old pine trees.

Three shallow wells at Cypress Creek could not be located in the field after considerable effort was expended. This effort included resurveying of the locations, use of a metal detector, and excavating the areas with a backhoe. It is likely the wells were not found due to their locations within areas 
disturbed by road construction and military maneuvers. In addition, a total of 12 wells ( 8 shallow wells and 4 deep water supply wells) were not to be plugged but turned over to the respective landowners. Specific wells are identified in Table 1. Six shallow wells (MCCG-108, 109, 116, 120, and MRIG-208 and 229) were identified from original LETCO field notes, logs, and reports (ONWI-165 and 167) as having been backfilled with soil immediately after drilling. No casing or PVC was left in these holes and no attempt was made to find them. The status of the nine wells that were not located in the field was discussed with the Bureau of Land and Water Resources and determined to require no further action (letter communication: $T$. Regan, SWEC, to J. Hoffman, MBLWR; September 28, 1988). A total of 67 wells remained to be plugged and abandoned as described below.

\subsection{Well Plugging}

\subsubsection{Deep Wells}

Procedures for plugging the deep wells are described in the Activity Plan. Plugging operations for the Mississippi deep wells commenced on September 14, 1988, with mobilization of a Challenger Deepwell Servicing workover rig to wellsite MH-6, north of Columbia, Mississippi. Western Company of North America provided the cementing services. No major problems or delays were encountered in the plugging of the four deep wells at this site.

The operations moved to wellsite $\mathrm{MH}-7$, north of Hattiesburg, on September 19. Preliminary information indicated three of the four wells at this site were pressurized. In actuality, only one well (7A) was under significant pressure and flowing, necessitating installation of a mechanical bridge plug prior to cementing. No significant problems or delays were encountered and plugging operations at $\mathrm{MH}-7$ were completed on September 22 .

The operation then moved to $\mathrm{MH}-8$, southeast of Columbia, where two, 60-ft conductor pipes and two deep wells were plugged; one of the deep wells quired a mechanical bridge plug due to pressurization. The conductor plugging is discussed in Section 3.2.2.3. No delays or problems were encountered and plugging was completed on September 26.

Mobilization to MH-5 occurred on September 27. Plugging and capping of the four wells at this site were completed on September 30 without any problems. The rig then moved to $\mathrm{MH}-4$, near New Augusta, where records indicated that two of the four wells were pressurized. Only one well (4A) needed a mechanical bridge plug and plugging operations progressed smoothly thereafter. The site was completed on October 4 and the operation then moved to $\mathrm{MCCH}-3$, inside Camp Shelby. Plugging of the four wells at this site experienced no problems or delays and was completed on 0ctober 7. The single-we11 sites MCCG-1 and MCCG-2 were both plugged on October 10 without incident.

Plugging operations then moved to MRIH-11 in the Richton area on October 11 , where two of the four wells were expected to be pressurized. Only one well (11B) actually flowed and a mechanical bridge plug was set in it before cementing. Operations were completed on October 13, and the rig then moved to the single-well site MRIG-9. The full calculated volume of cement was pumped into MRIG-9 on October 14 but failed to produce return of fluids or cement. After waiting for 2 hours for the cement to set-up, the hole was 
TABLE 1

WELL SUMMARY DATA SHEET

\begin{tabular}{|c|c|c|c|c|c|c|c|c|c|}
\hline $\begin{array}{l}\text { WELL } \\
\text { NAME \& NUMBER }\end{array}$ & $\begin{array}{c}\text { LOCATION } \\
\text { TOWNSHIP AND RANGE }\end{array}$ & LANDOWNER & OPERATOR & $\begin{array}{l}\text { P\&A } \\
\text { PERMIT } \\
\text { NUMBER }\end{array}$ & ACCESS & $\begin{array}{l}\text { AGREEMENT } \\
\text { TYPE }\end{array}$ & EXPIRES & $\begin{array}{l}\text { LANDOWNER } \\
\text { RELEASE } \\
\text { (DATE) }\end{array}$ & STATUS \\
\hline U.S.F.S. MCCG-1 & $\begin{array}{l}\text { S9,T2N,R1OW, } 2702 \text { FT. } \\
\text { EWL, } 2528 \text { FT. NSL }\end{array}$ & $\begin{array}{l}\text { U.S. } \\
\text { Forest Service }\end{array}$ & SWEC & 640 & DOE & MOU & OPEN END & $04 / 28 / 89$ & P\&A \\
\hline U.S.F.S. MCCG-IWS & $\begin{array}{l}\text { S9,T2N, R1OW, } 78 \text { FT. } \\
\text { N45W of MCCG-1 }\end{array}$ & $\begin{array}{l}\text { U.S. } \\
\text { Forest Service }\end{array}$ & NA & $\begin{array}{l}\text { NOT } \\
\text { REQU IRED }\end{array}$ & DOE & MOU & OPEN END & $04 / 28 / 89$ & $\overline{P \& A}$ \\
\hline U.S.F.S. MCCG-2 & $\begin{array}{l}\text { S33,T3N,RIOW, } 1119 \text { FT. } \\
\text { SNL, 1961 FT. WEL }\end{array}$ & $\begin{array}{l}\text { U.S. } \\
\text { Forest Service }\end{array}$ & SWEC & 641 & DOE & MOU & OPEN END & $04 / 28 / 89$ & P\&A \\
\hline U.S.F.S. MCCG-2WS & $\begin{array}{l}\text { S33,T3N,RIOW, } 94 \text { FT. } \\
\text { S47W of MCCG-2 }\end{array}$ & $\begin{array}{l}\text { U.S. } \\
\text { Forest Service }\end{array}$ & NA & $\begin{array}{l}\text { NOT } \\
\text { REQUTRED }\end{array}$ & DOE & MOU & OPEN END & $04 / 28 / 89$ & P\&A \\
\hline U.S.F.S. MCCH-3A & $\begin{array}{l}\text { S33,T2N, R1OW, 1084 FT. } \\
\text { WEL, 681 FT. NSL }\end{array}$ & $\begin{array}{l}\text { U.S. } \\
\text { Forest Service }\end{array}$ & SWEC & 642 & DOE & MOU & OPEN END & $04 / 28 / 89$ & P\&A \\
\hline U.S.F.S. MCCH-3B & $\begin{array}{l}\text { S33,T2N, R1OW, } 1030 \text { FT. } \\
\text { WEL, } 546 \text { FT NSL }\end{array}$ & $\begin{array}{l}\text { U.S. } \\
\text { Forest Service }\end{array}$ & SWEC & 636 & DOE & MOU & OPEN END & $04 / 28 / 89$ & P\&A \\
\hline U.S.F.S. MCCH-3C & $\begin{array}{l}\text { S33,T2N,RIOW, } 984 \text { FT. } \\
\text { WEL, G41 FT NSL }\end{array}$ & $\begin{array}{l}\text { U.S. } \\
\text { Forest Service }\end{array}$ & SWEC & 663 & DOE & MOU & OPEN END & $04 / 28 / 89$ & $P \& A$ \\
\hline U.S.F.S. MCCH-3D & $\begin{array}{l}\text { S33,T2N,R1OW, } 1129 \text { FT. } \\
\text { WEL, } 696 \text { FT NSL }\end{array}$ & $\begin{array}{l}\text { U.S. } \\
\text { Forest Service }\end{array}$ & SWEC & 664 & DOE & MOU & OPEN END & $04 / 28 / 89$ & P\&A \\
\hline U.S.F.S. $\mathrm{MCCH}-3 \mathrm{WS}$ & $\begin{array}{l}\text { S33,T2N, RIOW, } 110 \text { FT. } \\
\text { DUE WEST OF MCCH-3A }\end{array}$ & $\begin{array}{l}\text { U.S. } \\
\text { Forest Service }\end{array}$ & $\mathrm{NA}$ & $\begin{array}{l}\text { NOT } \\
\text { REQU IRED }\end{array}$ & DOE & MOU & OPEN END & $04 / 28 / 89$ & P\&A \\
\hline ROWELL MH-4A & $\begin{array}{l}\text { S3,T3N,R10W, } 1673 \text { FT } \\
\text { SNL, } 1465 \text { FT WEL }\end{array}$ & Reese Rowell & SWEC & 665 & SWEC & EASWEC & $02 / 09 / 89$ & $04 / 29 / 89$ & $P \& A$ \\
\hline ROWELL MH-4B & $\begin{array}{l}\text { S3,T3N,RIOW, } 1918 \text { FT } \\
\text { SNL, } 1467 \text { FT WEL }\end{array}$ & Reese Rowell & SWEC & 662 & SWEC & EASWEC & $02 / 09 / 89$ & $04 / 29 / 89$ & P\&A \\
\hline ROWELL MH-4C & $\begin{array}{l}\text { S3,T3N,R1OW, } 1793 \text { FT } \\
\text { SNL, 1465 F". WEL }\end{array}$ & Reese Rowell & SWEC & 661 & SWEC & EASWEC & $02 / 09 / 89$ & $04 / 29 / 89$ & P\&A \\
\hline ROWELL MH-4D & $\begin{array}{l}\text { S3,T3N,R1OW, } 2048 \mathrm{FT} \\
\text { SNL, } 1465 \mathrm{FT} \text {. WEL }\end{array}$ & Reese Rowell & SWEC & 660 & SWEC & EASWEC & $02 / 09 / 89$ & $04 / 29 / 89$ & P\&A \\
\hline
\end{tabular}


TABLE 1

WELL SUMMARY DATA SHEET

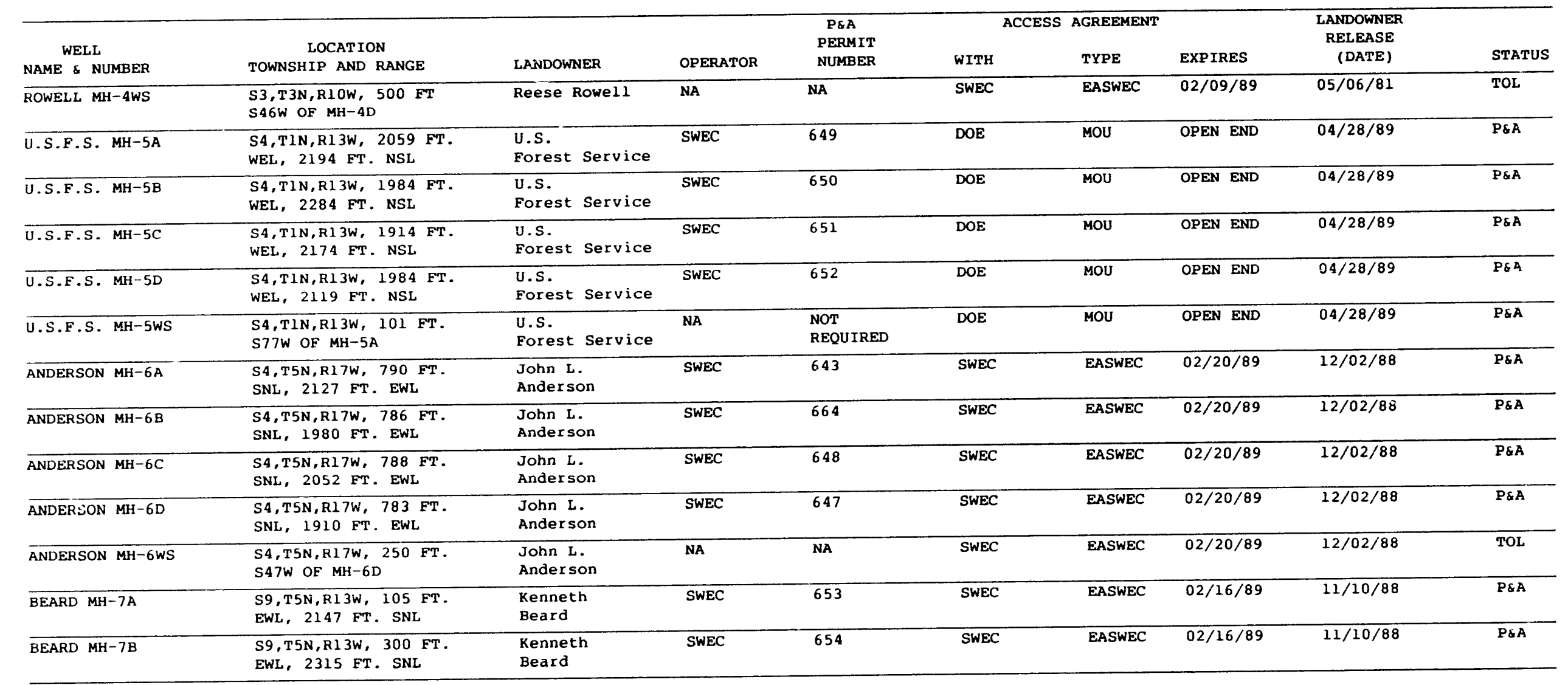


Page 3 of 7

TABLE 1

WELL SUMMARY DATA SHEET

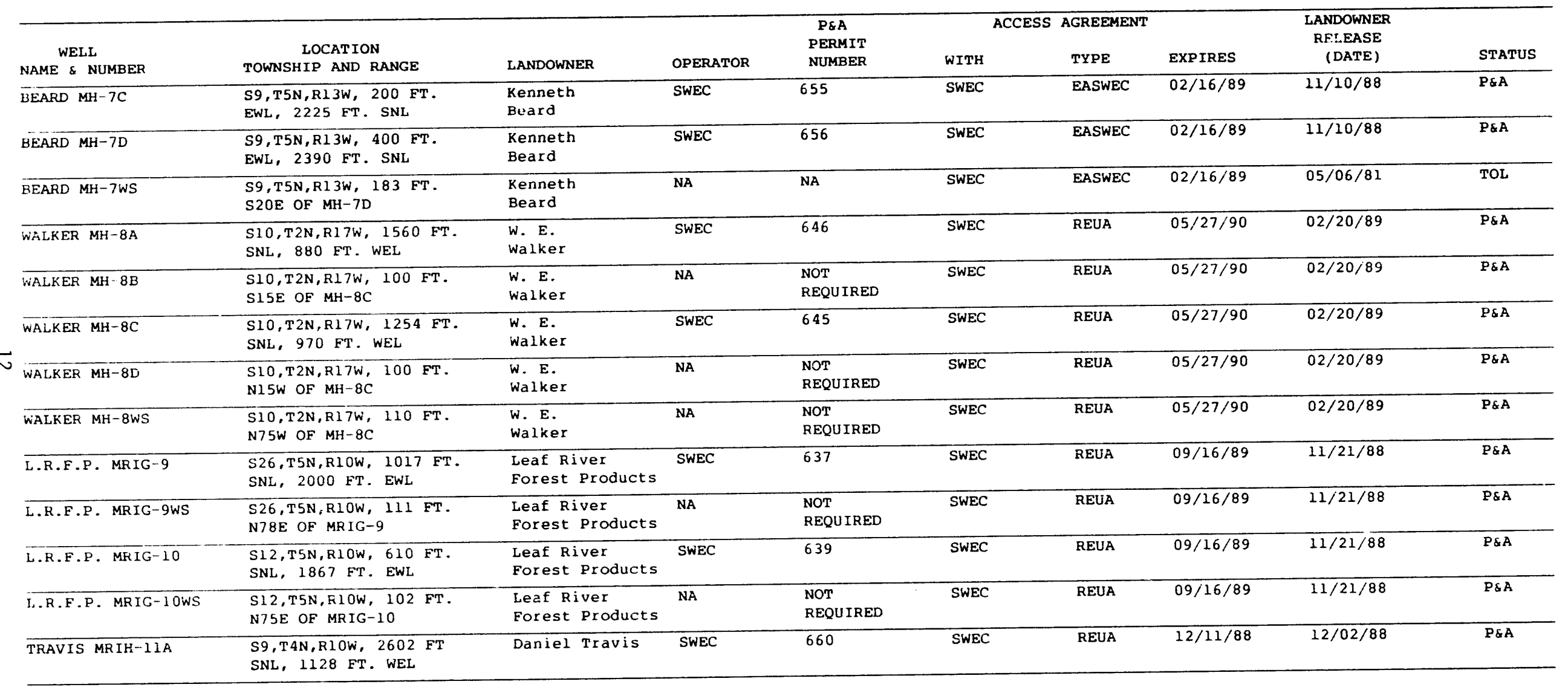


TABLE 1

WELL SUMMARY DATA SHEET

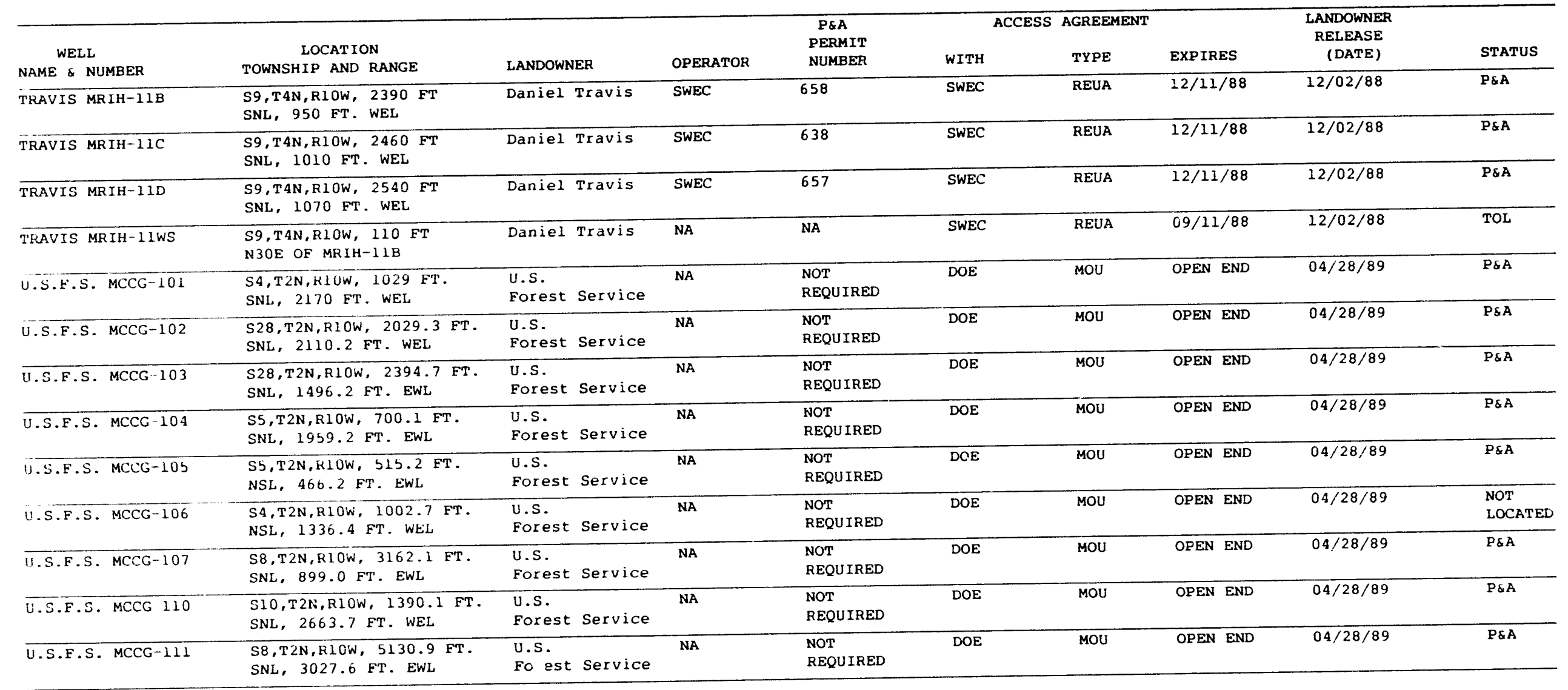


TABLE 1

WELL SUMMARY DATA SHEET

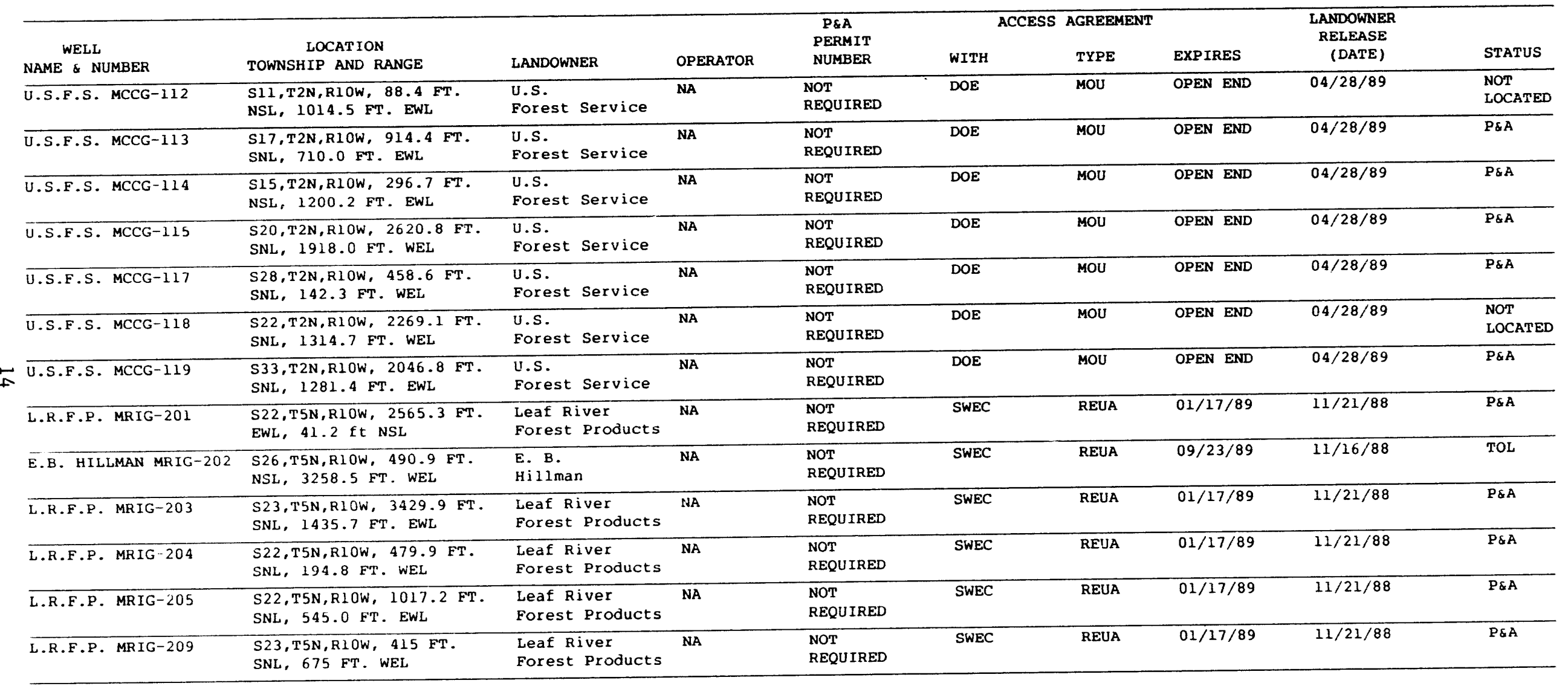


TABLE 1

WELL SUMMARY DATA SHEET

\begin{tabular}{|c|c|c|c|c|c|c|c|c|c|}
\hline $\begin{array}{l}\text { WELL } \\
\text { NAME \& NUMBER }\end{array}$ & $\begin{array}{c}\text { LOCATION } \\
\text { TOWNSHIP AND RANGE }\end{array}$ & LANDOWNER & OPERATOR & $\begin{array}{l}\text { P\&A } \\
\text { PERMIT } \\
\text { NUMBER }\end{array}$ & ACCESS & $\begin{array}{l}\text { AGREEMENT } \\
\text { TYPE }\end{array}$ & EXPIRES & $\begin{array}{l}\text { LANDOWNER } \\
\text { RELEASE } \\
\text { (DATE) }\end{array}$ & STATUS \\
\hline J.C. HILLMAN MRIG -210 & $\begin{array}{l}\text { S36.T5N,RIOW, } 1734.4 \text { FT. } \\
\text { SNL, } 1659.6 \text { FT. EWL }\end{array}$ & $\begin{array}{l}\text { J.C. } \\
\text { Hillman }\end{array}$ & NA & $\begin{array}{l}\text { NOT } \\
\text { REQUIRED } \\
\end{array}$ & SWEC & REUA & $09 / 23 / 89$ & $11 / 15 / 88$ & TOL \\
\hline E.B. MCCARDLE MRIG-211 & $\begin{array}{l}\text { S25,T5N,RIOW, } 2074.2 \text { FT. } \\
\text { EWL, 757.1 FT. NSL }\end{array}$ & $\begin{array}{l}\text { E. B. } \\
\text { McCardle }\end{array}$ & NA & $\begin{array}{l}\text { NOT } \\
\text { REQU IRED }\end{array}$ & SWEC & $\begin{array}{l}\text { VERBAL } \\
\text { RIGHT OF } \\
\text { ACCESS }\end{array}$ & $\begin{array}{l}\text { END } \\
\text { OF } \\
\text { P\&A }\end{array}$ & $11 / 15 / 88$ & TOL \\
\hline L. VALENTINE MRIG-212 & $\begin{array}{l}\text { S35, T5N,RIOW, } 848.8 \text { FT. } \\
\text { SNL, } 933.1 \text { FT. WEL }\end{array}$ & $\begin{array}{l}\text { Lloyd } \\
\text { Valent ine }\end{array}$ & NA & $\begin{array}{l}\text { NOT } \\
\text { REQUIRED }\end{array}$ & SWEC & REUA & $08 / 20 / 89$ & $04 / 14 / 89$ & $P \& A$ \\
\hline MCLENDON MRIG-213 & $\begin{array}{l}\text { S36,T5N,R1OW, } 28.3 \text { FT. } \\
\text { NSL, } 646 \text { FT. WEL }\end{array}$ & $\begin{array}{l}\text { John B. } \\
\text { McLendon }\end{array}$ & NA & $\begin{array}{l}\text { NOT } \\
\text { REQUIRED } \\
\end{array}$ & SWEC & REUA & $09 / 23 / 89$ & $11 / 15 / 88$ & $P \& A$ \\
\hline H.K. HILLMAN MRIG-215 & $\begin{array}{l}\text { Sl,T4N,RlOW, } 656.7 \text { FT. } \\
\text { SNL, 688.1 FT. EWL }\end{array}$ & $\begin{array}{l}\text { H. K. } \\
\text { Hillman }\end{array}$ & NA & $\begin{array}{l}\text { NOT } \\
\text { REQU IRED }\end{array}$ & SWEC & REUA & $09 / 23 / 89$ & $01 / 09 / 89$ & P\&A \\
\hline MCLENDON MRIG-216 & $\begin{array}{l}\text { S1,T4N,RIOW, } 2486 \text { FT. } \\
\text { NSL, 50 FT. EWL }\end{array}$ & $\begin{array}{l}\text { John B. } \\
\text { McLendon }\end{array}$ & NA & $\begin{array}{l}\text { NOT } \\
\text { REQUIRED }\end{array}$ & SWEC & REUA & $09 / 23 / 89$ & $11 / 15 / 88$ & $P \& A$ \\
\hline GODFREY MRIG-217 & $\begin{array}{l}\text { S11,T4N,R10W, } 1708.9 \text { FT. } \\
\text { SNL, } 1688.4 \text { FT. EWL }\end{array}$ & Godfrey & NA & $\begin{array}{l}\text { NOT } \\
\text { REQU IRED }\end{array}$ & $\begin{array}{l}\text { LEASE } \\
\text { EXPIRED }\end{array}$ & $\begin{array}{l}\text { NO } \\
\text { ACCESS } \\
\text { GRANTED }\end{array}$ & & $05 / 06 / 81$ & TOL \\
\hline E.B. HILLMAN MRIG-219 & $\begin{array}{l}\text { S27,T5N,R1OW, } 2010.5 \text { FT. } \\
\text { NSL, } 2228.4 \text { FT. WEL }\end{array}$ & $\begin{array}{l}\text { E. B. } \\
\text { Hilliman }\end{array}$ & $\mathrm{NA}$ & $\begin{array}{l}\text { NOT } \\
\text { REQU IRED }\end{array}$ & SWEC & REUA & $09 / 23 / 89$ & $11 / 10 / 88$ & P\&A \\
\hline OLIPHANT MRIG-220 & $\begin{array}{l}\text { S1,T4N,R10W, } 1170.9 \text { FT. } \\
\text { NSL, } 774.6 \text { FT. WEL }\end{array}$ & $\begin{array}{l}\text { G. R. } \\
\text { oliphant }\end{array}$ & NA & $\begin{array}{l}\text { NOT } \\
\text { REQUIRED }\end{array}$ & SWEC & LROE & $01 / 06 / 89$ & $11 / 15 / 88$ & P\&A \\
\hline$\overline{E . B . ~ H I L L M A N ~ M R I G-222 ~}$ & $\begin{array}{l}\text { S35, T5N,R1OW, } 730.5 \text { FT. } \\
\text { NSL, } 118.3 \text { FT. WEL }\end{array}$ & $\begin{array}{l}\text { E. B. } \\
\text { Hillman }\end{array}$ & $\mathrm{NA}$ & $\begin{array}{l}\text { NOT } \\
\text { REQU IRED }\end{array}$ & SWEC & REUA & $09 / 23 / 89$ & $11 / 16 / 88$ & TOL \\
\hline CLEARMAN MRIG-223 & $\begin{array}{l}\text { S2,T4N,R10W, } 105.0 \text { FT. } \\
\text { SNL, } 558.3 \mathrm{FT} \text {. EWL }\end{array}$ & $\begin{array}{l}\text { Herbert } \mathrm{C} . \\
\text { Clearman }\end{array}$ & NÁ & $\begin{array}{l}\text { NOT } \\
\text { REQUIRED }\end{array}$ & SWEC & REUA & $08 / 20 / 89$ & $11 / 16 / 88$ & TOL \\
\hline PHILLIPS MRIG-224 & $\begin{array}{l}\text { S3,T4N,RlOW, } 1925.7 \text { FT. } \\
\text { SNL, } 2036.8 \text { FT. WEL }\end{array}$ & Phillips & NA & $\begin{array}{l}\text { NOT } \\
\text { REQU IRED }\end{array}$ & SWEC & LROE & $11 / 30 / 88$ & $11 / 15 / 88$ & P\&A \\
\hline E.B. HILLMAN MRIG-220 & $\begin{array}{l}\text { S26,TSN,R1OW, 60.7 FT. } \\
\text { NSL, } 974.7 \text { FT. EWL }\end{array}$ & $\begin{array}{l}\text { E. B. } \\
\text { Hillman }\end{array}$ & $\mathrm{NA}$ & $\begin{array}{l}\text { NOT } \\
\text { REQUIRED } \\
\end{array}$ & SWEC & REUA & $09 / 23 / 89$ & $11 / 10 / 88$ & P\&A \\
\hline
\end{tabular}


TAELE 1

WELL SUMMARY DATA SHEET

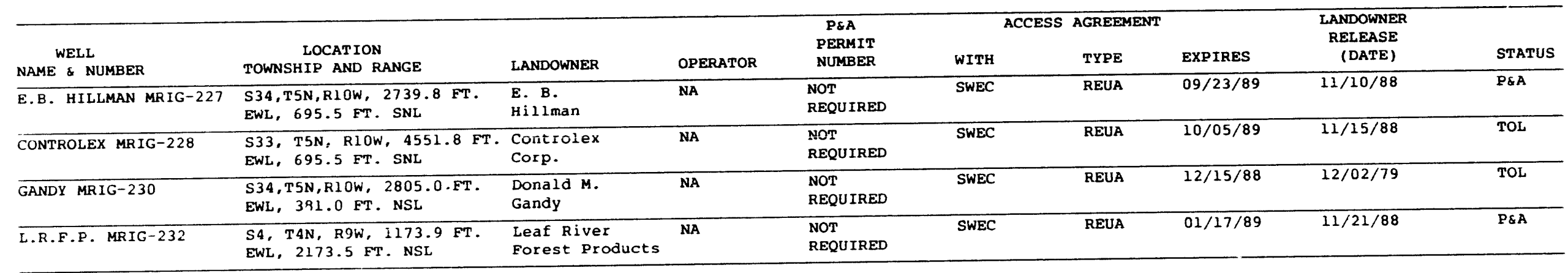

NOTES :

\section{Definition of Abbreviations Used}

EWL: East of the West Line.

East of the West hine.

WEL:

P\&A: Plugged and Abandoned.

TOL: Turned Over to Landowner

MOU: Memorandum of Understanding Between U.S. Forest Service and U.S. Department of Energy.

EASWEC: Exploration Agreement with Stone \& Webster Engineering Corp.
REUA: Right of Entry and Use Agreements (REUA)

LROE: Limited Right of Entry Agreement with SWEC

USFS: United States Forest Service

LRFP: Leaf River Forest Products

SWEC: Stone \& Webster Engineering Corporation

DOE: Department of Energy (U.S.)

NA. Not Applicable 
re-entered, and an attemot was made to establish circulation with water. Fluids again failed to return to the surface so it was decided to set a mechanical bridge plug in the $85 / 8 \mathrm{in}$. casing at $526 \mathrm{ft}$, above the open hole section ( 558 to $766 \mathrm{ft}$ ). This was done on 0ctober 17, and the casing was then cemented without further problems.

The rig then moved to the final site, MRIG-10, also a single-well site. Plugging was completed at this site on 0ctober 17, and the Challenger Deepwell Servicing rig was demobilized.

Al1 the wells were plugged from bottom to top in approximately $500 \mathrm{ft}$ stages, with cement being pumped from the surface down through $23 / 8$-in. tubing. At the completion of each stage, the tubing was withdrawn to the base of the next stage where pumping was again initiated. During the final stage, cement was pumped until it returned to the surface, indicating the well was completely filled with cement. The tops of the well casings were then cut off a minimum of $3 \mathrm{ft}$ below ground level, capped with a steel plate, and buried according to state requirements. Details on the cement mix, quantities, depths, and sequence of operations can be found in Appendix G, Plugging Reports. Sketches of the plugged wells are found in Appendix H.

All fluids displaced from the boreholes during plugging operations which contained TDS (Total Dissolved Solids) concentrations in excess of $1000 \mathrm{mg} / \mathrm{L}$ were collected and transported to a licensed disposal facility near Laurel, Mississippi, operated by G.B. Smith, Inc.

At the conclusion of plugging activity, Form 7 - Plugging Record was filed with the 0il and Gas Board, for each deep well, as required.

\subsubsection{Shallow Wells}

The general sequence of shallow well plugging activities is described herein. Due to the differing methods and materials of construction of these wells and to address concerns of state agencies, it was necessary to develop several plugging techniques. In addition, modifications and changes were required during the course of the work to respond to unanticipated conditions and inaccuracies in the original installation records. These methods and changes are discussed in Section 4.0, Modifications to Activity Plan.

\subsubsection{PVC Monitoring Wells}

Griner Drilling Service of Columbia, Mississippi mobilized on August 29, 1988, to the Richton area to commence plugging activities. Using a truck-mounted, Gardner-Denver 1000 drilling rig (rotary table drive), they successfully drilled out the PVC casing and screens in eight wells (MRIG-201, 204, 209, 213, 224, 226, 227, and 232) and carried the borings to their original total depths. The casing and screen of one well, MRIG-205, was removed intact by pulling with a cable and winch. After reaming and washing of the open holes, cement grout was placed through the drill rods, from bottom to top, until cement circulated to the surface. Boreholes were checked after a few days, and if settling of grout had occurred, the hole 
was topped off with additional cement until the level reached a depth of 3 to $5 \mathrm{ft}$ below ground. The holes were then backfilled with soil.

Three wells (MRIG-203, 216, and 219), which, according to project records, were constructed with a grouted annular space, were found to be open to depths of several tens of feet. The original plan required simply grouting the casing in place. In order to properly seal these wells, it was decided to drill out the PVC, similar to the method used previously. However, it was apparent during the drilling that the bit deviated off the original borehole when competent grout was encountered downhole. It seems probable, based on examination of the cuttings and drilling resistance, that the bit advanced along the side of the grouted portions of the well, leaving significant lengths of intact PVC, casing in the ground. The boreholes were subsequently grouted. Volumetric analysis of the cement slurry volumes used in grouting the holes indicates that more than adequate quantities of cement were used to fill the open hole and the casing remaining in place. It is SWEC's opinion that grouting of the deviated borehole would likely result in cement being pumped into the open casing as well. If this did not occur, the grout column created in the open hole would have effectively sealed off the casing at the point of departure, precluding the existence of a vertical hydraulic pathway from the subsurface. Since the original installation adequately sealed of $f$ the strata below that point, additional remediation of these three wells was unnecessary and not pursued. On September 9, plugging activities moved from Richton to the Cypress Creek Dome near New Augusta.

As a result of the difficulties experienced with the deviated holes at Richton, it was decided, with the concurrence of the Bureau of Land and Water Resources, to switch to a top head drive drilling rig in order to eliminate the need to pull the drill bit off the PVC while adding rods to the drill string. Also, a longer pilot would be used on the drill bit and the drilling rate would be reduced. A total of thirteen shallow wells were plugged at Cypress Creek Dome between September 9 and October 4, using the modified methods described above.

Two wells (MCCG-101 and 102), originally installed with a grouted annular seal, were grouted in place after verification of the seal was effected with a backhoe. The casing was cut off $3 \mathrm{ft}$ below ground, a cement pad ( $3 \mathrm{ft} \mathrm{x}$ $3 \mathrm{ft} \times 5 \mathrm{in}$ ) was poured over the casing tops, and the excavations were backfilled with soil.

During the course of the work at Cypress Creek Dome, several landowners in the Richton area, who had previously chosen to keep their wells, decided to have them plugged and abandoned. Between November 1 and November 11 , wells MRIG-212, 215, and 220 were plugged utilizing the top-drive rig system. No problems were encountered.

\subsubsection{Water Supply Wells}

Beginning October 5, seven water supply wells (MCCG-1WS and 2WS, MCCH-3WS, MH-5WS and 8WS, and MRIG-9WS and 10WS) associated with the regional we11 sites, were successfully plugged and abandoned. The methods used were similar to the shallow wells except that steel casing and screen were pulled out of the hole before redrilling to original total depth. Due to the larger size and greater depths involved in these wells, Western Company of 
North America was contracted to cement the holes, again utilizing Griner Drilling Service's rig. Cement was pumped in a single stage from bottom to top until it returned to the surface.

The only problem occurred at well MH-8WS. In contrast to the other wells, MH-8WS was originally constructed by grouting the annulus from the top of the screen to ground surface. In order to remove the casing, it was necessary to first remove the cement from around the casing. This was accomplished using a 6-in.-diameter, carbide over-coring bit. Individual joints of casing were then pulled out of the hole. The entire $219 \mathrm{ft}$ of casing was removed in this manner but the continuous-slot well screen proceeded to unravel; only about 20-30 ft of screen was removed, leaving 30-40 ft in the hole. Drilling continued past the screen to the original total depth and grouting occurred without further difficulty.

\subsubsection{Conductor Pipes}

Two 16-in. diameter, 60-ft-long conductor pipes were originally installed at site MH-8 in anticipation of two additional deep wells being drilled there. The deep wells were not drilled and the conductor pipes remained to be plugged as part of these activities. On September 23, Western Company of North America plugged $\mathrm{MH}-8 \mathrm{~B}$ and $\mathrm{MH}-8 \mathrm{D}$ by pumping cement-bentonite slurry from the surface into the open conductors. Visual inspection to determine the adequacy of the annular seals was performed in a 4-ft-deep excavation around each conductor. The casings were then cut off $3 \mathrm{ft}$ below ground, capped with a steel plate, and backfilled with soil.

At the conclusion of shallow well plugging activities, Well Decommissioning Forms were filed with the Bureau of Land and Water Resources, as required. These forms document the location of each well, the owner, the contractors used to install and plug the well, and the quantities and procedures used in plugging the well.

\subsubsection{Testing of Retained Water Wells}

Several landowners expressed interest in maintaining wells on their property as potential sources of potable water even though the wells were not installed for that purpose. SWEC, at the request of the Mississippi Bureau of Pollution Control and the Bureau of Land and Water Resources, agreed to test the quality of the water in these wells and to make the results available to the landowner. It was recommended that the water be tested for 19 parameters identified in the "EPA Primary Drinking Water Regulations" and 2 additional parameters in their "Secondary Drinking Water Regulations." The sampling and analysis plan became a part of Project Technical Procedure 1-18-1, "Documentation of Plugging and Abandonment of Holes and Restoration of Work Sites." The Mississippi Public Health Laboratory (PHL) was contracted to perform the analyses on samples that SWEC was to obtain.

The results from the first well analysis indicated that the coliform bacteria count was extremely high, so high that bacteria could not be counted, a condition called confluent growth (CG on Table 2). The PHL recommended chlorinating the wells prior to purging and sampling in order to obtain a sample representative of the natural ground-water conditions. 
TABLE 2

WATER QUALITY TESTING RESULTS

STONE \& WEBSTER ENGINEERING CORPORATIOH

MISSISSIPPI PUBLIC HEALTH LABS

\begin{tabular}{|c|c|c|c|c|c|c|c|c|c|c|c|c|}
\hline Parameter & $\begin{array}{l}\text { U.S.E.P.A. } \\
\text { Standard }\end{array}$ & $\begin{array}{l}\text { MH-4WS } \\
\text { Rowe11 }\end{array}$ & $\begin{array}{l}\text { MaH-6WS } \\
\text { Anderson }\end{array}$ & $\begin{array}{l}\text { Mat-7ws } \\
\text { Beard }\end{array}$ & $\begin{array}{l}\text { MRIH-11WS } \\
\text { Travis } \\
\end{array}$ & $\begin{array}{l}\text { HRIG-202 } \\
\text { E.B. Hillman }\end{array}$ & $\begin{array}{l}\text { MarG-210 } \\
\text { J.C. H121man }\end{array}$ & $\begin{array}{l}\text { MRIG-211 } \\
\text { McCardle }\end{array}$ & $\begin{array}{l}\text { MRIG-222 } \\
\text { E.B. Hillman }\end{array}$ & $\begin{array}{l}\text { MRIG-223 } \\
\text { Clearman }\end{array}$ & $\begin{array}{l}\text { MRIG-228 } \\
\text { Controlex }\end{array}$ & $\begin{array}{l}\text { MRIG-230 } \\
\text { Gandy }\end{array}$ \\
\hline Arsonic & $0.05 \mathrm{mg} / 1$ & $<0.001$ & 0.0021 & $<0.001$ & $<0.001$ & 0.002 & 0.0012 & 0.0013 & 0.0091 & $<0.001$ & 0.0015 & $<0.001$ \\
\hline Barlun & $1 \mathrm{mg} / 1$ & $<0.1$ & $<0.1$ & $<0.2$ & $<0.1$ & 0.175 & $<0.1$ & $<0.1$ & $<0.1$ & $<0.1$ & $<0.1$ & 0.126 \\
\hline Cadniun & $0.01 \mathrm{mg} / 1$ & $<0.001$ & $<0.001$ & $<0.001$ & $<0.001$ & $<0.001$ & 0.0054 & 0.0031 & $<0.001$ & $<0.001$ & $<0.001$ & $<0.001$ \\
\hline Chromiun & $0.05 \mathrm{mg} / 1$ & 0.0034 & 0.0025 & $<0.001$ & $<0.001$ & $<0.001$ & 0.0015 & $<0.001$ & 0.0017 & 0.0017 & $<0.001$ & 0.0033 \\
\hline Iron & $0.3 \mathrm{mg} / 1$ & 8.48 & 85 & 3.27 & 0.67 & 3.2 & 2.6 & 4.0 & 1.59 & 0.19 & 2.3 & 0.95 \\
\hline Load & $0.05 \mathrm{mg} / 1$ & 0.0152 & 0.0047 & 0.002 & 0.0081 & 0.0147 & 0.0309 & 0.0125 & 0.0174 & 0.0184 & 0.0134 & 0.0271 \\
\hline Mercury & $0.002 \mathrm{mg} / 1$ & $<0.0005$ & $<0.0005$ & $<0.0005$ & $<0.0005$ & $<0.0005$ & $<0.0005$ & $<0.0005$ & $<0.0005$ & 0.001 & $<0.0005$ & $<0.0005$ \\
\hline Seleniue & $0.01 \mathrm{mg} / 1$ & $<0.001$ & $<0.001$ & $<0.001$ & $<0.001$ & $<0.001$ & $<0.001$ & $<0.001$ & 0.0072 & $<0.001$ & $<0.001$ & $<0.001$ \\
\hline silver & $0.05 \mathrm{mg} / 1$ & $<0.001$ & $<0.001$ & $<0.001$ & $<0.001$ & $<0.001$ & $<0.001$ & $<0.001$ & $<0.001$ & $<0.001$ & $<0.001$ & $<0.001$ \\
\hline Mitrate-Hitrogen & $20 \mathrm{mg} / 1$ & $<0.1$ & $<0.1$ & $<0.1$ & $<0.11$ & $<0.1$ & $<0.96$ & $<0.15$ & $<0.1$ & 3 & $<0.1$ & 7.9 \\
\hline Haxdness & None & 4 & 14 & 30 & 10 & 34 & 38 & 40 & 36 & 16 & 58 & 36 \\
\hline Total Dissolved Solids & $500 \mathrm{mg} / 1$ & 112 & 76 & 85 & 38 & 239 & 198 & 123 & 8 & 139 & $180^{\circ}$ & 24 \\
\hline Gross Alpha Partlcle & $15 \mathrm{pCl} / 1$ & $<1 \pm 1.7$ & $<1 \pm 2.4$ & $<1 \pm 1.9$ & $<2 \pm 1.5$ & $3.1 \pm 2.9$ & $3.0 \pm 2.6$ & $7.9 \pm 4.2$ & $2.8 \pm 2.8$ & $<1 \pm 2.0$ & $<2 \pm 2.8$ & $<1 \pm 2.2$ \\
\hline Total collform & $1 / 100 \mathrm{ml}$ & CG & CG & 1 & 2 & CG & CG & $>80$ & CG & $<1$ & CG & $>80$ \\
\hline Benzene & $0.005 \mathrm{~g} / 1$ & $<0.0005$ & $<0.0005$ & $<0.0005$ & $<0.0005$ & $<0.0005$ & $<0.0605$ & $<0.0005$ & $<0.0005$ & $<0.0005$ & $<0.0005$ & $<0.0005$ \\
\hline Carbon Tetrachlor ide & $0.005 \mathrm{mg} / 1$ & $<0.0005$ & $<0.0005$ & $<0.0005$ & $<0.0005$ & $<0.0005$ & $<0.0005$ & $<0.0005$ & $<0.0005$ & $<0.0005$ & $<0.0005$ & $<0.0005$ \\
\hline 1,2-Dlchlorouthane & $0.005 \mathrm{mg} / 1$ & $<0.0005$ & $<0.0005$ & $<0.0005$ & $<0.0005$ & $<0.0005$ & $<0.0005$ & $<0.0005$ & $<0.0005$ & $<0.0005$ & $<0.0005$ & $<0.0005$ \\
\hline Irichloroethylene & $0.005 \mathrm{mg} / \mathrm{l}$ & $<0.0005$ & $<0.0005$ & $<0.0005$ & $<0.0005$ & $<0.0005$ & $<0.0005$ & $<0.0005$ & $<0.0005$ & $<0.0005$ & $<0.0005$ & $<0.0005$ \\
\hline Para-Dichlorobenzene & $0.075 \mathrm{mg} / 1$ & $<0.0005$ & $<0.0005$ & $<0.0005$ & $<0.0005$ & $<0.0005$ & $<0.0005$ & $<0.0005$ & $<0.0005$ & $<0.0005$ & $<0.0005$ & $<0.0005$ \\
\hline 1,1-Dichloroethylene & $0.00 ; \mathrm{mg} / 1$ & $<0.0005$ & $<0.0005$ & $<0.0005$ & $<0.0005$ & $<0.0005$ & $<0.0005$ & $<0.0005$ & $<0.0005$ & $<0.0005$ & $<0.0005$ & $<0.0005$ \\
\hline 1,1,1-Trichloronthane & $0.2 \mathrm{mg} / 1$ & $<0.0005$ & $<0.0005$ & $<0.0005$ & $<0.0005$ & $<0.0005$ & $<0.0005$ & $<0.0005$ & $<0.0005$ & $<0.0005$ & $<0.0005$ & $<0.0005$ \\
\hline vinyl chloxide & $0.002 \mathrm{mg} / 1$ & $<0.0005$ & $<0.0005$ & $<0.0005$ & $<0.0005$ & $<0.0005$ & $<0.0005$ & $<0.0005$ & $<0.0005$ & $<0.0005$ & $<0.0005$ & $<0.0005$ \\
\hline
\end{tabular}


Chlorination was performed in each of the wells with this procedure also being incorporated into PTP $1-18-1$, as required. The results of the sampling are found in Table 2 and indicate that 2 of the 22 parameters consistently exceed EPA standards. Total coliform levels exceed the regulations in 9 of 11 samples, with confluent growth occurring in 6 of these samples. Total iron also exceeds the EPA regulations in 10 of the 11 wells, but this parameter affects only the taste and appearance, not posing a health risk.

It is most likely that the chlorination and purging procedures were inadequate to remove all the coliform bacteria, but it is also possible that the groundwater is actually contaminated, especially in wells near where livestock are kept or septic systems are utilized. It is SWEC's opinion that with proper development and adequate chlorination, some of the transferred wells could be used as a source of potable water. However, it was apparent that most of the 2-in.-diameter PVC wells will not produce sufficient volumes of water to be of much use for any purpose.

The results of the water analysis and a cross-sectional sketch of each respective well have been provided to each landowner. We also pointed out the bacteria and iron problems with the wells and suggested further chlorination and well development prior to any usage.

\subsubsection{Site Restoration}

Restoration of all the well sites was subcontracted to Oddee Smith \& Sons, Inc. of Brookhaven, Mississippi and commenced on November 2, 1988, soon after plugging operations were completed. The procedure used at all the sites was that recommended by the U.S. Forest Service (personal communication: J. Duckworth, USFS, to D. Kistner, SWEC; July 27, 1988) for the sites on their land, and is described as follows:

1. Remove all debris, materials, and fencing

2. Rip or disc the site area to a depth of 8-12 in.

3. Broadcast 13-13-13 fertilizer at a rate of 800 pounds/acre and lime at a rate of 2 tons/acre

4 Apply seed at the following rates: bahia grass at 25 pounds/acre, rye grass at 20 pounds/acre, and bermuda grass at 5 pounds/acre.

5. Re-disc or harrow lightly and apply even cover of hay mulch.

Some sites and roads required construction of water bars to divert runoff and dirt barriers at the entrances to limit access. Top soil was hauled to site MH-7 to improve the existing soil conditions. In addition, three landowners chose to restore their sites themselves and accepted cash payment in lieu of restoration services. These sites were MH-4, MH-6, and MRIH-11. Photographs of sites after restoration can be found in Appendix E. Initial restoration was completed on November 11, 1988. Remedial work was required at MH-8 due to heavy rains and was accomplished between January 24-26, 1989.

Damage releases, liability releases, and well turnover agreements can be found in Appendix I. 


\subsection{MODIFICATIONS TO ACTIVITY PLAN}

The only modification to the deep well portion of the plugging plan of any significance related to the criterion for pumping cement from the bottom of the hole to the top in one operation. Due to the problems encountered in Louisiana where the 4-in. screen collapsed on the tubing, it was decided to start cementing operations above the 4-in. section of each well. In all cases except one, cementing was initiated within $150 \mathrm{ft}$ of the screened or open section of the borehole, in accordance with 0il and Gas Board recommendations. The exception involved well $\mathrm{MH}-5 \mathrm{~A}$, which contained $575 \mathrm{ft}$ of 4-in. riser pipe above the screen. Cementing began near the base of the $65 / 8$ casing at a depth of $2932.5 \mathrm{ft}, 588 \mathrm{ft}$ above the screen but significantly below the base of fresh water, located at a depth of about $1500 \mathrm{ft}$. The well, as plugged, is within the guidelines of the $0 i 1$ and Gas Board.

Changes to the shallow well plugging portion of the activity plan occurred prior to initiation of field work as a result of discussions with concerned state agencies and as a result of changed conditions encountered during the field work. The modifications are briefly summarized as follows:

1. PVC monitoring wells that were constructed without grouted annular seals were to be drilled out and carried to the original total depths. Neat cement grout would then be placed in the hole from bottom to top, to within $3 \mathrm{ft}$ of the ground surface. This procedure was successfully employed on 23 PVC wells. Success in removing the PVC was verified by the presence of PVC wel1-screen cuttings in the circulating fluids.

2. Five PVC wells were constructed with bentonite and cement grout seals between the wellscreen and the ground surface. Two of these wells were plugged by filling with cement and capping with a cement pad, after verifying the annular seal. The other three required drilling out due to inadequate annular seals. These wells are discussed in greater detail in Section 3.2.2.1.

3. The steel-casing in the water supply wells was physically removed from the ground and the hole was redrilled to the original total depth prior to cementing. This metho: was used on 6 of the 7 water supply wells which were plugged. The one exception is discussed in more detail in Section 3.2.2.2.

4. Two $60-\mathrm{ft}$ conductor pipes at site $\mathrm{MH}-8$ were plugged by pumping cement at the surface instead of pumping through pipe set on the bottom of the hole. The holes were essentially dry and a check of the original installation records indicated the volume of cement used exceeded the volume of the annular space.

5. A program of water sampling and analysis was incorporated into the field activities at the request of the Bureau of Land and Water Resources and the Bureau of Pollution Control. This program was instituted to collect and analyze well water from those wells which were being turned over to the respective landowners. The 
sampling and analysis procedure was, itself, modified to include chlorination of the wells prior to sampling.

\subsection{SUMMARY}

During the summer of 1988 , the location and condition of 79 wells drilled for the NWTS Southern Region Geologic Program were documented by SWEC. Nine additional wells were not located in the field, having either been backfilled soon after drilling or destroyed by subsequent surface activity. A total of 67 wells were successfully plugged and abandoned in the fall of 1988 as part of the site-closeout activities, under the regulations and guidelines of either the Mississippi 0il and Gas Board or the Bureau of Land and Water Resources. All of these well sites have been restored to the satisfaction of the respective landowners or controlling agencies. Damage payments were made to three landowners in lieu of restoration. Twelve separate wells have been released and transferred to the landowners for their use. All field related site-closeout activities in Mississippi have been completed. 
APFENDIX A

Weekly Reports 
WEEKLY PROGRESS REPORT

STONE \& WEBSTER ENGINEERING CORP.

\begin{tabular}{|l|l|l|}
\hline CLIENT DOE / SRPO & J.O. No. ${ }_{17500.25}$ & $\begin{array}{l}\text { PERIOD } \\
07 / 07 / 88 \text { TO 07/09/88 }\end{array}$ \\
\hline $\begin{array}{c}\text { STATE AND SITE } \\
\text { MISSISSIPPI, GENERAL }\end{array}$ & $\begin{array}{l}\text { SITE REPRESENTATIVE } \\
\text { T. REGAN, D. KISTNER, H. ABEDI }\end{array}$ \\
\hline
\end{tabular}

SUMMARY OF ACTIVITIES

PLUGGING

PERFORMED PRELIMINARY RECONNAISSANCE WORK; LOCATED WELLS AT RICHTON DOME AND

TALKED TO SEVERAL LANDOWNERS. COPIED RECORDS FROM MS OIL \& GAS BOARD AND MS

BUREAU OF GEOLOGY. TOOK INVENTORY OF MATERIALS LEFT BY ERTEC

AND LETCO IN HATTIESBURG OFFICE: CLEANED UP AND ORGANIZED OFFICE.

\section{RESTORATION}

NONE

\section{PROBLEMS}

NONE

\section{CHANGES TO ACTIVITY PLAN}

NONE

SITE VISITORS

NONE

WORK PLANNED FOR NEXT WEEK

MEET WITH USFS DISTRICT RANGER AND WITH CAMP SHELBY PERSONNEL.

BEGIN FIELD RECONNAISSANCE. 
WEEKLY PROGRESS REPORT

STONE \& WEBSTER ENGINEERING CORP.

\begin{tabular}{|c|c|c|}
\hline DOE / SRPO & J.O. No. ${ }_{17500.25}$ & $\begin{array}{l}\text { PERIOD } \\
07 / 10 / 88 \text { TO } 07 / 15 / 88\end{array}$ \\
\hline $\begin{array}{l}\text { STATE AND SITE } \\
\text { MISSISSIPPI, GENERAL }\end{array}$ & $\begin{array}{l}\text { SITE REPRESEN } \\
\text { D. LUTZ, H. ABE }\end{array}$ & $\begin{array}{l}\text { E REGAN, D. KISTNER } \\
\end{array}$ \\
\hline \multicolumn{3}{|l|}{ SUMMARY OF ACTIVITIES } \\
\hline \multicolumn{3}{|l|}{ PLUGGING } \\
\hline \multicolumn{3}{|c|}{ MRIG-9, 9WS, 10, 10WS, 201, 202, 203, 204, 210,211, 212, 215, 216, 219, 222 . } \\
\hline \multicolumn{3}{|c|}{$223,226,227,228,230$, AND 232. MH- $4 \mathrm{~A}, 4 \mathrm{~B}, 4 \mathrm{C}, 4 \mathrm{D}, 4 \mathrm{WS} ; \mathrm{MH}-5 \mathrm{~A}, 5 \mathrm{~B}, 5 \mathrm{C}, 5 \mathrm{D}$; } \\
\hline \multicolumn{3}{|c|}{ MH-6A, $5 B, 6 C, 6 D ; M H-7 A, 7 B, 7 C, 7 D, 7 W S ; \quad M H-8 A, 8 B, 8 C, 8 W S ; M C C G-1,1 W S$, } \\
\hline \multicolumn{3}{|c|}{$M C C G-2,2 W S ; M C C H-3 A, 3 B, 3 C, 3 D, 3 W S ; \quad M R I H-11 A, 11 B, 11 C, 11 D, 11 W S_{i}$} \\
\hline \multicolumn{3}{|c|}{$M C C G-101,102,104,105,107,110,111,113,114$ AND 115.} \\
\hline \multicolumn{3}{|l|}{ RESTORATION } \\
\hline
\end{tabular}

\section{PROBLEMS}

UNABLE TO LOCATE THE FOLLOWING WELLS DURING SITE RECONNAISSANCE: MH-5WS, MH-6WS, MH-8D, MCCG-106, 112,117, 118, 119, MRIG-205, 209,213, 224. DID NOT VISIT THE FOLLOWING SITES: MCCG-103 (DESTROYED), MCCG-108, 109, 116, 120, MRIG-229 (BACKFILLED), AND MRIG-217 AND 220 (NO ACCESS AGREEMENT).

\section{CHANGES TO ACTIVITY PLAN}

NONE

SITE VISITORS

NONE

WORK PLANNED FOR NEXT WEEK

RETURN TO BOSTON. FINALIZE DETAILS FOR PLUGGING AND ROADWORK CONTRACTS. 
WEEKLY PROGRESS REPORT

STONE \& WEBSTER ENGINEERING CORP.

\begin{tabular}{|c|c|c|}
\hline DOE / SRPO & J.O. No. ${ }_{17500.25}$ & $\begin{array}{l}\text { PERIOD } \\
08 / 02 / 88 \text { TO } 08 / 07 / 88\end{array}$ \\
\hline $\begin{array}{l}\text { STATE AND SITE } \\
\text { MISSISSIPPI, GENERAL }\end{array}$ & $\begin{array}{l}\text { SITE REPRESEN } \\
\text { T. REGAN, H. AE }\end{array}$ & J.GONZALES \\
\hline \multicolumn{3}{|l|}{ SUMMARY OF ACTIVITIES } \\
\hline \multicolumn{2}{|c|}{ H. ABEDI SUPERVISED ROAD RECONDITIONING WORK AT ALL SITES REQUIRING SAME. } & \\
\hline \multicolumn{3}{|c|}{ J. GONZALES AND T. REGAN SUPERVISED WIRELINE WORK AND SURVEYOR WORK. FILES } \\
\hline \multicolumn{3}{|c|}{ WERE SET UP FOR VARIOUS DOCUMENTATION FORMS AT HATTIESBURG OFFICE: SUPPLIES } \\
\hline
\end{tabular}

\section{RESTORATION}

NONE

\section{PROBLEMS}

DUE TO HEAVY RAINS AND TANK MANEUVERS AT CAMP SHELBY, SOME ROADS WILL REQUIRE ADDITIONAL REPAIRS WHEN MANEUVERS ARS COMPLETED AND PLUGGING IS TO BEGIN.

\section{CHANGES TO ACTIVITY PLAN}

SAMPLING OF WELL FLUIDS FOR TURN-OVER WELLS WILL REQUIRE AN ADDEIVDUM TO ACTIVITY PLAN FOR SAMPLING AND TESTING PROCEDURES.

SITE VISITORS

NONE 
WEEKLY PROGRESS REPORT

STONE \& WEBSTER ENGINEERING CORP.

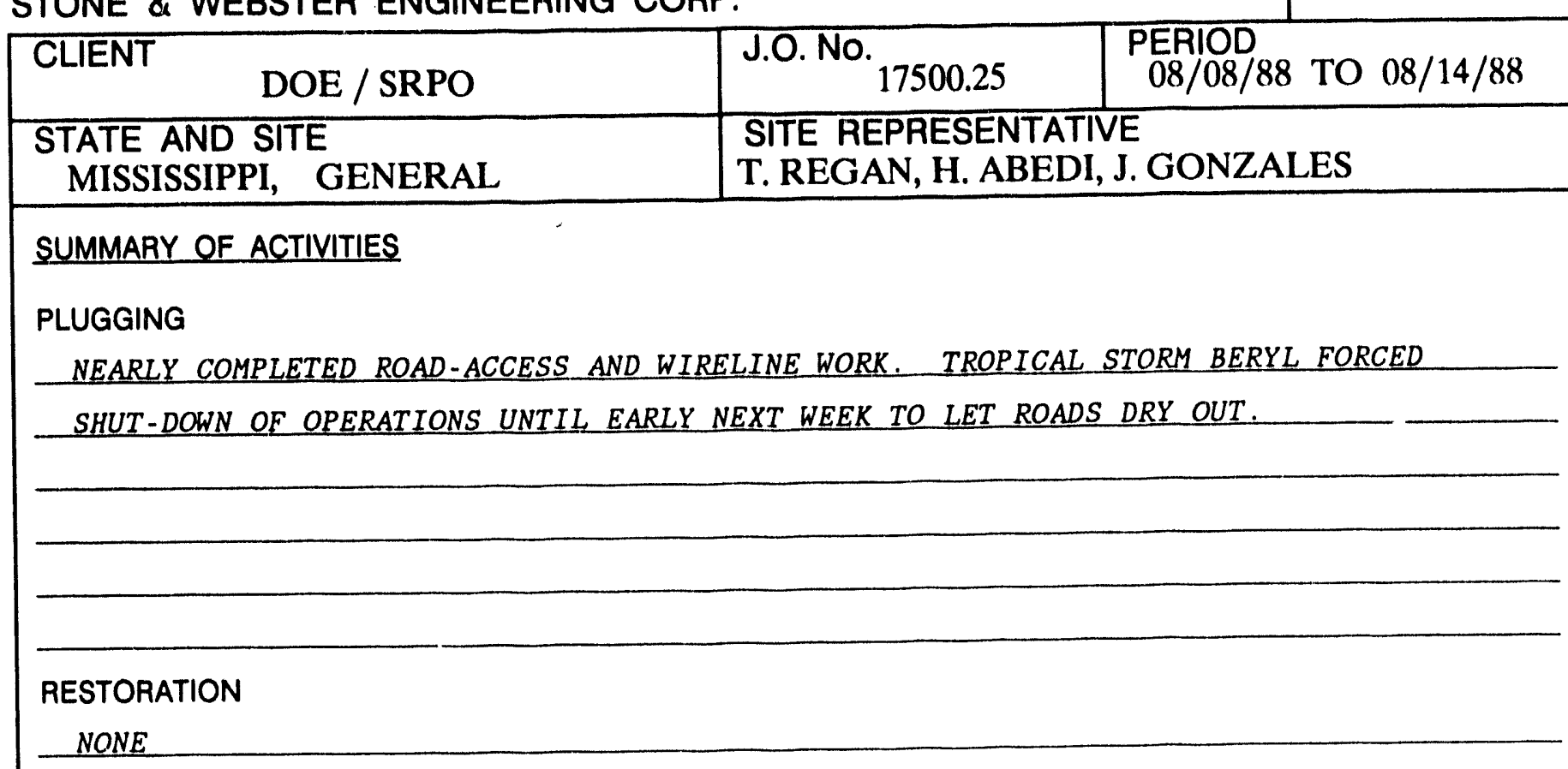

NONE

PROBLEMS

NONE

CHANGES TO ACTIVITY PLAN

NONE

SITE VISITORS

NONE

WORK PLANNED FOR NEXT WEEK

FINISH ROAD ACCESS, WIRELINE, SURVEYING. BEGIN LOGGING (3 WELLS) AND PLUGGING OF SHALLOW WELLS AT CAMP SHELBY (CYPRESS CREEK DOME). 
WEEKLY PROGRESS REPORT

STONE \& WEBSTER ENGINEERING CORP.

\begin{tabular}{|l|l|l|}
\hline CLIENT DOE / SRPO & $\begin{array}{l}\text { J.O. No. } \\
17500.25\end{array}$ & $\begin{array}{c}\text { PERIOD } \\
08 / 15 / 88 \text { TO } 08 / 21 / 88\end{array}$ \\
\hline $\begin{array}{c}\text { STATE AND SITE } \\
\text { MISSISSIPPI, GENERAL }\end{array}$ & $\begin{array}{l}\text { SITE REPRESENTATIVE } \\
\text { D. KISTNER, H. ABEDI }\end{array}$ \\
\hline
\end{tabular}

\section{SUMMARY OF ACTIVITIES}

PLUGGING

FINISHED UP SURVEY AND WIRELINE WORK. ROAD WORK IS COMPLETE FOR THE TIME

BEING. MAY NEED TO CALL OUT, AS-NEEDED, DEPENDING ON WEATHER CONDITIONS. LOCATED 6 OF THE 10 DESTROYED WELLS.

\section{RESTORATION}

NONE

\section{PROBLEMS}

THREE WELLS STILL NOT LOCATED: MCCG-106,112,118, MRIG-224 (PHILLIPS) STATUS NEEDS RESOLUTION (DO WE PLUG OR NOT ?). TWO WELLS MAY BE IN ENVIRONMENTALLY SENSITIVE AREAS, NEED TO WORK CAREFULLY AROUND THESE SITES: MRIG-205 (GOPHERTORTOISE BURROW) AND MCCG-118 (PITCHER-PLANT BOG).

\section{CHANGES TO ACTIVITY PLAN}

\section{NONE}

\section{SITE VISITORS}

NONE

\section{WORK PLANNED FOR NEXT WEEK}

BEGIN SAMPLING OF WELLS TO BE TURNED OVER TO LANDOWNERS. COMPLETE SAMPLING OF WELL-BORE FLUIDS FOR WELLS TO BE PLUGGED. MEET WITH SHALLOW-WELL CONTRACTOR, RUN CEMENT BOND LOGS IN 3 DEEP WELLS. 
WEEKLY PROGRESS REPORT

STONE \& WEBSTER ENGINEERING CORP.

\begin{tabular}{|l|l|l|}
\hline CLIENT DOE / SRPO & J.O. No. 17500.25 & $\begin{array}{c}\text { PERIOD } \\
08 / 22 / 88 \text { TO 08/28/88 }\end{array}$ \\
\hline $\begin{array}{l}\text { STATE AND SITE } \\
\text { MISSISSIPPI, GENERAL }\end{array}$ & $\begin{array}{l}\text { SITE REPRESENTATIVE } \\
\text { T. REGAN, D. KISTNER, R. GILLESPIE, H. ABEDI }\end{array}$ \\
\hline
\end{tabular}

SUMMARY OF ACTIVITIES

PLUGGING

TOOK SAMPLES OF WELL-BORE FLUIDS IN SHALLOW WELLS. TOOK FORMATION SAMPLE OF MRIG-210 (TO BE TURNED OVER) AND SENT TO MS PUBLIC HEALTH LABS FOR TESTING. MET WITH GRINER DRILLING REPRESENTATIVES TO PREPARE FOR PLUGGING SHALLOW WELLS, RAN CEMENT BOND LOGS IN MCCH-3A, MCCH-3D. AND MH-4B.

\section{RESTORATION}

NONE

\section{PROBLEMS}

ON ALL WATER-SUPPLY WELLS, JIM HOFFMAN OF BLWR WOULD LIKE US TO PULL OUT THE CASING. WE NEED TO DISCUSS THIS WITH CONTRACTORS TO SEE IF THIS IS FEASIBLE/ POSSIBLE, AND REVIEW POTENTIAL PROBLEMS WITH THIS PROCEDURE.

CHANGES TO ACTIVITY PLAN

ON SHALLOW WELLS IN WHICH PVC IS TO BE REMOVED. THERE IS NO LONGER A NEED TO EXCAVATE 3 FT. AND POUR CEMENT PAD (PER INSTRUCTIONS FROM J. HOFFMAN AT BLWR).

SITE VISITORS

J. DUCKWORTH (USFS), AND W. J. DUBOSE (MS OIL \& GAS BOARD) AT MCCH-3 AND MH -4.

WORK PLANNED FOR NEXT WEEK

BEGIN PLUGGING SHALLOW WELLS AT RICHTON. 
WEEKLY PROGRESS REPORT

STONE \& WEBSTER ENGINEERING CORP.

\begin{tabular}{|c|c|c|}
\hline DOE / SRPO & J.O. No. ${ }_{17500.25}$ & $\begin{array}{l}\text { PERIOD } \\
08 / 29 / 88 \text { TO } 09 / 04 / 88\end{array}$ \\
\hline $\begin{array}{l}\text { STATE AND SITE } \\
\text { MISSISSIPPI, GENERAL }\end{array}$ & $\begin{array}{l}\text { SITE REPRESEN } \\
\text { D. KIS'INER, } \mathrm{H} \text {. }\end{array}$ & $\begin{array}{l}\text { E. T. REGAN } \\
\text { DI, T }\end{array}$ \\
\hline \multicolumn{3}{|l|}{ SUMMARY OF ACTIVITIES } \\
\hline \multicolumn{3}{|l|}{ PLUGGING } \\
\hline \multicolumn{3}{|c|}{ SIX SHALLOW PVC WELLS DRILLED OUT AND GROUTED AT RICHTON DOME (MRIG 201, 203.} \\
\hline \multicolumn{3}{|c|}{ 204. 205, 226. AND 227): WORK IN PROGRESS ON MRIG-219. LOCATED AND SAMPLED } \\
\hline \multicolumn{3}{|c|}{ MRIG-224. SAMPLED MCCG-117. PVC WAS DRILLED OUT USING 4 1/2" OR $71 / 2 " 3-$} \\
\hline \multicolumn{3}{|c|}{ PRONG DRAG BIT, OR $7 "$ FISHTAIL BIT. MADE ARRANGEMENTS FOR DISPOSAL OF DRILL- } \\
\hline \multicolumn{3}{|c|}{ ING MUD AND SITE-RESTORATION DEBRIS AT PERRY CO. SANITARY LANDFILL. } \\
\hline \multicolumn{3}{|l|}{ RESTORATION } \\
\hline \multicolumn{3}{|l|}{ NONE } \\
\hline
\end{tabular}

\section{PROBLEMS}

DRILLING DEVIATED FROM ORIGINAL BOREHOLE AT MRIG-219 AND MRIG-203. PILOTS ON DRILL BITS ARE NOT KEEPING BIT ALIGNED ON PVC WHEN USING A KELLY-DRIVEN RIG. MRIG-219 AND 203 NOT GROUTED TO SURFACE AS REPORTED BY LETCO: GROUT APPARENTLY SETTLED TO SOME DEPTH, WHERE IT CAUSES BIT TO DEFLECT OFF OF ORIGINAL BORING.

CHANGES TO ACTIVITY PLAN NONE

SITE VISITORS

DAVE LOGAN (SWEC QA) ON $9 / 2 / 88$ AT MRIG-227

WORK PLANNED FOR NEXT WEEK

FINISH PLUGGING SHALLOW WELLS AT RICHTON, AND MOVE TO CAMP SHELBY BY $9 / 9 / 88$. 
WEEKLY PROGRESS REPORT

STONE \& WEBSTER ENGINEERING CORP.

\begin{tabular}{|l|l|l|}
\hline \multicolumn{1}{|c|}{ DOLIENT / SRPO } & J.O. No. 17500.25 & $\begin{array}{c}\text { PERIOD } \\
09 / 05 / 88 \text { TO 09/11/88 }\end{array}$ \\
\hline $\begin{array}{l}\text { STATE AND SITE } \\
\text { MISSISSIPPI, GENERAL }\end{array}$ & $\begin{array}{c}\text { SITE REPRESENTATIVE } \\
\text { D. KISTNER, H. ABEDI }\end{array}$ \\
\hline SUMMARY OF ACTIVITIES & \\
PLUGGING & \\
DRILLED OUT AND GROUTED SIX SHALLOW PVC WELLS AT RICHTON DOME; DRILLING \\
\hline RESULTS WERE SIMILAR TO LAST WEEK: TOP 20 FT OF PVC IS COMPLETELY DRILLED \\
\hline OUT. THEREAFTER GENERALLY DRILLED ALONGSIDE PVC PIPE TO T.D. PERFORATING \\
\hline AND/OR BREAKING PIPE OFF AT LENGTHS UP TO 35 FT WHICH RETURN WITH MUD. MOVED \\
\hline TO CYPRESS CREEK DOME ON 9/9/88 AND RIGGED UP ON MCCG-105. \\
\hline
\end{tabular}

RESTORATION

NONE

PROBLEMS

ON SEPT. 8. J. HOFFMAN (BLWR) CONTACTED T. REGAN TO EXPRESS CONCERNS REGARDING PVC REMAINING INTACT IN PREVIOUSLY-PLUGGED WELLS. DECIDED TO TRY USING A 30 FT. PILOT TO KEEP BIT ON PVC CASING: PILOT BROKE AT 5 FT AND FELL TO BOTTOM OF MCCG-105. UNABLE TO RETRIEVE AS OF 9/9; WILL RETURN ON 9/12 TO TRY AGAIN.

CHANGES TO ACTIVITY PLAN NONE

SITE VISITORS

NONE

WORK PLANNED FOR NEXT WEEK

WILL MEET WITH $J$. HOFFMAN OF MS BLWR TO DISCUSS PLUGGING PROCEDURES AND RESULTS SO FAR; WILL PROBABLY SWITCH TO A TOP-DRIVE RIG. CONTINUE PLUGGING SHALLOW WELLS. 
WEEKLY PROGRESS REPORT

STONE \& WEBSTER ENGINEERING CORP.

\begin{tabular}{|c|c|c|}
\hline DOE / SRPO & J.O. No. ${ }_{17500.25}$ & $\begin{array}{l}\text { PERIOD } \\
\text { 09/12/88 TO } 09 / 18 / 88\end{array}$ \\
\hline $\begin{array}{l}\text { STATE AND SITE } \\
\text { MISSISSIPPI, GENERAL }\end{array}$ & $\begin{array}{l}\text { SITE REPRESEN } \\
\text { R. GILLESPIE, D }\end{array}$ & NER, H. ABEDI \\
\hline \multicolumn{3}{|l|}{ SUMMARY OF ACTIVITIES } \\
\hline \multicolumn{3}{|c|}{ DEEP-WELL PLUGGING COMMENCED SEPTEMBER 14 AT SITE MH-6. PLUGGED MH-6A, 6B AND } \\
\hline \multicolumn{3}{|c|}{$6 C$ TO $+/-3$ FT DEEP WITH NO PROBLEMS. SHALLOW WELLS: DRILLED TO T.D. AND } \\
\hline \multicolumn{3}{|c|}{ PLUGGED MCCG-104, 105 AND 107 WITH NO PROBLEMS. NOW USING TOP-DRIVE RIG WITH } \\
\hline BETTER RESI'LTS THAN WITH & RIG. & \\
\hline
\end{tabular}

\section{RESTORATION}

NONE

\section{PROBLEMS}

NONE

\section{CHANGES TO ACTIVITY PLAN}

RESPONSIBILITY FOR PULLING CASING AND PLUGGING WATER-SUPPLY WELLS SHIFTED FROM DEEP-WELL CONTRACTOR TO GRINER DRILLING (SHALLOW-WELL CONTRACTOR).

\section{SITE VISITORS}

J. HOFFMAN (MS BLWR) \& M. MEADOWS (MS BOG) AT MCCG-104 ON $9 / 15$.

\section{WORK PLANNED FOR NEXT WEEK}

DEEP WELLS: FINISH MH-6 SITE, MOVE TO MH-7. SHALLOW WELLS: DRILL OUT PVC CASING AND PLUG MCCG-110,111, 114. AND 119. EXCAVATE TO DETERMINE WHETHER ANNULUS IS GROUTED AT MCCG-101 AND 102: IF SO. THEN GROUT PVC CASING IN PLACE. 
WEEKLY PROGRESS REPORT

STONE \& WEBSTER ENGINEERING CORP.

\begin{tabular}{|l|l|l|}
\hline \multicolumn{1}{|c|}{ DLIENT / SRPO } & J.O. No. 17500.25 & $\begin{array}{c}\text { PERIOD } \\
09 / 19 / 88 \text { TO 09/25/88 }\end{array}$ \\
\hline $\begin{array}{c}\text { STATE AND SITE } \\
\text { MISSISSIPPI, GENERAL }\end{array}$ & $\begin{array}{l}\text { SITE REPRESENTATIVE } \\
\text { D. KISTNER, H. ABEDI }\end{array}$ \\
\hline
\end{tabular}

\section{SUMMARY OF ACTIVITIES}

PLUGGING

SHALLOW WELLS: DRILLED OUT TO T.D. AND GROUTED FOUR PVC WELLS (MCCG-110, 111. 114. AND 119). ALSO GROUTED MCCG-101 WELL CASING IN PLACE AFTER VERIFYING

GOOD ANNULAR SEAL EXISTS.

DEEP WELLS: $M H-6 D, M H-7 A, 7 B, 7 C, 7 D, M H-8 A, 8 B$, AND 8D PLUGGED, AND DRILL RIG

MOVED TO MH-8C. CASINGS CUT OFF, PLATES WELDED ON AND PITS BACKFILLED AT

SITES MH-6 AND $M H-7$.

\section{RESTORATION}

NONE

\section{PROBLEMS}

SHALLOW WELLS: NONE

DEEP WELLS: NONE

CHANGES TO ACTIVITY PLAN

NONE

SITE VISITORS

J. HOFFMAN AND P. PHILLIPS (MS BLWR), AT MCCG-111 ON $9 / 21 / 88$.

WORK PLANNED FOR NEXT WEEK

SHALLOW WELLS: GROUT MCCG-102 IN PLACE, DRILL OUT AND GROUT MCCG-117, 103 AND 115. DEEP WELLS: FINISH SITE MH-8, AND MOVE TO SITE MH-5. 
WEEKLY PROGRESS REPORT

STONE \& WEBSTER ENGINEERING CORP.

CLIENT

DOE / SRPO

STATE AND SITE MISSISSIPPI, GENERAL
J.O. No.

17500.25

SITE REPRESENTATIVE

R. GILLESPIE, D. KISTNER

\section{SUMMARY OF ACTIVITIES}

PLUGGING

DEEP WELLS: COMPLETED PLUGGING OF MH-8C, MH-5A, 5B, 5C. AND 5D. CUT OFF

CASING AND WELDED PLATES ONTO CASINGS OF PLUGGED WELLS.

SHALLOW WELLS: DRILLED OUT PVC CASING AND CEMENTED THE FOLLOWING WELLS:

$M C C G-117, M C C G-103, M C C G-113$.

\section{RESTORATION}

NONE

\section{PROBLEMS}

DEEP WELLS: NONE

SHALLOW WELLS: NONE

\section{CHANGES TO ACTIVITY PLAN}

NONE

\section{SITE VISITORS}

NONE

WORK PLANNED FOR NEXT WEEK

DEEP WELLS: CONTINUE PLUGGING OPERATIONS AT MH-4. SHALLOW WELLS: DRILL OUT AND CEMENT MCCG-115; PULL STEEL CASING AND PLUG WATER-SUPPLY WELLS AT SEVEN DEEP-WELL SITES. 
WEEKLY PROGRESS REPORT

STONE \& WEBSTER ENGINEERING CORP.

\begin{tabular}{|l|l|l|}
\hline \multicolumn{1}{|c|}{ CLIENT } & J.O. No. ${ }^{17500.25}$ & $\begin{array}{c}\text { PERIOD } \\
10 / 03 / 88 \text { TO } 10 / 09 / 88\end{array}$ \\
\hline $\begin{array}{c}\text { STATE AND SITE } \\
\text { MISSISSIPPI, GENERAL }\end{array}$ & $\begin{array}{l}\text { SITE REPRESENTATIVE } \\
\text { W. BRANDON, H. ABEDI }\end{array}$ \\
\hline
\end{tabular}

\section{SUMMARY OF ACTIVITIES}

PLUGGING

DEEP WELLS - COMPLETED PLUGGING OF MH-4A, 4B, 4C, 4D; MCCH-3B, 3C, 3D, AND

3A. CUT OFF CASING AND WELDED STEEL PLATES ONTO CASINGS OF PLUGGED WELLS.

MOVED TO SITE MCCG-1.

SHALLOW WELLS: DRILLED OUT PVC AND GROUTED MCCG-115. PULLED 4" STEEL CASING

AND SCREEN, DRILLED TO TOTAL DEPTH AND CEMENTED MCCG-1WS AND

MCCG-2WS; PULLED 136 FT. OF 4" STEEL CASING IN MCCH-3WS.

RESTORATION

NONE

\section{PROBLEMS}

DEEP WELLS: NONE

SHALLOW WELLS: NONE

CHANGES TO ACTIVITY PLAN

NONE

$-$

SITE VISITORS

J. HOFFMAN AND P. PHILLIPS (MS BLW'R), AND M. MEADOWS (MS BOG) AT MCCG-1 ON 10/5

\section{WORK PLANNED FOR NEXT WEEK}

DEEP WELLS: CONTINUE PLUGGING OPERATIONS AT MCCG-1.

SHALLOW WELLS: PULL REMAINING STEEL CASING IN MCCG-3WS. PULL CASING, THEN

PLUG REMAINING WATER-SUPPLY WELLS NOT BEING TURNED OVER TO LANDOWNERS. 
WEEKLY PROGRESS REPORT

STONE \& WEBSTER ENGINEERING CORP.

\begin{tabular}{|l|l|l|}
\hline CLIENT DOE / SRPO & J.O. No. ${ }_{17500.25}$ & $\begin{array}{l}\text { PERIOD } \\
10 / 10 / 88 \text { TO } 10 / 16 / 88\end{array}$ \\
\hline $\begin{array}{c}\text { STATE AND SITE } \\
\text { MISSISSIPPI, GENERAL }\end{array}$ & $\begin{array}{l}\text { SITE REPRESENTATIVE } \\
\text { H. ABEDI, R. GILLESPIE, D. KISTNER }\end{array}$ \\
\hline
\end{tabular}

\section{SUMMARY OF ACTIVITIES}

PLUGGING

SHALLOW WELLS: PULLED REMAINING CASING AND GROUTED MCCH-3WS. PULLED 178 FT. OF CASING IN MH-8WS.

DEEP WELLS: PLUGGED MCCG-1, MCCG-2, MRIH-11A, 11B, 11C AND 11D, AND ATTEMPTED TO PLUG MRIG-9 WITH NO SUCCESS. WELL HEADS CUT OFF, CAPPED. AND BURIED ON ALL PLUGGED WELLS.

\section{RESTORATION}

NONE

\section{PROBLEMS}

SHALLOW WELLS: DUE TO CEMENTED ANNULUS IN MH-8WS, RATE OF DRILLING AND PULL-

ING CASING IS VERY SLOW AS CASING IS SEPARATING AT EVERY ONE OR TWO JOINTS.

DEEP WELLS: MRIG-9 TOOK FULL CALCULATED VOLUME OF CEMENT, WHICH APPARENTLY SUBSIDED \& SEEPED INTO FORMATION. WILL SET BRIDGE PLUG AND GROUT NEXT WEEK.

\section{CHANGES TO ACTIVITY PLAN}

NONE

\section{SITE VISITORS}

W. J. DUBOSE (MS OIL \& GAS BOARD) AT SITE MRIH-11 ON $10 / 12$.

\section{WORK PLANNED FOR NEXT WEEK}

SHALLOW WELLS: PULL REMAINING CASING AND PLUG MH-8WS. PLUG REMAINING WATERSUPPLY WELLS NOT BEING TURNED OVER TO LANDOWNERS. DEEP WELLS: SET BRIDGE PLUG AND GROUT MRIG-9, PLUG MRIG-10, FINISHING DEEP-WELL PLUGGING IN MISSISSIPPI. 
WEEKLY PROGRESS REPORT

STONE \& WEBSTER ENGINEERING CORP.

\begin{tabular}{|l|l|l|}
\hline CLIENT DOE / SRPO & \multicolumn{1}{|c|}{$\begin{array}{l}\text { J.O. No. } \\
17500.25\end{array}$} & $\begin{array}{l}\text { PERIOD } \\
10 / 17 / 88 \text { TO 10/23/88 }\end{array}$ \\
\hline $\begin{array}{c}\text { STATE AND SITE } \\
\text { MISSISSIPPI, GENERAL }\end{array}$ & $\begin{array}{l}\text { SITE REPRESENTATIVE } \\
\text { R. GILLESPIE, D. KISTNER, T. REGAN }\end{array}$ \\
\hline
\end{tabular}

\section{SUMMARY OF ACTIVITIES}

PLUGGING

DEEP WELLS: PLUGGED MRIG-9 AND 10, FINISHING ALL DEEP-WELL PLUGGING. ALL WELLS CAPPED $>3$ FT. BELOW GROUND. SHALLOW WELLS: MH-8WS - PULLED REMAINING 4 IN. STEEL CASING TO 220 FT. . PULLED 20-30 FT. OF UN-WOUND STEEL SCREEN, DRILLED PAST REMAINING SCREEN TO T.D. AT 425 FT. AND CEMENTED BOREHOLE.

\section{RESTORATION}

CLEANED UP ALL WELL SITES AT CYPRESS CREEK DOME; ALL ARE READY FOR RESEEDING AND MULCHING. FENCES REMAIN AT MCCG-2 AND MCCH-3 - TO BE DISMANTLED MONDAY \& TUESDAY NEXT WEEK. CLEANED UP SITES MRIG-201,203, 204 AND 205 AT RICHTON DOME.

\section{PROBLEMS}

DEEP WELLS: NONE

SHALLOW WELLS: DUE TO CEMENTED ANNULUS, MH-8WS REQUIRED OVERSHOOTING CASING TO DRILL OUT CEMENT, THEN PULLING CASING JOINT BY JOINT. CASING BROKE AT SCREEN, AND SCREEN UNWOUND. REMOVED SOME OF UNWOUND SCREEN, LEFT THE REST IN HOLE.

\section{CHANGES TO ACTIVITY PLAN}

NONE

SITE VISITORS

NONE

\section{WORK PLANNED FOR NEXT WEEK}

SHALLOW WELLS: SET UP ON MH-5WS ON 10/24/88. PLUG REMAINING WATER-SUPPLY WELLS (MH-5WS, MRIG-9WS AND MRIG-10WS) BY END OF NEXT WEEK. CONTINUE WITH SITE CLEAN-UP, BEGIN RESEEDING AT CYPRESS CREEK. 
WEEKLY PROGRESS REPORT

STONE \& WEBSTER ENGINEERING CORP.

\begin{tabular}{|c|l|l|}
\hline CLIENT DOE / SRPO & \multicolumn{1}{|c|}{$\begin{array}{l}\text { J.O. No. } \\
17500.25\end{array}$} & $\begin{array}{l}\text { PERIOD } \\
10 / 24 / 88 \text { TO 10/30/88 }\end{array}$ \\
\hline $\begin{array}{c}\text { STATE AND SITE } \\
\text { MISSISSIPPI, GENERAL }\end{array}$ & $\begin{array}{l}\text { SITE REPRESENTATIVE } \\
\text { D. KISTNER, T. REGAN }\end{array}$ \\
\hline
\end{tabular}

SUMMARY OF ACTIVITIES

PLUGGING

SHALLOW WELLS: PULLED CASING AND SCREEN, DRIILED TO T.D. AND GROUTED TO SUR-

FACE THE FOLLOWING WELLS: MH-5WS, MKIG-9WS, MRIG-10WS. THIS COMPLETES

PLUGGING OF WATER-SUPPLY WELLS.

\section{RESTORATION}

COMPLETED SITE CLEANUP, GRADING, AND REBUILDING ROADS WHERE REQUIRED AT ALL

(DEEP AND SHALLOW) SITES AT RICHTON AND CYPRESS CREEK DOMES.

\section{PROBLEMS}

NONE

CHANGES TO ACTIVITY PLAN

NONE

SITE VISITORS

NONE

\section{WORK PLANNED FOR NEXT WEEK}

DRILL OUT SHALLOW, PVC WELL MRIG-220: BEGIN GROUND-WATER SAMPLING FOR WELLS

BEING TURNED OVER TO LANDOWNERS. CONTINUE SITE RESTORATION: FERTILIZING,

LIMING, MULCHING, AND SEEDING OF ALL SITES. 
WEEKLY PROGRESS REPORT STONE \& WEBSTER ENGINEERING CORP.

\begin{tabular}{|c|c|l|}
\hline \multicolumn{1}{|c|}{ CLIENT } & J.O. No. ${ }^{17500.25}$ & $\begin{array}{l}\text { PERIOD } \\
10 / 31 / 88 \text { TO } 11 / 06 / 88\end{array}$ \\
\hline $\begin{array}{c}\text { STATE AND SITE } \\
\text { MISSISSIPPI, GENERAL }\end{array}$ & $\begin{array}{c}\text { SITE REPRESENTATIVE } \\
\text { D. KISTNER, R. GILLESPIE }\end{array}$ \\
\hline
\end{tabular}

\section{SUMMARY OF ACTIVITIES}

PLUGGING

DRILL OUT TO T.D. AND GROUT MRIG-220. CHLORINATED, PURGED AND SAMPLED THE

FOLLOWING 4" ID. WELLS: MH-4WS, MH-6WS, MH-TWS, MRIH-11WS, MRIG-222, MRIG-223

MRIG-230.

\section{RESTORATION}

THE FOLLOWING SITES HAVE BEEN RESTORED: MH-5, MCCG-2, 101, 104, 105, 107. 110, 111, 114, 115, 117, 113, AND 119. TOPSOIL HAS BEEN HAULED TO \& SPREAD AT SITE MH-7.

\section{PROBLEMS}

NONE

CHANGES TO ACTIVITY PLAN

NONE

SITE VISITORS

MR. GANDY (LANDOWNER) AT MRIG-230; MR. DAHMER, (LANDOWNER'S NEPHEW) AT MH-7.

WORK PLANNED FOR NEXT WEEK

CHLORINATE, PURGE AND SAMPLE THE 2" ID. WELLS TO BE TURNED OVER. RESTORATION OF ALL SITES SHOULD BE COMPLETED NEXT WEEK. 
WEEKLY PROGRESS REPORT

STONE \& WEBSTER ENGINEERING CORP.

\begin{tabular}{|c|c|c|}
\hline DOE / SRPO & J.O. No. ${ }_{17500.25}$ & $\begin{array}{l}\text { PERIOD } \\
11 / 07 / 88 \text { TO } 11 / 12 / 88\end{array}$ \\
\hline $\begin{array}{l}\text { STATE AND SITE } \\
\text { MISSISSIPPI, GENERAL }\end{array}$ & \multicolumn{2}{|c|}{$\begin{array}{l}\text { SITE REPRESENTATIVE } \\
\text { R. GILLESPIE, D. KISTNER, T. REGAN }\end{array}$} \\
\hline \multicolumn{3}{|l|}{ SUMMARY OF ACTIVITIES } \\
\hline \multicolumn{3}{|l|}{ PLUGGING } \\
\hline \multicolumn{3}{|c|}{ PLUGGED MRIG-212 AND 215, COMPLETING ALL PLUGGING ACTIVITIES IN MISSISSIPPI. } \\
\hline \multicolumn{3}{|c|}{ THE FOLLOWING 2" WELLS WERE CHLORINATED PRIOR TO SAMPLING: MRIG-202, $210,211}$. \\
\hline \multicolumn{3}{|c|}{ 212. 215, AND 228: ALL BUT MRIG-212 WERE PURGED AND SAMPLED. MRIG-212 WAS PLUG } \\
\hline \multicolumn{3}{|c|}{ GED AT LANDOWNER'S REQUEST DUE TO DAMAGED WELL CASING DOWNHOLE. MRIG-215 ALSO } \\
\hline \multicolumn{3}{|c|}{ PLUGGED (AFTER SAMPLING) AT LANDOWNER'S REQUEST. } \\
\hline \multicolumn{3}{|l|}{ RESTORATION } \\
\hline \multicolumn{3}{|c|}{ COMPLETED SITE RESTORATION AT THE FOLLOWING WELL SITES: MCCG-1, MRIG-9, 10,} \\
\hline \multicolumn{3}{|c|}{$201,203,204,205,209,213,216,219,226,227,232, M H-7$ AND MH-8. SWEC } \\
\hline \multicolumn{3}{|c|}{ PERSONNEL RESTORED SITES MRIG-212 AND MRIG-215 ON 11/12. THIS COMPLETES ALL } \\
\hline SITE-RESTORATION WORK IN & PENDING LANDOWNE & ROVAL OF WORK. \\
\hline
\end{tabular}

PROBLEMS

NONE

CHANGES TO ACTIVITY PLAN

NONE

SITE VISITORS

NONE

WORK PLANNED FOR NEXT WEEK

ALL FIELD WORK COMPLETED. TEST RESULTS FROM 11 SAMPLED WELLS TO BE SENT TO SWEC IN BOSTON BY MISSISSIPPI PUBLIC HEALTH LABS. 
WEEKLY PROGRESS REPORT

STONE \& WEBSTER ENGINEERING CORP.

\begin{tabular}{|l|l|l|}
\hline \multicolumn{1}{|c|}{ DOIENT / SRPO } & $\begin{array}{l}\text { J.O. NO. } 17500.25 \\
\text { PERIOD } \\
01 / 21 / 89 \text { TO 01/24/89 }\end{array}$ \\
\hline $\begin{array}{l}\text { STATE AND SITE } \\
\text { MISSISSIPPI, MH-8 AND GENERAL }\end{array}$ & $\begin{array}{c}\text { SITE REPRESENTATIVE } \\
\text { D. KISTNER }\end{array}$ \\
\hline $\begin{array}{l}\text { SUMMARY OF ACTIVITIES } \\
\begin{array}{l}\text { PLUGGING } \\
\text { NONE }\end{array}\end{array}$ \\
\hline
\end{tabular}

RESTORATION

PERFORMED REMEDIAL RESTORATION AT MH-8 (WALKER) WHICH CONSISTED OF STABILIZING GULLIES ON EAST AND SOUTH SIDE OF SITE USING HAY AND BOARDS (125 BALES AND 125 BOARDS). SUBCONTRACTOR PLANTED PINE SEEDLINGS OVER ENTIRE SITE ON 12 FT $\times 12$ FT SPACING. OWNER INSTALLED FENCE ALONG WEST SIDE TO LIMIT ACCESS. RE-PHOTOGRAPHED ALL DRILL SITES FOR INCLUSION IN FINAL REPORT. PROBLEMS NONE

CHANGES TO ACTIVITY PLAN

NONE

SITE VISITORS

MR. BOBY REED, FARM MANAGER FOR WALKER SITE. HE VERBALLY APPROVED THE SITE RESTORATION WORK.

WORK PLANNED FOR NEXT WEEK CONTINUE PREPARATION OF FINAL REPORT IN BOSTON. 


\section{APPENDIX B}

Site Reconnaissance Form 
SITE RECONNAISSANCE FORM

\begin{tabular}{|l|l|l|}
\hline CLIENT & DOE / SRPO \\
\hline $\begin{array}{l}\text { STATE } \\
\text { MISSISSIPPI }\end{array}$ & $\begin{array}{l}\text { WELL NAME \& NUMBER } \\
\text { U.S.F.S. MCCG-1 }\end{array}$ & TFS MC. \\
\hline
\end{tabular}

CATION

2702' EWL \& 2528' NSL" IN

SEC. 9-T2N-R10W -. PERRY COUNTY

J.O. No.

17500.25 DATE

$07 / 13 / 88$

TFSC FIELD REPRESENTATIVE

DESCRIPTION OF SITE ACCESS

1 MILE ON DIRT ROAD WITH NUMEROUS WATER BARS. DIFFICULT ACCESS DURING WET

WEATHER. ROAD REQUIRES GRADING.

DESCRIPTION OF SITE PAD AREA

OPEN, FLAT, SLIGHTLY GRASSY FIELD.

DESCRIPTION OF WELL HEAD

8-IN (ID) STEEL INNER CASING WITH LOCKING, HINGED CAP EXTENDS 2 FT ABOVE GROUND.

12-IN (ID) SURFACE CASING AND 20-IN (ID) CONDUCTOR PIPE, STICK-UP NOT MEASURED.

WELL HEAD IS SET IN A 10-15 FT DIA CEMENT PAD WHICH IS PARTIALLY BROKEN UP.

DESCRIPTION OF PITS

NA

DEPTH AND BOREHOLE - FLUID SURVEY:BY MASCO $(8 / 6 / 88)$

GROUND ELEVATION 221.0 FT (DATUM)

REPORTED DEPTH 1369.0 FT (SEE BOREHOLE COMPLETION SKETCH)

MEASURED DEPTH 1359.0 FT FTSTRUCTION

FLUID DEPTH $120.0 \quad$ FT $\quad \mathrm{pH} 10.55$

CONDUCTIVITY $376 \mathrm{mS} / \mathrm{cm} \quad$ TDS $188,800 \mathrm{mg} / \mathrm{L}$

TEMPERATURE

REMARKS

** 10 FT OF SILT AND PIPE SCALE IN BOTTOM OF HOLE, OR HOLE HAS CLOSED UP AT BOTTOM. OPEN BOREHOLE FROM BOTTOM OF CASING TO TOP OF CEMENT BACK-PLUG $(1369$ FT) 
SITE RECONNAISSANCE FORM

\begin{tabular}{|c|c|c|c|}
\hline \multirow{2}{*}{\multicolumn{2}{|c|}{$\begin{array}{ll}\text { CLIENT } & \text { DOE / SRPO }\end{array}$}} & \multicolumn{2}{|l|}{ SEC, $9-T 2 N-R 10$} \\
\hline & & J.O. No. 17500.25 & DATE $07 / 13 / 88$ \\
\hline $\begin{array}{l}\text { STATE } \\
\text { MISSISSIPPI }\end{array}$ & $\begin{array}{l}\text { WELL NAME \& NUMBER } \\
\text { U.S.F.S. MCCG-1WS }\end{array}$ & \multicolumn{2}{|c|}{$\begin{array}{l}\text { TFSC FIELD REPRESENTATIVE } \\
\text { T. REGAN, D. LUTZ }\end{array}$} \\
\hline
\end{tabular}

DESCRIPTION OF SITE PAD AREA

OPEN, FLAT, SLIGHTLY GRASSY FIELD.

WELL SURROUNDED BY $3 \times 3 \times 3$ ET PROTECTIVE BARRIER.

DESCRIPTION OF WELL HEAD

4-IN (ID) STEEL CASING WITH THREADED CAP EXTENDS 1.1 FT ABOVE GROUND.

SWEC REMOVED PUMP THAT HAD BEEN LODGED IN WELL (AT 17 FT) ON 10/5/88.

\section{DESCRIPTION OF PITS}

NA

DEPTH AND BOREHOLE - FLUID SURVEY:BY SWEC (10/05/88)

GROUND ELEVATION 219.9 FT (DATUM)

REPORTED DEPTH 100.0 FT (SEE BOREHOLE COMPLETION SKETCH)

MEASURED DEPTH $\frac{73.0}{2} \mathrm{FT}$ OBSTRUCTION $\ldots *$ FT

FLUID DEPTH $20.0 \quad \mathrm{FT} \quad \mathrm{pH}-6.06$

CONDUCTIVITY $0.058 \mathrm{~ms} / \mathrm{cm}$ TDS $29.0 \mathrm{mg} / \mathrm{L}$

TEMPERATURE $30.0-{ }^{\circ} \mathrm{C}$

\section{REMARKS}

OPEN HOLE BETWEEN BOTTOM OF WELL (77.4 FT) AND TOP OF CEMENT BACK-PLUG (100 FT). ** 4.4 FT OF SILT AT BOTTOM OF WELL. 
SITE RECONNAISSANCE FORM

$1119^{\prime}$ SNL \& 1961 ' WEL * IN

SEC. 33-T3N-R1OW -. PERRY COUNTY

\begin{tabular}{|l|l|l|l|}
\hline CLIENT & J.0. No. 17500.25 & DATE \\
\hline $\begin{array}{l}\text { STATE } \\
\text { MISSISSIPPI }\end{array}$ & $\begin{array}{l}\text { WELL NAME \& NUMBER } \\
\text { U.S.F.S. MCCG-2 }\end{array}$ & $\begin{array}{r}\text { TFSC FIELD REPRESENTATIVE } \\
\text { T. REGAN, D. LUTZ }\end{array}$ \\
\hline
\end{tabular}

DESCRIPTION OF SITE ACCESS

DIRECT ACCESS FROM GOOD GRAVEL ROAD. SITE IS SURROUNDED BY BARBED-WIRE FENCE.

SOME ROAD GRADING AND TREE REMOVAL REQUIRED FOR RIG ACCESS.

DESCRIPTION OF SITE PAD AREA

FLAT AREA WITH LOW BRUSH AND MANY 20 FT HIGH PINE TREES.

DESCRIPTION OF WELL HEAD

6-IN (ID) STEEL INNER CASING WITH LOCKING HINGED CAP IS BROKEN OFF AT FLANGE 0.2 FT ABOVE GROUND. STICK-UP OF 12 -IN (ID) SURFACE CASING AND 20-IN (ID) CONDUCTOR

PIPE WERE NOT MEASURED.

DESCRIPTION OF PITS

NA

DEPTH AND BOREHOLE - FLUID SURVEY:BY MASCO $(8 / 6 / 88)$

GROUND ELEVATION 196.9 FT (DATUM)

REPORTED DEPTH 2676.1 FT (SEE BOREHOLE COMPLETION SKETCH)

MEASURED DEPTH 2637.0 FT OBSTRUCTION

FLUID DEPTH $95.0 \quad$ FT $\quad \mathrm{PH}-9.19$

CONDUCTIVITY $180.4 \mathrm{~ms} / \mathrm{cm}$ TDS $89.800 \mathrm{mg} / \mathrm{L}$

TEMPERATURE $24.2{ }^{\circ} \mathrm{C}$

REMARKS

** 39.1 FT OF SILT AND PIPE SCALE IN BOTTOM WELL. 
SITE RECONNAISSANCE FORM

\begin{tabular}{|c|c|c|c|}
\hline CLIENT & DOE / SRPO & J.O. No. 17500.25 & DATE $07 / 13 / 88$ \\
\hline $\begin{array}{l}\text { STATE } \\
\text { MISSISSIPPI }\end{array}$ & $\begin{array}{l}\text { WELL NAME \& NUMBER } \\
\text { U.S.F.S. MCCG-2WS }\end{array}$ & \multicolumn{2}{|c|}{\begin{tabular}{|c|} 
TFSC FIELD REPRESENTATIVE \\
T. REGAN, D. LUTZ
\end{tabular}} \\
\hline \multicolumn{4}{|c|}{ DESCRIPTION OF SITE ACCESS } \\
\hline \multicolumn{4}{|c|}{ DIRECT ACCESS 50 FT EAST OF GOOD GRAVEL ROAD. } \\
\hline \multicolumn{4}{|c|}{ FENCE. } \\
\hline \multicolumn{4}{|c|}{ DESCRIPTION OF SITE PAD AREA } \\
\hline \multicolumn{4}{|c|}{ FLAT, GRASSY AREA WITH LOW BRUSH AND MANY 20-FT HIGH PINE TREES. } \\
\hline
\end{tabular}

DESCRIPTION OF WELL HEAD

4-IN (ID) STEEL CASING WITH LOCKING HINGED CAP EXTENDS 0.9 FT ABOVE GROUND.

\section{DESCRIPTION OF PITS}

$\underline{N A}$

DEPTH AND BOREHOLE - FLUID SURVEY:BY MASCO $(8 / 6 / 88)$

GROUND ELEVATION 196.5 FT (DATUM)

REPORTED DEPTH 248.3 FT (SEE BOREHOLE COMPLETION SKETCH)

MEASURED DEPTH 245.0 FT OBSTRUCTION

FLUID DEPTH $120.0 \quad$ FT $\quad \mathrm{PH}-8.00$

CONDUCTMITY $0.589 \mathrm{~ms} / \mathrm{cm} \mathrm{mg} / \mathrm{L}$

TEMPERATURE $24.5{ }^{\circ} \mathrm{C}$

\section{REMARKS}

OPEN HOLE BETWEEN BOTTOM OF WELL (248.3 FT) AND BOTTOM OF BOREHOLE (412 FT).

** 3.3 FT OF SILT AND PIPE SCALE AT BOTTOM OF WELL. 
SITE RECONNAISSANCE FORM

\begin{tabular}{|c|c|c|c|}
\hline \multicolumn{4}{|r|}{ Y COUNTY } \\
\hline \multicolumn{2}{|c|}{ CLIENT DOE / SRPO } & J.O. No. 17500.25 & DATE $07 / 13 / 88$ \\
\hline $\begin{array}{l}\text { STATE } \\
\text { MISSISSIPPI }\end{array}$ & $\begin{array}{l}\text { WELL NAME \& NUMBER } \\
\text { U.S.F.S. MCCH-3A } \\
\end{array}$ & \multicolumn{2}{|c|}{$\begin{array}{l}\text { TFSC FIELD REPRESENTATIVE } \\
\text { D. KISTNER, H. ABEDI }\end{array}$} \\
\hline \multicolumn{4}{|c|}{$\begin{array}{l}\text { DESCRIPTION OF SITE ACCESS } \\
0.1 \text { MILE ON PARET TOWER ROAD WEST OF STATE HWY 29, THEN LESS THAN } 0.1 \text { MILE SOUTH }\end{array}$} \\
\hline \multicolumn{4}{|c|}{ ON GOOD GRAVEL ROAD. SITE SURROUNDED BY 400-FT SQUARE BARBED-WIRE FENCE. } \\
\hline \multicolumn{4}{|c|}{ DESCRIPTION OF SITE PAD AREA } \\
\hline \multicolumn{4}{|c|}{ FLAT GRASSY AREA WITH NUMEROUS 10 TO 15-FT HIGH PINE SAPLINGS. } \\
\hline \multicolumn{4}{|c|}{ TREES WILL HAVE TO BE CLEARED FOR RIG ACCESS TO WELL HEAD. } \\
\hline \multicolumn{4}{|c|}{ DESCRIPTION OF WELL HEAD } \\
\hline \multicolumn{4}{|c|}{ 8-IN (ID) STEEL INNER CASING WITH LOCKING, HINGED CAP EXTENDS 1.1 FT ABOVE } \\
\hline \multicolumn{4}{|c|}{ GROUND. STICK-UP OF 10-IN (ID) SURFACE CASING AND 15.5-IN (ID) CONDUCTOR PIPE } \\
\hline \multicolumn{4}{|c|}{ WERE NOT MEASURED. } \\
\hline \multicolumn{4}{|c|}{ DESCRIPTION OF PITS } \\
\hline & & & \\
\hline
\end{tabular}

DEPTH AND BOREHOLE - FLUID SURVEY:BY MASCO (8/7/88)

GROUND ELEVATION 258.2 FT (DATUM)

REPORTED DEPTH 3098.6 FT (SEE BOREHOLE COMPLETION SKETCH)

MEASURED DEPTH 3098.0 FT OBSTRUCTION N/A FT

$\begin{array}{lll}\text { FLUID DEPTH } \frac{300.0}{2} \mathrm{FT} & \mathrm{PH}-\frac{8.68}{312} \mathrm{mS} / \mathrm{cm} & \text { TDS } 156.600 \\ \text { CONDUCTMITY } \mathrm{mg} / \mathrm{L}\end{array}$

TEMPERATURE $24.3{ }^{\circ} \mathrm{C}$

REMARKS

TOP 2 INNER-CASING JOINTS ARE 8" ID: REMAINING JOINTS ARE 6" ID. 
SITE RECONNAISSANCE FORM

\begin{tabular}{|c|c|c|c|}
\hline \multicolumn{2}{|c|}{ SIIE RECONNAISSANCE FORM } & \multicolumn{2}{|c|}{$\begin{array}{l}\text { LOCATION } \\
1030 \prime \text { WEL \& } 5461 \text { NSL* IN } \\
\text { SEC. 33-T2N-R1OW - P PERRY COUNTY }\end{array}$} \\
\hline \multicolumn{2}{|r|}{ DOE / SRPO } & J.O. No. 17500.25 & DATE $07 / 13 / 88$ \\
\hline $\begin{array}{l}\text { STATE } \\
\text { MISSISSIPPI }\end{array}$ & $\begin{array}{l}\text { WELL NAME \& NUMBER } \\
\text { U.S.F.S. MCCH-3B }\end{array}$ & \multicolumn{2}{|c|}{$\begin{array}{l}\text { TFSC FIELD REPRESENTATIVE } \\
\text { D. KISTNER, H. ABEDI }\end{array}$} \\
\hline \multicolumn{4}{|c|}{$\begin{array}{l}\text { DESCRIPTION OF SITE ACCESS } \\
0.1 \text { MILE ON PARET TOWER ROAD WEST OF STATE HWY 29, THEN LESS THAN } 0.1 \text { MILE SOUTH }\end{array}$} \\
\hline \multicolumn{4}{|c|}{ ON GOOD GRAVEL ROAD. SITE IS SURROUNDED BY A 400-FT SQUARE BARBED-WIRE FENCE. } \\
\hline \multicolumn{4}{|c|}{ DESCRIPTION OF SITE PAD AREA } \\
\hline \multicolumn{4}{|c|}{ TREE REMOVAL REQUIRED FOR RIG ACCESS. } \\
\hline \multicolumn{4}{|c|}{ DESCRIPTION OF WELL HEAD } \\
\hline \multicolumn{4}{|c|}{ GROUND: 10.25 -IN (ID) SURFACE CASING TO 0.6 FT ABOVE GROUND: 15.5 -IN (ID) } \\
\hline \multicolumn{4}{|c|}{ CONDUCTOR PIPE TO 0.35 FT ABOVE GROUND. } \\
\hline \multicolumn{4}{|c|}{ DESCRIPTION OF PITS } \\
\hline
\end{tabular}

DEPTH AND BOREHOLE - FLUID SURVEY:BY MASCO (8/7/88)

GROUND ELEVATION 257.9 FT (DATUM)

REPORTED DEPTH 3490.0 FT (SEE BOREHOLE COMPLETION SKETCH)

MEASURED DEPTH 3428.0 FT OBSTRUCTION

FLUID DEPTH $-300.0 \quad \mathrm{FT} \quad \mathrm{PH}-8.79$

CONDUCTIVITY $320 \quad \mathrm{mS} / \mathrm{cm} \quad$ TDS $160,000 \mathrm{mg} / \mathrm{h}$

TEMPERATURE $24.5{ }^{\circ} \mathrm{C}$

\section{REMARKS}

TOP 2 INNER-CASING JOINTS ARE 8" ID: REMAINING JOINTS ARE 6" ID.

** 62 FT SILT AND PIPE SCALE AT BOTTOM OF WELL. 
SITE RECONNAISSANCE FORM

\begin{tabular}{|c|c|c|c|}
\hline & & & \\
\hline CLIENT & DOE / SRPO & J.O. No. 17500.25 & DATE $\quad 07 / 13 / 88$ \\
\hline $\begin{array}{l}\text { STATE } \\
\text { MISSISSIPPI }\end{array}$ & $\begin{array}{l}\text { WELL NAME \& NUMBER } \\
\text { U.S.F.S. MCCH-3C }\end{array}$ & $\begin{array}{r}\text { TFSC FIELD REPRESEI } \\
\text { D.KIS }\end{array}$ & H. ABEDI \\
\hline DESCRIPTION OF & TE ACCESS & & \\
\hline 0.1 MILE ON PA & TOWER ROAD WEST OF STA & 29. THEN LESS TH & 1 MILE SOUTH \\
\hline ON GOOD GRAVEL & AD. SITE IS SURROUND & $T$ SQUARE BARE & FENCE. \\
\hline DESCRIPTION OF & TE PAD AREA & & \\
\hline FLAT GRASSY AR & UITH NUMEROUS PINE TRE & & \\
\hline SOME TREES WIL & VE TO BE CLEARED FOR & & \\
\hline
\end{tabular}

\section{DESCRIPTION OF WELL HEAD}

6-IN (ID) STEEL INNER CASING WITH LOCKING, HINGED CAP EXTENDS 0.7 FT ABOVE

GROUND. 12-IN (ID) CONDUCTOR PIPE IS LEVEL WITH GROUND. A 2-FT WIDE WASHOUT

AROUND CONDUCTOR HAS BEEN PARTIALLY COVERED WITH GRATING.

DESCRIPTION OF PITS

NA

DEPTH AND BOREHOLE - FLUID SURVEY:BY MASCO $(8 / 7 / 88)$

GROUND ELEVATION 258.3 FT (DATUM)

REPORTED DEPTH 1832.0 FT (SEE BOREHOLE COMPLETION SKETCH)

MEASURED DEPTH 1712.0 FT OBSTRUCTION $* *$ FT

FLUID DEPTH $250.0 \quad$ FT $\quad \mathrm{pH}-8.49$

CONDUCTIVITY $222 \mathrm{mS} / \mathrm{cm}$ TDS $111,300 \mathrm{mg} / \mathrm{L}$

TEMPERATURE $24.2{ }^{\circ} \mathrm{C}$

REMARKS

** 120 FT SILT AND PIPE SCALE AT BOTTOM OF WELL. 


\section{SITE RECONNAISSANCE FORM}

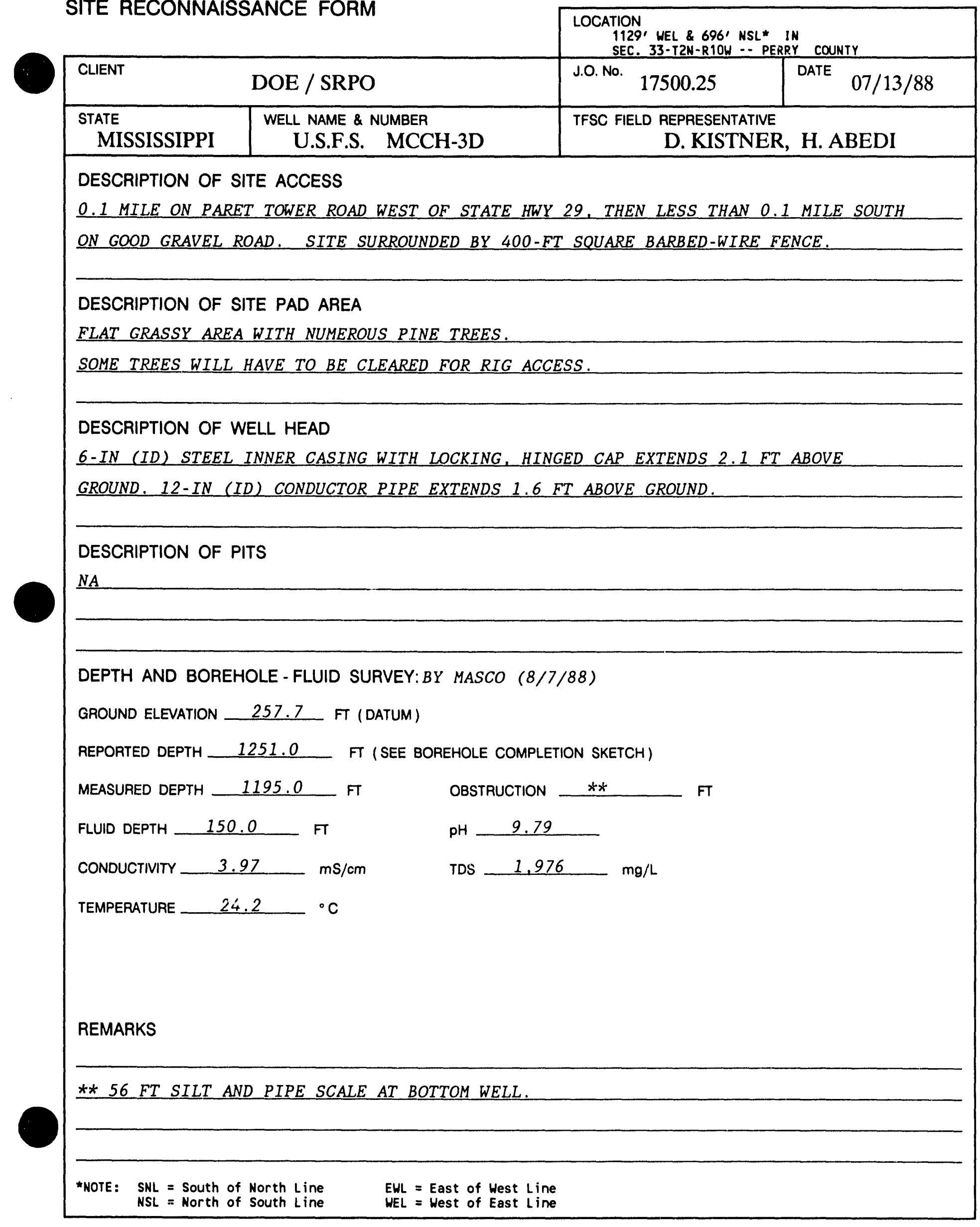


SITE RECONNAISSANCE FORM

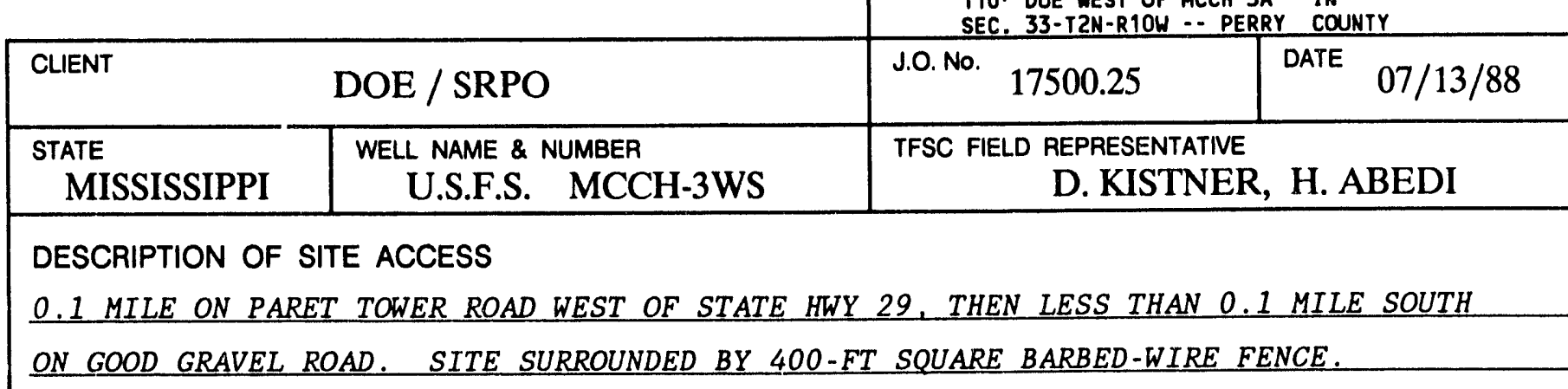

DESCRIPTION OF SITE PAD AREA

WELL HEAD IS OBSCURED BY SHRUBS AND TALL GRASS WHICH MUST BE CLEARED.

WELL IS LOCATED ALONG THE FENCE LINING THE WESTERN EDGE OF THE SITE.

DESCRIPTION OF WELL HEAD

4-IN (ID) STEEL CASING WITH LOCKING HINGED CAP EXTENDS 1 FT ABOVE GROUND.

DESCRIPTION OF PITS

NA

DEPTH AND BOREHOLE - FLUID SURVEY: $* * B Y$ MASCO $(8 / 7 / 88)$

GROUND ELEVATION 254.4 FT (DATUM)

REPORTED DEPTH 346.1 FT (SEE BOREHOLE COMPLETION SKETCH)

MEASURED DEPTH 345.0 FT OBSTRUCTION N/A TT

FLUID DEPTH 73.9 FT $\quad \mathrm{PH} \longrightarrow 7.61$

CONDUCTIVITY $0.380 \mathrm{~m} \mathrm{~cm}$ TDS $190.1 \mathrm{mg} / \mathrm{L}$

TEMPERATURE $24.3 \quad{ }^{\circ} \mathrm{C}$

\section{REMARKS}

OPEN HOLE BETWEEN BOTTOM OF WELL (346 FT) AND BOTTOM OF BOREHOLE (443 FT).

** ALL MEASUREMENTS BY MASCO $(8 / 7 / 88)$ EXCEPT FLUID DEPTH BY SWEC $(7 / 13 / 88)$. 
SITE RECONNAISSANCE FORM

\begin{tabular}{|c|c|c|c|}
\hline CLIENT & DOE / SRPO & J.O. No. 17500.25 & DATE $07 / 14 / 88$ \\
\hline $\begin{array}{l}\text { STATE } \\
\text { MISSISSIPPI } \\
\end{array}$ & $\begin{array}{c}\text { WELL NAME \& NUMBER } \\
\text { ROWELL MH-4A }\end{array}$ & \multicolumn{2}{|c|}{$\begin{array}{l}\text { TFSC FIELD REPRESENTATIVE } \\
\text { T.REGAN, D. LUTZ }\end{array}$} \\
\hline \multicolumn{4}{|c|}{ DESCRIPTION OF SITE ACCESS } \\
\hline \multicolumn{4}{|c|}{ BARN. DIRT ROAD UP HILL IS RUTTED BUT PASSABLE. } \\
\hline \multicolumn{4}{|c|}{$\begin{array}{l}\text { DESCRIPTION OF SITE PAD AREA } \\
\text { FLAT, GRASSY OPEN PASTURE. }\end{array}$} \\
\hline \multicolumn{4}{|c|}{ DESCRIPTION OF WELL HEAD } \\
\hline \multicolumn{4}{|c|}{ PRESSURIZED WEIL HEAD CAPPING 6-IN (ID) STEEL INNER CASING EXTENDS 2.2 FT ABOVE } \\
\hline \multicolumn{4}{|c|}{ GROUND. TWO CONTROL VALVES ON WELL HEAD. PRESSURE $=9.6$ PSI. } \\
\hline \multicolumn{4}{|c|}{ 10-IN (ID) SURFACE CASING AND 15.5-IN (ID) CONDUCTOR, STICK-UP NOT MEASURED. } \\
\hline
\end{tabular}

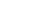

DEPTH AND BOREHOLE - FLUID SURVEY:BY MASCO $(8 / 15 / 88)$

GROUND ELEVATION 183.3 FT (DATUM)

REPORTED DEPTH 2524.6 FT (SEE BOREHOLE COMPLETION SKETCH)

MEASURED DEPTH 2490.0 FT OBSTRUCTION

FLUID DEPTH PRESSURIZED FT $\quad \mathrm{pH} \lcm{8.80}$

CONDUCTIVITY $38.52 \mathrm{mS} / \mathrm{cm} \quad$ TDS $19.260 \mathrm{mg} / \mathrm{L}$

TEMPERATURE $\quad 25.9-{ }^{\circ} \mathrm{C}$

REMARKS

NO ACCESS WORK REQUIRED.

** 34.6 FT SILT AND PIPE SCALE IN BOTTOM WELL. 
SITE RECONNAISSANCE FORM

LCATION

$1918^{\prime}$ SNL \& 1467' WEL* IN

SEC. 3-T3N-R1OW - - PERRY COUNTY

\begin{tabular}{|c|c|c|c|}
\hline CLIENT & DOE / SRPO & J.O. No. 17500.25 & DATE 07 \\
\hline $\begin{array}{l}\text { STATE } \\
\text { MISSISSIPPI }\end{array}$ & $\begin{array}{l}\text { WELL NAME \& NUMBER } \\
\text { ROWELL MH-4B }\end{array}$ & $\begin{array}{r}\text { TFSC FIELD REPRES } \\
\text { T. RI }\end{array}$ & LUTZ \\
\hline
\end{tabular}

DESCRIPTION OF SITE ACCESS

PAVED ROAD TO ROWELL PROPERTY. SITE IS ON TOP OF TERRACED HILL NORTHEAST OF BARN. DIRT ROAD UP HILL IS RUTTED BUT PASSABLE.

DESCRIPTION OF SITE PAD AREA

FLAT, GRASSY OPEN PASTURE.

DESCRIPTION OF WELL HEAD

WELL HEAD CAPPING 6-IN (ID) STEEL INNER CASING EXTENDS 3.1 FT ABOVE GROUND.

WELL HEAD CONSISTS OF 2-FT FITTING WITH 2 ACCESS PORTS, BOLTED ONTO ORIGINAL

FLANGE. STICK-UP OF 10-IN ID SURFACE CASING AND 15-IN ID CONDUCTOR NOT MEASURED

DESCRIPTION OF PITS

$\underline{N A}$

DEPTH AND BOREHOLE - FLUID SURVEY:BY MASCO $(8 / 6 / 88)$

GROUND ELEVATION 183.4 FT (DATUM)

REPORTED DEPTH 1971.4 FT (SEE BOREHOLE COMPLETION SKETCH)

MEASURED DEPTH 1918.0 FT OBSTRUCTION

FLUID DEPTH GROUND FT $\quad \mathrm{PH} \longrightarrow 9.28$

CONDUCTIVITY $41.4 \mathrm{~ms} / \mathrm{cm} \quad$ TDS $20.720 \quad \mathrm{mg} / \mathrm{L}$

TEMPERATURE $23.9-{ }^{\circ} \mathrm{C}$

REMARKS

WATER DEPTH $=$ GROUND LEVEL.

** 53.4 FT SILT AND PIPE SCALE AT BOTTOM OF WELL. 
SITE RECONNAISSANCE FORM

\begin{tabular}{|c|c|c|c|}
\hline CLIENT & DOE / SRPO & J.O. No. 17500.25 & $\begin{array}{ll}\text { DATE } & 07 / 14 / 88\end{array}$ \\
\hline $\begin{array}{l}\text { STATE } \\
\text { MISSISSIPPI }\end{array}$ & $\begin{array}{l}\text { WELL NAME \& NUMBER } \\
\text { ROWELL MH-4C }\end{array}$ & \multicolumn{2}{|c|}{$\begin{array}{l}\text { TFSC FIELD REPRESENTATIVE } \\
\text { T. REGAN, D. LUTZ }\end{array}$} \\
\hline \multicolumn{4}{|c|}{ DESCRIPTION OF SITE ACCESS } \\
\hline \multicolumn{4}{|c|}{ BARN. DIRT ROAD UP HILL IS RUTTED BUT PASSABLE. } \\
\hline \multicolumn{4}{|c|}{$\begin{array}{l}\text { DESCRIPTION OF SITE PAD AREA } \\
\text { FLAT, GRASSY OPEN PASTURE. }\end{array}$} \\
\hline \multicolumn{4}{|c|}{ DESCRIPTION OF WELL HEAD } \\
\hline \multicolumn{4}{|c|}{ PRESSURIZED WELL HEAD CAPPING 6-IN (ID) STEEL INNER CASING EXTENDS 4.1 FT ABOVE } \\
\hline \multicolumn{4}{|c|}{ GROUND. WELL HEAD HAS TWO CONTROL VALVES. PRESSURE $=16.3$ PSI } \\
\hline \multicolumn{4}{|c|}{ STICK-UP OF 15.5 -IN (ID) CONDUCTOR PIPE NOT MEASURED. } \\
\hline \multicolumn{4}{|c|}{$\begin{array}{l}\text { DESCRIPTION OF PITS } \\
\text { NA }\end{array}$} \\
\hline
\end{tabular}

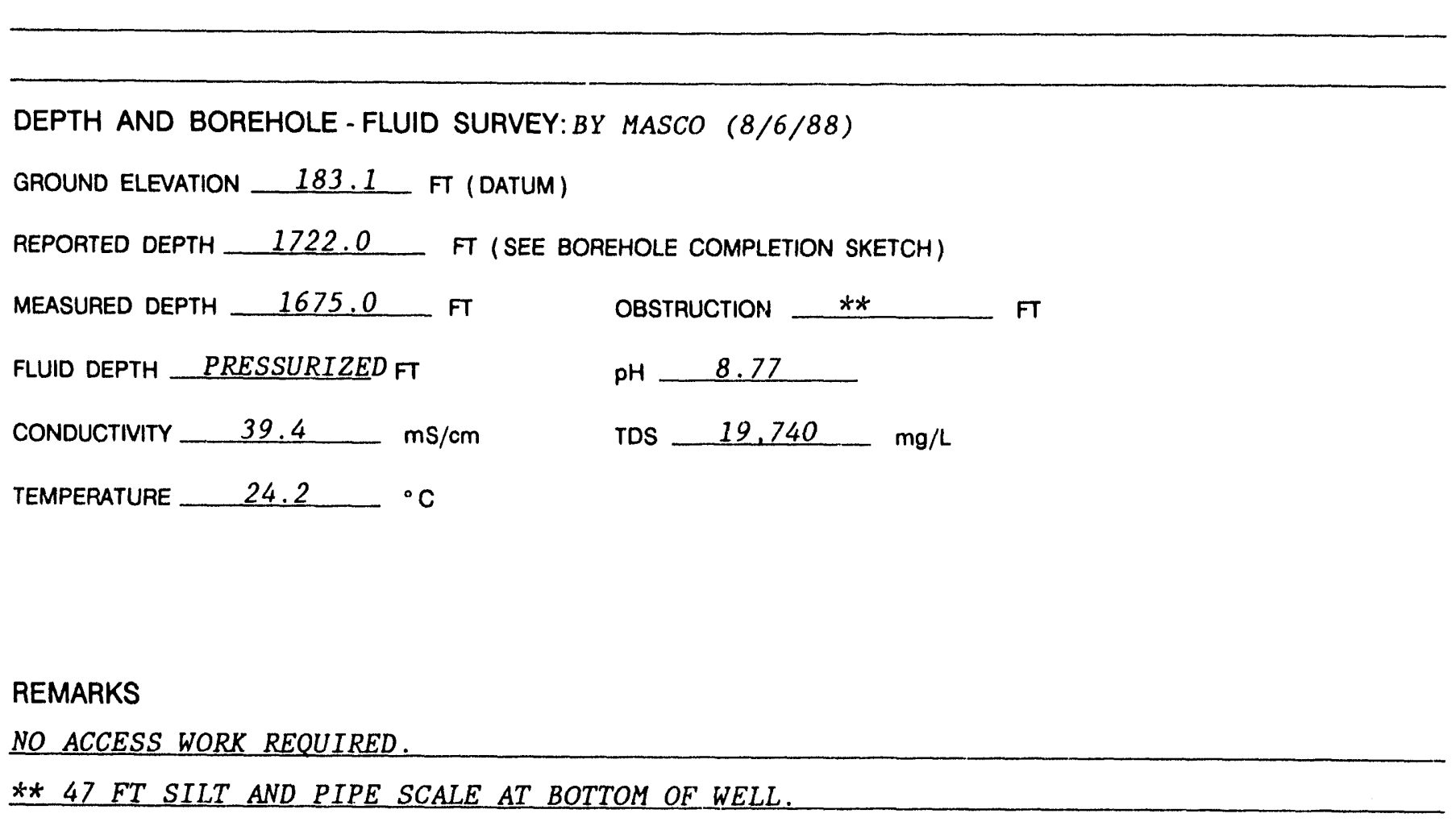




\begin{tabular}{|c|c|c|c|}
\hline & & SEC. 3-13N- & COUNTY \\
\hline CLIENT & DOE / SRPO & J.O. No. 17500.25 & $\begin{array}{ll}\text { DATE } & 07 / 14 / 88\end{array}$ \\
\hline $\begin{array}{l}\text { STATE } \\
\text { MISSISSIPPI }\end{array}$ & $\begin{array}{l}\text { WELL NAME \& NUMBER } \\
\text { ROWELL MH-4D }\end{array}$ & $\begin{array}{r}\text { TFSC FIELD REPRESE } \\
\text { T. RE }\end{array}$ & LUTZ \\
\hline $\begin{array}{l}\text { DESCRIPTION OF } \\
\text { PAVED ROAD TO }\end{array}$ & $\begin{array}{l}\text { TE ACCESS } \\
\text { VELL PROPERTY. SITE IS }\end{array}$ & OF TERRACED HILI & EAST OF \\
\hline BARN. DIRT RO & UP HILL IS RUTTED BUT & & \\
\hline NO ACCESS WOR & JIRED. & & \\
\hline $\begin{array}{l}\text { DESCRIPTION OF } \\
\text { FLAT, GRASSY O }\end{array}$ & $\begin{array}{l}\text { E PAD AREA } \\
\text { PASTURE. }\end{array}$ & & \\
\hline
\end{tabular}

\section{DESCRIPTION OF WELL HEAD}

8-IN (ID) STEEL INNER CASING WITH LOCKING, HINGED CAP EXTENDS 1.1' ABOVE GROUND. STICK-UP OF 15.5 -IN (ID) CONDUCTOR NOT MEASURED.

\section{DESCRIPTION OF PITS}

NA

DEPTH AND BOREHOLE - FLUID SURVEY: BY MASCO $(8 / 6 / 88)$

GROUND ELEVATION 183.1 FT (DATUM)

REPORTED DEPTH 1115.0 FT (SEE BOREHOLE COMPLETION SKETCH)

MEASURED DEPTH 1065.0 FT OBSTRUCTION

FLUID DEPTH 40.0 FT $\quad \mathrm{pH}-9.19$

CONDUCTIVITY $14.14 \mathrm{~ms} / \mathrm{cm}$ TDS $7.070 \mathrm{mg} / \mathrm{L}$

TEMPERATURE $23.8 \quad{ }^{\circ} \mathrm{C}$

\section{REMARKS}

TOP 2 JOINTS OF INNER CASING ARE 8-IN (ID): REMAINING JOINTS ARE 6-IN (ID).

* 50 FT SILT AND SCALE AT BOTTOM OF WELL. 
SITE RECONNAISSANCE FORM

\begin{tabular}{|c|c|c|c|}
\hline \multicolumn{2}{|c|}{ SITE RECONNAISSANCE FORM } & \multicolumn{2}{|c|}{$\begin{array}{l}\text { LOCATION } \\
5000^{\prime} \text { S46H OF MH-40 IN } \\
\text { SEC. 3-T3N-R1OH - PERY COUNTY }\end{array}$} \\
\hline CLIENT & DOE / SRPO & J.O. No. 17500.25 & DATE $\quad 07 / 14 / 88$ \\
\hline $\begin{array}{l}\text { STATE } \\
\text { MISSISSIPPI }\end{array}$ & $\begin{array}{l}\text { WELL NAME \& NUMBER } \\
\text { ROWELL MH-4WS }\end{array}$ & $\begin{array}{r}\text { TFSC FIELD REPRESE } \\
\text { T. RE }\end{array}$ & D. LUTZ \\
\hline \multicolumn{3}{|c|}{ DESCRIPTION OF SITE ACCESS } & THAN \\
\hline \multicolumn{4}{|c|}{0.1 MILE TO WELL BEHIND BARN. } \\
\hline $\begin{array}{l}\text { DESCRIPTION OF } \\
\text { WELL IS IN GRA }\end{array}$ & $\begin{array}{l}\text { TE PAD AREA } \\
\text { CLEARING AT BOTTOM OF }\end{array}$ & TWEEN BARN AND & LER SHED. \\
\hline \multicolumn{4}{|c|}{ DESCRIPTION OF WELL HEAD } \\
\hline \multicolumn{4}{|c|}{ TO AN ELBOW 0.7 FT ABOVE THE TOP OF CASING. THEN CONNECTING TO 2 STORAGE TANKS 5} \\
\hline \multicolumn{4}{|c|}{ FT FROM WELL. BROKEN PUMP AND 60 FT OF PIPE WERE REMOVED FROM WELL ON $8 / 06 / 88$. } \\
\hline \multicolumn{4}{|c|}{ DESCRIPTION OF PITS } \\
\hline
\end{tabular}

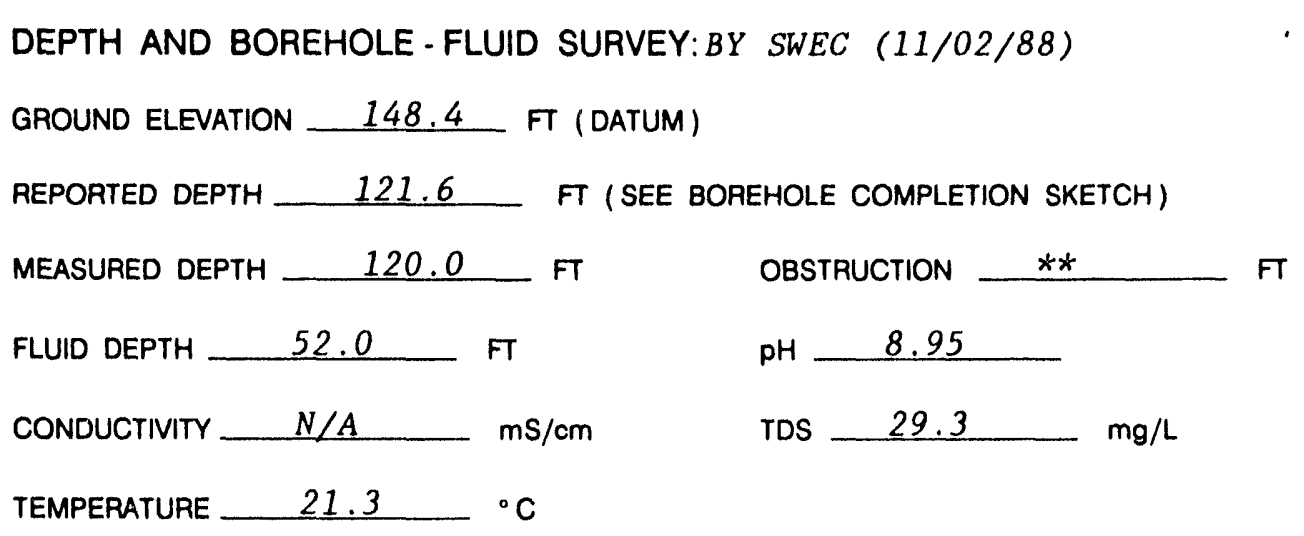

REMARKS

** $1.6 \mathrm{FT}$ OF SILT AND PIPE SCALE AT BOTTOM OF WELL. 
SITE RECONNAISSANCE FORM

\begin{tabular}{|c|c|c|c|}
\hline CLIENT & DOE / SRPO & $\begin{array}{l}\text { J.O. No. } 17500.25 \\
\end{array}$ & DATE $07 / 14 / 88$ \\
\hline $\begin{array}{l}\text { STATE } \\
\text { MISSISSIPPI }\end{array}$ & \begin{tabular}{|l} 
WELL NAME \& NUMBER \\
U.S.F.S. MH-5A
\end{tabular} & \multicolumn{2}{|c|}{$\begin{array}{l}\text { TFSC FIELD REPRESENTATIVE } \\
\text { T. REGAN, D. LUTZ }\end{array}$} \\
\hline
\end{tabular}

DESCRIPTION OF SITE PAD AREA

GRASSY FIELD WITH NUMEROUS 5 TO 15-FT HIGH PINE TREES

\section{DESCRIPTION OF WELL HEAD}

8-IN (ID) STEEL INNER CASING WITH LOCKING. HINGED CAP TO 1.7 FT ABOVE GROUND.

STICK-UP OF 10-IN (ID) SURFACE CASING AND 15.5-IN (ID) CONDUCTOR WERE NOT

MEASURED.

DESCRIPTION OF PITS

NA

DEPTH AND BOREHOLE - FLUID SURVEY:BY MASCO $(8 / 8 / 88)$

GROUND ELEVATION 315.8 FT (DATUM)

REPORTED DEPTH 3589.3 FT (SEE BOREHOLE COMPLETION SKETCH)

MEASURED DEPTH 3535.0 FT OBSTRUCTION

FLUID DEPTH $130.0 \quad$ FT $\quad \mathrm{pH}-7.07$

CONDUCTIVITY $122-\mathrm{mS} / \mathrm{cm}$ TDS $61.500-\mathrm{mg} / \mathrm{L}$

TEMPERATURE $24.6 \quad{ }^{\circ} \mathrm{C}$

\section{REMARKS}

TOP 2 JOINTS OF INNER CASING ARE 8-IN (ID): REMAINING JOINTS ARE 6-IN (ID).

** 54.3 FT OF SILT AND PIPE SCALE AT BOTTOM OF WELL. 


\section{SITE RECONNAISSANCE FORM}

\begin{tabular}{|c|c|c|c|}
\hline \multicolumn{2}{|c|}{ SIIE RECONNAISSANCE FORM } & \multicolumn{2}{|c|}{ 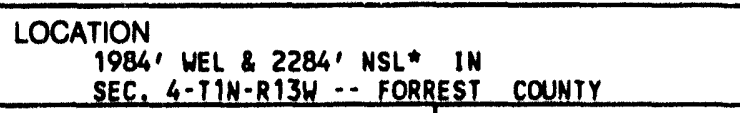 } \\
\hline CLIENT & DOE / SRPO & J.O. No. 17500.25 & DATE $07 / 14 / 88$ \\
\hline $\begin{array}{l}\text { STATE } \\
\text { MISSISSIPPI }\end{array}$ & $\begin{array}{l}\text { WELL NAME \& NUMBER } \\
\text { U.S.F.S. } \quad \text { MH-5B }\end{array}$ & $\begin{array}{r}\text { TFSC FIELD REPRESEN } \\
\text { T. REC }\end{array}$ & D. LUTZ \\
\hline \multicolumn{4}{|c|}{ DESCRIPTION OF SITE ACCESS } \\
\hline \multicolumn{4}{|c|}{ TO CLEARING IN DENSE WOODS. RIG ACCESS TO WELL HEAD REOUIRES TREE REMOVAL. } \\
\hline
\end{tabular}

\section{DESCRIPTION OF WELL HEAD}

8-IN (ID) STEEL INNER CASING WITH LOCKING HINGED CAP TO 1.1 FT ABOVE GROUND. STICK-UP OF 10-IN (ID) SURFACE CASING AND 15.5-IN (ID) CONDUCTOR WERE NOT MEASURED.

DESCRIPTION OF PITS

NA

DEPTH AND BOREHOLE - FLUID SURVEY:BY MASCO (8/8/88)

GROUND ELEVATION 315.8 FT (DATUM)

REPORTED DEPTH 2526.0 FT (SEE BOREHOLE COMPLETION SKETCH)

MEASURED DEPTH 2520.0 FT OBSTRUCTION

FLUID DEPTH $120.0 \quad \mathrm{FT} \quad \mathrm{pH}-\mathbf{8 . 8 5}$

CONDUCTIVITY $67.8 \mathrm{~ms} / \mathrm{cm}$ TDS $33.900 \mathrm{mg} / \mathrm{L}$

TEMPERATURE $-24.6 \quad{ }^{\circ} \mathrm{C}$

\section{REMARKS}

TOP 2 JOINTS OF INNER CASING ARE 8-IN (ID): REMAINING JOINTS ARE 6-IN (ID).

** 6 FT OF SILT AND PIPE SCALE AT BOTTOM OF WELL. 


\begin{tabular}{|c|c|c|c|}
\hline CLIENT & DOE / SRPO & J.O. No. 17500.25 & DATE $07 / 14 / 88$ \\
\hline $\begin{array}{l}\text { STATE } \\
\text { MISSISSIPPI }\end{array}$ & $\begin{array}{l}\text { WELL NAME \& NUMBER } \\
\text { U.S.F.S. MH-5C }\end{array}$ & \multicolumn{2}{|c|}{$\begin{array}{l}\text { TFSC FIELD REPRESENTATIVE } \\
\text { T. REGAN, D. LUTZ }\end{array}$} \\
\hline \multicolumn{4}{|c|}{ ACCESS BY PAVED ROAD FROM ROCK HILL, THEN 0.1 MILE EAST ON GOOD GRAVEL ROAD TO } \\
\hline \multicolumn{4}{|c|}{ CLEARING IN DENSE WOODS. RIG ACCESS TO WELL HEAD REQUIRES TREE REMOVAL. } \\
\hline \multicolumn{4}{|c|}{ DESCRIPTION OF SITE PAD AREA } \\
\hline \multicolumn{4}{|c|}{ FLAT, GRASSY CLEARING WITH LOW BRUSH AND NUMEROUS 10 TO 15-FT HIGH PINE TREES. } \\
\hline \multicolumn{4}{|c|}{ DESCRIPTION OF WELL HEAD } \\
\hline \multicolumn{4}{|c|}{ 8-IN (ID) STEEL INNER CASING WITH LOCKING, HINGED CAP TO 1.3 FT ABOVE GROUND. } \\
\hline \multicolumn{4}{|c|}{ ONE HINGE OF CAP IS RUSTED THROUGH. STICK -UP OF 10-IN (ID) SURFACE CASING AND } \\
\hline \multicolumn{4}{|c|}{ 15.5-IN (ID) CONDUCTOR PIPE WERE NOT MEASURED. } \\
\hline \multicolumn{4}{|c|}{ DESCRIPTION OF PITS } \\
\hline
\end{tabular}

\footnotetext{
DEPTH AND BOREHOLE - FLUID SURVEY: BY MASCO (8/8/88)

GROUND ELEVATION 315.7 FT (DATUM)

REPORTED DEPTH 2287.0 FT (SEE BOREHOLE COMPLETION SKETCH)

\section{REMARKS}

TOP 2 INNER CASING JOINTS ARE 8-IN (ID): REMAINING JOINTS ARE 6-IN (ID). ** 65 FT OF SILT AND PIPE SCALE AT BOTTOM OF WELL. 
SITE RECONNAISSANCE FORM

CLIENT

DOE / SRPO

LOCATION

1984' WEL \& $2119^{\prime}$ NSL* IN

SEC. 4 -T1N-R13W -. FORREST COUNTY

-

STATE

WELL NAME \& NUMBER

MISSISSIPPI

U.S.F.S. MH-5D

DATE

$07 / 14 / 88$

TFSC FIELD REPRESENTATIVE

T. REGAN, D. LUTZ

\section{DESCRIPTION OF SITE ACCESS}

ACCESS BY PAVED ROAD FROM ROCK HILL, THEN 0.1 MILE EAST ON GOOD GRAVEL ROAD TO

CLEARING IN DENSE WOODS. RIG ACCESS TO WELL HEAD WILL REQUIRE TREE REMOVAL.

DESCRIPTION OF SITE PAD AREA

FLAT GRASSY AREA WITH LOW BRUSH AND NUMEROUS 10 TO 15 -FT HIGH PINE TREES.

DESCRIPTION OF WELL HEAD

8-IN (ID) STEEL INNER CASING WITH LOCKING, HINGED CAP TO 1.5 FT ABOVE GROUND.

STICK-UP OF 15.5-IN (ID) CONDUCTOR PIPE WAS NOT MEASURED.

\section{DESCRIPTION OF PITS}

$\underline{N A}$

DEPTH AND BOREHOLE - FLUID SURVEY:BY MASCO (8/8/88)

GROUND ELEVATION 316.1 FT (DATUM)

REPORTEO OEPTH 1540.0 FT (SEE BOREHOLE COMPLETION SKETCH)

MEASURED DEPTH 1539.0 FT OBSTRUCTION

FLUID DEPTH $200.0 \quad$ FT $\quad \mathrm{pH}-9.46$

CONDUCTIVITY $0.99 \quad \mathrm{mS} / \mathrm{cm}$ TDS $494 \mathrm{mg} / \mathrm{L}$

TEMPERATURE $24.4 \quad{ }^{\circ} \mathrm{C}$

\section{REMARKS}

TOP 2 INNER CASING JOINTS ARE 8-IN (ID): REMAINING JOINTS ARE 6-IN (ID).

** 1 FT OF SILT AND PIPE SCALE AT BOTTOM OF WELL. 


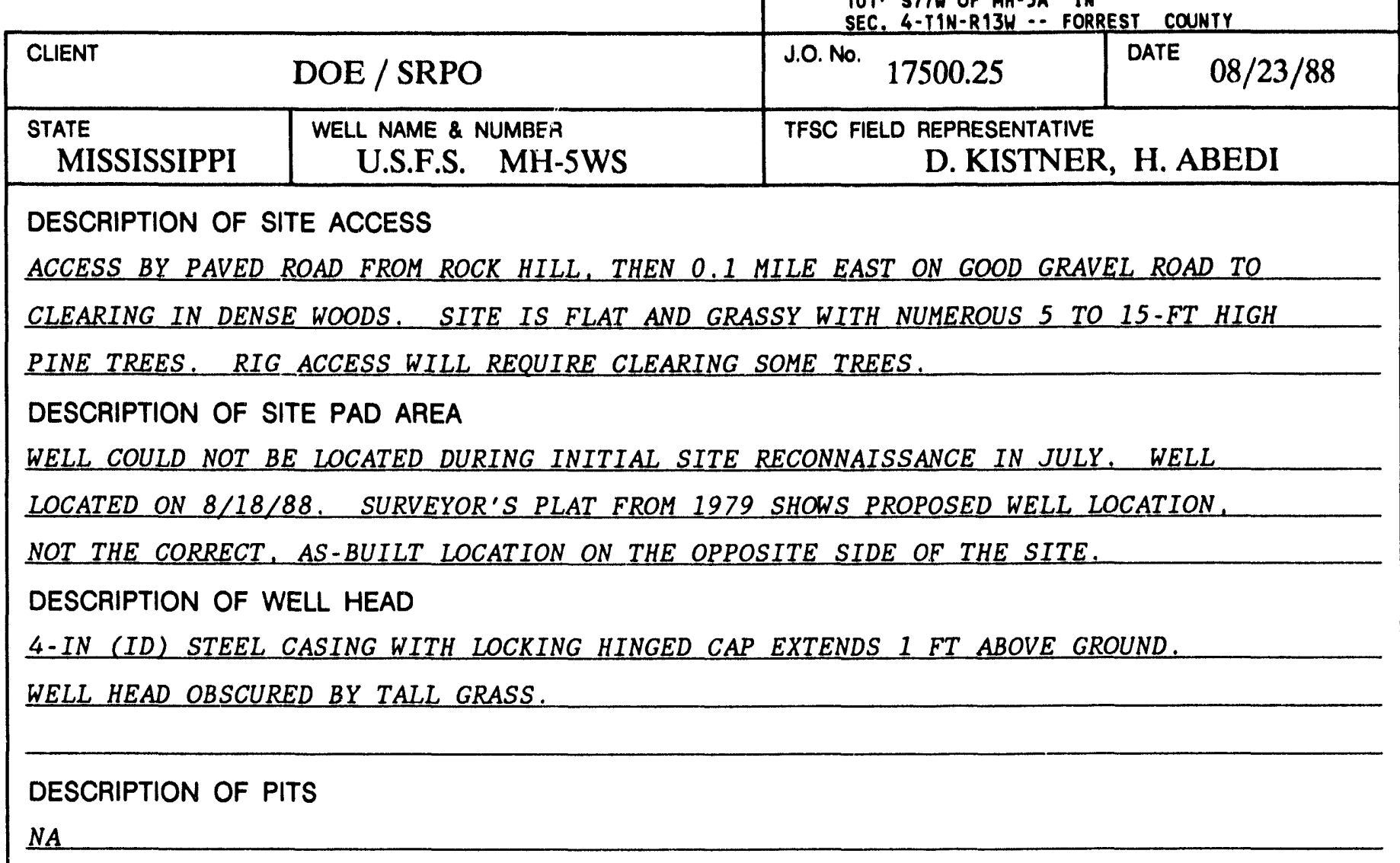

DEPTH AND BOREHOLE - FLUID SURVEY:BY SWEC (8/23/88)

GROUND ELEVATION 315.3 FT (DATUM)

REPORTED DEPTH 18.5 .0 FT (SEE BOREHOLE COMPLETION SKETCH)

MEASURED DEPTH 185.0 FT OBSTRUCTION N/A FT

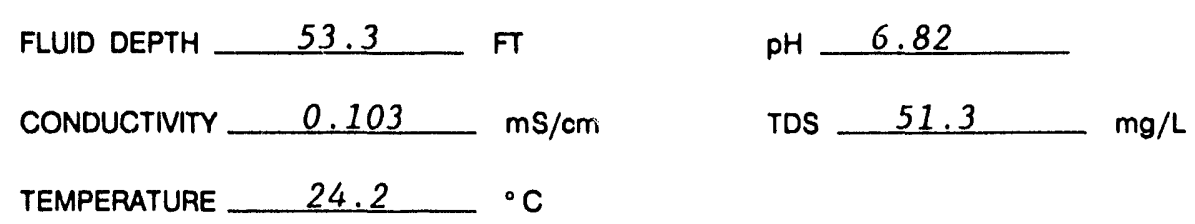

\section{REMARKS}

ACTUAL WELL LOCATION IS 101 FT STTW OF MH-5A.

OPEN HOLE BETWEEN BOTTOM OF WELL (185 FT) AND BOTTOM OF BOREHOLE (404 FT). 
SITE RECONNAISSANCE FORM

\begin{tabular}{|c|c|c|c|}
\hline \multicolumn{2}{|c|}{ SIIC MECUIVINAISSAIVUE FUMIVI } & \multicolumn{2}{|c|}{$\begin{array}{l}\text { LOCATION } \\
790^{\prime} \text { SNL \& } 21277^{\prime} \text { EWL" IN } \\
\text { SEC. 4-T5N-RITH - MARION COUNTY }\end{array}$} \\
\hline CLIENT & DOE / SRPO & J.O. No. 17500.25 & DATE $07 / 15 / 88$ \\
\hline $\begin{array}{l}\text { STATE } \\
\text { MISSISSIPPI }\end{array}$ & $\begin{array}{l}\text { WELL NAME \& NUMBER } \\
\text { ANDERSON MH-6A }\end{array}$ & \multicolumn{2}{|c|}{$\begin{array}{l}\text { TFSC FIELD REPRESENTATIVE } \\
\text { D. LUTZ, H. ABEDI }\end{array}$} \\
\hline \multicolumn{4}{|c|}{$\begin{array}{l}\text { DESCRIPTION OF SITE ACCESS } \\
\text { FROM CARTERVILLE ROAD, ACCESS THROUGH WOODEN HITCH GATE THEN } 0.1 \text { MILE NE ON DIRT }\end{array}$} \\
\hline \multicolumn{4}{|c|}{ ROAD THROUGH HEAVY UNDERBRUSH. ROAD WORK AND TREE REMOVAL REQUIRED FOR SITE } \\
\hline \multicolumn{4}{|c|}{ DESCRIPTION OF SITE PAD AREA } \\
\hline \multicolumn{4}{|c|}{ DESCRIPTION OF WELL HEAD } \\
\hline \multicolumn{4}{|c|}{ 8-IN (ID) STEEL INNER CASING WITH LOCKING HINGED CAP TO 1.5 FT ABOVE GROUND. } \\
\hline \multicolumn{4}{|c|}{ 10-IN (ID) SURFACE CASING TO 0.6 FT ABOVE GROUND. $15.6-$ IN (ID) CONDUCTOR PIPE } \\
\hline \multicolumn{4}{|c|}{ TO $0.3 \mathrm{FT}$ ABOVE GROUND. } \\
\hline \multicolumn{4}{|c|}{ DESCRIPTION OF' PITS } \\
\hline
\end{tabular}

DEPTH AND BOREHOLE - FLUID SURVEY:BY MASCO (8/8/88)

GROUND ELEVATION $\quad 444.6$ FT (DATUM)

REPORTED DEPTH $3293.0 \quad$ FT (SEE BOREHOLE COMPLETION SKETCH)

MEASURED DEPTH 3293.0 FT OBSTRUCTION N/A FT

FLUID DEPTH $180.0 \quad$ FT $\quad \mathrm{PH}-8.88$

CONDUCTIVITY $\frac{15.28}{28} \mathrm{~ms} / \mathrm{cm} \mathrm{mg} / \mathrm{L}$

TEMPERATURE $24.3 \quad{ }^{\circ} \mathrm{C}$

REMARKS

TOP 2 INNER CASING JOINTS ARE 8-IN (ID): REMAINING JOINTS ARE 6-IN (ID). 
SITE RECONNAISSANCE FORM

\begin{tabular}{|l|l|l}
\hline \multicolumn{2}{|l}{ LOCATION } \\
786' SNL \& $1980^{\prime}$ EWL* IN \\
SEC. 4-T5N-R17N .- MARION COUNTY
\end{tabular}

\begin{tabular}{|l|c|}
\hline CLIENT & DOE / SRPO \\
\hline $\begin{array}{l}\text { STATE } \\
\text { MISSISSIPPI }\end{array}$ & $\begin{array}{c}\text { WELL NAME \& NUMBER } \\
\text { ANDERSON MH-6B }\end{array}$ \\
\hline
\end{tabular}

TFSC FIELD REPRESENTATIVE

D. LUTZ, H. ABEDI

DESCRIPTION OF SITE ACCESS

FROM CARTERVILLE ROAD, ACCESS THROUGH A WOODEN HITCH GATE THEN 0.1 MILE NE ON A

DIRT ROAD THROUGH HEAVY UNDERBRUSH. ROAD WORK AND TREE REMOVAL REQUIRED FOR RIG ACCESS.

DESCRIPTION OF SITE PAD AREA

GRASSY FIELD WITH LOW BRUSH, 4 TO 15-FT HIGH PINE TREES. AND 1-FT DEEP GULLIES.

DESCRIPTION OF WELL HEAD

8-IN (ID) STEEL INNER CASING WITH LOCKING HINGED CAP EXTENDS 2 FT ABOVE GROUND.

ONE HINGE IS RUSTED THROUGH. 10-IN (ID) SURFACE CASING TO 0.25 FT ABOVE GROUND.

15.5 -IN (ID) CONDUCTOR PIPE TO $0.15 \mathrm{FT}$ ABOVE GROUND.

DESCRIPTION OF PITS

NA

DEPTH AND BOREHOLE - FLUID SURVEY:BY MASCO $(8 / 8 / 88)$

GROUND ELEVATION 446.1 FT (DATUM)

REPORTED DEPTH 2762.6 FT (SEE. BOREHOLE COMPLETION SKETCH)

MEASURED DEPTH 2709.0 FT OBSTRUCTION

FLUID DEPTH $250.0 \quad$ FT $\quad \mathrm{PH} \longrightarrow 9.44$

CONDUCTIVITY $12.28 \mathrm{~ms} / \mathrm{cm} \mathrm{mg} / \mathrm{L}$

TEMPERATURE $24.3-{ }^{\circ} \mathrm{C}$

REMARKS

TOP 2 INNER CASING JOINTS ARE 8-IN (ID): REMAINING JOINTS ARE 6-IN (ID).

** 53.6 FT OF SILT AND PIPE SCALE AT BOTTOM OF WELL. 
SITE RECONNAISSANCE FORM

\begin{tabular}{|c|c|c|}
\hline \multicolumn{2}{|c|}{ SITE RECONNAISSANCE FORM } & $\begin{array}{l}\text { LOCATION } \\
788^{\prime} \text { SNL \& 2052' EWL* IN } \\
\text { SEC. 4-TSN-R17H .-. MARION COUNTY }\end{array}$ \\
\hline CLIENT & DOE / SRPO & J.O. No. 17500.25 \\
\hline $\begin{array}{l}\text { STATE } \\
\text { MISSISSIPPI }\end{array}$ & $\begin{array}{l}\text { WELL NAME \& NUMBER } \\
\text { ANDERSON MH-6C }\end{array}$ & $\begin{array}{l}\text { TFSC FIELD REPRESENTATIVE } \\
\text { D. LUTZ, H. ABEDI }\end{array}$ \\
\hline \multicolumn{3}{|c|}{ DESCRIPTION OF SITE ACCESS } \\
\hline \multicolumn{3}{|c|}{ ROAD THROUGH HEAVY UNDERBRUSH. ROAD WORK AND TREE REMOVAL REQUIRED FOR RIG } \\
\hline \multicolumn{3}{|c|}{ ACCESS. } \\
\hline \multicolumn{3}{|c|}{ GRASSY FIELD WITH LOW BRUSH, 4 TO 15-FT HIGH PINE TREES, AND 1-FT DEEP GULLIES. } \\
\hline \multicolumn{3}{|c|}{ DESCRIPTION OF WELL HEAD } \\
\hline \multicolumn{3}{|c|}{ 6-IN (ID) STEEL INNER CASING WITH LOCKING HINGED CAP TO 0.3 FT ABOVE GROUND. } \\
\hline \multicolumn{3}{|c|}{ STICK-UP OF 10 -IN (ID) SURFACE CASING AND 15.5-IN (ID) CONDUCTOR PIPE NOT } \\
\hline \multicolumn{3}{|l|}{ MEASURED. } \\
\hline \multicolumn{3}{|c|}{ DESCRIPTION OF PITS } \\
\hline
\end{tabular}




\begin{tabular}{|c|c|c|c|}
\hline CLIENT & DOE / SRPO & J.O. No. 17500.25 & DATE $07 / 15 / 88$ \\
\hline $\begin{array}{l}\text { STATE } \\
\text { MISSISSIPPI } \\
\end{array}$ & $\begin{array}{l}\text { WELL NAME \& NUMBER } \\
\text { ANDERSON MH-6D }\end{array}$ & \multicolumn{2}{|c|}{$\begin{array}{l}\text { TFSC FIELD REPRESENTATIVE } \\
\text { D. LUTZ, H. ABEDI }\end{array}$} \\
\hline \multicolumn{4}{|c|}{ FROM CARTERVILLE ROAD, ACCESS THROUGH WOODEN HITCH GATE THEN 0.1 MILE NE ON DIRT } \\
\hline \multicolumn{4}{|c|}{ ROAD THROUGH HEAVY UNDERBRUSH. ROAD WORK AND TREE REMOVAL REQUIRED FOR RIG } \\
\hline \multicolumn{4}{|l|}{ ACCESS. } \\
\hline \multicolumn{4}{|c|}{ DESCRIPTION OF SITE PAD AREA } \\
\hline \multicolumn{4}{|c|}{ (2) } \\
\hline \multicolumn{4}{|c|}{ DESCRIPTION OF WELL HEAD } \\
\hline \multicolumn{4}{|c|}{ 6-IN (ID) STEEL INNER CASING WITH LOCKING HINGED CAP TO 3.3 FT ABOVE GROUND. } \\
\hline \multicolumn{4}{|c|}{ 15.5-IN (ID) CONDUCTOR PIPE EXTENDS 2.4 FT ABOVE GROUND. A 2-FT HIGH CEMENT PAD } \\
\hline \multicolumn{4}{|c|}{ SURROUNDS THE CONDUCTOR PIPE. } \\
\hline \multicolumn{4}{|c|}{ DESCRIPTION OF PITS } \\
\hline
\end{tabular}

DEPTH AND BOREHOLE - FLUID SURVEY:BY MASCO $(8 / 8 / 88)$

GROUND ELEVATION 446.2 FT (DATUM)

REPORTED DEPTH 1400.0 FT (SEE BOREHOLE COMPLETION SKETCH)

MEASURED DEPTH 1400.0 FT OBSTRUCTION N/A FT

FLUID DEPTH 260.0 FT $\quad$ PH 9.94

CONDUCTIVITY $1.3 \mathrm{~ms} / \mathrm{cm}$ TDS $650 \mathrm{mg} / \mathrm{h}$

TEMPERATURE $24.6-{ }^{\circ} \mathrm{C}$

REMARKS 
SITE RECONNAISSANCE FORM

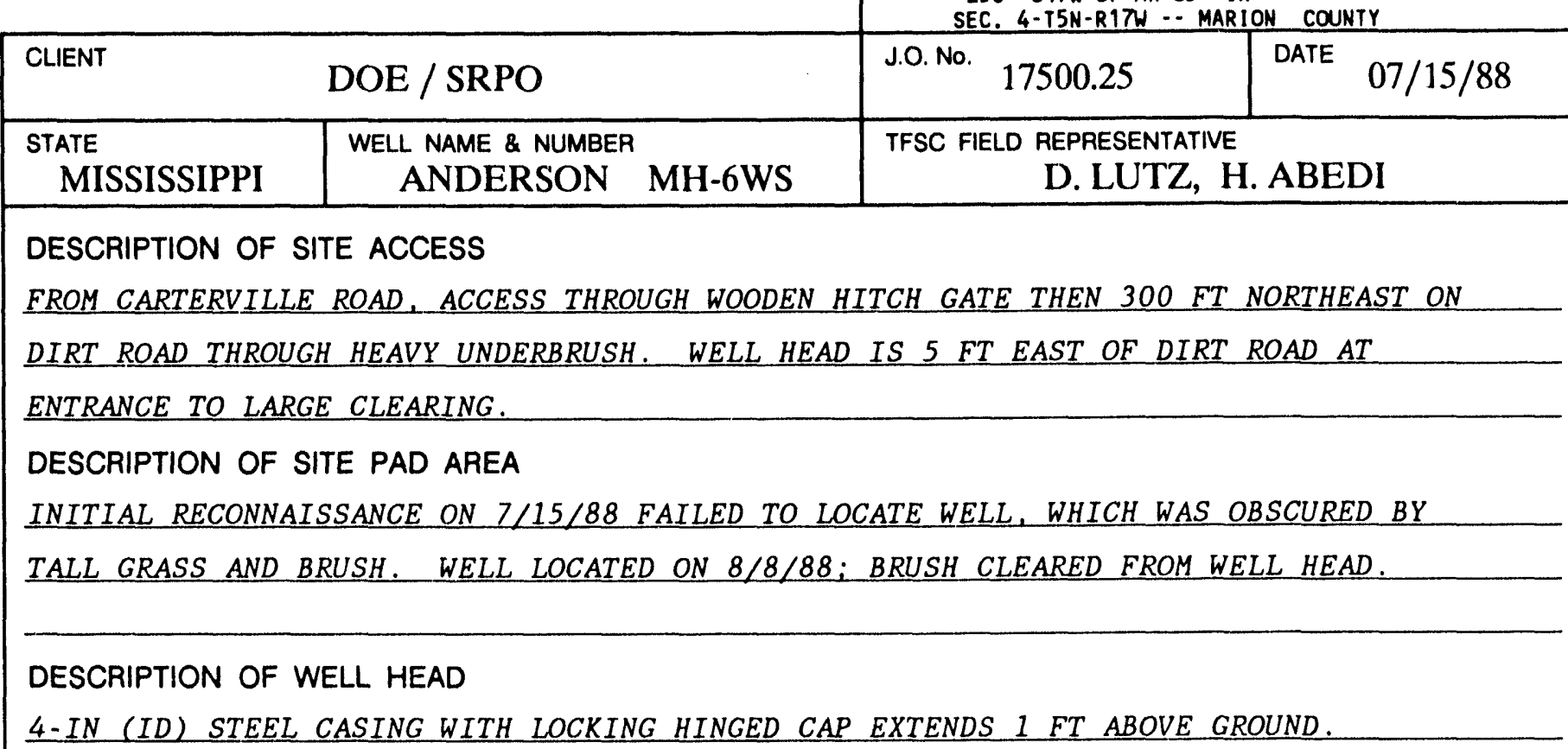

\section{DESCRIPTION OF PITS}

$\underline{N A}$

DEPTH AND BOREHOLE - FLUID SURVEY:BY SWEC (11/03/88)

GROUND ELEVATION 445.9 FT (DATUM)

REPORTED DEPTH 341.0 FT (SEE BOREHOLE COMPLETION SKETCH)

MEASURED DEPTH 341.0 FT OBSTRUCTION $-N / A \quad F T$

FLUID DEPTH $203.0 \quad \mathrm{FT} \quad \mathrm{pH} 6.63$

CONDUCTIVITY N $\mathrm{N} / \mathrm{A} \mathrm{mS} / \mathrm{cm} \quad$ TDS $39.7 \mathrm{mg} / \mathrm{L}$

TEMPERATURE $21.3{ }^{\circ} \mathrm{C}$

REMARKS 
SITE RECONNAISSANCE FORM

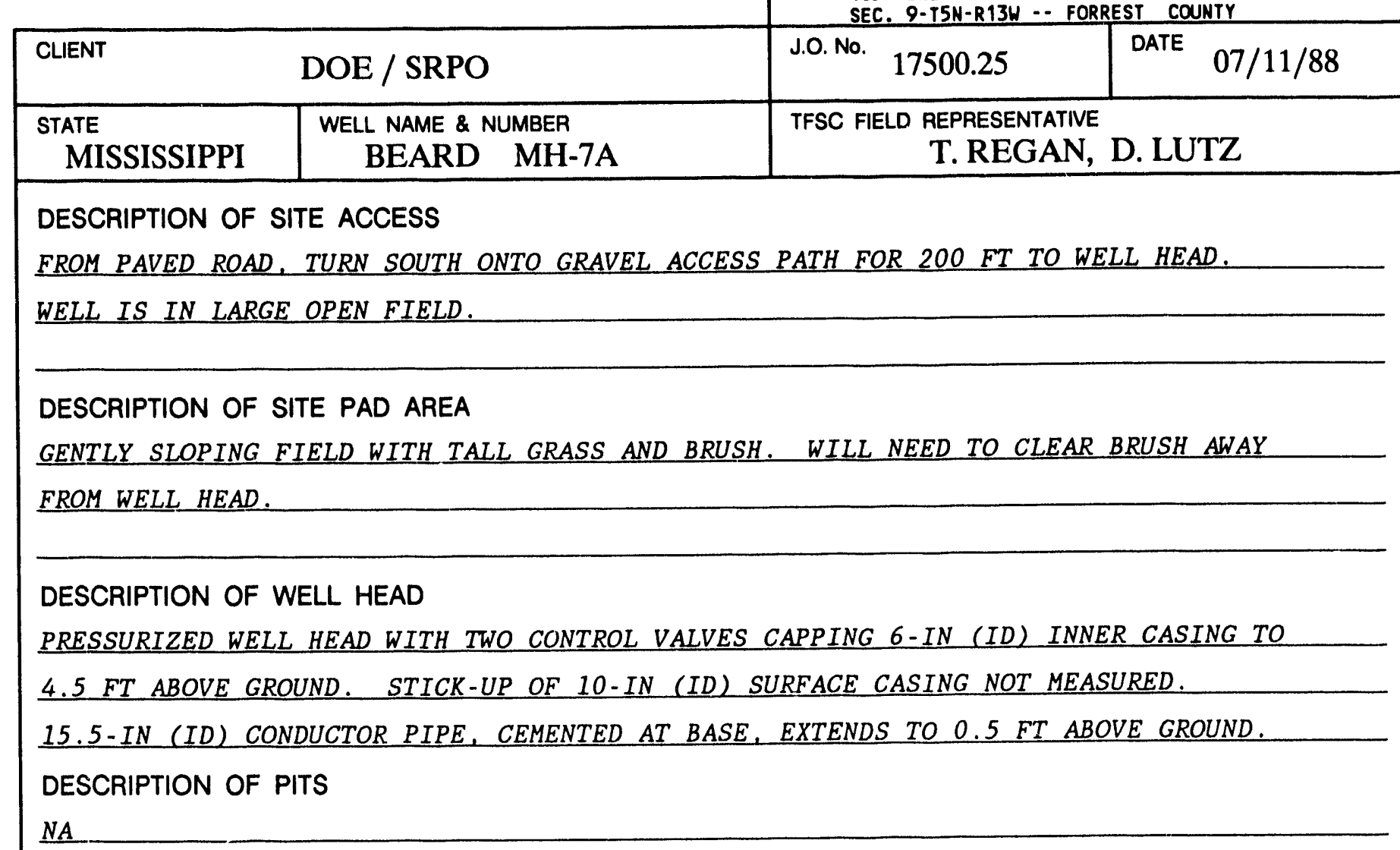

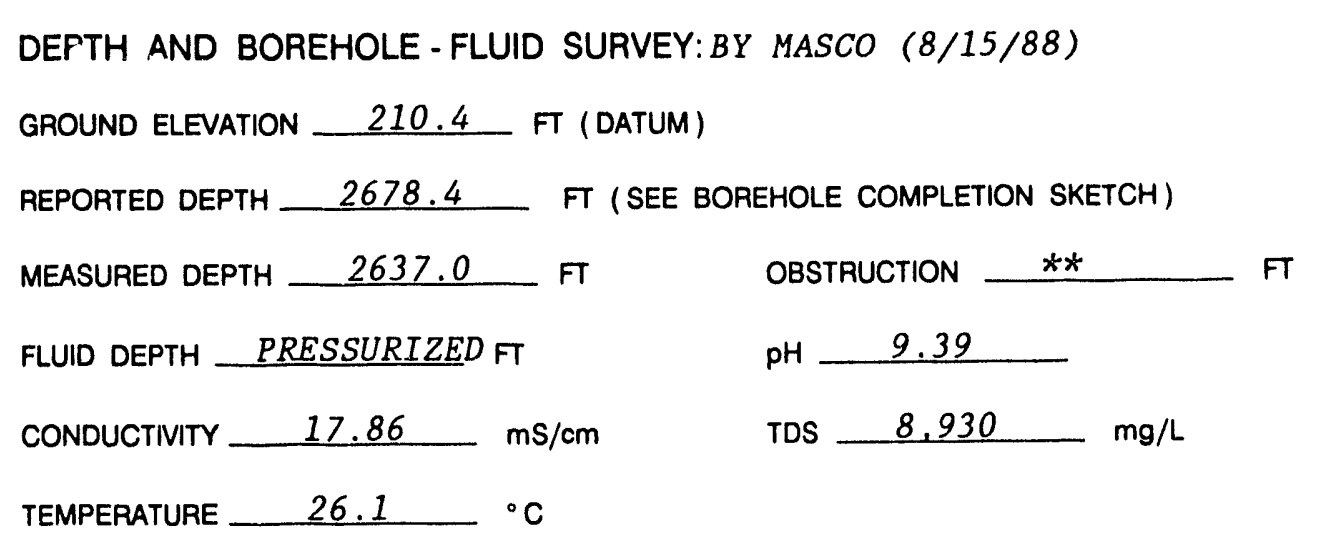

REMARKS

PRESSURE $=10$ PSI.

** 41.4 FT OF SILT AND PIPE SCALE AT BOTTOM OF WELL. 
SITE RECONNAISSANCE FORM

\begin{tabular}{|c|c|c|}
\hline \multicolumn{2}{|c|}{ SIIE RECUNINAISSANCE FOKM } & 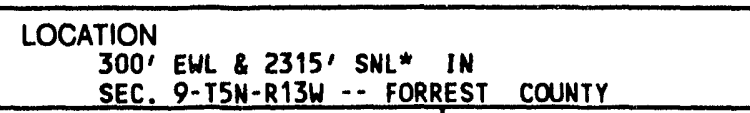 \\
\hline CLIENT & DOE / SRPO & 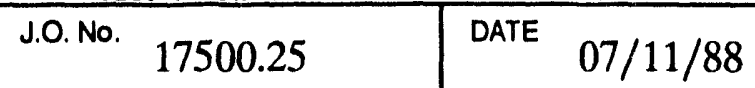 \\
\hline $\begin{array}{l}\text { STATE } \\
\text { MISSISSIPPI }\end{array}$ & $\begin{array}{l}\text { WELL NAME \& NUMBER } \\
\text { BEARD } \text { MH-7B }\end{array}$ & $\begin{array}{l}\text { TFSC FIELD REPRESENTATIVE } \\
\text { T. REGAN, D. LUTZ }\end{array}$ \\
\hline \multicolumn{3}{|c|}{$\begin{array}{l}\text { DESCRIPTION OF SITE ACCESS } \\
\text { FROM PAVED ROAD, TURN SOUTH ON GRAVEL }\end{array}$} \\
\hline \multicolumn{3}{|c|}{ SITE IS LARGE OPEN FIELD. } \\
\hline \multicolumn{3}{|c|}{$\begin{array}{l}\text { DESCRIPTION OF SITE PAD AREA } \\
\text { GENTLY SLOPING FIELD WITH BRUSH AND TALL GRASS. BRUSH NEEDS TO BE CLEARED }\end{array}$} \\
\hline \multicolumn{3}{|c|}{ AWAY FROM WELL HEAD. } \\
\hline \multicolumn{3}{|c|}{ DESCRIPTION OF WELL HEAD } \\
\hline \multicolumn{3}{|c|}{ WELL HEAD HAS TWO CONTROL VALVES AND ACCESS PORTS. STICK-UP OF 10-IN (ID) SUR- } \\
\hline \multicolumn{3}{|c|}{ FACE CASING AND 15.5 -IN (ID) CONDUCTOR PIPE NOT MEASURED. I FT OF SOIL AT BASE. } \\
\hline \multicolumn{3}{|c|}{ DESCRIPTION OF PITS } \\
\hline
\end{tabular}

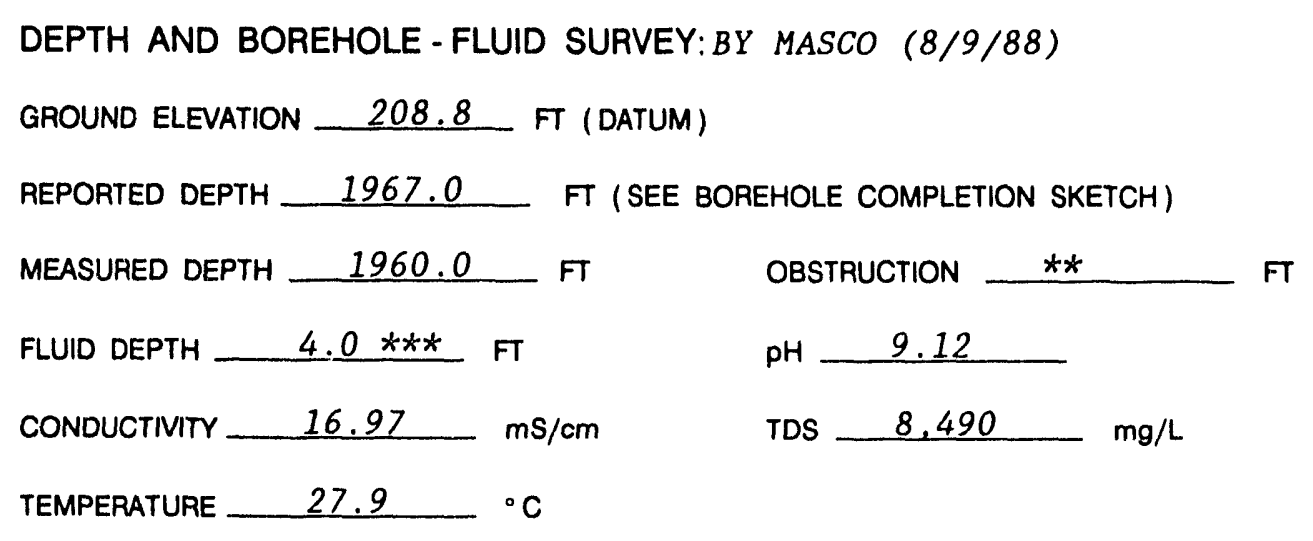

\section{REMARKS}

** 7 FT OF SILT AND PIPE SCALE AT BOTTOM OF WELL. ***DEPTH TO WATER IS 4 FT: WATER LEVEL ROSE VERY SLOWLY WHILE SOUNDING THE WELL. 
SITE RECONNAISSANCE FORM

\begin{tabular}{|l|c|}
\hline CLIENT & DOE / SRPO \\
\hline $\begin{array}{l}\text { STATE } \\
\text { MISSISSIPPI }\end{array}$ & $\begin{array}{c}\text { WELL NAME \& NUMBER } \\
\text { BEARD MH-7C }\end{array}$ \\
\hline
\end{tabular}

LOCATION

200' EWL \& 2225' SNL* IN

SEC. 9-T5N-R13W -. FORREST COUNTY

J.O. No.

17500.25

DATE

TFSC FIELD REPRESENTATIVE

T. REGAN, D. LUTZ

DESCRIPTION OF SITE ACCESS

FROM PAVED ROAD, TURN SOUTH ONTO GRAVEL ACCESS PATH FOR 200 FT TO WELL HEAD.

SITE IS LARGE OPEN FIELD.

DESCRIPTION OF SITE PAD AREA

GENTLY SLOPING FIELD WITH BRUSH AND TALL GRASS. BRUSH NEEDS TO BE CLEARED AWAY

FROM WELL HEAD.

DESCRIPTION OF WELL HEAD

PRESSURIZED WELL HEAD CAPPING 6-IN (ID) INNER CASING EXTENDS 4 FT ABOVE GROUND.

WELL, HEAD HAS TWO CONTROL VALVES AND ACCESS PORTS. STICK-UP OF 15.5-IN (ID)

CONDUCTOR PIPE NOT MEASURED. 1-FT HIGH CEMENT APRON AT BASE.

DESCRIPTION OF PITS

NA

DEPTH AND BOREHOLE - FLUID SURVEY:BY MASCO (8/9/88)

GROUND ElEVATION -210.1 FT (DATUM)

REPORTED DEPTH 1661.5 FT (SEE BOREHOLE COMPLETION SKETCH)

MEASURED DEPTH 1661.0 FT OBSTRUCTION N/A FT

FLUID DEPTH GROUND FT $\quad \mathrm{PH}-9.19$

CONDUCTIVITY $6.46 \quad \mathrm{~ms} / \mathrm{cm} \quad$ TDS $3.230 \mathrm{mg} / \mathrm{L}$

TEMPERATURE $\quad 28.0$

REMARKS

PRESSURE -8 PSI. 
SITE RECONNAISSANCE FORM

LOCATION

400' EWL \& 2390' SNL* IN

SEC. 9-T5H-R13W -. FORREST COUNTY

\begin{tabular}{|l|l|l|}
\hline \multicolumn{1}{|c|}{ DLIENT } & J.0. No. 17500.25 & DATE $07 / 11 / 88$ \\
\hline $\begin{array}{l}\text { STATE } \\
\text { MISSISSIPPI }\end{array}$ & $\begin{array}{c}\text { WELL NAME \& NUMBER } \\
\text { BEARD MH-7D }\end{array}$ & $\begin{array}{c}\text { TFSC FIELD REPRESENTATIVE } \\
\text { T. REGAN, H. ABEDI }\end{array}$ \\
\hline $\begin{array}{l}\text { DESCRIPTION OF SITE ACCESS } \\
\text { FROM PAVED ROAD, TURN SOUTH ON GRAVEL ACCESS PATH FOR 200 FT TO WELL HEAD. }\end{array}$ \\
\hline
\end{tabular}

DESCRIPTION OF SITE PAD AREA

GENTLY SLOPING FIELD WITH BRUSH AND TALL GRASS. BRUSH NEEDS TO BE CLEARED AWAY

FROM WELL HEAD.

DESCRIPTION OF WELL HEAD

6-IN (ID) INNER CASING WITH LOCKING HINGED CAP EXTENDS 3 FT ABOVE GROUND.

STICK-UP OF 15.5-IN (ID) CONDUCTOR NOT MEASURED. 2-FT HIGH CEMENT APRON AT BASE

OF CONDUCTOR.

DESCRIPTION OF PITS

$\underline{N A}$

DEPTH AND BOREHOLE - FLUID SURVEY:BY MASCO (8/7/88)

GROUND ELEVATION 207.9 FT (DATUM)

REPORTED DEPTH 805.5 FT (SEE BOREHOLE COMPLETION SKETCH)

MEASURED DEPTH 802.0 FT OBSTRUCTION

FLUID DEPTH $60.0 \quad$ FT $\quad \mathrm{pH}-9.19$

CONDUCTIVITY $0.357 \mathrm{mS} / \mathrm{cm} \quad$ TDS $178.6 \mathrm{mg} / \mathrm{L}$

TEMPERATURE $23.3 \quad{ }^{\circ} \mathrm{C}$

\section{REMARKS}

** 3.5 FT OF SILT AND PIPE SCALE AT BOTTOM OF WELL. 
SITE RECONNAISSANCE FORM

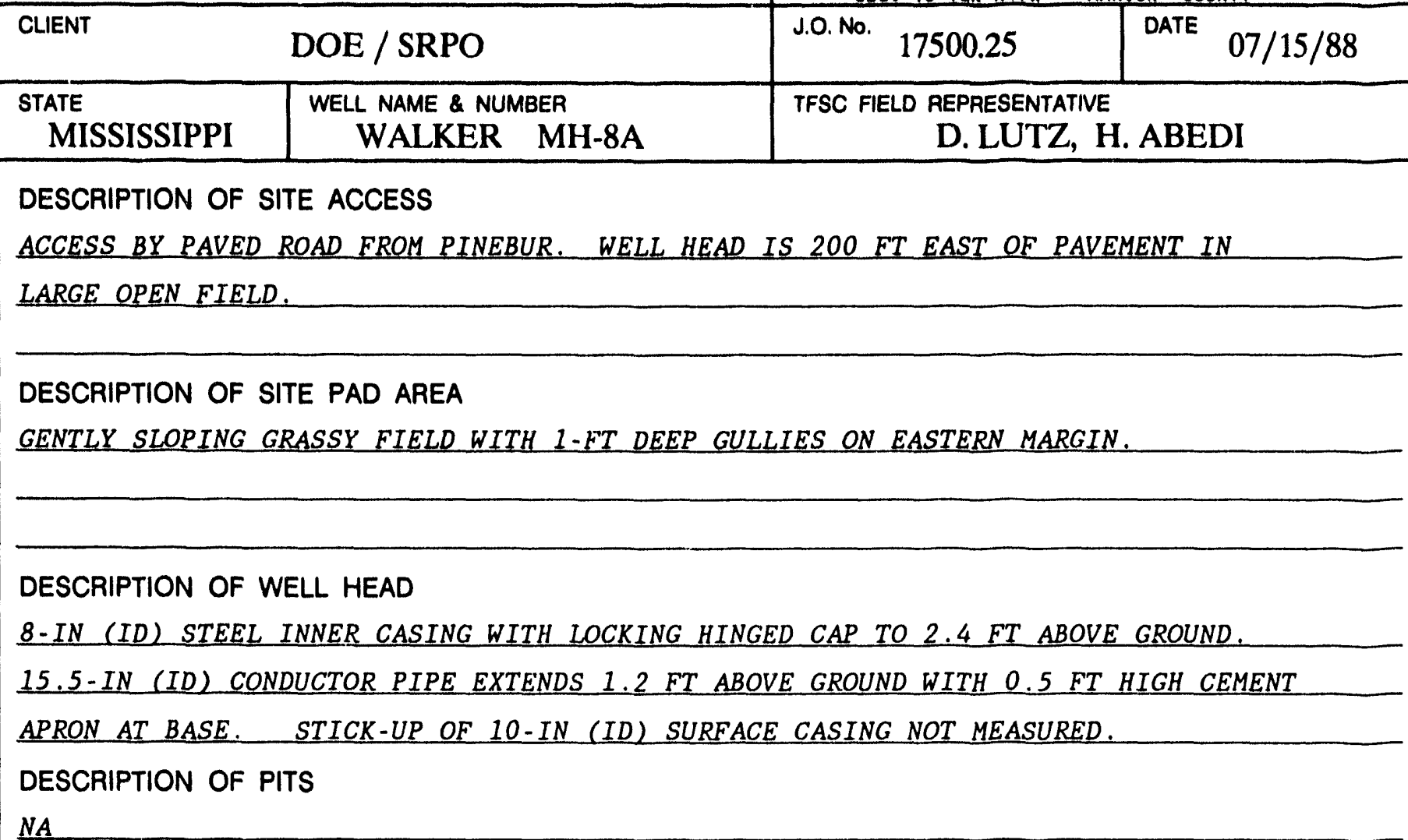

NA

DEPTH AND BOREHOLE - FLUID SURVEY:BY MASCO $(8 / 15 / 88)$

GROUND ELEVATION 244.3 FT (DATUM)

REPORTED DEPTH 3194.4 FT (SEE BOREHOLE COMPLETION SKETCH)

MEASURED DEPTH 3194.4 FT OBSTRUCTION N N

FLUID DEPTH $43.0 \quad \mathrm{FT} \quad \mathrm{pH}-8.64$

CONDUCTIVITY $70.8 \mathrm{mS} / \mathrm{cm} \quad$ TDS $35,400 \mathrm{mg} / \mathrm{L}$

TEMPERATURE $25.9 \quad{ }^{\circ} \mathrm{C}$

REMARKS

TOP 2 INNER CASING JOINTS ARE 8-IN (ID): REMAINING JOINTS ARE 6-IN (ID). 
SITE RECONNAISSANCE FORM

\begin{tabular}{|c|c|c|c|}
\hline CLIENT & DOE / SRPO & J.O. No. 17500.25 & DATE $07 / 15 / 88$ \\
\hline $\begin{array}{l}\text { STATE } \\
\text { MISSISSIPPI }\end{array}$ & $\begin{array}{l}\text { WELL NAME \& NUMBER } \\
\text { WALKER MH-8B }\end{array}$ & \multicolumn{2}{|c|}{$\begin{array}{l}\text { TFSC FIELD REPRESENTATIVE } \\
\text { D. LUTZ, H. ABEDI }\end{array}$} \\
\hline \multicolumn{4}{|c|}{$\begin{array}{l}\text { DESCRIPTION OF SITE ACCESS } \\
\text { ACCESS BY PAVED ROAD FROM PINEBUR. PAD AREA IS } 200 \text { FT EAST OF PAVEMENT IN LARGE }\end{array}$} \\
\hline
\end{tabular}

\section{DESCRIPTION OF SITE PAD AREA}

GENTLY SLOPING GRASSY FIELD WITH 1-FT DEEP GULLIES AT EASTERN MARGIN.

DESCRIPTION OF WELL HEAD

15.5-IN (ID) CONDUCTOR, CAPPED WITH WELDED METAL PLATE, EXTENDS 0.5 FT ABOVE GROUND. 3-IN HIGH CEMENT APRON AT BASE.

\section{DESCRIPTION OF PITS}

NA

DEPTH AND BOREHOLE - FLUID SURVEY: $N / A$

GROUND ELEVATION N/A FT (DATUM)

REPORTED DEPTH N/A FT (SEE BOREHOLE COMPLETION SKETCH)

MEASURED DEPTH N N F OBSTRUCTION N N N

$\begin{array}{lll}\text { FLUID DEPTH } \frac{D R Y}{\mathrm{FT}} & \mathrm{PH} \frac{\mathrm{N} / \mathrm{A}}{\mathrm{N}} \mathrm{\textrm {NS } / \mathrm { cm }} & \mathrm{TDS} N \mathrm{~N} / \mathrm{Ag} / \mathrm{L} \\ \text { CONDUCTIVIT } & \end{array}$

\section{REMARKS}




\begin{tabular}{|c|c|c|c|}
\hline & & SEC. $10-T 2 N-R 1$ & ON COUNTY \\
\hline CLIENT & DOE / SRPO & J.O. No. 17500.25 & DATE $07 / 15 / 88$ \\
\hline $\begin{array}{l}\text { STATE } \\
\text { MISSISSIPPI }\end{array}$ & $\begin{array}{l}\text { WELL NAME \& NUMBER } \\
\text { WALKER } \quad \text { MH-8D }\end{array}$ & $\begin{array}{r}\text { TFSC FIELD REPRESEI } \\
\text { D. LU' } \\
\end{array}$ & ABEDI \\
\hline $\begin{array}{l}\text { DESCRIPTION OF } \\
\underline{A C C E S S ~ B Y ~ P A V E}\end{array}$ & $\begin{array}{l}\text { TE ACCESS } \\
\text { ROAD FROM PINEBUR. PAD }\end{array}$ & 200 FT EAST OF & NT IN LARGE \\
\hline OPEN FIELD. & & & \\
\hline
\end{tabular}

\section{DESCRIPTION OF WELL HEAD}

15.5-IN (ID) CONDUCTOR PIPE, CAPPED BY WELDED METAL PLATE, WAS BURIED 1 FT BELOW GROUND. LOCATED WITH METAL DETECTOR ON $8 / 18 / 88$.

\section{DESCRIPTION OF PITS}

$\underline{N A}$

DEPTH AND BOREHOLE - FLUID SURVEY: $N / A$

GROUND ELEVATION $N / A$

REPORTED DEPTH N/A FT (SEE BOREHOLE COMPLETION SKETCH)

MEASURED DEPTH N N N OBSTRUCTION N N

FLUID DEPTH DRY FT $\quad \mathrm{PH} N$ N

CONDUCTIVITY N N/A $\mathrm{mS} / \mathrm{cm} \quad$ TDS N/A $\mathrm{Ng} / \mathrm{L}$

TEMPERATURE $N$ N ${ }^{\circ} \mathrm{C}$

REMARKS 
SITE RECONNAISSANCE FORM

\begin{tabular}{|l|l|l|l|}
\hline CLIENT & DOE / NRPO & 17500.25 & DATE $07 / 15 / 88$ \\
\hline $\begin{array}{l}\text { STATE } \\
\text { MISSISSIPPI }\end{array}$ & $\begin{array}{c}\text { WELL NAME \& NUMBER } \\
\text { WALKER MH-8WS }\end{array}$ & $\begin{array}{c}\text { TFSC FIELD REPRESENTATIVE } \\
\text { D. LUTZ, H. ABEDI }\end{array}$ \\
\hline $\begin{array}{l}\text { DESCRIPTION OF SITE ACCESS } \\
\text { ACCESS BY PAVED ROAD FROM PINEBUR. WELL HEAD IS 75 FT EAST OF PAVEMENT AT EDGE }\end{array}$ \\
\hline
\end{tabular}

DESCRIPTION OF SITE PAD AREA

GENTLY SLOPING GRASSY FIELD.

DESCRIPTION OF WELL HEAD

4-IN (ID) STEEL CASING WITH LOCKING HINGED CAP EXTENDS 2.7 FT ABOVE GROUND.

\section{DESCRIPTION OF PITS}

NA

DEPTH AND BOREHOLE - FLUID SL

: MASCO $(8 / 15 / 88)$

GROUND ELEVATION 247.3 FT (DATUM)

REPORTED DEPTH 286.0 FT (SEE BOREHOLE COMPLETION SKETCH)

MEASURED DEPTH 295.0 FT OBSTRUCTION

FLUID DEPTH $\frac{90.0}{2}$ FT $\quad$ PH 10.23

CONDUCTIVITY $0.498 \mathrm{~ms} / \mathrm{cm}$ TDS $249 \mathrm{mg} / \mathrm{L}$

TEMPERATURE

\section{REMARKS}

OPEN HOLE BETWEEN BOTTOM OF TAIL PIPE (286 FT) AND BOTTOM OF BOREHOLE (402.5 FT). * 1 FT OF SILT AND PIPE SCALE AT BOTTOM OF TAIL PIPE. 
SITE RECONNAISSANCE FORM

\begin{tabular}{|c|c|c|c|}
\hline \multirow{2}{*}{\multicolumn{2}{|c|}{ DOE / SRPO }} & \multicolumn{2}{|r|}{ If counir } \\
\hline & & J.0. No. 17500.25 & DATE $07 / 11 / 88$ \\
\hline \begin{tabular}{|l} 
STATE \\
MISSISSIPPI
\end{tabular} & $\begin{array}{l}\text { WELL NAME \& NUMBER } \\
\text { L.R.F.P } \\
\text { MRIG-9 }\end{array}$ & \multicolumn{2}{|c|}{$\begin{array}{l}\text { TFSC FIELD REPRESENTATIVE } \\
\text { D. KISTNER, H. ABEDI }\end{array}$} \\
\hline
\end{tabular}

DESCRIPTION OF SITE PAD AREA

FLAT CLEARING. SPARSE VEGETATION.

DESCRIPTION OF WELL HEAD

8-IN (ID) STEEL INNER CASING WITH LOCKING HINGED CAP EXTENDS 1 FT ABOVE GROUND. 10-IN (ID) SURFACE CASING EXTENDS 0.7 FT ABOVE GROUND. 15.5-IN (ID) CONDUCTOR PIPE EXTENDS 0.5 FT ABOVE GROUND.

DESCRIPTION OF PITS

NA

DEPTH AND BOREHOLE - FLUID SURVEY:BY MASCO (8/16/88)

GROUND ELEVATION 256.0 FT (DATUM)

REPORTED DEPTH 766.0 FT (SEE BOREHOLE COMPLETION SKETCH)

MEASURED DEPTH 756.0 FT OBSTRUCTION $\star * *$ FT

FLUID DEPTH 200.0 FT $\quad \mathrm{PH}-10.03$

CONDUCTIVITY $208 \mathrm{mS} / \mathrm{cm}$ TDS $104.500 \mathrm{mg} / \mathrm{L}$

TEMPERATURE $26.4{ }^{\circ} \mathrm{C}$

\section{REMARKS}

OPEN BOREHOLE FROM BOTTOM OF CASING TO TOP OF CEMENT BACK-PLUG (766 FT).

$\star \star 10$ FT OF SILT AND PIPE SCALE AT BOTTOM OF BOREHOLE, OR BOTTOM HAS CLOSED UP. 
SITE RECONNAISSANCE FORM

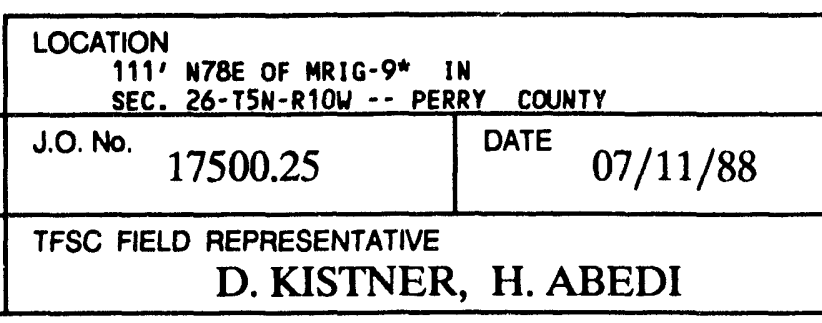

DESCRIPTION OF SITE ACCESS

DIRECT ACCESS 81 FT WEST OF PAVEMENT (LOOP RD).

DESCRIPTION OF SITE PAD AREA

NARROW DIRT PATH LEADS FROM PAVEMENT TO WELL. NUMEROUS PINE SAPLINGS AND

TALL GRASS OBSCURE WELL HEAD.

DESCRIPTION OF WELL HEAD

4-IN (ID) STEEL CASING WITH LOCKING HINGED CAP EXTENDS 0.7 FT ABOVE GROUND.

\section{DESCRIPTION OF PITS}

NA

DEPTH AND BOREHOLE - FLUID SURVEY: **BY MASCO (8/5/88)

GROUND ELEVATION 256.2 FT (DATUM)

REPORTED DEPTH 384.3 FI (SEE BOREHOLE COMPLETION SKETCH)

MEASURED DEPTH 380.0 FT OBSTRUCTION $* * *$ FT

FLUID DEPTH $107.8 \quad \mathrm{FT} \quad \mathrm{PH}-9.46$

CONDUCTMIT $0.67 \mathrm{~ms} / \mathrm{cm}$ TDS $320 \mathrm{mg} / \mathrm{L}$

TEMPERATURE $26.2{ }^{\circ} \mathrm{C}$

REMARKS

** ALL MEASUREMENTS BY MASCO (8/5/88) EXCEPT FLUID DEPTH BY SWEC $(\neg / 11 / 88)$.

*** 4.3 FT OF SILT AND PIPE SCALE AT BOTTOM OF WELL. 
SITE RECONNAISSANCE FORM

\begin{tabular}{|c|c|c|c|}
\hline \multirow{2}{*}{\multicolumn{2}{|c|}{ CLIENT $\quad$ DOE / SRPO }} & \multicolumn{2}{|c|}{ SEC. 12 -TSN-R1OH $\cdots$ PERRY COUNTY } \\
\hline & & J.O. No. 17500.25 & $\begin{array}{ll}\text { DATE } & 07 / 12 / 88\end{array}$ \\
\hline $\begin{array}{l}\text { STATE } \\
\text { MISSISSIPPI }\end{array}$ & $\begin{array}{l}\text { WELL NAME \& NUMBER } \\
\text { L.R.F.P. } \quad \text { MRIG-10 }\end{array}$ & \multicolumn{2}{|c|}{$\begin{array}{l}\text { TFSC FIELD REPRESENTATINE } \\
\text { D. KISTNER, H. ABEDI }\end{array}$} \\
\hline
\end{tabular}

DESCRIPTION OF SITE PAD AREA

FLAT GRASSY FIELD WITH SCATTERED 5 TO 8-FT HIGH TREES.

DESCRIPTION OF WELL HEAD

8-IN (ID) STEEL INNER CASING WITH LOCKING HINGED CAP EXTENDS 1 FT ABOVE GROUND.

STICK-UP OF 10-IN (ID) SURFACE CASING AND 15.5-IN (ID) CONDUCTOR PIPE NOT

MEASURED.

DESCRIPTION OF PITS

NA

DEPTH AND BOREHOLE - FLUID SURVEY:BY MASCO (8/5/88)

GROUND ELEVATION 284.6 FT (DATUM)

REPORTED DEPTH 2640.5 FT (SEE BOREHOLE COMPLETION SKETCH)

MEASURED DEPTH 2640.0 FT OBSTRUCTION N/A FT

FLUID DEPTH 30.0 FT $\quad \mathrm{PH}-8.75$

CONDUCTIVITY $22.1 \mathrm{~ms} / \mathrm{cm}$ TDS $11.050 \mathrm{mg} / \mathrm{L}$

TEMPERATURE $26.9-{ }^{\circ} \mathrm{C}$

REMARKS

TOP 2 INNER CASING JOINTS ARE 8-IN (ID): REMAINING JOINTS ARE 6-IN (ID). 
SITE RECONNAISSANCE FORM

\begin{tabular}{|l|l|}
\hline CLIENT & \\
\hline STATE & DOE / SRPO \\
MISSISSIPPI & WELL NAME \& NUMBER \\
L.R.F.P. MRIG-10WS & \\
\hline
\end{tabular}

LOCATION

102' NTSE OF MRIG-10" IN

SEC. 12-T5N-R1OW - P PERRY COUNTY

J.O. No.

17500.25

DATE

$07 / 12 / 88$

TFSC FIELD REPRESENTATIVE

DESCRIPTION OF SITE ACCESS

WELL HEAD IS IN GRASSY FIELD 75 FT NORTH OF GOOD GRAVEL ROAD. KEY TO GATE

AT ENTRANCE OF ACCESS ROAD CAN BE OBTAINED FROM LEAF RIVER FOREST PRODUCTS.

DESCRIPTION OF SITE PAD AREA

FLAT GRASSY FIELD WITH SCATTERED 5 TO 8-FT HIGH TREES.

DESCRIPTION OF WELL HEAD

4-IN (ID) STEEL CASING WITH LOCKING HINGED CAP EXTENDS 2.3 FT ABOVE GROUND.

DESCRIPTION OF PITS

$\underline{N A}$

DEPTH AND BOREHOLE - FLUID SURVEY: $* *$ BY MASCO $(8 / 5 / 88)$

GROUND ELEVATION 282.6 FT (DATUM)

REPORTED DEPTH 295.4 FT (SEE BOREHOLE COMPLETION SKETCH)

MEASURED DEPTH 286.0 FT OBSTRUCTION _

FLUID DEPTH 111.6 FT $\quad \mathrm{PH}-9.58$

CONDUCTIVITY $0.115 \mathrm{mS} / \mathrm{cm}$ TDS $57 \quad \mathrm{mg} / \mathrm{L}$

TEMPERATURE $26.4{ }^{\circ} \mathrm{C}$

REMARKS

** ALL MEASUREMENTS BY MASCO (8/5/88) EXCEPT FLUID DEPTH BY SWEC $(7 / 12 / 88)$

*** 9.4 FT OF SILT AND PIPE SCALE AT BOTTOM OF WELL. 
SITE RECONNAISSANCE FORM

\begin{tabular}{|c|c|c|}
\hline CLIENT & DOE / SRPO & J.O. No. 17500.25 \\
\hline $\begin{array}{l}\text { STATE } \\
\text { MISSISSIPPI }\end{array}$ & $\begin{array}{l}\text { WELL NAME \& NUMBER } \\
\text { TRAVIS MRIH-11A }\end{array}$ & $\begin{array}{l}\text { TFSC FIELD REPRESENTATIVE } \\
\text { D. KISTNER, H. ABEDI }\end{array}$ \\
\hline \multicolumn{3}{|c|}{ DESCRIPTION OF SITE ACCESS } \\
\hline \multicolumn{3}{|c|}{ RESIDES IN TRAILER AT GATE), THEN TURN SOUTHEAST FOR 100 FT ON DIRT PATH TO } \\
\hline \multicolumn{3}{|c|}{ SITE. GRAVEL ACCESS ROAD IS BADLY RUTTED AND NEEDS GRADING. } \\
\hline \multicolumn{3}{|c|}{ DESCRIPTION OF SITE PAD AREA } \\
\hline
\end{tabular}

DESCRIPTION OF WELL HEAD

8-IN (ID) STEEL INNER CASING WITH LOCKING HINGED CAP EXTENDS 1.8 FT ABOVE GROUND. 10-IN (ID) SURFACE CASING EXTENDS 1.0 FT ABOVE GROUND. 15.5-IN (ID) CONDUCTOR PIPE TO 0.4 FT ABOVE GROUND.

DESCRIPTION OF PITS

NA
DEPTH AND BOREHOLE - FLUID SURVEY:BY MASCO $(8 / 6 / 88)$.
GROUND ELEVATION 180.4 FT (DATUM)
REPORTED DEPTH 4231.4 FT (SEE BOREHOLE COMPLETION SKETCH)
MEASURED DEPTH 4160.0 FT OBSTRUCTION $\_*$ FT
FLUID DEPTH 75.0 FT $\quad \mathrm{PH}-9.11$
CONDUCTIVITY $22.8 \mathrm{~ms} / \mathrm{cm}$ TDS $11.400 \mathrm{mg} / \mathrm{L}$
TEMPERATURE $23.5{ }^{\circ} \mathrm{C}$

REMARKS

TOP 2 INNER CASING JOINTS ARE 8-IN (ID): REMAINING JOINTS ARE 6-IN (ID).

** 71.4 FT OF SILT AND PIPE SCALE AT BOTTOM OF WELL. 
SITE RECONNAISSANCE FORM

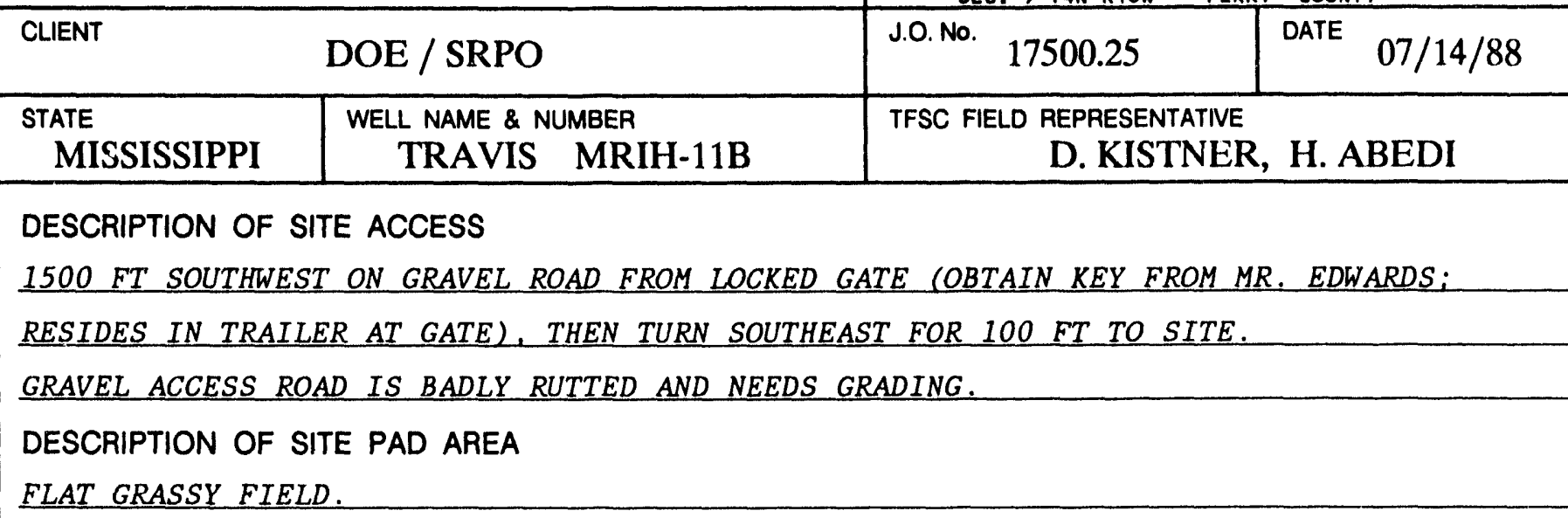

\section{DESCRIPTION OF WELL HEAD}

PRESSURIZED WELL HEAD CAPPING 6-IN (ID) INNER CASING, TO 3.1 FT ABOVE GROUND.

WELL HEAD HAS TWO CONTROL VALVES AND ACCESS PORTS. PRESSURE $=9.5$ PSI.

STICK-UP OF 10-IN (ID) SURFACE CASING AND 15.5-IN (ID) CONDUCTOR NOT MEASURED.

DESCRIPTION OF PITS

$\underline{N A}$

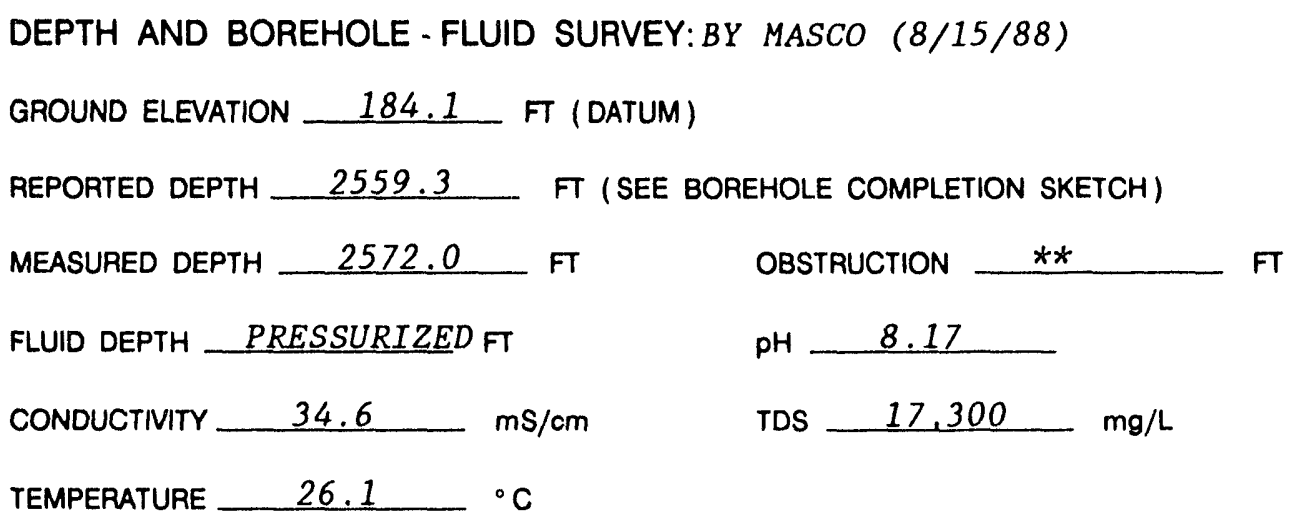

REMARKS

** MEASURED WELL DEPTH IS 13 FT DEEPER THAN REPORTED DEPTH OF TAIL PIPE. 
SITE RECONNAISSANCE FORM

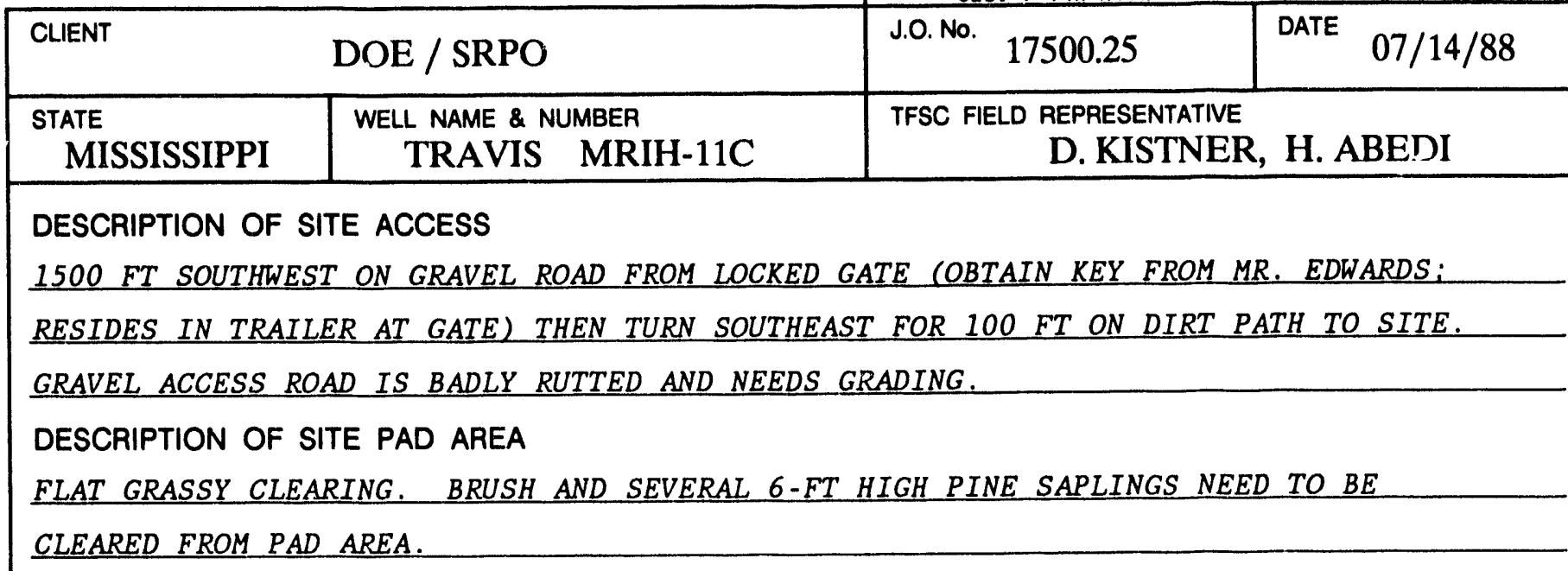

\section{DESCRIPTION OF WELL HEAD}

PRESSURIZED WELL HEAD CAPPING 6-IN (ID) INNER CASING, TO 2.8 FT ABOVE GROUND.

WELL HEAD HAS TWO CONTROL VALVES AND ACCESS PORTS. PRESSURE $=4$ PSI.

STICK -UP OF 15.5-IN (ID) CONDUCTOR NOT MEASURED.

DESCRIPTION OF PITS

NA

DEPTH AND BOREHOLE - FLUID SURVEY:BY MASCO (8/15/88)

GROUND ELEVATION 181.7 FT (DATUM)

REPORTED DEPTH 1616.4 FT (SEE BOREHOLE COMPLETION SKETCH)

MEASURED DEPTH 1620.0 FT OBSTRUCTION

FLUID DEPTH PRESSURIZED FT $\quad \mathrm{PH} \quad 8.42$

CONDUCTIVITY $11.68 \mathrm{mS} / \mathrm{cm} \quad$ TDS $5.840 \mathrm{mg} / \mathrm{L}$

TEMPERATURE $25.7 \quad{ }^{\circ} \mathrm{C}$

REMARKS

** MEASURED WELL DEPTH IS 3.6 FT DEEPER THAN REPORTED DEPTH. 
SITE RECONNAISSANCE FORM

\begin{tabular}{|c|c|c|c|}
\hline CLIENT & DOE / SRPO & J.O. No. 17500.25 & DATE $07 / 14 / 88$ \\
\hline $\begin{array}{l}\text { STATE } \\
\text { MISSISSIPPI }\end{array}$ & $\begin{array}{l}\text { WELL NAME \& NUMBER } \\
\text { TRAVIS MRIH-11D }\end{array}$ & \multicolumn{2}{|c|}{$\begin{array}{l}\text { TFSC FIELD REPRESENTATIVE } \\
\text { D. KISTNER, H. ABEDI }\end{array}$} \\
\hline \multicolumn{4}{|c|}{ DESCRIPTION OF SITE ACCESS } \\
\hline \multicolumn{4}{|c|}{ RESIDES IN TRAILER AT GATE), THEN IURN SOUTHEAST FOR 100 FT ON DIRT PATH TO } \\
\hline \multicolumn{4}{|c|}{ SITE. GRAVEL ACCESS ROAD IS BADLY RUTTED AND NEEDS GRADING. } \\
\hline \multicolumn{4}{|c|}{ DESCRIPTION OF SITE PAD AREA } \\
\hline \multicolumn{4}{|c|}{ FLAT GRASSY CLEARING. BRUSH AND SEVERAL 6-FT HIGH PINE SAPLINGS NEED TO BE } \\
\hline
\end{tabular}

DESCRIPTION OF WELL HEAD

8-IN (ID) STEEL INNER CASING WITH LOCKING HINGED CAP TO 0.6 FT ABOVE GROUND. STICK -UP OF 15.5-IN (ID) CONDUCTOR PIPE NOT MEASURED.

DESCRIPTION OF PITS

$\underline{N A}$

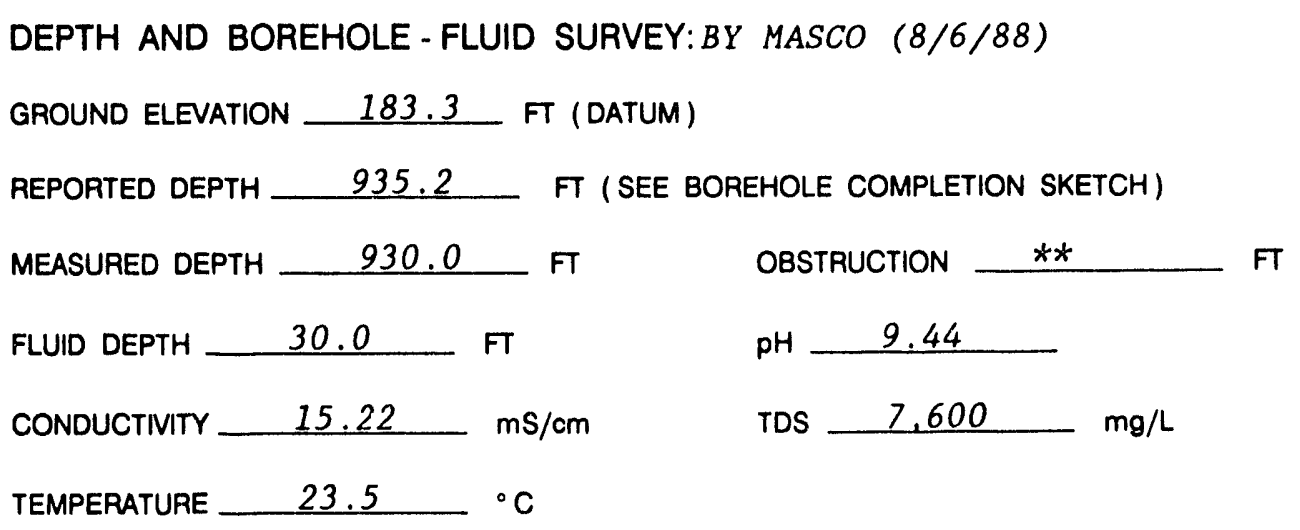

REMARKS

TOP 2 INNER CASING JOINTS ARE 8-IN (ID): REMAINING JOINTS ARE 6-IN (ID).

** 5.2 FT OF SILT AND PIPE SCALE AT BOTTOM OF WELL. 
SITE RECONNAISSANCE FORM

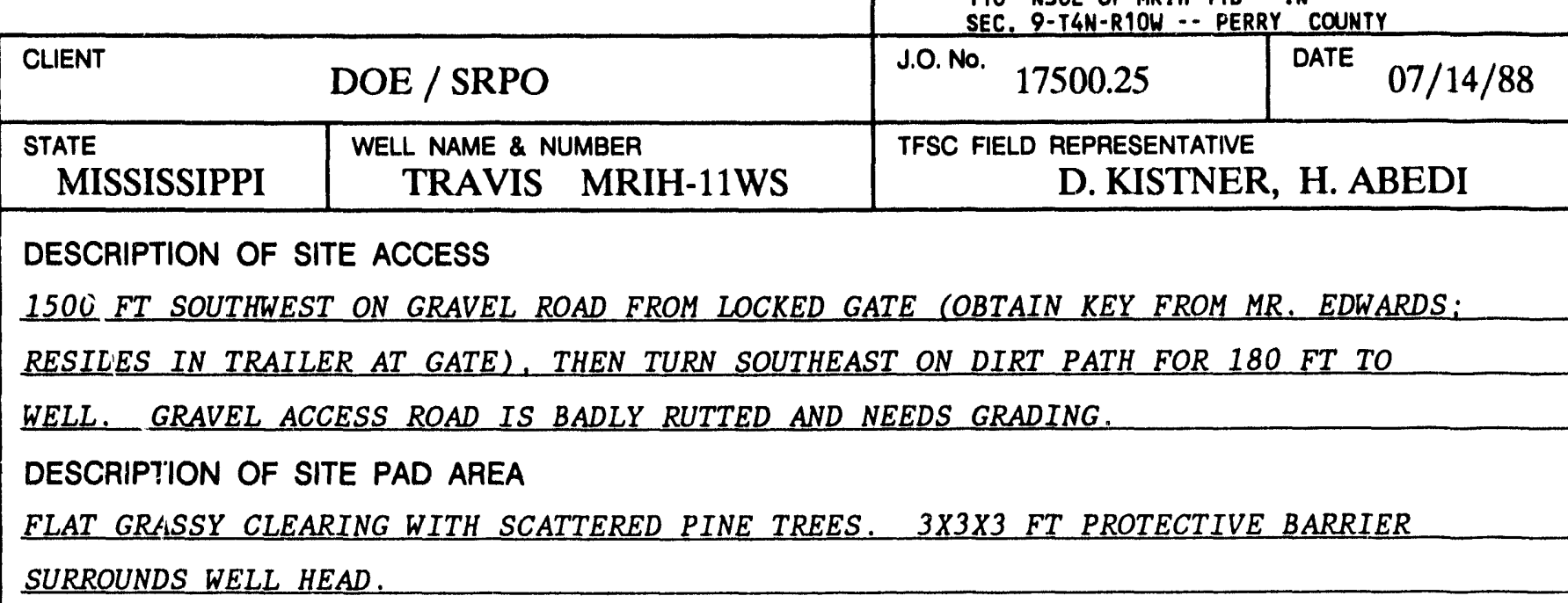

\section{DESCRIPTION OF WELL HEAD}

4-IN (ID) STEEL CASING, THREADED ID WITHOUT CAP, EXTENDS 1.6 FT ABOVE GROUND.

\section{DESCRIPTION OF PITS}

NA

DEPTH AND BOREHOLE - FLUID SURVEY:BY SWEC $(11 / 01 / 88)$

GROUND ELEVATION 188.3 FT (DATUM)

REPORTED DEPTH 159.4 FT (SEE BOREHOLE COMPLETION SKETCH)

MEASURED DEPTH 157.3 FT OBSTRUCTION

FLUID DEPTH $77.3 \quad$ FT $\quad \mathrm{pH}-9.50$

CONDUCTIVITY N/A $\mathrm{NS} / \mathrm{cm} \mathrm{TDS} 17.2 \mathrm{mg} / \mathrm{L}$

TEMPERATURE $21.2{ }^{\circ} \mathrm{C}$

\section{REMARKS}

** I FT OF SILT AND PIPE SCALE AT BOTTOM OF WELL. 
SITE RECONNAISSANCE FORM

\begin{tabular}{|c|c|c|c|}
\hline \multicolumn{2}{|c|}{ SITE RECONNAISSANCE FORM } & \multicolumn{2}{|c|}{$\begin{array}{l}\text { LOCATION } \\
\text { 1029.0' SHL \& } 2170.0^{\prime} \text { WEL* IN } \\
\text { SEC. 4-T2N-R1OH - PERRY COUNTY }\end{array}$} \\
\hline CLIENT & DOE / SRPO & $\begin{array}{ll}\text { J.O. No. } 17500.25 \\
\end{array}$ & $\begin{array}{ll}\text { DATE } & 07 / 13 / 88\end{array}$ \\
\hline $\begin{array}{l}\text { STATE } \\
\text { MISSISSIPPI }\end{array}$ & $\begin{array}{l}\text { WELL NAME \& NUMBER } \\
\text { U.S.F.S. MCCG-101 }\end{array}$ & \multicolumn{2}{|c|}{$\begin{array}{l}\text { TFSC FIELD REPRESENTATIVE } \\
\text { T. REGAN, D. LUTZ }\end{array}$} \\
\hline
\end{tabular}

DESCRIPTION OF SITE ACCESS

WELL HEAD IS 34 FT EAST OF DIRT ROAD. EASY ACCESS.

\section{DESCRIPTION OF SITE PAD AREA}

PAD AREA IS GRASSY FIELD. WELL HEAD IS SURROUNDED BY $3 \times 3 \times 3$ FT PROTECTIVE

BARRIER.

DESCRIPTION OF WELL HEAD

2-IN (ID) PVC CASING, UNCAPPED, EXTENDS 0.8 FT ABOVE GL, 4-IN (ID) STEEL GUARD

PIPE WITH LOCKING HINGED CAP EXTENDS 0.9 FT ABOVE GL.

\section{DESCRIPTION OF PITS}

$\underline{N A}$

DEPTH AND BOREHOLE - FLUID SURVEY:BY SWEC (8/25/88)

GROUND ELEVATION 234.5 FT (DATUM)

REPORTED DEPTH 165.0 FT (SEE BOREHOLE COMPLETION SKETCH)

MEASURED DEPTH 163.1 FT OBSTRUCTION NA FT

FLUID DEPTH 71.1 FT $\mathrm{PH} 7.30$

CONDUCTIVITY $0.189 \mathrm{mS} / \mathrm{cm} \quad$ TDS $95.0-\mathrm{mg} / \mathrm{h}$

TEMPERATURE $31.8-{ }^{\circ} \mathrm{C}$

REMARKS

NO . ACCESS PROBLEMS. 1.1 FT OF SILT AT BOTTOM OF WELL. 
SITE RECONNAISSANCE FORM

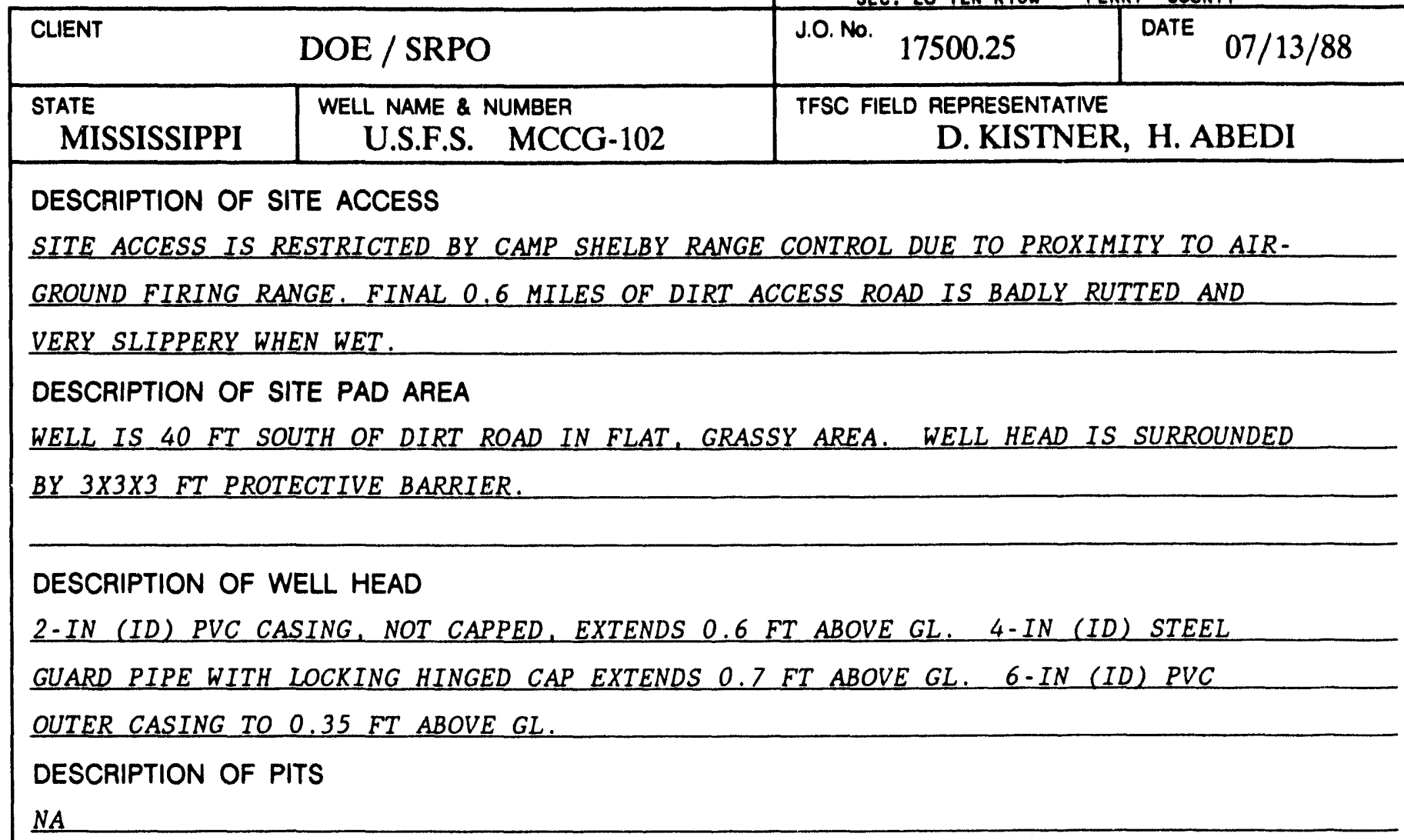

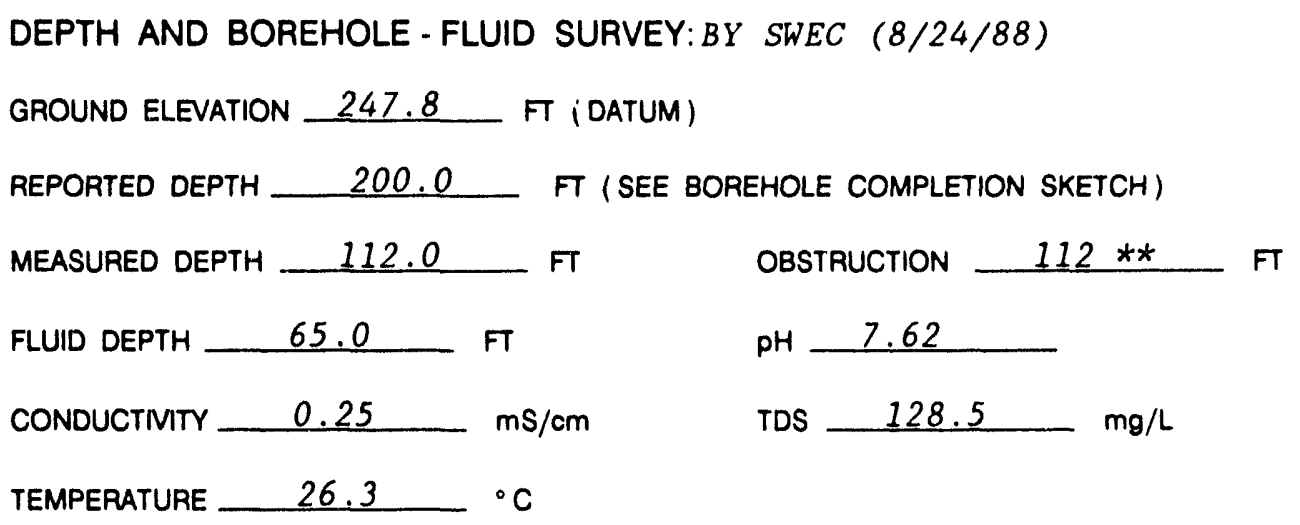

REMARKS

* 42 FT OF NYLON ROPE FISHED FROM WELL ON 9/25/88. NEW MEASURED DEPTH IS 196 FT. 
SITE RECONNAISSANCE FORM

2394.7' SNL \& 1496.2' EWL* IN

SEC. 28-T2N-R10W -. PERRY COUNTY

CLIENT

DOE / SRPO

J.O. No.

17500.25

DATE

\begin{tabular}{l|c}
\hline STATE & WELL NAME \& NUMBER \\
MISSISSIPPI & U.S.F.S. MCC \\
\hline
\end{tabular}

U.S.F.S. MCCG-103

TFSC FIELD REPRESENTATIVE

$08 / 19 / 88$

DESCRIPTION OF SITE ACCESS

ACCESS TO SITE WEST OF STATE HWY 29 RESTRICTED BY CAMP SHELBY RANGE CONTROL DUE

TO PROXIMITY OF TANK-FIRING RANGE. ACCESS VIA GOOD GRAVEL ROAD, 0.2 MILES WEST

FROM STATE HIGHWAY 29

DESCRIPTION OF SITE PAD AREA

NO SURFACE EVIDENCE OF WELL HEAD ON 7-13-88. LOCATION RESURVEYED ON 8-17-88,

EXCAVATION ON 8-19-88 REVEALS PVC WELL BURIED IN MIDDLE OF DIRT ROAD TURNOFF

FROM ACCESS ROAD.

DESCRIPTION OF WELL HEAD

2-IN (ID) PVC CASING, TOP PLUGGED BY SOIL, 2 FT BELOW GL.

DESCRIPTION OF PITS

NA

DEPTH AND BOREHOLE - FLUID SURVEY:BY SWEC (9/28/88)

GROUND ELEVATION 248.5 FT (DATUM)

REPORTED DEPTH 270.0 FT (SEE BOREHOLE COMPLETION SKETCH)

MEASURED DEPTH 270.0 FT OBSTRUCTION NA FT

FLUID DEPTH $66.5 \quad \mathrm{PT} \quad \mathrm{PH}-7.74$

CONDUCTIVITY $0.202 \mathrm{mS} / \mathrm{cm}$ TDS $101.3-\mathrm{mg} / \mathrm{L}$

TEMPERATURE $21.2{ }^{\circ} \mathrm{C}$

REMARKS

SCHEDULE ACCESS WITH CAMP SHELBY RANGE CONTROL. 
SITE RECONNAISSANCE FORM

\begin{tabular}{|c|c|c|c|}
\hline \multicolumn{2}{|c|}{ SITE RECONNAISSANCE FOAM } & \multicolumn{2}{|c|}{$\begin{array}{l}\text { LOCATION } \\
700.11 \text { SNL \& } 1959.21 \text { EUL" IN } \\
\text { SEC. 5-T2N-R1OH - PERRY COUNYY }\end{array}$} \\
\hline CLIENT & DOE / SRPO & J.O. No. 17500.25 & DATE $07 / 13 / 88$ \\
\hline $\begin{array}{l}\text { STATE } \\
\text { MISSISSIPPI }\end{array}$ & $\begin{array}{l}\text { WELL NAME \& NUMBER } \\
\text { U.S.F.S. MCCG-104 }\end{array}$ & $\begin{array}{r}\text { TFSC FIELD REPRESEI } \\
\text { T. REC }\end{array}$ & D. LUTZ \\
\hline \multicolumn{4}{|c|}{$\begin{array}{l}\text { DESCRIPTION OF SITE ACCESS } \\
\text { WELL IS } 28 \text { FT NORTH OF GOOD GRAVEL ROAD, } 0.4 \text { MI EAST OF STATE HWY } 29 \text {. DIRECT }\end{array}$} \\
\hline \multicolumn{4}{|c|}{$\begin{array}{l}\text { DESCRIPTION OF SITE PAD AREA } \\
\text { NO PROTECTIVE BARRIER AROUND WELL. DIFFICULT TO LOCATE IN LOW BRUSH. BROKEN CON- }\end{array}$} \\
\hline \multicolumn{4}{|c|}{ CRETE AND POST HOLES NEAR WELL INDICATE THAT PROTECTIVE BARRIER WAS REMOVED } \\
\hline \multicolumn{4}{|c|}{ SUBSEQUENT TO INSTALLATION. } \\
\hline \multicolumn{4}{|c|}{$\begin{array}{l}\text { DESCRIPTION OF WELL HEAD } \\
2-I N \text { (ID) PVC CASING WITH }\end{array}$} \\
\hline
\end{tabular}

\section{DESCRIPTION OF PITS}

NA

DEPTH AND BOREHOLE - FLUID SURVEY:BY MASCO $(8 / 6 / 88)$

GROUND ELEVATION 241.0 FT (DATUM)

REPORTED DEPTH $95.0 \quad$ FT (SEE BOREHOLE COMPLETION SKETCH)

MEASURED DEPTH

FLUID DEPTH $34.1 \quad$ FT $\quad \mathrm{pH}-6.78$

CONDUCTIVITY $0.164 \quad \mathrm{mS} / \mathrm{cm} \quad \mathrm{mg} / \mathrm{h}$

TEMPERATURE _. $24.1 \quad{ }^{\circ} \mathrm{C}$

REMARKS 


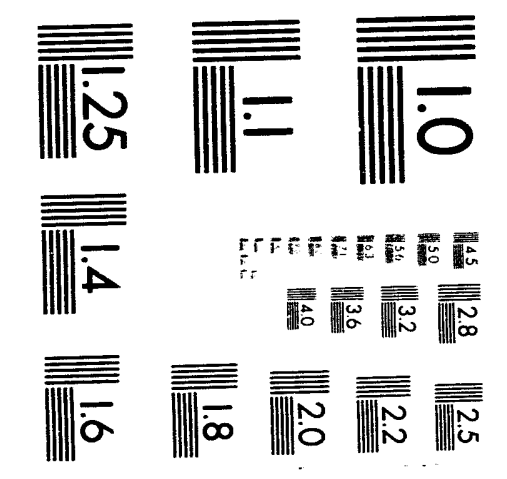



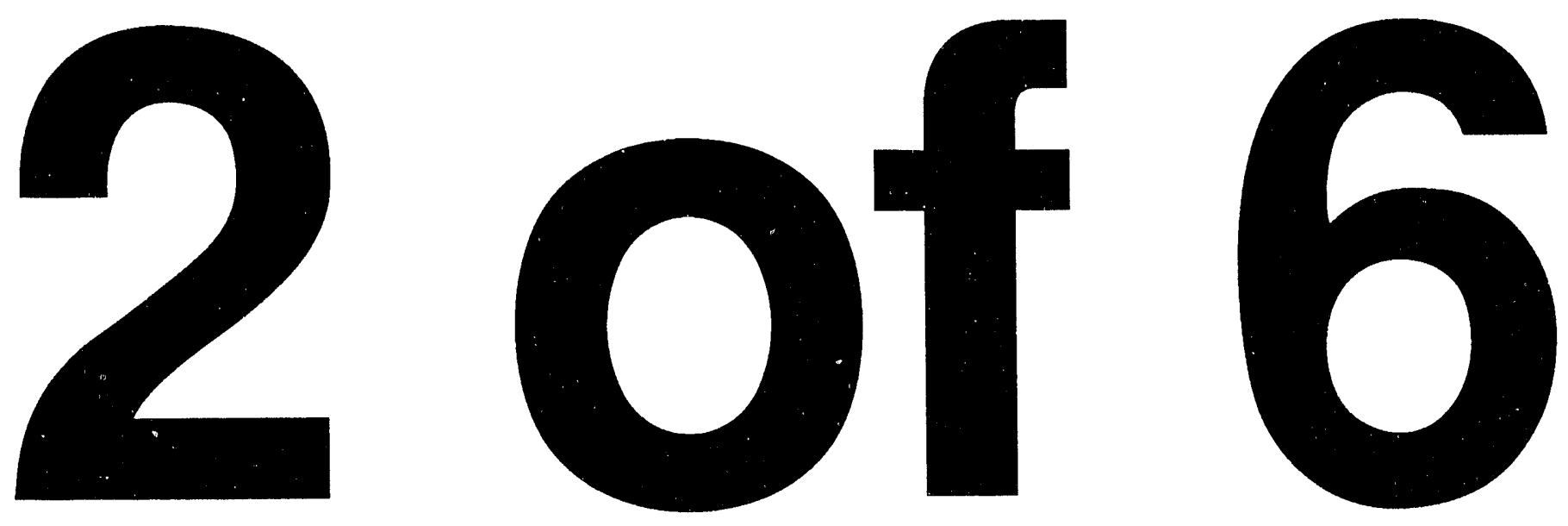
SITE RECONNAISSANCE FORM

\begin{tabular}{|l|l|l|l|}
\hline CLIENT & DOE / SRPO & J.0. No. 17500.25 & DATE $07 / 13 / 88$ \\
\hline $\begin{array}{l}\text { STATE } \\
\text { MISSISSIPPI }\end{array}$ & $\begin{array}{l}\text { WELL NAME \& NUMBER } \\
\text { U.S.F.S. MCCG-105 }\end{array}$ & $\begin{array}{r}\text { TFSC FIELD REPRESENTATIVE } \\
\text { D. KISTNER, H. ABEDI }\end{array}$ \\
\hline $\begin{array}{l}\text { DESCRIPTION OF SITE ACCESS } \\
\text { WELL IS 230 FT EAST OF STATE HWY 29. DIRECT ACCESS. }\end{array}$ \\
\hline
\end{tabular}

DESCRIPTION OF SITE PAD AREA

3X3X3 FT PROTECTIVE BARRIER SURROUNDS WELL. PAD AREA IS FLAT AND OPEN,

SURROUNDED BY PINES.

DESCRIPTION OF WELL HEAD

2-IN (ID) PVC CASING, UNCAPPED, EXTENDS 0.85 FT ABOVE GL. 4-IN (ID) STEEL GUARD

PIPE WITH LOCKING HINGED CAP EXTENDS I FT ABOVE GL. 6-IN (ID) PVC OUTER CASING

TO 0.7 FT ABOVE GL.

DESCRIPTION OF PITS

NA

DEPTH AND BOREHOLE - FLUID SURVEY:BY MASCO $(8 / 7 / 88)$

GROUND ELEVATION 272.9 FT (DATUM)

REPORTED DEPTH 90.0 FT (SEE BOREHOLE COMPLETION SKETCH)

MEASURED DEPTH 87.0 TT OBSTRUCTION NA FT

FLUID DEPTH 41.0 FT $\quad \mathrm{pH}-6.00$

CONDUCTINITY $0.180 \mathrm{~ms} / \mathrm{cm}$ TDS $90.0 \mathrm{mg} / \mathrm{L}$

TEMPERATURE $23.7{ }^{\circ} \mathrm{C}$

\section{REMARKS}

3 FT SILT AT BOTTOM OF WELL. 
SITE RECONNAISSANCE FORM

\begin{tabular}{|c|c|c|c|}
\hline \multicolumn{2}{|c|}{ CLIENT DOE / SRPO } & J.O. No. 17500.25 & DATE $07 / 13 / 88$ \\
\hline $\begin{array}{l}\text { STATE } \\
\text { MISSISSIPPI }\end{array}$ & $\begin{array}{l}\text { WELL NAME \& NUMBER } \\
\text { U.S.F.S. MCCG-107 }\end{array}$ & \multicolumn{2}{|c|}{$\begin{array}{l}\text { TFSC FIELD REPRESENTATIVE } \\
\text { D. KISTNER, H. ABEDI }\end{array}$} \\
\hline \multicolumn{4}{|c|}{ DESCRIPTION OF SITE ACCESS } \\
\hline \multicolumn{4}{|c|}{ HWY 29. WELL IS LOCATED 36 FT NORTH OF GRAVEL ROAD. } \\
\hline \multicolumn{4}{|c|}{ DESCRIPTION OF SITE PAD AREA } \\
\hline \multicolumn{4}{|c|}{ DESCRIPTION OF WELL HEAD } \\
\hline \multicolumn{4}{|c|}{ 2-IN (ID) PVC CASING, UNCAPPED, EXTENDS 0.45 FT ABOVE GL. 4-IN (ID) STEEL } \\
\hline \multicolumn{4}{|c|}{ GUARD PIPE WITH LOCKING HINGED CAP EXTENDS 0.6 FT ABOVE GL. 6-IN (ID) PVC OUTER } \\
\hline \multicolumn{4}{|c|}{ CASING TO 0.2 FT ABOVE GL. } \\
\hline \multicolumn{4}{|c|}{ DESCRIPTION OF PITS } \\
\hline & & & \\
\hline
\end{tabular}

DEPTH AND BOREHOLE - FLUID SURVEY:BY MASCO $(8 / 7 / 88)$

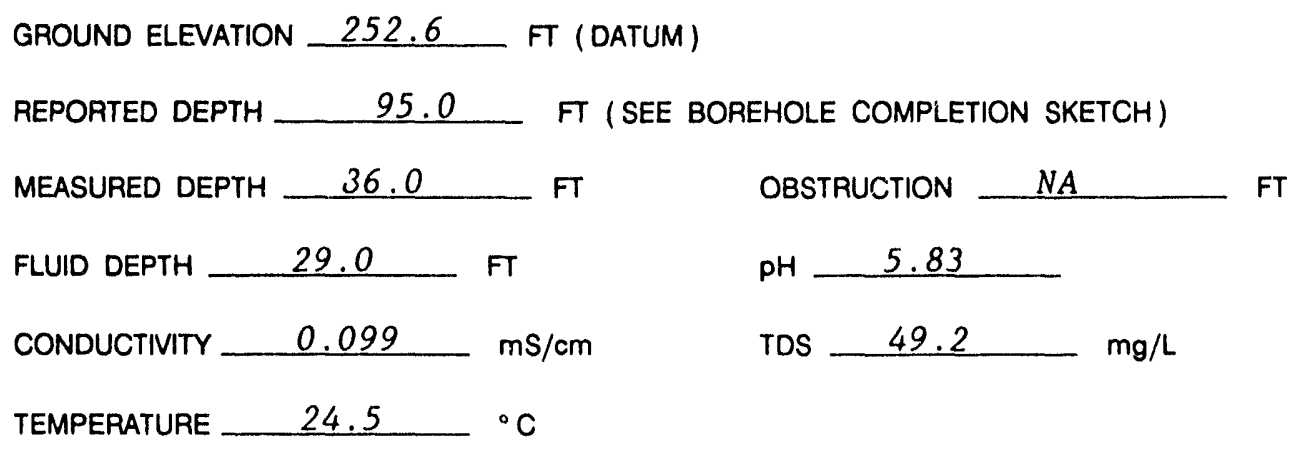

\section{REMARKS}

9 FT OF SILT AT BOTTOM OF WELL. 


\section{SITE RECONNAISSANCE FORM}

LOCATION

2457.2' NSL \& 1816.5' EWL* IN

SEC. 9-T2N-R10W - . PERRY COUNTY

\begin{tabular}{|l|l|l|l|}
\hline CLIENT DOE / SRPO & J.O. No. 17500.25 & DATE $07 / 13 / 88$ \\
\hline
\end{tabular}

STATE

WELL NAME \& NUMBER

MISSISSIPPI

U.S.F.S. MCCG-108

TFSC FIELD REPRESENTATIVE

T. REGAN, D. LUTZ.

DESCRIPTION OF SITE ACCESS

SITE NOT VISITED DURING SITE RECONNAISSANCE, UNCASED BOREHOLE WAS BACKFILLED

IMMEDIATELY FOLLOWING DRILLING BY LETCO

DESCRIPTION OF SITE PAD AREA

NA

DESCRIPTION OF WELL HEAD

NA

DESCRIPTION OF PITS

NA

DEPTH AND BOREHOLE - FLUID SURVEY:

GROUND ELEVATION NA FT (DATUM)

REPORTED DEPTH $20.5 \quad$ FT (SEE BOREHOLE COMPLETION SKETCH)

MEASURED DEPTH NA FT OBSTRUCTION NA FT

FLUID DEPTH NA

$\mathrm{pH}$

CONDUCTIVITY N NA

$\mathrm{mS} / \mathrm{cm}$

TDS NA

$\mathrm{mg} / \mathrm{L}$

TEMPERATURE

REMARKS 


\section{SITE RECONNAISSANCE FORM}

$1867.4^{\prime}$ NSL \& 3430.5' EWL* IN

SEC. 9-T2N-R10W - . PERRY COUNTY

\begin{tabular}{|l|l|l|l|}
\hline CLIENT & J.0. No. 17500.25 & \multicolumn{1}{c}{ DATE $07 / 13$} \\
\hline $\begin{array}{l}\text { STATE } \\
\text { MISSISSIPPI }\end{array}$ & $\begin{array}{l}\text { WELL NAME \& NUMBER } \\
\text { U.S.F.S. MCCG-109 }\end{array}$ & TFSC FIELD REPRESENTATIVE \\
\end{tabular}

DESCRIPTION OF SITE ACCESS

SITE NOT VISITED DURING SITE RECONNAISSANCE, UNCASED BOREHOLE WAS BACKFILLED

IMMEDIATELY FOLLOWING DRILLING BY LETCO.

\section{DESCRIPTION OF SITE PAD AREA}

$\underline{N A}$

DESCRIPTION OF WELL HEAD

NA

DESCRIPTION OF PITS

$\underline{N A}$

DEPTH AND BOREHOLE - FLUID SURVEY:

GROUND ELEVATION $\sim 205$ FT (DATUM)

REPORTED DEPTH $20.5 \quad$ FT (SEE BOREHOLE COMPLETION SKETCH)

MEASURED DEPTH NA FT OBSTRUCTION NA FT

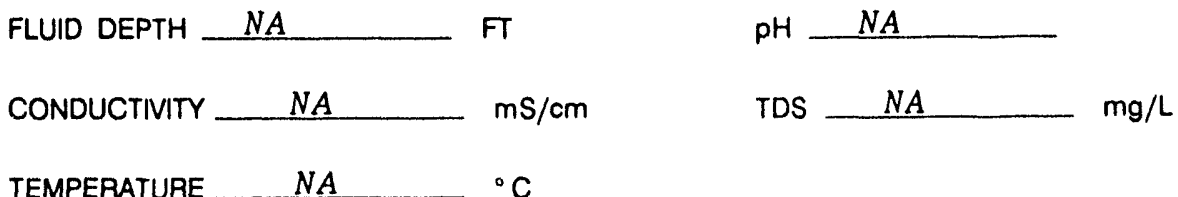

REMARKS 
SITE RECONNAISSANCE FORM

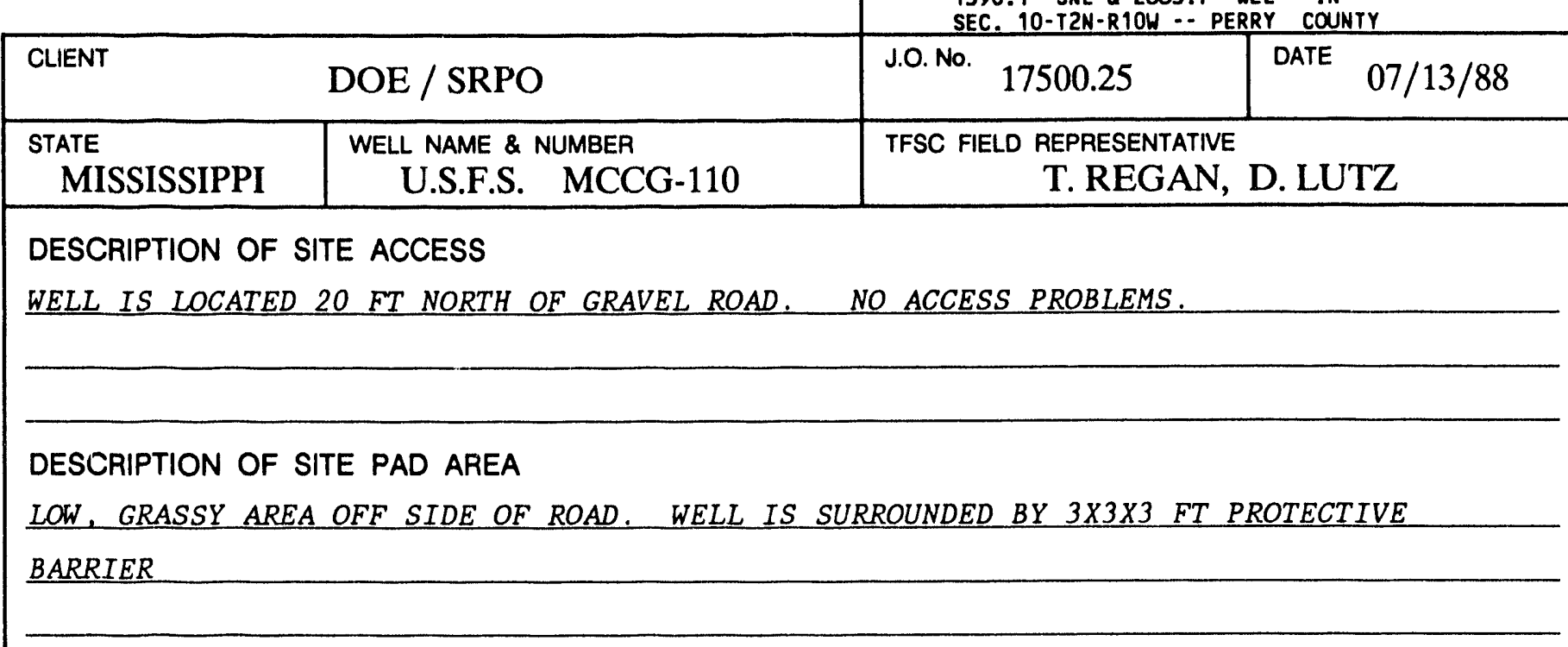

DESCRIPTION OF WELL HEAD

2-IN (ID) PVC CASING, UNCAPPED, EXTENDS 0.3 FT ABOVE GL. 4-IN (ID) STEEL GUARD

PIPE WITH LOCKING HINGED CAP EXTENDS 0.9 FT ABOVE GL.

\section{DESCRIPTION OF PITS}

NA

DEPTH AND BOREHOLE - FLUID SURVEY:BY SWEC (09/14/88)

GROUND ELEVATION 240.8 FT (DATUM)

REPORTED DEPTH $\quad 86.0$ FT (SEE BOREHOLE COMPLETION SKETCH)

MEASURED DEPTH 80.7 FT OBSTRUCTION NA FT

FLUID DEPTH $52.5 \quad \mathrm{FT} \quad \mathrm{pH} \quad 5.81$

CONDUCTIVITY $0.060 \mathrm{mS} / \mathrm{cm} \quad$ TDS $30.0 \quad \mathrm{mg} / \mathrm{L}$

TEMPERATURE $23.1-{ }^{\circ} \mathrm{C}$

\section{REMARKS}

5 FT OF SILT AT BOTTOM OF WELL. 
SITE RECONNAISSANCE FORM

CLIENT

DOE / SRPO

J.O. No.

LOCATION

$5130.9^{\prime}$ SNL \& 3027.6' EHL* IN

SEC. 8-T2N-R1OW - - PERRY COUNTY

STATE

WELL NAME \& NUMBER

MISSISSIPPI

U.S.F.S. MCCG-111

TFSC FIEL.D REPRESENTATIVE

DATE

$07 / 13 / 88$

DESCRIPTION OF SITE ACCESS

WELL LOCATED 1400 FT DOWN UNIMPROVED DIRT ROAD WHICH FORKS OFF OF GOOD GRAVEL

ROAD 0.2 MI EAST OF HWY 29. ROAD IS PASSABLE AFTER REMOVAL OF FALLEN TREE

TRUNKS.

DESCRIPTION OF SITE PAD AREA

WELL LOCATED IN CLEARING BESIDE UNIMPROVED DIRT ROAD, SURROUNDED BY $3 \times 3 \times 3$ FT

PROTECTIVE BARRIER.

DESCRIPTION OF WELL HEAD

2-IN (ID) CAPPED PVC CASING EXTENDS 2.7 FT ABOVE GL. 4-IN (ID) LOCKING STEEL

GUARD PIPE EXTENDS 2.9 FT ABOVE GL. 6-IN (ID) PVC OUTER CASING TO 0.2 FT ABOVE

GL.

DESCRIPTION OF PITS

NA

DEPTH AND BOREHOLE - FLUID SURVEY:BY MASCO $(8 / 7 / 88)$

GROUND ELEVATION 207.9 FT (DATUM)

REPORTED DEPTH 120.0 FT (SEE BOREHOLE COMPLETION SKETCH)

MEASURED DEPTH 113.6 FT OBSTRUCTION NA FT

FLUID DEPTH $1.2 \quad \mathrm{FT} \quad \mathrm{PH} \quad 5.95$

CONDUCTIVITY $0.192 \mathrm{mS} / \mathrm{cm} \quad$ TDS $96.3 \mathrm{mg} / \mathrm{L}$

TEMPERATURE $24.2 \quad{ }^{\circ} \mathrm{C}$

REMARKS

6.4 FT OF SILT AT BOTTOM OF WELL. ROAD WORK REQUIRED FOR RIG ACCESS 
SITE RECONNAISSANCE FORM

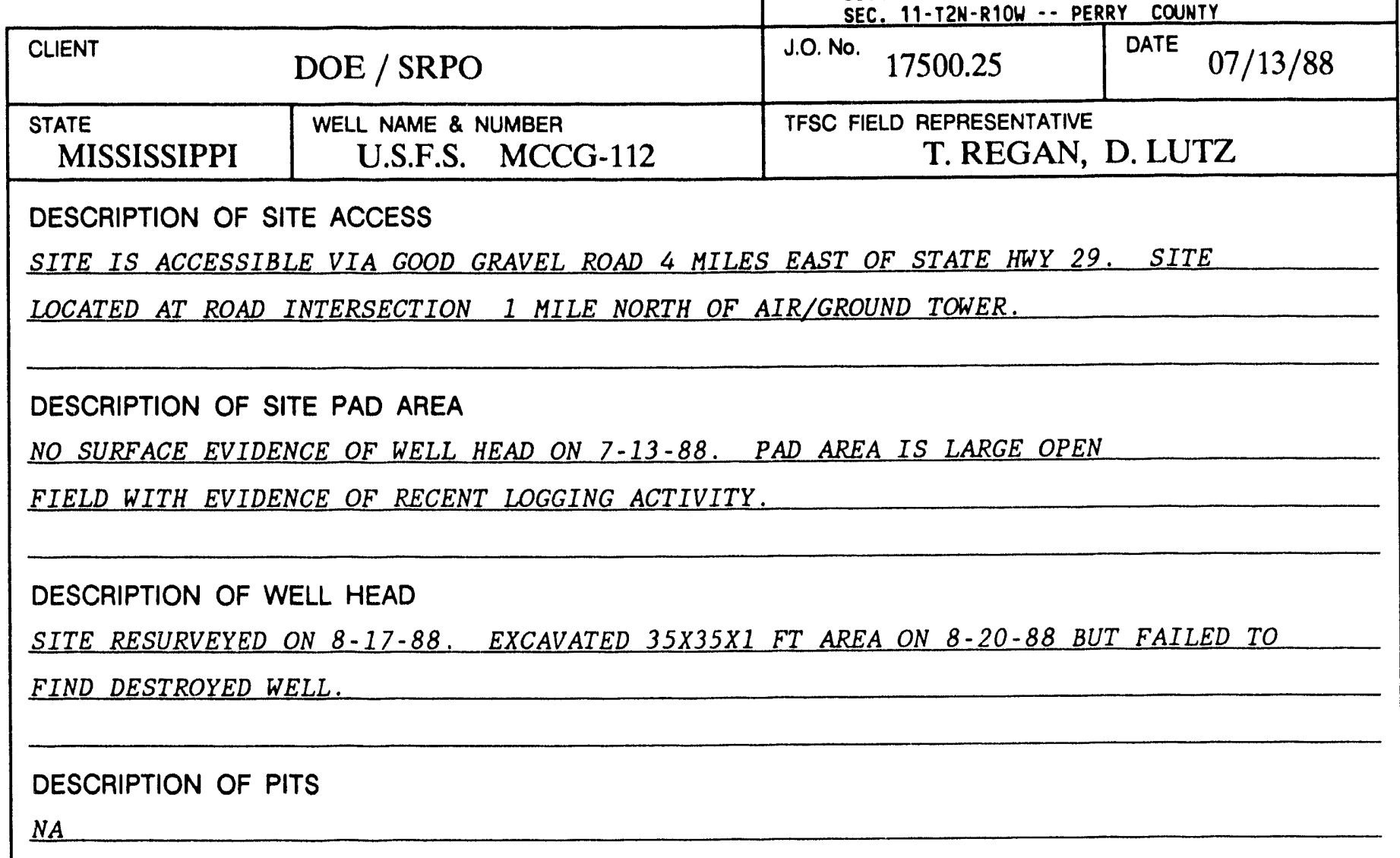

DEPTH AND BOREHOLE - FLUID SURVEY:

GROUND ELEVATION 317.7 FT (DATUM)

REPORTED UEPTH 180.5 FT (SEE BOREHOLE COMPLETION SKETCH)

MEASURED DEPTH NA OBSTRUCTION NT NA FT

$\begin{array}{lll}\text { FLUID DEPTH } & \mathrm{NA} & \mathrm{PH} \\ \text { CONDUCTIVITY } & \mathrm{NA} & \mathrm{NA} \\ \text { TEMPERATURE } & \mathrm{mS} / \mathrm{cm} & \mathrm{TDS} N \mathrm{NA} \\ \mathrm{N} & \mathrm{C} & \end{array}$

\section{REMARKS}

DESTRUCTION OF ORIGINAL SURVEY MONUMENTS SINCE 1979 PREVENTS ACCURATE RESURVEY OF WELL SITE. 
SITE RECONNAISSANCE FORM

914.4' SNL \& 710.0' EWL* IN

SEC. $17-$ T2N-R1OW - P PERRY COUNTY

\begin{tabular}{|l|c|c|c|}
\hline \multicolumn{2}{|c|}{ DLIENT } & J.0. No. 17500.25 & $\begin{array}{c}\text { DATE } \\
07 / 13 / 88\end{array}$ \\
\hline $\begin{array}{l}\text { STATE } \\
\text { MISSISSIPPI }\end{array}$ & $\begin{array}{c}\text { WELL NAME \& NUMBER } \\
\text { U.S.F.S. MCCG-113 }\end{array}$ & $\begin{array}{r}\text { TFSC FIELD REPRESENTATIVE } \\
\text { D. KISTNER, H. ABEDI }\end{array}$ \\
\hline $\begin{array}{l}\text { DESCRIPTION OF SITE ACCESS } \\
\text { WELL SITE IS 350 FT WEST OF STATE HWY 29 OFF OF RUTTED, BUT PASSABLE DIRT ROAD. }\end{array}$ \\
\hline
\end{tabular}

DESCRIPTION OF SITE PAD AREA

FLAT, OPEN AREA. WELL SURROUNDED BY $3 \times 3 \times 3$ FT PROTECTIVE BARRIER.

\section{DESCRIPTION OF WELL. HEAD}

2-IN (ID) CAPPED PVC CASING EXTENDS 0.5 FT ABOVE GL. 4-IN (ID) LOCKING STEEL GUARD PIPE EXTENDS 0.9 FT ABOVE GL. 6-IN (ID) PVC OUTER CASING TO 0.6 FT ABOVE GL.

\section{DESCRIPTION OF PITS}

NA

DEPTH AND BOREHOLE - FLUID SURVEY:BY MASCO (8/7/88)

GROUND ELEVATION 269.4 FT (DATUM)

REPORTED DEPTH 85.0 FT (SEE BOREHOLE COMPLETION SKETCH)

MEASURED DEPTH $82.0 \quad$ FT OBSTRUCTION NA FT

FLUID DEPTH $60.0 \quad$ FT $\quad \mathrm{pH}-9.76$

CONDUCTIVITY $0.140 \quad \mathrm{~ms} / \mathrm{cm} \quad$ TDS $70.0 \quad \mathrm{mg} / \mathrm{L}$

TEMPERATURE $24.1-{ }^{\circ} \mathrm{C}$

\section{REMARKS}

COORDINATE SITE ACCESS WITH CAMP SHELBY RANGE CONTROL. 3 FT OF SILT AT BOTTOM OF WELL. 
SITE RECONNAISSANCE FORM

LOCATION

296.7' NSL \& 1200.2' EWL* IN

SEC. 15-T2N-R1OW -. PERRY COUNTY

\begin{tabular}{|l|l|l|l|}
\hline \multicolumn{2}{|c|}{ DLIENT } & J.0. No. 17500.25 & DATE $/$ SRPO \\
\hline $\begin{array}{c}\text { STATE } \\
\text { MISSISSIPPI }\end{array}$ & $\begin{array}{l}\text { WELL NAME \& NUMBER } \\
\text { U.S.F.S. MCCG-114 }\end{array}$ & $\begin{array}{r}\text { TFSC FIELD REPRESENTATIVE } \\
\text { T. REGAN, D. LUTZ }\end{array}$ \\
\hline
\end{tabular}

DESCRIPTION OF SITE ACCESS

SITE LOCATED 32 FT NORTH OF DIRT ROAD. SITE ACCESS RESTRICTED BY CAMP SHELBY

RANGE CONTROL DUE TO PROXIMITY OF AIR/GROUND FIRING RANGE.

DESCRIPTION OF SITE PAD AREA

FLAT GRASSY FIELD WITH LOW BRUSH.

DESCRIPTION OF WELL HEAD

WELL HEAD BROKEN OFF AT GROUND LEVEL. WELL HEAD CONSISTS OF 2 -IN (ID) PVC

CASING CEMENTED INSIDE 4-IN (ID) STEEL GUARD PIPE, CEMENTED INSIDE 6-IN (ID) PVC

OUTER CASING.

DESCRIPTION OF PITS

NA

DEPTH AND BOREHOLE - FLUID SURVEY:BY SWEC $(8 / 24 / 88)$

GROUND ELEVATION 231.9 FT (DATUM)

REPORTED DEPTH

MEASURED DEPTH $72.0 \quad$ FT OBSTRUCTION NA

FLUID DEPTH $30.0 \quad$ FT $\quad \mathrm{pH}-6.32$

CONDUCTIVITY $0.033 \mathrm{mS} / \mathrm{cm} \quad \mathrm{mg} \quad 16.8 \mathrm{~L}$

TEMPERATURE $\quad 28.2-{ }^{\circ} \mathrm{C}$

REMARKS

ACCESS RESTRICTED, COORDINATE WITH RANGE CONTROL OFFICE. 3 FT OF SILT AT BOTTOM OF WELL. 
SITE RECONNAISSANCE FORM

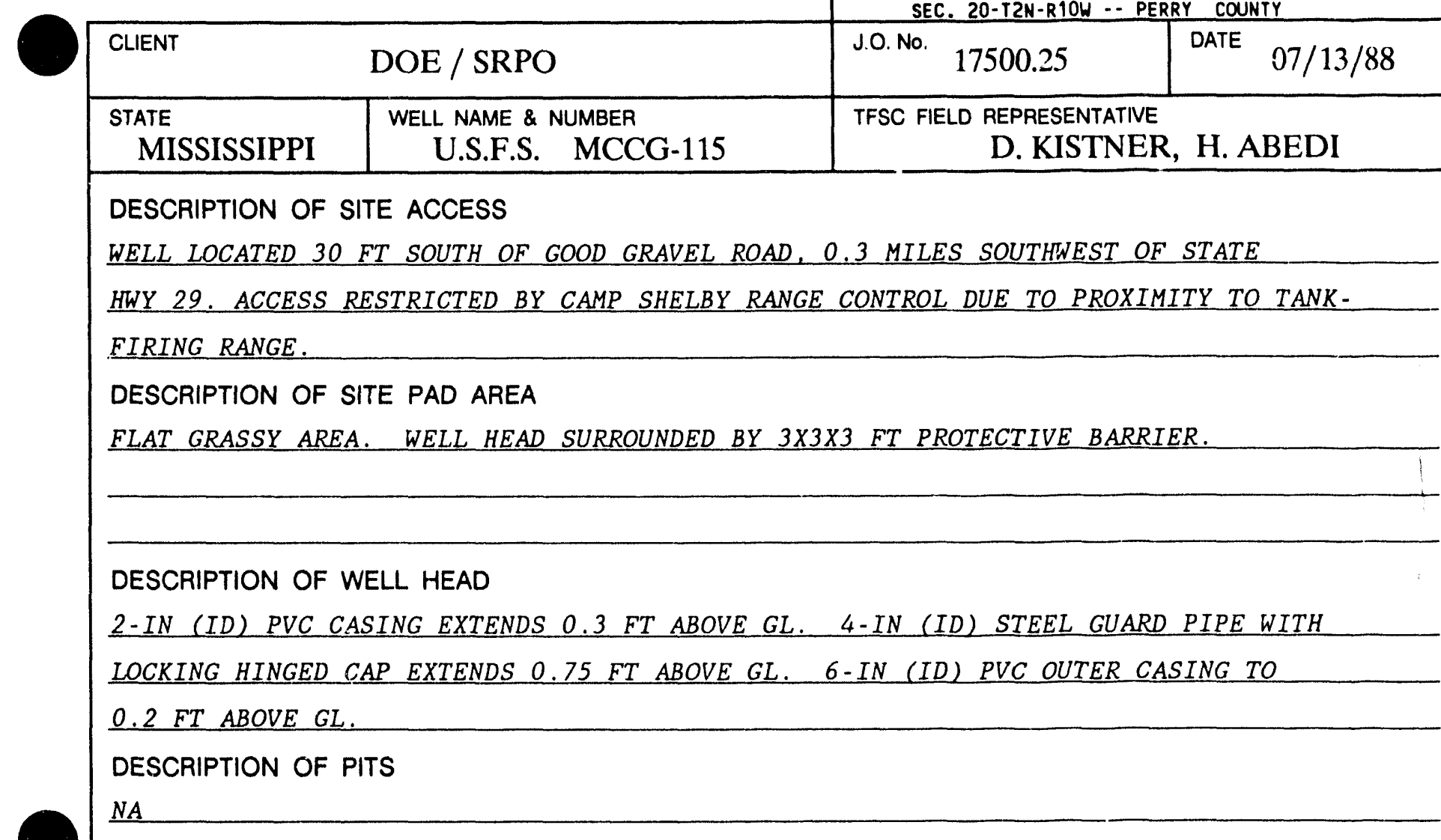

SEC. 20-T2N-R1OW - PERRY COUNTY

DEPTH AND BOREHOLE - FLUID SURVEY:BY MASCO $(8 / 7 / 88)$

GROUND ELEVATION 189.7 FT (DATUM)

REPORTED DEPTH 155.0 FT (SEE BOREHOLE COMPLETION SKETCH)

MEASURED DEPTH 150.0 FT OBSTRUCTION NA TT

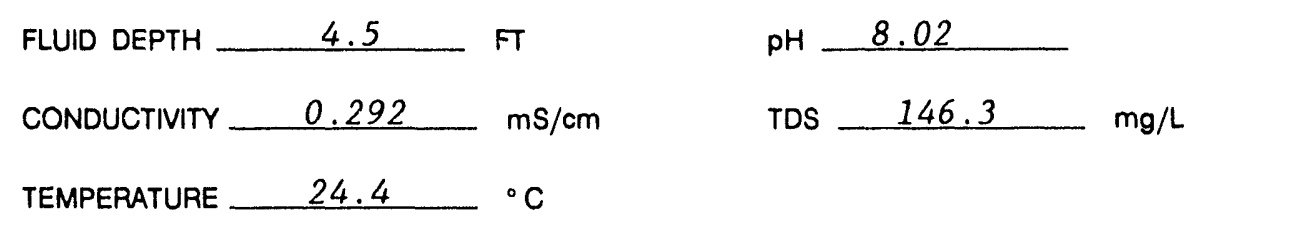

REMARKS

COORDINATE ACCESS WITH RANGE CONTROL OFFICE. 5.5 FT OF SILT AT BOTTOM OF WELL. 
SITE RECONNAISSANCE FORM

\begin{tabular}{|c|c|c|c|}
\hline CLIENT & DOE / SRPO & J.O. No. 17500.25 & DATE $07 / 13 / 88$ \\
\hline $\begin{array}{l}\text { STATE } \\
\text { MISSISSIPPI }\end{array}$ & $\begin{array}{l}\text { WELL NAME \& NUMBER } \\
\text { U.S.F.S. MCCG-116 }\end{array}$ & \multicolumn{2}{|c|}{$\begin{array}{l}\text { TFSC FIELD REPRESENTATIVE } \\
\text { T. REGAN, D. LUTZ }\end{array}$} \\
\hline \multicolumn{4}{|c|}{ DESCRIPTION OF SITE ACCESS } \\
\hline
\end{tabular}

\section{DESCRIPTION OF SITE PAD AREA}

NA

\section{DESCRIPTION OF WELL HEAD \\ $\underline{N A}$}

\section{DESCRIPTION OF PITS}

$\underline{N A}$

\section{DEPTH AND BOREHOLE - FLUID SURVEY:}

GROUND ELEVATION NA FT (DATUM)

REPORTED DEPTH 25.5 FT (SEE BOREHOLE COMPLETION SKETCH)

MEASURED DEPTH NA FT OBSTRUCTION NA FT

FLUID DEPTH

CONDUCTIVITY NA $\mathrm{NS} / \mathrm{cm} \mathrm{mg} \_\mathrm{NA} \mathrm{mg} / \mathrm{L}$

TEMPERATURE

REMARKS 
SITE RECONNAISSANCE FORM

\begin{tabular}{|c|c|c|c|}
\hline CLIENT & DOE / SRPO & J.O. No. 17500.25 & DATE $08 / 16 / 88$ \\
\hline $\begin{array}{l}\text { STATE } \\
\text { MISSISSIPPI }\end{array}$ & $\begin{array}{l}\text { WELL NAME \& NUMBER } \\
\text { U.S.F.S. MCCG-117 }\end{array}$ & \multicolumn{2}{|c|}{$\begin{array}{r}\text { TFSC FIELD REPRESENTATIVE } \\
\text { D. KISTNER } \\
\end{array}$} \\
\hline
\end{tabular}

\section{DESCRIPTION OF SITE PAD AREA}

WELL HEAD NOT LOCATED DURING INITIAL RECONNAISSANCE ON 7-13-88. RESURVEYED SITE

AND LOCATED WELL ON 8-16-88. TOP OF GUARD PIPE IS AT GROUND LEVEL, HIDDEN BY

TALL GRASS.

DESCRIPTION OF WELL HEAD

2-IN (ID) PVC CASING BROKEN OFF 0.5 FT BELOW GL, CLOGGED WITH DIRT AND CEMENT.

4-IN (ID) LOCKING STEEL GUARD PIPE IS LEVEL WITH GROUND SURFACE.

\section{DESCRIPTION OF PITS}

NA

DEPTH AND BOREHOLE - FLUID SURVEY:BY SWEC (9/1/88)

GROUND ELEVATION 246.2 FT (DATUM)

REPORTED DEPTH 110.0 FT (SEE BOREHOLE COMPLETION SKETCH)

MEASURED DEPTH 97.4 FT OBSTRUCTION NA FT

FLUID DEPTH $45.3 \quad \mathrm{FT} \quad \mathrm{PH}-6.22$

CONDUCTIVITY $0.088 \mathrm{~ms} / \mathrm{cm} \quad$ TDS $44.2 \mathrm{mg} / \mathrm{L}$

TEMPERATURE $22.1 \quad{ }^{\circ} \mathrm{C}$

\section{REMARKS}

12.6 FT OF SILT AT BOTTOM OF WELL. 
SITE RECONNAISSANCE FORM

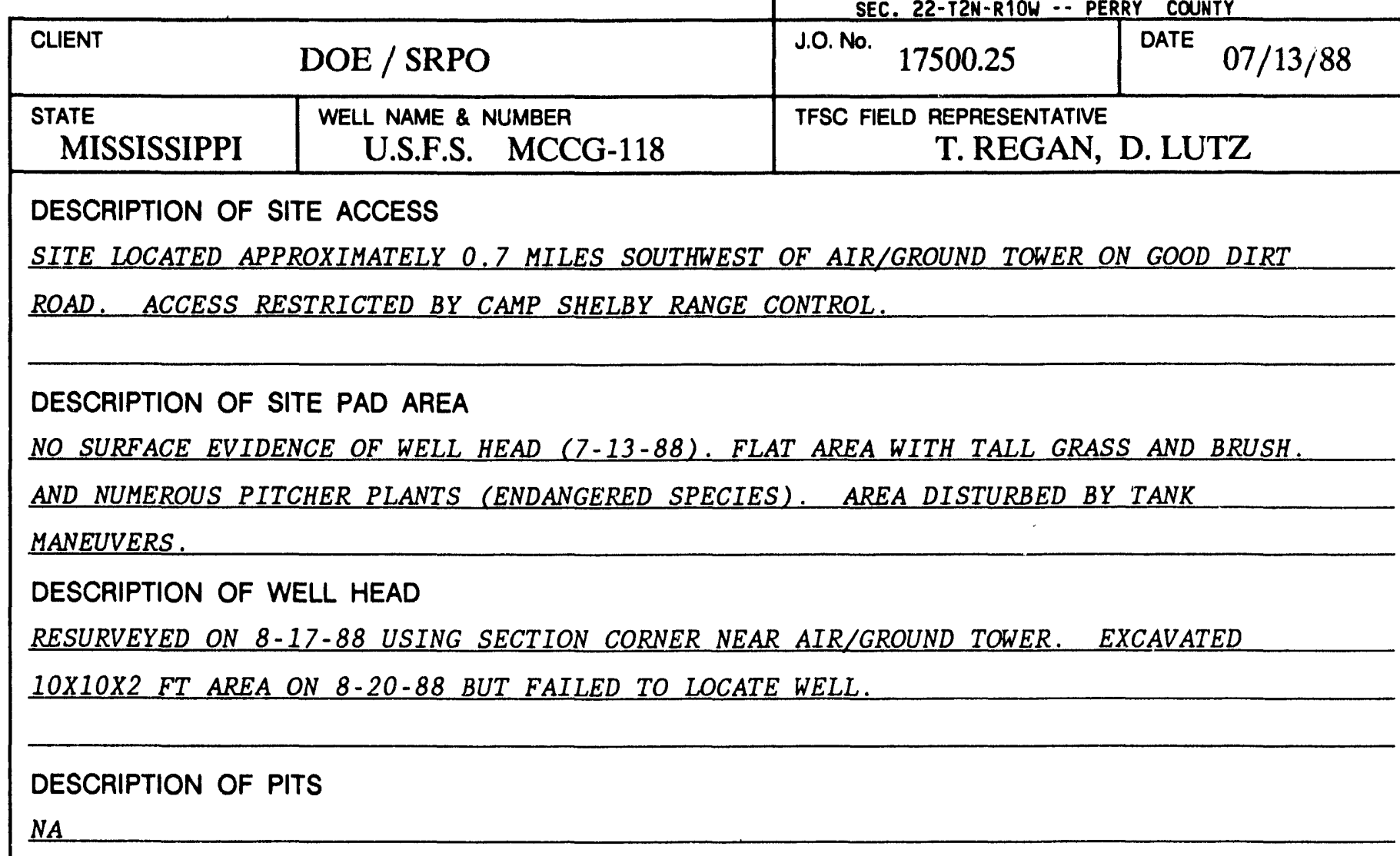

\footnotetext{
DEPTH AND BOREHOLE - FLUID SURVEY:

GROUND ELEVATION 235.3 FT (DATUM)

REPORTED DEPTH 100.5 FT (SEE BOREHOLE COMPLETION SKETCH)

MEASURED DEPTH NA FT OBSTRUCTION NA FT
FLUID DEPTH NA FT $\quad \mathrm{PH} \longrightarrow \mathrm{NA}$
CONDUCTIVITY NA $\mathrm{NA} \mathrm{ms} / \mathrm{cm} \mathrm{mg} / \mathrm{L}$

TEMPERATURE

\section{REMARKS}

PRESENCE OF PITCHER PLANT BOG AND POSSIBILITY OF UNEXPLODED SHELLS IN SUBSURFACE PRECLUDES FURTHER EXCAVATION.
} 
SITE RECONNAISSANCE FORM

LOCATION

2046.8' SNL \& 1281.4' EWL* IN

SEC. 33-T3N-R1OW - P PERRY COUNTY

CLIENT

DOE / SRPO

J.O. No.

17500.25

DATE

$08 / 19 / 88$

STATE

MISSISSIPPI
WELL NAME \& NUMBER

U.S.F.S. MCCG-119

TFSC FIELD REPRESENTATIVE

D. KISTNER

SITE IS 10 FT SOUTH OF GRAVEL ROAD.

\section{DESCRIPTION OF SITE PAD AREA}

WELL HEAD NOT LOCATED DURING RECONNAISSANCE OF 7-13-88. ON 8-18-88 BROKEN PVC CASING AND GUARD PIPE FOUND IN BLADED AREA OFF ROAD.

DESCRIPTION OF WELL HEAD

EXCAVATED AND FOUND BURIED WELL ON 8-19-88. 2-IN (ID) PVC CASING IS NOT CAPPED

BUT STILL OPEN.

DESCRIPTION OF PITS

NA

DEPTH AND BOREHOLE - FLUID SURVEY:BY SWEC (9/14/88)

GROUND ELEVATION 200.3 FT (DATUM)

REPORTED DEPTH $\quad 80.0 \quad$ FT (SEE BOREHOLE COMPLETION SKETCH)

MEASURED DEPTH 51.0 FT OBSTRUCTION $51 * *$ FT

FLUID DEPTH $11.4 \quad \mathrm{FT} \quad \mathrm{pH}-4.68$

CONDUCTIVITY $0.025 \mathrm{mS} / \mathrm{cm} \quad$ TDS $12.4 \mathrm{mg} / \mathrm{L}$

TEMPERATURE $\quad 26.4 \quad{ }^{\circ} \mathrm{C}$

\section{REMARKS}

** OBSTRUCTION DRILLED OUT ALONG WITH PVC CASING DURING PLUGGING. ACCESS ROAD NEEDS GRADING. 
SITE RECONNAISSANCE FORM

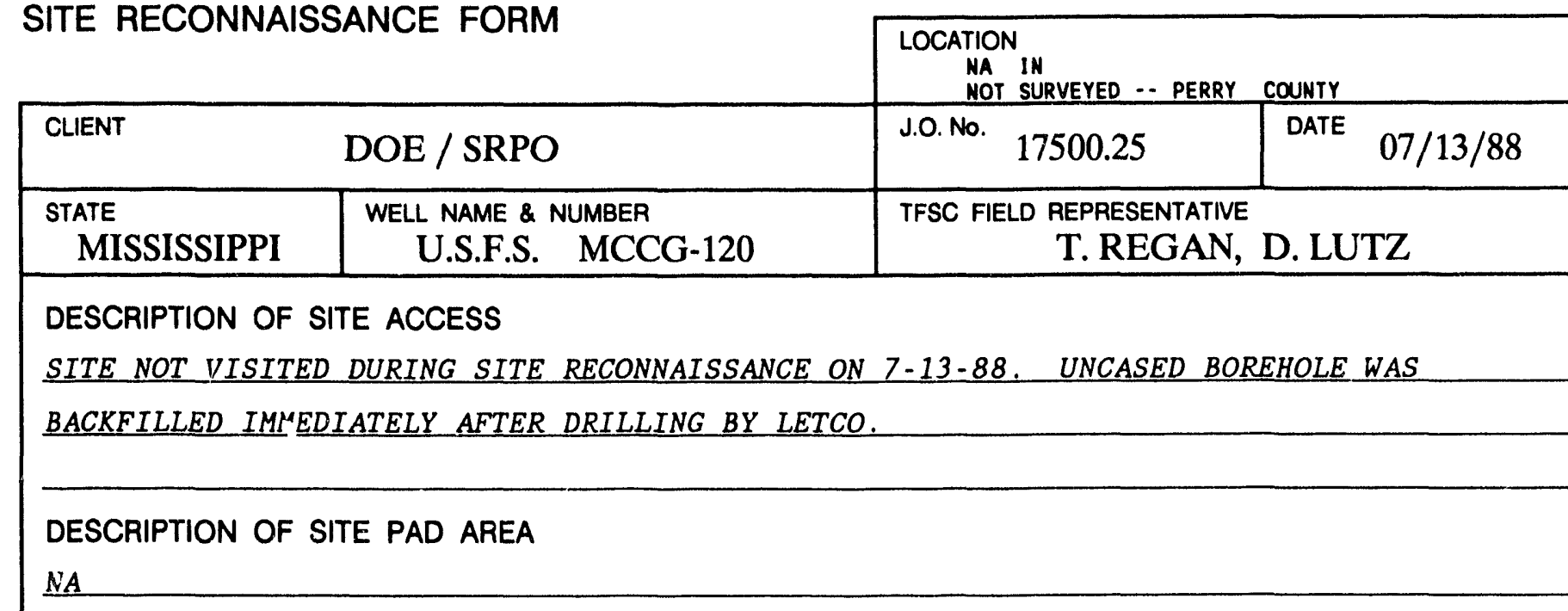

\section{DESCRIPTION OF WELL HEAD}

NA

\section{DESCRIPTION OF PITS}

NA

DEPTH AND BOREHOLE - FLUID SURVEY:

GROUND ELEVATION NA FT (DATUM)

REPORTED DEPTH 13.0 - FT (SEE BOREHOLE COMPLETION SKETCH)

MEASURED DEPTH NA FT OBSTRUCTION NA FT

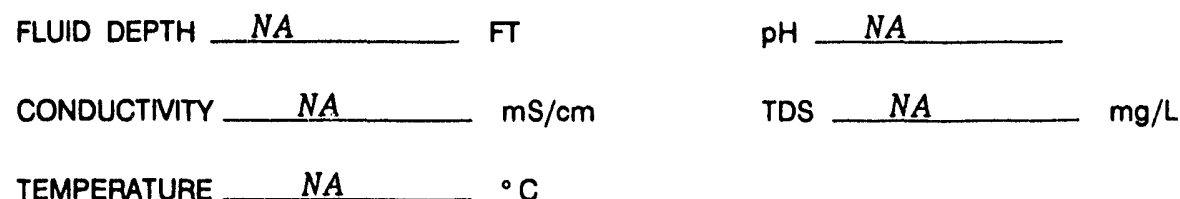

REMARKS 
SITE RECONNAISSANCE FORM

\begin{tabular}{|c|c|c|c|}
\hline \multicolumn{2}{|c|}{ SITE RECONNAISSANCE FORM } & \multicolumn{2}{|c|}{$\begin{array}{l}\text { LOCATION } \\
2565.3 \text { ENL \& } 41.2 \text { NSL* IN } \\
\text { SEC. } 22-T 5 N-R 10 W \text {.. PERRY COUNTY }\end{array}$} \\
\hline CLIENT & DOE / SRPO & J.O. No. 17500.25 & DATE $07 / 11 / 88$ \\
\hline $\begin{array}{l}\text { STATE } \\
\text { MISSISSIPPI }\end{array}$ & $\begin{array}{l}\text { WELL NAME \& NUMBER } \\
\text { L.R.F.P. MRIG-201 }\end{array}$ & $\begin{array}{r}\text { TFSC FIELD REPRESEN } \\
\text { D. KIS } \\
\end{array}$ & H. ABEDI \\
\hline \multicolumn{4}{|c|}{$\begin{array}{l}\text { DESCRIPTION OF SITE ACCESS } \\
\text { LOOP ROAD NORTH FROM HWY } 422.3 \mathrm{MI} \text {, THEN } 1.25 \mathrm{MI} \text { SW ON DIRT ROAD TO "T" }\end{array}$} \\
\hline \multicolumn{4}{|c|}{ INTERSECTION. WELL SITE IS 300 FT EAST OF INTERSECTION. } \\
\hline \multicolumn{4}{|c|}{$\begin{array}{l}\text { DESCRIPTION OF SITE PAD AREA } \\
\text { FLAT AREA WITH OVERHANGING TREES. WELL HEAD SURROUNDED BY } 3 \times 3 \times 3 \text { FT PROTECTIVE }\end{array}$} \\
\hline \multicolumn{4}{|l|}{ BARRIER. } \\
\hline \multicolumn{4}{|c|}{$\begin{array}{l}\text { DESCRIPTION OF WELL HEAD } \\
2-I N \text { (ID) PVC CASING, NOT CAPPED, EXTENDS } 0.85 \text { FT ABOVE GL. 4-IN (ID) LOCKING }\end{array}$} \\
\hline \multicolumn{4}{|c|}{ STEEL GUARD PIPE EXTENDS 0.8 FT ABOVE GL. PVC STICKUP MAKES IT DIFFICULT TO } \\
\hline \multicolumn{4}{|c|}{ LOCK CAP. } \\
\hline \multicolumn{4}{|c|}{$\begin{array}{l}\text { DESCRIPTION OF PITS } \\
N A\end{array}$} \\
\hline
\end{tabular}

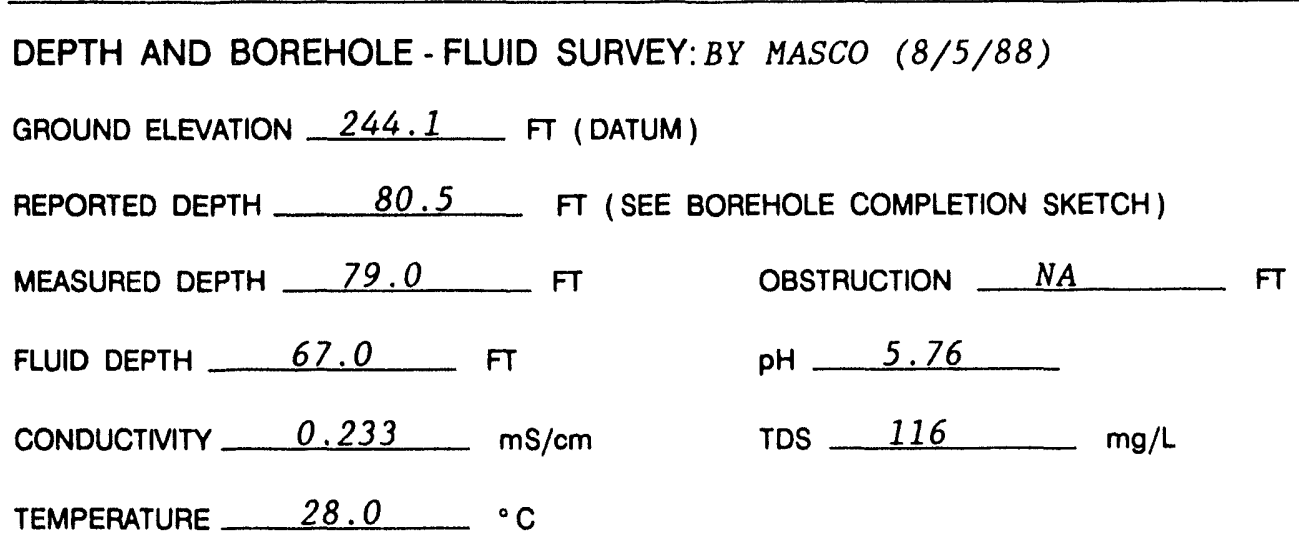

REMARKS

FINAL $1 / 2$ MILE TO SITE REQUIRES GRADING. 


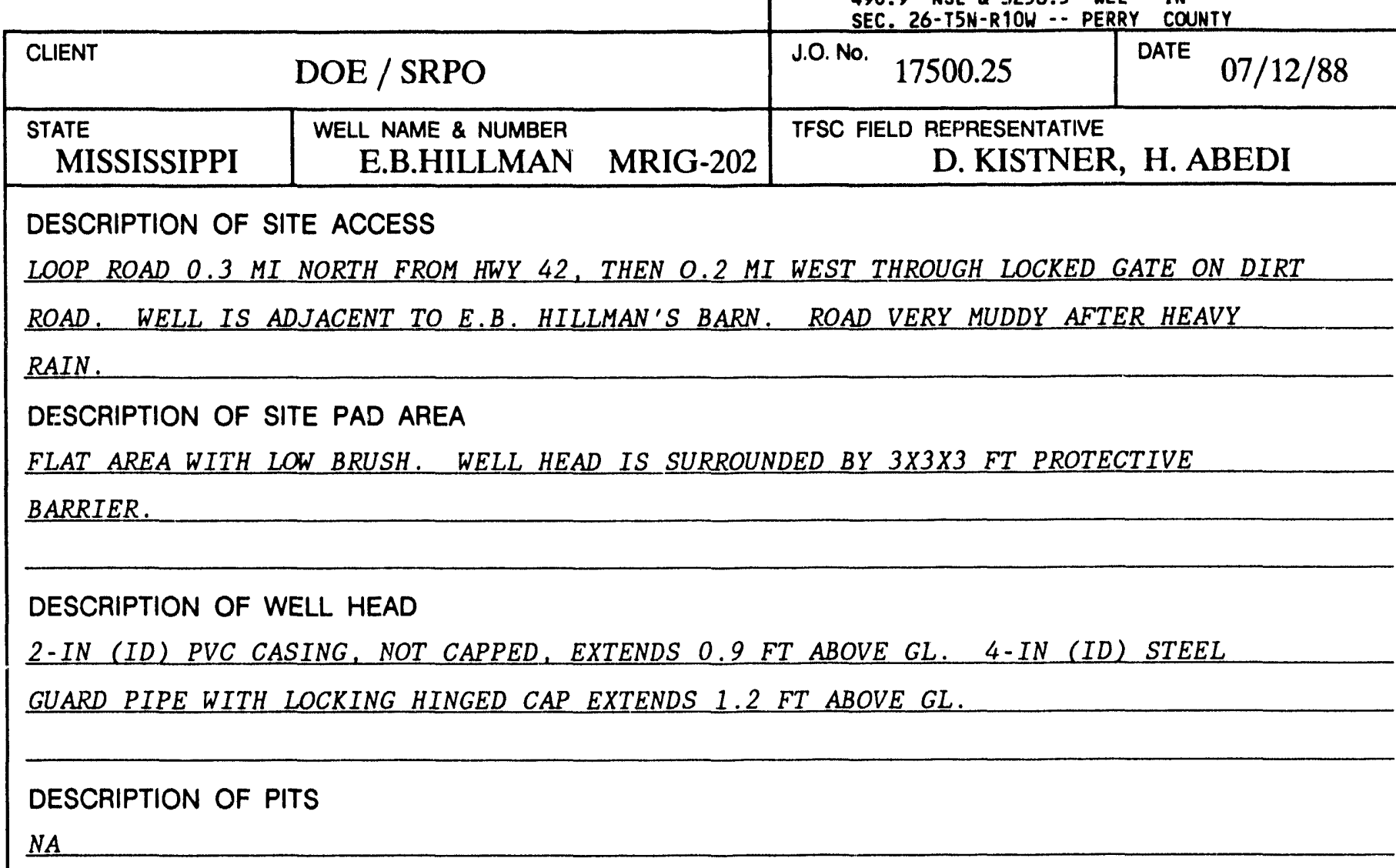

DEPTH AND BOREHOLE - FLUID SURVEY:BY SWEC (11/7/88)

GROUND ELEVATION _-281.5 FT (DATUM)

REPORTED DEPTH 200.0 FT (SEE BOREHOLE COMPLETION SKETCH)

MEASURED DEPTH 195.6 FT OBSTRUCTION NA FT

$\begin{array}{lll}\text { FLUID DEPTH } \frac{42.8}{\mathrm{~F}} \mathrm{FT} & \mathrm{PH} \frac{7.12}{\mathrm{0}} \mathrm{mS} / \mathrm{cm} & \mathrm{TDS} \frac{139.5}{\mathrm{mg} / \mathrm{L}}\end{array}$

TEMPERATURE

\section{REMARKS}

TO BE TURNED OVER TO LANDOWNER. RUTS IN ROAD REQUIRE BACKFILL WITH GRAVEL. 
SITE RECONNAISSANCE FORM

\begin{tabular}{|c|c|c|c|}
\hline \multicolumn{2}{|c|}{ SITE RECONNAISSANCE FORM } & \multicolumn{2}{|c|}{$\begin{array}{l}\text { LOCATION } \\
3429.91 \text { SNL \& } 1435.71 \text { ENL* IN } \\
\text { SEC. 23-T5N-R1OW } \because \text { PERRY COUNTY }\end{array}$} \\
\hline CLIENT & DOE / SRPO & J.O. No. 17500.25 & DATE $07 / 11 / 88$ \\
\hline $\begin{array}{l}\text { STATE } \\
\text { MISSISSIPPI }\end{array}$ & $\begin{array}{l}\text { WELL NAME \& NUMBER } \\
\text { L.R.F.P. } \quad \text { MRIG-203 }\end{array}$ & $\begin{array}{r}\text { TFSC FIELD REPRESEN } \\
\text { D. KIS }\end{array}$ & H. ABEDI \\
\hline \multicolumn{4}{|c|}{$\begin{array}{l}\text { DESCRIPTION OF SITE ACCESS } \\
\text { LOOP ROAD } 1.6 \mathrm{MI} \text { NORTH OF HWY 42. NELL IS } 66 \text { FT WEST OF PAVEMENT. SHALLOW }\end{array}$} \\
\hline \multicolumn{4}{|c|}{ GULLY MAY REQUIRE GRADING FOR RIG ACCESS. } \\
\hline \multicolumn{4}{|c|}{$\begin{array}{l}\text { DESCRIPTION OF SITE PAD AREA } \\
\text { WELL HEAD SURROUNDED BY } 3 \times 3 \times 3 \text { FT PROTECTIVE BARRIER AND BY OVERHANGING PINE }\end{array}$} \\
\hline \multicolumn{4}{|c|}{ SAPLINGS. } \\
\hline \multicolumn{4}{|c|}{ DESCRIPTION OF WELL. HEAD } \\
\hline \multicolumn{4}{|c|}{ 2-IN (ID) PVC CASING, NOT CAPPED, EXTENDS 0.35 FT ABOVE GL. 4-IN (ID) STEEL } \\
\hline \multicolumn{4}{|c|}{ GUARD PIPE WITH LOCKING HINGED CAP EXTENDS 1.0 FT ABOVE GL. } \\
\hline $\begin{array}{l}\text { DESCRIPTION OF } \\
N A\end{array}$ & & & \\
\hline
\end{tabular}
DEPTH AND BOREHOLE - FLUID SURVEY: BY MASCO (8/5/88)
GROUND ELEVATION 211.1 FT (DATUM)
REPORTED DEPTH $\frac{120.0}{2}$ FT (SEE BOREHOLE COMPLETION SKETCH)
MEASURED DEPTH $86.5 \quad$ FT OBSTRUCTION 86.5 FT
FLUID DEPTH $28.9 \quad \mathrm{FT} \quad \mathrm{PH}-6.69$
CONDUCTIVITY $0.515 \mathrm{~ms} / \mathrm{cm} \quad$ TDS $256 \quad \mathrm{mg} / \mathrm{L}$
TEMPERATURE $\quad 27.9{ }^{\circ} \mathrm{C}$

REMARKS

GRADE ROAD, REMOVE SAPLINGS. 
SITE RECONNAISSANCE FORM

\begin{tabular}{|l|l|l|l|}
\hline CLIENT & J.0. No. 17500.25 & DATE $07 / 11 / 88$ \\
\hline $\begin{array}{l}\text { STATE } \\
\text { MISSISSIPPI }\end{array}$ & $\begin{array}{r}\text { WELL NAME \& NUMBER } \\
\text { L.R.F.P. MRIG-204 }\end{array}$ & $\begin{array}{l}\text { TFSC FIELD REPRESENTATIVE } \\
\text { D. KISTNER, H. ABEDI }\end{array}$ \\
\hline $\begin{array}{l}\text { DESCRIPTION OF SITE ACCESS } \\
\text { LOOP ROAD 2.3 MI NORTH FROM HWY 42. WELL IS 46 FT EAST OF PAVEMENT. }\end{array}$
\end{tabular}

\section{DESCRIPTION OF SITE PAD AREA}

FLAT, OPEN AREA. WELL HEAD SUROUNDED BY $3 \times 3 \times 3$ FT PROTECTIVE BARRIER.

\section{DESCRIPTION OF WELL HEAD}

2-IN (ID) PVC CASING, NOT CAPPED, EXTENDS 0.4 FT ABOVE GL. 4-IN (ID) STEEL

GUARD PIPE, HINGED CAP RUSTED OFF, EXTENDS 0.6 FT ABOVE GL.

\section{IESCRIPTION OF PITS}

NA

DEPTH AND BOREHOLE - FLUID SURVEY:BY SWEC $(8 / 29 / 88)$

GROUND ELEVATION 275.1 FT (DATUM)

REPORTED DEPTH 65.0 FT (SEE BOREHOLE COMPLETION SKETCH)

MEASURED DEPTH 61.9 FT OBSTRUCTION NA FT

FLUID DEPTH $16.0 \quad$ FT $\quad \mathrm{PH}-5.65$

CONDUCTIVITY $0.061 \mathrm{~ms} / \mathrm{cm} \mathrm{mg} / \mathrm{L}$

TEMPERATURE $24.2{ }^{\circ} \mathrm{C}$

\section{REMARKS}

3.1 FT OF SILT AT BOTTOM OF WELL. DIRECT RIG ACCESS. 
SITE RECONNAISSANCE FORM

\begin{tabular}{|c|c|c|c|}
\hline \multicolumn{2}{|c|}{ SITE RECONNAISSANCE FORM } & \multicolumn{2}{|c|}{$\begin{array}{l}\text { LOCATION } \\
\text { 1017.2' SNL \& 545' EWL* IN } \\
\text { SEC. 22-T5N-R10W -.. PERRY COUNTY }\end{array}$} \\
\hline CLIENT & DOE / SRPO & I.O. No. 17500.25 & DATE $\quad 08 / 22 / 88$ \\
\hline $\begin{array}{l}\text { STATE } \\
\text { MISSISSIPPI }\end{array}$ & $\begin{array}{l}\text { WELL NAME \& NUMBER } \\
\text { L.R.F.P. MRIG-205 }\end{array}$ & $\begin{array}{r}\text { TFSC FIELD REPRESEN } \\
\text { D. KIS }\end{array}$ & H. ABEDI \\
\hline \multicolumn{4}{|c|}{ DESCRIPTION OF SITE ACCESS } \\
\hline \multicolumn{4}{|c|}{ DESCRIPTION OF SITE PAD AREA } \\
\hline \multicolumn{4}{|c|}{ PRESUMABLY WAS DESTROYED BY LOGGING TRUCKS. LOCATION RESURVEYED $(8-13-88)$. } \\
\hline \multicolumn{4}{|c|}{ DESCRIPTION OF WELL HEAD } \\
\hline \multicolumn{4}{|c|}{ EXCAVATED SITE AND LOCATED BURIED WELL ON 8-15-88. SITE IS DIRECTLY IN FRONT OF } \\
\hline \multicolumn{4}{|c|}{ GOPHER-TORTOISE BURROW. 2-IN (ID) CASING, NOT CAPPED, BURIED 2 FT. BELOW GL. } \\
\hline $\begin{array}{l}\text { DESCRIPTION OF } \\
\text { NA }\end{array}$ & & & \\
\hline
\end{tabular}

DEPTH AND BOREHOLE - FLUID SURVEY:BY SWEC (8/29/88)

GROUND ELEVATION 222.4 FT (DATUM)

REPORTED DEPTH 38.5 FT (SEE BOREHOLE COMPLETION SKETCH)

MEASURED DEPTH $33.0 \quad$ FT OBSTRUCTION NA FT

FLUID DEPTH $12.9 \quad \mathrm{FT} \quad \mathrm{PH} \_6.07$

CONDUCTIVITY $0.071 \mathrm{~ms} / \mathrm{cm} \quad$ TDS $35.6 \mathrm{mg} / \mathrm{L}$

TEMPERATURE $22.4{ }^{\circ} \mathrm{C}$

REMARKS

MUST PROTECT TORTOISE BURROW (ENDANGERED SPECIES). 5.5 FT OF SILT AT BOTTOM OF WELL. 
SITE RECONNAISSANCE FORM

\begin{tabular}{|l|l|l|l|}
\hline CLIENT & \multicolumn{2}{c|}{ SEC. 24-T5N-R10W - PERRY COUNTY } \\
\hline STATE & J.0. No. 17500.25 & DATE $08 / 22 / 88$ \\
MISSISSIPPI & $\begin{array}{r}\text { WELL NAME \& NUMBER } \\
\text { L.R.F.P. MRIG-208 }\end{array}$ & $\begin{array}{l}\text { TFSC FIELD REPRESENTATIVE } \\
\text { H. ABEDI }\end{array}$ \\
\hline
\end{tabular}

\section{DESCRIPTION OF SITE ACCESS}

STATE HWY 152.5 MI NORTH OF RICHTON, THEN 1.35 MI WEST ON GOOD GRAVEL ROAD TO

SITE. UNCASED BOREHOLE IMMEDIATELY BACKFILLED AFTER COMPLETION BY LETCO.

\section{DESCRIPTION OF SITE PAD AREA}

NO VISIBLE SIGN OF BACKFILLED BOREHOLE. AREA IS FLAT AND HEAVILY VEGETATED.

\section{DESCRIPTION OF WELL HEAD}

NA

\section{DESCRIPTION OF PITS}

NA

DEPTH AND BOREHOLE - FLUID SURVEY:

GROUND ELEVATION 223.4 FT (DATUM)

REPORTED DEPTH $75.5 \quad$ FT (SEE BOREHOLE COMPLETION SKETCH)

MEASURED DEPTH NA FT OBSTRUCTION NA FT

FLUID DEPTH NA FT PH NA

CONDUCTIVITY NA $\mathrm{NS} / \mathrm{cm} \mathrm{mg} / \mathrm{L}$

TEMPERATURE _ NA ${ }^{\circ} \mathrm{C}$

REMARKS 
SITE RECONNAISSANCE FORM

\begin{tabular}{|c|c|c|c|}
\hline \multicolumn{2}{|c|}{ SITE RECONNAISSANCE FORM } & \multicolumn{2}{|c|}{$\begin{array}{l}\text { LOCATION } \\
415, \text { SNL \& 675' WEL* IN } \\
\text { SEC. 23-T5N-R1OW -. PERRY COUNTY }\end{array}$} \\
\hline CLIENT & DOE / SRPO & J.O. No. 17500.25 & DATE $08 / 22 / 88$ \\
\hline $\begin{array}{l}\text { STATE } \\
\text { MISSISSIPPI }\end{array}$ & $\begin{array}{l}\text { WELL NAME \& NUMBER } \\
\text { L.R.F.P. } \quad \text { MRIG-209 }\end{array}$ & $\begin{array}{r}\text { TFSC FIELD REPRESE } \\
\mathrm{H} . \mathrm{AB}\end{array}$ & \\
\hline $\begin{array}{l}\text { DESCRIPTION OF } \\
\text { STATE HWY } 15 \quad 2\end{array}$ & $\begin{array}{l}\text { TE ACCESS } \\
\text { MI NORTH FROM RICHTON, }\end{array}$ & 5 MILES WEST ON & RAVEL ROAD \\
\hline \multicolumn{4}{|c|}{ (PAST GRAVEL PIT) TO SITE. ROADS SLIPPERY WHEN WET. } \\
\hline \multicolumn{4}{|c|}{ DESCRIPTION OF SITE PAD AREA } \\
\hline \multicolumn{4}{|c|}{ SECTION CORNER (8-13-88). EXCAVATED AND FOUND BURIED WELL $(8-13-88)$. } \\
\hline \multicolumn{4}{|c|}{ DESCRIPTION OF WELL HEAD } \\
\hline \multicolumn{4}{|c|}{ 2-IN (ID) PVC CASING, NOT CAPPED, BURIED 1.5 FT BELOW GL. } \\
\hline DESCRIPTION OF & & & \\
\hline
\end{tabular}


SITE RECONNAISSANCE FORM

\begin{tabular}{|l|r|}
\hline \multicolumn{3}{|c|}{ CLIENT } \\
\hline $\begin{array}{c}\text { STATE } \\
\text { MISSISSIPPI }\end{array}$ & $\begin{array}{l}\text { WELL NAME \& NUMBER } \\
\text { J.C.HILLMAN } \quad \text { MRIG-210 }\end{array}$ \\
\hline
\end{tabular}

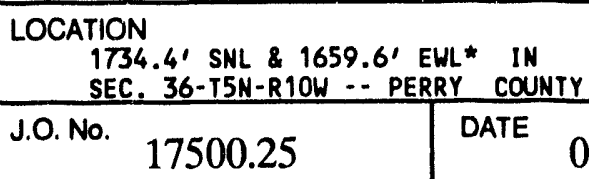

D. KISTNER, H. ABEDI

DESCRIPTION OF SITE ACCESS

STATE HWY 421.5 MI WEST OF RICHTON. WELL IS 450 FT NORTH OF HIGHWAY. EASY RIG ACCESS THROUGH LOCKED GATE.

\section{DESCRIPTION OF SITE PAD AREA}

FLAT, GRASSY COW PASTURE. WELL HEAD IS SURROUNDED BY $3 \times 3 \times 3$ FT PROTECTIVE BARRIER

DESCRIPTION OF WELL HEAD

2-IN (ID) PVC CASING, NOT CAPPED, EXTENDS 1.1 FT ABOVE GL. 4-IN (ID) STEEL GUARD PIPE WITH LOCKING HINGED CAP EXTENDS 1.2 FT ABOVE GL.

\section{DESCRIPTION OF PITS}

$\underline{N A}$

DEPTH AND BOREHOLE - FLUID SURVEY:BY SWEC (8/24/88)

GROUND ELEVATION 245.1 FT (DATUM)

REPORTED DEPTH 175.0 FT (SEE BOREHOLE COMPLETION SKETCH)

MEASURED DEPTH 174.8 FT OBSTRUCTION NA FT

FLUID DEPTH $\frac{51.5}{2}$ FT $\quad \mathrm{PH}-6.51$

CONDUCTIVITY $0.096-\mathrm{ms} / \mathrm{cm} \quad$ TDS $47.4 \quad \mathrm{mg} / \mathrm{L}$

TEMPERATURE $24.8-{ }^{\circ} \mathrm{C}$

\section{REMARKS}

WELL TO BE TURNED OVER TO LANDOWNER. 
SITE RECONNAISSANCE FORM

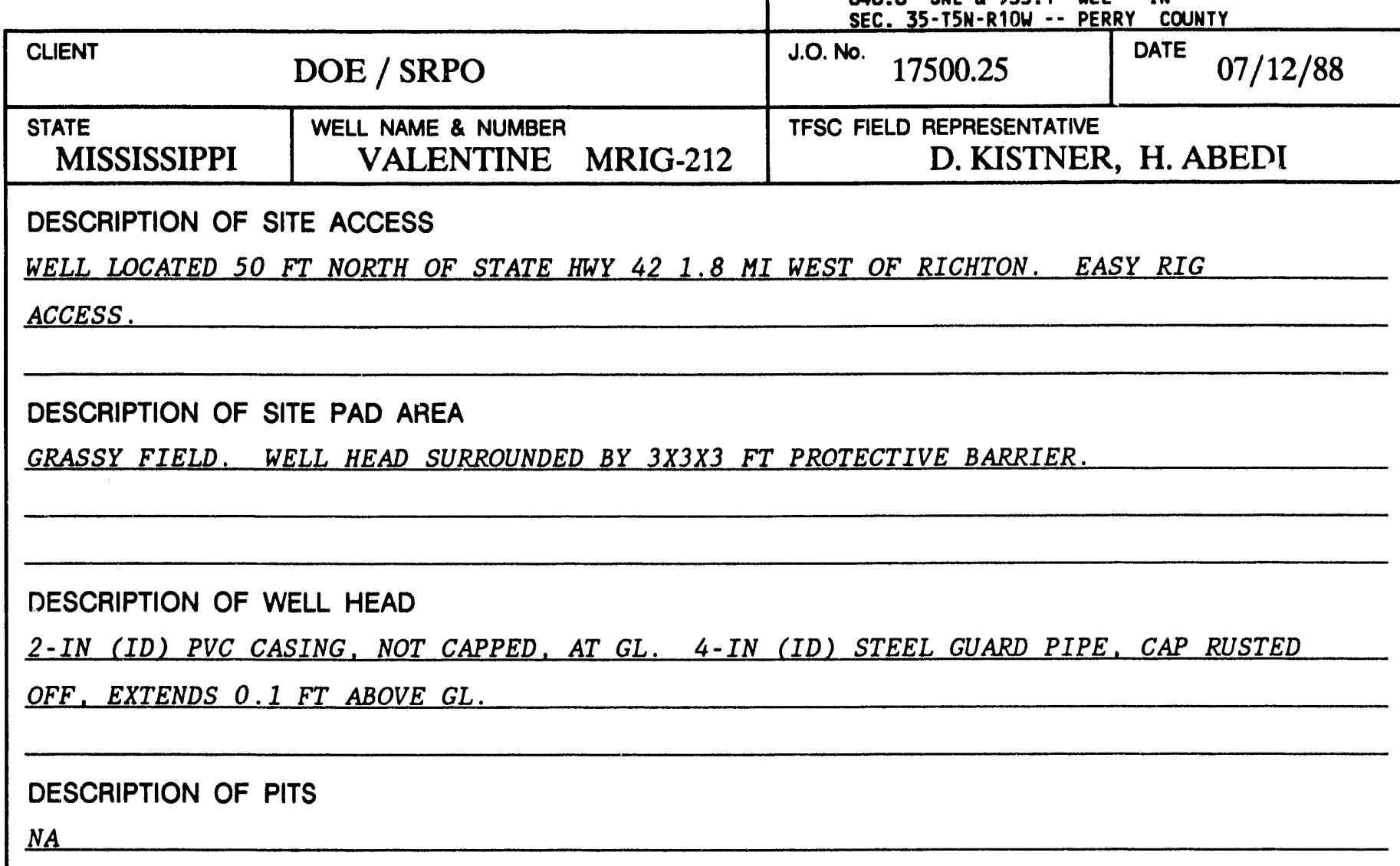

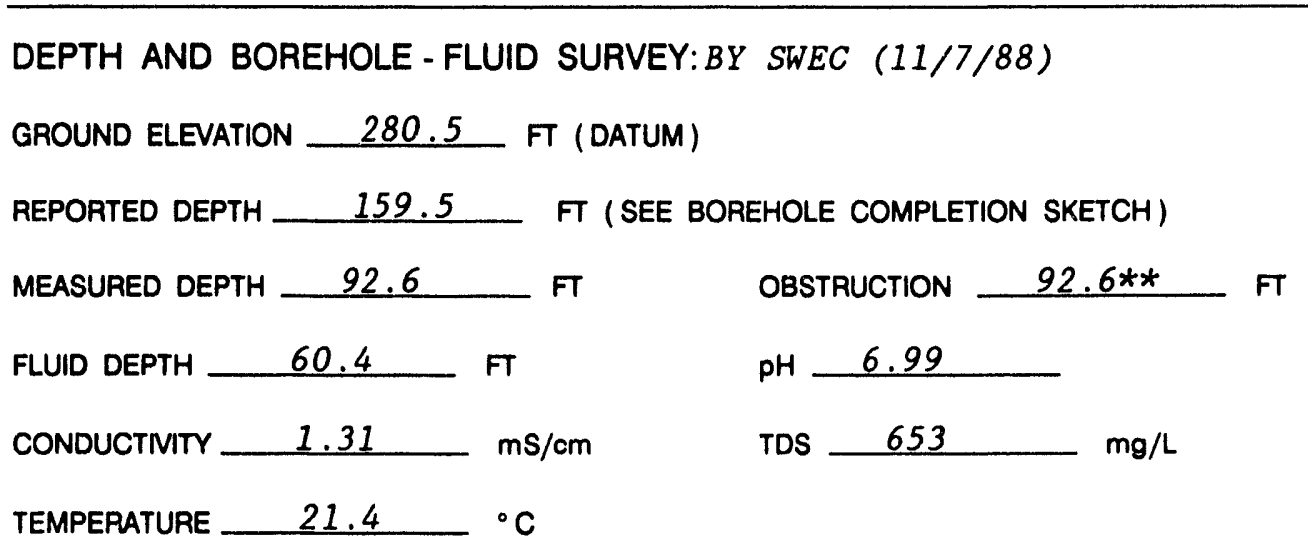

REMARKS

** CASING APPARENTLY DAMAGED DOWNHOLE. OBSTRUCTION DRILLED OUT ALONG WITH PVC CASING DURING PLUGGING. 
SITE RECONNAISSANCE FORM

CLIENT DOE / SRPO

OCATION

28.3' NSL \& 646! WEL" IN

SEC. 36-T5N-R10W -. PERRY COUNTY

P STATE WELL NAME \& NUMBER MISSISSIPPI MCLENDON MRIG-213 J.O. No. 17500.25 DATE $08 / 22 / 88$ TFSC FIELD REPRESENTATIVE DESCRIPTION OF SITE ACCESS WELL LOCATED IN VACANT DIRT LOT ADJACENT TO BUILDING OWNED BY MR. JOHN MCLENDON. DIRECT RIG ACCESS 38 FT. NORTH OF PAVEMENT AT OLD AUGUSTA ROAD AND ASH STREET.

DESCRIPTION OF SITE PAD AREA

NO SURFACE EVIDENCE OF WELL HEAD ON 7-12-88. WELL EXCAVATED AND REPAIRED

ON 8-20-88.

DESCRIPTION OF WELL HEAD

2-IN (ID) PVC CASING BURIED 1 FT BELOW GL. CASING NOT CAPPED BUT STILL OPEN TO SCREEN.

DESCRIPTION OF PITS

NA

DEPTH AND BOREHOLE - FLUID SURVEY:BY SWEC $(8 / 22 / 88)$

GROUND ELEVATION 178.3 FT (DATUM)

REPORTED DEPTH $\quad 84.0$ FT (SEE BOREHOLE COMPLETION SKETCH)

MEASURED DEPTH 73.8 FT OBSTRUCTION NA FT

FLUID DEPTH $11.6 \quad \mathrm{FT} \quad \mathrm{PH} \quad 5.95$

CONDUCTIVITY $0.168 \mathrm{~ms} / \mathrm{cm}$ TDS $84.2 \mathrm{mg} / \mathrm{L}$

TEMPERATURE

REMARKS

8.2. FT OF SILT AT BOTTOM OF WELL. 


\section{SITE RECONNAISSANCE FORM}

\begin{tabular}{|l|l|}
\hline CLIENT & DOE / SRPO \\
\hline STATE & WELL NAME \& NUMBER \\
MISSISSIPPI & H.K.HILLMAN MRIG-215 \\
\hline
\end{tabular}

\section{DESCRIPTION OF SITE ACCESS}

FROM DRIVEWAY OF MR. H.K. HILLMAN, WELL IS 1500 FT SOUTH ALONG FENCE MARKING THE WEST EDGE OF A LARGE CULTIVATED FIELD. ACCESS DIFFICULT WHEN WET.

\section{DESCRIPTION OF SITE PAD AREA}

WELL HEAD OBSCURED BY OVERHANGING BRUSH. WELL SURROUNDED BY $3 \times 3 \times 3$ FT PROTECTIVE

BARRIER.

\section{DESCRIPTION OF WELL HEAD}

2-IN (ID) PVC CASING, NOT CAPPED, EXTENDS 1.2 FT ABOVE GL. 4-IN (ID) STEEL GUARD PIPE WITH LOCKING HINGED CAP EXTENDS 1.4 FT ABOVE GL.

\section{DESCRIPTION OF PITS}

NA

DEPTH AND BOREHOLE - FLUID SURVEY:BY SWEC (11/7/88)

GROUND ELEVATION 220.2 FT (DATUM)

REPORTED DEPTH 110.0 FT (SEE BOREHOLE COMPLETION SKETCH)

MEASURED DEPTH 103.6 FT OBSTRUCTION NA

FLUID DEPTH $27.7 \quad \mathrm{FT} \quad \mathrm{pH} \quad 6.78$

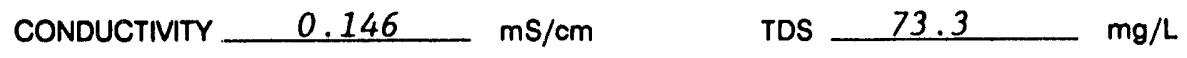

TEMPERATURE 21.1

REMARKS

6.4 FT OF SILT AT BOTTOM OF WELL. 
SITE RECONNAISSANCE FORM

\begin{tabular}{|c|c|c|c|}
\hline \multicolumn{2}{|c|}{ SITE RECONNAISSANCE FORM } & \multicolumn{2}{|c|}{ 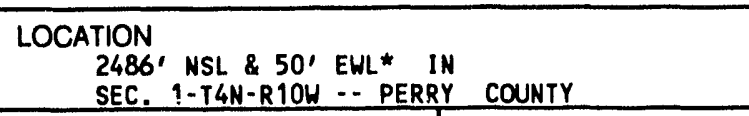 } \\
\hline CLIENT & DOE / SRPO & J.O. No. $\quad 17500.25$ & DATE $07 / 12 / 88$ \\
\hline $\begin{array}{l}\text { STATE } \\
\text { MISSISSIPPI }\end{array}$ & $\begin{array}{l}\text { WELL. NAME \& NUMBER } \\
\text { MCLENDON MRIG-216 }\end{array}$ & $\begin{array}{r}\text { TFSC FIELD REPRESEN } \\
\text { T. REC }\end{array}$ & LUTZ \\
\hline \multicolumn{4}{|c|}{$\begin{array}{l}\text { DESCRIPTION OF SITE ACCESS } \\
\text { FROM OLD AUGUSTA ROAD TURN SE ON DIRT ROAD FOR } 170 \text { FT THROUGH GATE, THEN TURN SW }\end{array}$} \\
\hline \multicolumn{4}{|c|}{ FOR 175 FT TO WELL. WELL IS UPHILL FROM FIELD LITTERED WITH AUTO PARTS. } \\
\hline \multicolumn{4}{|c|}{ DESCRIPTION OF SITE PAD AREA } \\
\hline
\end{tabular}

DESCRIPTION OF WELL HEAD

2-IN (ID) OPEN PVC CASING AND 4-IN (ID) LOCKING STEEL GUARD PIPE BOTH EXTEND

1.8 FT ABOVE GL.

DESCRIPTION OF PITS

NA

DEPTH AND BOREHOLE - FLUID SURVEY:BY MASCO (8/5/88)

GROUND ELEVATION 218.5 FT (DATUM)

REPORTED DEPTH 109.0 FT (SEE BOREHOLE COMPLETION SKETCH)

MEASURED DEPTH 106.2 FT OBSTRUCTION NA FT

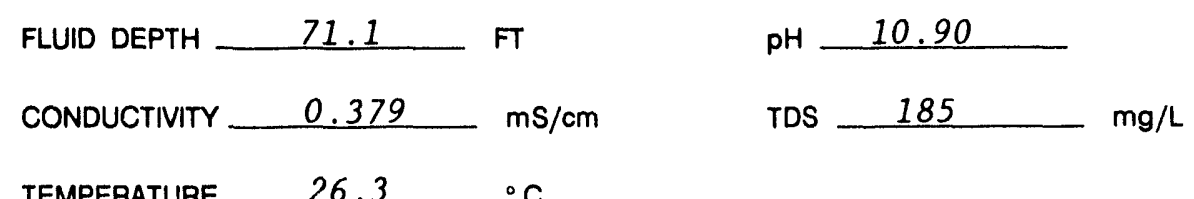

TEMPERATURE $26.3 \quad{ }^{\circ} \mathrm{C}$

REMARKS

2.8 FT OF SILT AT BOTTOM OF WELL. 
SITE RECONNAISSANCE FORM

\begin{tabular}{|c|c|c|c|}
\hline & & \multicolumn{2}{|c|}{ SEC. $11-T 4 N-R 1 O W \cdots$ PERRY COUNTY } \\
\hline \multicolumn{2}{|c|}{ CLIENT $\quad$ DOE / SRPO } & J.O. No. 17500.25 & DATE $07 / 14 / 88$ \\
\hline $\begin{array}{l}\text { STATE } \\
\text { MISSISSIPPI }\end{array}$ & $\begin{array}{l}\text { WELL NAME \& NUMBER } \\
\text { GODFREY MRIG-217 }\end{array}$ & \multicolumn{2}{|c|}{$\begin{array}{l}\text { TFSC FIELD REPRESENTATIVE } \\
\text { D. KISTNER, H. ABEDI }\end{array}$} \\
\hline \multicolumn{4}{|c|}{$\begin{array}{l}\text { DESCRIPTION OF SITE ACCESS } \\
\text { NOT VISITED DURING SITE RECONNAISSANCE ON 7-12-88. NO CURRENT ACCESS AGREEMENT. }\end{array}$} \\
\hline \multicolumn{4}{|c|}{ LANDOWNER DENIED SWEC SITE ACCESS FOR RECON, SAMPLE OR PHOTOGRAPHS. } \\
\hline
\end{tabular}

DESCRIPTION OF WELL HEAD

NA

\section{DESCRIPTION OF PITS}

NA

DEPTH AND BOREHOLE - FLUID SURVEY:

GROUND ELEVATION 269.8 FT (DATUM)

REPORTED DEPTH 169.0 FT (SEE BOREHOLE COMPLETION SKETCH)

MEASURED DEPTH NA FT OBSTRUCTION NA F F

FLUID DEPTH NA FT $\quad \mathrm{PH} \perp \mathrm{NA}$

CONDUCTIVITY NA $\mathrm{ms} / \mathrm{cm} \quad$ TDS NA $\mathrm{Ng} / \mathrm{L}$

TEMPERATURE

\section{REMARKS}

WELL TURNED OVER TO LANDOWNER BY LETCO IN 1980. 
SITE RECONNAISSANCE FORM

\begin{tabular}{|c|c|c|c|}
\hline CLIENT & DOE / SRPO & J.O. No. 17500.25 & DATE $07 / 12 / 88$ \\
\hline $\begin{array}{l}\text { STATE } \\
\text { MISSISSIPPI }\end{array}$ & $\begin{array}{l}\text { WELL NAME \& NUMBER } \\
\text { E.B.HILLMAN } \quad \text { MRIG-219 }\end{array}$ & \multicolumn{2}{|c|}{$\begin{array}{l}\text { TFSC FIELD REPRESENTATIVE } \\
\text { D. KISTNER, H. ABEDI }\end{array}$} \\
\hline \multicolumn{4}{|c|}{$\begin{array}{l}\text { DESCRIPTION OF SITE ACCESS } \\
\text { STATE HWY } 42 \quad 3.1 \mathrm{MI} \text { WEST OF RICHTON, THEN THROUGH LOCKED GATE FOR } 0.55 \mathrm{MI} \text { ON }\end{array}$} \\
\hline \multicolumn{4}{|c|}{$\begin{array}{l}\text { DIRT ROAD WEST OF CULTIVATED FIELD. ROAD IS BADLY RUTTED IN SPOTS. THEN TURN } \\
\text { NW FOR } 300 \mathrm{FT} \text { ON ROAD THROUGH WOODS. }\end{array}$} \\
\hline
\end{tabular}

\section{DESCRIPTION OF WELL HEAD}

2-IN (ID) OPEN PVC CASING EXTENDS 1.2 FT ABOVE CONCRETE APRON. 4-IN (ID) STEEL GUARD PIPE WITH LOCKING HINGED CAP EXTENDS 1.4 FT ABOVE CONCRETE.

\section{DESCRIPTION OF PITS}

$\underline{N A}$

DEPTH AND BOREHOLE - FLUID SURVEY:BY MASCO $(8 / 5 / 88)$

GROUND ELEVATION 239.7 FT (DATUM)

REPORTED DEPTH 106.0 FT (SEE BOREHOLE COMPLETION SKETCH)

MEASURED DEPTH 96.0 FT OBSTRUCTION NA FT

FLUID DEPTH $50.5 \quad \mathrm{FT} \quad \mathrm{PH}-9.02$

CONDUCTIVITY $0.193 \mathrm{mS} / \mathrm{cm} \quad$ TDS $95.9 \quad \mathrm{mg} / \mathrm{L}$

TEMPERATURE $25.0 \quad{ }^{\circ} \mathrm{C}$

\section{REMARKS}

NOTIFY LANDOWNER BEFORE ENTERING THROUGH LOCKED GATE. 10 FT OF SILT AT BOTTOM OF WELL. 
SITE RECONNAISSANCE FORM

\begin{tabular}{|c|c|c|c|}
\hline CLIENT & DOE / SRPO & J.O. No. 17500.25 & DATE $10 / 06 / 88$ \\
\hline $\begin{array}{l}\text { STATE } \\
\text { MISSISSIPPI }\end{array}$ & $\begin{array}{l}\text { WELL NAME \& NUMBER } \\
\text { OLIPHANT MRIG-220 }\end{array}$ & \multicolumn{2}{|c|}{$\begin{array}{c}\text { TFSC FIELD REPRESENTATIVE } \\
\text { T. REGAN }\end{array}$} \\
\hline \multicolumn{4}{|c|}{$\begin{array}{l}\text { DESCRIPTION OF SITE ACCESS } \\
\text { FROM INTERSECTION OF OAK AND PECAN STS CONTINUE SOUTH ON PECAN ST (DIRT ROAD) }\end{array}$} \\
\hline \multicolumn{4}{|c|}{ FOR O.Z MILES. WELL IS 200 FT WEST OF ROAD THROUGH WOODEN GATE. } \\
\hline
\end{tabular}

DESCRIPTION OF WELL HEAD

2-IN (ID) OPEN PVC CASING AND 4-IN (ID) LOCKING STEEL GUARD PIPE BOTH EXTEND

1.0 FT ABOVE GL.

\section{DESCRIPTION OF PITS}

NA

DEPTH AND BOREHOLE - FLUID SURVEY:BY SWEC $(10 / 6 / 88)$

GROUND ELEVATION 215.7 FT (DATUM)

REPORTED DEPTH $\quad 78.5$ FT (SEE BOREHOLE COMPLETION SKETCH)

MEASURED DEPTH 74.0 FT OBSTRUCTION NA FT

FLUID DEPTH $64.9 \quad \mathrm{FT} \quad \mathrm{pH}+6.64$

CONDUCTIVITY $0.121-\mathrm{mS} / \mathrm{cm} \quad$ TDS 61.1

TEMPERATURE $11.2{ }^{\circ} \mathrm{C}$

\section{REMARKS}

LANDOWNER MUST ACCOMPANY ALL VISITORS TO SITE. 4 FT OF SILT AT BOTTOM OF WELL. 
SITE RECONNAISSANCE FORM

LOCATION

$730.5^{\prime}$ NSL \& 118.3' WEL* IN

SEC. $35-T 5 N-R 10 \mathrm{H}$ - P PERRY COUNTY

CLIENT

DOE / SRPO

J.O. No.

17500.25

DATE

$07 / 12 / 88$

STATE

WELL NAME \& NUMBER

TFSC FIELD REPRESENTATIVE

MISSISSIPPI

E.B.HILLMAN MRIG-222

D. KISTNER, H. ABEDI

DESCRIPTION OF SITE ACCESS

SITE ACCESSIBLE FROM HICKORY LANE (BEFORE TURNOFF TO E.B. HILLMAN'S HOME). IURN SOUTH ON GRASSY TRAIL FOR 550 FT TO CORRAL AND SMALL BARN. WELL IS 50 FT EAST THROUGH 2 GATES.

DESCRIPTION OF SITE PAD AREA

WELL OBSCURED BY HEAVY UNDERBRUSH AND OVERHANGING TREES. WELL HEAD SURROUNDED

BY $3 \times 3 \times 3$ FT PROTECTIVE BARRIER.

DESCRIPTION OF WELL HEAD

4-IN (ID) CAPPED PVC CASING EXTENDS 3 FT ABOVE GL.

DESCRIPTION OF PITS

NA

DEPTH AND BOREHOLE - FLUID SURVEY:BY SWEC (11/2/88)

GROUND ELEVATION 209.4 FT (DATUM)

REPORTED DEPTH $\quad 52.0 \quad$ FT (SEE BOREHOLE COMPLETION SKETCH)

MEASURED DEPTH $50.0 \quad$ FT OBSTRUCTION NA

FLUID DEPTH $15.0 \quad$ FT $\quad \mathrm{pH} \quad 5.19$

CONDUCTIVITY $-0.089-\mathrm{ms} / \mathrm{cm} \quad$ TDS $-44.6-\mathrm{mg} / \mathrm{L}$

TEMPERATURE

REMARKS

TO BE TURNED OVER TO LANDOWNER. 
SITE RECONNAISSANCE FORM

\begin{tabular}{|c|c|c|c|}
\hline CLIENT & DOE / SRPO & J.O. No. 17500.25 & DATE $07 / 12 / 88$ \\
\hline $\begin{array}{l}\text { STATE } \\
\text { MISSISSIPPI }\end{array}$ & $\begin{array}{l}\text { WELL NAME \& NUMBER } \\
\text { CLEARMAN MRIG-223 }\end{array}$ & \multicolumn{2}{|c|}{$\begin{array}{l}\text { TFSC FIELD REPRESENTATIVE } \\
\text { T. REGAN, D. LUTZ }\end{array}$} \\
\hline \multicolumn{4}{|c|}{ DESCRIPTION OF SITE ACCESS } \\
\hline \multicolumn{4}{|c|}{ SITE AT HUNTING CAMP. WELL IS SOUTH OF ROAD, EAST OF CAMP IN VEGETATED AREA, } \\
\hline \multicolumn{4}{|c|}{ ENCLOSED BY WOODEN FENCE. } \\
\hline \multicolumn{4}{|c|}{ DESCRIPTION OF SITE PAD AREA } \\
\hline \multicolumn{4}{|c|}{ HEAVY UNDERBRUSH. 1.5 FT HIGH WOODEN BARRIER AROUND WELL. } \\
\hline \multicolumn{4}{|c|}{ DESCRIPTION OF WELL HEAD } \\
\hline \multicolumn{4}{|c|}{ 4-IN (ID) PVC CASING WITH THREADED CAP EXTENDS 0.7 FT ABOVE GL. } \\
\hline \multicolumn{4}{|c|}{ DESCRIPTION OF PITS } \\
\hline \multicolumn{4}{|l|}{$\underline{N A}$} \\
\hline
\end{tabular}

DEPTH AND BOREHOLE - FLUID SURVEY:BY SWEC $(11 / 1 / 88)$

GROUND ELEVATION 265.5 FT (DATUM)

REPORTED DEPTH 125.0 FT (SEE BOREHOLE COMPLETION SKETCH)

MEASURED DEPTH 125.0 FT OBSTRUCTION NA FT

FLUID DEPTH 77.1 FT $\quad \mathrm{PH}-5.5$

CONDUCTIVITY $0.076 \mathrm{~ms} / \mathrm{cm} \quad$ TDS $38.2 \mathrm{mg} / \mathrm{L}$

TEMPERATURE $19.1 \quad{ }^{\circ} \mathrm{C}$

REMARKS

TO BE TURNED OVER TO LANDOWNER. 
SITE RECONNAISSANCE FORM

\begin{tabular}{|c|c|c|c|}
\hline CLIENT & DOE / SRPO & J.O. No. 17500.25 & DATE $09 / 01 / 88$ \\
\hline $\begin{array}{l}\text { STATE } \\
\text { MISSISSIPPI }\end{array}$ & $\begin{array}{l}\text { WELL NAME \& NUMBER } \\
\text { PHILLIPS MRIG-224 }\end{array}$ & \multicolumn{2}{|c|}{$\begin{array}{c}\text { TFSC FIELD REPRESENTATIVE } \\
\text { T.REGAN }\end{array}$} \\
\hline \multicolumn{4}{|c|}{ DESCRIPTION OF SITE ACCESS } \\
\hline \multicolumn{4}{|c|}{ J.P. HINTON ROAD. NO ACCESS PROBLEMS, BUT NEED TO RENEW AGREEMENT } \\
\hline \multicolumn{4}{|c|}{ DESCRIPTION OF SITE PAD AREA } \\
\hline \multicolumn{4}{|c|}{ MR PHILLIPS HAD CUT OFF CASING I FT BELOW GL, CAPPED AND BURIED WELL. PAD IS } \\
\hline
\end{tabular}

DESCRIPTION OF WELL HEAD

WELL LOCATED WITH METAL DETECTOR AND EXCAVATED ON $9 / 1 / 88$

\section{DESCRIPTION OF PITS}

NA

DEPTH AND BOREHOLE - FLUID SURVEY:BY SWEC $(9 / 1 / 88)$

GROUND ELEVATION 261.8 FT (DATUM)

REPORTED DEPTH 130.0 FT (SEE BOREHOLE COMPLETION SKETCH)

MEASURED DEPTH

FLUID DEPTH DRY FT $\quad \mathrm{pH}-\mathrm{NA}$

CONDUCTIVITY

TEMPERATURE

REMARKS

ACCESS AGREEMENT RENEWED ON $8 / 31 / 88$. 
SITE RECONNAISSANCE FORM

\begin{tabular}{|c|c|c|c|c|}
\hline \multirow{2}{*}{\multicolumn{3}{|c|}{$\begin{array}{ll}\text { CLIENT } & \text { DOE / SRPO }\end{array}$}} & \multicolumn{2}{|r|}{ RY COUNTY } \\
\hline & & & J.O. No. 17500.25 & DATE $07 / 12 / 88$ \\
\hline $\begin{array}{l}\text { STATE } \\
\text { MISSISSIPPI }\end{array}$ & $\begin{array}{r}\text { WELL NAME \& NUMBER } \\
\text { E.B.HILLMAN }\end{array}$ & MRIG-226 & \multicolumn{2}{|c|}{$\begin{array}{l}\text { TFSC FIELD REPRESENTATIVE } \\
\text { D. KISTNER, H. ABEDI }\end{array}$} \\
\hline
\end{tabular}

\section{DESCRIPTION OF SITE PAD AREA}

WELL LOCATED IN CLEARING WITHIN DENSE FOREST. $3 \times 3 \times 3$ FT PROTECTIVE BARRIER

AROUND WELLHEAD.

DESCRIPTION OF WELL HEAD

2-IN (ID) PVC CASING EXTENDS APPROX. 1.6 FT ABOVE GL. 4-IN (ID) LOCKING STEEL

GUARD PIPE AROUND PVC EXTENDS TO 1.7 FT ABOVE GL.

DESCRIPTION OF PITS

$\underline{N A}$

DEPTH AND BOREHOLE - FLUID SURVEY:BY MASCO (8/5/88)

GROUND ELEVATION 262.6 FT (DATUM)

REPORTED DEPTH 154.5 FT (SEE BOREHOLE COMPLETION SKETCH)

MEASURED DEPTH 151.6 FT OBSTRUCTION NA FT

FLUID DEPTH 29.8 FT $\quad \mathrm{PH}-6.72$

CONDUCTIVIT $0.210 \mathrm{~ms} / \mathrm{cm}$ TDS $107.0 \mathrm{mg} / \mathrm{L}$

TEMPERATURE $26.5{ }^{\circ} \mathrm{C}$

REMARKS

2.9 FT OF SILT AT BOTTOM OF WELL. 
SITE RECONNAISSANCE FORM

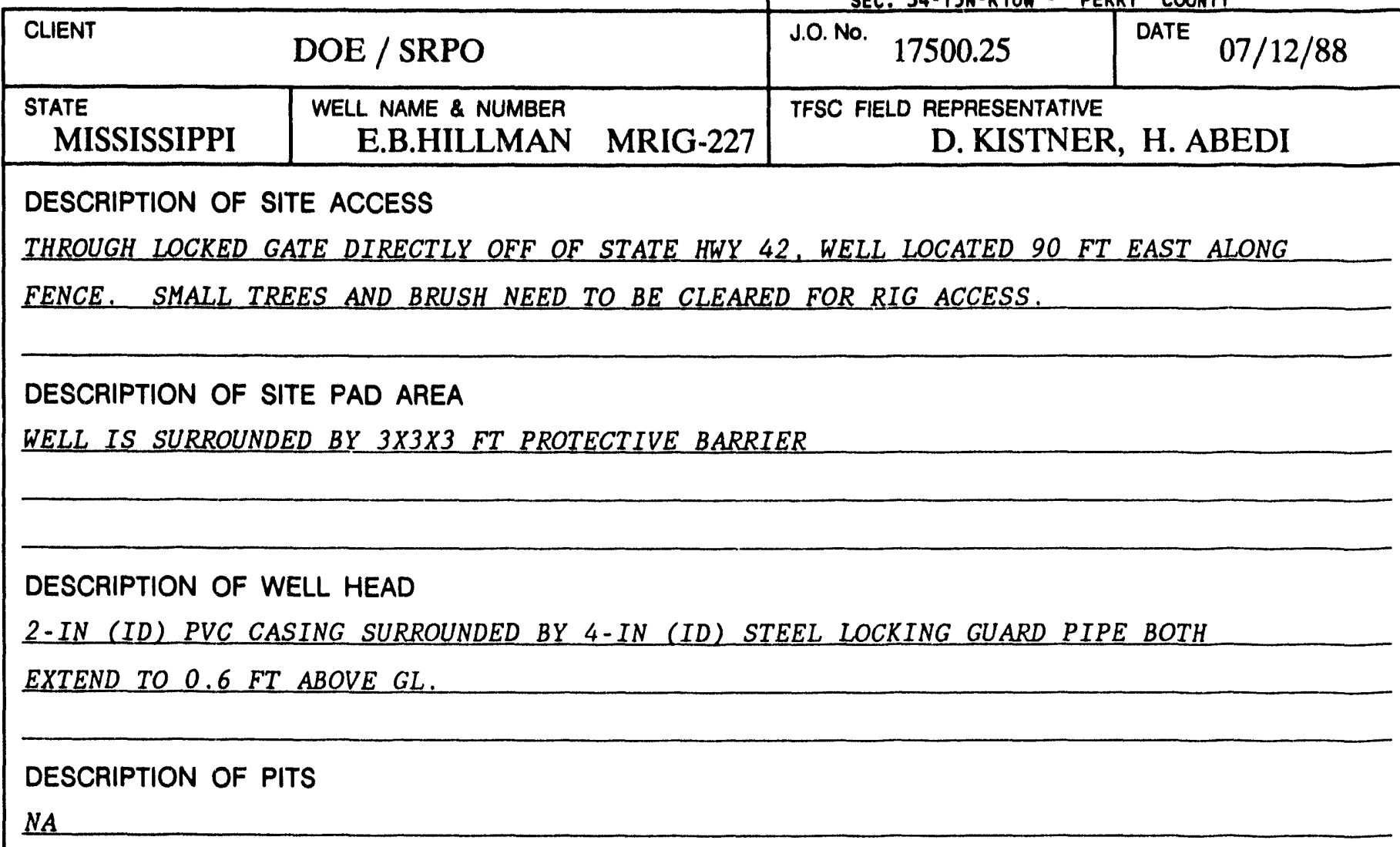

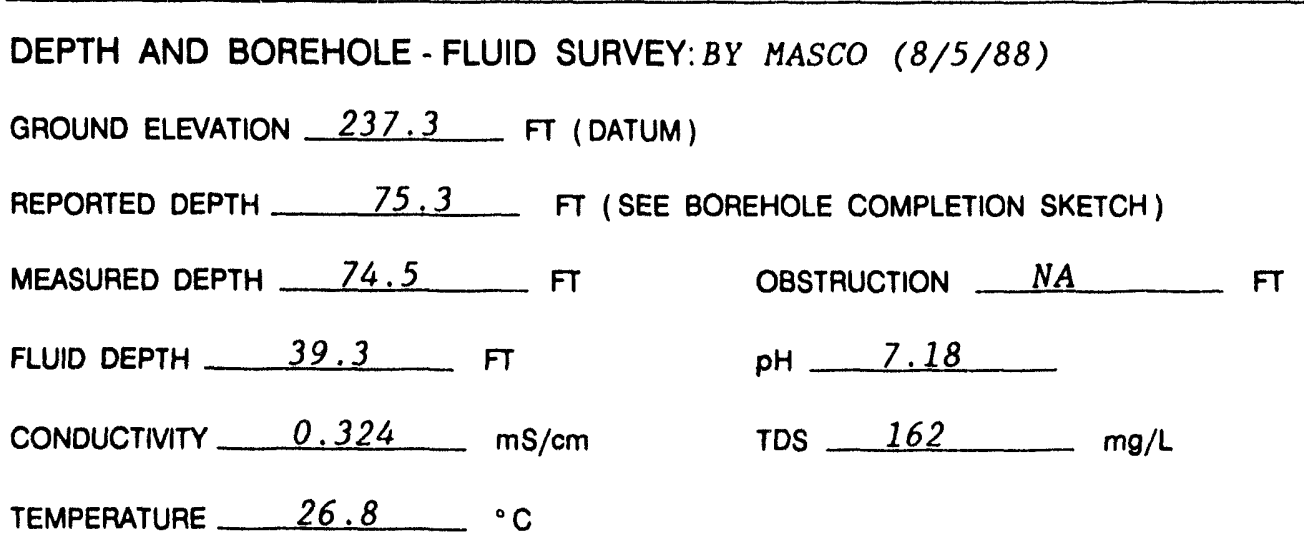

REMARKS 
SITE RECONNAISSANCE FORM

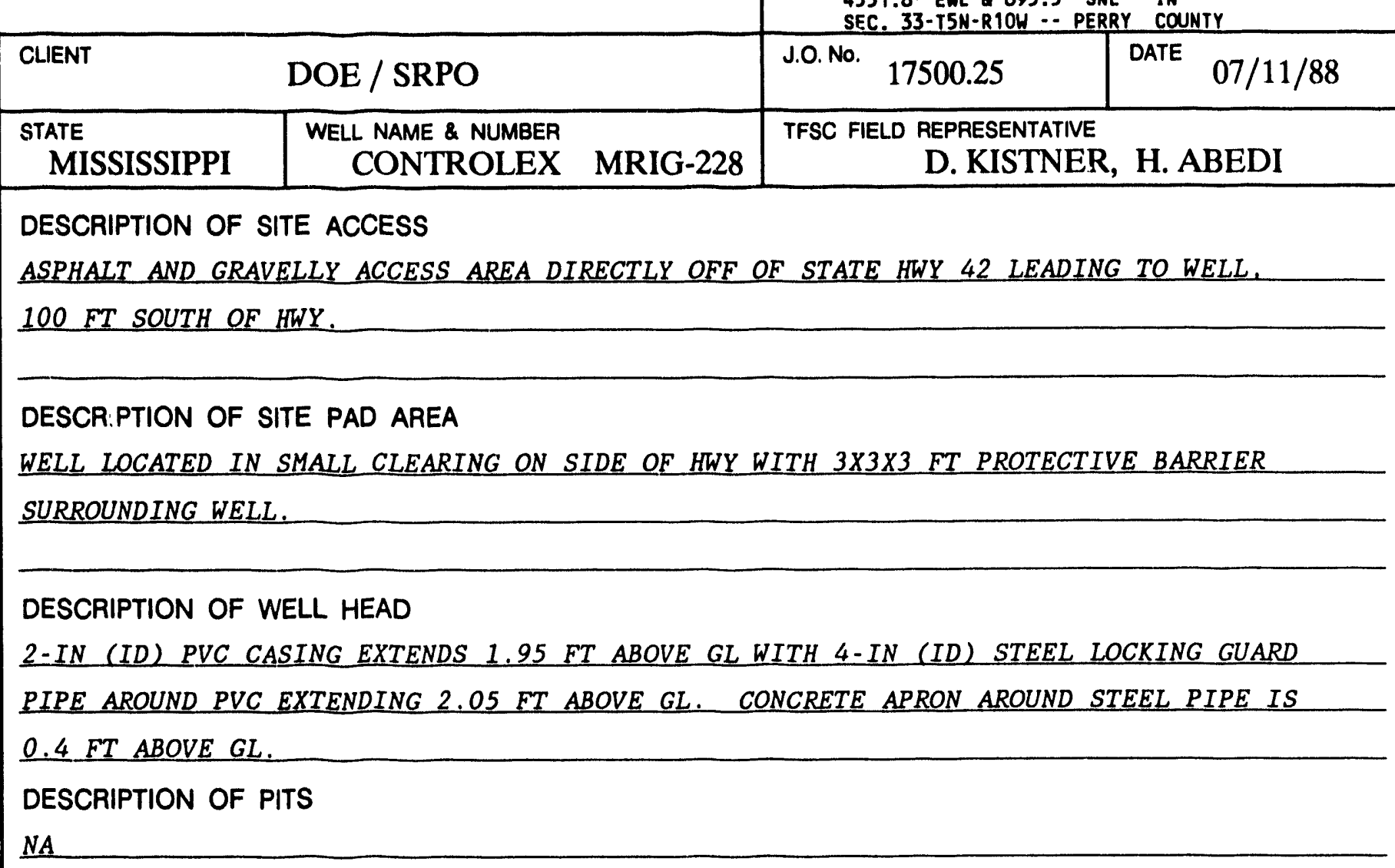

DEPTH AND BOREHOLE - FLUID SURVEY: BY SWEC $(11 / 7 / 88)$

GROUND ELEVATION 185.1 FT (DATUM)

REPORTED DEPTH 108.4 FT (SEE BOREHOLE COMPLETION SKETCH)

MEASURED DEPTH 105.3 FT OBSTRUCTION NA FT

FLUID DEPTH 21.9 FT $\quad \mathrm{pH}-6.30$

CONDUCTIVITY $0.182 \mathrm{~ms} / \mathrm{cm}$ TDS $91.2 \mathrm{mg} / \mathrm{L}$

TEMPERATURE $18.2{ }^{\circ} \mathrm{C}$

\section{REMARKS}

WELL TO BE TURNED OVER TO LANDOWNER (MR. CLARK, OWNER OF CONTROLEX). 
SITE RECONNAISSANCE FORM

CLENT

DOE / SRPO

LOCATION

1380.5' WEL \& 1341.3' SNL* IN

SEC. 32-T5N-R10W $\cdots$ PERRY COUNTY

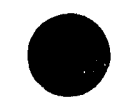

STATE MISSISSIPPI WELL NAME \& NUMBER SOWARD MRIG-229
J.O. No. 17500.25 DATE

TFSC FIELD REPRESENTATIVE

D. KISTNER, H. ABEDI

DESCRIPTION OF SITE ACCESS

SITE IS 60 FT WEST OF PAVED ROAD, 0.1 MILE SOUTH OF STATE HWY 42 IN OVERGROWN AREA. UNCASED BOREIIOLE BACKFILLED IMMEDIATELY AFTER DRILLING BY LETCO IN 1979.

DESCRIPTION OF SITE PAD AREA

NO EVIDENCE OF BACKFILLED BOREHOLE AT SURFACE.

\section{DESCRIPTION OF WELL HEAD}

NA

DESCRIPTION OF PITS

NA

DEPTH AND BOREHOLE - FLUID SURVEY: NA

GROUND ELEVATION 144.7 FT (DATUM)

REPORTED DEPTH 99.5 FT (SEE BOREHOLE COMPLETION SKETCH)

MEASURED DEPTH NA FT OBSTRUCTION NA FT

FLUID DEPTH NA FT $\quad \mathrm{PH} \perp$ NA

CONDUCTIVITY NA TDS NA $\mathrm{NS} / \mathrm{cm} \mathrm{mg} / \mathrm{L}$

TEMPERATURE

REMARKS

*NOTE: SNL $=$ South of North Line 
SITE RECONNAISSANCE FORM

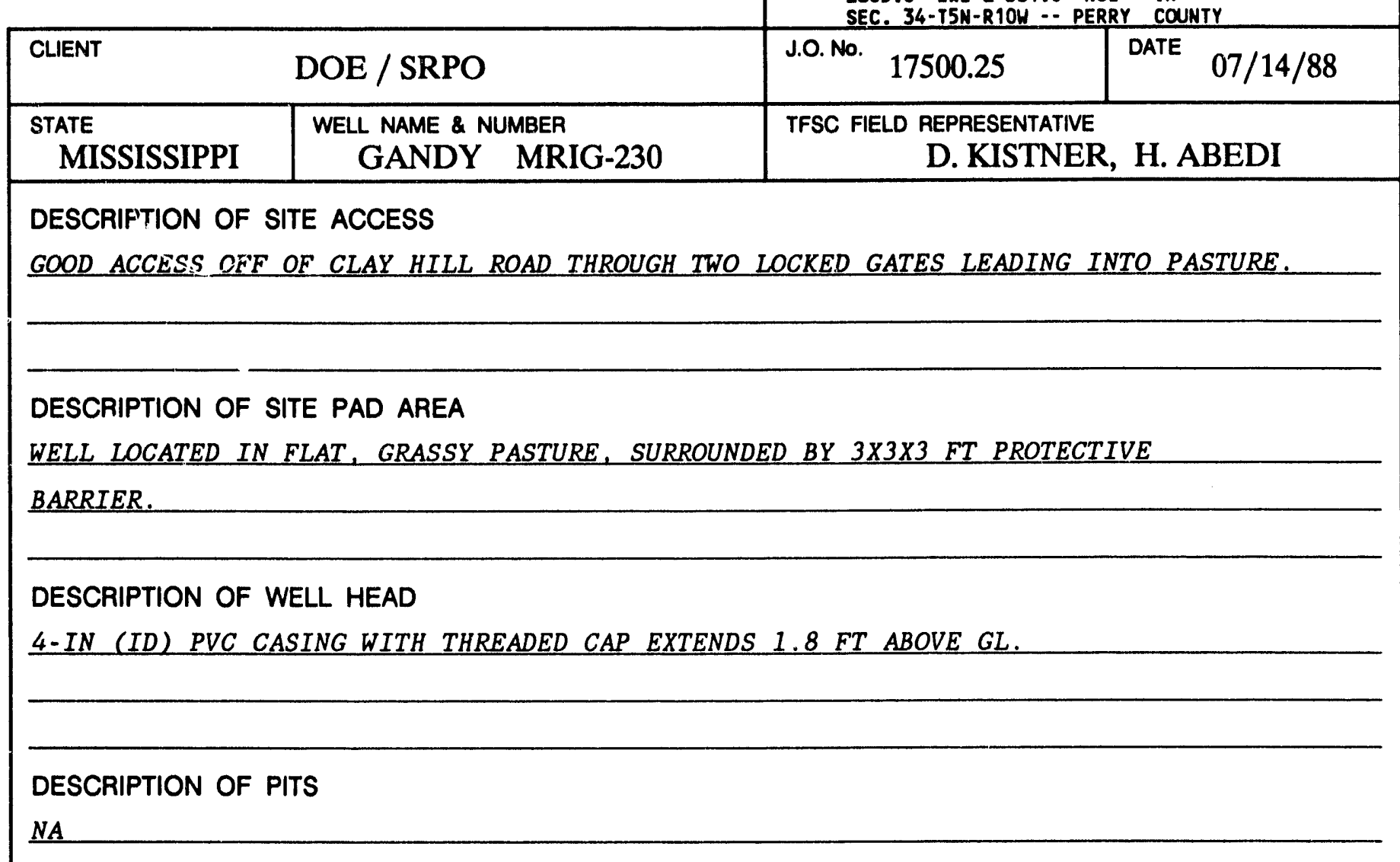

DEPTH AND BOREHOLE - FLUID SURVEY:BY SWEC $(11 / 1 / 88)$

GROUND ELEVATION 257.8 FT (DATUM)

REPORTED DEPTH 135.0 FT (SEE BOREHOLE COMPLETION SKETCH)

MEASURED DEPTH 131.2 FT OBSTRUCTION NA FT

FLUID DEPTH $116.5 \quad$ FT $\quad \mathrm{PH} \quad 5.5$

CONDUCTIVITY $0.070 \mathrm{~ms} / \mathrm{cm} \quad$ TDS $35.0 \quad \mathrm{mg} / \mathrm{L}$

TEMPERATURE $22.0 \quad{ }^{\circ} \mathrm{C}$

REMARKS

WELL TO BE TURNED OVER TO LANDOWNER. 
SITE RECONNAISSANCE FORM

LOCATION

$1173.9^{\prime}$ EWL \& 2173.5' NSL * IN

SEC. 4-TAN-RQW - - PERRY CQUNTY

\begin{tabular}{|l|l|l|l|}
\hline CLIENT & J.0. No. 17500.25 & DATE $07 / 14 / 88$ \\
\hline $\begin{array}{l}\text { STATE } \\
\text { MISSISSIPPI }\end{array}$ & $\begin{array}{l}\text { WELL NAME \& NUMBER } \\
\text { L.R.F.P. MRIG-232 }\end{array}$ & $\begin{array}{r}\text { TFSC FIELD REPRESENTATIVE } \\
\text { D. KISTNER, H. ABEDI }\end{array}$ \\
\hline
\end{tabular}

DESCRIPTION OF SITE ACCESS

WELL IS LOCATED 60 FT NW OF DEEPLY-RUTTED LOGGING RD. SOME PINE SEEDLINGS MAY

NEED TO BE CLEARED FOR RIG ACCESS.

DESCRIPTION OF SITE PAD AREA

WELL IS SURROUNDED BY $3 \times 3 \times 3$ FT PROTECTIVE BARRIER IN AREA REPLANTED WITH

PINE TREES.

DESCRIPTION OF WELL HEAD

2-IN (ID) PVC CASING SURROUNDED BY 4-IN (ID) STEEL LOCKING GUARD PIPE BOTH

EXTENDING 1.1 FT ABOVE GL. 0.5 FT CONCRETE APRON SURROUNDS GUARD PIPE.

DESCRIPTION OF PITS

NA

DEPTH AND BOREHOLE - FLUID SURVEY: BY MASCO $(8 / 5 / 88)$

GROUND ELEVATION 223.5 FT (DATUM)

REPORTED DEPTH 118.5 FT (SEE BOREHOLE COMPLETION SKETCH)

MEASURED DEPTH 110.5 FT OBSTRUCTION NA FT

FLUID DEPTH $32.1 \quad \mathrm{FT} \quad \mathrm{PH}-6.57$

CONDUCTIVTY $0.140 \mathrm{~ms} / \mathrm{cm} \quad$ TDS $70.4 \mathrm{mg} / \mathrm{L}$

TEMPERATURE $-26.9 \quad{ }^{\circ} \mathrm{C}$

REMARKS

8.0 FT OF SILT AT BOTTOM OF WELL. 
APPENDIX C

Schematic of Preabandonment Site Status 
SCHEMATIC OF PREABANDONMENT SITE STATUS

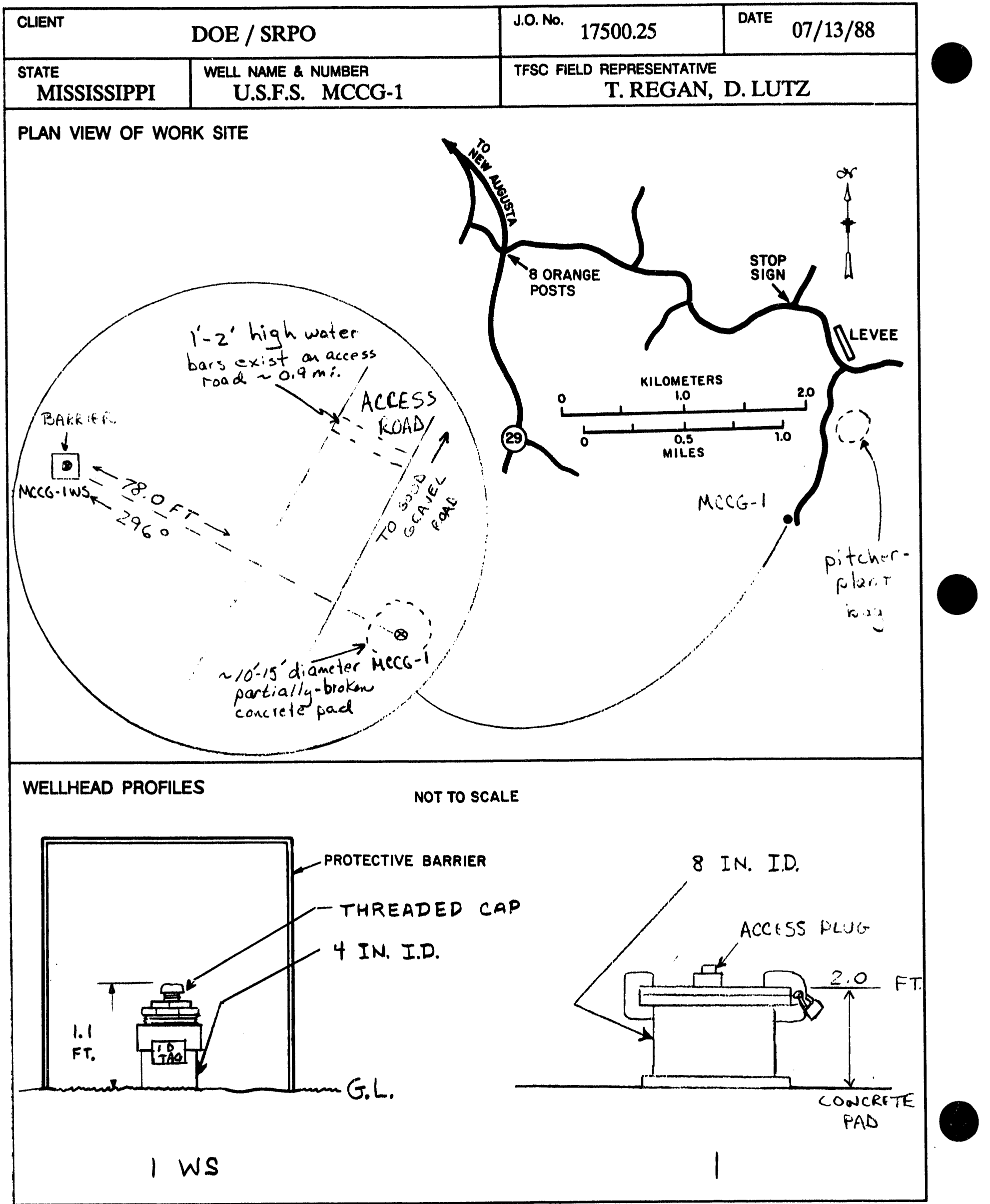




\section{SCHEMATIC OF PREABANDONMENT SITE STATUS}

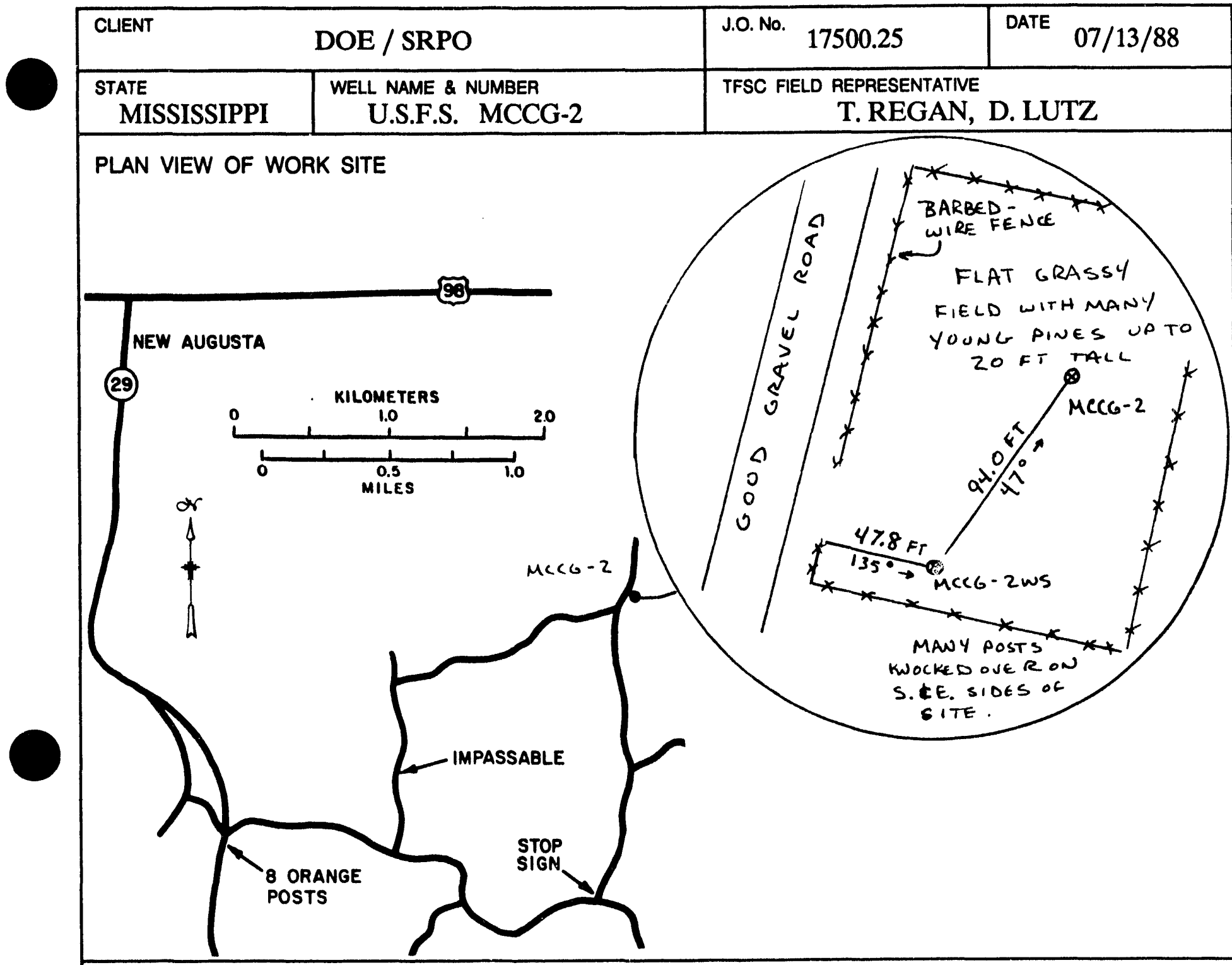

WELLHEAD PROFILE

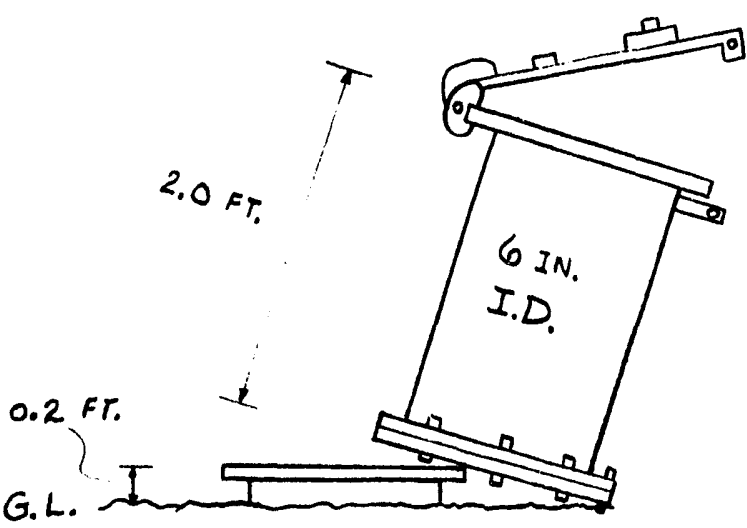

2
NOT TO SCALE

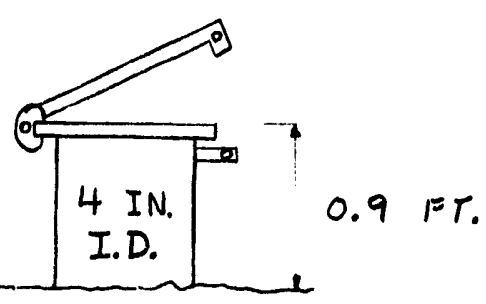

$2 W S$ 
SCHEMATIC OF PREABANDONMENT SITE STATUS

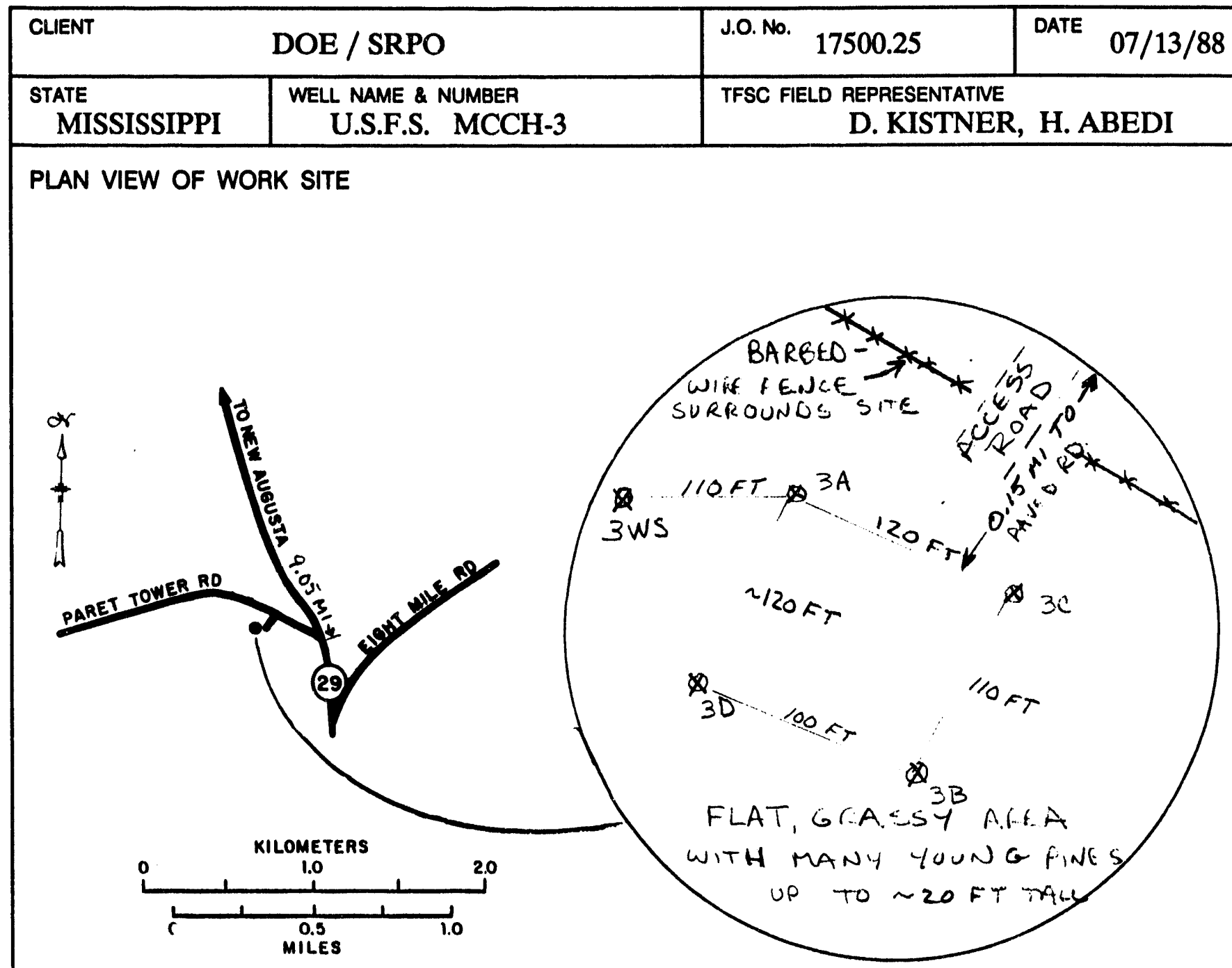

WELLHEAD PROFILE

NOT TO SCALE
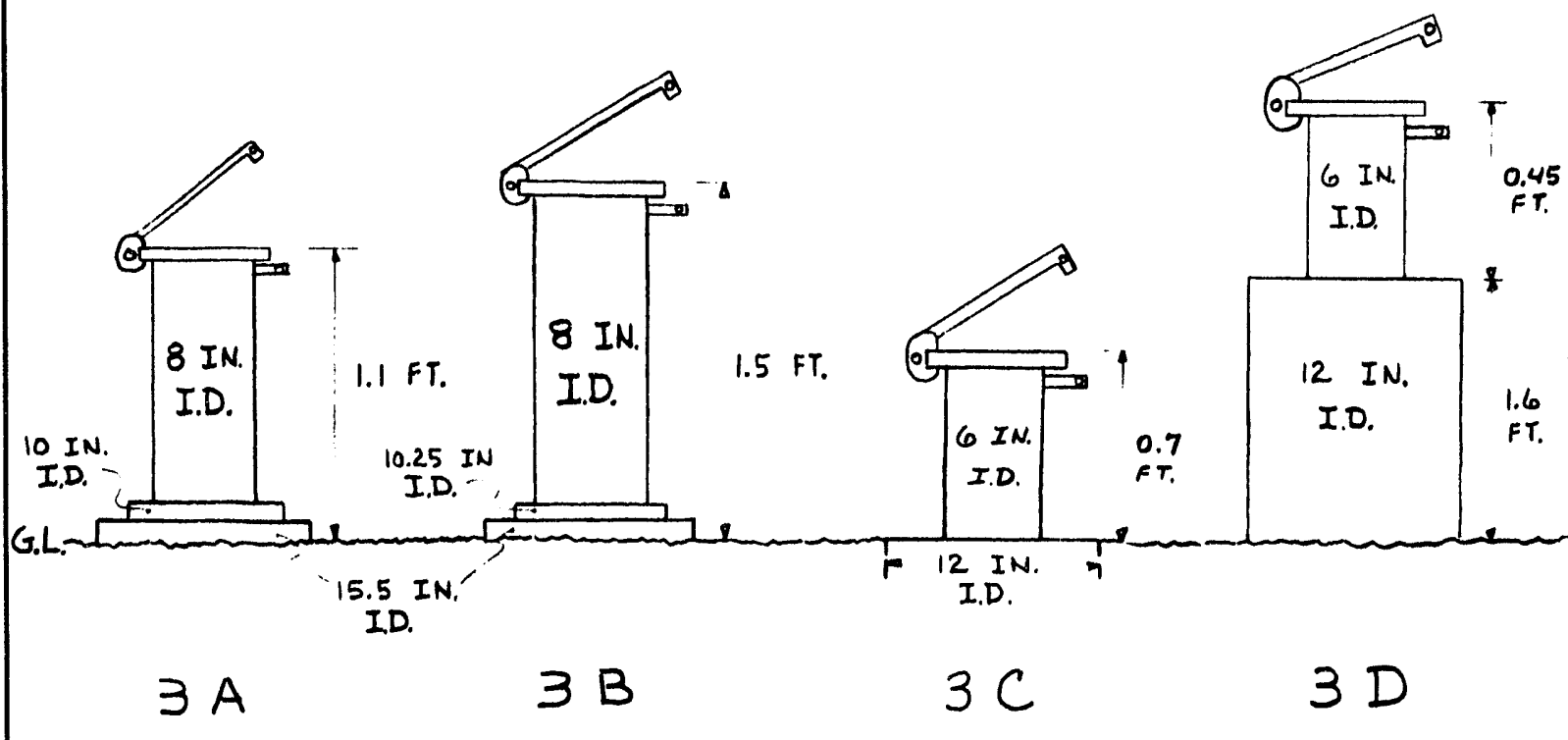

2 IN.

I.D.

$3 C$

$3 D$

$3 W S$ 
SCHEMATIC OF PREABANDONMENT SITE STATUS

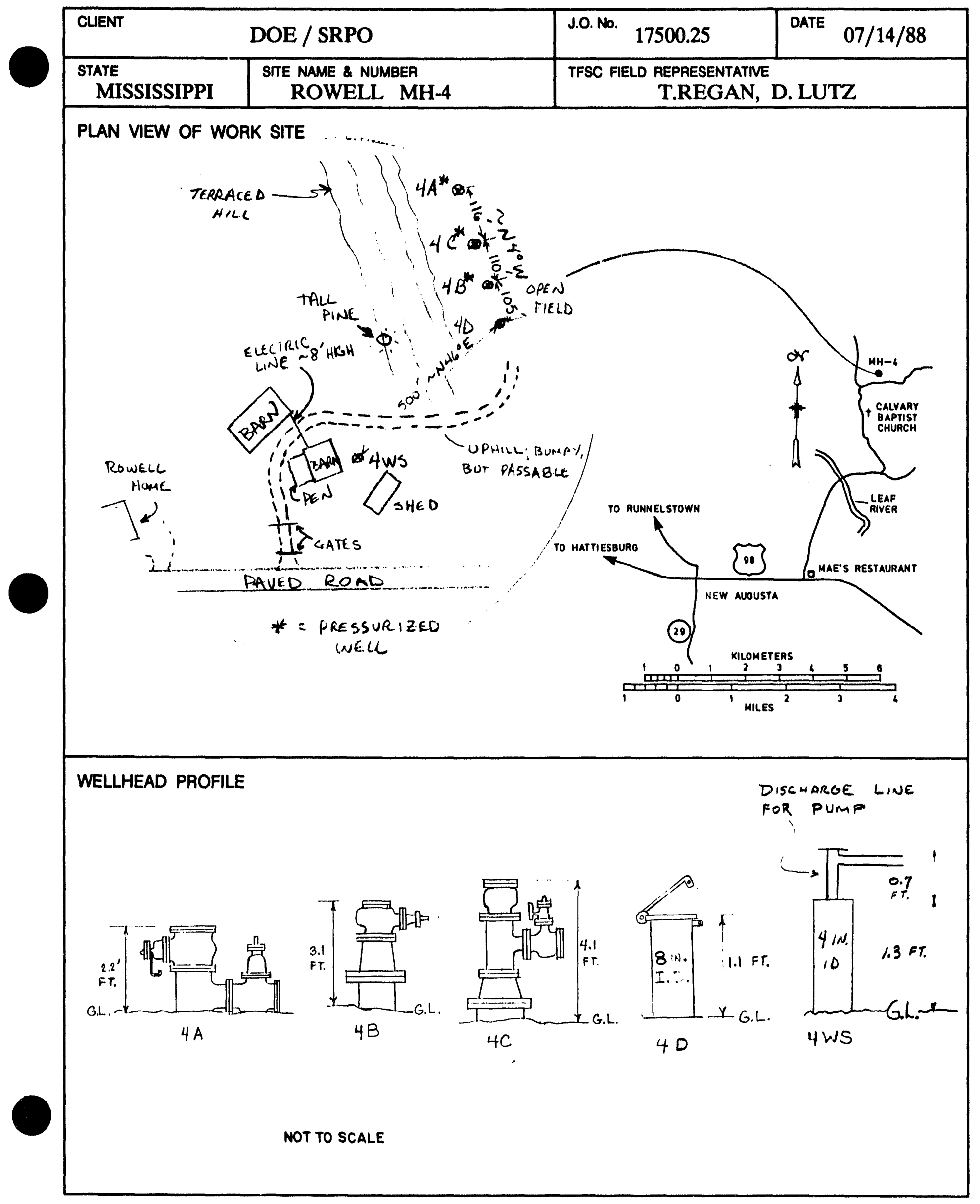




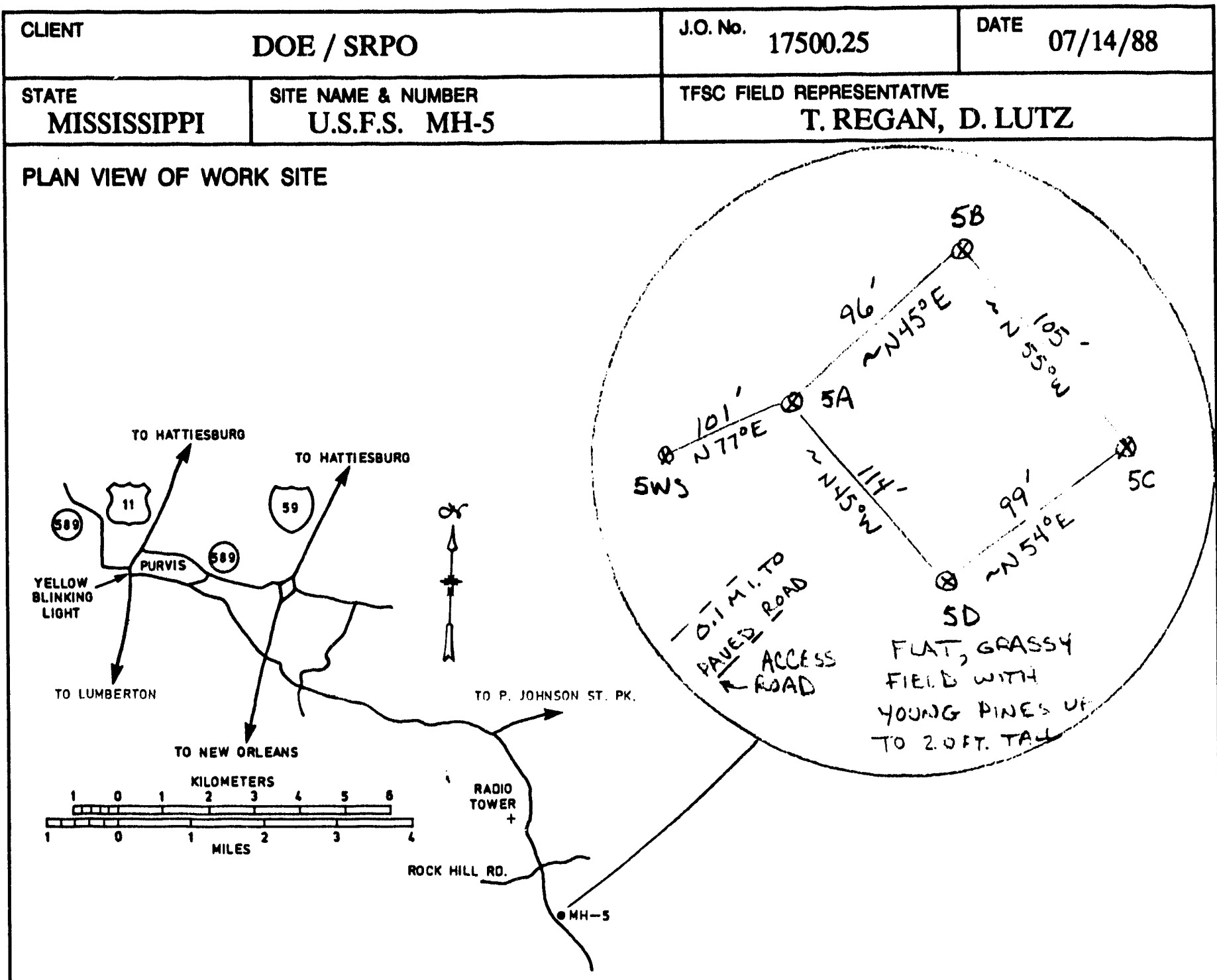

\section{WELLHEAD PROFILE}

I.D.

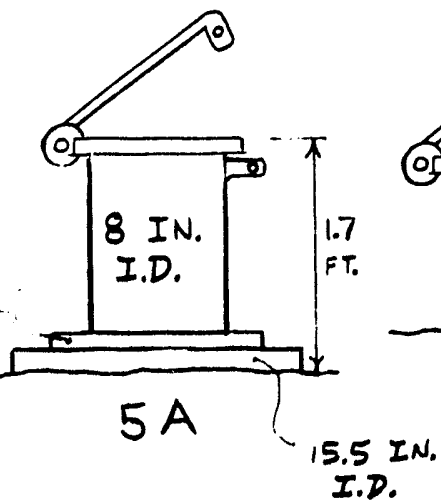

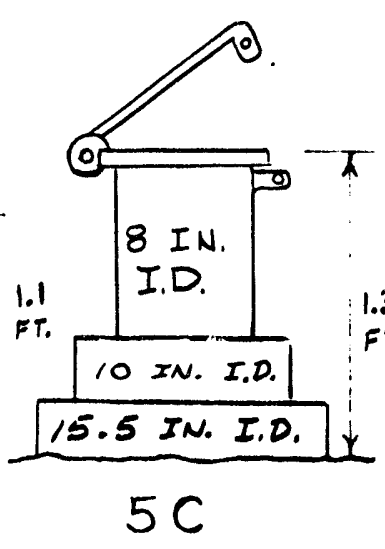

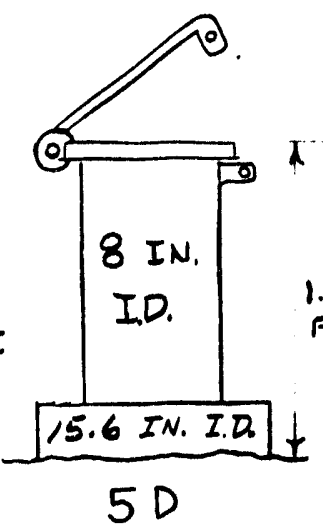

$x$

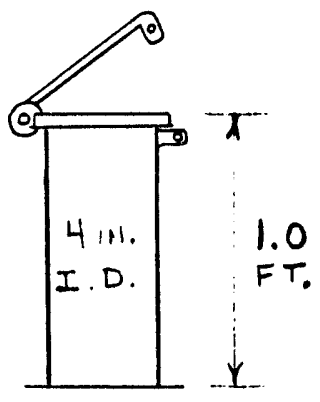

5WS 
SCHEMATIC OF PREABANDONMENT SITE STATUS

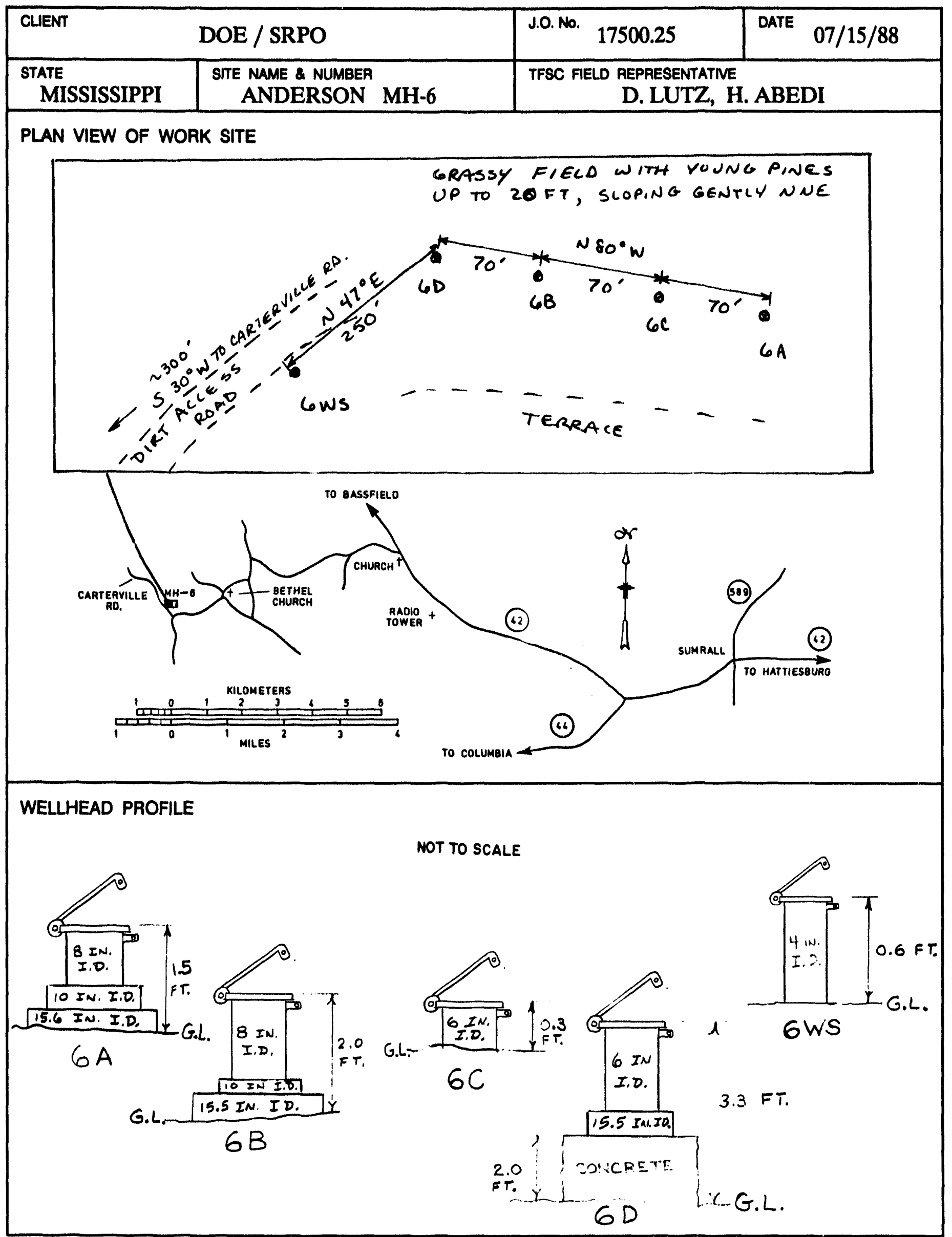


SCHEMATIC OF PREABANDONMENT SITE STATUS

\begin{tabular}{|l|c|l|l|}
\hline CLIENT & DOE / SRPO & J.0. No. 17500.25 & DATE $07 / 11 / 88$ \\
\hline $\begin{array}{c}\text { STATE } \\
\text { MISSISSIPPI }\end{array}$ & $\begin{array}{c}\text { SITE NAME \& NUMBER } \\
\text { BEARD MH-7 }\end{array}$ & $\begin{array}{c}\text { TFSC FIELD REPRESENTATINE } \\
\text { T. REGAN, D. LUTZ }\end{array}$ \\
\hline
\end{tabular}

PLAN VIEW OF WORK SITE

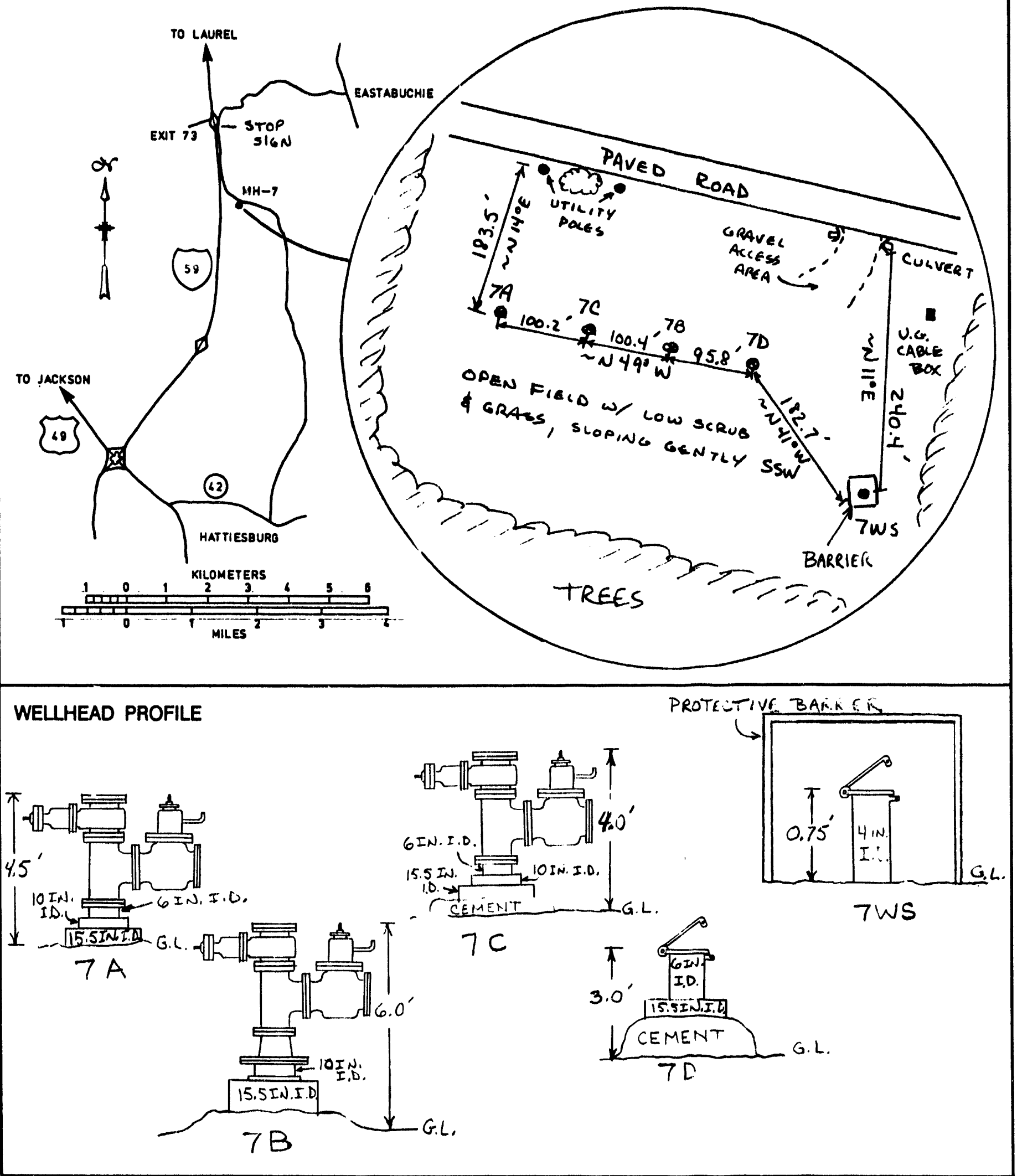


SCHEMATIC OF PREABANDONMENT SITE STATUS

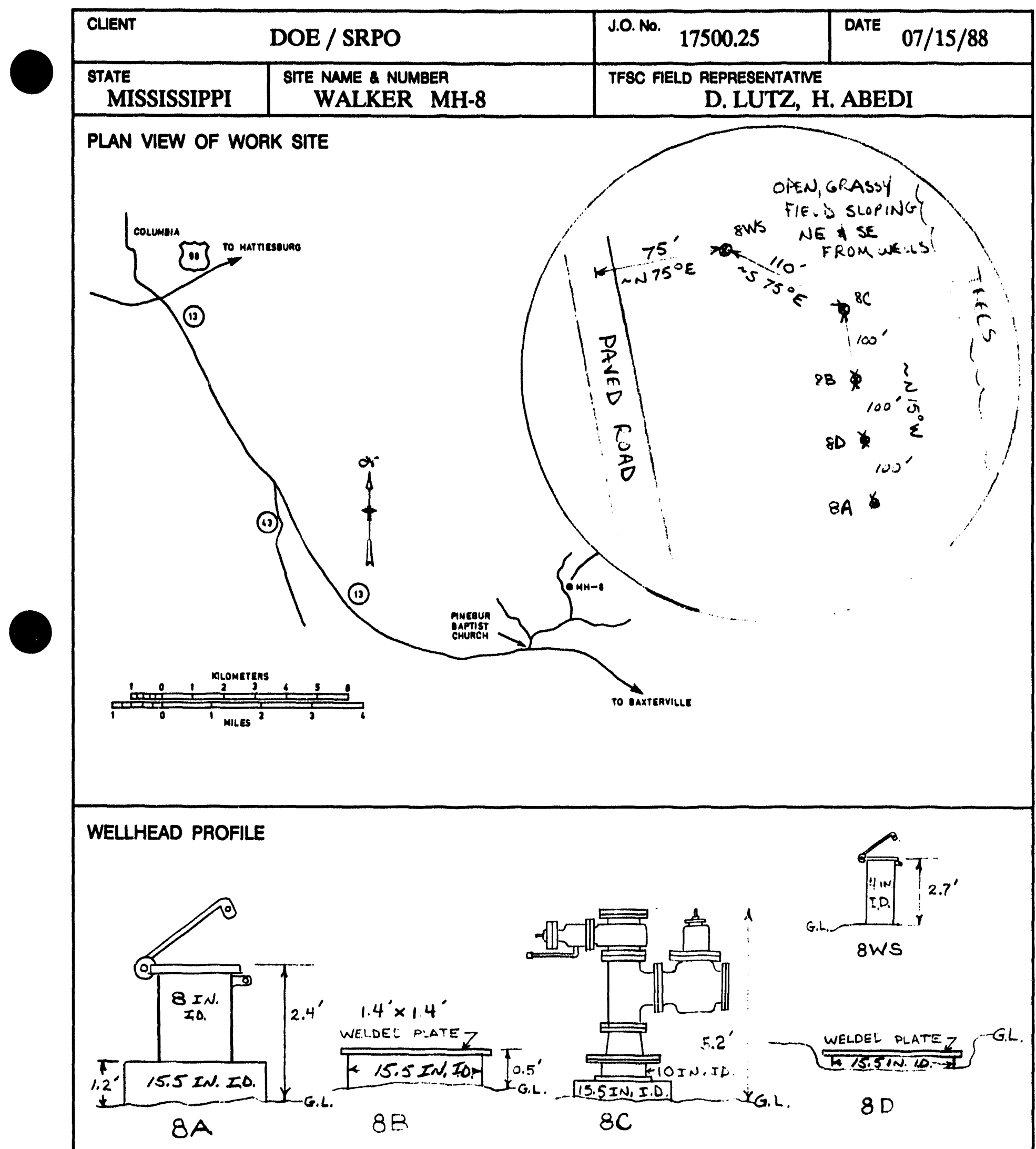




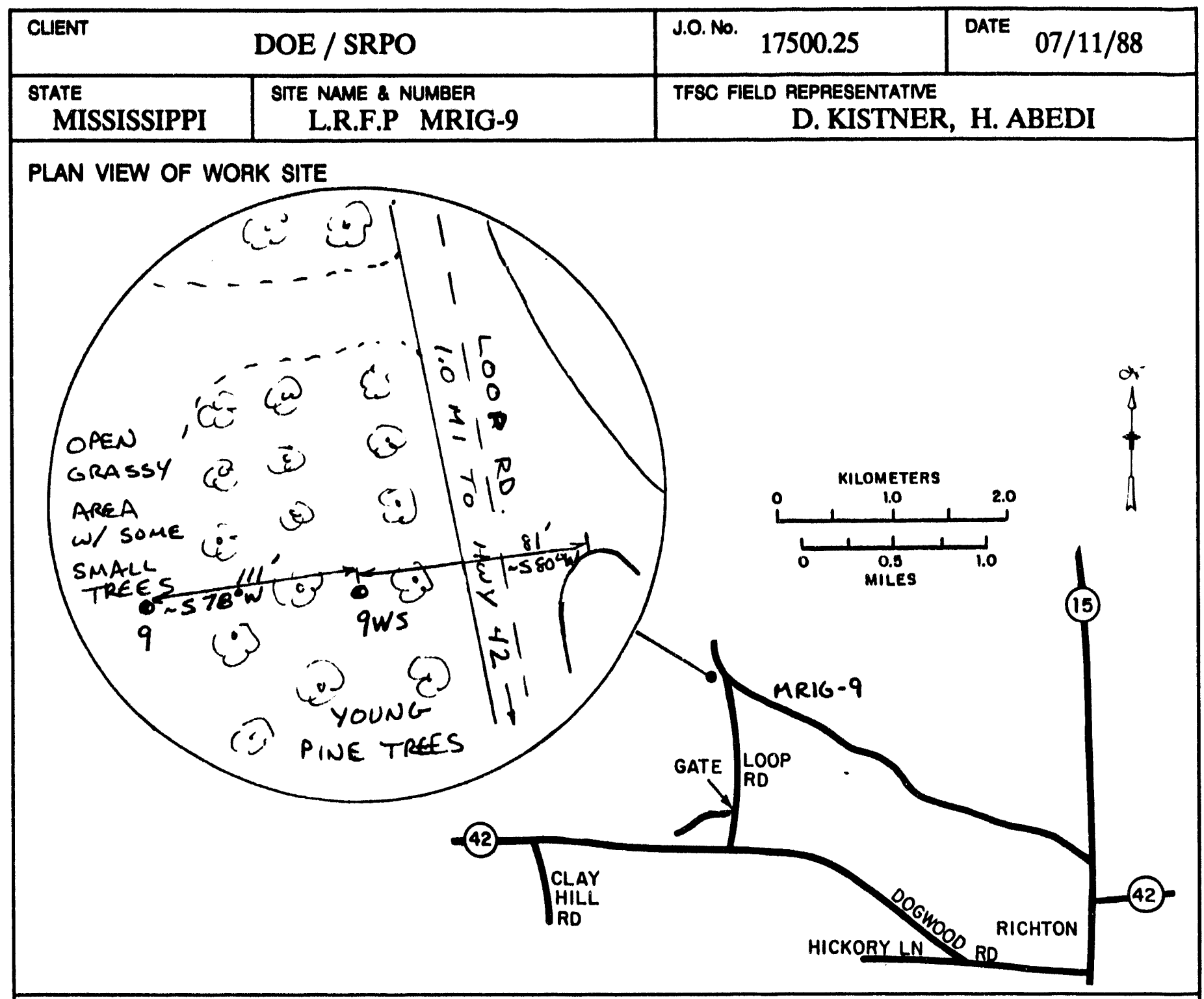

WELLHEAD PROFILE
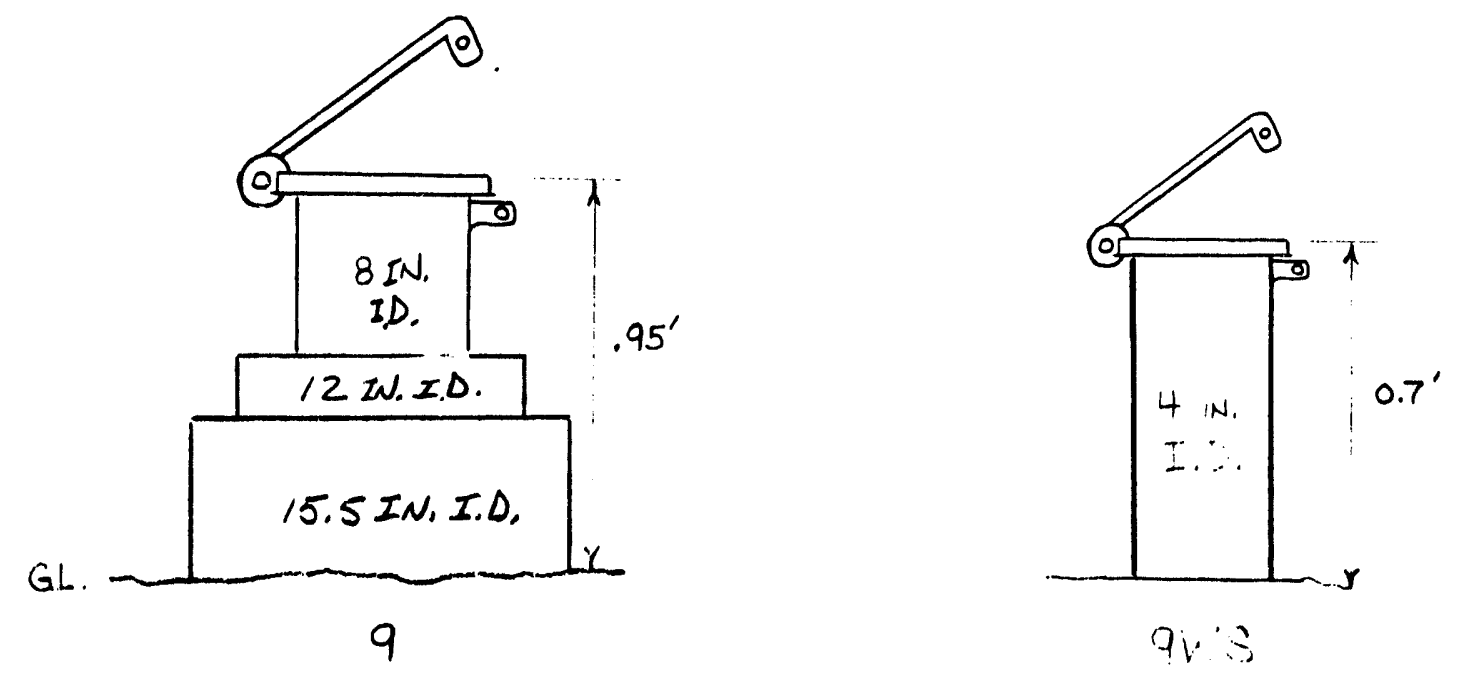
SCHEMATIC OF PREABANDONMENT SITE STATUS

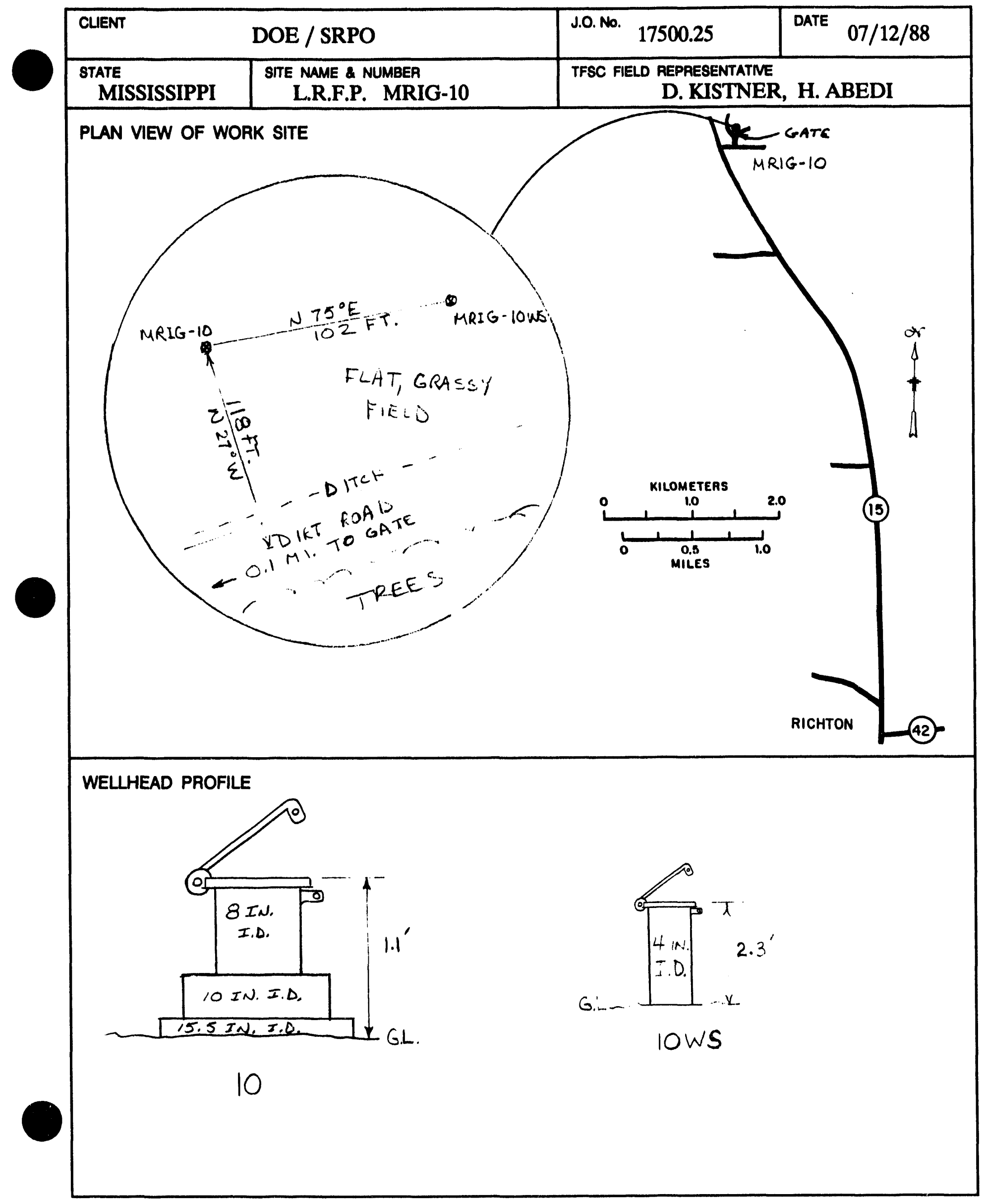




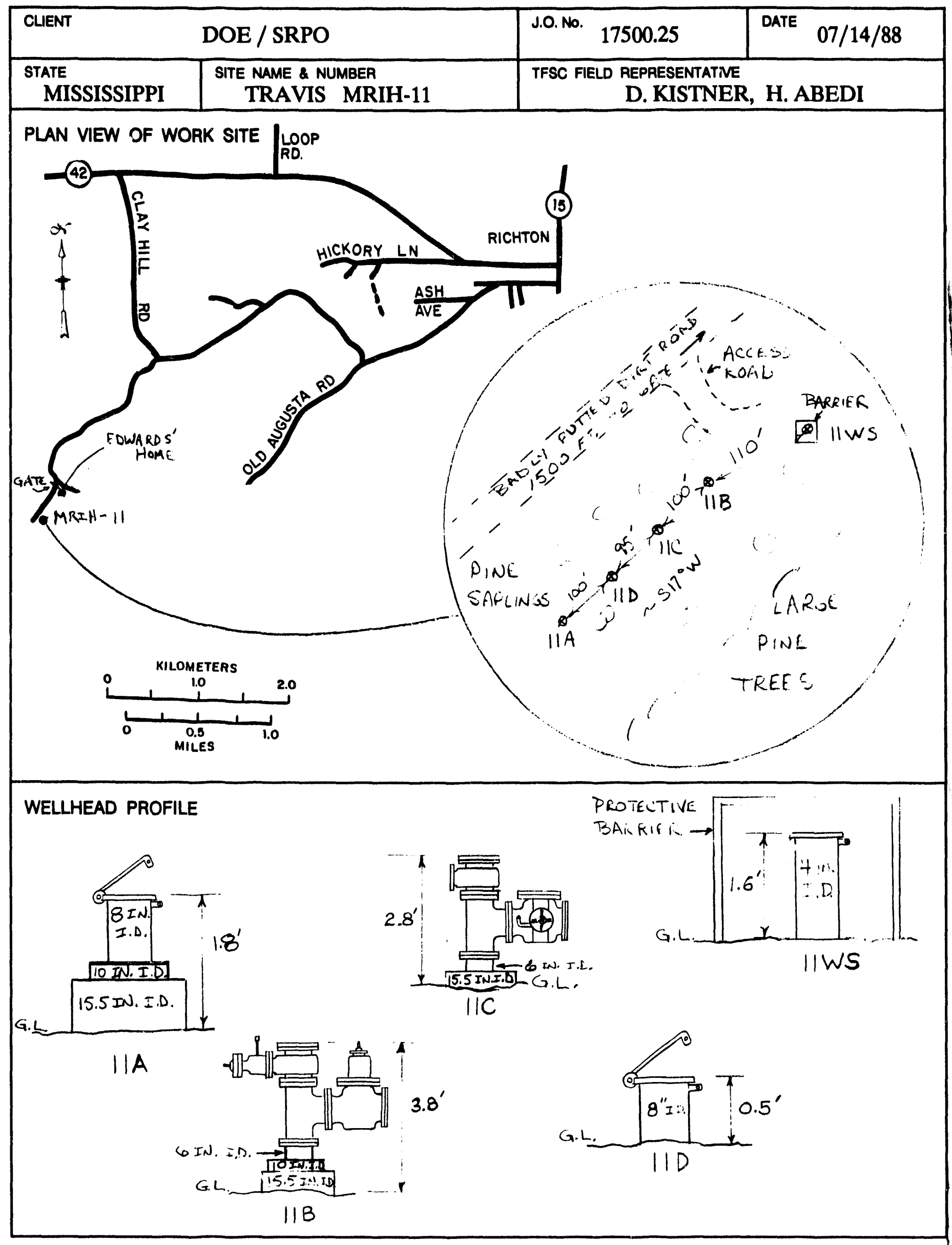


SCHEMATIC OF PREABANDONMENT SITE STATUS

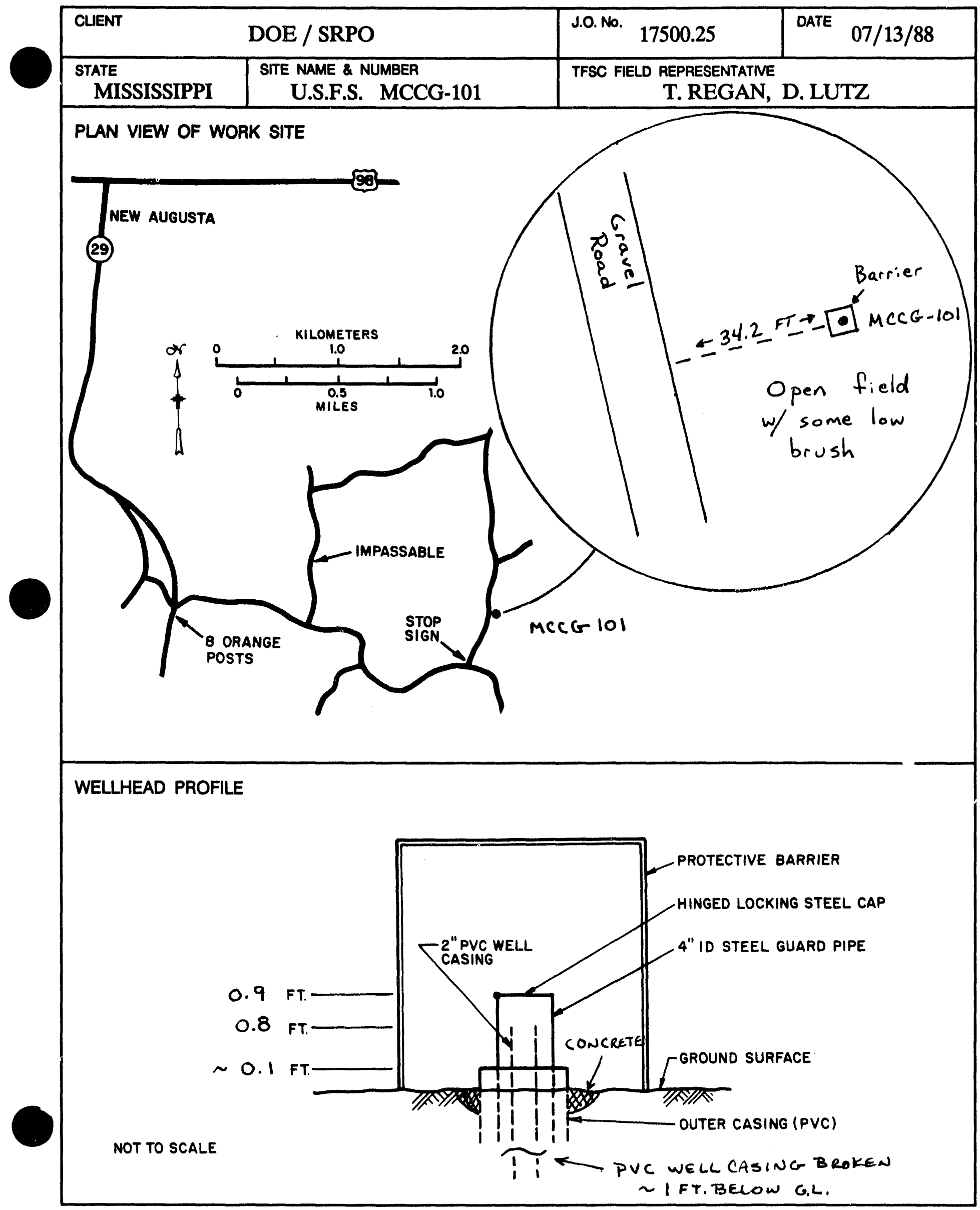




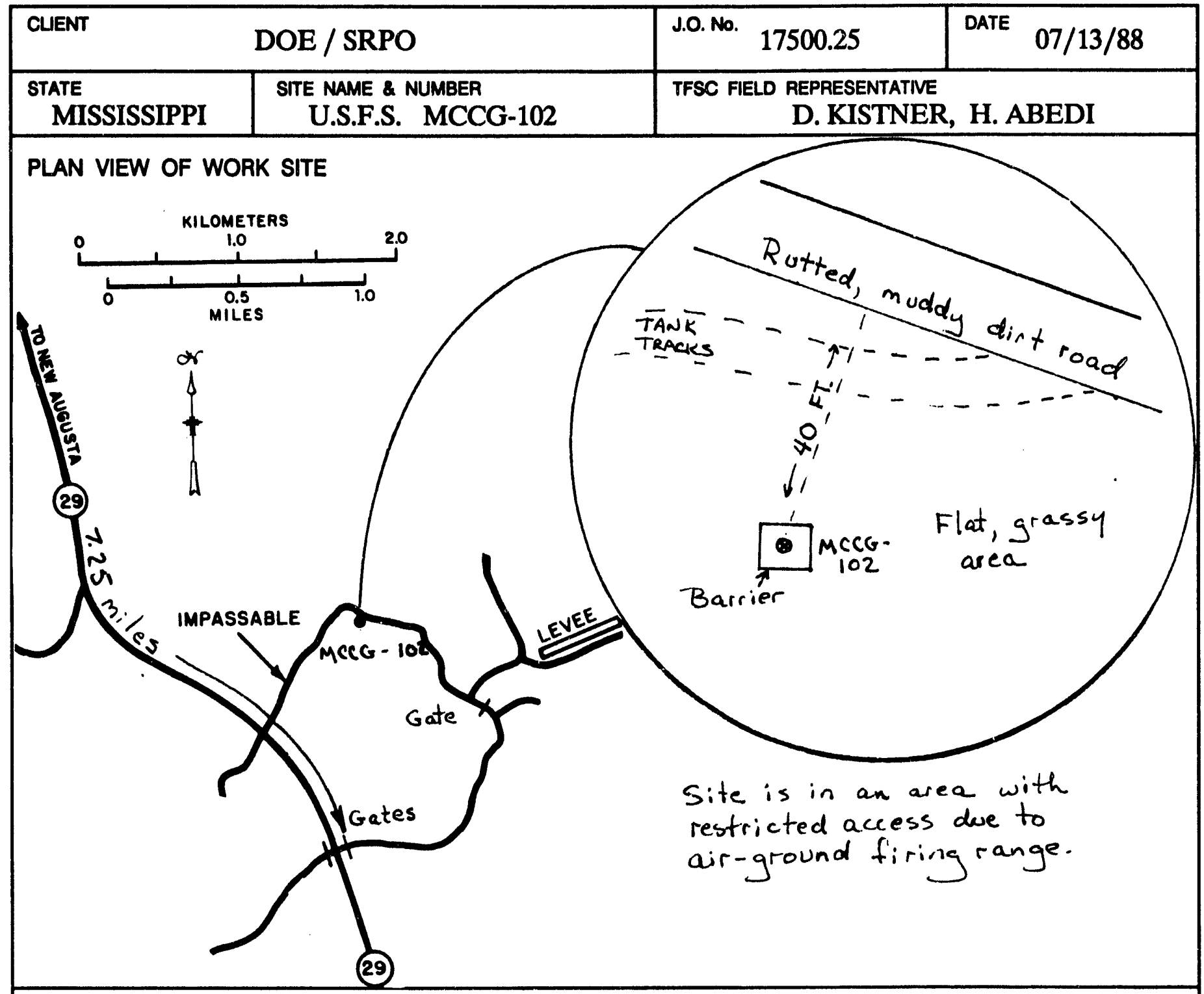

WELLHEAD PROFILE

NOT TO SCALE

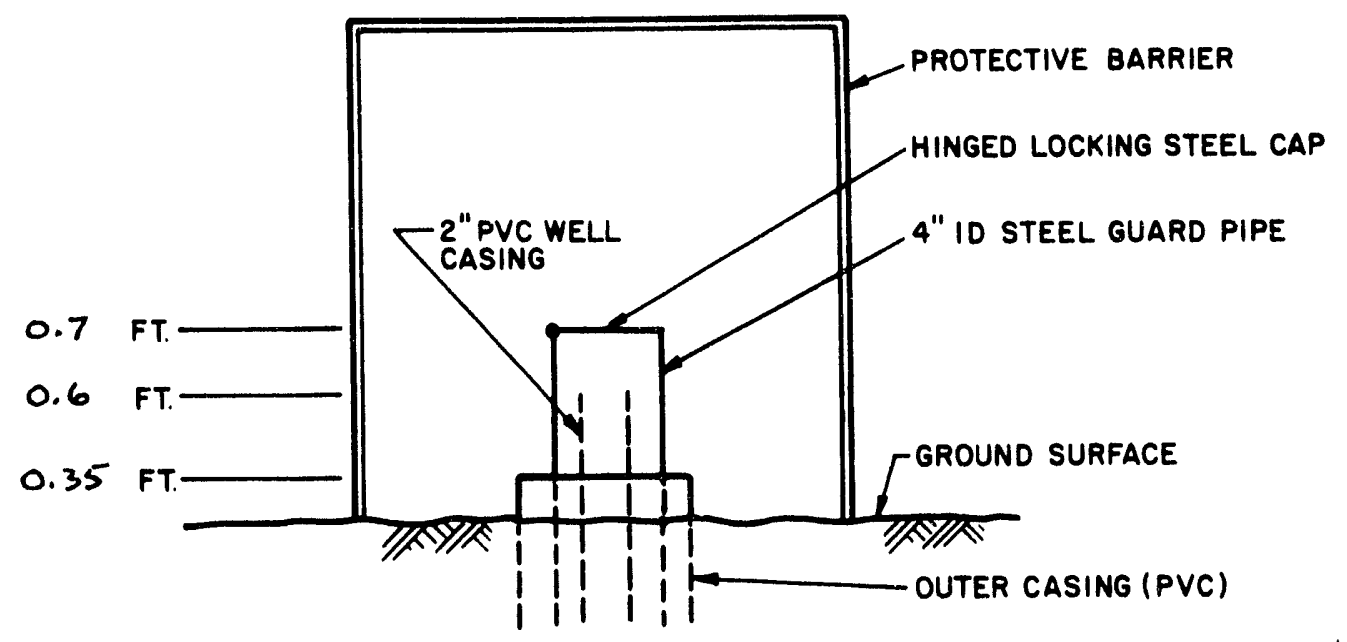


SCHEMATIC OF PREABANDONMENT SITE STATUS

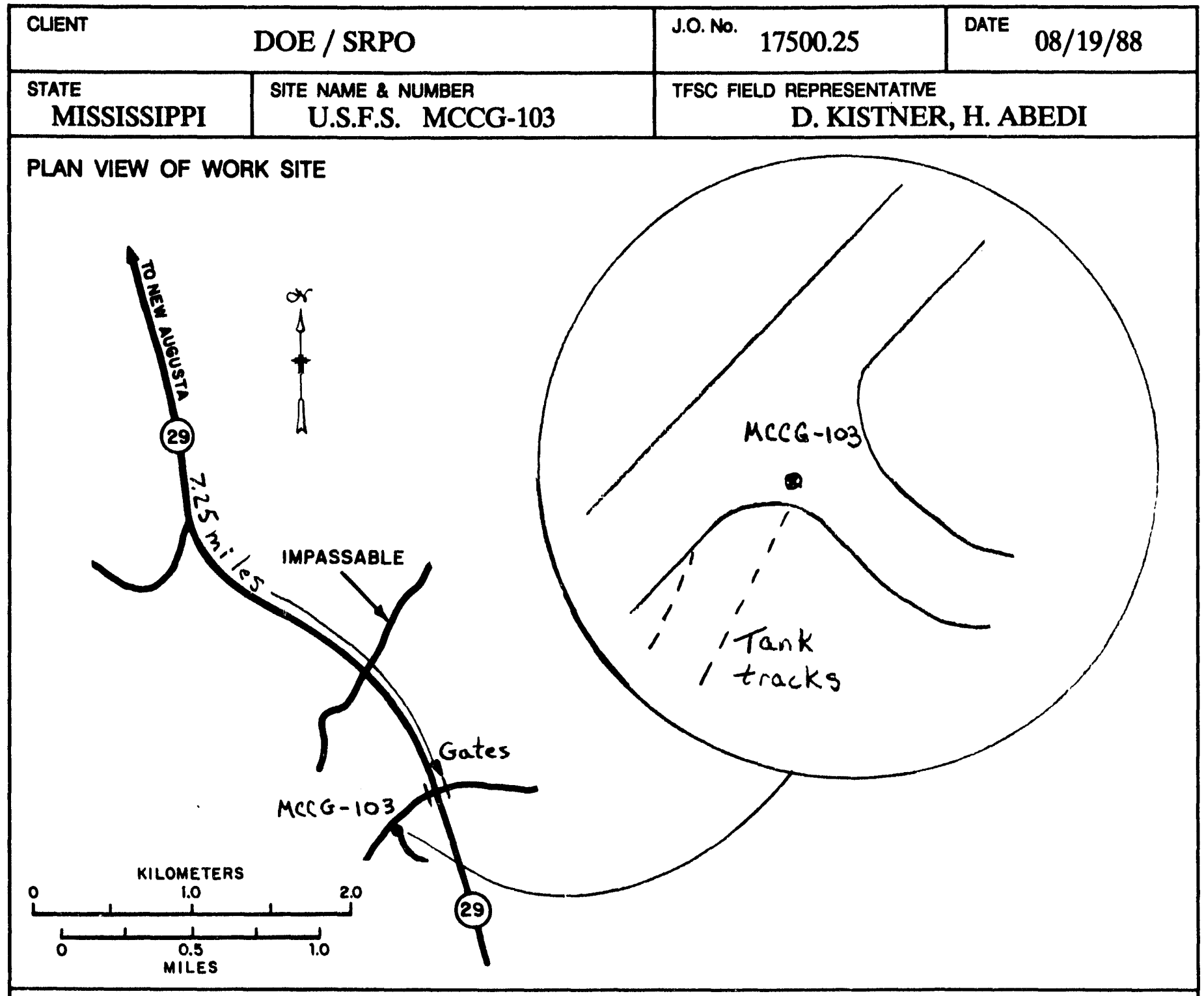

WELLHEAD PROFILE

Well head apparently destroyed during tank maneuvers

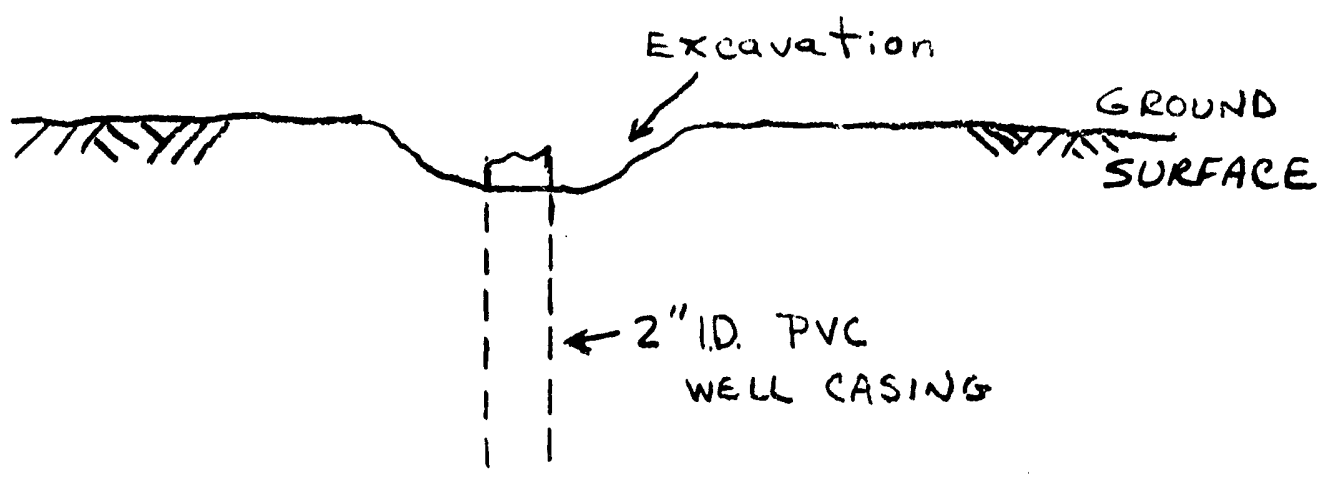

NOT TO SCALE 
SCHEMATIC OF PREABANDONMENT SITE STATUS

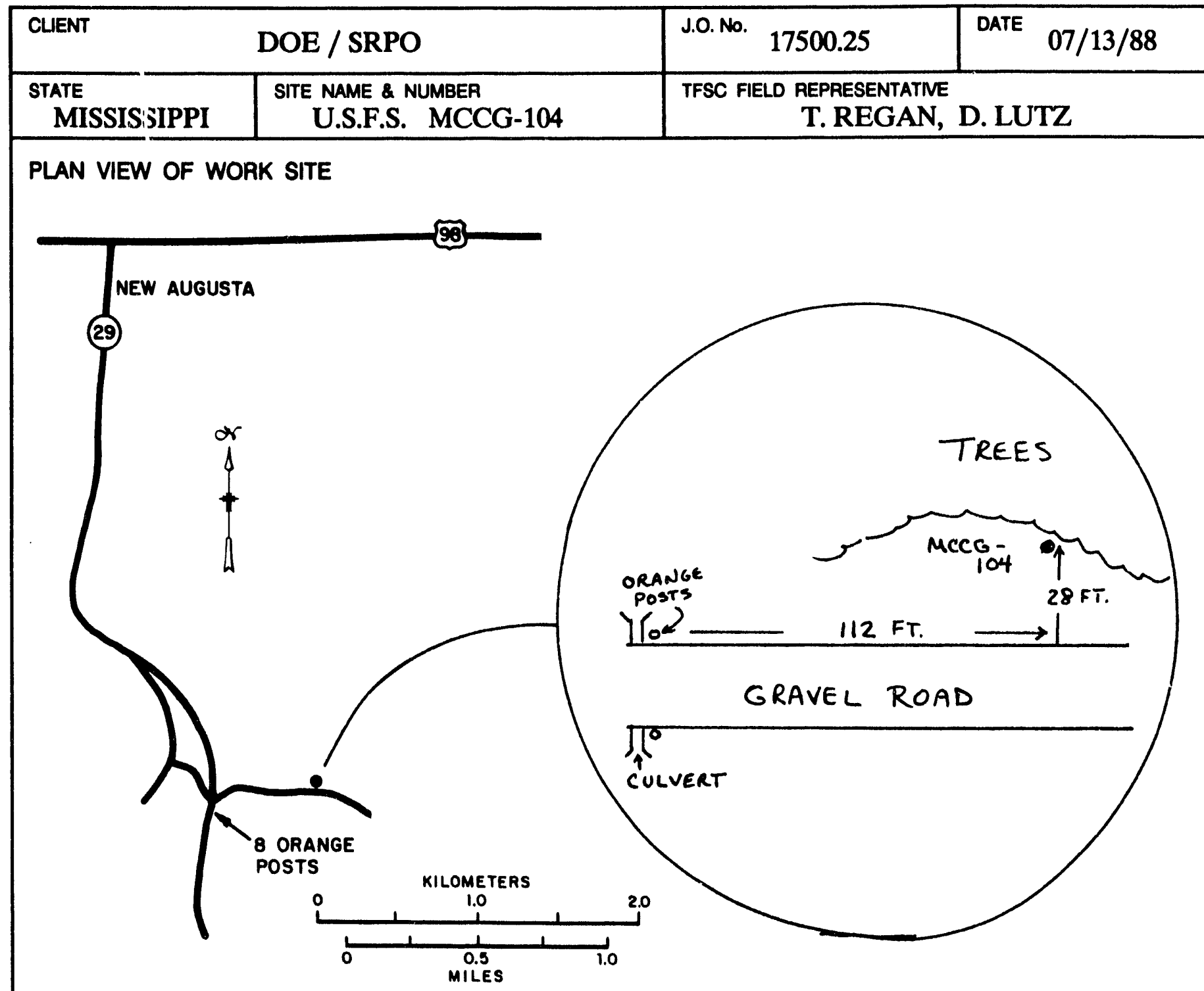

WELLHEAD PROFILE

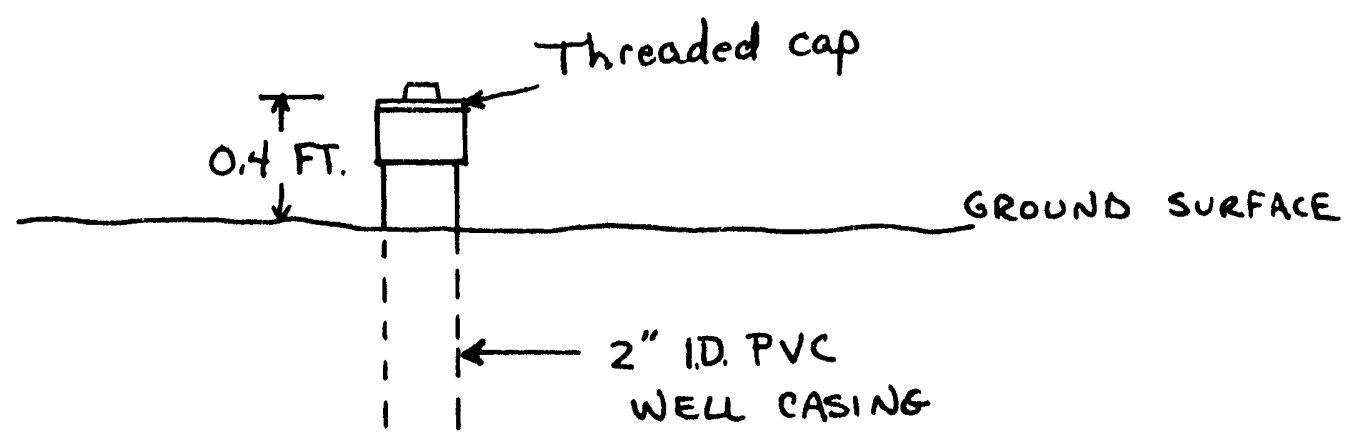

NOT TO SCALE 
SCHEMATIC OF PREABANDONMENT SITE STATUS

\begin{tabular}{|l|l|l|l|}
\hline CLIENT & DOE / SRPO & J.0. No. 17500.25 & DATE $07 / 13 / 88$ \\
\hline $\begin{array}{c}\text { STATE } \\
\text { MISSISSIPPI }\end{array}$ & $\begin{array}{l}\text { SITE NAME \& NUMBER } \\
\text { U.S.F.S. MCCG-105 }\end{array}$ & $\begin{array}{r}\text { TFSC FIELD REPRESENTATME } \\
\text { D. KISTNER, H. ABEDI }\end{array}$ \\
\hline
\end{tabular}

PLAN VIEW OF WORK SITE

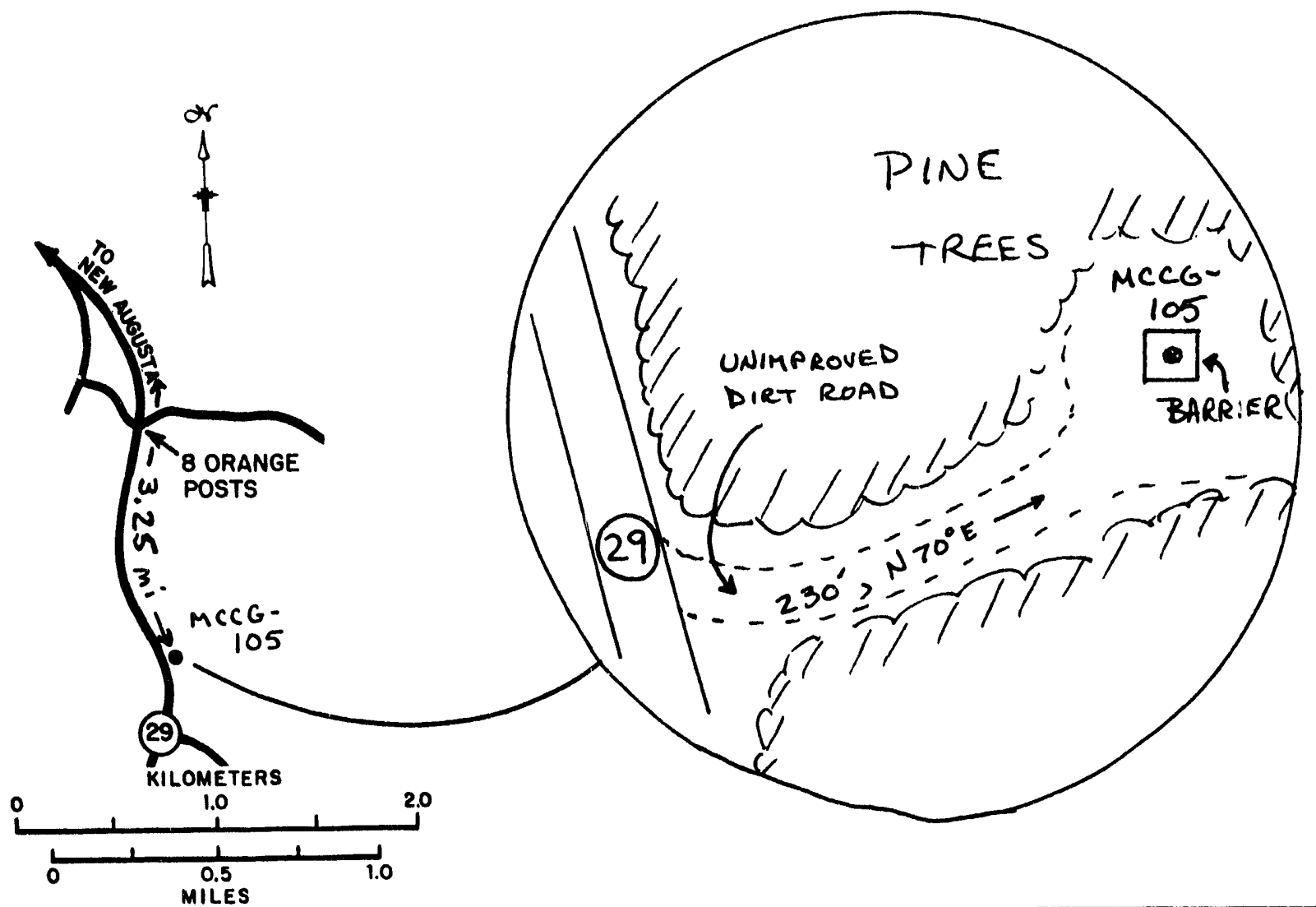

WELLHEAD PROFILE

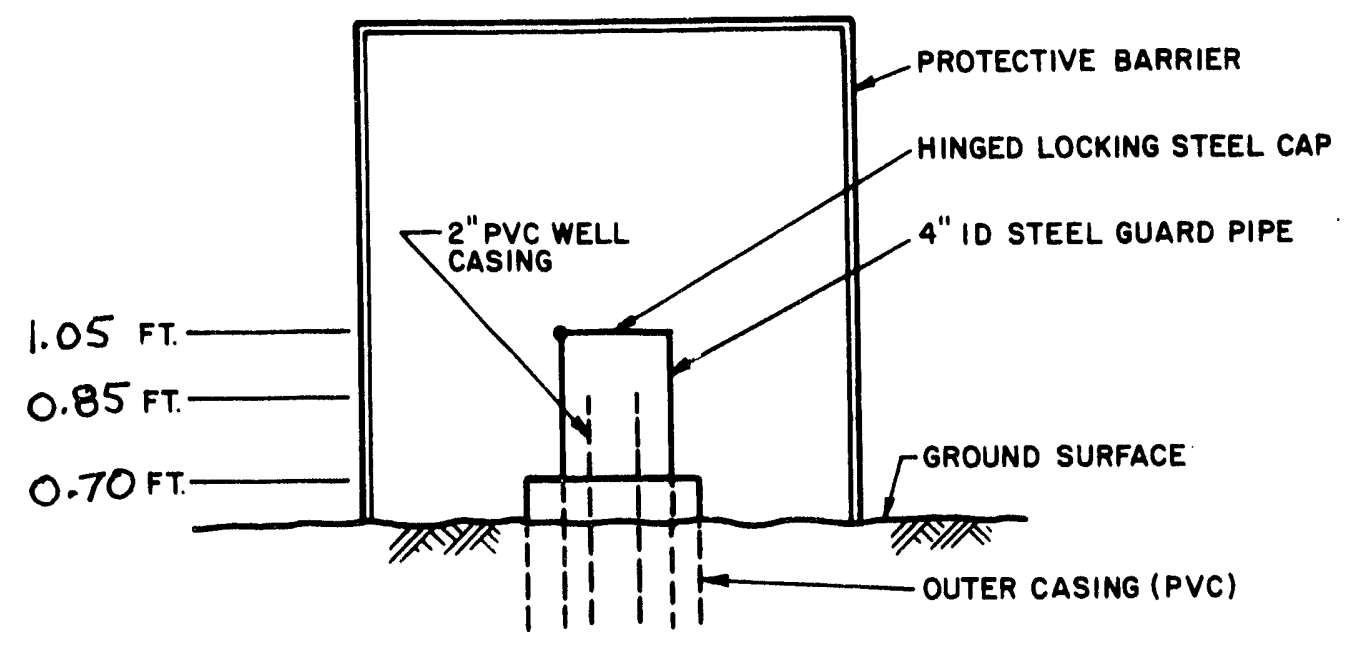

NOT TO SCALE 
SCHEMATIC OF PREABANDONMENT SITE STATUS

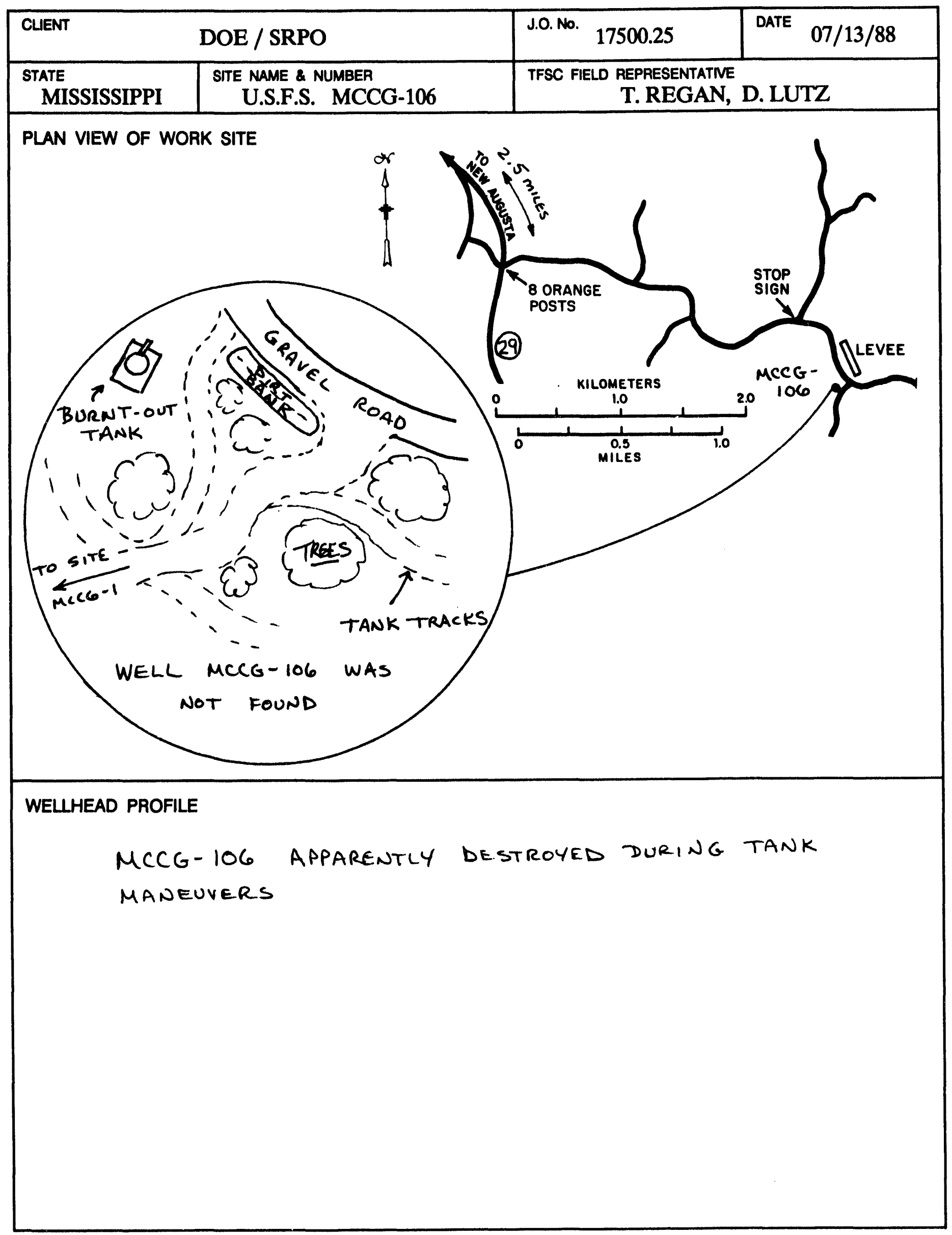




\section{SCHEMATIC OF PREABANDONMENT SITE STATUS}

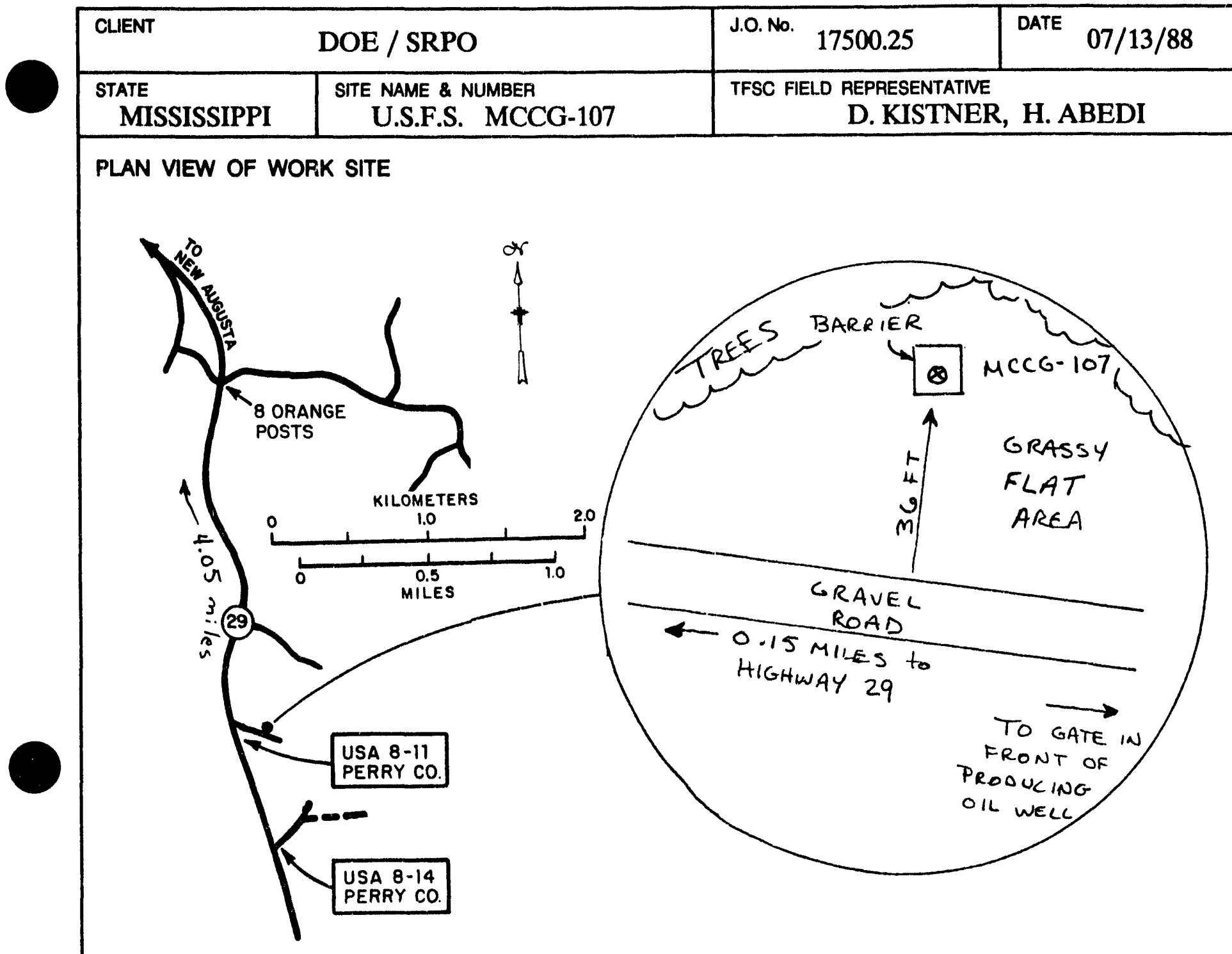

WELLHEAD PROFILE

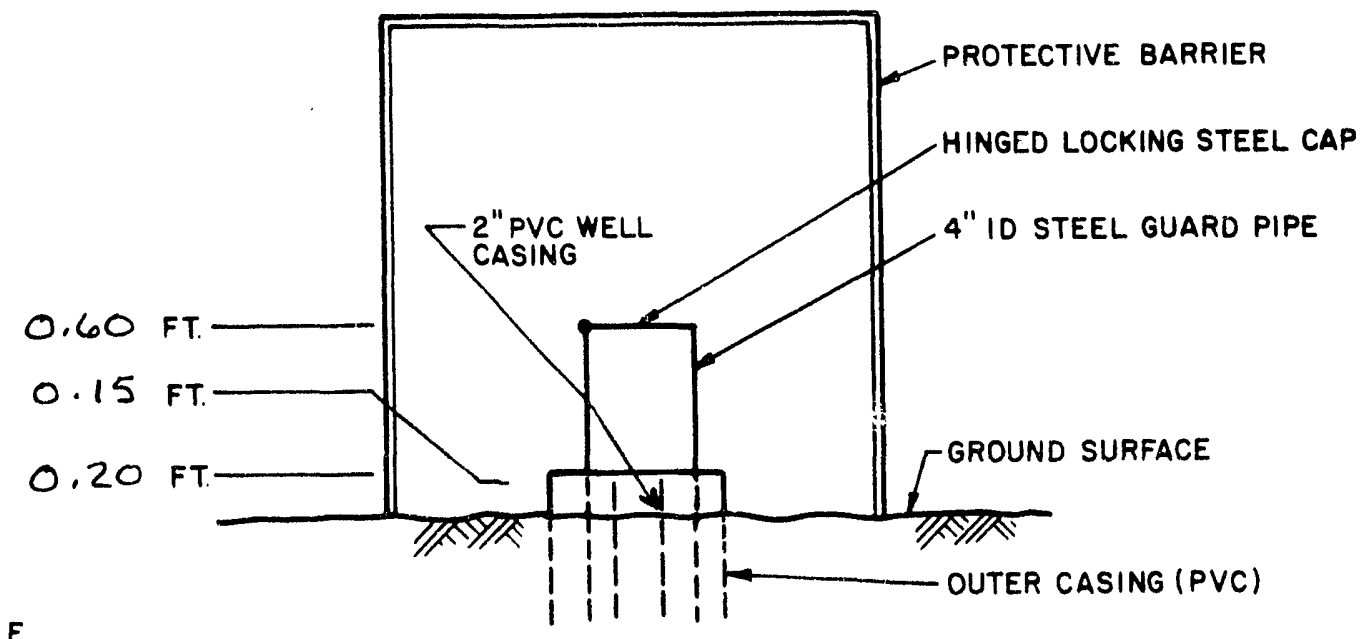

NOT TO SCALE 
SCHEMATIC OF PREABANDONMENT SITE STATUS

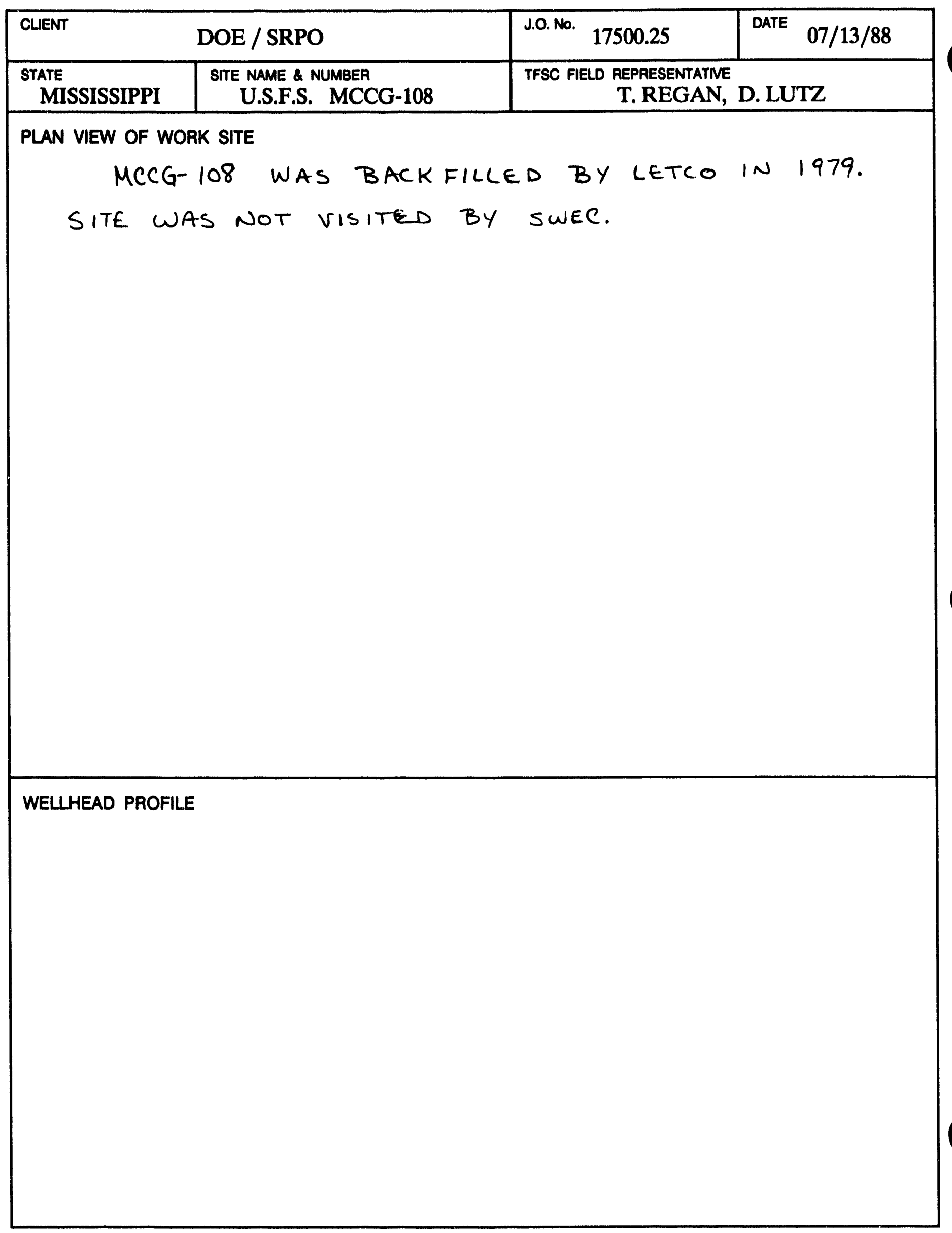


SCHEMATIC OF PREABANDONMENT SITE STATUS

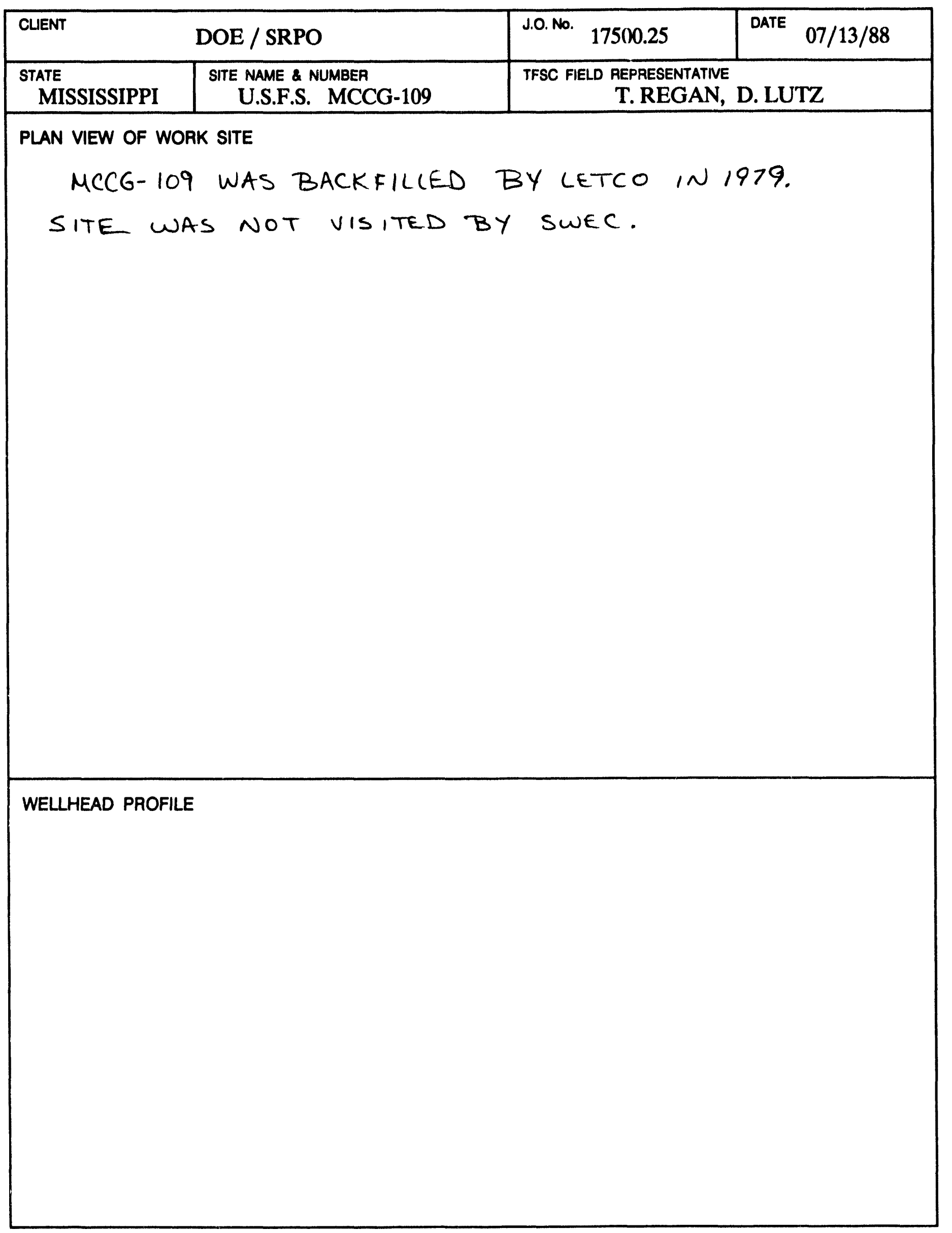


SCHEMATIC OF PREABANDONMENT SITE STATUS

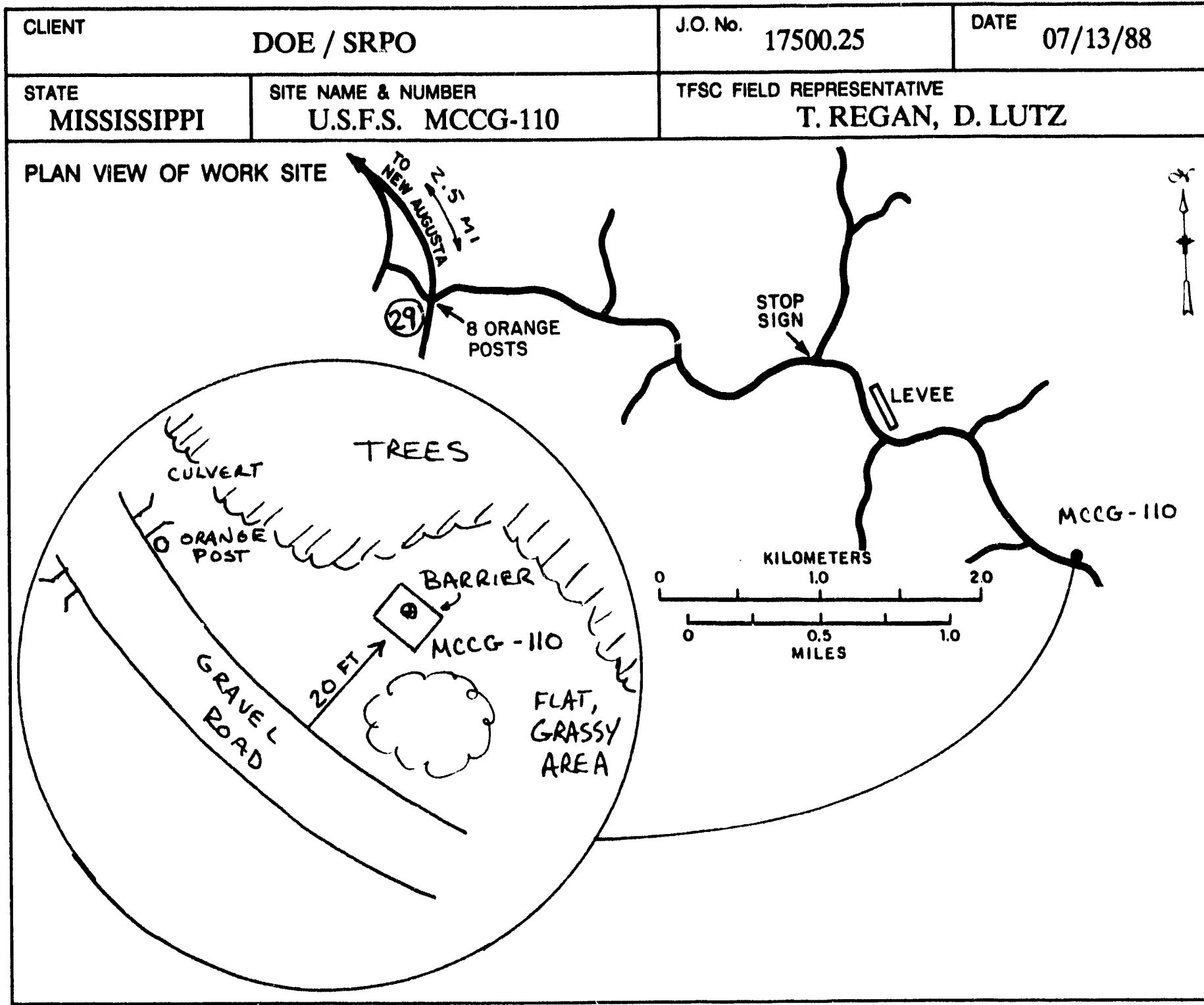

WELLHEAD PROFILE

NOT TO SCALE

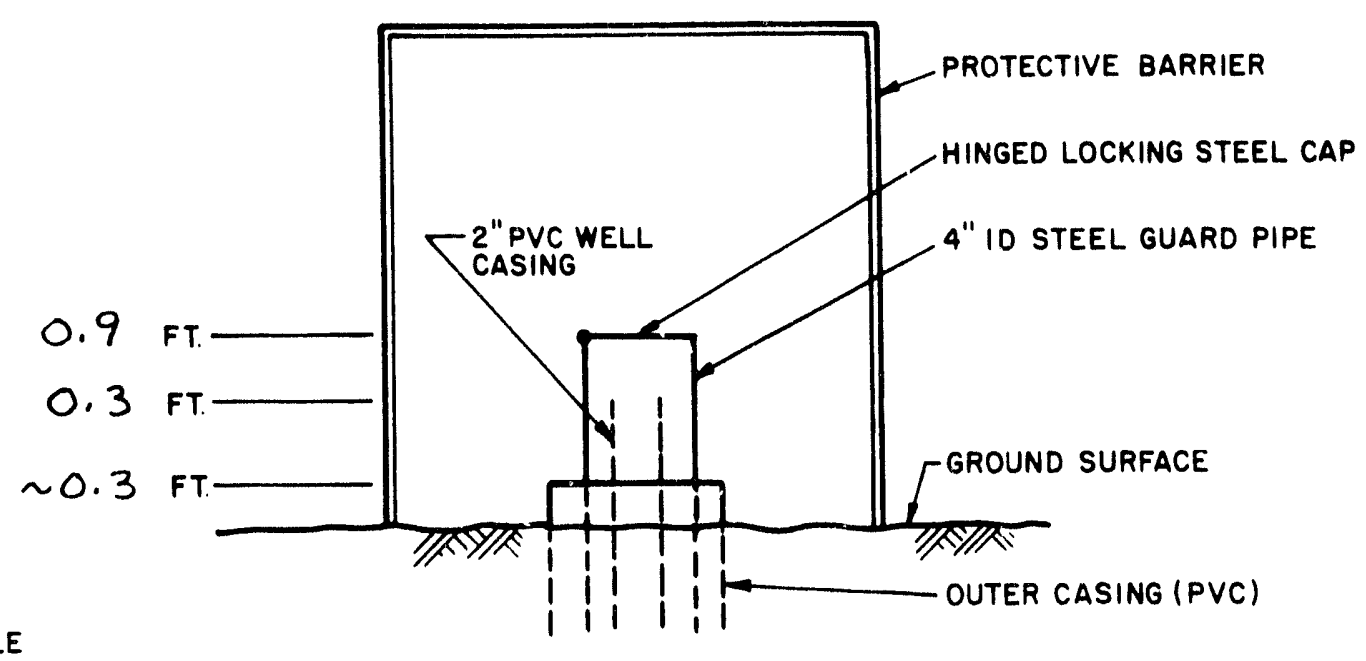


SCHEMATIC OF PREABANDONMENT SITE STATUS

\begin{tabular}{|l|l|l|l|}
\hline CLIENT & J.0. No. 17500.25 & DATE & $07 / 13 / 88$ \\
\hline $\begin{array}{c}\text { STATE } \\
\text { MISSISSIPPI }\end{array}$ & $\begin{array}{c}\text { SITE NAME \& NUMBER } \\
\text { U.S.F.S. MCCG-111 }\end{array}$ & $\begin{array}{r}\text { TFSC FIELD REPPESENTATNE } \\
\text { D. KISTNER, H. ABEDI }\end{array}$ \\
\hline
\end{tabular}

PLAN VIEW OF WORK SITE
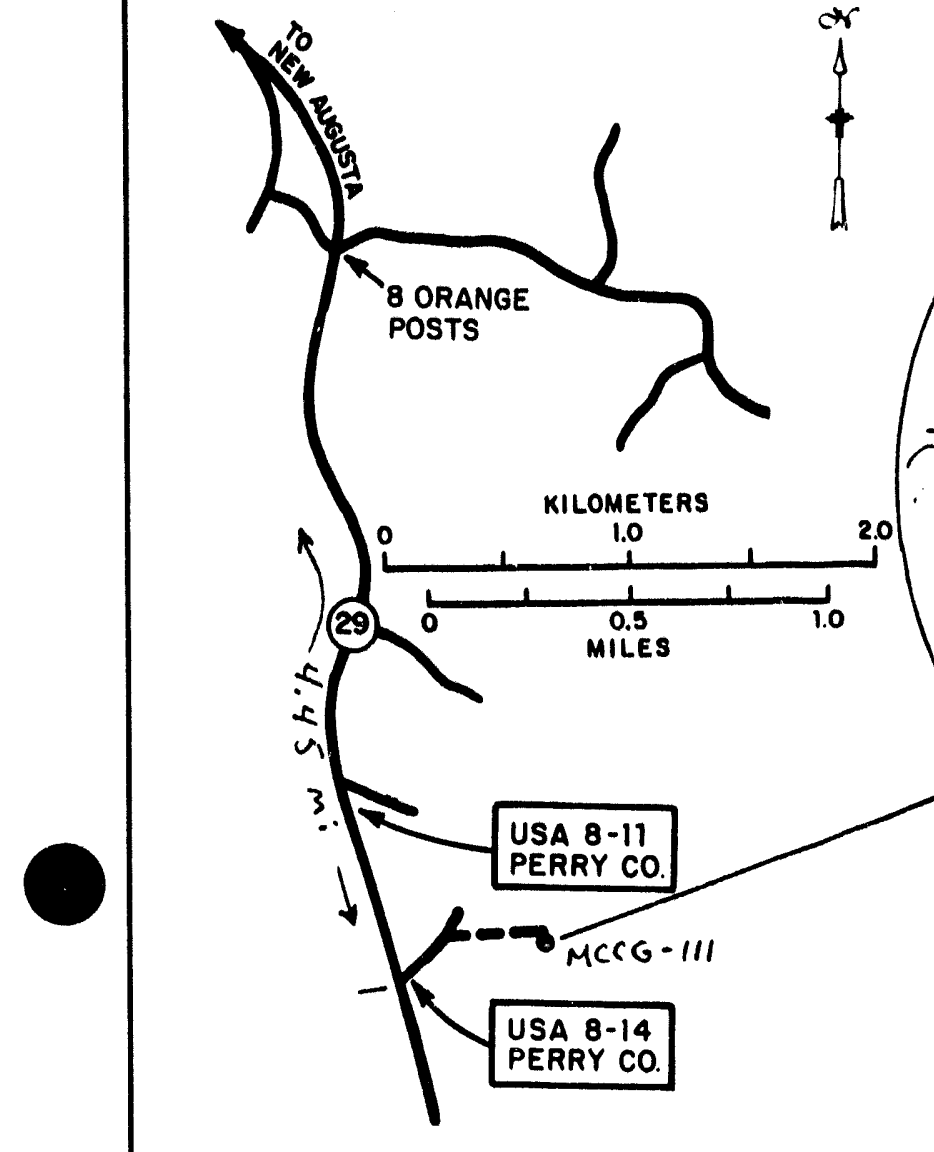

WELLHEAD PROFILE

NOT TO SCALE

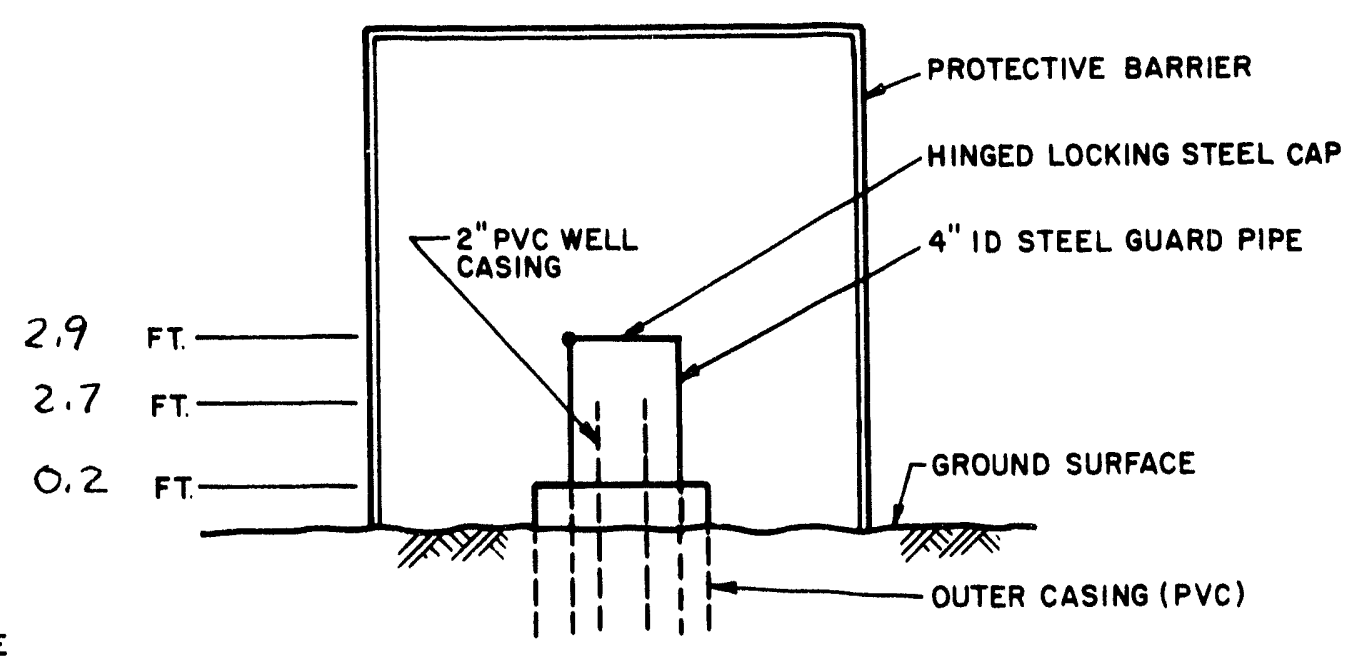
GOODGRAVEL ROAO FROM HWY 29 TO
PRODUCING OIL WELL SITE. UNIMPROVEO
DIRT ROAD OFF OF GRAVEL ROAD, O.2MI.
FROM HWY 29 , SLOPES STEADILY DOWN -
HILL FROM GRAVE L ROAD TOSITE. GOODGRAVEL ROAO FROM HWY 29 TO
PRODUCING OIL WELL SITE. UNIMPROVED
DIRT ROAD OFF OF GRAVEL ROAD, O.2MI.
FROM HWY 29 , SLOPES STEADILY DOWN -
HILL FROM GRAVE L ROAD TOSITE. GOODGRAVEL ROAO FROM HWY 29 TO
PRODUCING OIL WELL SITE. UNIMPROVED
DIRT ROAD OFF OF GRAVEL ROAD, O.2MI.
FROM HWY 29 , SLOPES STEADILY DOWN -
HILL FROM GRAVE L ROAD TOSITE. GOODGRAVEL ROAO FROM HWY 29 TO
PRODUCING OIL WELL SITE. UNIMPROVEO
DIRT ROAD OFF OF GRAVEL ROAD, O.2MI.
FROM HWY 29 , SLOPES STEADILY DOWN -
HILL FROM GRAVE L ROAD TOSITE.

- Unimproved DIRT ROAD

SITE HOOFT N $80^{\circ} E$ FROM GOOD

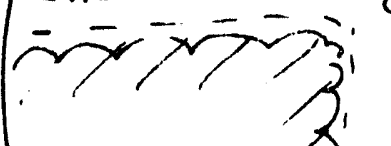
TREESS
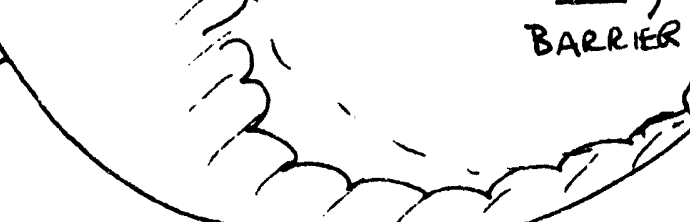


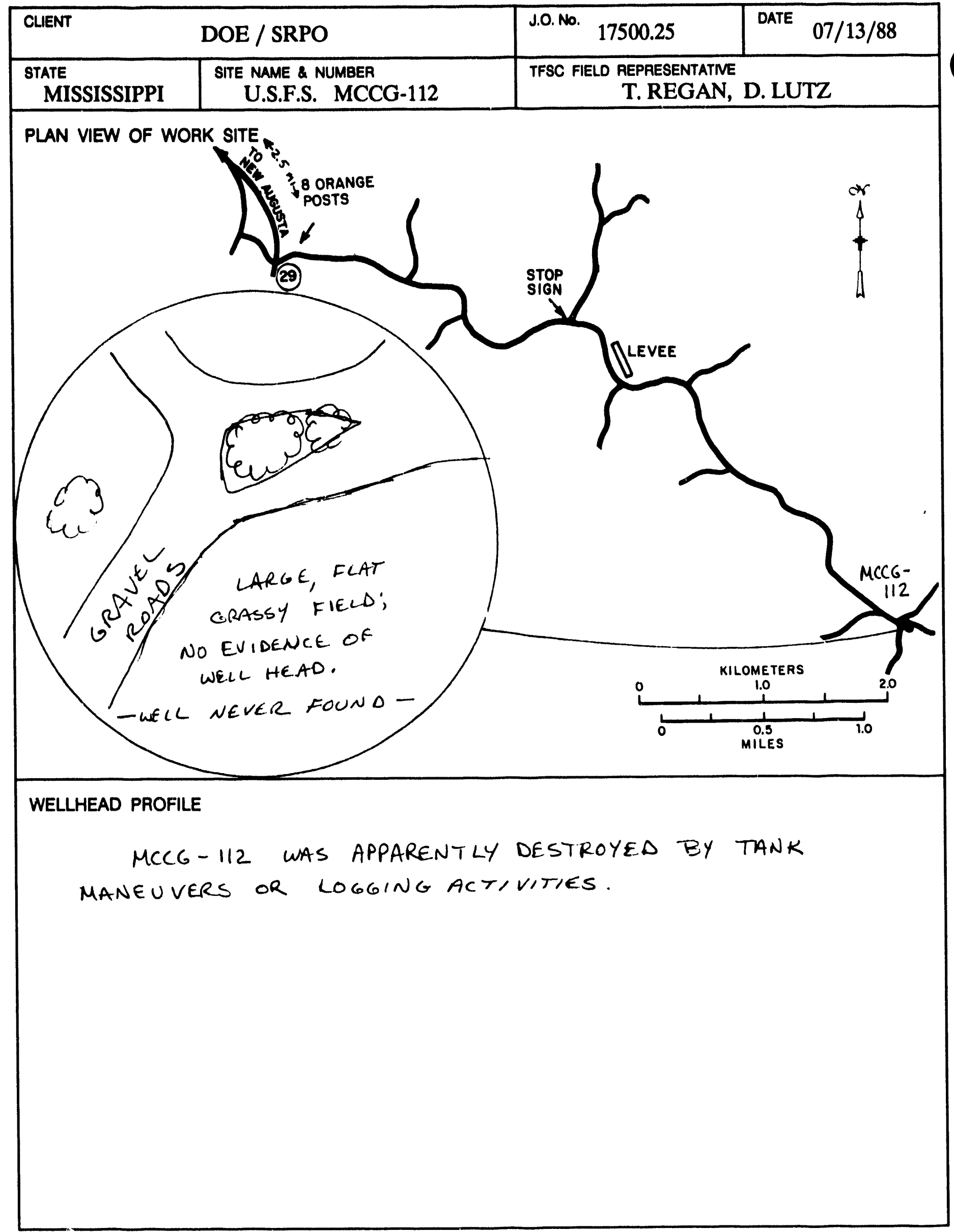


SCHEMATIC OF PREABANDONMENT SITE STATUS

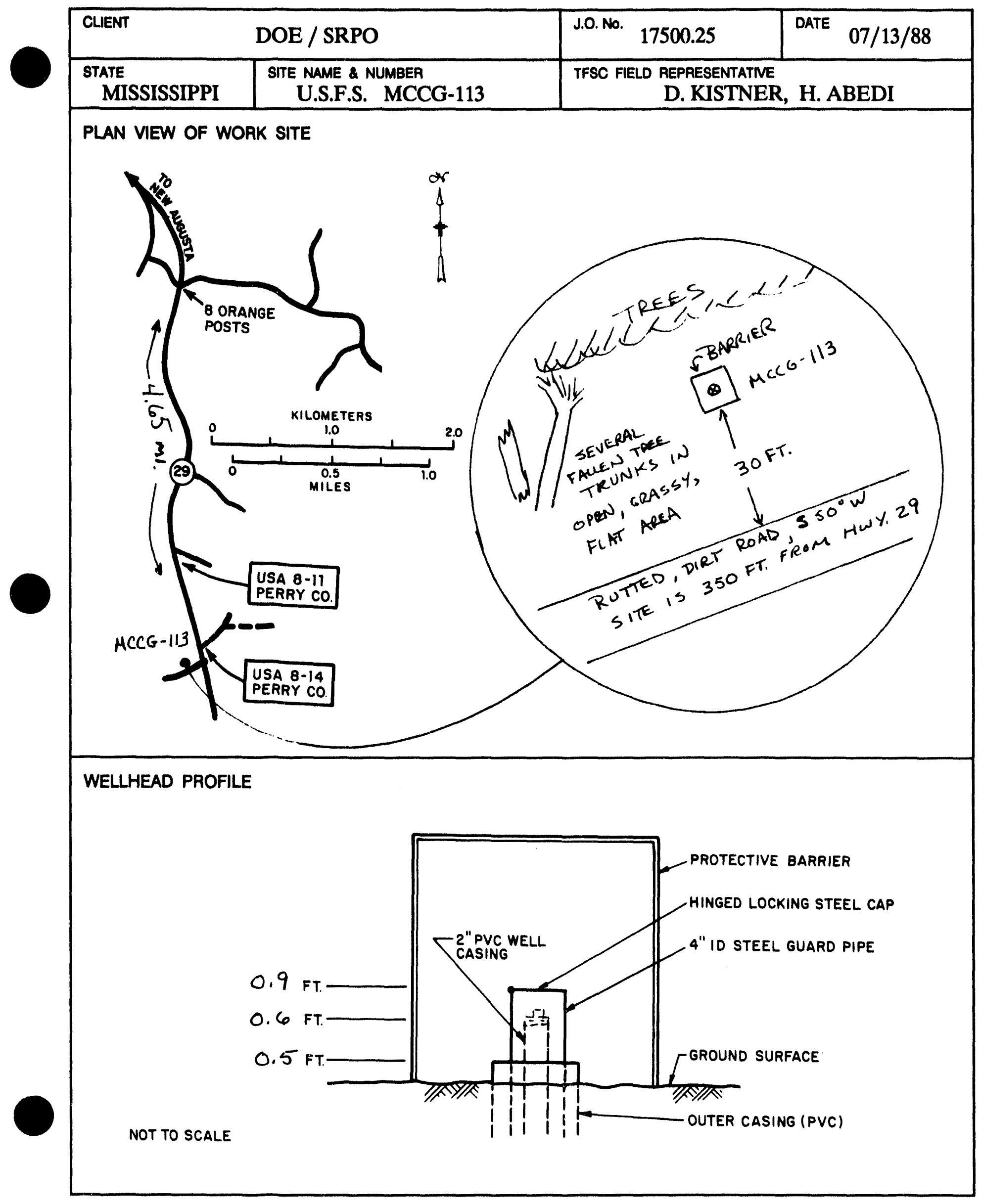




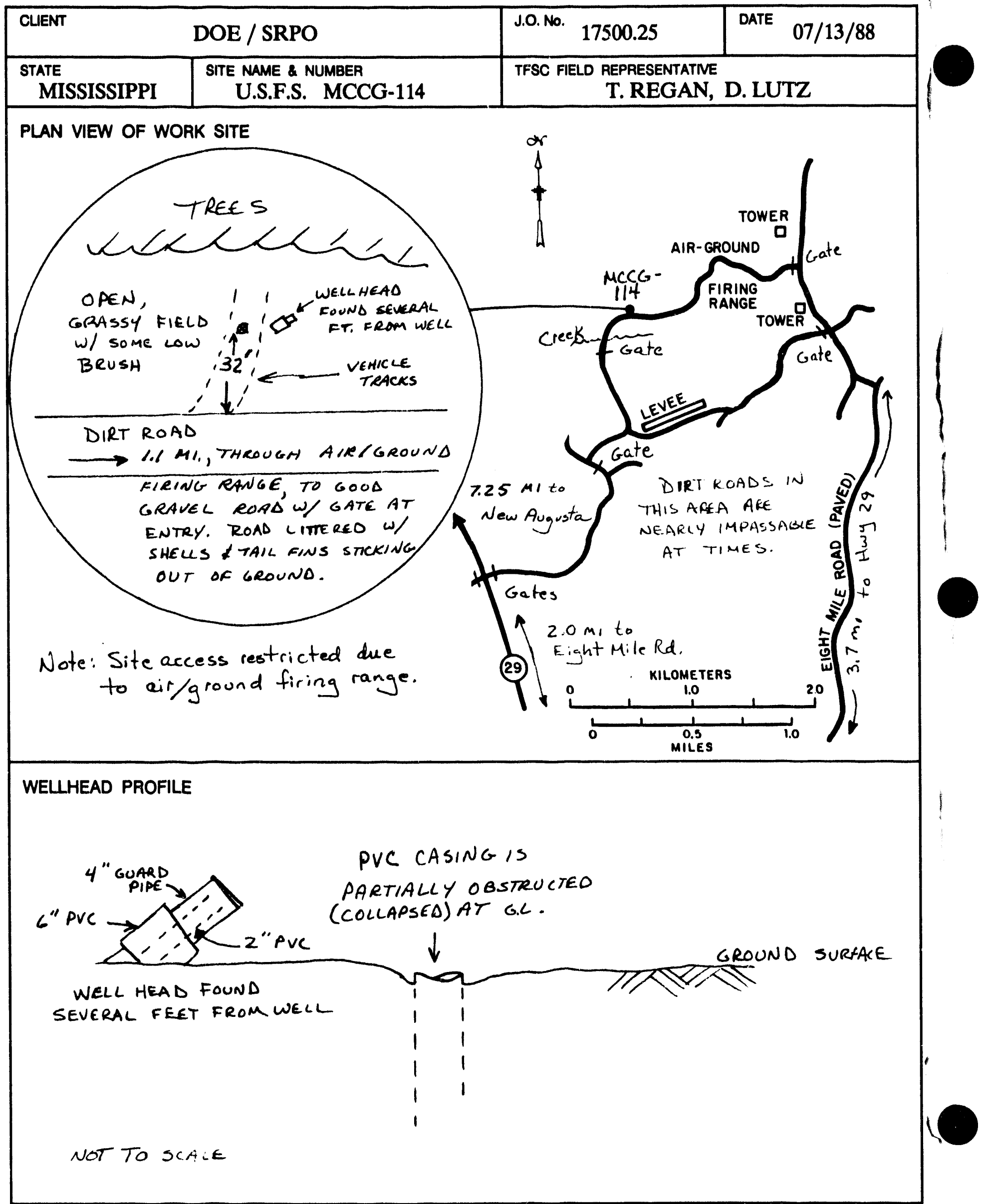


SCHEMATIC OF PREABANDONMENT SITE STATUS

\begin{tabular}{|l|l|l|l|}
\hline CLIENT & J.0. No. 17500.25 & DATE & $07 / 13 / 88$ \\
\hline $\begin{array}{c}\text { STATE } \\
\text { MISSISSIPPI }\end{array}$ & $\begin{array}{r}\text { SITE NAME \& NUMBER } \\
\text { U.S.F.S. MCCG-115 }\end{array}$ & $\begin{array}{r}\text { TFSC FIELD REPRESENTATNE } \\
\text { D. KISTNER, H. ABEDI }\end{array}$ \\
\hline
\end{tabular}

PLAN VIEW OF WORK SITE

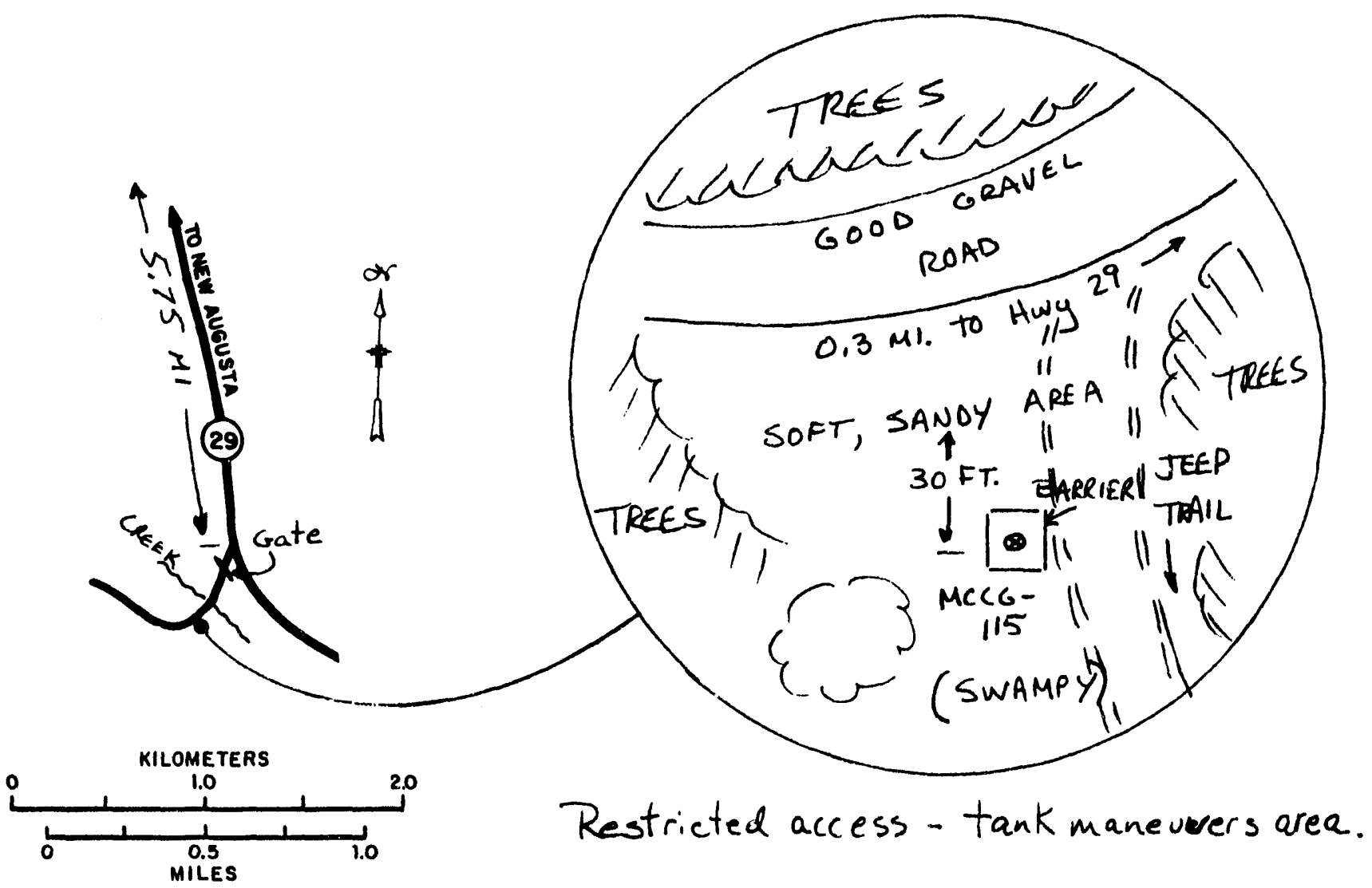

WELLHEAD PROFILE

NOT TO SCALE

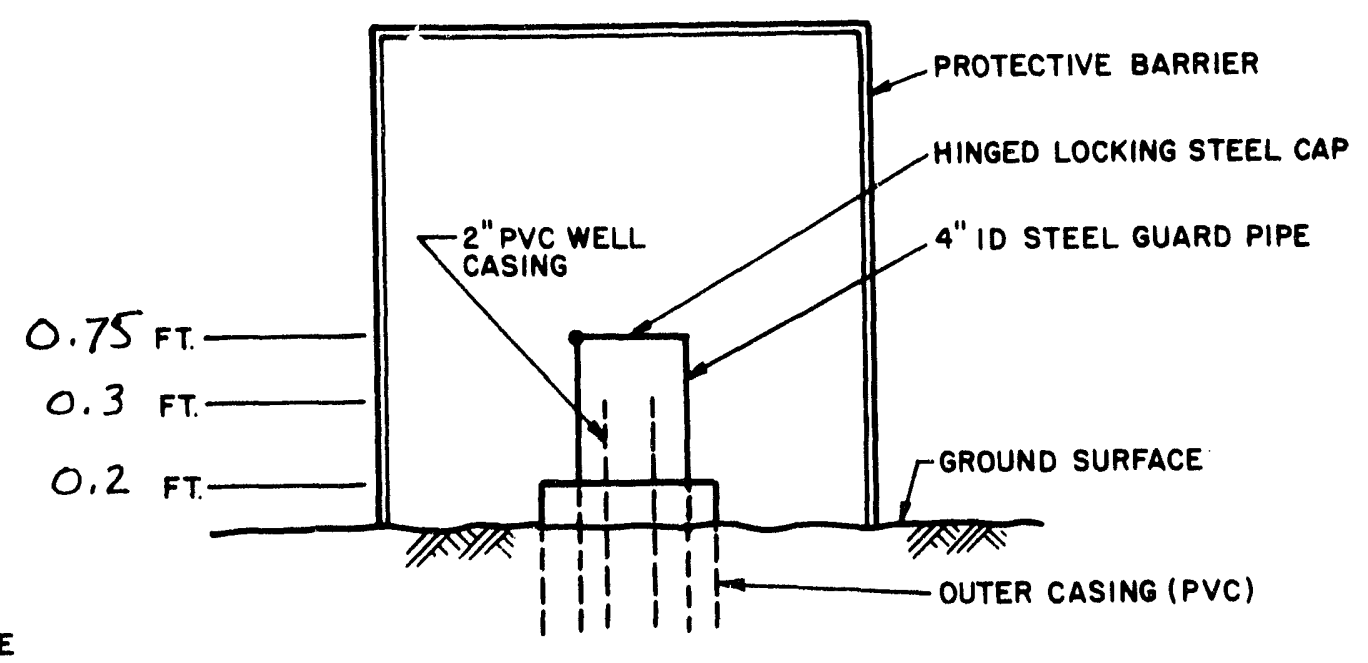


SCHEMATIC OF PREABANDONMENT SITE STATUS

\begin{tabular}{|l|l|l|l|}
\hline CLIENT & DOE / SRPO & J.0. No. 17500.25 & DATE $07 / 13 / 88$ \\
\hline $\begin{array}{l}\text { STATE } \\
\text { MISSISSIPPI }\end{array}$ & $\begin{array}{l}\text { SITE NAME \& NUMBER } \\
\text { U.S.F.S. MCCG-116 }\end{array}$ & $\begin{array}{r}\text { TFSC FIELD REPRESENTATNE } \\
\text { T. REGAN, D. LUTZ }\end{array}$ \\
\hline
\end{tabular}

PLAN VIEW OF WORK SITE

MCCG - 116 WAS BACKFILLED BY LETCO IN 1979.

SITE WAS NOT VISITED BY SWEC.

WELLHEAD PROFILE 
SCHEMATIC OF PREABANDONMENT SITE STATUS

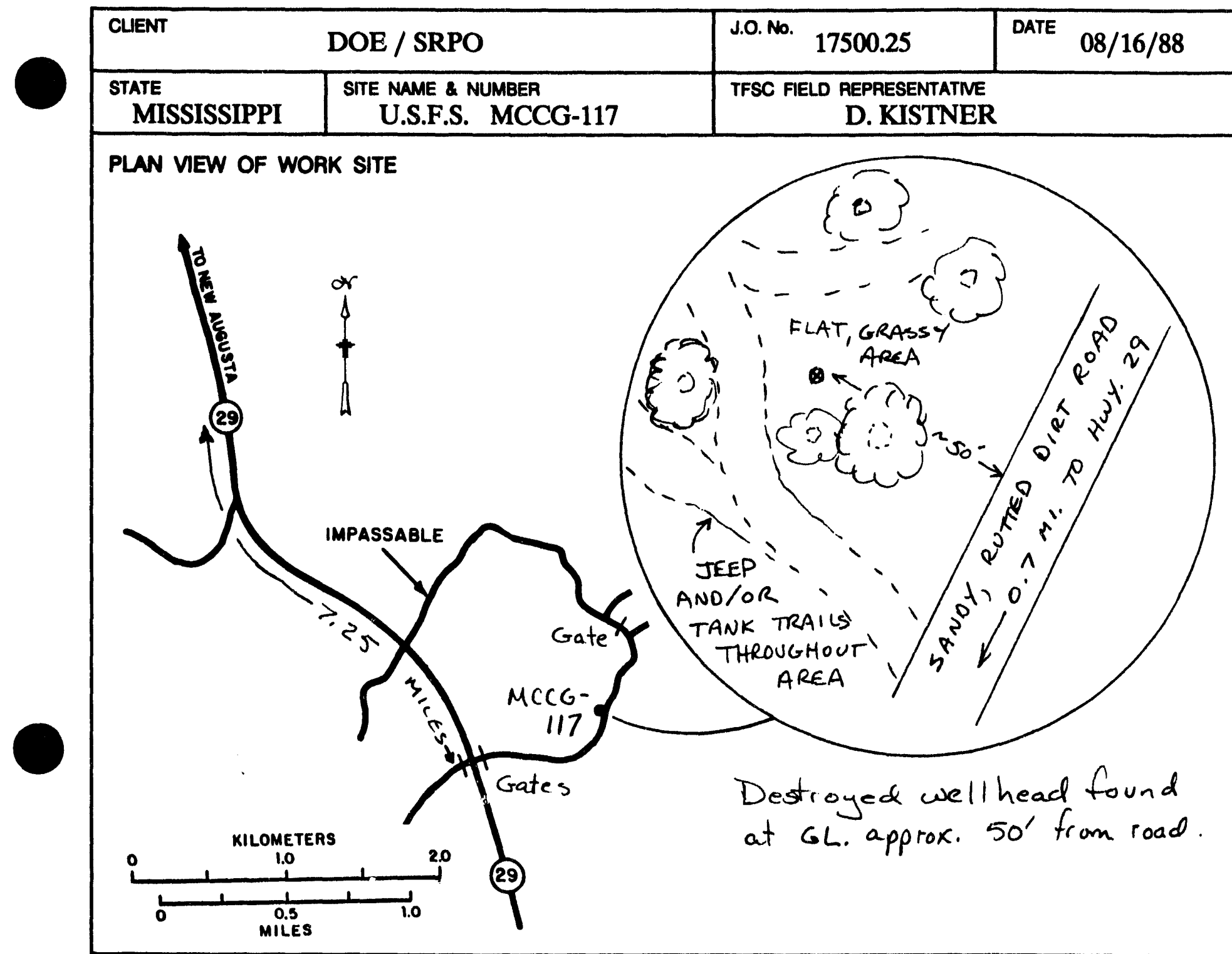

WEUHEAD PROFILE

NOT TO SCALE

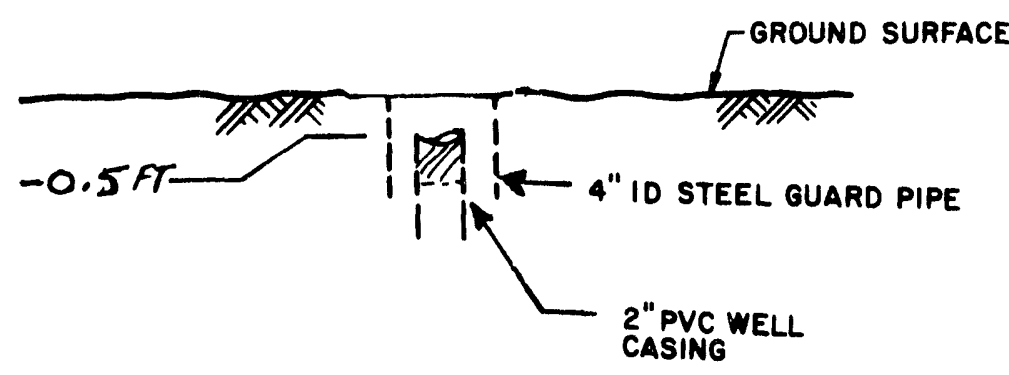

2 " CASING o bStructeo

AT 0.5 FT. BELOW GL. 
SCHEMATIC OF PREABANDONMENT SITE STATUS

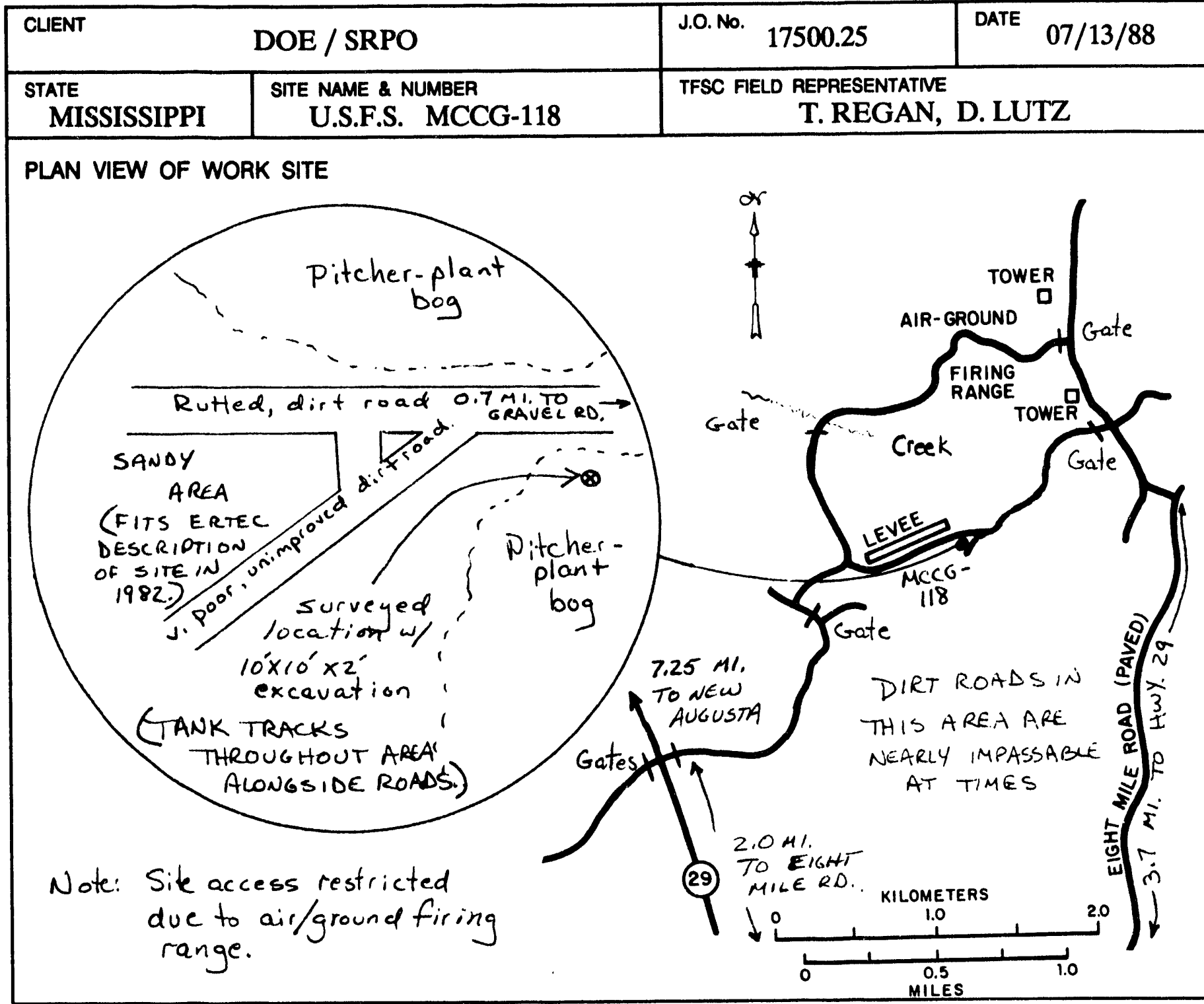

WELLHEAD PROFILE

WELL MCCG-118 WAS APPARENTLY DESTROYED BY TANK MANEUVERS. WELl WAS NEVER FOUND. 
SCHEMATIC OF PREABANDONMENT SITE STATUS

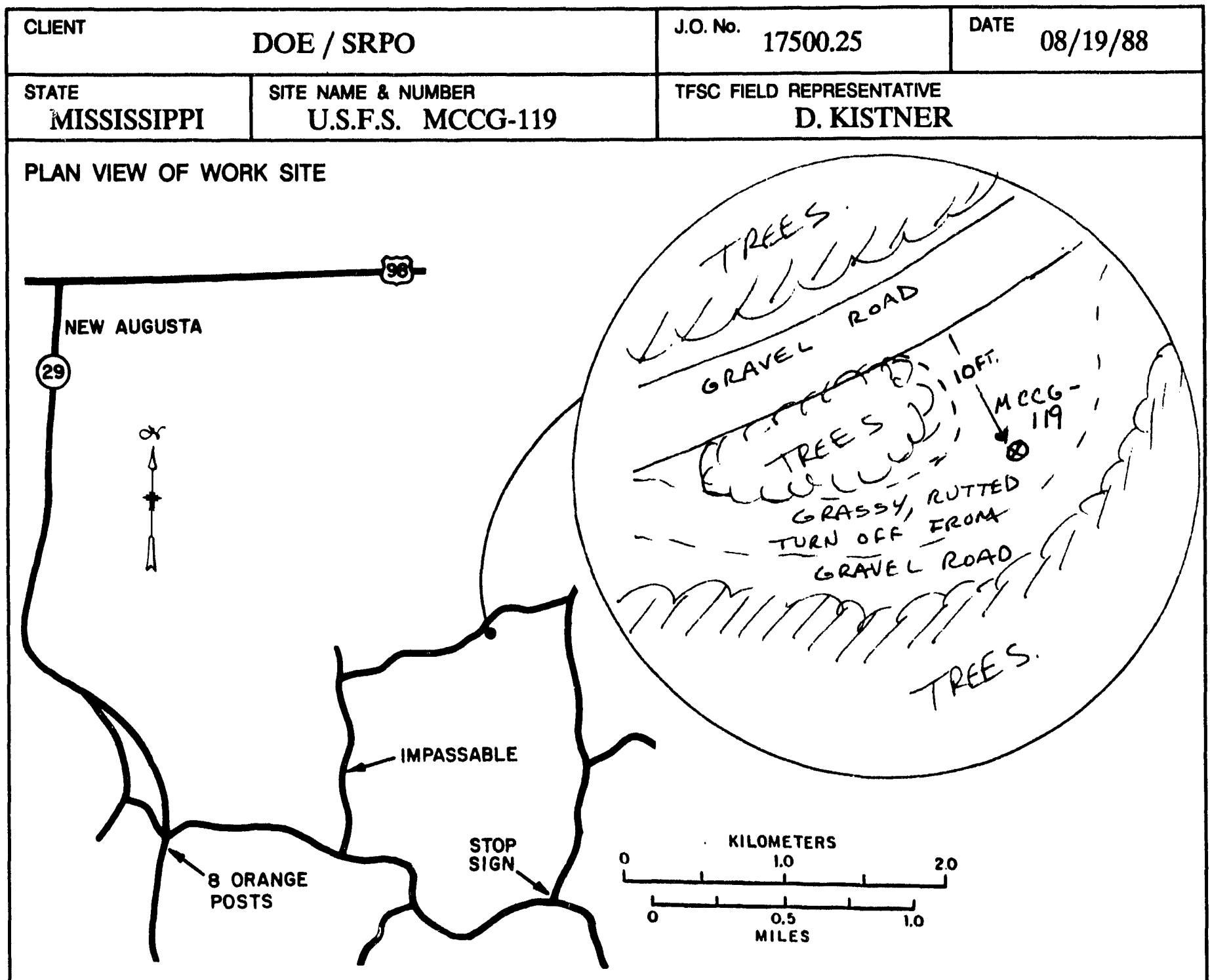

WELLHEAD PROFILE

OUTER CASING (PVC)

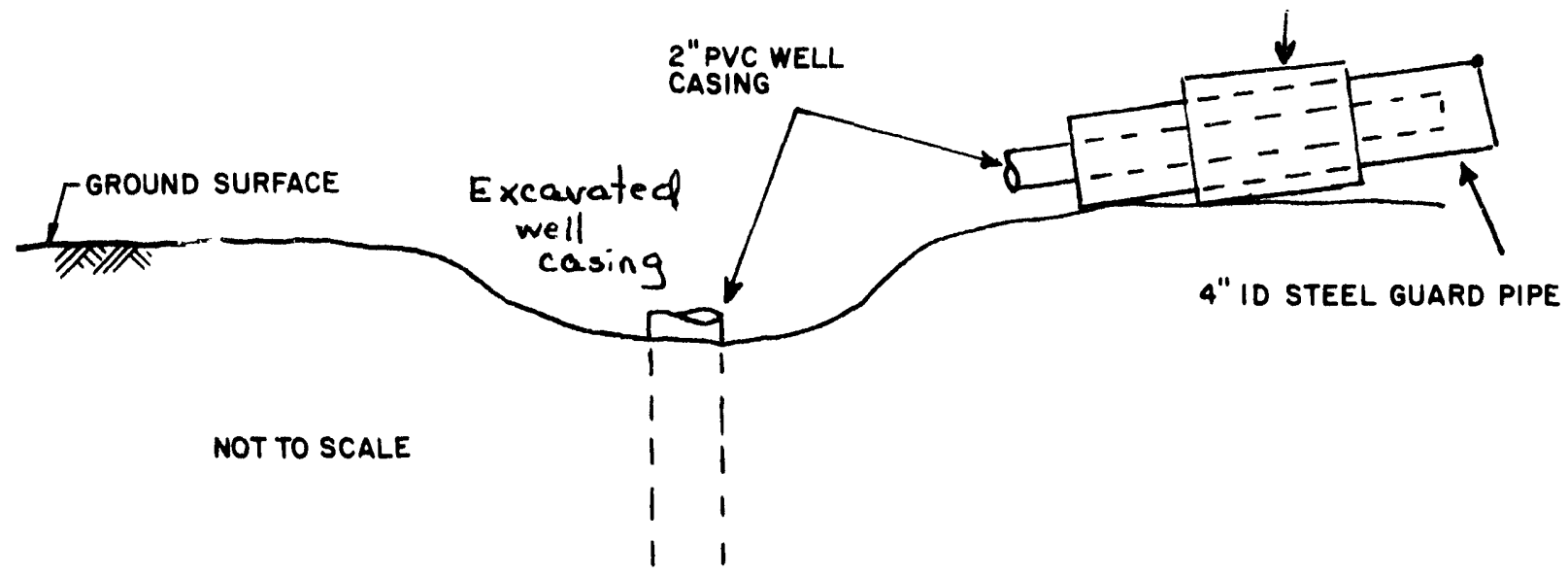


SCHEMATIC OF PREABANDONMENT SITE STATUS

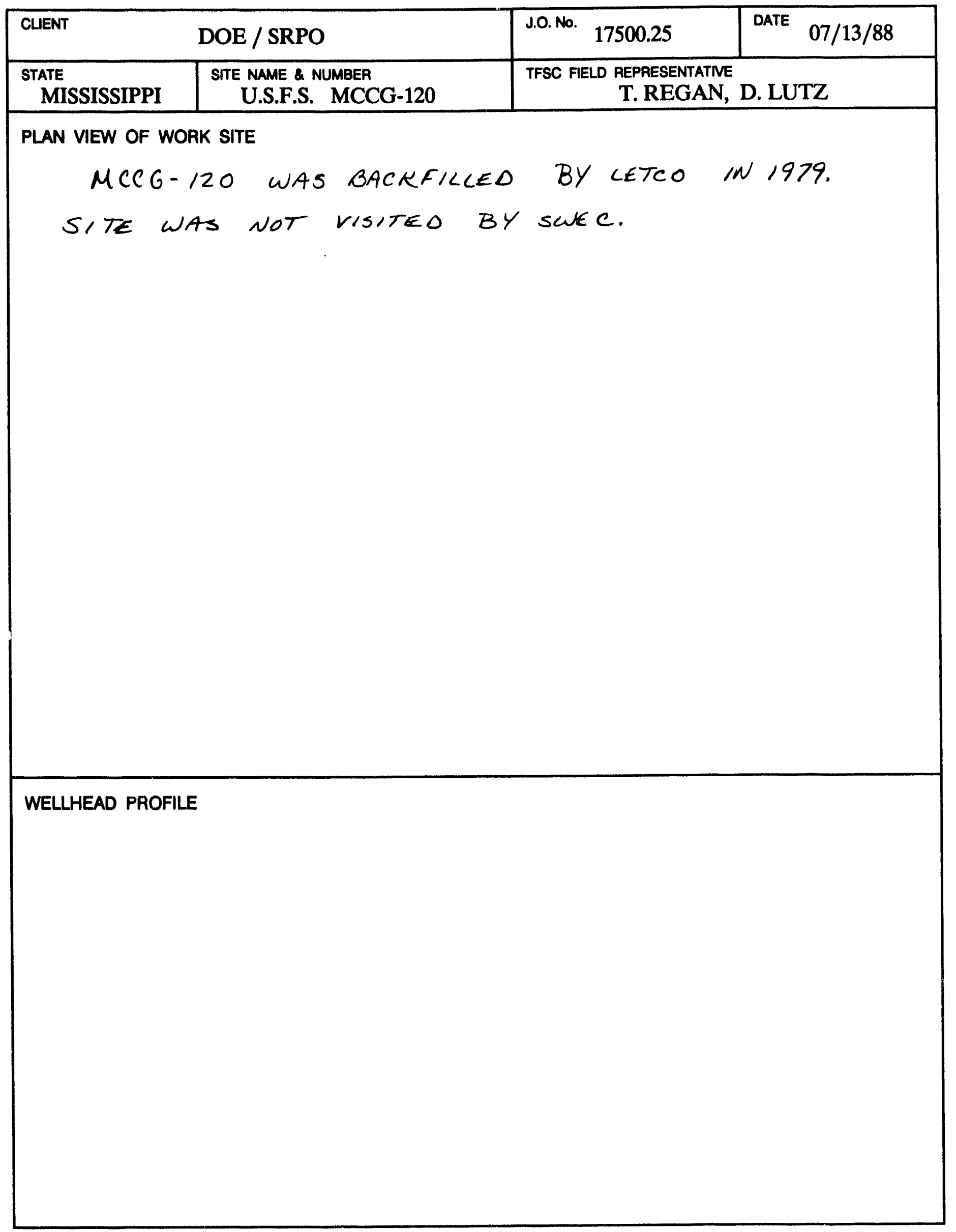


SCHEMATIC OF PREABANDONMENT SITE STATUS

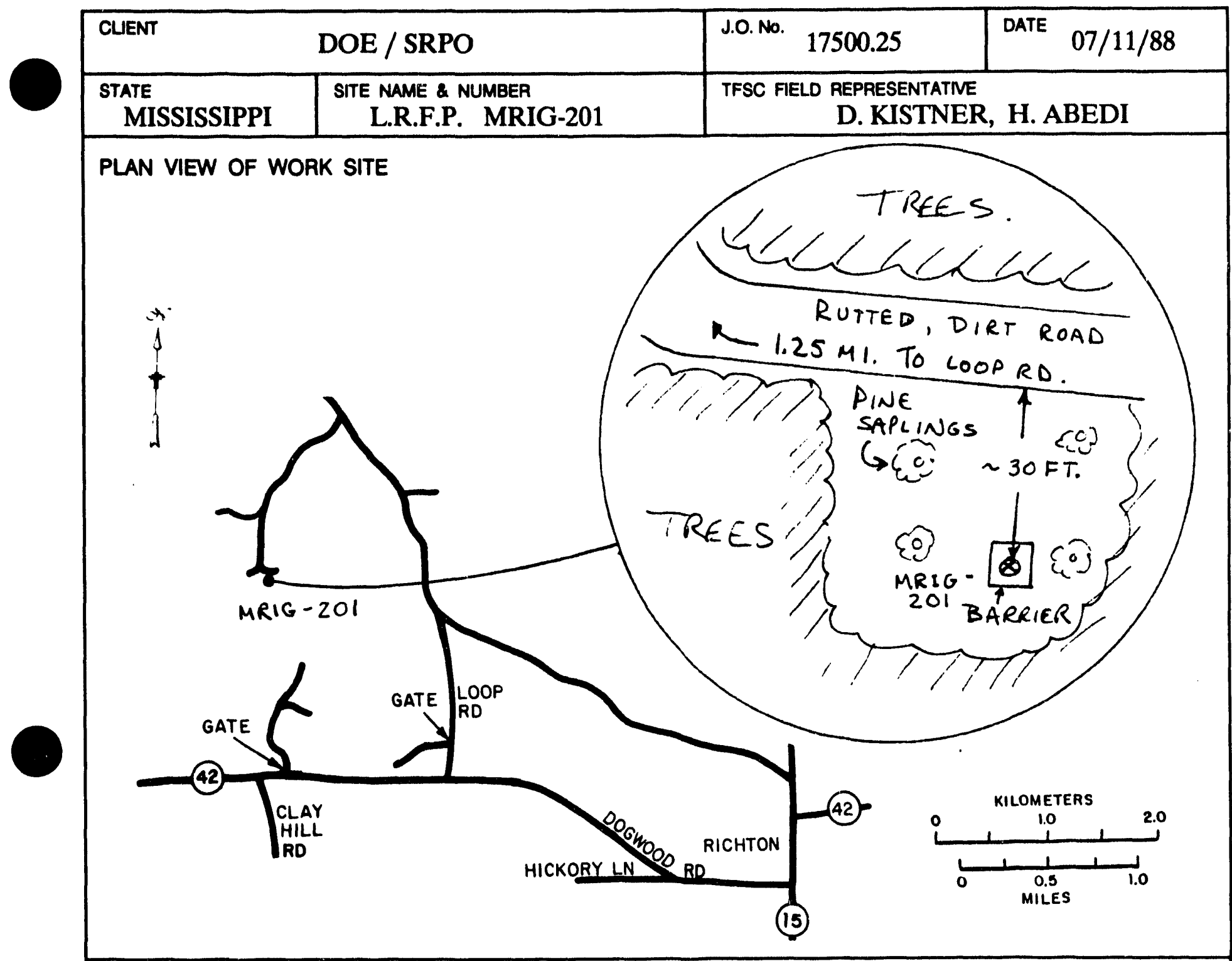

WELLHEAD PROFILE

NOT TO SCALE

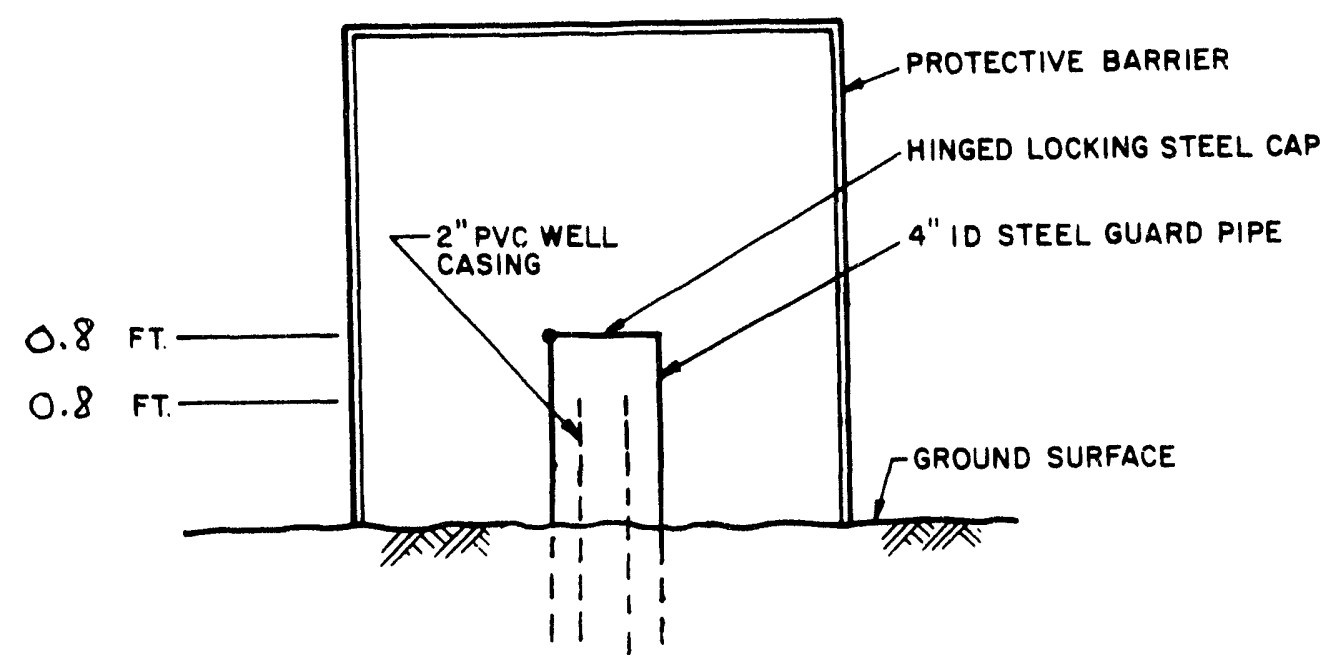


SCHEMATIC OF PREABANDONMENT SITE STATUS

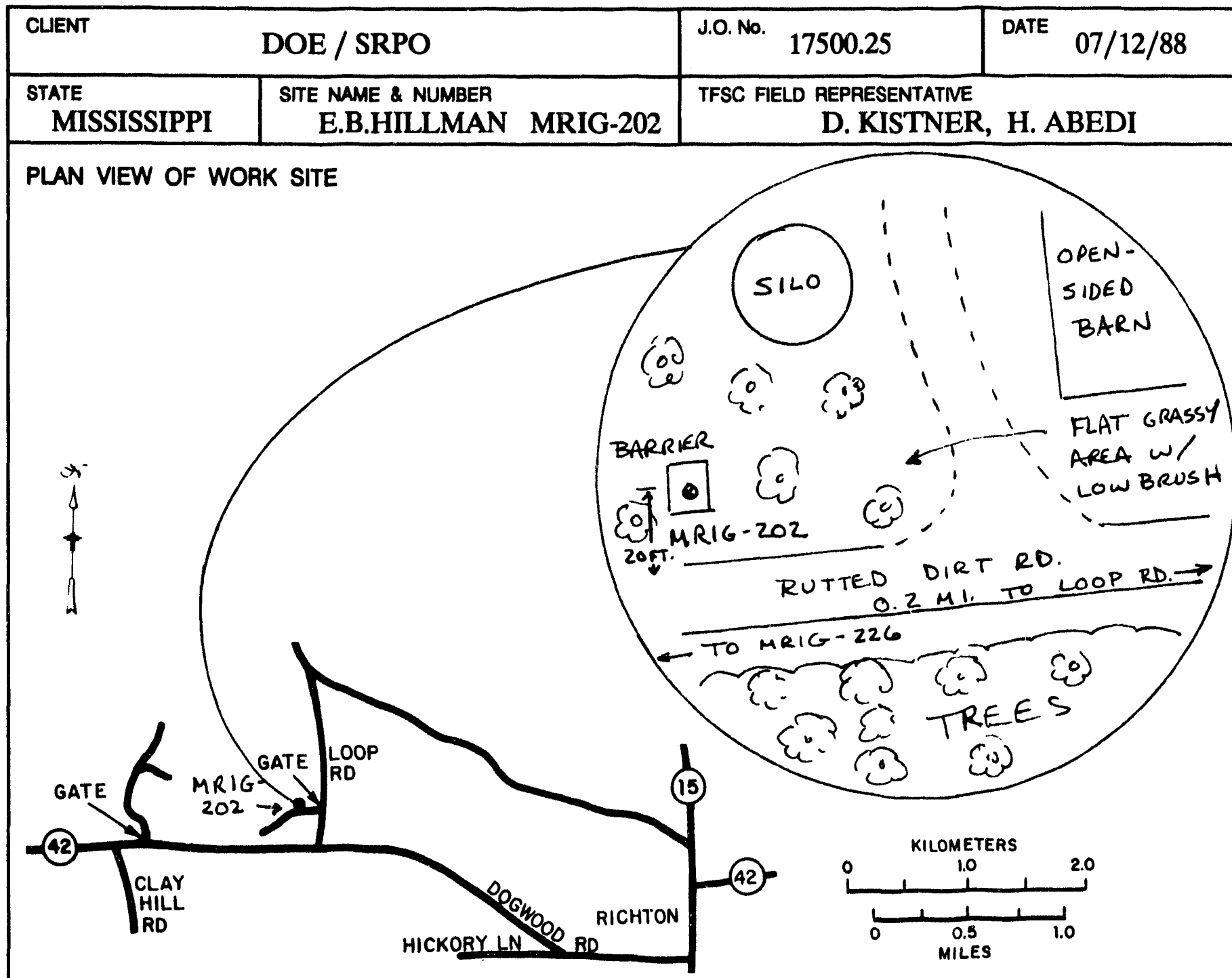

WELLHEAD PROFILE

NOT TO SCALE

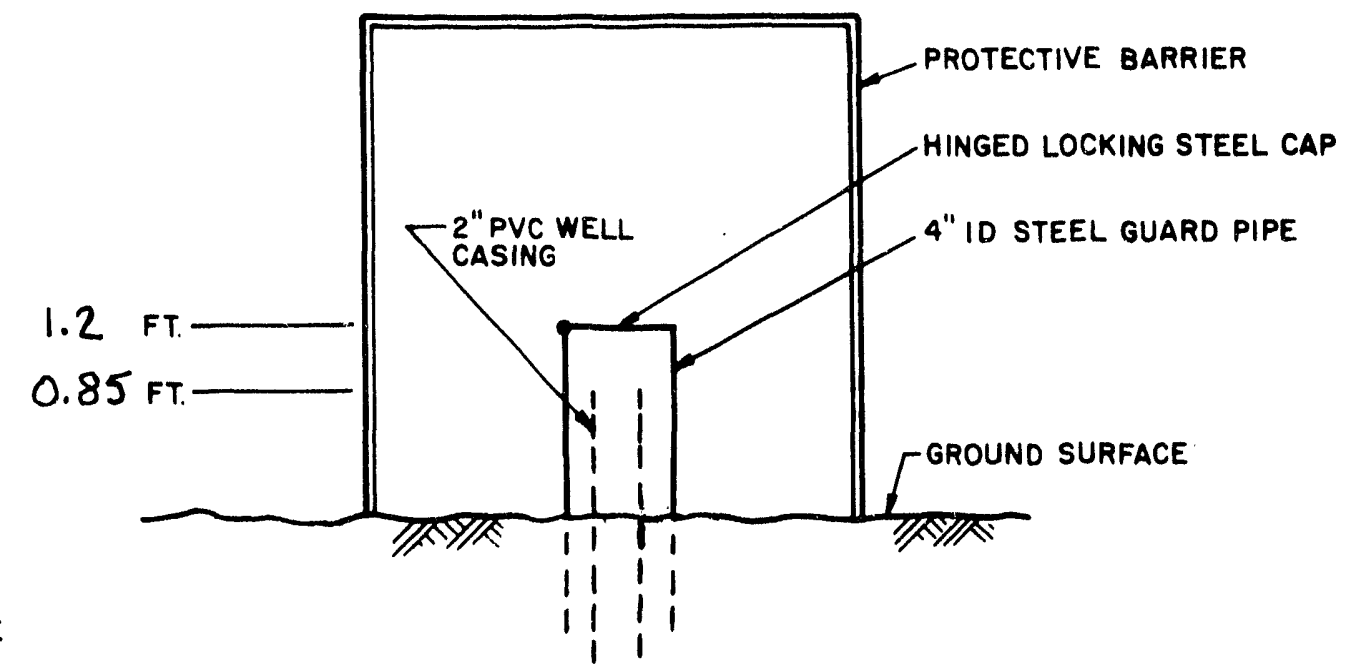


SCHEMATIC OF PREABANDONMENT SITE STATUS

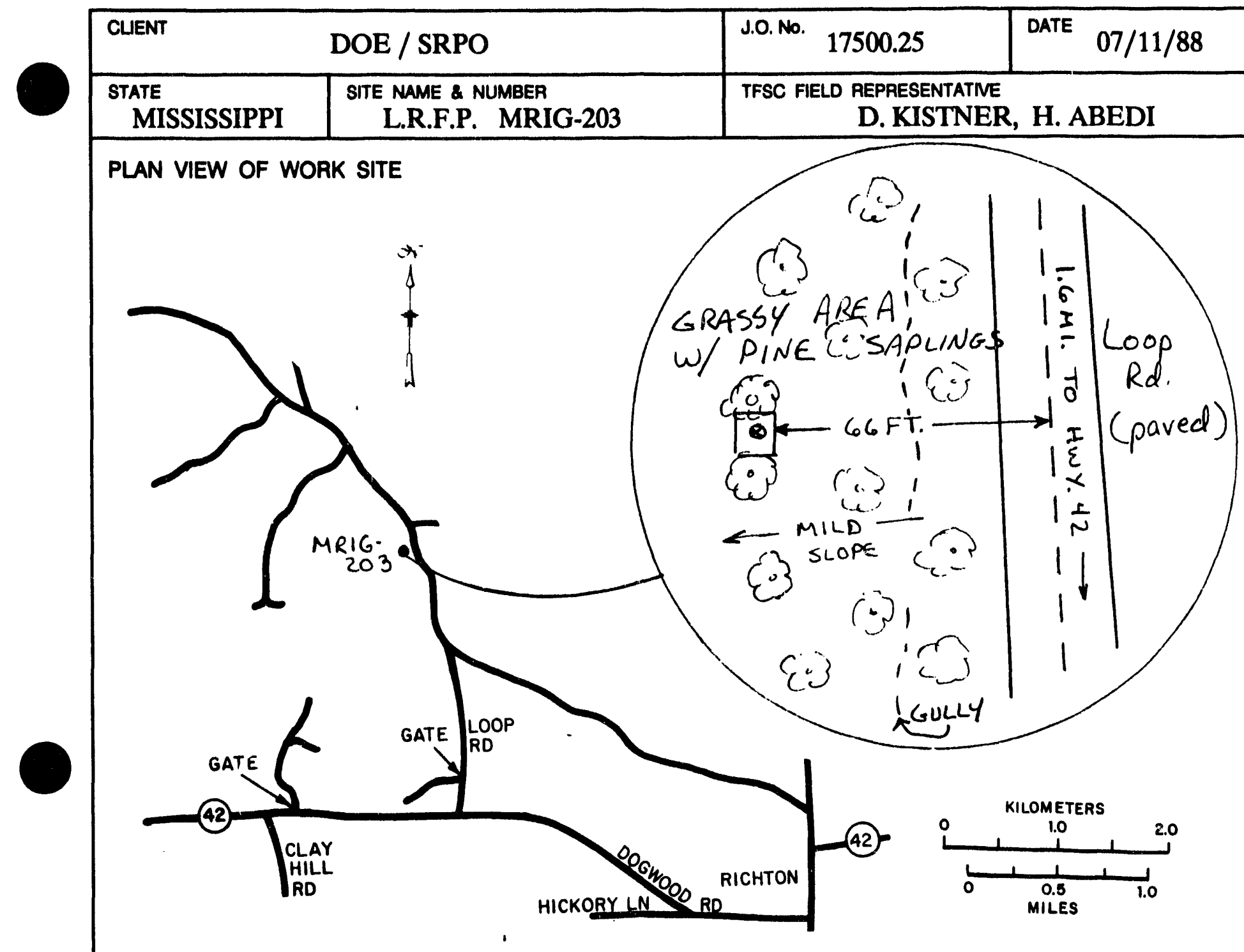

WELLHEAD PROFILE

NOT TO SCALE

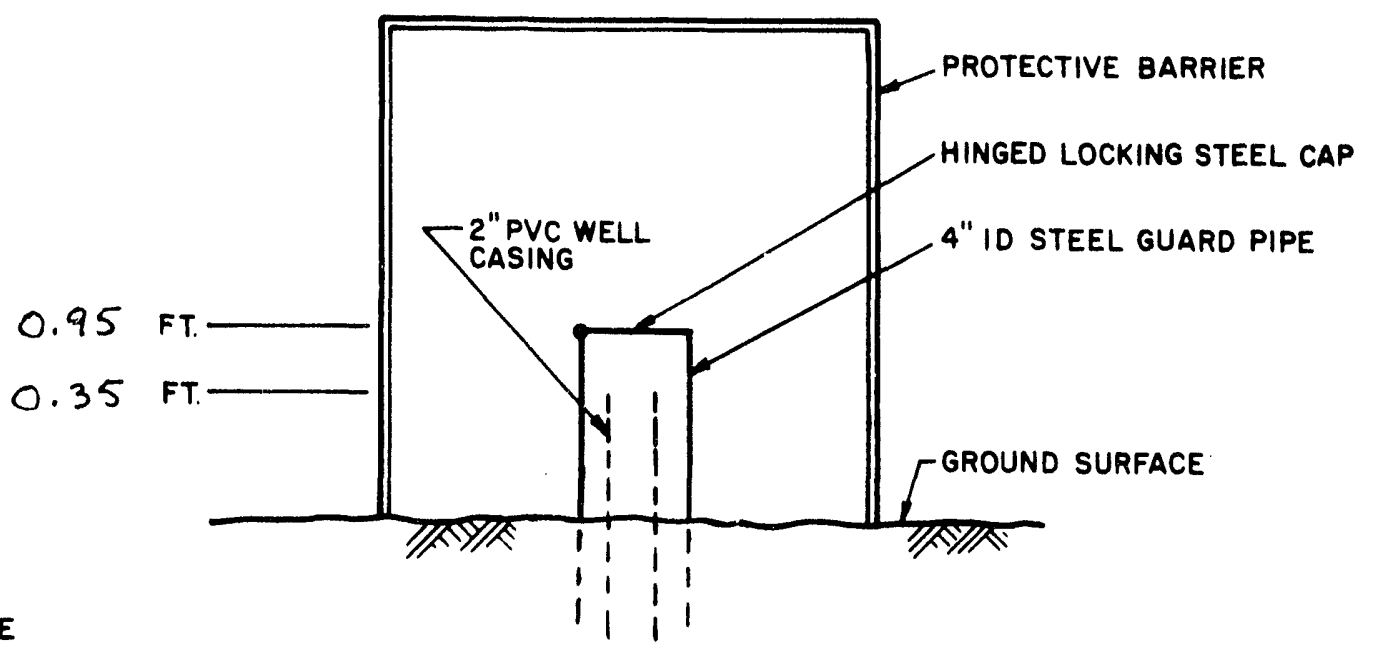


SCHEMATIC OF PREABANDONMENT SITE STATUS

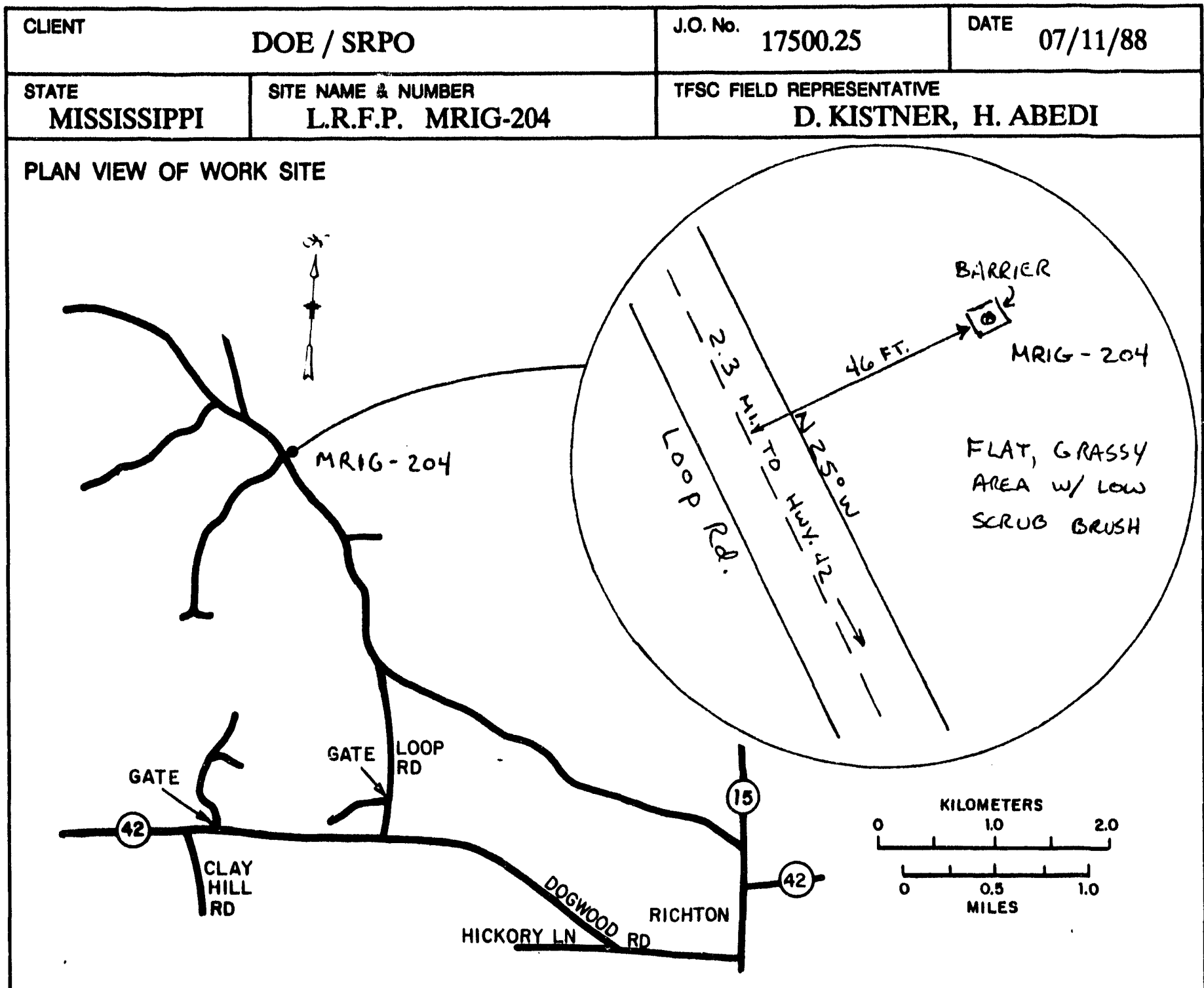

WELLHEAD PROFILE

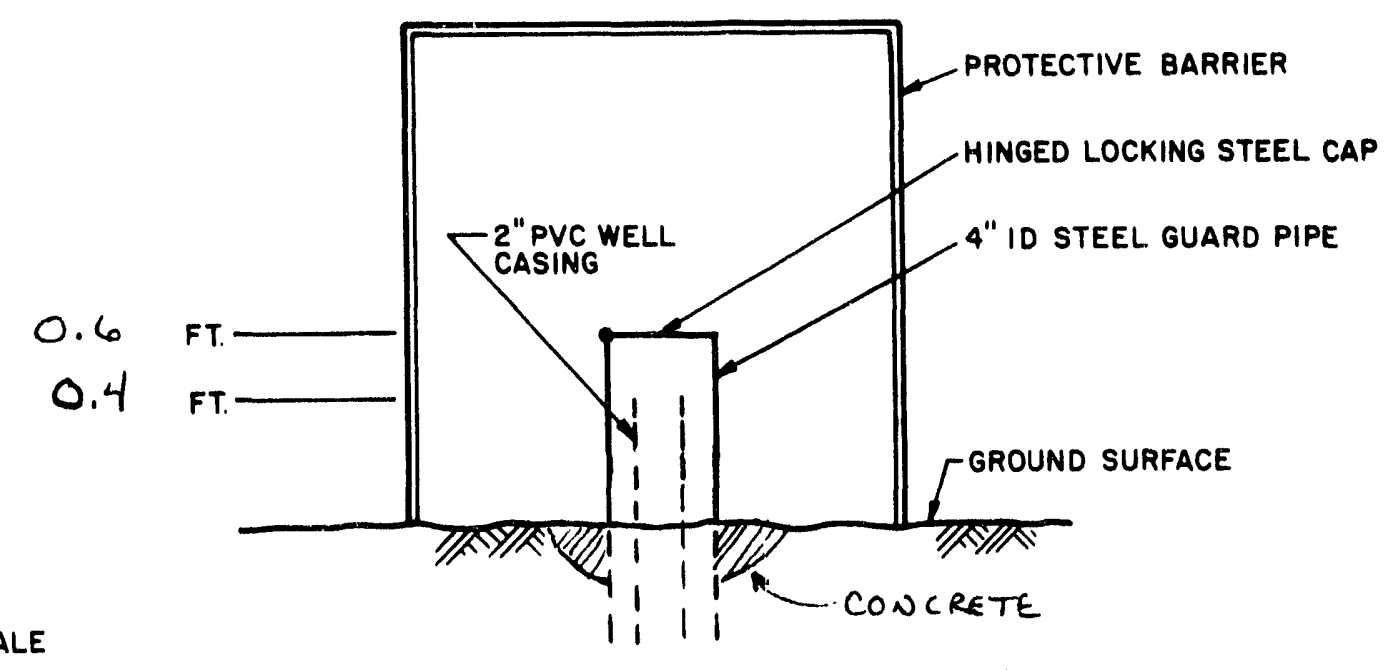

NOT TO SCALE

PVE CASING BENT AT HFT. 
SCHEMATIC OF PREABANDONMENT SITE STATUS

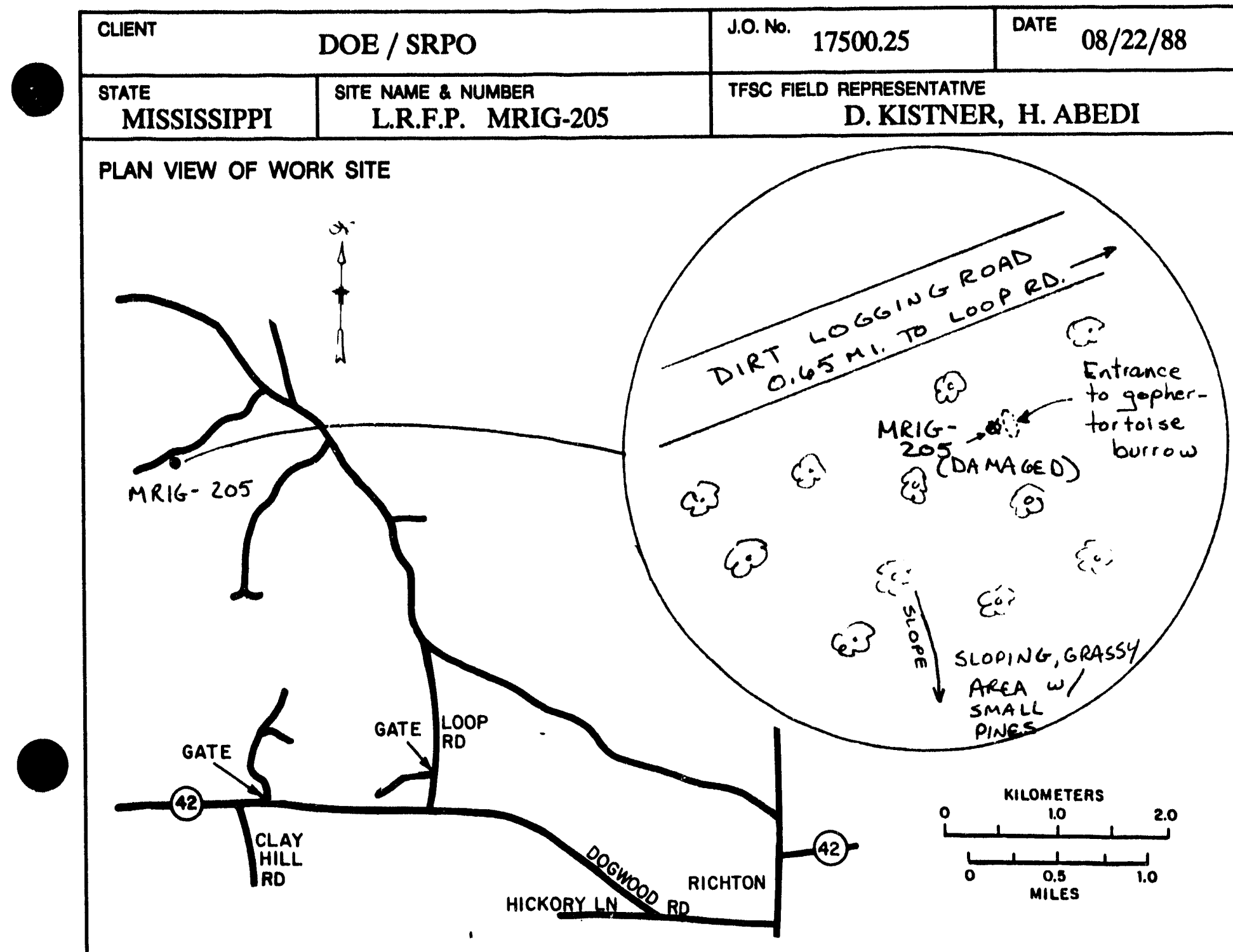

WELLHEAD PROFILE

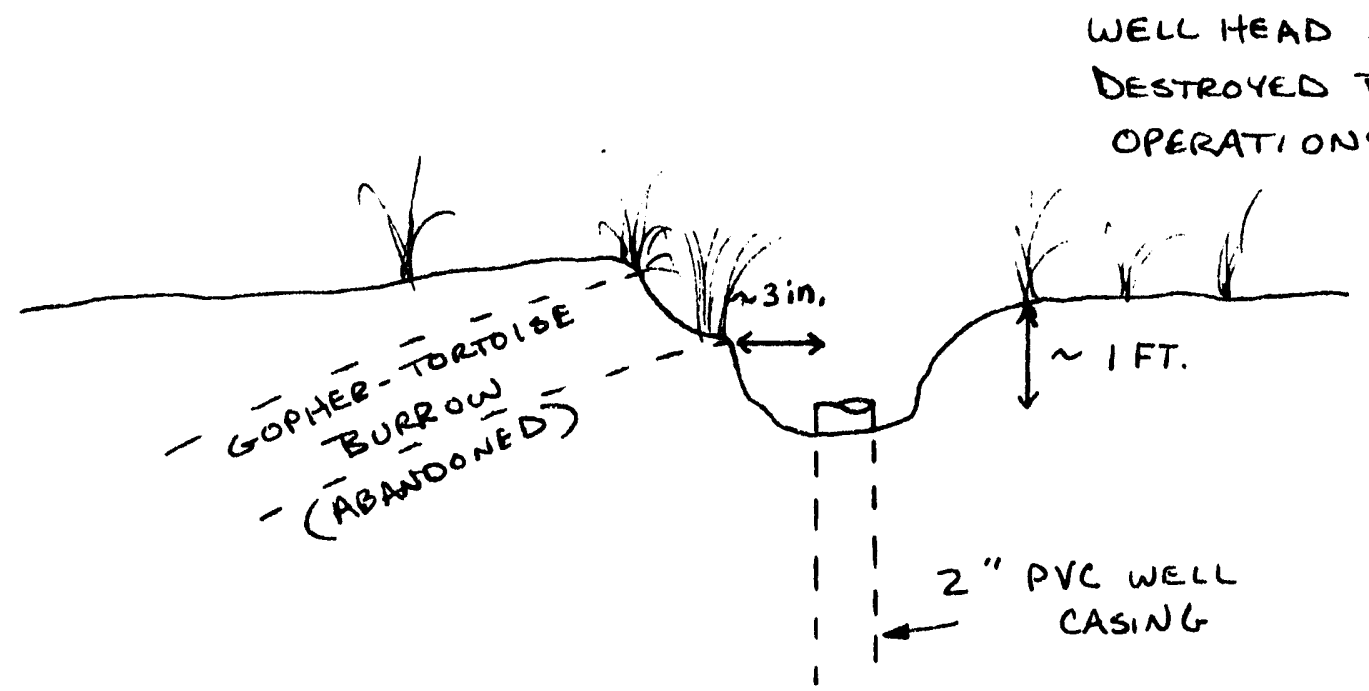

NOT TO SCALE 
SCHEMATIC OF PREABANDONMENT SITE STATUS

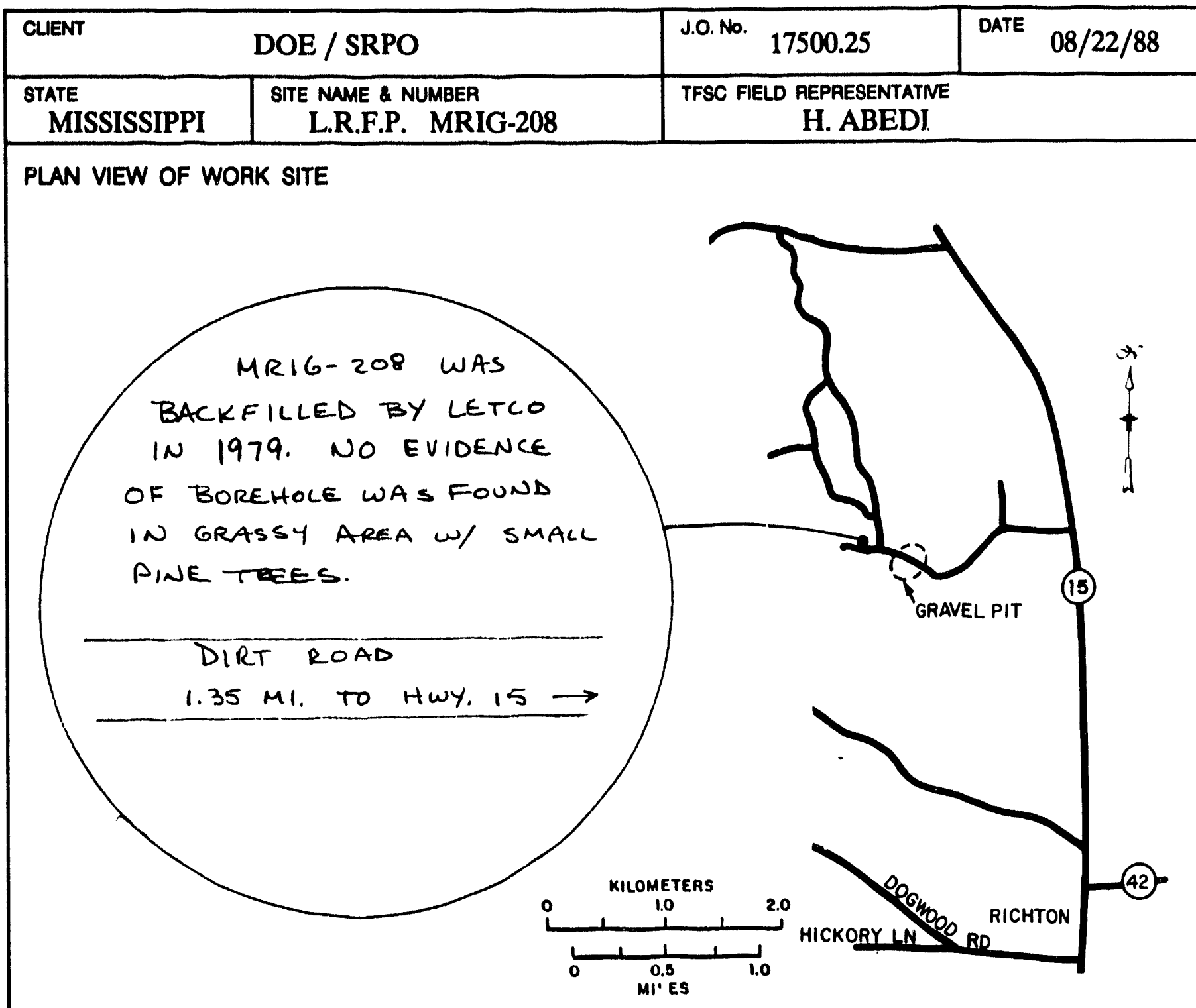

WELLHEAD PROFILE NA 
SCHEMATIC OF PREABANDONMENT SITE STATUS

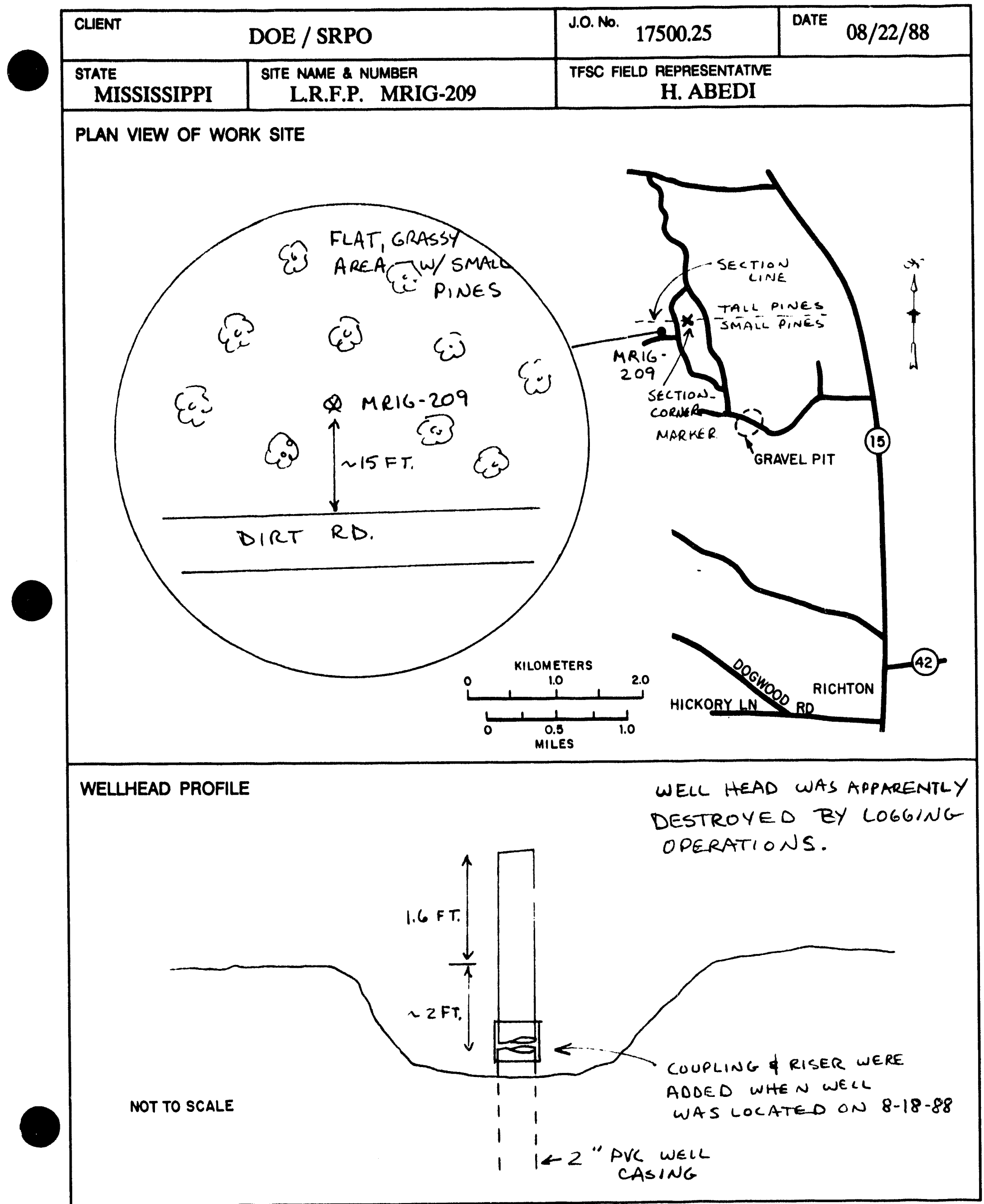




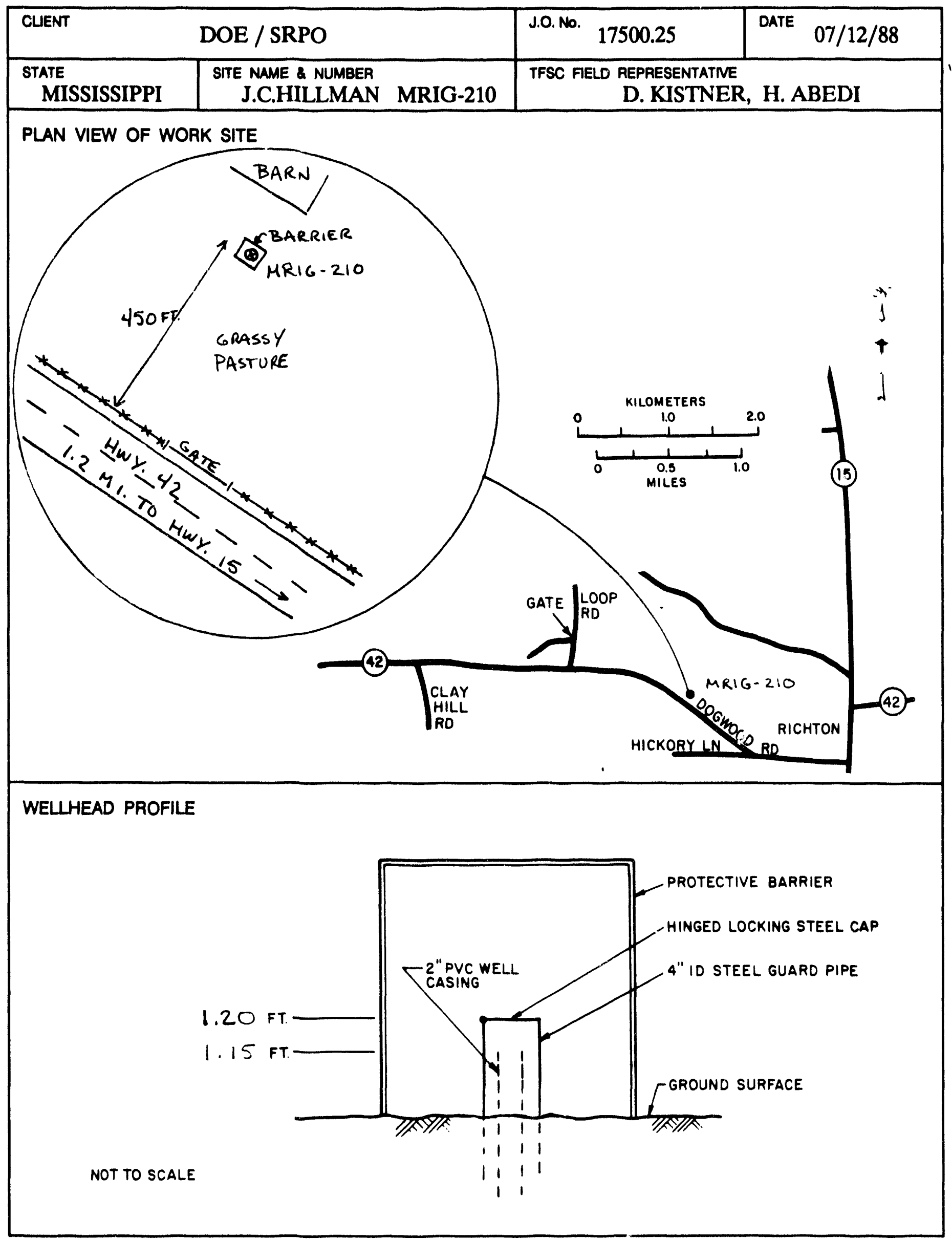


SCHEMATIC :F PREABANDONMENT SITE STATUS

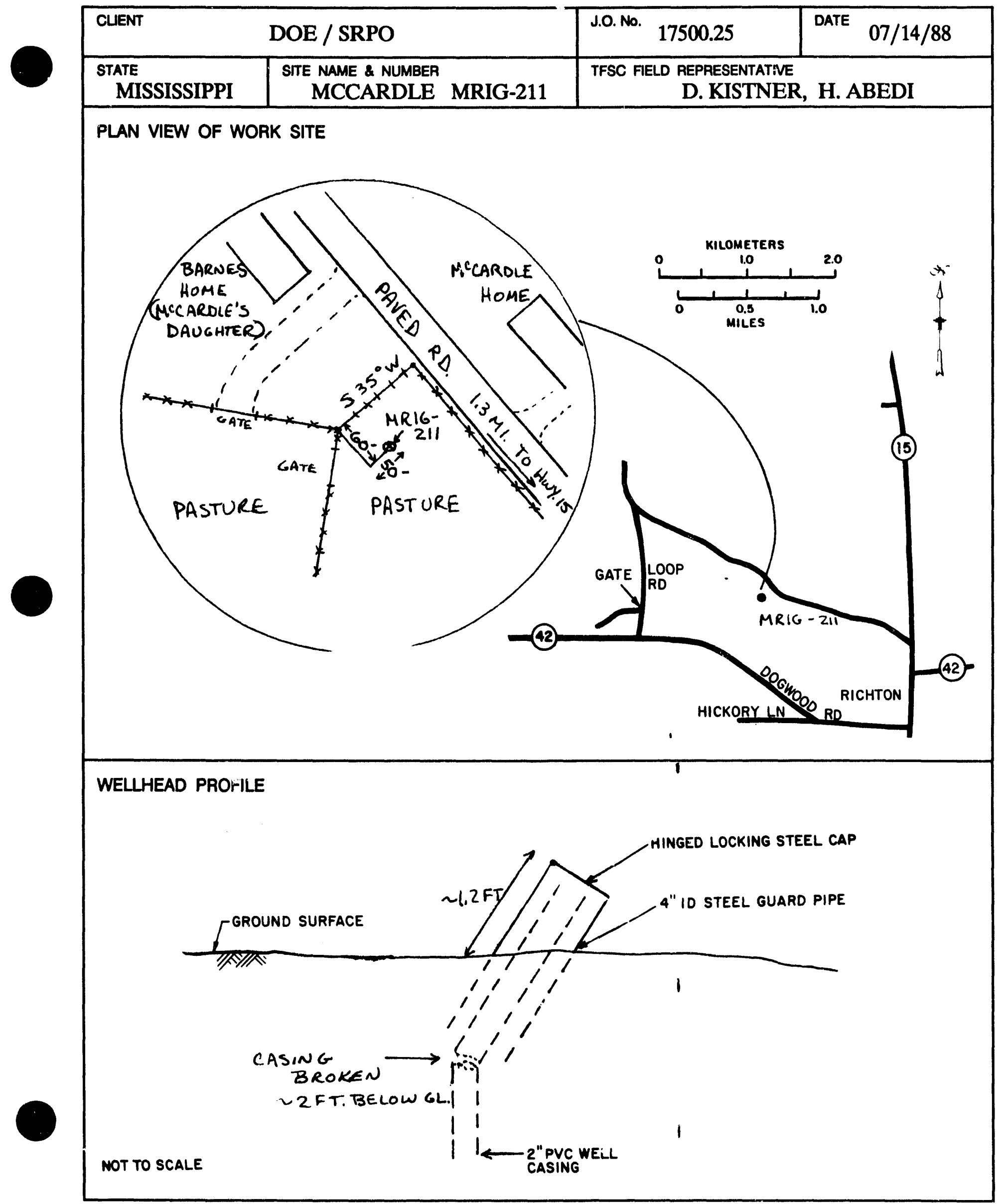


SCHEMATIC OF PREABANDONMENT SITE STATUS

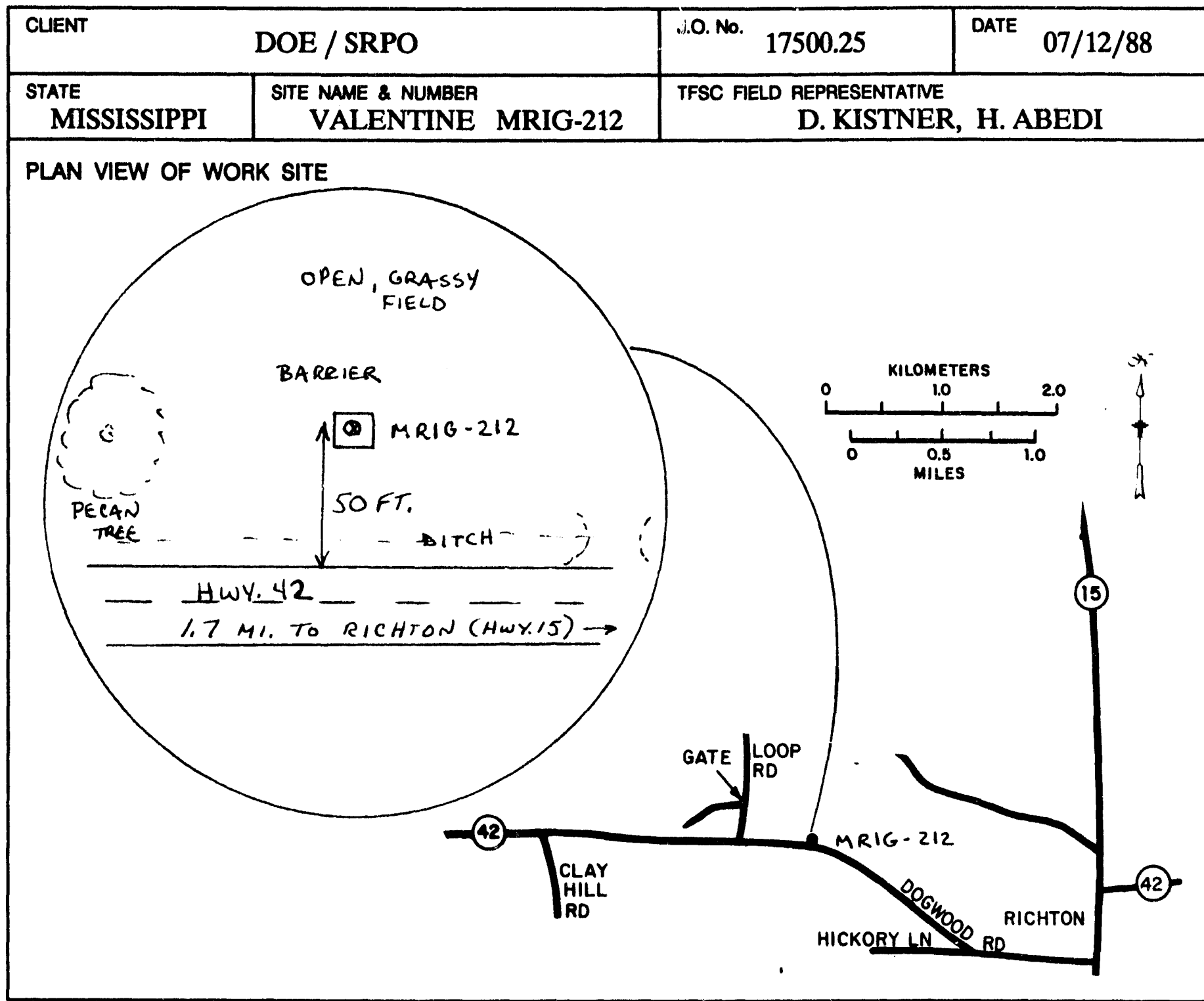

WEUHEAD PROFILE

NOT TO SCALE

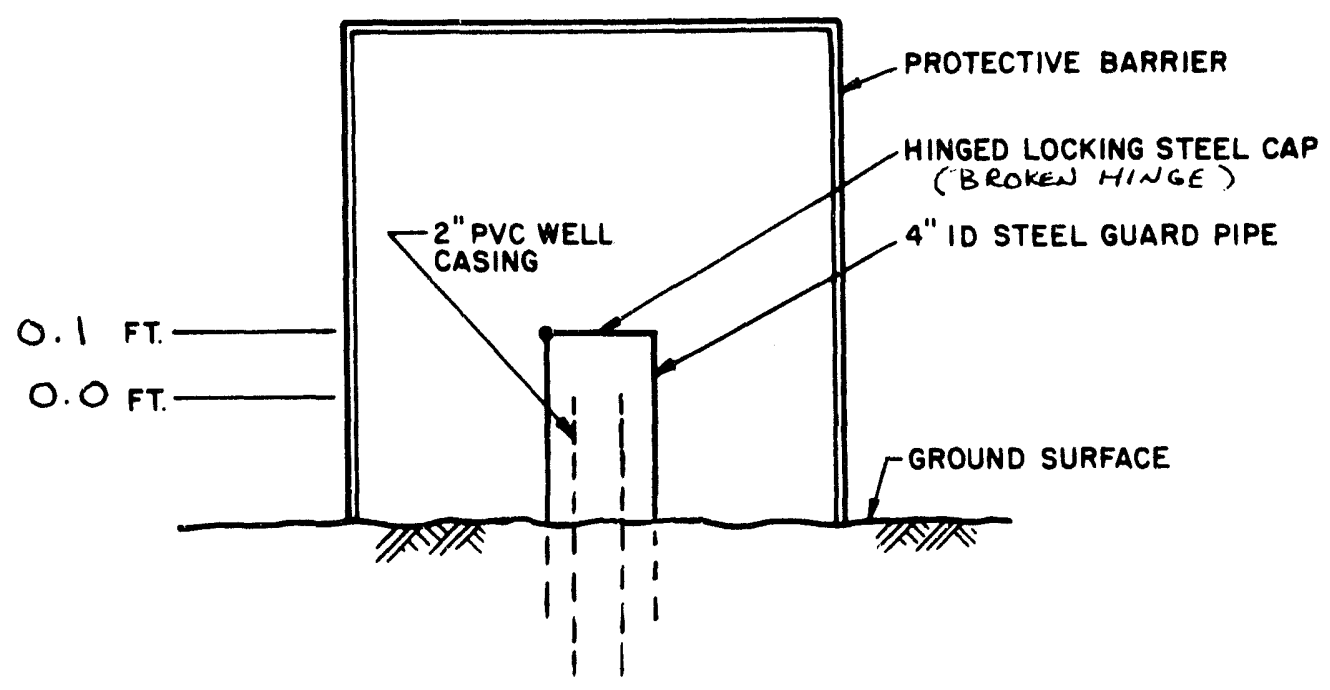


SCHEMATIC OF PREABANDONMENT SITE STATUS

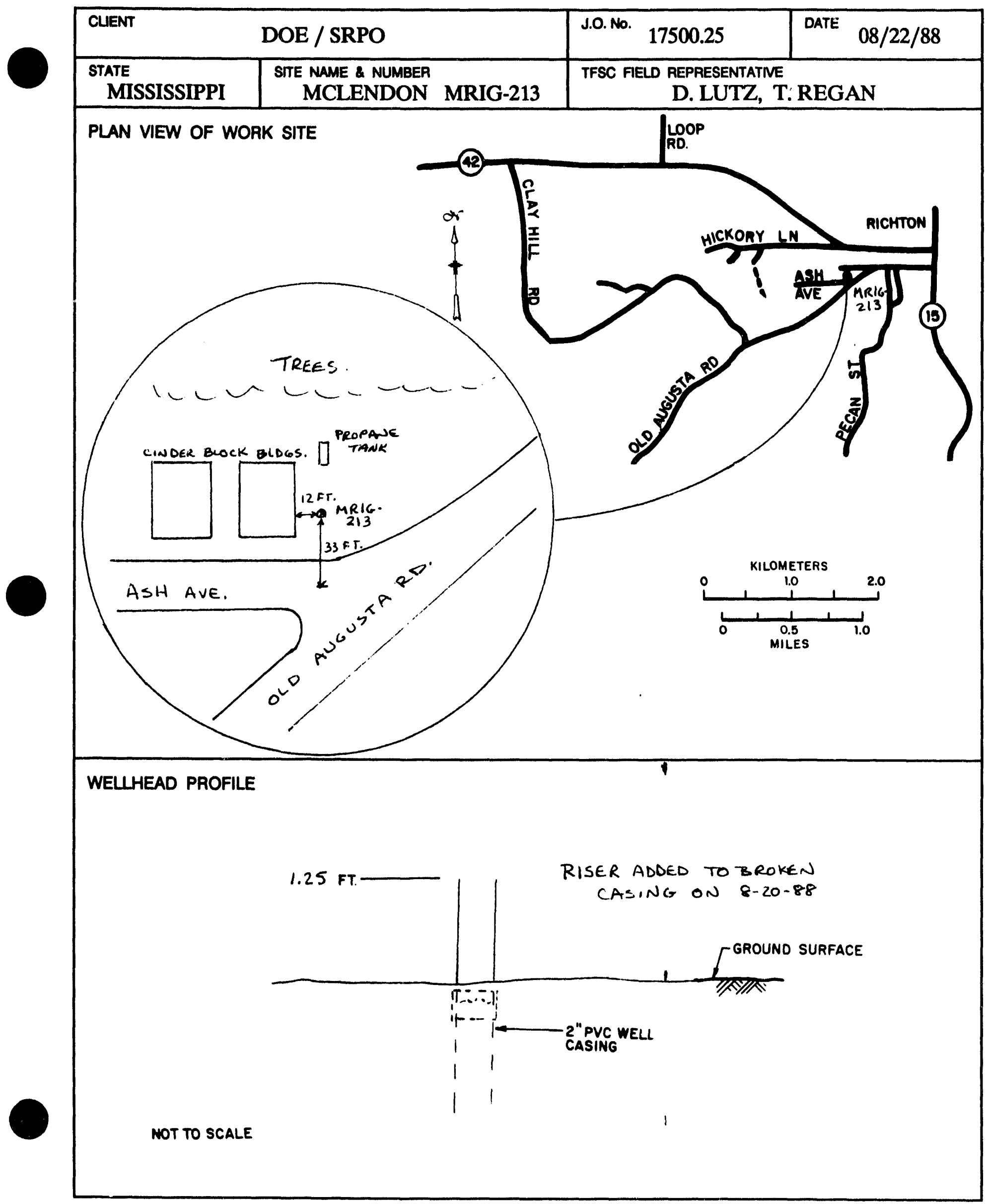


SCHEMATIC OF PREABANDONMENT SITE STATUS

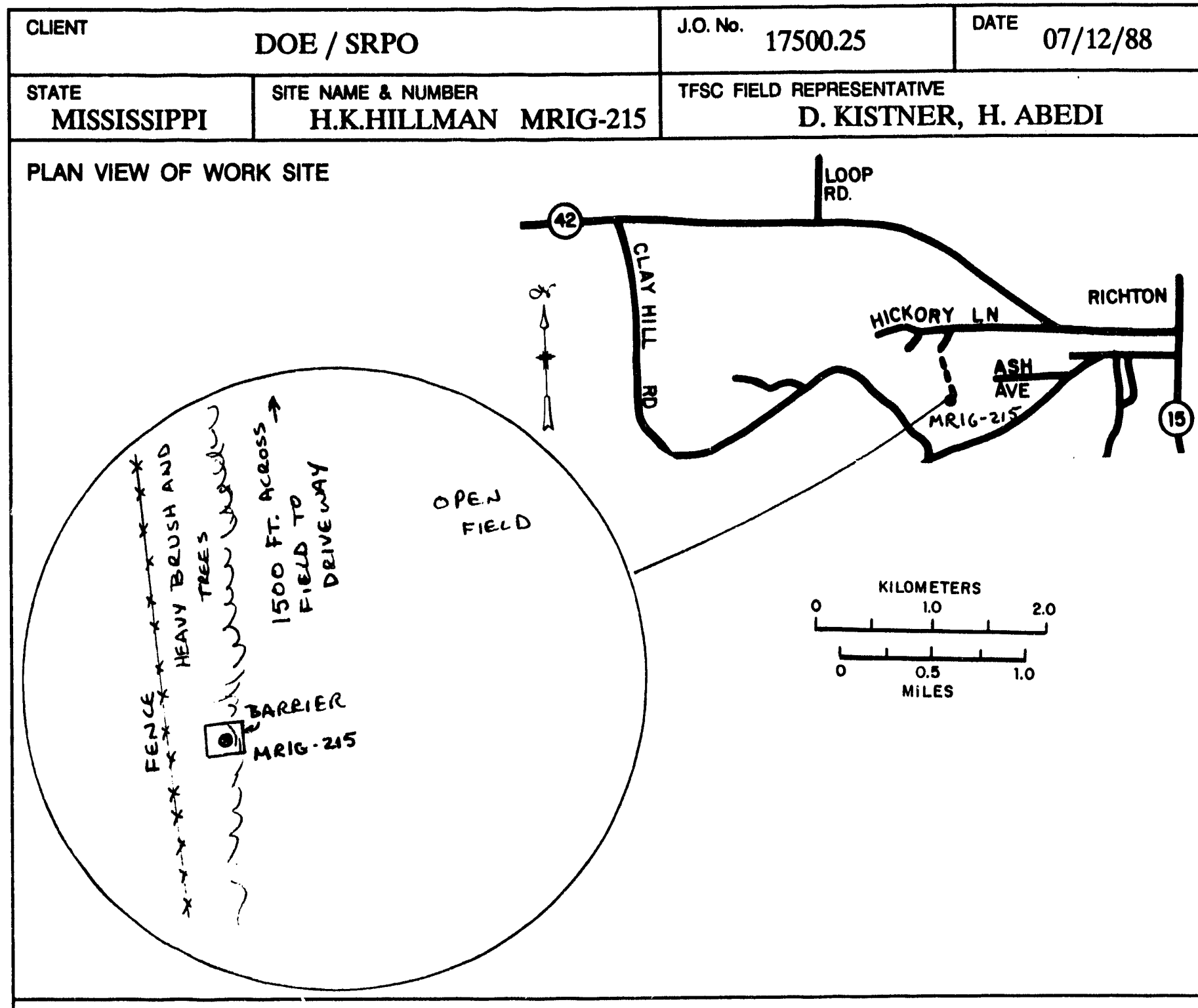

WELLHEAD PROFILE

NOT TO SCALE

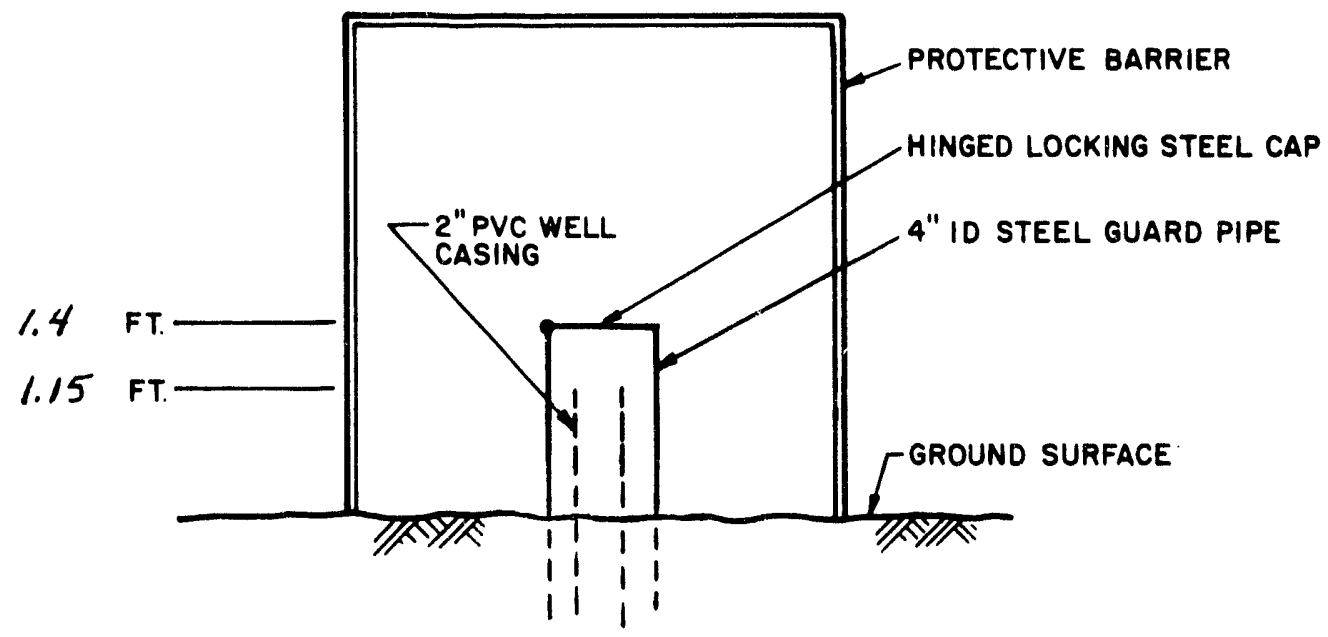


SCHEMATIC OF PREABANDONMENT SITE STATUS

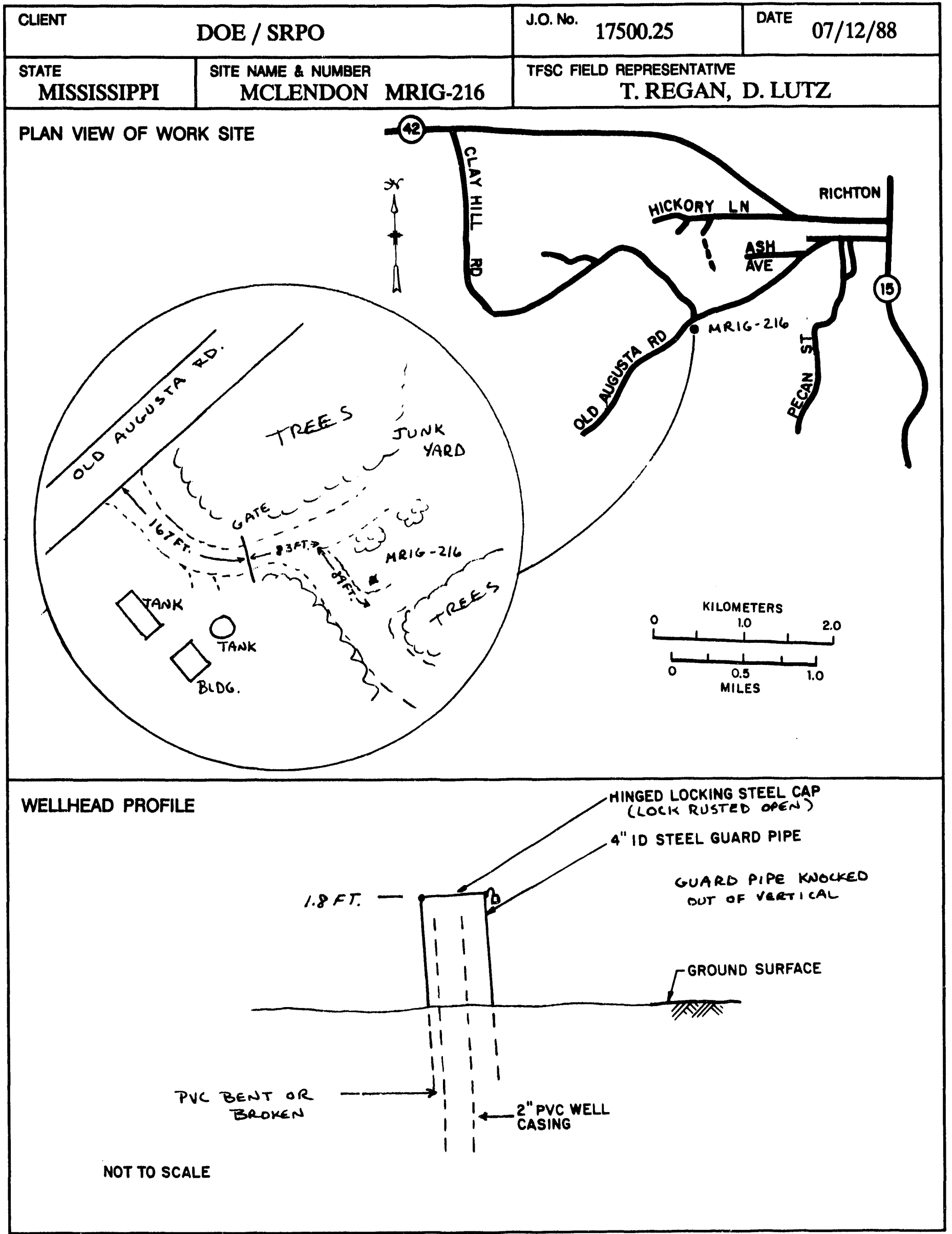


SCHEMATIC OF PREABANDONMENT SITE STATUS

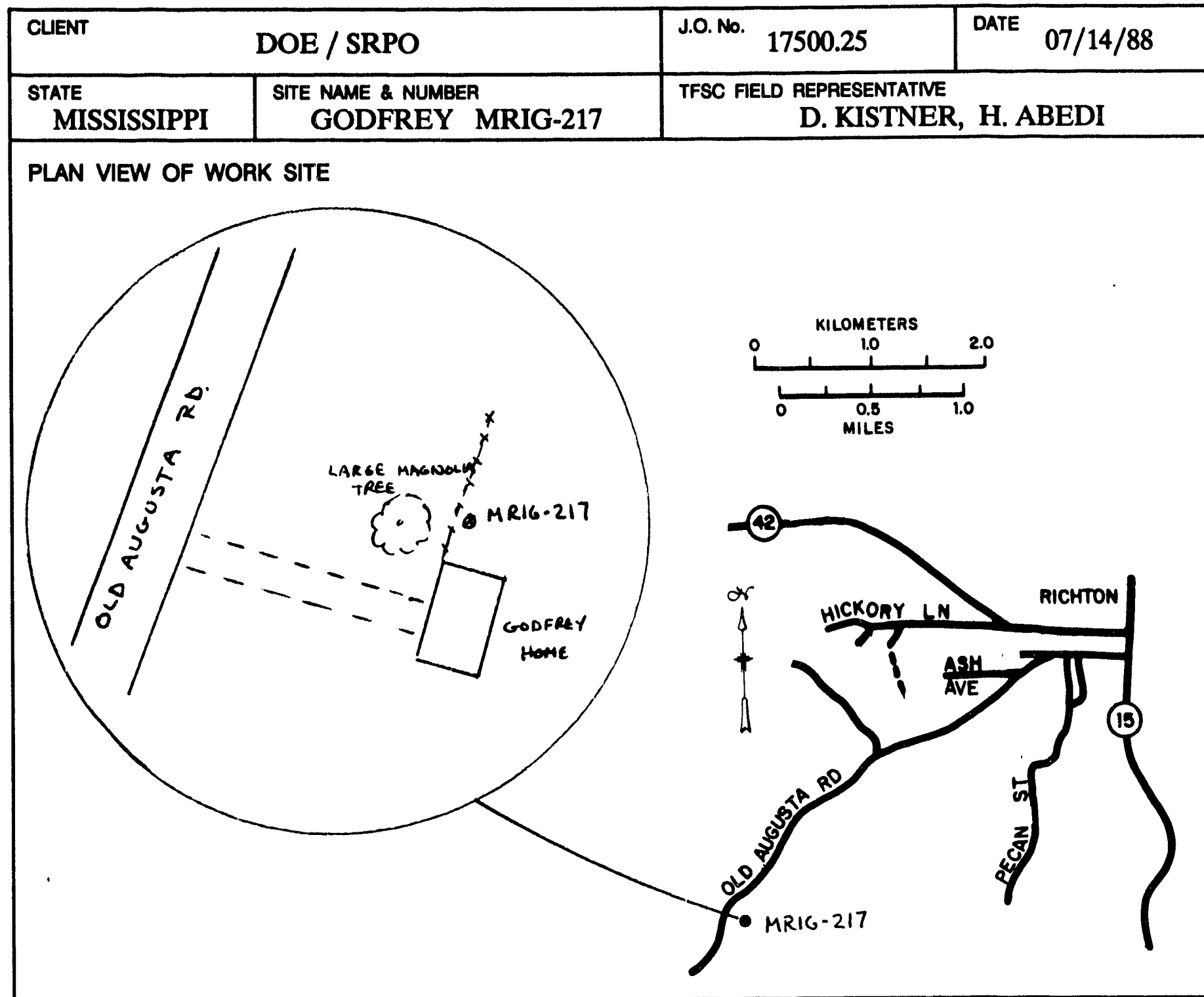

WELLHEAD PROFILE

NO ACCESS AGREEMENT - SITE NOT VISITED DURING RECONNAISSANRE. 


\section{SCHEMATIC OF PREABANDONMENT SITE STATUS}

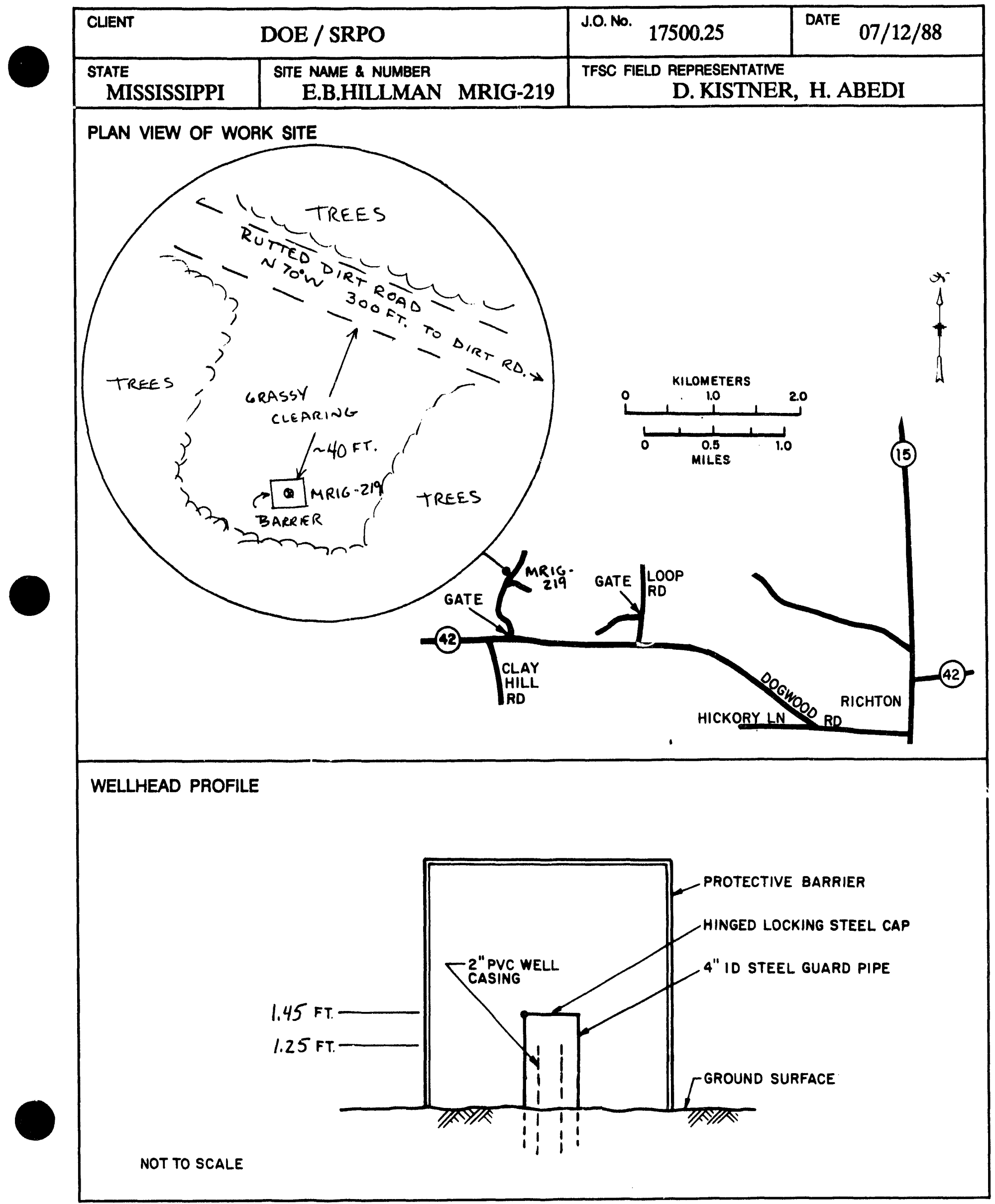


SCHEMATIC OF PREABANDONMENT SITE STATUS

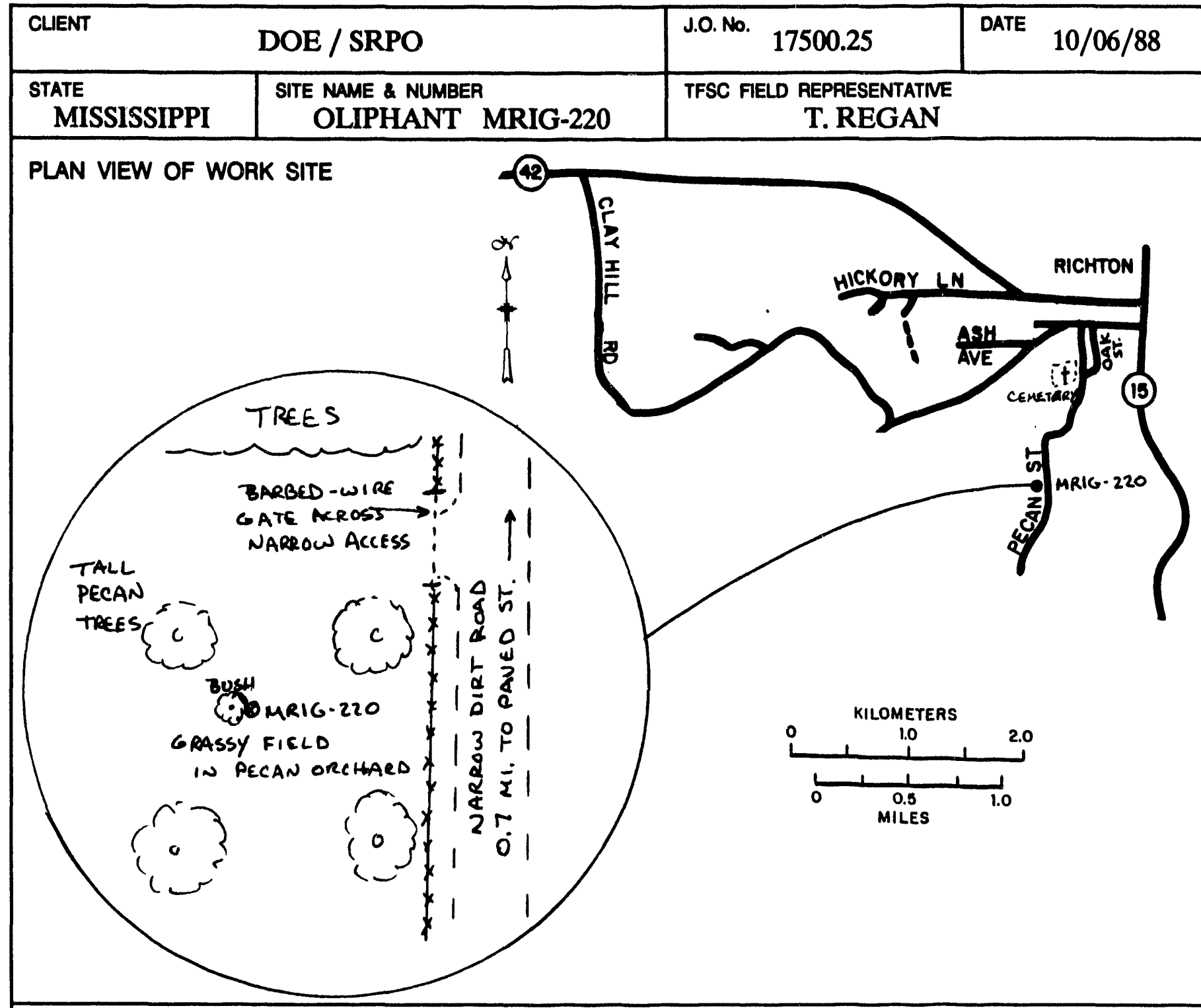

WELLHEAD PROFILE

NOT TO SCALE

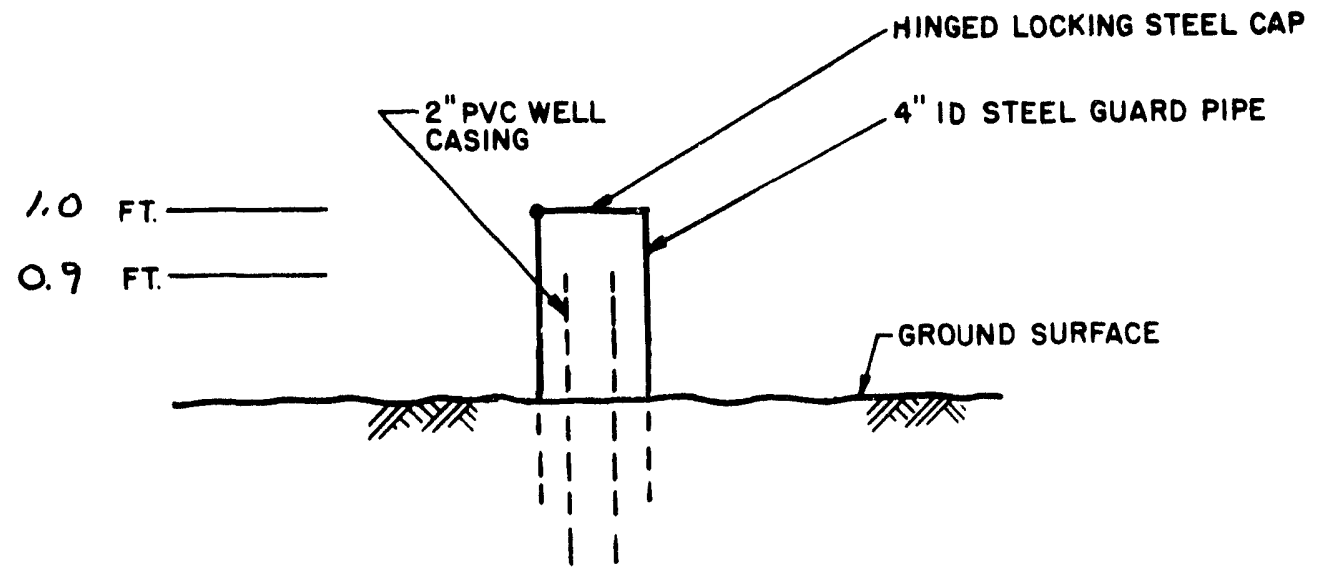


SCHEMATIC OF PREABANDONMENT SITE STATUS

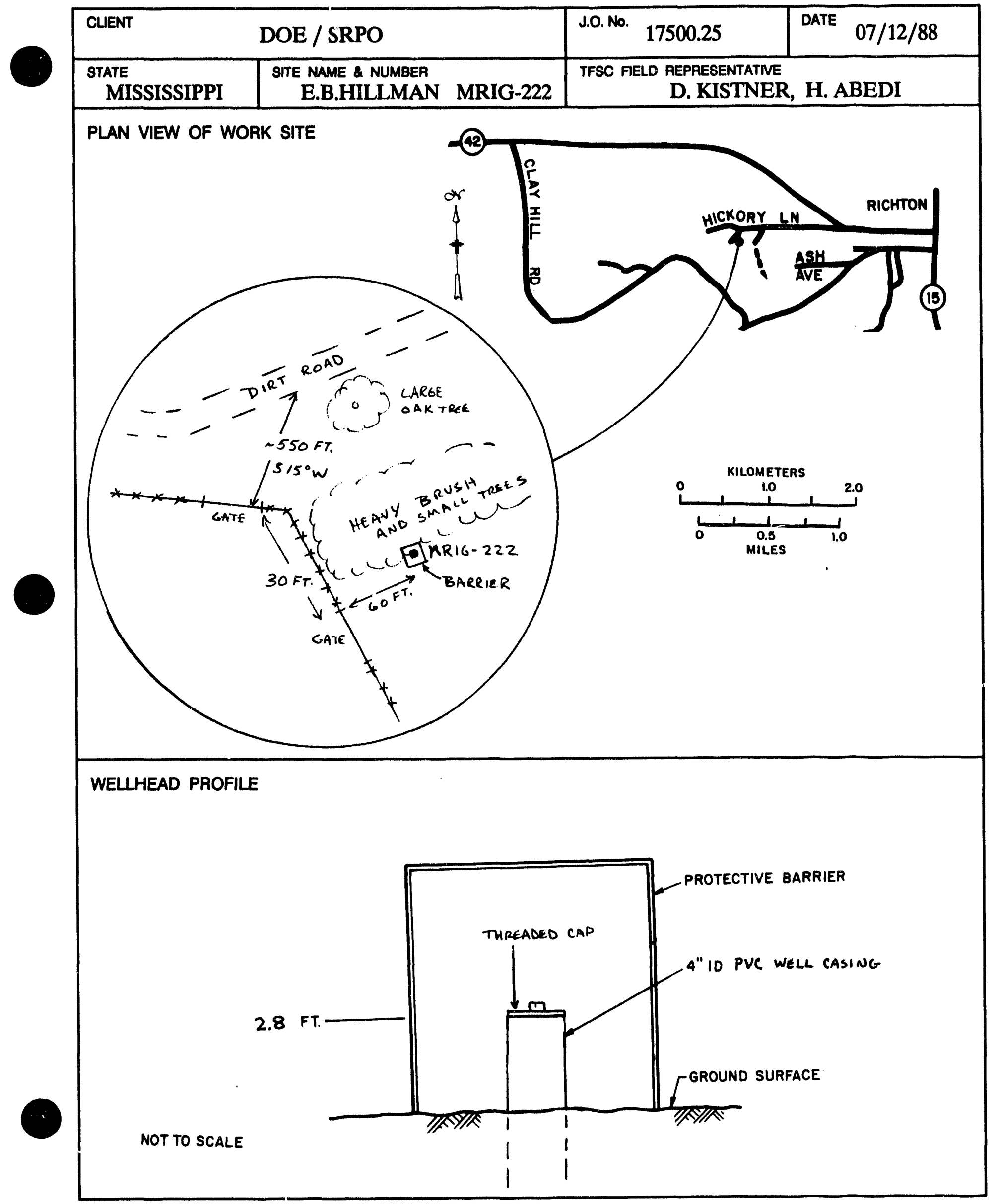


SCHEMATIC OF PREABANDONMENT SITE STATUS

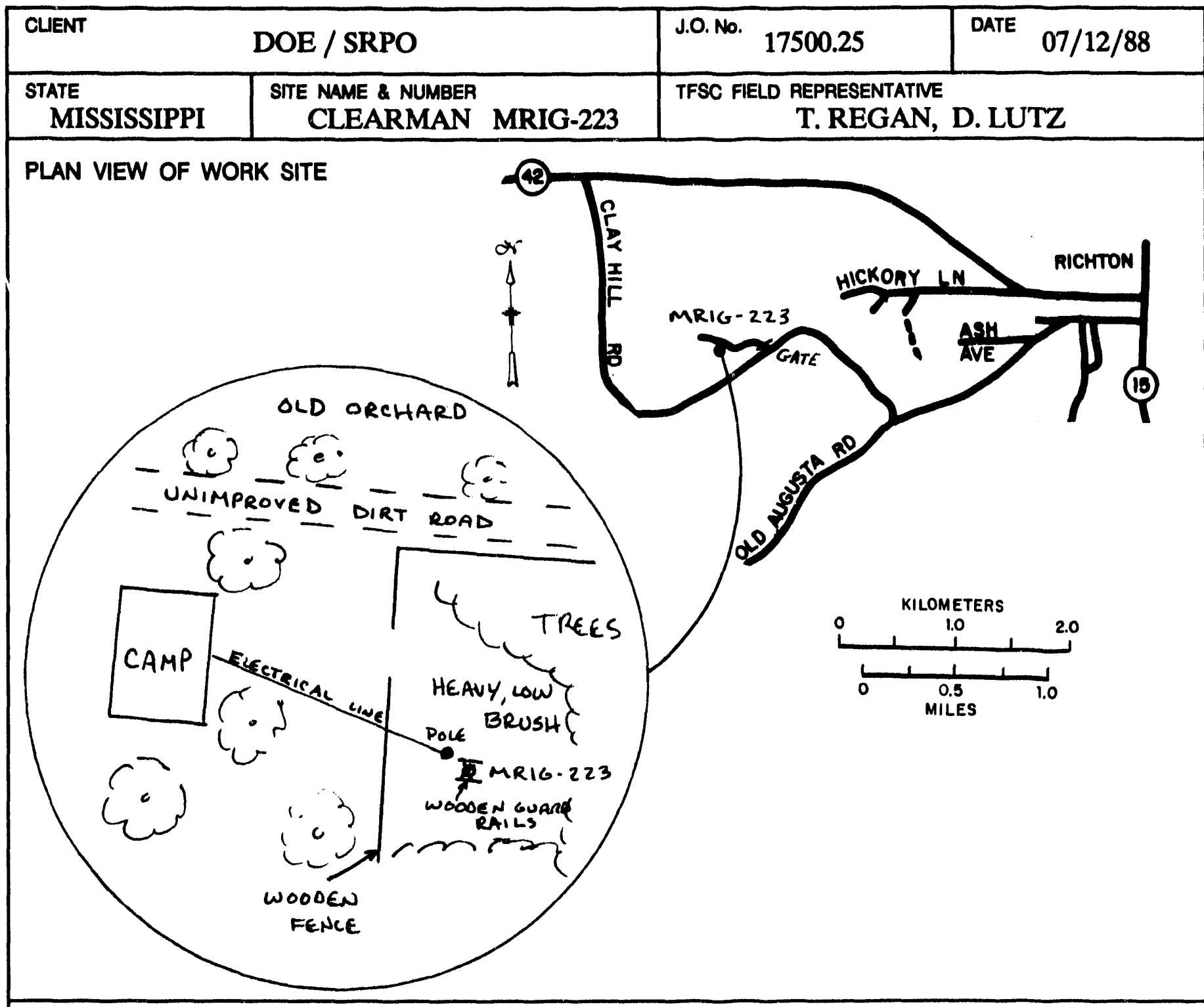

WELLHEAD PROFILE

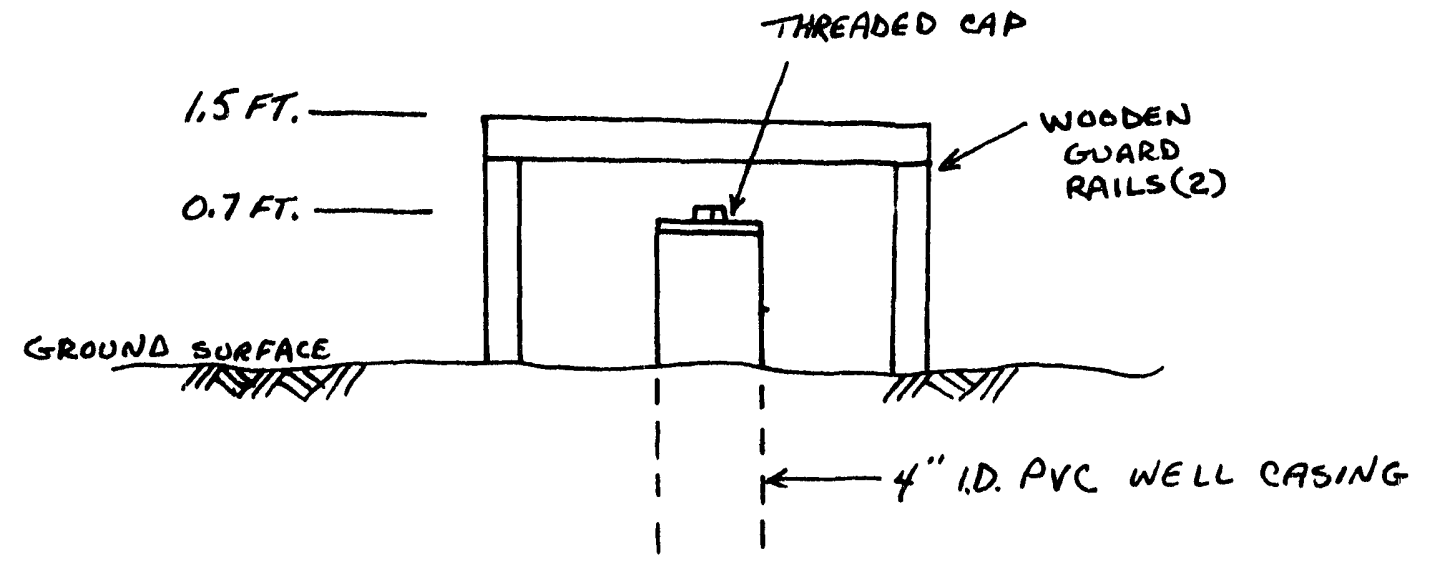

NOT TO SCALE 
SCHEMATIC OF PREABANDONMENT SITE STATUS

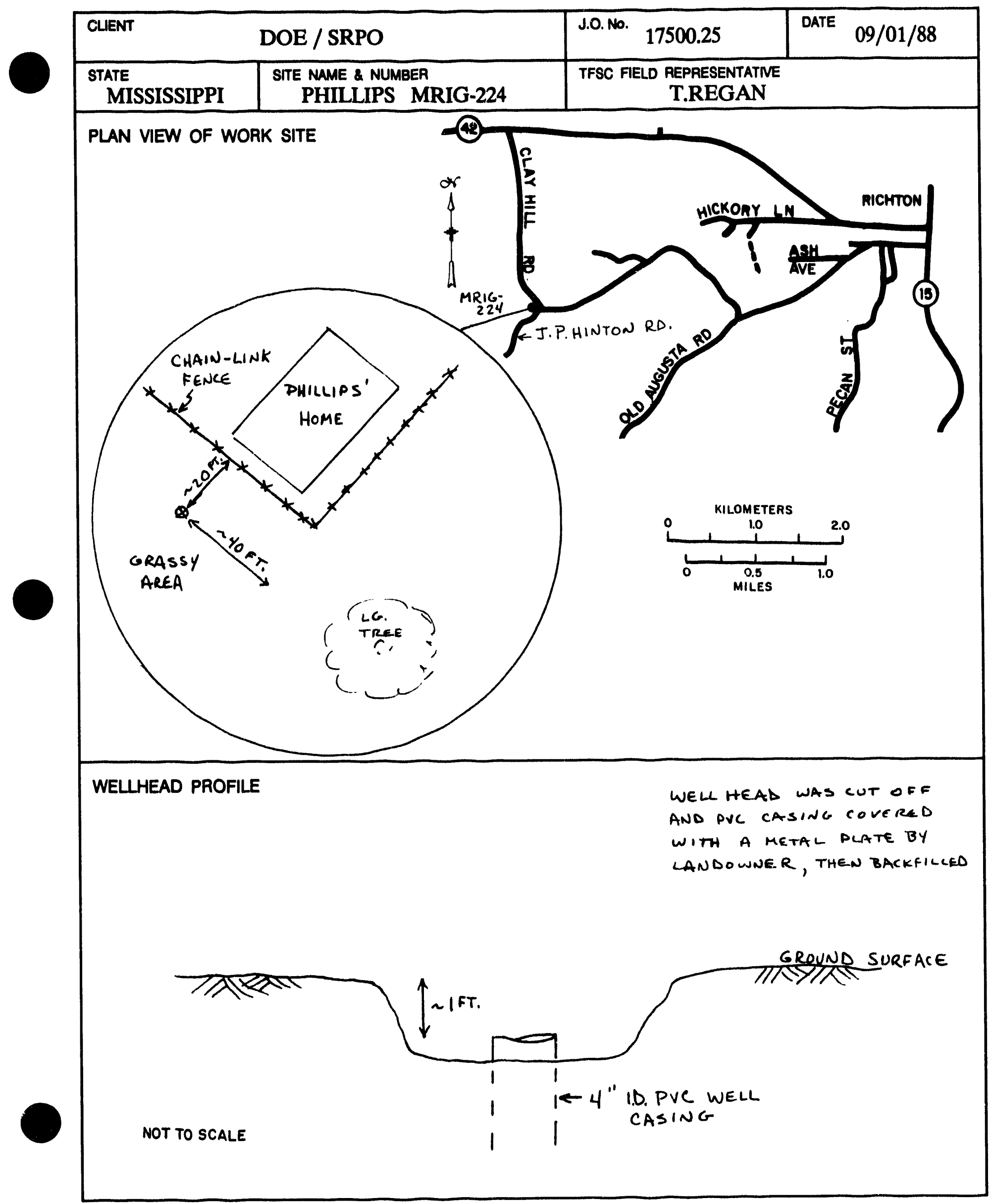


SCHEMATIC OF PREABANDONMENT SITE STATUS

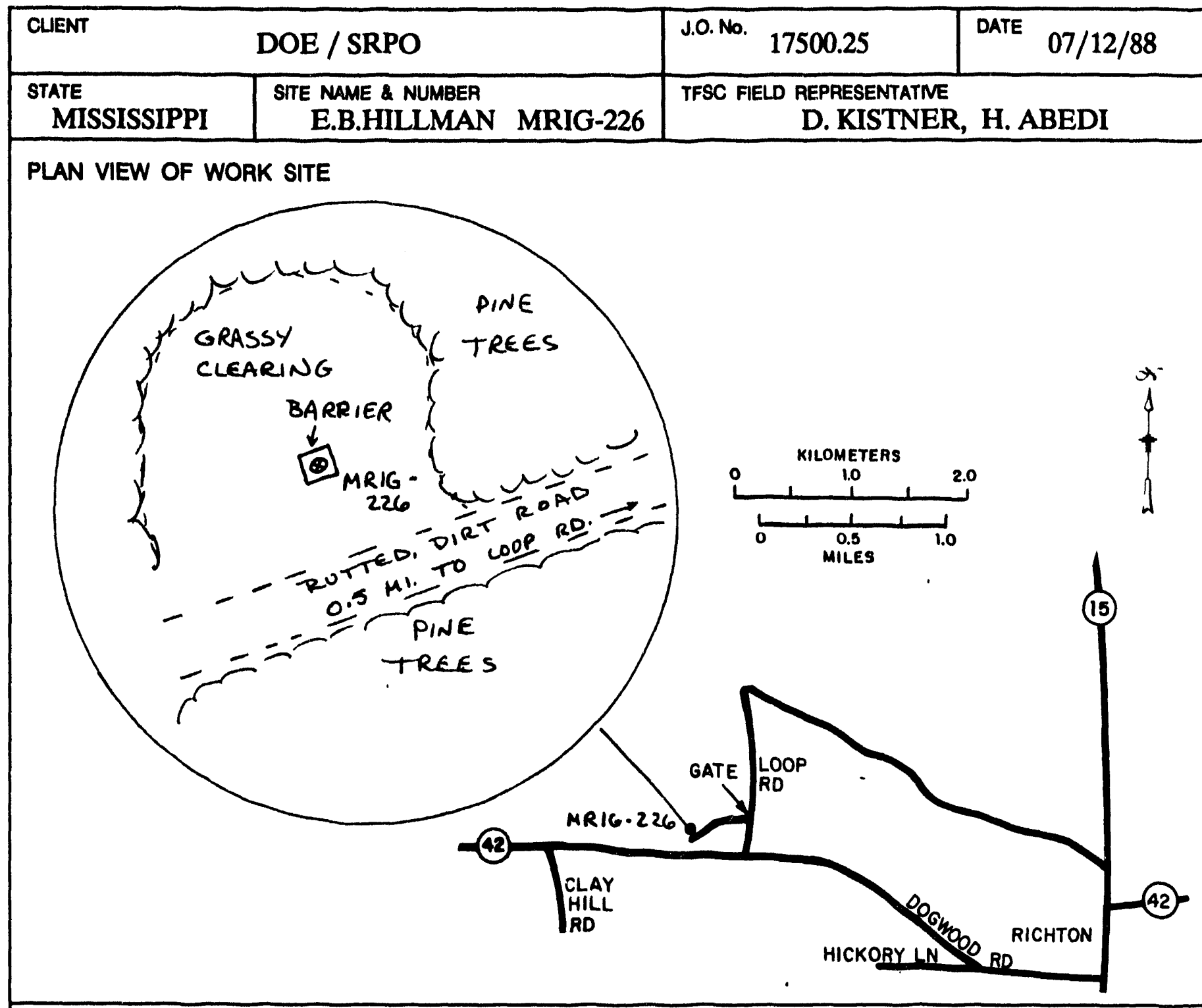

WELLHEAD PROFILE

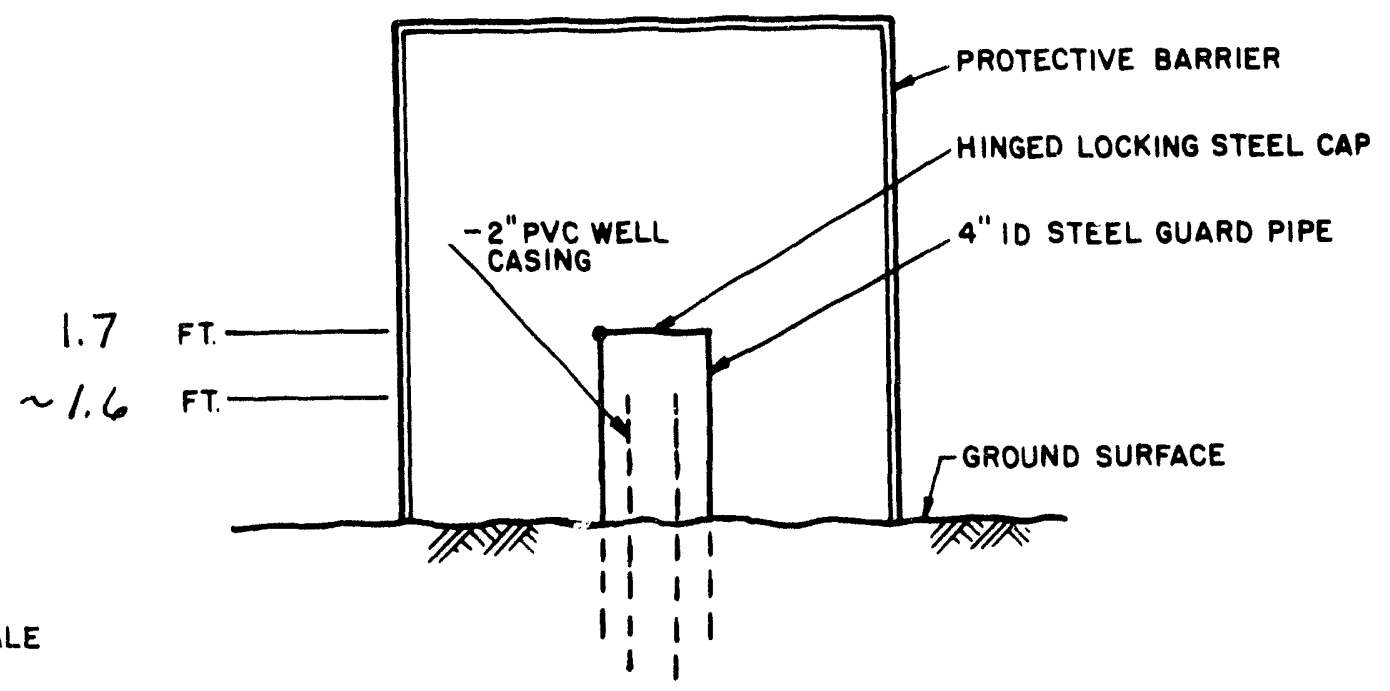

NOT TO SCALE 
SCHEMATIC OF PREABANDONMENT SITE STATUS

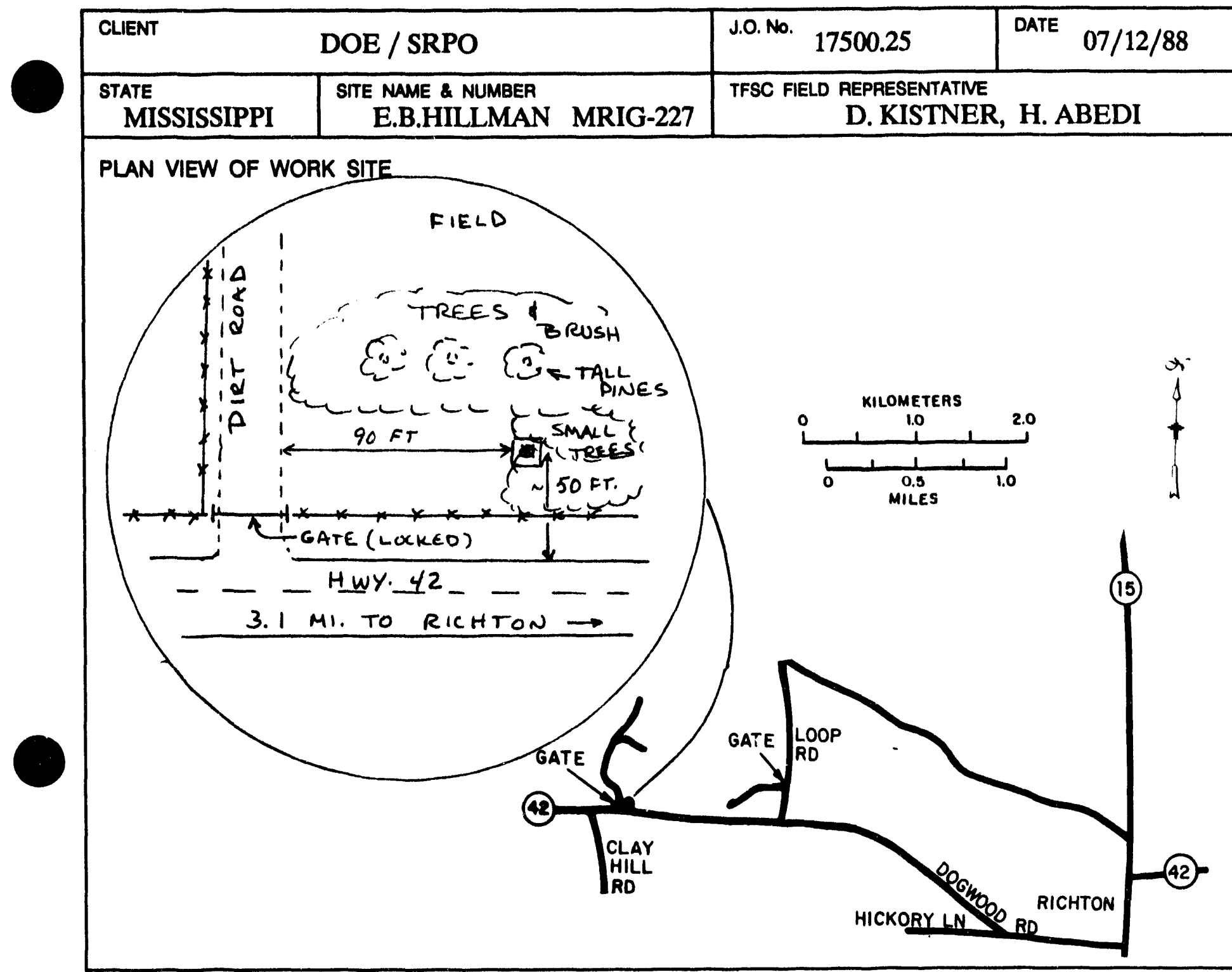

WELLHEAD PROFILE

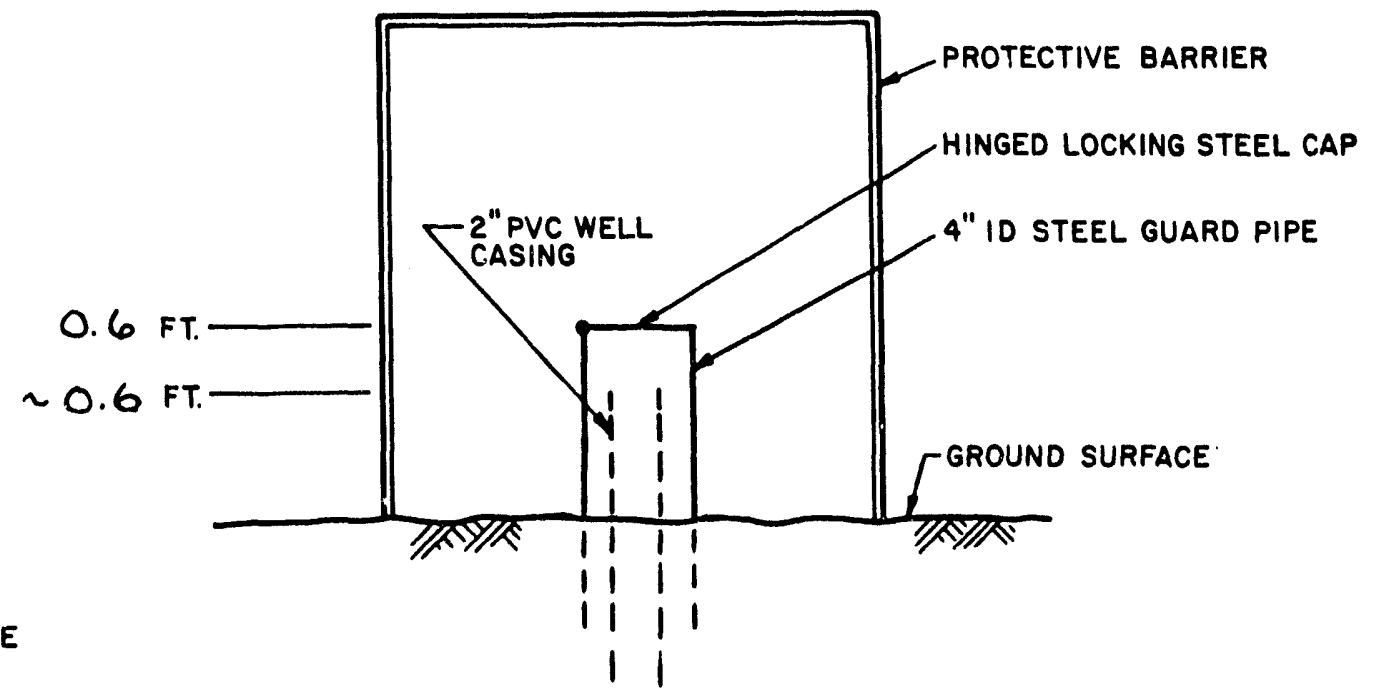

NOT TO SCALE 
SCHEMATIC OF PREABANDONMENT SITE STATUS

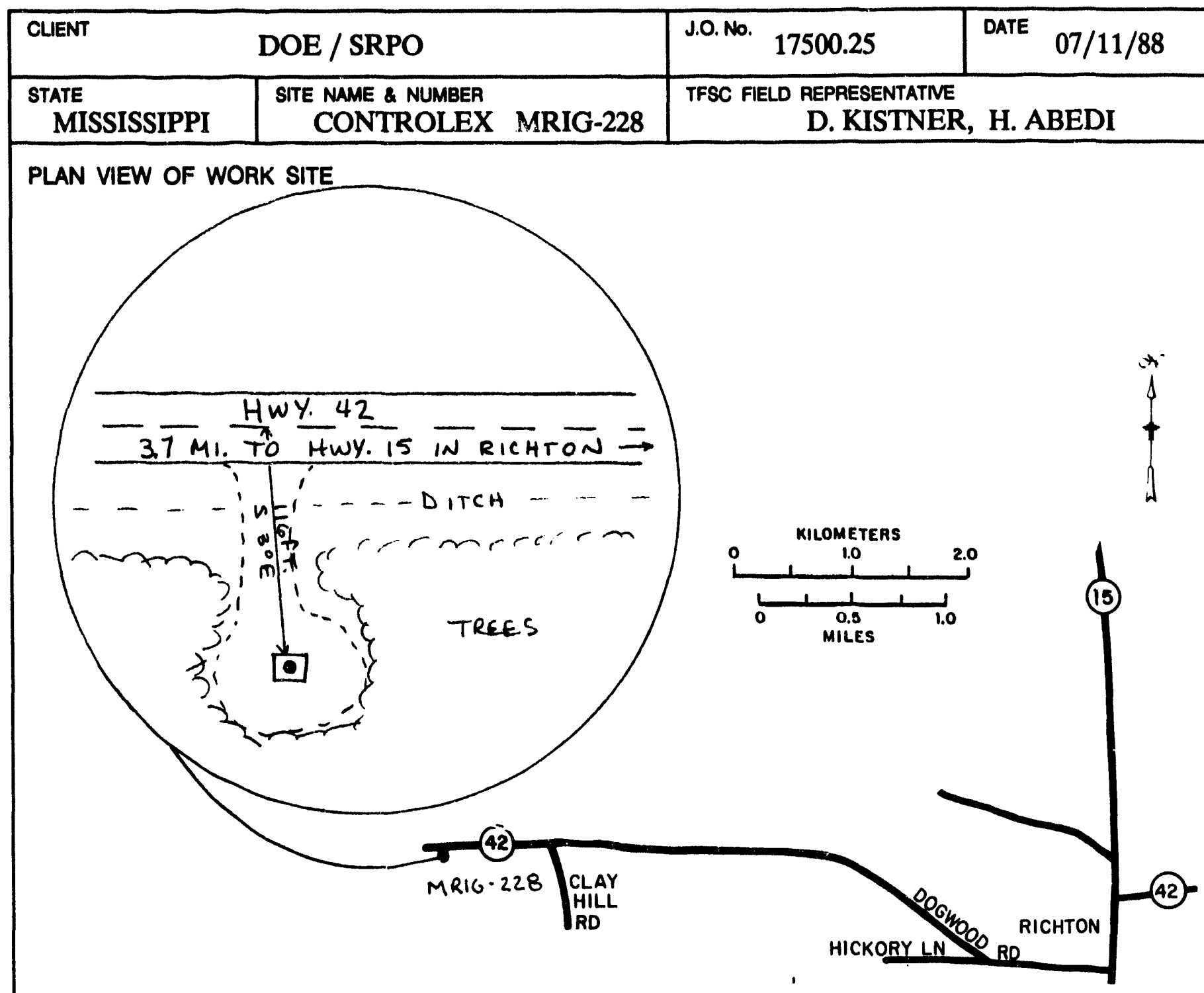

WELLHEAD PROFILE

NOT TO SCALE

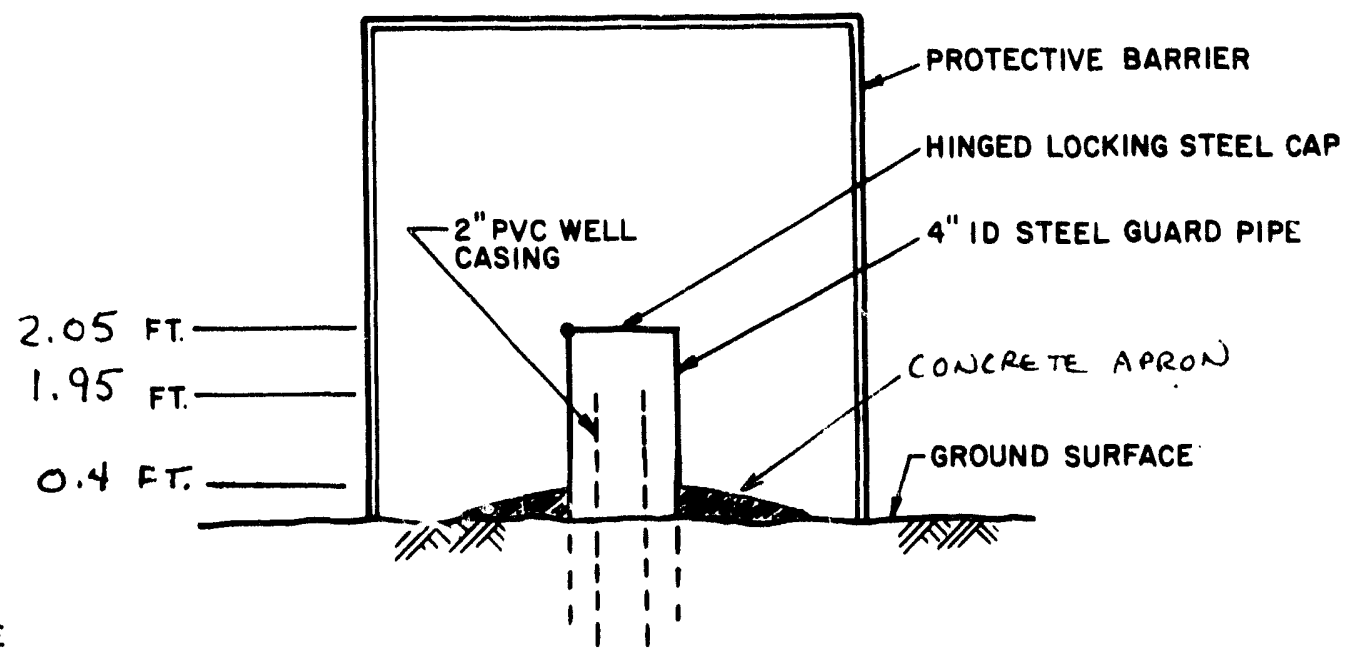




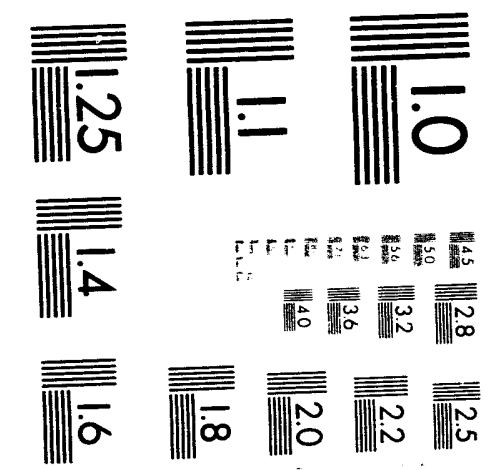



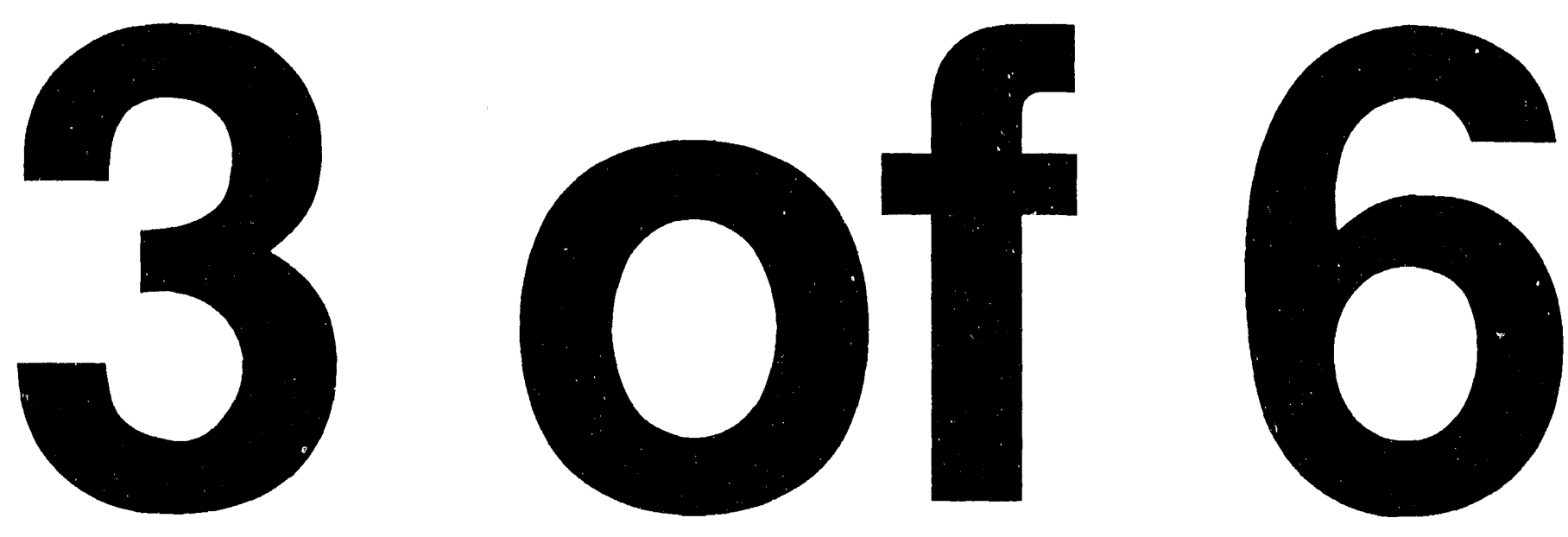
SCHEMATIC OF PREABANDONMENT SITE STATUS

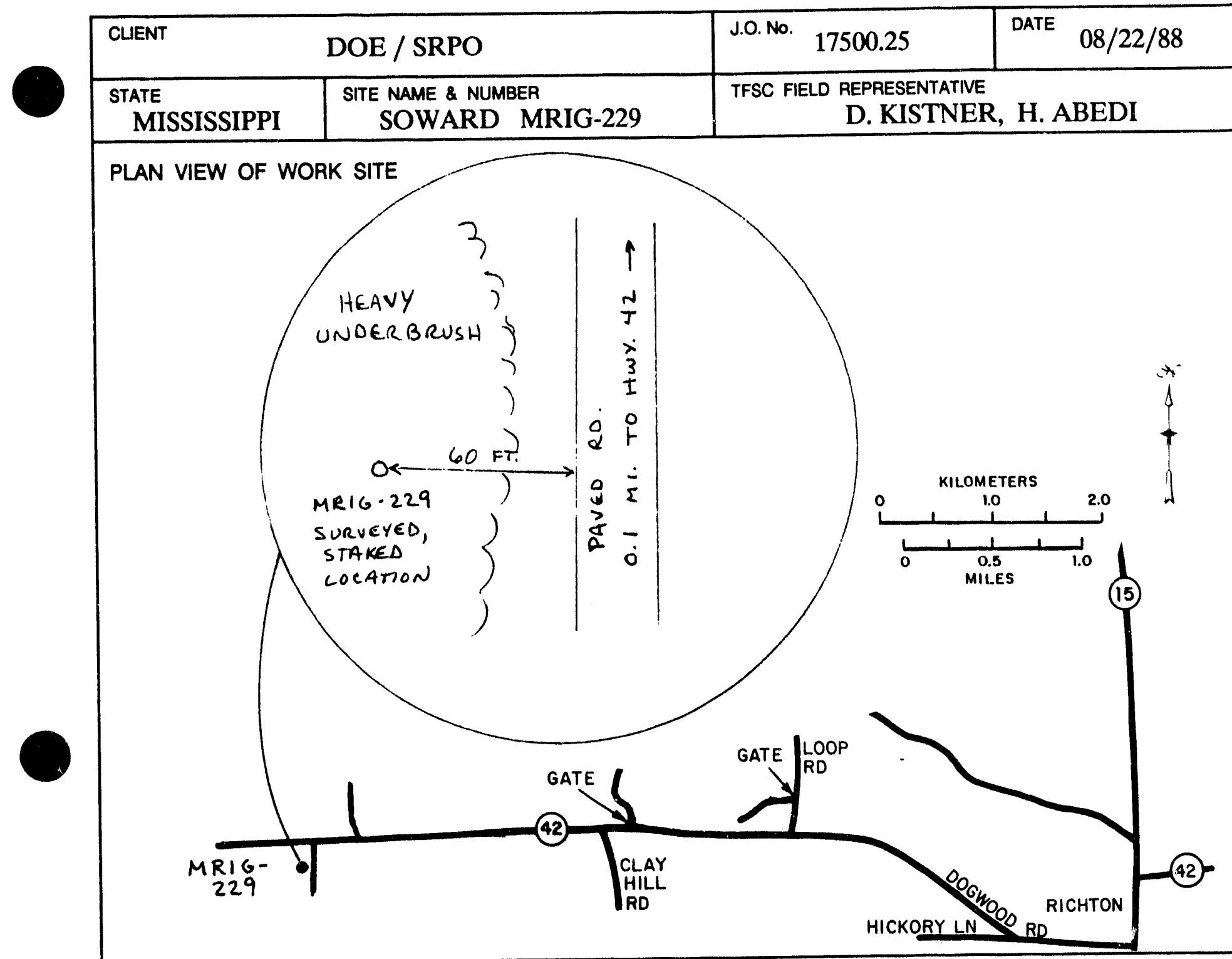

WELLHEAD PROFILE

BOREHOLE WAS BACKFILLEO BY LETCO IN 1979. NO EVIDENCE OF BOREHOLE WAS FOUND AT SURFACE IN AREA OF TALL GRASSES AND LOW BRUSH ANO TREES. 


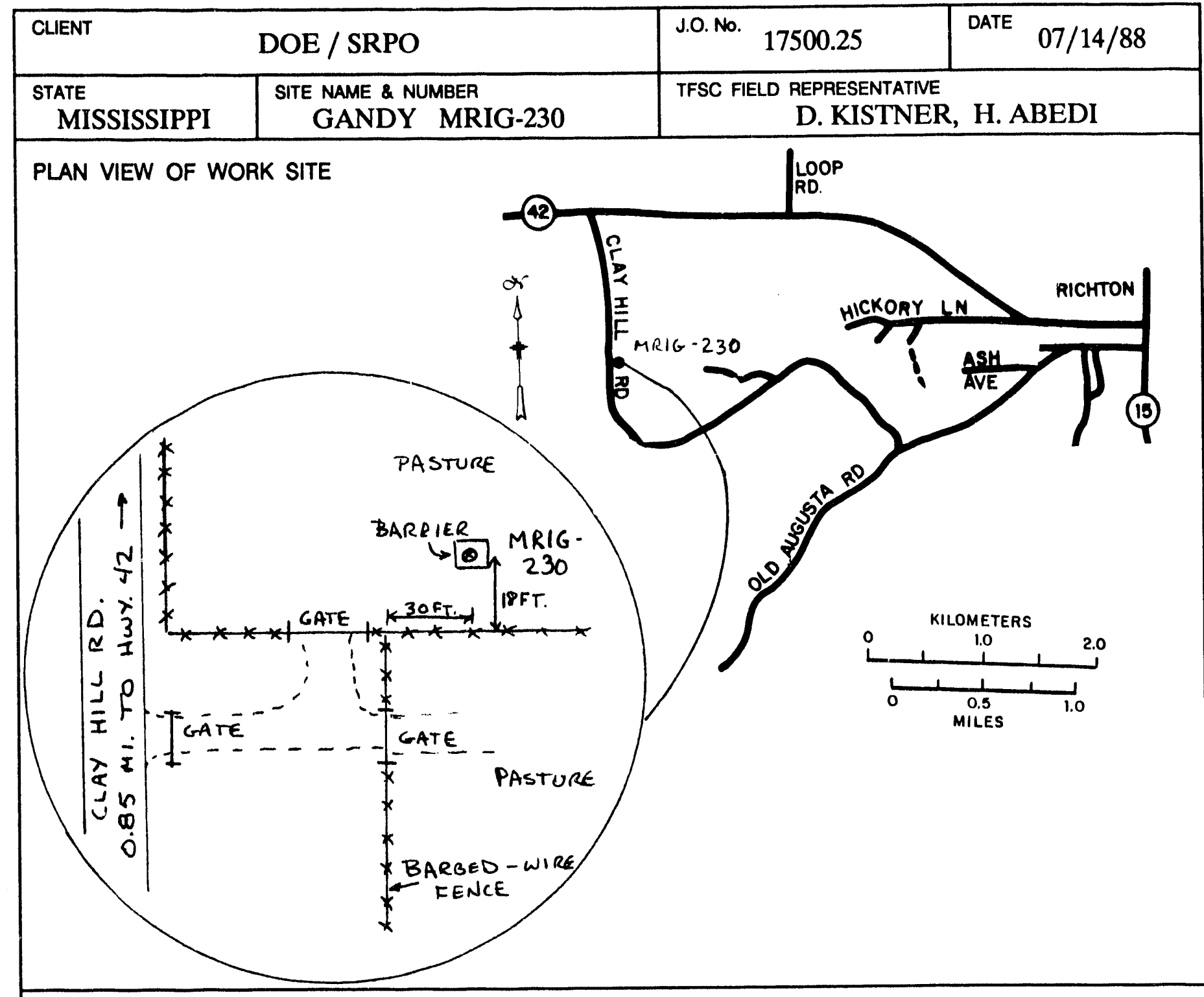

WELLHEAD PROFILE

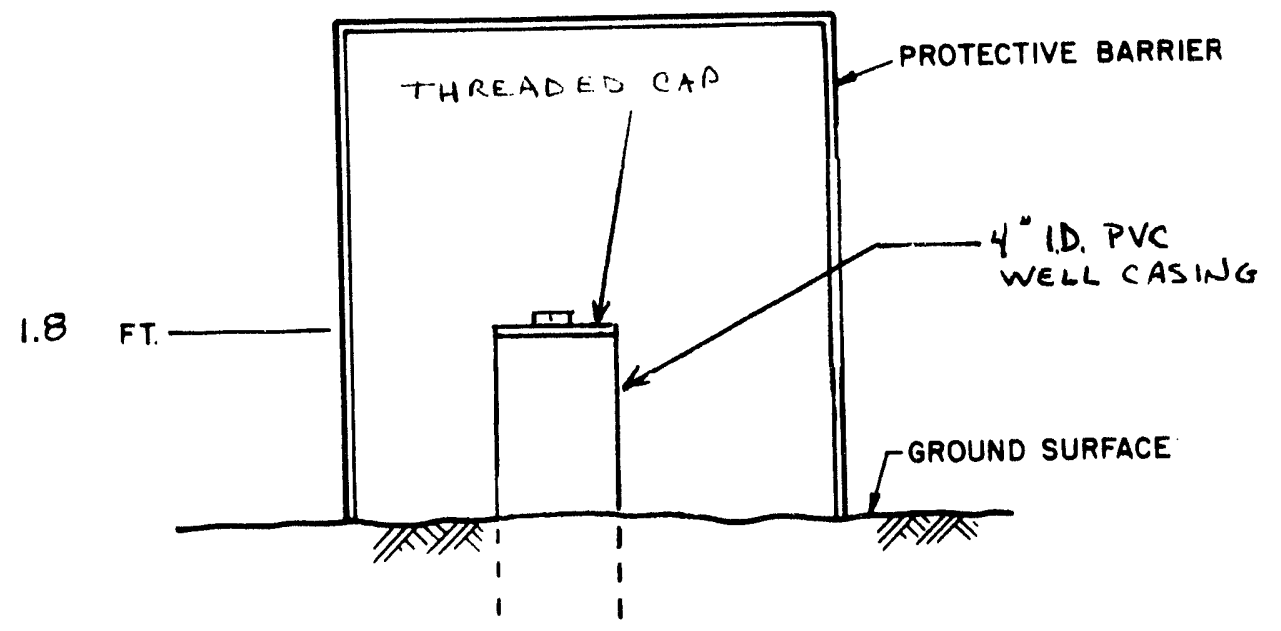

NOT TO SCALE 
SCHEMATIC OF PREABANDONMENT SITE STATUS

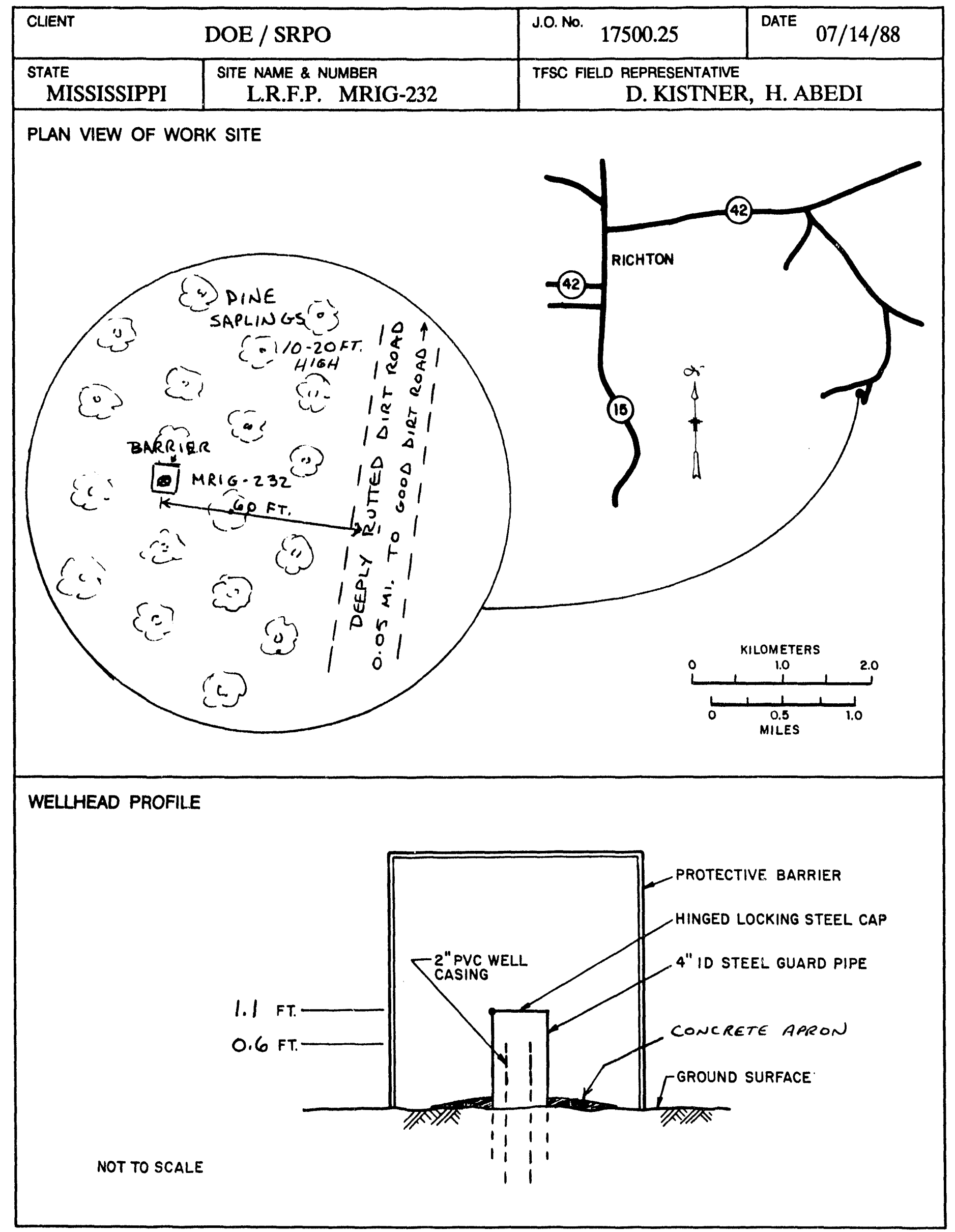




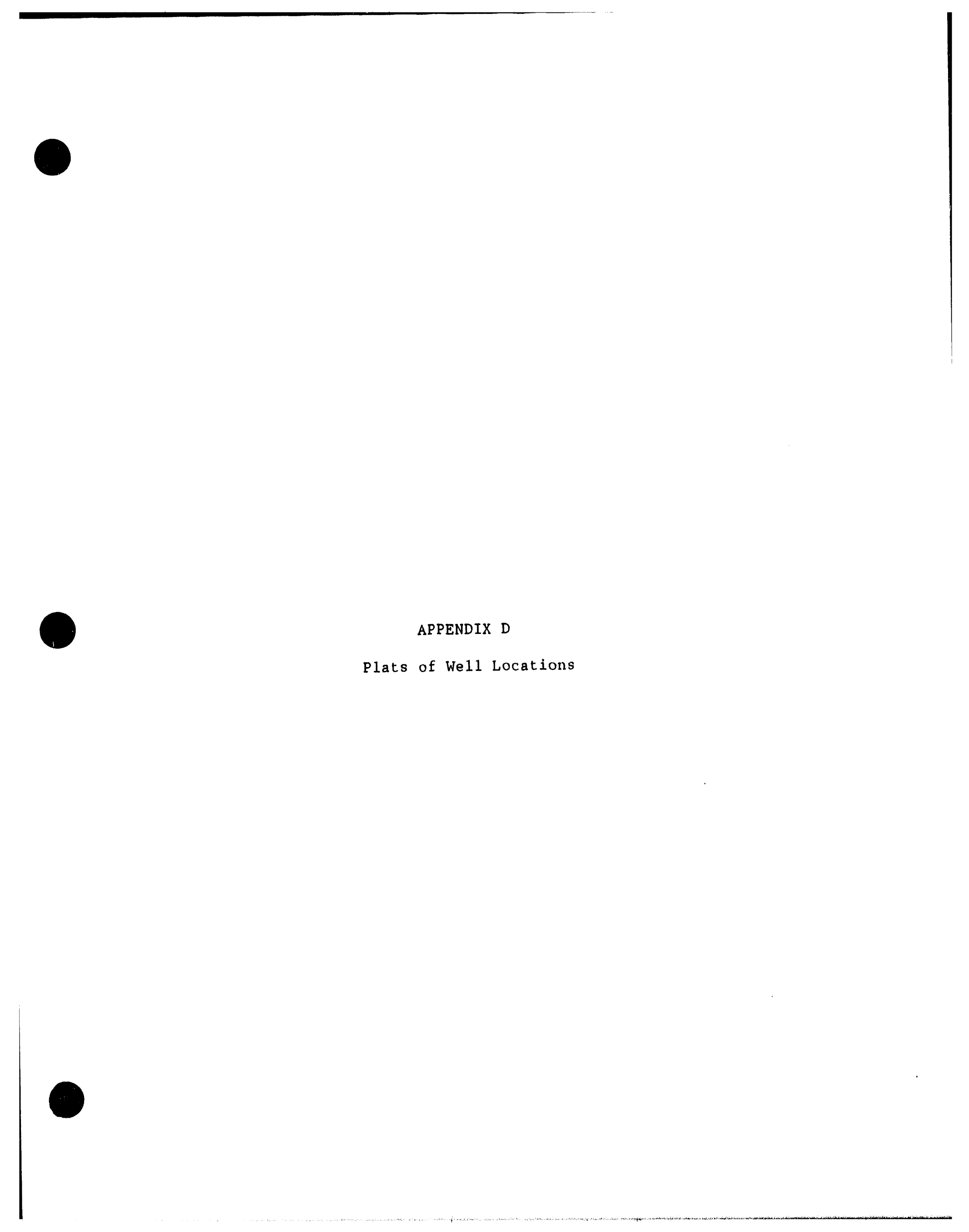

\section{Plats of We 11 Locations}




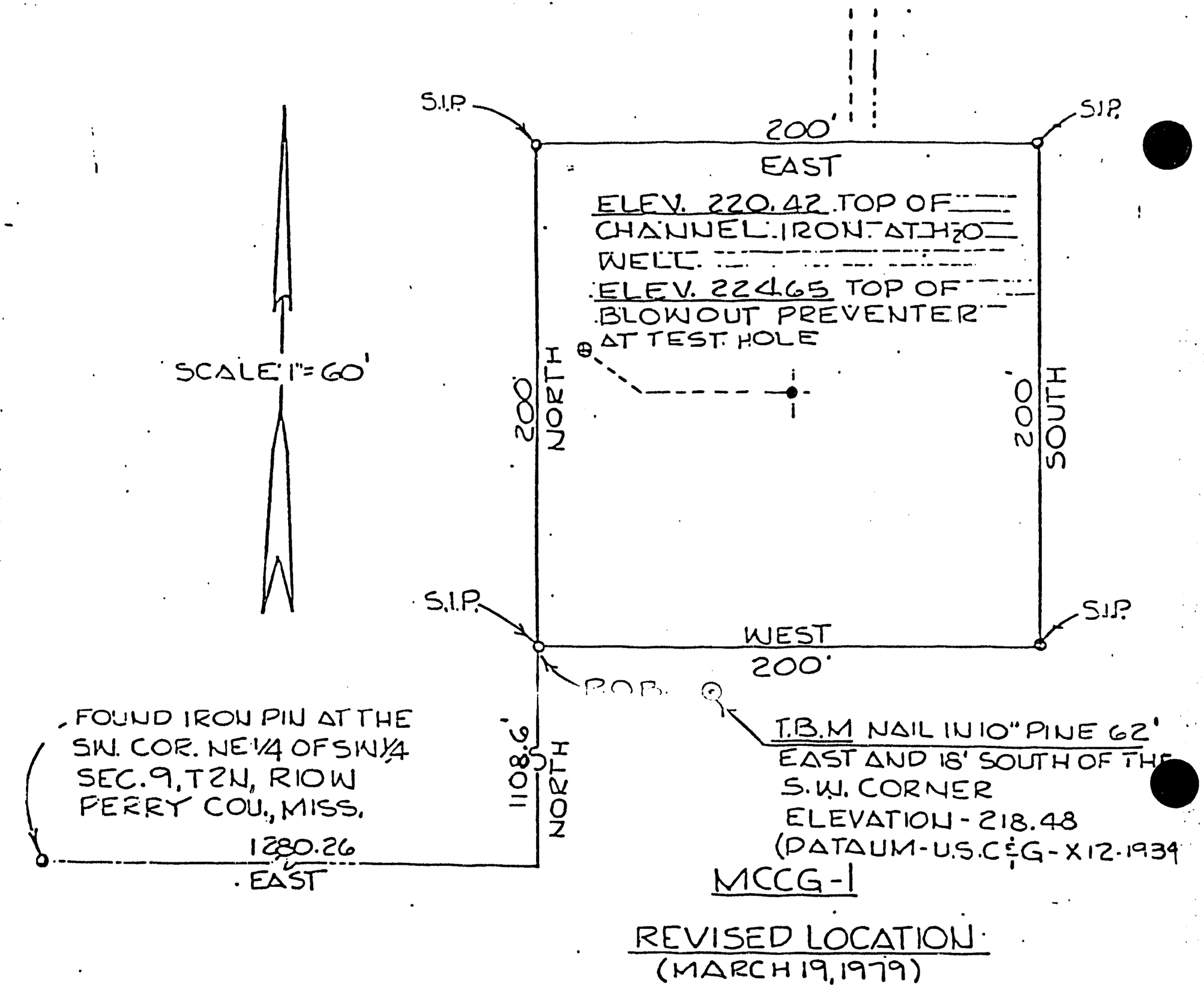

PESCRIPfIOli: A percel of land in the Northeast $1 / 4$ of the southwest $1 / 4$ and in the licrthi:est $1 / 4$ or the southeast 1/4, Section 9, Tolnship 2 jiorth, Range 10 West, Perry County; lis, and more particularly described as commencing et the Southweat Conner or the above said Northcast lily of line southwest $1 / 4$ and run East 1280.26 reet; thence North 1208.6 reet to the Point or Beginning; thence continue North 200 reet; thence East 200 reet; tinence south 200 reet; thence west $200 \mathrm{r} \in \in \mathrm{t}$ to the point or Bcginning.

\section{LEGEND}

S.I.P. SET IRON PIN

- hole location

* WATER SUPPLT WELL

- - - W NTER SUPPLT LINE

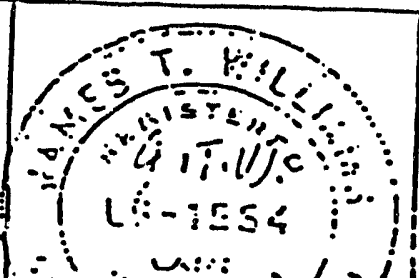

SURVEY OFAPART OFTHE NE 1/4 OF SWV $1 / 4$ ND A PART OFTHE NW1/4 OF S.E Y4, SEC.9, T2N, RIOW, PEERY COUNTT, MISS.

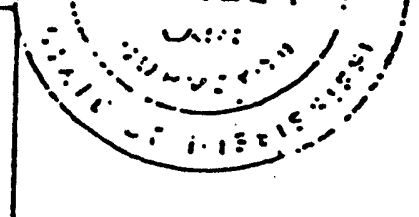
in WILLIAMSEYBROOM-SURVEYORS SN.391 78

DN.88.3 


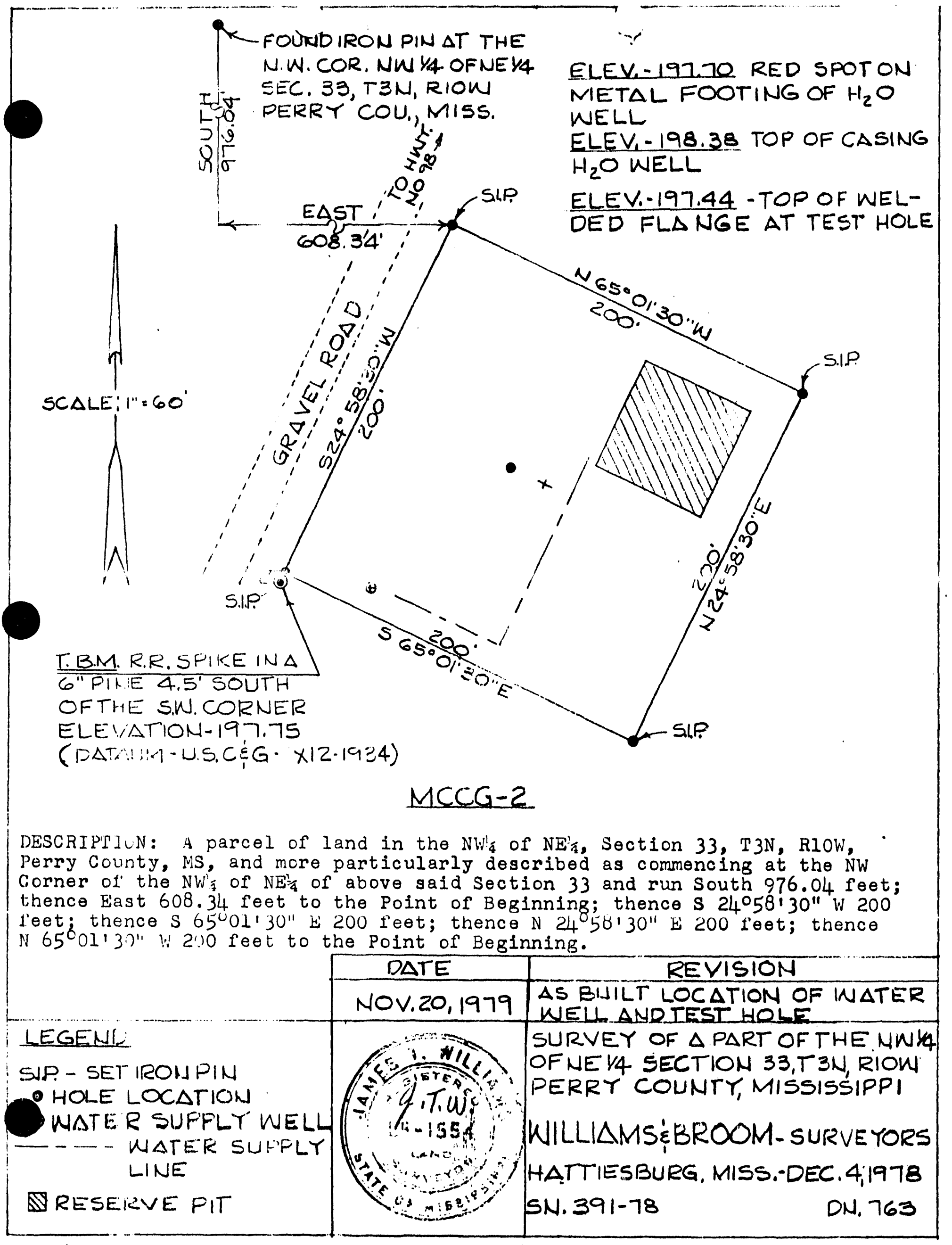




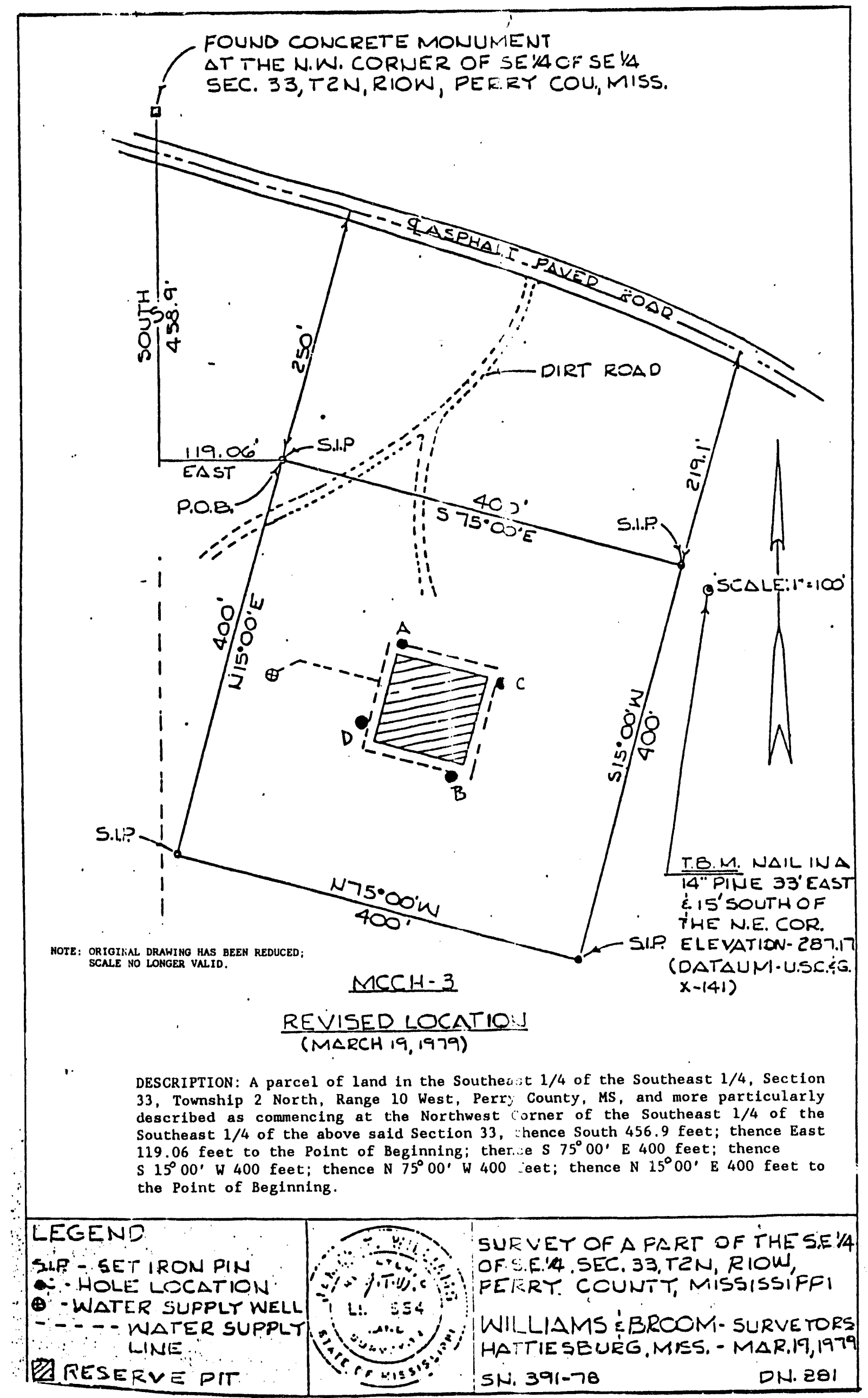




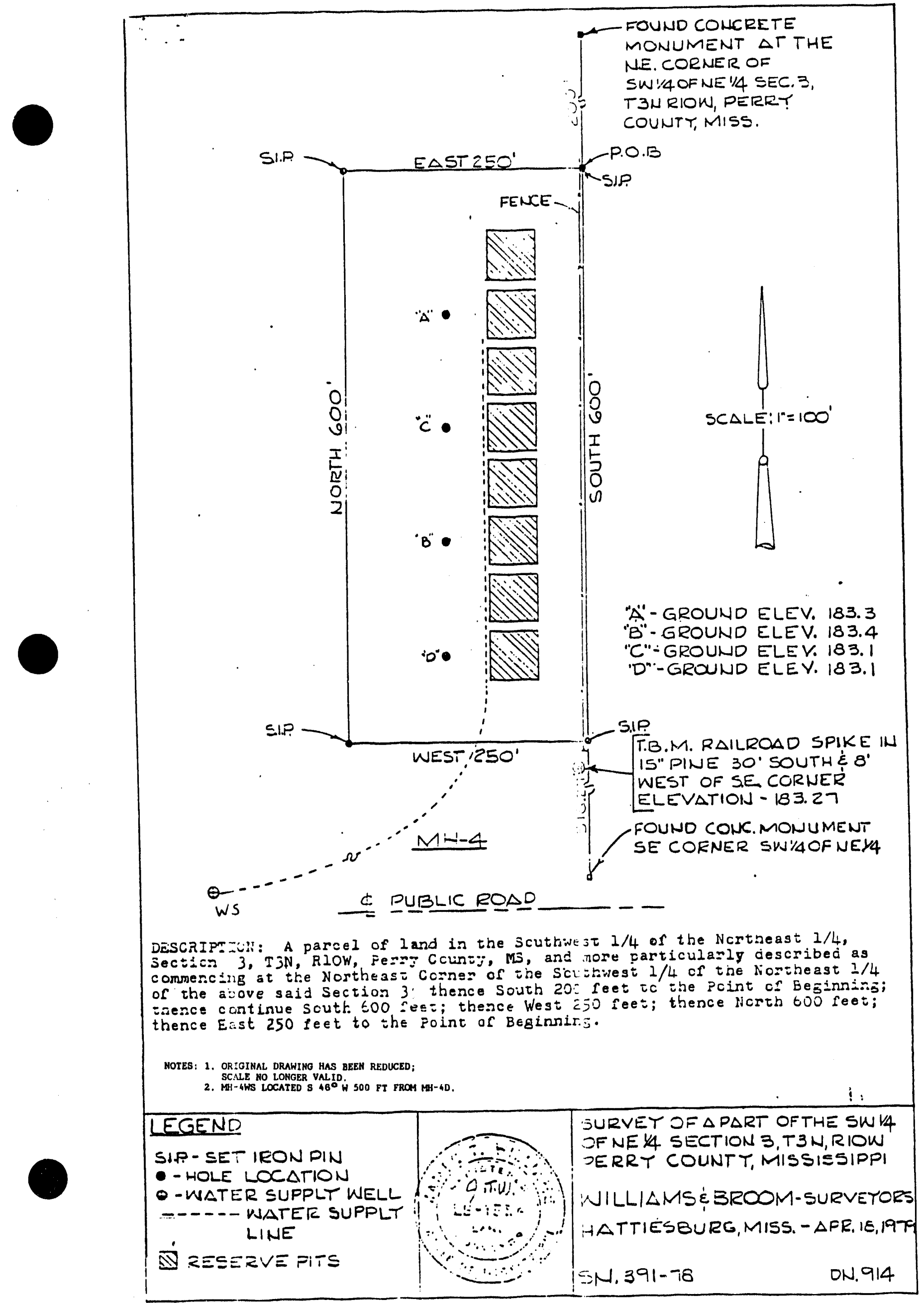




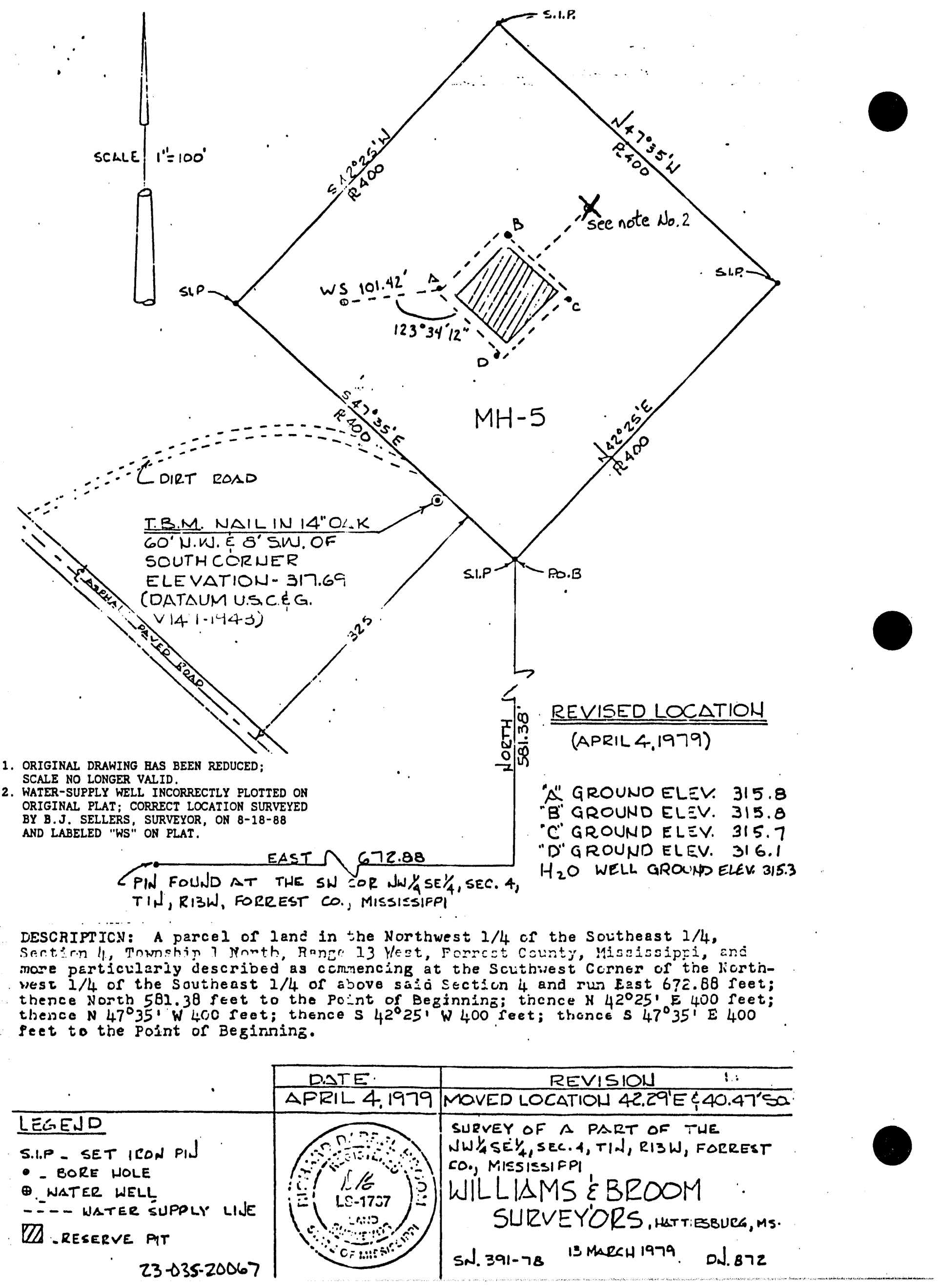




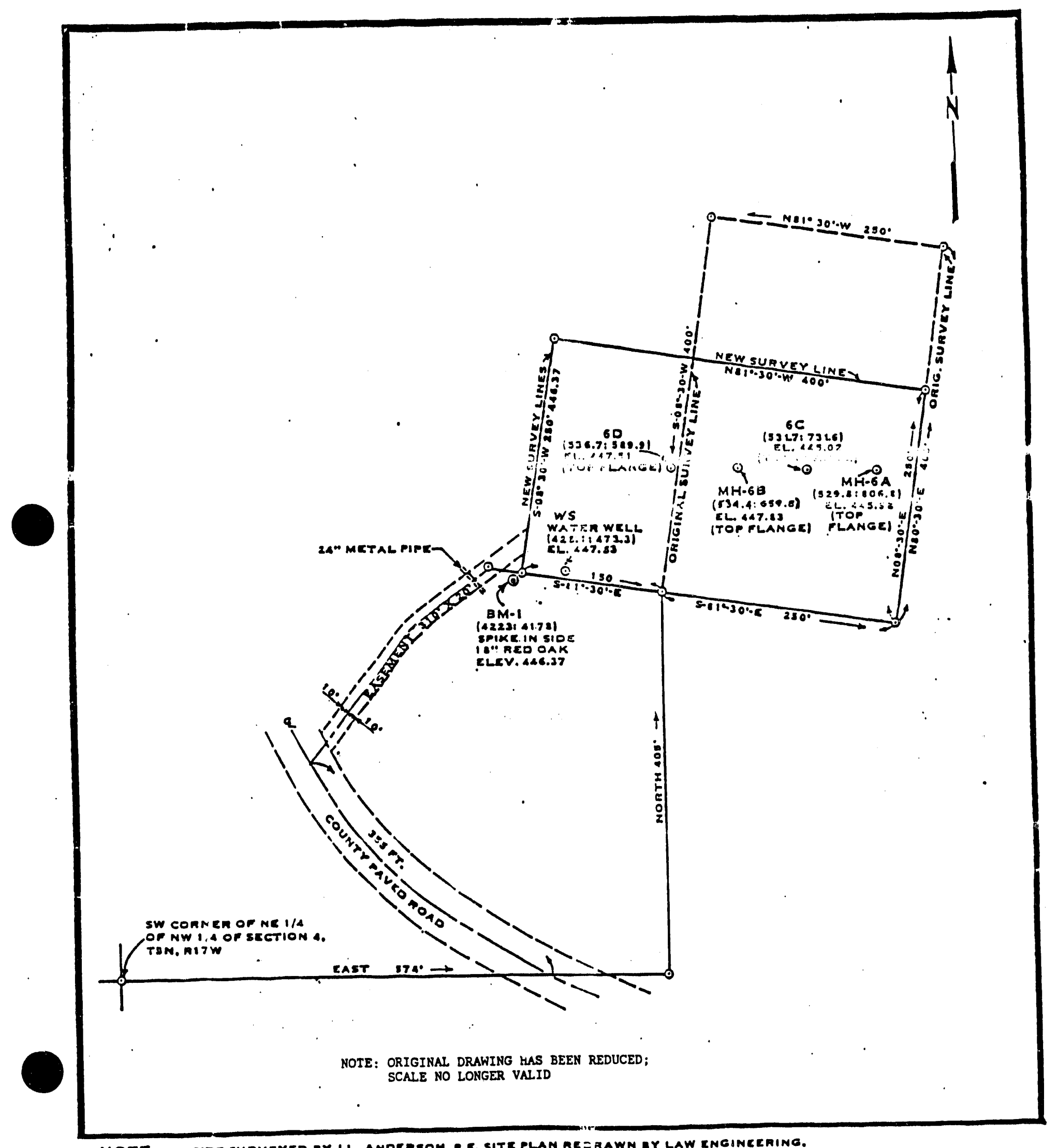




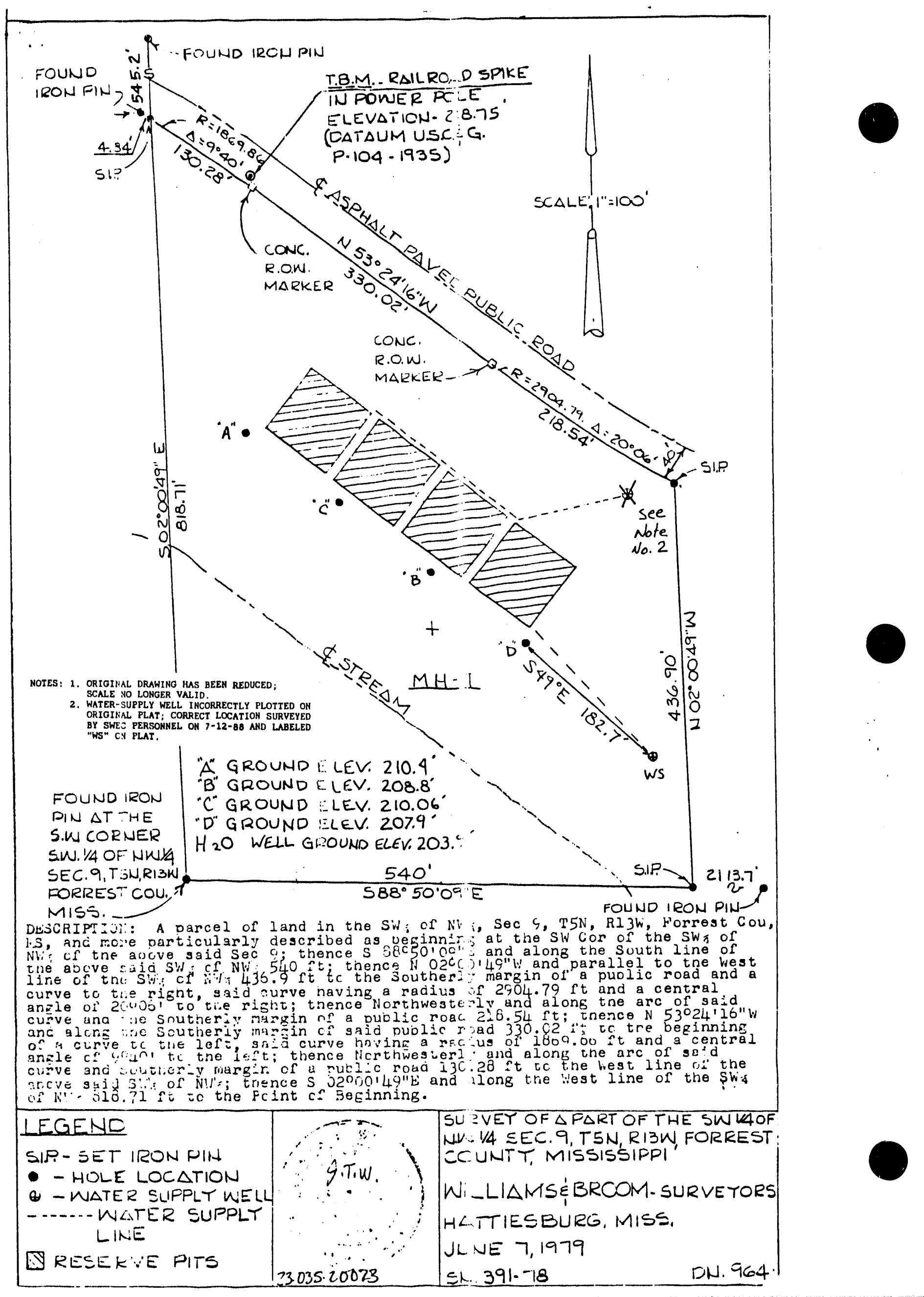




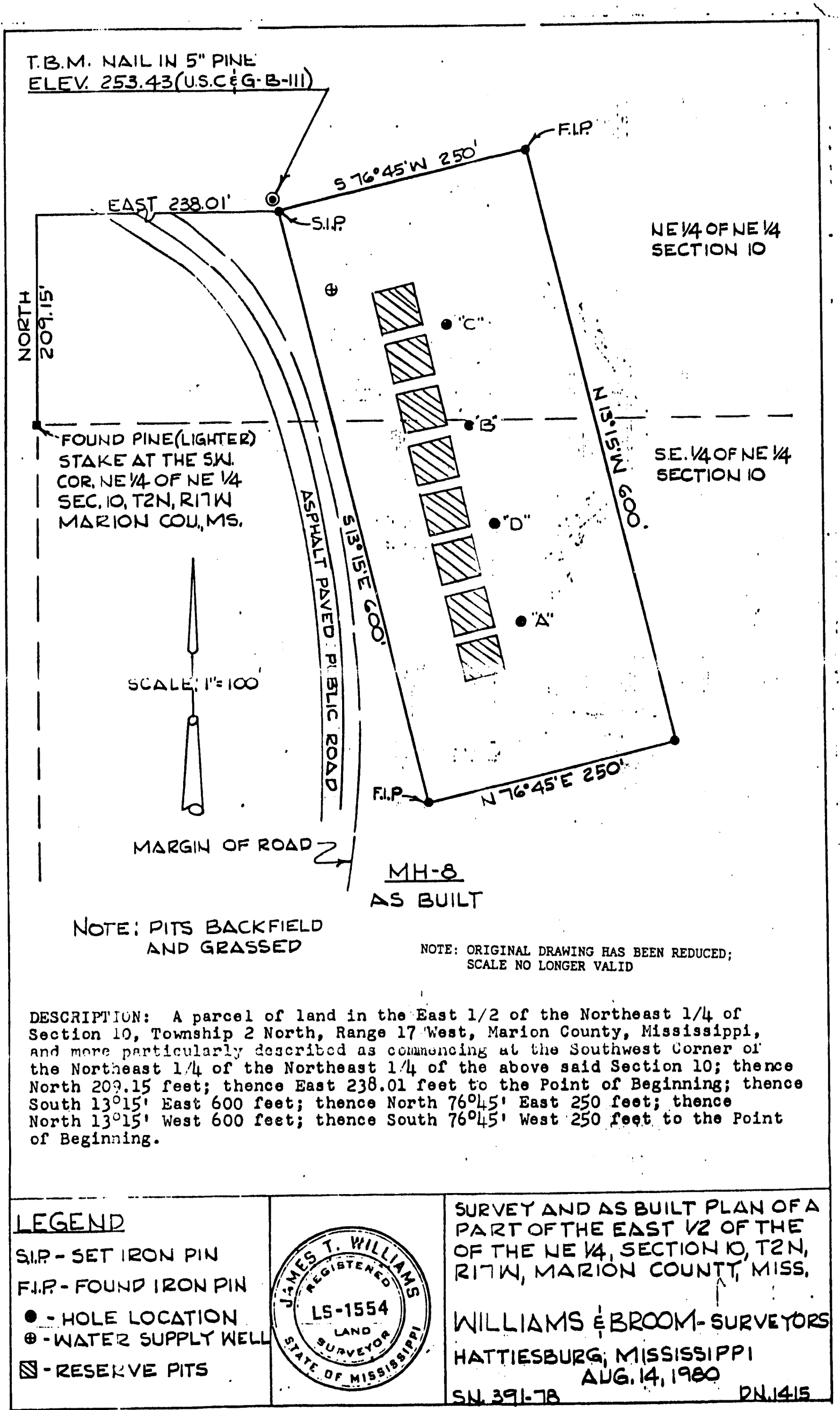




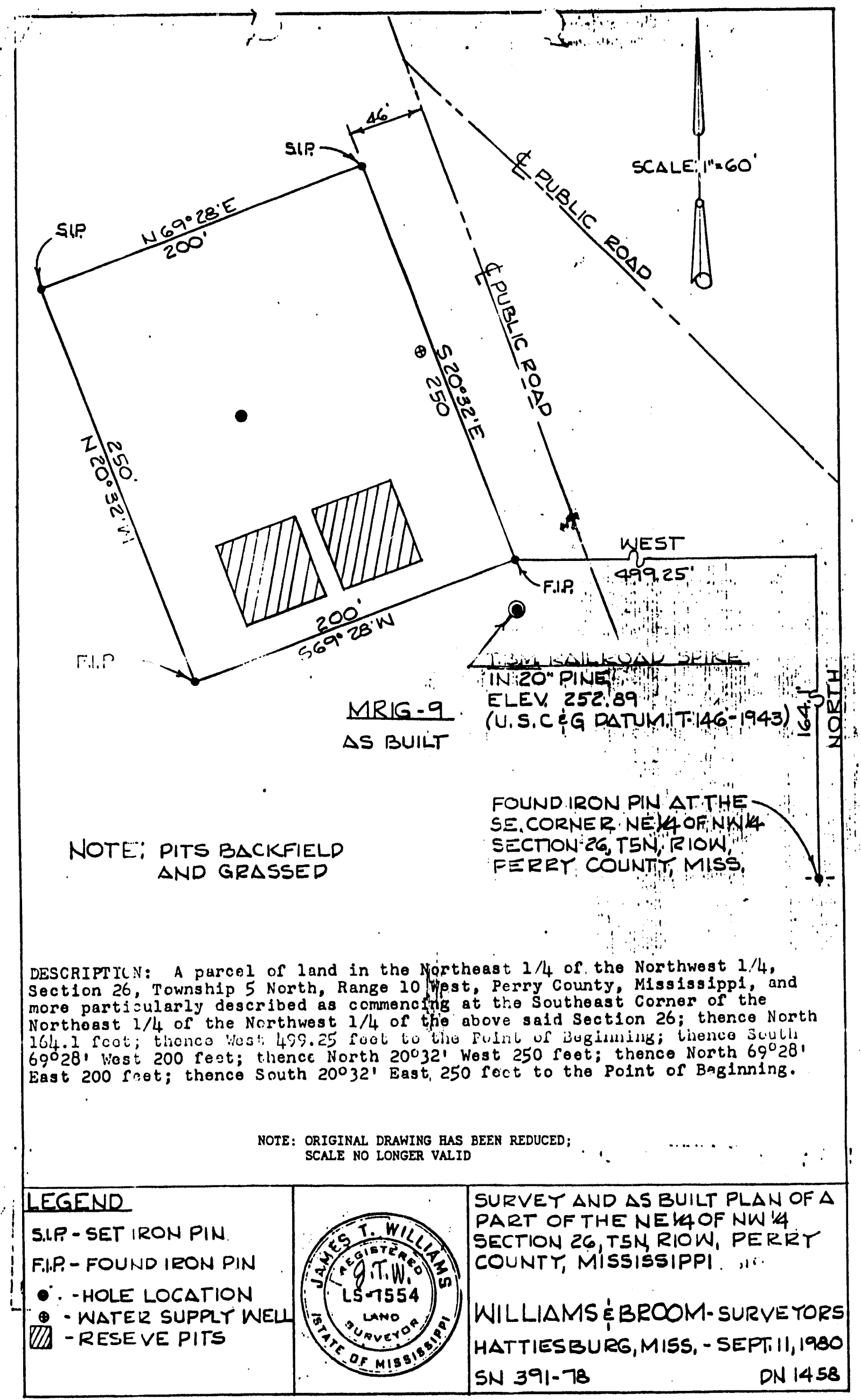




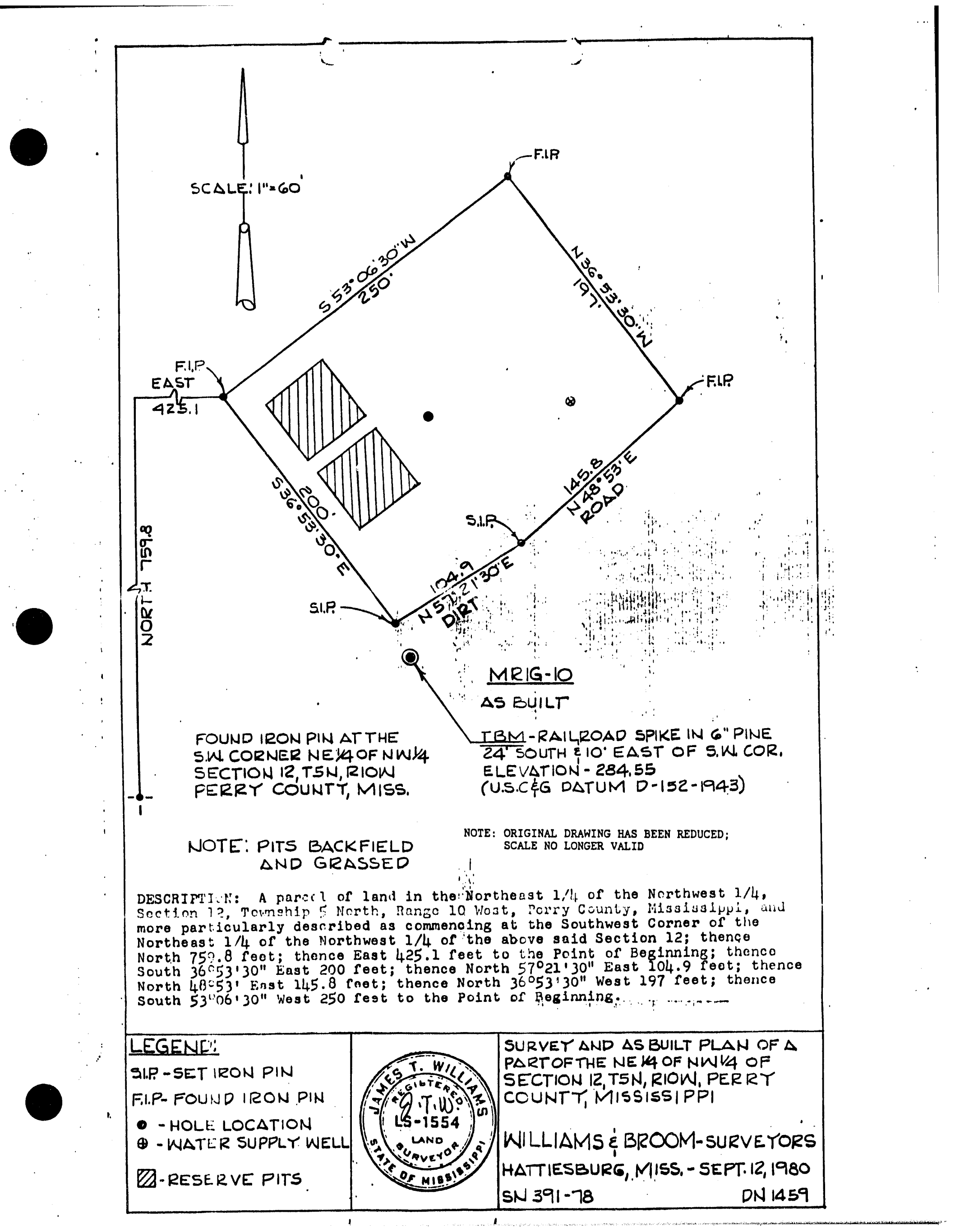




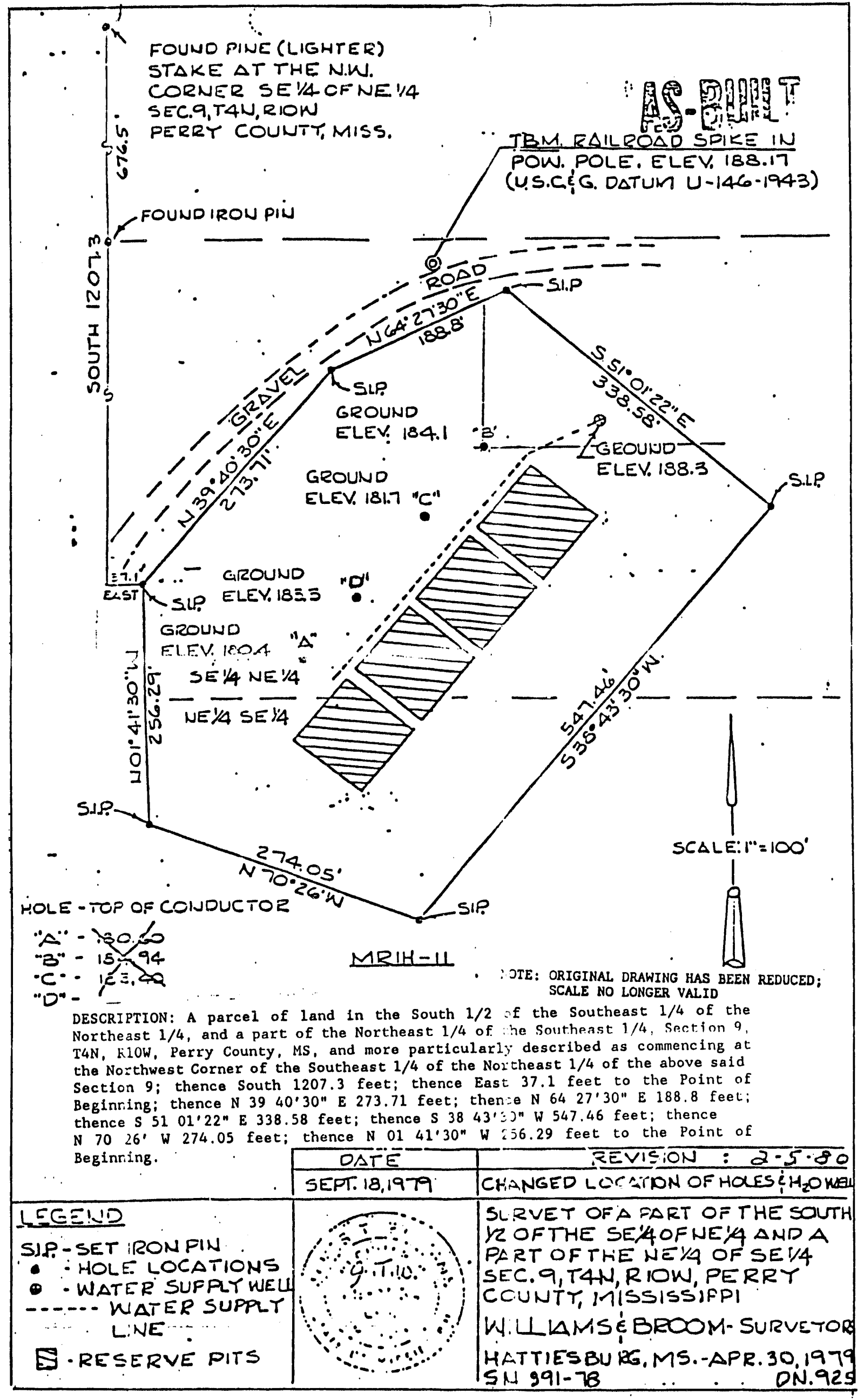


ub

-

$\because \ldots$

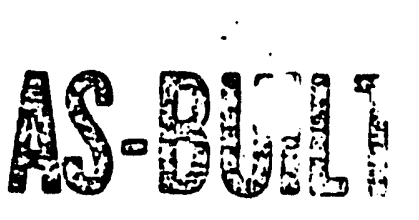

1. Weacteptide
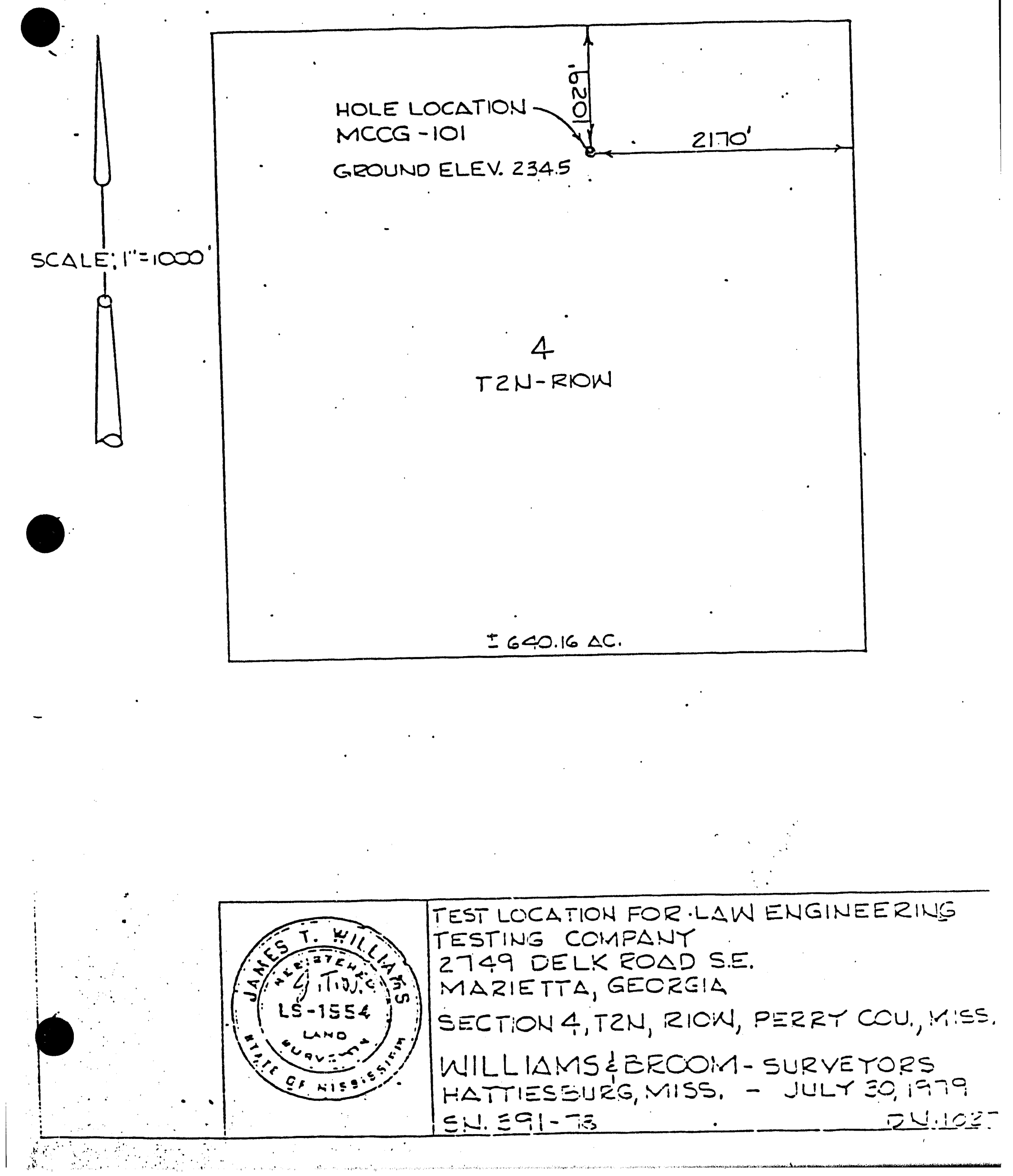

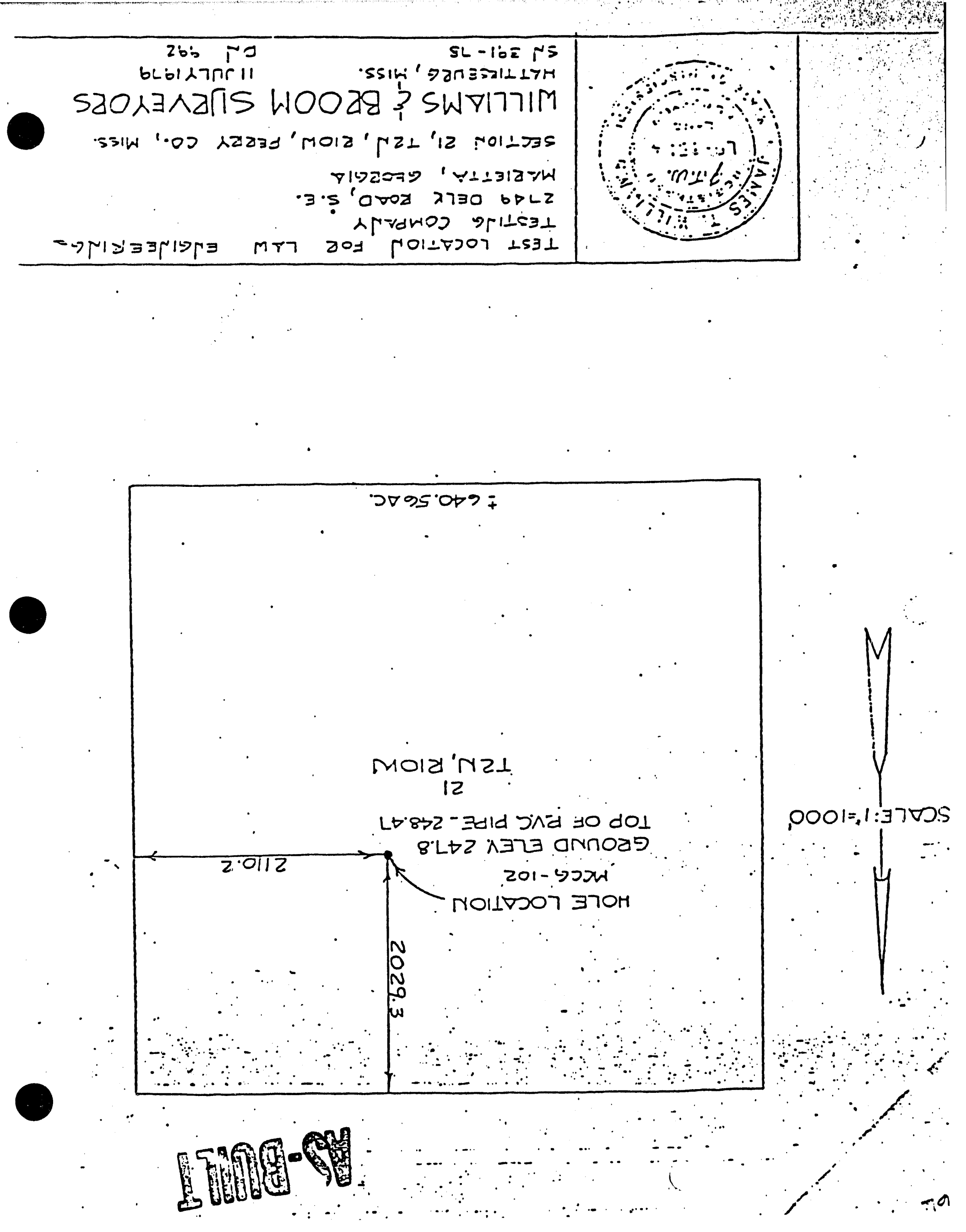

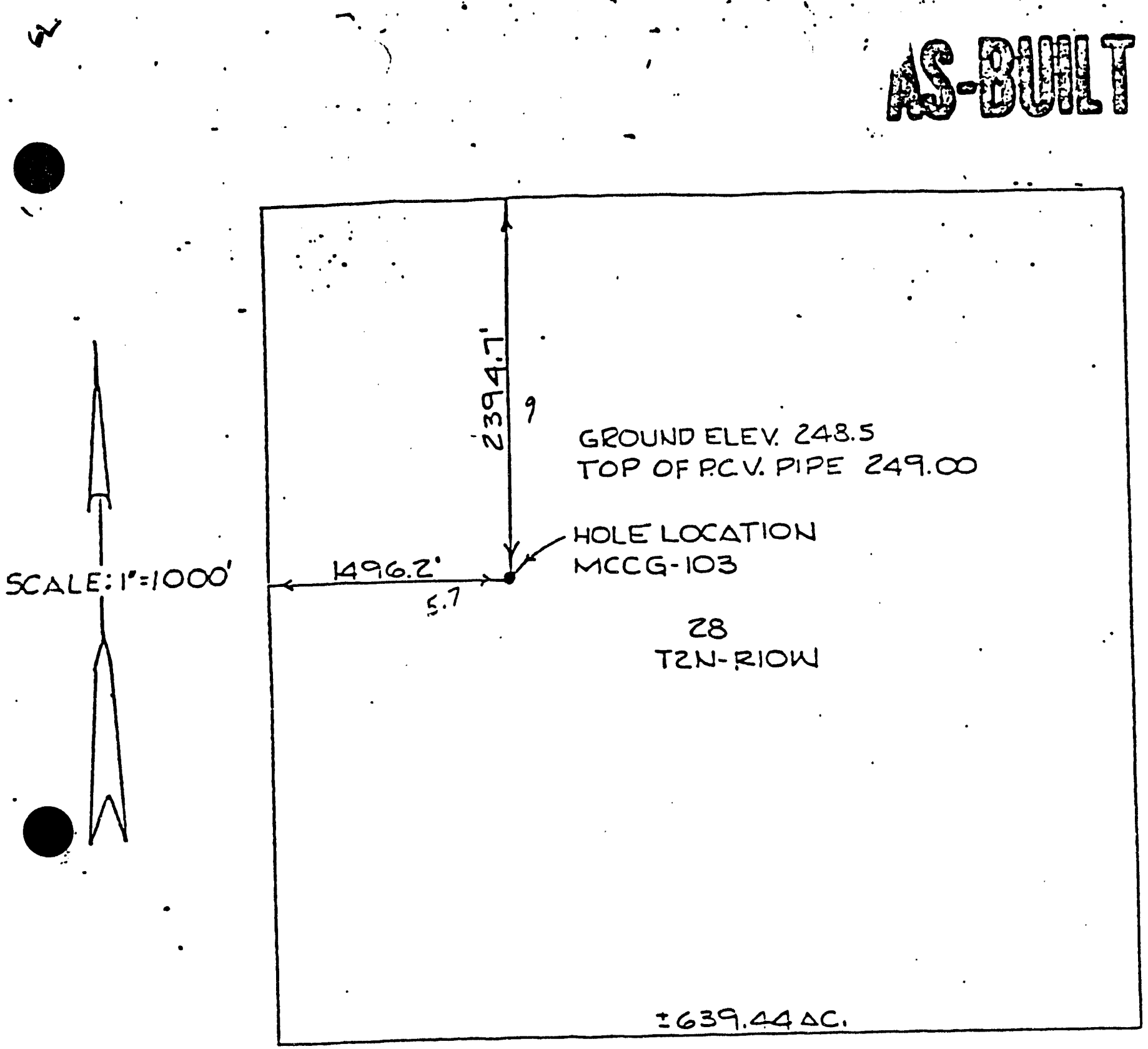

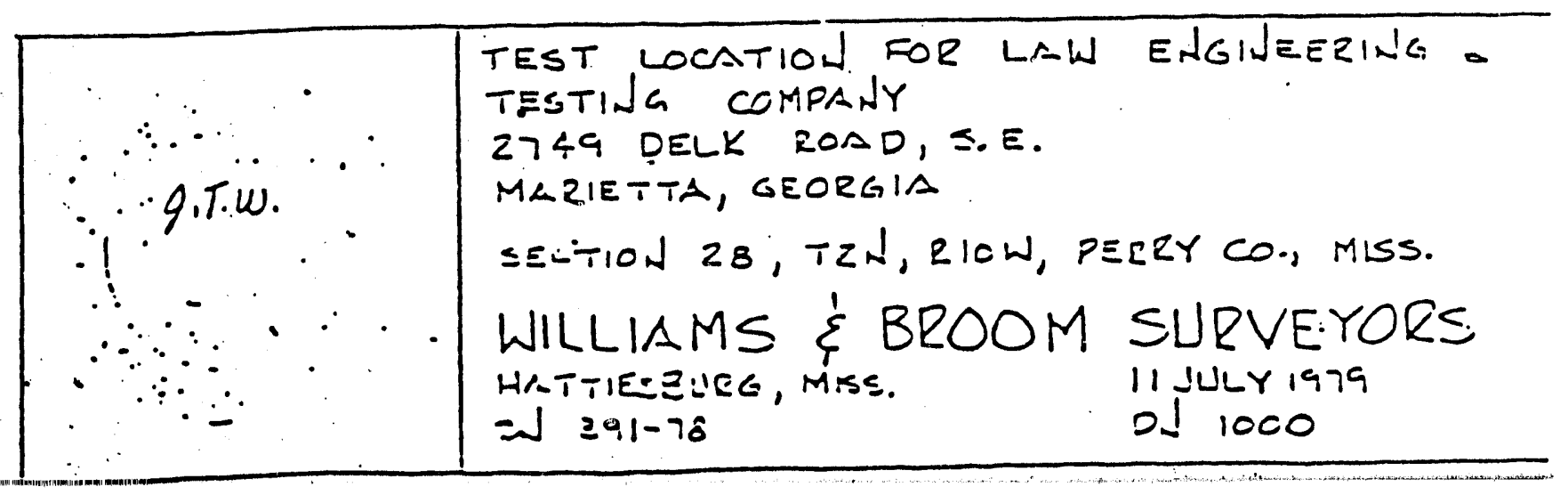



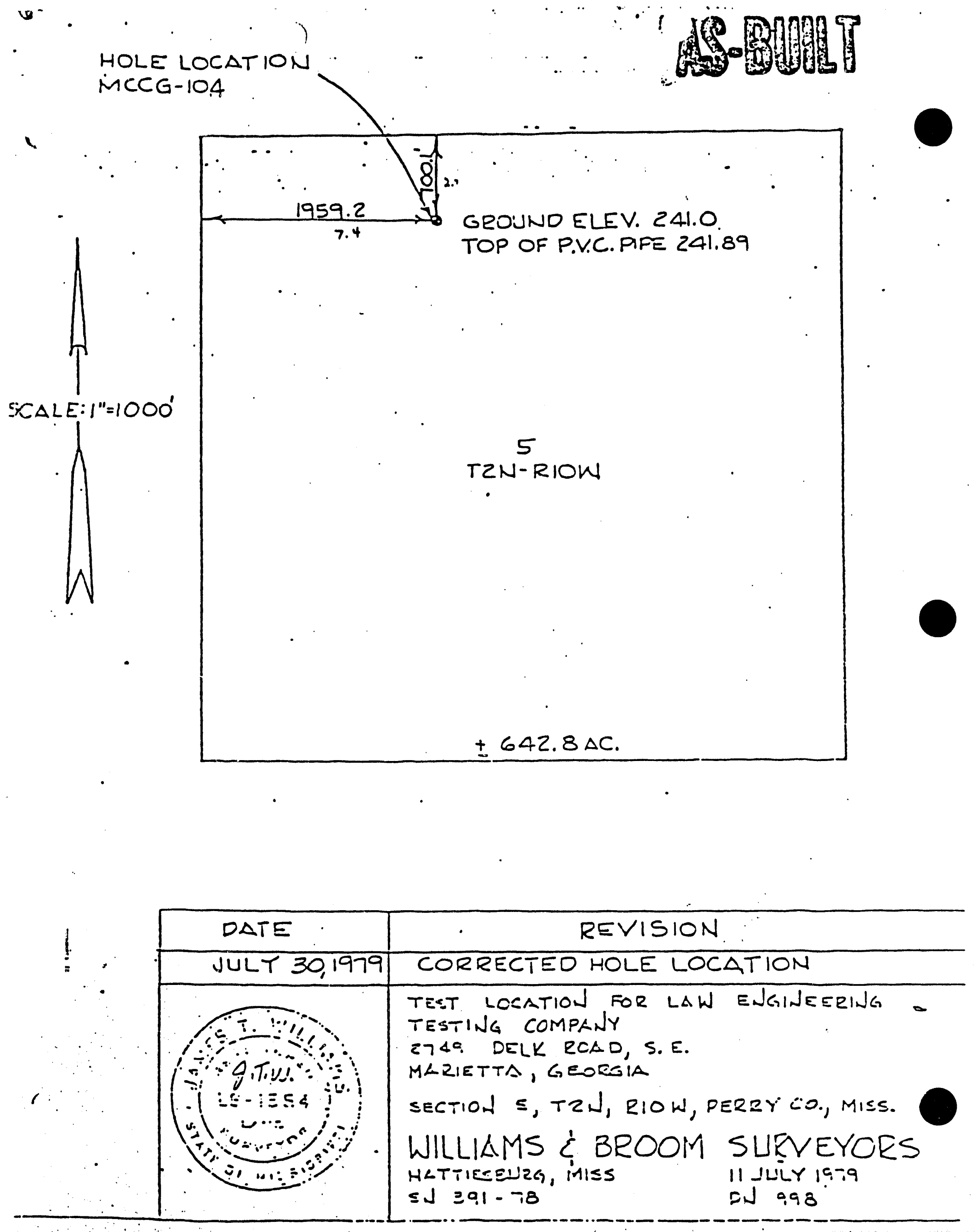


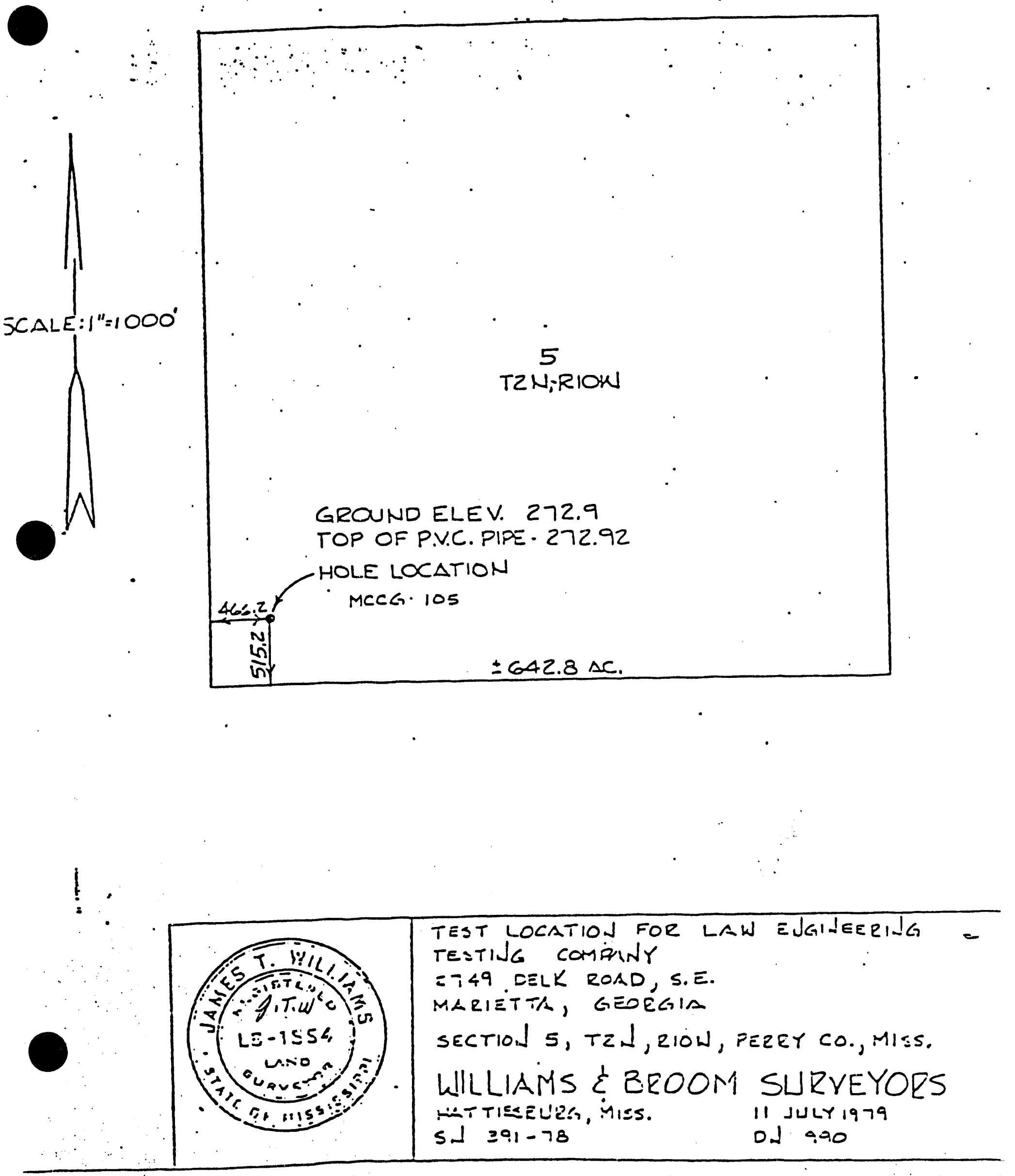




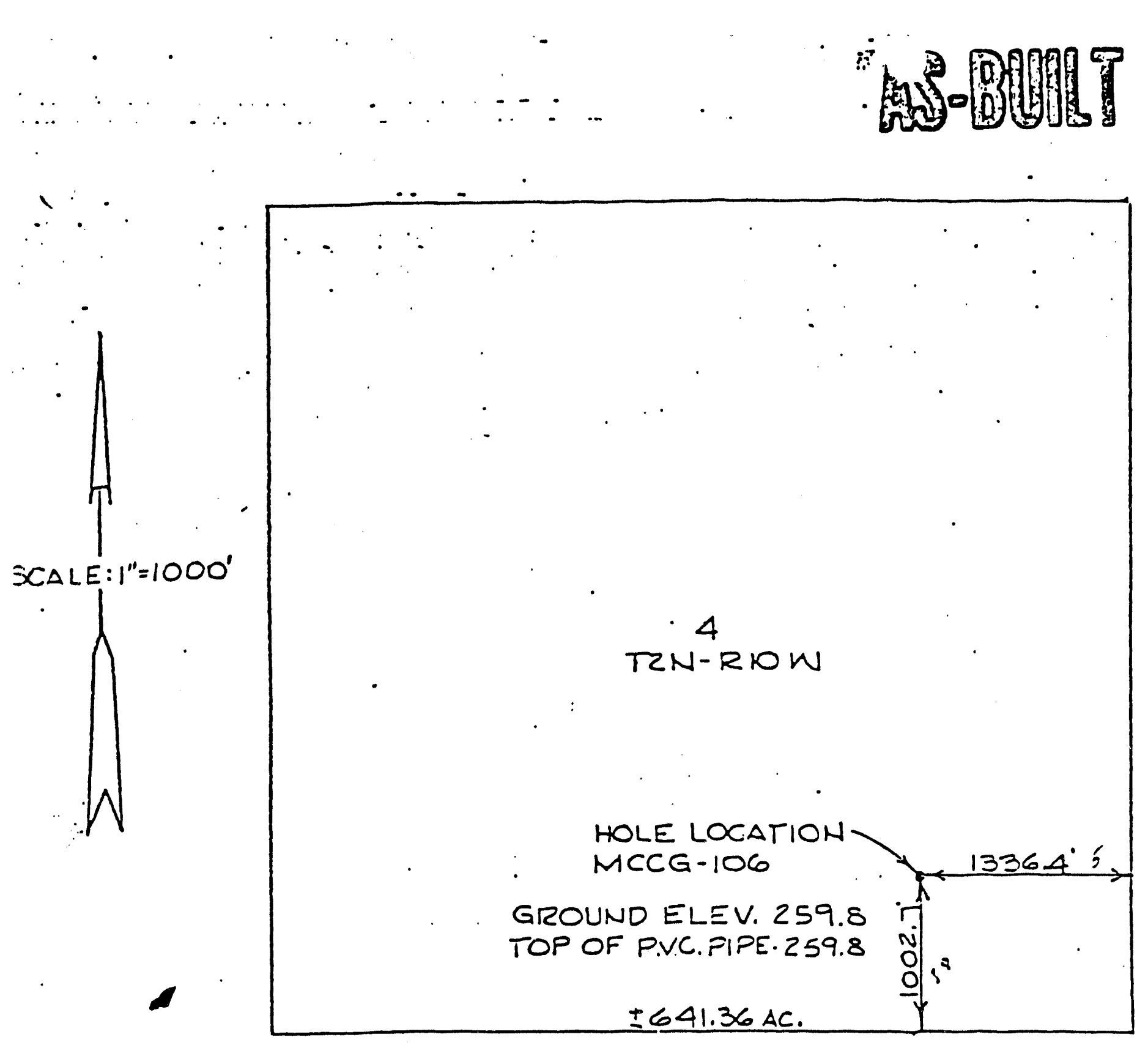

1

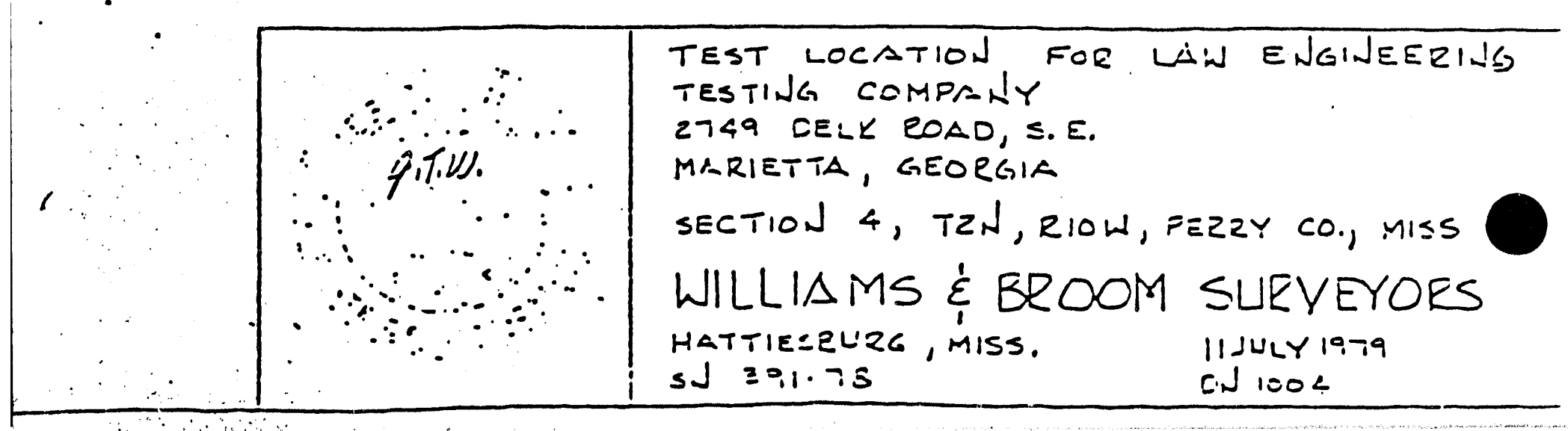




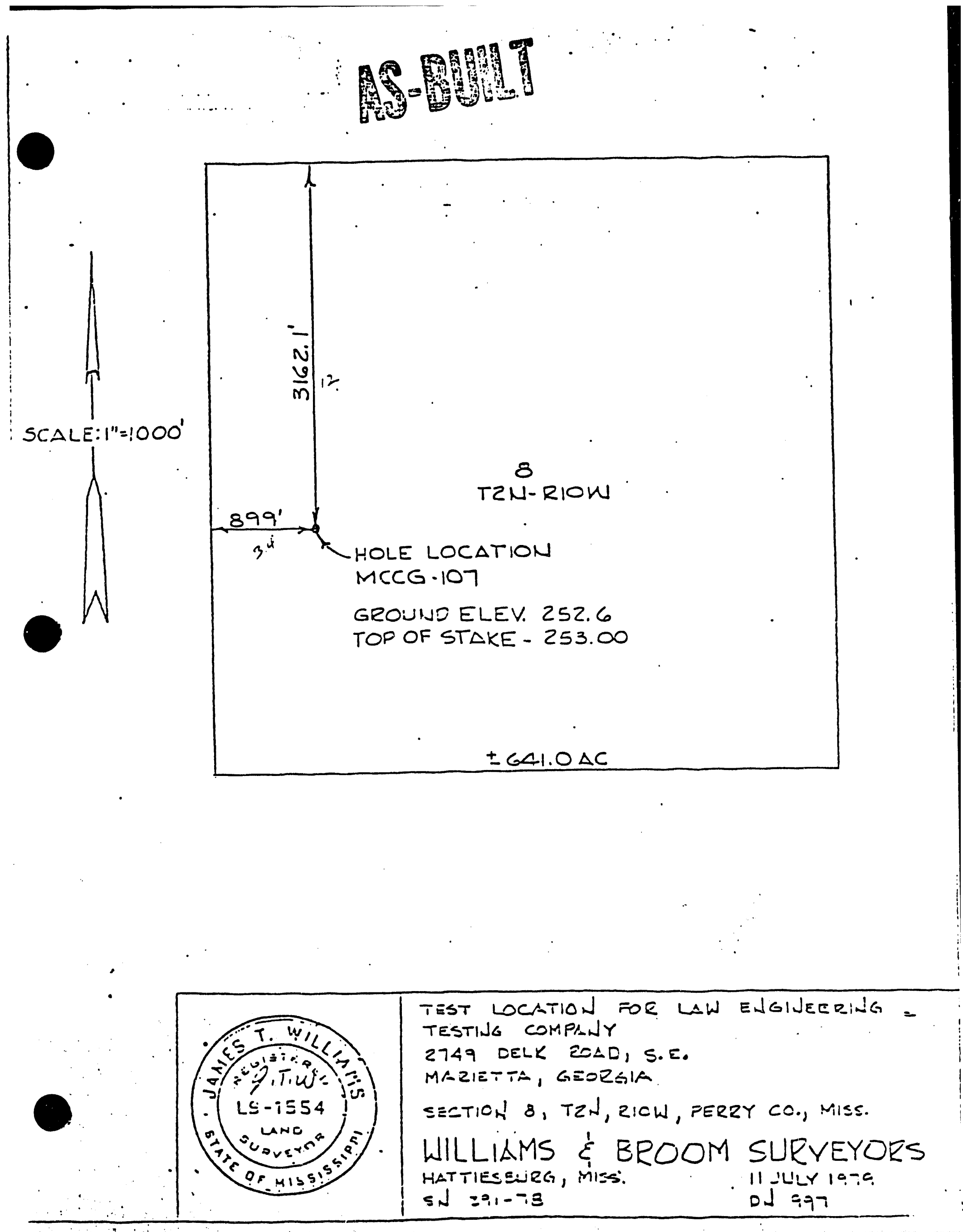



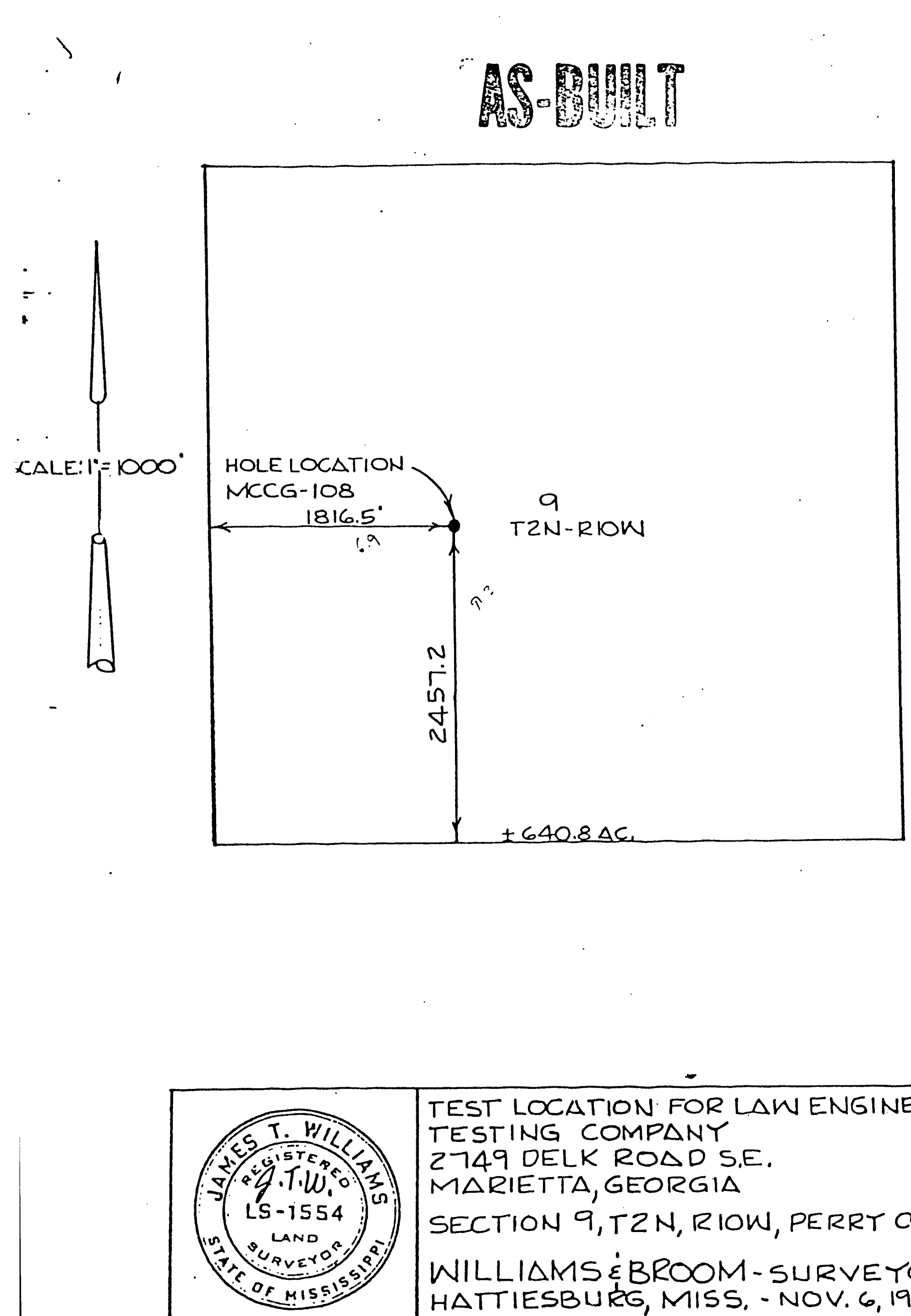

TEST LOCATION FOR LAW ENGINEERING TESTING COMPANY 2749 DELK ROAD S.E. MARIETTA, GEORGIA SECTION 9, T2N, RIOW, PERRT COU, MIS WILLIAMSËBROOM - SURVEYORS HATTIESBUKG, MISS. - NOV. 6,1979 

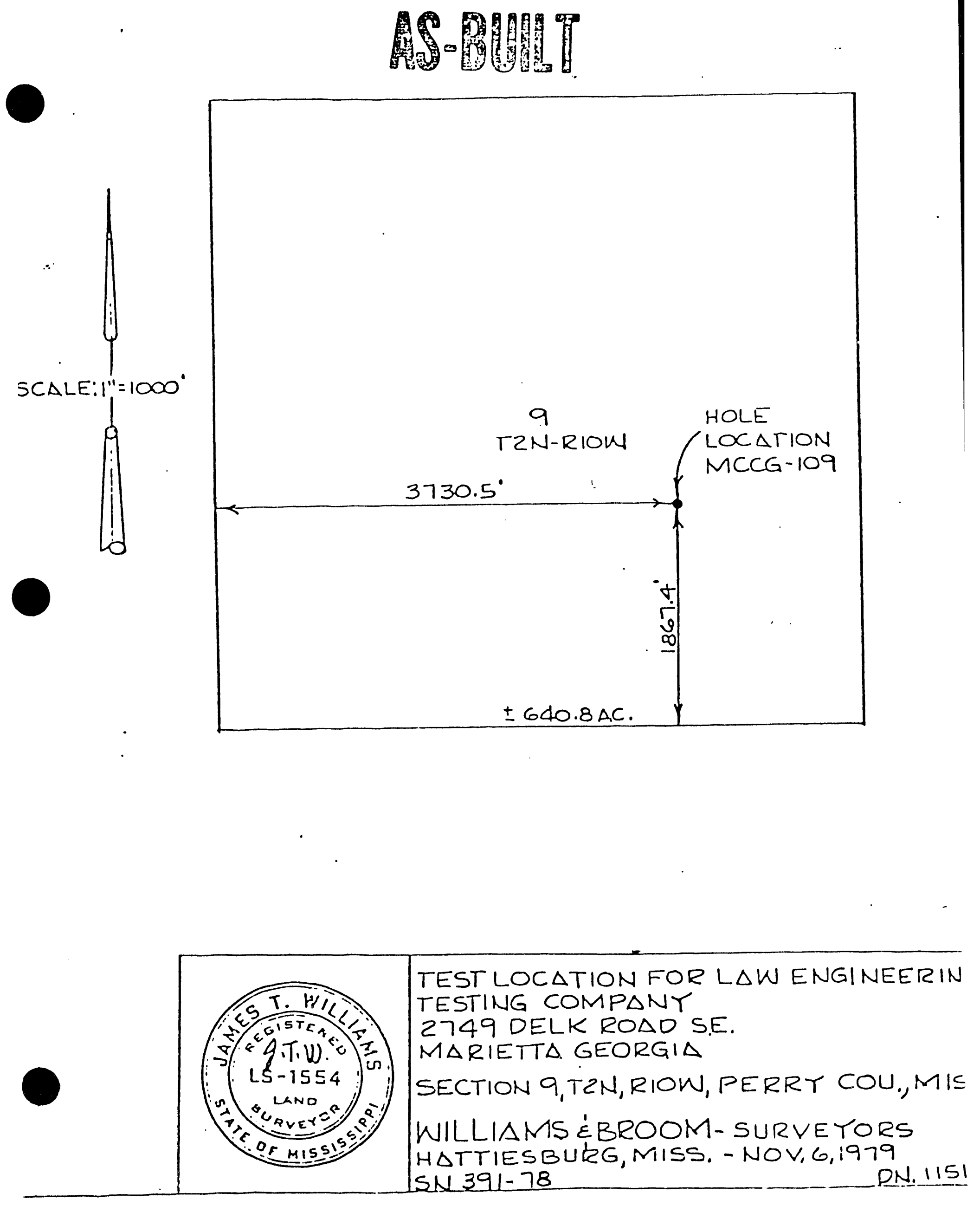


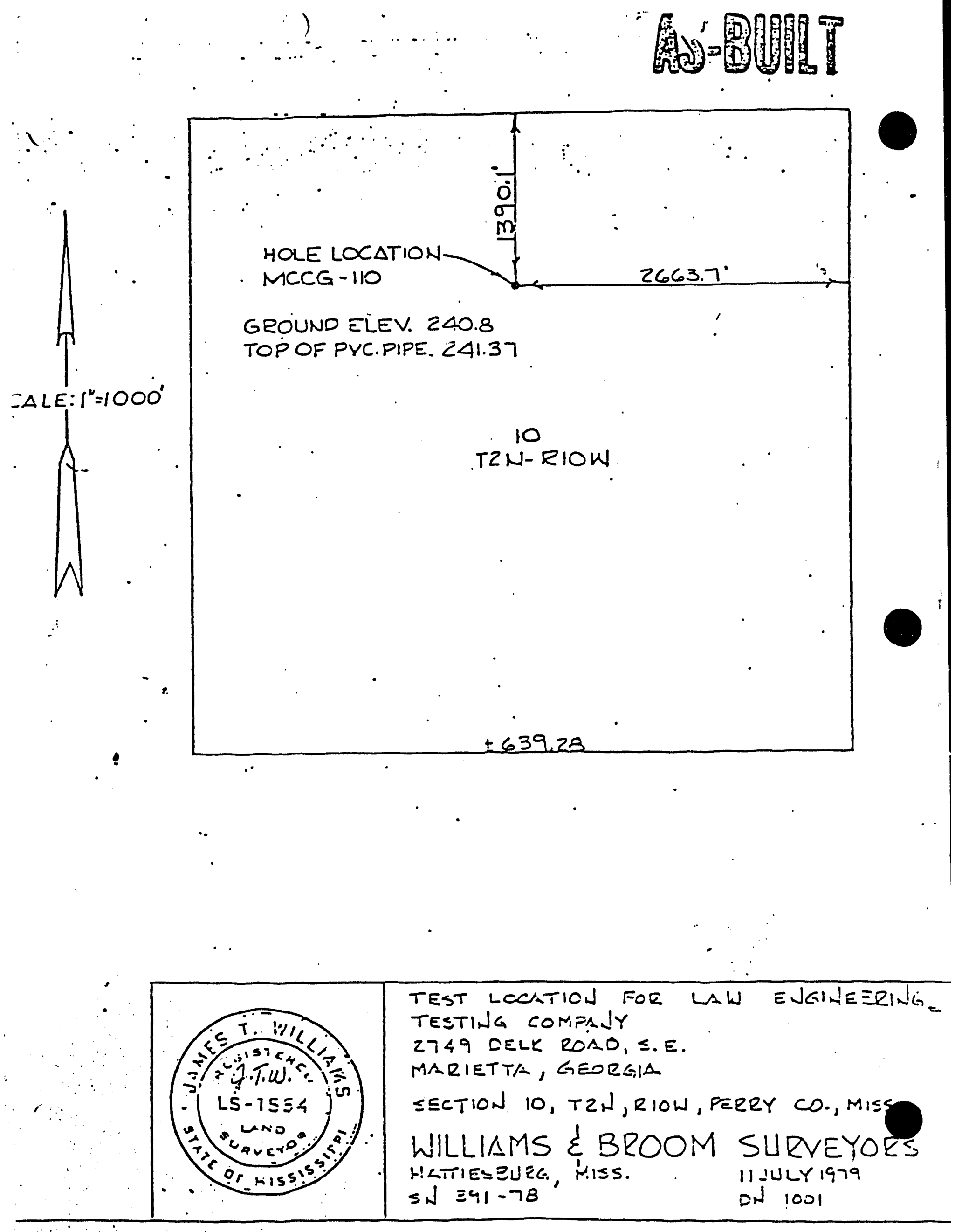




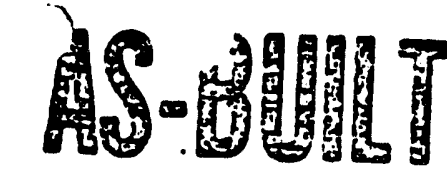
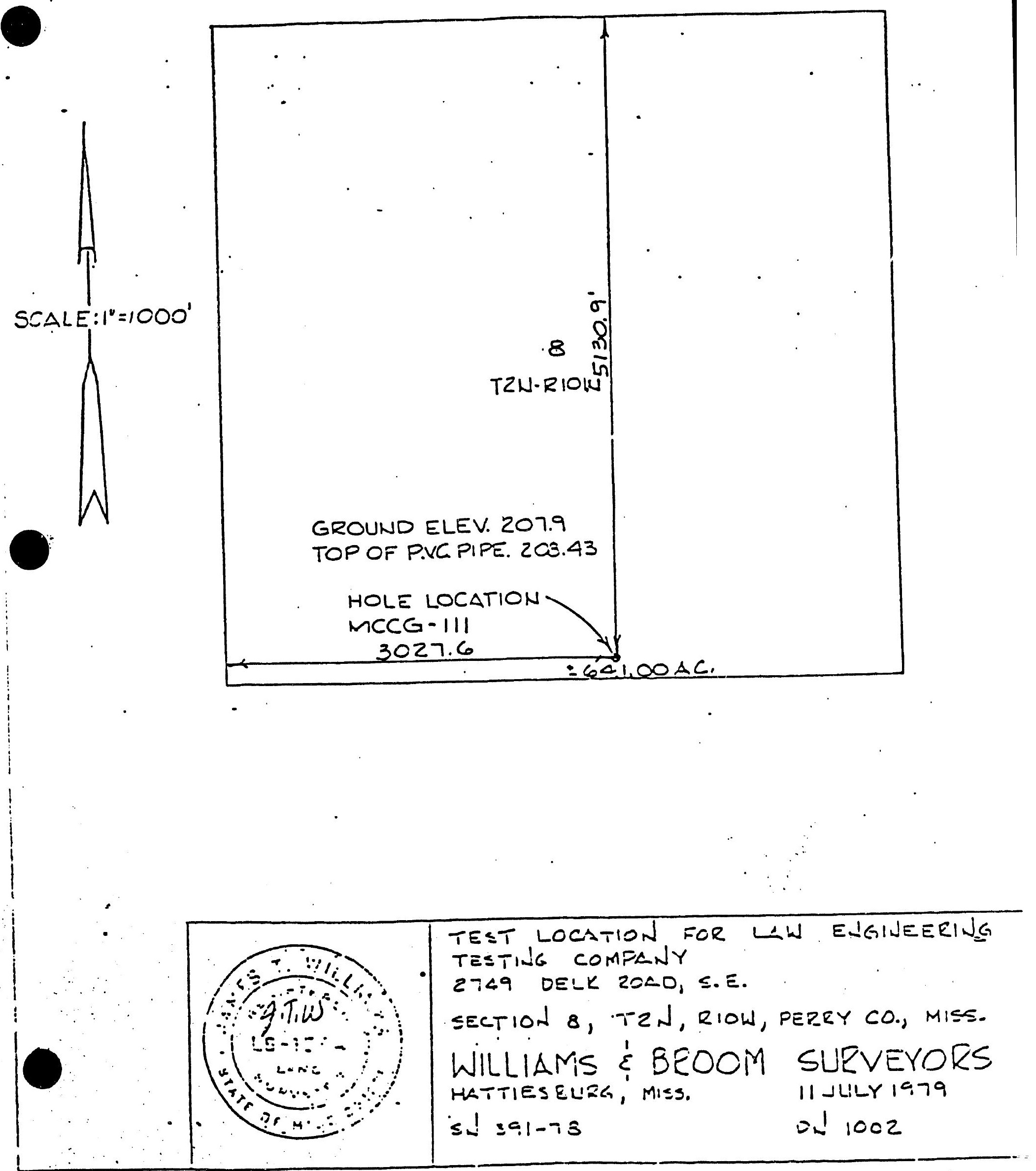

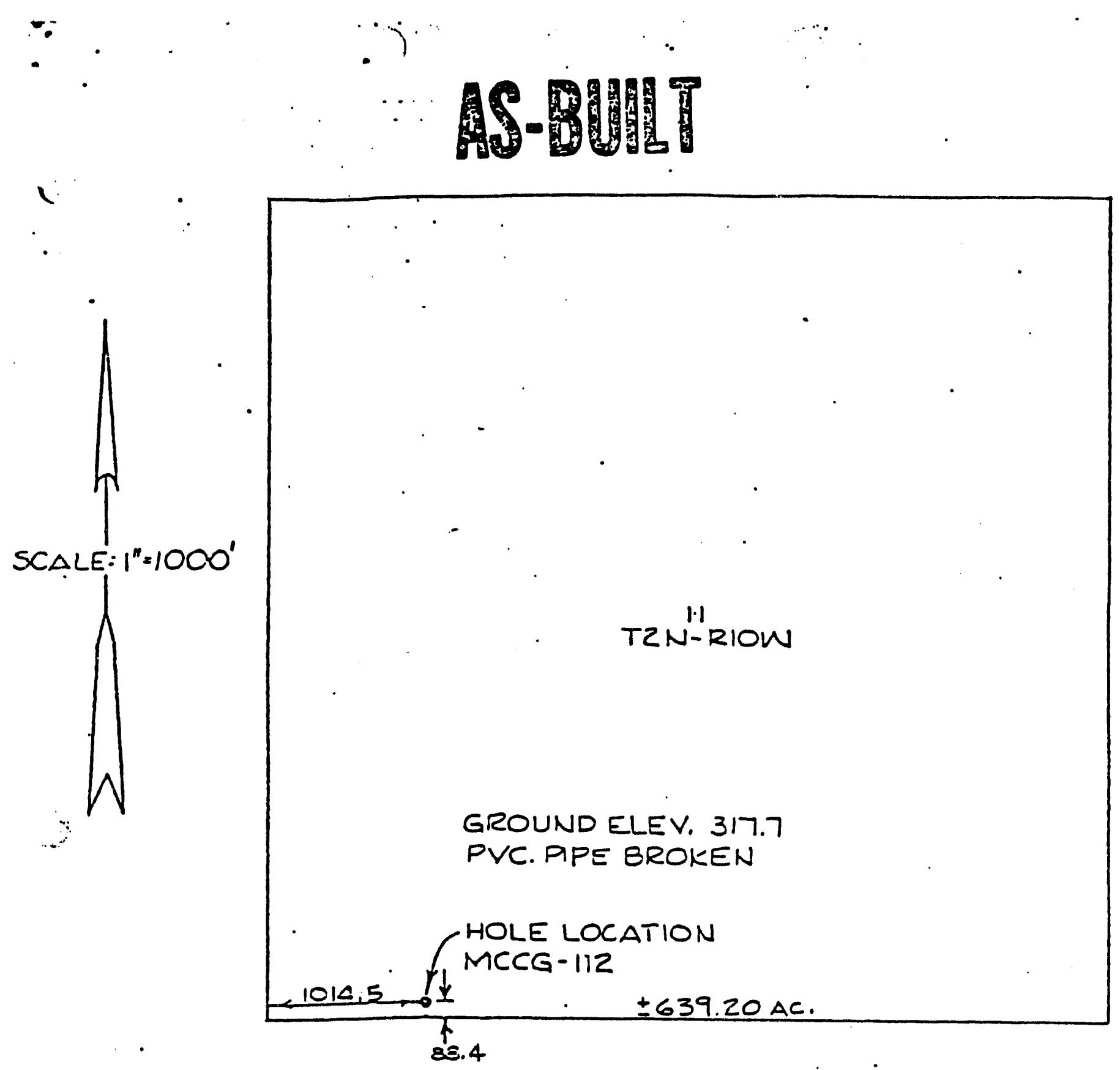

GROUND ELEV. 317.7

PVC. PIPE BROKEN

HOLE LOCATION

$\pm 639.20 \mathrm{AC}$.

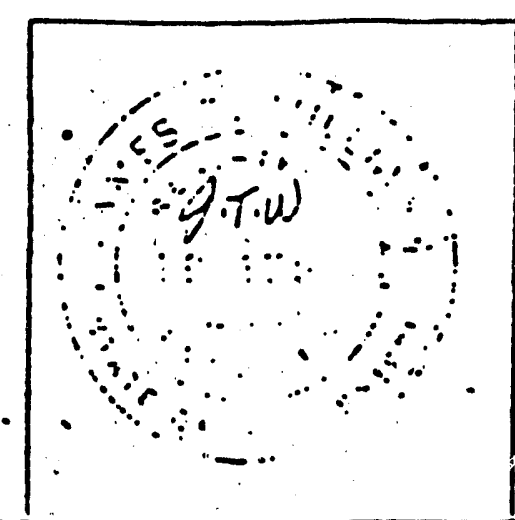

TEUT LOCATION. FOR LAW EJGINEERING TESTING COMFANY $2 T 49$ CELK ROAD, S..

MARIETTA , GEORGIA

SECTIOJ II, TZN, RIOW, FEREY CO.; MISS.

WILLILMS \& EROOM SLRVEYORS

HAT TIEEEHEG, MISS. HULY I979

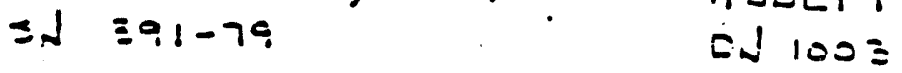




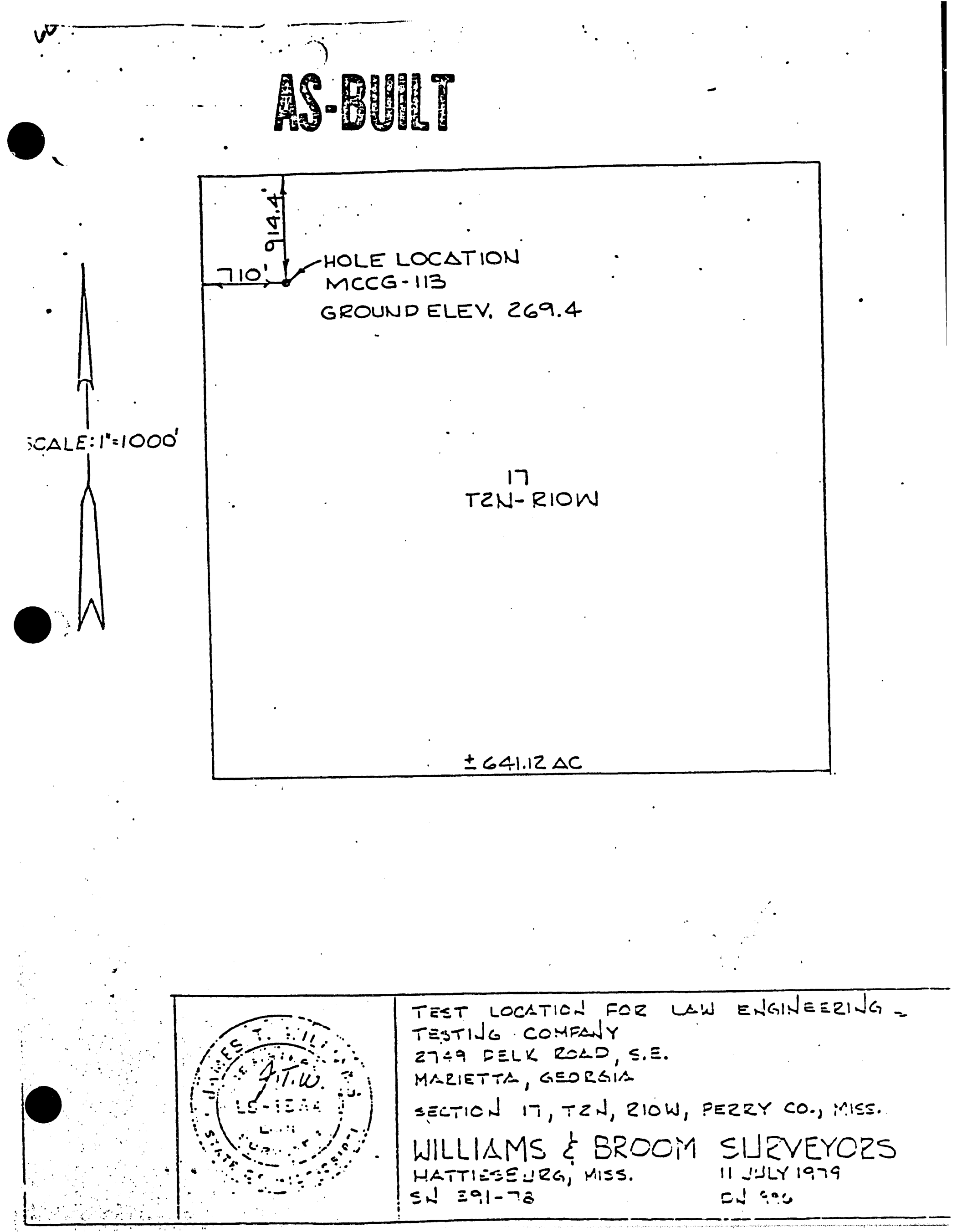



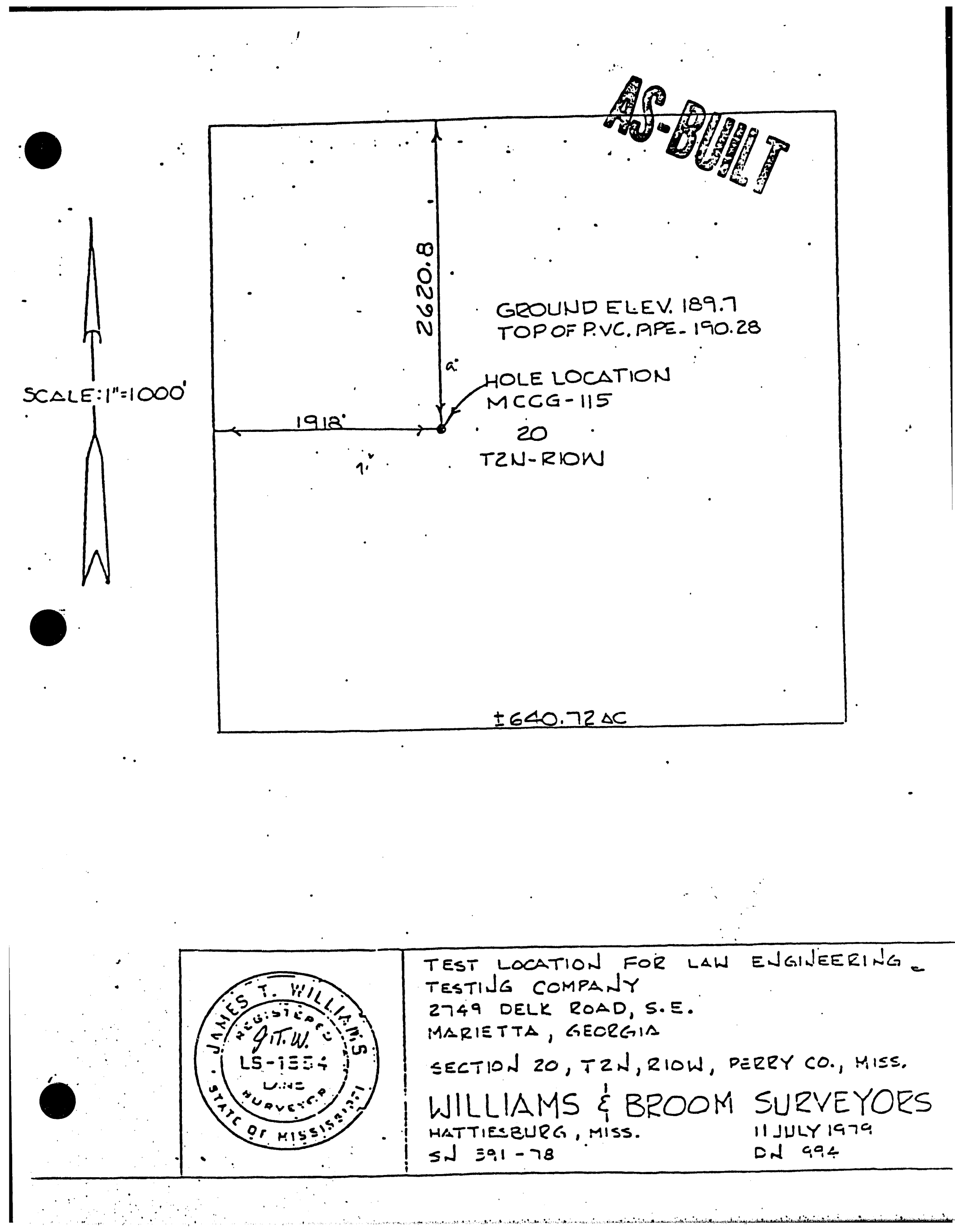


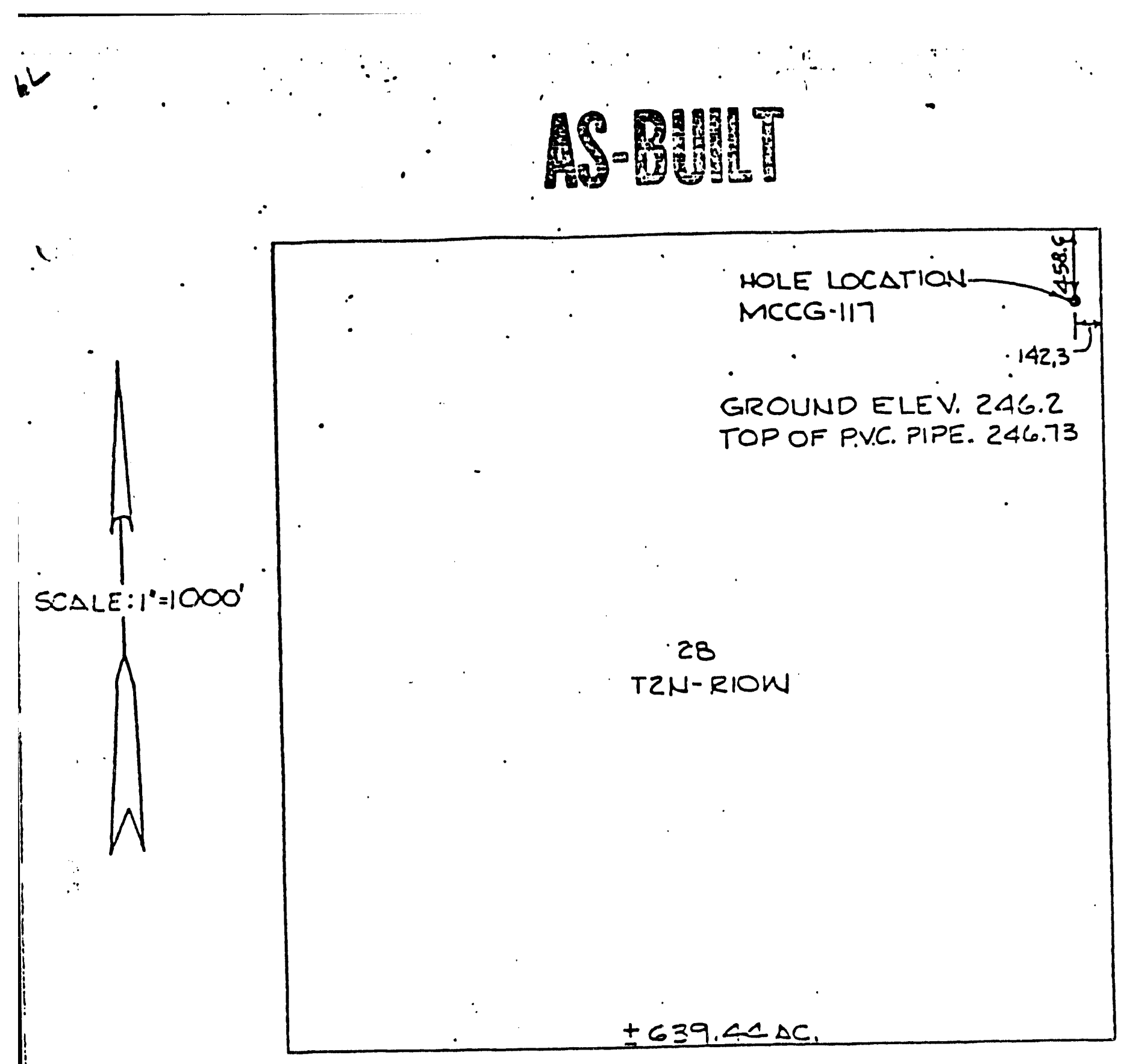



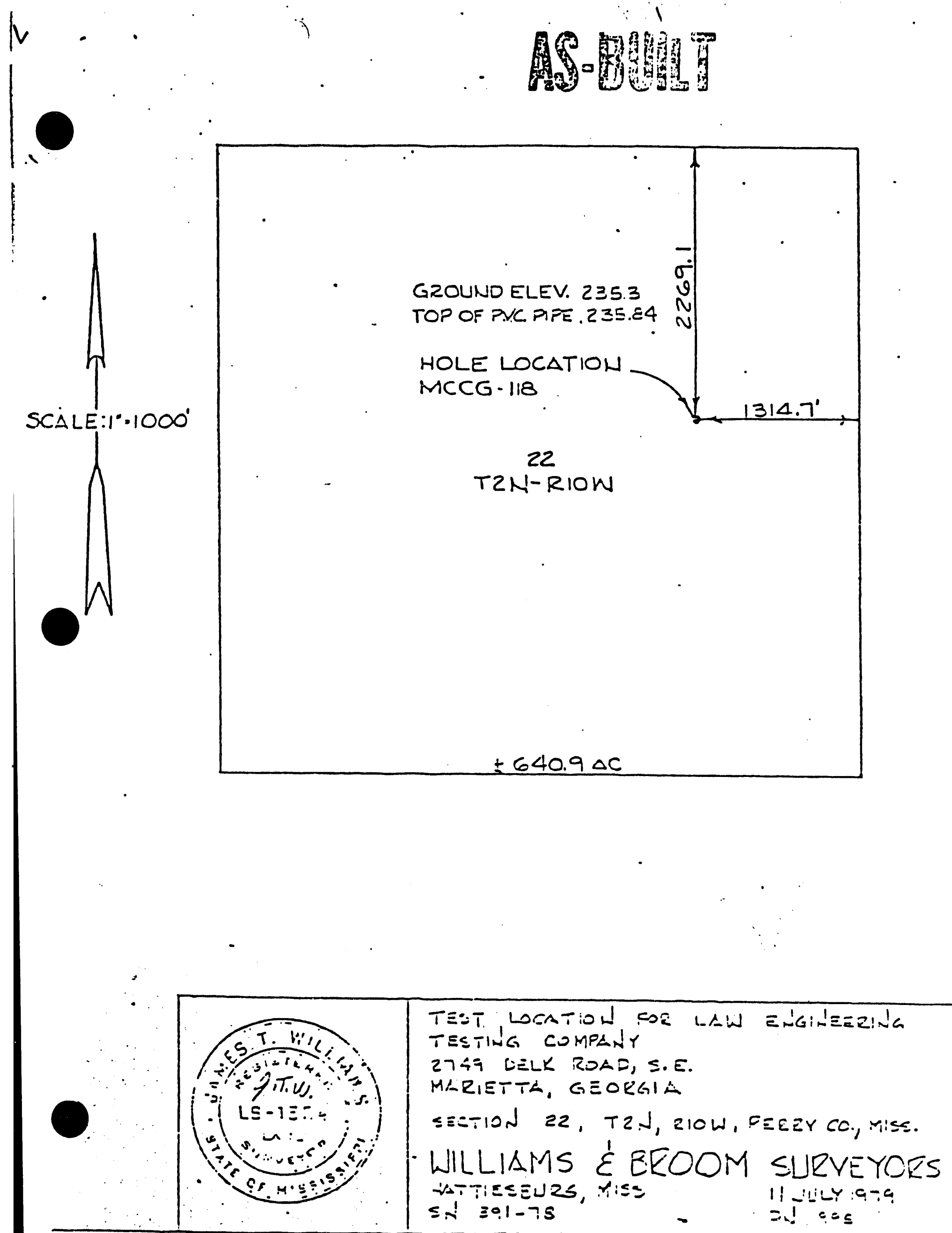

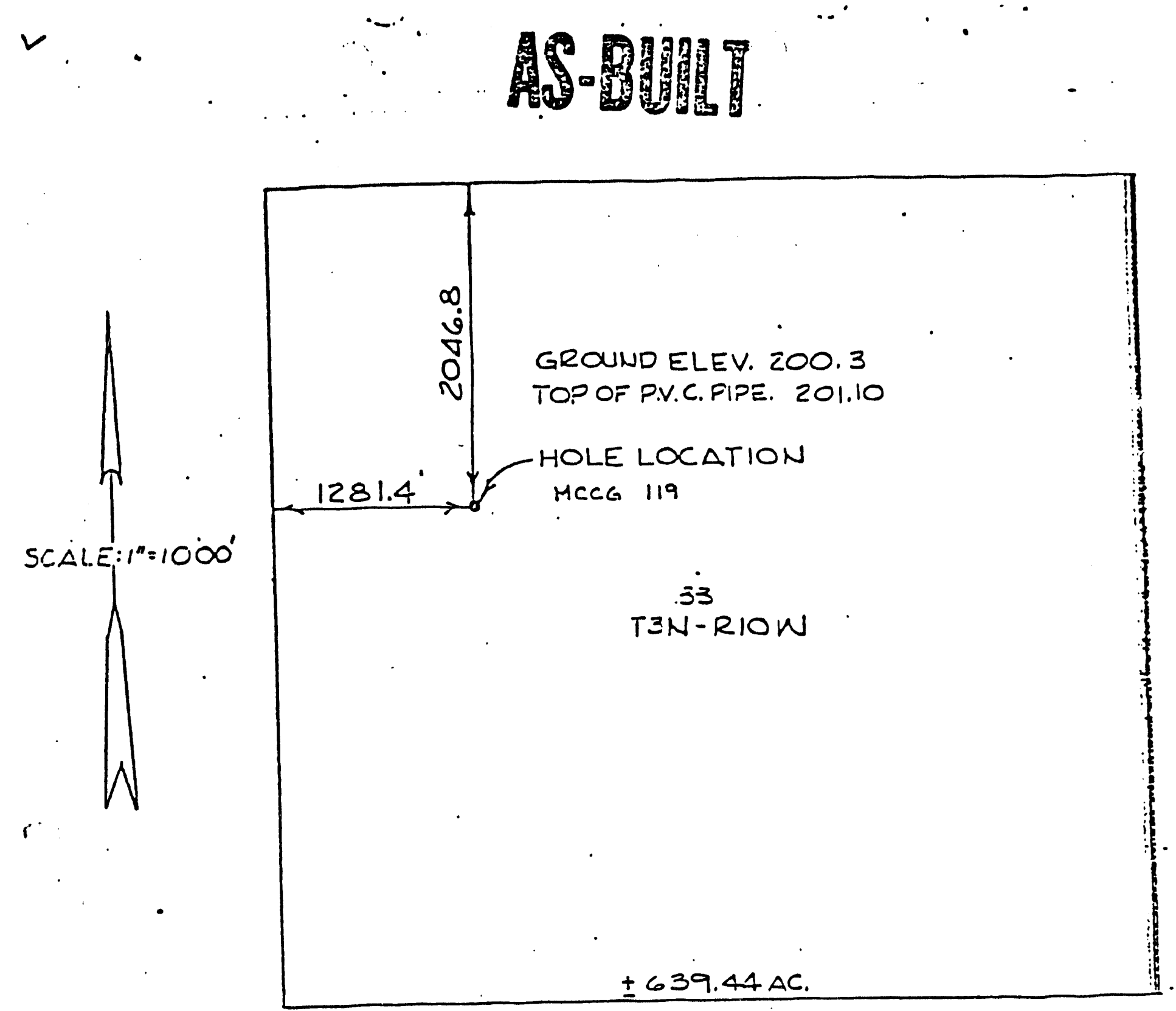


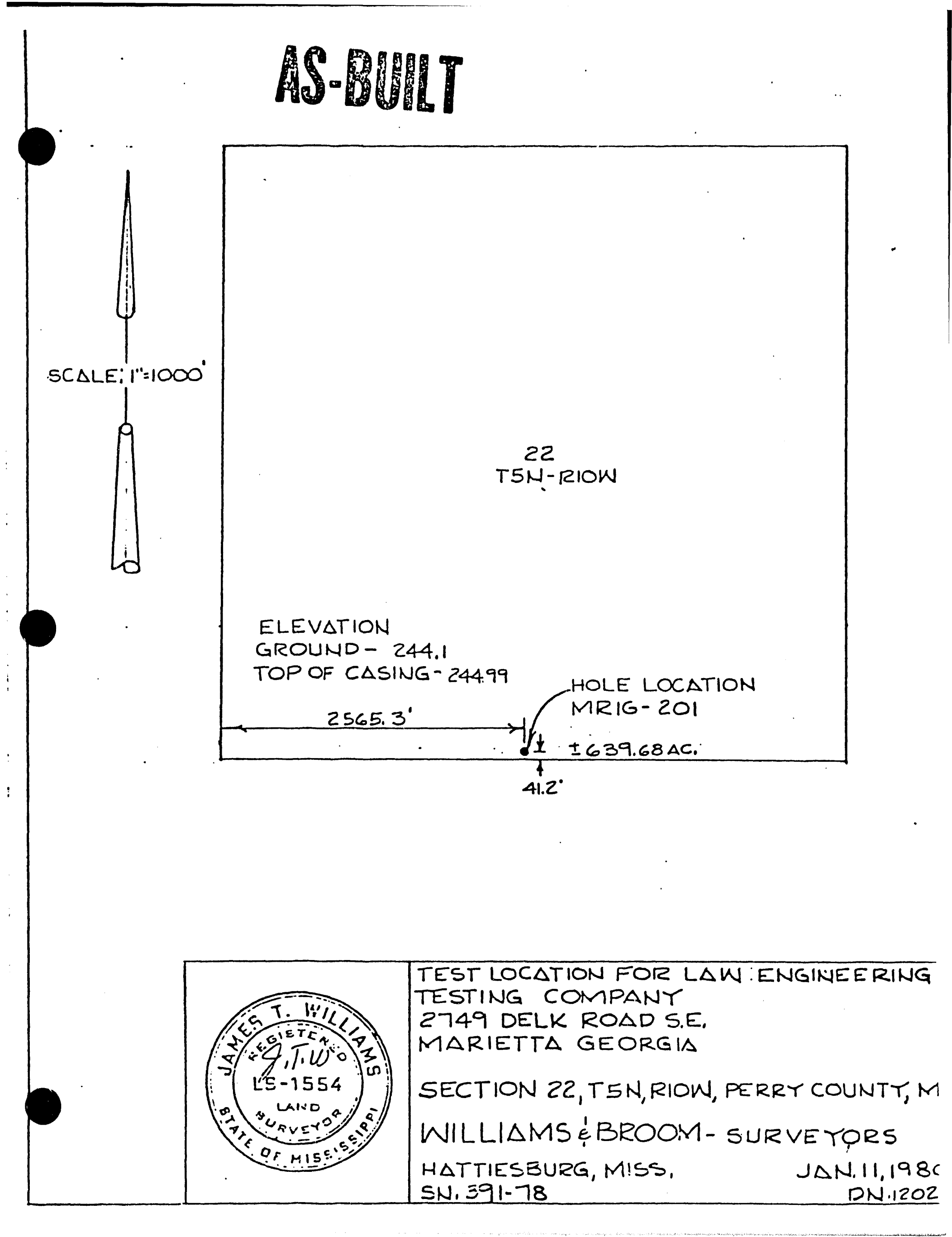




\section{AS-BULLT}

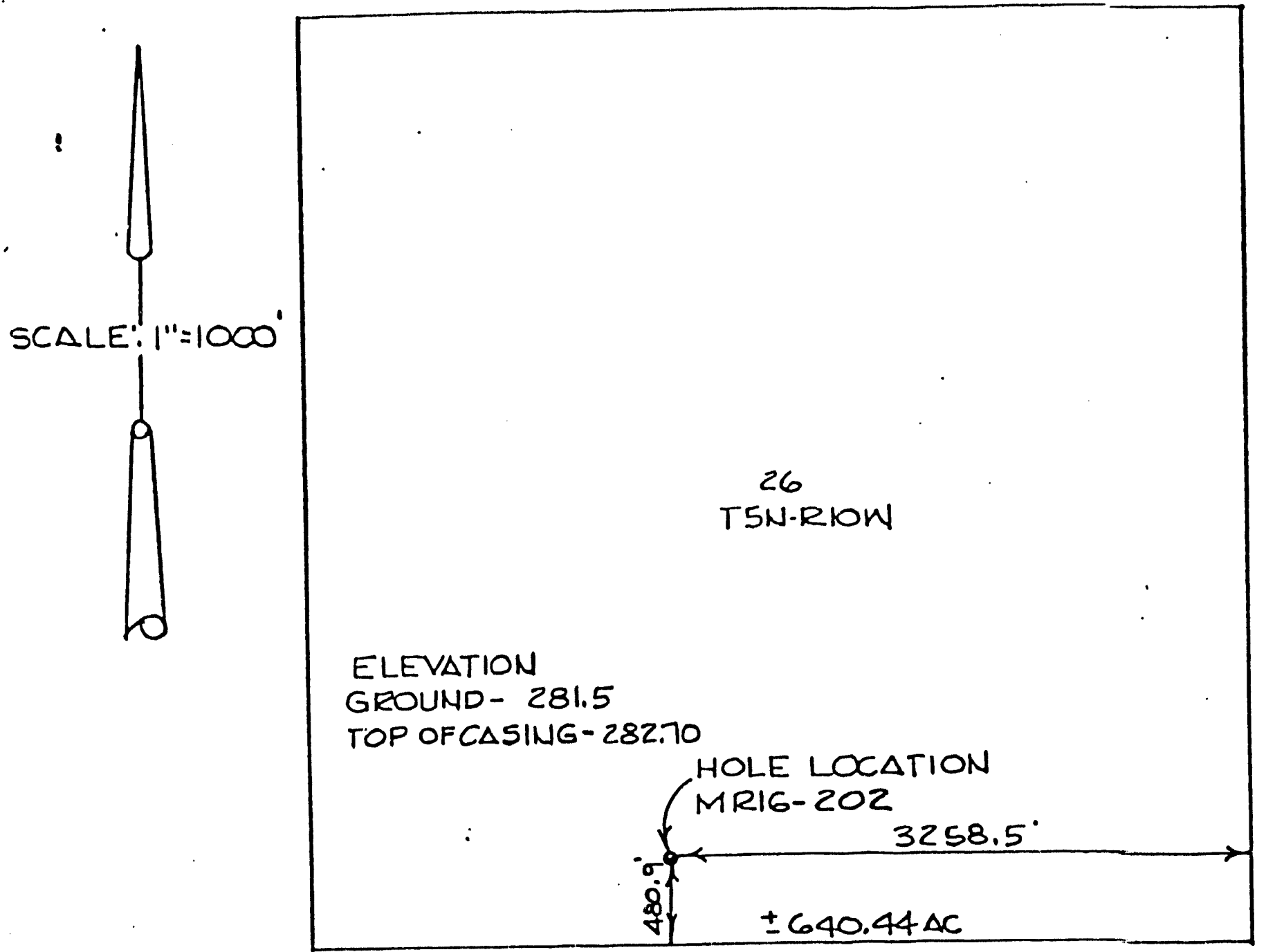

ELEVATION

GROUND - 281.5

TOP OFC $\triangle$ SING -282.70

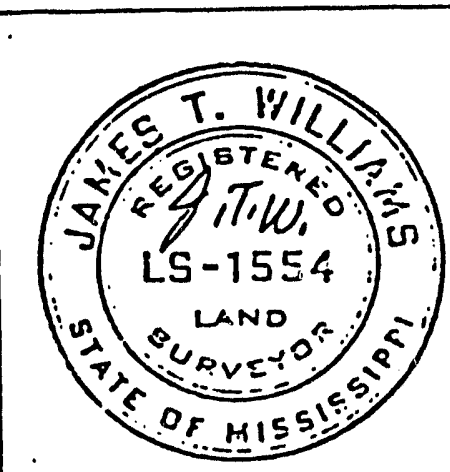

TEST LOCATION FOE

ENGEERING

TESTIJG COMPANY

2749 DELK ROAD S.E.

marietTA, GEORGIA

SECTION 26, TSN,RIOW, PERRY CO., MIS

KIILLIAMS \&' BROOM SLRVEYORS HaTTIESBURG, MISS. NOV 19, 1979 


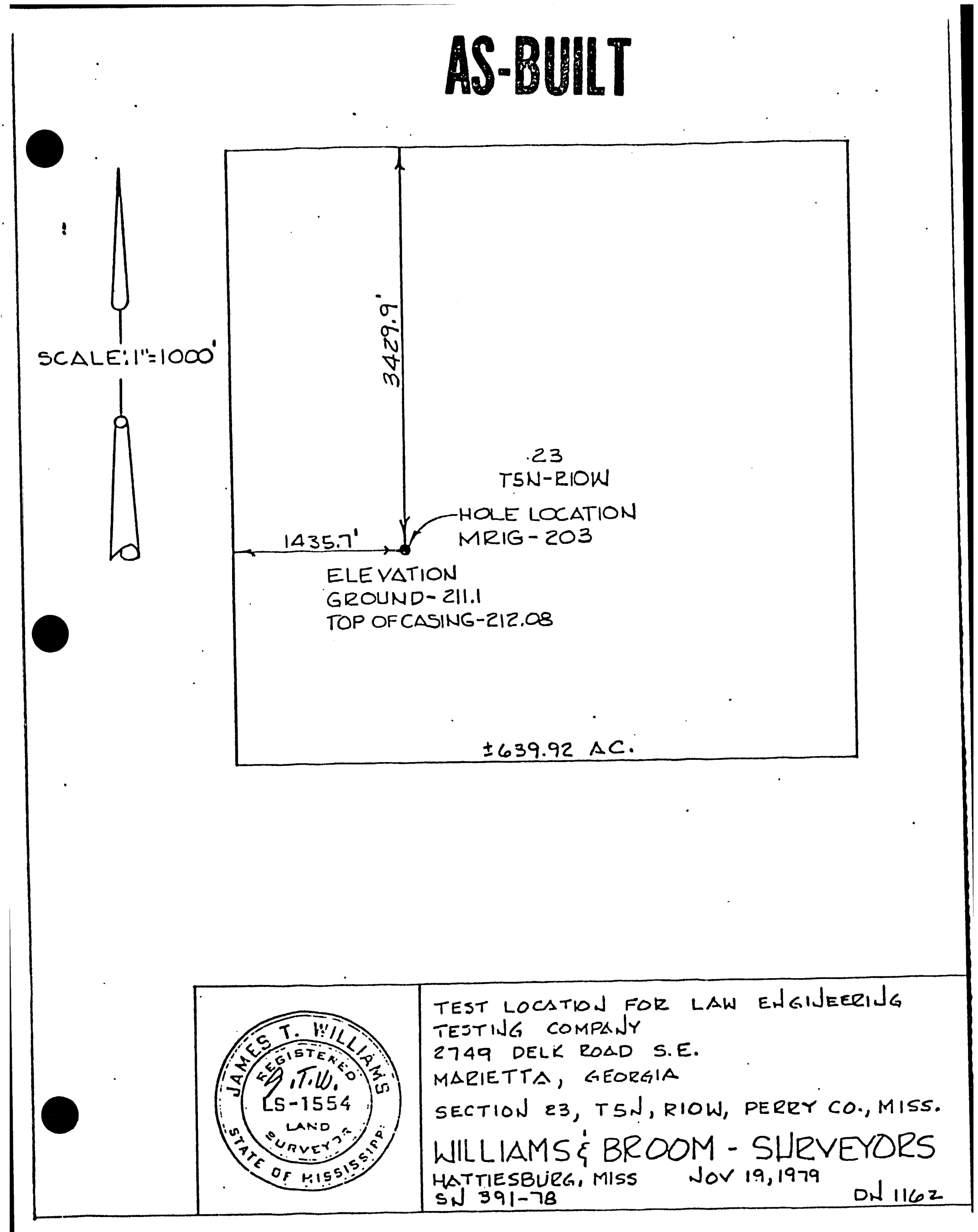




\section{AS-BULLT}
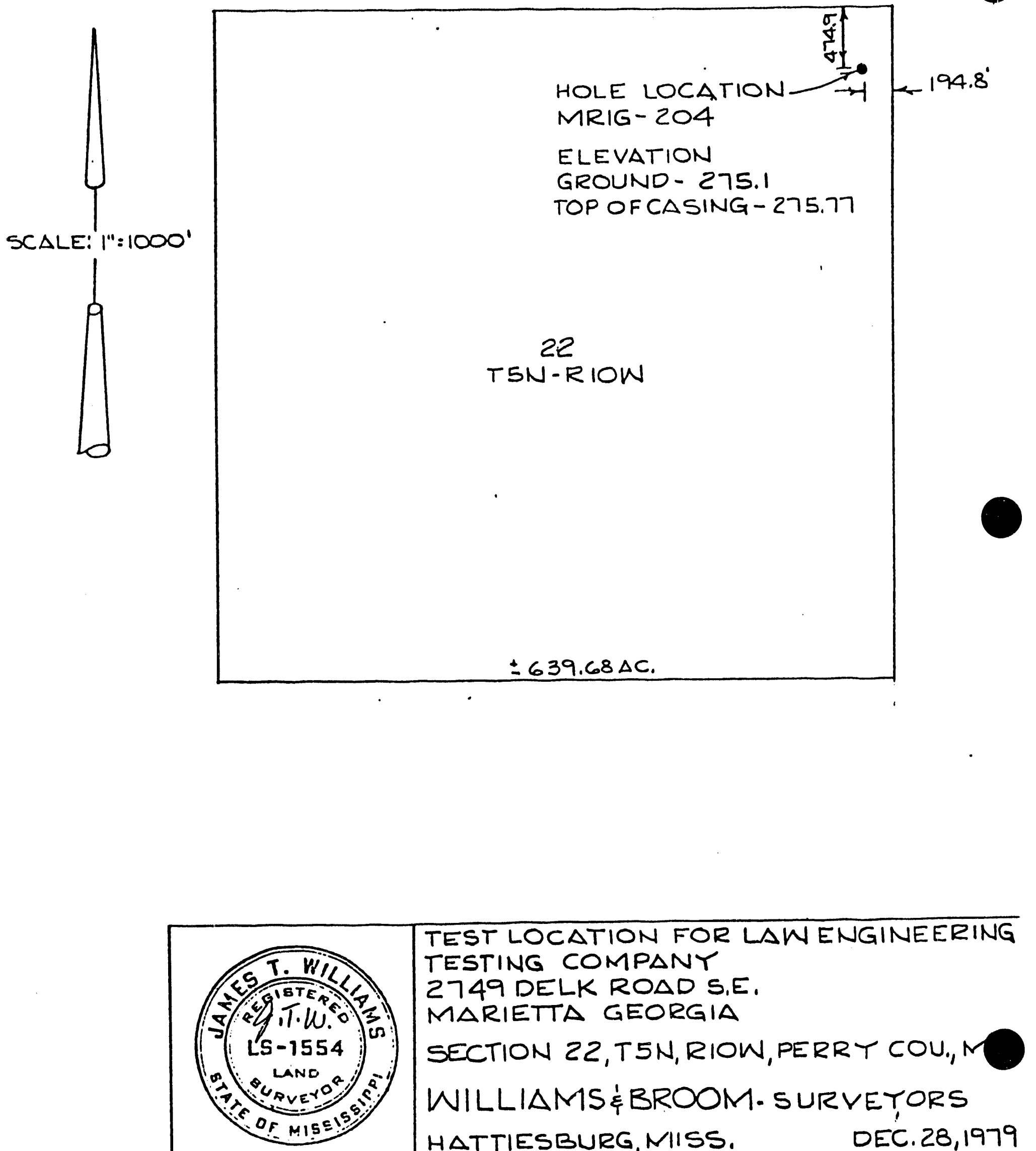

TEST LOCATION FOR LAWENGINEERING TESTING COMPANY

2749 DELK ROAD S.E.

MARIETTA GEORGIA

SECTION 22,T5N,RIOW,PERRY COU,M

WILLIAMIS\&BBROOM. SURVEYORS

HATTIESBURG, MIISS.

$S N .391-78$

DEC. 28,1979 


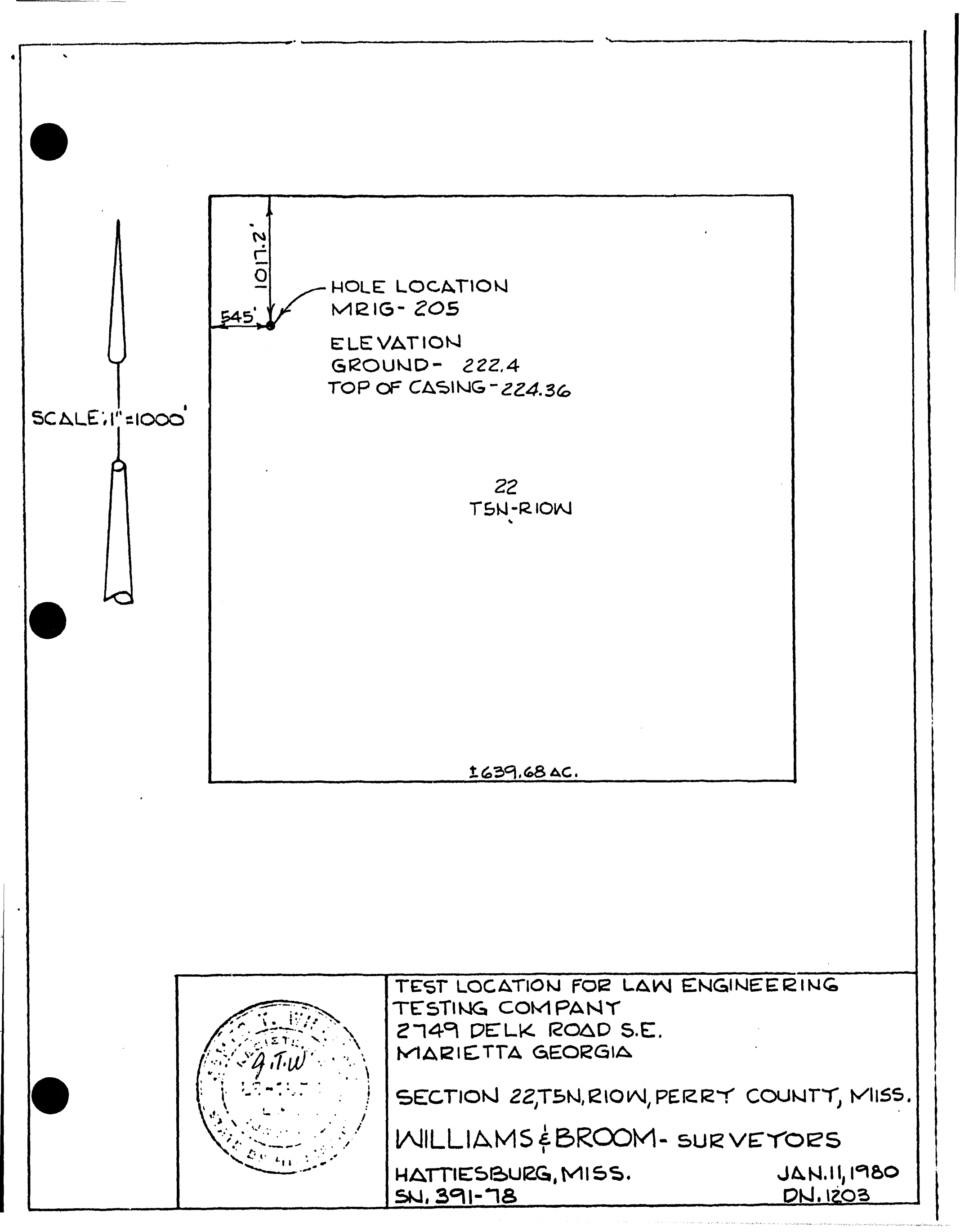




\section{AS-BUILT}
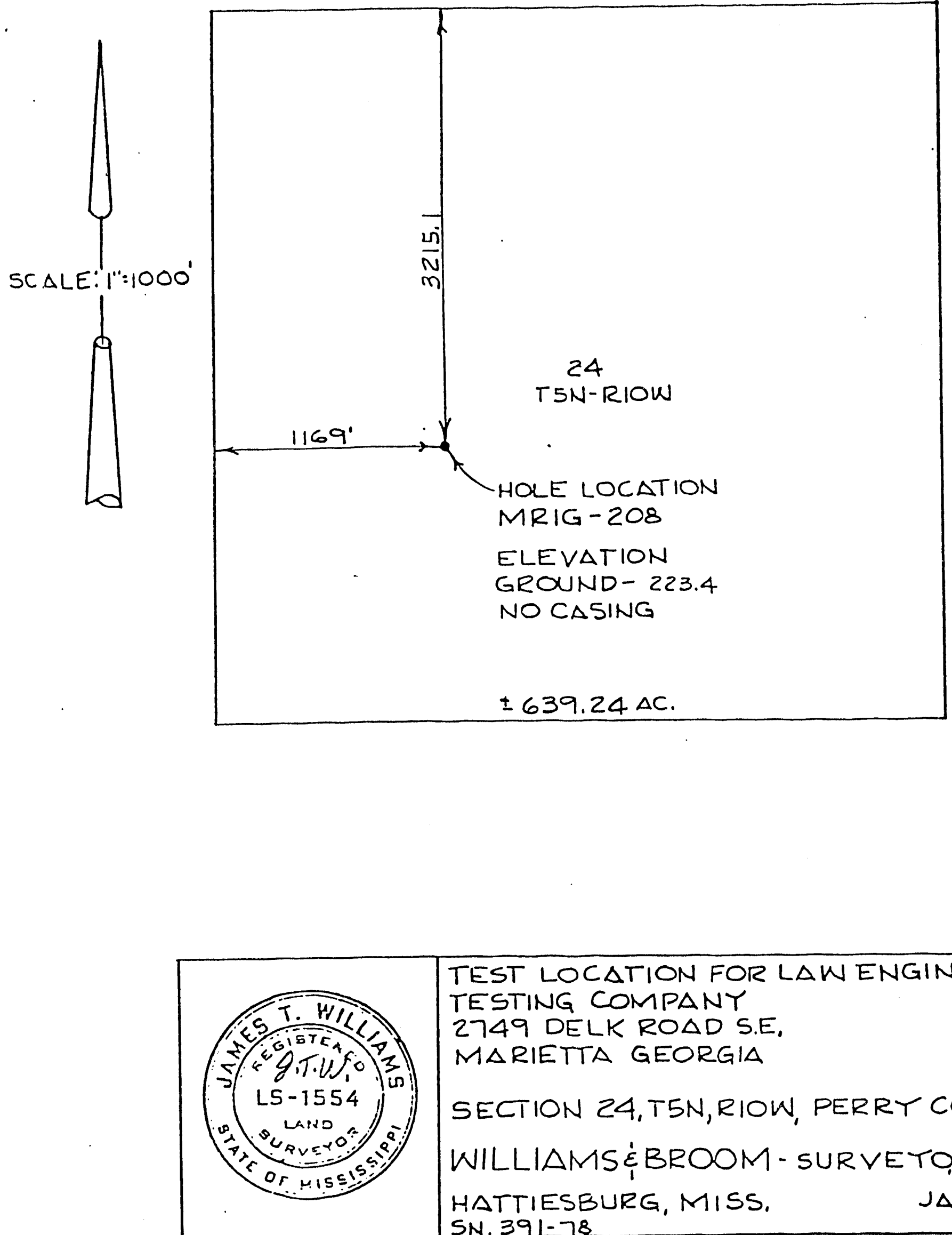

TEST LOCATION FOR LAW ENGINEERING TESTING COMPANY 2749 DELK ROAD S.E. MARIETTA GEORGIA

SECTION 24,T5N, RIOW, PERRY COUNTT, WILLIAMSĖBROOM - SURVETOPRS 


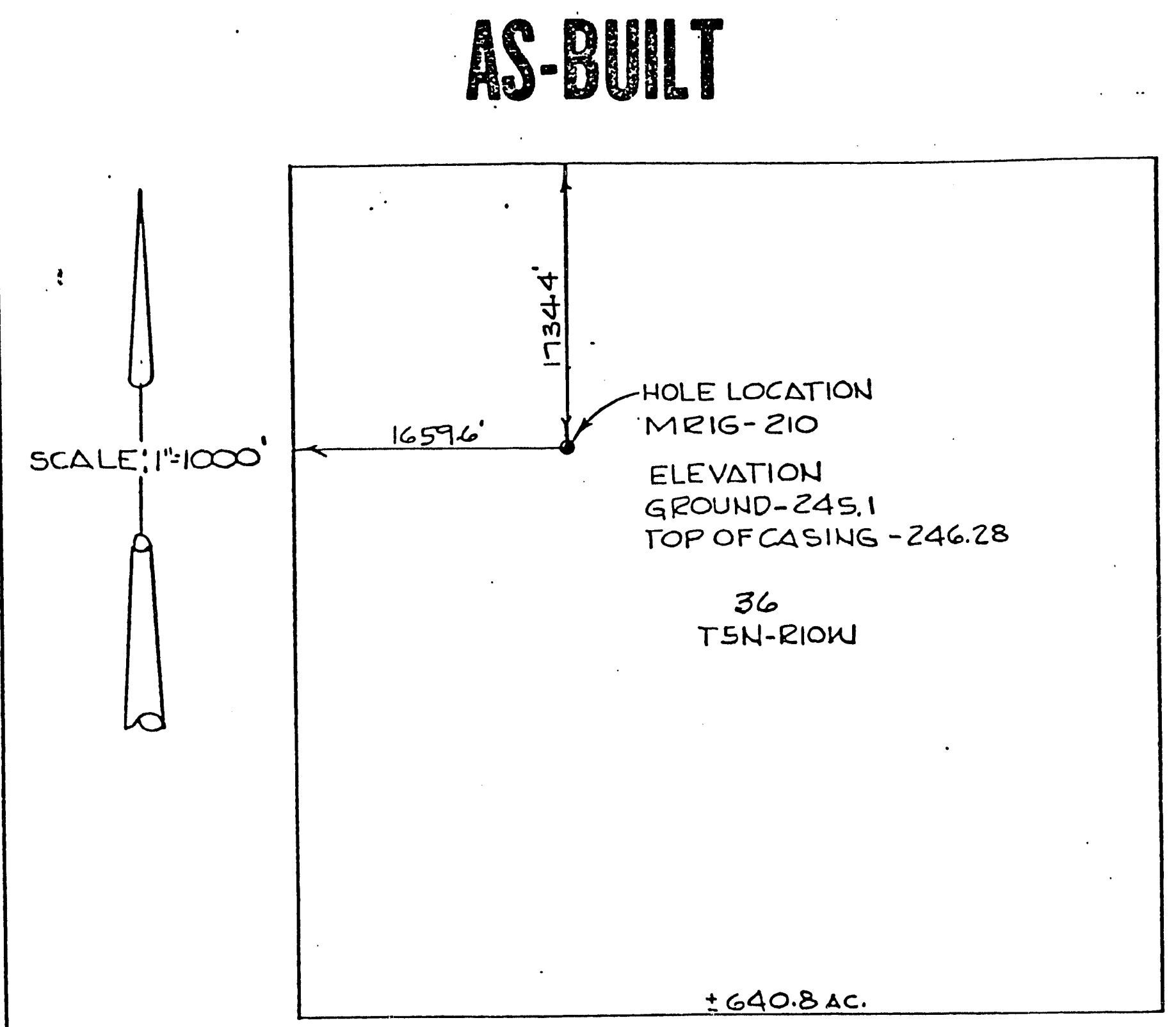

$\pm 640.8 \triangle C$

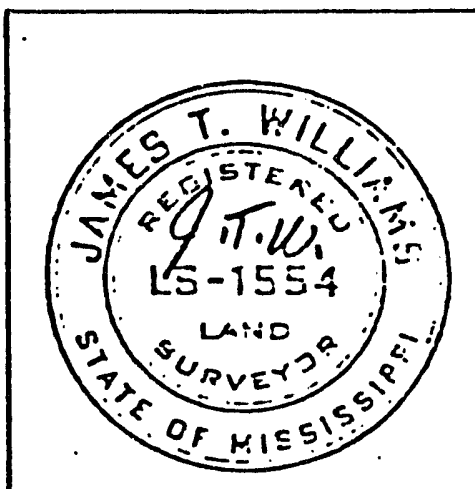

TEST LOCATION FOR LAW ENGINEERING TESTING COMPANY 2ᄀ49 DELK ROAD S.E. MARIETTA, GEORGIA SECTION 36, TSN, RIOW, PERRY CO., MISS. WILLIAMS É BROOM SURVEYORS HATTIESGURG, MISS. SN $391-78$ 

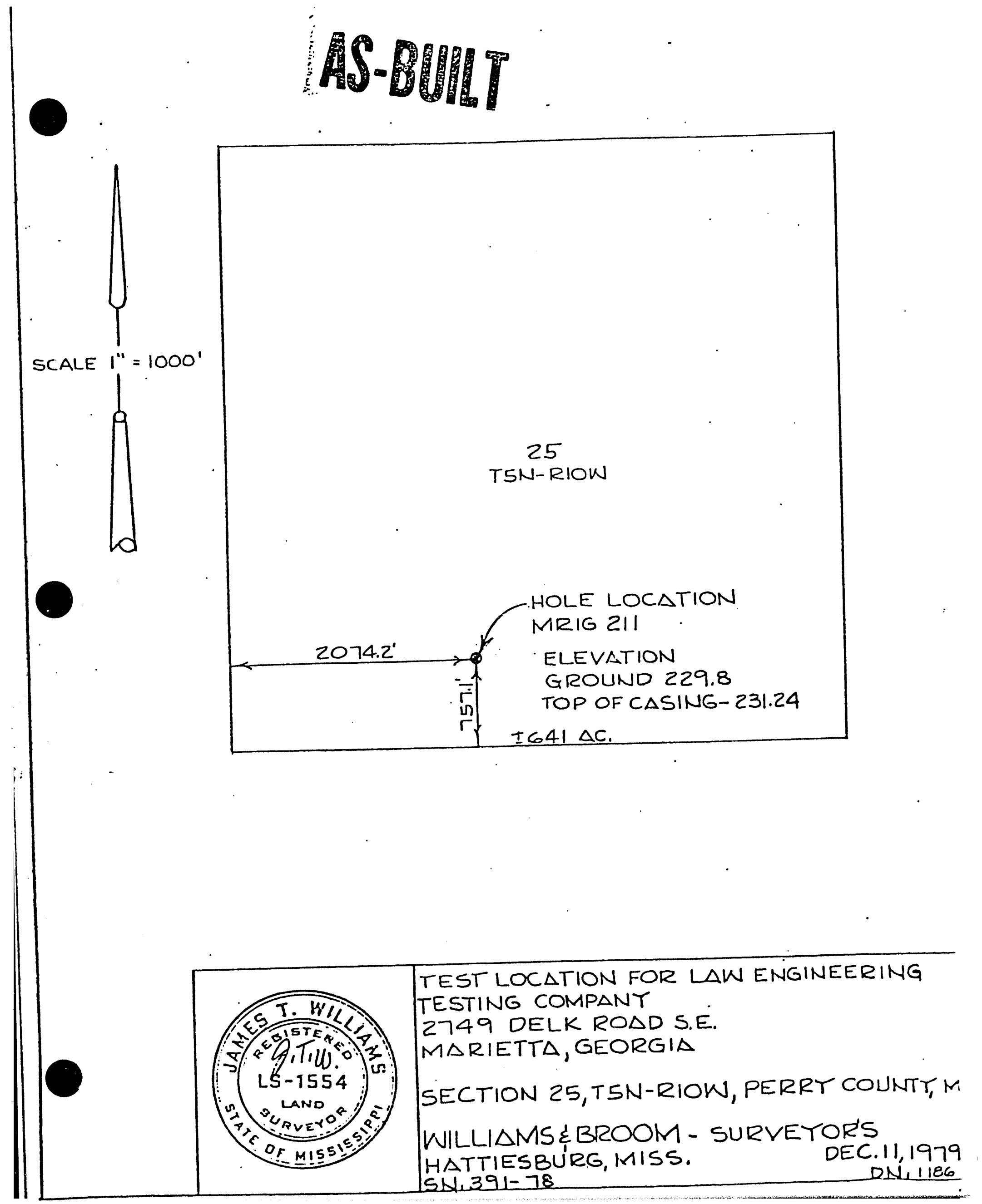


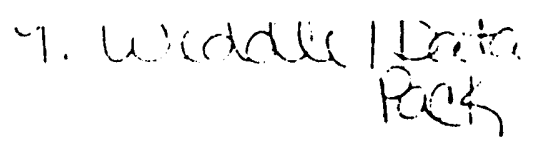
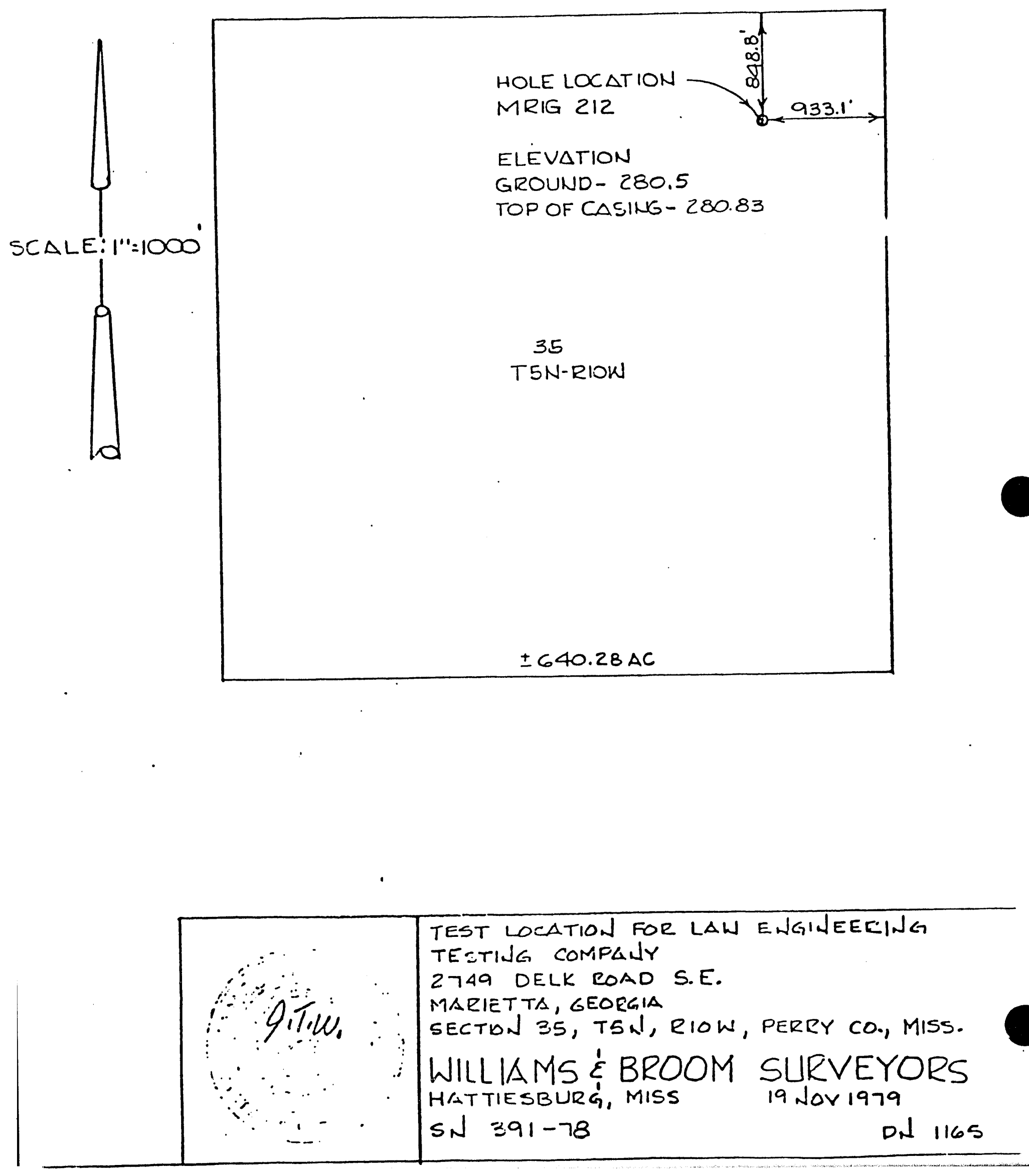


\section{6 \\ T5N-RIOW}

$\pm 640.8 \mathrm{AC}$

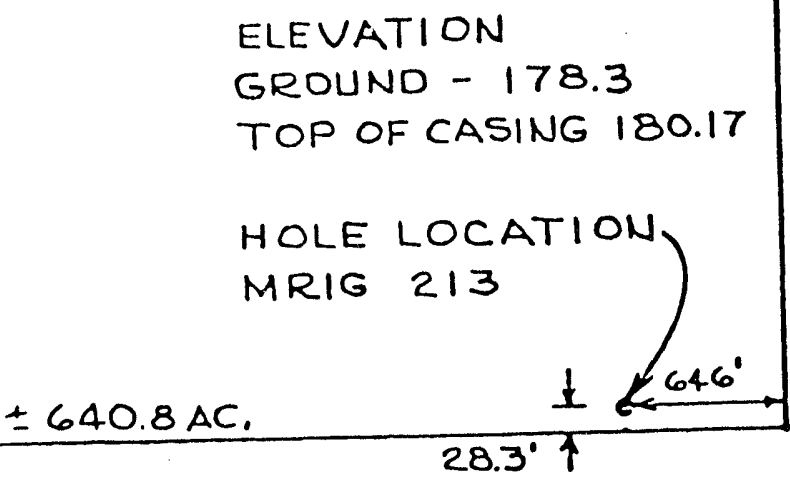

SCALE $I^{\prime \prime}=1000^{\circ}$

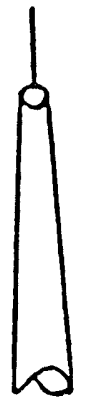

TEST LOCATION FOR LAW ENGINEERING TESTING COMPANY

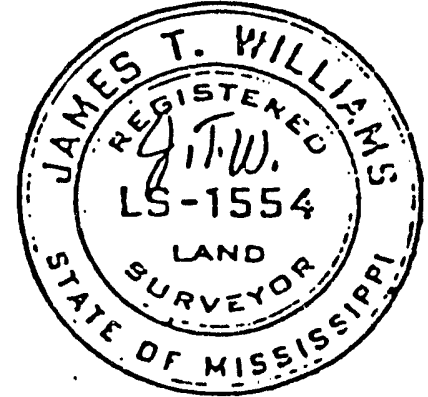

2749 DELK ROAD S.E.

MARIETTA, GEORGIA

SECTION 36,TSN, RIOW, PERRY COU., MS WILLIAMS E BROOM - SURVEYORS HATTIESBURG, MS DEC. 10, 1979 

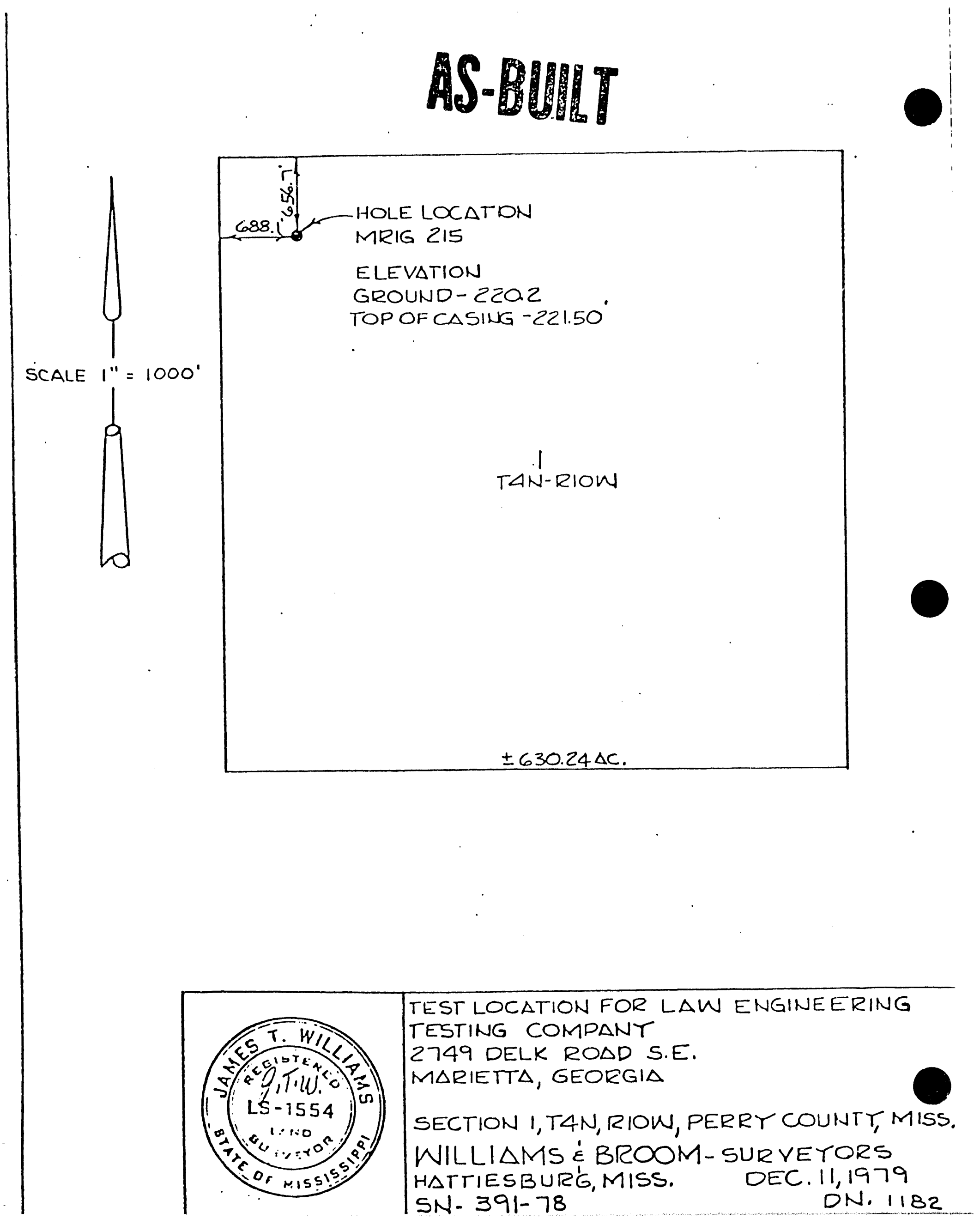

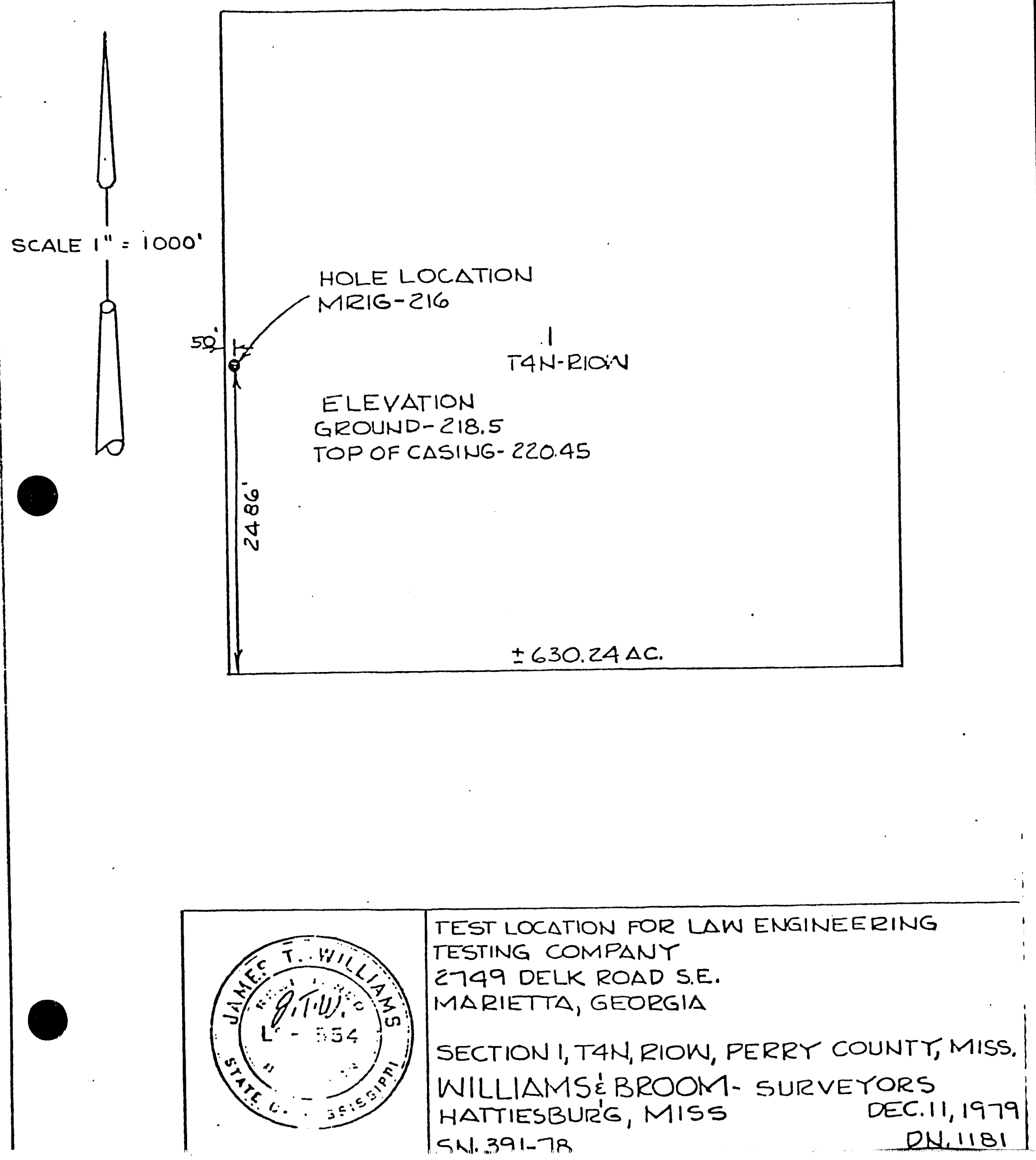

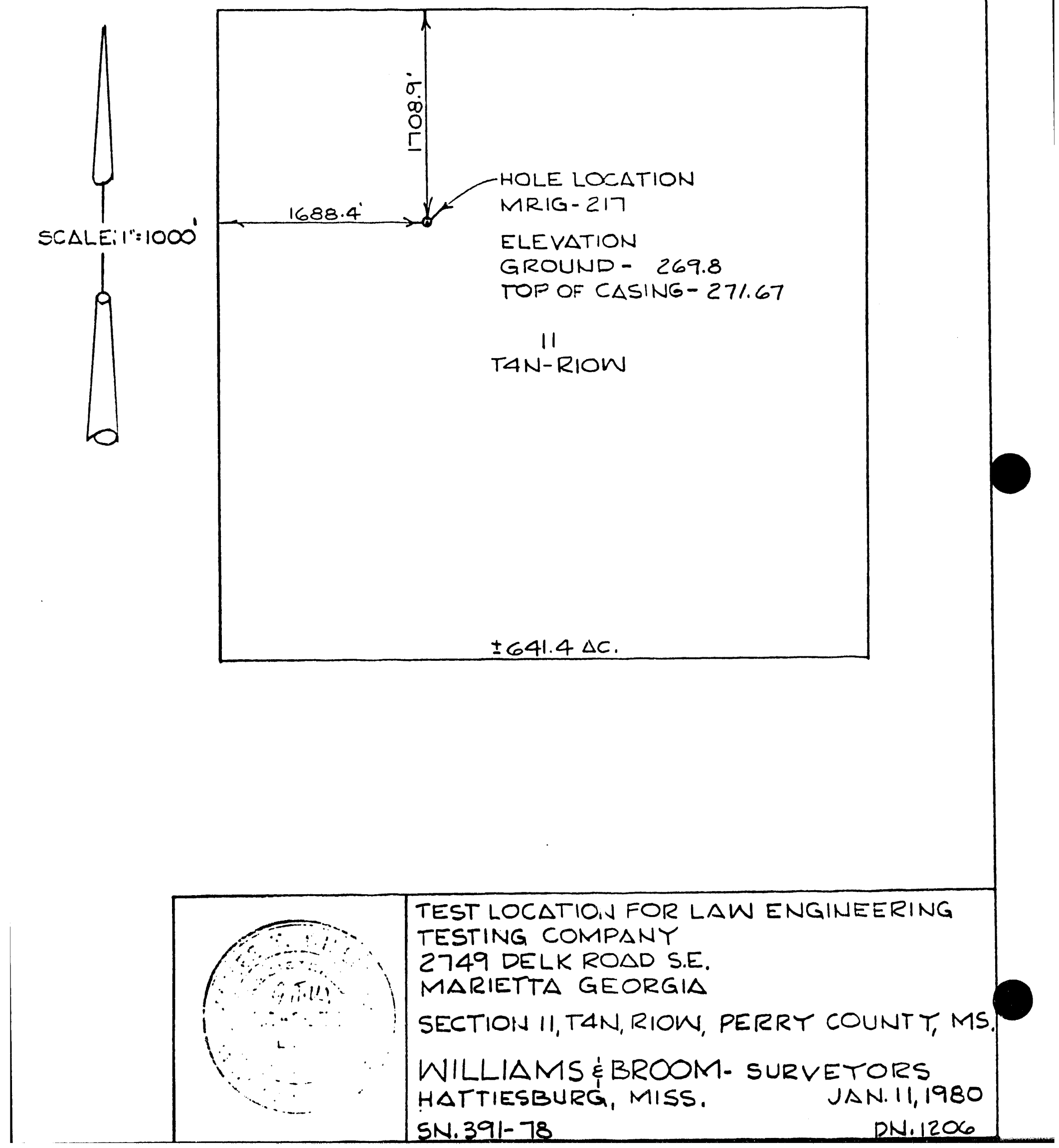


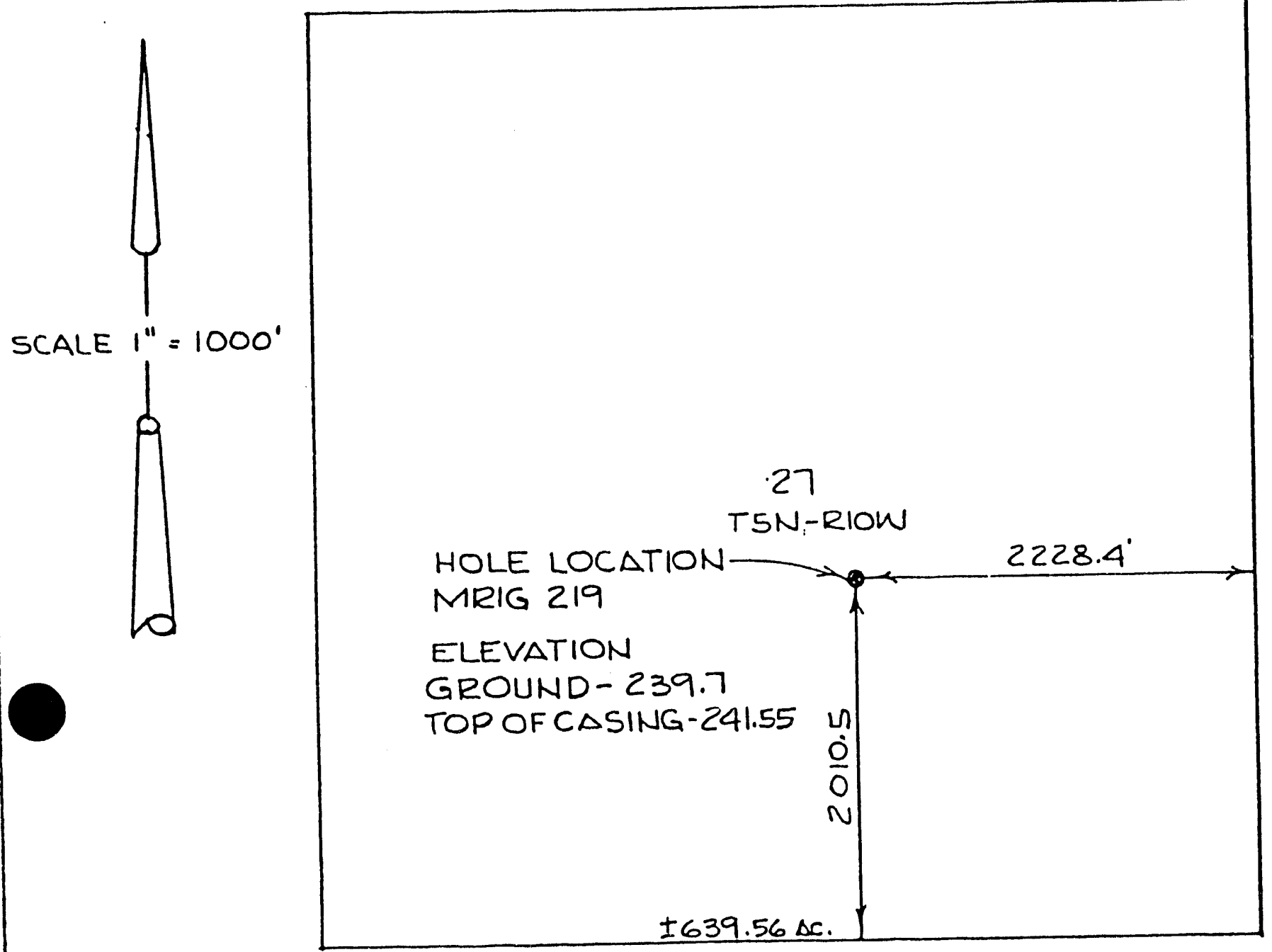

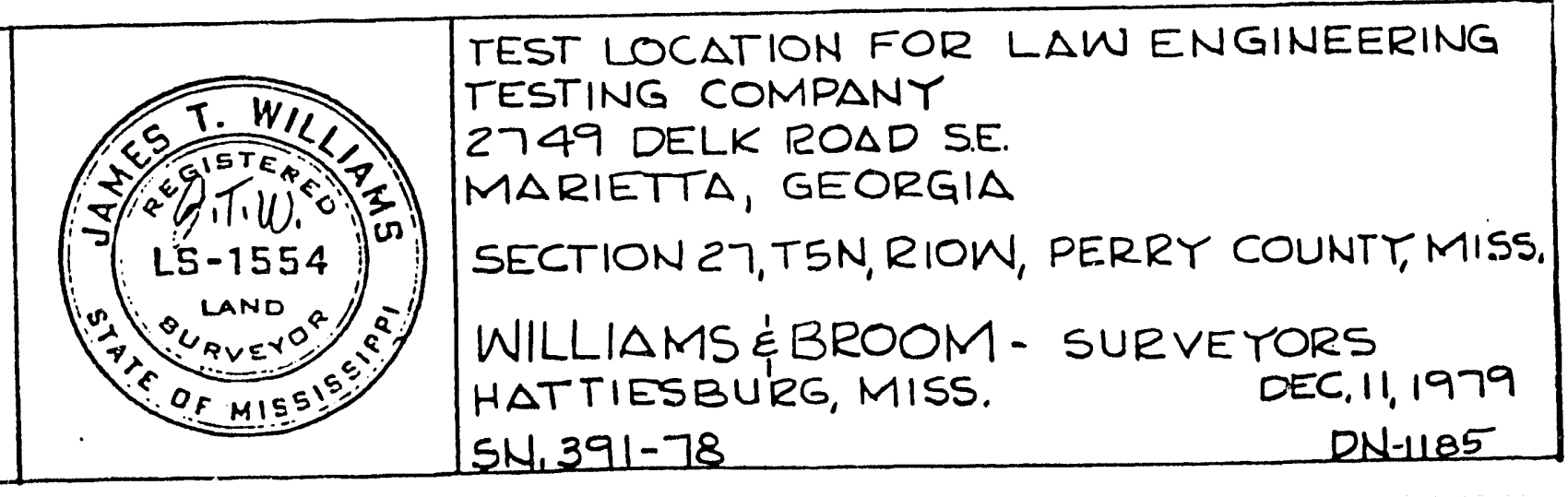




\section{AS-BULLT}

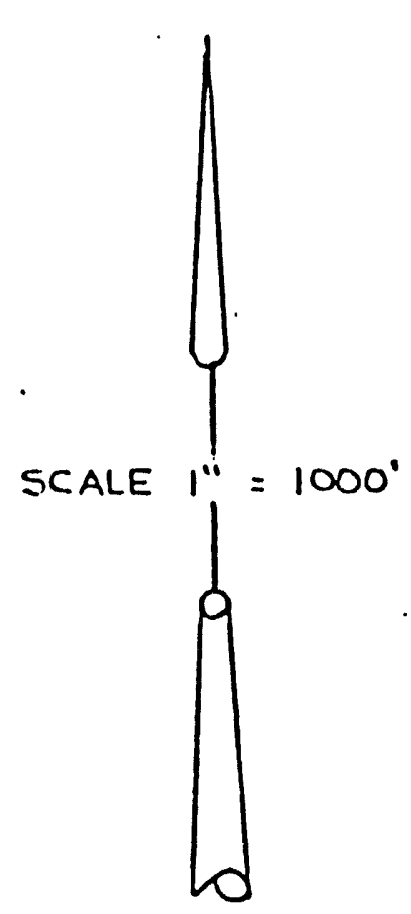

\section{T4N-RIOW}

ELEVATION

GROUND - 215.7

TOP OF CASING - 216.68
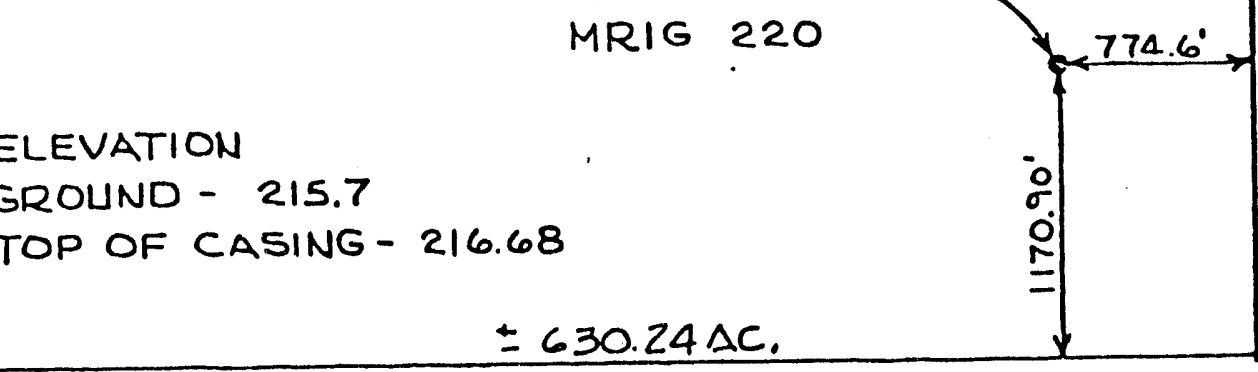

$\pm 630.24 \Delta C$.

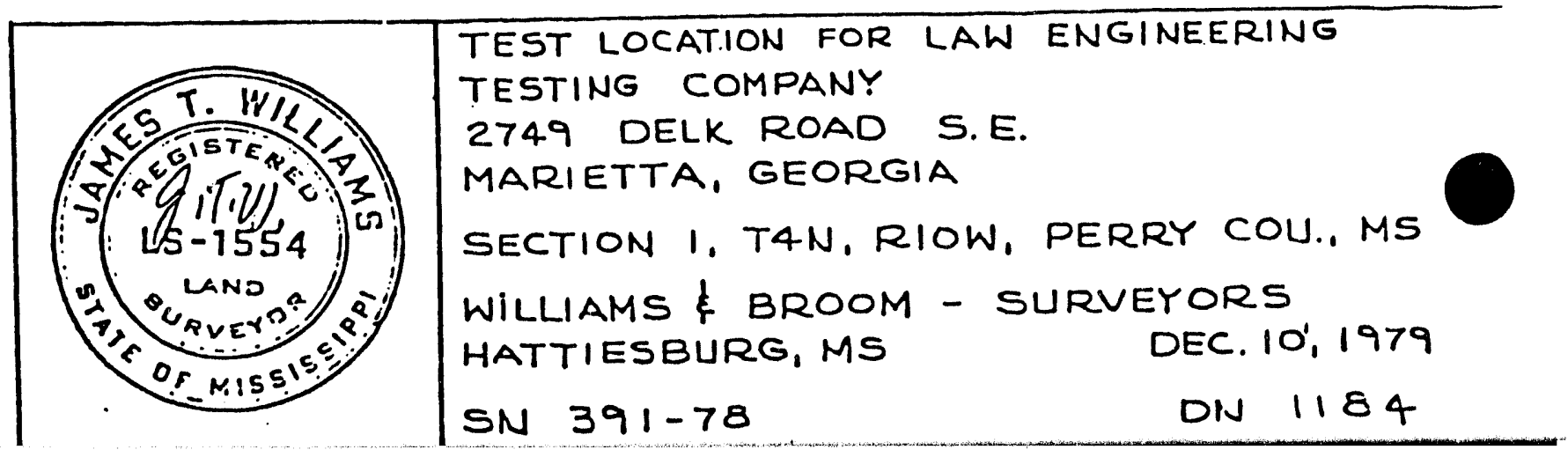




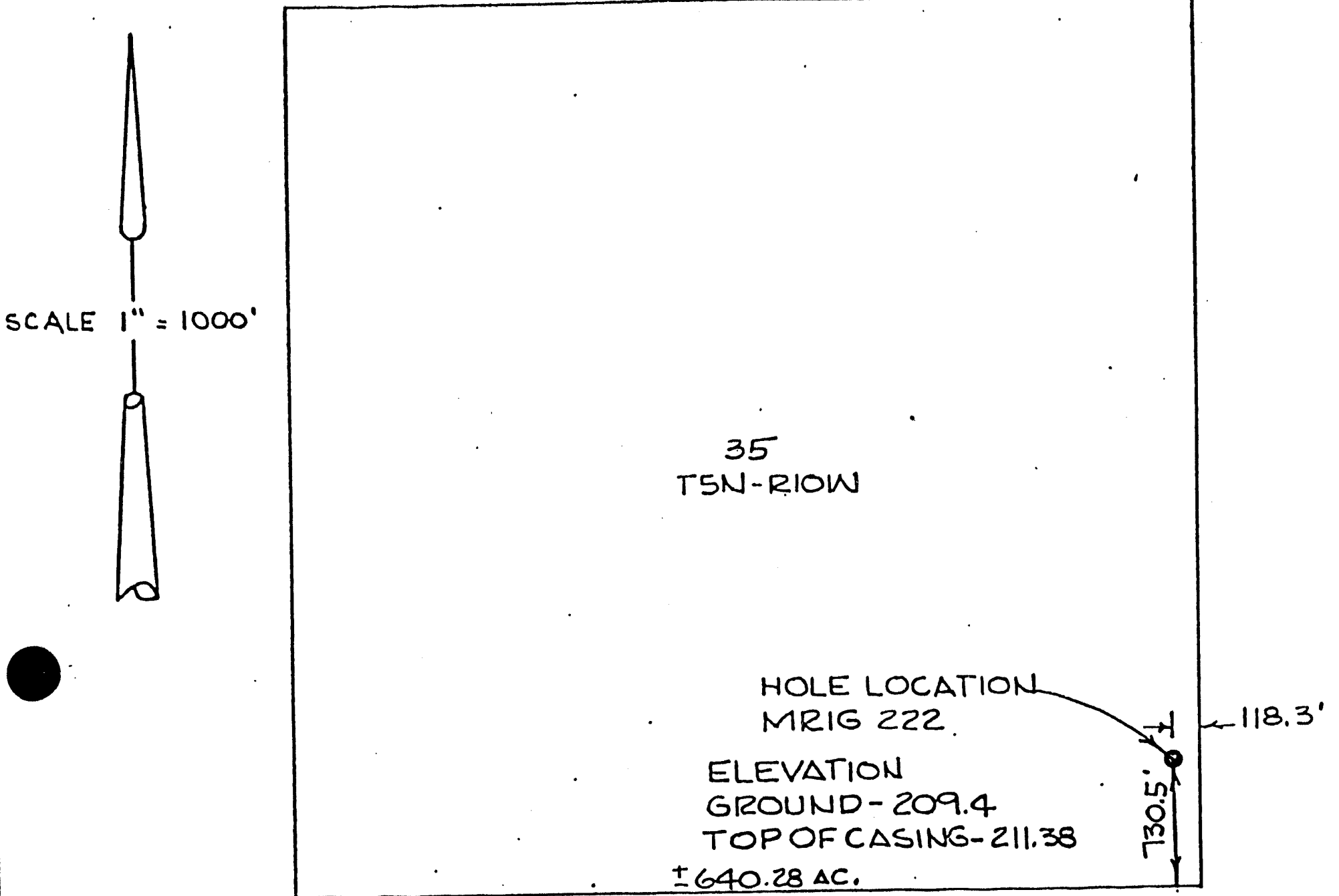

$\pm 640.28 \Delta C$.

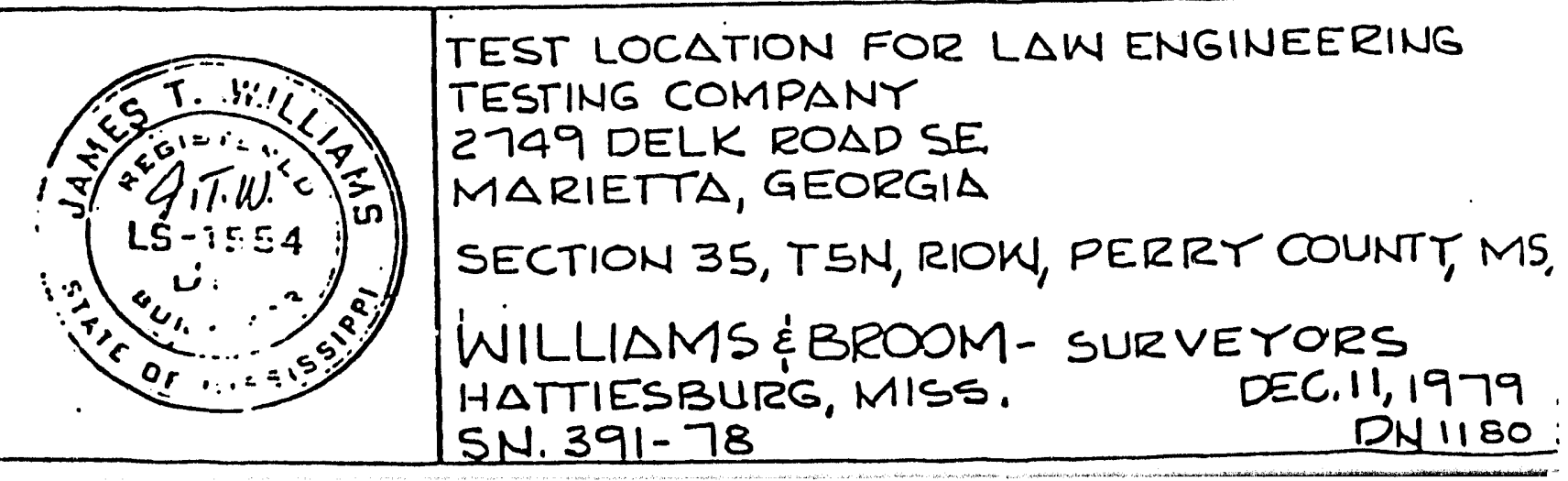



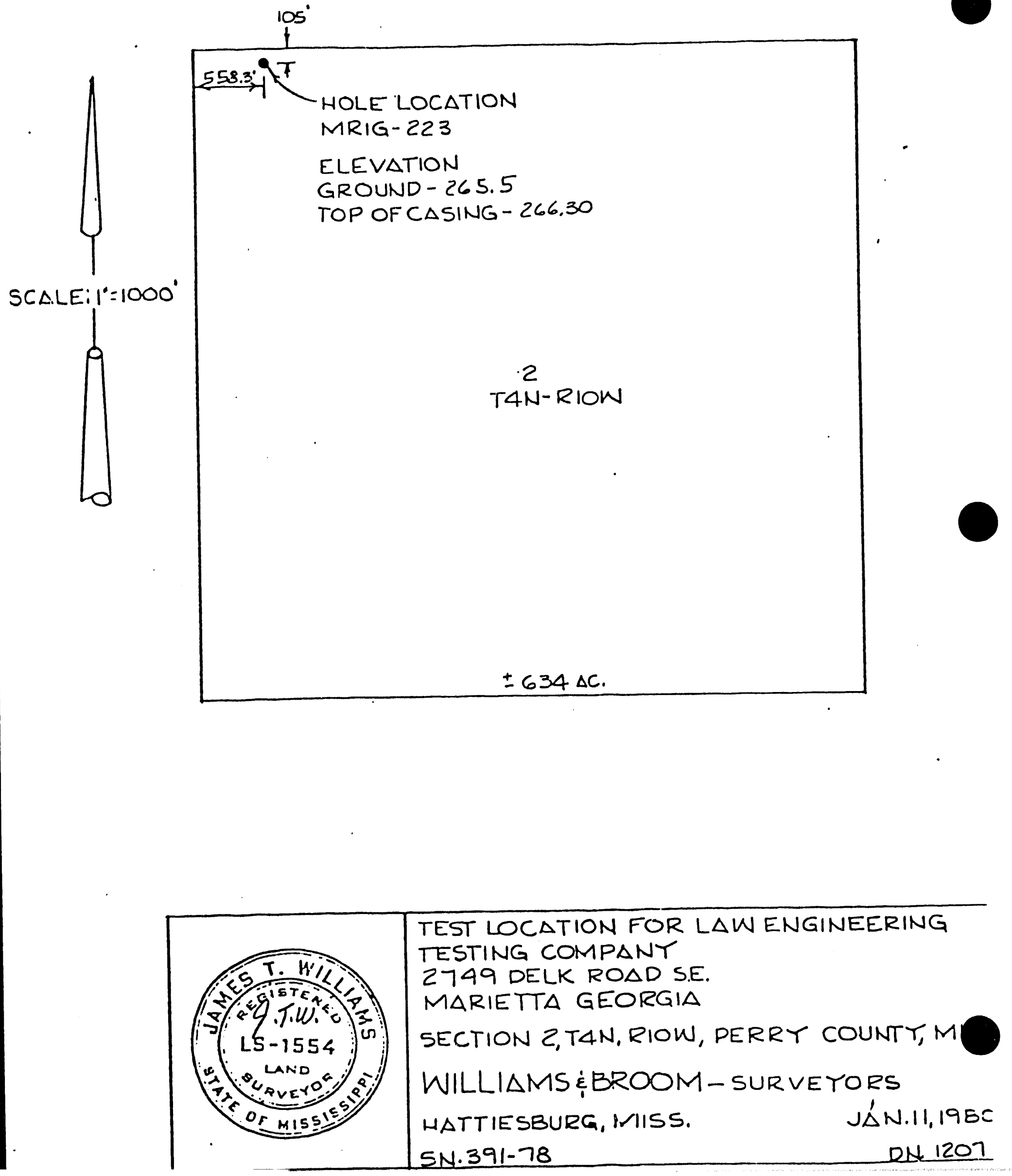
AS BUILT 


\section{AS-BULLT}

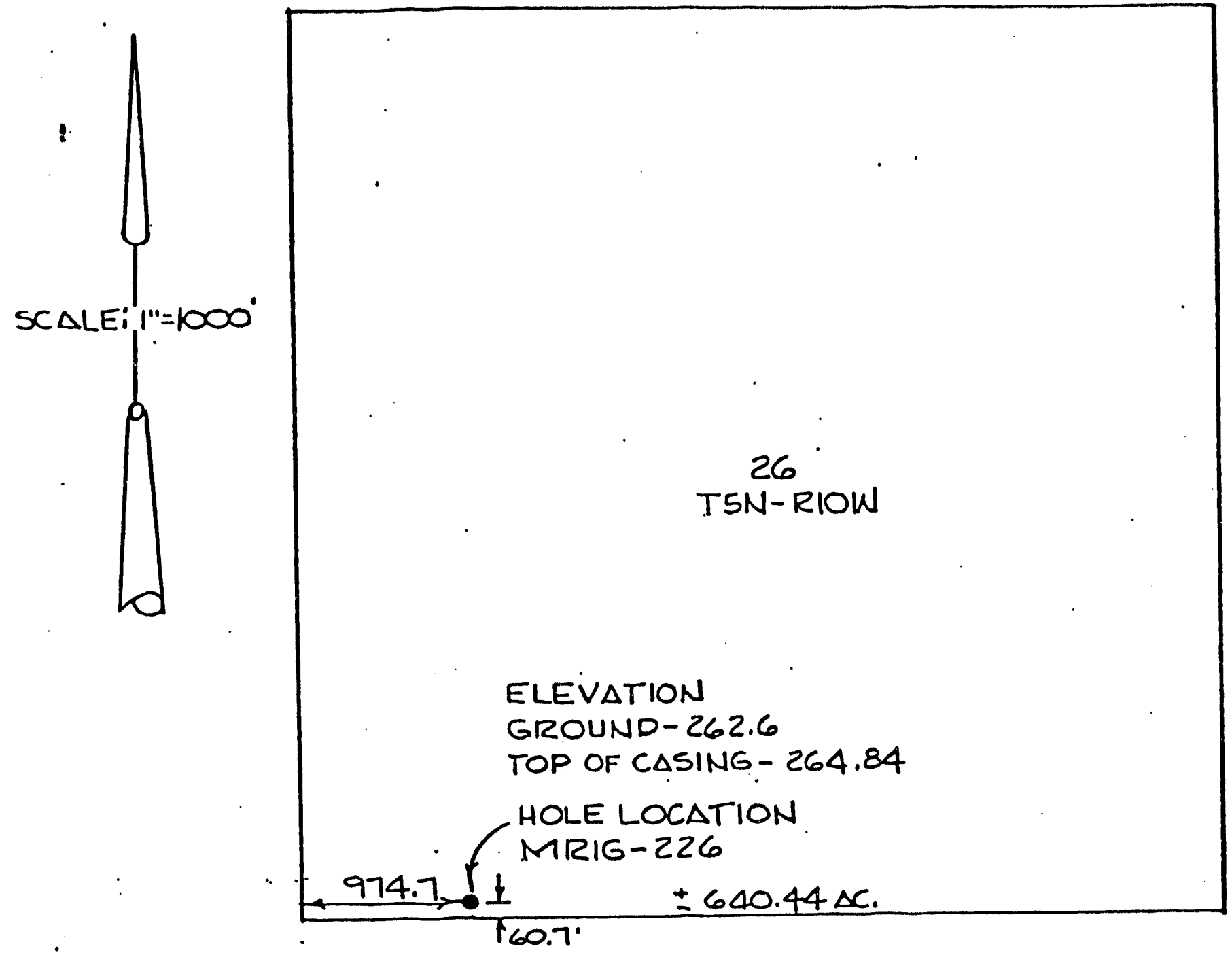

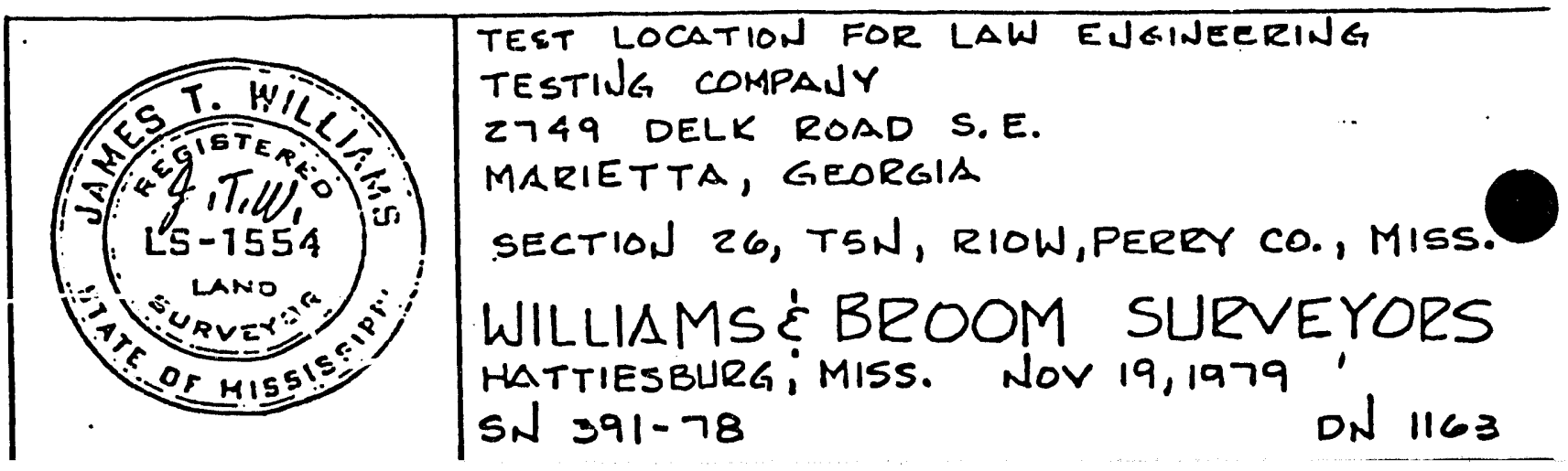



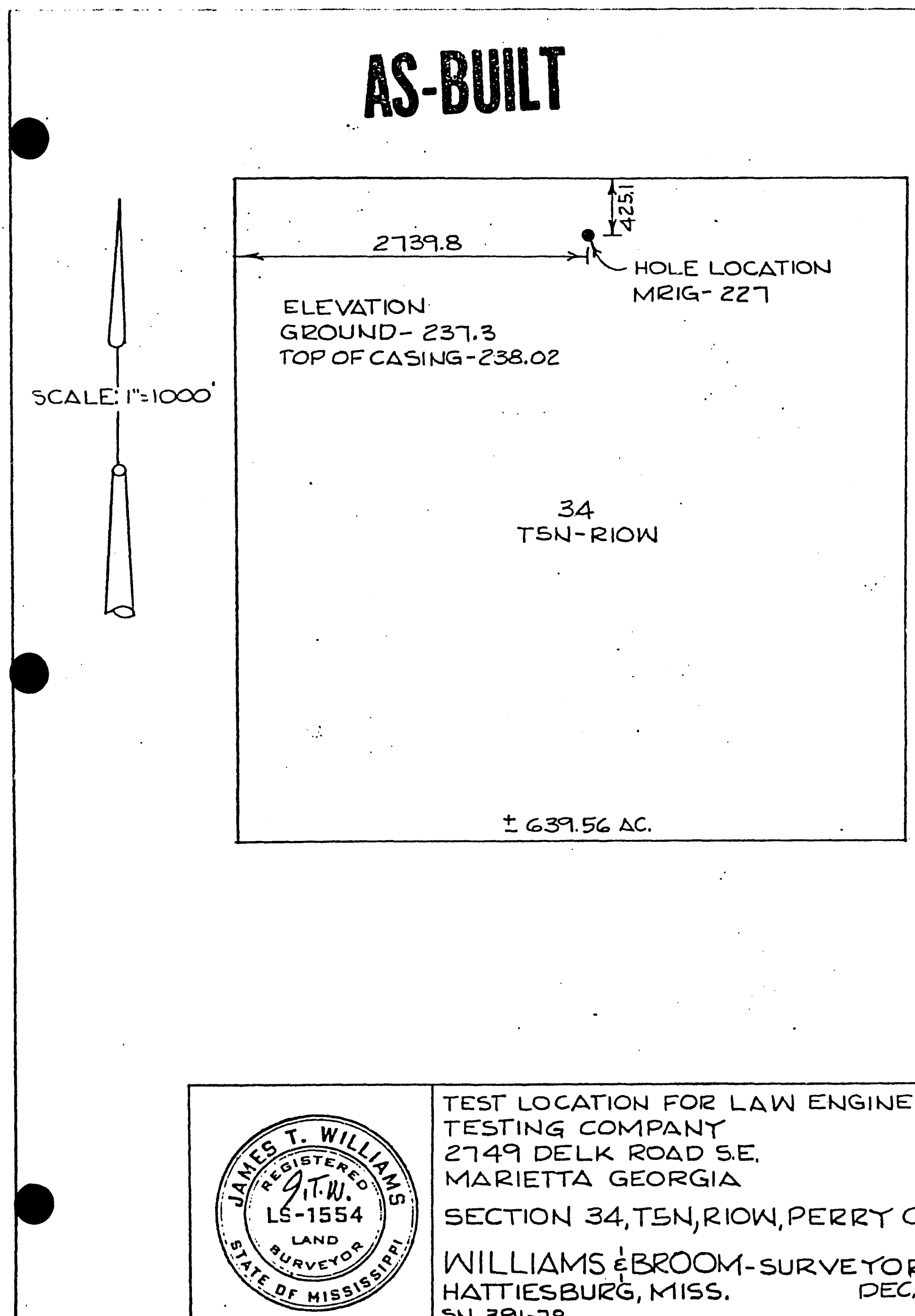

TEST LOCATION FOR LAW ENGINEERING TESTING COMPANY 2749 DELK ROAD S.E. MARIETTA GEORGIA SECTION 34, TSN, RIOW, PERRT COU, MISS WILLIAMS ÉBROOM-SURVETORS HATTIESBURG, MISS. $S N .391-78$ DEC. 28, 1979 DN1195 


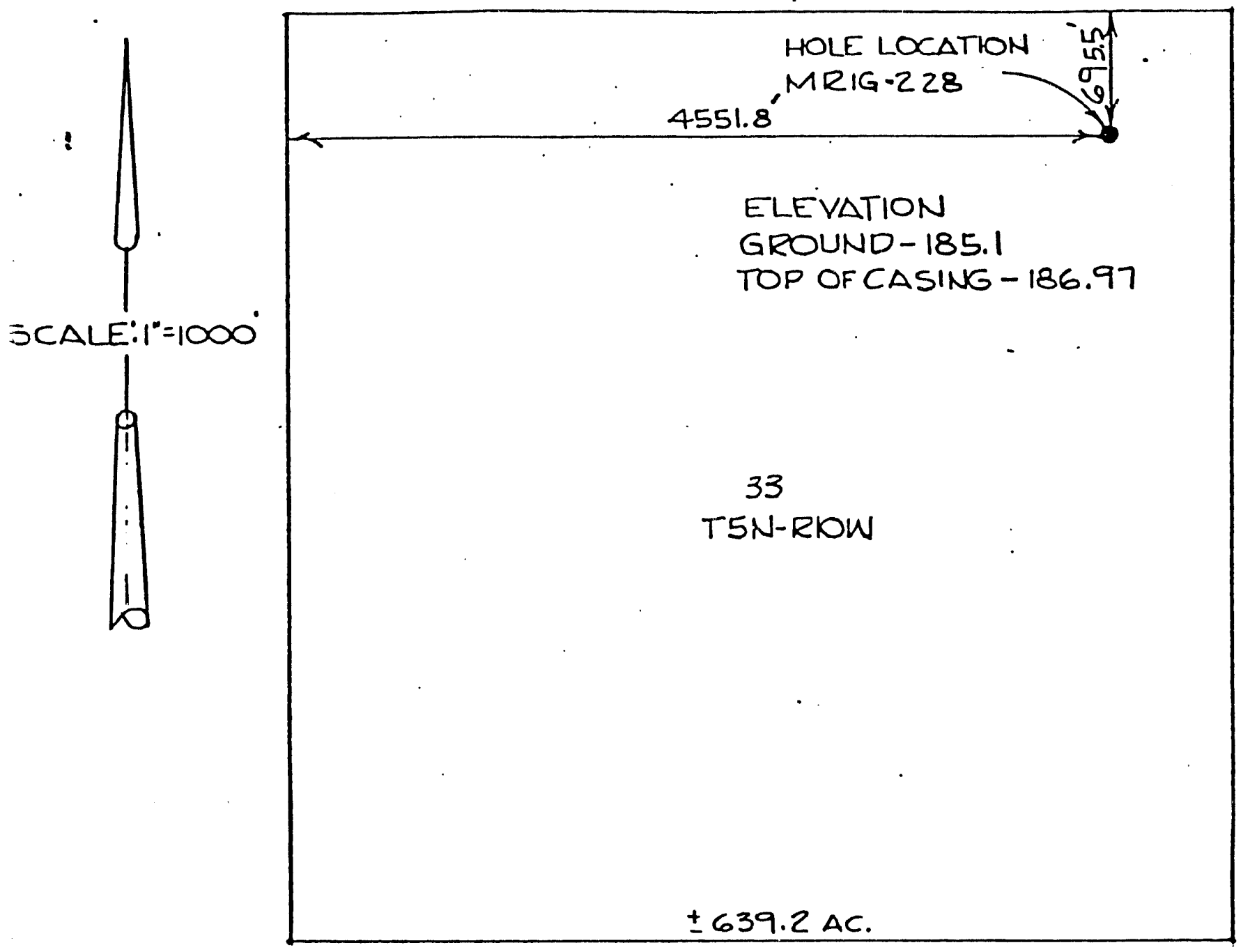

$A C$.

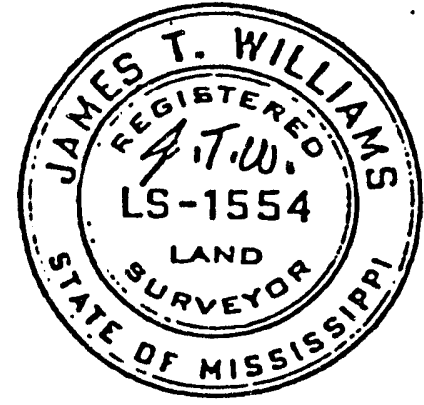

TEST LOCATION FOR LAW ENGINEERING TESTING COMPANY 2749 DELK ROAD S.E. MARIETTA,GEORGIA SECTION 33, TSN, RIOW, PERRY COU., MISS, WILLIAMS Ė BROOM- SURVETORS HATTIESBUEG, MISS, NOV.20.1979 SN.391-78 DN. 1168 

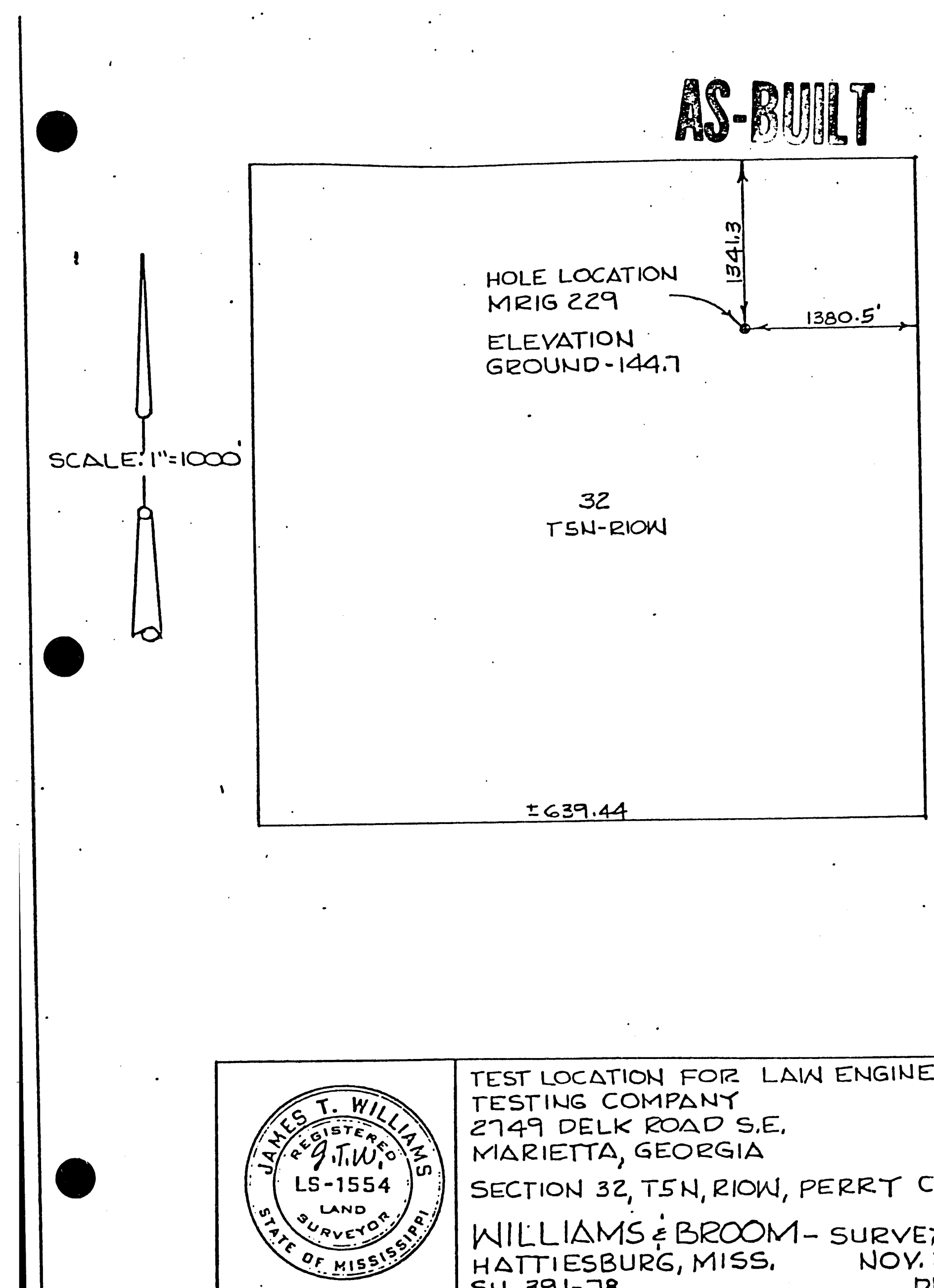

TEST LOCATION FOR LAIN ENGINEERING TESTING COMPANY 2749 DELK ROAD S.E. MIARIETTA, GEORGIA SECTION 32, T5N,RIOW, PERRT COU, MISS WILLIAMS \&BROOM - SURVETORS HATTIESBURG, MISS. NOV. 20,1979 SN.391-78 DN 1167 

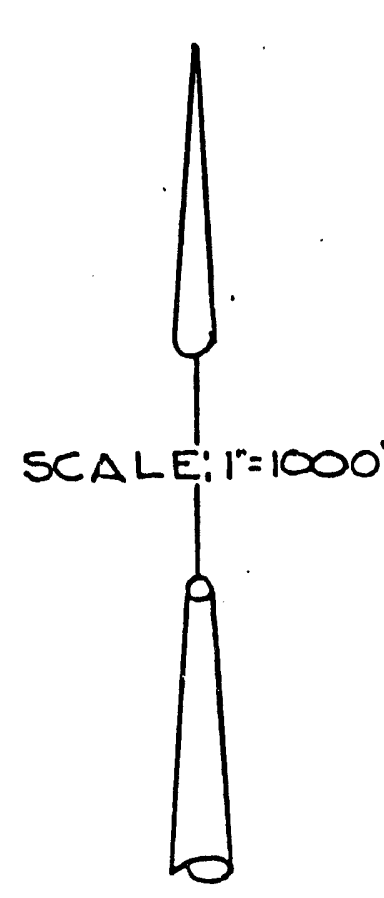
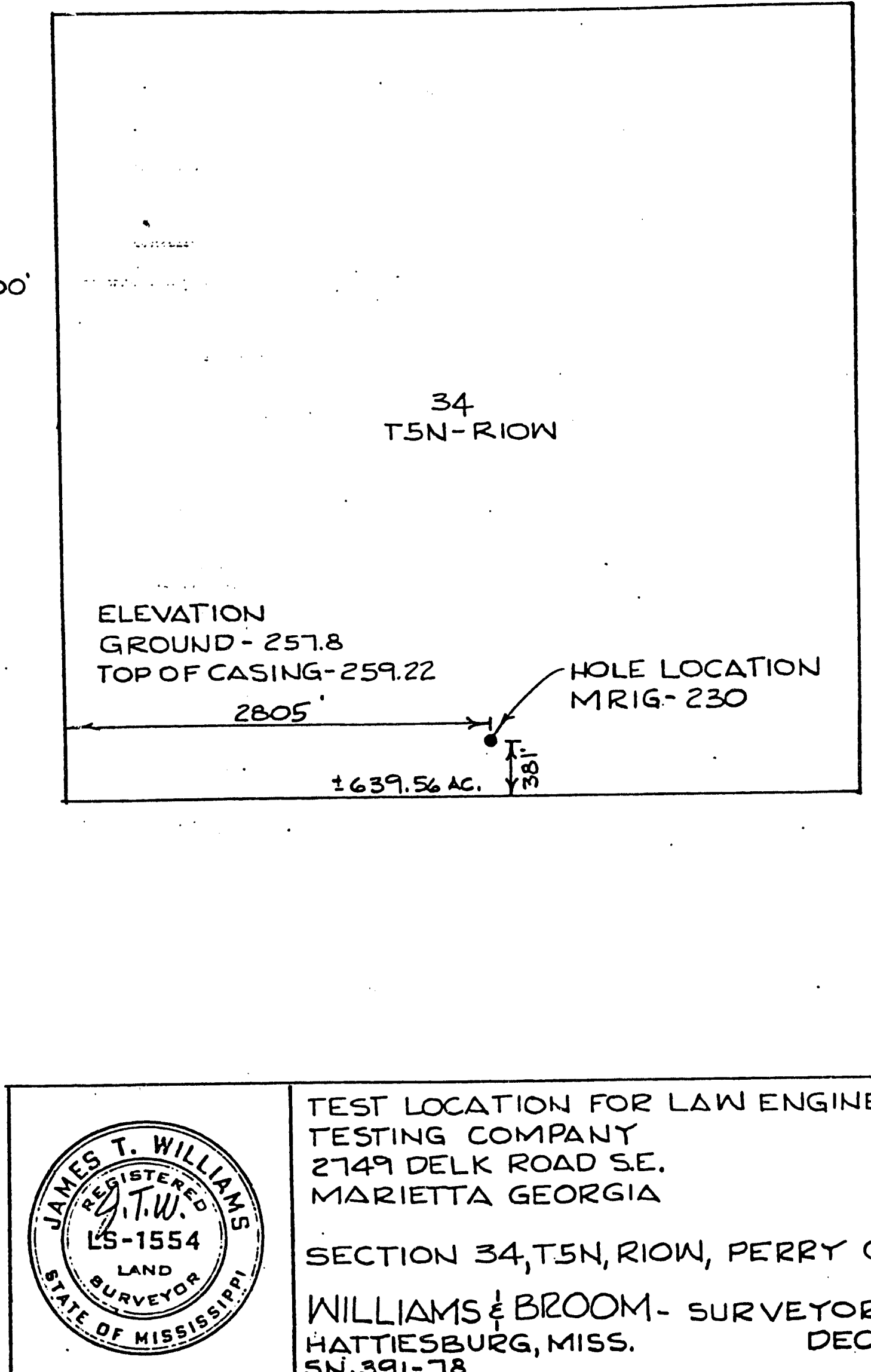

TEST LOCATION FOR LAW ENGINEERING

TESTING COMPANY

2749 DELK ROAD SE.

MARIETTA GEORGIA

SECTION 34, T.5N, RIOW, PERRY COU, MS,

WILLIAMS \& BROOM - SURVEYORS

HATTIESBURG, MISS.

DEC. 28,1979

5N.391-78 DNila4 


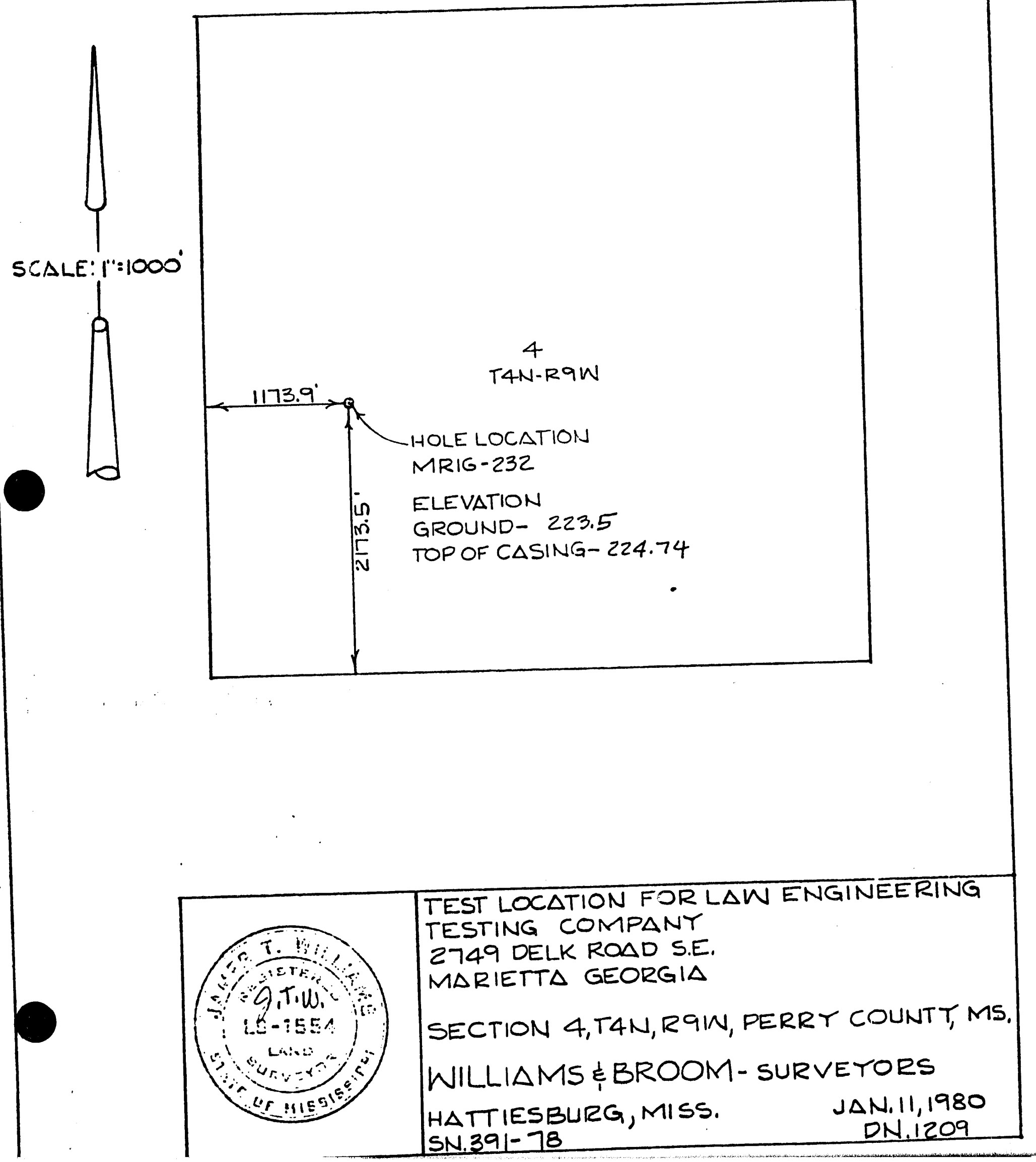




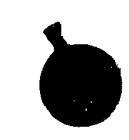

APPENDIX E

Site Restoration Form 


\section{SITE RESTORATION FORM}

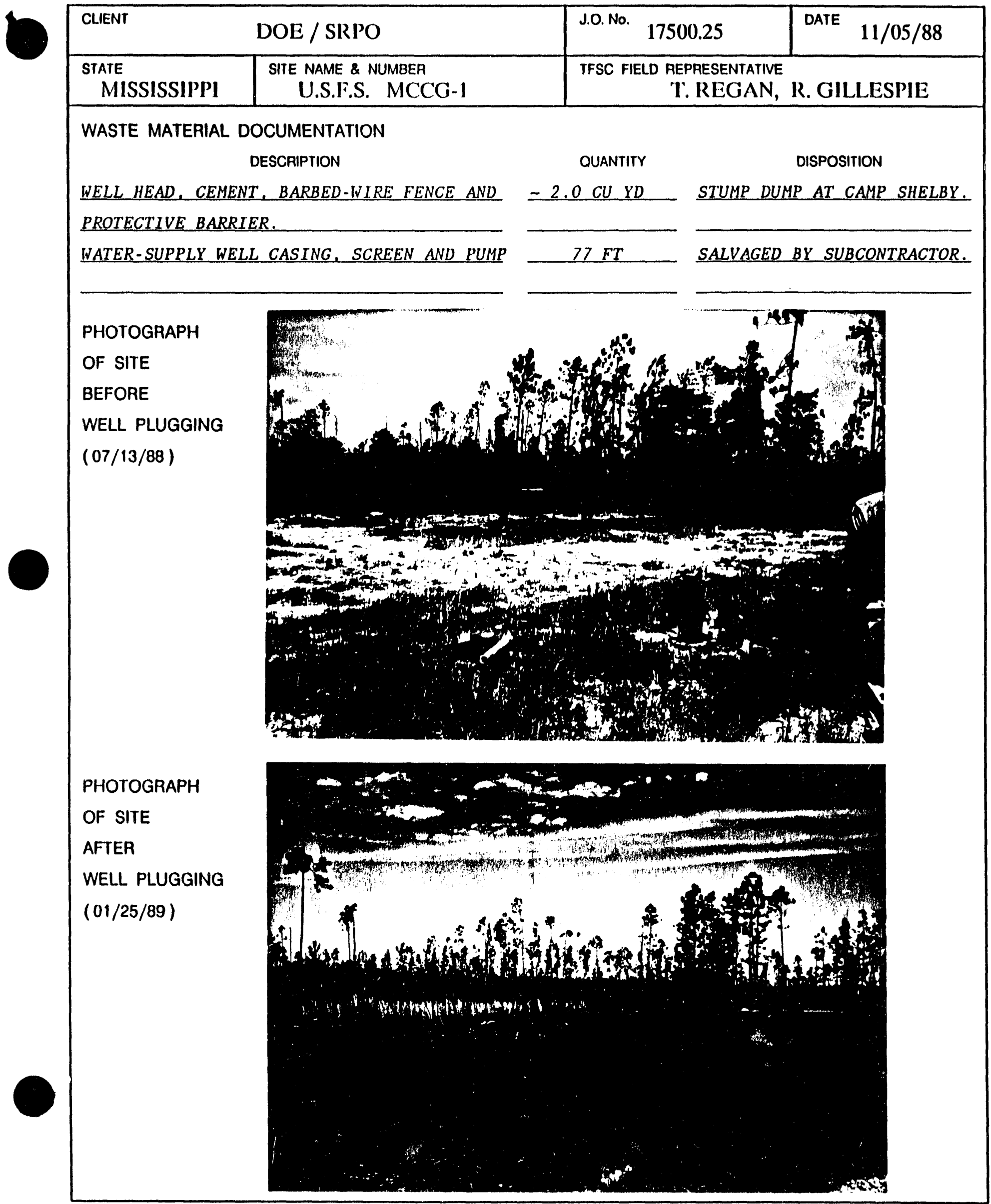




\section{SITE RESTORATION FORM}

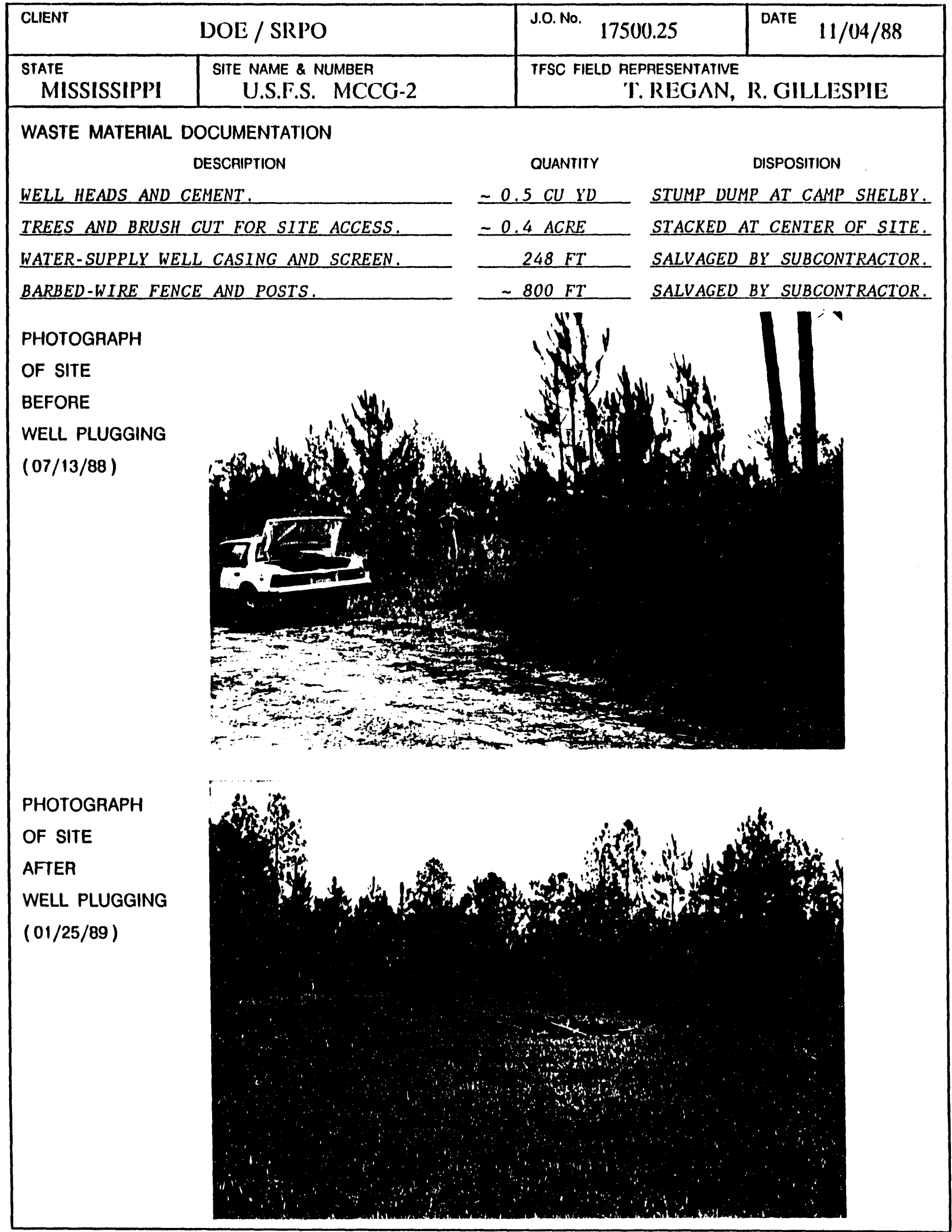


SITE RESTORATION FORM

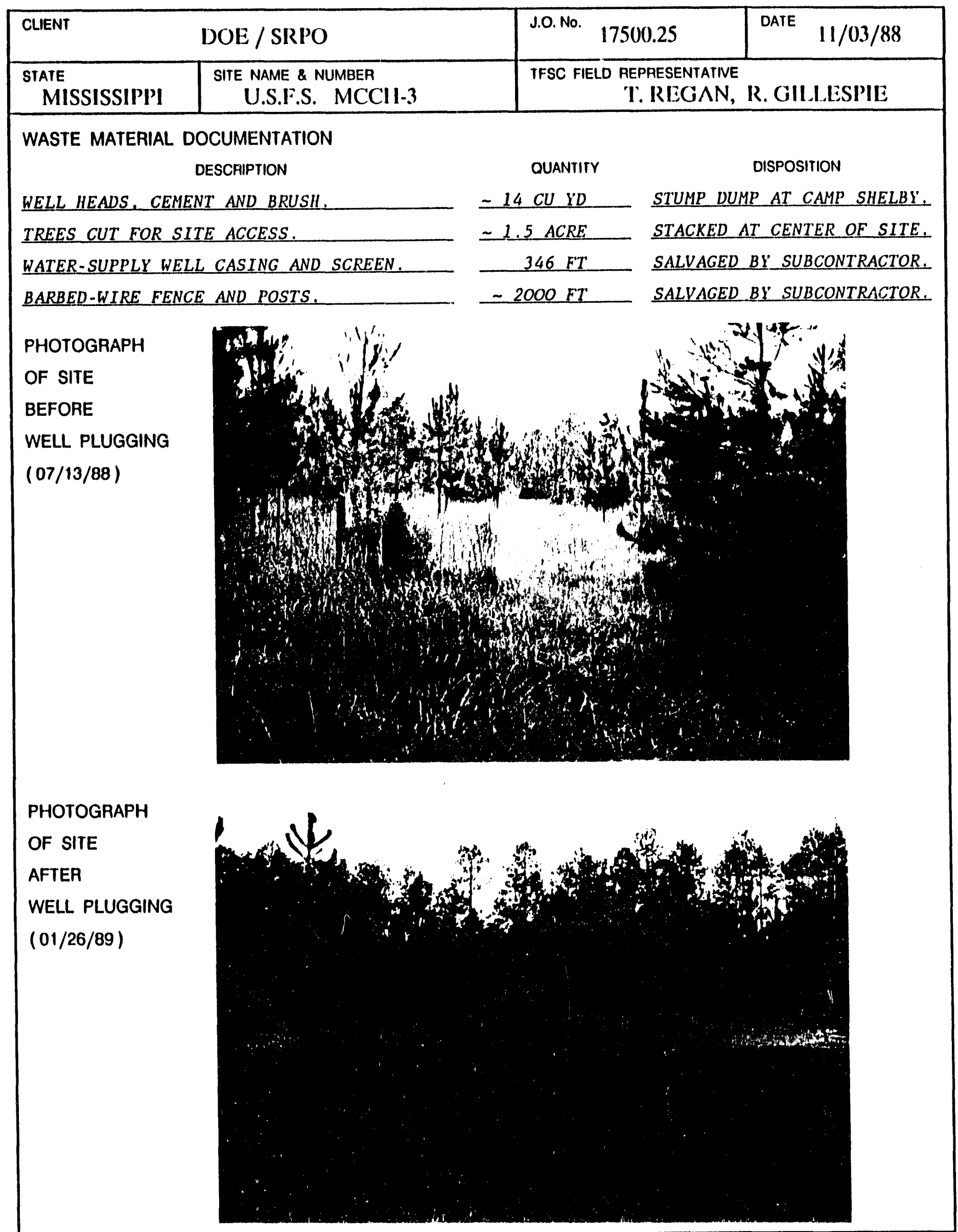


SITE RESTORATION FORM

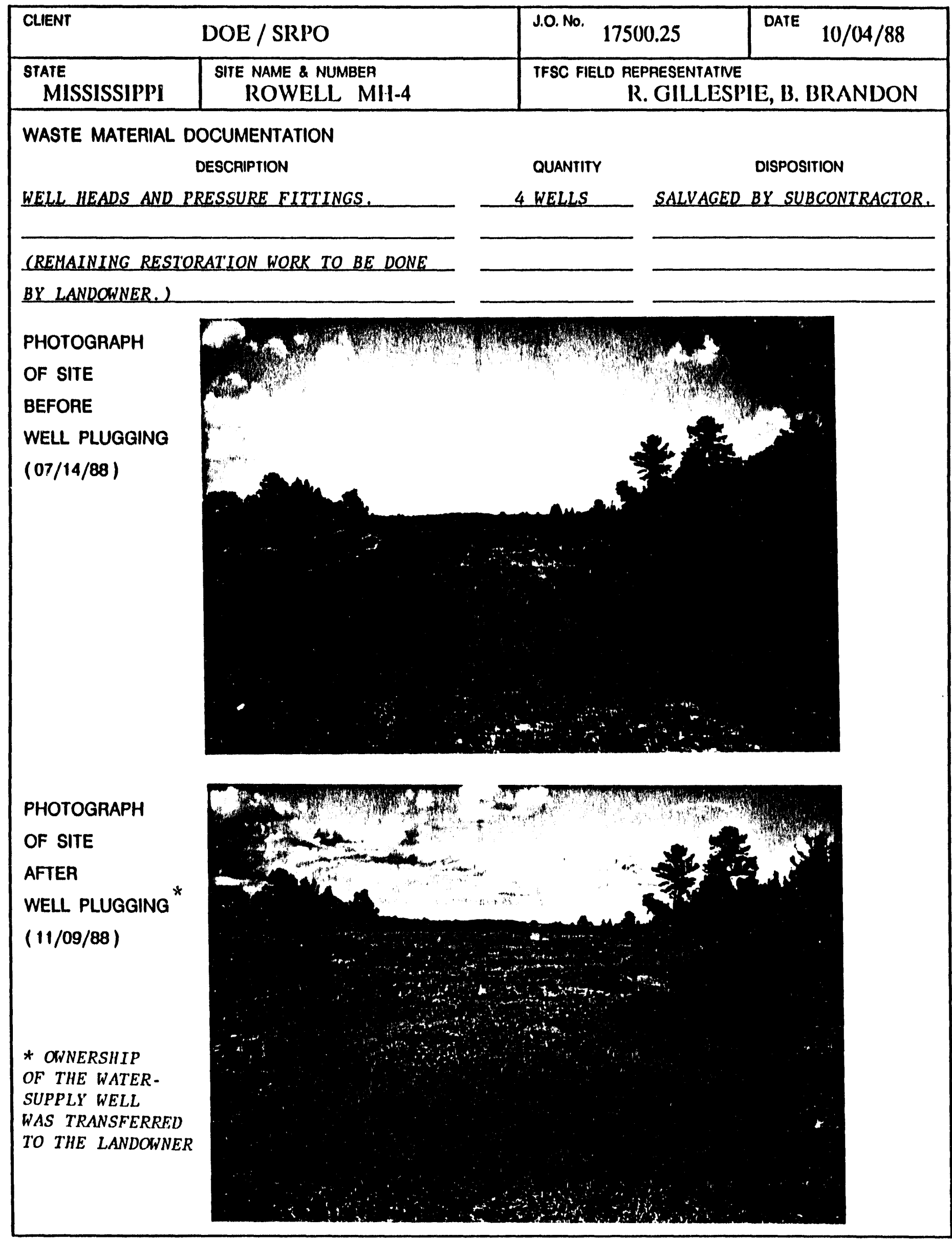




\section{SITE RESTORATION FORM}

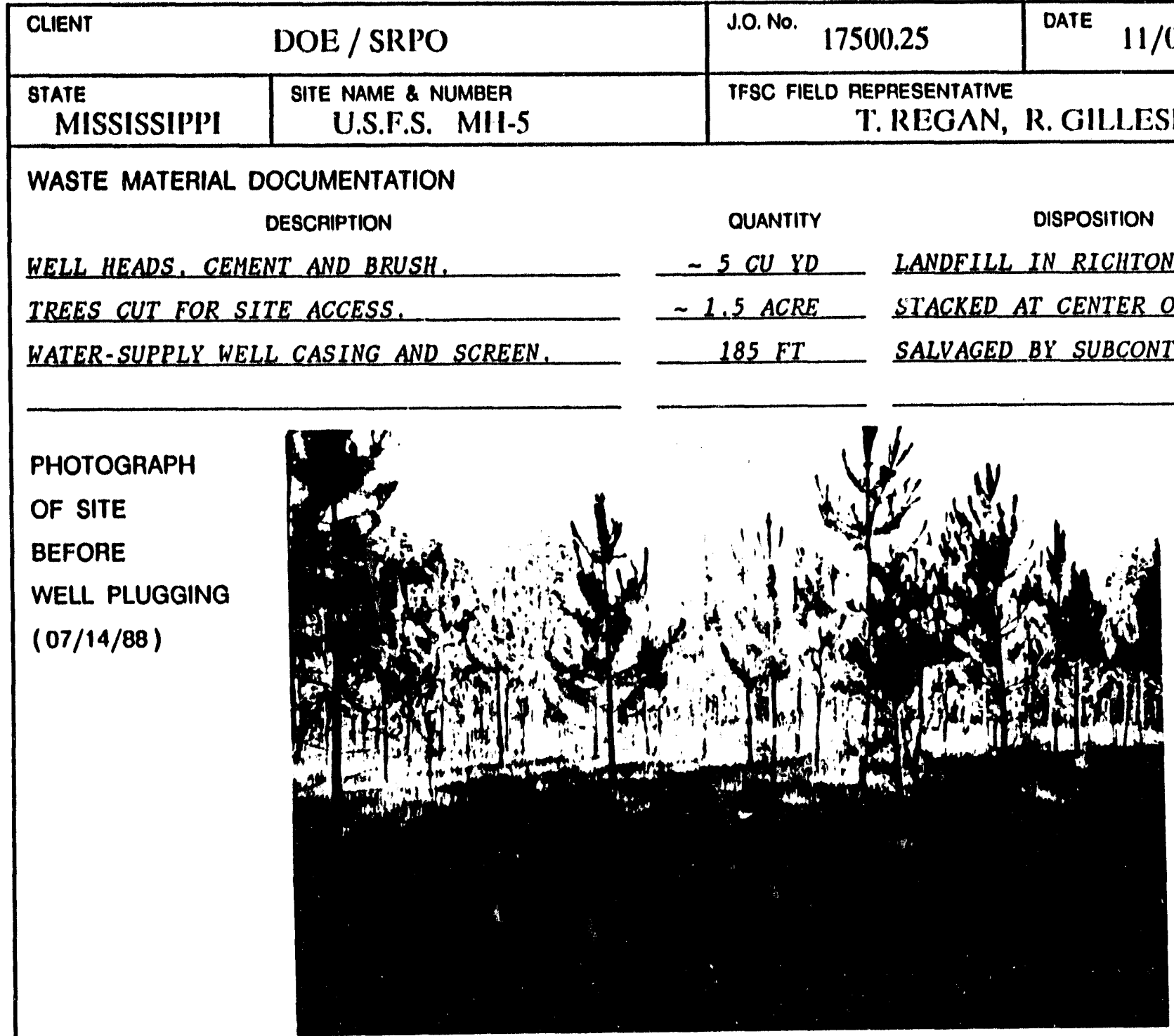

PHOTOGRAPH

OF SITE

AFTER

WELL PLIJGGING

$(01 / 23 / 89)$

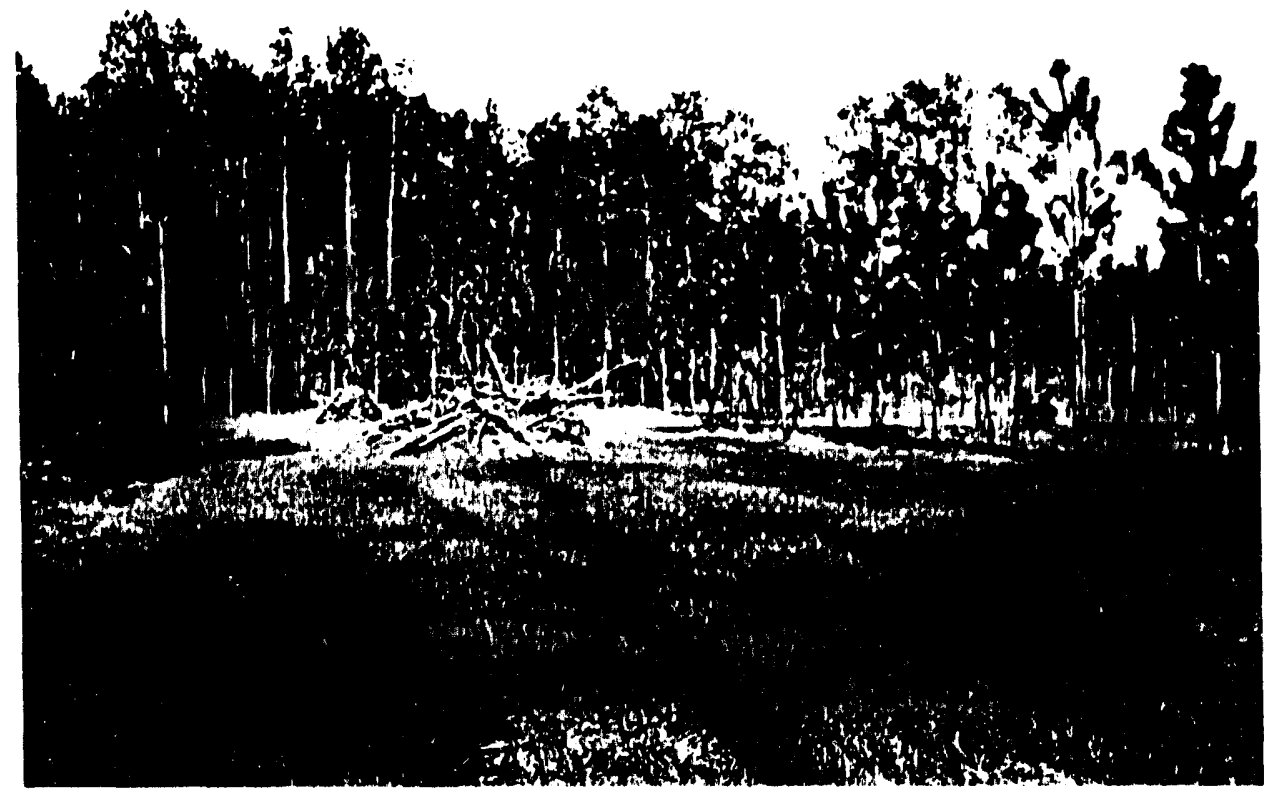




\section{SITE RESTORATION FORM}

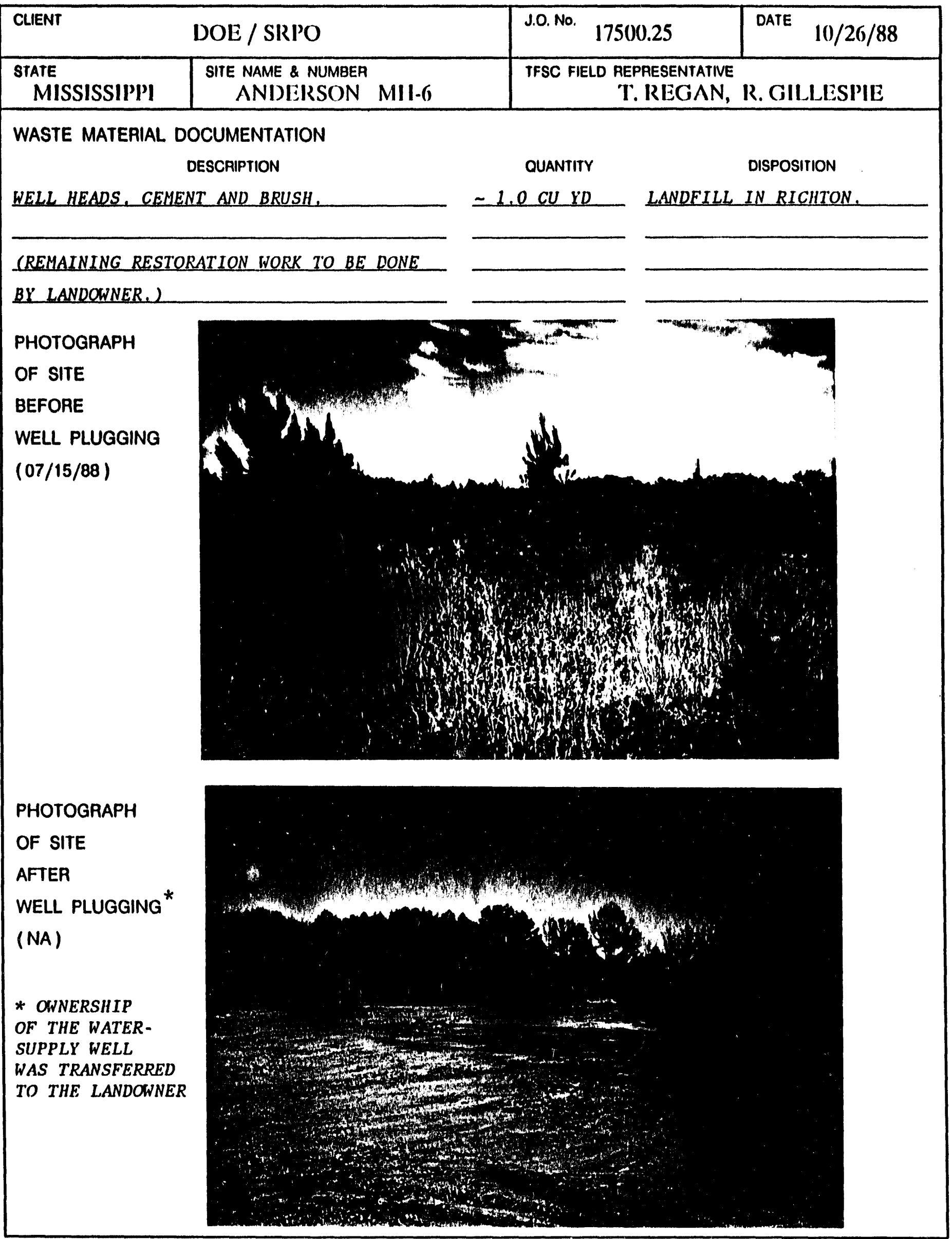




\section{SITE RESTORATION FORM}

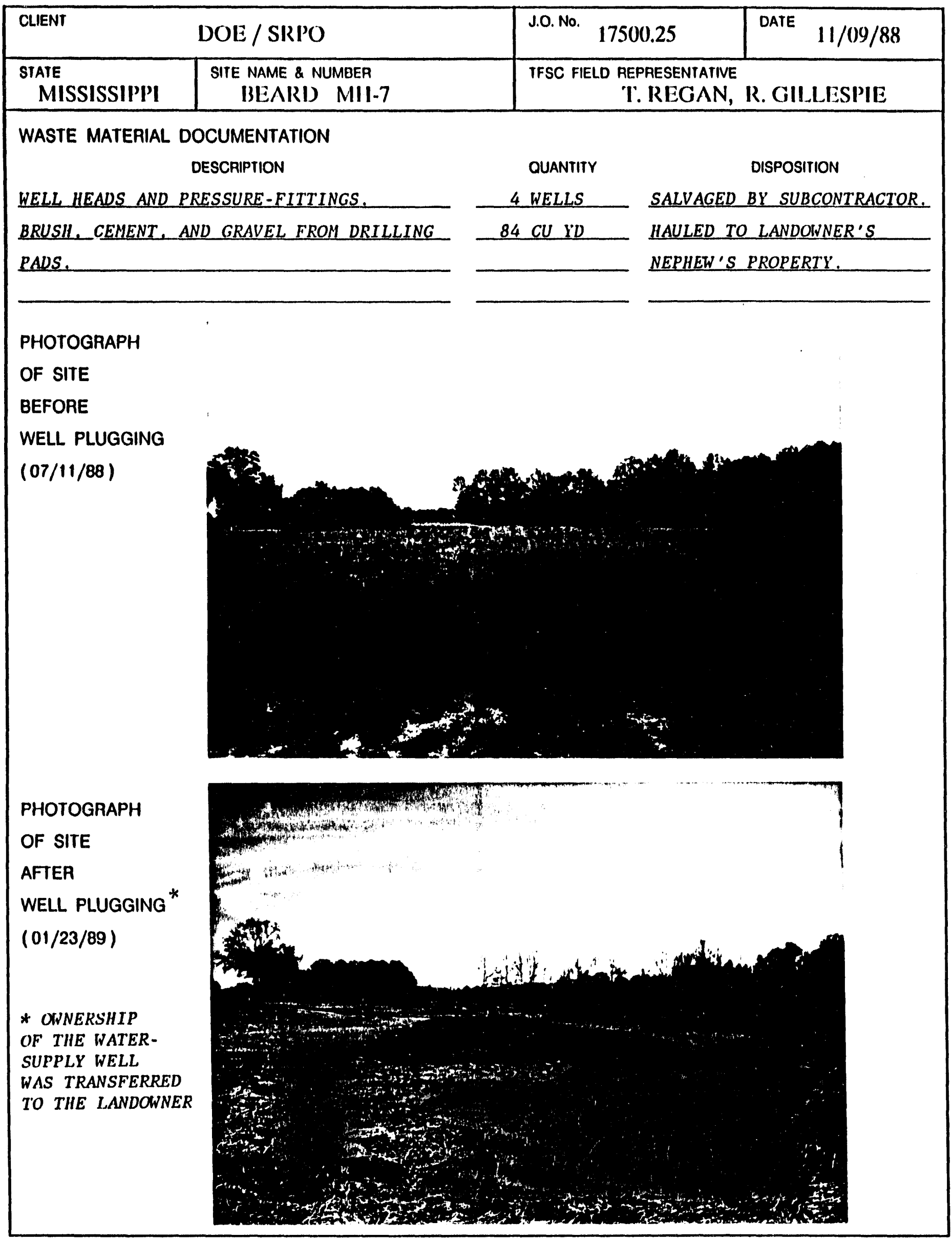


SITE RESTORATION FORM

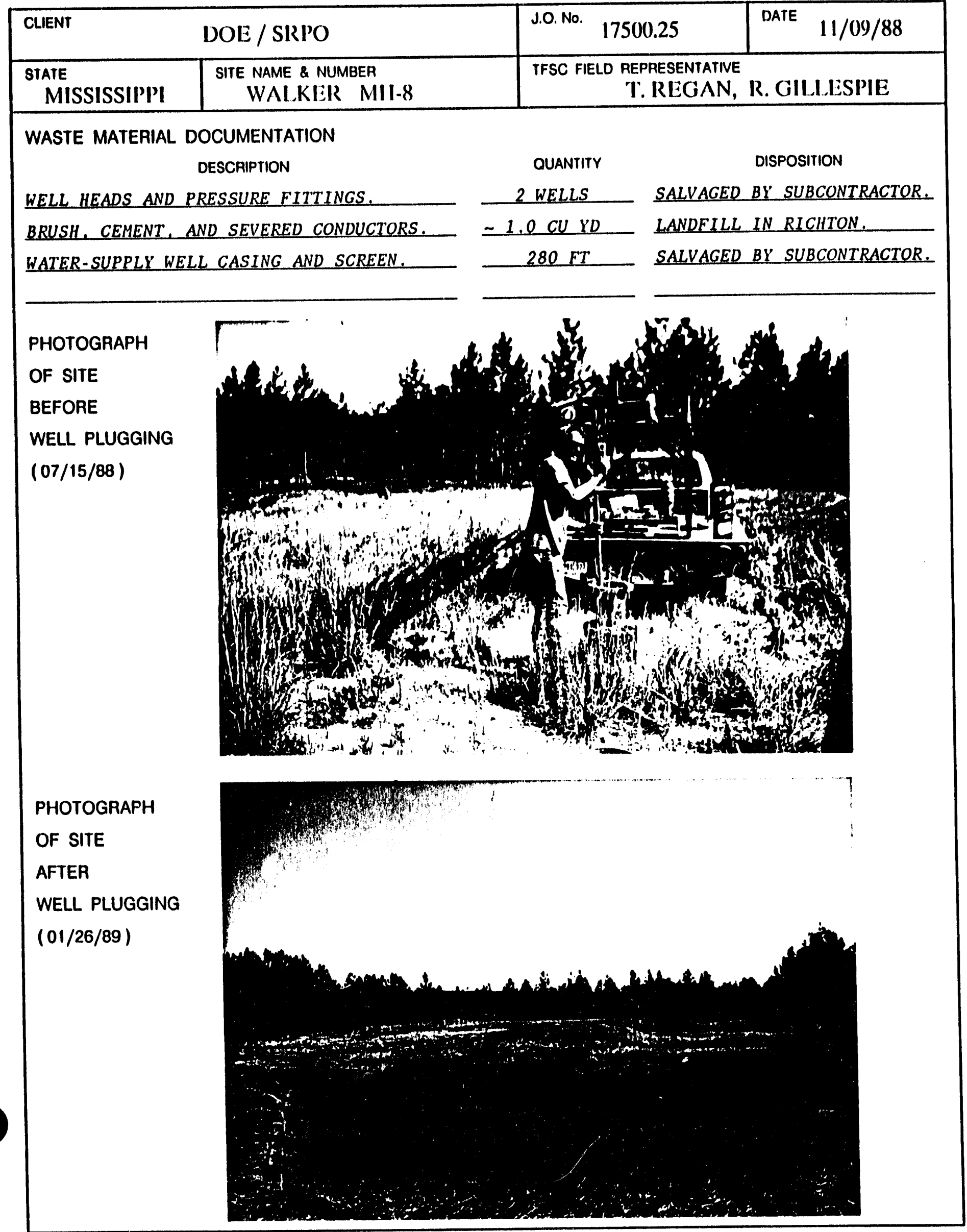


SITE RESTORATION FORM

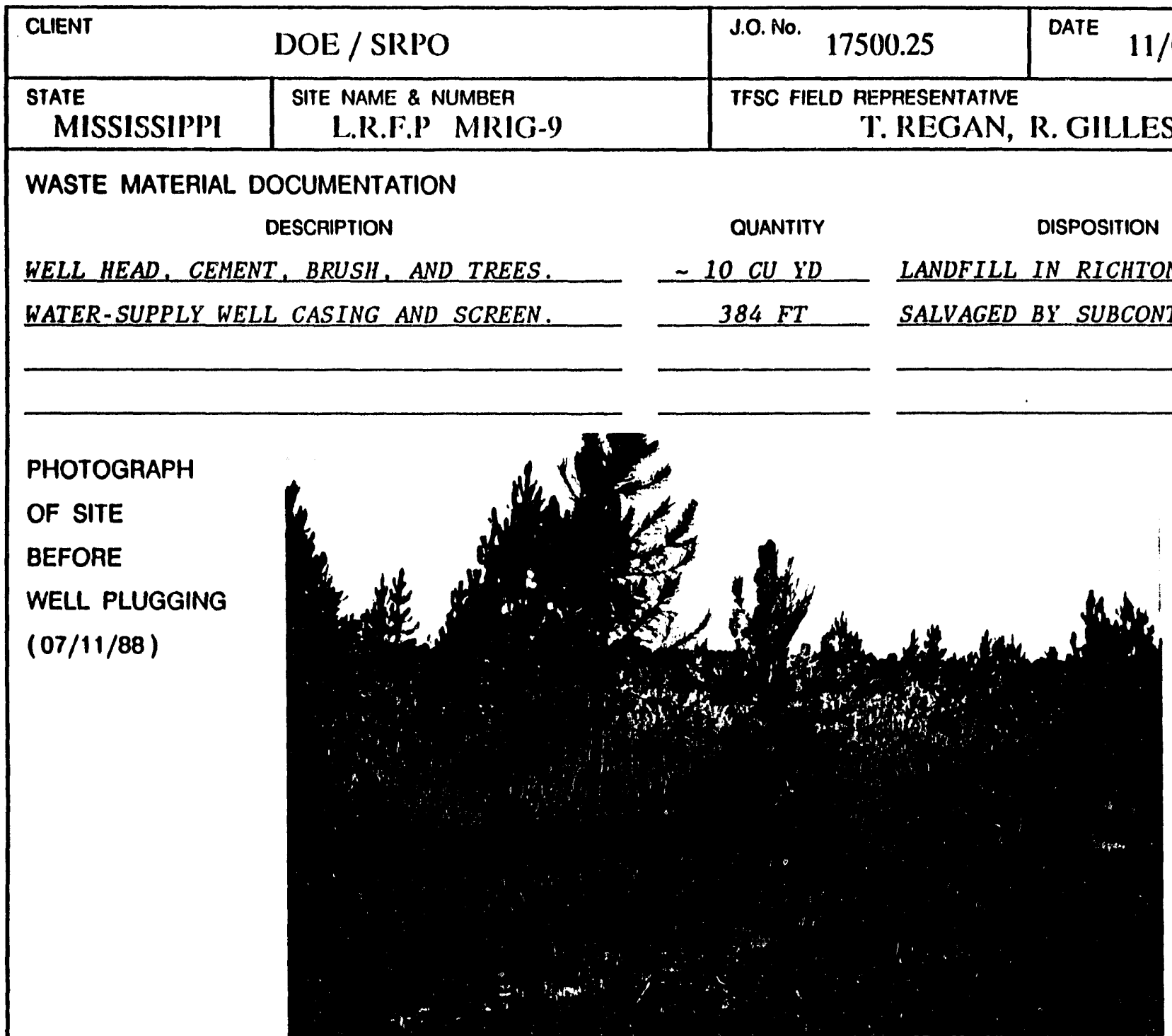

PHOTOGRAPH

OF SITE

AFTER

WELL PLUGGING

(01/26/89)

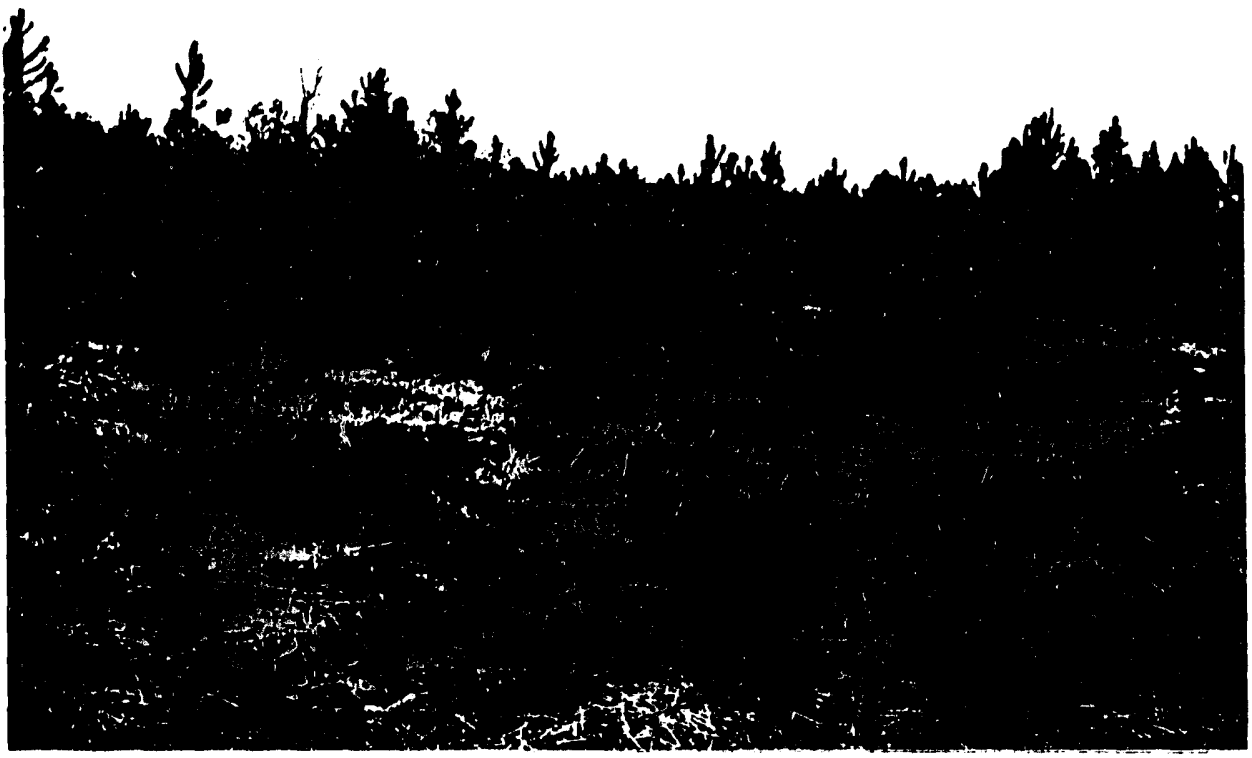


SITE RESTORATION FORM

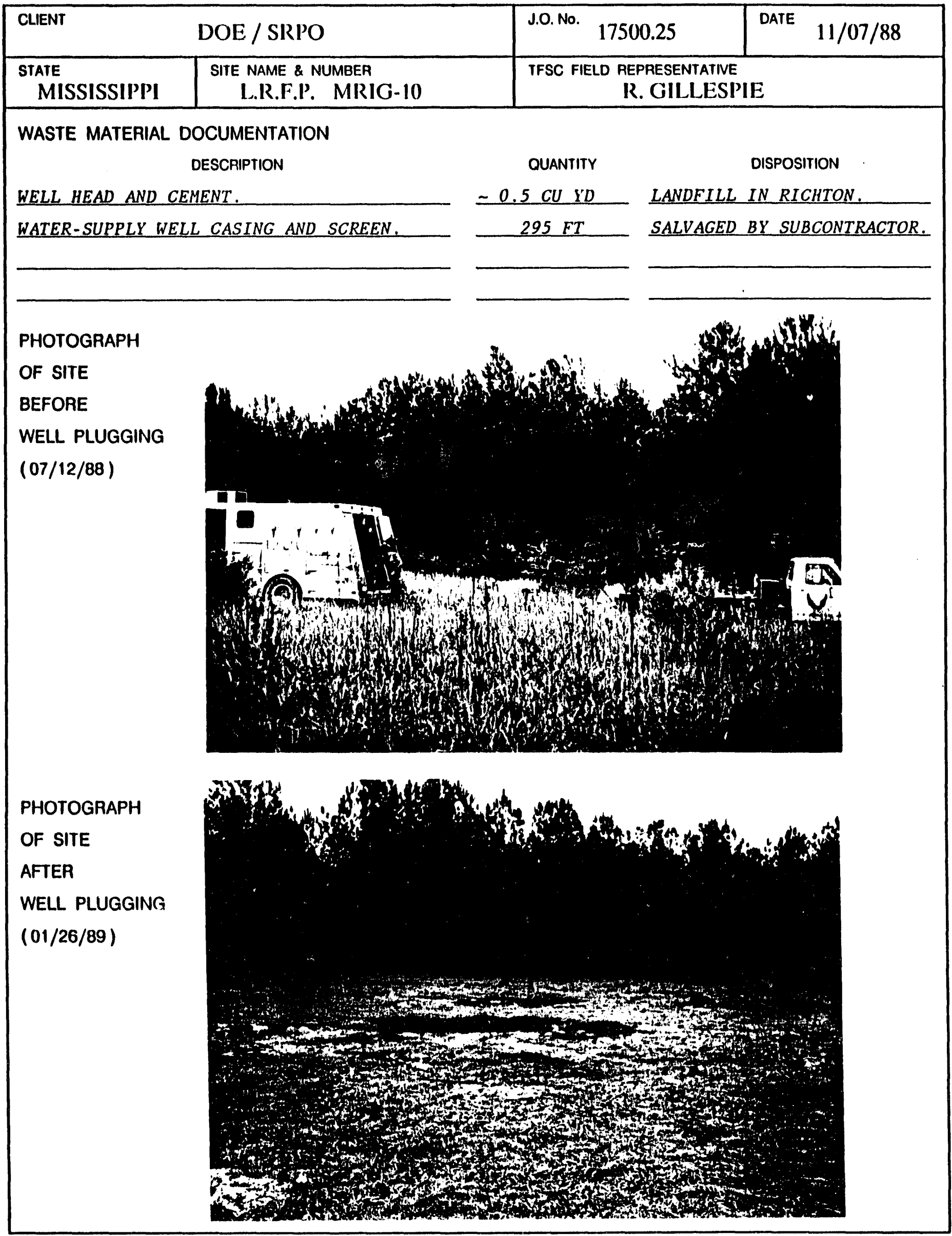




\section{SITE RESTORATION FORM}

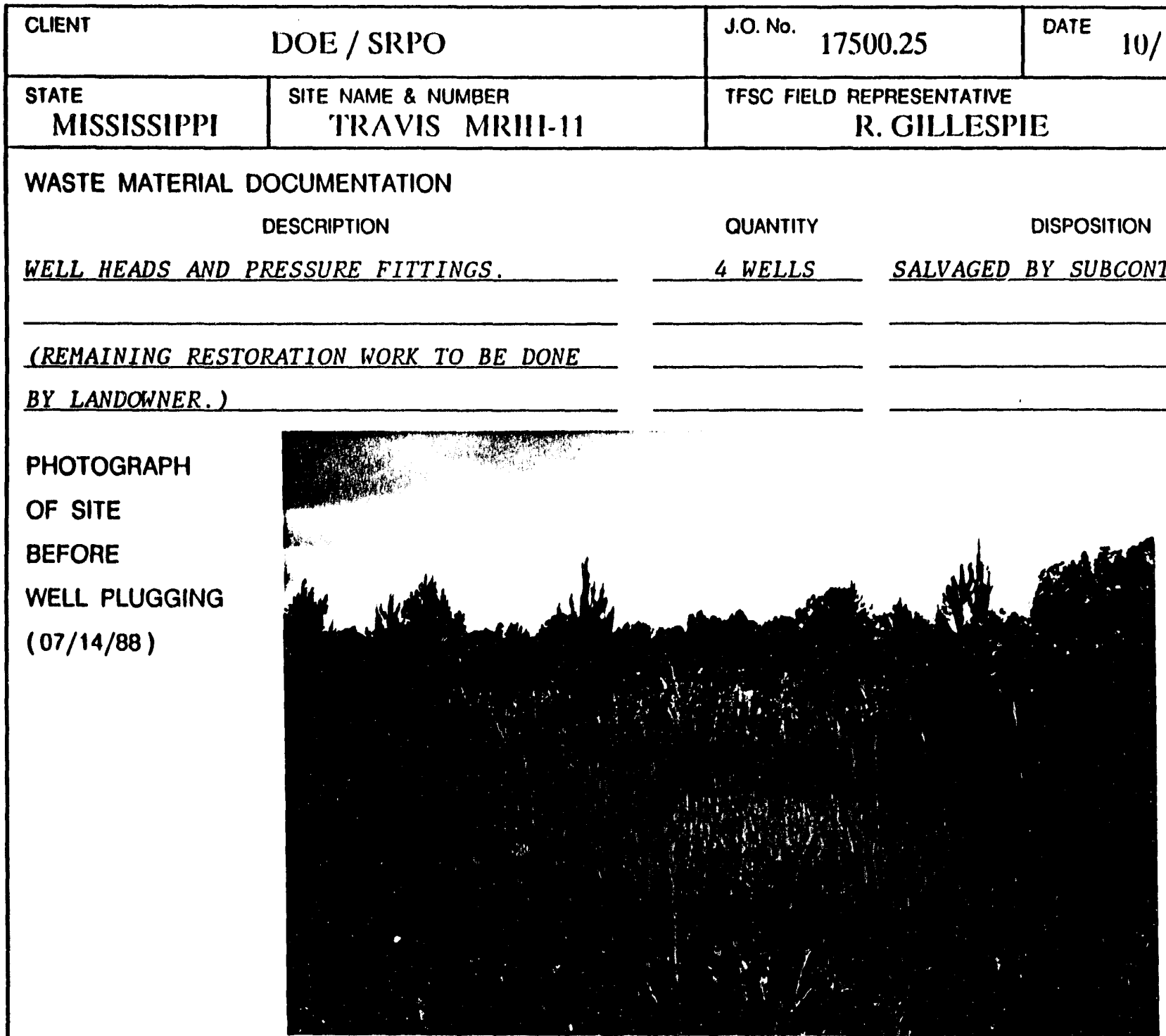

PHOTOGRAPH

OF SITE

AFTER

WELL PLUGGING * (NA)

* OWNERSHIP OF THE WATERSUPPLY WELL WAS TRANSFERRED TO THE LANDOWNER

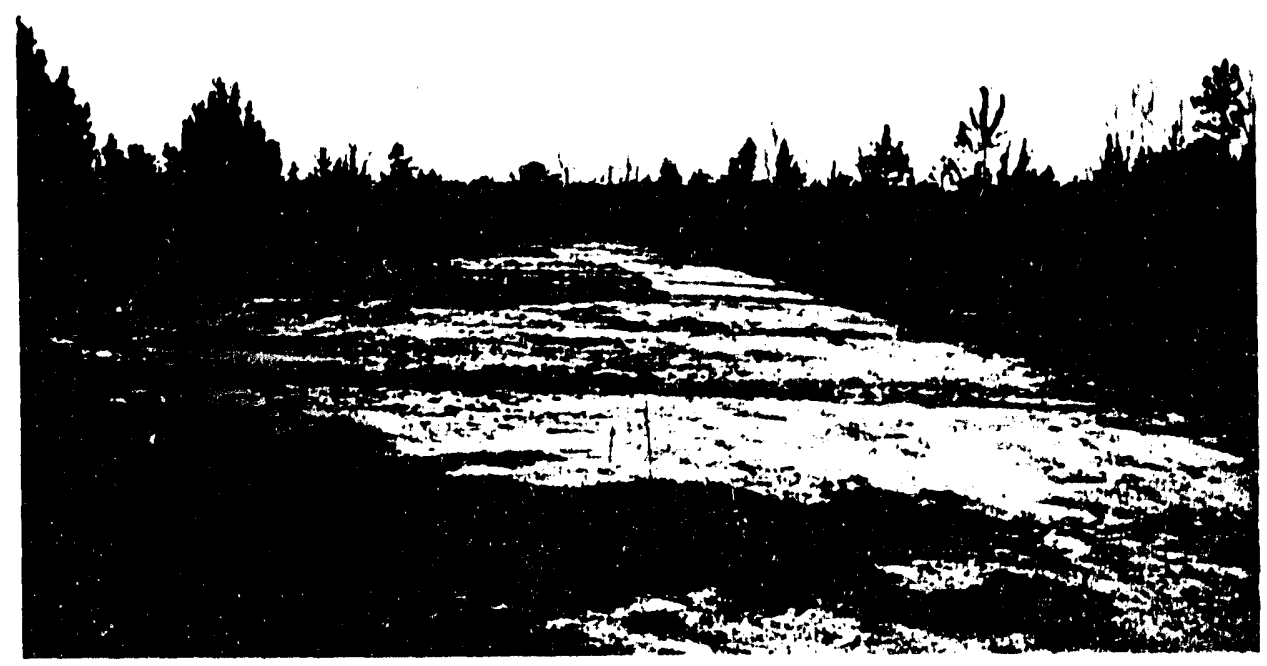


SITE RESTORATION FORM

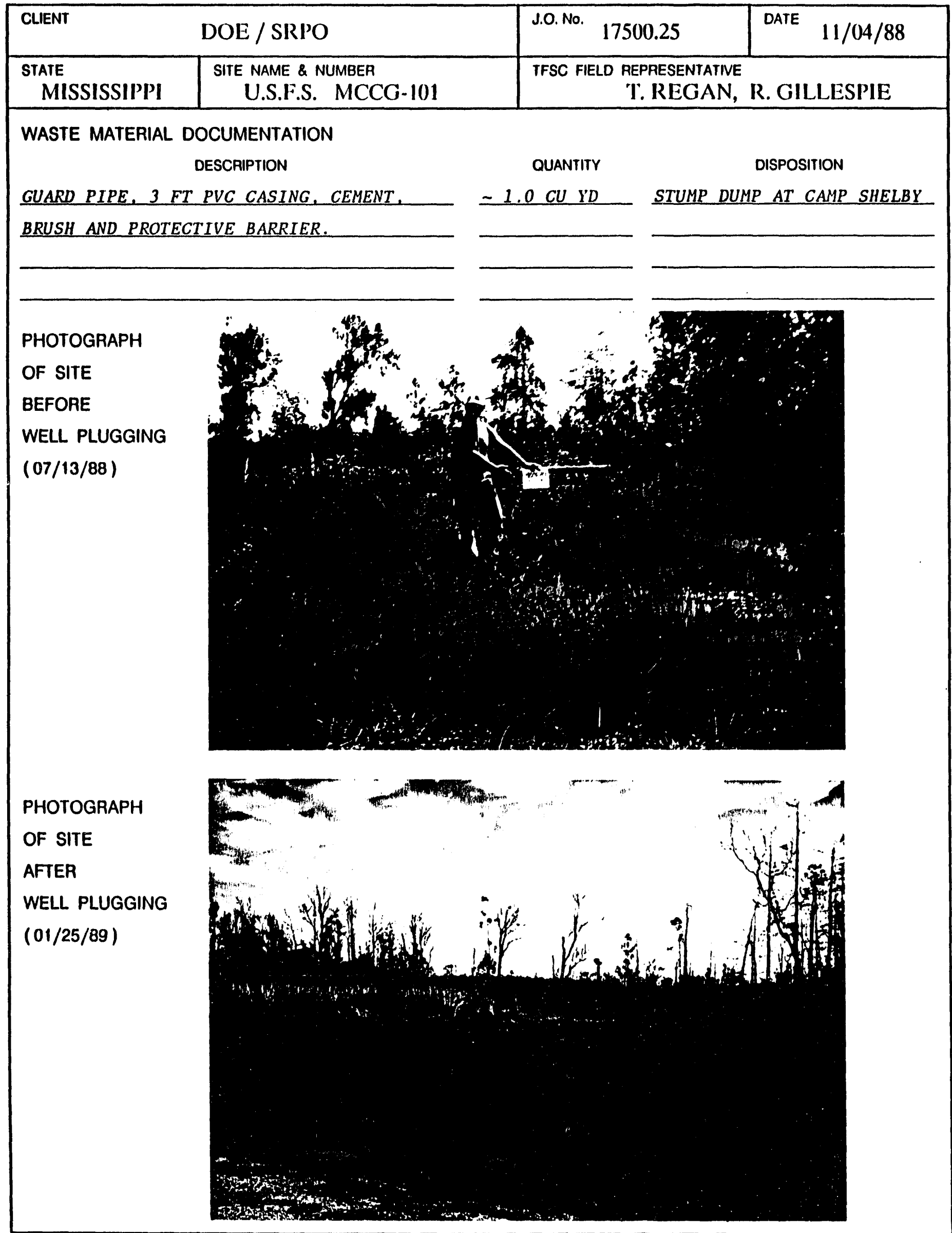


SITE RESTORATION FORM

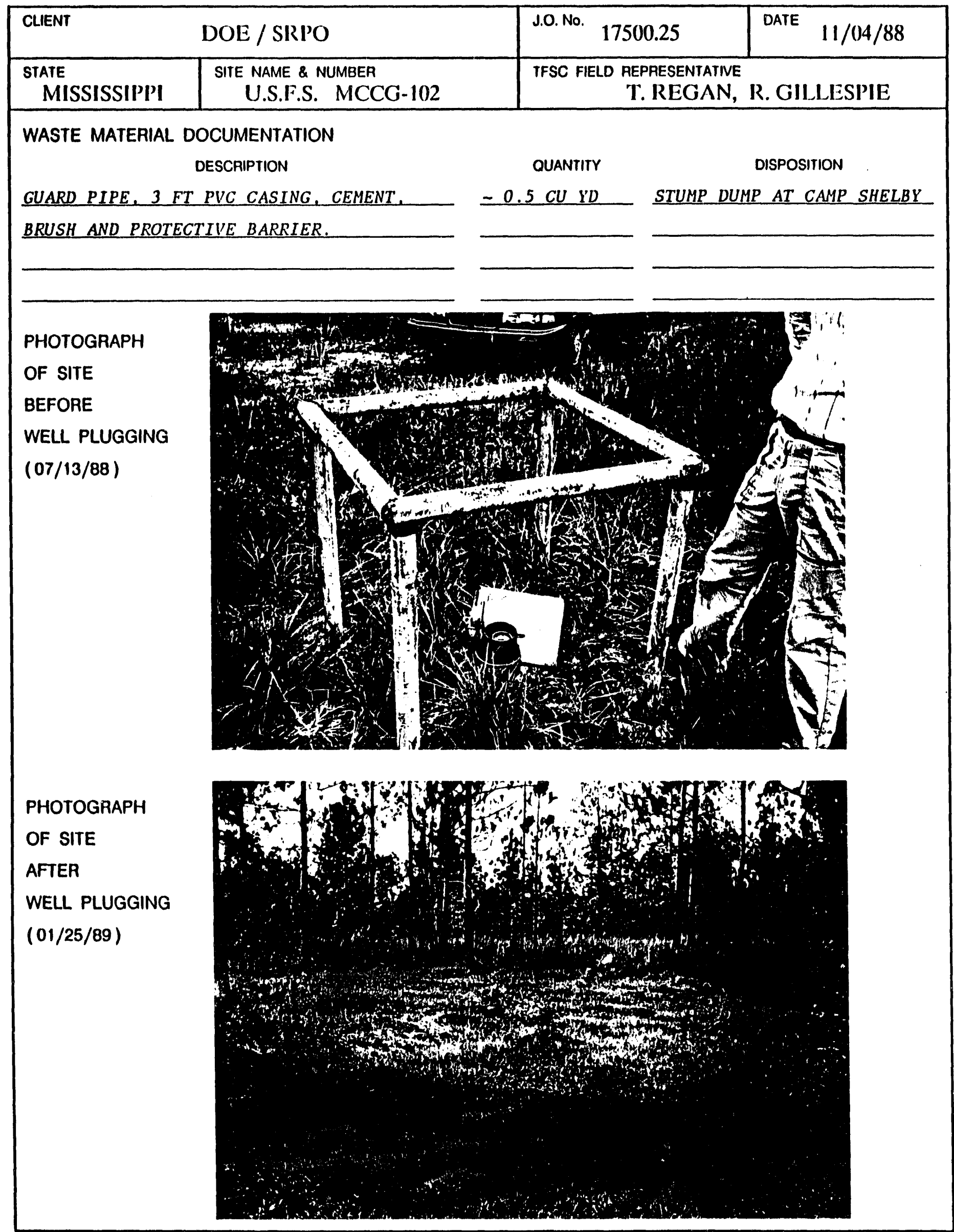




\section{SITE RESTORATION FORM}

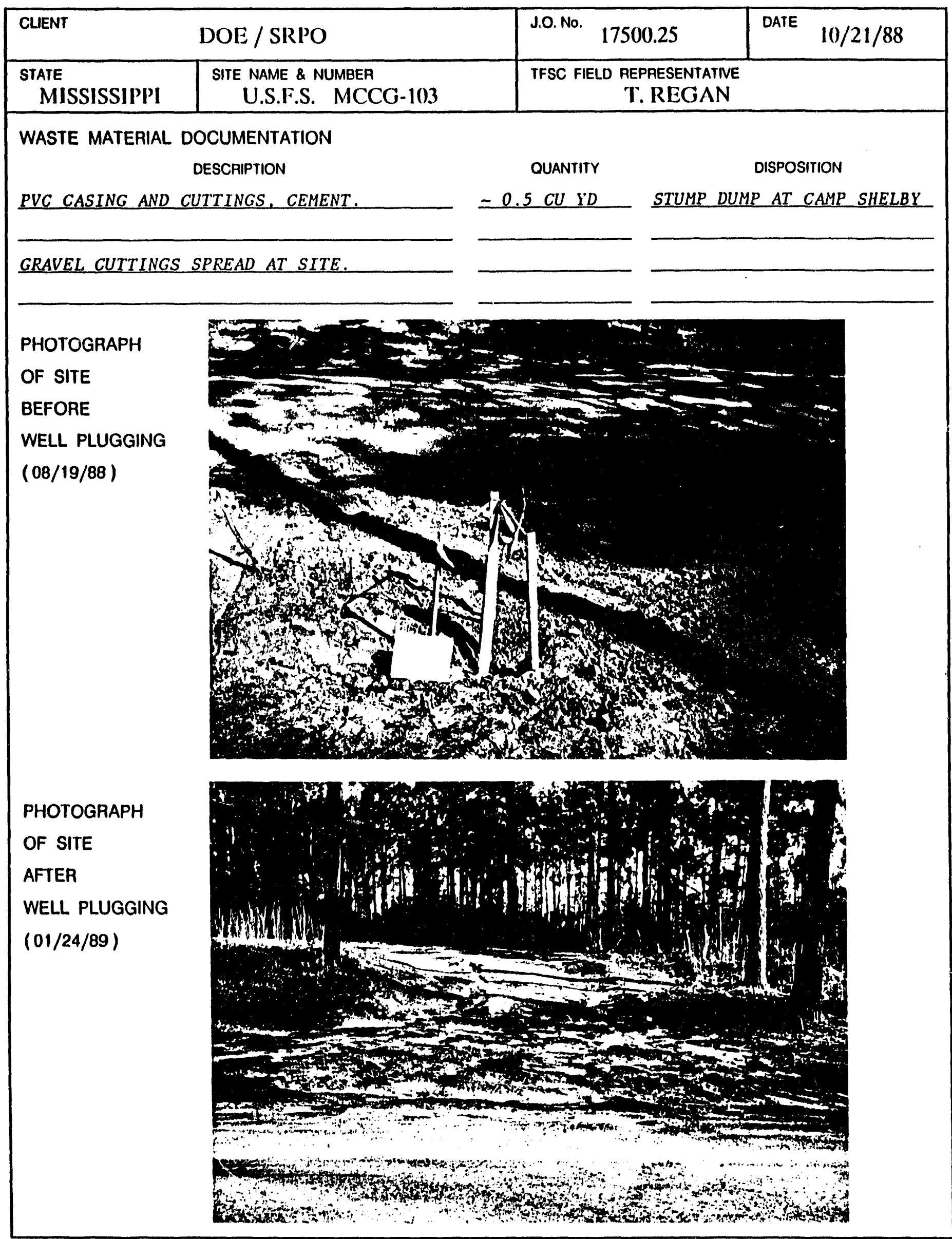


SITE RESTORATION FORM

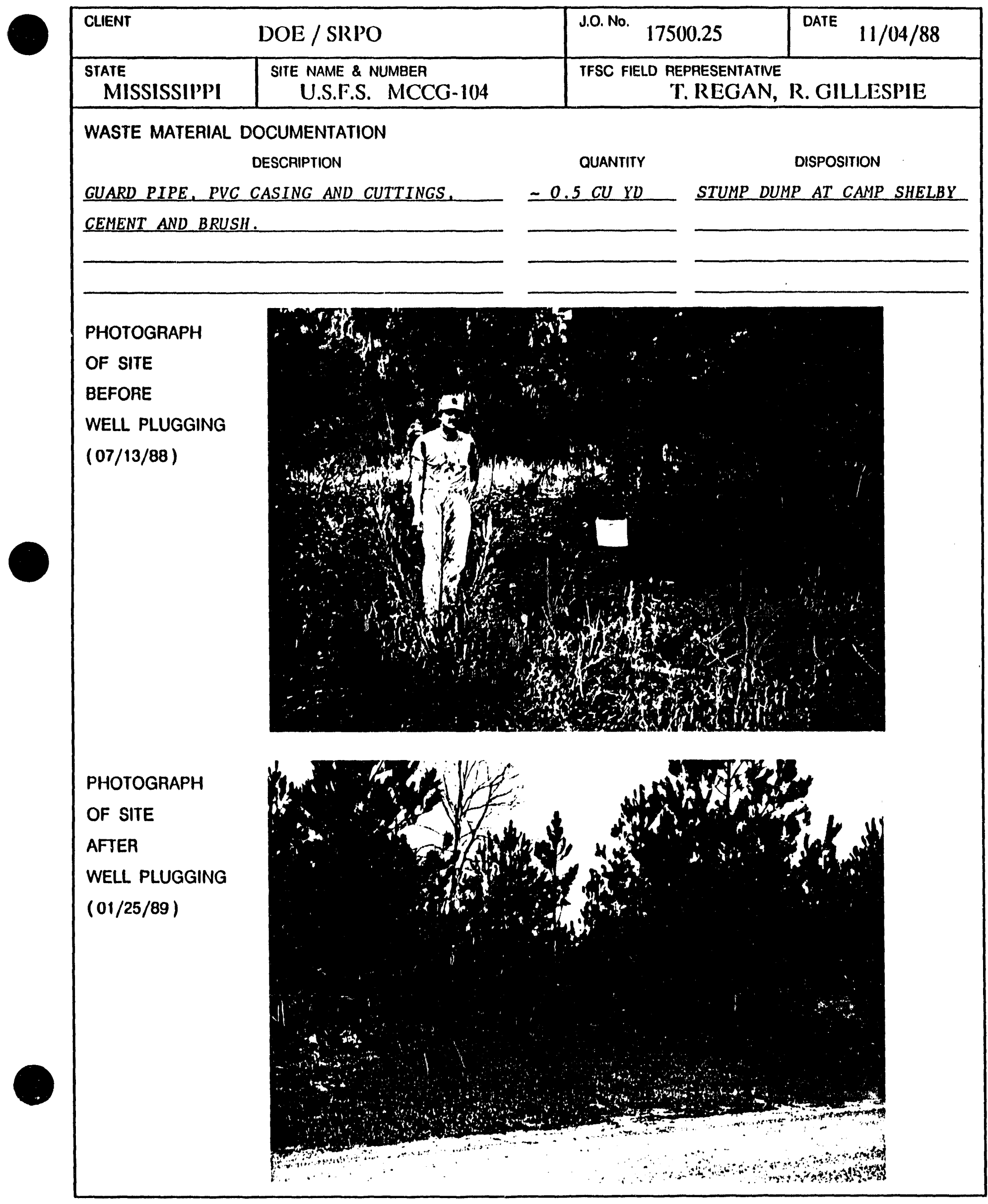




\section{SITE RESTORATION FORM}

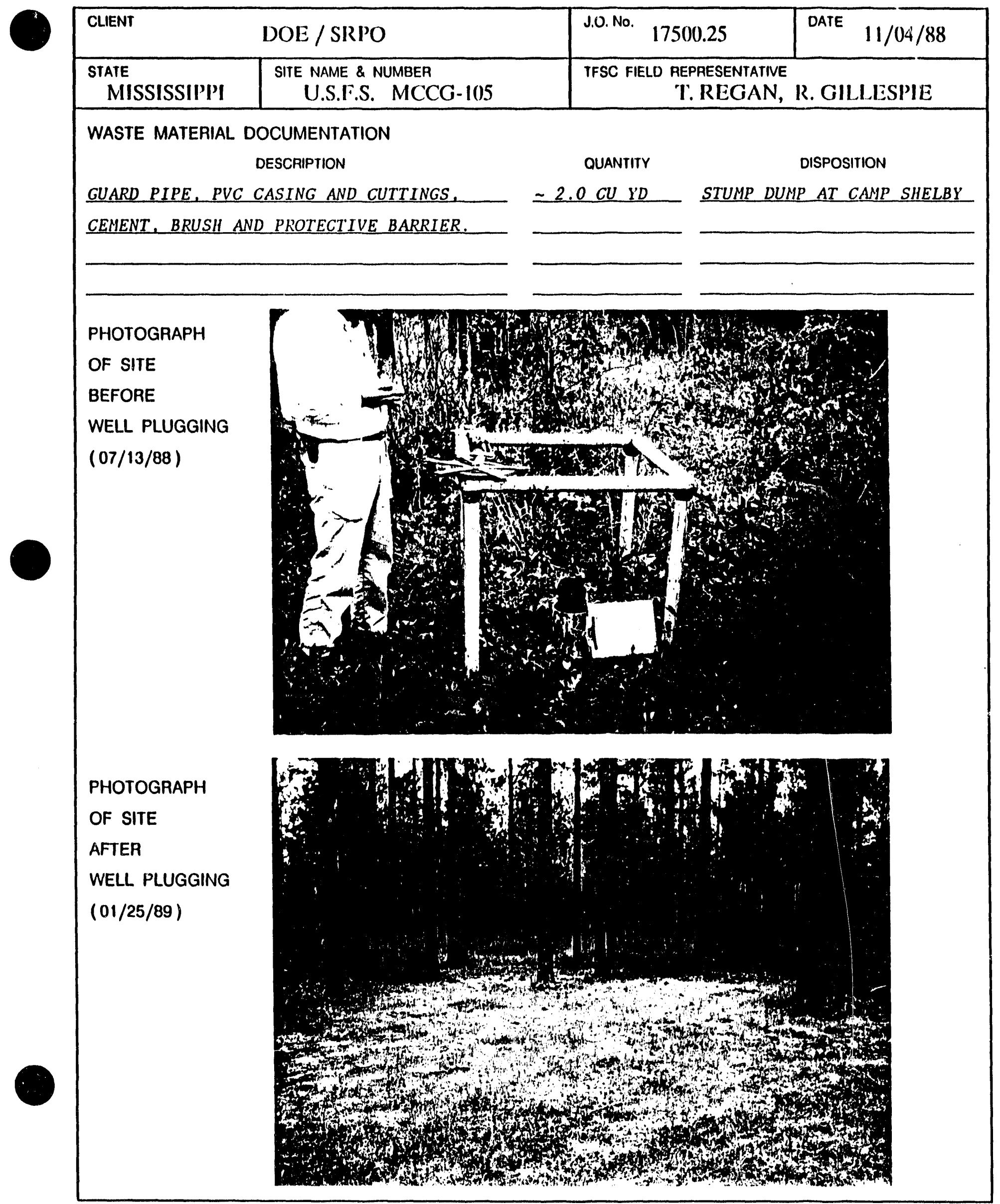


SITE RESTORATION FORM

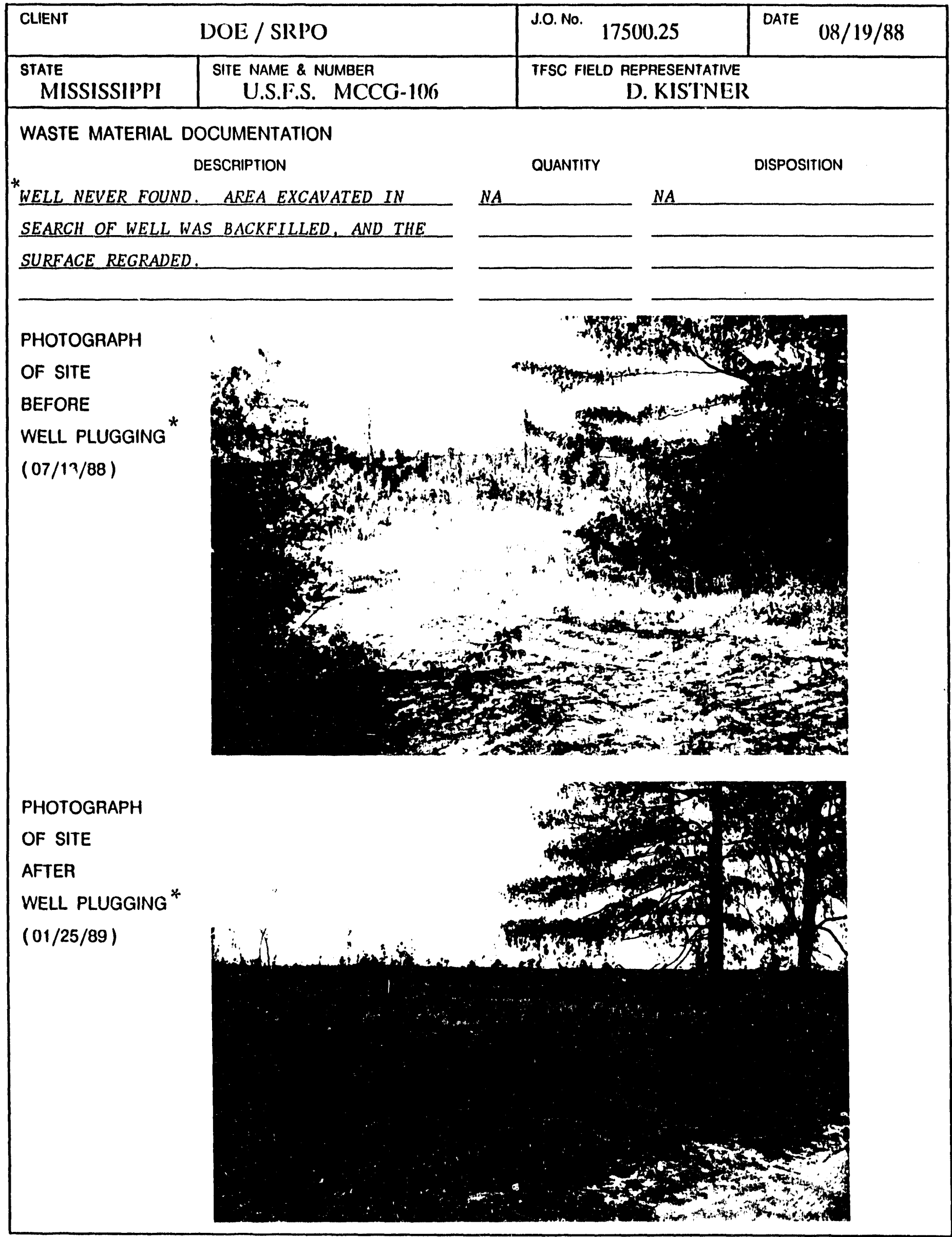


SITE RESTORATION FORM

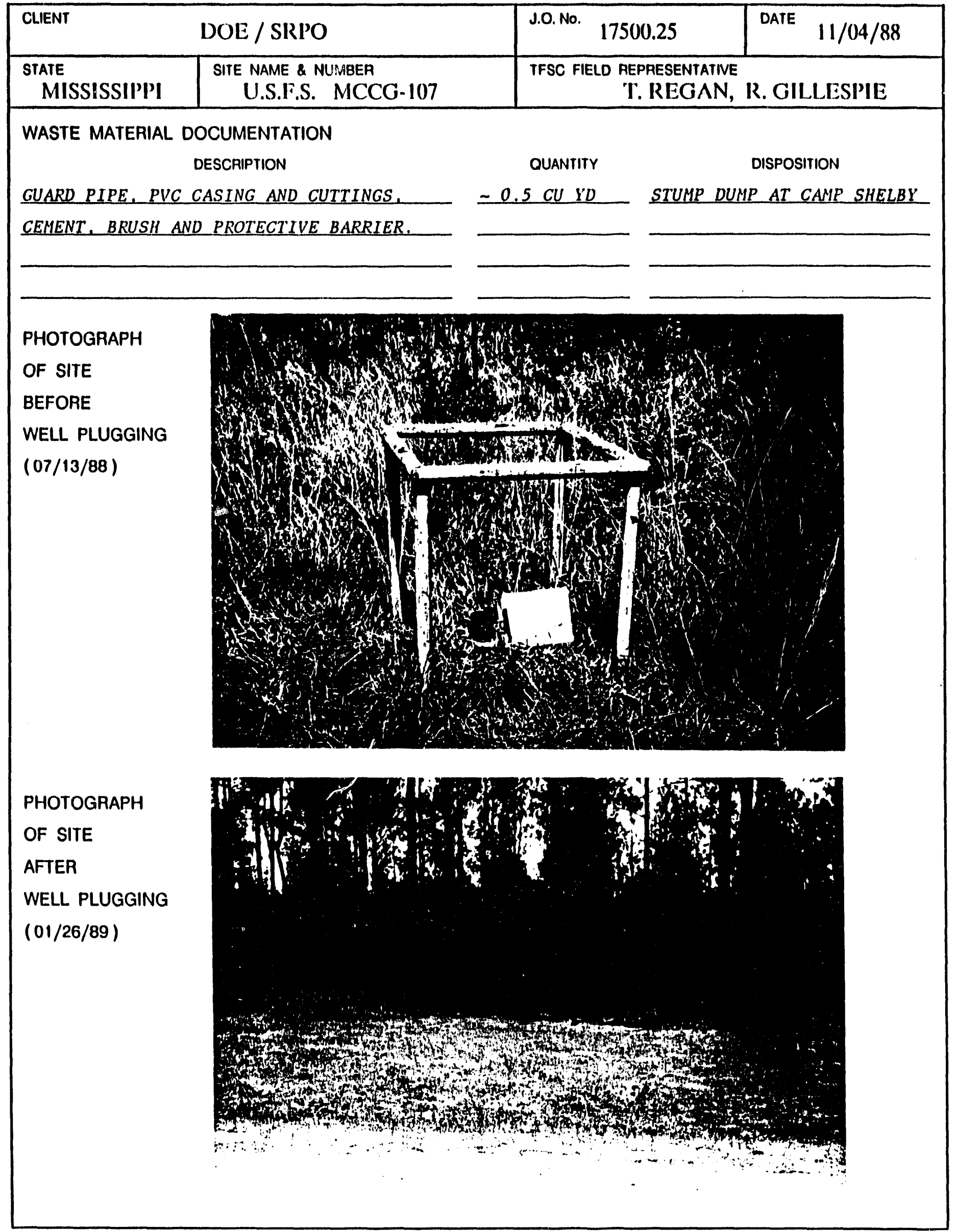




\section{SITE RESTORATION FORM}

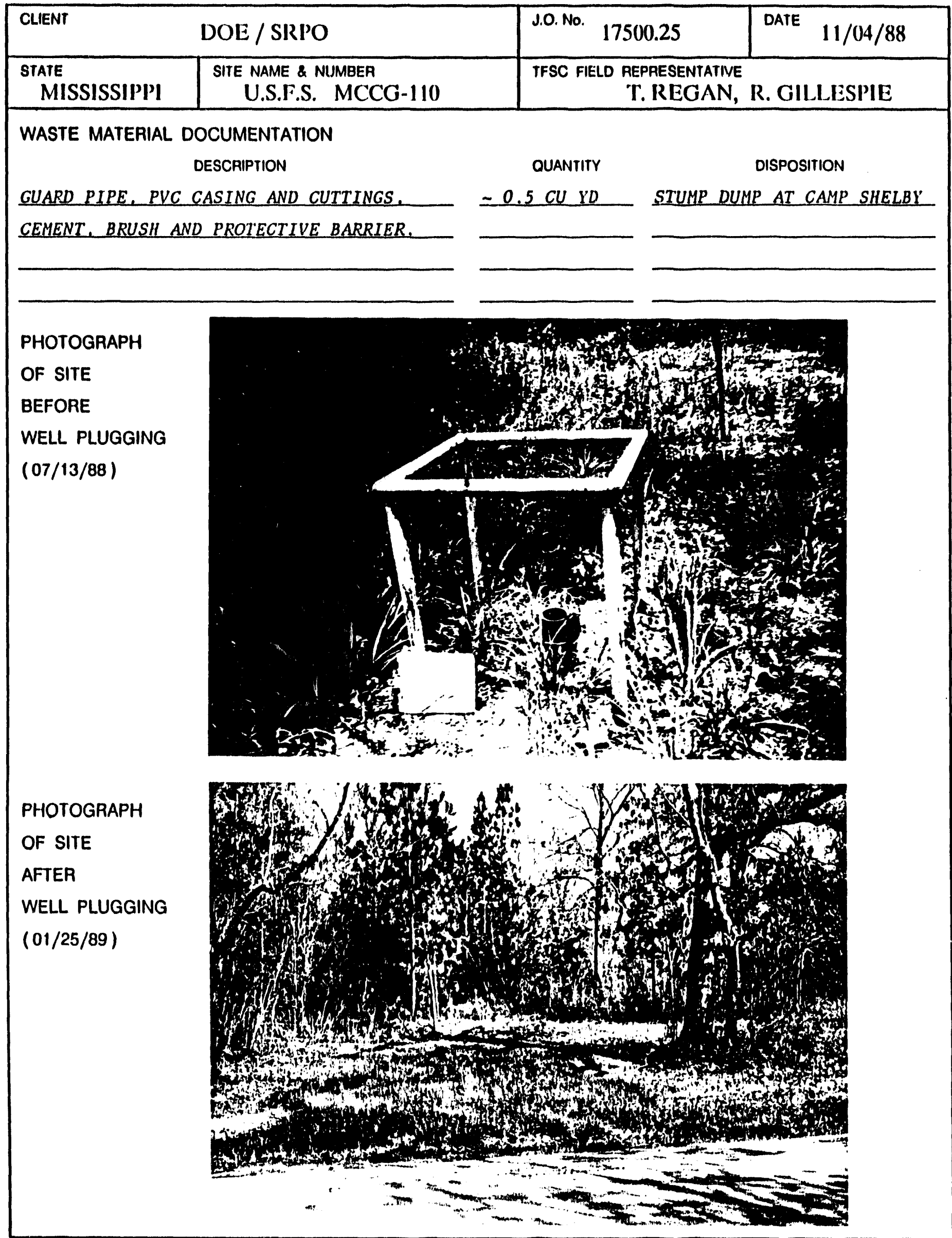


SITE RESTORATION FORM

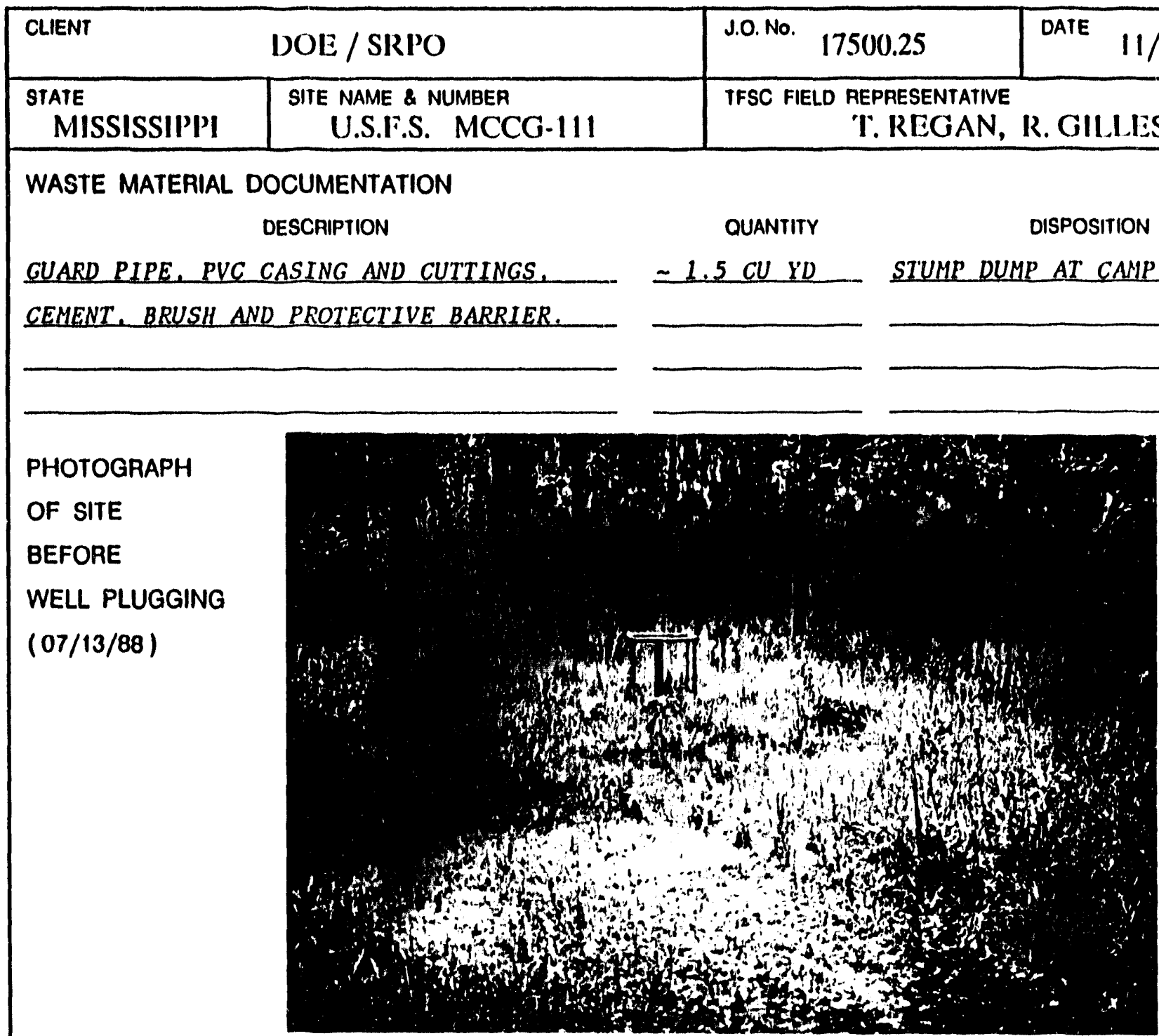

PHOTOGRAPH

OF SITE

AFTER

WELL PLUGGING

$(01 / 26 / 89)$

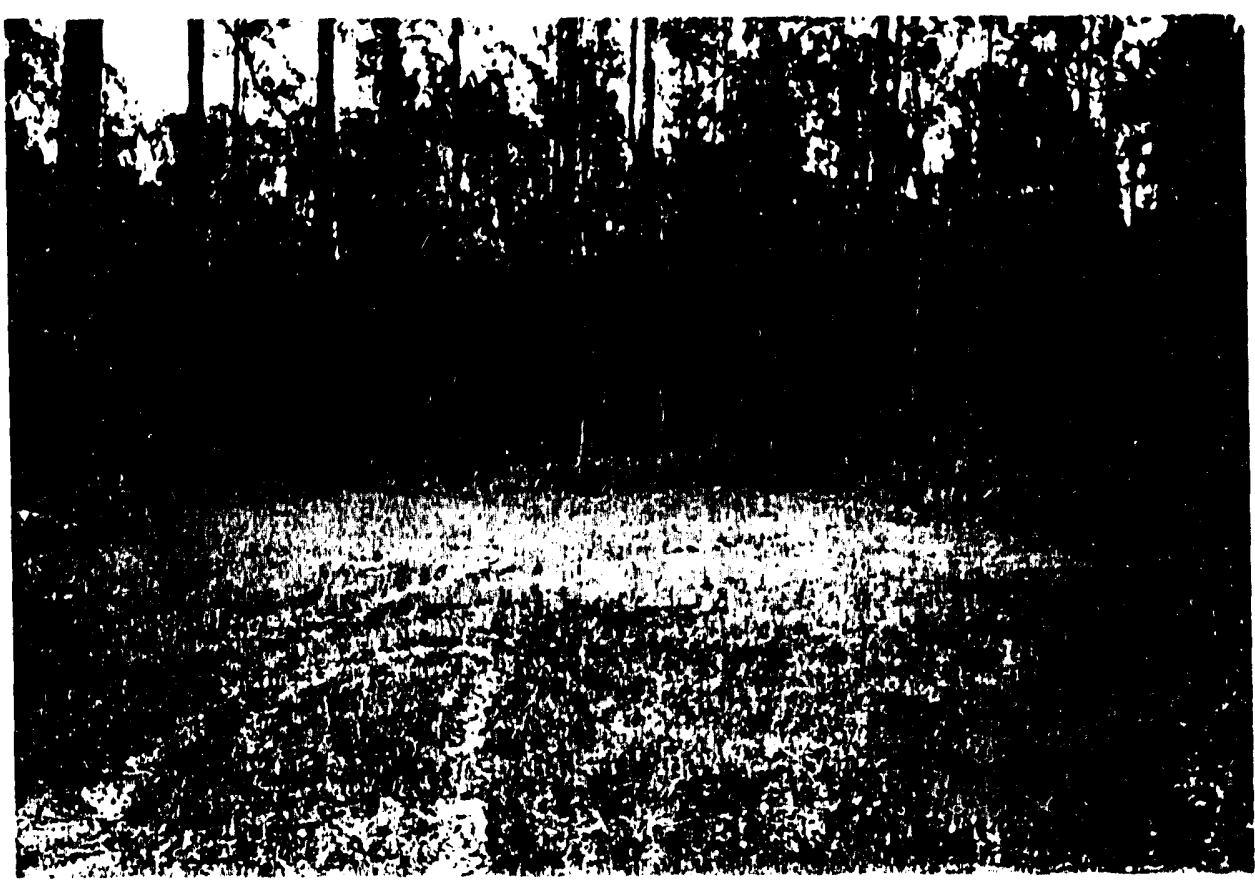


SITE RESTORATION FORM

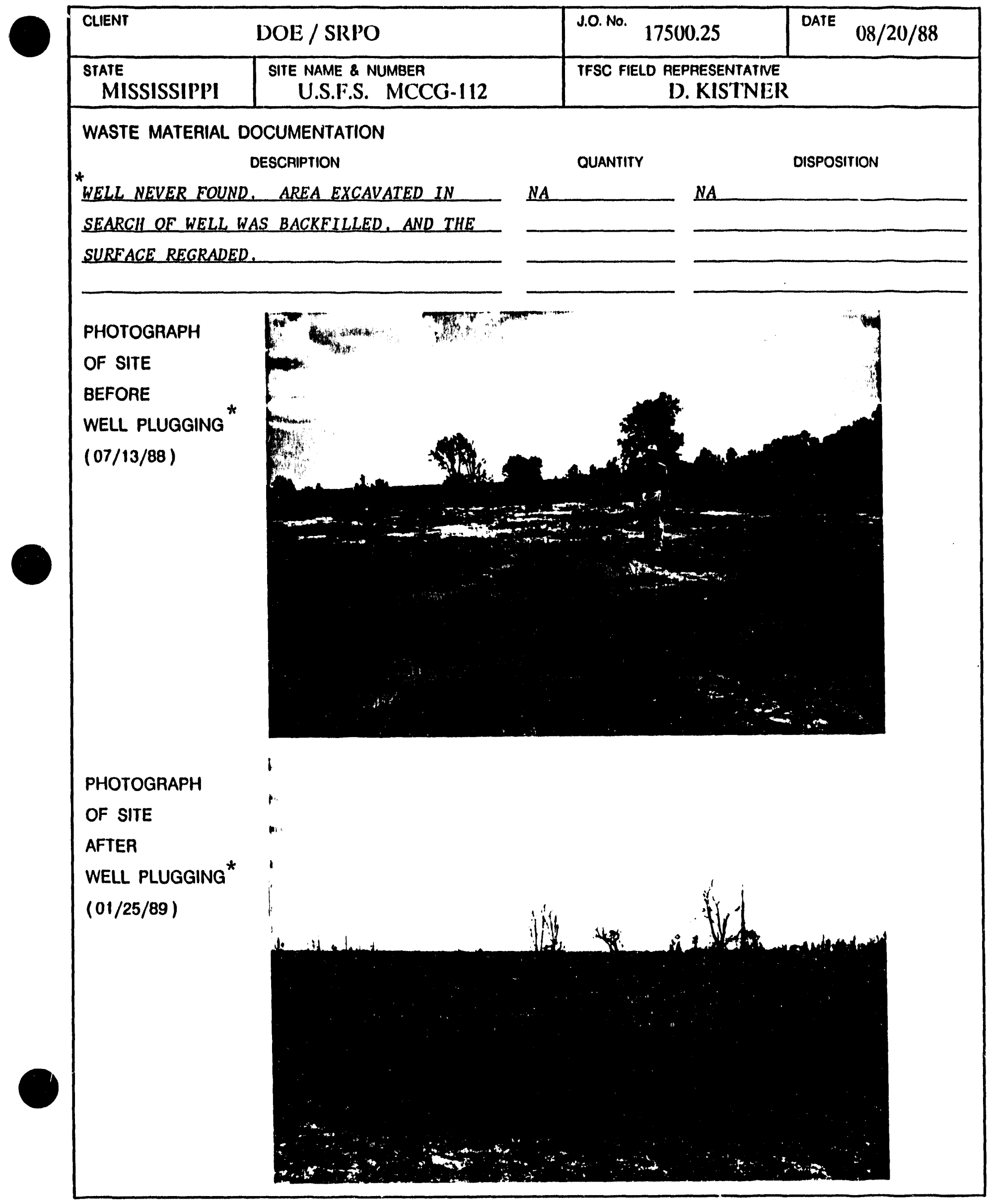




\section{SITE RESTORATION FORM}

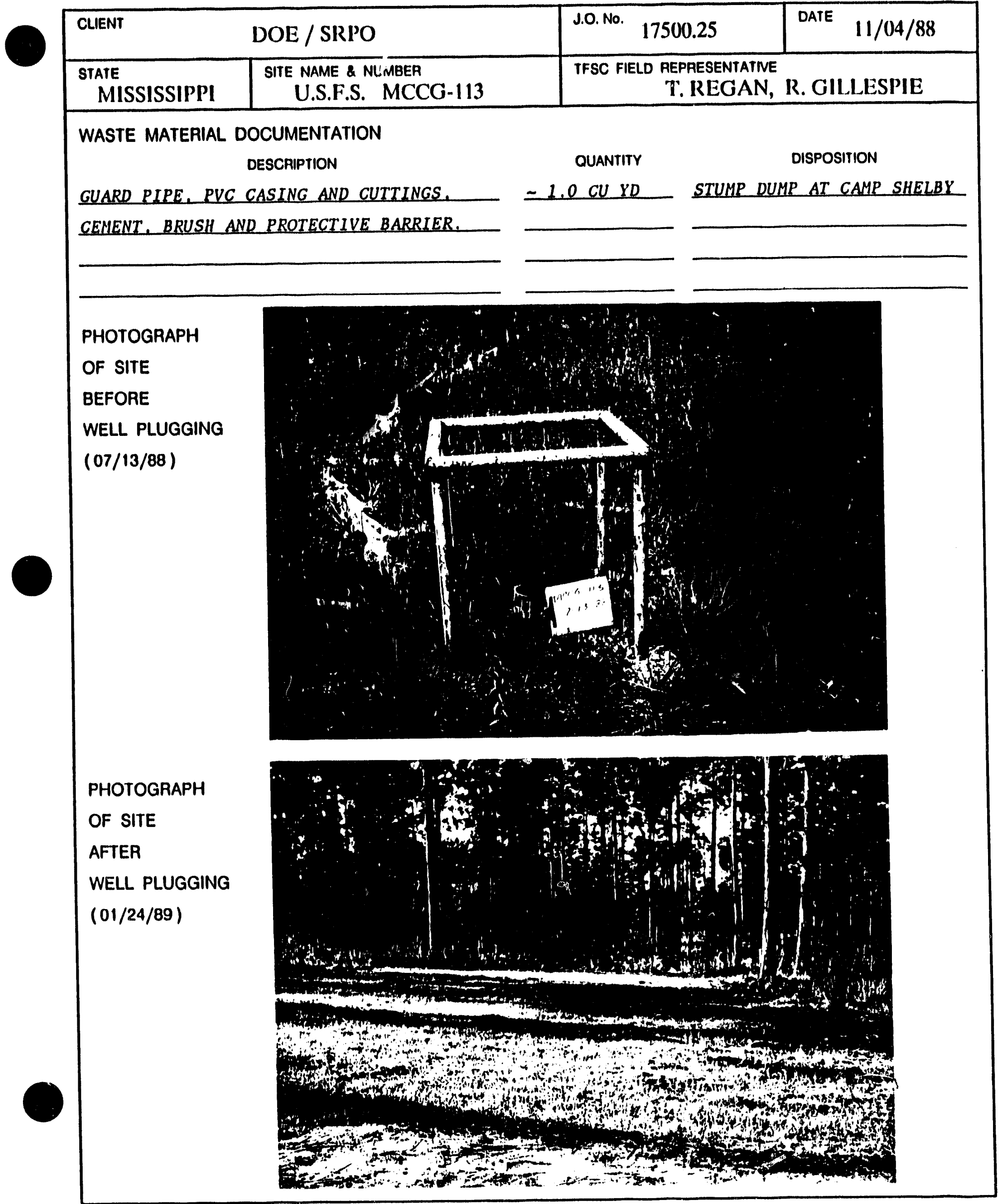


SITE RESTORATION FORM

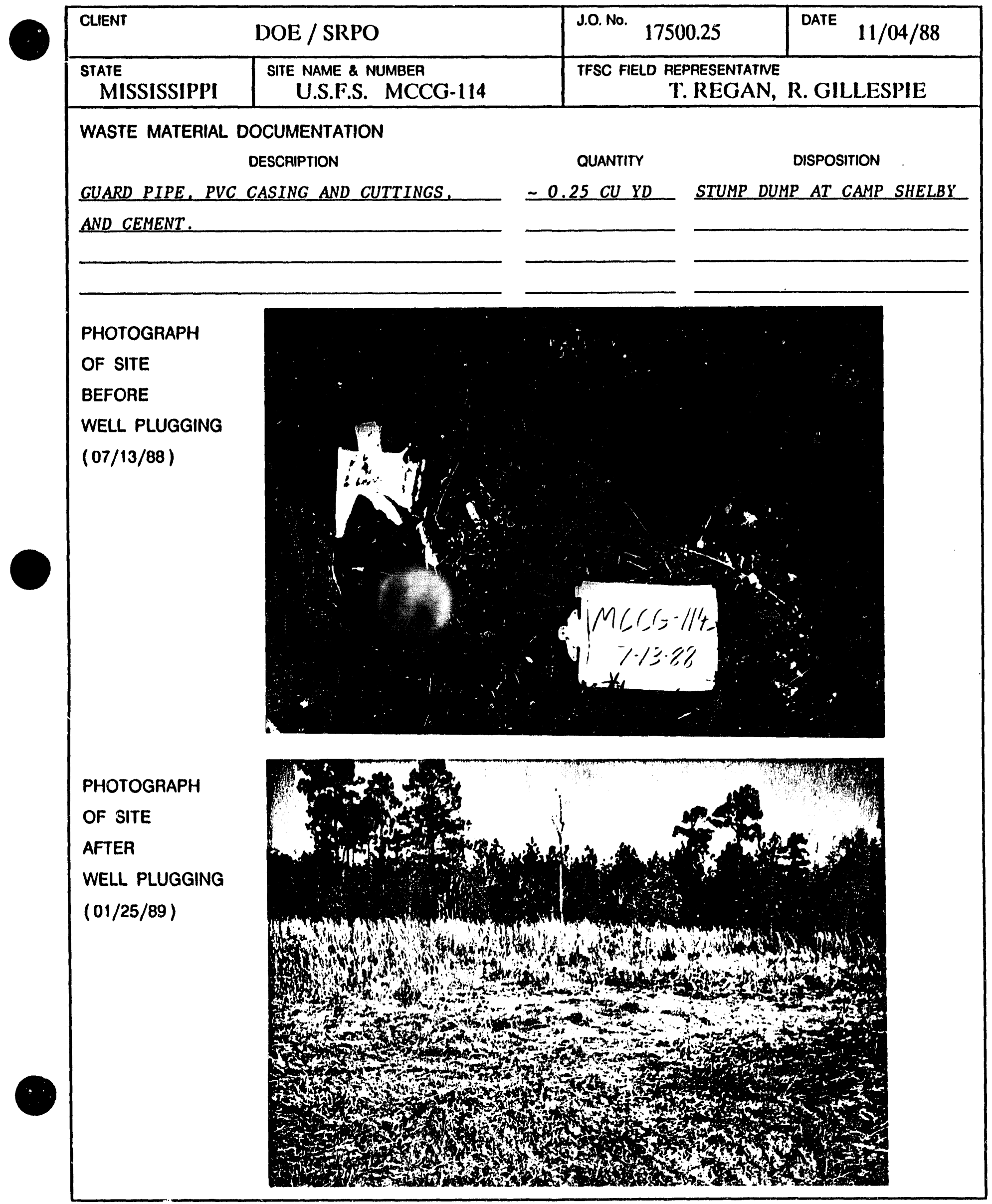


SITE RESTORATION FORM

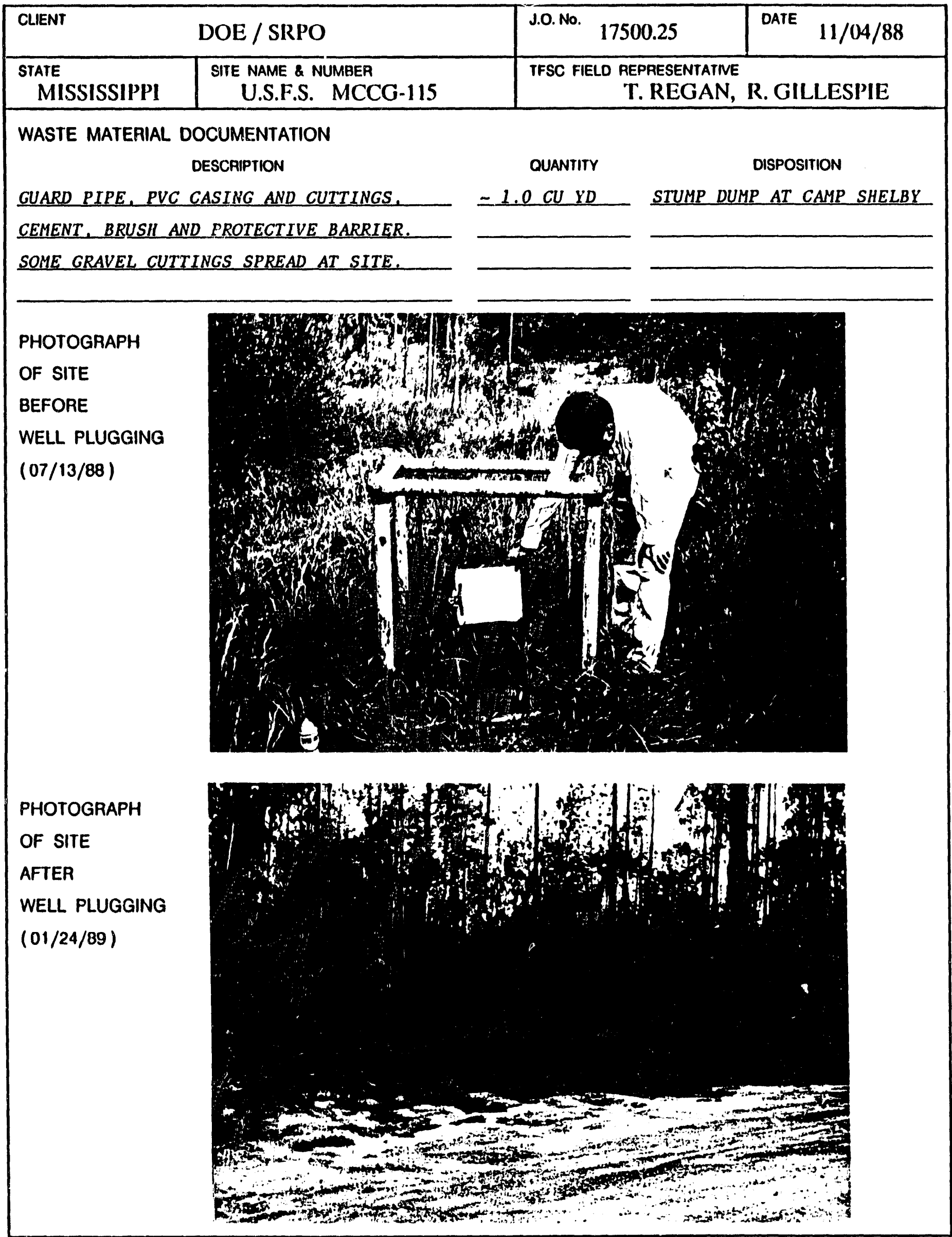




\section{SITE RESTORATION FORM}

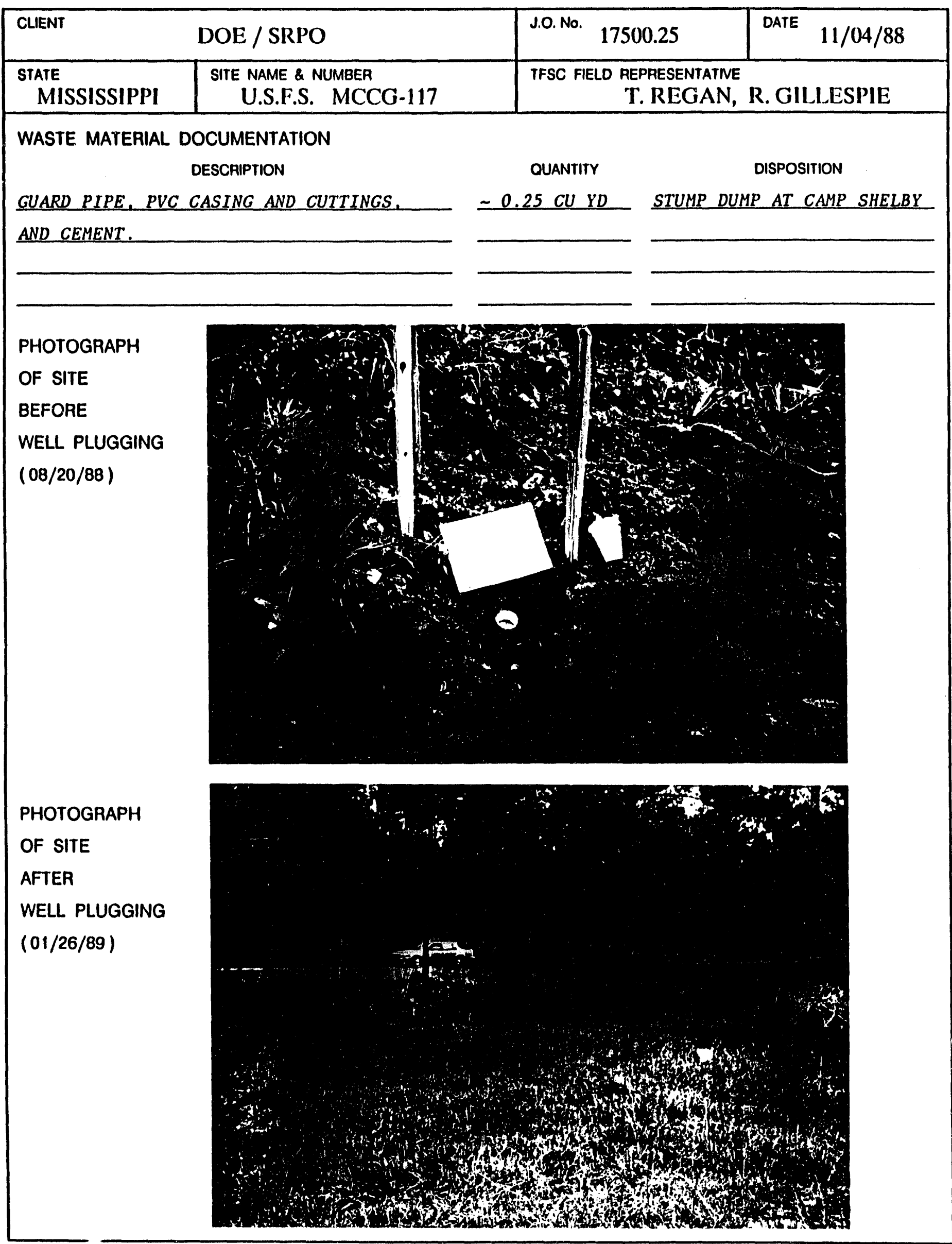


SITE RESTORATION FORM

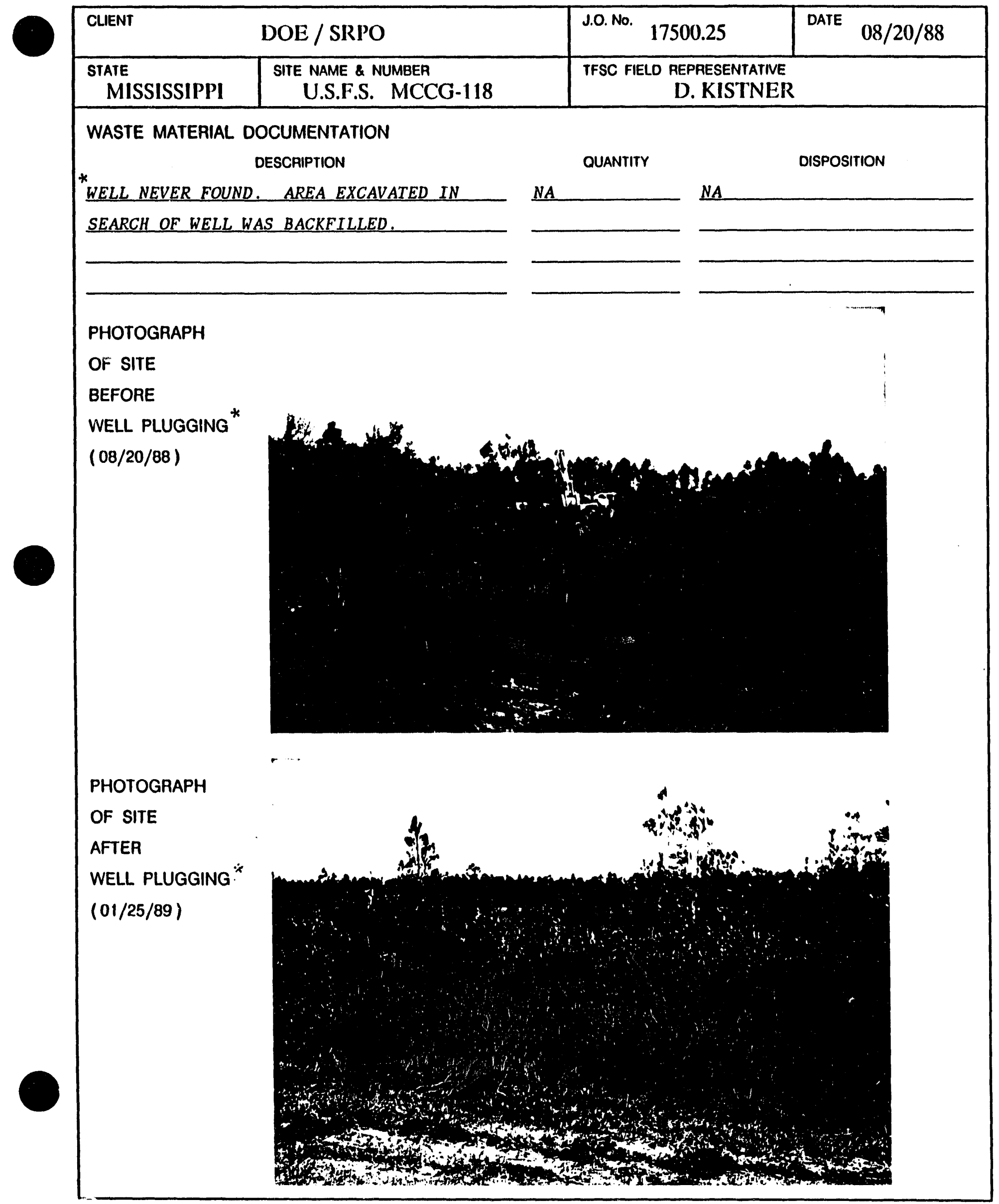




\section{SITE RESTORATION FORM}

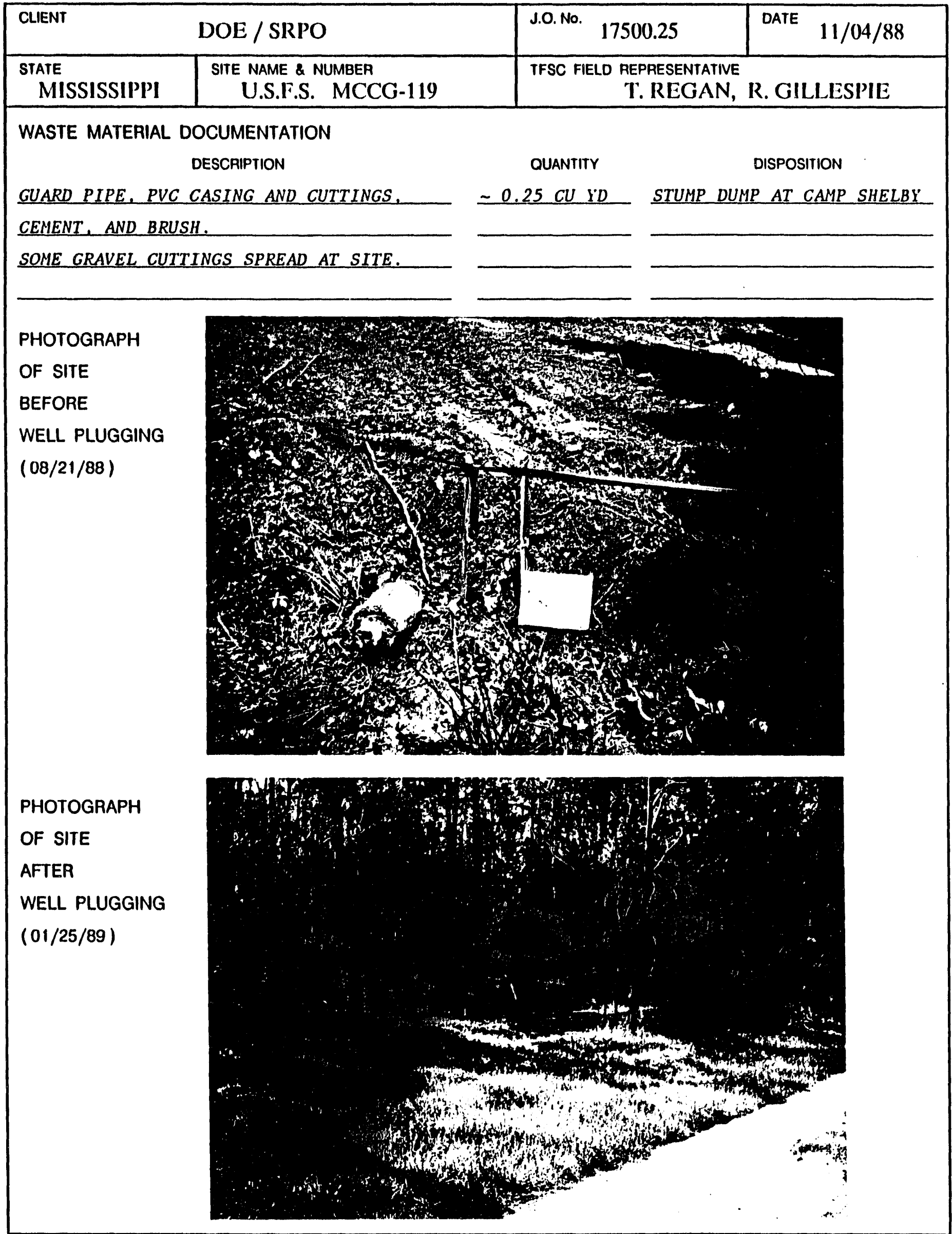




\section{SITE RESTORATION FORM}

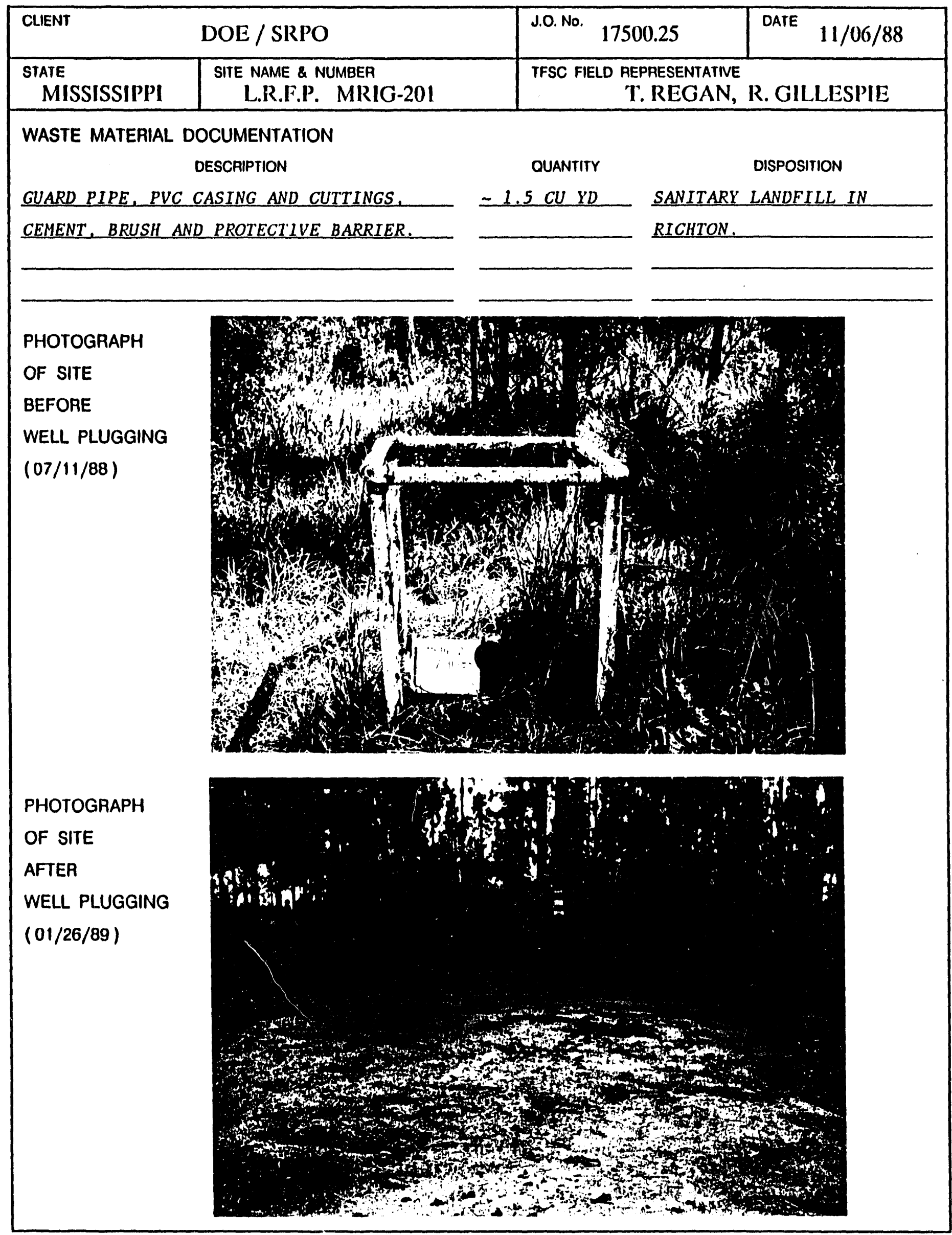


SITE RESTORATION FORM

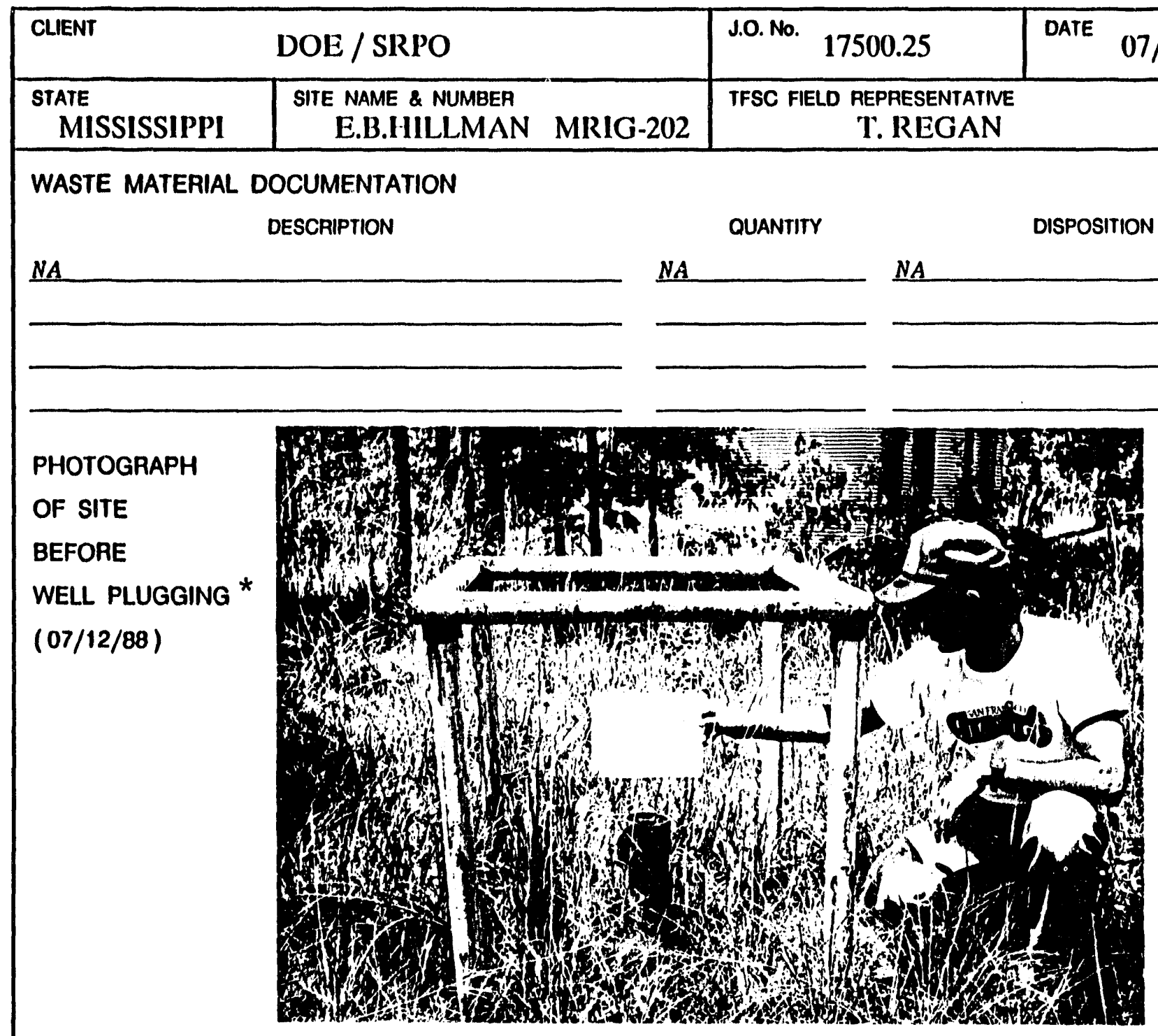

* ThIS WELL WAS NOT PLUGGED;

OWNERSHIP OF THE WELL WAS TRANSFERRED

TO THE CURRENT LANDOWNER IN THE CONDITION

SHOWN IN THE ABOVE PHOTOGRAPH. 


\section{SITE RESTORATION FORM}

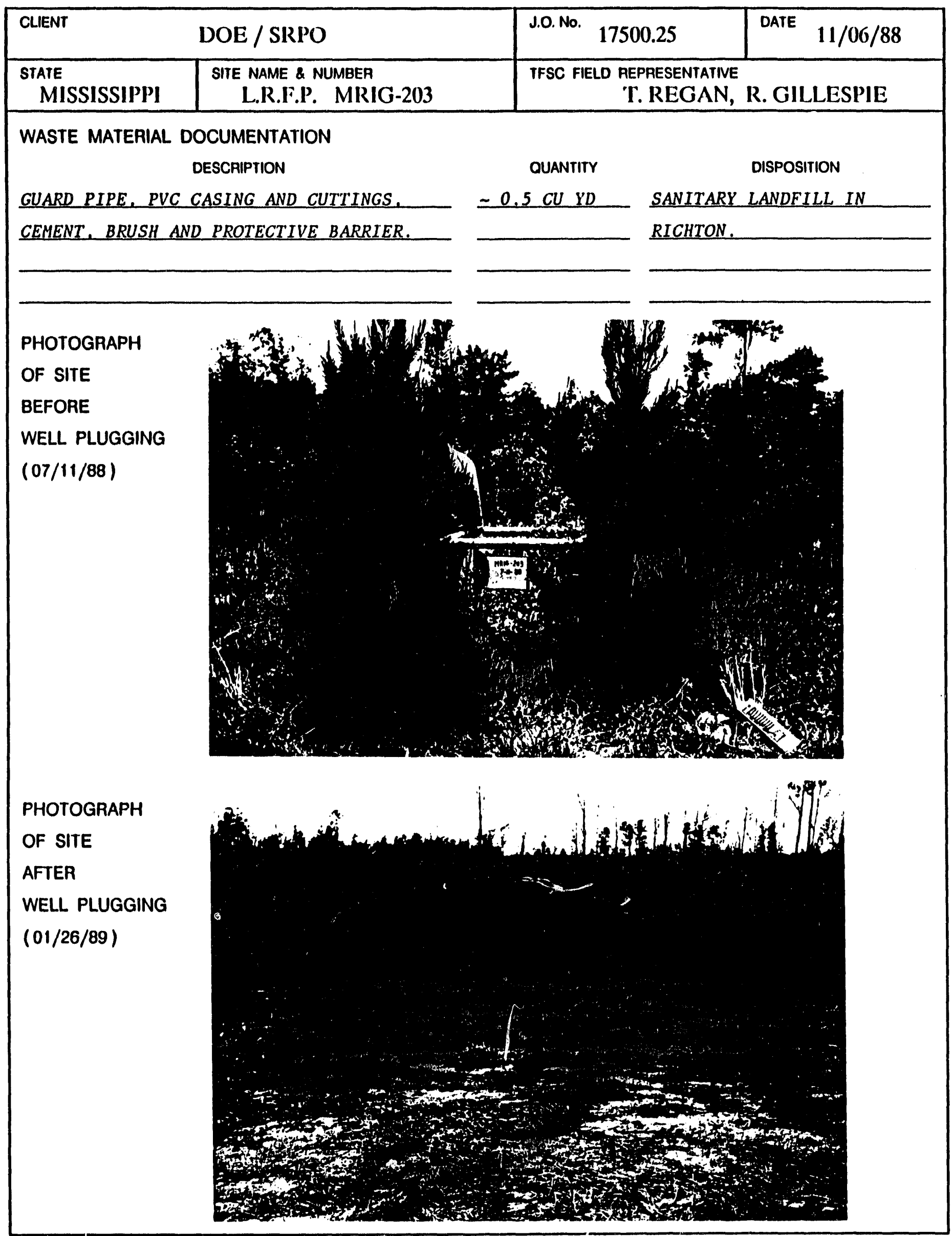




\section{SITE RESTORATION FORM}

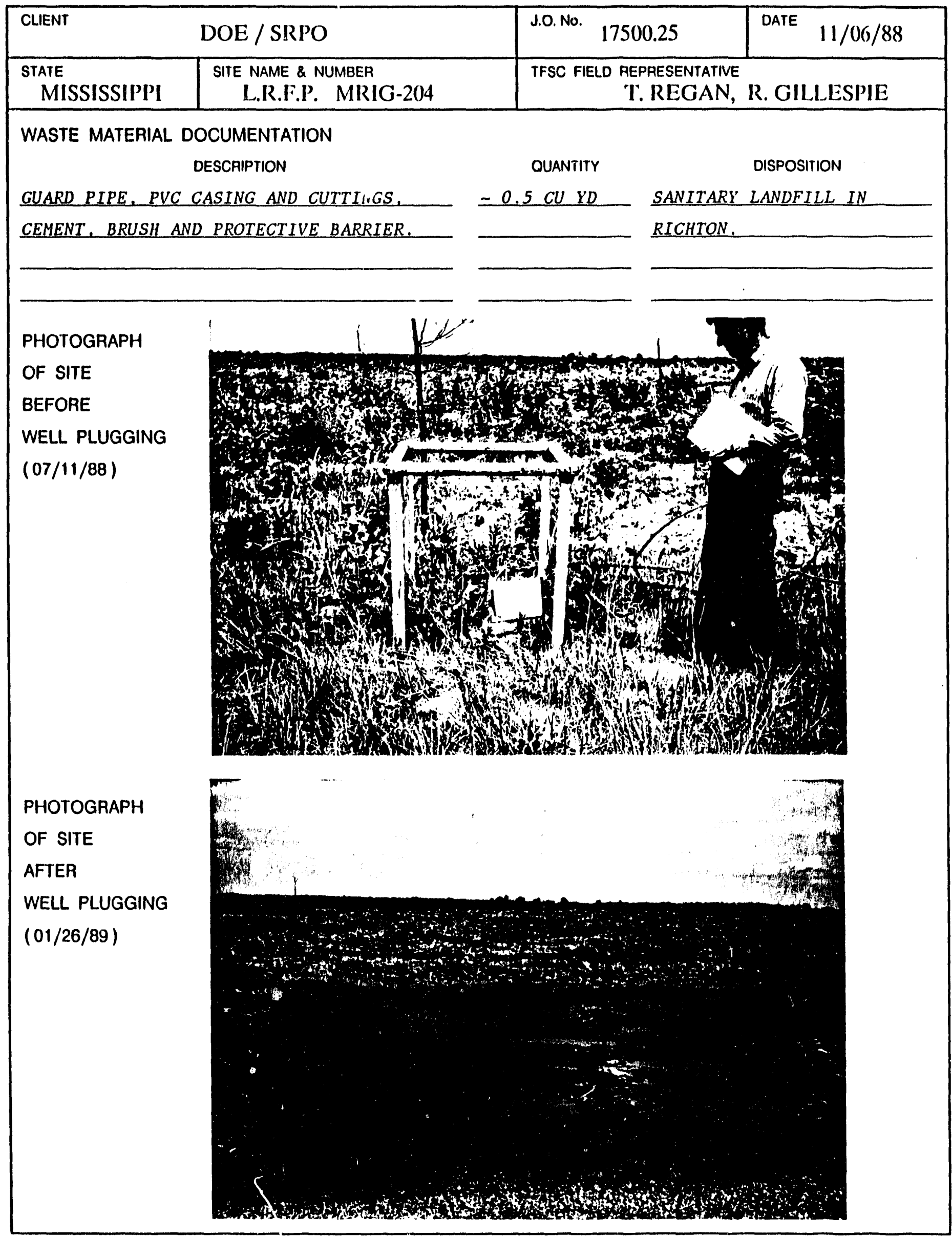




\section{SITE RESTORATION FORM}

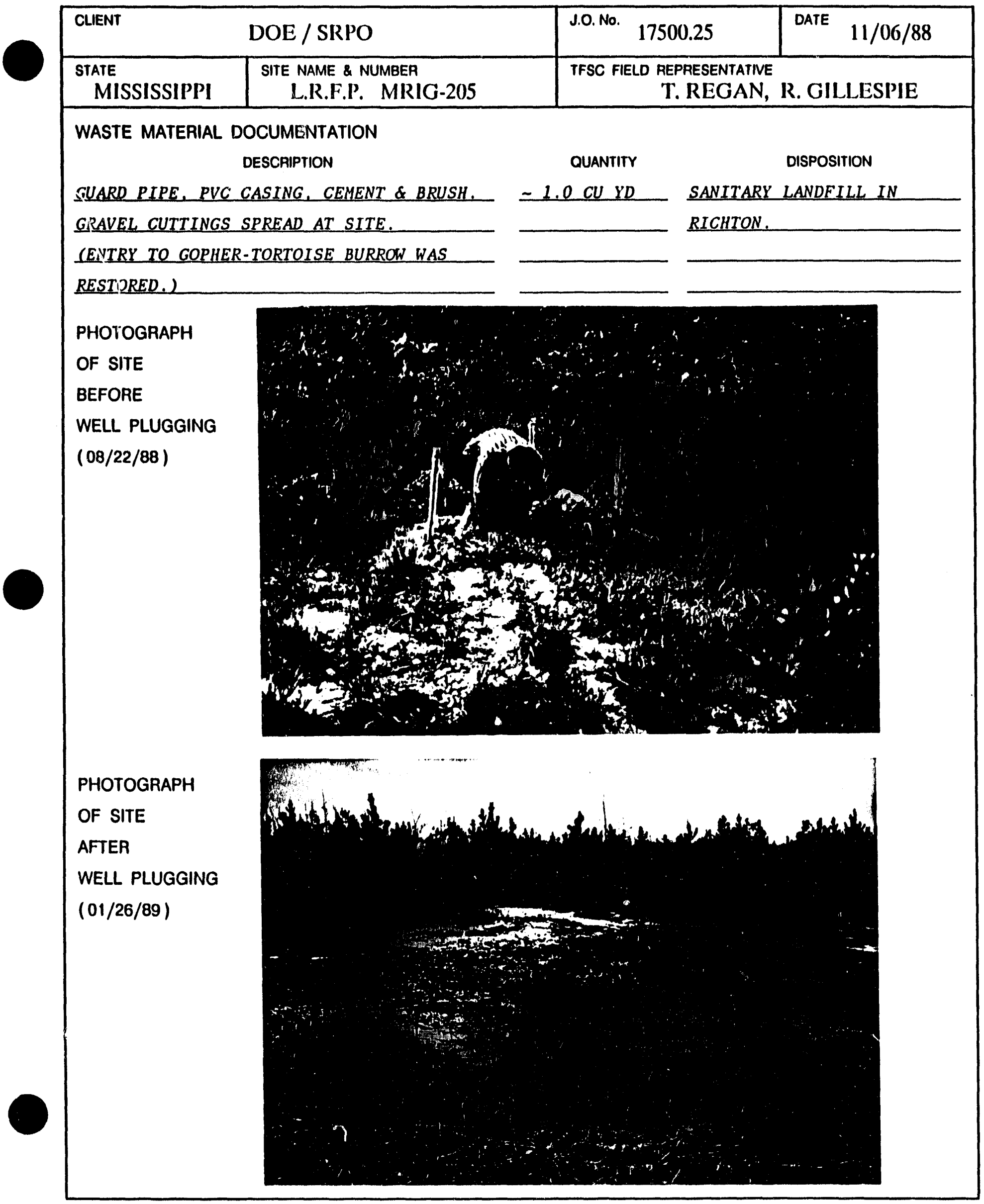


SITE RESTORATION FORM

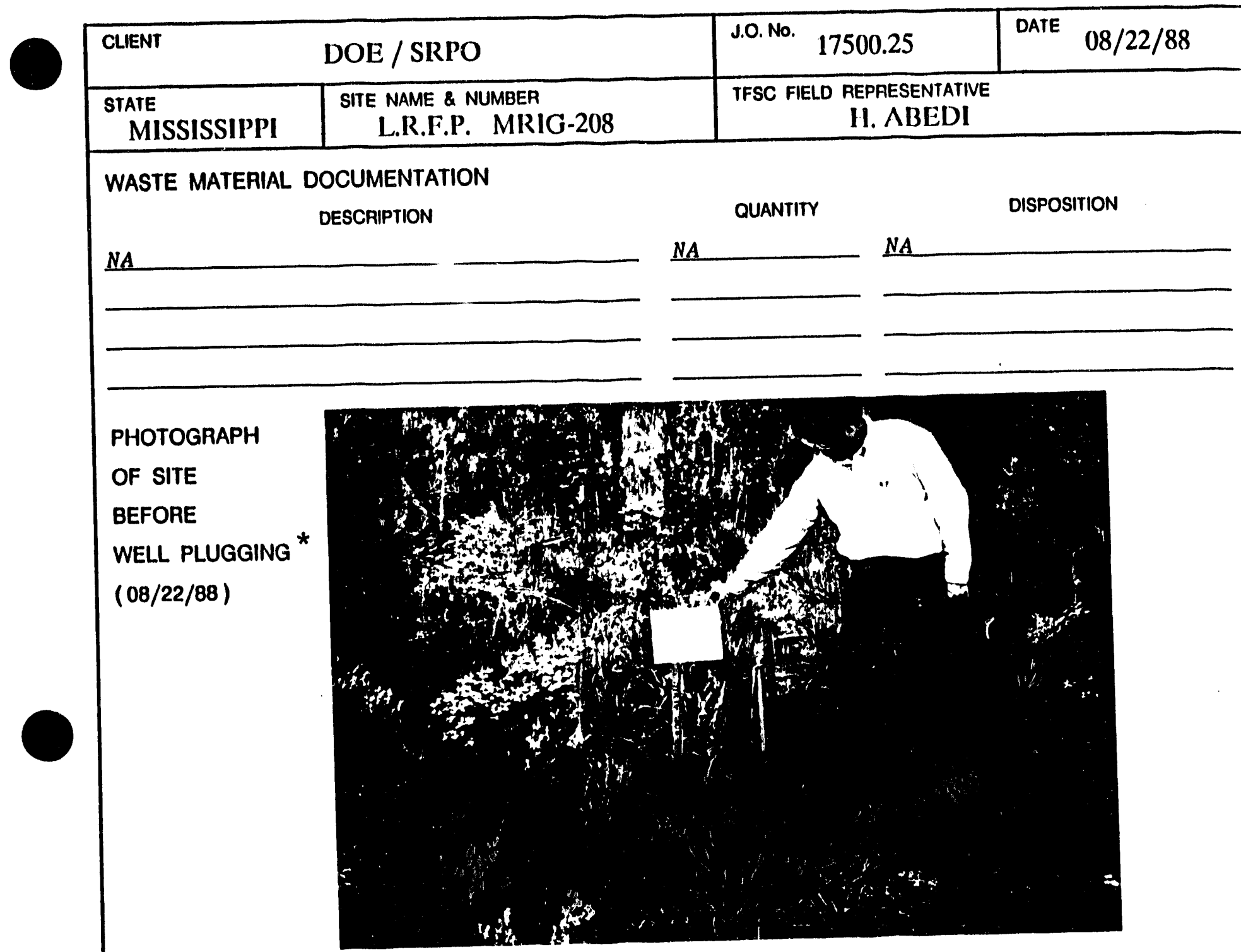

* BOREHOLE WAS BACKFILLED BY LETCO IN 1979. THERE WAS NO PLUGGING OR RESTORATION WORK PERFORMED AT THIS SITE 


\section{SITE RESTORATION FORM}

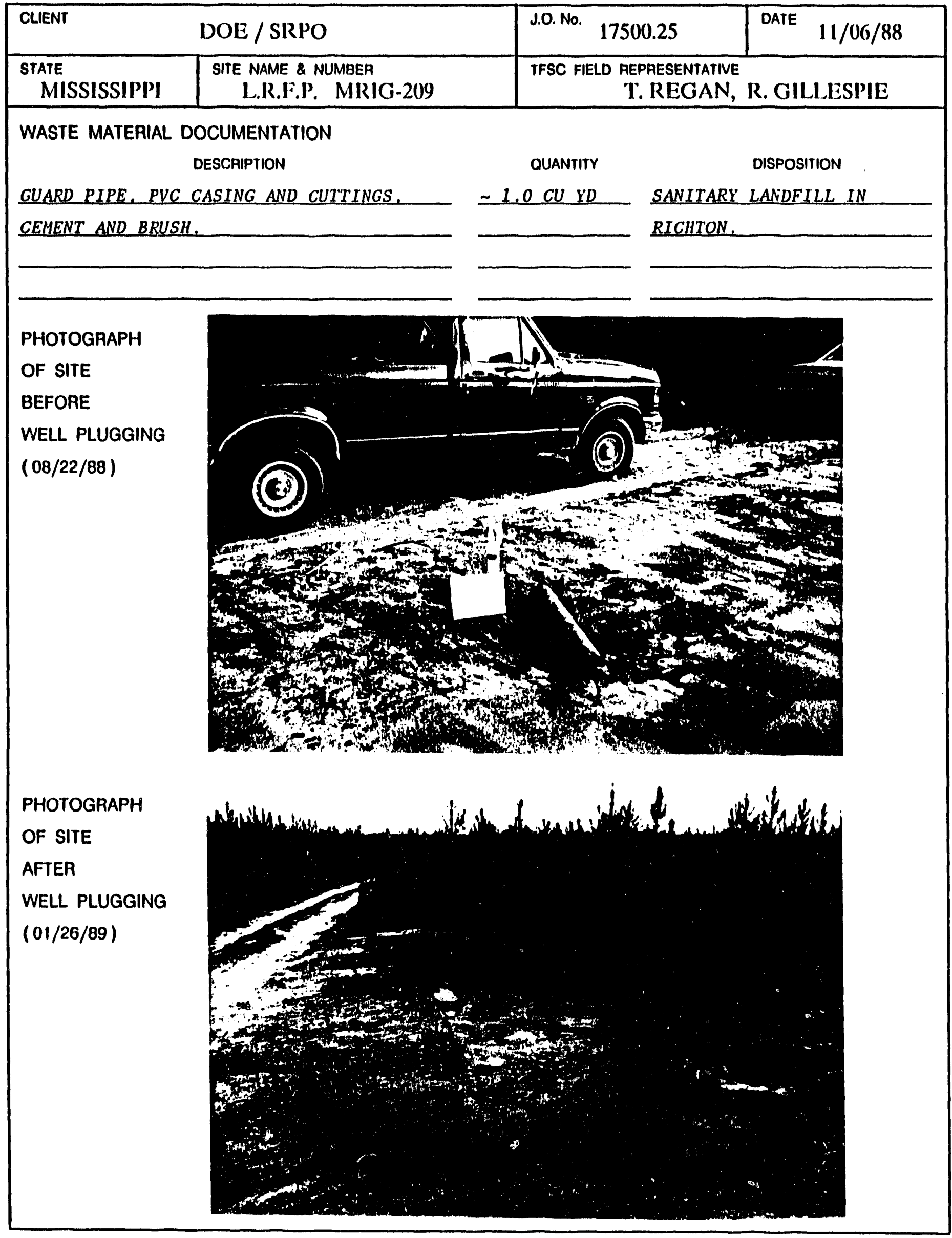




\section{SITE RESTORATION FORM}

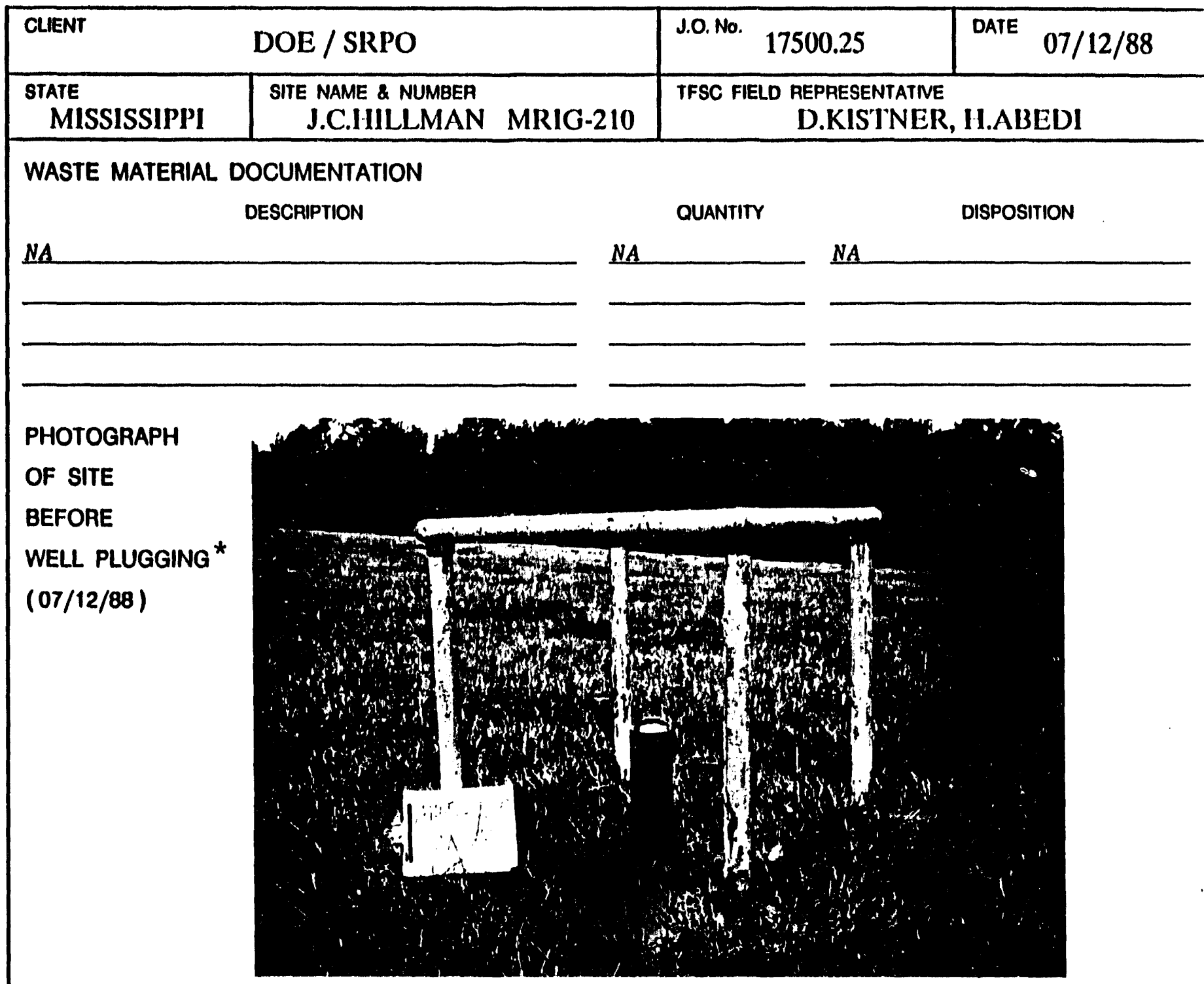

* THIS WELL WAS NOT PLUGGED;

ONNERSHIP OF THE WELL WAS TRANSFERRED

TO THE CURRENT LANDOWNER IN THE CONDITION

SHOWN IN THE ABOVE PHOTOGRAPH. 
SITE RESTORATION FORM

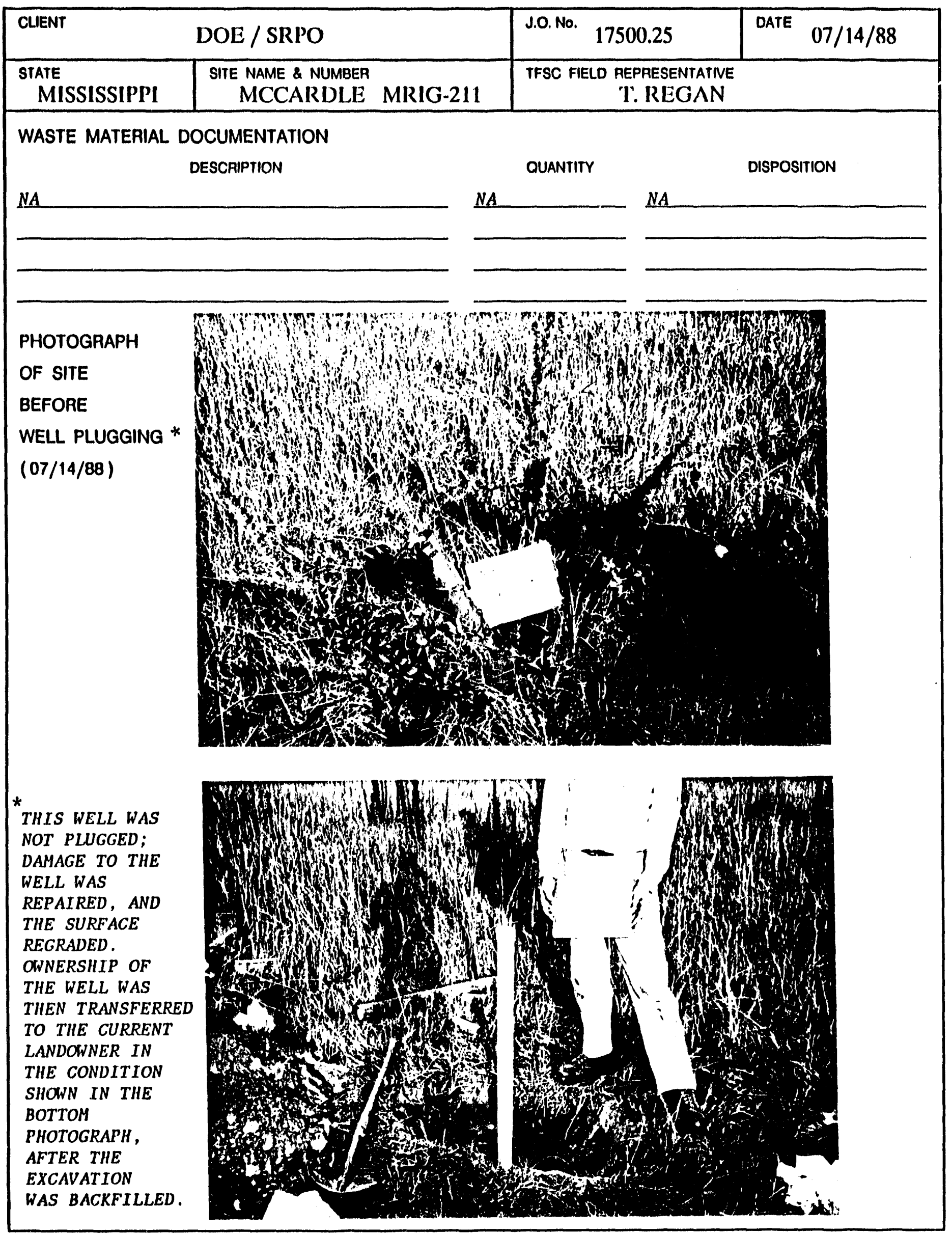




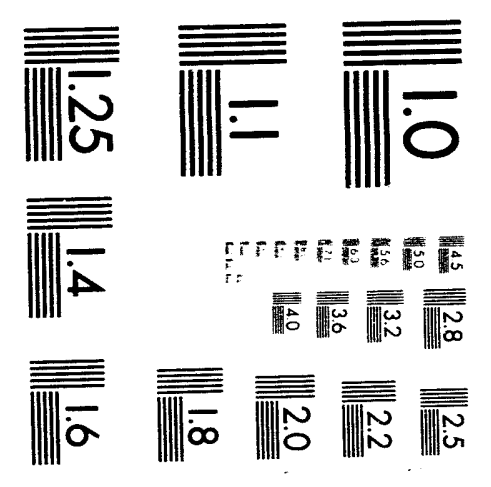



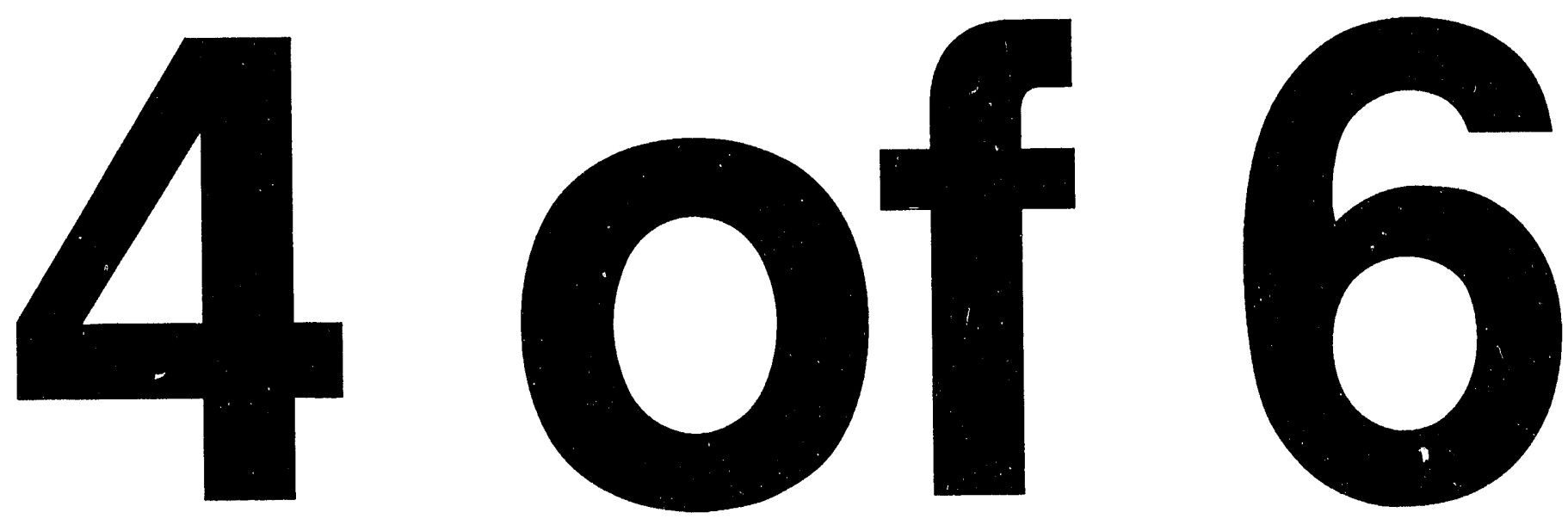


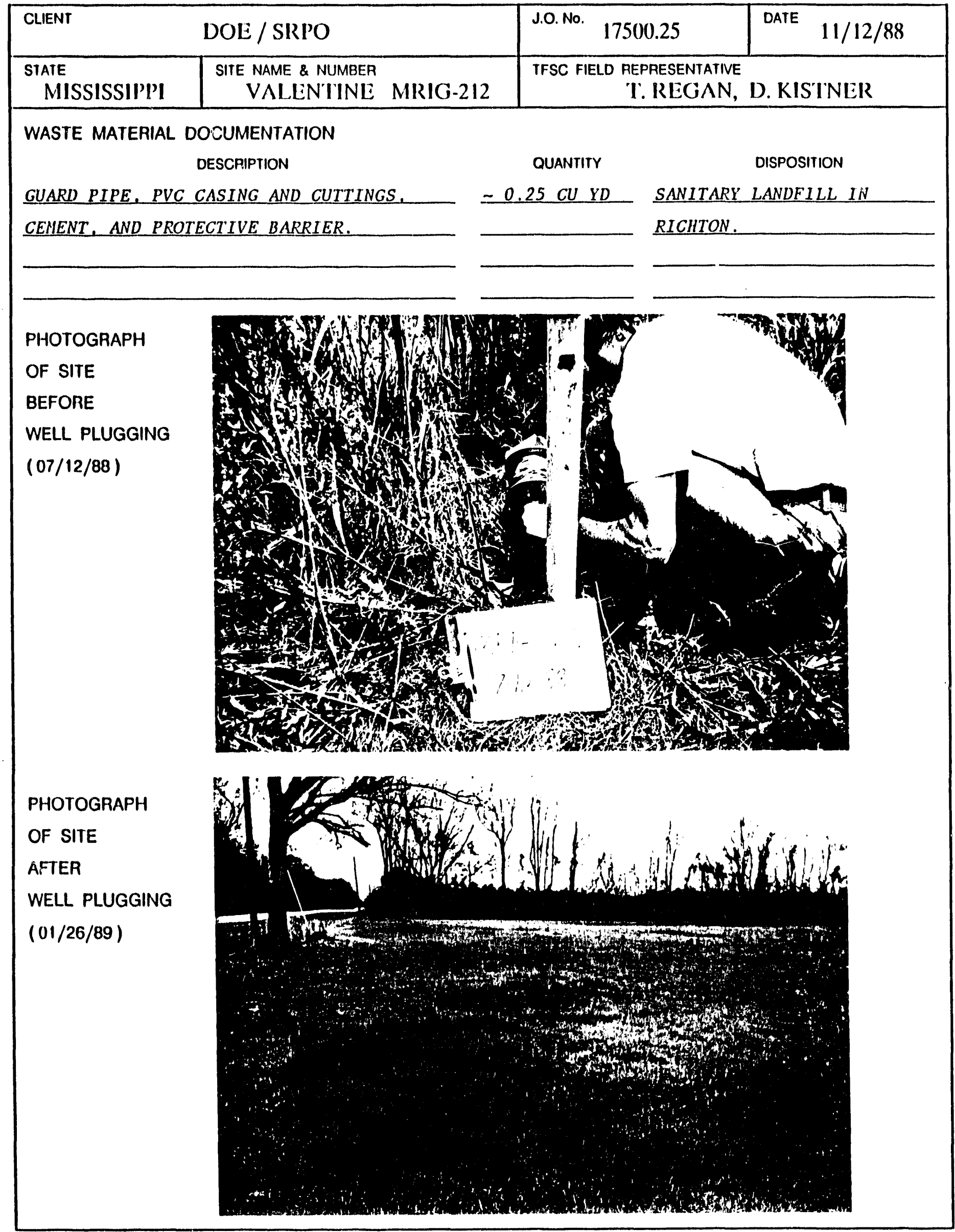




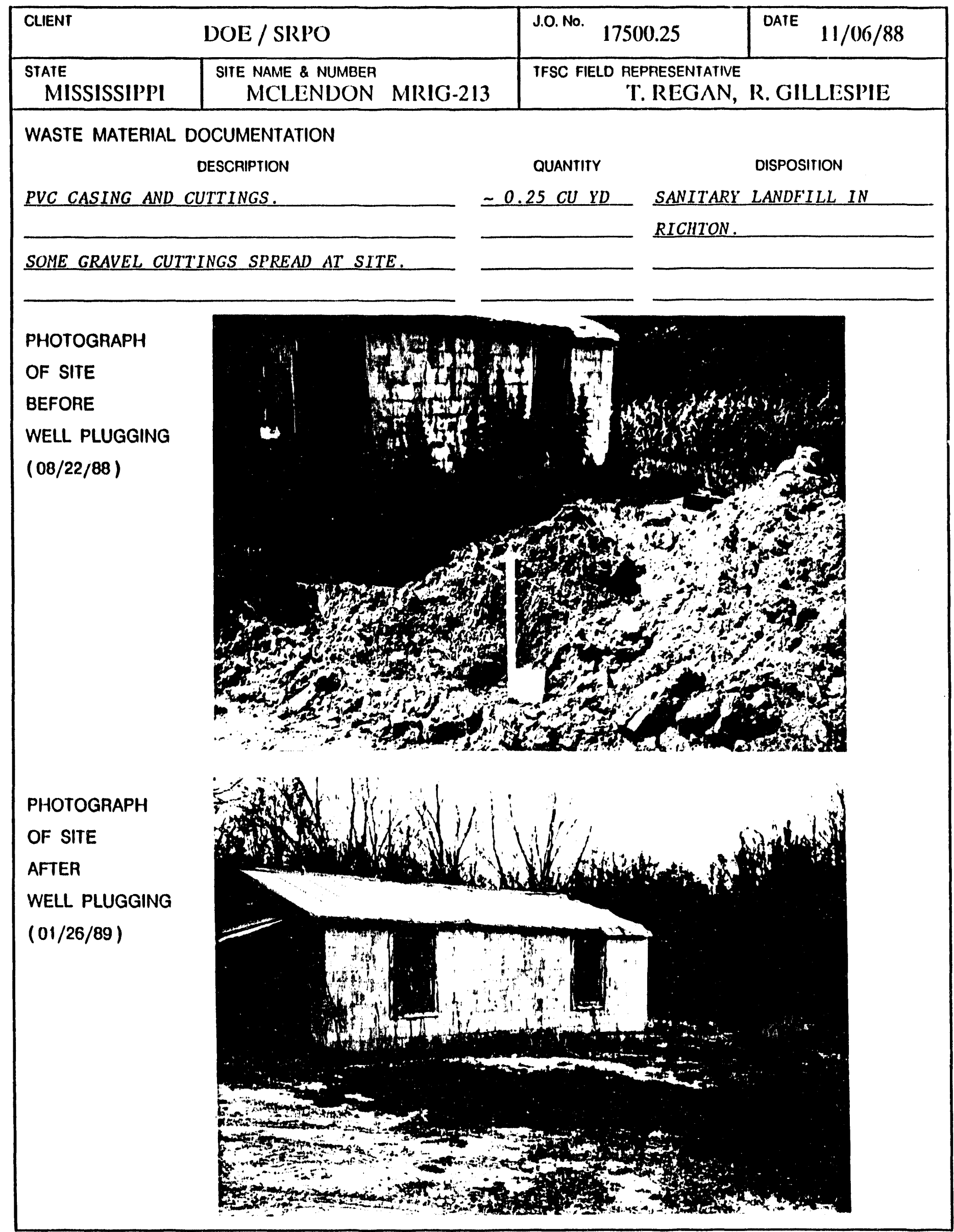


SITE RESTORATION FORM

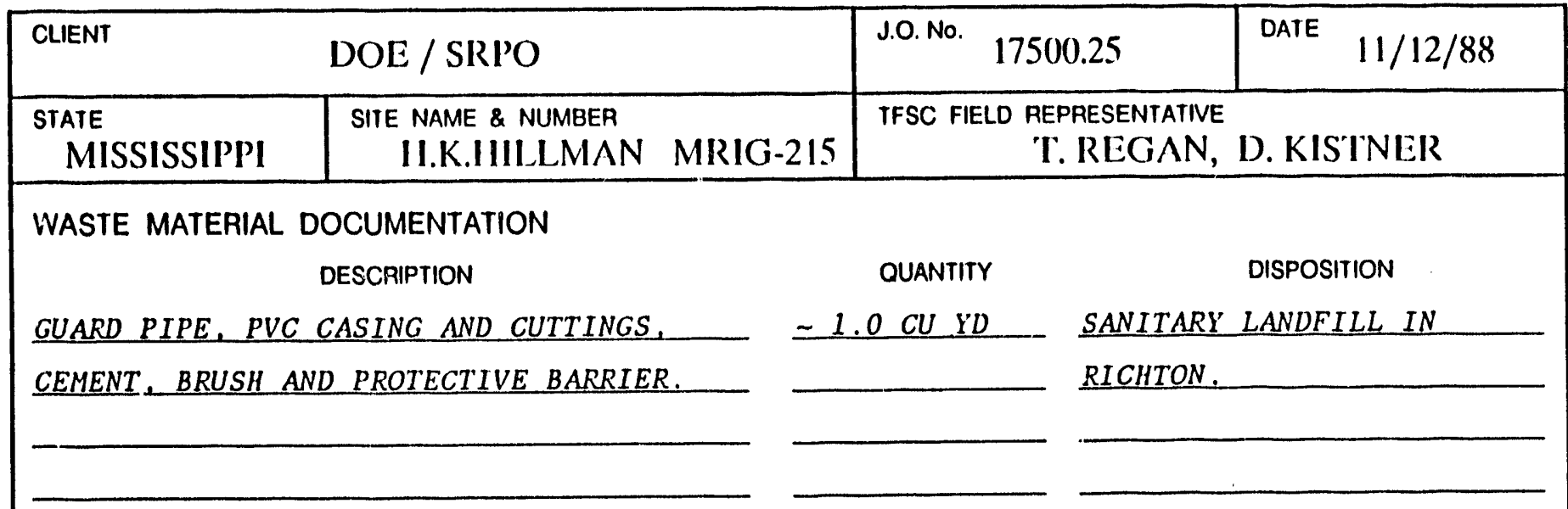

PHOTOGRAPH

OF SITE

BEFORE

WELL PLUGGING

$(07 / 12 / 88)$

PHOTOGRAPH

OF SITE

AFTER

WELL PLUGGING

$(01 / 26 / 89)$
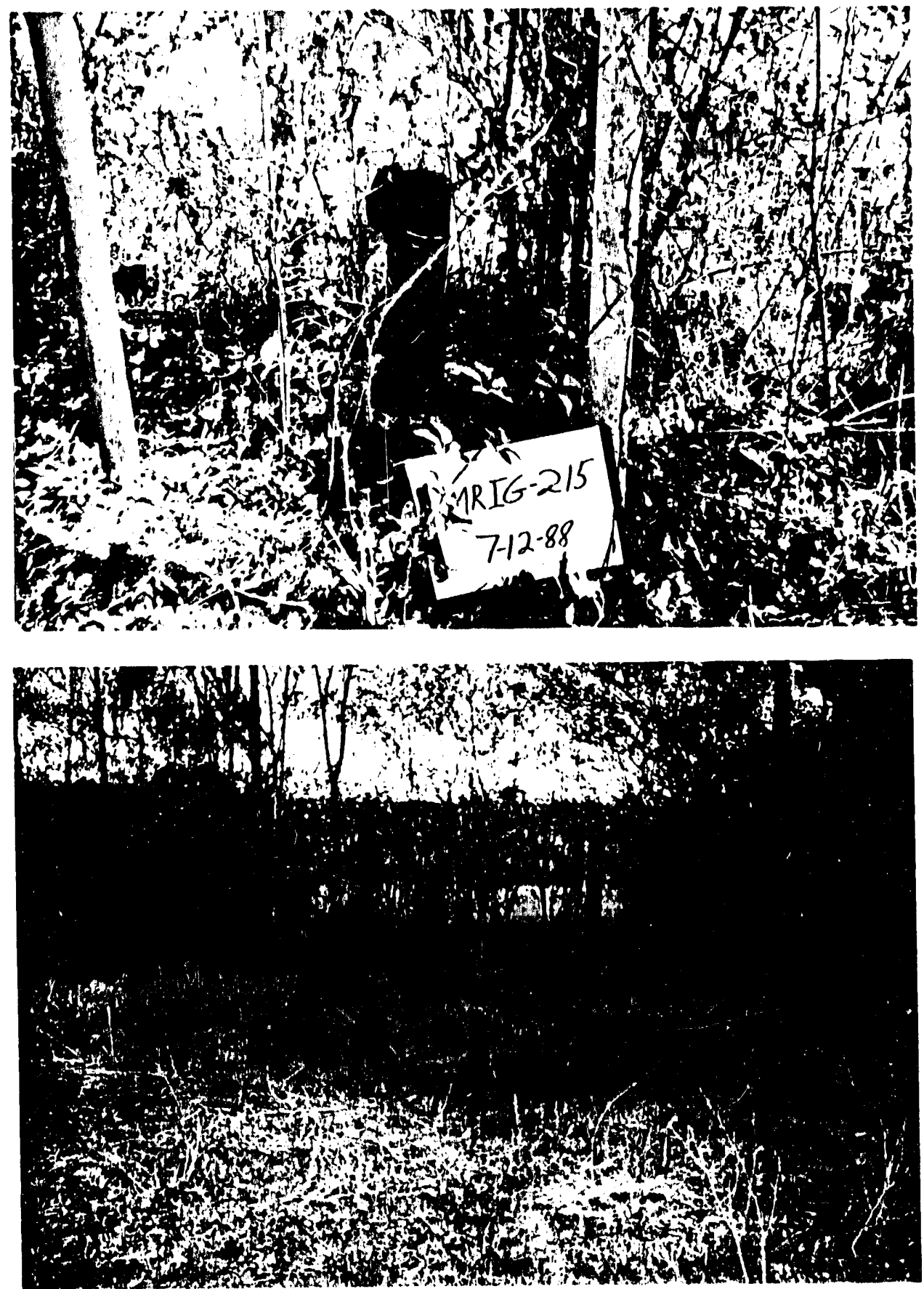


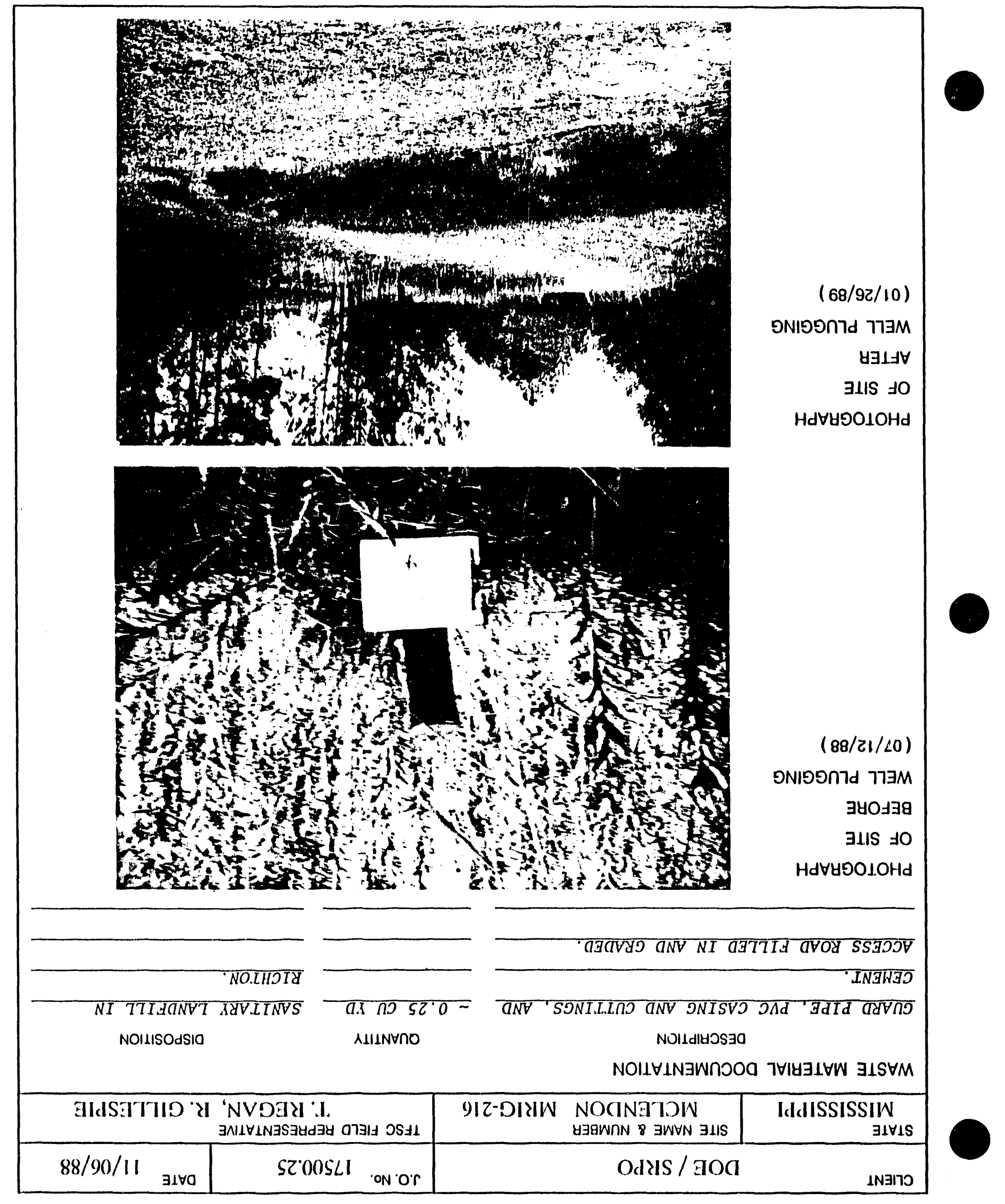




\section{SITE RESTORATION FORM}

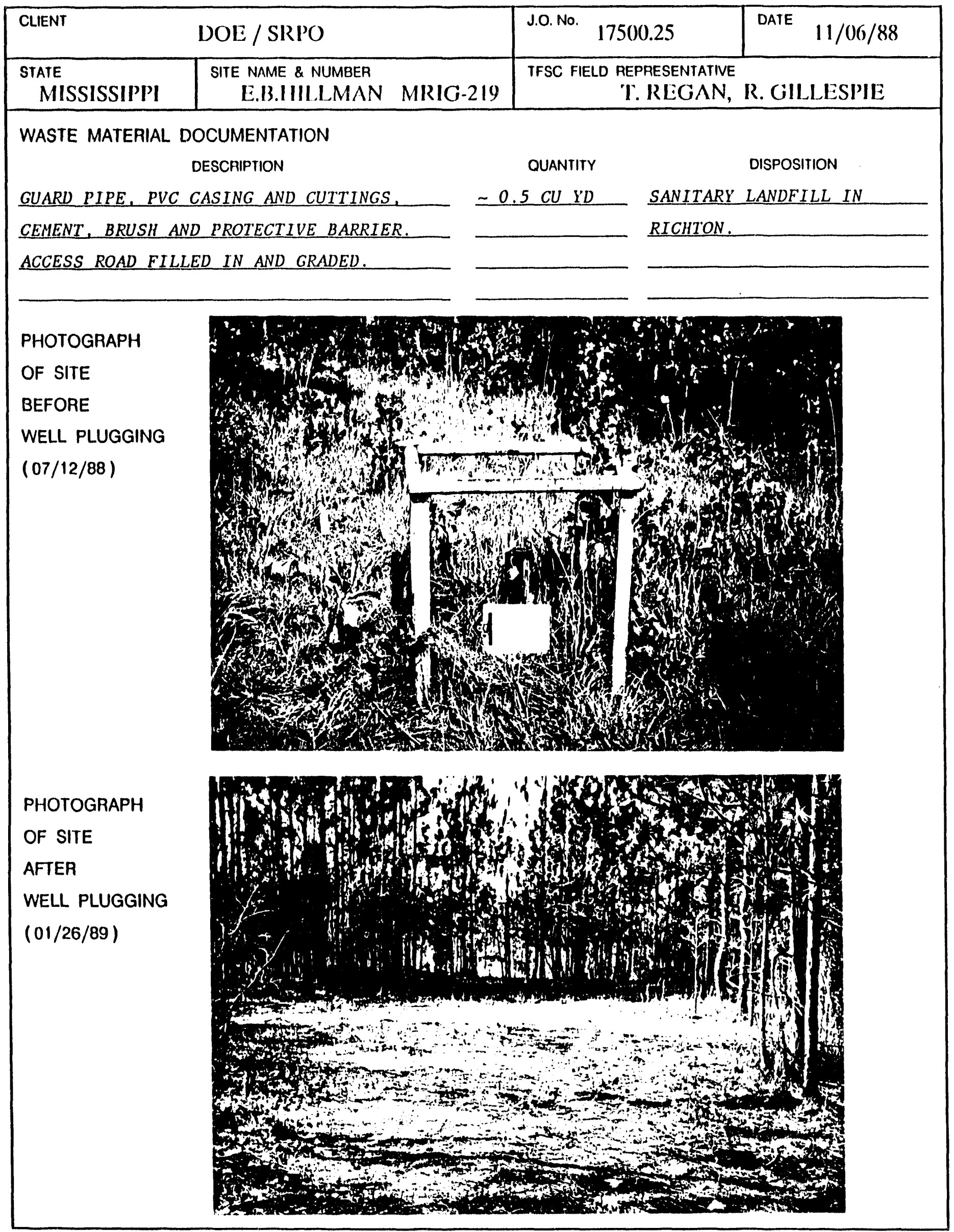




\section{SITE RESTORATION FORM}

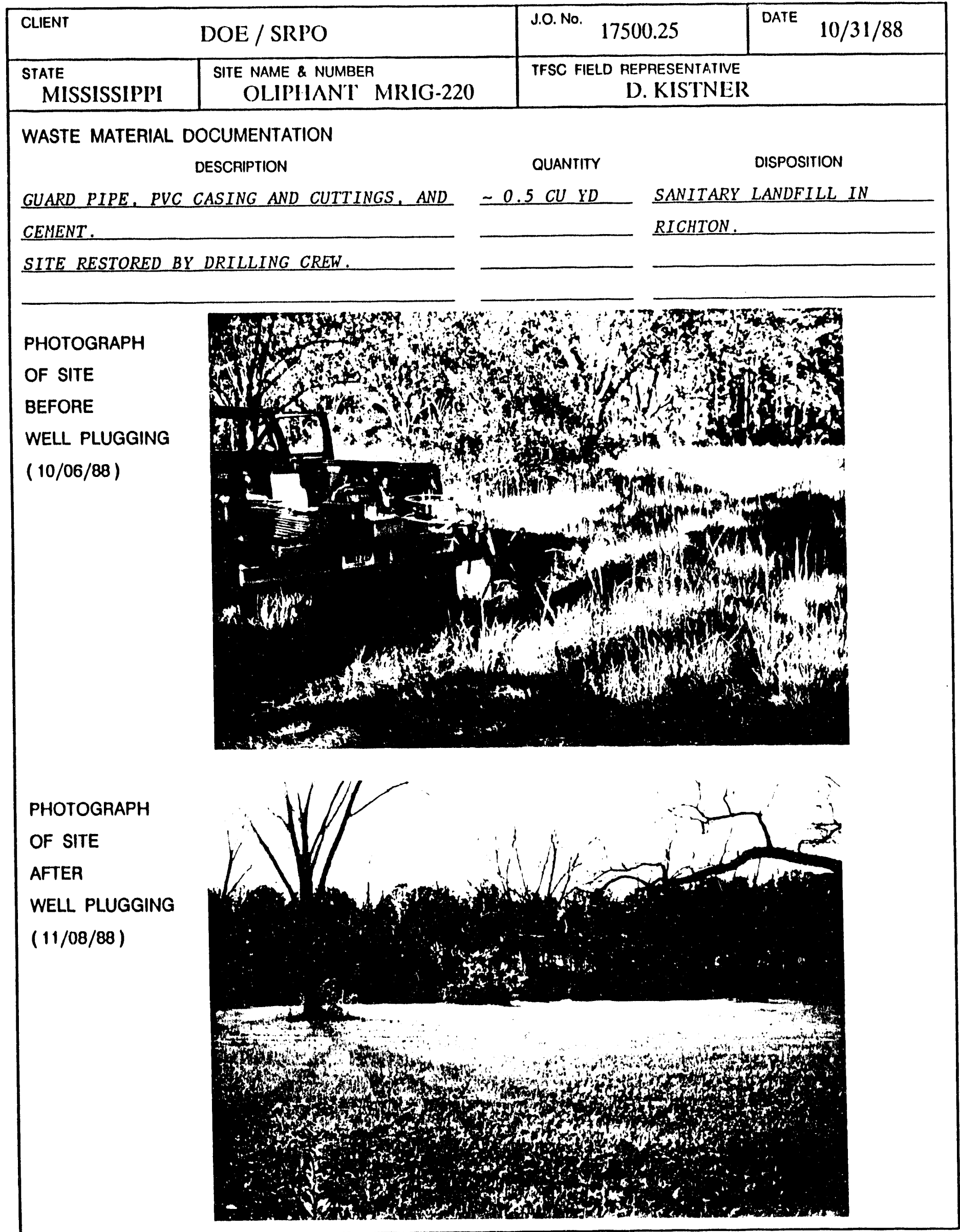




\section{SITE RESTORATION FORM}

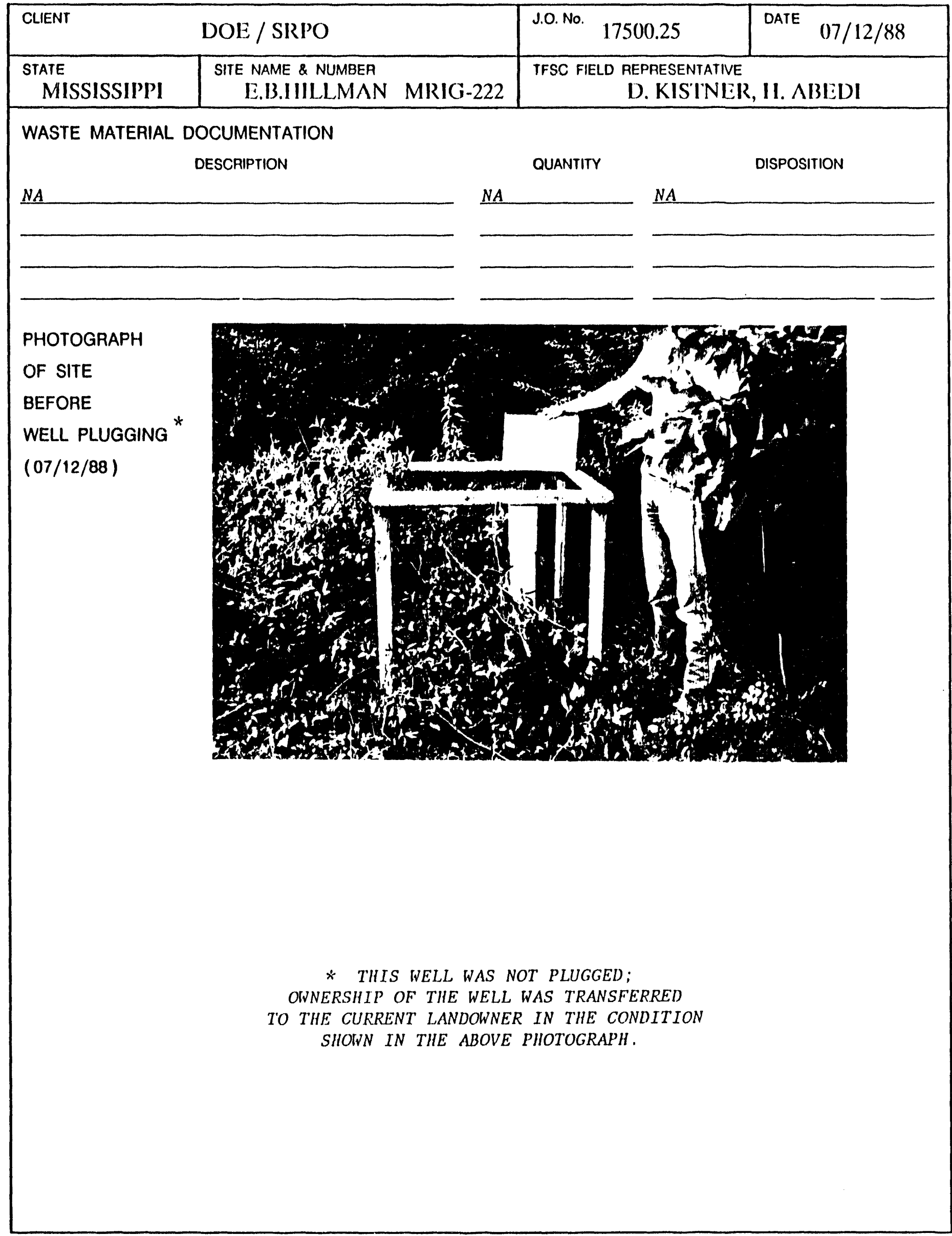


SITE HESTORATION FOHM

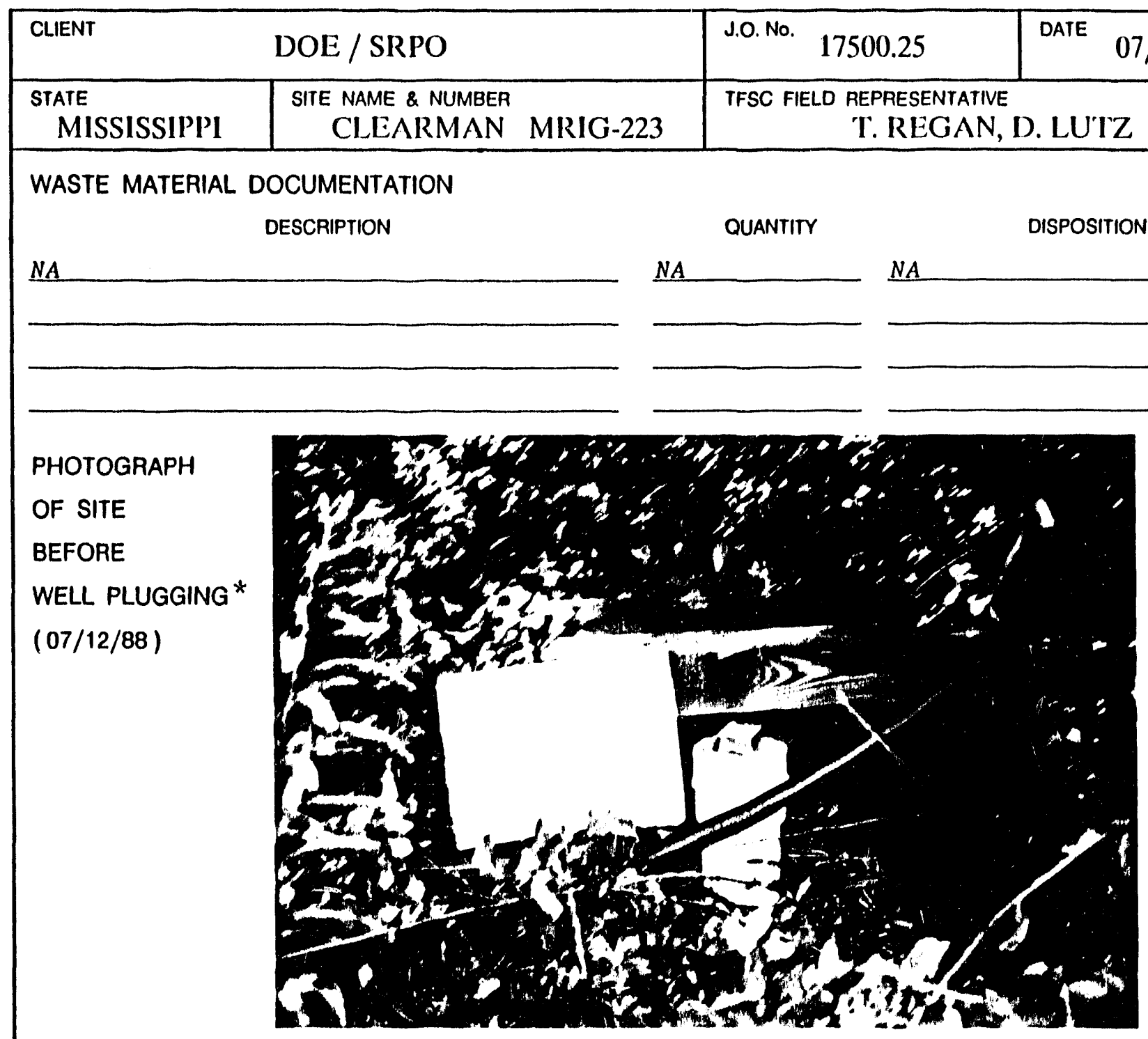

* THIS WELL WAS NOT PLUGGED;

OWNERSHIP OF THE WELL WAS TRANSFERRED

TO THE CURRENT LANDOINER IN THE CONDITION

SHOWN IN THE ABOVE PHOTOGRAPH. 


\section{SITE RESTORATION FORM}

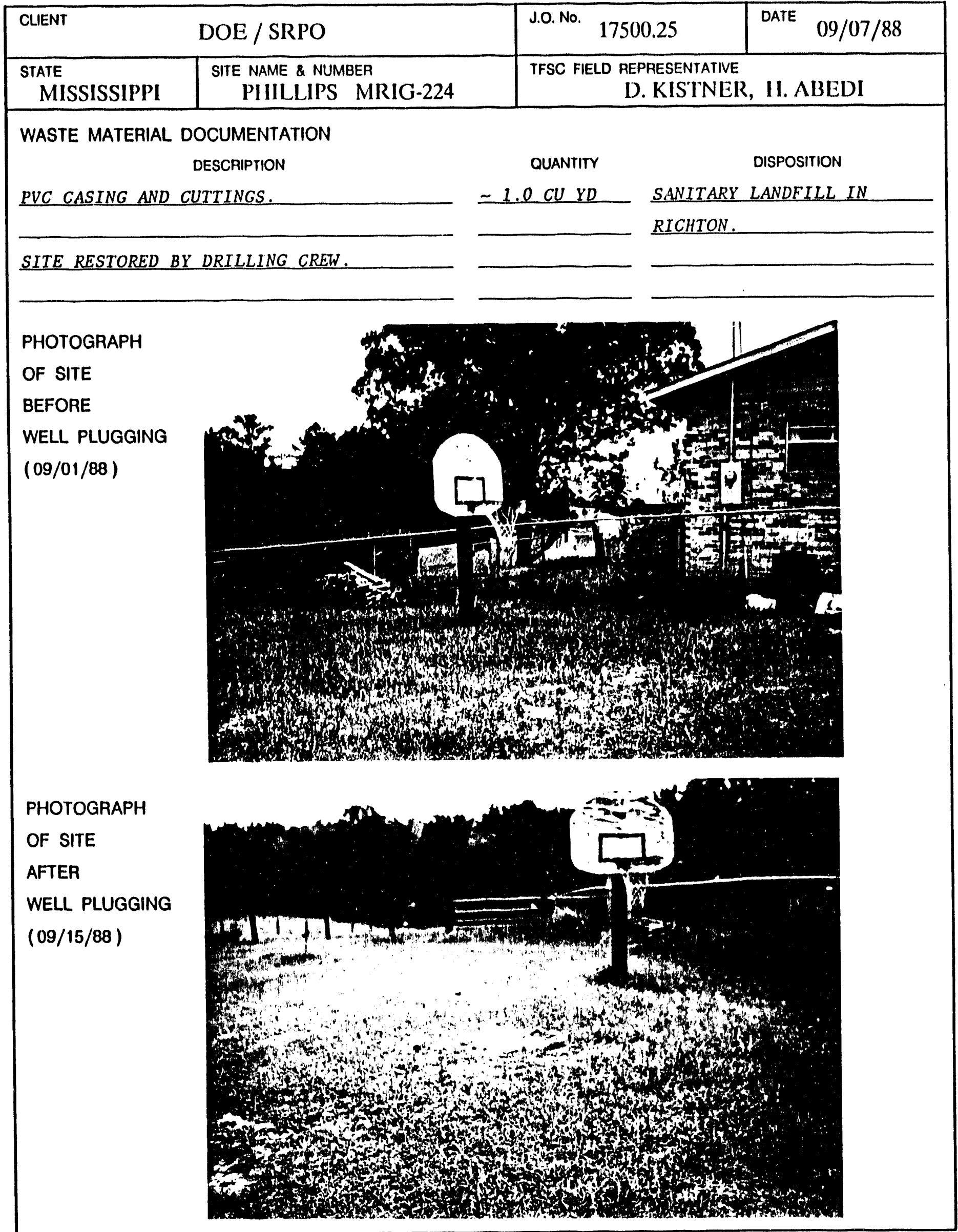




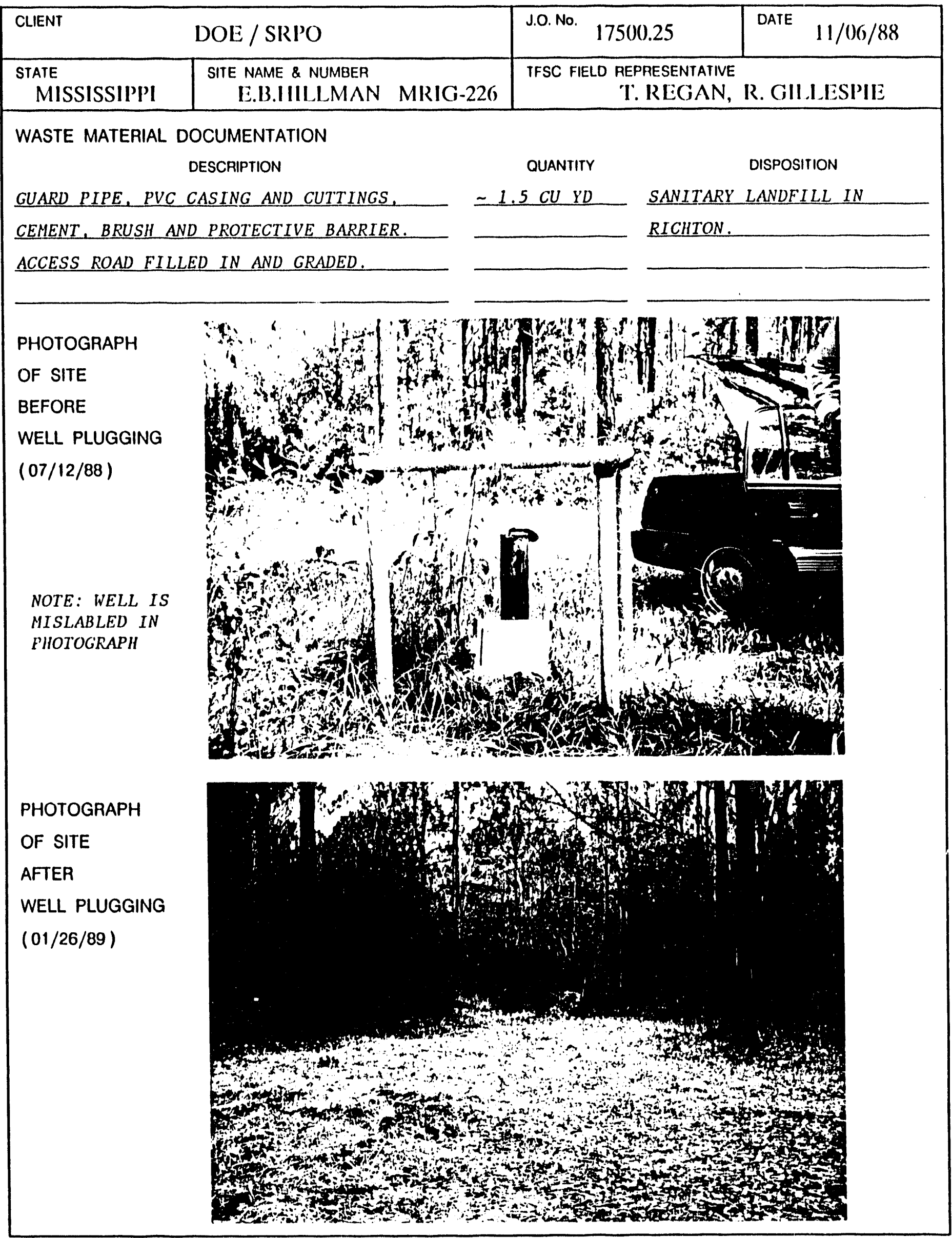




\section{SITE RESTORATION FORM}

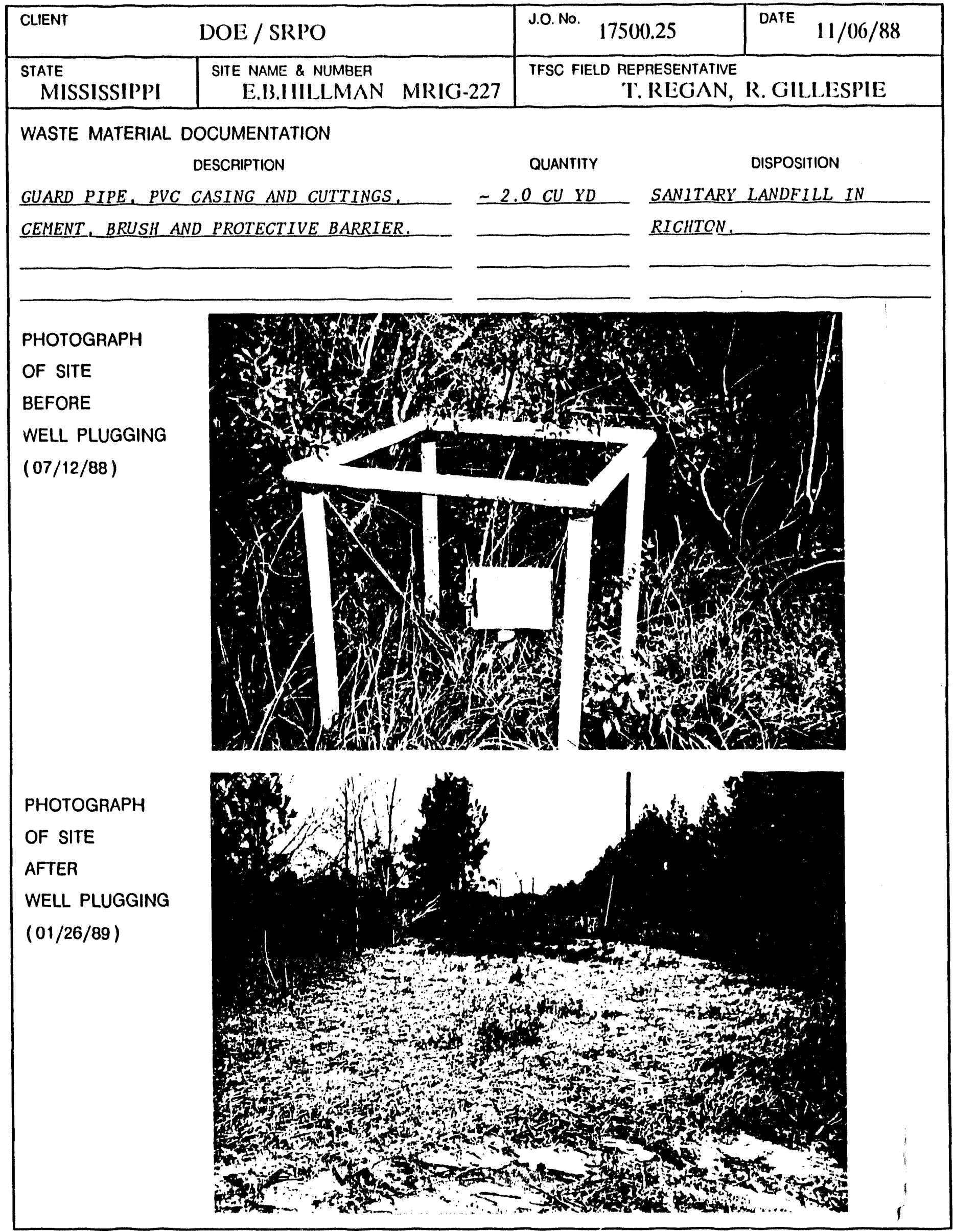


SITE RESTORATION FORM

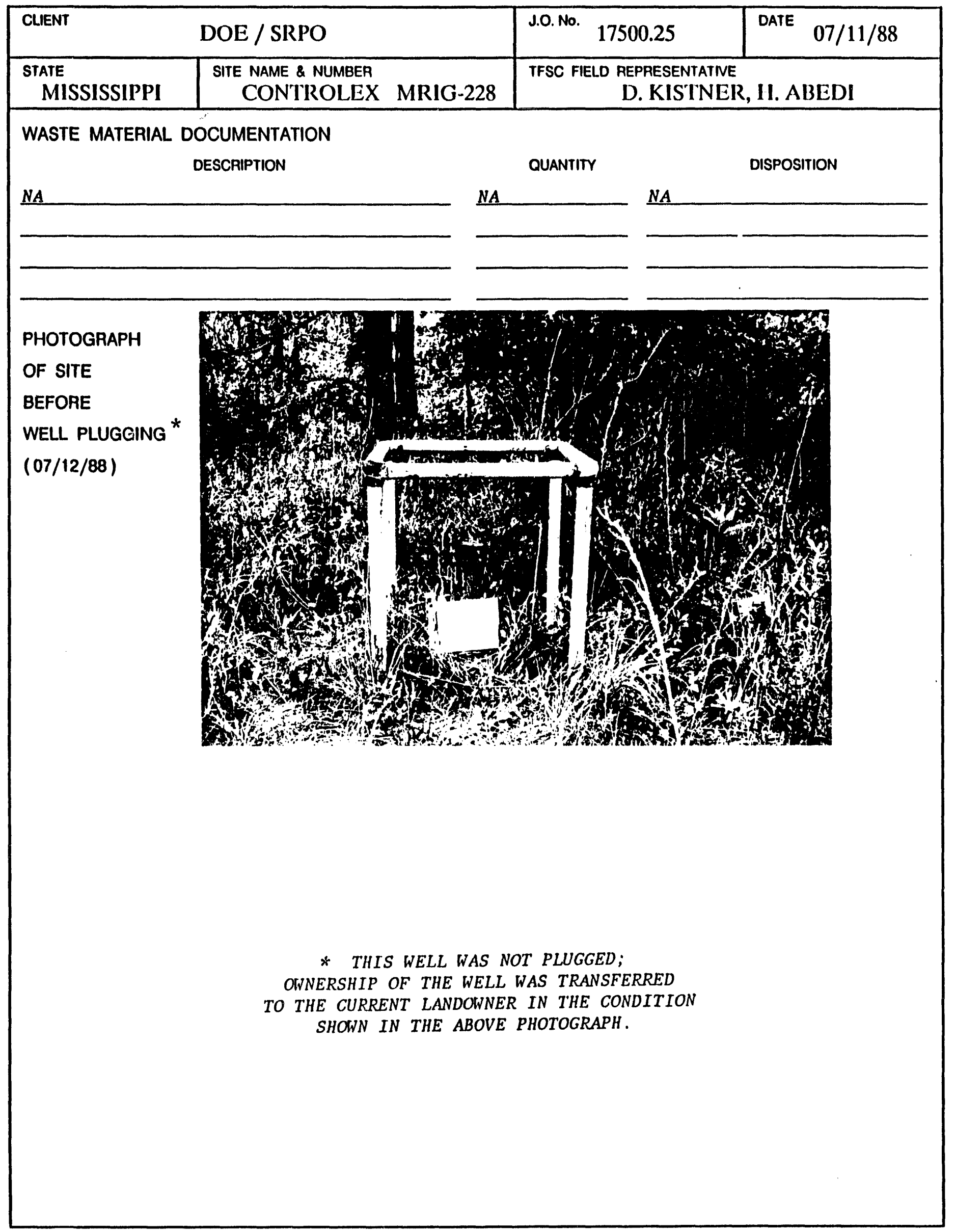


SITE RESTORATION FORM

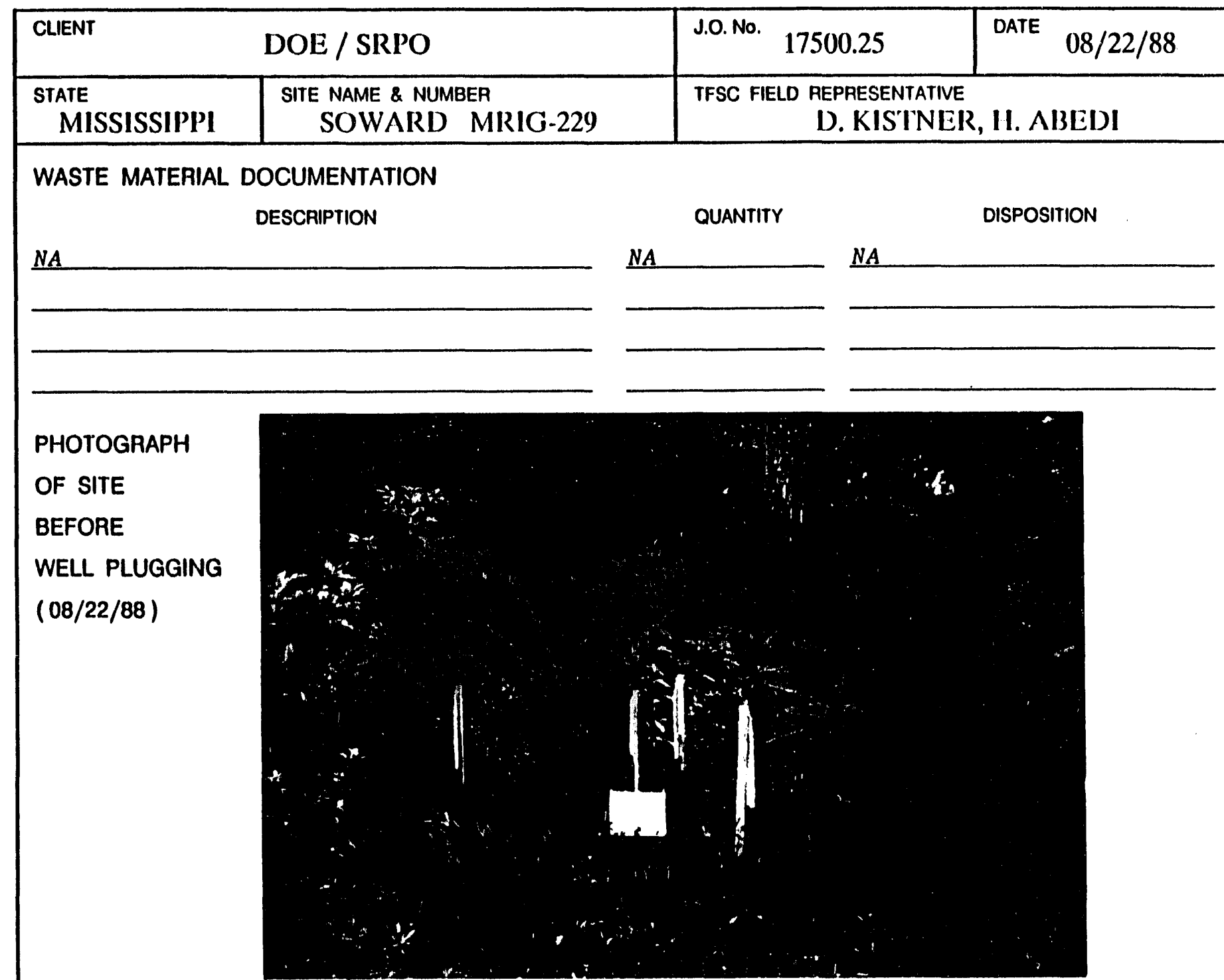

* boREHOLE WAS baCKFILLED bY LETCO IN 1979. THERE WAS NO PLUGGING OR RESTORATION WORK PERFORMED AT THIS SITE 
SITE RESTORATION FORM

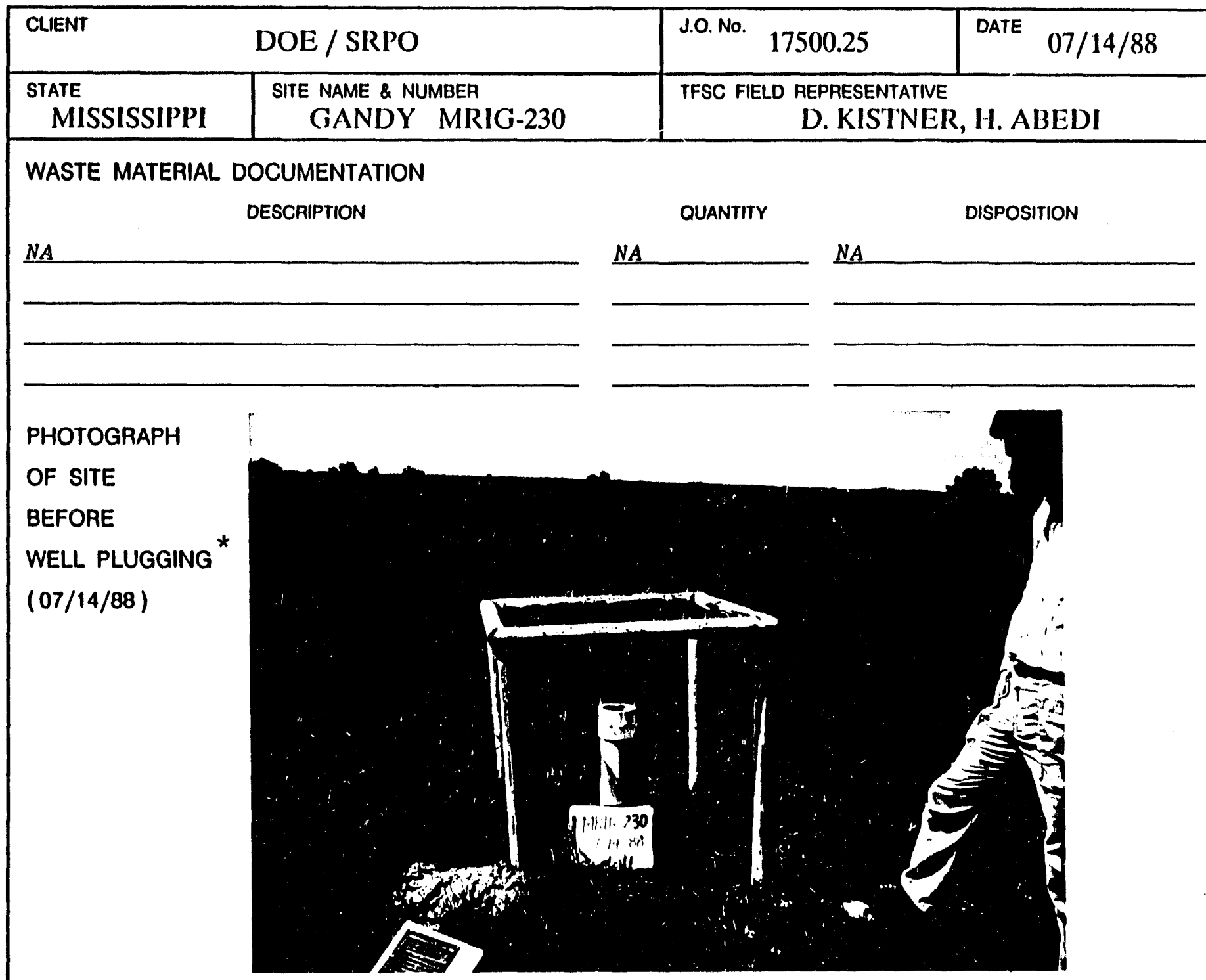

* THIS WELL WAS NOT PLUGGED;

OWNERSHIP OF THE WELL WAS TRANSFERRED

TO THE CURRENT LANDOWNER IN THE CONDITION

SHOWN IN THE ABOVE PHOTOGRAPH. 


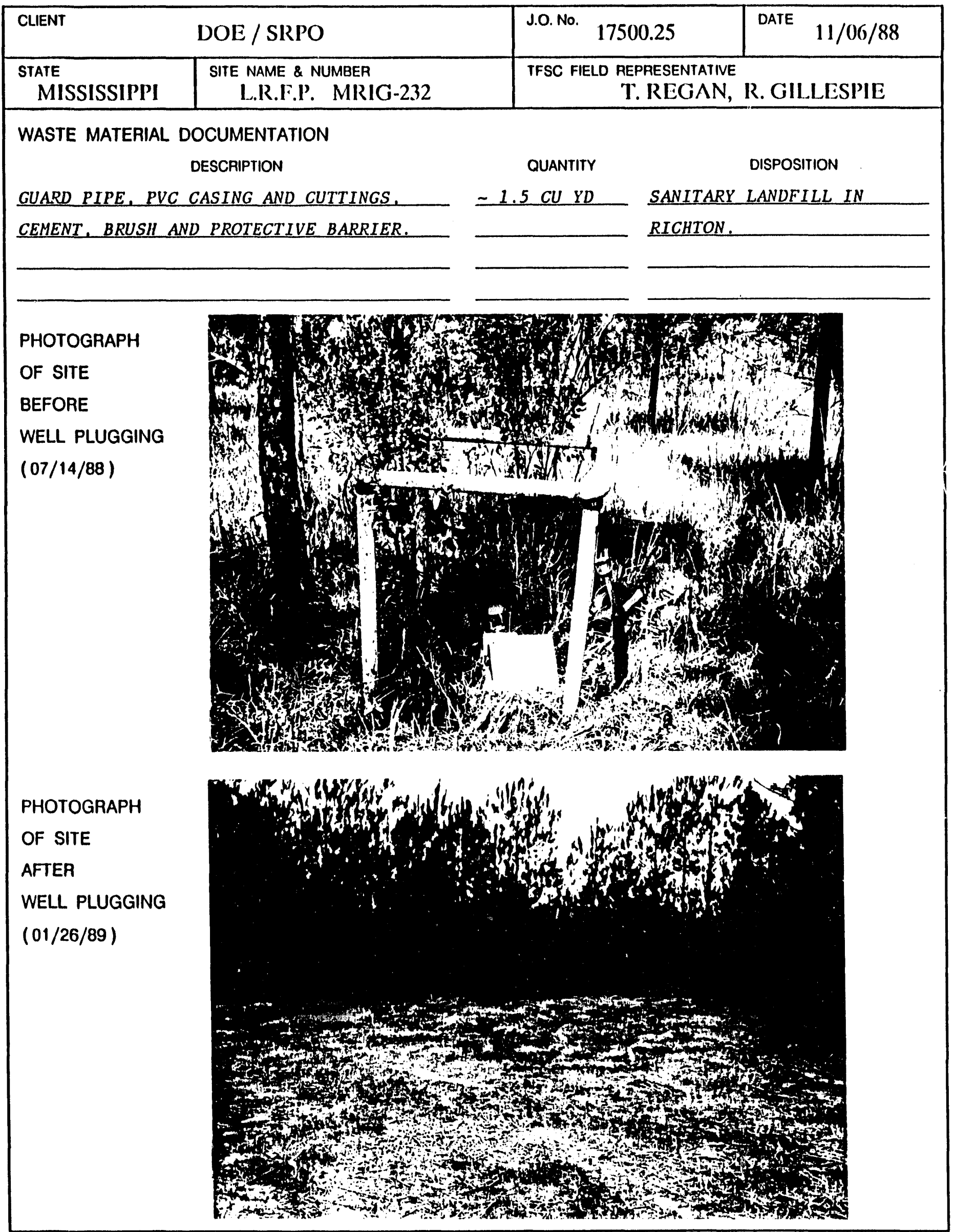


APPENDIX F

Waste Hauler's Manifests 


\section{OILFIELD WASTE SHIPPING CONTROL TICKET}

FILE COPY

J.0.10. 17011

Troctono

P.O: 0085

DOC. IO $M \cdot 2$
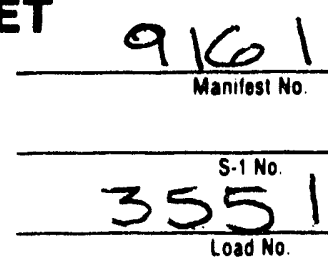

PART I: TO BE COMPLETED BY GENERATOR

Company name STONE \&WEBSTER ENGR CORP Code No. Business Address P.O. BOX 2325 BOSTON MA 02107 Telephone No. 617 58P-2098 Well Name AWDERSON-DOE MH GD

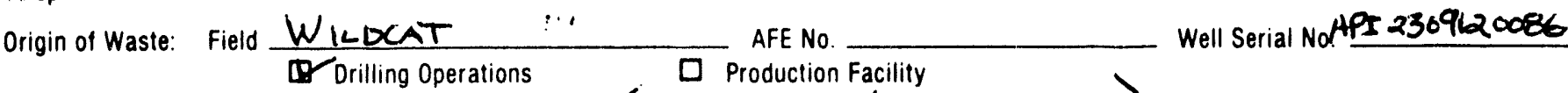
Description of Waste SALT WATER (TDS- $600 \mathrm{mg} / \mathrm{L}, \mathrm{PH}-9.95$ ) Code No Quantity of Waste 120 BBLS

\section{DESTINATION:}

Disposer's Name G. B. Boots" Smith Code No.

Business address P. Drawer i987

Disposal Site Address H. HCREST DR

IERTIFICATION: The waste described above was consigned to the carrier named below. I certify that the oregoing is true and correct to the best of my knowledge.

Xulan of fichs pie

$$
9 \cdot 16-88 \quad 5: 00 \mathrm{pm}
$$

PART II: TO BE COMPLETED BY TRANSPORTER (Note: To be completed in presence of Generator, before removing Generator's copy) Transporter's Name G.B." Beots" Smith

Business Address P. DRAw R 1987

Telephone No. $\mathrm{GO} / 649-1220$

License Plate of Truck $\$ \mathrm{SO} 1426 \mathrm{H}$

License Plate of Trailer $\angle 0062 T L$

If Transportation by Barge, Barge and Tug Identification N/A

CERTIFICATION: I certify that the waste in the quantity above was received by me for shipment to the above destination.
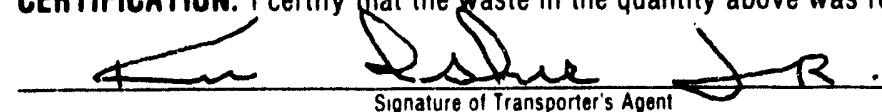
$9-162-88$

\section{PART III: TO BE COMPLETEO BY DISPOSER}

Disposer's Name G.B." BooTs" Smith Code No. $\operatorname{cocswa}$ Disposal Site Address HilleresT DR Telephone No. $601 / 649-1220$

'.icense Plate of Truck $801426 \mathrm{H}$ License Plate of Trailer $40602+5$

If received by Barge, Barge and Tug Identification CERTIFICATION: I certify that the waste in the quantify and description in Part I was received by me for proper disposal.
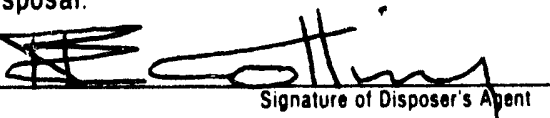


\section{G.B. "BOOTS" SMITH CORP.}

\section{LAUREL, MISSISSIPPI 39441}

\section{FILE COPY \\ J:0.10. 17500 \\ TrEC-SPpo \\ PO- 0085 \\ DOC I.D M-Z}

OILFIELD WASTE SHIPPING CONTROL TICKET $920 /$

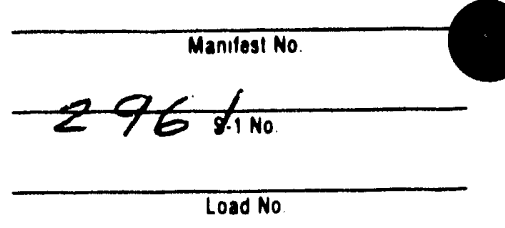

PART I: TO BE COMPLETED BY GENERATOR

Company name STONE \& WEBETER ENGR. CORP Code No

8usiness Address $P O$. BCX 2325

Telephone No. $617589-2098$

Origin of Waste: Field $\frac{W_{I L D C A T}}{\square \text { Drilling Operations }}$ AFE No.

Origin of Waste: Field $\frac{W_{I L D C A T}}{\square \text { Drilling Operations AFE No. }}$ Well Name MH-7A DOE

Description of Waste SALT WATER TRS $=8930 \mathrm{mg} / \mathrm{f}, \mathrm{PH}=9.39$ Code No.

Quantity of Waste 120 BBL'S

Seal On

\section{OESTINATION:}

Disposer's Name G.G. BOOT'S Smith Code No.

Business Address $P O . \angle Q Z 7 \angle A M R E L M I S S$.

Disposal Site Address LAUREL MLS.

CERTIFICATION: The waste described above was consigned to the carrier named below. I certify that the

foregoing is true and correct to the best of my knowledge.

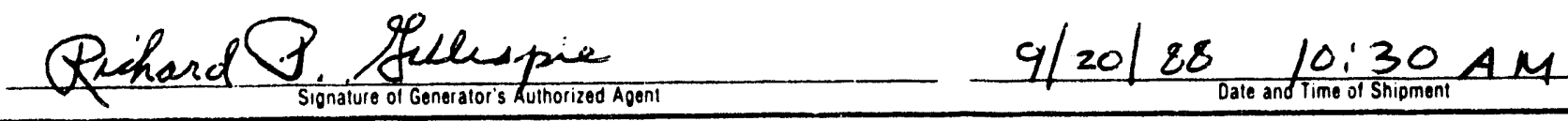

PART II: TO BE COMPLETED BY TRANSPORTER (Note: To be completed in presence of Generator, before removing Generator's copy) Transporter's Name Mllllllll \& 13 BOOTS Smill

Business Address PO Drawer 1987 LAUREL MS.

Telephone No. $649 \cdot 1220$

License Plate of Truck 8014464

License Plate of Trailer $-3941 T C$

If Transportation by Barge, Barge and Tug Identification

CERTIFICATION: I certify that the waste in the quantity above was received by me for shipment to the above destination.

TO BE COMPLETED BY DISPOSER

\section{PART III: TO BE COMPLETED BY DISPOSER}

Disposer's Name G.B. $1300 \mathrm{~T}$ 'S

Smith Code No.

Disposal Site Address $\angle A M R E L M S$.

Telephone No. $\quad 249-1220$

Seal Off

License Plate of Truck $80 / 446 \mathrm{H}$

icense Plate of Trailer $39415 \angle$

' received by Barge. Barge and Tug Identification

'ERTIFICATION: I certify that the waste in the quantity and description in Part I was received by me for

roper disposal.

DISTRIBUTION:

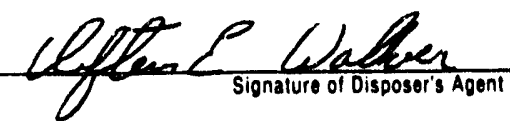


G.B. "BOOTS" SMITH CORP.

$3 / 29$

LAUREL, MISSISSIPPI 39441

OILFIELD WASTE SHIPPING CONTROL TICKET FILE COPY J.0.10. 17500

Ifsconpo

P. 0.0085

DOC. I.D. NorM. 2

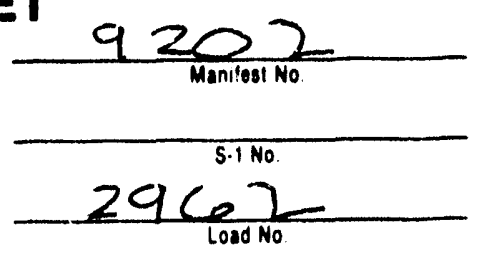

PART I: TO BE COMPLETED BY GENERATOR

company name STONE ? WEBSTER ENGR CORP Code No. Business Address PO. BOX 2325 BOSTON MA OZ 107

Telephone No. $617589-2098$

Origin of Waste: Field WICDCAT Well Name DOE $\mathrm{MH}-7 \mathrm{~A}$

$\lambda$ Drilling Operations

AFE No Well Serial No.

Description of Waste SALT WATER $8930 \mathrm{mg} / L$-TDS, pH 9.39 Code No. Quantity of Waste 120 BELS

Seal on

DESTINATION:

Disposer's Name G.B. "Boots" Smith_ Code No.

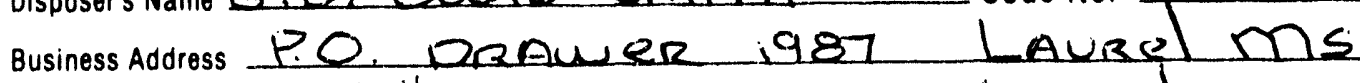

Disposal Site Address Hillckest DR Laurel no

CERTIFICATION: The waste described above was consigned to the carrier named below. I certify that the foregoing is true and correct to the best of my knowledge.

Qunanald Sellespice

$$
9 \cdot 20-88
$$

Date and Time of Shipment

PART II: TO BE COMPLETE O BY TRANSPORTER (Note: To be completed in presence of Generator, before removing Generator's copy) Transporter's Name G.B."Boots" Sa i th

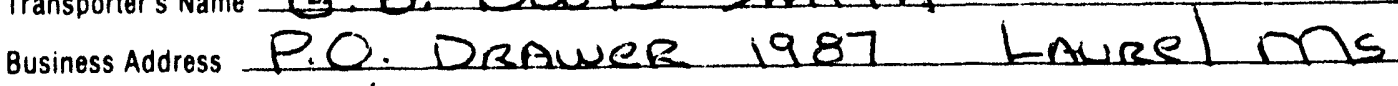

Telephone No. $601 / 649-1220$

License Plate of Truck

License Plate of Trailer

If Transportation by Barge, Barge and Tug Identification

CERTIFICATION: L certify that the waste in the quantity above was received by me for shipment to the above destination.

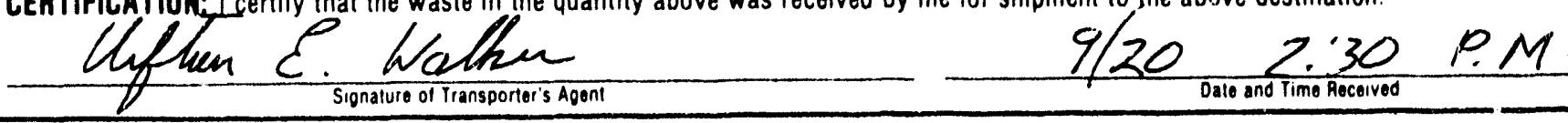

PART III: TO BE COMPLETED BY DISPOSER

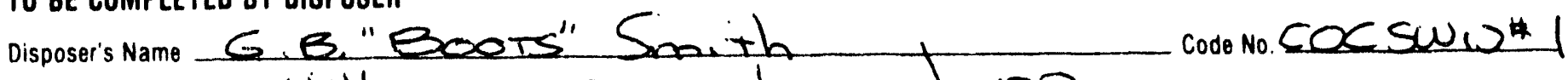

Disposal Site Address Hill CREST DR LAurel $D$ NS

Telephone No. Col /644-1220

Seal off

License Plate of Truck

License Plate of Trailer

If received by Barge, Barge and Tug Identification $N / A$

CERTIFICATION: I certify that the waste in the quantity and description in Part I was received by me for proper disposal.

DISTRIBUTION:

Signature of Disposer's Agent

Dale and Time Receiver

Original: G.B. "Boots" Smith Corp. - Green: Office of Conservation - Yellow: Disposer - PInk: Transporter - Bold: Generator's Pst Copy 
G.B. "BOOTS" SMITH CORP.

LAUREL, MISSISSIPPI 39441

OILFIELD WASTE SHIPPING CONTROL TICKET

FILE COPY

J.O. NO. 1TiNo

TFECSRPO

P. O 0085

rou I. N NO M-Z

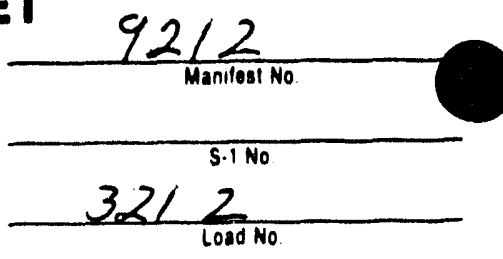

PART I: TO BF COMPLETED BY GENERATOR

Company Name STONE \& WEBSTER ENGINEERING CORP. Business Address PO BOX 2325 BOSTON.MA O2107 Code No. Telephone No. El 7-5E9-2098: Well Name BIAER DOE MH.7B Origin of Waste: Field WILDCAT AFE No. Well Serial No. 2303520075

$\checkmark$ Drilling Operations $\square$ Production Facility Description of WasteSALT WATER TOS $8,490 \mathrm{mg} / \mathrm{L} 9.12 \mathrm{ptt}$ code No. Quantity of Waste $80 \mathrm{BB} L$

\section{DESTIMATION:}

Disposer's Name G.B.BOOT's Smille Code No. Business Address P.O Orame 1987 TALREL MS

Disposal Site Address Hell liest Dive LAUREL MS

CERTIFICATION: The waste described above was consigned to the carrier named below. I certify that the toregoing is true and correct to the best of my knowledge.

1017 .

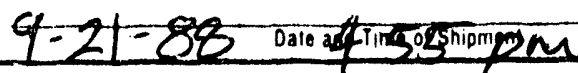

PART II: TO BE COMPLETED BY TRANSPORTER (Note: To be complet in presence of Generator, before removing Generator's copy)

Transporter's Name \&.

Business Address PQ DRAWER 1987

Telephone No. $-849-1220$

License Plate of Truck $801415 \%$

License Plate of Trailer $394 \angle C$

If Transportation by Barge. Barge and Tug Identification

CERTIFICATION: I certify that the waste in the quantity above was received by me for shipment to the above destination.

Clyfex

\section{PART III: TO BE CORPLETEO BY DISPOSER}

Disposer's Name $G B$. BOOT'S Smilh

Disposal Site Address Hill Great Dawe $\angle A C B E L M S$.

Telephone No. $649-1220$ Code No.COS SWD $\$ 1$

License Plate of Truck

License Plate of Trailer

If received by Barge. Barge and Tug Identification

CERTIFICATION: I certify that the waste in the quantity and description in Part I was received by me for proper disposal.

DISTRIBUTION:

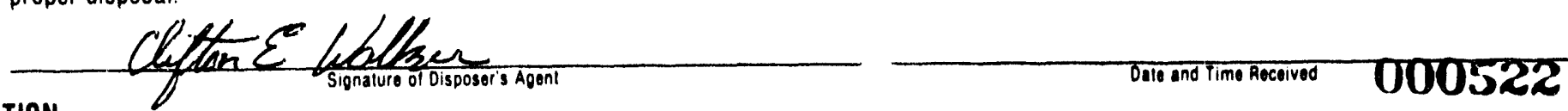

Original: G.B. "Boots" Smith Corp. - Green: Office of Conservation - Yellow: Disposer - PInk: Transporter - Gold: Generator's 1st Copy 
G.B. "BOOTS" SMITH CORP.

LAUREL, MISSISSIPPI 39441

\section{OILFIELD WASTE SHIPPING CONTROL TICKET}

FILE COPY

J.0. No. 17v00

Trsasipo

P.O No. $00^{\circ} 85$

DOC. J.O. No. M-Z

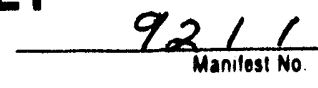

$321_{\text {Load no }}^{\text {s.1 }}$

PART I: TO BE COMPLETED BY GENERATOR

Company Name STOME \&WEBSTER EMSR CORP Code No. Business Address Po. Box 2325 Telephone No. $617587-2098$ Origin of Waste: Field WILDCAT Well Name BEARD-DOE NO.7C

\section{Drilling Operations $\square$ Production Facility}

Description of Waste SAGTWATER (TOS:3230mg/L, pH-9.A) $65 \quad B B<$ is

Quantity of Waste $65 \quad 3 B<5$

Seal On

\section{DESTINATION:}

Disposer's Name G.B BOOT'S Smith Code No.

Business Address P.ODRAWER 1987

Disposal Site Address Hill Crest or LAURAL MS

CERTIFICATION: The waste described above was consigned to the carrier named below. I certify that the foregoing is true and correct to the best of my knowledge.

\section{Xuchancf Jieles pie

PART II: TO BE COMPLETED BY TRANSPORTER (Note: To be completed in gresence of Generator, betore removing Generator's copy) Transporter's Name G.B. BOOT'S smith

Business Address P.O Drower 1987

Telephone No. $644-1220$

License Plate of Truck $8014 / 5 / 4$

License Plate of Trailer $394 / T L$

If Transportation by Barge. Barge and Tug Identification

CERTIFICATION: I certify that the waste in the quantity above was received by me for shipment to the pbove destination.
Whem \& wathece

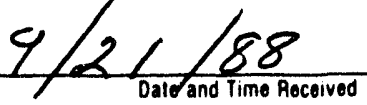

\section{PART III: TO BE COMPLETED BY DISPOSER}

Disposer's Name G.B BOOTS Smith

Disposal Site Address Hill hesP Dive LAUAEL NS.

Telephone No. $649-1220$

Seal 0tf

License Plate of Truck $801415 \mathrm{H}$

License Plate of Traile

$$
394 \angle T L
$$

If received by Barge, Barge and Tug Identification

CERTIFICATION: I certify that the waste in the quantity and description in Part I was received by me for proper disposal.

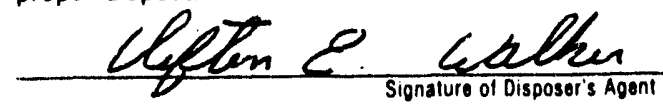

DISTRIBUTION: 


\section{G.B. "BOOTS" SMITH CORP. LAUREL, MISSISSIPPI 39441 \\ OILFIELD WASTE SHIPPING CONTROL TICKET \\ FILE COPY \\ J.0.NO. 1T500 \\ TFSCSRPO \\ P. O. 00 का \\ DoS I.D.NO M.2}

PART I: TO BE COMPLETED BY GEMERATOR

Company Name STONE \&WEBSTER ENGR CORP Business Address PO.BOX 2325 BOSTON MA O2107 Telephone No. 617-589-2098 Well Name BEARD-DOE NO.7B Origin of Waste: Field LUKDCAT $\triangle$ Drilling Operations AFE No. $\square$ Production Facility

Description of Waste SALT WATER - TDS $=7490, \mathrm{PH}=9.12$ Quantity of Waste $20 \mathrm{bb} / \mathrm{s}$

DESTINATION:

Dispnser's Name GB, BOOT'S Smill Code No.

Business Address PQ Dacuer 1987

Disposal Site Address HILL Reat Daine LAUREL MS,

CERTIFICATION: The waste described above was consigned to the carrier named below. I certify that the toregoing is true and correct to the best of my knowledge.

Prichur of P. Sillespie.

PART II: TO BE COMPLETEO BY JRANSPORTER (Note: To, be completed in presence of Generator, before removing Generator's copy) Transporter's Name G.B. BOOTg Santh

Business Address Po Draun 1987

Telephone No. $649-1230$

License Plate of Truck $8014 \angle 5 / 1$

License Plate of Trailer $394 / 7 L$

If Transportation by Barge, Barge ard Tug Identification

CERTIFICATION: I certiiv that the waste in the quantity above was received by me for shipment to the above destination.

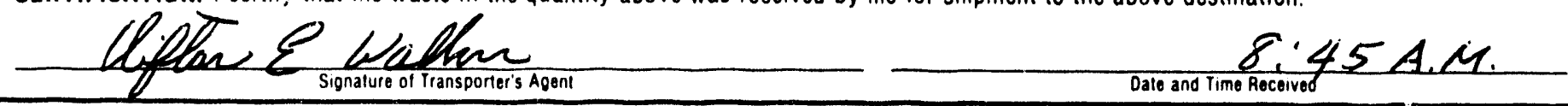

\section{PART III: TO BE COMPLETED BY OISPOSER}

Disposer's Name Code No

Disposal Site Address

Telephone No.

License Plate of Truck

License Plate of Trailer

If received by Barge. Barge and Tug Identification

CERTIFICATION: I certify that the waste in the quantity and description in Part I was received by me for proper disposal. 


\title{
G.B. "BOOTS" SMITH CORP.
}

\author{
LAUREL, MISSISSIPPI 39441
}

\section{OILFIELD WASTE SHIPPING CONTROL TICKET FILE COPY \\ J.0. Mo. ITwe \\ Trecenpo \\ Po. 0085 \\ DOC I.D. No M-Z}

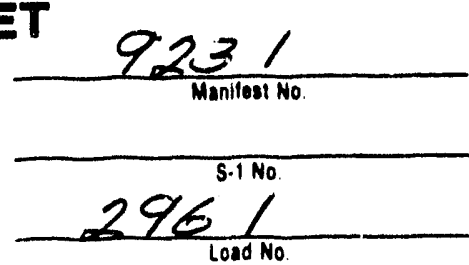

PART I: TO BE COMPLETED BY GENERATOR

Company Name STONE \& WEBSTER ENG INEERING CORP. Business Address 245 SUMmER ST, BOSRIN MA C2107

Telephone No. $617-589-2098$ Well Name $\mathrm{MH}-8 \mathrm{~A}$

Origin of Waste: Field WILDCAT AFE No.

Production Facility Code No.

$\square$ Drilling Operations

Description of Waste SALTWATER Well Serial No. 2.1309120095 Quantity of Waste $120 \mathrm{BBL}$

Seal on

DESTIMATION:

Disposer's Name G.B. BooTs Smithe Code No.

Business Address P.O. Drames 1987 LAUREL 15.

Disposal Site Address ItiL Coeat Dine LAURE L MS.

CERTIFICATION: The waste described above was consigned to the carrier named below. I certify that the foregoing is true and correct to the best of my knowledge.
n rand Signalitire of Generalor's Authorized Agent

PART II: TO BE COMPLETED BY TRANSPORTER (Note: To be completed in presence of Generator, before removing Generator's copy) Transporter's Name G. B. B OTS.Smithe

Business address PO. Drawer 1987

Telephone No. $\quad 649-1220$

License Plate of Truck $801446 / 4$

License Plate of Trailer $394 / T L$

If Transportation by Barge. Barge and Tug Identification

CERTIFICATION: I certify that the waste in the quantity above was received by me for shipment to the above destination.
Lefar \& La bee

\section{PART III: TO BE COMPLETED GY OISPOSER}

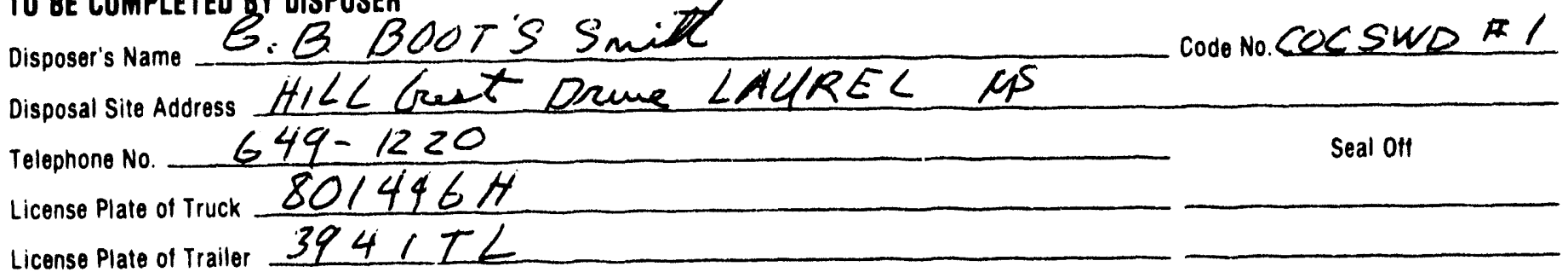

If received by Barge, Barge and Tug Identification

CERTIFICATION: I certify that the waste in the quantity and description in Part I was received by me for

proper disposal.

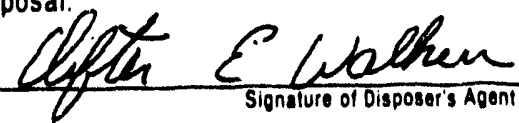

DISTAIBUTION:

Orialnal: G.B. "Boots" Smith Coro. - Green: Office of Conservation - Yollow: Disposer - PInk: Transoorter - Gald: Generator's 1st Cony 


\section{OILFIELD WASTE SHIPPING CONTROL TICKET FILE COPY \\ J.O. 10.17500 \\ TFSCSPPO \\ PO. No 0085 \\ DOC. I.D.MO M.M.Z}

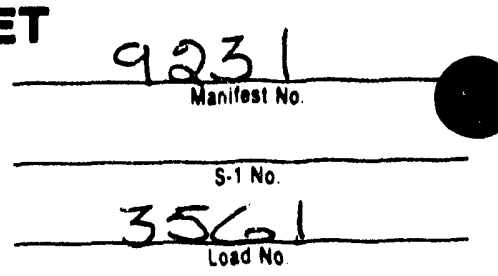

PART I: TO BE COMPLETED BY GENERATOR

company name STONE \&WEBSTER ENGINEERING CORP, INK Business Address 245 SU MMMER ST. BESTON MA OZ107 Telephone No 6 TI $\quad 589-2094$ Origin of Waste: Field WKD CAT Well Name $M H-8 A$ OFE No.

\section{Drilling Operations}

Description of Waste SALT WATRR $35,400 \mathrm{mg} / 8$ TDS pH 8.64 Well Serial No 2309120095 Quantity of Waste 30 BBL

DESTIMATION:

Disposer's name Ei, B. Boots Smith Code No. Business Address P.O. Drawer 1987 Disposal Site Address Hillsrest DR

CERTIFICATION: The waste described above was consigned to the carrier named below. I certify that :he foregoing is true and correct to the best of my knowledge.
wC
Bra
he
9-23-88 $3: 30 \mathrm{PM}$

PART II: TO BE COMPLETEO BY TRAKSPORTER (Note: To be completed in presence of Generator, before removing Generator's copy)

Transporter's Name G. "Boor" Smith

Business Address P.C. DRAWER 1987

Telephone No. $\mathrm{C}_{201 / 649-1220}$

License Plate of Truck

License Plate of Trailer

If Transportation by Barge, Barge and Tug Identification

CERTIFICATION: I certify that the waste in the quantity above was received by me for shipment to the above destination.

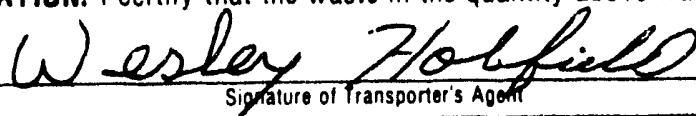
Q-23-88
3.25 P.M

\section{PART III: TO BE COMPLETED BY OISPOSER}

Disposer's Name - G. B" Exots" Smai Th

Disposal Site Address HillCR RST DR

Telephone No. $601 / 649-1226)$

Seal Off

License Plate of Truck License Plate of Trailer

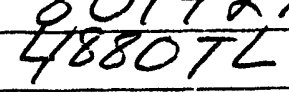

If received by Barge, Barge and Tug Identification $N$

CERTIFICATION: I certify that the waste in the quantity and description in Part I was received by me for proper disposal.

\section{OISTRIBUTION:}

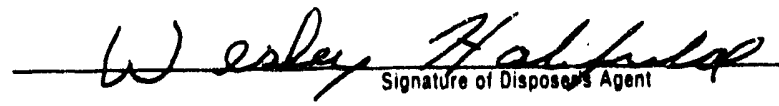

\section{$923-88 \quad 3 i 30 \mathrm{pm}$}


G.B. "BOOTS" SMITH CORP.

$9 / 29$

LAUREL, MISSISSIPPI 39441

OILFIELD WASTE SHIPPING CONTROL TICKET

FILE COPY

J.0. NO. 17201

Trepogion

P.O. No OOBS

DOC. ID. No M-2

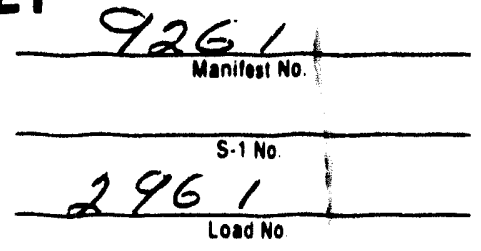

PART I: TO BE COMPLETED BY GENERATOR

Company Name SPNE \& WEBSTER

Code No.

Business Address 245 Summer ST DOSTON, MA 02107

Telephone No. $617-589-2098$ Well Name $\mathrm{MH}-8 \mathrm{C}$

Origin of Waste: Field $\frac{W 1 L D C A T}{\text { Drilling operations }}$

AFE No.

Well Serial No. APT 2309120097

Description of Waste SALT WATER

Code No.

Quantity of Waste $120 B B L$

Seal pin

DESTINATION:

Disposer's Name Code No.

Business Address

Disposal Site Address

CERTIFICATION: The waste described above was consigned to the carrier named below. I certify that the foregoing is true and correct to the best of my knowledge.

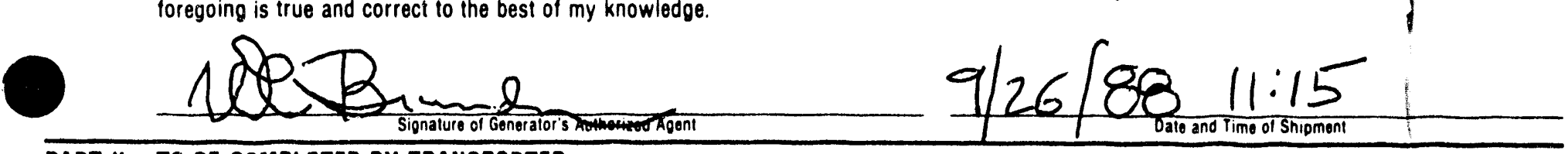

PART II: TO BE COMPLETEO BY TRANSPORTER (Note: To be completed in presence of Generator, before removing Generator's copy) Transporter's Name $-\mathrm{G}, \mathrm{B} \quad 300 \mathrm{TS} \mathrm{Sm}$ Th

Business Address P. Drawer 1987 LAUREL AS.

Telephone No. 649 - 10220

License Plate of Truck $80 / 4464$

License Plate of Trailer $394<T$

If Transportation by Barge, Barge and Tug Identification

CERTIFICATION: I certify that the waste in the quantity above was received by me for shipment to the above destination.

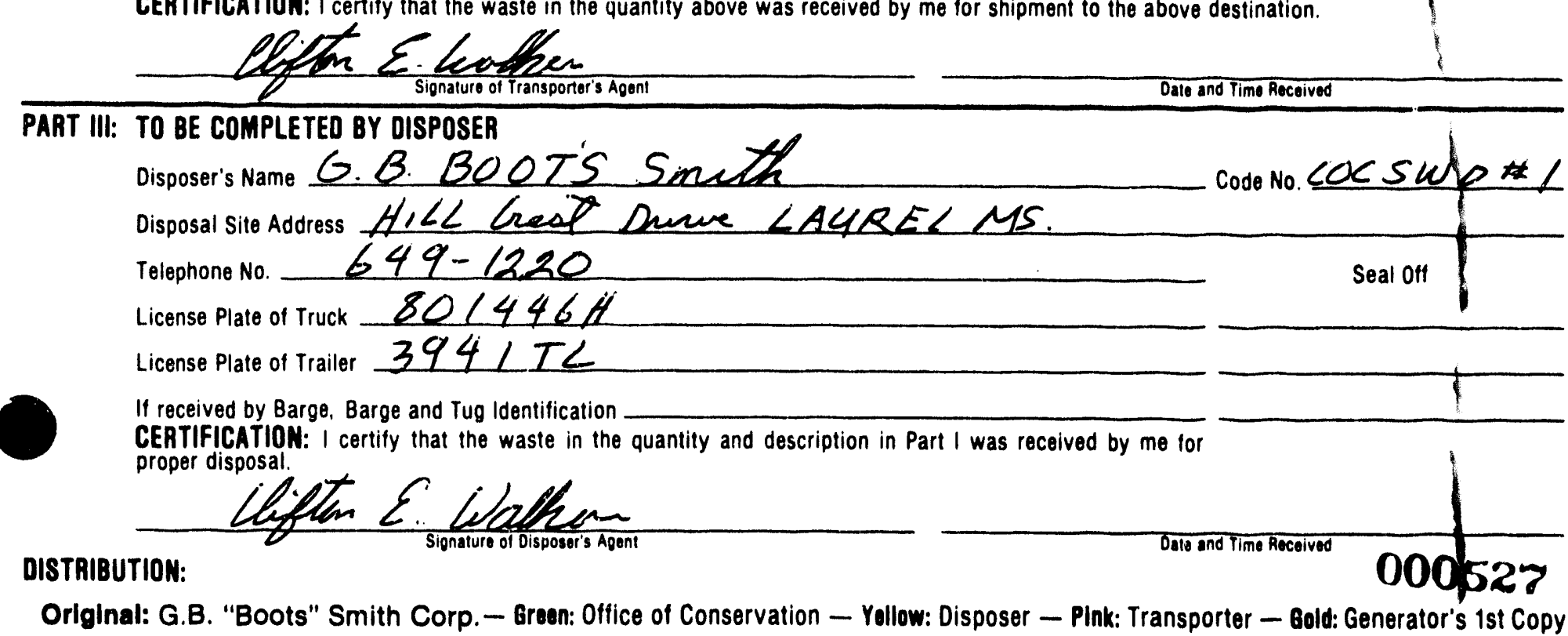


G.B. "BOOTS" SMITH CORP.

LAUREL, MISSISSIPPI 39441

\section{OILFIELD WASTE SHIPPING CONTROL TICKET FILE COPY \\ J.0.NO.17500 \\ Trocsion \\ P.O. No.00\%5 \\ Doe I.D. No M.Z}

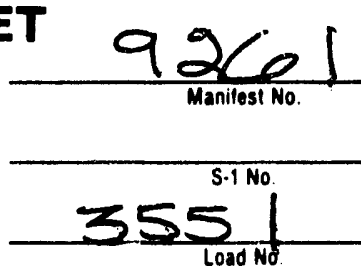

PART I: TO BE COMPLETED BY GENERATOR Company Name STONE \& WEBSTER

Business Address 245 Summer BOSTON,MA

Code No.

Telephone No. $617-589-2098$

Origin of Waste: Field WILDCAT

Description of Waste $\frac{\text { Drilling Operations }}{S 4 L T W A T E R}$

Description of Waste $\frac{\text { SALT WATER }}{\text { SAlling Operations }}$

Well Name $M H-8 C$

Quantity of Waste 1020 Code No.

DESTIMATION:

Disposer's Name

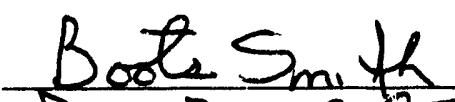

Business Address

D.

O. Bx

1987

Code No.

Disposal Site Address

\section{LAurel Ms}

CERTIFICATION: The waste described above was consigned to the carrier named below. I certify that the foregoing is true and correct to the best of my knowledge.

WBRo

Signature of Generator's Authorized Agent

Seal On

PART II: TO BE COMPLETEO BY TRAMSPORTER (Note: To be completed in presence of Generator, betore removing Generator's copy)

Transporter's Name $B$ Bocts smith Camp

Business Address $P$ O. $D$ r 987

Telephone No. $60 x-649-1220$

License Plate of Truck $80142 \mathrm{CH}$

License Plate of Trailer $3460 T L$

If Transportation by Barge. Barge and Tug Identification

CERTIFICAFION: I certify that the waste.in the quantity above was received by me for shipment to the above destination.

\section{PART III: TO BE COMPLETED BY DISPOSER}
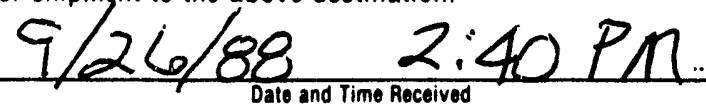

Disposer's Name Code No.

Disposal Site Address

Telephone No.

Seal Off

License Plate of Truck

License Plate of Trailer

If received by Barge. Barge and Tug Identification

CERTIFICATION: I certify that the waste in the quantity and description in Part I was received by me for proper disposal. 
G.B. "BOOTS" SMITH CORP.

LAUREL, MISSISSIPPI 39441

OILFIELD WASTE SHIPPING CONTROL TICKET

FILE COPY

J.0.NO. 17500

Mo. No. 0085
DOC. I.D No. M. 2

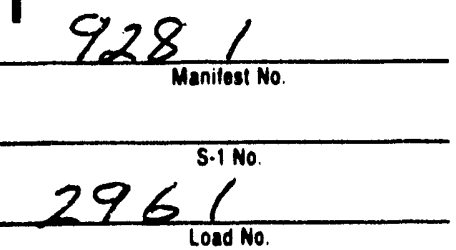

PART I: TO BE COMPLETED BY GENERATOR

Company Name STONE WNEBSTER ENGR CORP Business Address 245 SUM MER ST BOSTON, MA

Telephone No. $\leqslant 617-589-2098$ well Name $M H-513$

Origin of Waste: Field wiLCAT $_{\text {AFE No. Well Serial No. } 2303520067}$

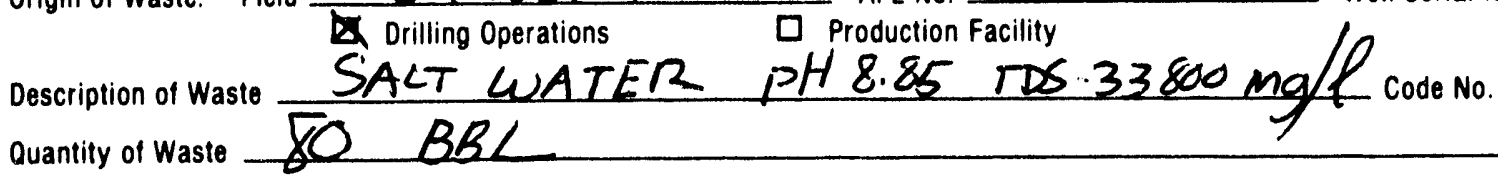

Seal On

\section{DESTINATION:}

Disposer's Name Code No.

Business Address

Disposal Site Address

CERTIFICATION: The waste described above was consigned to the carrier named below. I certify that the foregoing is true and correct to the best of my knowledge.

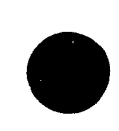

(1) Signature of Generator's Authorizes weant

Code No.

PART II: TO BE COMPLETEO BY TRANSPORTER (Note: To be completed in presence of Generator, betore removing Generator's copy)

Transporter's Name G.B BOOTS Smith

Business Address PO. DRAWER 1987 LAYRAC MS

Telephone No. $649-1220$

License Plate of Truck $801446 / t$

License Plate of Trailer $394 / 7<$

If Transportation by Barge. Barge and Tug Identification

CERTIFICATIQN: I certify that the waste in the quantity above was received by me for shipment to the above destination.

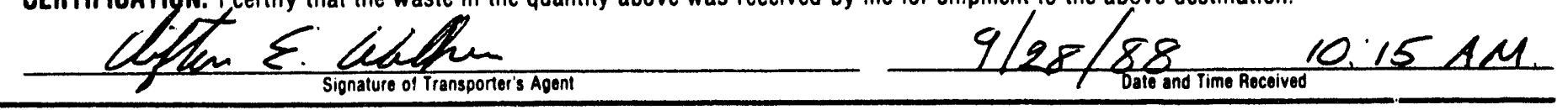

\section{PART III: TO BE COMPLETED BY DISPOSER}

Disposer's Name G.B Boots Smith

Disposal Site Address $4 \mathrm{KL}$ Geat Drive $\angle A 4 R E L M S$.

Telephone No. $649 \cdot 1220$

License Plate of Truck $801446 \mathrm{~A}$

License Plate of Trailer $3941 \mathrm{TL}$

If received by Barge, Barge and Tug Identification

CERTIFICATION: I certify that the waste in the quantity and description in Part I was received by me for proper disposal.

DISTRIBUTION: 
G.B. "BOOTS" SMITH CORP.

\author{
LAUREL, MISSISSIPPI 39441
}

OILFIELD WASTE SHIPPING CONTROL TICKET

FILE COPY

J.O.NO. 17500

TFSC-SpPO

P.O. 0.885

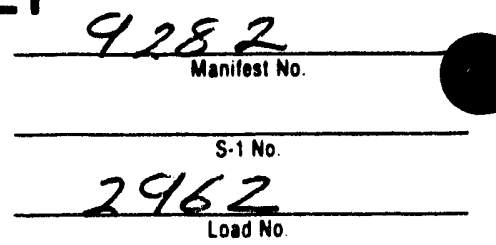

PART I: TO BE COMPLETED BY GENERATOR

Company Name STONE\& WEBSTER ENGR CORP Business Address Po BOX 2325 BOSTON, MA Telephone No. 617589-2098 Well Name US FarEST SERVICE - DOE MH - 5A Origin of Waste: Field WILDCAT AFE No. Well Serial No. 2303520066 Description of Waste SALT WATER (TDS $=6,500 \mathrm{mg} / \mathrm{L}, \mathrm{pH} 7.07)$ Code No.

Quantity of Waste

Seal On

DESTINATION:

Disposer's Name Code No.

Business Address

Disposal Site Address

CERTIFICATION: The waste described above was consigned to the carrier named below. I certify that the

foregoing is true and correct to the best of my knowledge.

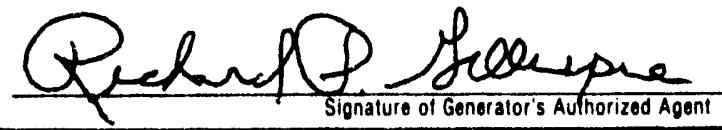

$9 / 28 / 88 \quad 4: 45 \mathrm{PM}$

PART II: TO BE COMPLETED BY TRANSPORTER (Note: TO be completed in presence of Generator, before removing Generator's copy)

Transporter's Name G.B BOOTS Smith

Business Address PO. DRAWER 1987 LACR HL MS.

Telephone No. $649-1220$

License Plate of Truck $801446 \mu$

License Plate of Trailer $394 / T<$

If Transportation by Barge, Barge and Tug Identification

CERTIFICATION: I certify that the waste in the quantity above was received by me for shipment to the above destination.

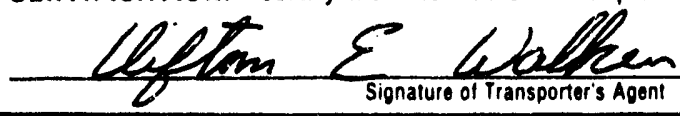

Date and Time Received

PART III: TO BE COMPLETED BY DISPOSER

Disposer's Name G. B. BOOTS Smeth

Disposal Site Address $\$ 1 / L L$ Creat Onive LAUREL MS

Telephone No. $649-1220$

License Plate of Truck $80 / 4464$

License Plate of Trailer $3941 T \angle$

If received by Barge, Barge and Tug Identification

CERTIFICATION: I certify that the waste in the quantity and description in Part I was received by me for

proper disposal.

DISTRIBUTION:

Signature of Disposer's Agent

Date and Time Received

Seal off

scswo Code No.

Original: G.B. "Boots" Smith Corp. - Green: Office of Conservation - Yollow: Disposer — PInk: Transporter — Gold: Generator's 1st Copy 
G.B. "BOOTS" SMITH CORP.

LAUREL, MISSISSIPPI 39441

\section{OILFIELD WASTE SHIPPING CONTROL TICKET FILE COPY \\ J.0.NO. 17500 \\ TFSCSRPO \\ P.O. No 0085 \\ DOC.I.D. NO M-2}

PART I: TO BE COMPLETEO BY GEMERATOR

Company Name STONE \$WEester ENer. Core Business Address P.O BOX 2325 BOSTON, MA

Telephone No. $617589-2098$

Origin of Waste: Field W/LOCAT

Dorilling Operations

Description of Waste SALTWATER (TDS $=17,300 ; P H=9.7$

Quantity of Waste liO BBC'S Well Name US Forest SERVICE DOE MH-SC.

AFE No.

Code No

\section{DESTINATION:}

Disposer's Name

Code No.

Business Address

Disposal Site Address

CERTIFICATION: The waste described above was consigned to the carrier named below. I certify that the foregoing is true and correct to the best of my knowledge.

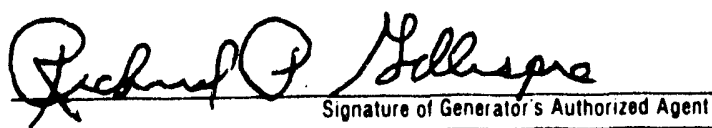

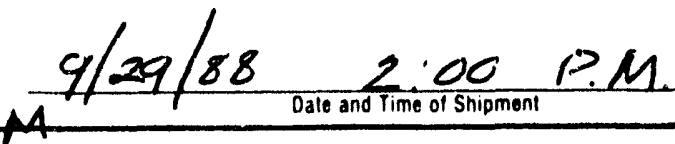

PART II: TO BE COMPLETED BY TRANSPORTER (Note: To be completgd in presence of Generator, before removing Generator's copy)

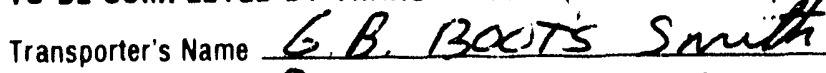

Business Address Po Drasuen 1984 LAYREL MS

Telephone No. $649-1230$

License Plate of Truck $801446 \mathrm{H}$

License Plate of Trailer $394 \angle T L$

If Transportation by Barge, Barge and Tug Identification

CERTIFICATION: I certify that the waste in the quantity above was received by me for shipment to the above destination.

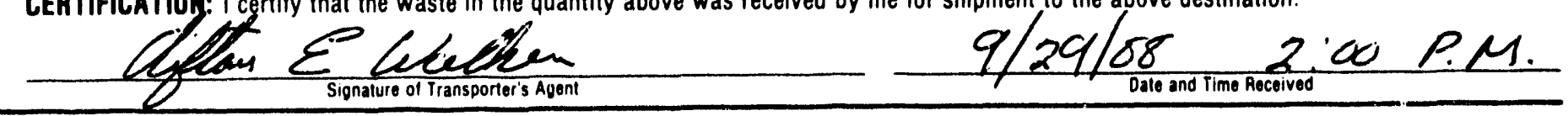

\section{PART III: TO BE COMPLETED BY DISPOSER} Code No.

Disposer's Name

Disposal Site Address

Telephone No.

License Plate of Truck

License Plate of Trailer

If received by Barge, Barge and Tug Identification

CERTIFICATION: I certify that the waste in the quantity and description in Part I was received by me for proper disposal.

\section{DISTRIBUTION:}


PART I: TO BF COMPLETED BY GENERATOR

Company name STONE \$WEBSTER ENGR CORP Code No.

Business Address Po Bax 2325 Boston MA

Telephone No. $617589-2.207$ Well Name DOE-REXUELL MH-4A

Origin of Waste: Field WILDCAT AFE No.

Well Serial APT 23 Macour

Origin or Waste. Filling Operations $\square$ production Facility

Description of Waste SALT WATER (TDS $=29 \mathrm{mg} / \mathrm{L}$, pH 8.83 code No

Quantity of Waste $30 \mathrm{bb} / \mathrm{s}$

Seal on

DESTIMATION:

Disposer's Name

Code No.

Business Address

Disposal Site Address

CERTIFICATION: The waste described above was consigned to the carrier named below. I certify that the toregoing is true and correct to the best of my knowledge.

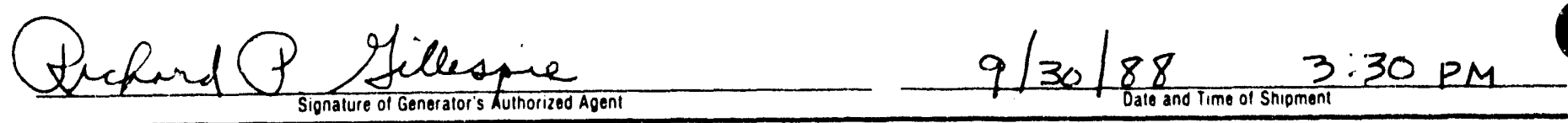

PART II: TO BE COMPLETEO BY TRANSPORTER (Note: To be completed in presence of Generator, before removing Generator's copy) Transporter's Name $\angle B D O 0 T S$ Sinith

Business Address $\frac{P C D R A W E R / 987}{649-1220}$ Telephone No.

License Plate of Truck $80 ; 4464$

License Plate of Trailer $394 / T<$

If Transportation by Barge, Barge and Tug Identification

CERTIFICATION: I certify that the waste in the quantity above was received by me for shipment to the above destination.

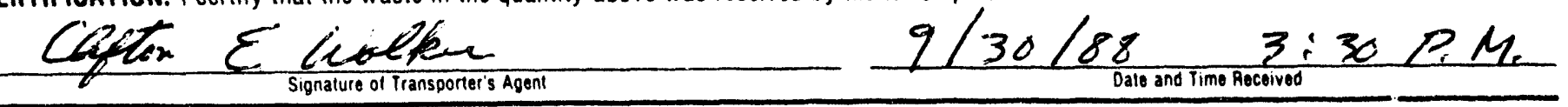

PART III: TO BE COMPLETEO BY DISPOSER

Disposer's Name G. B BOOTS Smith

Disposal Site Address HKL Ciest Deur LALREZ MS

Telephone No. $64 q-1220$

License Plate of Truck 801446.19

License Plate of Trailer $39 j 4 T L$

If received by Barge. Barge and Tug Identification

CERTIFICATION: I certify that the waste in the quantity and description in Part I was received by me for

proper disposal.

DISTRIBUTION:

Original: G.B. "Boots" Smith Coro. - Graen: Office of Conservation - Yollow: Disposer - Pink; Transnortar - Gald: Ganaratnr's 1st C.nnv 
G.B. "BOOTS" SMITH CORP.

$15 / 29$

LAUREL, MISSISSIPPI 39441

OILFIELD WASTE SHIPPING CONTROL TICKET

FILE COPY

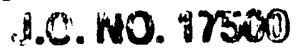

TFBCARSO

P.O. No 0085

DOC .ID. Mr
$16 \cdot 92$

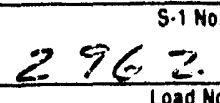

PART I: TO BE COMPLETED BY GENERATOR

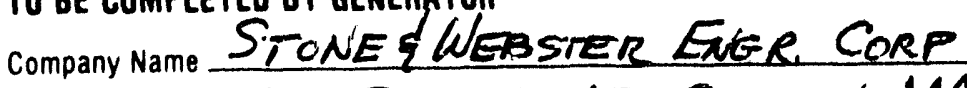

Code No.

Business Address PO BOX 2325 BOSTON, $M 4$

Telephone No. $617-589-2098$

Origin of Waste: Field W/LDCAT

Well NameDOE-ROWELC. $M H-4 D$

Well Serial Na23/1120094

AFE No.

Description of Waste SALTWATER (TDS $=7070 \mathrm{mg} / \mathrm{L} ; p H=q .2)$

Code No.

Quantity of Waste

$2020 \mathrm{cis}$

Seal on

DESTINATION:

Disposer's Name

Code No.

Business Address

Disposal Site Address

CERTIFICATION: The waste described above was consigned to the carrier named below. I certify that the foregoing is true and correct to the best of my knowledge.

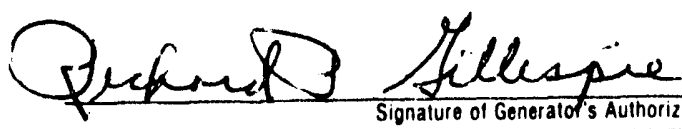

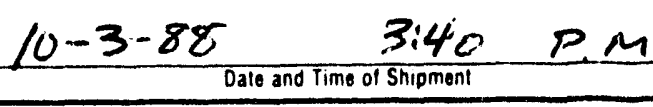

PART II: TO BE COMPLETED BY TRANSPORTER (Note: To be completed in presence of Generator, before removing Generator's copy)

Transporter's Name G CB BAD Ts Smith

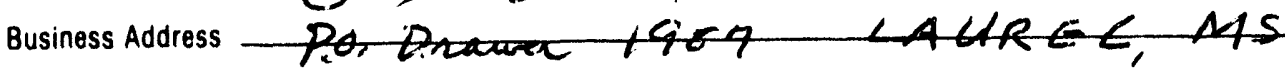

Telephone No.

$6419+220$

License Plate of Truck 80 fy et it

License Plate of Trailer $344+T L$

If Transportation by Barge, Barge and Tug Identification

CERTIFICATION: I certify that the waste in the quantity above was received by me for shipment to the above destination.

before $\sum_{\text {signature of transporter's agon }}$

$$
\frac{10-3-88 \quad 3: 40 \quad P, M}{\text { Dale and Time Received }}
$$

PART III: TO BE COMPLETED BY DISPOSER

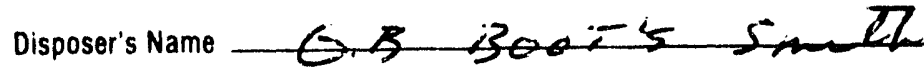
Code No

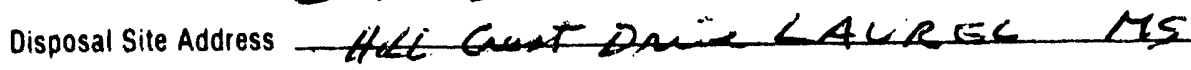

Telephone No. $649-1220$

Seal Off

License Plate of Truck Soche 6 T

License Plate of Trailer $397+\div-2$

If received by Barge. Barge and Tug Identification

CERTIFICATION: I certify that the waste in the quantity and description in Part I was received by me for proper disposal.

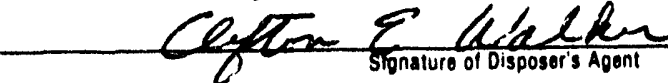

Date and Time Received 00053

DISTRIBUTION:

Original: G.B. "Boots" Smith Corp. - Green: Office of Conservation - Yalow: Disposer - PInk: Transporter - Gold: Generator's 1st Copy 
G.B. "BOOTS" SMITH CORP.

OILFIELD WASTE SHIPPING CONTROL TICKET
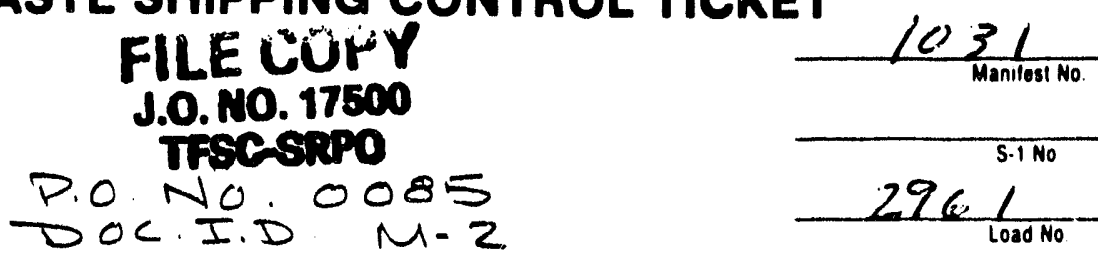

PART I: TO BE COMPLETED BY GENERATOR

Company name STONE \& W'EBSTER

Business Address PO. BOX 2325 BOSTON MA

Telephone No. $617589-2098$ Well Name DoE-RoweLL 44

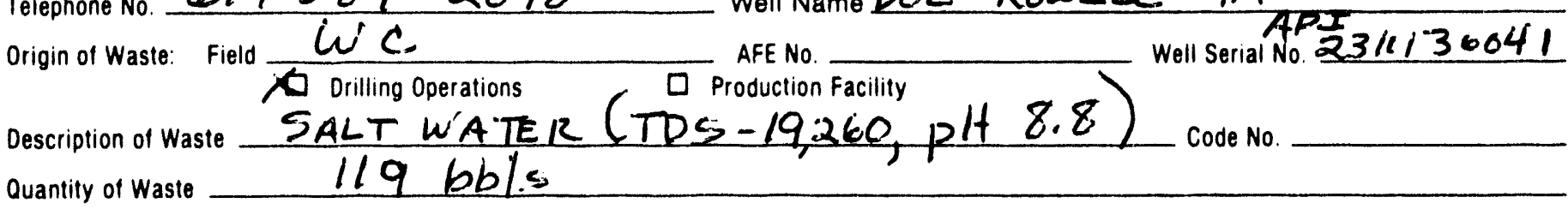

Seal On

\section{DESTINATION:}

Disposer's Name

Code No.

Business Address

Disposal Site Address

CERTIFICATION: The waste described above was consigned to the carrier named below. I certify that the

foregoing is true and correct to the best of my knowledge.

QRechend P. Aclespu

Signalure of Generator's Authorized Agent

$30 x-788$

$10: 15 \mathrm{Am}$ Dale and Time of Shipment

PART II: TO BE COMPLETED BY TRANSPORTER (Note: To be completed in presence of Generator, before removing Generator's copy)

Transporter's Name 6.8 BCOTS Senth

Business Address P.O. RMawed 1987 LAUREL. MS

Telephone No. $649-1220$

License Plate of Truck $80 / 446.4$

License Plate of Trailer $3941 T L$

If Transportation by Barge, Barge and Tug Identification

CERTIFICATION: I certify that the waste in the quantity above was received by me for shipment to the above destination.

Quese \& Cadlere

Date and Time Received

\section{PART III: TO BE COMPLETED BY DISPOSER}

Disposer's Name G.B BUOTS Sinith

Disposal Site Address HlleClent DMire LAUREL MS.

Telephone No. $6.49-1220$

License Plate of Truck $80<4464$

License Plate of Trailer $394 i T L$

If received by Barge, Barge and Tug Identification

CERTIFICATION: I certify that the waste in the quantity and description in Part I was received by me for proper disposal. 
G.B. "BOOTS" SMITH CORP.

$17 / 29$

LAUREL, MISSISSIPPI 39441

OILFIELD WASTE SHIPPING CONTROL TICKET

FILE COPY

Le. 10.17500

P.O. No. 0085

DOC ID. NO Mr

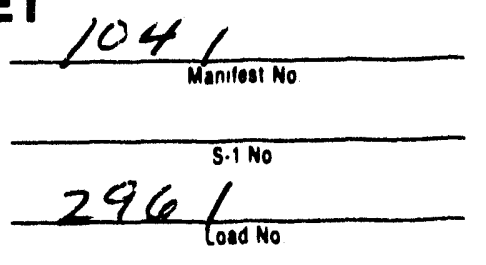

PART I: TO BE COMPLETED BY GENERATOR

company Name STONE tWEeter Ever Core

Code No

Business Address P.O. Box 2325 BOston MA

Telephone No. 6 -17 $589-2207$

Well Name DOE-ROWELL MH-4B

Origin of Waste: Field $\frac{\omega(\angle D C A T}{D \text { Drilling Ope rat }}$

AFE No.

Well Serial No $23 / 1 / 20042$

Description of Waste SALT K/ATER $20720 \mathrm{mg} / L=T 25 ; \quad p H=9.28$

Code No.

Quantity of Waste

$80 B 8 \angle 5$

Seal On

DESTINATION:

Disposer's Name

Code No.

Business Address

Disposal Site Address

CERTIFICATION: The waste described above was consigned to the carrier named below. I certify that the foregoing is true and correct to the best of my knowledge.

Pectoral. gillespie

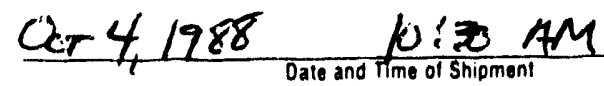

PART II: TO BE COMPLETED BY TRANSPORTER (Note: To be completed in presence of Generator, before removing Generator's copy) Transporter's Name $G$ B. BOOT'S Santa

Business Address P.O. Darer 1987 MU.

Telephone No. $\quad 649-122 \mathrm{C}$

License Plate of Truck $80 / 446 H$

License Plate of Trailer $394 / 7 L$

If Transportation by Barge. Barge and Tug Identification

CERTIFICATION: I certify that the waste in the quantity above was received by me for shipment to the above destination.

Signature of transporter's Agent

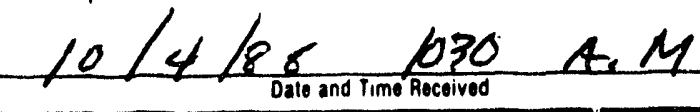

PART III: TO BE COMPLETED BY DISPOSER

Disposer's Name G. B. $200>5$ Sixth

Code No.ecsuep if I

Disposal Site Address HLL Coat Dree $\angle 40 R E L ~ M S$.

Telephone No. $649 .<2.20$

Seal off

License Plate of Truck $80 / 446 / 4$

License Plate of Trailer $394<T L$

If received by Barge. Barge and Tug Identification

CERTIFICATION: I certify that the waste in the quantity and description in Part I was received by me for proper disposal.

DISTRIBUTION:

Dale and time Received

000535

Original: G.B. "Boots" Smith Corp. - Green: Office of Conservation - Yalow: Disposer - PInk: Transporter - Gold: Generator's Pst Copy 
G.B. "BOOTS" SMITH CORP.

$18 / 29$

LAUREL, MISSISSIPPI 39441

OILFIELD WASTE SHIPPING CONTROL TICKET

FILE COPY

wa 10.17000

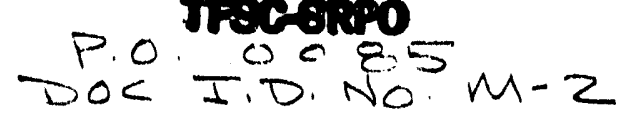

$\frac{\frac{1042}{296 \frac{2}{\text { Mannitosi No }}}}{\frac{2.1 \mathrm{No}}{\log \mathrm{N} \text { No }}}$

PART I: TO BE COMPLETED BY GENERATOR

Company Name STONE \& WEBSTER ENGR CorP Code No. Business Address PO. BOX 2325 BOSTON, MA Telephone No. $617589-2098$ Well NameDOE-ROWELL MH-4C

Origin of Waste: Field U/LDCAT AFE No Well Serial No .23/1/20043

2 Drilling Operations Description of Waste S4LTWATER (TDS $=19740 \mathrm{mg} / \mathrm{pH}$, 8.7$)$ Quantity of Waste 75 BBC's Code No. DESTINATION:

Disposer's Name Code No.

Business Address

Disposal Site Address

CERTIFICATION: The waste described above was consigned to the carrier named below. I certify that the foregoing is true and correct to the best of my knowledge.

Richard H. Helesipe

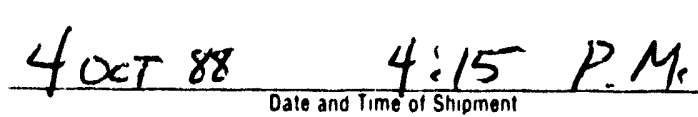

PART II: TO BE COMPLETED BY TRANSPORTER (Note: To be completed in presence of Generator, before removing Generator's copy)

Seal On Transporter's Name C. B. BOOT'S Business Address Pl. D awe 1987 LALREL 15. Telephone No. 649.1220

License Plate of Truck $\frac{801446 \pi}{344.74}$

License Plate of Trailer 394172

If Transportation by Barge, Barge and Tug Identification

CERTIFICATIQN: I certify that the waste in the quantity above was received by me for shipment to the above destination.

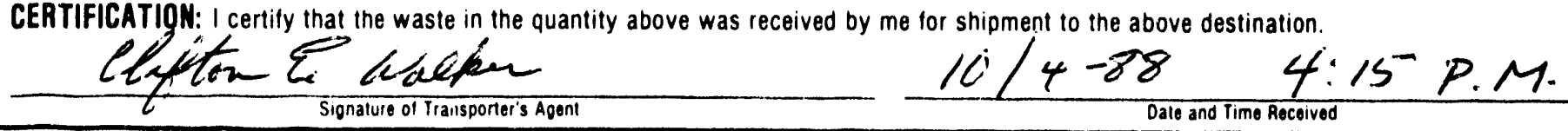

PART III: TO BE COMPLETED BY DISPOSER

Disposer's name 6 . BO

Code No. COC Sits

Disposal Site Address HL L Croat D. LALREL MS:

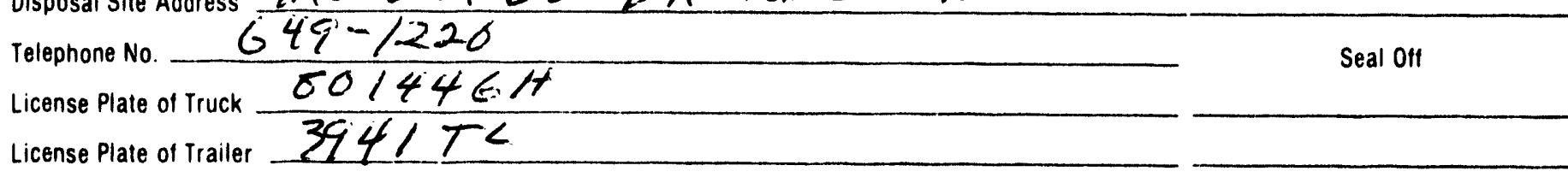

If received by Barge, Barge and Tug Identification

CERTIFICATION: I certify that the waste in the quantity and description in Part I was received by me for

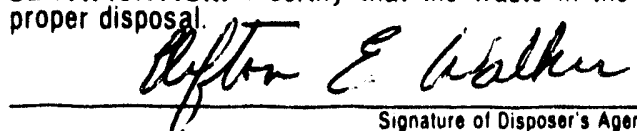

DISTRIBUTION:

Date and Time Received

000536

Original: G.B. "Boots" Smith Corp. - Green: Office of Conservation - Yellow: Disposer - PInk: Transporter - Gold: Generator's 1st Copy 
G.B. "BOOTS" SMITH CORP.

$19 / 29$

LAUREL, MISSISSIPPI 39441

OILFIELD WASTE SHIPPING CONTROL TICKET

FILE COPY

10.110.17500

P.O No .0085

DOC. ID. $M-2$

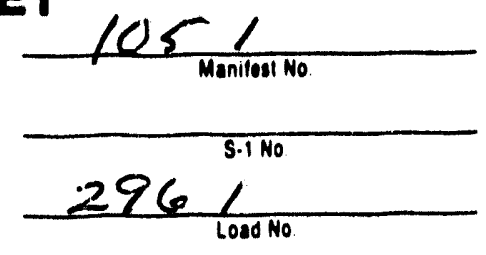

Code No.

Company Name STONES WeBSTER ENGR

Business Address P.O. Bor 2325 Boston MA

Telephone No. $617 \quad 589-2098$

Well Name MCCH - 3B

AFE No.

Well Serial No .2311120038

Origin of Waste: Field Whepcar

Production Facility

Description of waste SALT WATER (TDS $=160,000 \mathrm{mg} / \mathrm{h}, p H=9.39$ Code No.

Quantify of Waste

$120 B B<' s$

Seal On

DESTINATION:

Disposer's Name

Code No.

Business Address

Disposal Site Address

CERTIFICATION: The waste described above was consigned to the carrier named below. I certify that the foregoing is true and correct to the best of my knowledge.

Ruched. gillespie

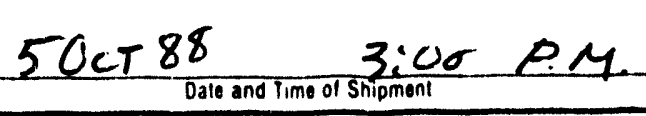

PART II: TO BE COMPLETED BY TRAMSPORTER (Note: TO be completed in presence of Generator, before removing Generator's copy)

Transporter's Name 3 B BQO TS smith

Business Address R.O PRALUER AI E7 LACREL MS

Telephone No. $649-1220$

License Plate of Truck $-201446 / 4$

License Plate of Trailer $3941 Z L$

If Transportation by Barge, Barge and Tug Identification

CERTIFICATION: I certify that the waste in the quantity above was received by me for shipment to the above destination.

Defier \& Ne lew

$10 / 5 / 88$

Bios P.M.

PART III: TO BE COMPLETED BY DISPOSER

Dale and Time Received

Disposer's Name G. B. BOOTS Smith Code No. $\operatorname{coc} \sin D \neq 1$

Disposal Site Address H/LLCreat De LACIREL MS.

Telephone No. $649-1220$

Seal off

License Plate of Truck 8014461

License Plate of Trailer $394 \angle I L$

If received by Barge, Barge and Tug Identification

CERTIFICATION: I certify that the waste in the quantity and description in Part I was received by me for proper disposal.

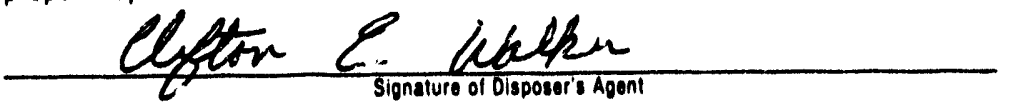

Date and Time Received

DISTRIBUTION:

000537

Original: G.B. "Boots" Smith Corp. - Groan: Office of Conservation - Yalow: Disposer - PInk: Transporter - Gold: Generator's 1st Copy 
G.B. "BOOTS" SMITH CORP.

LAUREL, MISSISSIPPI 39441

OILFIELD WASTE SHIPPING CONTROL TICKET
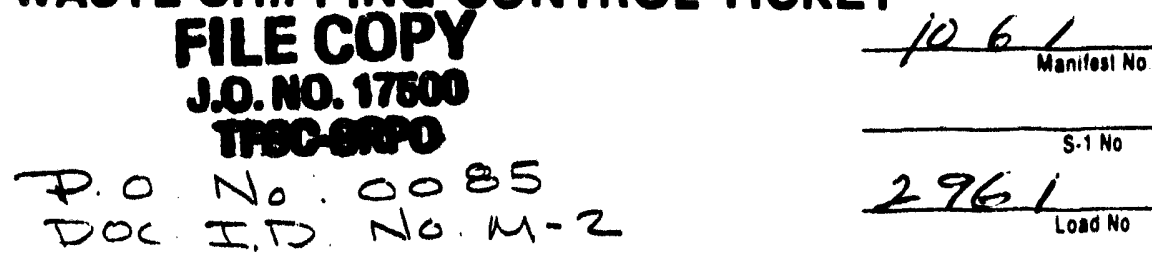

$226 i_{\text {LOAO NO }}^{\text {S.1 NO }}$

PART I: TO BF COMPLETEO BY GENERATOR

Company Name STONE $\{$ WERSTI: ENGX. CORP Business Address $P_{0 .}$ Bar 2325 Baston MA

Telephone No. $617589-2098$

Origin of Waste: Field $\frac{\text { Whocar }}{\text { Drilling Operations }}$ Well Name MCCH - 3C \& MCCH -3D

Code No.

Description of Waste SALTWATER (TDS-111,300 mg/L, $\mathrm{pH}=8.49$ )

Quantity of Waste $60 \mathrm{bbls}-3 C \quad 50 \mathrm{bb} / \mathrm{s}-3 D$

HOTOTAL

Seal On

OESTINATION:

Disposer's Name

Code No.

Business Address

Disposal Site Address

CERTIFICATION: The waste described above was consigned to the carrier named bolow. I certify that the

foregoing is true and correct to the best of my knowledge.

Prakent. Sillospie Signalure of denerator's Authorizod Agent

3i 30 pan

PART II: TO BE COMPLETED BY TRANSPORTER (Note: TO be completed in presence of Generator, before removing Generator's copy)

Transporter's Name GB. BOOTS Sme th

Business Address P.O. Drowa 1987 LALREC MS

Telephone No. G49-1220

License Plate of Truck $80 / 4464$

License Plate of Trailer $344 L I L$

If Transportation by Barge. Barge and Tug Identification

CERTIFICATION: I certify that the waste in the quantity above was received by me for shipment to the above destination.

Eifter E. Cuxtein

$10 / 8 / 88$ 3:35 P.M.

\section{PART III: TO BE COMPLETED BY DISPOSER}

Disposer's Name G.B. BOQ is SanZz

Code No. COCSLLO \#1

Disposal Site Address HILC Cnest De LALLREL MS.

Telephone No. $649-1220$

Seal off

License Plate of Truck 8014464

License Plate of Trailer $3941 T L$

If received by Barge, Barge and Tug Identification

CERTIFICATION: I certify that the waste in the quantity and description in Part I was received by me for proper disposal. 
G.B. "BOOTS" SMITH CORP.

$21 / 29$

LAUREL, MISSISSIPPI 39441

OILFIELD WASTE SHIPPING CONTROL TICKET

FILE COPY

JoT. NO. 17500

TFBC-8RPo

D. O. 0085

DoC .I.T. No. M.2
107

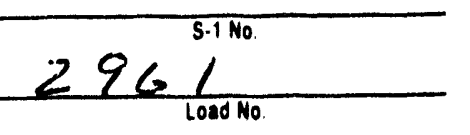

Code No.

TO BE COMPLETED BY GENERATOR

Company Name STONE \& WEB si: FVGINIRERNG-CORP.

Bo stor, me

Business Address $\frac{245 \text { SUMMER }}{67-589-2098}$

Well Name MC C $-1 /-34$

Origin of Waste:

Field $\frac{\text { WICCA }}{O}$

AFE No.

Well Serial AOP 2311120037

Description of Waste SALT WATER

Code No.

Quantity of Waste $120 \mathrm{BBL}$

Seal on

DESTINATION:

Disposer's Name

Code No.

Business Address

Disposal Site Address

CERTIFICATION: The waste described above was consigned to the carrier named below. I certify that the foregoing is true and correct to the best of my knowledge.

WC Bras

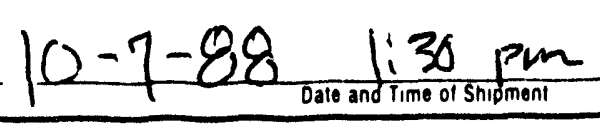

PART II: TO BE COMPLETED BY TRANSPORTER (Note: To be completed in presence of Generator, before removing Generator's copy) Transporter's Name G.B. BOOTS Seth

Business Address P.O. DRAWEE ME 7 LAUREL MS.

Telephone No. $E 49-1228$

License Plate of Truck $80 / 446 \%$

License Plate of Trailer $394 / 2 L$

If Transportation by Barge, Barge and Tug Identification

CERTIFICATION: I certify that the waste in the quantity above was received by me for shipment to the above destination.

leveler

$10 / 7 / 88$

PART III: TO BE COMPLETED BY DISPOSER

Disposer's Name G.B. Boo T's Smith

Code No. COC SND

Disposal site Address H/LCwest $D_{n}$. LAUREL NS.

Telephone No. $649-1220$

Seal Oft

License Plate of Truck $50<4464$

License Plate of Trailer $3941 T \angle$

If received by Barge, Barge and Tug Identification

CERTIFICATION: I certify that the waste in the quantity and description in Part I was received by me for proper disposal.

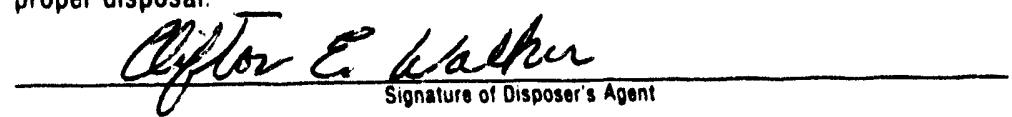

Date and Time Received DISTRIBUTION:

000539

Original: G.B. "Boots" Smith Corp. - Green: Office of Conservation - Yellow: Disposer - Pink: Transporter - Gold: Generator's 1st Copy 
G.B. "BOOTS" SMITH CORP.

OILFIELD WASTE SHIPPING CONTROL TICKET

FIE SOCPY"

J.0. NO. 17500

TFSCORPO

P.O. No. 0085

DOC.I.D. No M-2

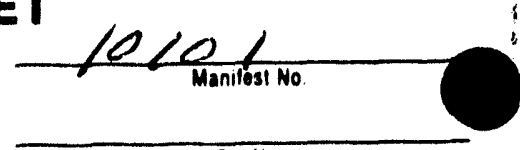

$296 \int_{\text {lote No }}^{5.1 N_{0}}$

PART I: TO BE COMPLETEO BY GENERATOR

Company Name Stove thesstar ENar Cone Code No.

Business Address PO Box 2325 Buston MA 02107

Telephone No. $617589-2098$ Well Name MCCG-1

Origin of Waste: Field UN LOCAT

AFE No.

Drilling Operations

$\square$ Production Facility

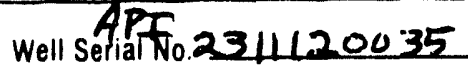

Description of Waste SALT WATER (TDS $=188,800 \mathrm{hgh}$, pH 10.55 Code No.

Quantity of Waste 75 BBtis

Seal On

\section{DESTIMATION:}

Disposer's Name Code No.

Business Address

Disposal Site Address

CERTIFICATION: The waste described above was consigned to the carrier named below. I certify that the foregoing is true and correct to the best of my knowledge.

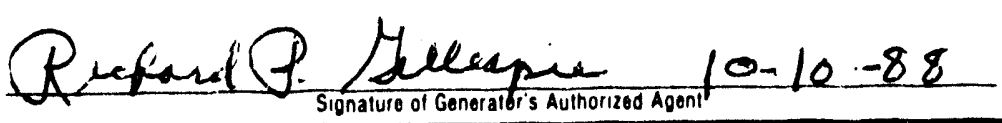

PART II: TO BE COMPLETEO BY TRAMSPORTER (Note: To be completed in presence of Generator, before removing Generator's copy) Transporter's Name GB. BOOTS smite

Business Address P.Q. Diewer 1987 LALREL MS.

Telephone No. $649-1220$

License Plate of Truck $80<4464$

License Plate of Trailer $34 ; T L$

If Transportation by Barge, Barge and Tug Identification

CERTIFICATION: I certify that the waste in the quantity above was received by me for shipment to the above destination.

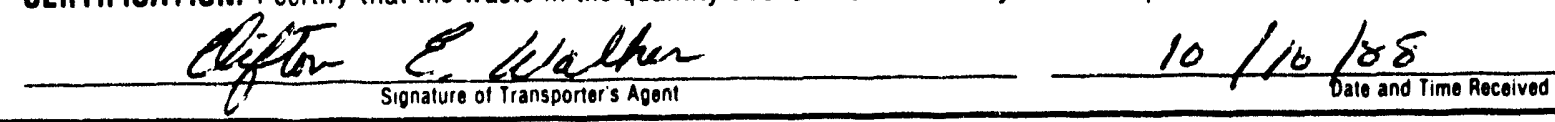

PART III: TO BE COMPLETED BY DISPOSER

Disposer's Name GB. BOOT's Simitz Code No. COCSWD

Disposal Site Address HILLCest De $\angle A U R E L M S$.

Telephone No.

$$
649-1220
$$

Seal 0ft

License Plate of Truck 8014464

License Plate of Trailer $3941 T L$

If received by Barge, Barge and Tug Identification

CERTIFICATION: I certify that the waste in the quantity and description in Part I was received by me for

proper disposal.

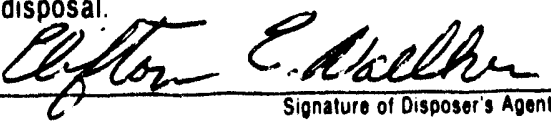

DISTRIBUTION:

Signature of Disposer's Agen

$10 / 10 / 88$

Original: G.B. "Boots" Smith Corp. - Green: Office of Conservation - Yollow: Disposer - PInk: Transporter - Bold: Generator's 1st Copy 
G.B. "BOOTS" SMITH CORP. LAUREL, MISSISSIPPI 39441

\section{FILE COPY J.0.NO.1700 \\ Tropsion \\ P.O. No. 0085 \\ DOC I.D.NO M-2

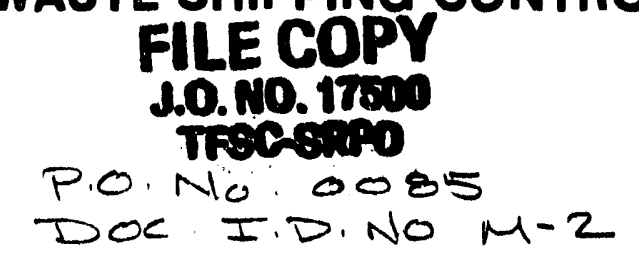

OILFIELD WASTE SHIPPING CONTROL TICKET

PART I: TO BE COMPLETED BY GENERATOR

company name stone lovaestar Ener Corp. Business Address PO BOX 2325. Boston, MA Telephone No. $627589-2098$ Well Name DaE MCCG-2

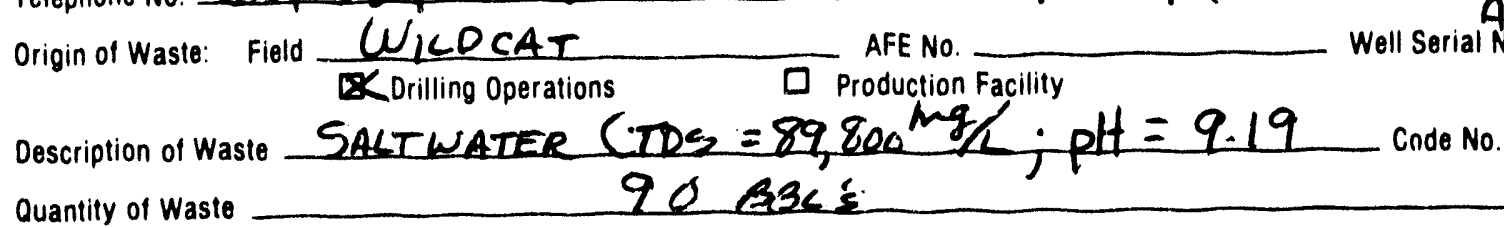
Well Serial APL API 2311120036 DESTINATION: Disposer's Name Code No.

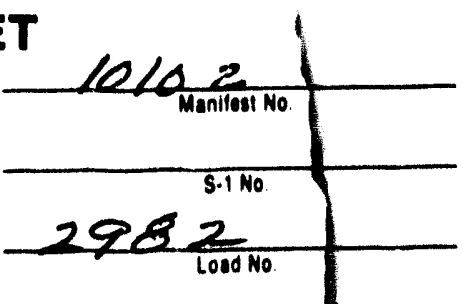

Business Address

Disposal Site Address

CERTIFICATION: The waste described above was consigned to the carrier named below. 1 certify that the foregoing is true and correct to the best of my knowledge.

Seal On Pechard A Lillezpie

$p-10 \cdot 88 \quad 5: 30 \mathrm{PM}$

PART II: TO BE COMPLETED BY TRANSPORTER (Note: To be completed in presence of Generator, before removing Generator's copy) Transporter's Name E. B. B Q T TE suill Business Address $P Q$ Rawer 1987 LAAREC ME Telephone No. $649-1220$ License Plate of Truck $2 \mathrm{CL4} / \mathrm{GC}$ License Plate of Trailer $394 \angle L$

If Transportation by Barge, Barge and Tug Identification

CERTIFICATION: I certify that the waste in the quantity above was received by me for shipment to the above destination.
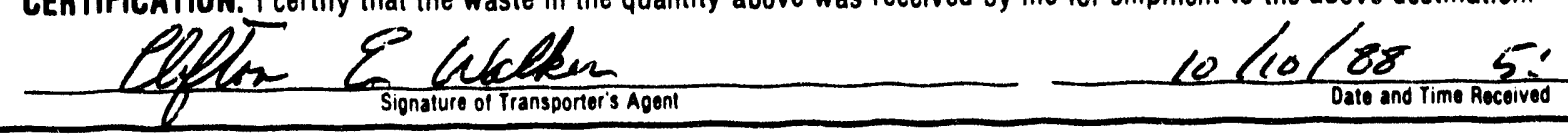

30 PAL

\section{PART III: TO BE COMPLETED BY DISPOSER}

Disposer's Name E.B. BeOT 5 simith Code No.

Disposal Site Address H.LL Crent prine LAUREL ME

Telephone No. $649-1220$

License Plate of Truck $\mathrm{Eos} 14464$

License Plate of Trailer 39 if $T$

If received by Barge, Barge and Tug Identification

CERTIFICATION: I certify that the waste in the quantity and description in Part I was received by me for proper disposal.

DISTRIBUTION:
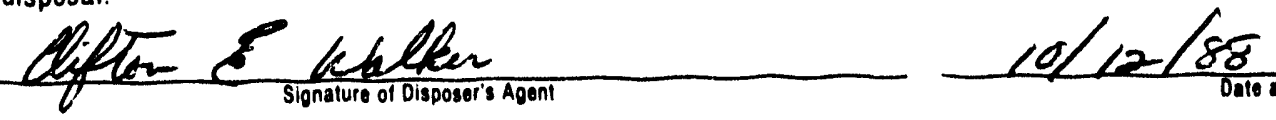

Oplalnal: G.B. "Boots" Smith Coro. - Groen: Office of Conservation - Yellow: Disposer - PInk: Transporter - Bold: Generator's 1st Copy 
G.B. "BOOTS" SMITH CORP.

$24 / 29$

LAUREL, MISSISSIPPI 39441

OILFIELD WASTE SHIPPING CONTROL TICKET

FILE COPY

JO. NO. 17500

TFSC-SRPO

P.O. No .0085

DOC. I. D. NO MiL

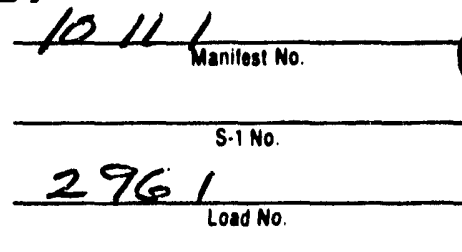

PART I: TO BE COMPLETED BY GENERATOR

Company Marne 5 TONE \& WEBSTER ENGR CORP.

Code No.

Business Address PO BoX 2325 BOstoN MA

Telephone No. $617589-2098$

Well Name DOE -SMITH MRI G - ILC

Origin of Waste: Field WiLDCAT

AFE No. Well Serial No. 2311120047

Drilling Operations

Production Facility

Description of Waste SALT WATER (TDS $=5840 ; \mathrm{pH}=8.42)$ Code No.

Quantity of Waste

70 BBC's

Seal On

DESTINATION:

Disposer's Name Code No.

Business Address

Disposal Site Address

CERTIFICATION: The waste described above was consigned to the carrier named below. I certify that the foregoing is true and correct to the best of my knowledge.

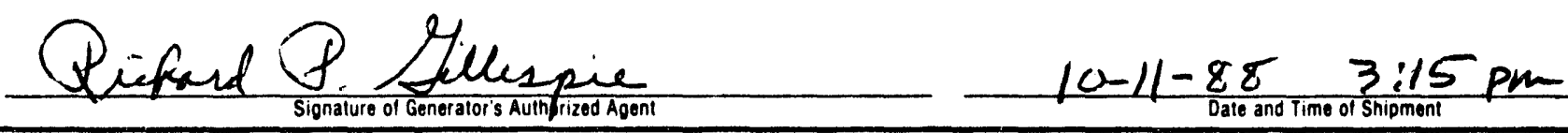

PART II: TO BE COMPLETE O BY TRANSPORTER (Note: To be completed in presence of Generator, before removing Generator's copy)

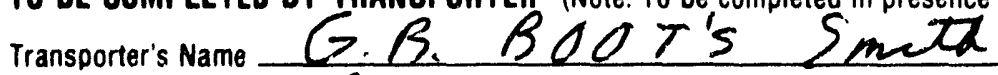

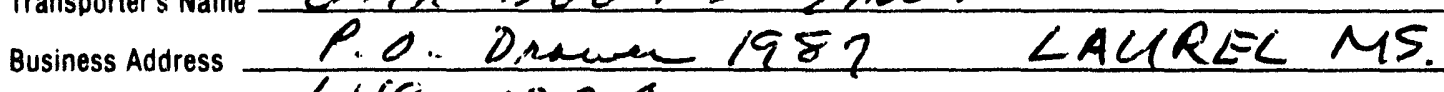

Telephone No. $649-1220$

License Plate of Truck 8014461

License Plate of Trailer $394 \mathrm{LL}$

If Transportation by Barge, Barge and Tug Identification

CERTIFICATION: I certify that the waste in the quantity above was received by me for shipment to the above destination.

$10 / 1 / 88$ 3:15 P.4.

PART III: TO BE COMPLETE O BY DISPOSER

Disposer's Name G-B. BOOT5 Smith

Code No. $\operatorname{coc} \operatorname{sen} \neq 1$

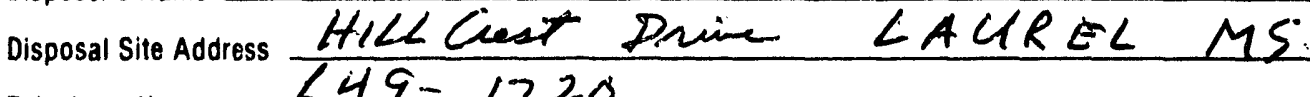

Telephone No. $\frac{649-1220}{80}$ Seal off

License Plate of Truck $80 / 4464$

License Plate of Trailer $3941 \mathrm{TL}$

If received by Barge, Barge and Tug Identification

CERTIFICATION: I certify that the waste in the quantity and description in Part I was received by me for proper disposal.

Lather

DISTRIBUTION:

Signature oi Disposer's Agent

Date and Time Received 000542

Original: G.B. "Boots" Smith Corp. - Green: Office of Conservation - Yellow: Disposer - PInk: Transporter - Gold: Generator's 1st Copy 
G.B. "BOOTS" SMITH CORP. LAUREL, MISSISSIPPI 39441

\section{OILFIELD WASTE SHIPPING CONTROL TICKET}

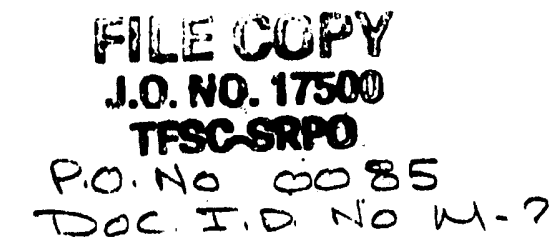

\begin{tabular}{l}
\hline Manilost No. \\
\hline S.1 No. \\
\hline Load No.
\end{tabular}

PART I: TO BE COMPLETED BY GEMERATOR

Company Name STONE \&WEB STER ENGR CORP Code No. Business Address PO. BOX 2325 BOsiton MA Telephone No. $617589-2098$ Well Name DaE/SMITH MRIH - IIA Origin of Waste: Field W/LPCAT AFE No. Well Serial No. Description of Waste SALT WATER (TDS $=180,000 \mathrm{mg} / \mathrm{L}, \mathrm{pH}=8.8)_{1}^{\prime}$ Code No. Quantity of Waste 100 BELS.

DESTIMATION:

Disposer's Name Code No.

Business Address

Disposal Site Address

CERTIFICATION: The waste described above was consigned to the carrier named below. I certify that the foregoing is true and correct to the best of my knowledge.

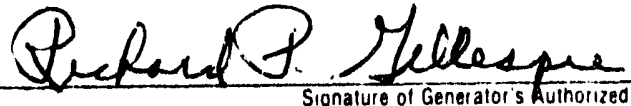
$10 / 12 / 88$
$1: 45 P M$

PART II: TO BE COMPLETED BY TRANSPORTER (Note. 10 be completed in presence of Generator, betore removing Generator's copy)

Transporter's Name

Business Address

Telephone No.

License Plate of Truck 80142714

License Plate of Trailer 195

If Transportation by Barge. Barge and Tug Identification

CERTIFICATION: I certify that the waste in the quantity above was received by me for shipment to the above destination.
Saylor Rewed

PART III: TO BE COMPLETEO BY DISPOSER

Disposer's Name Code No.

Disposal Site Address

Telephone No.

License Plate of Truck $801422 \mathrm{Ht}$

License Plate of Trailer $7 / 95$

If received by Barge, 8arge and Tug Identification

CERTIFICATION: I certify that the waste in the quantity and description in Part I was received by me for proper disposal.

OISTRIBUTION:

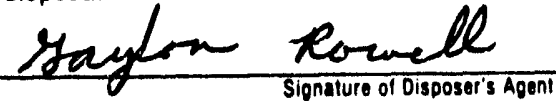




\section{G.B. "BOOTS" SMITH CORP.}

LAUREL, MISSISSIPPI 39441

OILFIELD WASTE SHIPPING CONTROL TICKET

FILE COPY

J.0. 110. 1460

TFocesivo

P.O. No:0085

DOC I.D. NO.M-2

10121 Manilest No.

$296 /_{\text {Load No. }}^{\text {S.1No. }}$

\section{PART I: TO BE COMPLETED BY GENERATOR}

Company name STONE $\{$ WeBster ENGR, CORP Code No.

Business Address PO BOX 2325 Busre

Telephone No. $617589-2098$

Origin of Waste: Field WILDCAT

Origin of Waste. Field Drilling Operations

Well Name DOE/SMITH'MRIH - IIA

AFE No.

Well Serial No.

Description of Waste $\frac{S_{A 4 T} W_{A T E R}(T D S=180,000 \mathrm{mg} / \mathrm{L}, \mathrm{pH}=8.8) !}{110 \mathrm{BBLS}}$ Quantity of Waste

\section{OESTIMATION:}

Disposer's Name

Code No.

Business Address

Disposal Site Address

CERTIFICATION: The waste described above was consigned to the carrier named below. I certify that the

foregoing is true and correct to the best of my knowledge.

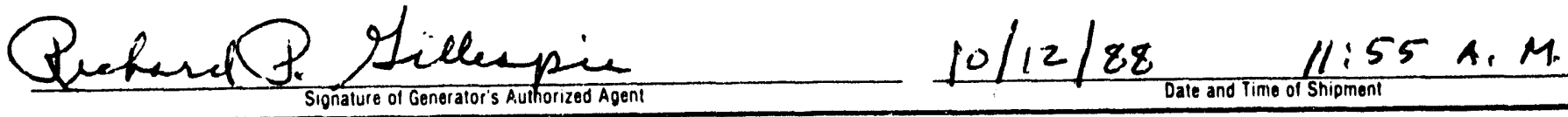

PART II: TO BE COMPLETEO BY TRANSPORTER (Note: To be completed in presence of Generator, before removing Generator's copy)

Transporter's Name

Business Address

Telephone No.

License Plate of Truck

License Plate of Trailer

If Transportation by Barge. Barge and Tug Identification

CERTIFICATION: I certify that the waste in the quantity above was received by me for shipment to the above destination.
Clear
E.
chelew
$10 / 12 / 85$
$11: 55$ A.

PART III: TO BE COMPLETED BY DISPOSER

Disposer's Name Codeno.cocswD

Disposal Site Address

Telephone No.

License Plate of Truck

License Plate of Trailer

If received by Barge, Barge and Tug Identification

41

CERTIFICATION: I certify that the waste in the quantity and description in Part I was received by me for proper disposal.

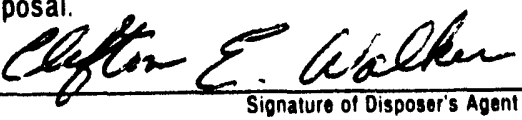




\section{G.B. "BOOTS" SMITH CORP. \\ LAUREL, MISSISSIPPI 39441}

\section{FILE COPY \\ J.U. Na. 1750 . \\ TFSCSTPO \\ P.O. No. 0085 \\ DOC. I.D. NO M-2}

OILFIELD WASTE SHIPPING CONTROL TICKET

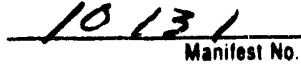

Manilesi No

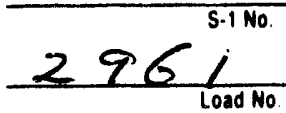

PART I: TO BE COMPLETED BY GENERATOR Company Name STONE\& WEBETER ENGR CORP Business Address PO BuX 2325 BOETON MA Telephone No. $617589-2098$ Well Name DoE $/ \mathrm{sm}$ TH MRIH- II DD Origin of Waste: Field WILDCAT AFE No. Quantity of Waste 80 sec's

\section{DESTIMATION:}

Disposer's Name Code No.

Business Address

Disposal Site Address

CERTIFICATION: The waste described above was consigned to the carrier named below. I certity that the foregoing is true and correct to the best of my knowledge.

QP. Rillepiniv

$$
10-13-88 \text { Date and time of Shipment }
$$

PART II: TO BE COMPLETED BY TRANSPORTER (Note: To be completed in presence of Generator, before removing Generator's copy)

Transporter's Name

Business Address

Telephone No.

License Plate of Truck

License Plate of Trailer

If Transportation by Barge. Barge and Tug Identification

CERTIFICATION: f certify that the waste in the quantity above was received by me for shipment to the above destination.

Cefor

PART III: TO BE COMPLETED BY DISPOSER

Disposer's Name

Disposal Site Address

Telephone No.

License Plate of Truck

License Plate of Trailer

If received by Barge, Barge and Tug Identification

CERTIFICATION: I certify that the waste in the quantity and description in Part I was received by me for proper disposal. 
G.B. "BOOTS" SMITH CORP.

\section{LAUREL, MISSISSIPPI 39441}

OILFIELD WASTE SHIPPING CONTROL TICKET

FILE COPY

J.0. Na 1700

TFsosivo

P.O. No. 0085

DOC ID. No M-2

\section{2}

$2982^{\text {S.1 No }}$

PART I: TO BE COMPLETED BY GENERATOR

Company Name STONE \& WEBSTER ENa Corp Code No.

Business Address PO BOX 2325 BOSTON $M A$

Telephone No. 617589-2098 Well Name DoE/SMITH MR/1t-IIB

Origin of Waste: Field W/LPCAT

AFE No.

Well Serial No. $23 / 1 / 20046$

$\triangle$ Drilling Operations $\square$ Production Facility

Description of Waste SALT WATER $\left(T D C_{2}=17,300 ; P H=8.1\right) 1$ Code No.

Quantity of Waste 100661

Seal On

\section{DESTINATION:}

Disposer's Name

Code No.

Business Address

Disposal Site Address

CERTIFICATION: The waste described above was consigned to the carrier named below. I certify that the foregoing is true and correct to the best of my knowledge.

\section{Qfutar P. Sillengie $10 / 13 / 88$} Date and Time of Shipment

PART II: TO BE COMPLETED BY TRANSPORTER (Note: To be completed in presence of Generator, before removing Generator's copy)

Transporter's Name

Business Address

Telephone No.

License Plate of Truck

License Plate of Trailer

If Transportation by Barge. Barge and Tug Identification

CERTIFICATION: I certify that the waste in the quantity above was received by me for shipment to the above destination.

Signature of Transporter's Agent

\section{PART III: TO BE COMPLETED BY DISPOSER}

Disposer's Name Code No.

Disposal Site Address

Telephone No.

License Plate of Truck

License Plate of Trailer

If received by Barge. Barge and Tug Identification

CERTIFICATION: I certify that the waste in the quantity and description in Part I was received by me for proper disposal.

DISTRIBUTION:

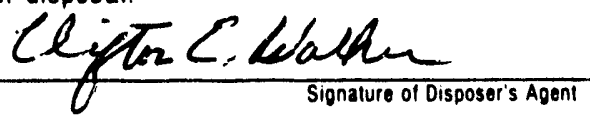




\section{G.B. "BOOTS" SMITH CORP.}

\section{LAUREL, MISSISSIPPI 39441}

\section{OILFIELD WASTE SHIPPING CONTROL TICKET FILE COPY \\ d.0.MO. 1700 \\ Tranino \\ P.O. Nu:0085 \\ Do. I.D. No, M-2}

\begin{tabular}{c}
\hline Manitest No. \\
\hline S-1 No. \\
\hline Load No
\end{tabular}

\section{PART I: TO BE COMPLETED BY GENERATOR}

Company name STONE \&WESSTER ENer. CORP Business Address PO BOX 2325 BOSTON MA. 02107 Telephone No. $6 i 7589-2098$ Well Name DOE/MASONITE MRIC-9 Origin of Waste: Field W/LDCAT AFE No. Well Serial No. 2311120049 Quantity of Waste

1 Drilling Operations $\square$ Production Facility

Description of Waste

SALT WATER (TDE $=104,5$ $25 \mathrm{BB} \angle \mathrm{S}$

\section{DESTINATION:}

Disposer's Name Code No.

Business Address

Disposal Site Address

CERTIFICATION: The waste described above was consigned to the carrier named below. I certify that the foregoing is true and correct to the best of my knowledge. Quiband. Heleypu

PART II: TO BE COMPLETED BY TRANSPORTER (Note: To be completed in presence of Generator, before removing Generator's copy)

Transporter's Name

Business Address

Telephone No.

License Plate of Truck

License Plate of Trailer

If Transportation by Barge. Barge and Tug Identification

CERTIFICATION: I gertify that the waste in the quantity above was received by me for shipment to the above destination

CERTIFICATION: I gertify that the waste in the quantity above was received by me for shipment to the above destination.

\section{PART III: TO BE COMPLETED BY DISPOSER}

Disposer's Name Code No

Disposal Site Address

Telephone No.

License Plate of Truck

License Plate of Trailer

If received by Barge, Barge and Tug Identification

CERTIFICATION: I certify that the waste in the quantity and description in Part I was received by me for proper disposal. 
APPENDIX G

Plugging Reports 
PLUGGING REPORT

\begin{tabular}{|c|c|c|}
\hline & & $\begin{array}{c}\text { DATUM ELEVATION } \\
219.91 \\
\end{array}$ \\
\hline J.O. No. & 17500.25 & DATE $\quad 10 / 05 / 88$ \\
\hline
\end{tabular}

\section{NOTIFICATION OF REGULATORY AGENCY}

DATE $10 / 05 / 88$

AGENCY MS BUREAU OF LAND \& WATER RESOURCES REPRESENTATIVE JAMES HOFFMAN

CEMENTING COMPANY GRINER DRILLING

SERVICE ENGINEER / REP. JERRY BEACH

DISPOSAL OF FLUIDS

BY GRINER DRILLING

QUANTITY NO EST.

DATE 11

FLUID TYPE DRILLING MUD

REMARKS

EXCESS DRILLING MUD IS HAULED TO CAMP SHELBY LANDFILL AS NECESSARY.

ZONE 1

TYPE PLUG CONTINUOUS

INTERVAL $120 \mathrm{FT}$ TO $2.0 \mathrm{FT}$

DIAMETER OF CASING/HOLE $\quad 6 \quad 3 / 4$ IN

BOTTOM OF TUBING OR PIPE 120 FT CEMENT/MUD TYPE CLASS A, NEAT

NO. OF SACKS 51 YIELD 1.17 CU.FT/SK. VOL. PUMPED 59.7 CU.FT

SLURRY WEIGHT 15.6 LBS/GAL TOP OF PLUG 2.0 FT

BRIDGE PLUG TYPE NA N DEPTH NA

REMARKS

PULLED ALL CASING AND SCREEN OUT OF HOLE. REAMED HOLE TO $120 \mathrm{FT}$, ENCOUNTERED CEMENT

PLUG AT $110 \mathrm{FT}$. CEMENT CIRCULATED TO SURFACE. TOP 2 FT BACKFILLED WITH SOIL.

ZONE

TYPE PLUG

INTERVAL

DIAMETER OF CASING/HOLE IN

BOTTOM OF TUBING OR PIPE FT CEMENT/MUD TYPE

NO. OF SACKS

YIELD

CU. FT/SK.

VOL. PUMPED

CU. FT

SLURRY WEIGHT LBS/GAL

TOP OF PLUG FT

BRIDGE PLUG TYPE

DEPTH

REMARKS 
PLUGGING REPORT

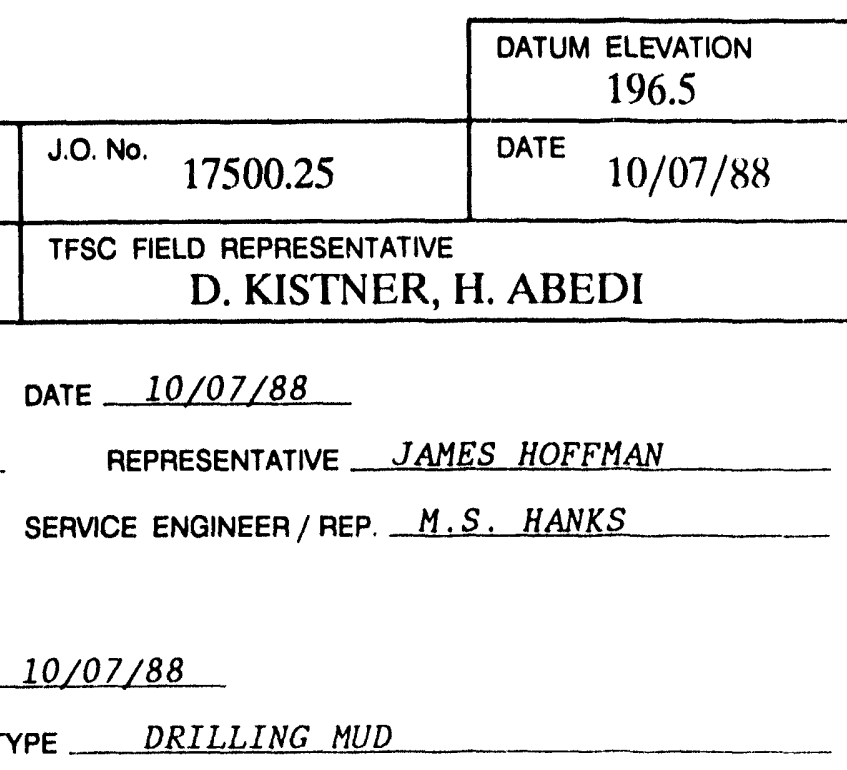

\section{REMARKS}

EXCESS DRILLING MUD HAULED TO CAMP SHELBY LANDFILL AS NECESSARY.

ZONE 1

INTERVAL 415 TO $3.0 \mathrm{FT}$

BOTTOM OF TUBING OR PIPE

NO. OF SACKS 105

SLURRY WEIGHT 13.1 LBS/GAL

BRIDGE PLUG TYPE _ NA

REMARKS

PULLED ALL CASING AND SCREEN OUT OF HOLE. REAMED HOLE TO 415 FT. CEMENT CIRCULATED TO SURFACE, TOP 3 FT BACKFILLED WITH SOIL.

ZONE

INTERVAL FT

YIELD

SLURRY WEIGHT

BRIDGE PLUG TYPE

REMARKS
TYPE PLUG CONTINUOUS

DIAMETER OF CASING/HOLE $\quad 6 \quad 3 / 4$ IN CEMENT/MUD TYPE CLASS A. 88 BENTONITE

VOL. PUMPED 201.6 CU.FT TOP OF PLUG 3.0

DEPTH NA LBS/GAL

BOTTOM OF TUBING OR PIPE

NO. OF SACKS
TYPE PLUG

DIAMETER OF CASING/HOLE IN CEMENT/MUD TYPE
CU. FT/SK. VOL. PUMPED

TOP OF PLUG DEPTH 
PLUGGING REPORT

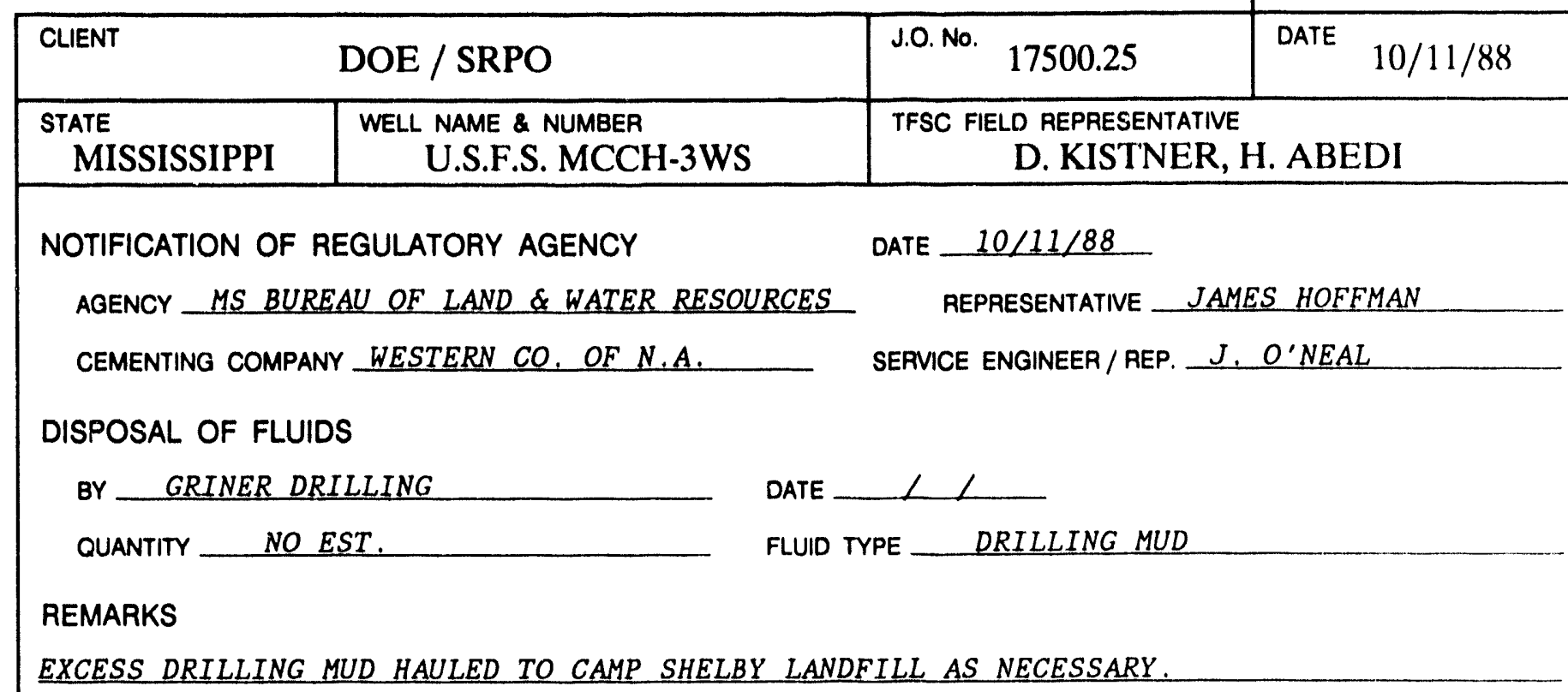

ZONE 1

TYPE PLUG CONTINUOUS

INTERVAL $\quad 444$ TO $5.0 \mathrm{FT}$

DIAMETER OF CASING/HOLE 7.0 IN

BOTTOM OF TUBING OR PIPE 441 FT CEMENT/MUD TYPE CLASS A. 88 BENTONITE

NO. OF SACKS $76 \quad$ YIELD 1.92 CU.FT/SK. VOL. PUMPED 146 CU. FT

SLURRY WEIGHT 13.1 LBS/GAL TOP OF PLUG 5.0 FT

BRIDGE PLUG TYPE NA DEPTH NA

REMARKS

PULLED ALL CASING AND SCREEN OUT OF HOLE. REAMED HOLE TO $444 \mathrm{FT}$. CEMENT CIRCULATED TO SURFACE, TOP 5 FT BACKFILLED WITH SOIL.

ZONE

INTERVAL

BOTTOM OF TUBING OR PIPE

NO. OF SACKS

SLURRY WEIGHT

BRIDGE PLUG TYPE

REMARKS
TYPE PLUG

DIAMETER OF CASING/HOLE IN FT CEMENT/MUD TYPE

YIELD CU. FT/SK. VOL. PUMPED CU.FT LBS/GAL TOP OF PLUG FT DEPTH 
PLUGGING REPORT

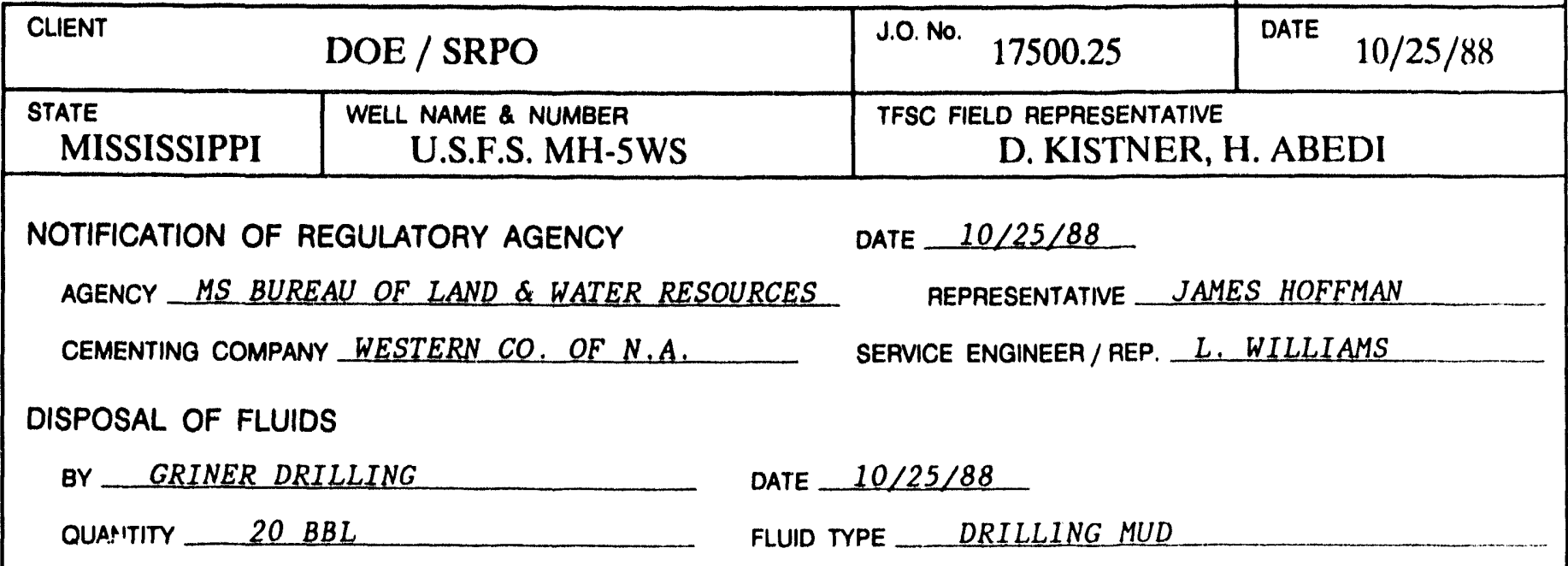

\section{REMARKS}

EXCESS DRILLING MUD HAULED TO SUBCONTRACTOR'S YARD FOR DISPOSAL.

ZONE 1

INTERVAL 405 TO $4.0 \mathrm{FT}$

BOTTOM OF TUBING OR PIPE $\quad 40$

NO. OF SACKS $100 \quad$ YIELD 1.92 CU.FT/SK.

SLURAY WEIGHT 13.1 LBS/GAL

BRIDGE PLUG TYPE NA

REMARKS

PULLED ALL CASING AND SCREEN OUT OF HOLE. REAMED HOLE TO 405 FT. CEMENT CIRCULATED TO SURFACE. TOP 4 FT BACKFILLED WITH SOIL.
TYPE PLUG CONTINUOUS

DIAMETER OF CASING/HOLE $6 \quad 3 / 4$ IN 


\begin{tabular}{|c|c|c|c|c|}
\hline CLIENT & \multicolumn{2}{|l|}{ DOE / SRPO } & J.O. No. 17500.25 & DATE $10 / 21 / 88$ \\
\hline $\begin{array}{l}\text { STATE } \\
\text { MISSISSIPPI } \\
\end{array}$ & \multicolumn{2}{|l|}{$\begin{array}{l}\text { WELL NAME \& NUMBER } \\
\text { WALKER MH-8WS }\end{array}$} & \multicolumn{2}{|c|}{$\begin{array}{l}\text { TFSC FIELD REPRESENTATIVE } \\
\text { D. KISTNER, H. ABEDI }\end{array}$} \\
\hline \multirow{3}{*}{\multicolumn{3}{|c|}{$\begin{array}{l}\text { NOTIFICATION OF REGULATORY AGENCY } \\
\text { AGENCY MS BUREAU OF LAND \& WATER RESOURCES } \\
\text { CEMENTING COMPANY WESTERN CO. OF N.A. }\end{array}$}} & \multirow{2}{*}{\multicolumn{2}{|c|}{ DATE $\frac{10 / 21 / 88}{\text { REPRESENTATIVE JAMES HOFFM }}$}} \\
\hline & & & & \\
\hline & & & \multicolumn{2}{|c|}{ SERVICE ENGINEER / REP. W. HARRIS } \\
\hline \multicolumn{5}{|c|}{ DISPOSAL OF FLUIDS } \\
\hline \multicolumn{2}{|c|}{ BY GRINER DRILLING } & \multicolumn{2}{|c|}{ DATE $10 / 21 / 88$} & \\
\hline QUANTITY. & & FLUID & YPE $\_$DRILLING & \\
\hline
\end{tabular}

\section{REMARKS}

EXCESS DRILLING MUD HAULED TO SUBCONTRACTOR'S YARD FOR DISPOSAL.

ZONE 1

INTERVAL 425 TO $4.0 \mathrm{FT}$

BOTTOM OF TUBING OR PIPE _ 420

NO. OF SACKS 102.5 YIELD 1.92 CU. FT/SK.

SLURRY WEIGHT $\frac{13.1}{1}$ LBS/GAL

BRIDGE PLUG TYPE NA

REMARKS

PULLED OUT 220 FT OF CASING AND 20-30 FT OF SCREEN. REAMED HOLE TO 425 FT. CEMENT CIRCULATED TO SURFACE, TOP 4 FT BACKFILLED WITH SOIL.

ZONE

INTERVAL

BOTTOM OF TUBING OR PIPE

NO. OF SACKS

SLURRY WEIGHT

BRIDGE PLUG TYPE

REMARKS
TYPE PLUG CONTINUOUS

DIAMETER OF CASING/HOLE $65 / 8$ IN CEMENT/MUD TYPE CLASS A, $8 \%$ BENTONITE

VOL. PUMPED 195 CU. FT TOP OF PLUG 4.0 FT DEPTH _ NA 
PLUGGING REPORT

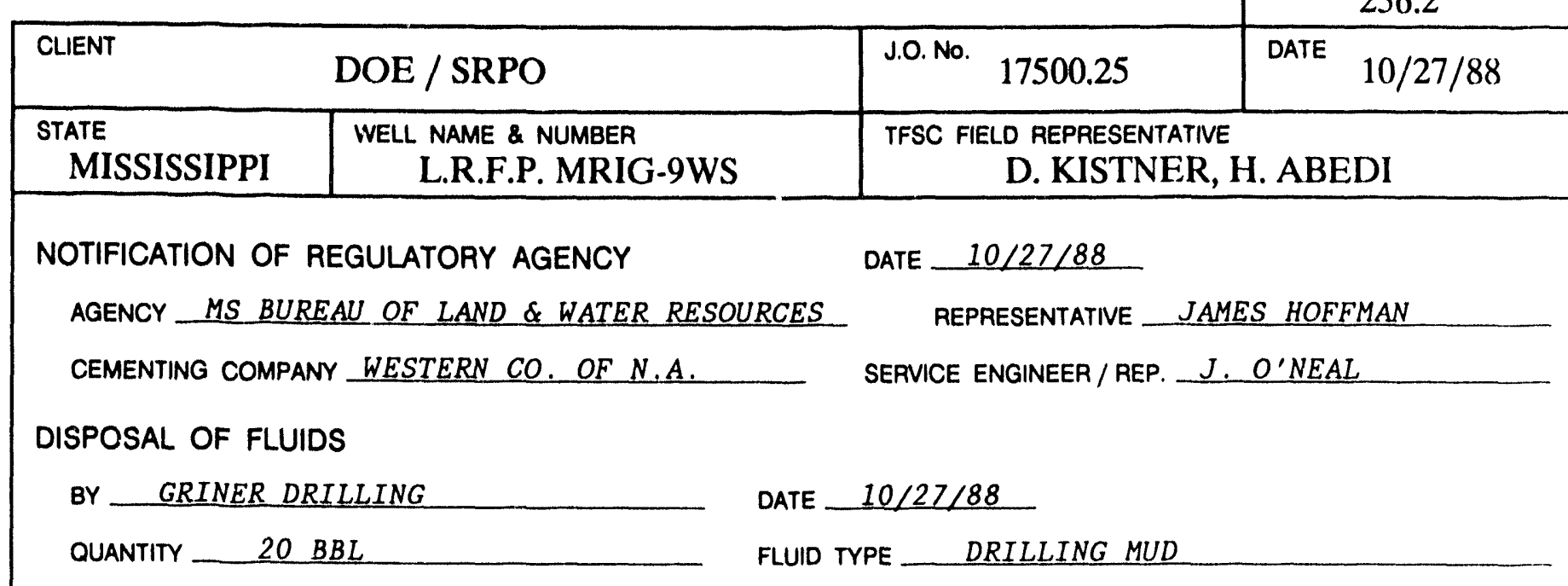

\section{REMARKS}

EXCESS DRILLING MUD HAULED TO RICHTON LANDFILL AS NECESSARY.

ZONE 1

INTERVAL 403 TO 5 FT

BOTTOM OF TUBING OR PIPE 395

NO. OF SACKS $100 \quad$ YIELD 1.92

SLURAY WEIGHT 13.1 LBS/GAL

BRIDGE PLUG TYPE

REMARKS

PULLED ALL CASING AND SCREEN OUT OF HOLE. REAMED HOLE TO 403 FT. CEMENT CIRCULATED TO SURFACE, TOP 5 FT BACKFILLED WITH SOIL.

ZONE

INTERVAL

BOTTOM OF TUBING OR PIPE

NO. OF SACKS

YIELD FT

SLURRY WEIGHT LBS/GAL

BRIDGE PLUG TYPE

TYPE PLUG

DIAMETER OF CASING/HOLE IN

TPE PLUG CONTINUOUS

DIAMETER OF CASING/HOLE $61 / 2$ IN CEMENT/MUD TYPE CLASS A, 8 \% BENTONITE

CU. FT/SK.

TOP OF PLUG 5.0 FT

DEPTH NA

\section{REMARKS}


PLUGGING REPORT

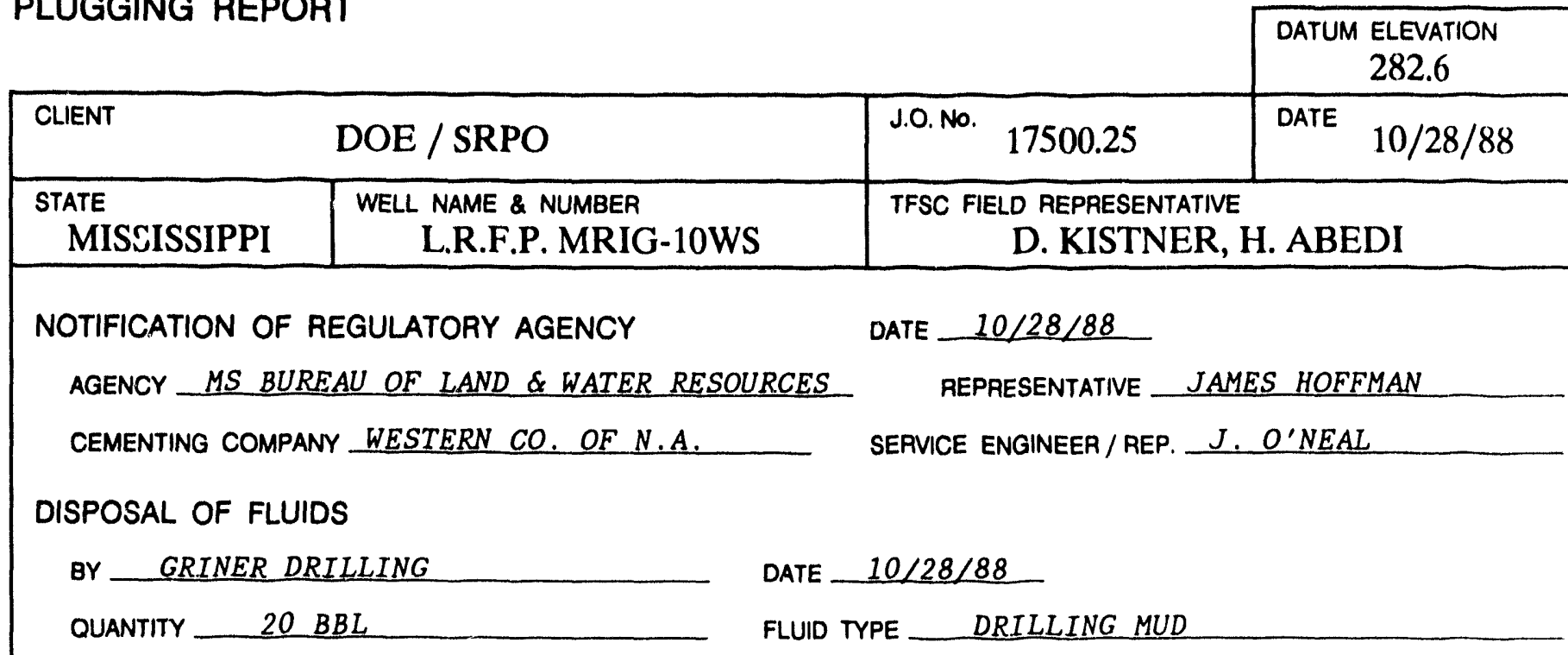

\section{REMARKS}

EXCESS DRILLING MUD HAULED TO RICHTON LANDFILL AS NECESSARY.

ZONE 1

INTERVAL 405 TO $3.0 \mathrm{FT}$

BOTTOM OF TUBING OR PIPE 39

NO. OF SACKS 85 YIELD 1.92 CU. FT/SK.

SLURRY WEIGHT 13.1 LBS/GAL

BRIDGE PLUG TYPE N NA

REMARKS

PULLED ALL CASING AND SCREEN OUT OF HOLE. REAMED HOLE TO 405 FT. CEMENT CIRCULATED

TO SURFACE, TOP 3 FT BACKFILLED WITH SOIL.
TYPE PLUG CONTINUOUS

DIAMETER OF CASING/HOLE $61 / 2$ IN CEMENT/MUD TYPE CLASS A, $8 \%$ BENTONITE
TOP OF PLUG 3.0

DEPTH

\section{ZONE}

INTERVAL

BOTTOM OF TUBING OR PIPE

NO. OF SACKS

SLURRY WEIGHT

BRIDGE PLUG TYPE

REMARKS
TYPE PLUG

DIAMETER OF CASING/HOLE IN FT CEMENT/MUD TYPE

YIELD CU. FT/SK.

VOL. PUMPED CU. FT LBS/GAL

TOP OF PLUG FT DEPTH 
PLUGGING REPORT

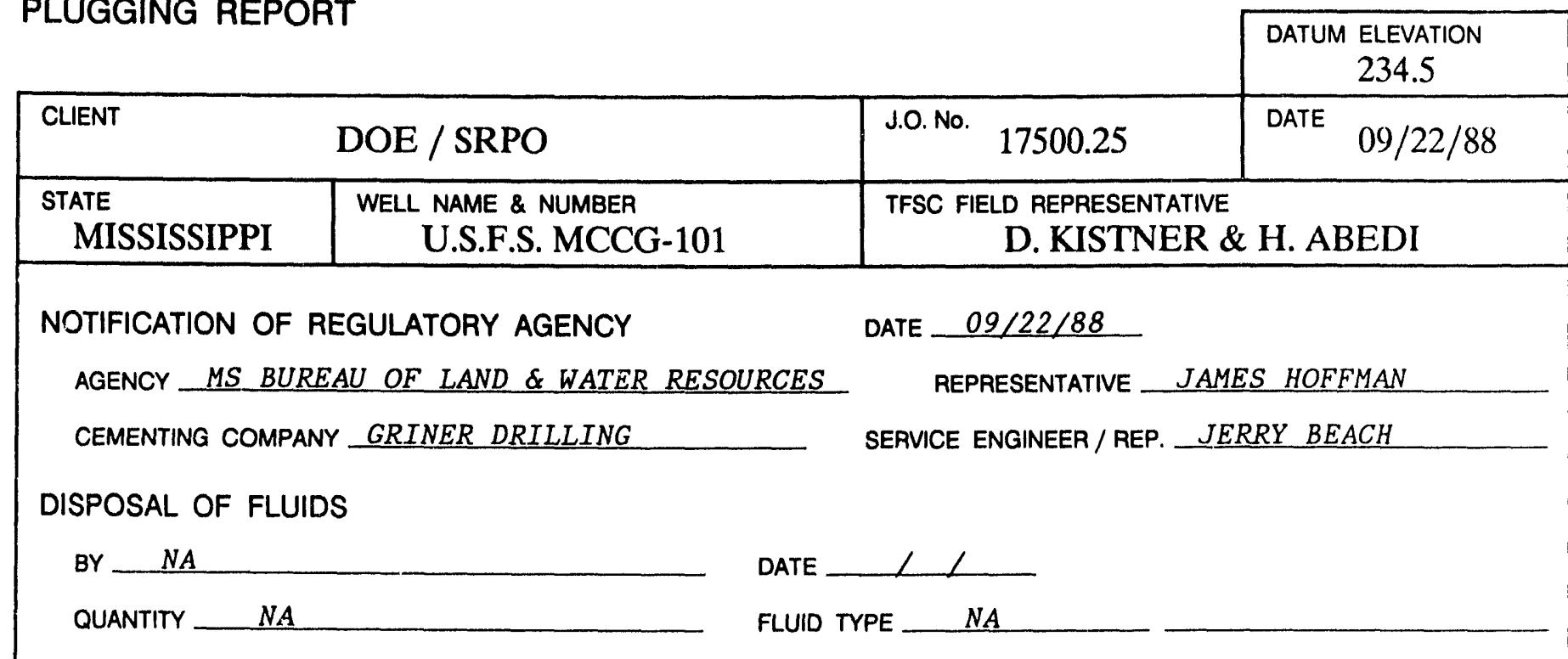

REMARKS

ZONE 1

TYPE PLUG CONTINUOUS

INTERVAL 164 TO 3 FT

DIAMETER OF CASING/HOLE _ 2 IN

BOTTOM OF TUBING OR PIPE 164 FT CEMENT/MUD TYPE CLASS A, NEAT

NO. OF SACKS 4 YIELD 1.17 CU.FT/SK. VOL. PUMPED 4.68 CU.FT

SLURRY WEIGHT 15.6 LBS/GAL TOP OF PLUG 3 FT

BRIDGE PLUG TYPE _ NA DEPTH NA

REMARKS

SCREEN FILLED WITH SILT (164-170 FT). PVC RISER AND SCREEN CEMENTED IN F ICE FROM BOTTOM TO TOP. RISER CUT OFF 3 FT BELOW SURFACE. 5 IN. CEMENT PAD (7 CU.FT.) POURED AT TOP OF PVC. BACKFILLED WITH SOIL.

ZONE

INTERVAL

BOTTOM OF TUBING OR PIPE

NO. OF SACKS

SLURRY WEIGHT LBS/GAL

BRIDGE PLUG TYPE REMARKS
TYPE PLUG

DIAMETER OF CASING/HOLE IN FT CEMENT/MUD TYPE

YIELD CU. FT/SK.

VOL. PUMPED CU. FT TOP OF PLUG FT

DEPTH 
PLUGGING REPORT

\begin{tabular}{|c|c|}
\hline & $\begin{array}{c}\text { DATUM ELEVATION } \\
247.8\end{array}$ \\
\hline J.O. No. 17500.25 & $\begin{array}{ll}\text { DATE } & 09 / 26 / 88\end{array}$ \\
\hline
\end{tabular}

\begin{tabular}{|c|c|c|c|c|}
\hline \multicolumn{3}{|c|}{ DOE / SRPO } & $\begin{array}{ll}\text { J.O. No. } & 17500.25\end{array}$ & $\begin{array}{ll}\text { DATE } & 09 / 26 / 88\end{array}$ \\
\hline $\begin{array}{l}\text { STATE } \\
\text { MISSISSIPPI }\end{array}$ & \multicolumn{2}{|l|}{$\begin{array}{l}\text { WELL NAME \& NUMBER } \\
\text { U.S.F.S. MCCG-102 }\end{array}$} & \multicolumn{2}{|c|}{$\begin{array}{l}\text { TFSC FIELD REPRESENTATIVE } \\
\text { D. KISTNER, H. ABEDI }\end{array}$} \\
\hline \multirow{3}{*}{\multicolumn{3}{|c|}{$\begin{array}{l}\text { NOTIFICATION OF REGULATORY AGENCY } \\
\text { AGENCY MS BUREAU OF LAND \& WATER RESOURCES } \\
\text { CEMENTING COMPANY GRINER DRILLING }\end{array}$}} & \multicolumn{2}{|l|}{ DATE $09 / 26 / 88$} \\
\hline & & & \multicolumn{2}{|c|}{ REPRESENTATIVE JAMES HOFFMAN } \\
\hline & & & \multicolumn{2}{|c|}{ SERVICE ENGINEER / REP. JERRY BEACH } \\
\hline \multicolumn{5}{|c|}{ DISPOSAL OF FLUIDS } \\
\hline \multicolumn{2}{|l|}{ BY NA } & \multirow{2}{*}{\multicolumn{3}{|c|}{$\begin{array}{l}\text { DATE } 11 \\
\text { FLUID TYPE }- \text { FRESH WAT }\end{array}$}} \\
\hline QUANTITY & & & & \\
\hline
\end{tabular}

\section{REMARKS}

ZONE 1

TYPE PLUG CONTINUOUS

INTERVAL 200 TO $3 \mathrm{FT}$

DIAMETER OF CASING/HOLE 2

BOTTOM OF TUBING OR PIPE 196 FT CEMENT/MLD TYPE CLASS A, NEAT

NO. OF SACKS 13 YIELD 1.17 CU.FT/SK. VOL. PUMPED 15.2 CU.FT

SLURRY WEIGHT 15.6 LBS/GAL TOP OF PLUG 3.0 FT

BRIDGE PLUG TYPE NA NA DEPTH_NA

REMARKS

CEMENT CIRCULATED TO SURFACE. PVC RISER CUT OFF 3.0 FT BELOW SURFACE. 2x2x.5 FT CEMENT PAD POURED AT TOP OF PVC. BACKFILLED TO SURFACE WITH SOIL.

ZONE

INTERVAL

BOTTOM OF TUBING OR PIPE

NO. OF SACKS

SLURRY WEIGHT

BRIDGE PLUG TYPE

REMARKS
TYPE PLUG

DIAMETER OF CASING/HOLE IN CEMENT/MUD TYPE

YIELD CU. FT/SK.

VOL. PUMPED CU.FT LBS/GAL TOP OF PLUG FT DEPTH 
PLUGGING REPORT

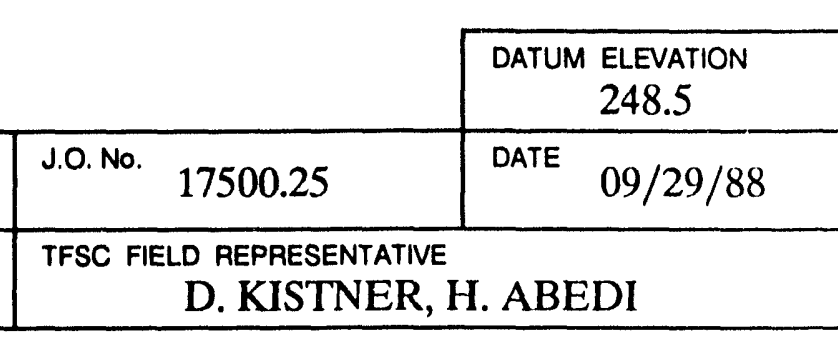

NOTIFICATION OF REGULATORY AGENCY

AGENCY MS BUREAU OF LAND \& WATER RESOURCES

CEMENTING COMPANY GRINER DRILLING
DATE $09 / 29 / 88$

REPRESENTATIVE JAMES HOFFMAN

SERVICE ENGINEER / REP. JERRY BEACH

\section{DISPOSAL OF FLUIDS}

BY GRINER DRILLING

QUANTITY $20 B B L$

DATE $09 / 29 / 88$

FLUID TYPE _ DRILLING MUD

REMARKS

DRILLING MUD DISPOSED OF IN CAMP SHELBY LANDFILL.

ZONE 1

INTERVAL 270 TO $4.7 \mathrm{FT}$

BOTTOM OF TUBING OR PIPE 270

NO. OF SACKS 65 YIELD $\quad 1.17$

SLURRY WEIGHT 15.6 LBS/GAL

BRIDGE PLUG TYPE _ NA

REMARKS

PVC DRILLED OUT TO 270 FT WITH 4.5 IN. DRAG BIT. CEMENT CIRCULATED TO SURFACE. TOP

4.7 FT BACKFILLED WITH SOIL.

\section{ZONE}

INTERVAL

BOTTOM OF TUBING OR PIPE NO. OF SACKS YIELD CU. FT/SK. LBS/GAL

SLURRY WEIGHT

BRIDGE PLUG TYPE

TYPE PLUG

DIAMETER OF CASING/HOLE IN FT CEMENT/MUD TYPE CU. FT/SK. TOP OF PLUG VOL. PUMPED CU. FT CEMENT/MUD TYPE CLASS A, NEAT

VOL. PUMPED CU. FT TOP OF PLUG 4.7 DEPTH NA REMARKS 
PLUGGING REPORT

DATUM ELEVATION

241.0

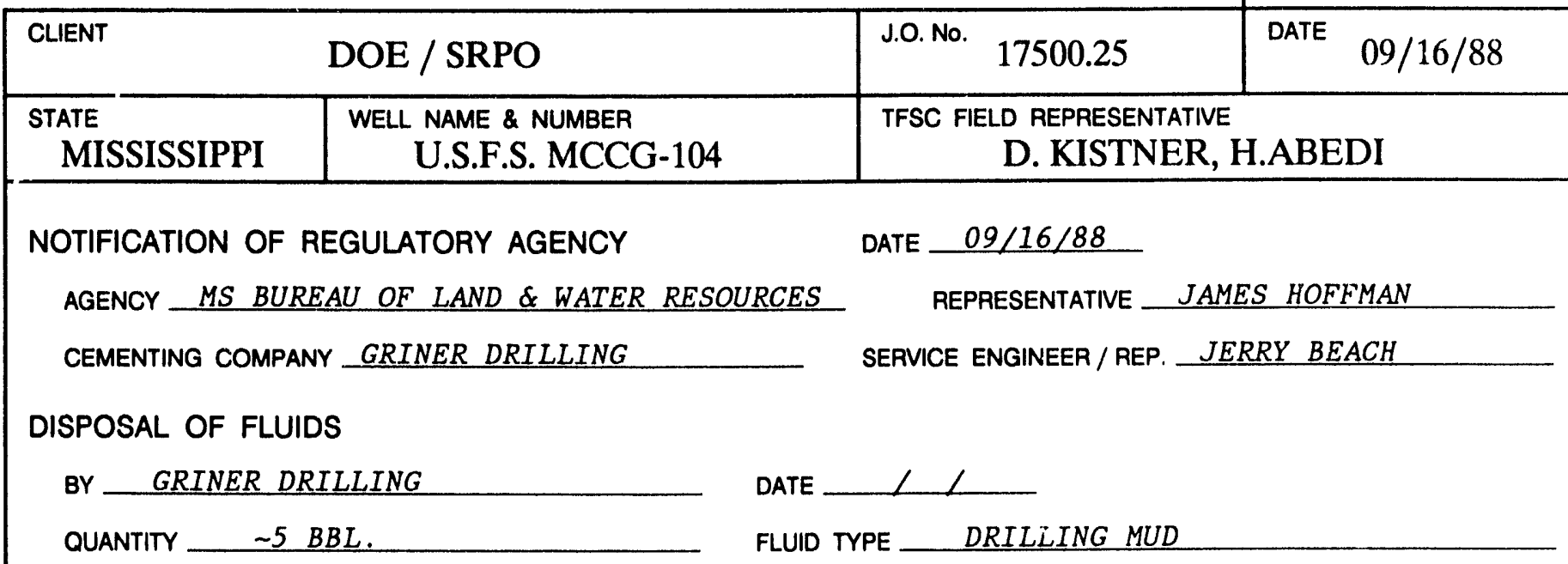

REMARKS

EXCESS MUD HAULED TO CAMP SHELBY LANDFILL AS NECESSARY.

ZONE 1

INTERVAL 95.5 TO 3.6 FT

BOTTOM OF TUBING OR PIPE 95 .

NO. OF SACKS 21 YIELD 1.17

SLURRY WEIGHT 15.6 LBS/GAL

BRIDGE PLUG TYPE

REMARKS

PVC DRILLED OUT TO $95.5 \mathrm{FT}$. CEMENT CIRCULATED TO SURFACE. TOP $3.6 \mathrm{FT}$. BACKFILLED WITH SOIL.

ZONE

TYPE PLUG

INTERVAL

DIAMETER OF CASING/HOLE IN

BOTTOM OF TUBING OR PIPE FT

YIELD CU. FT/SK.

VOL. PUMPED CU. FT 
PLUGGING REPORT

\begin{tabular}{|c|c|c|}
\cline { 3 - 3 } \multicolumn{2}{c|}{} & $\begin{array}{c}\text { DATUM ELEVATION } \\
272.9\end{array}$ \\
\hline $\begin{array}{l}\text { J.O. No. } \\
17500.25\end{array}$ & DATE $09 / 13 / 88$ \\
\hline $\begin{array}{l}\text { TFSC FIELD REPRESENTATIVE } \\
\text { D. KISTNER, H. ABEDI }\end{array}$ \\
\hline
\end{tabular}

\section{NOTIFICATION OF REGULATORY AGENCY \\ AGENCY MS BUREAU OF LAND \& WATER RESOURCES \\ CEMENTING COMPANY GRINER DRILLING}

DATE $09 / 13 / 88$

REPRESENTATIVE JAMES HOFFMAN

SERVICE ENGINEER / REP. JERRY BEACH

DISPOSAL OF FLUIDS

BY GRINER DRILLING

QUANTITY 20 BBL

DATE $09 / 13 / 88$

FLUID TYPE_DRILLING MUD

\section{REMARKS}

EXCESS DRILLING MUD HAULED TO CAMP SHELBY LANDFILL

ZONE 1

INTERVAL 90.5 TO $2.5 \mathrm{FT}$.
TYPE PLUG CONTINUOUS

DIAMETER OF CASING/HOLE $\quad 7.5$ IN

BOTTOM OF TUBING OR PIPE 90.5 FT CEMENT/MUD TYPE CLASS A, NEAT

NO. OF SACKS 44 YIELD 1.17 CU.FT/SK. VOL. PUMPED 51.5 CU. FT

SLURRY WEIGHT 15.6 LBS/GAL TOP OF PLUG 2.5 FT

BRIDGE PLUG TYPE N NA DEPTH _ NA

REMARKS

PVC DRILLED OUT TO 90.5 FT. CEMENT CIRCULATED TO SURFACE. TOP 2.5 FT BACKFILLED WITH SOIL.

ZONE

INTERVAL

BOTTOM OF TUBING OR PIPE

NO. OF SACKS LBS/GAL

BRIDGE PLUG TYPE

REMARKS
TYPE PLUG

DIAMETER OF CASING/HOLE IN FT CEMENT/MUD TYPE CU. FT/SK. VOL. PUMPED CU. FT

TOP OF PLUG FT DEPTH 


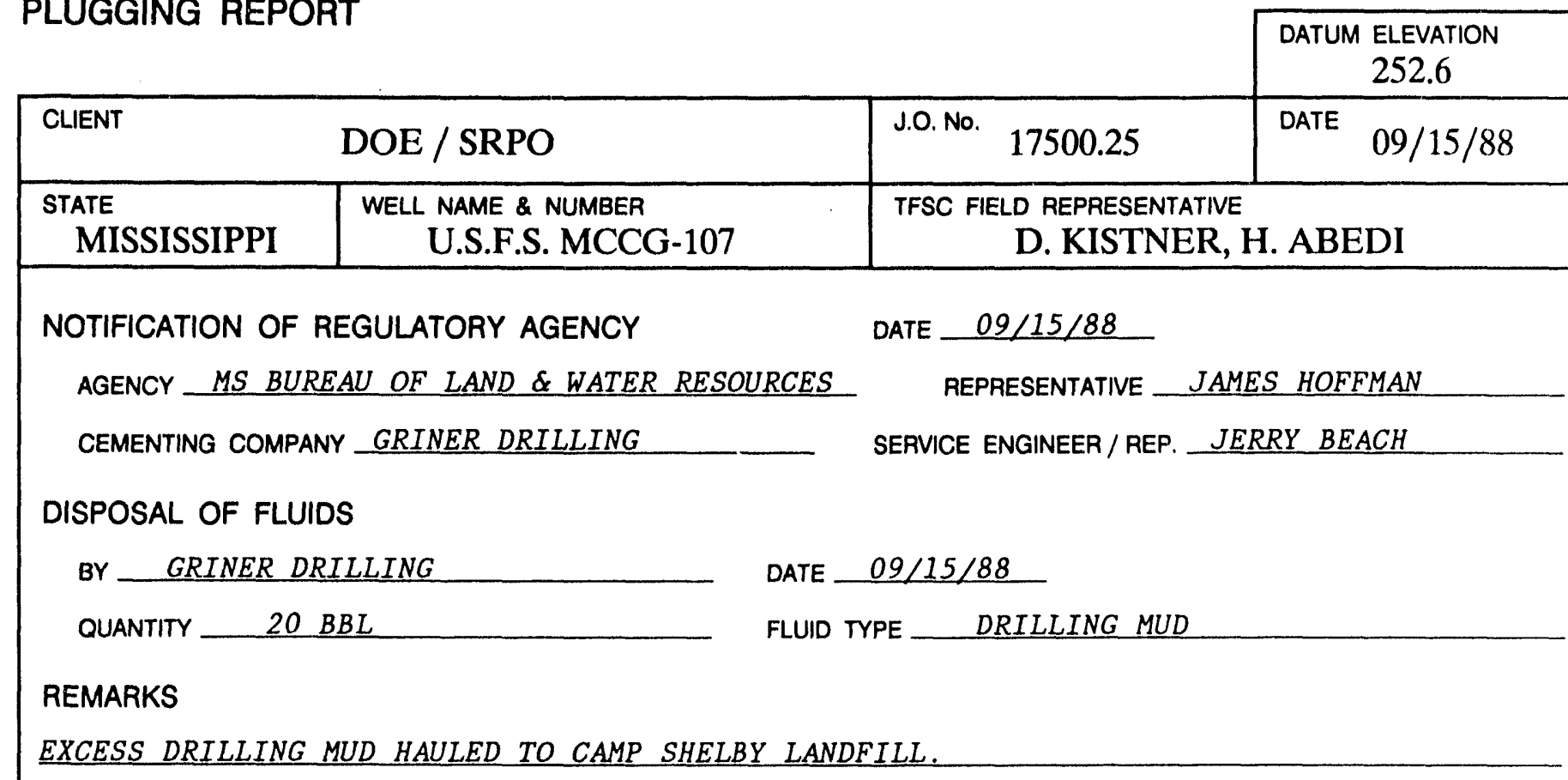

ZONE 1

INTERVAL 95.5 '1O $3.5 \mathrm{FT}$

BOTTOM OF TUBING OR PIPE 95.5
TYPE PLUG CONTINUOUS

DIAMETER OF CASING/HOLE $5 \quad 7 / 8$
SLURRY WEIGHT 15.6 YIELD $\frac{1.17}{21}$ LBS/GAL $\quad$ VOL. PUMPED $\frac{24}{24}$

BRIDGE PLUG TYPE _ NA

DEPTH

REMARKS

PVC DRILLED OUT TO 95.5 FT. CEMENT CIRCULATED TO SURFACE. TOP 3.5 FT BACKFILLED WITH SOIL.

ZONE

INTERVAL

BOTTOM OF TUBING OR PIPE

NO. OF SACKS

SLURPY WEIGHT

BRIDGE PLUG TYPE

REMARKS
TYPE PLUG

DIAMETER OF CASING/HOLE IN FT CEMENT/MUD TYPE YIELD _ CU. FT/SK. VOL. PUMPED CU.FT LBS/GAL

TOP OF PLUG FT DEPTH 
PLUGGING REPORT

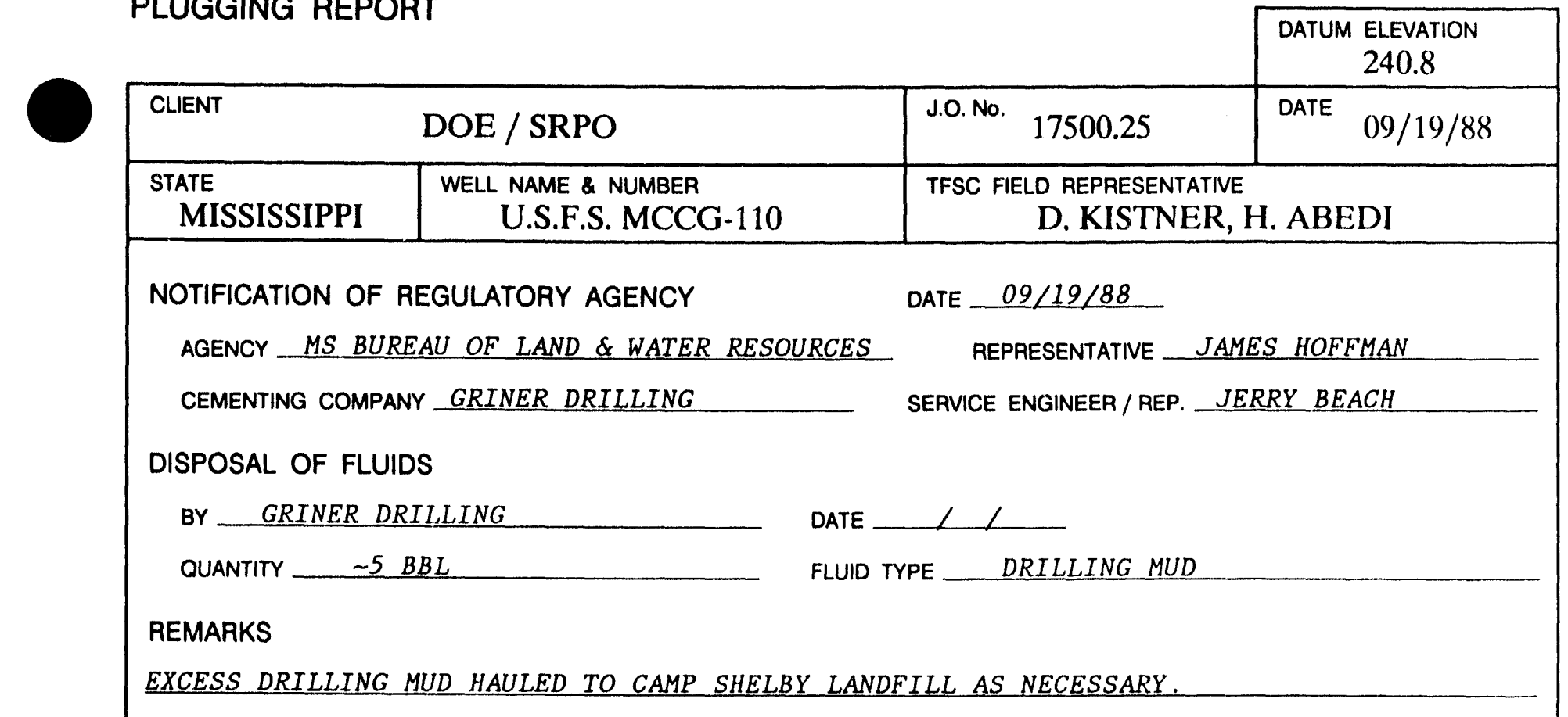

ZONE 1

TYPE PLUG CONTINUOUS

INTERVAL 86 TO 3.7 FT

DIAMETER OF CASING/HOLE $\quad 5 \quad 7 / 8$ IN

BOTTOM OF TUBING OR PIPE 86 . FT CEMENT/MUD TYPE_CLASS A, NEAT

NO. OF SACKS 21 YIELD 1.17 CU.FT/SK. VOL. PUMPED 24.6 CU.FT

SLURRY WEIGHT 15.6 LBS/GAL TOP OF PLUG $3.7 \quad$ FT

BRIDGE PLUG TYPE _NA DEPTH NA NA

REMARKS

PVC DRILLED OUT TO 86 FT. CEMENT CIRCULATED TO SURFACE. TOP 3.7 FT BACKFILLED WITH SOIL.

ZONE

TYPE PLUG

INTERVAL

DIAMETER OF CASING/HOLE IN

BOTTOM OF TUBING OR PIPE FT CEMENT/MUD TYPE

NO. OF SACKS

YIELD CU. FT/SK.

VOL. PUMPED CU. FT

SLURRY WEIGHT LBS/GAL

TOP OF PLUG FT

BRIDGE PLUG TYPE

DEPTH REMARKS 


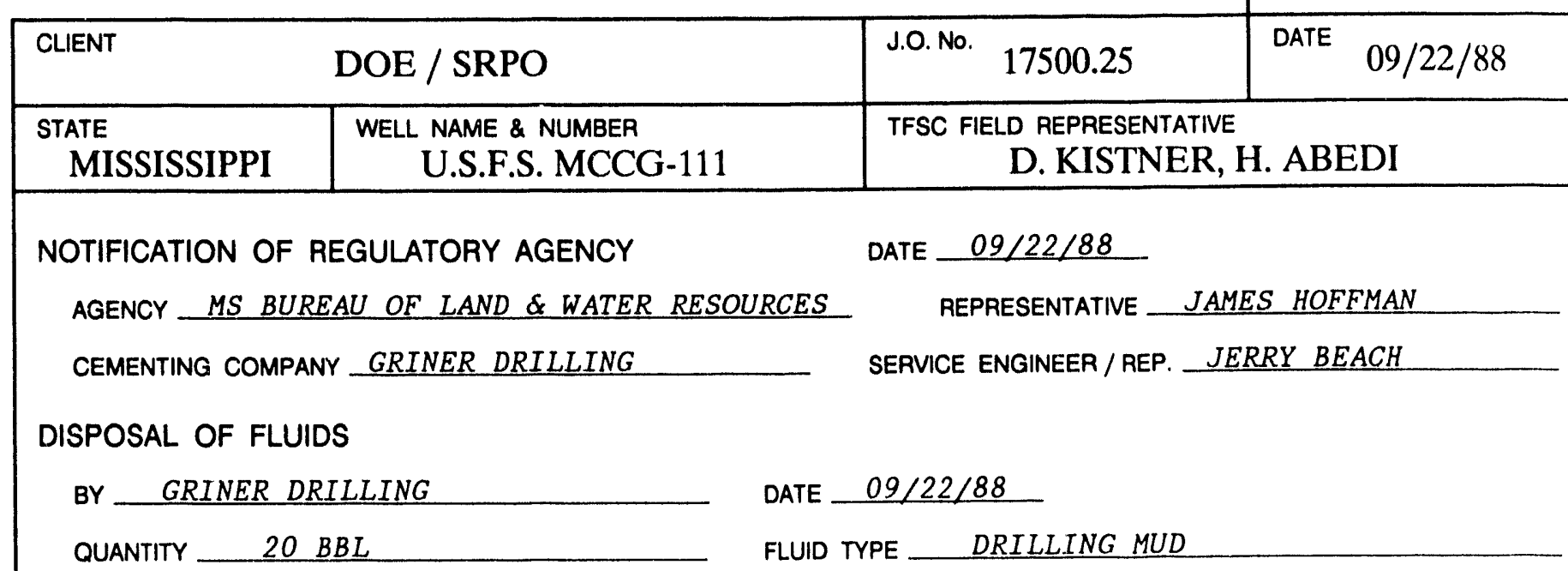

\section{REMARKS}

EXCESS DRILLING MUD HAULED TO CAMP SHELBY LANDFILL.

ZONE 1

INTERVAL $\quad 120.5$ TO $4.5 \mathrm{FT}$
TYPE PLUG CONTINUOUS

DIAMETER OF CASING/HOLE $\quad 5 \quad 7 / 8$ IN

BOTTOM OF TUBING OR PIPE 120.5 FT CEMENT/MUD TYPE CLASS A, NEAT

NO. OF SACKS 32 YIELD 1.17 CU.FT/SK. VOL. PUMPED 37.4 CU.FT

SLURRY WEIGHT 15.6 LBS/GAL TOP OF PLUG 4.5 FT

BRIDGE PLUG TYPE N NA DEPTH N N N

REMARKS

PVC DRILLED OUT TO 120.5 FT. CEMENT CIRCULATED TO SURFACE. TOP 4.5 FT BACKFILLED WITH SOIL.

ZONE

INTERVAL

BOTTOM OF TUBING OR PIPE

NO. OF SACKS

SLURRY WEIGHT

BRIDGE PLUG TYPE

REMARKS
TYPE PLUG

DIAMETER OF CASING/HOLE $\mathbb{I N}$ FT CEMENT/MUD TYPE YIELD CU.FT/SK. VOL. PUMPED C CU.FT LBS/GAL TOP OF PLUG FT DEPTH 
PLUGGING REPORT

\begin{tabular}{|c|c|c|c|}
\hline $\begin{array}{ll}\text { CLIENT } \\
\end{array}$ & DOE / SRPO & J.O. No. 17500.25 & DATE $09 / 30 / 88$ \\
\hline $\begin{array}{l}\text { STATE } \\
\text { MISSISSIPPI }\end{array}$ & $\begin{array}{l}\text { WELL NAME \& NUMBER } \\
\text { U.S.F.S. MCCG-113 }\end{array}$ & \multicolumn{2}{|c|}{\begin{tabular}{|l} 
TFSC FIELD REPRESENTATIVE \\
D. KISTNER, H. ABEDI
\end{tabular}} \\
\hline \multirow{3}{*}{\multicolumn{2}{|c|}{$\begin{array}{l}\text { NOTIFICATION OF REGULATORY AGENCY } \\
\text { AGENCY MS BUREAU OF LAND \& WATER RESOURCES } \\
\text { CEMENTING COMPANY GRINER DRILLING }\end{array}$}} & \multicolumn{2}{|l|}{ DATE $09 / 30 / 88$} \\
\hline & & \multicolumn{2}{|c|}{ REPRESENTATIVE JAMES HOFFMAN } \\
\hline & & \multicolumn{2}{|c|}{ SERVICE ENGINEER / REP. JERRY BEACH } \\
\hline \multicolumn{4}{|c|}{ DISPOSAL OF FLUIDS } \\
\hline \multicolumn{2}{|c|}{ BY GRINER DRILLING } & \multicolumn{2}{|c|}{ DATE $09 / 30 / 88$} \\
\hline \multicolumn{2}{|c|}{ QUANTITY $20 \mathrm{BBL}$} & \multicolumn{2}{|c|}{ FLUID TYPE DRILLING MUD } \\
\hline
\end{tabular}

\section{REMARKS}

EXCESS DRILLING MUD HAULED TO CAMP SHELBY LANDFILL.

ZONE 1

INTERVAL $\quad 85.5$ TO 3.5 FT
TYPE PLUG CONTINUOUS

DIAMETER OF CASING/HOLE $\quad 57 / 8$
IN

BOTTOM OF TUBING OR PIPE $\quad 85.5$ FT CEMENT/MUD TYPE _ CLASS A. NEAT

NO. OF SACKS 21.5 YIELD 1.17 CU. FT/SK. VOL. PUMPED _ 25.2 _ CU.FT

SLURAY WEIGHT 15.6 LBS/GAL TOP OF PLUG 3.5 FT

BRIDGE PLUG TYPE N NA DEPTH N N N

REMARKS

PVC DRILLED OUT TO 85.5 FT. CEMENT CIRCULATED TO SURFACE. TOP 3.5 FT BACKFILLED

WITH SOIL.

ZONE

INTERVAL

BOTTOM OF TUBING OR PIPE

NO. OF SACKS

SLURRY WEIGHT

YIELD LBS/GAL

BRIDGE PLUG TYPE

REMARKS
TYPE PLUG

DIAMETER OF CASING/HOLE IN FT CEMENT/MUD TYPE CU. FT/SK. VOL. PUMPED CU. FT TOP OF PLUG FT DEPTH 
PLUGGING REPORT

\begin{tabular}{|c|c|c|}
\cline { 3 - 3 } \multicolumn{2}{c|}{} & $\begin{array}{c}\text { DATUM ELEVATION } \\
231.9\end{array}$ \\
\hline $\begin{array}{c}\text { J.O. No. } \\
17500.25\end{array}$ & DATE $09 / 18 / 88$ \\
\hline $\begin{array}{c}\text { TFSC FIELD REPRESENTATIVE } \\
\text { D. KISTNER, H. ABEDI }\end{array}$ \\
\hline
\end{tabular}

NOTIFICATION OF REGULATORY AGENCY

AGENCY MS BUREAU OF LAND \& WATER RESOURCES

CEMENTING COMPANY GRINER DRILLING

\section{DATE $09 / 18 / 88$}

REPRESENTATIVE JAMES HOFFMAN

SERVICE ENGINEER / REP. JERRY BEACH

DISPOSAL OF FLUIDS

BY GRINER DRILLING

QUANTITY $\quad-5 B B L$
DATE $L 1$

FLUID TYPE _ DRILLING MUD

REMARKS

EXCESS DRILLING MUD HAULED TO CAMP SHELBY LANDFILL AS NECESSARY.

ZONE 1

INTERVAL $\quad 75.5$ TO $3.5 \mathrm{FT}$
TYPE PLUG CONTINUOUS

DIAMETER OF CASING/HOLE $57 / 8$ IN CEMENT/MUD TYPE CLASS $A, N E A T$

NO. OF SACKS $20 \quad$ YIELD $\quad 1.17$ CU.FT/SK. VOL. PUMPED $23.4 \quad$ CU.FT SLURRY WEIGHT 15.6 LBS/GAL TOP OF PLUG 3.5

BRIDGE PLUG TYPE N NA DEPTH _ NA

REMARKS

PVC DRILLED OUT TO $75.5 \mathrm{FT}$. CEMENT CIRCULATED TO SURFACE. TOP 3.5 FT BACKFILLED WITH SOIL.

ZONE

INTERVAL

BOTTOM OF TUBING OR PIPE

NO. OF SACKS

SLURRY WEIGHT

BRIDGE PLUG TYPE

REMARKS
TYPE PLUG

DIAMETER OF CASING/HOLE IN FT CEMENT/MUD TYPE YIELD CU. FT/SK. VOL. PUMPED CU.FT TOP OF PLUG FT DEPTH 


\begin{tabular}{|l|l|l|}
\cline { 3 - 3 } \multicolumn{2}{c|}{} & $\begin{array}{c}\text { DATUM ELEVATION } \\
189.7\end{array}$ \\
\hline J.O. No. & 17500.25 & DATE $10 / 04 / 88$ \\
\hline
\end{tabular}

\begin{tabular}{|l|l|l}
\hline $\begin{array}{l}\text { STATE } \\
\text { MISSISSIPPI }\end{array}$ & WELL NAME \& NUMBER \\
U.S.F.S. MCCG-115 & \\
\hline
\end{tabular}

NOTIFICATION OF REGULATORY AGENCY

AGENCY MS BUREAU OF LAND \& WATER RESOURCES

CEMENTING COMPANY GRINER DRILLING

TFSC FIELD REPRESENTATIVE

D. KISTNER, H. ABEDI

DATE $10 / 04 / 88$

REPRESENTATIVE J JAMES HOFFMAN

SERVICE ENGINEER / REP. JERRY BEACH

DISPOSAL OF FLUIDS

BY GRINER DRILLING - DATE L L

QUANTITY _ _ NO EST.

FLUID TYPE _ D DRILLING MUD

REMARKS

EXCESS DRILLING MUD HAULED TO CAMP SHELBY LANDFILL AS NECESSARY.

ZONE 1

TYPE PLUG CONTINUOUS

INTERVAL 155 TO $3.0 \mathrm{FT}$

DIAMETER OF CASING/HOLE 7.0 $\mathbb{N}$

BOTTOM OF TUBING OR PIPE 155 FT CEMENT/MUD TYPE CLASS A, NEAT

NO. OF SACKS 46 YIELD 1.17 CU.FT/SK. VOL. PUMPED 53.8 CU.FT

SLURPY WEIGHT 15.6 LBS/GAL TOP OF PLUG $3.0 \quad \mathrm{FT}$

BRIDGE PLUG TYPE N NA DEPTH_NA

REMARKS

PVC DRILLED OUT TO 155 FT. CEMENT CIRCULATED TO SURFACE. TOP 3 FT BACKFILLED WITH SOIL.

ZONE

INTERVAL

BOTTOM OF TUBING OR PIPE

NO. OF SACKS

SLURRY WEIGHT

BRIDGE PLUG TYPE

REMARKS
TYPE PLUG

DIAMETER OF CASING/HOLE IN FT CEMENT/MUD TYPE

YIELD CU. FT/SK.

VOL. PUMPED CU. FT LBS/GAL TOP OF PLUG FT DEPTH 


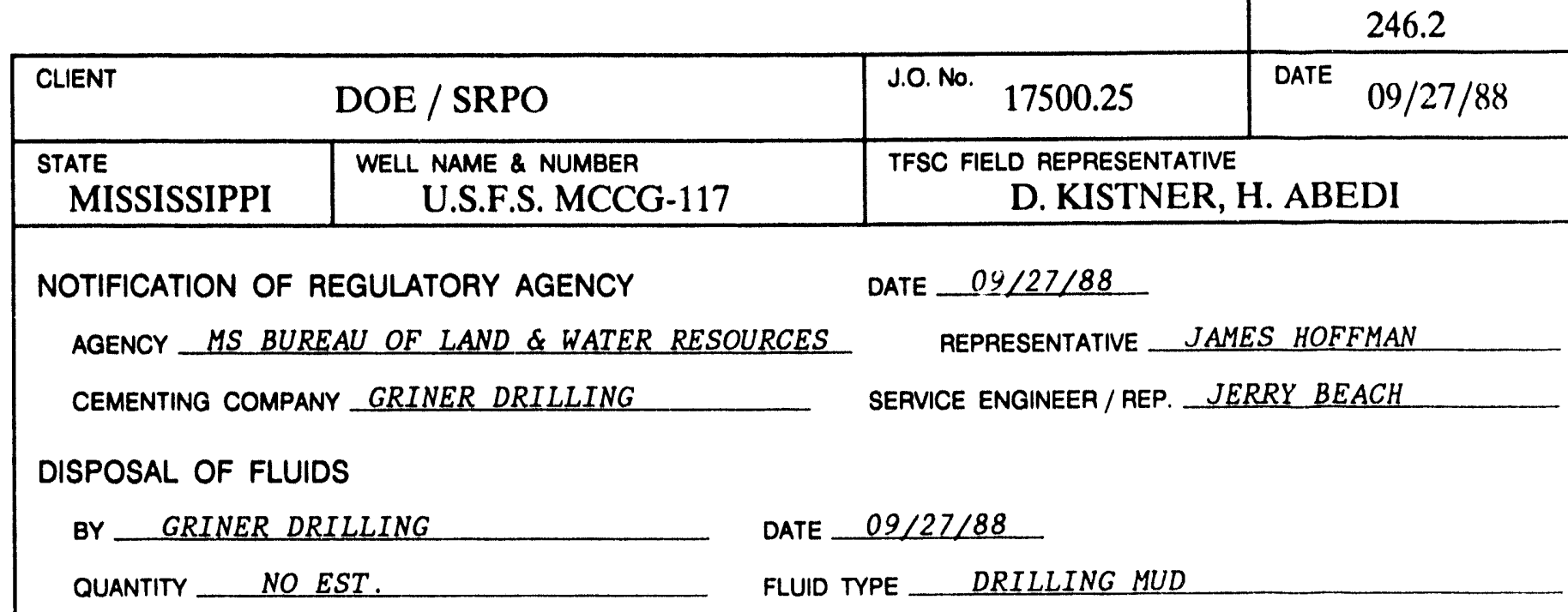

\section{REMARKS}

EXCESS DRILLING MUD HAULED TO CAMP SHELBY LANDFILL AS NECESSARY.

ZONE 1

INTERVAL 107 TO $3.0 \mathrm{FT}$

BOTTOM OF TUBING OR PIPE 107
TYPE PLUG CONTINUOUS

DIAMETER OF CASING/HOLE _ $57 / 8$ CEMENT/MUD TYPE CLASS A, NEAT

NO. OF SACKS 24 YIELD 1.17 CU.FT/SK. VOL. PUMPED 28 CU.FT

SLURRY WEIGHT 15.6 LBS/GAL TOP OF PLUG $3.0 \quad \mathrm{FT}$

BRIDGE PLUG TYPE _ NA DEPTH N NA

REMARKS

PVC DRILLED OUT TO 107 FT. CEMENT CIRCULATED TO SURFACE. TOP 3.0 FT BACKFILLED WITH SOIL.

ZONE

INTERVAL

BOTTOM OF TUBING OR PIPE

NO. OF SACKS

SLURRY WEIGHT

BRIDGE PLUG TYPE

REMARKS
TYPE PLUG

DIAMETER OF CASING/HOLE IN FT CEMENT/MUD TYPE YIELD CU.FT/SK. VOL. PUMPED _ CU.FT LBS/GAL

TOP OF PLUG FT DEPTH 
PLUGGING REPORT

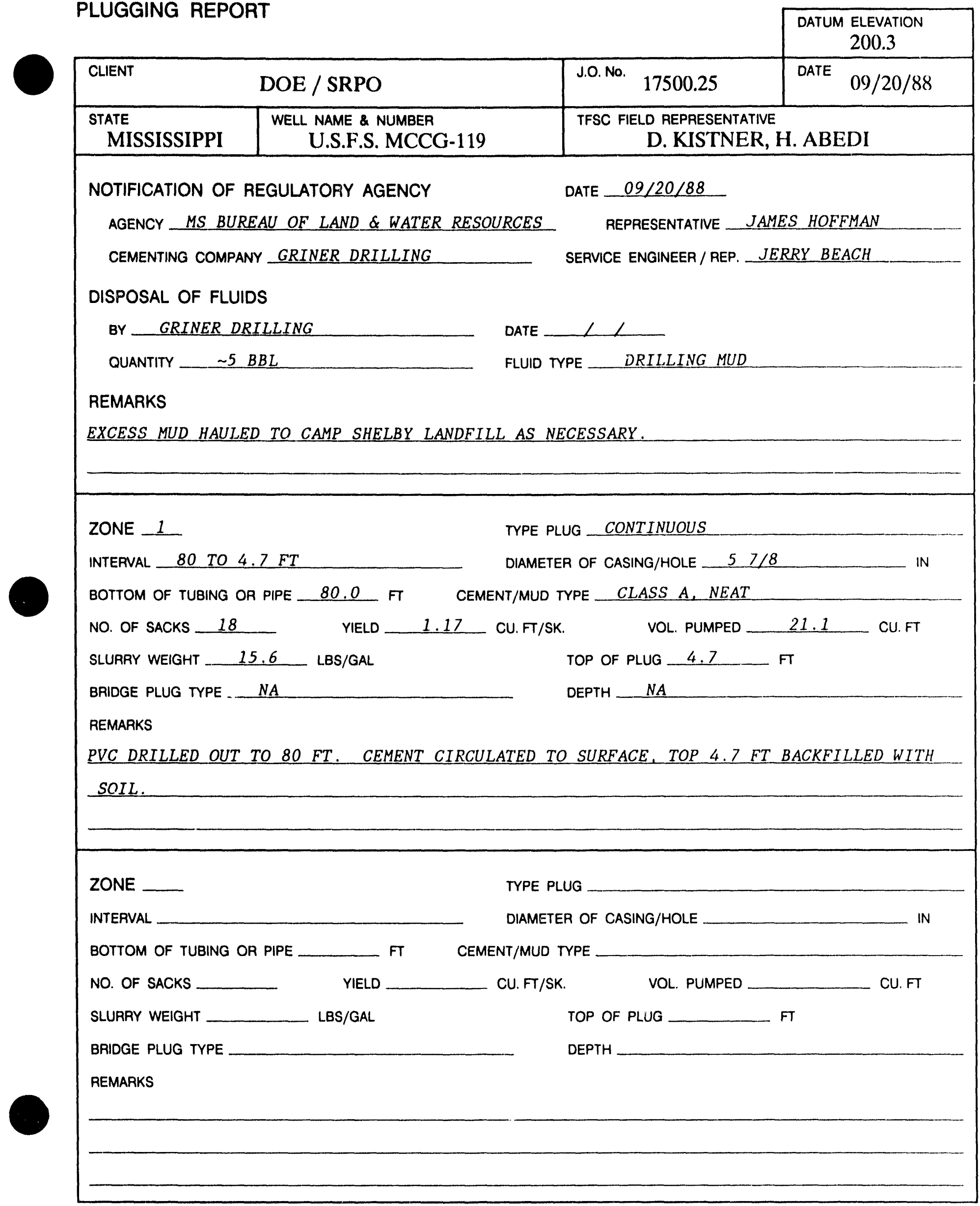




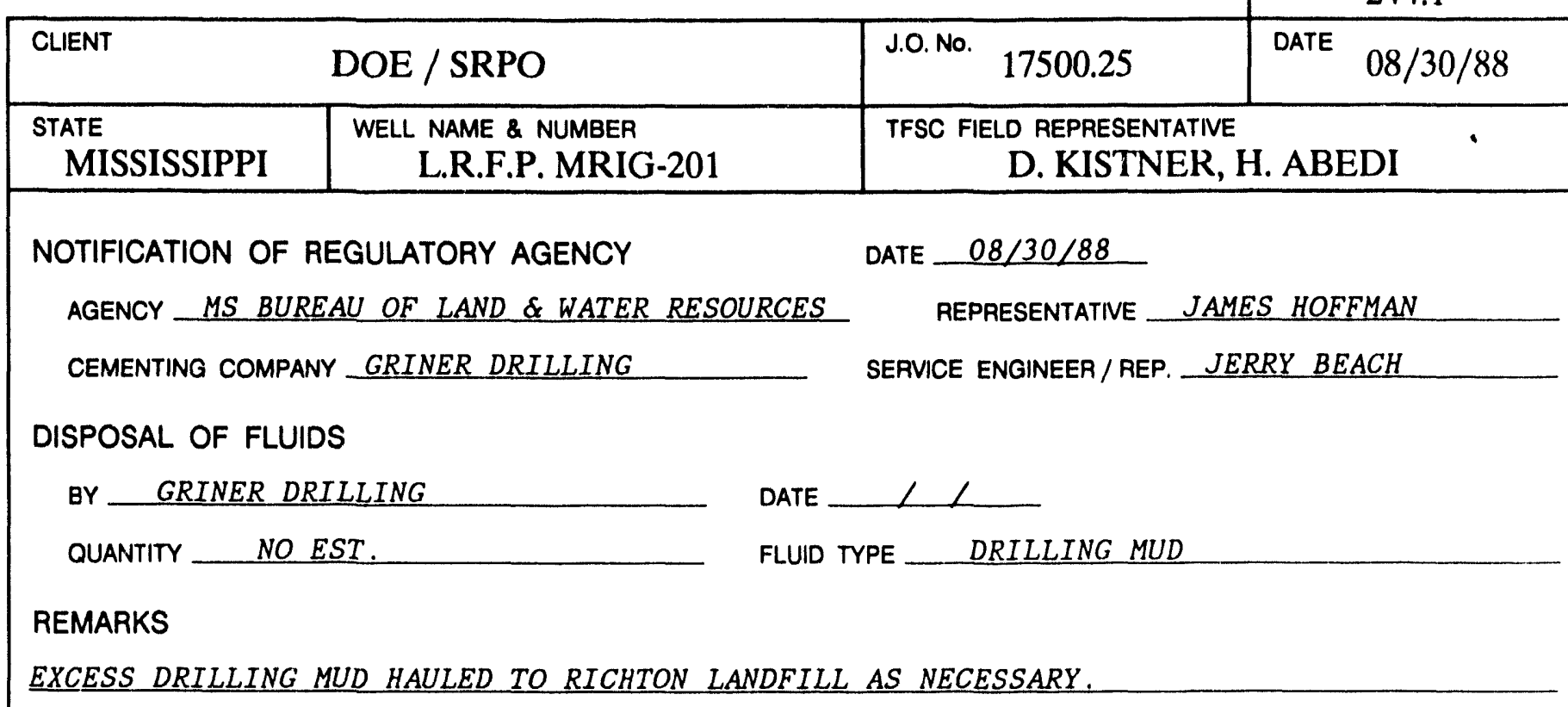

ZONE 1

INTERVAL 80.5 TO $3.0 \mathrm{FT}$
TYPE PLUG CONTINUOUS

DIAMETER OF CASING/HOLE $\quad 41 / 2$ IN

BOTTOM OF TUBING OR PIPE 80.5 FT CEMENT/MUD TYPE CLASS A, NEAT

NO. OF SACKS 22 YIELD 1.17 CU.FT/SK. VOL. PUMPED 25.7 CU. FT SLURRY WEIGHT 15.6 LBS/GAL TOP OF PLUG 3.0

BRIDGE PLUG TYPE _ NA DEPTH_NA

REMARKS

PVC DRILLED OUT TO 79 FT. CARRIED HOLE TO ORIGINAL T.D. A 80.5 FT, CEMENT CIRCULATED TO SURFACE. TOP 3.0 FT BACKFILLED WITH SOIL.

ZONE

INTERVAL

BOTTOM OF TUBING OR PIPE

NO. OF SACKS

SLURRY WEIGHT

BRIDGE PLUG TYPE

REMARKS
TYPE PLUG

DIAMETER OF CASING/HOLE IN FT CEMENT/MUD TYPE

YIELD CU. FT/SK. VOL. PUMPED CU. FT LBS/GAL TOP OF PLUG FT DEPTH 
PLUGGING REPORT

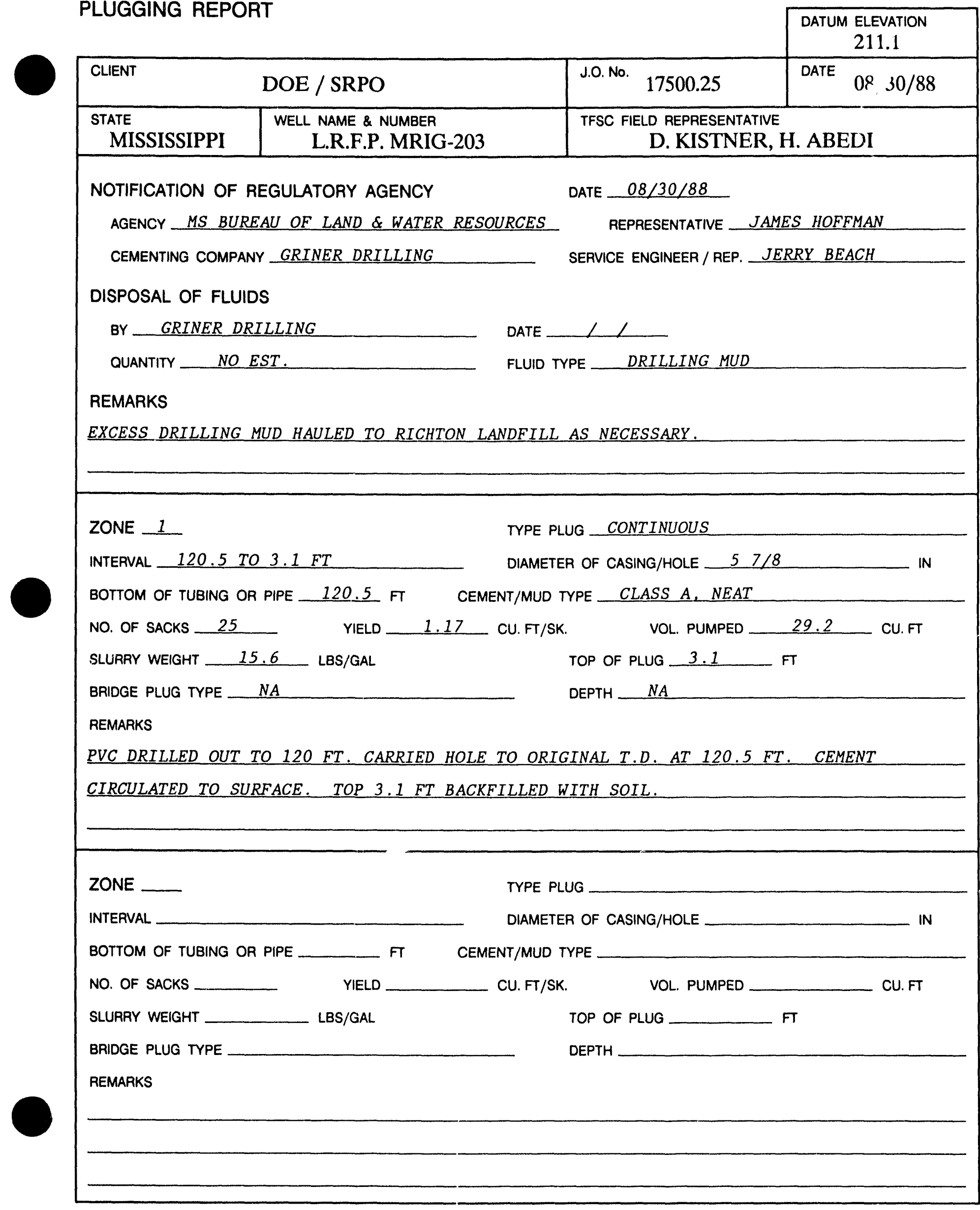


PLUGGING REPORT

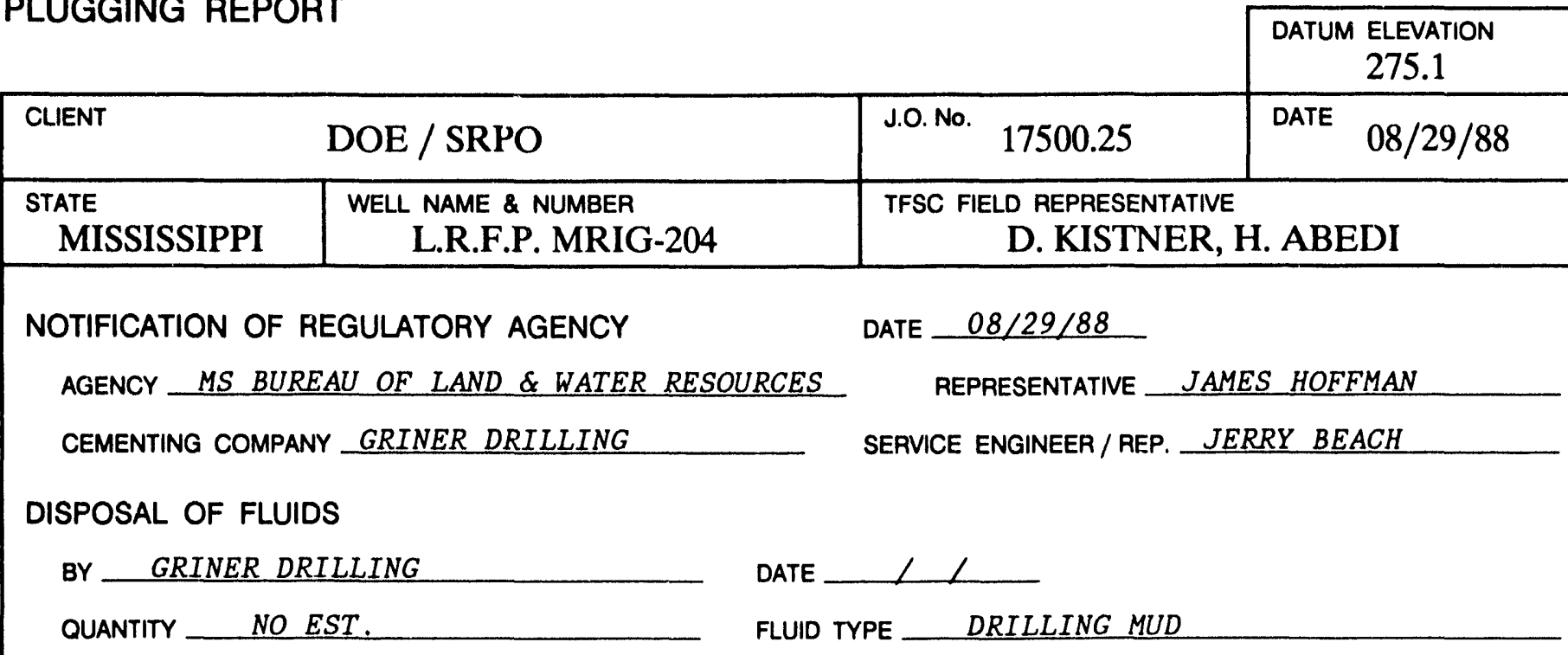

\section{REMARKS}

EXCESS DRILLING MUD HAULED TO RICHTON LANDFILL AS NECESSARY.

ZONE 1

INTERVAL 180 TO $3.9 \quad \mathrm{FT}$

BOTTOM OF TUBING OR PIPE 180

NO. OF SACKS $24 \quad$ YIELD 1.1

SLURRY WEIGHT 15.6 LBS/GAL

BRIDGE PLUG TYPE _ NA

REMARKS

PVC DRILLED OUT TO 65 FT. CARRIED HOLE TO ORIGINAL T.D. AT 180 FT. CEMENT WAS

CIRCULATED TO SURFACE, TOP 3.9 FT BACKFILLED WITH SOIL.

\section{ZONE}

INTERVAL

BOTTOM OF TUBING OR PIPE

NO. OF SACKS

SLURRY WEIGHT

YIELD FI LBS/GAL

BRIDGE PLUG TYPE REMARKS
TYPE PLUG CONTINUOUS

DIAMETER OF CASING/HOLE 7.0 IN CEMENT/MUD TYPE CLASS A, NEAT

CU. FT/SK.

VOL. PUMPED 26.4 CU. FT TOP OF PLUG 3.9 DEPTH 
PLUGGING REPORT

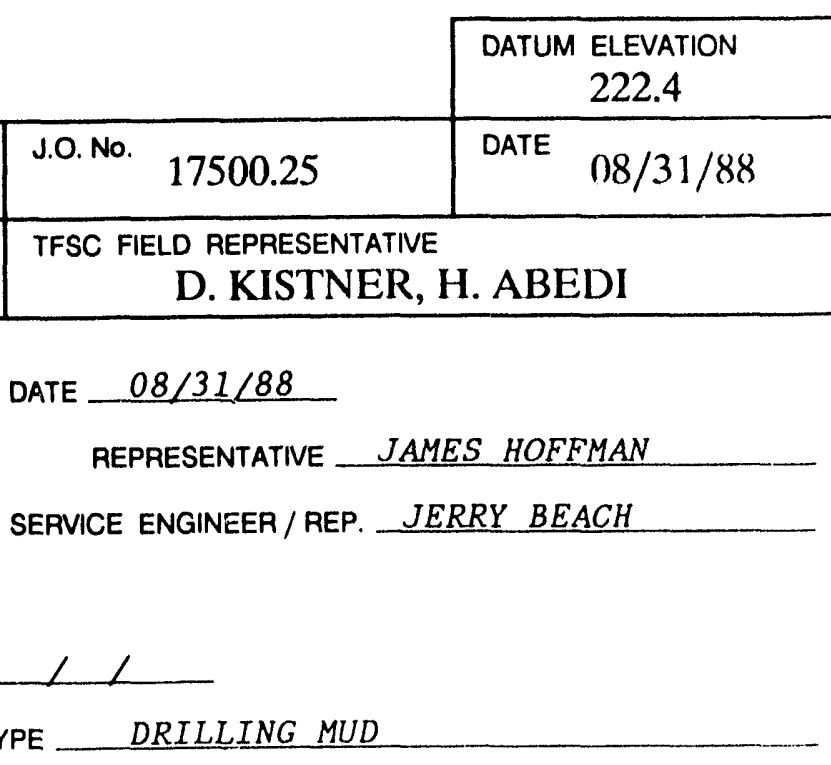

\section{REMARKS}

EXCESS DRILLING MUD HAULED TO RICHTON LANDFILL AS NECESSARY.

ZONE 1

INTERVAL 140.5 TO $2.7 \mathrm{FT}$

BOTTOM OF TUBING OR PIPE 140.5 FT
TYPE PLUG CONTINUOUS

DIAMETER OF CASING/HOLE _ $\quad 6.0$ IN

NO. OF SACKS 25 YIELD 1.17 CU. FT/SK. VOL. PUMPED 29.3 CU.FT

SLURRY WEIGHT 15.6 LBS/GAL TOP OF PLUG 2.7 FT

BRIDGE PLUG TYPE _ NA DEPTH N NA

REMARKS

PVC DRILLED OUT TO 38 FT. CARRIED HOLE TO ORIGINAL T.D. AT 140.5 FT. CEMENT CIRCULATED TO SURFACE. TOP 2.7 FT BACKFILLED WITH SOIL.

ZONE

INTERVAL BOTTOM OF TUBING OR PIPE NO. OF SACKS YIELD FT

SLURRY WEIGHT LBS/GAL

BRIDGE PLUG TYPE REMARKS
TYPE PLUG

DIAMETER OF CASING/HOLE IN CEMENT/MUD TYPE CU. FT/SK. VOL. PUMPED CU. FT TOP OF PLUG FT DEPTH 
PLUGGING REPORT

\begin{tabular}{|c|c|c|}
\hline & & $\begin{array}{c}\text { DATUM ELEVATION } \\
240.6\end{array}$ \\
\hline J.O. No & 17500.25 & $\begin{array}{ll}\text { DATE } & 09 / 06 / 88\end{array}$ \\
\hline
\end{tabular}

\section{NOTIFICATION OF REGULATORY AGENCY}

AGENCY MS BUREAU OF LAND \& WATER RESOURCES

CEMENTING COMPANY GRINER DRILLING
DATE $-09 / 06 / 88$

REPRESENTATIVE JAMES HOFFMAN

SERVICE ENGINEER / REP. JERRY BEACH

DISPOSAL OF FLUIDS

BY GRINER DRILLING

QUANTITY 20 BBL
DATE $\quad 06 / 09 / 88$

FLUID TYPE DRILLING MUD

\section{REMARKS}

EXCESS DRILLING MUD HAULED TO RICHTON LANDFILL AS NECESSARY.

ZONE 1

INTERVAL 80.5 TO $3.0 \mathrm{FT}$

BOTTOM OF TUBING OR PIPE 80.5 FT
TYPE PLUG _ CONTINUOUS

DIAMETER OF CASING/HOLE $71 / 2$
IN
NO. OF SACKS 29 YIELD 1.17 CU.FT/SK. VOL. PUMPED 33.9 CU. FT

SLURRY WEIGHT 15.6 LBS/GAL

TOP OF PLUG 3.0

BRIDGE PLUG TYPE NA

DEPTH N NA

REMARKS

PVC DRILLED OUT TO 58 FT. CARRIED HOLE TO ORIGINAL T.D. AT 80.5 FT. CEMENT CIRCULATED TO SURFACE. TOP 3.0 FT BACKFILLED WITH SOIL.

ZONE

INTFRVAL

BOTTOM OF TUBING OR PIPE

NO. OF SACKS

SLURRY WEIGHT LBS/GAL

BRIDGE PLUG TYPE REMARKS

YIELD
TYPE PLUG

DIAMETER OF CASING/HOLE IN FT CEMENT/MUD TYPE CU. FT/SK.

VOL. PUMPED CU. FT

TOP OF PLUG FT

DEPTH 
PLUGGING REPORT

\begin{tabular}{|c|c|c|c|}
\hline \multicolumn{3}{|c|}{ PLUGGING REPORT } & $\begin{array}{c}\text { DATUM ELEVATION } \\
280.5 \\
\end{array}$ \\
\hline CLIENT & DOE / SRPO & J.O. No. 17500.25 & DATE $11 / 10 / 88$ \\
\hline $\begin{array}{l}\text { STATE } \\
\text { MISSISSIPPI }\end{array}$ & $\begin{array}{l}\text { WELL NAME \& NUMBER } \\
\text { VALENTINE MRIG-212 }\end{array}$ & \multicolumn{2}{|c|}{$\begin{array}{l}\text { TFSC FIELD REPRESENTATIVE } \\
\text { D. KISTNER, H. ABEDI }\end{array}$} \\
\hline \multirow{3}{*}{\multicolumn{2}{|c|}{$\begin{array}{l}\text { NOTIFICATION OF REGULATORY AGENCY } \\
\text { AGENCY MS BUREAU OF LAND \& WATER RESOURCES } \\
\text { CEMENTING COMPANY GRINER DRILLING }\end{array}$}} & \multirow{2}{*}{\multicolumn{2}{|c|}{ DATE $\frac{11 / 10 / 88}{\text { REPRESENTATIVE JAMES HOFFMAN }}$}} \\
\hline & & & \\
\hline & & \multicolumn{2}{|c|}{ SERVICE ENGINEER / REP. JERRY BEACH } \\
\hline \multicolumn{4}{|c|}{ DISPOSAL OF FLUIDS } \\
\hline \multicolumn{2}{|c|}{ BY GRINER DRILLING } & $11 / 11 / 88$ & \\
\hline \multicolumn{2}{|c|}{ QUANTITY 20 BBL } & YPE DRILLING M & \\
\hline
\end{tabular}

\section{REMARKS}

EXCESS DRILLING MUD HAULED TO RICHTON LANDFILL AS NECESSARY.

ZONE 1

INTERVAL 180.5 TO $3.0 \mathrm{FT}$

BOTTOM OF TUBING OR PIPE 177

NO. OF SACKS $44.5 \quad$ YIELD 1.17 CIJ.FT/SK.

SLURRY WEIGHT 15.6 LBS/GAL

BRIDGE PLUG TYPE _ NA

REMARKS

PVC DRILLED OUT TO 130 FT. CARRIED HOLE TO ORIGINAL T.D. AT 180.5 FT. CEMENT CIRCULATED TO SURFACE. TOP 3.0 FT BACKFILLED WITH SOIL.

\section{ZONE}

INTERVAL

BOTTOM OF TUBING OR PIPE

NO. OF SA:KS

YIELD

SLURRY WEIGHT LBS/GAL

BRIDGE PLUG TYPE REMARKS
TYPE PLUG CONTINUOUS

DIAMETER OF CASING/HOLE $\quad 7.0$ IN CEMENT/MUD TYPE CLASS A. NEAT 
PLUGGING REPORT

\begin{tabular}{|c|c|c|c|}
\hline CLIENT & DOE / SRPO & J.O. No. 17500.25 & DATE $\quad 09 / 05 / 88$ \\
\hline $\begin{array}{l}\text { STATE } \\
\text { MISSISSIPPI }\end{array}$ & $\begin{array}{l}\text { WELL NAME \& NUMBER } \\
\text { MCLENDON MRIG-213 }\end{array}$ & \multicolumn{2}{|c|}{$\begin{array}{l}\text { TFSC FIELD REPRESENTATIVE } \\
\text { D. KISTNER, H. ABEDI }\end{array}$} \\
\hline \multicolumn{2}{|c|}{ NOTIFICATION OF REGULATORY AGENCY } & \multicolumn{2}{|l|}{ DATE $\quad 09 / 05 / 88$} \\
\hline \multicolumn{2}{|c|}{ AGENCY MS BUREAU OF LAND \& WATER RESOURCES } & \multicolumn{2}{|c|}{ REPRESENTATIVE JAMES HOFFMAN } \\
\hline \multicolumn{2}{|c|}{ CEMENTING COMPANY GRINER DRILLING } & \multicolumn{2}{|c|}{ SERVICE ENGINEER / REP. JERRY BEACH } \\
\hline \multicolumn{4}{|c|}{ DISPOSAL OF FLUIDS } \\
\hline \multicolumn{2}{|c|}{ BY GRINER DRILLING } & $09 / 05 / 88$ & \\
\hline QUANTITY & FLI & LING M & \\
\hline
\end{tabular}

\section{REMARKS}

EXCESS DRILLING MUD HAULED TO RICHTON LANDFILL AS NECESSARY.

ZONE 1

INTERVAL 100.5 TO $3.2 \mathrm{FT}$

BOTTOM OF TUBING OR PIPE $100.5 \mathrm{FT}$
TYPE PLUG CONTINUOUS

DIAMETER OF CASING/HOLE $71 / 2$ IN CEMENT/MUD TYPE CLASS A, NEAT
SLURRY WEIGHT

BRIDGE PLUG TYPE

DEPTH

PVC DRILLED OUT TO BOTTOM OF WELL AT 84 FT, CARRIED HOLE TO ORIGINAL T.D. A 100.5 FT. CEMENT CIRCULATED TO SURFACE. TOP 3.2 FT BACKFILLED WITH SOIL.

ZONE

INTERVAL

BOTTOM OF TUBING OR PIPE NO. OF SACKS

SLURRY WEIGHT

BRIDGE PLUG TYPE

REMARKS
TYPE PLUG

DIAMETER OF CASING/HOLE IN FT CEMENT/MUD TYPE

YIELD CU. FT/SK.

VOL. PUMPED CU. FT FT DEPTH 
PLUGGING REPORT

\begin{tabular}{|c|c|c|c|}
\hline CLIENT & DOE / SRPO & $\begin{array}{ll}\text { J.O. No. } 17500.25 \\
\end{array}$ & DATE $11 / 11 / 88$ \\
\hline $\begin{array}{l}\text { STATE } \\
\text { MISSISSIPPI }\end{array}$ & $\begin{array}{l}\text { WELL NAME \& NUMBER } \\
\text { H.K. HILLMAN MRIG-215 }\end{array}$ & \multicolumn{2}{|c|}{$\begin{array}{l}\text { TFSC FIELD REPRESENTATIVE } \\
\text { D. KISTNER, } \mathrm{H} \text {. ABEDI }\end{array}$} \\
\hline \multirow{3}{*}{\multicolumn{2}{|c|}{$\begin{array}{l}\text { NOTIFICATION OF REGULATORY AGENCY } \\
\text { AGENCY MS BUREAU OF LAND \& WATER RESOURCES } \\
\text { CEMENTING COMPANY GRINER DRILLING }\end{array}$}} & \multicolumn{2}{|l|}{ DATE $11 / 11 / 88$} \\
\hline & & \multicolumn{2}{|c|}{ REPRESENTATIVE JAMES HOFFMAN } \\
\hline & & \multicolumn{2}{|c|}{ SERVICE ENGINEER / REP. JERRY BEACH } \\
\hline \multicolumn{4}{|c|}{ DISPOSAL OF FLUIDS } \\
\hline \multicolumn{2}{|c|}{ BY GRINER DRILLING } & $11 / 11 / 88$ & \\
\hline QUANTITY. & FLUIL & DRILLING $M$ & \\
\hline
\end{tabular}

\section{REMARKS}

EXCESS DRILLING MUD HAULED TO RICHTON LANDFILL AS NECESSARY.

ZONE 1

INTERVAL 120.5 TO $4.5 \mathrm{FT}$
TYPE PLUG CONTINUOUS

DIAMETER OF CASING/HOLE 7.0 IN BOTTOM OF TUBING OR PIPE 117 FT CEMENT/MUD TYPE CLASS A, NEAT NO. OF SACKS 32 YIELD 1.17 CU.FT/SK. VOL. PUMPED 37.4 CU. FT SLURRY WEIGHT 15.6 LBS/GAL TOP OF PLUG 4.5 FT BRIDGE PLUG TYPE NA DEPTH NA REMARKS

PVC DRILLED OUT TO $120.5 \mathrm{FT}$. CEMENT CIRCULATED TO SURFACE. TOP 4.5 FT BACKFILLED WITH SOIL.

\section{ZONE}

INTERVAL

BOTTOM OF TUBING OR PIPE

NO. OF SACKS

SLURRY WEIGHT

BRIDGE PLUG TYPE REMARKS
TYPE PLUG

DIAMETER OF CASING/HOLE IN FT CEMENT/MUD TYPE YIELD CU. FT/SK.

VOL. PUMPED CU. FT LBS/GAL

TOP OF PLUG FT 
PLUGGING REPORT

\begin{tabular}{|l|l|l|}
\cline { 3 - 3 } \multicolumn{2}{c|}{} & $\begin{array}{c}\text { DATUM ELEVATION } \\
218.5\end{array}$ \\
\hline J.O. No. 17500.25 & DATE $09 / 06 / 88$ \\
\hline
\end{tabular}

\begin{tabular}{|c|c|c|c|}
\hline \multicolumn{2}{|r|}{ DOE / SRPO } & J.0. No. 17500.25 & $\begin{array}{ll}\text { DATE } & 09 / 06 / 88\end{array}$ \\
\hline $\begin{array}{l}\text { STATE } \\
\text { MISSISSIPPI }\end{array}$ & $\begin{array}{l}\text { WELL NAME \& NUMBER } \\
\text { MCLENDON MRIG-216 }\end{array}$ & \multicolumn{2}{|c|}{$\begin{array}{l}\text { TFSC FIELD REPRESENTATIVE } \\
\text { D. KISTNER, H. ABEDI }\end{array}$} \\
\hline \multirow{3}{*}{\multicolumn{2}{|c|}{$\begin{array}{l}\text { NOTIFICATION OF REGULATORY AGENCY } \\
\text { AGENCY MS BUREAU OF LAND\& WATER RESOURCES } \\
\text { CEMENTING COMPANY GRINER DRILLING }\end{array}$}} & \multicolumn{2}{|l|}{ DATE $09 / 06 / 88$} \\
\hline & & \multicolumn{2}{|c|}{ REPRESENTATIVE JAMES HOFFMAN } \\
\hline & & \multicolumn{2}{|c|}{ SERVCE ENGINEER / REP. JERRY BEACH } \\
\hline \multicolumn{4}{|c|}{ DISPOSAL OF FLUIDS } \\
\hline \multicolumn{2}{|c|}{ BY GRINER DRILLING } & \multicolumn{2}{|c|}{ ATE $09 / 06 / 88$} \\
\hline \multicolumn{2}{|c|}{ QUANTITY 20 BBL } & PE DRILLING & \\
\hline
\end{tabular}

REMARKS

EXCESS DRILLING MUD HAULED TO RICHTON LANDFILL.

ZONE 1

INTERVAL 120.5 TO $2.9 \mathrm{FT}$

BOTTOM OF TUBING OR PIPE 120.5 FT

NO. OF SACKS 36 YIELD 1.17 CU. FT/SK.

SLURRY WEIGHT 15.6 LBS/GAL

BRIDGE PLUG TYPE NA

REMARKS

PVC DRILLED OUT TO 109 FT. CARRIED HOLE TO ORIGINAL T.D. C 120.5 FT. CIRCULATED TO SURFACE. TOP 2.9 FT BACKFILLED WITH SOIL.
TYPE PLUG CONTINUOUS

DIAMETER OF CASING/HOLE IN CEMENT/MUD TYPE CLASS A. NEAT

VOL. PUMPED 42.1 CU. FT TOP OF PLUG 2.9 DEPTH NA

\section{ZONE}

INTERVAL BOTTOM OF TUBING OR PIPE NO. OF SACKS YIELD LBS/GAL

SLURRY WEIGHT BRIDGE PLUG TYPE REMARKS
TYPE PLUG

DIAMETER OF CASING/HOLE IN CEMENT/MUD TYPE CU. FT/SK. VOL. PUMPED CU. FT TOP OF PLUG FT DEPTH 
PLUGGING REPORT

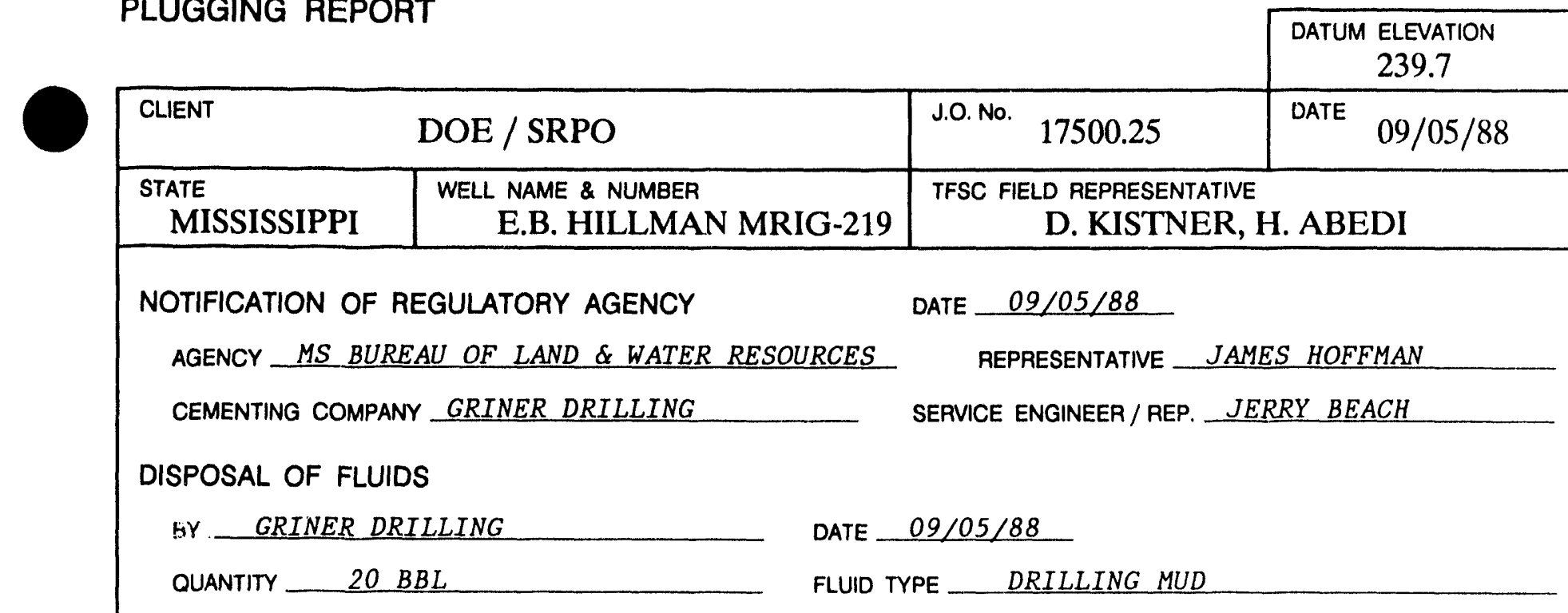

\section{REMARKS}

EXCESS DRILLING MUD HAULED TO RICHTON LANDFILL.

ZONE 1

INTERVAL 140.9 TO $2.7 \mathrm{FT}$
TYPE PLUG CONTINUOUS

DIAMETER OF CASING/HOLE _ $71 / 2$
IN

BOTTOM OF TUBING OR PIPE 140.9 FT CEMFNT/MUD TYPE CLASS A. NEAT

NO. OF SACKS 46 YIELD 1.17 CU.FT/SK. VOL. PUMPED 53.8 CU.FT

SLURRY WEIGHT -15.6 LBS/GAL TOP OF PLUG 2.7 FT

BRIDGE PLUG TYPE

REMARKS

PVC DRILLED OUT TO 106 FT. CARRIED HOLE TO ORIGINAL T.D. A 140.9 FT. CEMENT

CIRCULATED TO SURFACE. TOP 2.7 FT BACKFILLED WITH SOIL.

ZONE

INTERVAL

BOTTOM OF TUBING OR PIPE

NO. OF SACKS

SLURRY WEIGHT

YIELD LBS/GAL

BRIDGE PLUG TYPE

REMARKS
TYPE PLUG

DIAMETER OF CASING/HOLE IN CEMENT/MUD TYPE CU. FT/SK. VOL. PUMPED CU. FT TOP OF PLUG FT DEPTH 
PLUGGING REPORT

\begin{tabular}{|c|c|}
\hline & $\begin{array}{c}\text { DATUM ELEVATION } \\
215.7 \\
\end{array}$ \\
\hline J.O. No. 17500.25 & DATE $11 / 01 / 88$ \\
\hline
\end{tabular}

TFSC FIELD REPRESENTATIVE

D. KISTNER, H. ABEDI

\section{NOTIFICATION OF REGULATORY AGENCY \\ AGENCY MS BUREAU OF LAND \& WATER RESOURCES \\ CEMENTING COMPANY GRINER DRILLING}

DATE $11 / 01 / 88$

REPRESENTATIVE JAMES HOFFMAN

SERVICE ENGINEER / REP. JERRY BEACH

DISPOSAL OF FLUIDS

BY GRINER DRILLING

DATE $11 / 01 / 88$

QUANTITY 20 BBL

FLUID TYPE _ DRILLING MUD

\section{REMARKS}

EXCESS DRILLING MUD HAULED TO RICHTON LANDFILL.

ZONE 1

INTERVAL 120.5 TO $3.0 \mathrm{FT}$

\begin{tabular}{l} 
BOTTOM OF TUBING OR PIPE $\frac{117}{\text { NT }} \begin{array}{r}\text { CEMENT/MUD TY } \\
\text { NO. OF SACKS } 36\end{array}$ YIELD 1.17 CU. FT/SK. \\
\hline
\end{tabular}

SLURRY WEIGHT 15.6 LBS/GAL

BRIDGE PLUG TYPE _ NA

REMARKS

PVC DRILLED OUT TO 78.5 FT. CARRIED HOLE TO ORIGINAL T.D. Q 120.5 FT. CEMENT

CIRCULATED TO SURFACE. TOP 3 FT BACKFILLED WITH SOIL.
TYPE PLUG CONTINUOUS

DIAMETER OF CASING/HOLE 7.0 IN 
PLUGGING REPORT

\begin{tabular}{|c|c|}
\hline & $\begin{array}{l}\text { DATUM ELEVATION } \\
261.8\end{array}$ \\
\hline J.O. No. 17500.25 & $\begin{array}{l}\text { DATE } \quad 09 / 07 / 88\end{array}$ \\
\hline
\end{tabular}

NOTIFICATION OF REGULATORY AGENCY

DATE $09 / 07 / 88$

AGENCY MS BUREAU OF LAND \& WATER RESOURCES REPRESENTATIVE — JAMES HOFFMAN

CEMENTING COMPANY GRINER DRILLING

SERVICE ENGINEER / REP. JERRY BEACH

DISPOSAL OF FLUIDS

BY GRINER DRILLING

DATE $09 / 07 / 88$

QUANTITY 25 BBL

FLUID TYPE _ DRILLING MUD

\section{REMARKS}

EXCESS DRILLING MUD HAULED TO RICHTON LANDFILL.

ZONE 1

INTERVAL 130 TO $3.25 \mathrm{FT}$.

BOTTOM OF TUBING OR PIPE 130 FT

NO. OF SACKS 43 YIELD 1.17 CU. FT/SK.

SLURAY WEIGHT 15.6 LBS/GAL

BRIDGE PLUG TYPE

REMARKS

PVC DRILLED OUT TO $130 \mathrm{FT}$. CEMENT CIRCULATED TO SURFACE. TOP 3.25 FT BACKFILLED WITH SOIL.

ZONE

INTERVAL

TYPE PLUG

BOTTOM OF TUBING OR PIPE

NO. OF SACKS FT

DIAMETER OF CASING/HOLE IN

SLURRY WEIGHT LBS/GAL

BRIDGE PLUG TYPE

REMARKS
TYPE PLUG CONTINUOUS

DIAMETER OF CASING/HOLE _ 8.0 IN CEMENT/MUD TYPE CLASS A. NEAT

TOP OF PLUG 3.25

DEPTH _ NA 
PLUGGING REPORT

\begin{tabular}{|c|c|c|c|}
\hline \multicolumn{3}{|l|}{ DLUGGING REPO } & $\begin{array}{c}\text { DATUM ELEVATION } \\
237.3 \\
\end{array}$ \\
\hline CLIENT & DOE / SRPO & J.O. No. 17500.25 & DATE $09 / 02 / 88$ \\
\hline $\begin{array}{l}\text { STATE } \\
\text { MISSISSIPPI }\end{array}$ & $\begin{array}{l}\text { WELL NAME \& NUMBER } \\
\text { E.B. HILLMAN MRIG-227 }\end{array}$ & \multicolumn{2}{|c|}{$\begin{array}{l}\text { TFSC FIELD REPRESENTATIVE } \\
\text { D. KISTNER, H. ABEDI }\end{array}$} \\
\hline \multicolumn{2}{|c|}{$\begin{array}{l}\text { NOTIFICATION OF REGULATORY AGENCY } \\
\text { AGENCY MS BUREAU OF LAND \& WATER RESOURCES }\end{array}$} & \multicolumn{2}{|l|}{ DATE $\quad 09 / 02 / 88$} \\
\hline \multicolumn{2}{|c|}{ CEMENTING COMPANY GRINER DRILLING } & \multicolumn{2}{|c|}{ SERVICE ENGINEER / REP. JERRY BEACH } \\
\hline \multicolumn{4}{|c|}{ DISPOSAL OF FLUIDS } \\
\hline \multicolumn{2}{|c|}{ BY GRINER DRILLING } & \multicolumn{2}{|l|}{$09 / 02 / 88$} \\
\hline \multicolumn{2}{|c|}{ QUANTITY 20 BBL } & \multicolumn{2}{|c|}{ DRILLING MUD } \\
\hline \multicolumn{4}{|l|}{ REMARKS } \\
\hline \multicolumn{4}{|l|}{ SS } \\
\hline
\end{tabular}

ZONE 1

INTERVAL 140.5 TO $5.0 \mathrm{FT}$.
TYPE PLUG CONTINUOUS

DIAMETER OF CASING/HOLE 7.0 IN

BOTTOM OF TUBING OR PIPE 140.5 FT CEMENT/MUD TYPE CLASS A, NEAT

NO. OF SACKS 40 YIELD 1.17 CU.FT/SK. VOL. PUMPED 46.8 CU.FT

SLURRY WEIGHT 15.6 LBS/GAL TOP OF PLUG 5.0 FT

BRIDGE PLUG TYPE _ NA DEPTH NA

REMARKS

PVC DRILLED OUT TO $75 \mathrm{FT}$. HOLE CARRIED TO ORIGINAL T.D. OF $140.5 \mathrm{FT}$. CEMENT CIRCULATED TO SURFACE. TOP 5.0 FT BACKFILLED WITH SOIL.

ZONE

INTERVAL

BOTTOM OF TUBING OR PIPE NO. OF SACKS

SLURRY WEIGHT

BRIDGE PLUG TYPE

REMARKS
TYPE PLUG

DIAMETER OF CASING/HOLE IN CEMENT/MUD TYPE

YIELD CU. FT/SK. VOL. PUMPED CU. FT TOP OF PLUG FT DEPTH 
PLUGGING REPORT

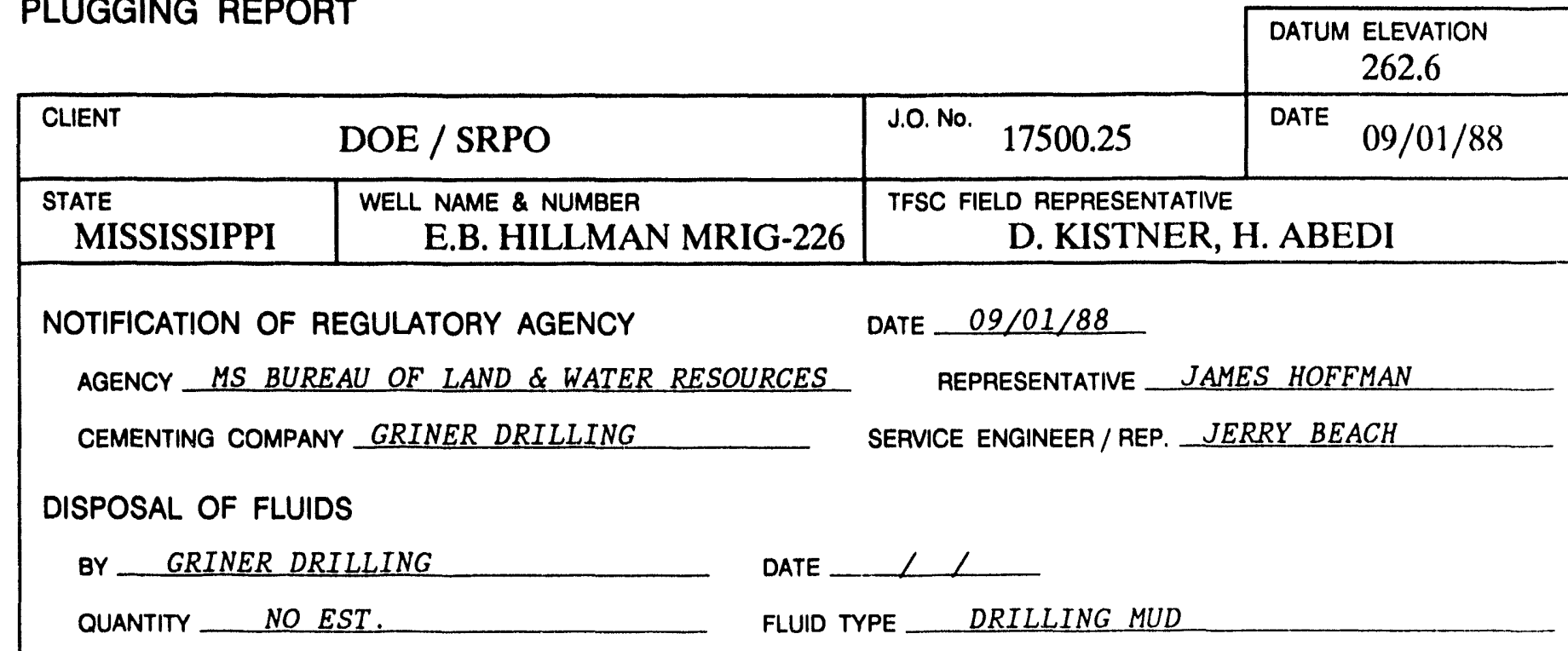

\section{REMARKS}

EXCESS DRILLING MUD HAULED TO RICHTON LANDFILL AS NECESSARY.

ZONE 1

INTERVAL 160.5 TO $2.9 \mathrm{FT}$
TYPE PLUG CONTINUOUS

DIAMETER OF CASING/HOLE 7.0 IN BOTTOM OF TUBING OR PIPE 160.5 FT CEMENT/MUD TYPE CLASS A, NEAT NO. OF SACKS 46 YIELD 1.17 CU.FT/SK. VOL. PUMPED 53.8 CU. FT SLURRY WEIGHT 15.6 LBS/GAL TOP OF PLUG 2.9 BRIDGE PLUG TYPE NA DEPTH NA REMARKS

PVC DRILLED OUT TO 154.5 FT. CARRIED HOLE TO ORIGINAL T.D. OF $160.5 \mathrm{FT}$. CEMENT CIRCULATED TO SURFACE. TOP 2.9 FT BACXFILLED WITH SOIL.

ZONE

INTERVAL BOTTOM OF TUBING OR PIPE NO. OF SACKS SLURRY WEIGHT BRIDGE PLUG TYPE REMARKS
TYPE PLUG

DIAMETER OF CASING/HOLE $\mathbb{N}$ FT CEMENT/MUD TYPE YIELD CU. FT/SK. VOL. PUMPED CU. FT LBS/GAL

TOP OF PLUG FT DEPTH 


\section{PLUGGING REPORT}

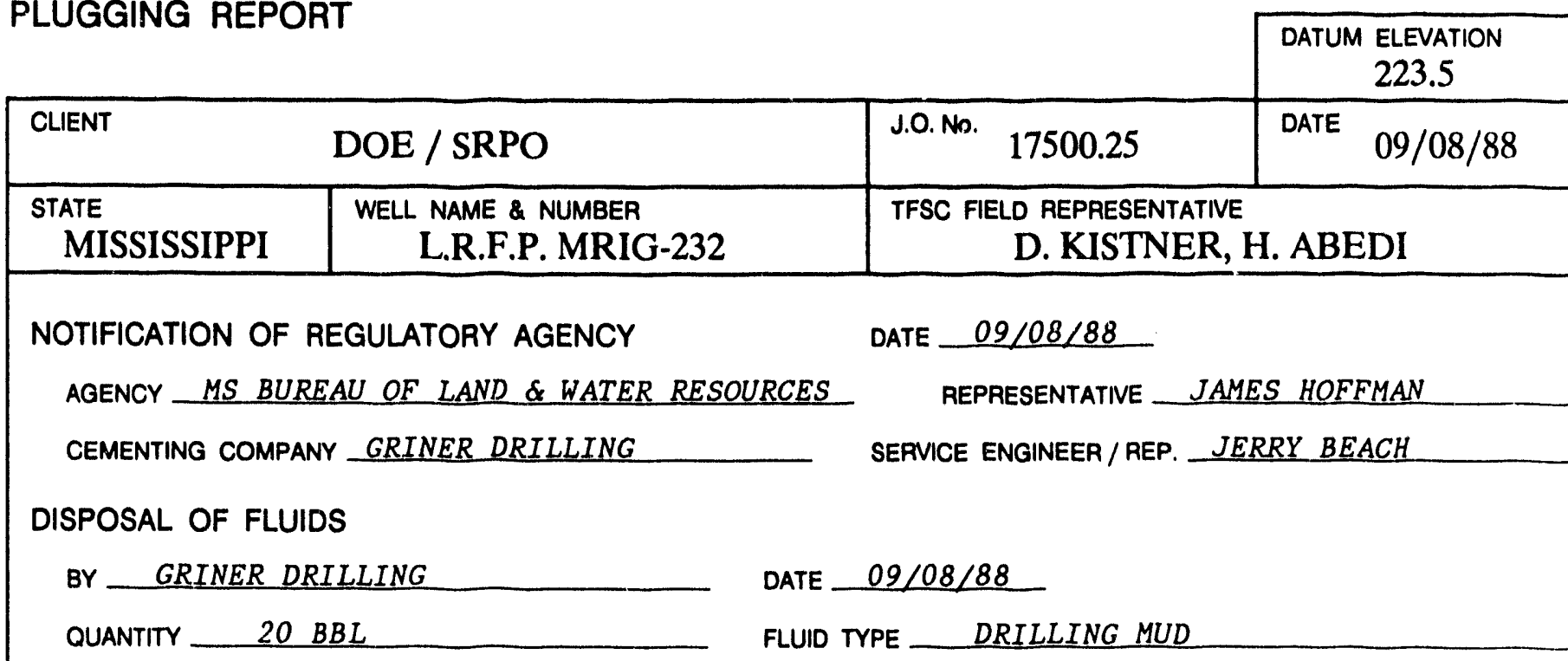

\section{REMARKS}

EXCESS DRILLING MUD HAULED TO RICHTON LANDFILL.

ZONE 1

INTERVAL 120.5 TO $3.0 \mathrm{FT}$
TYPE PLUG CONTINUOUS

DIAMETER OF CASING/HOLE _ 7.5
IN

BOTTOM OF TUBING OR PIPE 120 FT CEMENT/MUD TYPE_ CLASS A, NEAT

NO. OF SACKS 36 YIELD 1.17 CU.FT/SK. VOL. PUMPED 42.1 CU.FT

SLURRY WEIGHT 15.6 LBS/GAL TOP OF PLUG 3.0

BRIDGE PLUG TYPE NA DEPTH _ NA

REMARKS

DRILLED OUT PVC TO 120.5 FT. CEMENT CIRCULATED TO SURFACE. TOP 3.0 FT BACKFILLED WITH SOIL.

ZONE

INTERVAL

BOTTOM OF TUBING OR PIPE

NO. OF SACKS

YIELD LBS/GAL

SLURRY WEIGHT

BRIDGE PLUG TYPE

REMARKS
TYPE PLUG

DIAMETER OF CASING/HOLE IN CEMENT/MUD TYPE CU. FT/SK. VOL. PUMPED CU. FT TOP OF PLUG FT DEPTH 
PLUGGING REPORT

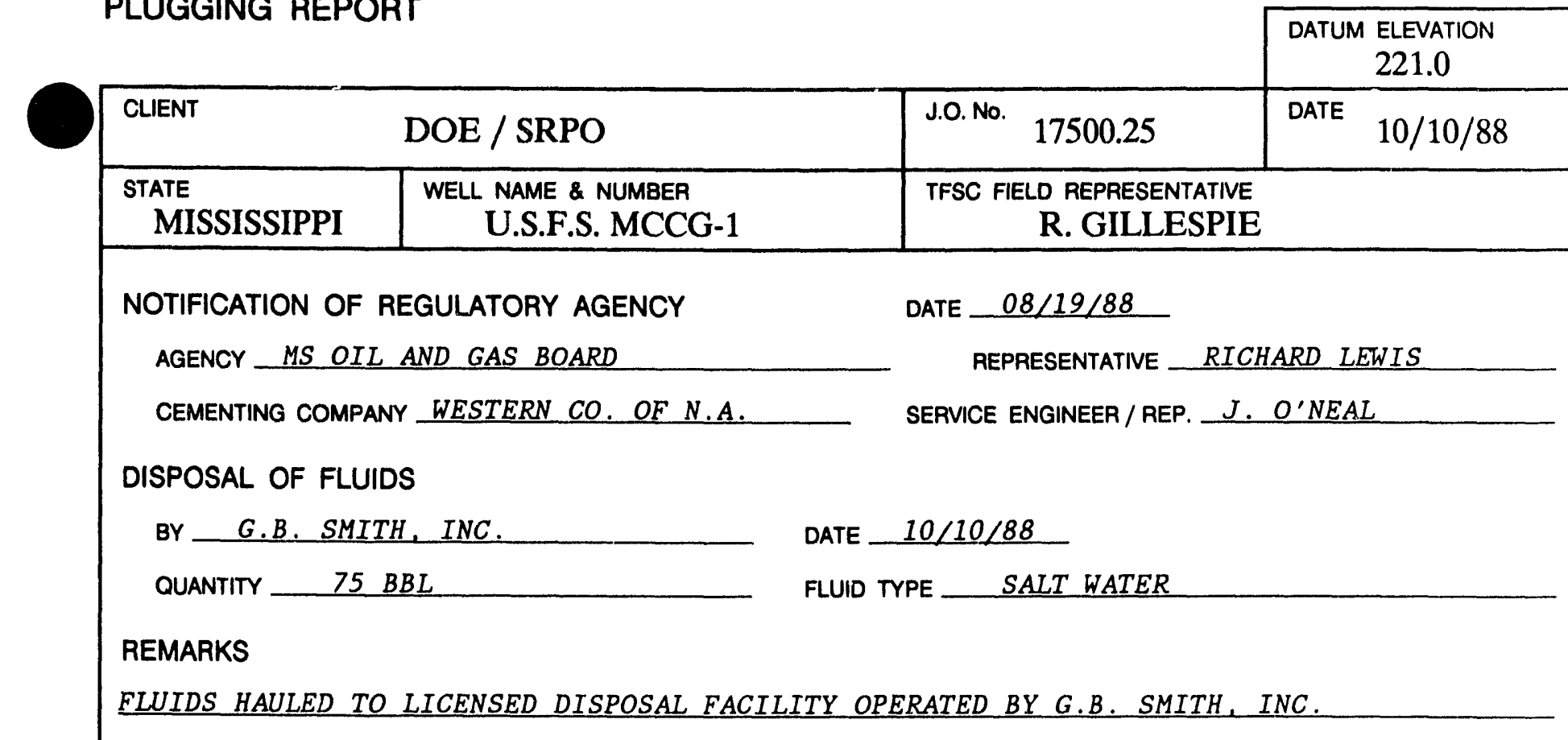

ZONE 1

TYPE PLUG CONTINUOUS

INTERVAL 1369 FT TO SURFACE

DIAMETER OF CASING/HOLE $77 / 8 \& 8 \quad 5 / 8$ IN

BOTTOM OF TUBING OR PIPE $1321.3 \mathrm{FT}$ CEMENT/MUD TYPE CLASS A, 88 BENTONITE

NO. OF SACKS $237 \quad \mathrm{LD} \quad 1.92$ CU.FT/SK. VOL. PLi:MPED 455 CU.FT

SLURRY WEIGHT 13.1 TOP OF PLUG 4.0 FT

BRIDGE PLUG TYPE NA DEPTH NA

REMARKS

CEMENT PUMPED IN STAGES WITH TUBING SET AT 1321 AND 671 FT. CEMENT CIRCULATED TO SURFACE. LASING CUT OFF AND CAPPED 4 FT BELOW SURFACE, TOP 4 FT BACKFILLED WITH SOIL.

ZONE

INTERVAL

BOTTOM OF TUBING OR PIPE

NO. OF SACKS

SLURR!" WEIGHT

BRIDGE PLUG TYPE REMARKS
TYPE PLUG

DIAMETER OF CASING/HOLE IN FT CEMENT/MUD TYPE

YIELD CU. FT/SK.

VOL. PUMPED CU. FT LBS/GAL TOP OF PLUG FT DEPTH 


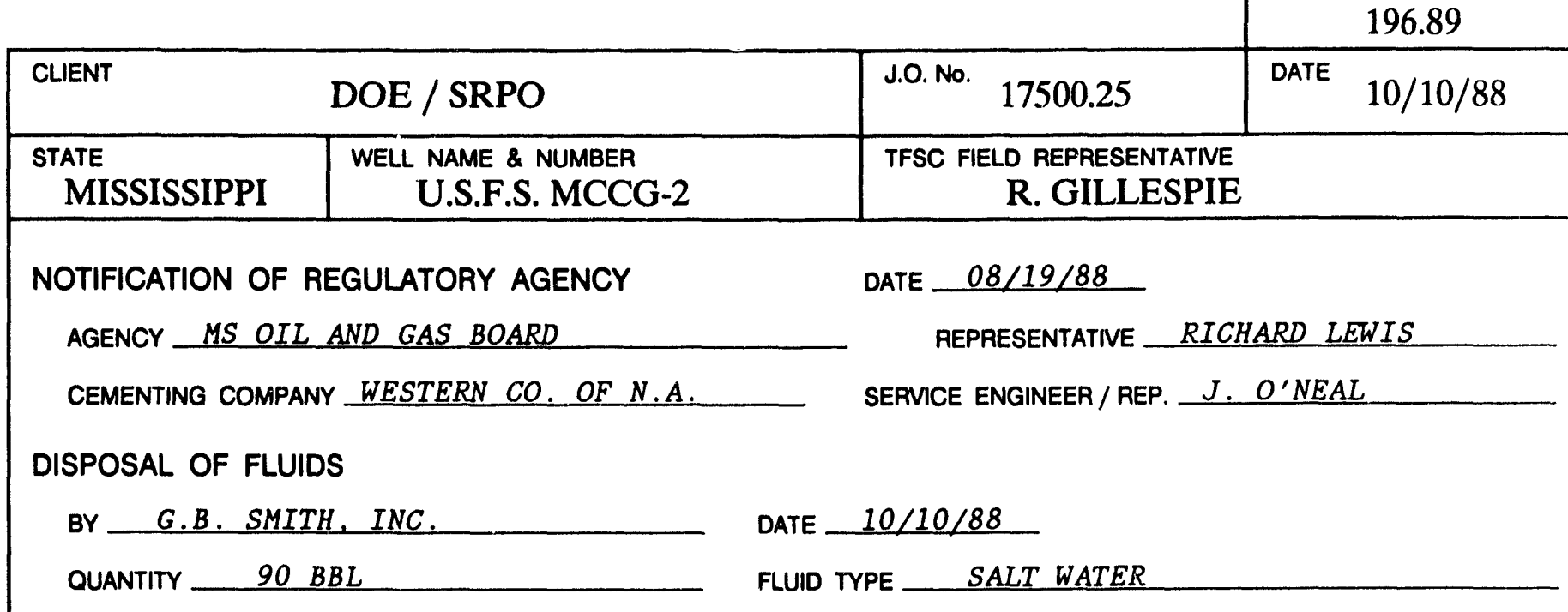

\section{REMARKS}

FLUIDS HAULED TO LICENSED DISPOSAL FACILITY OPERATED BY G.B. SMITH, INC.

ZONE 1

INTERVAL 2683.5 FT TO SURFACE
TYPE PLUG CONTINUOUS

DIAMETER OF CASING/HOLE $4.0 \& 65 / 8$ IN

BOTTOM OF TUBING OR PIPE 2622 FT CEMENT/MUD TYPE CLASS A, 88 BENTONITE

NO. OF SACKS 263 YIELD 1.92 CU.FT/SK. VOL. PUMPED 505 CU. FT

SLURRY WEIGHT 13.1 LBS/GAL TOP OF PLUG 3.5 FT

BRIDGE PLUG TYPE NA DEPTH NA

REMARKS

CEMENT CIRCULATED TO SURFACE, PUMPED IN STAGES WITH TUBING SET AT 2622, 2096, 1568 , 1042. AND $525 \mathrm{FT}$. CASING CUT OFF AND CAPPED 3.5 FT BELOW SURFACE, TOP 3.5 FT BACK.

FILLED WITH SOIL.

ZONE

INTERVAL

BOTTOM OF TUBING OR PIPE

NO. OF SACKS

SLURAY WEIGHT

BRIDGE PLUG TYPE

REMARKS
TYPE PLUG

DIAMETER OF CASING/HOLE IN FT CEMENT/MUD TYPE

YIELD CU. FT/SK.

VOL. PUMPED CU. FT LBS/GAL

TOP OF PLUG $F T$

DEPTH 
PLUGGING REPORT

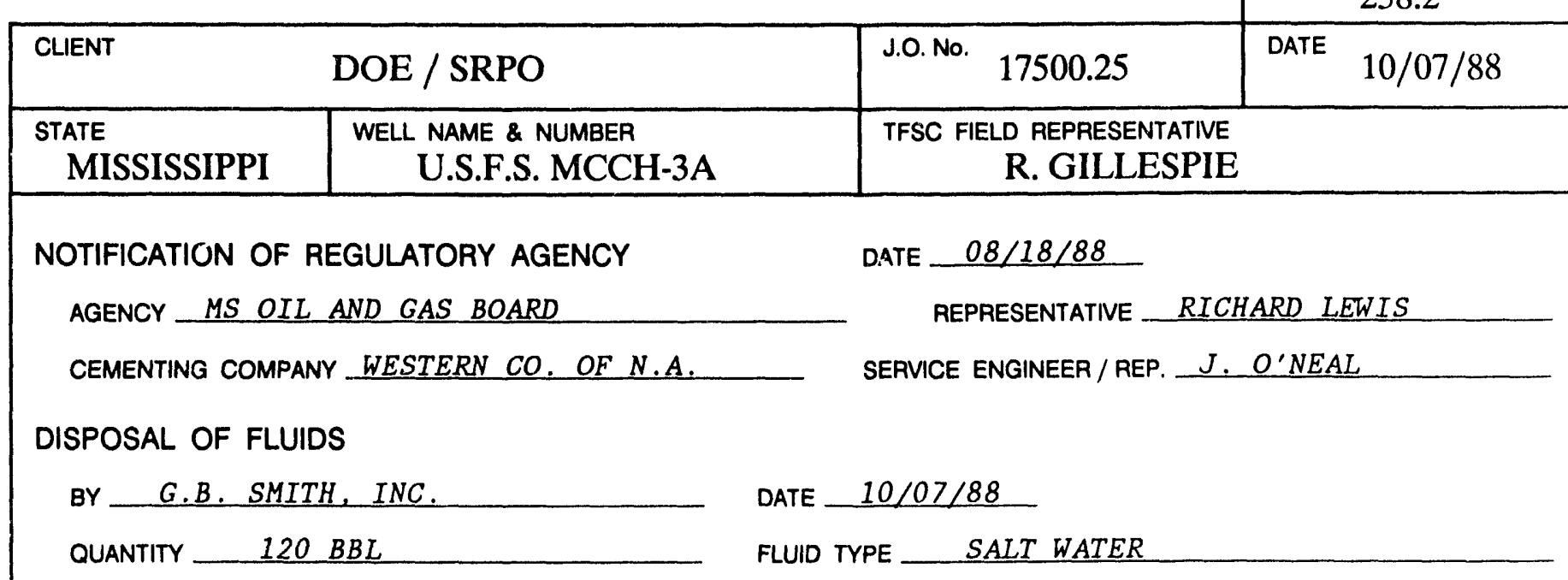

\section{REMARKS}

FLUIDS HAULED TO LICENSED DISPOSAL FACILITY OPERATED BY G.B. SMITH, INC.

ZONE 1

INTERVAL 3098.6 FT TO SURFACE
TYPE PLUG CONTINUOUS

DIAMETER OF CASING/HOLE $4.0,65 / 8, \& 8.0 \mathrm{IN}$ BOTTOM OF TUBING OR PIPE 2993 FT CEMENT/MUD TYPE CLASS A, 88 BENTONITE NO. OF SACKS _ 309 YIELD 1.92 CU.FT/SK. VOL. PUMPED 593 CU.FT SLURRY WEIGHT 13.1 LBS/GAL TOP OF PLUG 3.0 FT

BRIDGE PLUG TYPE _ NA DEPTH

REMARKS

CEMENT PUMPED IN STAGES WITH TUBING SET AT 2993, 2498, 2002, 1507, 1011, AND 516 FT. CEMENT CIRCULATED TO SURFACE, CASING CUT OFF AND CAPPED 3.5 FT BELOW SURFACE, TOP 3.5 FT BACKFILLED WITH SOIL.

ZONE

INTERVAL

BOTTOM OF TUBING OR PIPE NO. OF SACKS

SLURRY WEIGHT

BRIDGE PLUG TYPE REMARKS
TYPE PLUG

DIAMETER OF CASING/HOLE IN CEMENT/MUD TYPE

YIELD CU. FT/SK.

VOL. PUMPED CU. FT FT LBS/GAL TOP OF PLUG DEPTH 
PLUGGING REPORT

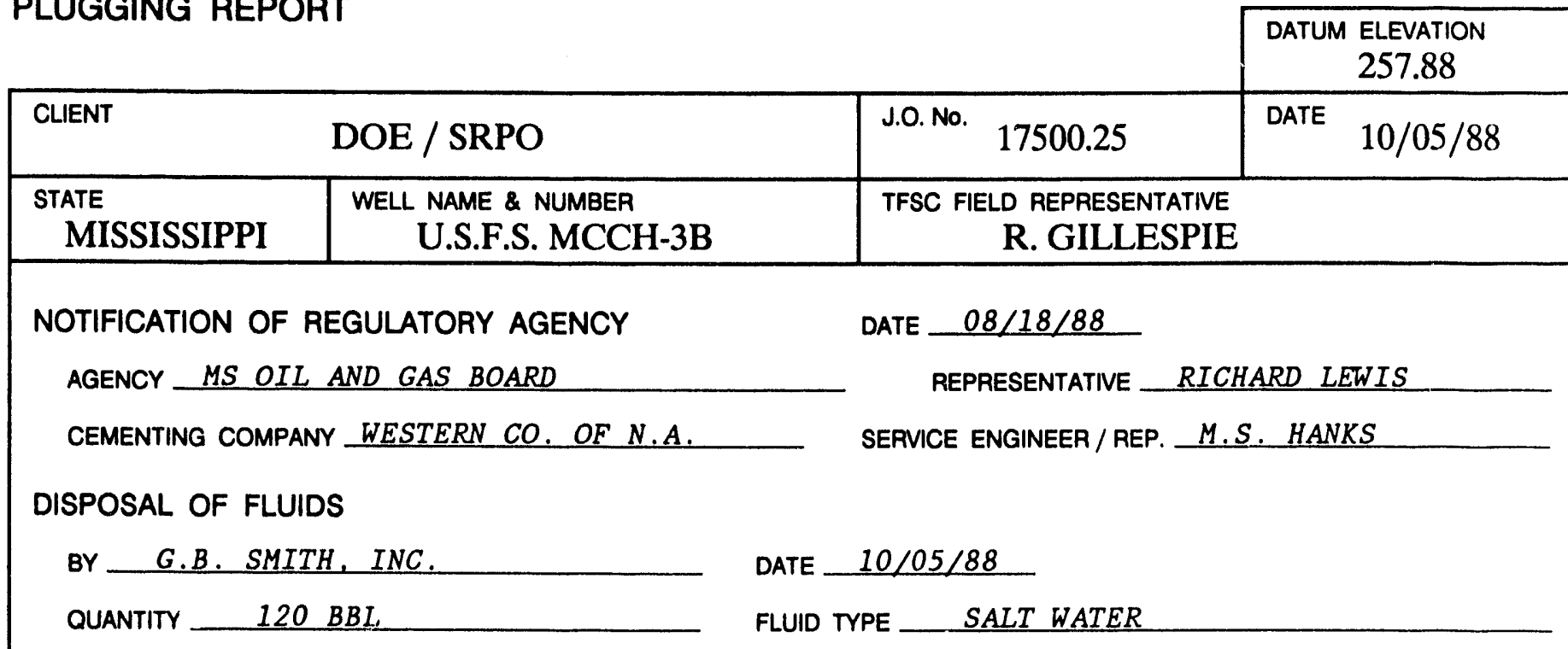

\section{REMARKS}

FLUIDS HAULED TO LICENSED DISPOSAL FACILITY OPERATED BY G.B. SMITH, INC.

ZONE 1

INTERVAL 3490.1 FT TO SURFACE
TYPE PLUG CONTINUOUS

DIAMETER OF CASING/HOLE $4.0,65 / 8, \& 8.0 \mathrm{~N}$ BOTTOM OF TUBING OR PIPE $3427.4 \mathrm{FT}$ CEMENT/MUD TYPE CLASS A. 88 BENTONITE NO. OF SACKS _ 333 YIELD 1.92 CU.FT/SK. VOL. PUMPED 639 CU.FT SLURRY WEIGHT 13.1 LBS/GAL TOP OF PLUG 4.0 FT BRIDGE PLUG TYPE N NA DEPTH NA

REMARKS

CEMENT PUMPED IN STAGES WITH TUBING SET \& 3427, 2870, 2313, 1757, 1198, AND 609.5 FT. CEMENT CIRCULATED TO SURFACE, CASING CUT OFF AND CAPPED 4 FT BELOW SURFACE, AND TOP 4.O FT BACKFILLED WITH SOIL.

ZONE

INTERVAL BOTTOM OF TUBING OR PIPE NO. OF SACKS SLURRY WEIGHT BRIDGE PLUG TYPE REMARKS
TYPE PLUG

DIAMETER OF CASING/HOLE IN FT CEMENT/MUD TYPE

YIELD CU. FT/SK. VOL. PUMPED CU. FT LBS/GAL TOP OF PLUG FT DEPTH 
PLUGGING REPORT

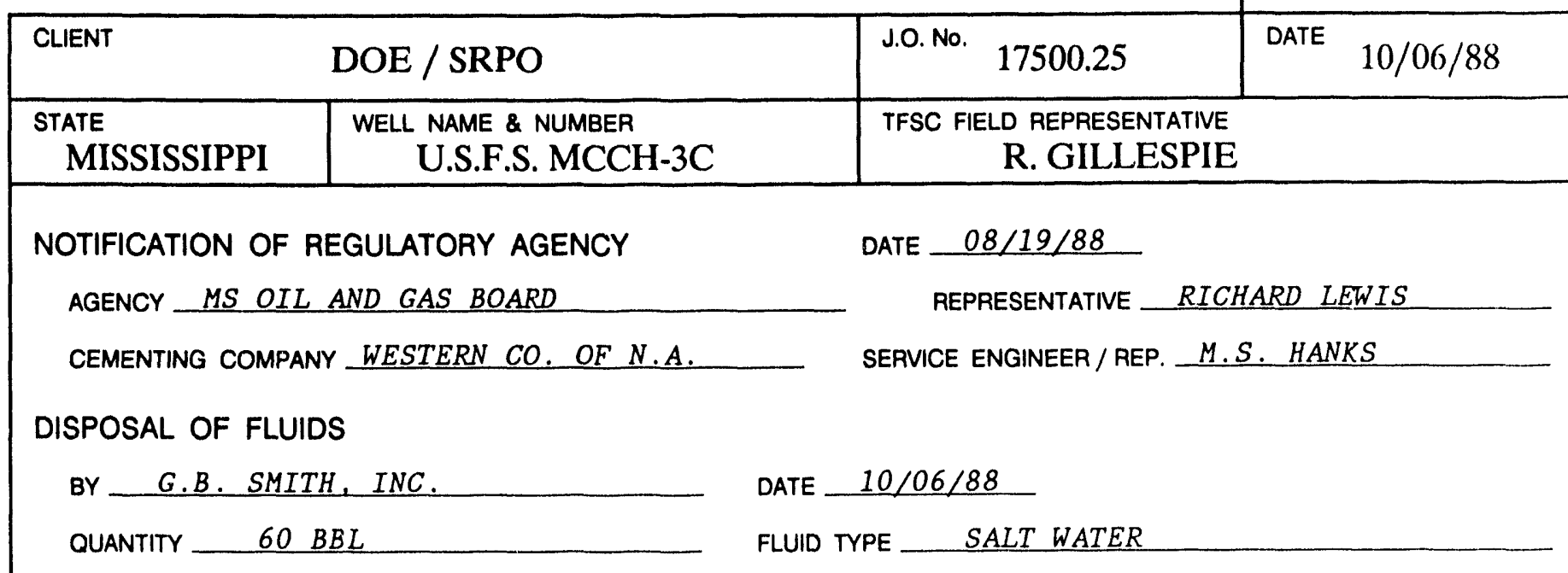

\section{REMARKS}

FLUIDS HAULED TO LICENSED DISPOSAL FACILITY OPERATED BY G.B. SMITH, INC.

ZONE 1 TYPE PLUG CONTINUOUS

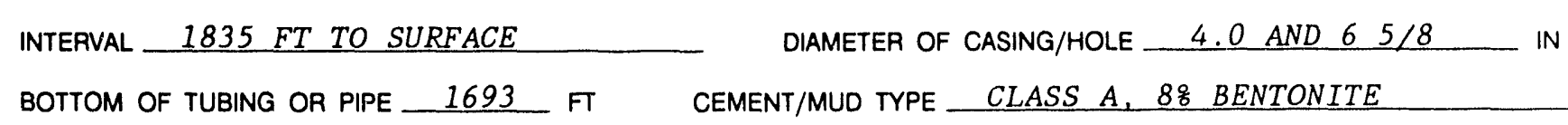

NO. OF SACKS 176 YIELD 1.92 CU.FT/SK. VOL. PUMPED 338 CU.FT

SLURRY WEIGHT 13.1 LBS/GAL TOP OF PLUG 4.0 FT

BRIDGE PLUG TYPE NA DEPTH_NA

REMARKS

CEMENT PUMPED IN STAGES WITH TUBING SET AT 1693, 1135, AND 577 FT. CEMENT CIRCULATED TO SURFACE, CASING CUT OFF 4 FT BELOW SURFACE AND CAPPED, TOP 4 FT BACKFILLED WITH SOIL.

ZONE

INTERVAL

BOTTOM OF TUBING OR PIPE

NO. OF SACKS

SLURAY WEIGHT LBS/GAL

BRIDGE PLUG TYPE REMARKS
TYPE PLUG

DIAMETER OF CASING/HOLE IN CEMENT/MUD TYPE CU. FT/SK. VOL. PUMPED CU. FT YIELD TOP OF PLUG FT DEPTH 
PLUGGING REPORT

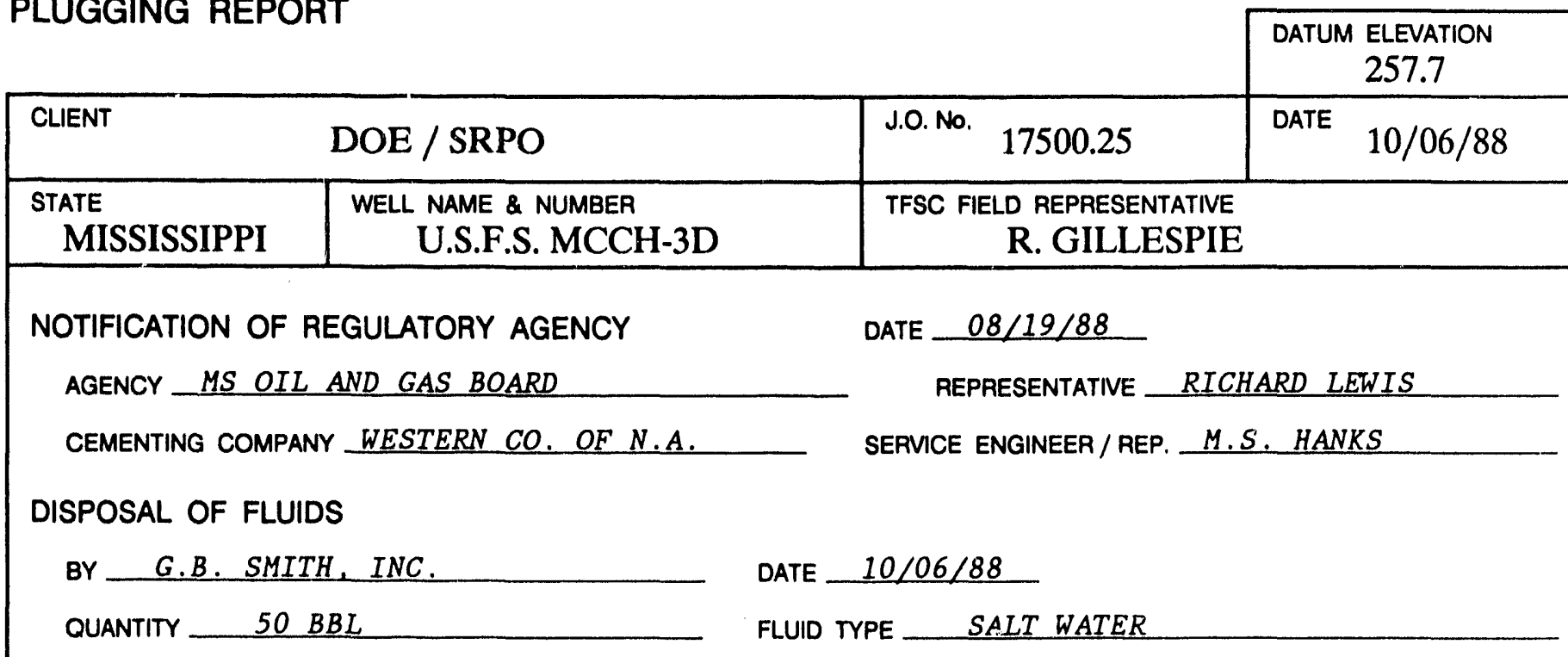

\section{REMARKS}

ELUIDS HAULED TO LICENSED DISPOSAL FACILITY OPERATED BY G.B. SMITH, INC.

ZONE 1

INTERVAL 1251 FT TO SURFACE

BOTTOM OF TUBING OR PIPE 1195.6 FT

NO. OF SACKS 123 YIELD 1.92

SLURRY WEIGHT $\frac{13.1}{1}$ LBS/GAL

BRIDGE PLUG TYPE NA

REMARKS

CEMENT PUMPED IN STAGES WITH TUBING SET AT 1195.6 AND 607.5 FT. CEMENT CIRCULATED TO SURFACE. CASING CUT OFF 3.5 FT BELOW GROUND AND CAPPED, TOP 3.5 FT BACKFILLED WITH SOIL.

ZONE

INTERVAL

TYPE PLUG

DIAMETER OF CASING/HOLE IN

BOTTOM OF TUBING OR PIPE

NO. OF SACKS

YIELD FT CEMENT/MUD TYPE LBS/GAL CU. FT/SK. TOP OF PLUG VOL. PUMPED CU. FT TYPE PLUG CONTINUOUS

DIAMETER OF CASING/HOLE 4.0 AND $6 \quad 5 / 8$ IN CEMENT/MUD TYPE CLASS A, $8 \%$ BENTONITE

SLURRY WEIGHT

BRIDGE PLUG TYPE DEPTH REMARKS 
PLUGGING REPORT

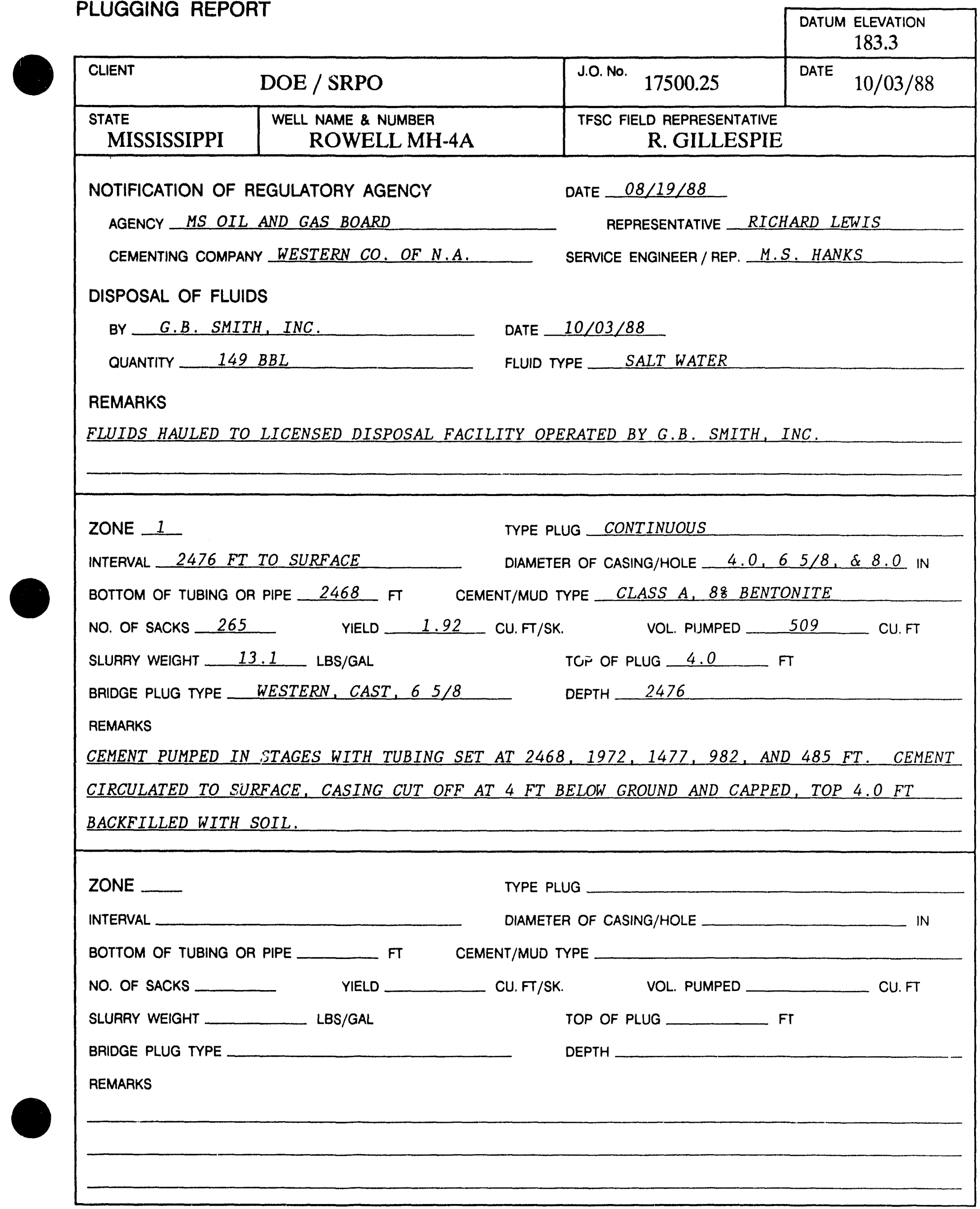


PLUGGING REPORT

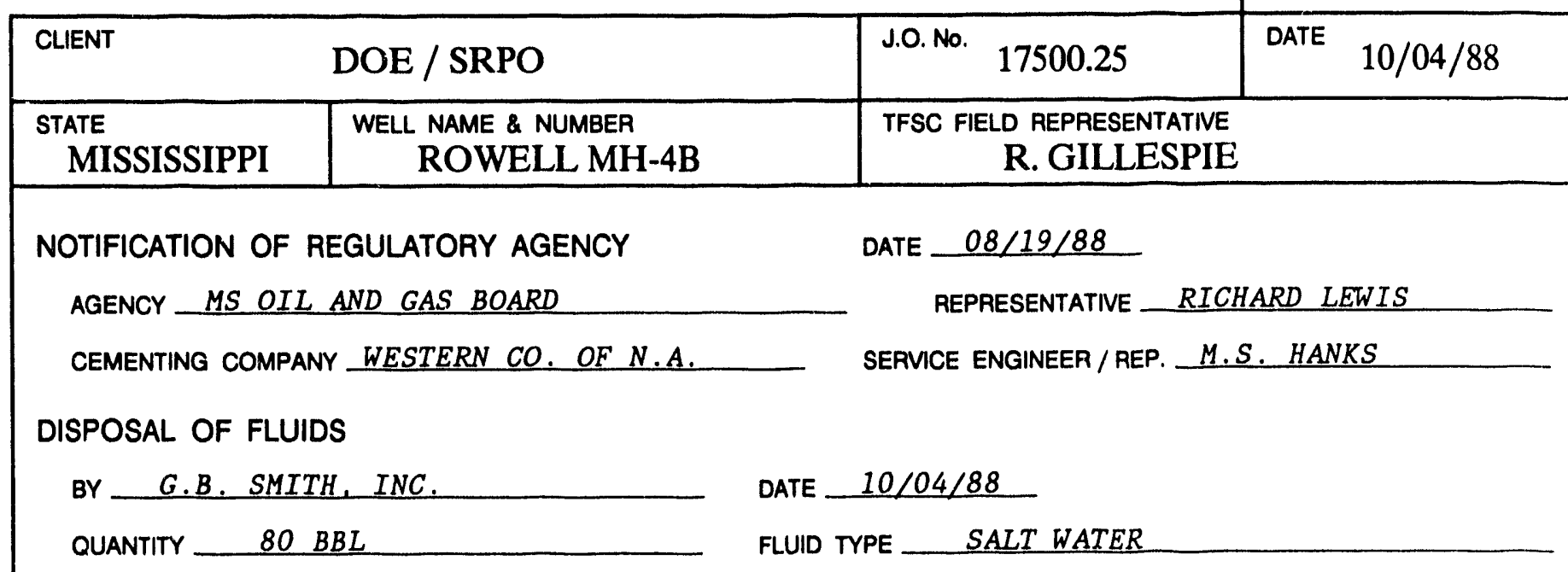

\section{REMARKS}

FLUIDS HAULED TO LICENSED DISPOSAL FACILITY OPERATED BY G.B. SMITH, INC.

ZONE 1

INTERVAL 1971.4 FT TO SURFACE

BOTTOM OF TUBING OR PIPE 1910.5 FT
TYPE PLUG CONTINUOUS

DIAMETER OF CASING/HOLE $4.0,65 / 8, \& 8.0 \mathrm{IN}$ CEMENT/MUD TYPE CLASS A. 88 BENTONITE

SLURRY WEIGHT 13.1 LBS/GAL TOP OF PLUG 4.0 FT

BRIDGE PLUG TYPE

REMARKS

CEMENT PUMPED IN STAGES WITH TUBING SET AT 1910.5, 1446.3,982.3. AND 517 FT. CEMENT CIRCULATED TO SURFACE. CASING CUT OFF AT 4 FT BELOW GROUND LEVEL AND CAPPED. TOP 4.0 FT BACKFILLED WITH SOIL.

\section{ZONE}

INTERVAL

BOTTOM OF TUBING OR PIPE NO. OF SACKS SLURRY WEIGHT BRIDGE PLUG TYPE REMARKS
TYPE PLUG

DIAMETER OF CASING/HOLE IN FT CEMENT/MUD TYPE

YIELD SU. FT/SK.

VOL. PUMPED CU. FT 


\section{PLUGGING REPORT}

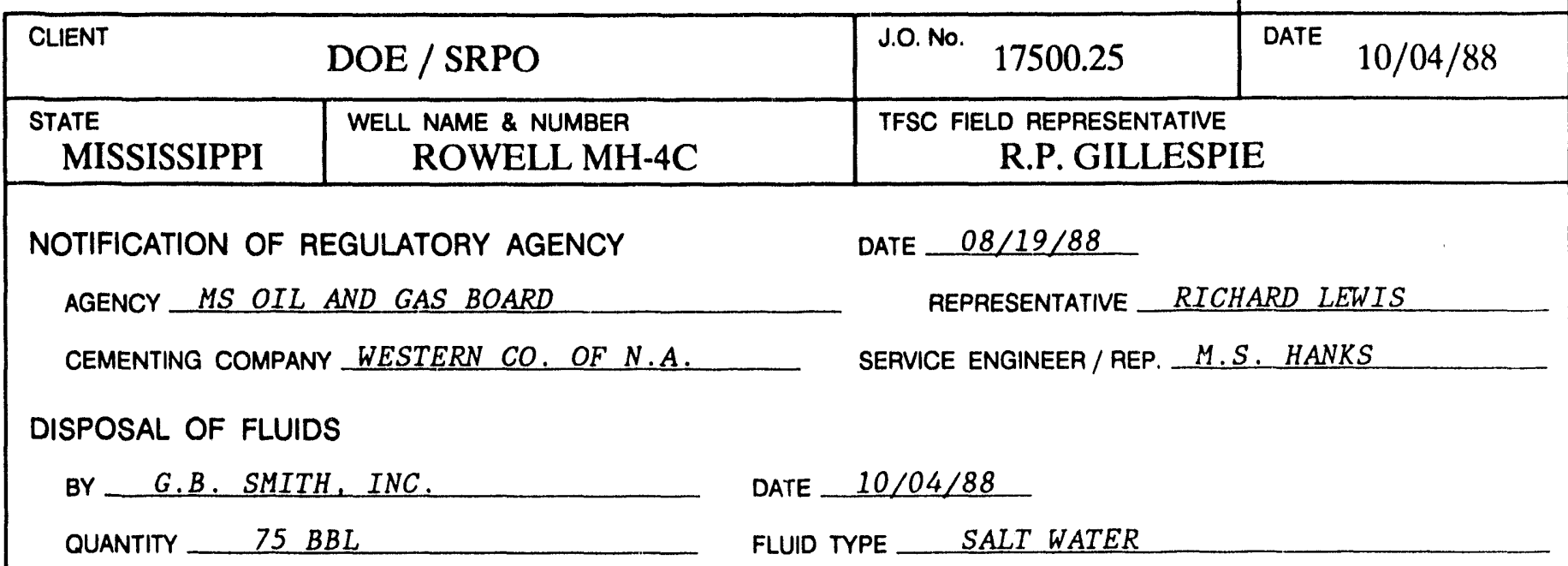

\section{REMARKS}

FLUIDS HAULED TO LICENSED DISPOSAL FACILITY OPERATED BY G.B. SMITH, INC.

ZONE 1

INTERVAL 1722.6 FT TO SURFACE
TYPE PLUG CONTINUOUS

DIAMETER OF CASING/HOLE $\quad 4.0$ AND $6 \quad 5 / 8 \quad$ IN

BOTTOM OF TUBING OR PIPE 1660 FT CEMENT/MUD TYPE CLASS A, $8 \%$ BENTONITE

NO. OF SACKS 167 YIELD 1.92 CU.FT/SK. VOL. PUMPED 320 CU.FT

SLURRY WEIGHT 13.1 LBS/GAL TOP OF PLUG 3.5

BRIDGE PLUG TYPE NA NEPTH_NA

REMARKS

CEMENT PUMPED IN STAGES WITH TUBING SET AT 1660, 1103, AND $545 \mathrm{FT}$. CEMENT CIRCULATED TO SURFACE. CASING CUT OFF AND CAPPED 3.5 FT BELOW SURFACE, TOP 3.5 FT BACKFILLED WITH SOIL.

ZONE

INTERVAL

BOTTOM OF TUBING OR PIPE

NO. OF SACKS

SLURRY WEIGHT

BRIDGE PLUG TYPE

REMARKS
TYPE PLUG

DIAMETER OF CASING/HOLE IN FT CEMENT/MUD TYPE YIELD _ CU.FT/SK. VOL. PUMPED CU. FT LBS/GAL TOP OF PLUG FT DEPTH 
PLUGGING REPORT

\begin{tabular}{|c|c|}
\hline & $\begin{array}{c}\text { DATUM ELEVATION } \\
183.1\end{array}$ \\
\hline J.O. No. 17500.25 & DATE $\quad 10 / 03 / 88$ \\
\hline \multicolumn{2}{|c|}{$\begin{array}{l}\text { TFSC FIELD REPRESENTATIVE } \\
\text { R.P. GILLESPIE }\end{array}$} \\
\hline \multicolumn{2}{|l|}{ DATE $\quad 08 / 19 / 88$} \\
\hline \multicolumn{2}{|c|}{ REPRESENTATIVE RICHARD LEWIS } \\
\hline \multicolumn{2}{|c|}{ SERVICE ENGINEER / REP. M.S. HANKS } \\
\hline $10 / 03 / 88$ & \\
\hline
\end{tabular}

\section{REMARKS}

FLUIDS HAULED TO LICENSED DISPOSAL FACILITY OPERATED BY G.B. SMITH. INC.

ZONE 1

INTERVAL 1115 FT TO SURFACE

BOTTOM OF TUBING OR PIPE 1045 FT

NO. OF SACKS 106 YIELD

SLURRY WEIGHT 13.1 LBS/GAL

BRIDGE PLUG TYPE _ NA

REMARKS

CEMENT PUMPED IN STAGES WITH TUBING SET AT 1045 AND 517.5 FT. CEMENT CIRCULATED TO SURFACE, CASING CUT OFF AND CAPPED 4 FT BELOW SURFACE, TOP 4.0 FT BACKFILLED WITH SOIL.

ZONE

INTERVAL

BOTTOM OF TUBING OR PIPE

NO. OF SACKS

YIELD FT LBS/GAL

SLURRY WEIGHT

BRIDGE PLUG TYPE
TYPE PLUG

DIAMETER OF CASING/HOLE IN

TYPE PLUG CONTINUOUS

DIAMETER OF CASING/HOLE 4.0 AND $65 / 8$ iN CEMENT/MUD TYPE CLASS A, 88 BENTONITE

1.92 CU.FT/SK.

TOP OF PLUG $4.0 \quad \mathrm{FT}$

DEPTH NA CEMENT/MUD TYPE

CU. FT/SK.

VOL. PUMPED CU. FT
DEPTH FT

REMARKS 
PLUGGING REPORT

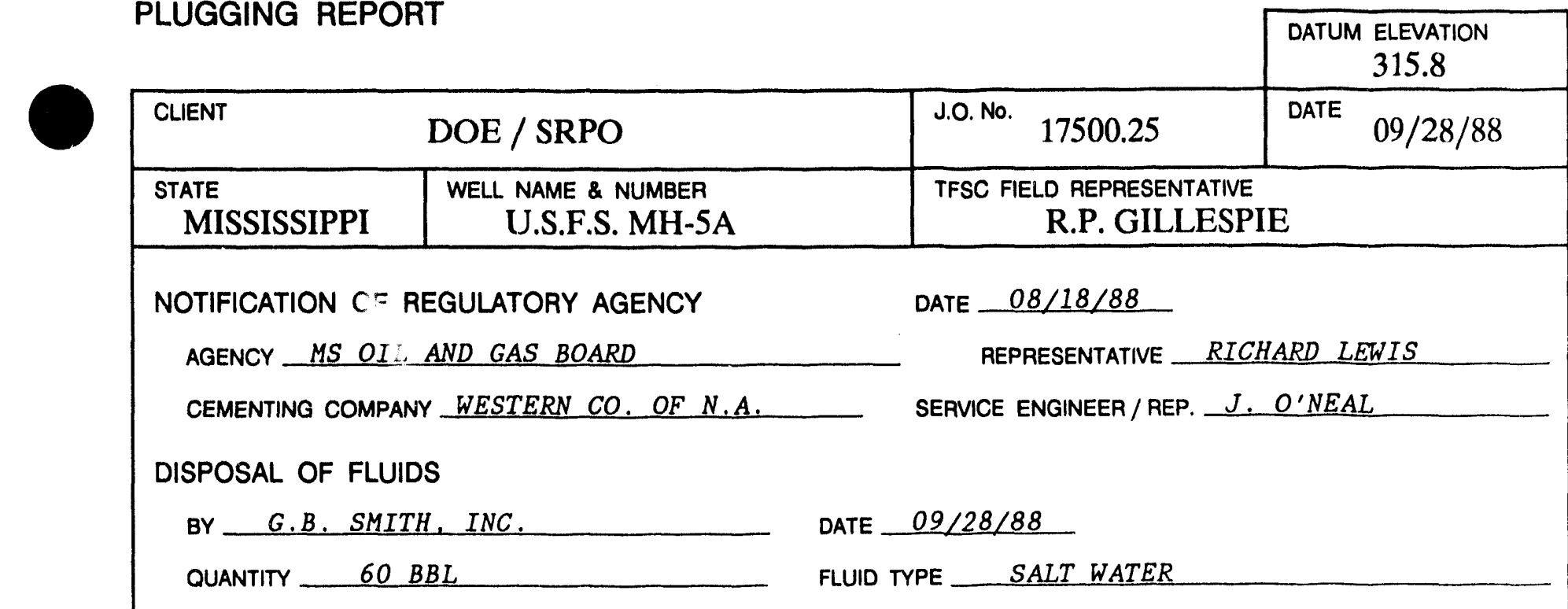

\section{REMARKS}

FLUIDS HAULED TO LICENSED DISPOSAL FACILITY OPERATED BY G.B. SMITH, INC.

ZONE 1

INTERVAL 3589 FT TO SURFACE

BOTTOM OF TUBING OR PIPE $2932.5 \mathrm{FT}$

NO. OF SACKS 305 YIELD

SLURRY WEIGHT 13.1 LBS/GAL

BRIDGE PLUG TYPE _ NA

REMARKS

CEMENT PUMPED IN STAGES WITH TUBING SET AT 2932.5, 2343.5, 1755.5, 1167.5, AND 578.5 FT. CEMENT CIRCULATED TO SURFACE. CASING CUT OFF AND CAPPED 4 FT BELOW GROUND LEVEL, TOP 4 FT BACKFILLED WITH SOIL.

ZONE

INTERVAL

TYPE PLUG

BOTTOM OF TUBING OR PIPE

NO. OF SACKS

YIELD FT

DIAMETER OF CASING/HOLE $\mathbb{N}$

SLURRY WEIGHT LBS/GAL

BRIDGE PLUG TYPE

REMARKS
TYPE PLUG CONTINUOUS

DIAMETER OF CASING/HOLE $4.0,65 / 8, \& 8.0 \mathrm{IN}$ CEMENT/MUD TYPE CLASS A, $8 \%$ BENTONITE

TOP OF PLUG 4.0

DEPTH NA 


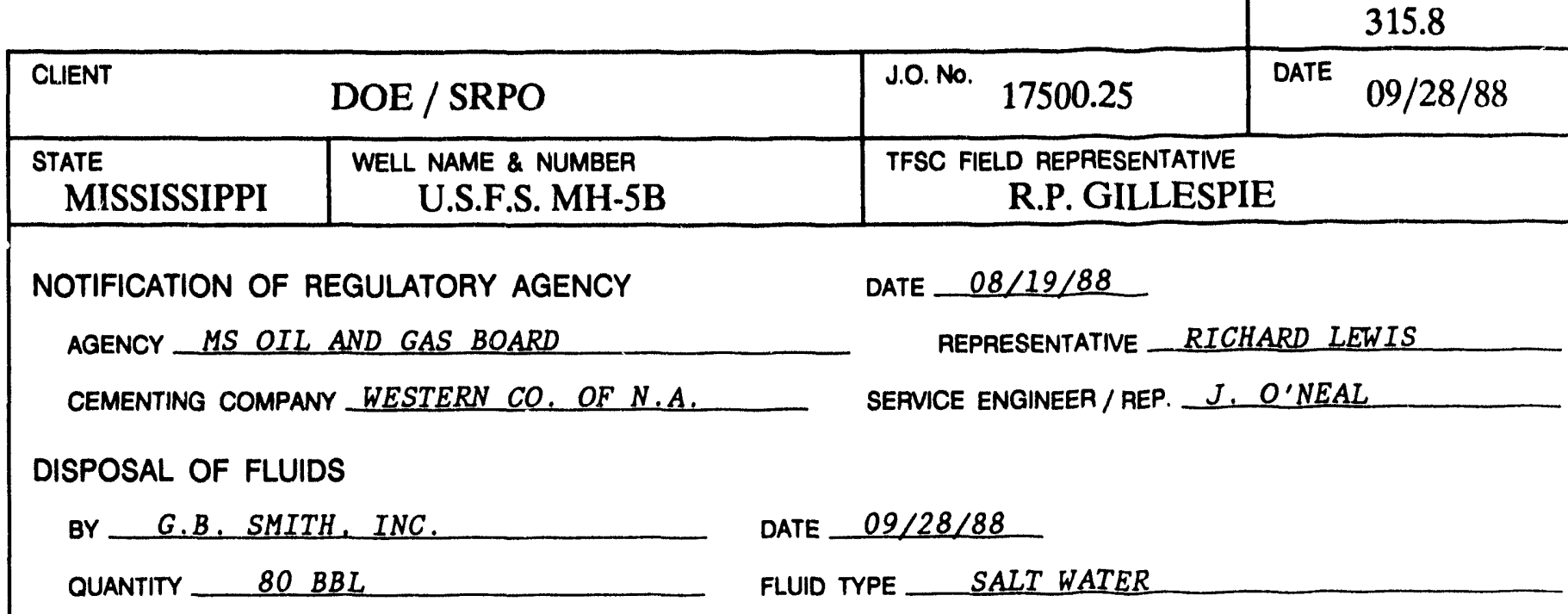

\section{REMARKS}

FLUIDS HAULED TO LICENSED DISPOSAL FACILITY OPERATED BY G.B. SMITH, INC.

ZONE 1

INTERVAL 2526 FT TO SURFACE
TYPE PLUG CONTINUOUS

DIAMETER OF CASING/HOLE $\quad 4.0,65 / 8, \& 8.0 \mathrm{~N}$ BOTTOM OF TUBING OR PIPE 2468 FT CEMENT/MUD TYPE CLASS A, 88 BENTONITE NO. OF SACKS 244 YIELD 1.92 CU.FT/SK. VOL. PUMPED 468 CU.FT SLURRY WEIGHT 13.1 LBS/GAL TOP OF PLUG 4.0 FT BRIDGE PLUG TYPE NA NA DEPTH NA

REMARKS

CEMENT PUMPED IN STAGES WITH TUBING SET AT 2468, 1879, 1292, AND 703 FT. CEMENT CIRCULATED TO SURFACE. CASING CUT OFF AND CAPPED 4 FT BELOW SURFACE. TOP 4.0 FT BACKFILLED WITH SOIL.

ZONE

TYPE PLUG

INTERVAL

DIAMETER OF CASING/HOLE IN

BOTTOM OF TUBING OR PIPE FT CEMENT/MUD TYPE

NO. OF SACKS

YIELD CU. FT/SK.

VOL. PUMPED CU. FT SLURAY WEIGHT LBS/GAL TOP OF PLUG FT BRIDGE PLUG TYPE DEPTH 
PLUGGING REPORT

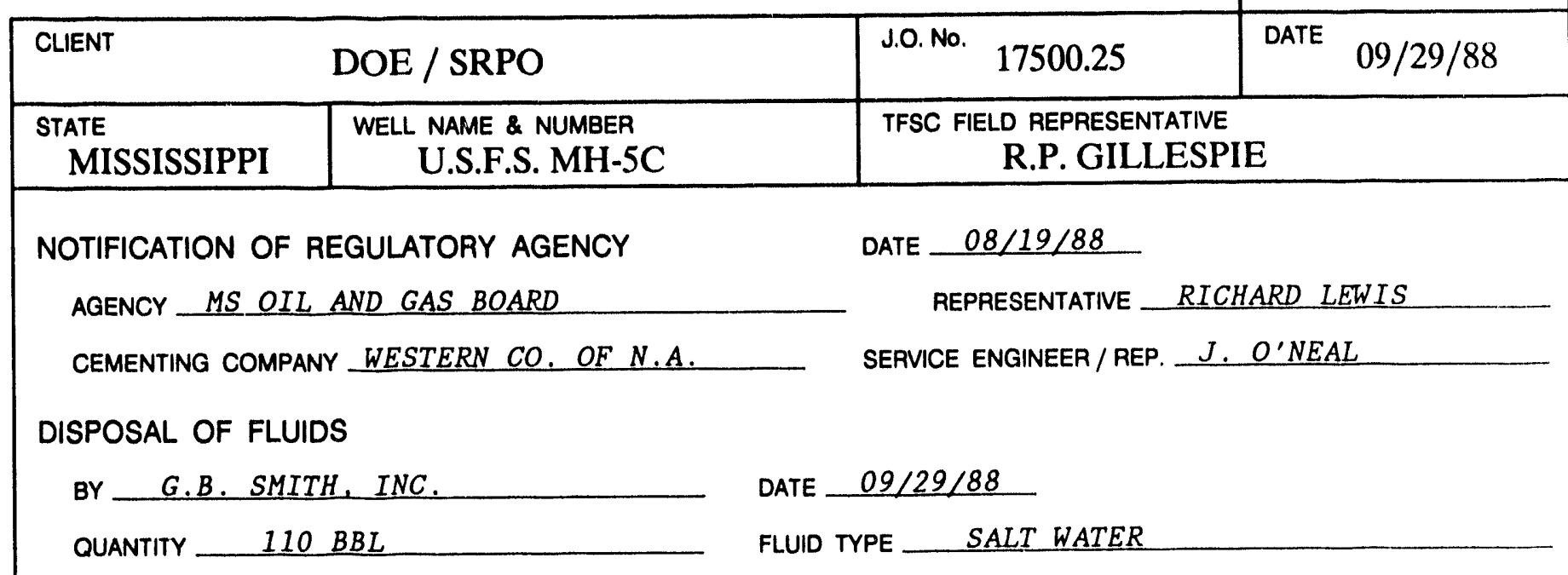

\section{REMARKS}

FLUIDS HAULED TO LICENSED DISPOSAL FACILITY OPERATED BY G.B. SMITH, INC.

ZONE 1

INTERVAL 2287 FT TO SURFACE

BOTTOM OF TUBING OR PIPE 2220

NO. OF SACKS 249 YIELD 1.92 CU. FT/SK.

SLURRY WEIGHT 13.1 LBS/GAL

BRIDGE PLUG TYPE _NA

REMARKS

CEMENT PUMPED IN STAGES WITH TUBING SET AT 2220, 1663, 1106, AND 548 FT. CEMENT CIRCULATED TO SURFACE. CASING CUT OFF AND CAPPED 4.5 FT BELOW GROUND, TOP 4.5 FT BACKFILLED WITH SOIL.

ZONE

INTERVAL

BOTTOM OF TUBING OR PIPE

NO. OF SACKS

SLURRY WEIGHT LBS/GAL

BRIDGE PLUG TYPE REMARKS
TYPE PLUG CONTINUOUS

DIAMETER OF CASING/HOLE $\quad 4.0,65 / 8, \& 8.0 \mathrm{IN}$ CEMENT/MUD TYPE CLASS A, $8 \%$ BENTONITE

TOP OF PLUG 4.5

DEPTH _ N 


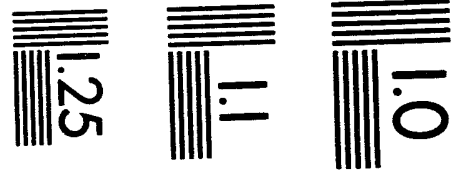

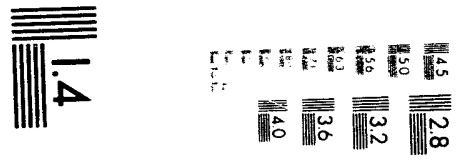

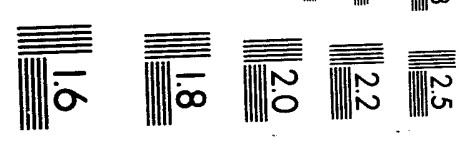



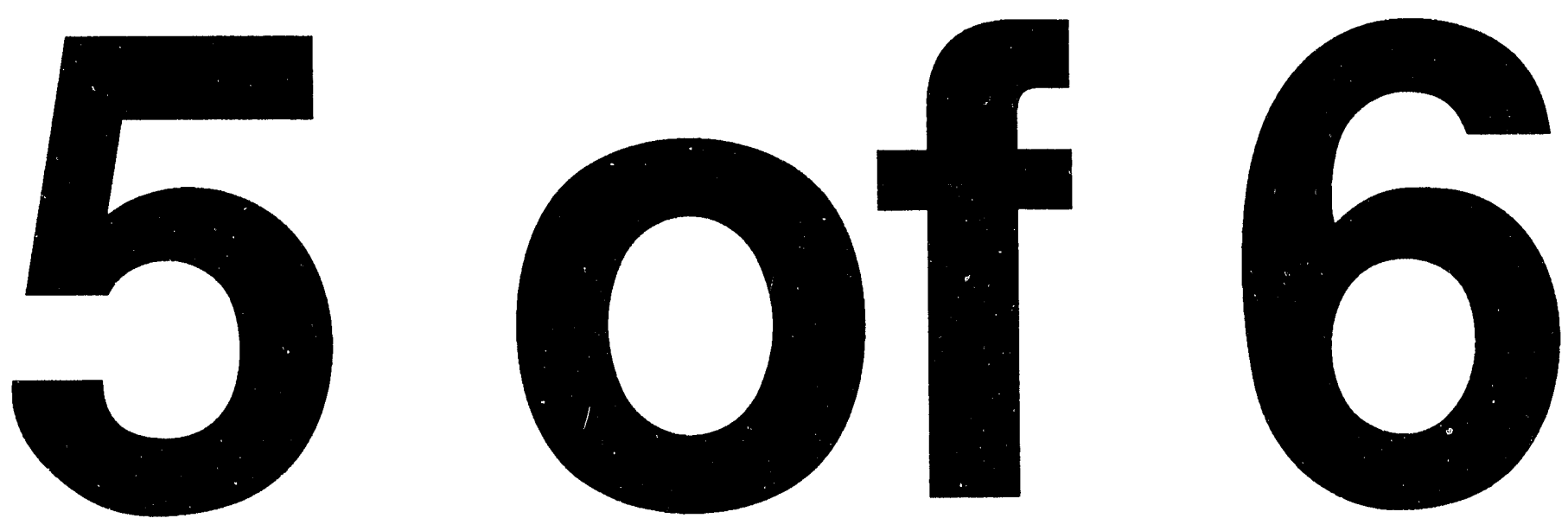
PLUGGING REPORT

\begin{tabular}{|c|c|c|c|}
\hline CLIENT & DOE / SRPO & J.O. No. 17500.25 & $\begin{array}{ll}\text { DATE } & 09 / 15 / 88\end{array}$ \\
\hline $\begin{array}{l}\text { STATE } \\
\text { MISSISSIPPI } \\
\end{array}$ & $\begin{array}{l}\text { WELL NAME \& NUMBER } \\
\text { ANDERSON MH-6A }\end{array}$ & \multicolumn{2}{|c|}{$\begin{array}{l}\text { TFSC FIELD REPRESENTATIVE } \\
\text { R.P. GILLESPIE } \\
\end{array}$} \\
\hline \multicolumn{2}{|c|}{$\begin{array}{l}\text { NOTIFICATION OF REGULATORY AGENCY } \\
\text { AGENCY MS OIL AND GAS BOARD }\end{array}$} & DATE $\quad 08 / 19 / 88$ & ARD IFWIS \\
\hline \multicolumn{2}{|c|}{ CEMENTING COMPANY WESTERN CO. OF N.A. } & \multicolumn{2}{|c|}{ SERVICE ENGINEER / REP. M.S. HANKS } \\
\hline \multicolumn{4}{|c|}{ DISPOSAL OF FLUIDS } \\
\hline \multicolumn{2}{|c|}{ BY G.B.SMITH. INC. } & \multicolumn{2}{|l|}{$09 / 15 / 88$} \\
\hline \multicolumn{2}{|c|}{ QUANTITY 120 BBL } & YPE SALT WATER & \\
\hline
\end{tabular}

REMARKS

FLUIDS HAULED TO LICENSED DISPOSAL FACILITY OPERATED BY G.B. SMITH, INC.

ZONE 1

INTERVAL 3293 FT TO SURFACE
TYPE PLUG CONTINUOUS

DIAMETER OF CASING/HOLE $\quad 4.0,6 \quad 5 / 8, \& 8.0 \mathrm{IN}$

BOTTOM OF TUBING OR PIPE $3242.5 \mathrm{FT}$ CEMENT/MUD TYPE CLASS A, $8 \%$ BENTONITE

NO. OF SACKS 318 YIELD 1.92 CU.FT/SK.

SLURRY WEIGHT 13.1 LBS/GAL

VOL. PUMPED 610 CU. FT

BRIDGE PLUG TYPE _ NA

TOP OF PLUG 3.5 FT

DEPTH

REMARKS

CEMENT PUMPED IN STAGES WITH TUBING SET AT 3242.5, 2715.5, 2189.5, 1653.8, 1127.7. AND 601.7 FT. CEMENT CIRCULATED TO SURFACE, CASING CUT OFF AND CAPPED 3.5 FT BELOW GROUND, TOP 3.5 FT BACKFILLED WITH SOIL.

ZONE

INTERVAL

BOTTOM OF TUBING OR PIPE

NO. OF SACKS

SLURRY WEIGHT

BRIDGE PLUG TYPE REMARKS
TYPE PLUG

DIAMETER OF CASING/HOLE IN FT CEMENT/MUD TYPE

YIELD CU. FT/SK.

VOL. PUMPED CU. FT LBS/GAL

TOP OF PLUG FT DEPTH 


\section{PLUGGING REPORT}

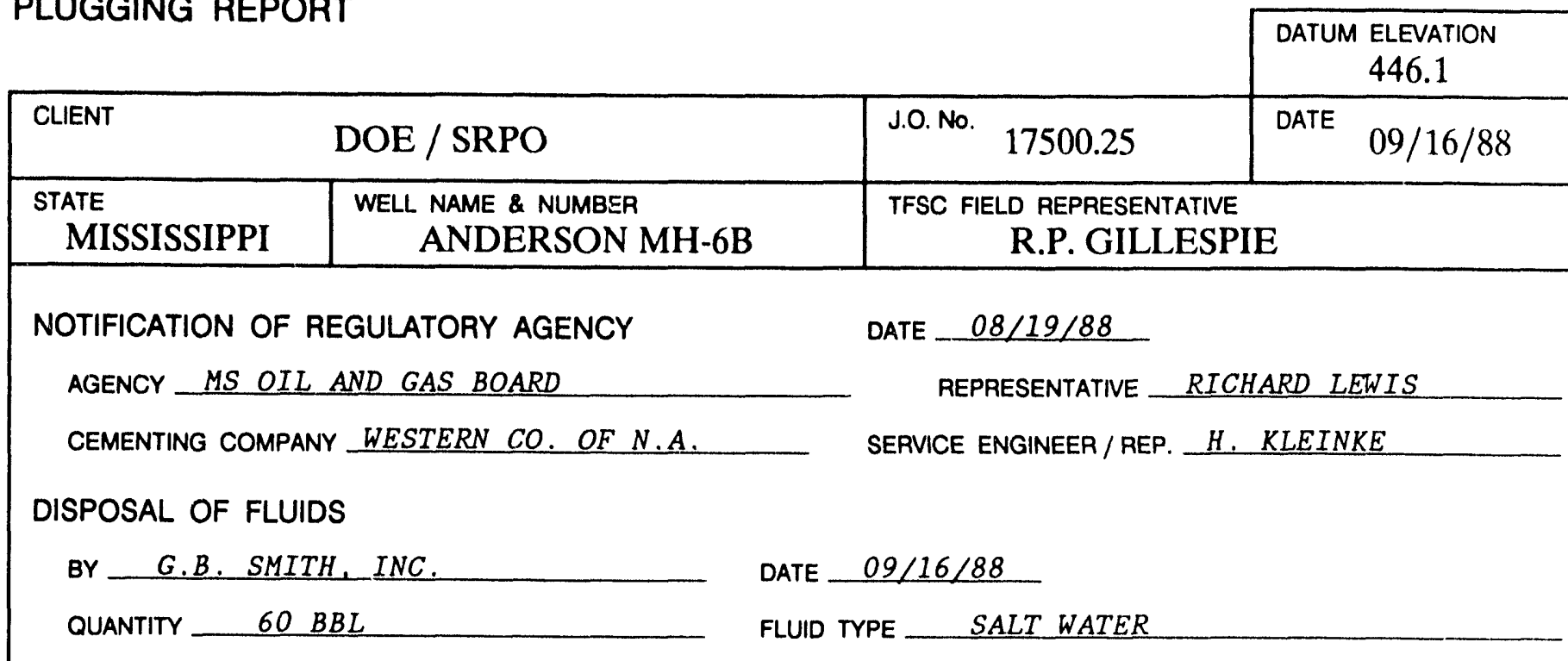

\section{REMARKS}

FLUIDS HAULED TO LICENSED DISPOSAL FACILITY OPERATED BY G.B. SMITH, INC.

ZONE 1

INTERVAL 2762.6 FT TO SURFACE
TYPE PLUG CONTINUOUS

DIAMETER OF CASING/HOLE 4.0 AND $6 \quad 5 / 8$
IN

BOTTOM OF TUBING OR PIPE 2682 FT CEMENT/MUD TYPE CLASS A, 88 BENTONITE
NO. OF SACKS 265
YIELD 1.92
CU. FT/SK.
VOL. PUMPED
509 CU. FT

SLURRY WEIGHT 13.1 LBS/GAL

TOP OF PLUG 4.0

BRIDGE PLUG TYPE _ NA

CEPTH

REMARKS

CEMENT PUMPED IN STAGES WITH TUBING SET AT 2682, 2167.6, 1630, 1103.6, AND 577.5 FT. CEMENT CIRCULATED TO SURFACE, CASING CUT OFF AND CAPPED 4.0 FT BELOW GROUND, TOP 4 FT BACKFILLED WITH SOIL.

ZONE

INTERVAL

BOTTOM OF TUBING OR PIPE NO. OF SACKS

SLURRY WEIGHT

BRIDGE PLUG TYPE REMARKS
TYPE PLUG

DIAMETER OF CASING/HOLE IN FT CEMENT/MUD TYPE

YIELD CU. FT/SK.

VOL. PUMPED CU. FT FT LBS/GAL

TOP OF PLUG DEPTH 
PLUGGING REPORT

\begin{tabular}{|c|c|c|c|}
\hline \multicolumn{2}{|r|}{ DOE / SRPO } & $\begin{array}{ll}\text { J.O. No. } & 17500.25\end{array}$ & DATE $09 / 15 / 88$ \\
\hline $\begin{array}{l}\text { STATE } \\
\text { MISSISSIPPI }\end{array}$ & $\begin{array}{l}\text { WELL NAME \& NUMBER } \\
\text { ANDERSON MH-6C }\end{array}$ & \multicolumn{2}{|c|}{$\begin{array}{c}\text { TFSC FIELD REPRESENTATIVE } \\
\text { R.P. GILLESPIE }\end{array}$} \\
\hline \multicolumn{2}{|c|}{ NOTIFICATION OF REGULATORY AGENCY } & \multicolumn{2}{|l|}{ DATE $08 / 19 / 88$} \\
\hline \multicolumn{2}{|c|}{ CEMENTING COMPANY WESTERN CO. OF N.A. } & \multicolumn{2}{|c|}{ SERVICE ENGINEER/REP. M.S. HANKS } \\
\hline \multicolumn{4}{|c|}{ DISPOSAL OF FLUIDS } \\
\hline \multicolumn{2}{|c|}{$\mathrm{BY}$ G.B. SMITH, INC. } & \multicolumn{2}{|l|}{$09 / 15 / 88$} \\
\hline \multicolumn{2}{|c|}{ QUANTITY $\quad 60 \quad B B L$} & SALT WATER & \\
\hline
\end{tabular}

\section{REMARKS}

FLUIDS HAULED TO LICENSED DISPOSAL FACILITY OPERATED BY G.B. SMITH, INC.

ZONE 1 TYPE PLUG CONTINUOUS

INTERVAL 2422.8 FT TO SURFACE DIAMETER OF CASING/HOLE $4.0,65 / 8, \& 8.0 \mathrm{IN}$ BOTTOM OF TUBING OR PIPE $2311.7 \mathrm{FT}$ CEMENT/MUD TYPE CLASS A, $8 \%$ BENTONITE NO. OF SACKS 248.5 YIELD 1.92 CU.FT/SK. VOL. PUMPED 477 CU.FT SLURRY WEIGHT 13.1 LBS/GAL TOP OF PLUG 4.5 FT BRIDGE PLUG TYPE NA NA DEPTH_NA REMARKS

CEMENT PUMPED IN STAGES WITH TUBING SET AT 2311.7, 1723.2, 1134.6, AND 546.4 FT. CEMENT CIRCULATED TO SURFACE, CASING CUT OFF AND CAPPED 4.5 FT BELOW GROUND, AND TOP 4.5 FT BACKFILLED WITH SOIL.

ZONE

INTERVAL

BOTTOM OF TUBING OR PIPE

NO. OF SACKS

SLURRY WEIGHT

BRIDGE PLUG TYPE

REMARKS
TYPE PLUG

DIAMETER OF CASING/HOLE IN CEMENT/MUD TYPE

YIELD CU. FT/SK.

VOL. PUMPED CU. FT FT LBS/GAL

TOP OF PLUG DEPTH 
PLUGGING REPORT

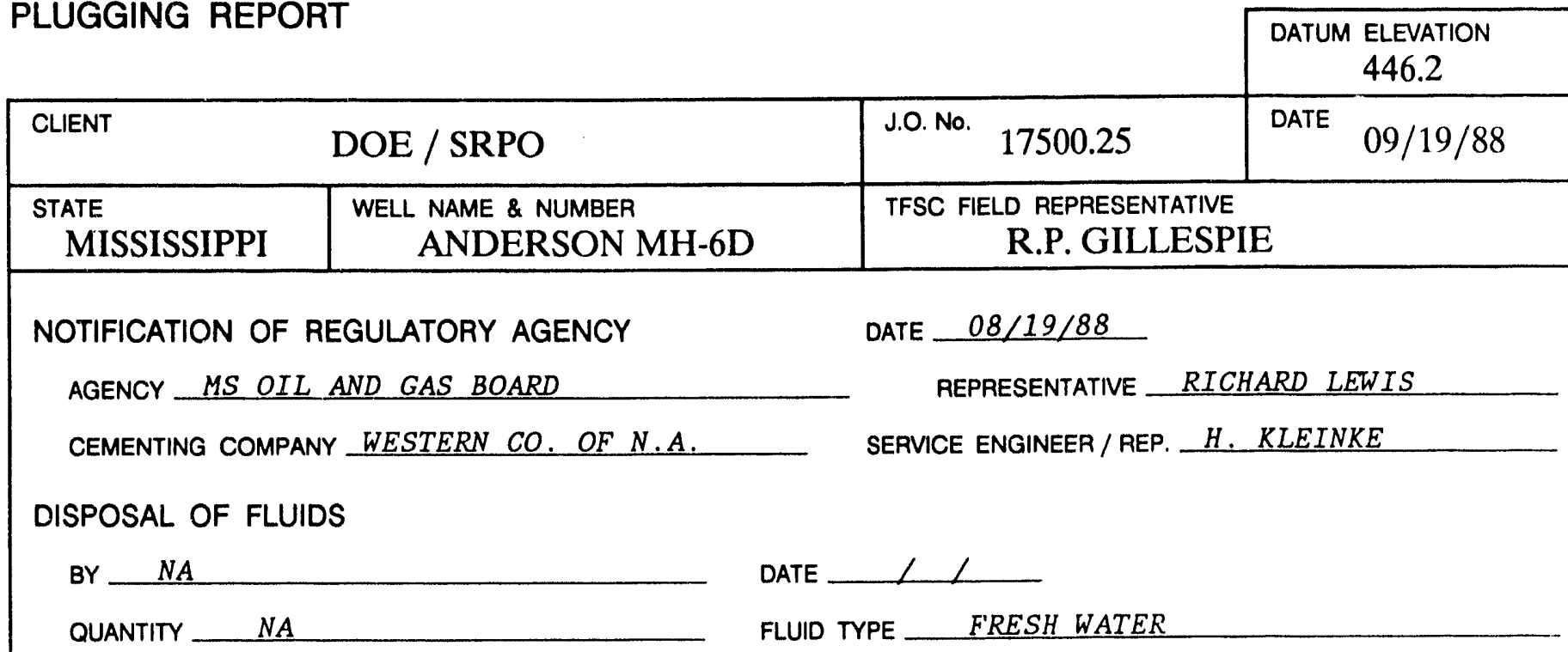

REMARKS

ZONE 1

INTERVAL 1400 FT TO SURFACE

BOTTOM OF TUBING OR PIPE $1288.5 \mathrm{FT}$

NO. OF SACKS $134 \quad$ YIELD 1.92 CU. FT/SK.

SLURRY WEIGHT 13.1 LBS/GAL

BRIDGE PLUG TYPE _ NA

REMARKS

CEMENT PUMPED IN STAGES WITH TUBING SET AT 1288.5 AND 638.3 FT. CEMENT CIRCULATED TO SURFACE, CASING CUT OFF AND CAPPED 4.0 FT BELOW GROUND, TOP 4 FT BACKFILLED WITH SOIL.

ZONE

INTERVAL

TYPE PLUG

DIAMETER OF CASING/HOLE IN

BOTTOM OF TUBING OR PIPE

NO. OF SACKS

YIELD FT CEMENT/MUD TYPE

SLURRY WEIGHT LBS/GAL CU. FT/SK.

TOP OF PLUG

VOL. PUMPED CU. FT

BRIDGE PLUG TYPE DEPTH

REMARKS 
PLUGGING REPORT

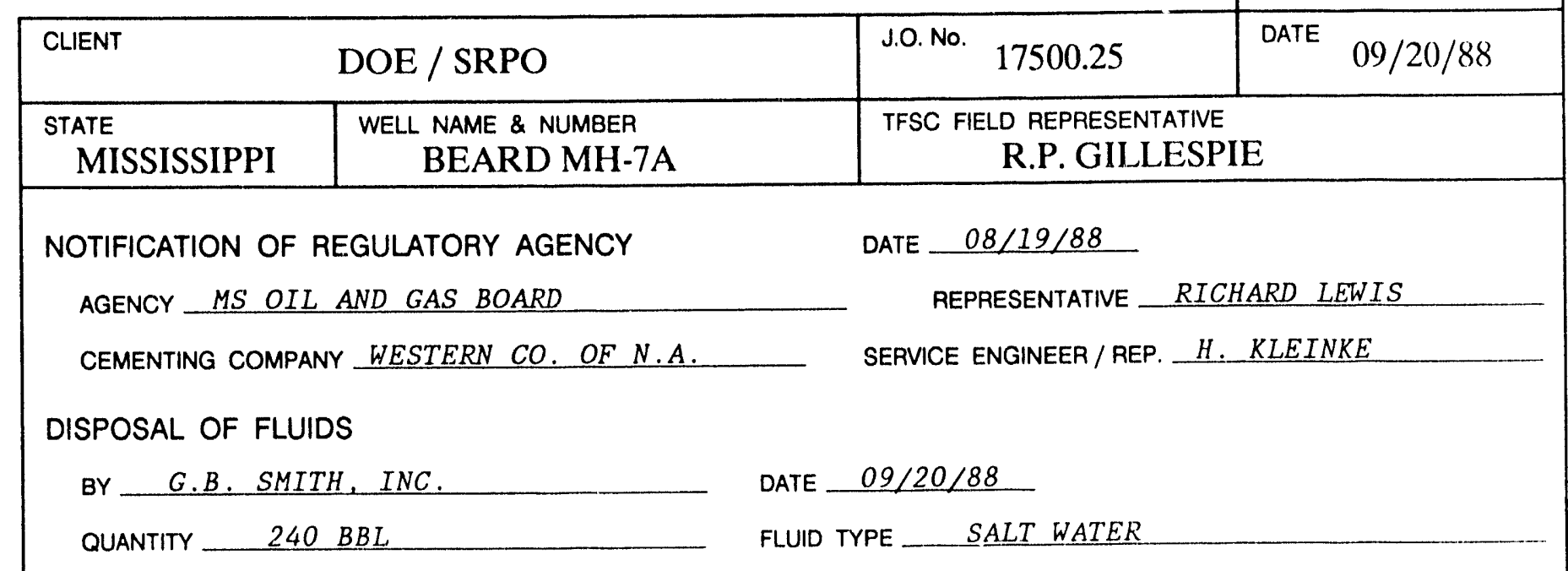

\section{REMARKS}

FLUIDS HAULED TO LICENSED DISPOSAL FACILITY OPERATED BY G.B. SMITH, INC.

ZONE 1

INTERVAL 2609 FT TO SURFACE

BOTTOM OF TUBING OR PIPE 2589 FT

TYPE PLUG CONTINUOUS

NO. OF SACKS $270 \quad$ YIELD 1.92 CU. FT/SK.

SLURRY WEIGHT 13.1 LBS/GAL

DIAMETER OF CASING/HOLE 4.0 AND $6 \quad 5 / 8$ IN

BRIDGE PLUG TYPE WESTERN, CAST, $65 / 8$ DEPTH 2609 FT

REMARKS

CEMENT PUMPED IN STAGES WITH TUBING SET AT 2589, 2062, 1535, 1010, AND 484 FT. CEMENT CIRCULATED TO SURFACE, CASING CUT OFF AND CAPPED 4.5 FT BELOW GROUND, AND TOP 4.5 FT BACKFILLED WITH SOIL.

ZONE

INTERVAL

BOTTOM OF TUBING OR PIPE

NO. OF SACKS

SLURRY WEIGHT

BRIDGE PLUG TYPE

REMARKS
TYPE PLUG

DIAMETER OF CASING/HOLE IN CEMENT/MUD TYPE

YIELD CU. FT/SK.

VOL. PUMPED CU. FT LBS/GAL

TOP OF PLUG FT 
PLUGGING REPORT

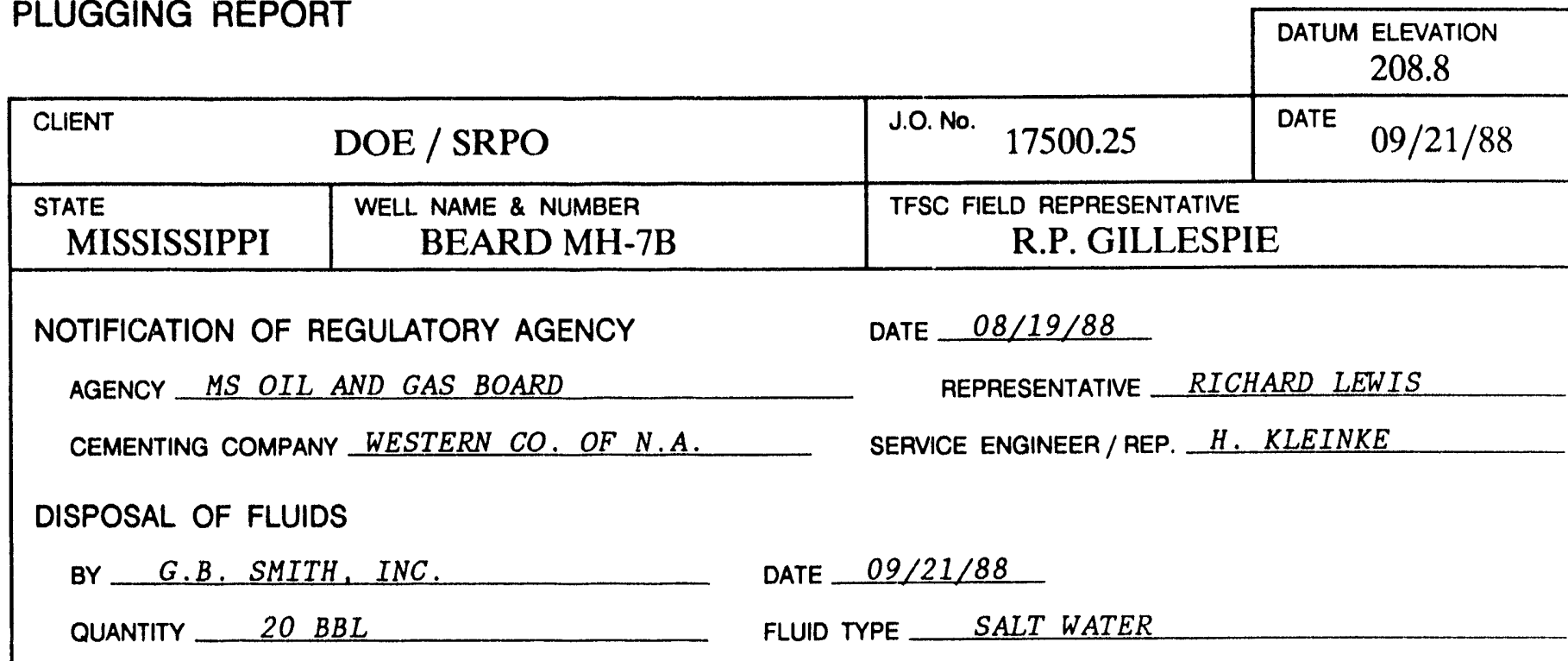

\section{REMARKS}

FLUIDS HAULED TO LICENSED DISPOSAL FACILITY OPERATED BY G.B. SMITH. INC.

ZONE 1

INTERVAL 1976 FT TO SURFACE

BOTTOM OF TUBING OR PIPE 1909
TYPE PLUG CONTINUOUS

DIAMETER OF CASING/HOLE $4.0,65 / 8, \& 8.0 \mathrm{IN}$ CEMENT/MUD TYPE CLASS A, 88 BENTONITE
NO. OF SACKS 192 YIELD 1.92 CU.FT/SK.

SLURAY WEIGHT 13.1 LBS/GAL

BRIDGE PLUG TYPE N.A

\section{REMARKS}

CEMENT PUMPED IN STAGES WITH TUBING SET AT 1909, 1263, AND 671 FT. CEMENT CIRCULATED TO SURFACE, CASING CUT OFF AND CAPPED 4.5 FT BELOW GROUND, TOP 4.5 FT BACKFILLED WITH SOIL.

ZONE

INTERVAL

BOTTOM OF TUBING OR PIPE

NO. OF SACKS

YIELD FT

TOP OF PLUG 4.5 FT

DEPTH
TYPE PLUG

DIAMETER OF CASING/HOLE IN CEMENT/MUD TYPE CU. FT/SK.

VOL. PUMPED CU. FT
SLURRY WEIGHT

BRIDGE PLUG TYPE LBS/GAL FT
TOP OF PLUG REMARKS 
PLUGGING REPORT

\begin{tabular}{|c|c|}
\hline & $\begin{array}{c}\text { DATUM ELEVATION } \\
210.1\end{array}$ \\
\hline J.O. No. $\quad 17500.25$ & DATE $\quad 09 / 21 / 88$ \\
\hline
\end{tabular}

\begin{tabular}{|l|r|}
\hline $\begin{array}{l}\text { STATE } \\
\text { MISSISSIPPI }\end{array}$ & $\begin{array}{r}\text { WELL NAME \& NUMBER } \\
\text { BEARD MH-7C }\end{array}$ \\
\hline
\end{tabular}

\section{NOTIFICATION OF REGULATORY AGENCY}

AGENCY MS OIL AND GAS BOARD

TFSC FIELD REPRESENTATIVE

R.P. GILLESPIE

CEMENTING COMPANY WESTERN CO. OF N.A.

DATE $08 / 19 / 88$

REPRESENTATIVE RICHARD LEWIS

SERVICE ENGINEER / REP. H. KLEINKE

DISPOSAL OF FL.UIDS

BY G.B. SMITH, INC.

DATE $09 / 21 / 88$

QUANTITY $145 \mathrm{BBL}$

FLUID TYPE SALT WATER

\section{REMARKS}

FLUIDS HAULED TO LICENSED DISPOSAL FACILITY OPERATED BY G.B. SMITH, INC.

ZONE 1

INTERVAL 1661.5 FT TO SURFACE

BOTTOM OF TUBING OR PIPE 1599 FT

NO. OF SACKS 165 YIELD 1.92 CU.FT/SK.

SLURRY WEIGHT 13.1 LBS/GAL

BRIDGE PLUG TYPE _ NA

REMARKS

CEMENT PUMPED IN STAGES WITH TUBING SET AT 1599, 1072, AND 546 FT. CEMENT CIRCULATED TO SURFACE, CASING CUT OFF AND CAPPED 4.0 FT BELOW GROUND, TOP 4.0 FT BACKFILLED WITH SOIL.

ZONE

INTERVAL

BOTTOM OF TUBING OR PIPE

NO. OF SACKS

YIELD FT

SLURRY WEIGHT LBS/GAL

BRIDGE PLUG TYPE

TYPE PLUG

DIAMETER OF CASING/HOLE IN CEMENT/MUD TYPE

CU. FT/SK.

VOL. PUMPED CU. FT

\section{REMARKS}


PLUGGING REPORT

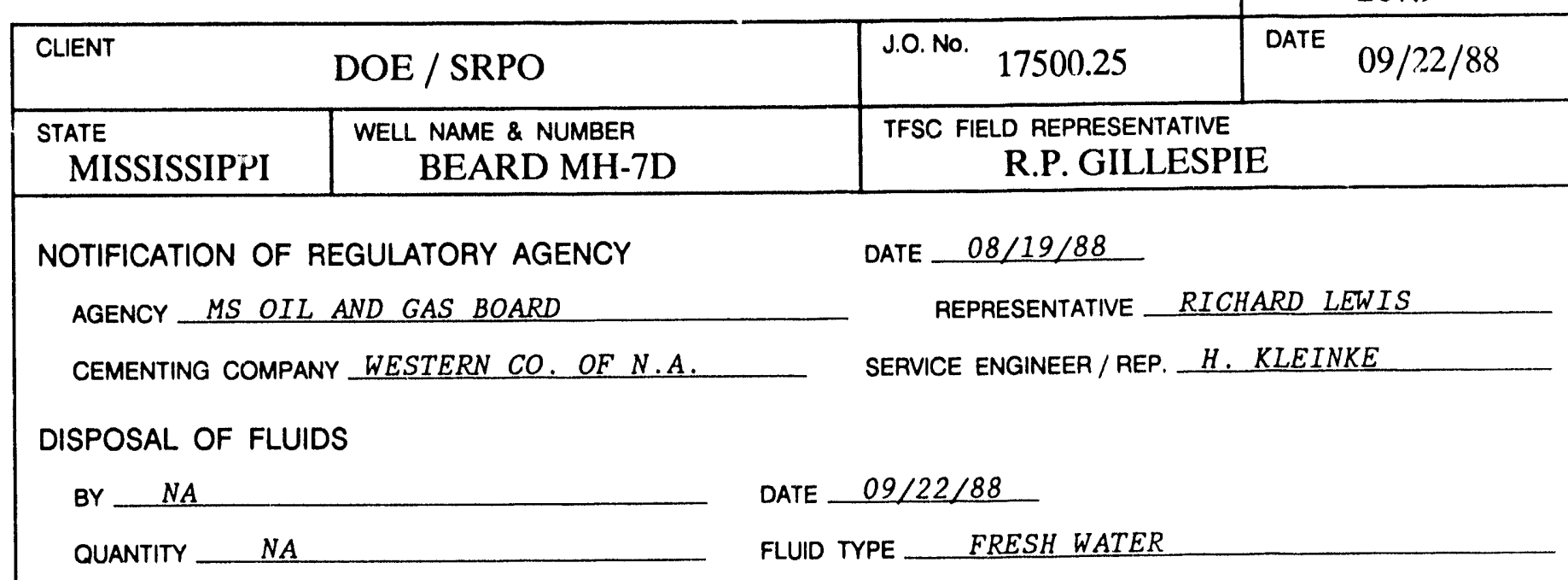

\section{REMARKS}

ZONE 1

INTERVAL 805.5 FT TO SURFACE

BOTTOM OF TUBING OR PIPE 732

NO. OF SACKS 79

SLURRY WEIGHT 13.1

BRIDGE PLUG TYPE

REMARKS

CEMENT CIRCULATED TO SURFACE, CASING CUT OFF AND CAPPED 4.5 FT BELOW GROUND SURFACE, TOP 4.5 FT BACKFILLED WITH SOIL.

ZONE

INTERVAL YIELD $\quad 1.92$ LBS/GAL CU. FT

TYPE PLUG CONTINUOUS

DIAMETER OF CASING/HOLE \begin{tabular}{l}
4.0 AND $6 \quad 5 / 8$ \\
\hline
\end{tabular} IN CEMENT/MUD TYPE CLASS A, $8 \%$ BENTONITE

VOL. PUMPED 152 FT

DEPTH

BOTTOM OF TUBING OR PIPE FT CEMENT/MUD TYPE

NO. OF SACKS

YIELD CU. FT/SK.

VOL. PUMPED CU. FT

SLURRY WEIGHT LBS/GAL

TOP OF PLUG FT

BRIDGE PLUG TYPE

DEPTH 
PLUGGING REPORT

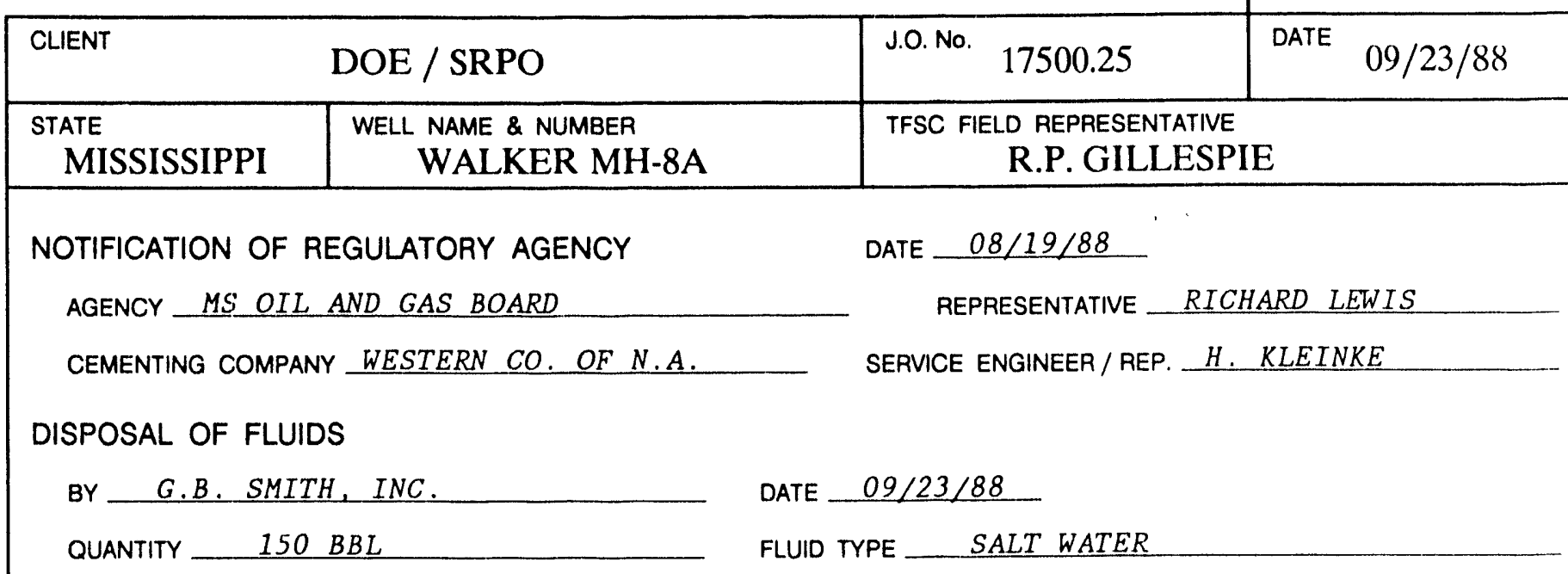

\section{REMARKS}

FLUIDS HAULED TO LICENSED DISPOSAL FACILITY OPERATED BY G.B. SMITH, INC.

ZONE 1

INTERVAL 3194 FT TO SURFACE
TYPE PLUG CONTINUOUS

DIAMETER OF CASING/HOLE $4.0,65 / 8, \& 8.0 \mathrm{IN}$ BOTTOM OF TUBING OR PIPE 3119 FT CEMENT/MUD TYPE CLASS A, 88 BENTONITE NO. OF SACKS 323 YIELD 1.92 CU.FT/SK. VOL. PUMPED 620 CU. FT SLURAY WEIGHT 13.1 LBS/GAL TOP OF PLUG 4.5 FT BRIDGE PLUG TYPE _NA NEPTH NA N N N REMARKS

CEMENT PUMPED IN STAGES WITH TUBING SET AT 3119,2591,2065, 1539, 1011, AND 485 FT. CEMENT CIRCULATED TO SURFACE, CASING CUT OFF AND CAPPED 4.5 FT BELOW GROUND SURFACE,_TOP 4.5 FT BACKFILLED WITH SOIL.

ZONE

INTERVAL

BOTTOM OF TUBING OR PIPE

NO. OF SACKS

SLURRY WEIGHT

BRIDGE PLUG TYPE REMARKS
TYPE PLUG

DIAMETER OF CASING/HOLE IN FT CEMENT/MUD TYPE

YIELD CU. FT/SK. VOL. PUMPED CU. FT TOP OF PLUG FT DEPTH 
PLUGGING REPORT

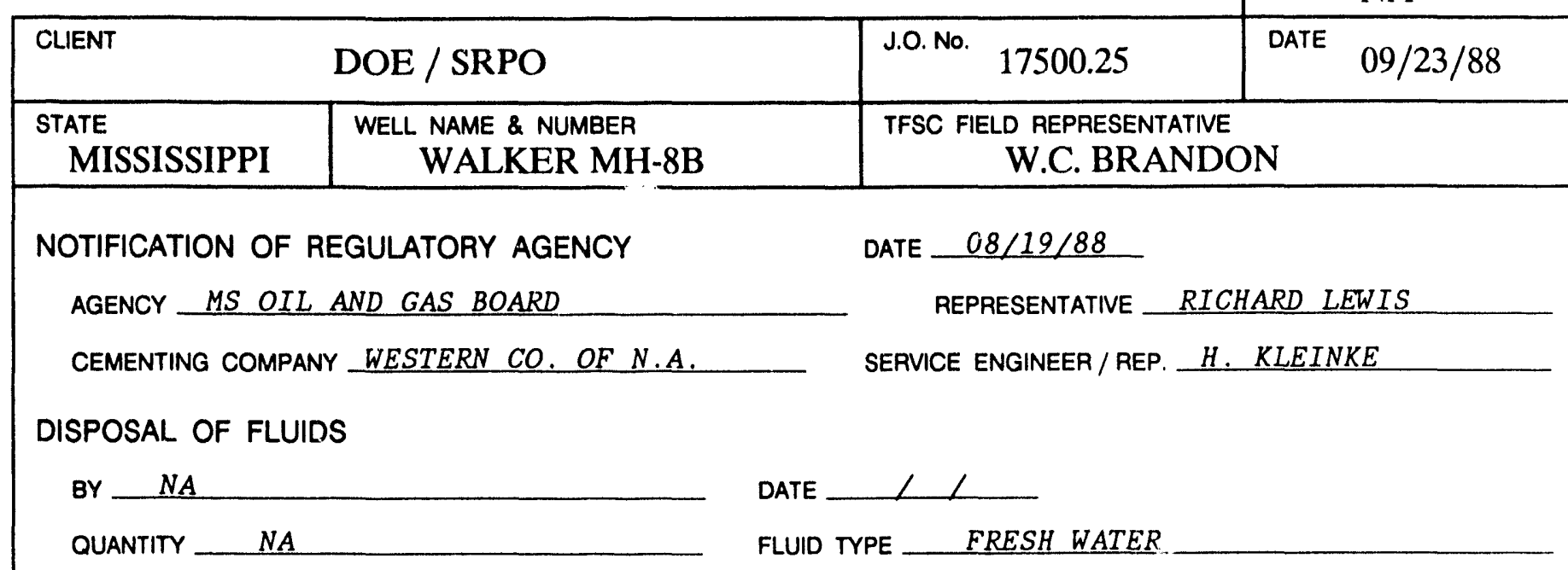

REMARKS

ZONE 1

TYPE PLUG CONTINUOUS

INTERVAL 60 FT TO SURFACE

DIAMETER OF CASING/HOLE 16.0 $\mathbb{N}$

BOTTOM OF TUBING OR PIPE 3.0 FT FT CEMENT/MUD TYPE CLASS A, 88 BENTONITE

NO. OF SACKS 32 YIELD 1.92 CU.FT/SK. VOL. PUMPED 61 CU. FT

SLURAY WEIGHT 13.1 LBS/GAL TOP OF PLUG 0 FT FT

BRIDGE PLUG TYPE _ NA DEPTH_NA

REMARKS

CEMENT TREMIED FROM SURFACE. CASING CUT OFF AND CAPPED 3.0 FT BELOW SURFACE, AND TOP

3.0 FT BACKFILLED WITH SOIL.

ZONE

INTERVAL

BOTTOM OF TUBING OR PIPE

NO. OF SACKS LBS/GAL

SLURRY WEIGHT

BRIDGE PLUG TYPE

REMARKS
TYPE PLUG

DIAMETER OF CASING/HOLE IN FT CEMENT/MUD TYPE CU. FT/SK. VOL. PUMPED CU. FT TOP OF PLUG DEPTH 
PLUGGING REPORT

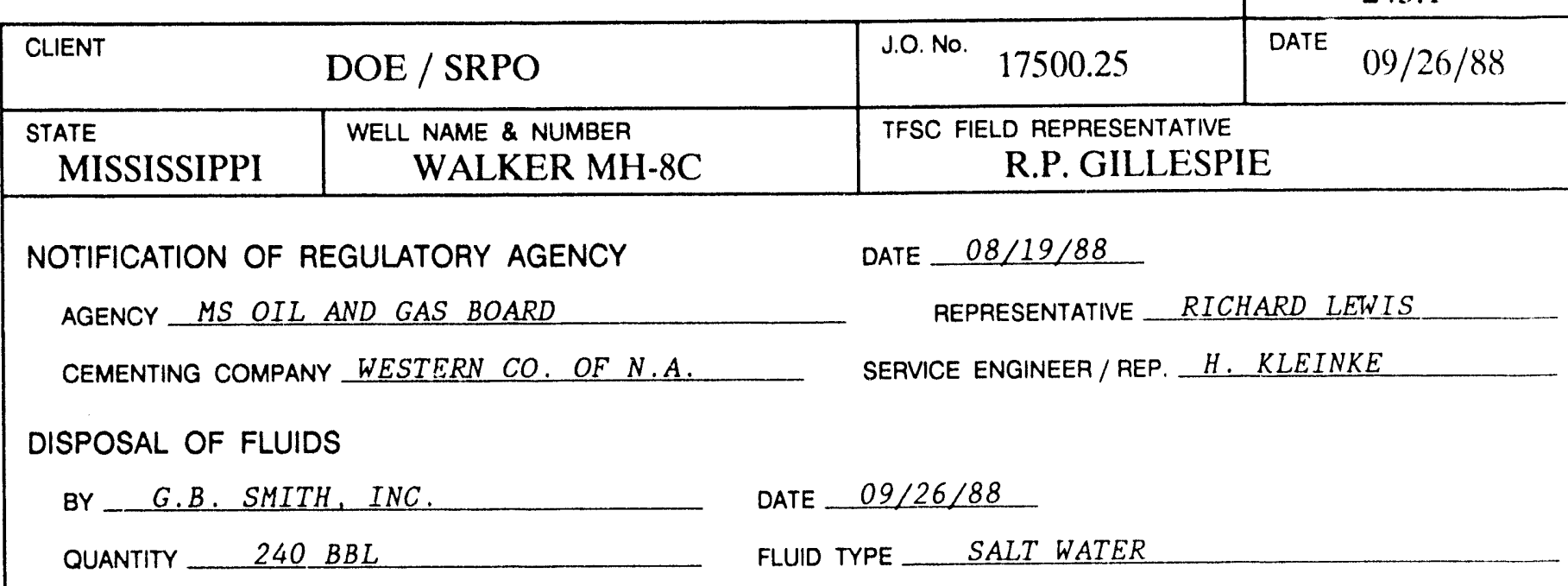

\section{REMARKS}

FLUIDS HAULED TO LICENSED DISPOSAL FACILITY OPERATED BY G.B. SMITH, INC.

ZONE 1

INTERVAL 2374 FT TO SURFACE

BOTTOM OF TUBING OR PIPE 2372 FT

NO. OF SACKS 247 YIELD 1.92

SLURRY WEIGHT 13.1 LBS/GAL

BRIDGE PLUG TYPE WESTERN, CAST, $65 / 8$

REMARKS

CEMENT PUMPED IN STAGES WITH TUBING SET AT 2372, 1784, 1196, AND 609 FT. CEMENT WAS CIRCULATED TO SURFACE, CASING CUT OFF AND CAPPED 4 FT BELOW GROUND, TOP 4.0 FT BACK.

FILLED WITH SOIL.

ZONE

INTERVAL

TYPE PLUG

BOTTOM OF TUBING OR PIPE

NO. OF SACKS

SLURRY WEIGHT

BRIDGE PLUG TYPE

REMARKS
TYPE PLUG CONTINUOUS

DIAMETER OF CASING/HOLE $\quad 65 / 8, \& 8.0 \quad$ IN CEMENT/MUD TYPE CLASS A. 88 BENTONITE

CU. FT/SK.

VOL. PUMPED 474 CU. FT TOP OF PLUG 4.0 DEPTH 2374 FT FT CEMENT/MUD TYPE
YIELD LBS/GAL CU. FT/SK.

VOL. PUMPED CU. FT FT

DEPTH 


\begin{tabular}{|c|c|c|c|c|}
\hline \multicolumn{3}{|c|}{ DOE / SRPO } & J.O. No. 17500.25 & DATE $09 / 23 / 88$ \\
\hline $\begin{array}{l}\text { STATE } \\
\text { MISSISSIPPI }\end{array}$ & $\begin{array}{l}\text { WELL NAME \& NUMBER } \\
\text { WALKER MH-8D }\end{array}$ & & \multicolumn{2}{|c|}{$\begin{array}{l}\text { TFSC FIELD REPRESENTATIVE } \\
\text { W.C. BRANDON }\end{array}$} \\
\hline \multirow{2}{*}{\multicolumn{3}{|c|}{$\begin{array}{l}\text { NOTIFICATION OF REGULATORY AGENCY } \\
\text { AGENCY MS OIL AND GAS BOARD }\end{array}$}} & DATE $08 / 19 / 88$ & \\
\hline & & & \multicolumn{2}{|c|}{ REPRESENTATIVE RICHARD LEWIS } \\
\hline \multicolumn{3}{|c|}{ CEMENTING COMPANY WESTERN CO. OF N.A. } & \multicolumn{2}{|c|}{ SERVICE ENGINEER / REP. H. KLEINKE } \\
\hline \multicolumn{5}{|c|}{ DISPOSAL OF FLUIDS } \\
\hline \multicolumn{2}{|l|}{ BY } & DATE & 11 & \\
\hline QUANTITY __ NA & - & FLUID & TYPE _ FRESH WATER & \\
\hline
\end{tabular}

REMARKS

ZONE 1

TYPE PLUG CONTINUOUS

INTERVAL 12 FT TO SURFACE

DIAMETER OF CASING/HOLE 16.0 IN

BOTTOM OF TUBING OR PIPE 3.0 FT CEMENT/MUD TYPE CLASS A, $8 \%$ BENTONITE

NO. OF SACKS 6 YIELD 1.92 CU. FT/SK. VOL. PUMPED 11.5 CU. FT

SLURRY WEIGHT 13.1 LBS/GAL TOP OF PLUG O FT FT

BRIDGE PLUG TYPE _ NA DEPTH N N N

REMARKS

CEMENT TREMIED FROM SURFACE. CASING CUT OFF AND CAPPED 3.0 FT BELOW GROUND, TOP 3.0

FT BACKFILLED WITH SOIL.

ZONE

INTERVAL

BOTTOM OF TUBING OR PIPE

NO. OF SACKS

SLURRY WEIGHT LBS/GAL

BRIDGE PLUG TYPE

REMARKS
TYPE PLUG

DIAMETER OF CASING/HOLE IN FT CEMENT/MUD TYPE

YIELD CU. FT/SK.

VOL. PUMPED CU. FT
TOP OF PLUG FT 
PLUGGING REPORT

\begin{tabular}{|c|c|c|c|c|}
\hline \multicolumn{4}{|c|}{ PLUGGING REPORT } & $\begin{array}{c}\text { DATUM ELEVATION } \\
256.0 \\
\end{array}$ \\
\hline CLIENT & DOE / SRPO & & J.O. No. 17500.25 & DATE $10 / 17 / 88$ \\
\hline $\begin{array}{l}\text { STATE } \\
\text { MISSISSIPPI }\end{array}$ & $\begin{array}{l}\text { WELL NAME \& NUMBER } \\
\text { L.R.F.P. MRIG-9 }\end{array}$ & & \multicolumn{2}{|c|}{$\begin{array}{l}\text { TFSC FIELD REPRESENTATIVE } \\
\text { R.P. GILLESPIE } \\
\end{array}$} \\
\hline \multicolumn{3}{|c|}{$\begin{array}{l}\text { NOTIFICATION OF REGULATORY AGENCY } \\
\text { AGENCY MS OIL AND GAS BOARD }\end{array}$} & \multicolumn{2}{|c|}{ REPRESENTATIVE RICHARD LEWIS } \\
\hline \multicolumn{3}{|c|}{ CEMENTING COMPANY WESTERN CO. OF N.A. } & \multicolumn{2}{|c|}{ SERVICE ENGINEER / REP. L. WILLIAMS } \\
\hline \multicolumn{2}{|c|}{ BY G.B. SMITH. INC. } & DATE & $10 / 17 / 88$ & \\
\hline QUANTITY & & FLUID & YPE SALT WATER & \\
\hline
\end{tabular}

\section{REMARKS \\ FLUIDS HAULED TO LICENSED DISPOSAL FACILITY OPERATED BY G.B. SMITH, INC.}

ZONE 1

INTERVAL 525.6 FT TO SURFACE

BOTTOM OF TUBING OR PIPE 518

NO. OF SACKS $114 \quad$ YIELD $\quad 1.92$

SLURRY WEIGHT 13.1 LBS/GAL

BRIDGE PLUG TYPE WESTERN, CAST, $85 / 8$

TYPE PLUG CONTINUOUS

DIAMETER OF CASING/HOLE $713 / 16 \& 8 \quad 5 / 8$ IN CEMENT/MUD TYPE CLASS A, 88 BENTONITE

\section{REMARKS}

CEMENT CIRCULATED TO SURFACE, CASING CUT OFF AND CAPPED 4 FT BELOW GROUND, TOP 4.0 FT BACKFILLED WITH SOIL.

ZONE

INTERVAL

BOTTOM OF TUBING OR PIPE

NO. OF SACKS

SLURRY WEIGHT

BRIDGE PLUG TYPE REMARKS
TYPE PLUG

DIAMETER OF CASING/HOLE IN FT CEMENT/MUD TYPE

YIELD CU. FT/SK.

VOL. PUMPED CU. FT

TOP OF PLUG FT DEPTH 
PLUGGING REPORT

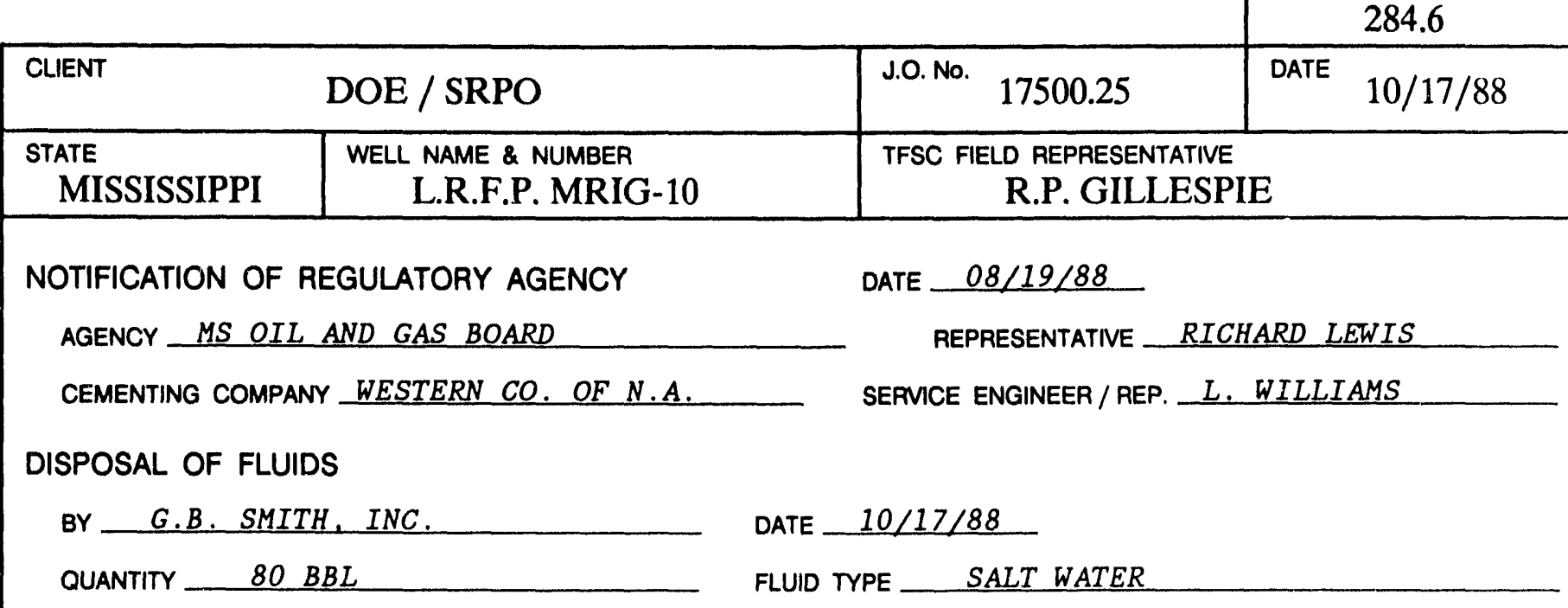

\section{REMARKS}

FLUIDS HAULED TO LICENSED DISPOSAL FACILITY OPERATED BY G.B. SMITH, INC.

ZONE 1

INTERVAL 2640.5 FT TO SURFACE
TYPE PLUG CONTINUOUS

DIAMETER OF CASING/HOLE $4.0,65 / 8, \& 8.0 \mathrm{IN}$ BOTTOM OF TUBING OR PIPE $2529.1 \mathrm{FT}$ CEMENT/MUD TYPE CLASS A, 88 BENTONITE
NO. OF SACKS 262.5
YIELD $\quad 1.92$
CU. FT/SK.
VOL. PUMPED CU. FT

SLURRY WEIGHT 13.1

BRIDGE PLUG TYPE NA LBS/GAL
TOP OF PLUG 4.5

DEPTH N

REMARKS

CEMENT PUMPED IN STAGES WITH TUBING SET AT 2529.1, 2033.9, 1538.3, 1042.3,\& 515.6 FT CEMENT CIRCULATED TO SURFACE, CASING CUT OFF AND CAPPED 4.5 FT BELOW GROUND, TOP 4.5 FT BACKFILLED WITH SOIL.

ZONE

INTERVAL

BOTTOM OF TUBING OR PIPE

NO. OF SACKS

SLURRY WEIGHT

BRIDGE PLUG TYPE REMARKS
TYPE PLUG

DIAMETER OF CASING/HOLE IN FT CEMENT/MUD TYPE YIELD CU.FT/SK. VOL. PUMPED _ CU.FT LBS/GAL TOP OF PLUG FT DEPTH 


\section{PLUGGING REPORT}

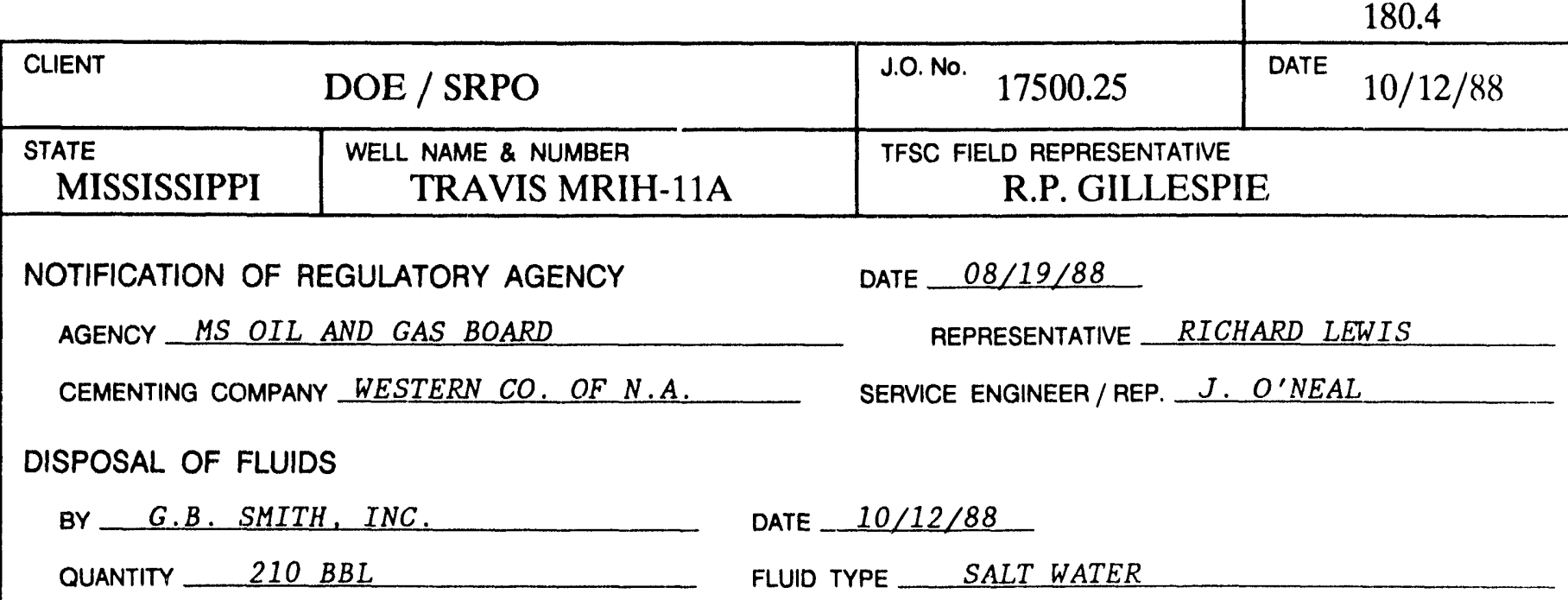

\section{REMARKS}

FLUIDS HAULED TO LICENSED DISPOSAL FACILITY OPERATED BY G.B. SMITH, INC.

ZONE 1

INTERVAL 4231.4 FT TO SURFACE
TYPE PLUG CONTINUOUS

DIAMETER OF CASING/HOLE $4.0,65 / 8, \& 8.0 \mathrm{IN}$ BOTTOM OF TUBING OR PIPE $\quad 4140.2$ FT CEMENT/MUD TYPE CLASS A. $8 \%$ BENTONITE NO. OF SACKS _ 429.5 YIELD 1.92 CU.FT/SK. VOL. PUMPED 825 CU.FT SLURRY WEIGHT 13.1 LBS/GAL TOP OF PLUG 4.0 BRIDGE PLUG TYPE _ NA DEPTH N NA REMARKS

CEMENT PUMPED IN STAGES WITH TUBING SET AT 4140.2, 3613.3, 3086.9, 2561.2, 2034.9, 1508.2, 981.4. AND 454.7 FT. CEMENT CIRCULATED TO SURFACE, CASING CUT OFF AND CAPPED 4 FT BELOW GROUND, TOP 4.0 FT BACKFILLED WITH SOIL.

ZONE

INTERVAL BOTTOM OF TUBING OR PIPE NO. OF SACKS YIELD FT SLURRY WEIGHT LBS/GAL

BRIDGE PLUG TYPE REMARKS
TYPE PLUG

DIAMETER OF CASING/HOLE IN CEMENT/MUD TYPE CU. FT/SK. VOL. PUMPED CU. FT TOP OF PLUG FT DEPTH 
PLUGGING REPORT

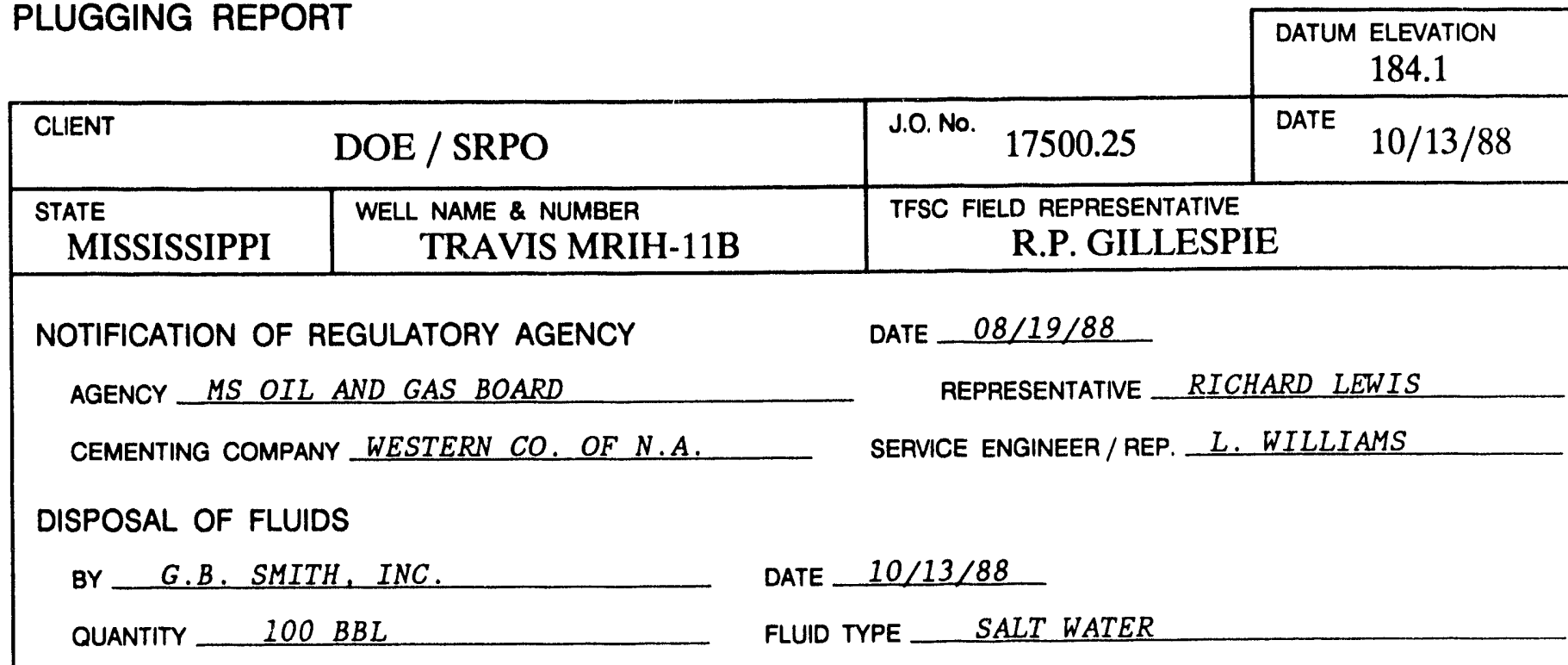

\section{REMARKS}

FLUIDS HAULED TO LICENSED DISPOSAL FACILITY OPERATED BY G.B. SMITH, INC.

ZONE 1

INTERVAL 2445 FT TO SURFACE

BOTTOM OF TUBING OR PIPE 2436 FT CEMENT/MUD TYPE_CLASS A, $8 \%$ BENTONITE
NO. OF SACKS 292.5 YIELD 1.92 CU. FT/SK.

SLURRY WEIGHT 13.1 LBS/GAL

BRIDGE PLUG TYPE WESTERN, CAST, $65 / 8 \quad$ DEPTH -2445
TYPE PLUG CONTINUOUS

DIAMETER OF CASING/HOLE 4.0 AND $6 \quad 5 / 8 \quad$ IN

REMARKS

CEMENT PUMPED IN STAGES WITH TUBING SET AT 2436, 1944, 1445, 949, AND 454 FT. CEMENT CIRCULATED TO SURFACE, CASING CUT OFF AND CAPPED 4.5 FT BELOW GROUND, TOP 4.5 FT BACKFILLED WITH SOIL.

ZONE

INTERVAL

BOTTOM OF TUBING OR PIPE

NO. OF SACKS

YIELD LBS/GAL SLURRY WEIGHT
TYPE PLUG

DIAMETER OF CASING/HOLE IN FT CEMENT/MUD TYPE

BRIDGE PLUG TYPE CU. FT/SK.

VOL. PUMPED CU. FT CU. FT FT REMARKS FT

TOP OF PLUG DEPTH 
PLUGGING REPORT

\begin{tabular}{|c|c|c|}
\hline & & $\begin{array}{c}\text { DATUM ELEVATION } \\
181.7\end{array}$ \\
\hline J.O. No. & 17500.25 & DATE $\quad 10 / 11 / 88$ \\
\hline
\end{tabular}

NOTIFICATION OF REGULATORY AGENCY

AGENCY MS OIL AND GAS BOARD

CEMENTING COMPANY WESTERN CO. OF N.A.
DATE $08 / 19 / 88$

REPRESENTATIVE RICHARD LEWIS

SERVICE ENGINEER / REP. J. O'NEAL

DISPOSAL OF FLUIDS

BY G.B. SMITH, INC

DATE $10 / 11 / 88$

QUANTITY $70 \mathrm{BBL}$

FLUID TYPE

SALT WATER

\section{REMARKS}

FLUIDS HAULED TO LICENSED DISPOSAL FACILITY OPERATED BY G.B. SMITH, INC.

ZONE 1

INTERVAL 1616.4 FT TO SURFACE

BOTTOM OF TUBING OR PIPE $1507.2 \mathrm{FT}$

NO. OF SACKS $154.5 \quad$ YIELD $\quad 1.92$

SLURRY WEIGHT 13.1 LBS/GAL

BRIDGE PLUG TYPE _ NA

REMARKS

CEMENT PUMPED IN STAGES WITH TUBING SET AT 1507.2, 1011.3, AND 515.6 FT. CEMENT WAS CIRCULATED TO SURFACE, CASING CUT OFF GIND CAPPED 4 FT BELOW GROUND, TOP 4.0 FT BACK -

FILLED WITH SOIL.

ZONE

INTERVAL

BOTTOM OF TUBING OR PIPE

NO. OF SACKS

SLURRY WEIGHT

BRIDGE PLUG TYPE

REMARKS
TYPE PLUG CONTINUOUS

DIAMETER OF CASING/HOLE 4.0 AND $6 \quad 5 / 8$ IN CEMENT/MUD TYPE CLASS A, $8 \%$ BENTONITE

VOL. PUMPED 297 CU. FT TOP OF PLUG 5.0 DEPTH 


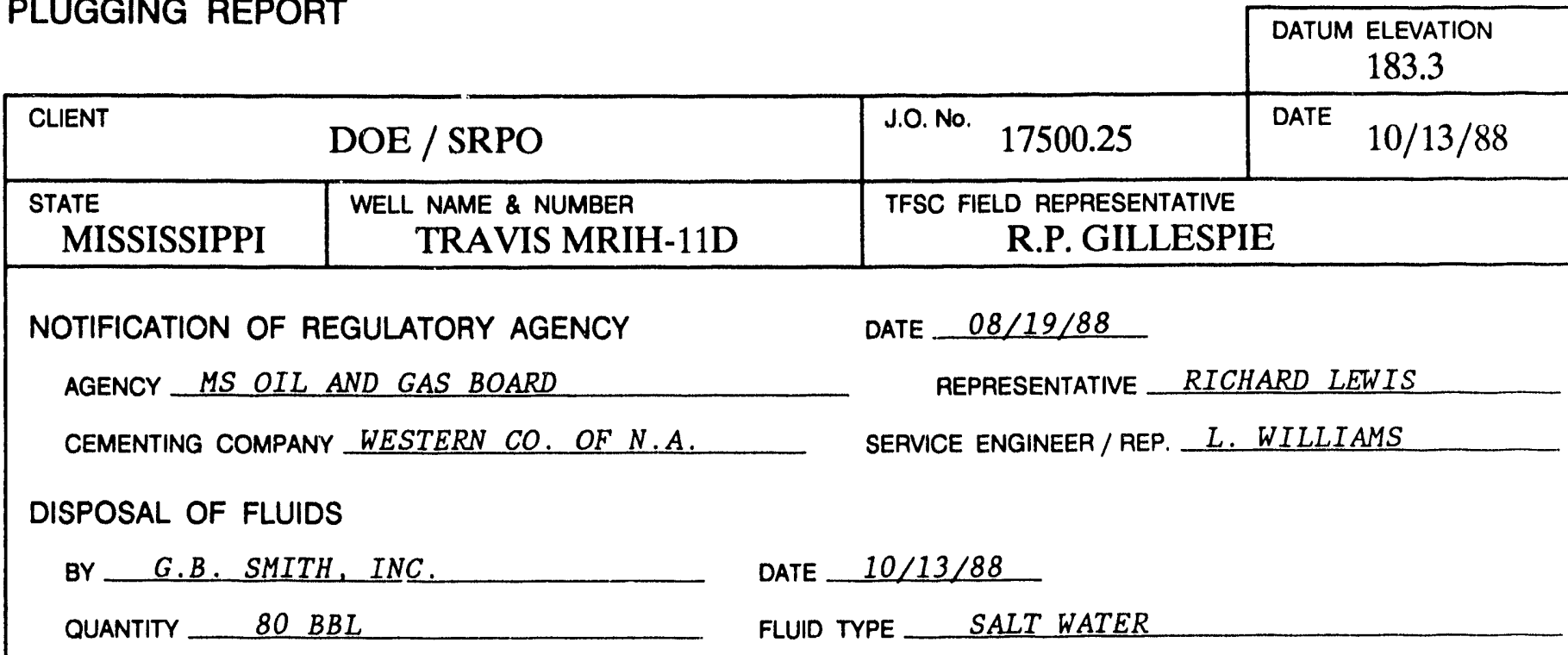

\section{REMARKS}

FLUIDS HAULED TO LICENSED DISPOSAL FACILITY OPERATED BY G.B. SMITH, INC.

ZONE 1

INTERVAL 935.2 FT TO SURFACE
TYPE PLUG CONTINUOUS

DIAMETER OF CASING/HOLE $4.0,65 / 8, \& 8.0 \mathrm{IN}$ BOTTOM OF TUBING OR PIPE $\quad 825.8$ FT CEMENT/MUD TYPE CLASS A, $8 \%$ BENTONITE

NO. OF SACKS $94 \quad$ YIELD 1.92 CU.FT/SK.

SLURRY WEIGHT 13.1 LBS/GAL

BRIDGE PLUG TYPE _ NA
VOL. PUMPED 180 CU. FT

REMARKS

CEMENT PUMPED IN STAGES WITH TUBING SET AT 825.8 AND 392.8 FT. CEMENT CIRCULATED TO SURFACE, CASING CUT OFF AND CAPPED 4 FT BELOW GROUND, TOP 4.0 FT BACKFILLED WITH SOIL.

ZONE

INTERVAL

BOTTOM OF TUBING OR PIPE

NO. OF SACKS

SLURRY WEIGHT

BRIDGE PLUG TYPE

REMARKS
TYPE PLUG

DIAMETER OF CASING/HOLE IN CEMENT/MUD TYPE

YIELD CU. FT/SK.

VOL. PUMPED CU. FT LBS/GAL TOP OF PLUG FT DEPTH 
APPENDIX H

Borehole Completion Sketches 
BOREHOLE COMPLETION SKETCH

\begin{tabular}{|c|c|c|c|}
\hline \multicolumn{2}{|c|}{ CLIENT DOE / SRPO } & J.O. No. 17500.25 & $\begin{array}{l}\text { DATE } \\
10-20-88\end{array}$ \\
\hline $\begin{array}{l}\text { STATE } \\
\text { MISSISSIPPI }\end{array}$ & $\begin{array}{l}\text { WELL NAME \& NUMBER } \\
\text { U.S.F.S. MCCG-1 }\end{array}$ & $\begin{array}{r}\text { TFSC FIELD REPRESE } \\
\text { R.P. GILL }\end{array}$ & \\
\hline
\end{tabular}

All Depths Meosured From

Ground Level

Boring Diameter, Conductor Pipe 30 in

गात्राए

Tack-Welded Steel Plate

Boring Diameter,

Surface Casing $17 \mathrm{in.}$

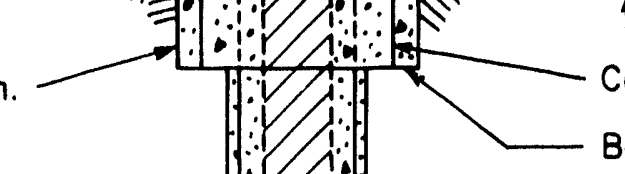

$t_{23 \mathrm{ft}}$ Elevation $221.0 \mathrm{ft}$. $\geq 3 \mathrm{ff}$.

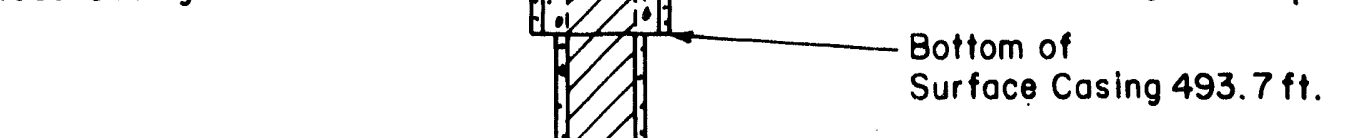

Boring Diameter, Inner Cosing 11 in.

Explanation

Open Hole $7 \frac{7}{8}$ in

\section{Bottom of}

Inney Cosing $1195.0 \mathrm{ft}$.

Inner Casing $8 \frac{5}{8}$ in.

20 in.

Bottom of

Conductor Pipe 59.0 ft.

Surface Cosing $123 / 4$ in.

Bottom of

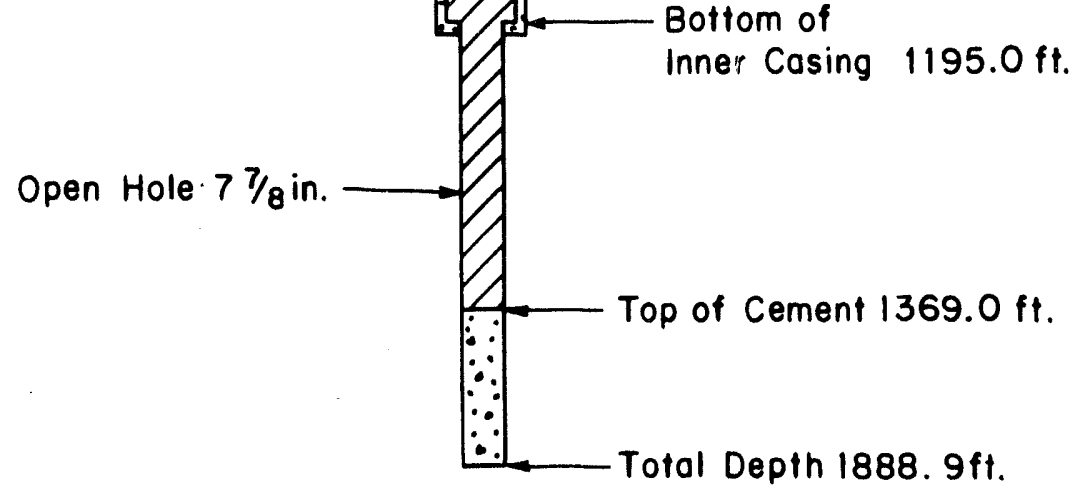

$\because \because$ Cement Placed by Others

27 cement-Bentonite Plug Placed for Abandonment

Backfilled Soil

Note: Not to Scale. 
BOREHOLE COMPLETION SKETCH

\begin{tabular}{|c|c|c|c|}
\hline $\begin{array}{l}\text { CLIENT DOE /SRPO } \\
\text { WELL NAME \& NUMBER }\end{array}$ & $\begin{array}{l}\text { J.0. No. } \\
17500.25\end{array}$ & 12-21-88 \\
\hline $\begin{array}{c}\text { STATE } \\
\text { MISSISSIPPI }\end{array}$ & U.S.F.S. MCCG-1WS & TFSC FIELD REPRESENTATIVE \\
D. KISTNER / H. ABEDI
\end{tabular}

Ground Level

219.9 ft. (msl)
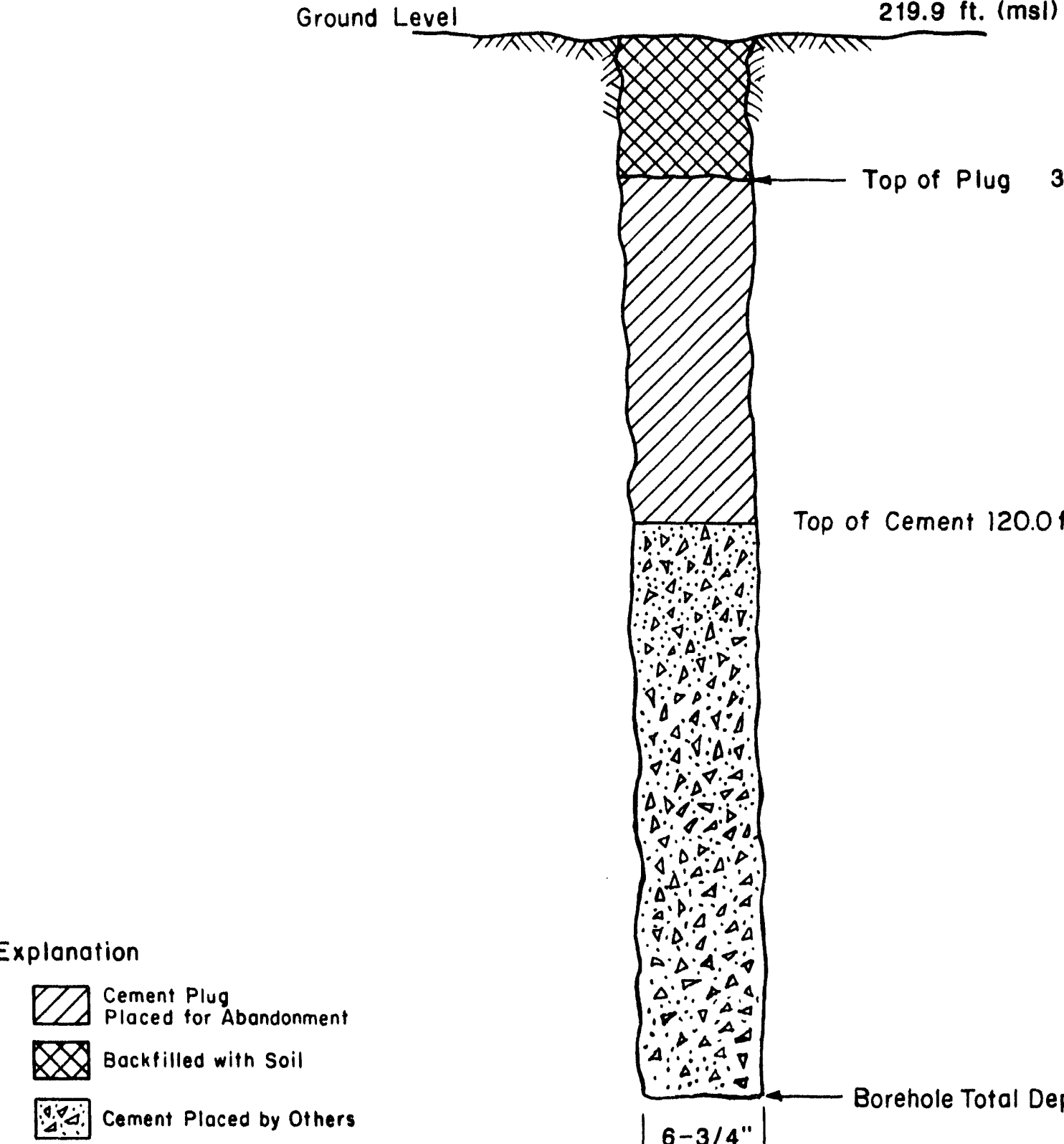

Top of Plug $3.0 \mathrm{ft}$.

Top of Cement $120.0 \mathrm{ft}$.

0 i.

$\because 4: 4$

a d a

0 a

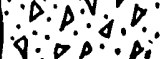

$\because 4.40$

r.sid.

a

a sidid

- 40

$\therefore: 4: 4$

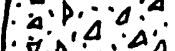

จ. 0.4 .4

$\therefore \cdot \Delta \cdot 4 \cdot \dot{0}$

$\therefore \square \Delta$

$\therefore \nabla \nabla \Delta$

$\therefore \Delta \Delta \Delta \Delta$

$\Delta \because \therefore \Delta$

6-3/4"

Borehole Total Depth $514.0 \mathrm{ft}$

Borehole Diameter

Note:

Not to scale.

All depths measured from Ground Level Elevation $219.9 \mathrm{ft}$. 


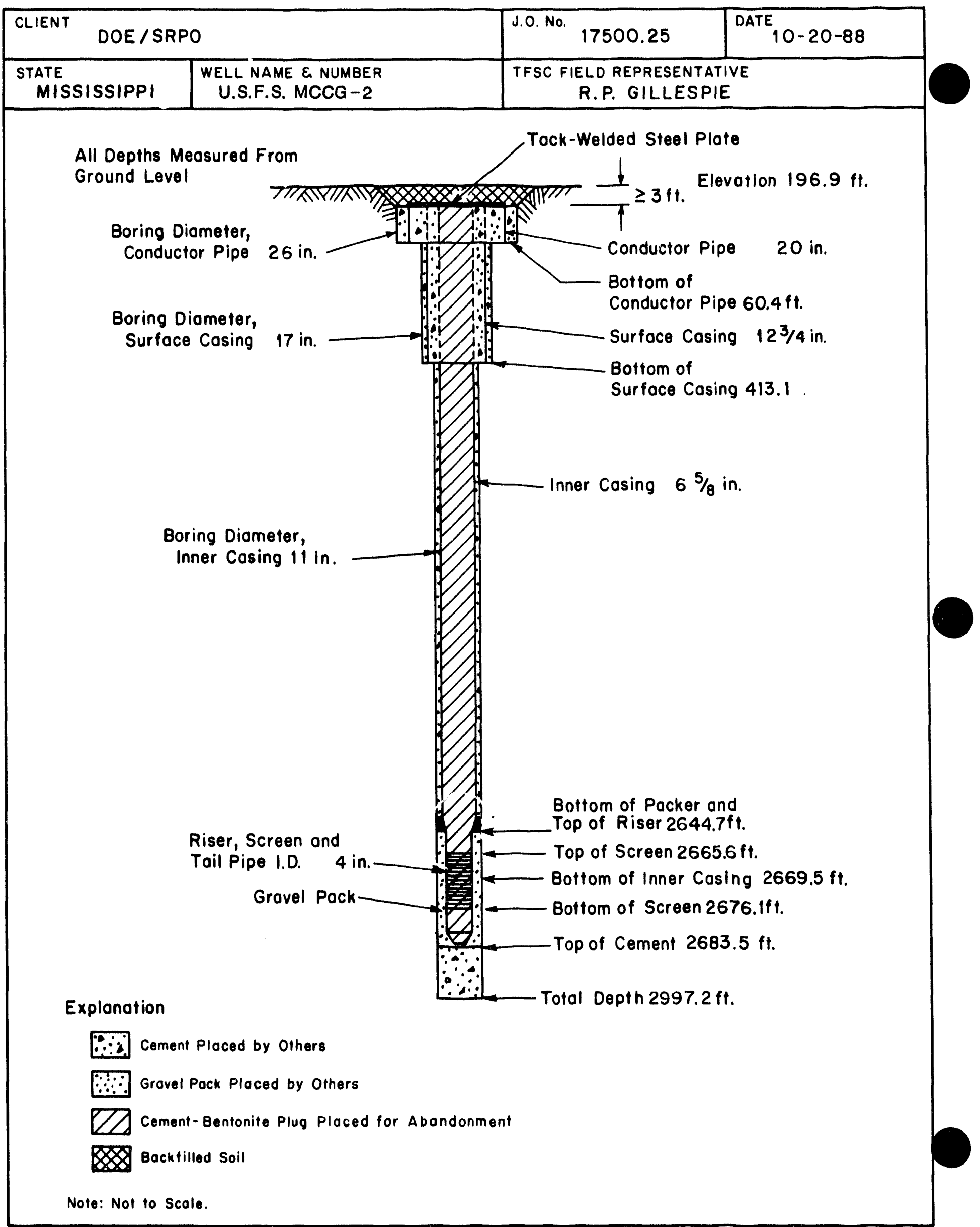


BOREHOLE COMPLETION SKETCH

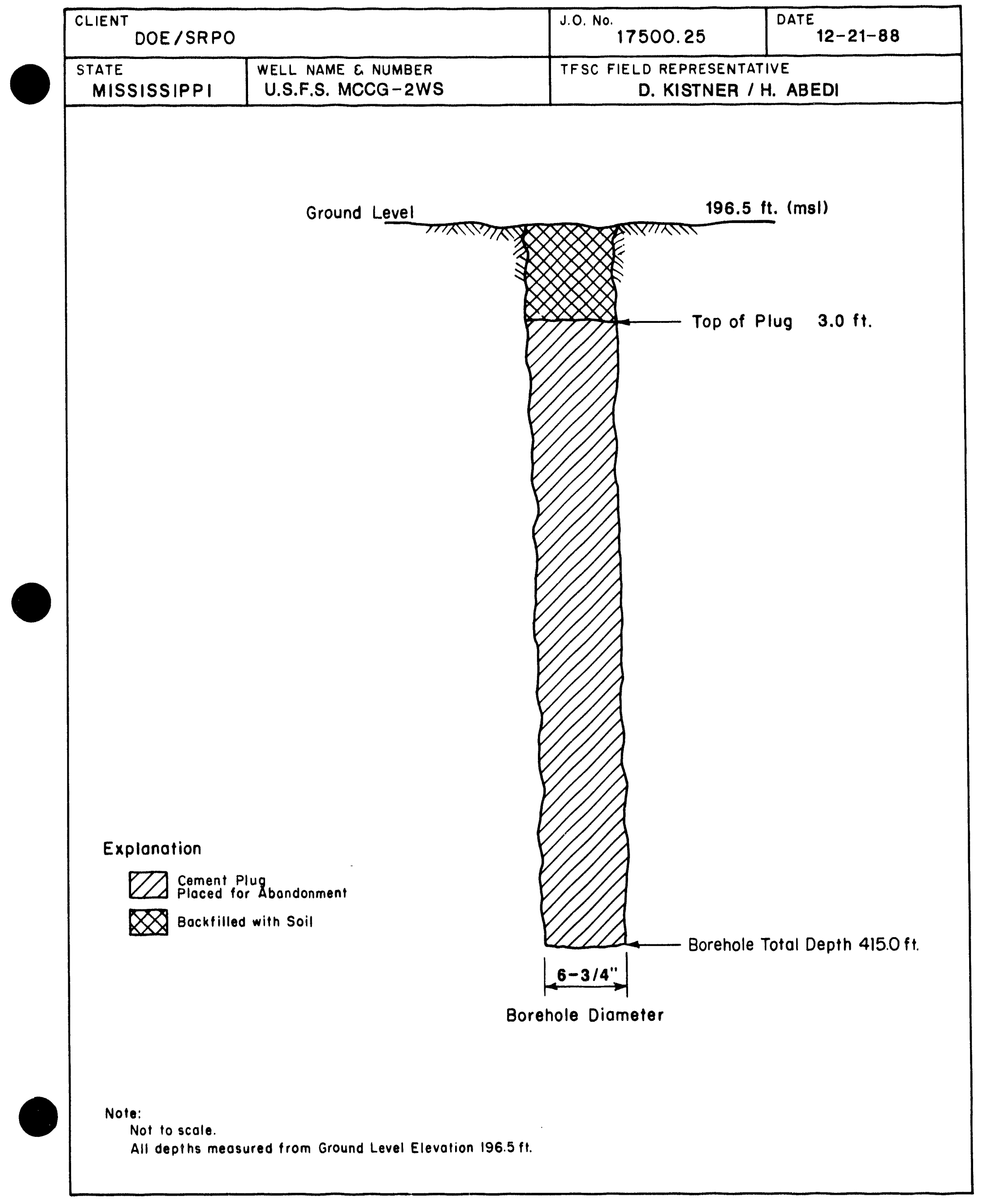


BOREHOLE COMPLETION SKETCH

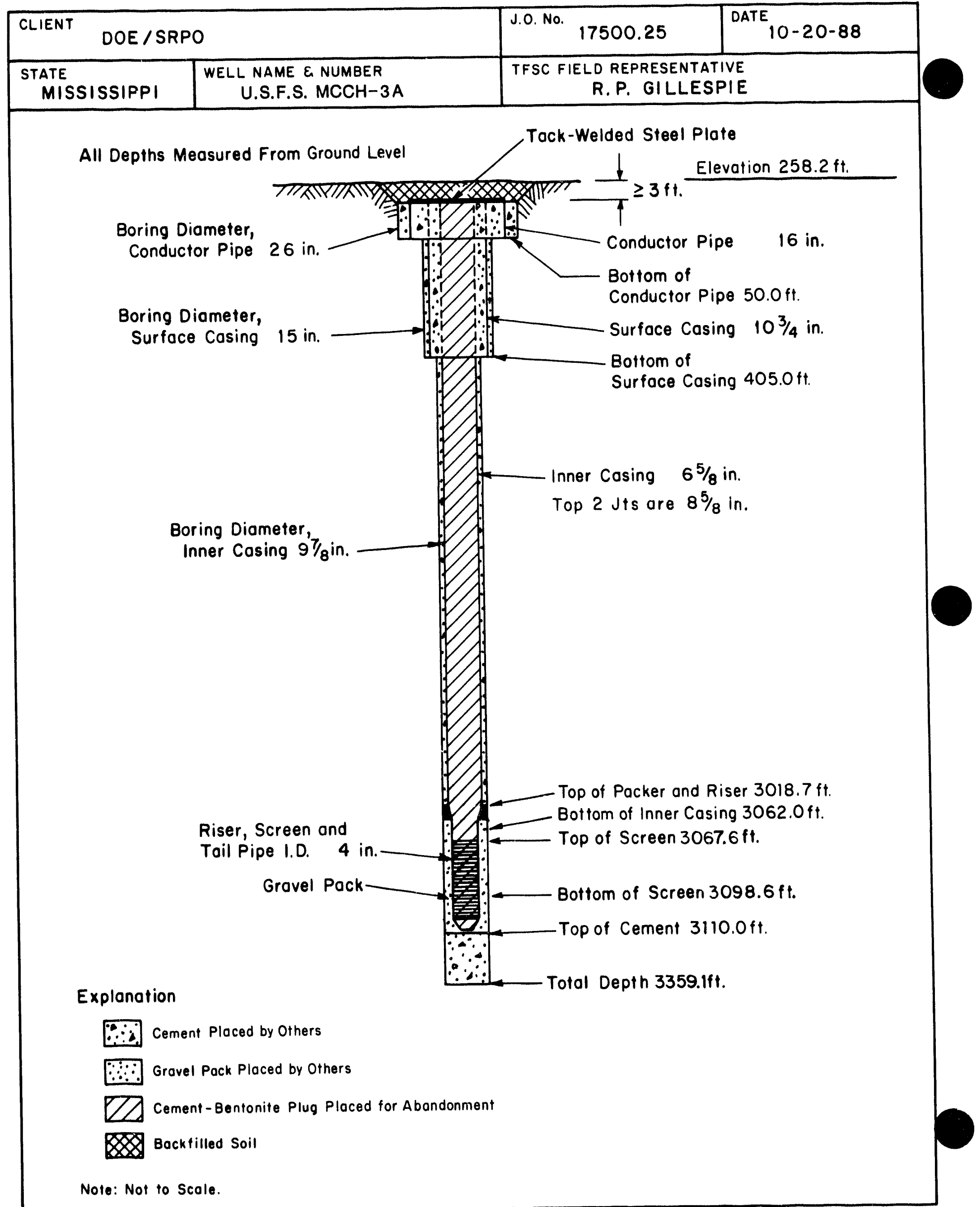


BOREHOLE COMPLETION SKETCH

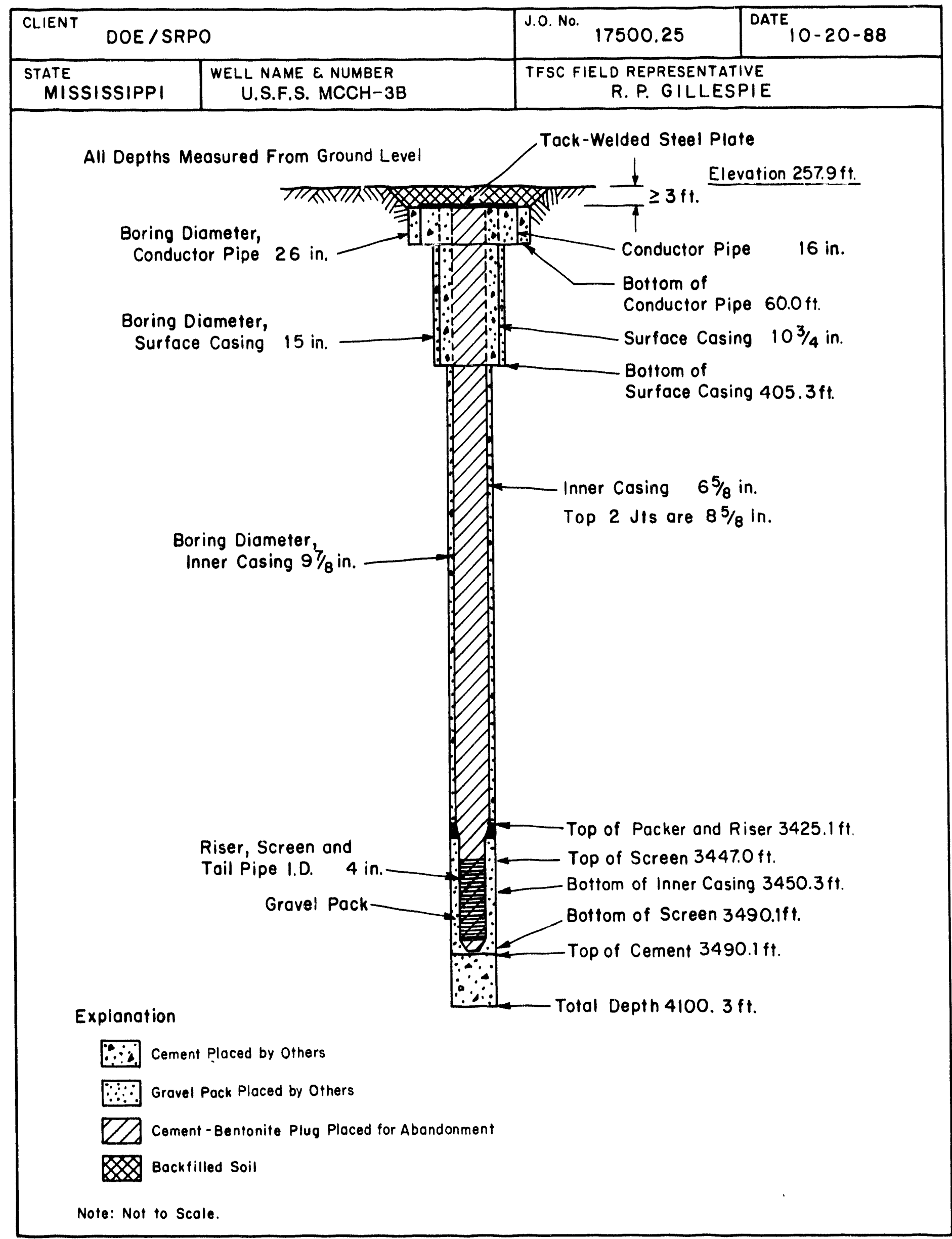


BOREHOLE COMPLETION SKETCH

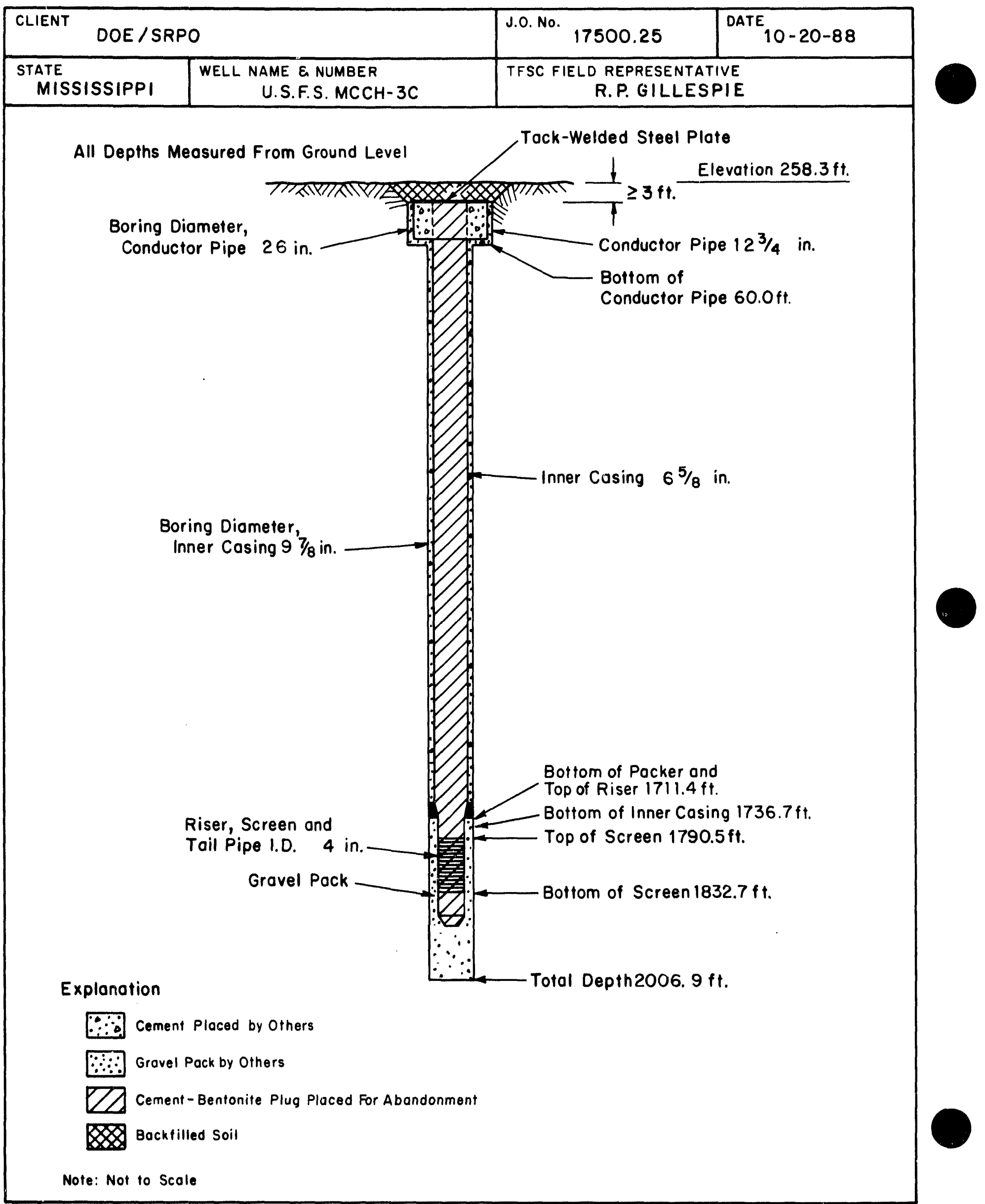


BOREHOLE COMPLETION SKETCH

\begin{tabular}{|l|l|l|l|}
\hline \multicolumn{2}{|c|}{ CLIENT DOE 'SRPO } & $\begin{array}{l}\text { J.0. No. } 17500.25 \\
\text { DATE }\end{array}$ & 10-20-88 \\
\hline $\begin{array}{c}\text { STATE } \\
\text { MISSISSIPPI }\end{array}$ & $\begin{array}{c}\text { WELL NAME \& NUMBER } \\
\text { U.S.F.S. MCCH-3D }\end{array}$ & $\begin{array}{c}\text { TFSC FIELD REPRESENTATIVE } \\
\text { R.P. GI LLESPIE }\end{array}$ \\
\hline
\end{tabular}

All Depths Measured From Ground Level

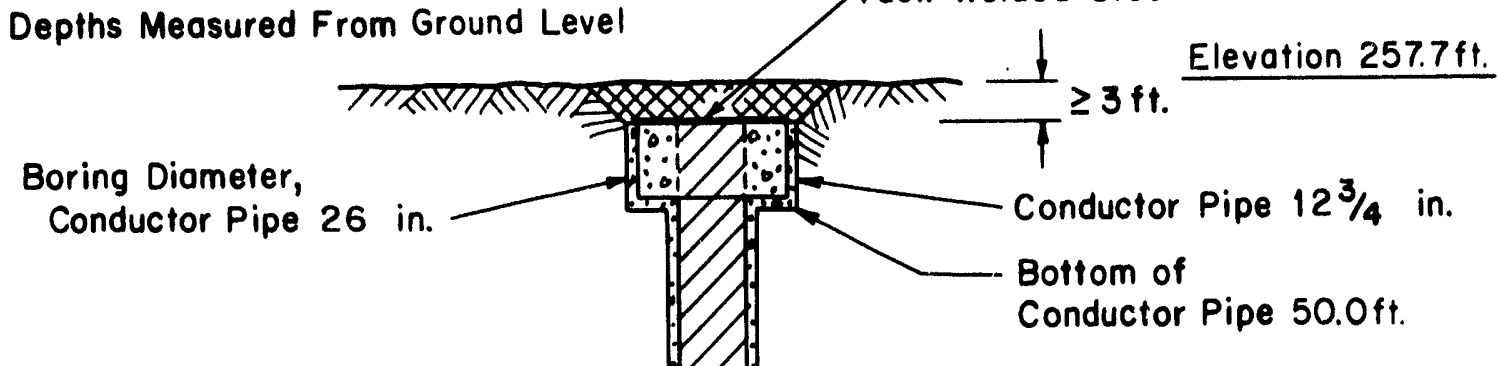

Boring Diameter,

Inner Cosing $9 \% 8$ in.

Riser, Screen and

Tail Pipe I.D. 4 in. Bottom of Inner Casing $1210.0 \mathrm{ft}$

Boring Diameter $5-7 / 8$ in

Top of Screen $1219.6 \mathrm{ft}$.

Gravel Pack Bottom of Screen 1251.0ft.

Total Depth

\section{Explanation}

$\because \because$ Cement Placed by Others

$\because \because \because$ Grovel Pack Placed by Others

7 Cement-Bentonite Plug Placed for Abandonment

Q8 Backfilled Soil

Note: Not to Gcale 
BOREHOLE COMPLETION SKETCH

\begin{tabular}{|l|l|l|l|}
\hline $\begin{array}{l}\text { CLIENT DOE / SRPO } \\
\text { STATE }\end{array}$ & $\begin{array}{l}\text { J.0. No. } 17500.25 \\
\text { MISSISSIPPI }\end{array}$ & $\begin{array}{c}\text { WELL NAME \& NUMBER } \\
\text { U.S.F.S. MCCH-3WS }\end{array}$ & $\begin{array}{r}\text { TFSC FIELD REPRESENTATIVE } \\
\text { D. KISTNER / H. ABEDI }\end{array}$ \\
\hline
\end{tabular}

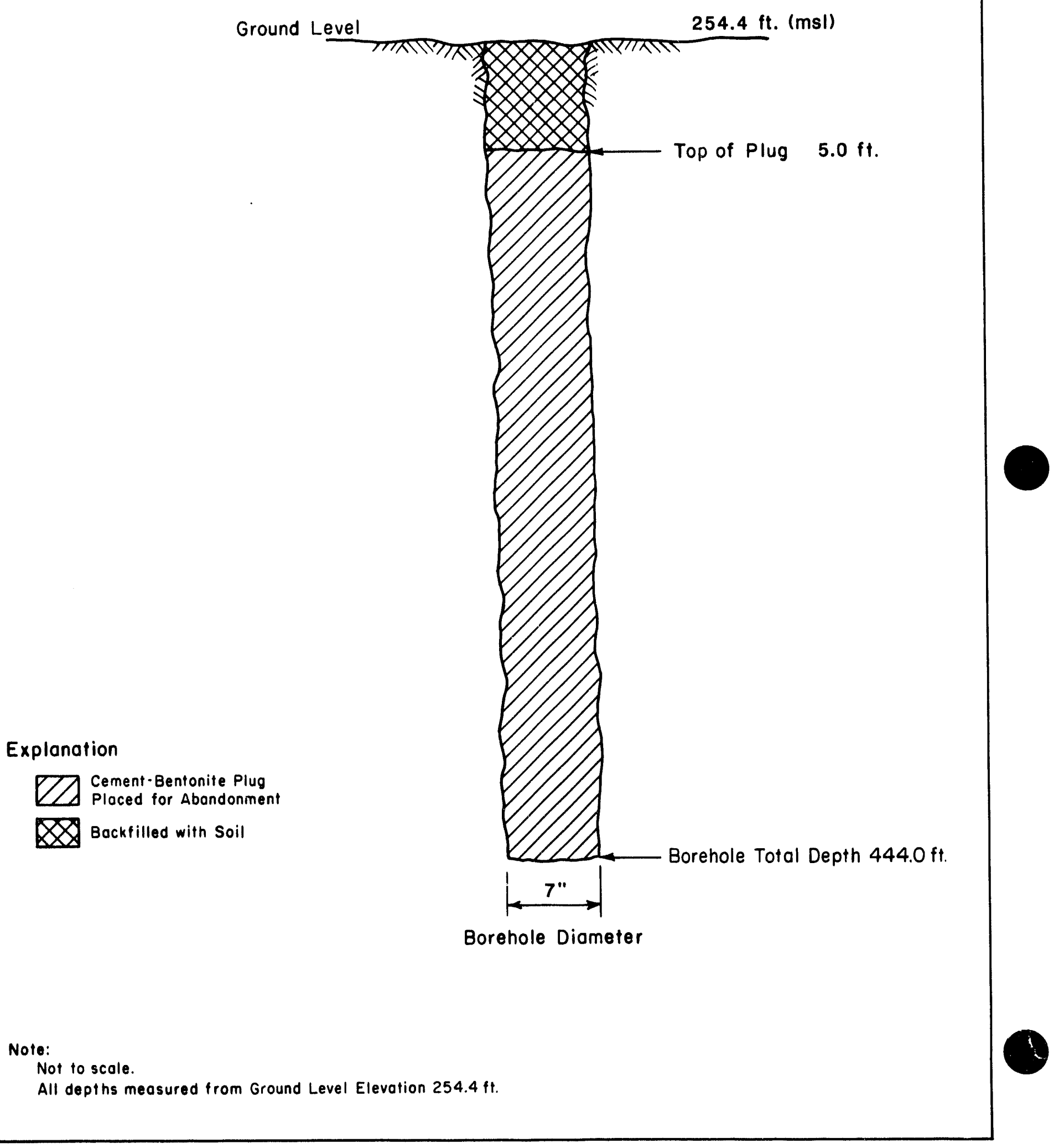


BOREHOLE COMPLETION SKETCH

\begin{tabular}{|l|c|c|c|}
\hline CLIENT DOE/SRPO & $\begin{array}{l}\text { J.0. No. } 17500.25 \\
\text { DATE }\end{array}$ & $10-20-88$ \\
\hline $\begin{array}{c}\text { STATE } \\
\text { MISSISSIPPI }\end{array}$ & $\begin{array}{r}\text { WELL NAME E NUMBER } \\
\text { ROIVELL MH-4A }\end{array}$ & $\begin{array}{c}\text { TFSC FIELO REPRESENTATIVE } \\
\text { R.P. GILLESPIE }\end{array}$ \\
\hline
\end{tabular}

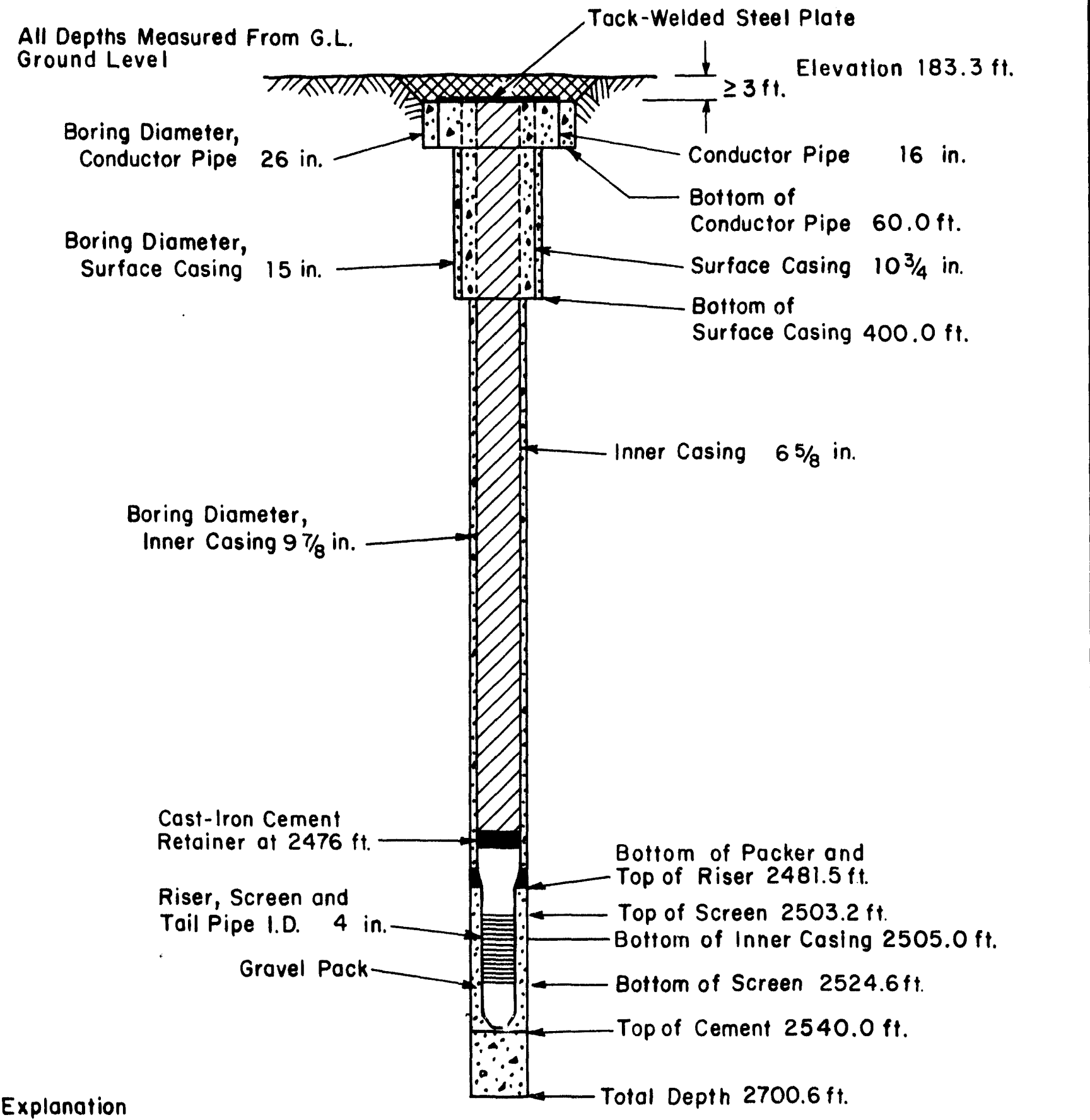

$\therefore \therefore$ Cement Ploced by Others

$\because \therefore$ Gravel Pack Placed by Others

Z Cement-Bentonite Plug Placed for Abandonment

Backfilled Soil

Note: Not to Scale. 
BOREHOLE COMPLETION SKETCH

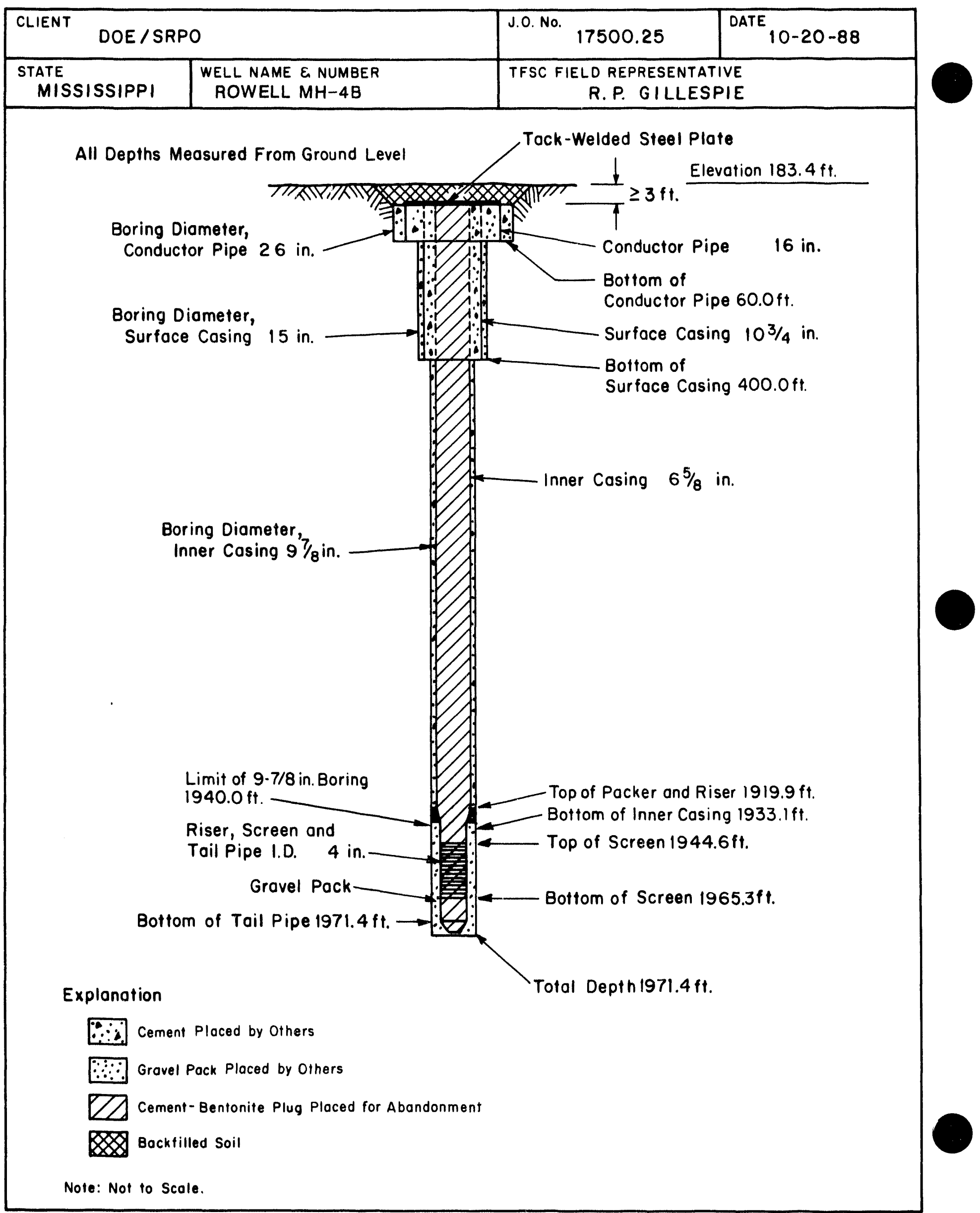


BOREHOLE COMPLETION SKETCH

\begin{tabular}{|c|l|l|l|}
\hline CLIENT DOE / SRPO & $\begin{array}{l}\text { J.0. No. } 17500.25 \\
\text { DATE }\end{array}$ & $10-20-88$ \\
\hline $\begin{array}{c}\text { STATE } \\
\text { MISSISSIPPI }\end{array}$ & $\begin{array}{l}\text { WELL NAME \& NUMBER } \\
\text { ROWELL MH-4C }\end{array}$ & $\begin{array}{c}\text { TFSC FIELD REPRESENTATIVE } \\
\text { R. P. GILLESPIE }\end{array}$ \\
\hline
\end{tabular}

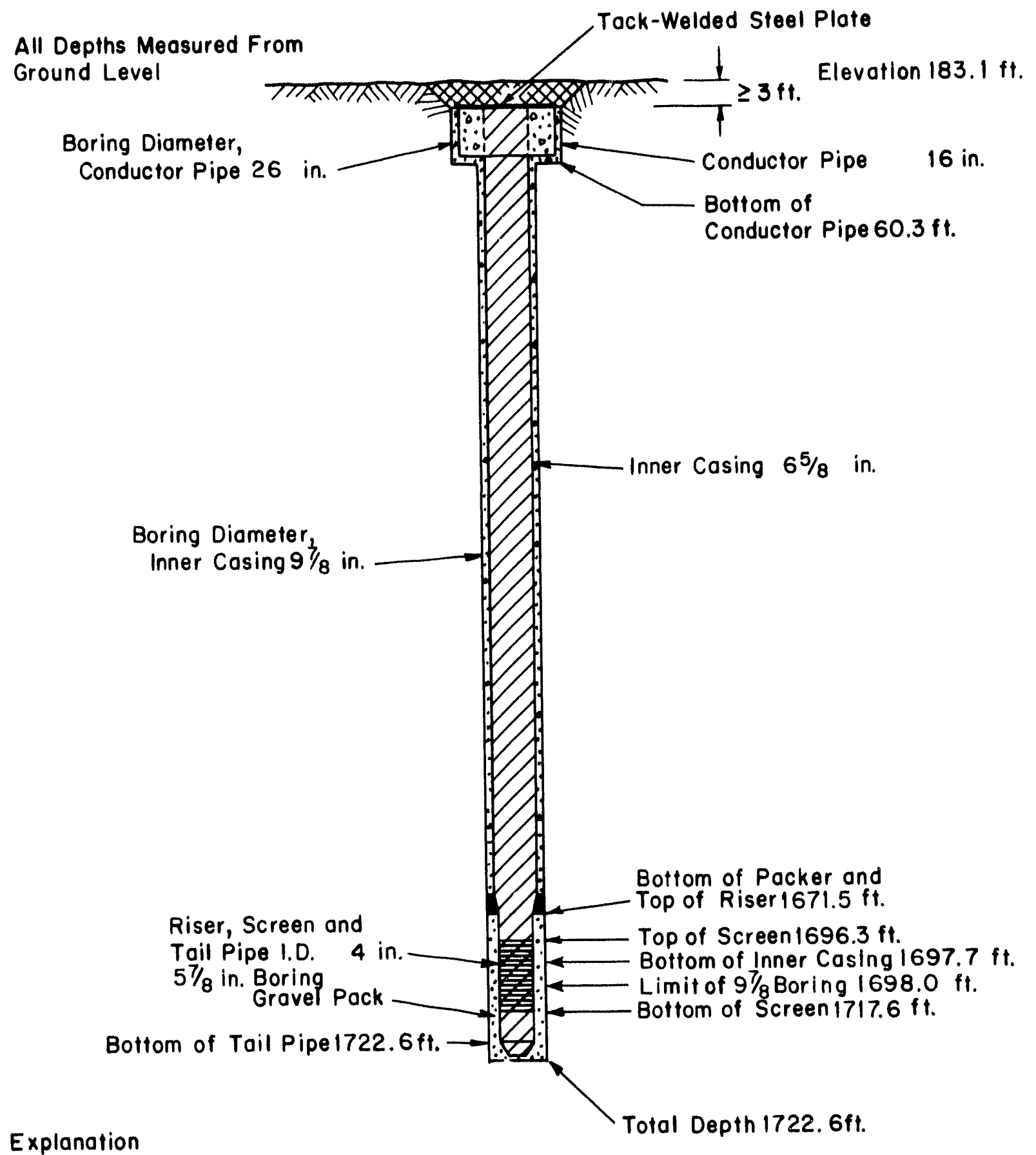

$\because \therefore$ Cement Placed by Others

$\because \because \therefore$ Grovel Pack Placed by Others

C Cement-Bentonite Plug Placed for Abandonment

EX8 Bockfilled Soil

Note: Not to Scale 
BOREHOLE COMPLETION SKETCH

\begin{tabular}{|l|l|l|l|}
\hline \multicolumn{2}{|c|}{ CLIENT DOE / SRPO } & $\begin{array}{l}\text { J.0. No. } 17500.25 \\
\text { DATE }\end{array}$ & 10-19-88 \\
\hline $\begin{array}{c}\text { STATE } \\
\text { MISSISSIPPI }\end{array}$ & $\begin{array}{c}\text { WELL NAME E NUMBER } \\
\text { ROWELL MH-4D }\end{array}$ & $\begin{array}{r}\text { TFSC FIELD REPRESENTATIVE } \\
\text { R.P. GILLESPIE }\end{array}$ \\
\hline
\end{tabular}

\section{All Depths Measured From}

Ground Level

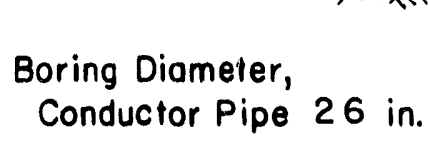

Boring Diameter,

Inner Casing $9 \% 8$ in.
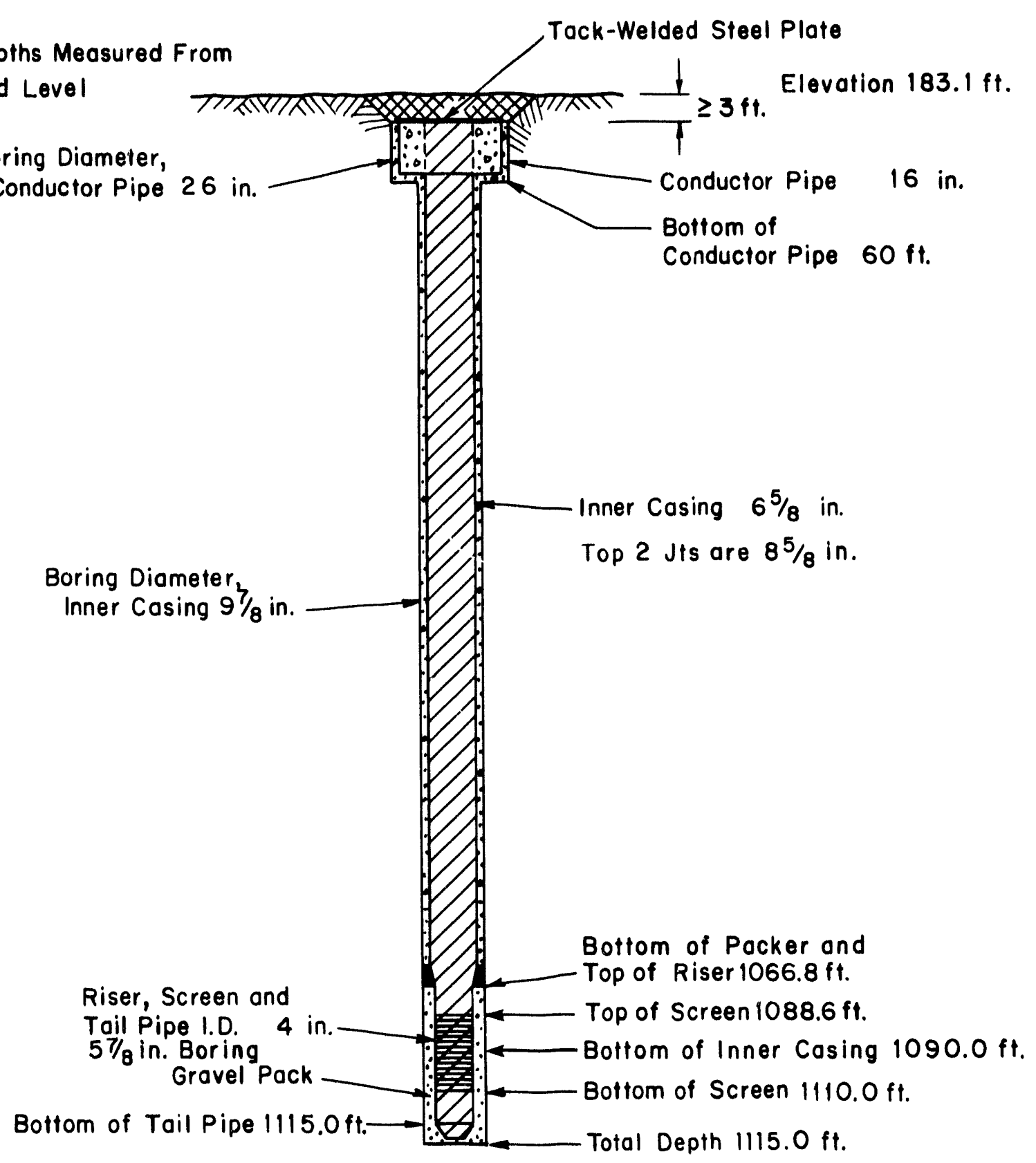

Explanation

$\therefore \therefore$ Cement Placed by Others

$\because \because$ Gravel Pack Placed by Others

27 Cement-Bentonite Plug Placed for Abandonment

$8 x$

Backfilled Soil 
BOREHOLE COMPLETION SKETCH

\begin{tabular}{|c|c|c|c|}
\hline \multicolumn{2}{|c|}{ CLIENT DOE / SRPO } & J.0. No. 17500.25 & DATE $12-13-88$ \\
\hline $\begin{array}{c}\text { STATE } \\
\text { MISSISSIPPI }\end{array}$ & $\begin{array}{l}\text { WELL NAME E NUMBER } \\
\text { ROWELL MH-4WS }\end{array}$ & TFSC FIELD REPESENTATIVE \\
\hline
\end{tabular}

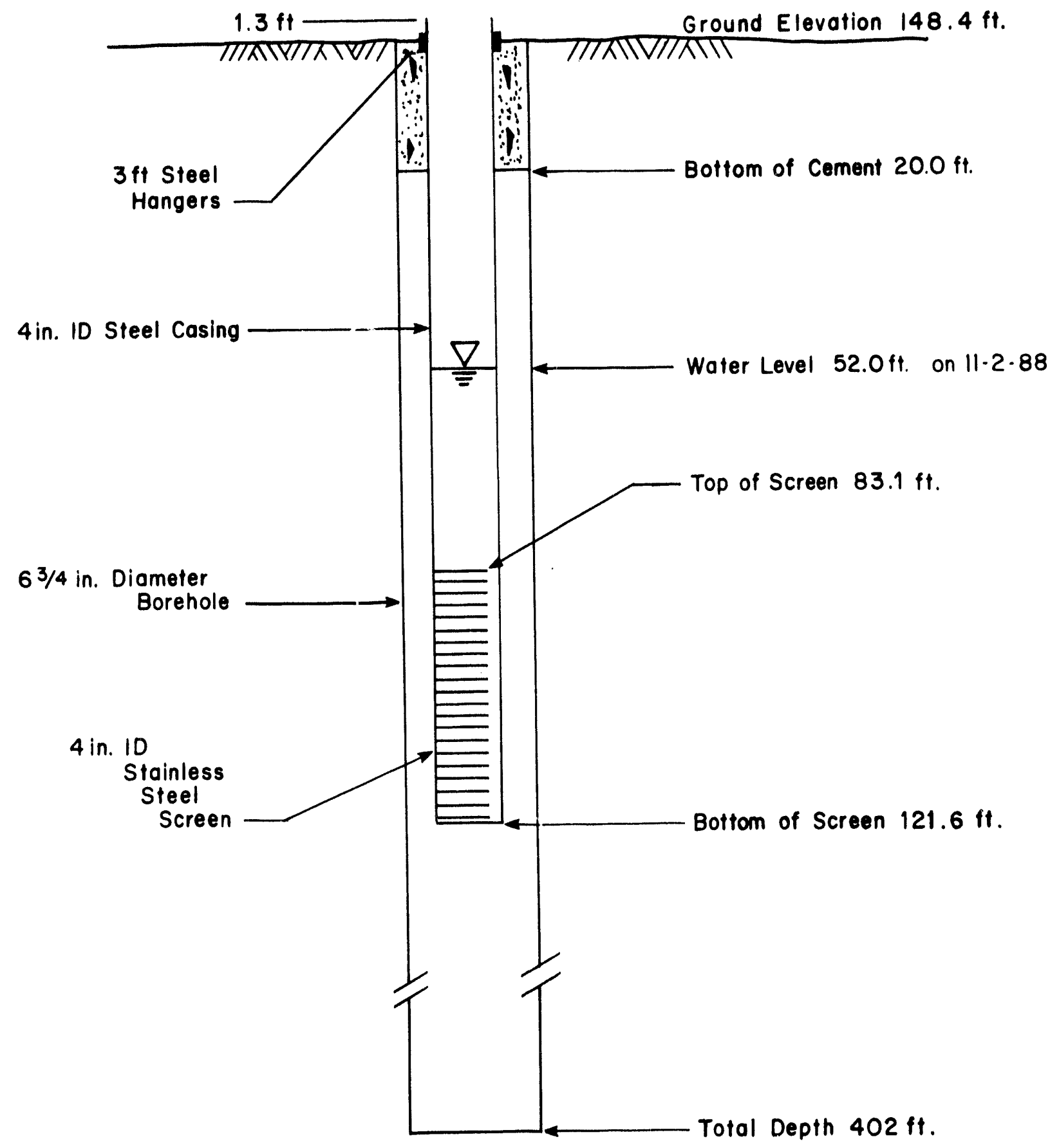

\section{Explanation}

Cement Placed by Others

1. All Depth Measurements From Ground Level

2. Not to Scale

3. Ownership of Well Transferred to Land Owner 
BOREHOLE COMPLETION SKETCH

\begin{tabular}{|l|l|l|l|}
\hline \multicolumn{2}{|c|}{ CLIENT DOE / SRPO } & J.0. No. 17500.25 & DATE $10-19-88$ \\
\hline $\begin{array}{c}\text { STATE } \\
\text { MISSISSIPPI }\end{array}$ & $\begin{array}{c}\text { WELL NAME E NUMBER } \\
\text { U.S.F.S. MH-5A }\end{array}$ & $\begin{array}{r}\text { TFSC FIELD REPRESENTATIVE } \\
\text { R.P. GILLESPIE }\end{array}$ \\
\hline
\end{tabular}

All Depths Measured From Ground Level

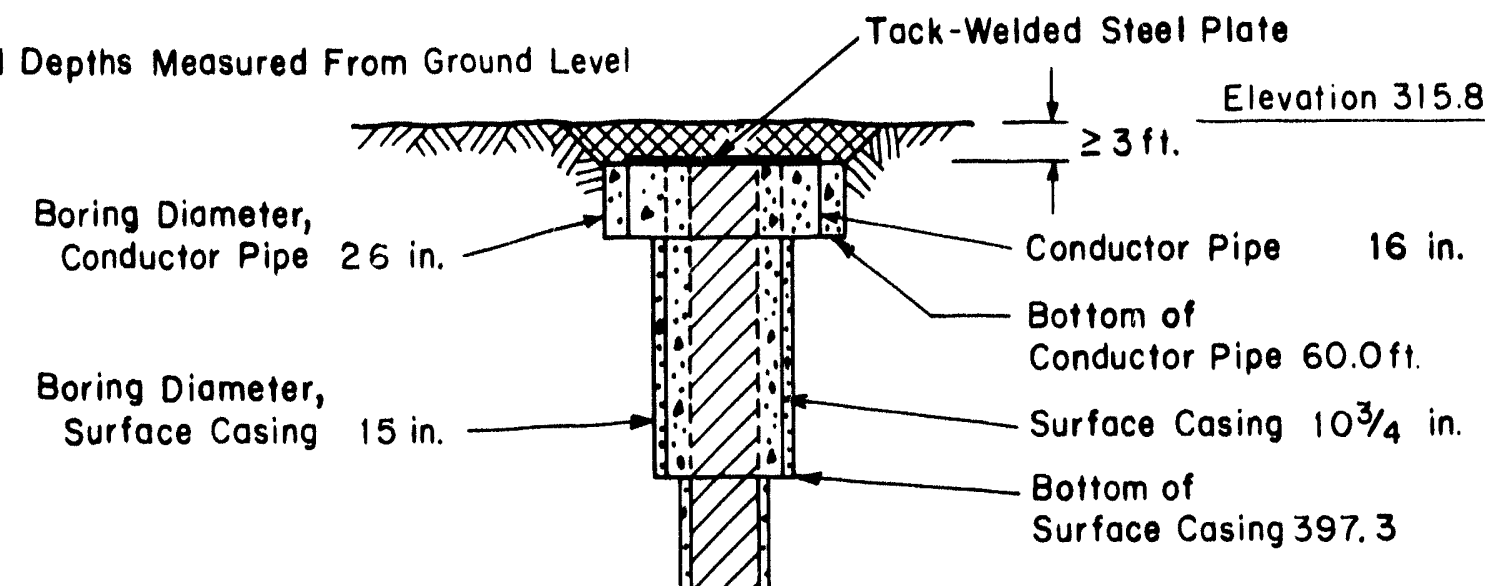

Boring Diameter,

Inner Cosing $9 \frac{7}{8}$ in.

Inner Casing $65 / 8$ in.

Explanation

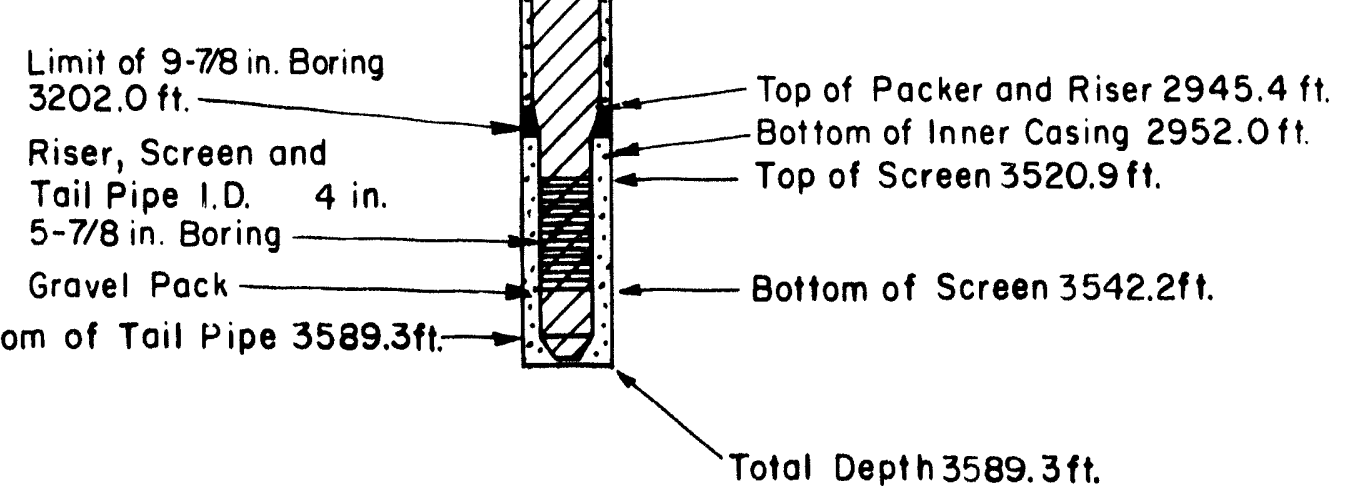

$\because \therefore$ Cement Placed by Others
$\because \because:$ Gravel Pack Placed by Others
8 Cement-Bentonite Plug Placed for Abandonment
88 Backtilled Soil

Note: Not to Scale. 
BOREHOLE COMPLETION SKETCH

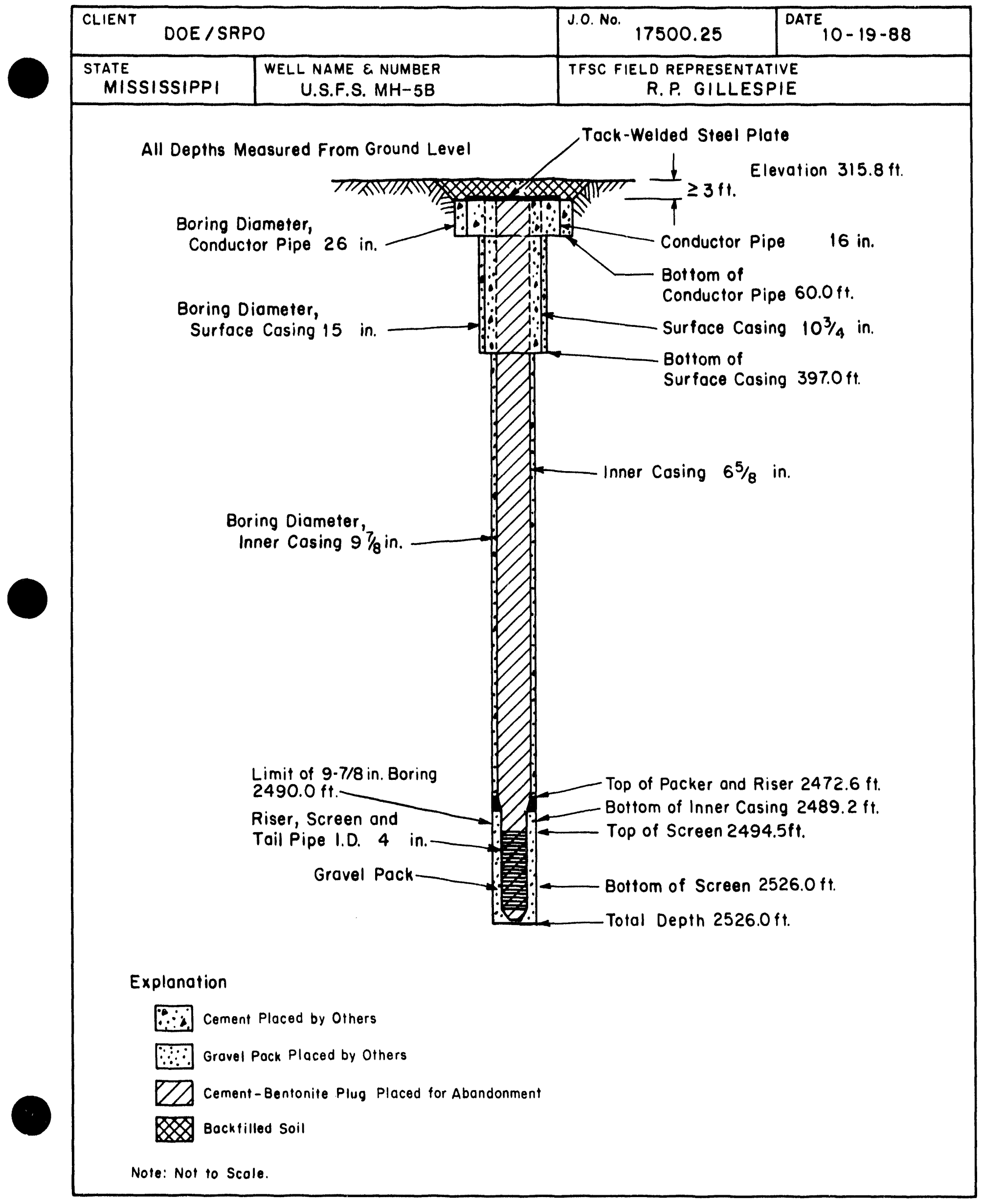


BOREHOLE COMPLETION SKETCH

\begin{tabular}{|l|l|l|l|}
\hline CLIENT DOE / SRPO & $\begin{array}{l}\text { J. No. } \\
17500.25\end{array}$ & DATE $10-19-88$ \\
\hline $\begin{array}{c}\text { STATE } \\
\text { MISSISSIPPI }\end{array}$ & $\begin{array}{c}\text { WELL NAME E NUMBER } \\
\text { U.S.F.S. MH-5C }\end{array}$ & $\begin{array}{c}\text { TFSC FIELD REPRESENTATIVE } \\
\text { R. P. GILLESPIE }\end{array}$ \\
\hline
\end{tabular}

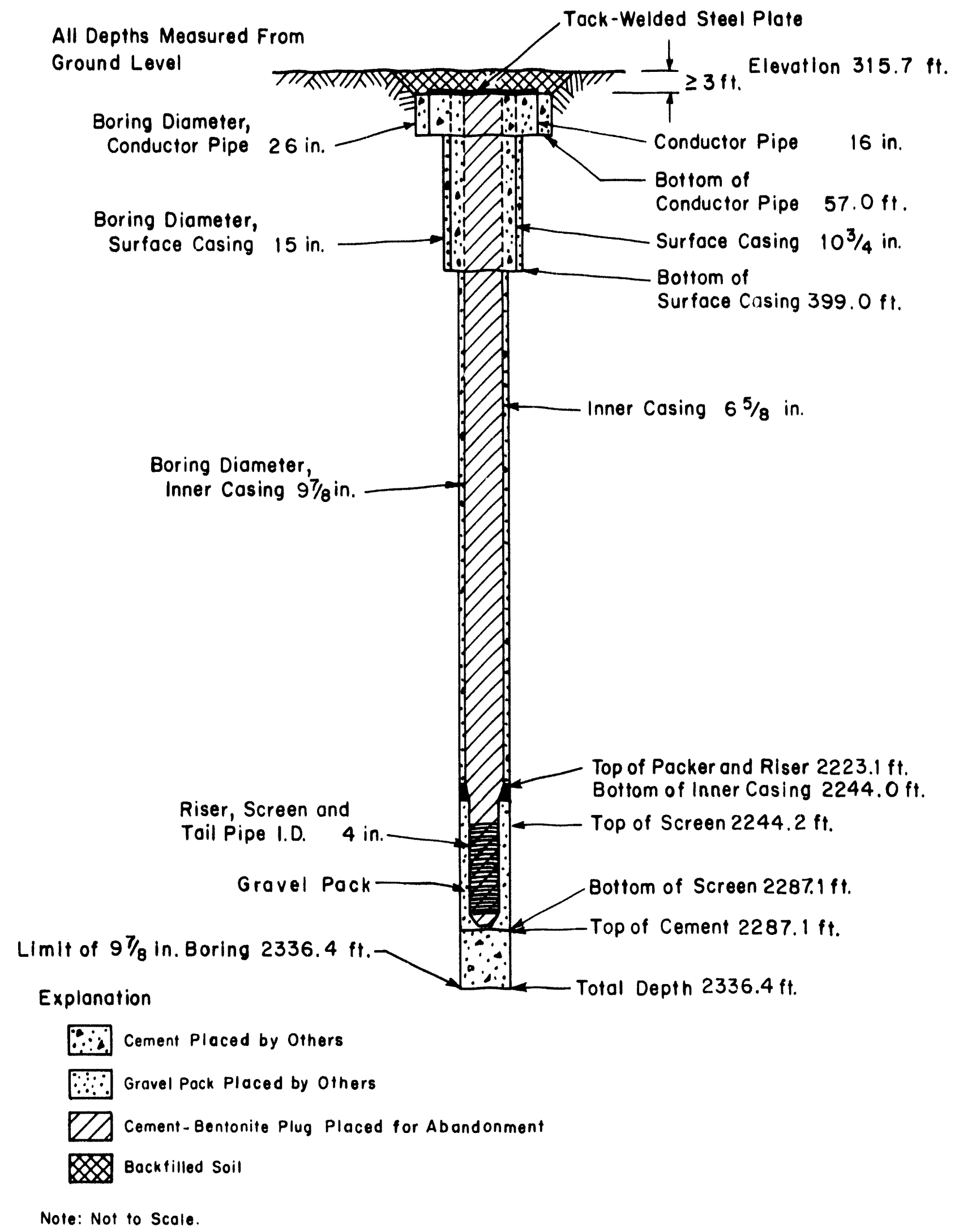


BOREHOLE COMPLETION SKETCH

\begin{tabular}{|l|l|l|l|}
\hline \multicolumn{2}{|c|}{ CLIENT DOE / SRPO } & J.0. No. 17500.25 & DATE $10-19-88$ \\
\hline $\begin{array}{l}\text { STATE } \\
\text { MISSISSIPPI }\end{array}$ & $\begin{array}{c}\text { WELL NAME E NUMEER } \\
\text { U.S.F.S. MH-5D }\end{array}$ & $\begin{array}{c}\text { TFSC FIELD REPRESENTATIVE } \\
\text { R.P. GILLESPIE }\end{array}$ \\
\hline
\end{tabular}

All Depths Measured From Ground Level

Tock-Welded Steel Plate

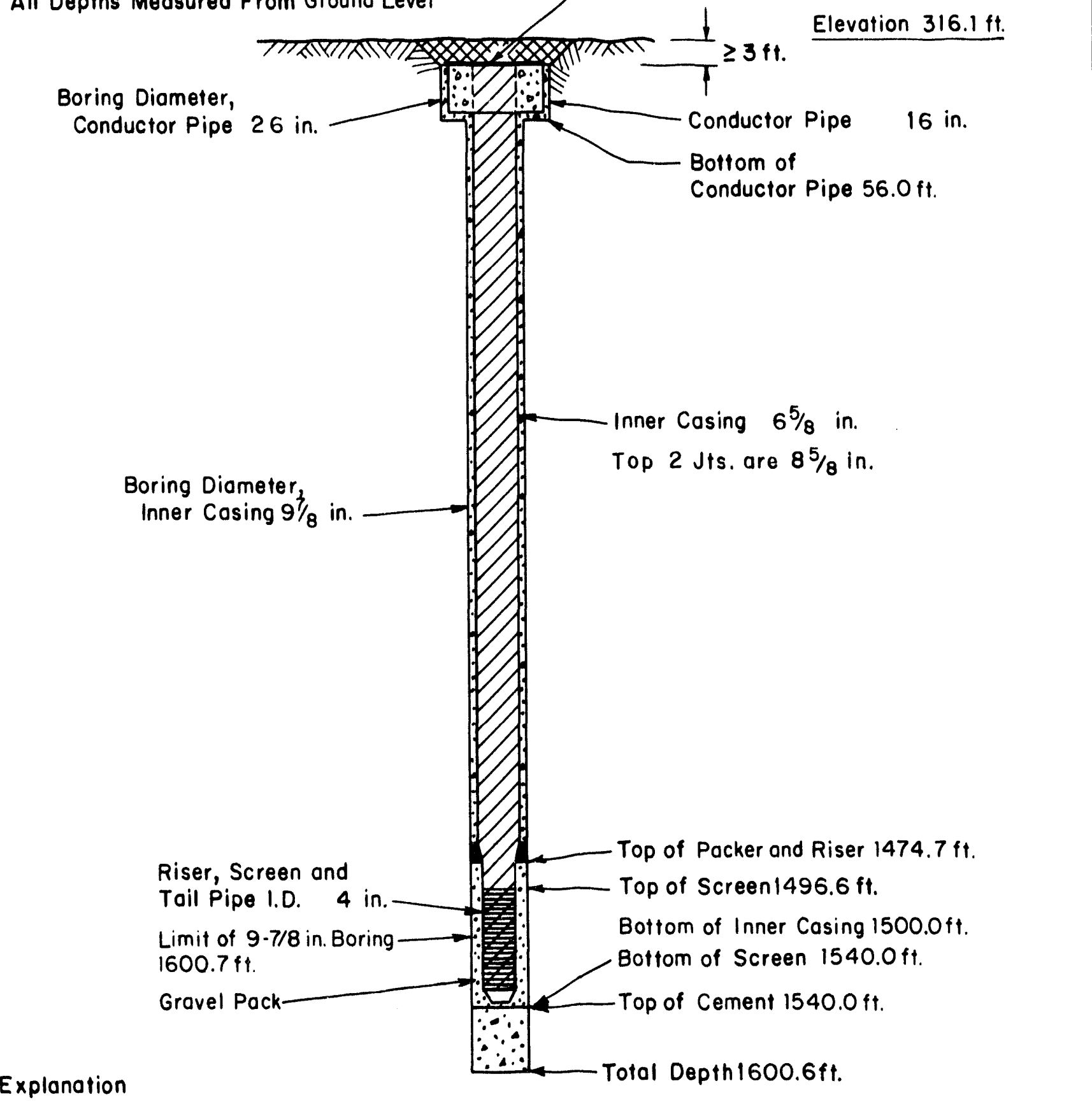

$\therefore \therefore$ Cement Placed by Others

$\because \because$ Gravel Pack Placed by Others

ZCement-Bentonite Plug Placed for Abandonment

Backfilled Soil

Note: Not to Scale 


\section{BOREHOLE COMPLETION SKETCH}

\begin{tabular}{|l|l|l|l|}
\hline $\begin{array}{l}\text { CLIENT DOE / SRPO } \\
\text { STATE }\end{array}$ & $\begin{array}{l}\text { J. No. } 17500.25 \\
\text { DATE } \\
\text { MISSISSIPPI }\end{array}$ & $\begin{array}{r}\text { TFSC FIELD REPRESENTATIVE } \\
\text { D. KISTNER / H. ABEDI }\end{array}$ \\
\hline
\end{tabular}

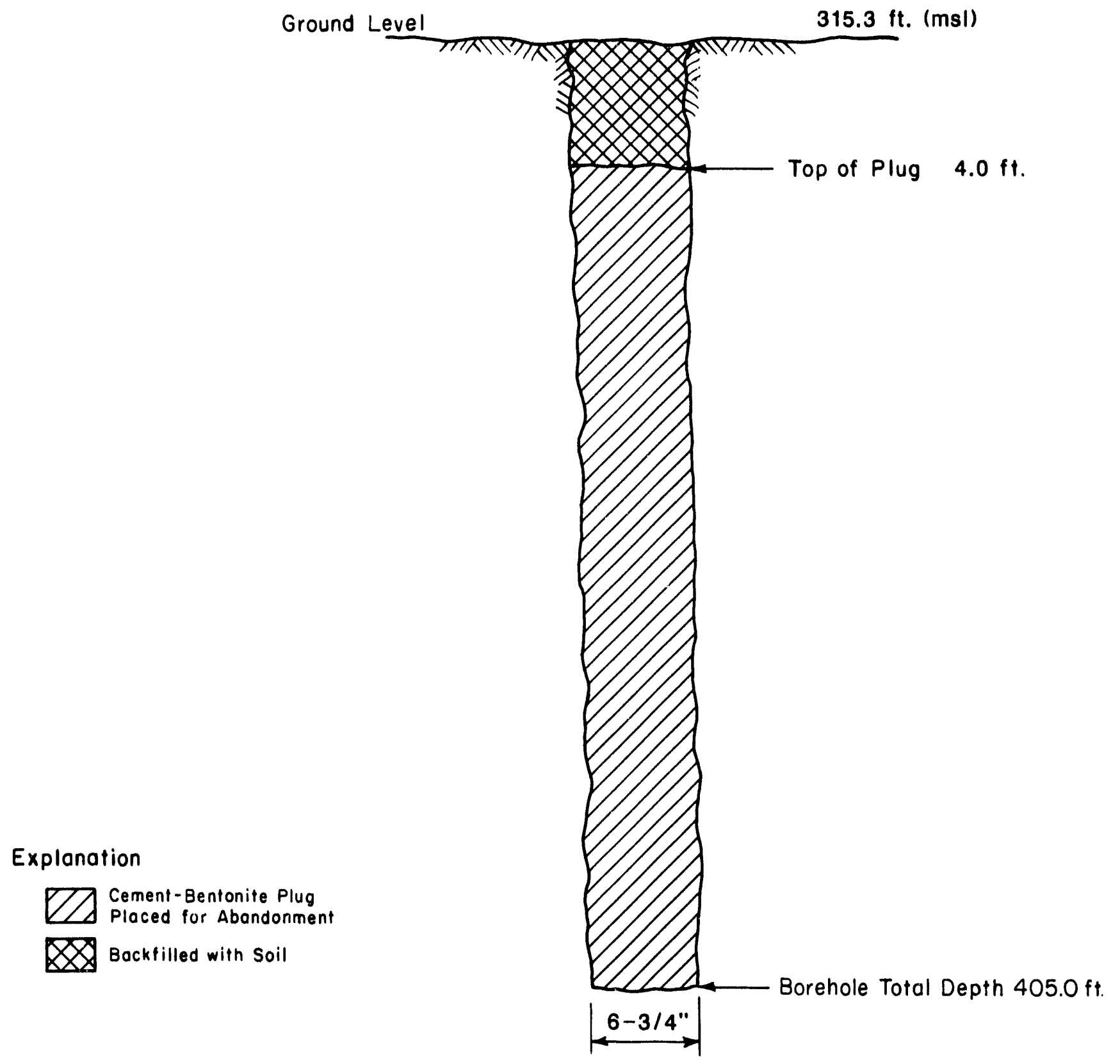

Borehole Diameter

Note:

Not to scale.

All depths measured from Ground Level Elevation $315.3 \mathrm{ft}$. 
BOREHOLE COMPLETION SKETCH

\begin{tabular}{|l|l|l|l|}
\hline $\begin{array}{l}\text { CLIENT DOE / SRPO } \\
\text { STATE }\end{array}$ & $\begin{array}{l}\text { J.0. No. } 17500.25 \\
\text { MISSISSIPPI }\end{array}$ & $\begin{array}{c}\text { WELL NAME E NUMBER } \\
\text { ANDERSON MH-6A }\end{array}$ & $\begin{array}{c}\text { TFSC FIELD REPRESENTATIVE } \\
\text { R.P. GILLESPIE }\end{array}$ \\
\hline
\end{tabular}

All Depths Measured From Ground Level

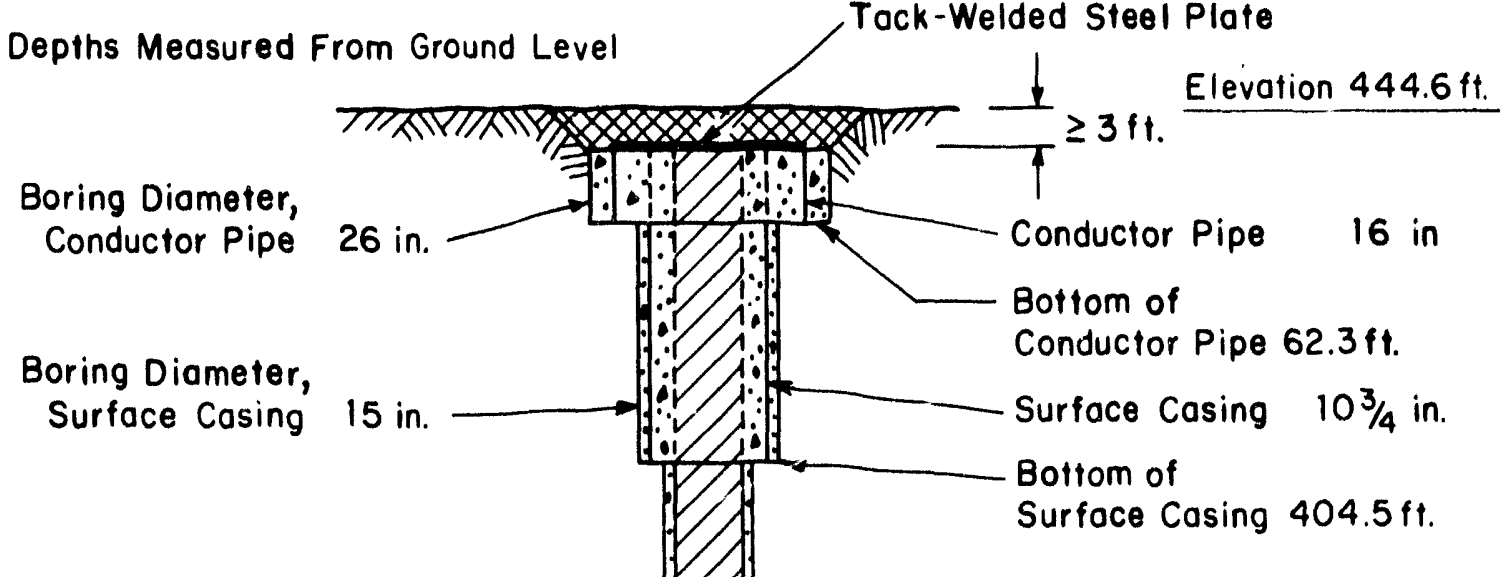

Boring Diameter,

Inner Cosing 9\% in.

Explanation

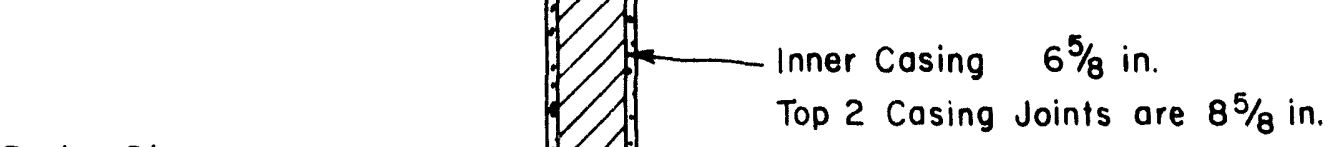

$\because \therefore$ Cement Placed by Others

$\because \because \because$ Gravel Pack Placed by Others

PCement-Bentonite Plug Placed for Abandonment

Backfilled Soil

Note: Not to Scale. 
BOREHOLE COMPLETION SKETCH

\begin{tabular}{|l|l|l|l|}
\hline \multicolumn{2}{|c|}{ CLIENT DOE / SRPO } & $\begin{array}{l}\text { J.0. No. } 17500.25 \\
\text { DATE }\end{array}$ & $10-11-88$ \\
\hline $\begin{array}{l}\text { STATE } \\
\text { MISSISSIPPI }\end{array}$ & $\begin{array}{c}\text { WELL NAME \& NUMBER } \\
\text { ANDERSON MH-6B }\end{array}$ & $\begin{array}{c}\text { TFSC FIELD REPRESENTATIVE } \\
\text { R.P. GILLESPIE }\end{array}$ \\
\hline
\end{tabular}

All Depths Measured From Ground Level

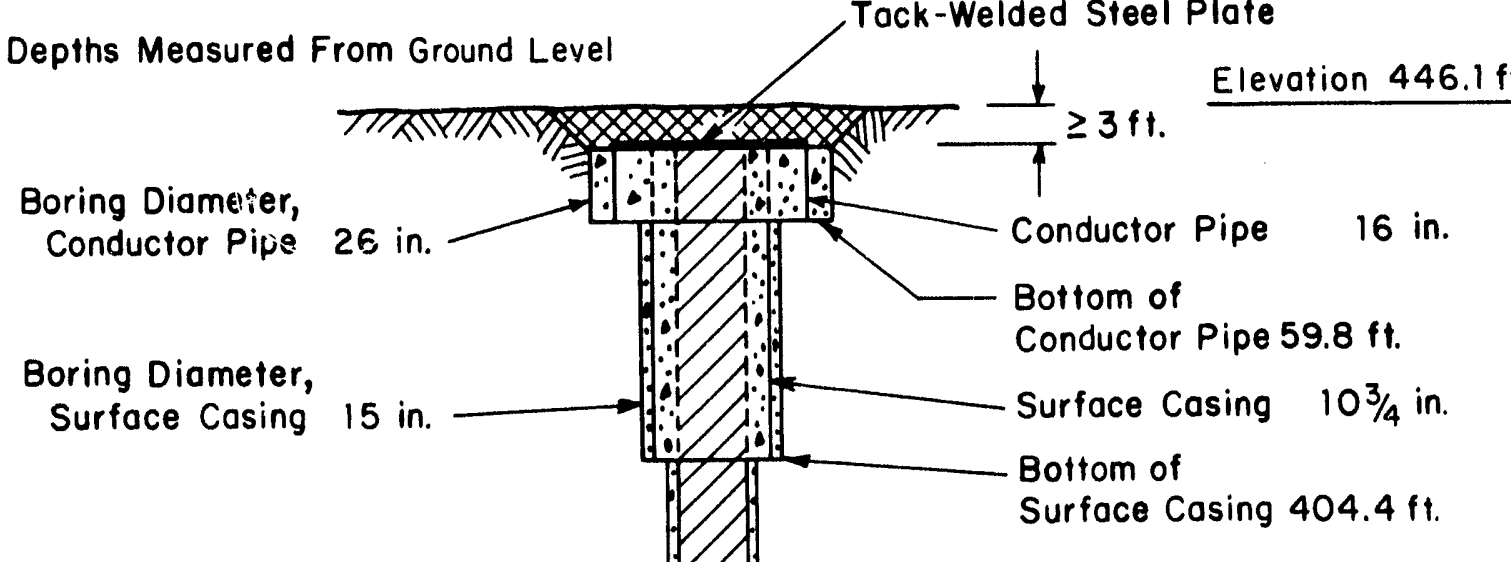

Boring Diometer,

Inner Cosing 97/8 in.

Inner Casing 65/8 in.

Top 2 Cosing Joints are $8 \frac{5}{8}$ in.

Explanation

Top of Packer and Riser 2702.4ft.

Bottom of Inner Casing $2720.7 \mathrm{ft}$.

Top of Screen $2724.2 \mathrm{ft}$.

Bottom of Screen $2756.3 \mathrm{ft}$.

Botfom of Tail Pipe 2762.6ft. $\rightarrow:$

$\because \therefore$ Cement Placed by Others

$\because \because \because$ Gravel Pack Placed by Others

DCement-Bentonite Plug Placed for Abandonment

BQS Backfilled Soil

Note: Not to Scale. 
BOREHOLE COMPLETION SKETCH

\begin{tabular}{|l|c|c|c|}
\hline \multicolumn{2}{|c|}{ CLIENT DOE / SRPO } & J.0. No. 17500.25 & DATE $10-11-88$ \\
\hline $\begin{array}{c}\text { STATE } \\
\text { MISSISSIPPI }\end{array}$ & $\begin{array}{c}\text { WELL NAME E NUMBER } \\
\text { ANDERSON MH-6C }\end{array}$ & $\begin{array}{c}\text { TFSC FIELD REPRESENTATIVE } \\
\text { R.P. GILLESPIE }\end{array}$ \\
\hline
\end{tabular}

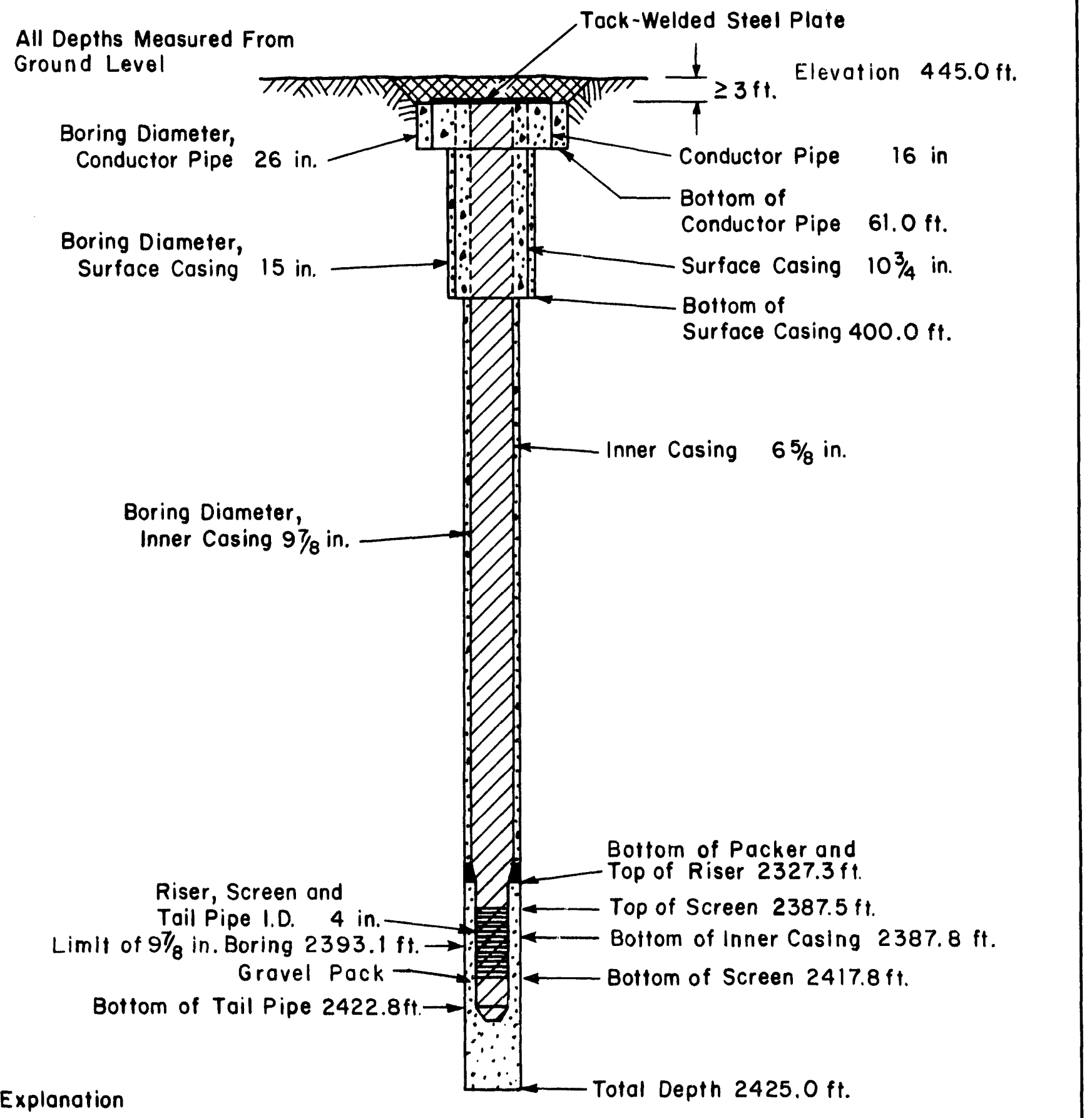

$\because \therefore$ Cement Placed by Others

$\because \because$ Gravel Pack Placed by Others

27 cement-Bentonite Plug Placed for Abandonment

Backfilled Soil

Note: Not to Scale. 
BOREHOLE COMPLETION SKETCH

\begin{tabular}{|c|c|c|c|}
\hline \multicolumn{2}{|c|}{ CLIENT DOE / SRPO } & J.0. No. 17500.25 & DATE $10-11-88$ \\
\hline $\begin{array}{c}\text { STATE } \\
\text { MISSISSIPPI }\end{array}$ & $\begin{array}{c}\text { WELL NAME \& NUMBER } \\
\text { ANDERSON MH-60 }\end{array}$ & $\begin{array}{c}\text { TFSC FIELD REPRESENTATIVE } \\
\text { R.P. GILLESPIE }\end{array}$ \\
\hline
\end{tabular}

All Depths Measured From Ground Level

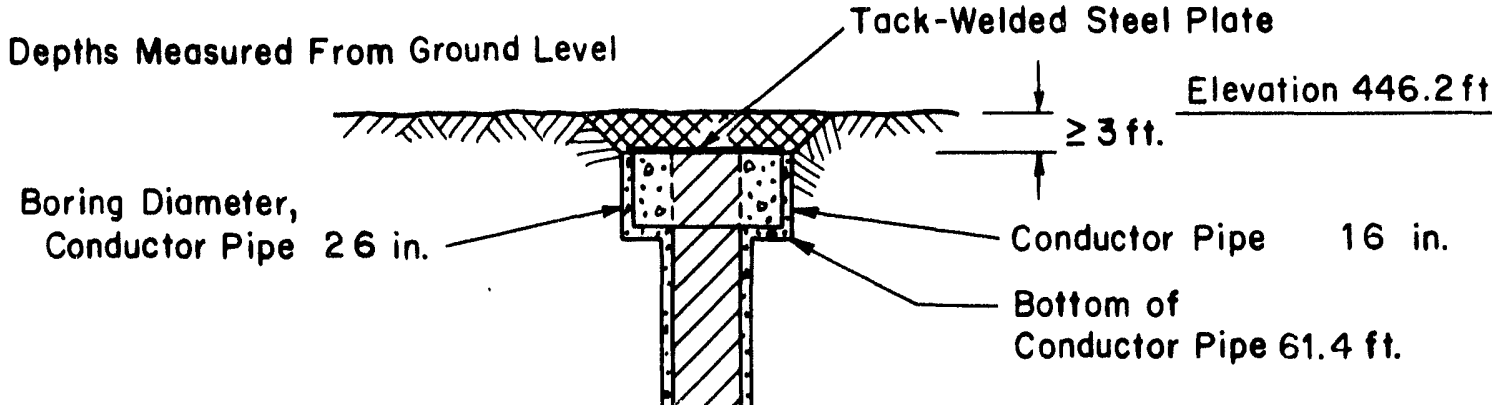

Boring Diameter, Inner Cosing $9 \% 8$ in.

Explanation

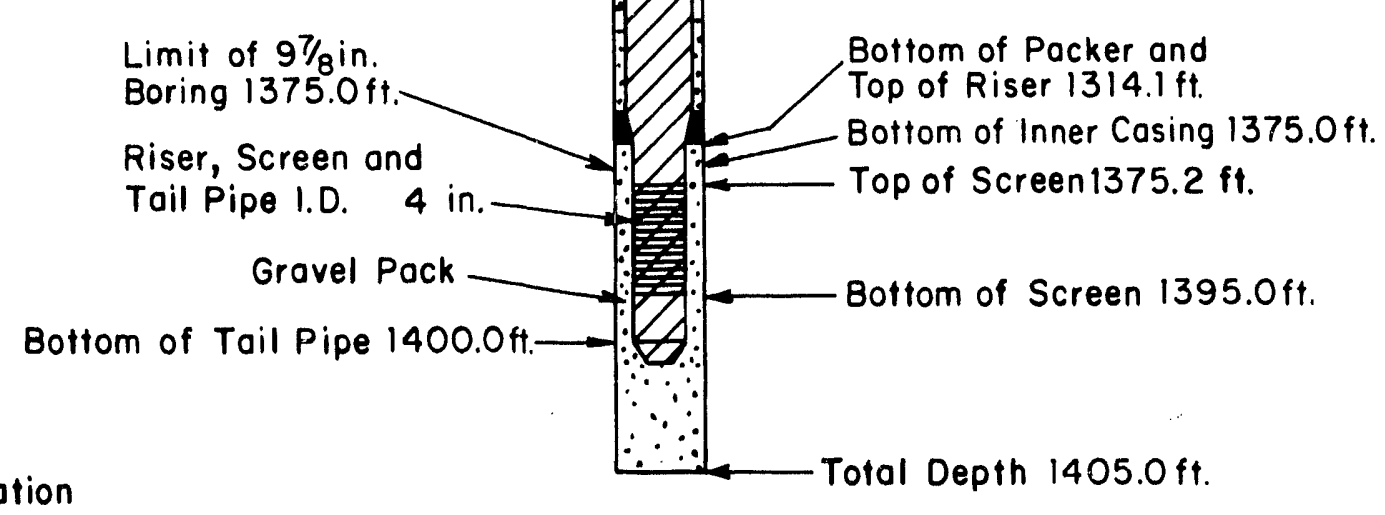

$\therefore 0$ Cement Placed by Others

$\because \because$ Grovel Pack Placed by Others

Z2 Cement-Bentonite Plug Placed For Abandonment

Backfilled Soil

Note: Not to Scole 
BOREHOLE COMPLETION SKETCH

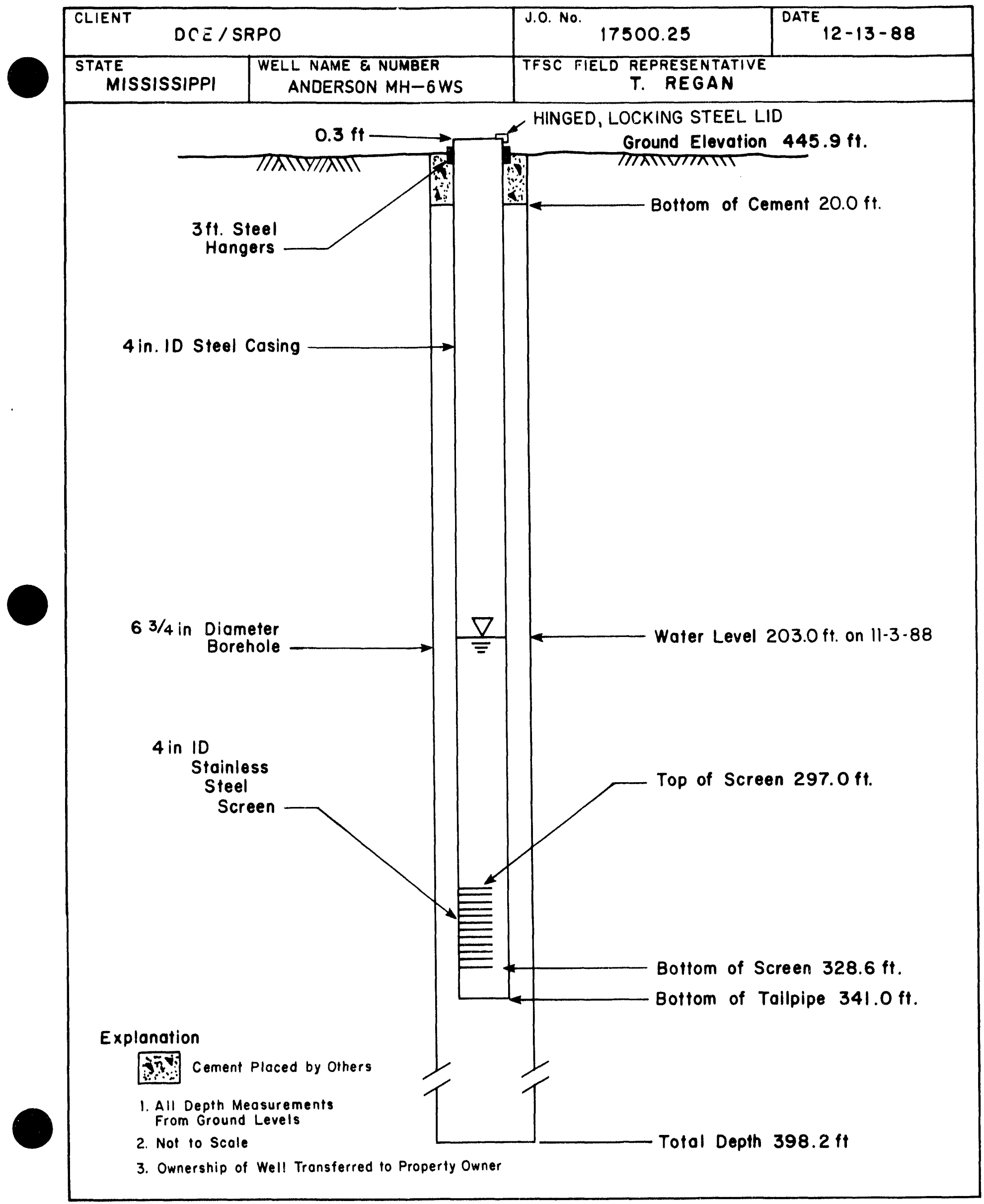


BOREHOLE COMPLETION SKETCH

\begin{tabular}{|l|l|l|l|}
\hline CLIENT DOE / SRPO & J.0. No. 17500.25 & DATE & $10-14-88$ \\
\hline $\begin{array}{c}\text { STATE } \\
\text { MISSISSIPPI }\end{array}$ & $\begin{array}{c}\text { WELL NAME E NUMBER } \\
\text { BEARD MH-7A }\end{array}$ & $\begin{array}{r}\text { TFSC FIELD REPRESENTATIVE } \\
\text { R.P. GILLESPIE }\end{array}$ \\
\hline
\end{tabular}

All Depths Measured From Ground Level

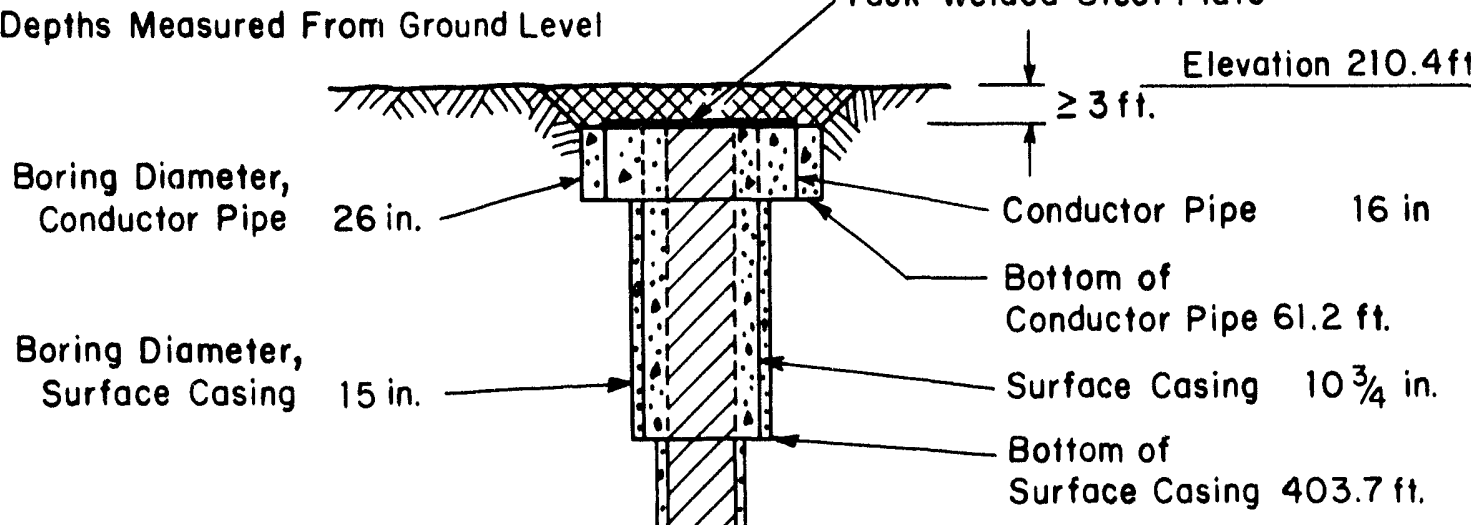

Boring Diameter,

Inner Casing $9 \frac{7}{8}$ in.

\section{Explanation}

Cast-Iron Cement

Retainer at $2609 \mathrm{ft}$.

Inner Casing $6 \frac{5}{8}$ in.

$\therefore \therefore$ Cement Placed by Others

$\because \because$ Gravel Pack Place by Others

ZCement-Bentonite Plug Placed For Abondonment

Backtilled Soil

Note: Not to Scole. 
BOREHOLE COMPLETION SKETCH

\begin{tabular}{|l|c|c|c|}
\hline CLIENT DOE/SRPO & J.0. No. 17500.25 & DATE $10-14-88$ \\
\hline $\begin{array}{c}\text { STATE } \\
\text { MISSISSIPPI }\end{array}$ & $\begin{array}{c}\text { WELL NAME E NUMBER } \\
\text { BEARD MH-7B }\end{array}$ & $\begin{array}{c}\text { TFSC FIELD REPRESENTATIVE } \\
\text { R.P. GILLESPIE }\end{array}$ \\
\hline
\end{tabular}
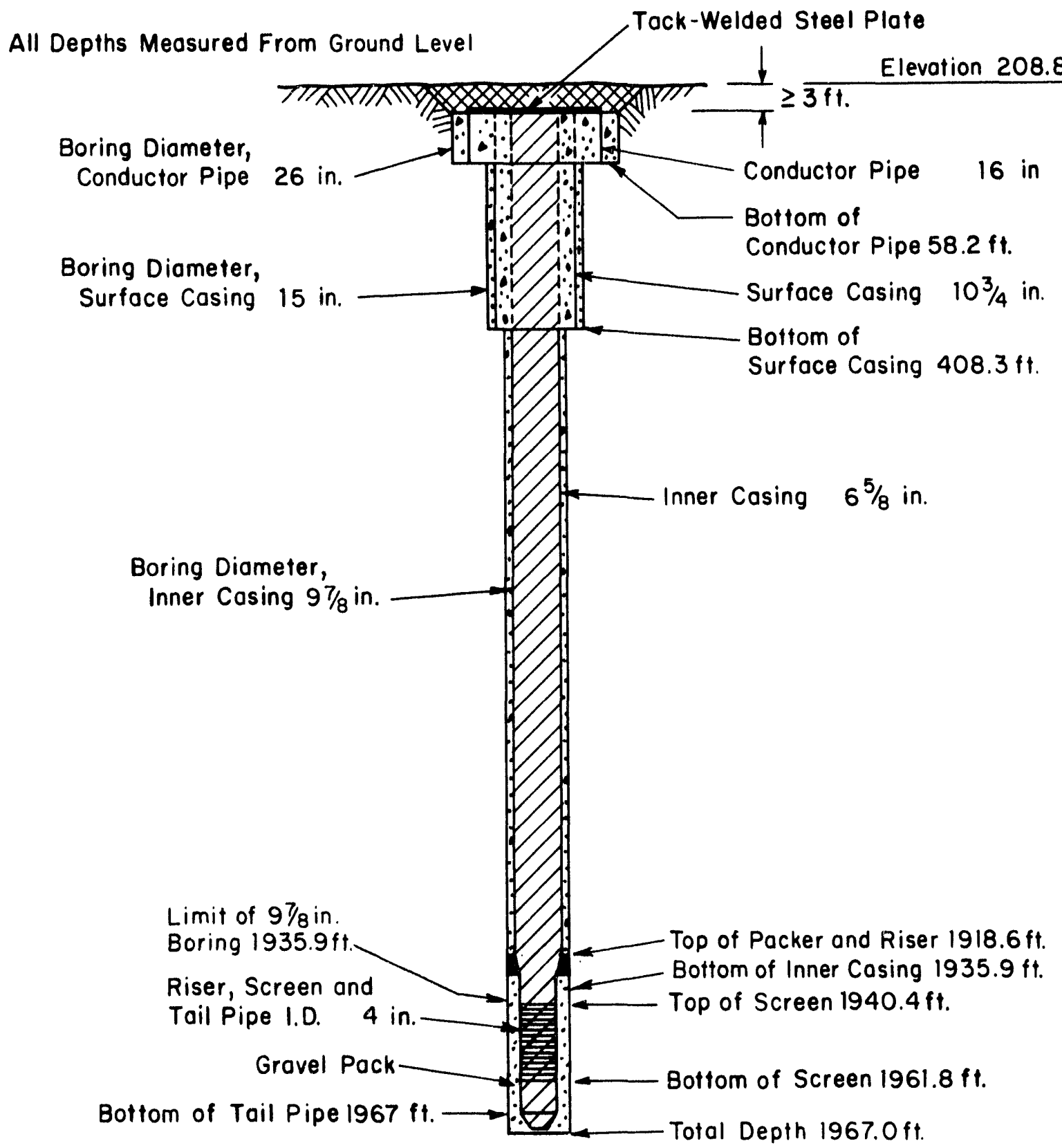

\section{Explanation}

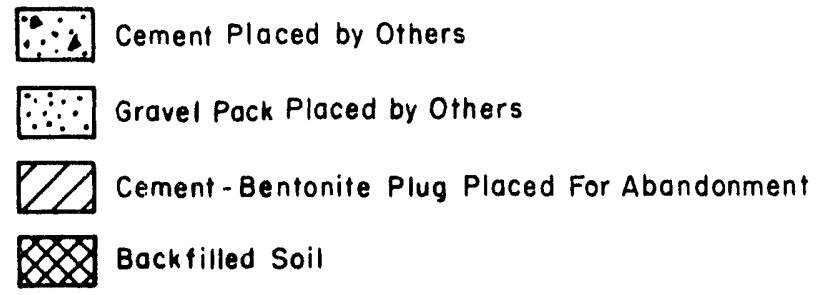

Note: Not to Scale. 
BOREHOLE COMPLETION SKETCH

\begin{tabular}{|l|l|l|l|}
\hline CLIENT DOE / SRPO & J.0. No. 17500.25 & DATE $10-14-88$ \\
\hline $\begin{array}{c}\text { STATE } \\
\text { MISSISSIPPI }\end{array}$ & $\begin{array}{l}\text { WELL NAME \& NUMBER } \\
\text { BEARD MH-7C }\end{array}$ & $\begin{array}{c}\text { TFSC FIELD REPRESENTATIVE } \\
\text { R.P. GILLESPIE }\end{array}$ \\
\hline
\end{tabular}

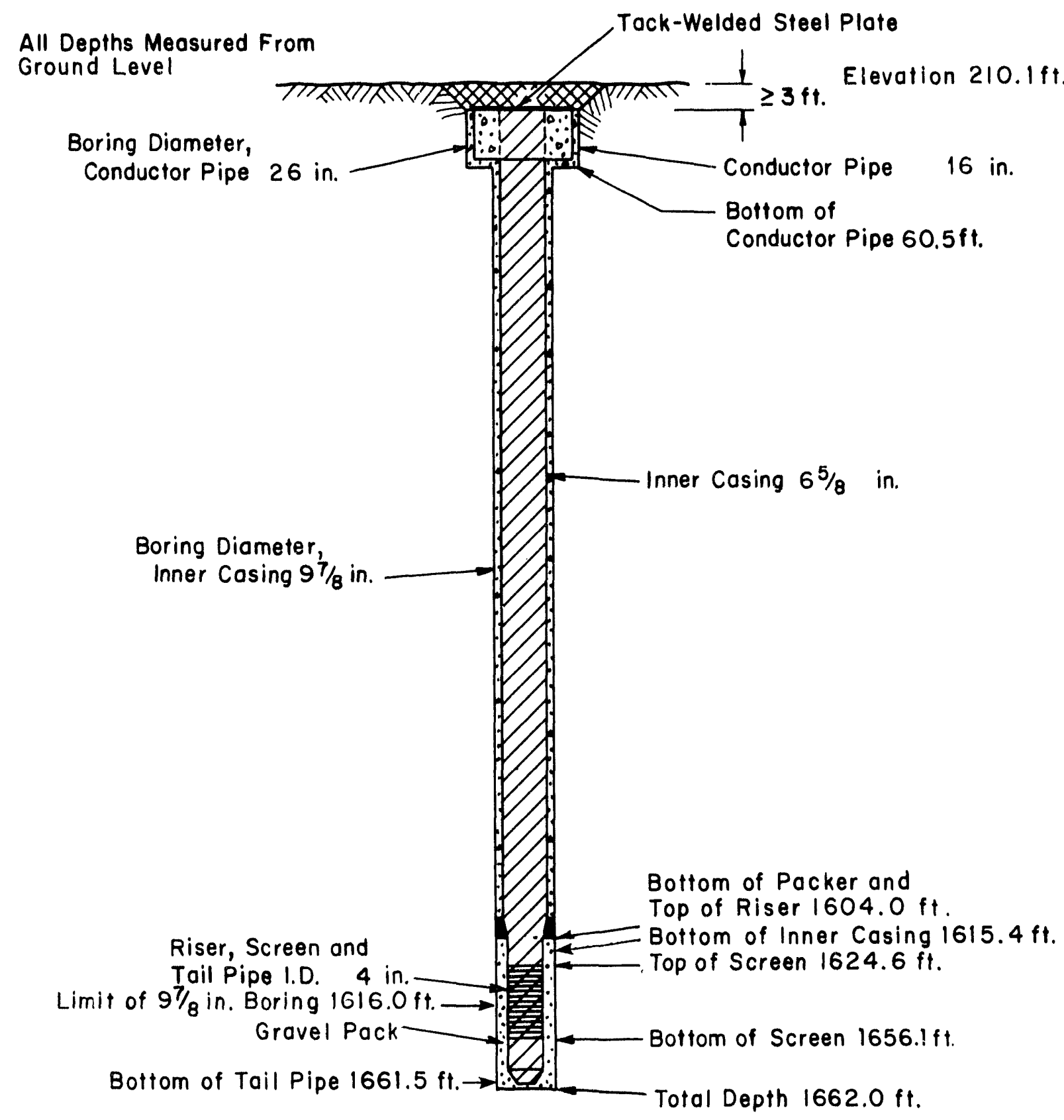

\section{Explanation}

$\because \therefore$ Cement Placed by Others

$\because \therefore$ Gravel Pack Placed by Others

D Cement-Bentonite Plug Placed for Abandonment

888 Backfilled Soil

Note: Not to Scale 
BOREHOLE COMPLETION SKETCH

\begin{tabular}{|c|c|c|c|}
\hline \multicolumn{2}{|c|}{ CLIENT DOE / SRPO } & J.0. No. 17500.25 & DATE 10-14-88 \\
\hline $\begin{array}{c}\text { STATE } \\
\text { MISSISSIPPI }\end{array}$ & $\begin{array}{c}\text { WELL NAME E NUMBER } \\
\text { BEARD MH-7D }\end{array}$ & $\begin{array}{r}\text { TFSC FIELD REPRESENTATIVE } \\
\text { R.P. GILLESPIE }\end{array}$ \\
\hline
\end{tabular}

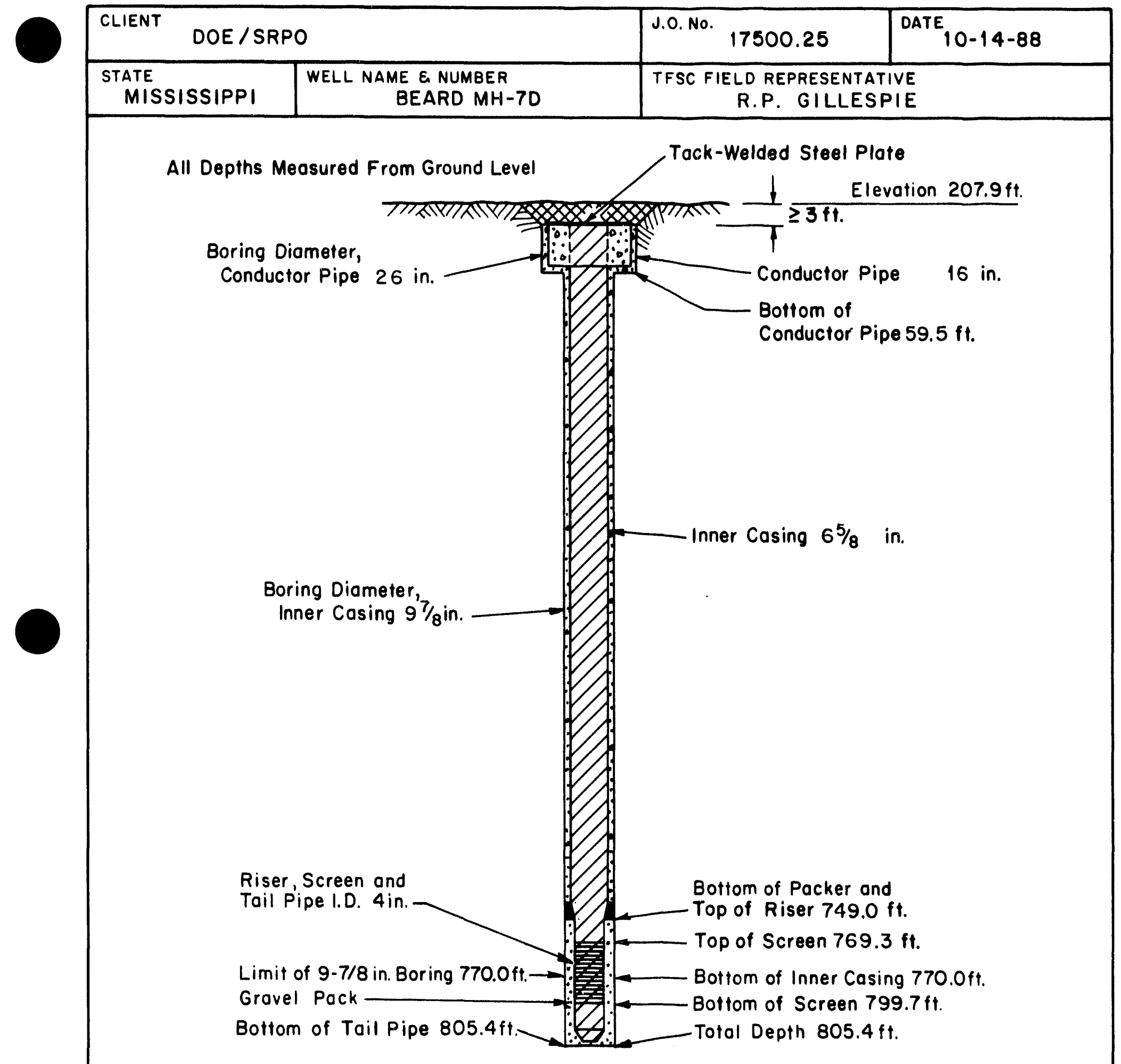

\section{Explanation}

$\therefore \therefore$ Cement Placed by Others

$\because \because$ Gravel Pack Placed by Others

Z2 Cement-Bentonite Plug Placed For Abandonment

Backfilled Soil 
BOREHOLE COMPLETION SKETCH

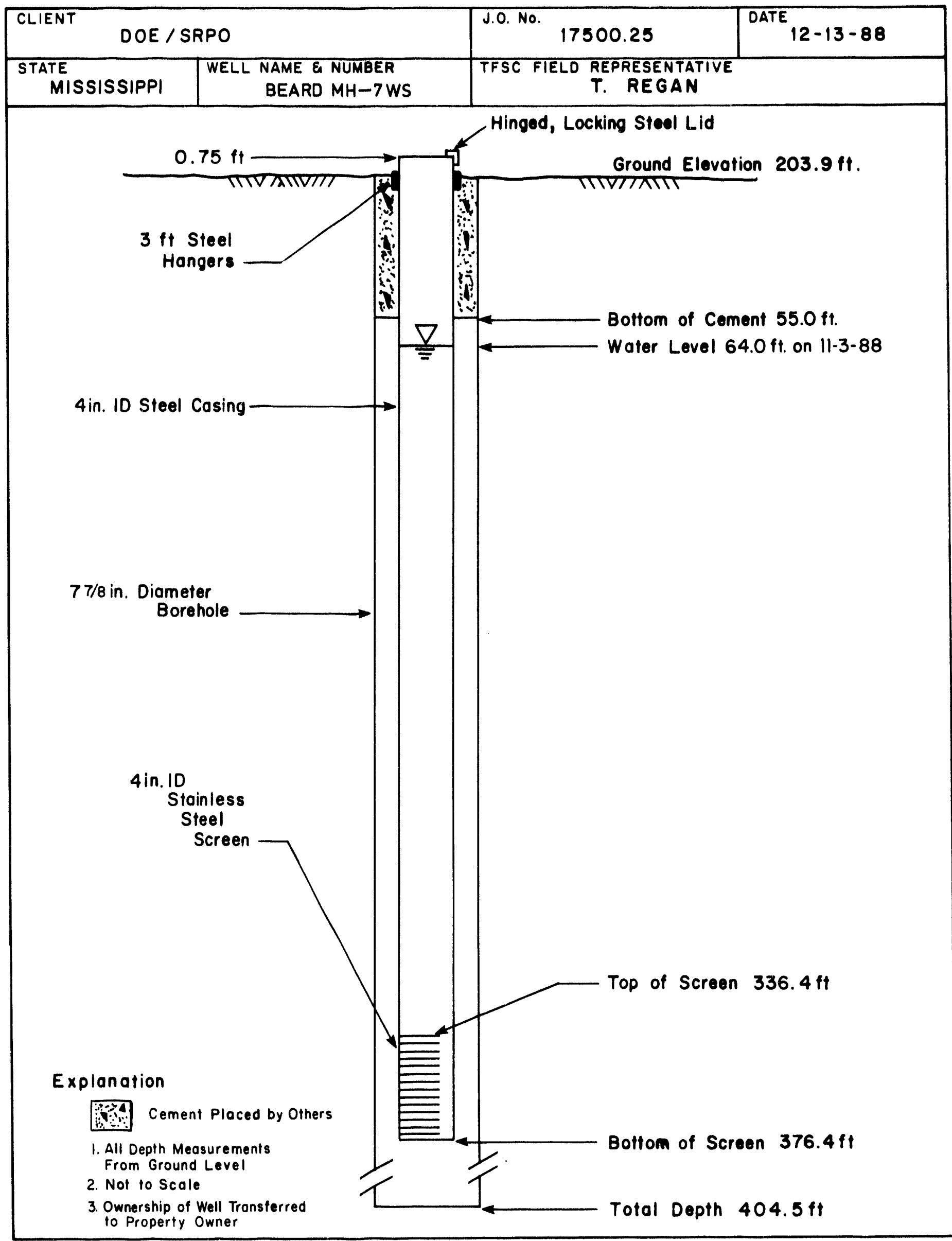


BOREHOLE COMPLETION SKETCH

\begin{tabular}{|l|c|c|c|}
\hline CLIENT DOE / SRPO & J.0. No. 17500.25 & DATE 10-14-88 \\
\hline $\begin{array}{l}\text { STATE } \\
\text { MISSISSIPPI }\end{array}$ & WELL NAME E NUMBER \\
WALKER MH-8A & TFSC FIELD REPRESENTATIVE \\
R.P. GILLESPIE
\end{tabular}

All Depths Meosured From Ground Level

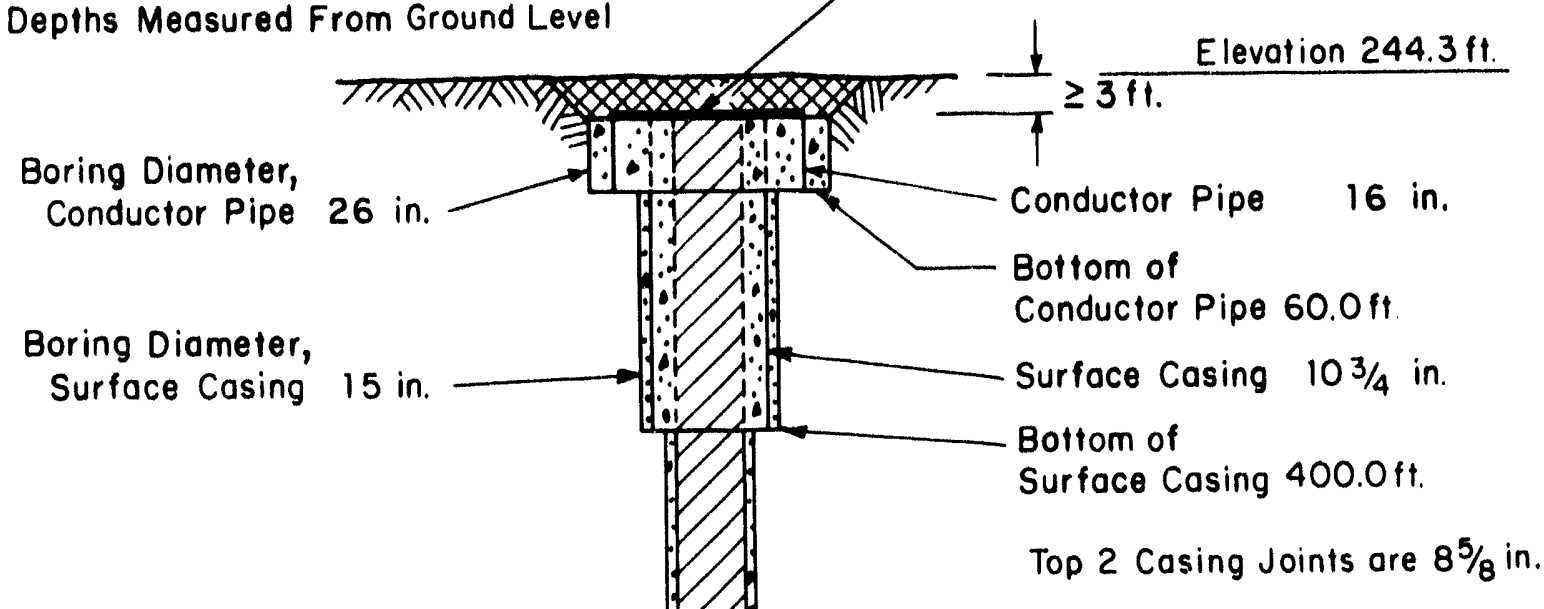

Boring Diameter, Inner Cosing 97/8 in.

Inner Cosing $6 \frac{5}{8}$ in.

Explanation

Bottom of Packer and

Top of Riser $3116.7 \mathrm{ft}$.

Riser, Screen and

Tail Pipe I.D. 4 in.

Top of Screen $3179.4 \mathrm{ft}$.

Gravel Pack Bottom of Inner Casing $3180.0 \mathrm{ft}$. Bottom of Screen $3190.0 \mathrm{ft}$.

Bottom of Tail Pipe 3194.4ft. Top of Cement Plug $3200.0 \mathrm{ft}$.

$\because \because \therefore$

$\therefore \therefore$ Cement Placed by Others

$\because \therefore$ Gravel Pack Placed by Others

D Cement - Bentonite Plug Placed For Abandonment

Backtilled Soil

Note: Not to Scale. 
BOREHOLE COMPLETION SKETCH

\begin{tabular}{|l|l|l|l|}
\hline $\begin{array}{l}\text { CLIENT DOE / SRPO } \\
\text { STATE } \\
\text { MISSISSIPPI }\end{array}$ & $\begin{array}{c}\text { W.0. No. } 17500.25 \\
\text { DATE NAME E NUMBER } \\
\text { WALKER MH-8B }\end{array}$ & $\begin{array}{c}\text { TFSC FIELD REPRESENTATIVE } \\
\text { W. C. BRANDON }\end{array}$ \\
\hline
\end{tabular}

Existing Ground Surfoce

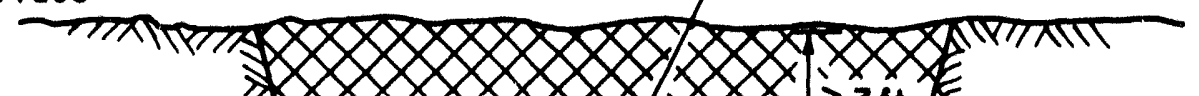

Explanation

$\because \because$. Cement Placed by Others
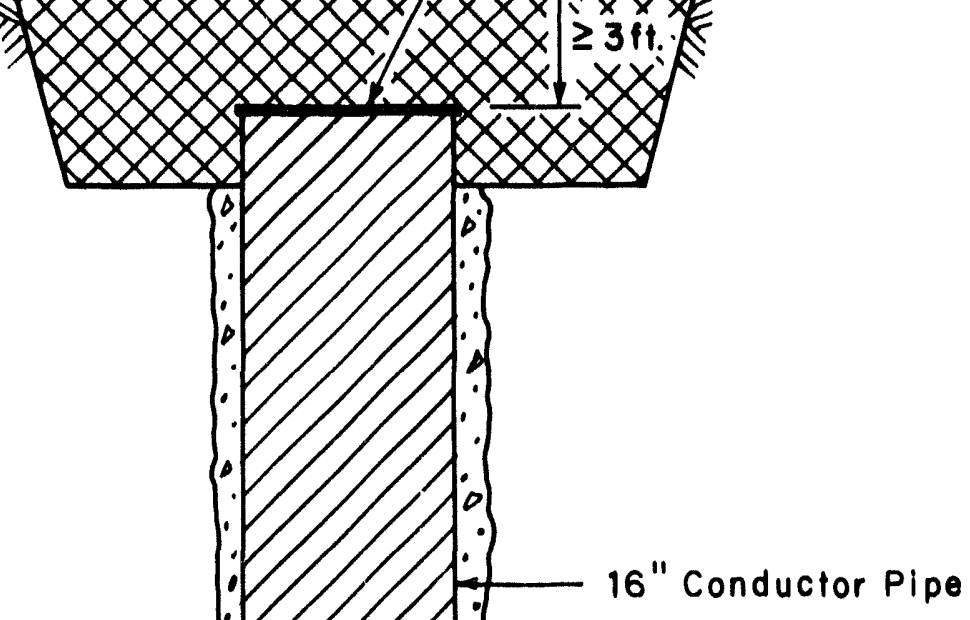

7 Cement - Bentonite Plug

Placed for Abandonment

$\bigotimes$ Backfilled Soil

Note:

Not to scale.

All depths meosured from

Existing Ground Surface 
BOREHOLE COMPLETION SKETCH

\begin{tabular}{|l|l|l|l|}
\hline CLIENT DOE/SRPO & $\begin{array}{l}\text { J.0. No. } 17500.25 \\
\text { DATE }\end{array}$ & $10-14-88$ \\
\hline $\begin{array}{c}\text { STATE } \\
\text { MISSISSIPPI }\end{array}$ & $\begin{array}{c}\text { WELL NAME E NUMBER } \\
\text { WALKER MH-8C }\end{array}$ & $\begin{array}{c}\text { TFSC FIELD REPRESENTATIVE } \\
\text { R.P. GILLESPIE }\end{array}$ \\
\hline
\end{tabular}

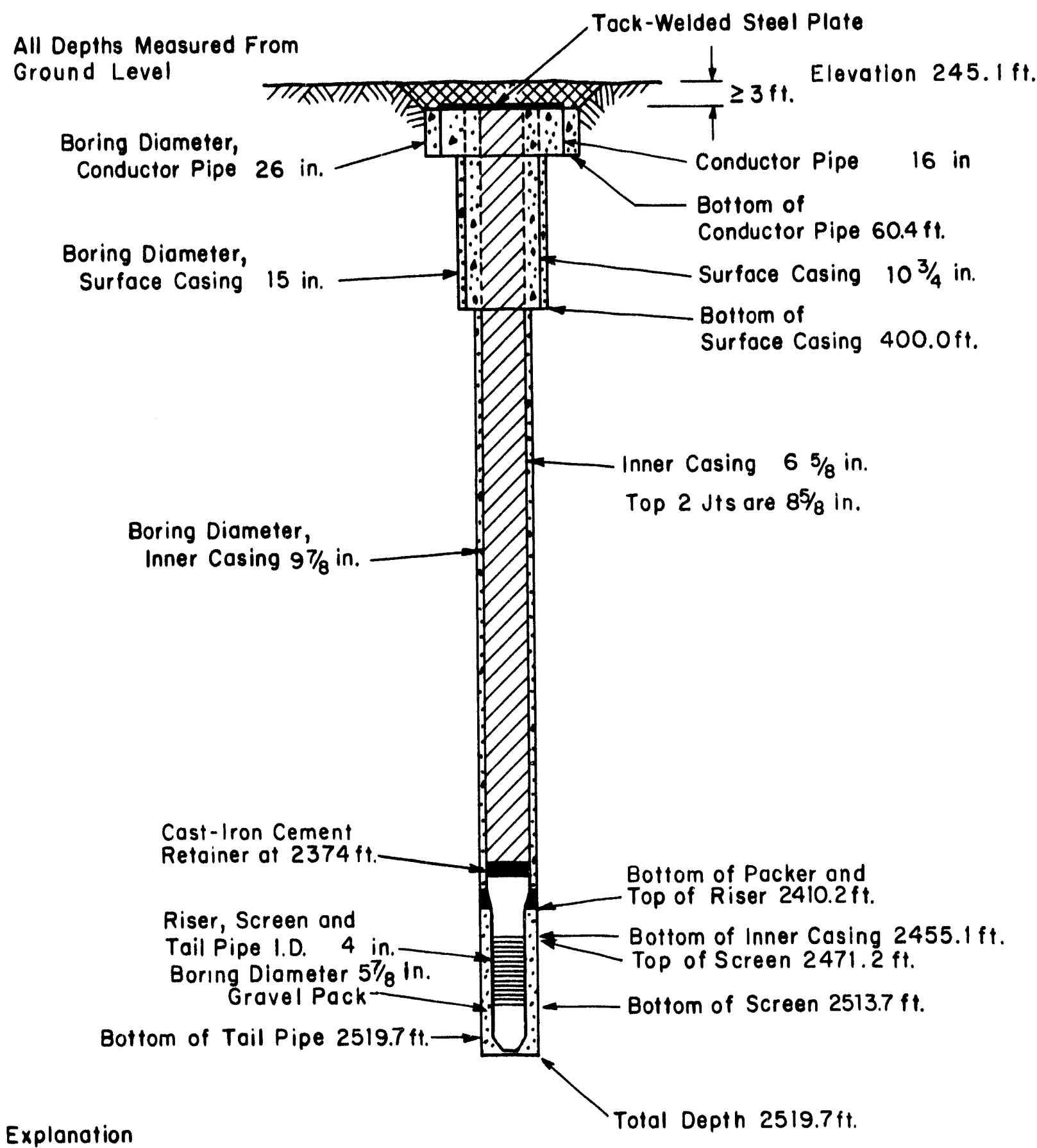

$\because \therefore$ Cement Placed by Others

$\because \therefore$ Gravel Pack Placed by Others

D Cement-Bentonite Plug Placed for Abandonment

Backfilled Soil

Note: Not to Scale. 
BOREHOLE COMPLETION SKETCH

\begin{tabular}{|l|l|l|l|}
\hline $\begin{array}{l}\text { CLIENT DOE / SRPO } \\
\text { STATE }\end{array}$ & $\begin{array}{l}\text { J.0. No. } 17500.25 \\
12-21-88\end{array}$ \\
MISSISSIPPI & $\begin{array}{l}\text { WELL NAME \& NUMBER } \\
\text { WALKER MH-8D }\end{array}$ & $\begin{array}{c}\text { TFSC FIELD REPRESENTATIVE } \\
\text { W.C. BRANDON }\end{array}$ \\
\hline
\end{tabular}

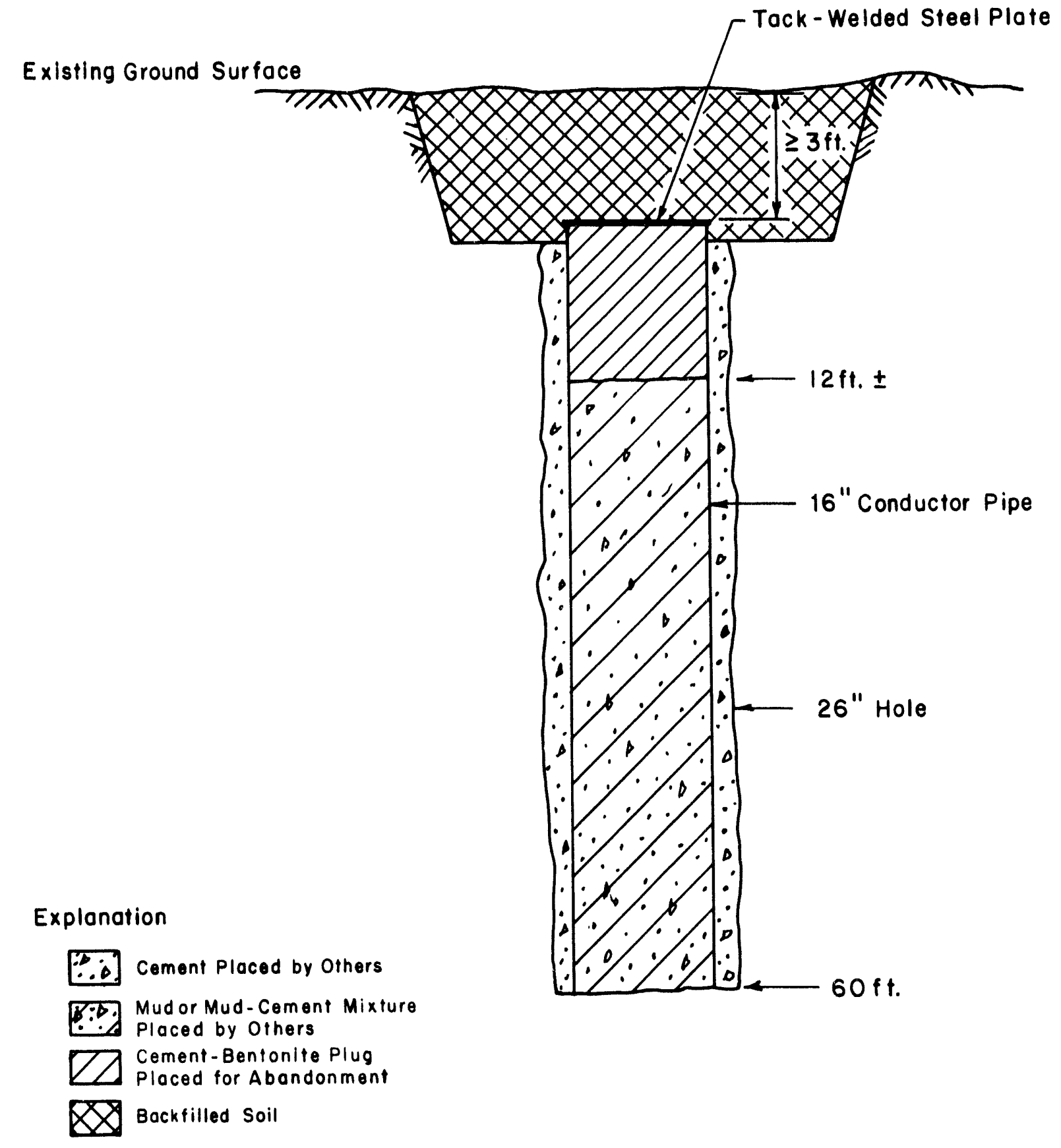

Note:

Not to scale.

All depths measured from

Existing Ground Surface 


\section{BOREHOLF COMPLETION SKETCH}

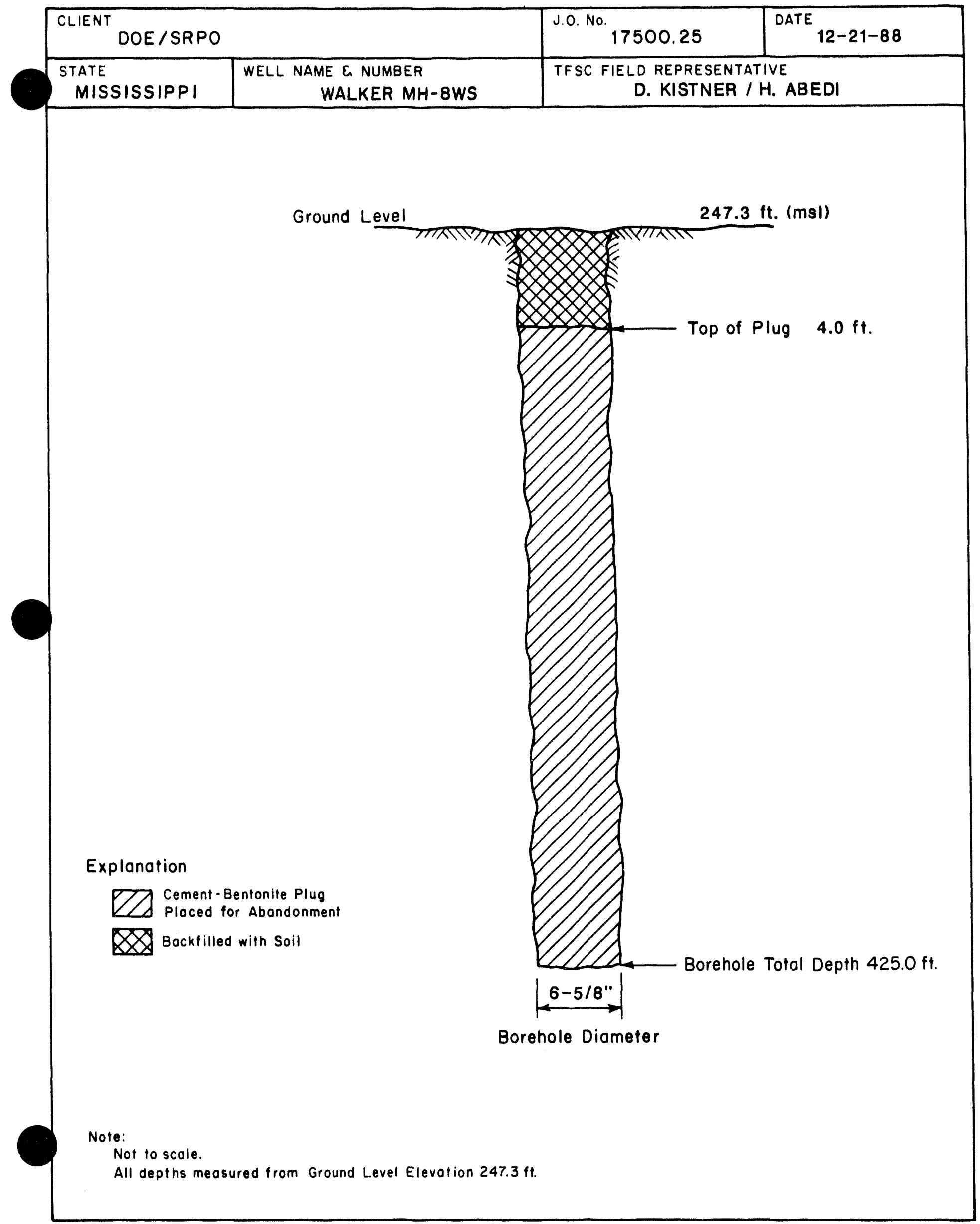


BOREHOLE COMPLETION SKETCH

\begin{tabular}{|l|c|c|c|}
\hline \multicolumn{2}{|c|}{ CLIENT DOE / SRPO } & $\begin{array}{l}\text { J.0. No. } 17500.25 \\
\text { DATE }\end{array}$ & 10-20-88 \\
\hline $\begin{array}{c}\text { STATE } \\
\text { MISSISSIPPI }\end{array}$ & $\begin{array}{c}\text { WELL NAME \& NUMBER } \\
\text { L.R.F.P. MRIG-9 }\end{array}$ & $\begin{array}{c}\text { TFSC FIELO REPRESENTATIVE } \\
\text { R.P. GI LLESPIE }\end{array}$ \\
\hline
\end{tabular}

All Depths Measured From Ground Level

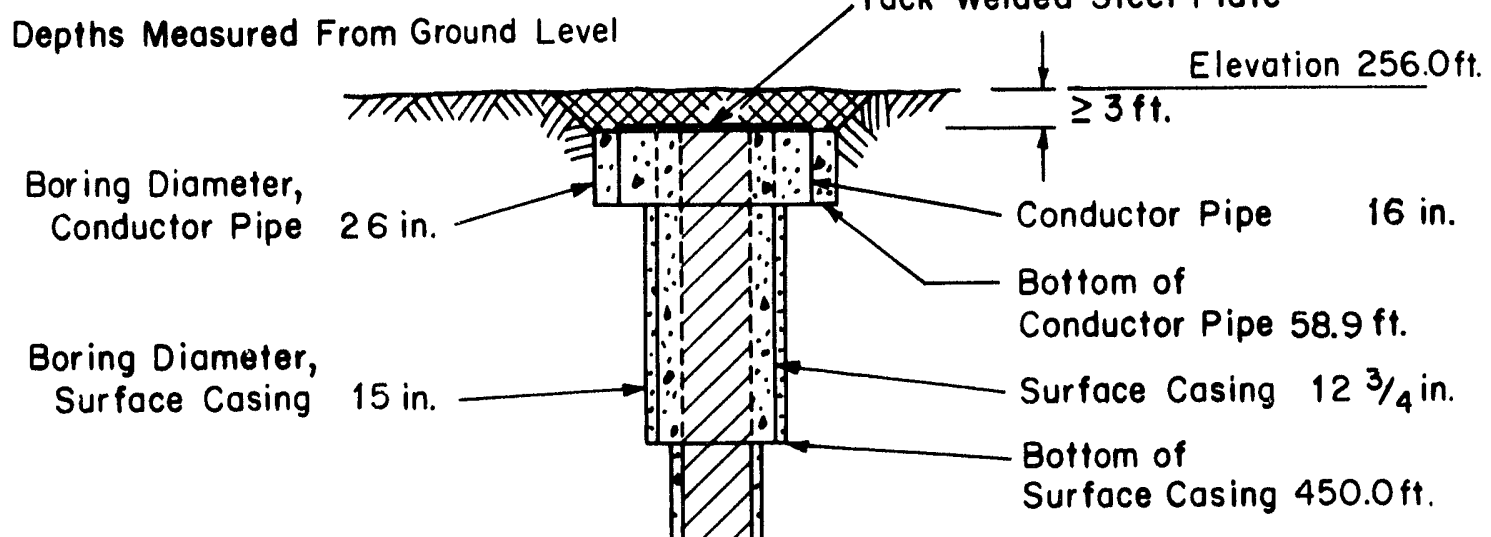

Boring Diameter, Inner Cosing $11 \mathrm{in}$.

\section{Explanation}

8 in. Cast Iron Cement Retainer at $525.6 \mathrm{ft}$.

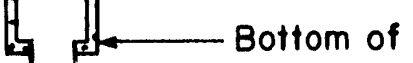
Inner Cosing 558.4ft.

Open Hole $73 / 16$ in. Inner Casing $8 \frac{5}{8}$ in.

$\because \because$ Cement Placed by Others

7 cement-Bentonite Plug Placed for Abandonment

88 Backfilled Soil 
BOREHOLE COMPLETION SKETCH

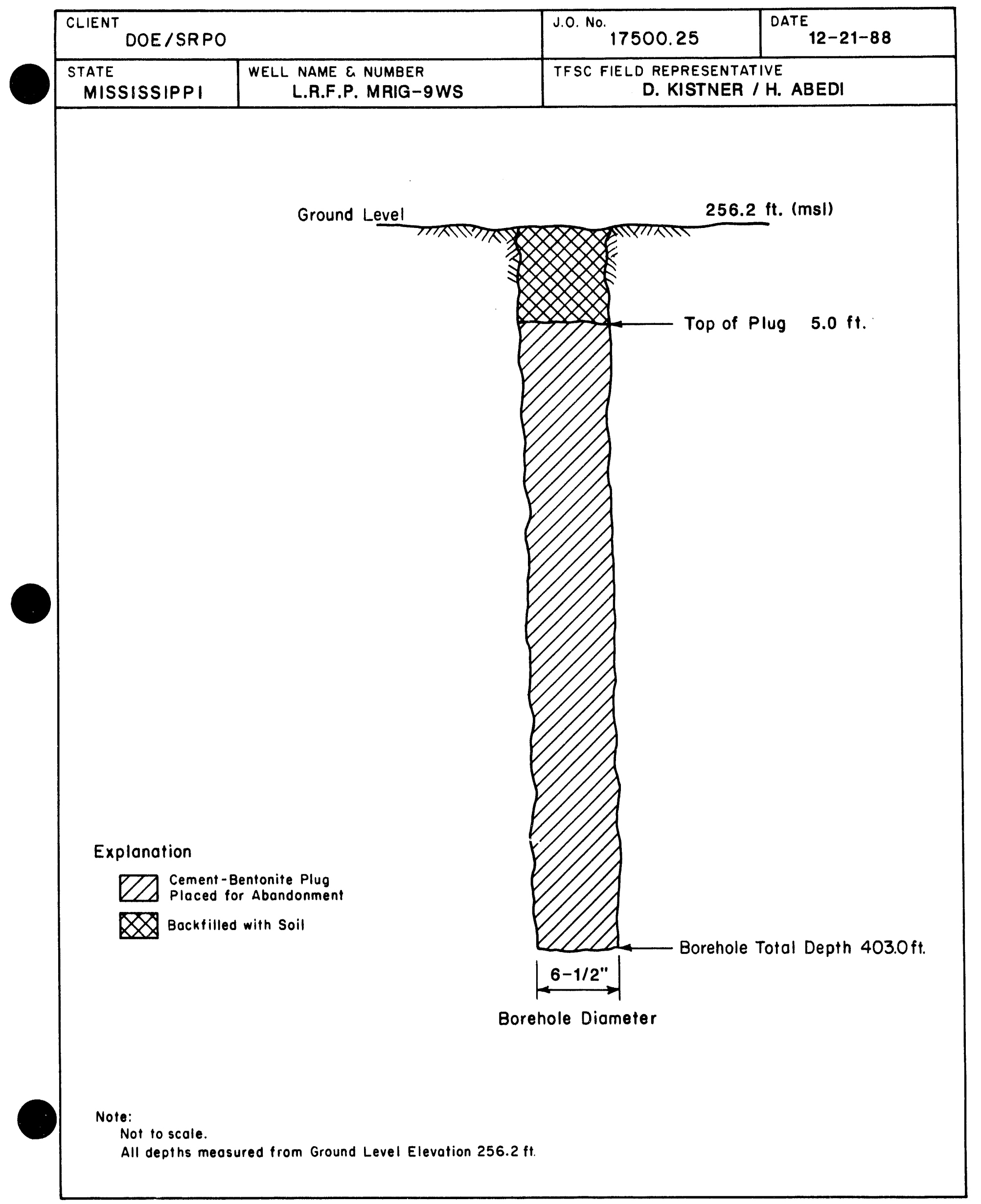


BOREHOLE COMPLETION SKETCH

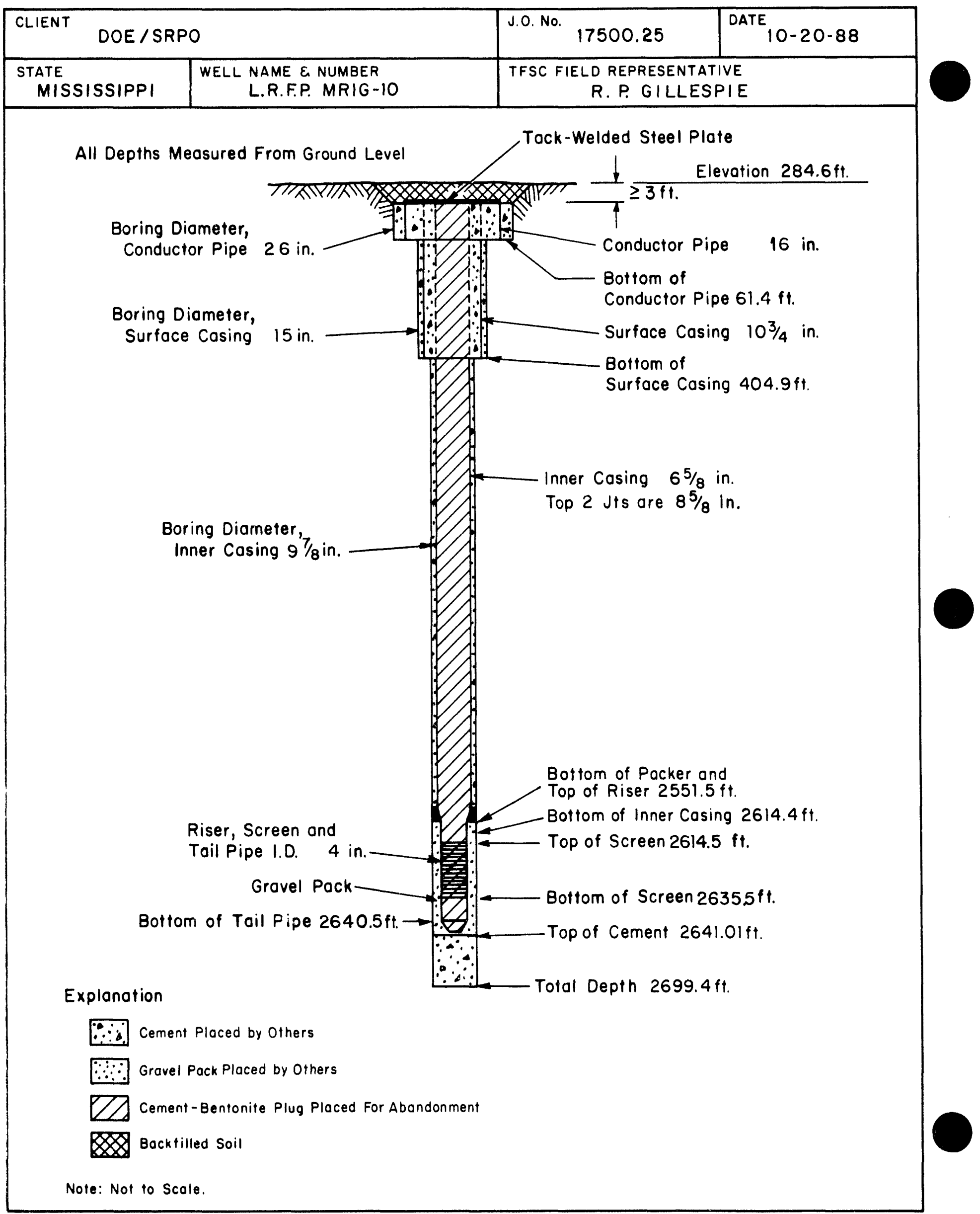


BOREHOLE COMPLETION SKETCH

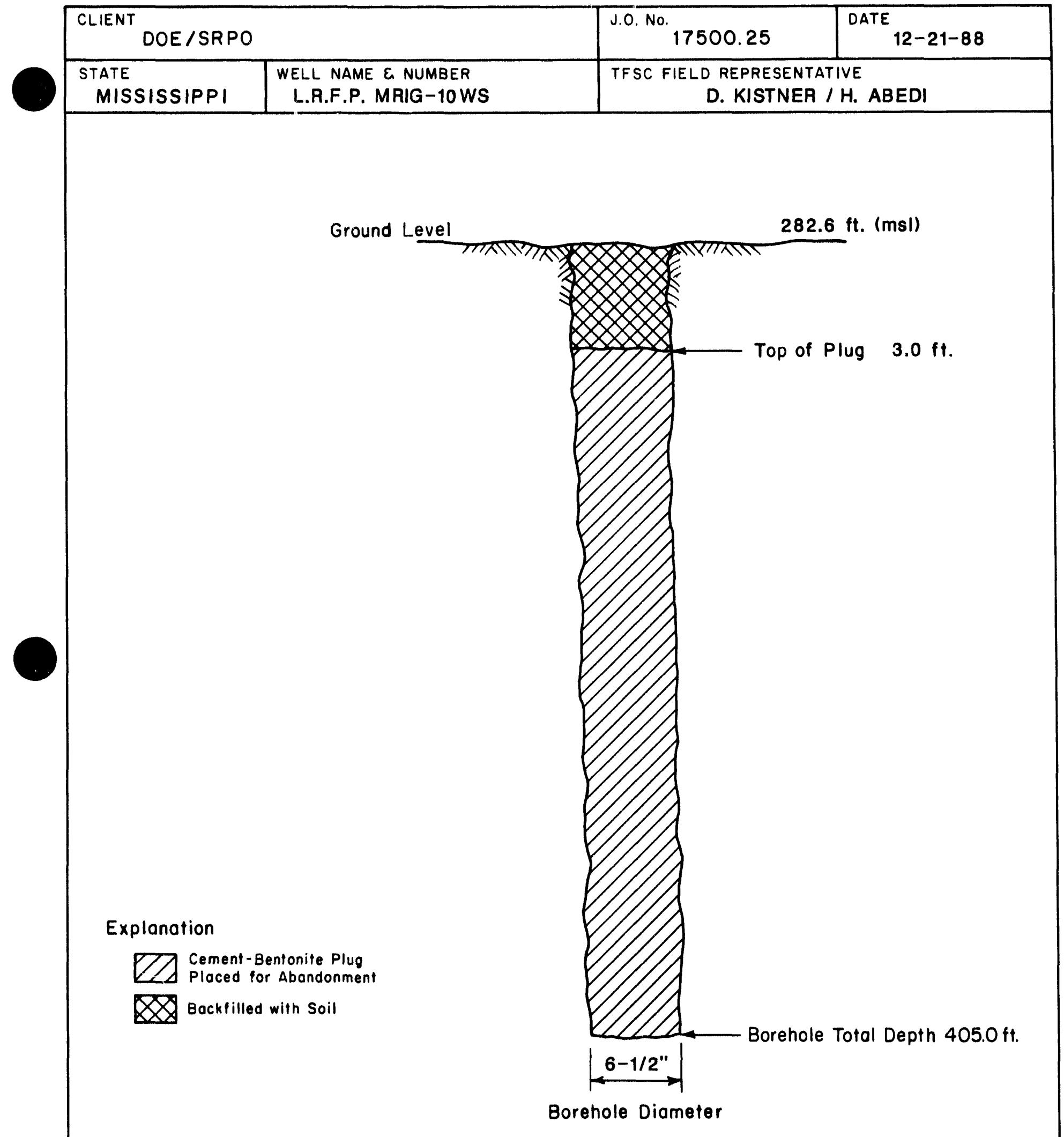

Note:

Not to scole.

All depths meosured from Ground Level Elevation $282.6 \mathrm{ft}$. 
BOREHOLE COMPLETION SKETCH

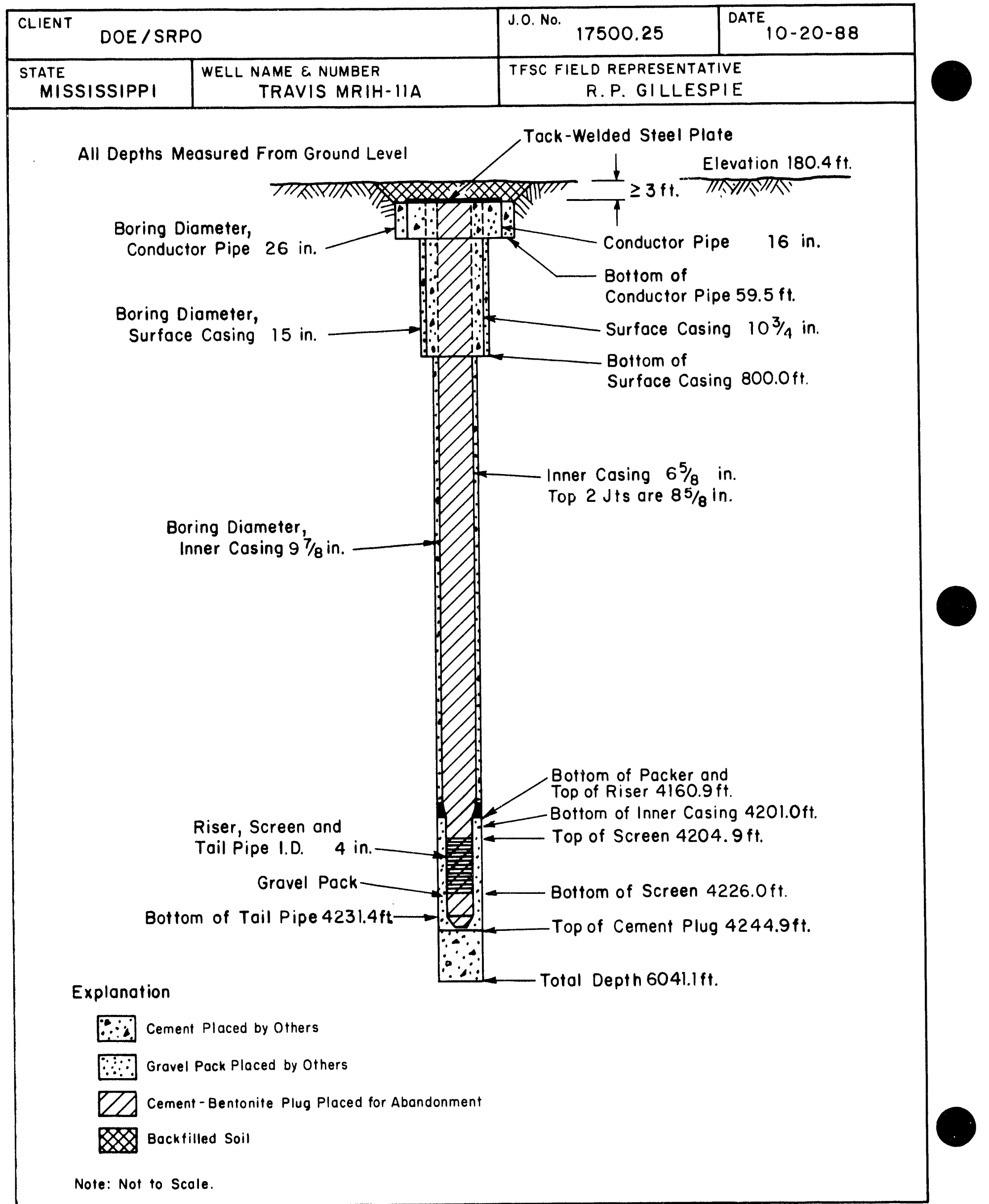


BOREHOLE COMPLETION SKETCH

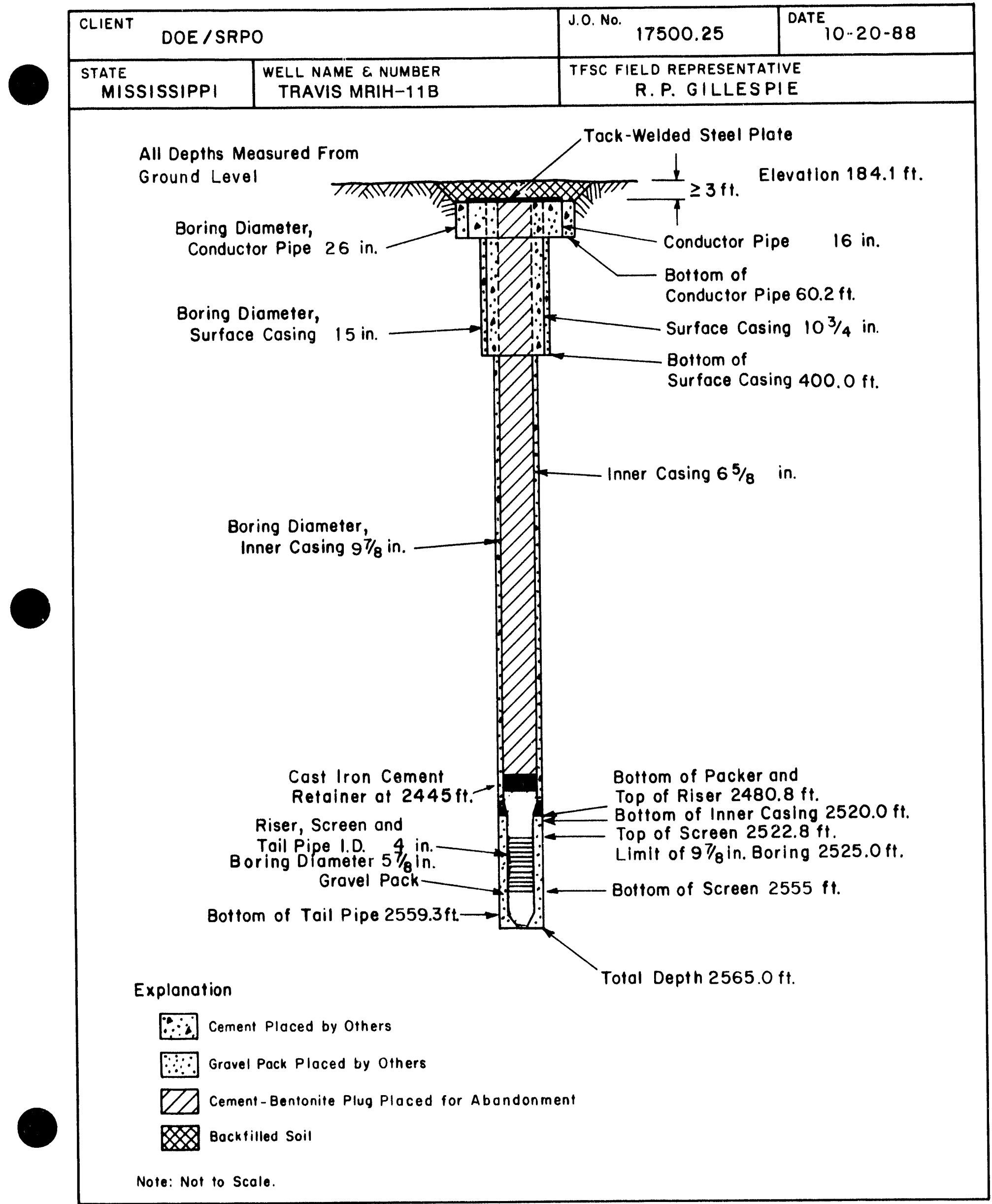


BOREHOLE COMPLETION SKETCH

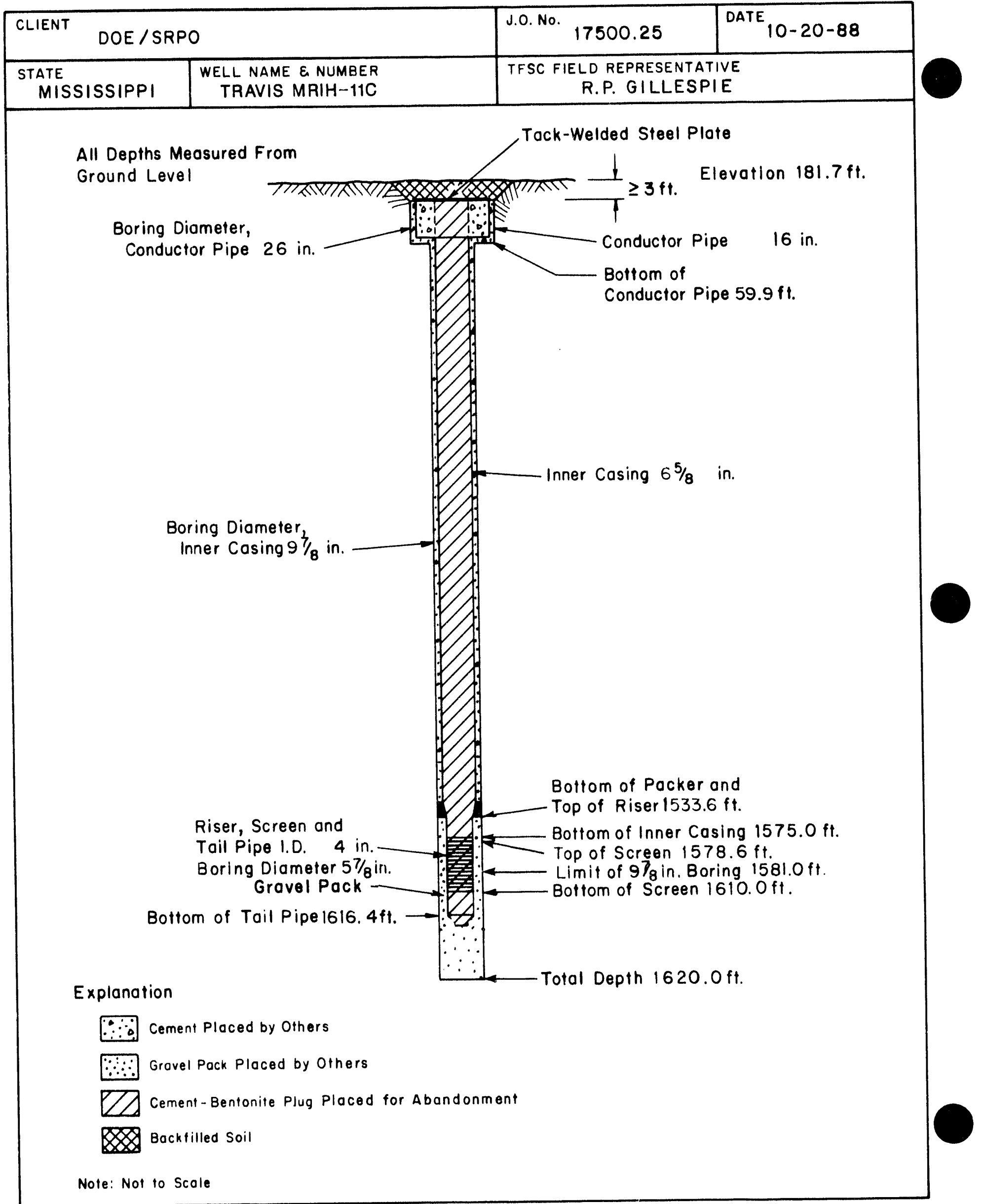


BOREHOLE COMPLETION SKETCH

\begin{tabular}{|l|l|l|c|}
\hline \multicolumn{2}{|c|}{ CLIENT DOE / SRPO } & J.0. No. 17500.25 & DATE 10-20-88 \\
\hline $\begin{array}{c}\text { STATE } \\
\text { MISSISSIPPI }\end{array}$ & $\begin{array}{c}\text { WELL NAME E NUMBER } \\
\text { TRAVIS MRIH-11D }\end{array}$ & $\begin{array}{c}\text { TFSC FIELD REPRESENTATIVE } \\
\text { R.P. GI LLESPIE }\end{array}$ \\
\hline
\end{tabular}

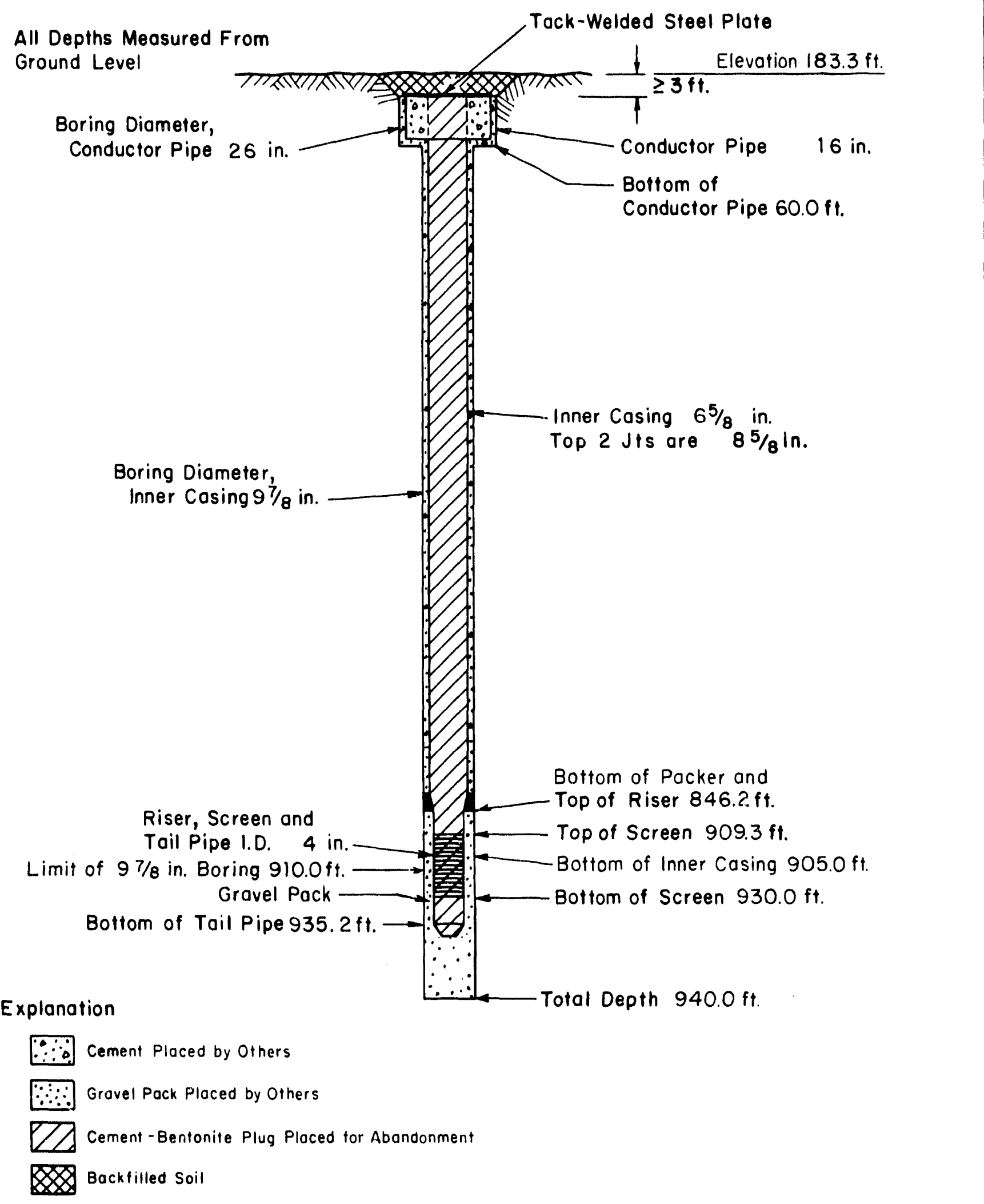

Note: Not to Scole 
BOREHOLE COMPLETION SKETCH

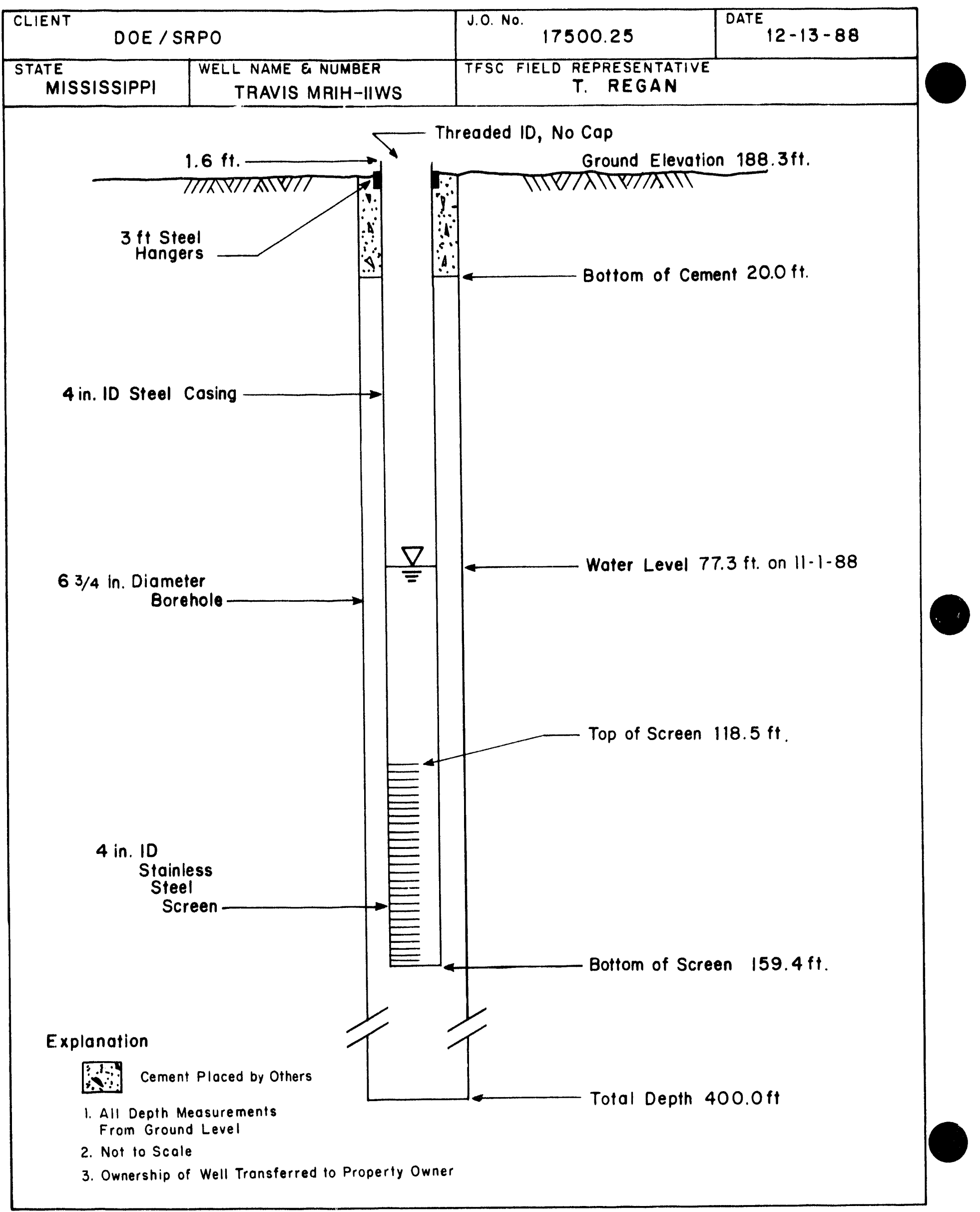


BOREHOLE COMPLETION SKETCH

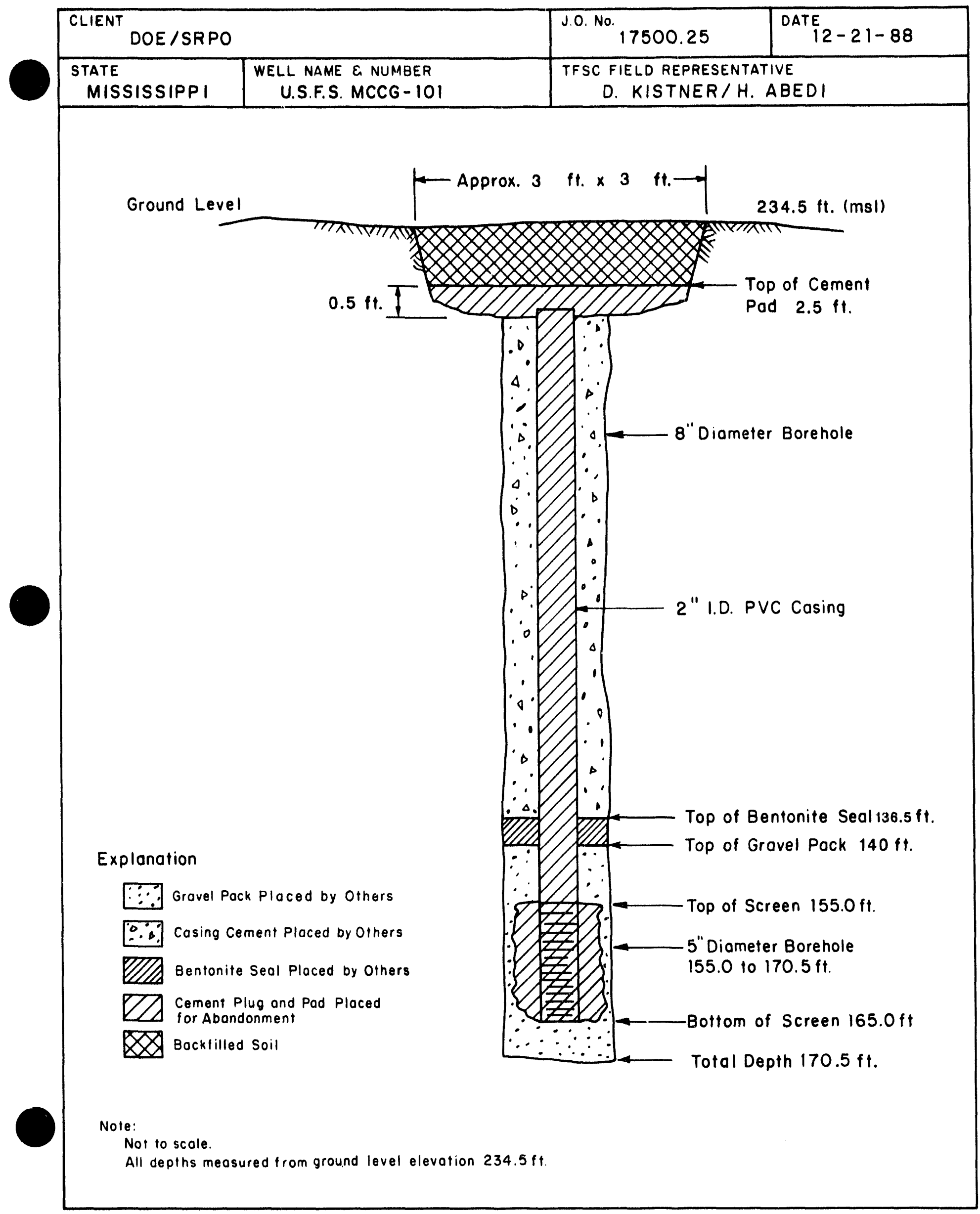


BOREHOLE COMPLETION SKETCH

\begin{tabular}{|l|l|l|l|}
\hline $\begin{array}{l}\text { CLIENT DOE / SRPO } \\
\text { STATE }\end{array}$ & $\begin{array}{l}\text { J. No. } 17500.25 \\
\text { MISSISSIPPI }\end{array}$ & $\begin{array}{r}\text { WELL NAME E. NUMBER } \\
\text { U.S.F.S. MCCG - } 102\end{array}$ & $\begin{array}{c}\text { TFSC FIELD REPRESENTATIVE } \\
\text { D. KISTNER/H. ABEDI }\end{array}$ \\
\hline
\end{tabular}

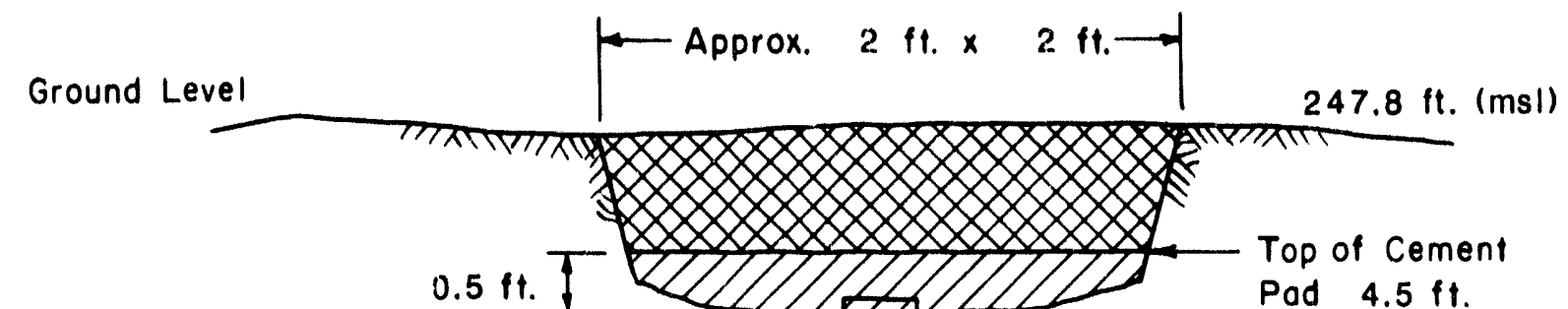

$0.5 \mathrm{ft}$

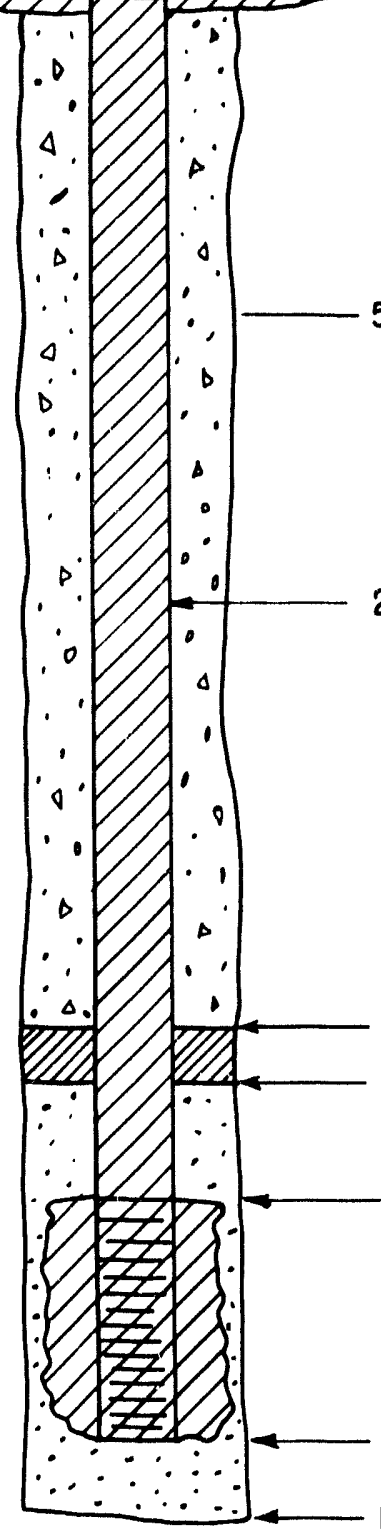

$5-7 / 8$ in. Diameter Borehole

2 " I.D. PVC Cosing

Explanation

$\because \because$ Gravel Pack Placed by Others

:.B. Casing Cement Placed by Others

QDA Bentonite Seal Placed by Others

7 Cement Plug and Pad Placed for Abandonment

Backfilled Soil

Pad $4.5 \mathrm{ft}$.

Top of Bentonite Seal $156.5 \mathrm{ft}$

Top of Gravel Pack $160 \mathrm{ft}$.

Top of Screen $190.0 \mathrm{ft}$.

Bottom of Screen $200.0 \mathrm{ft}$.

Borehole Total Depth $200.5 \mathrm{ft}$.

Note:

Not to scole.

All depths measured from ground level elevation $247.8 \mathrm{ft}$ 
BOREHOLE COMPLETION SKETCH

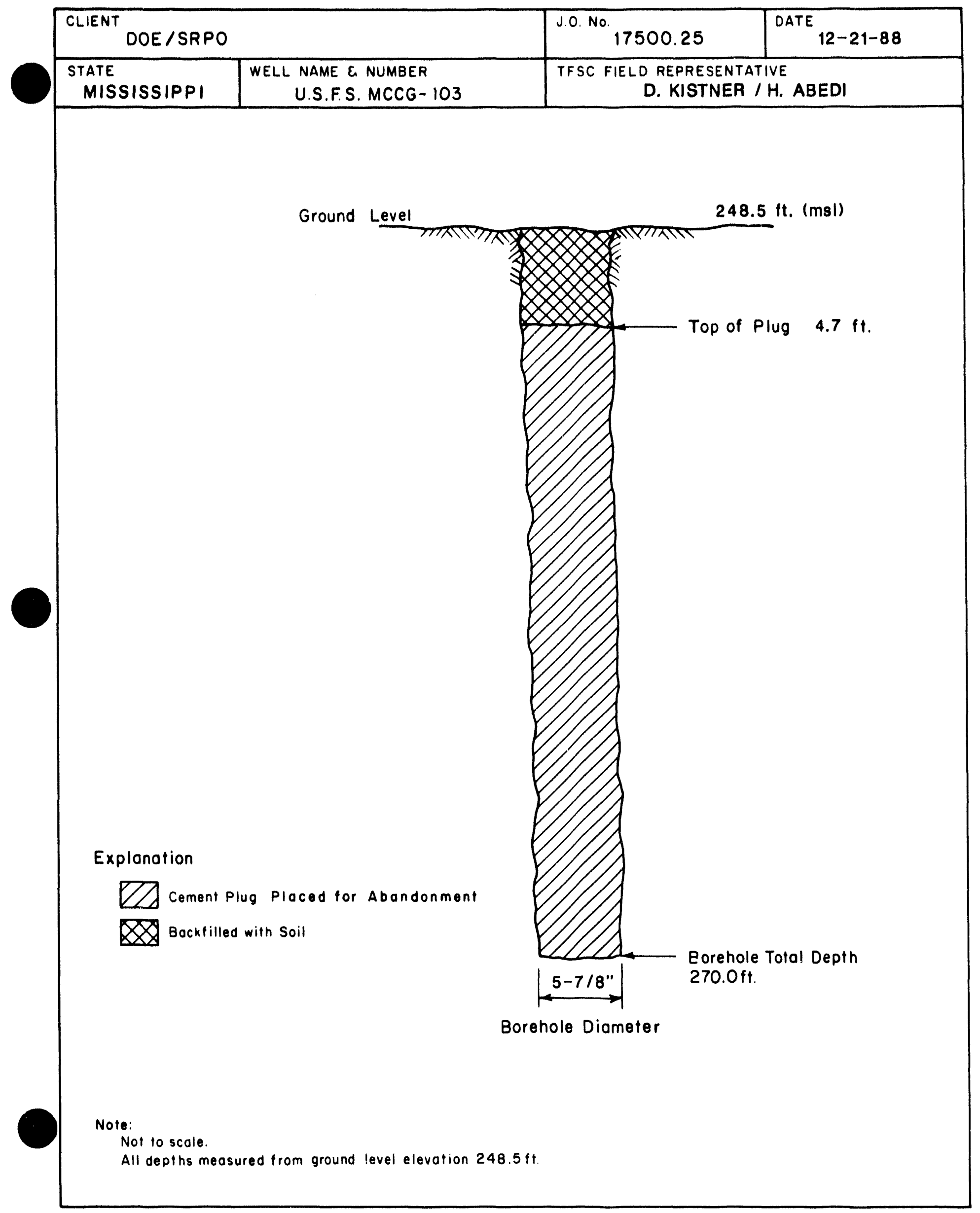


BOREHOLE COMPLETION SKETCH

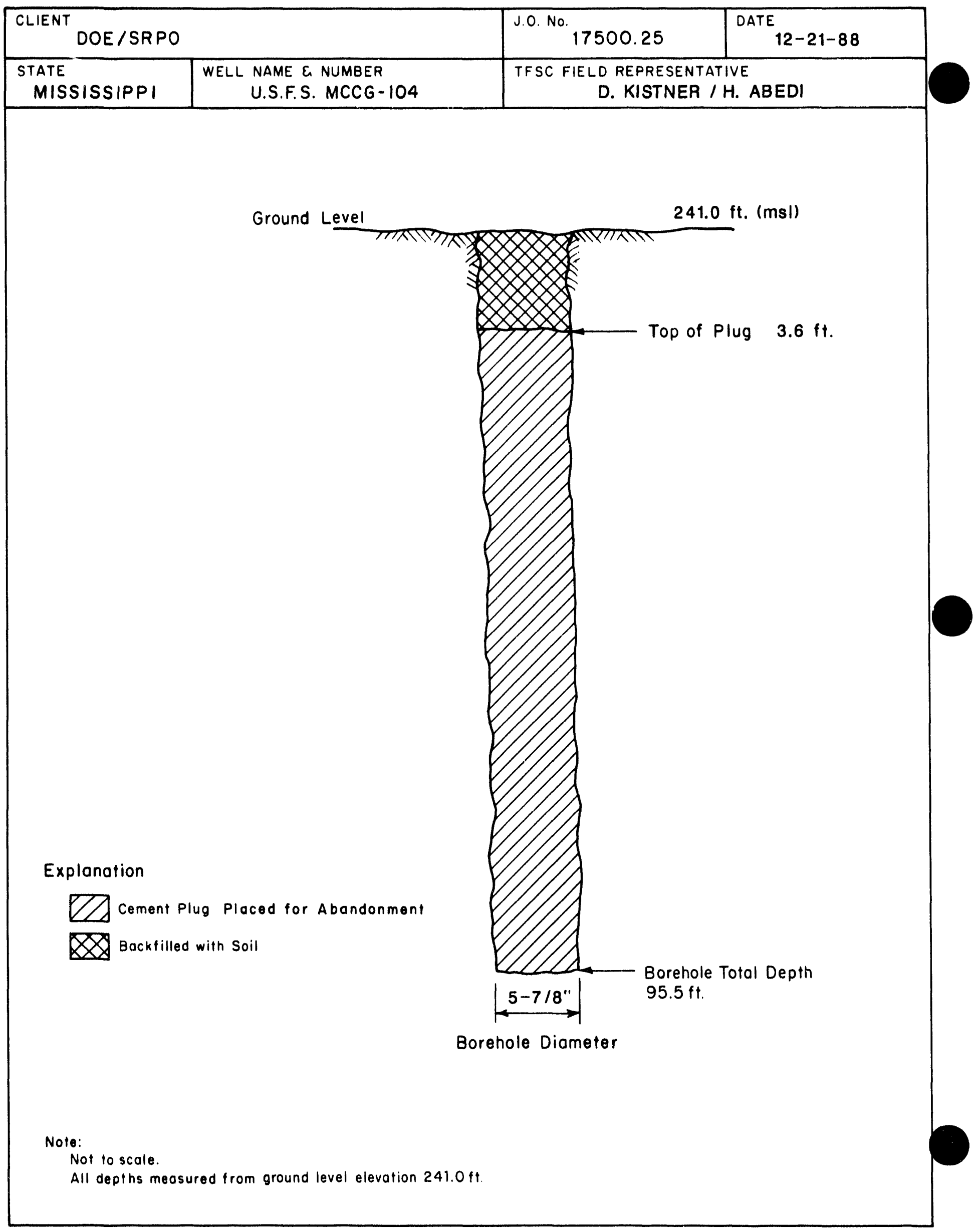


BOREHOLE COMPLETION SKETCH

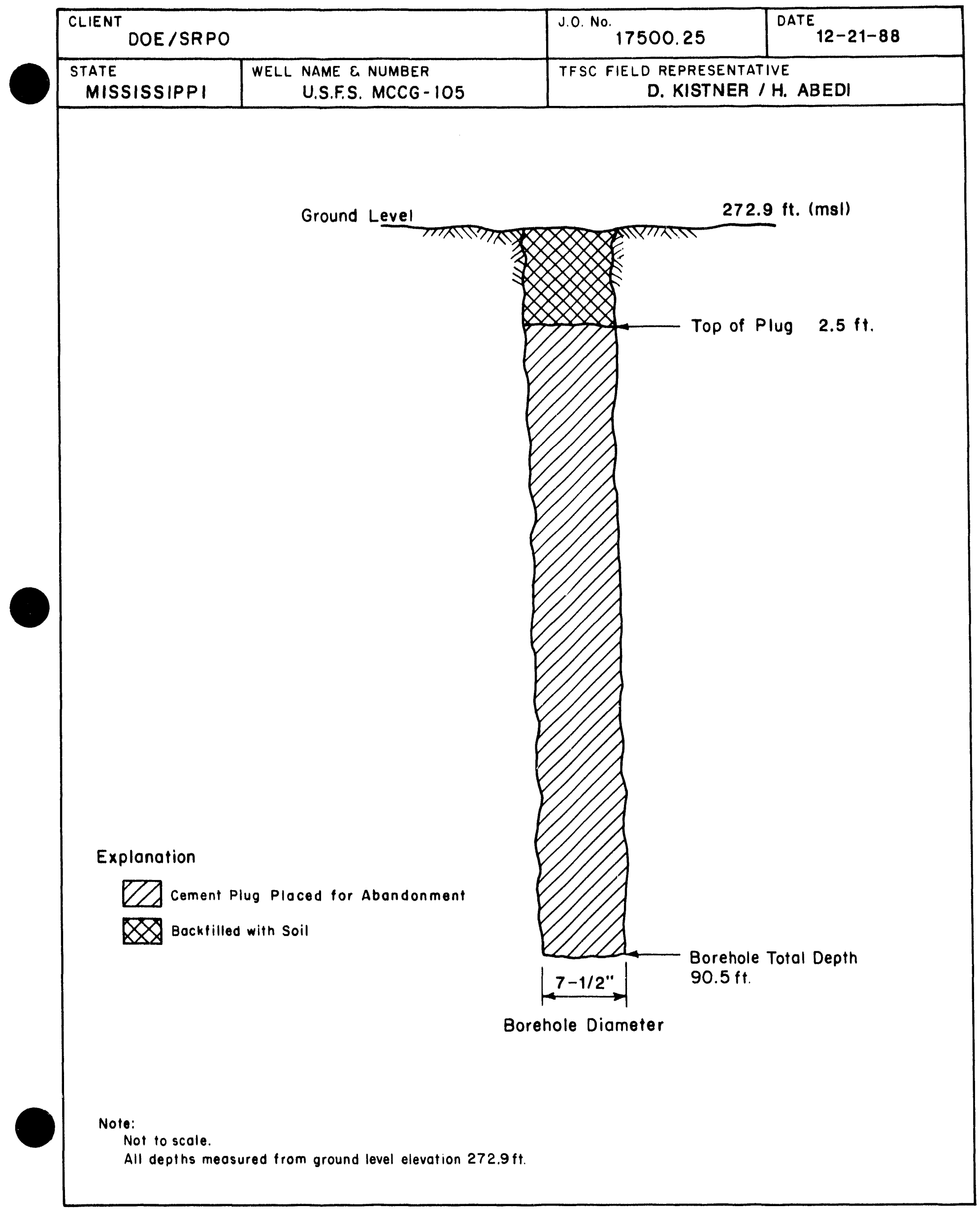


BOREHOLE COMPLETION SKETCH

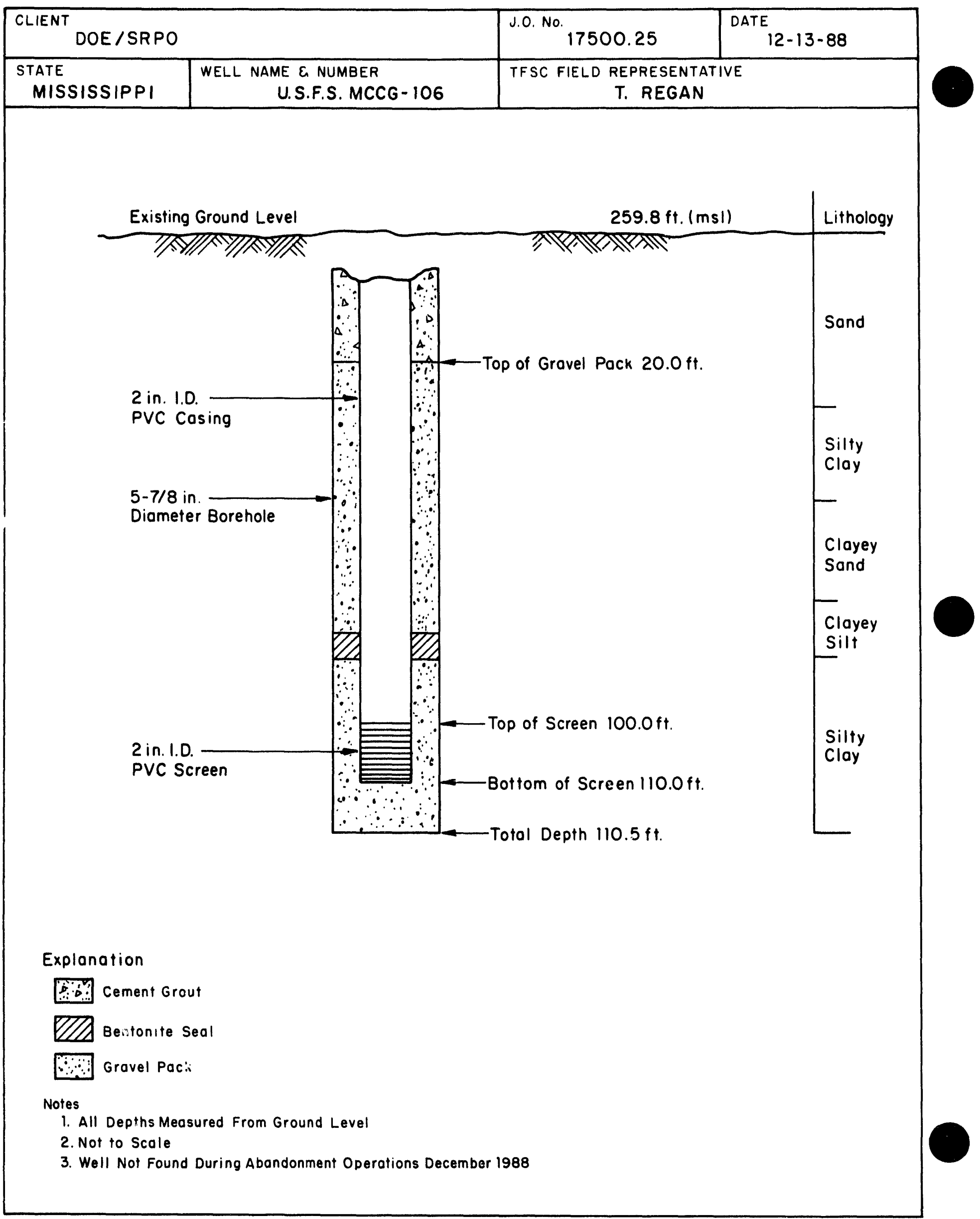


BOREHOLE COMPLETION SKETCH

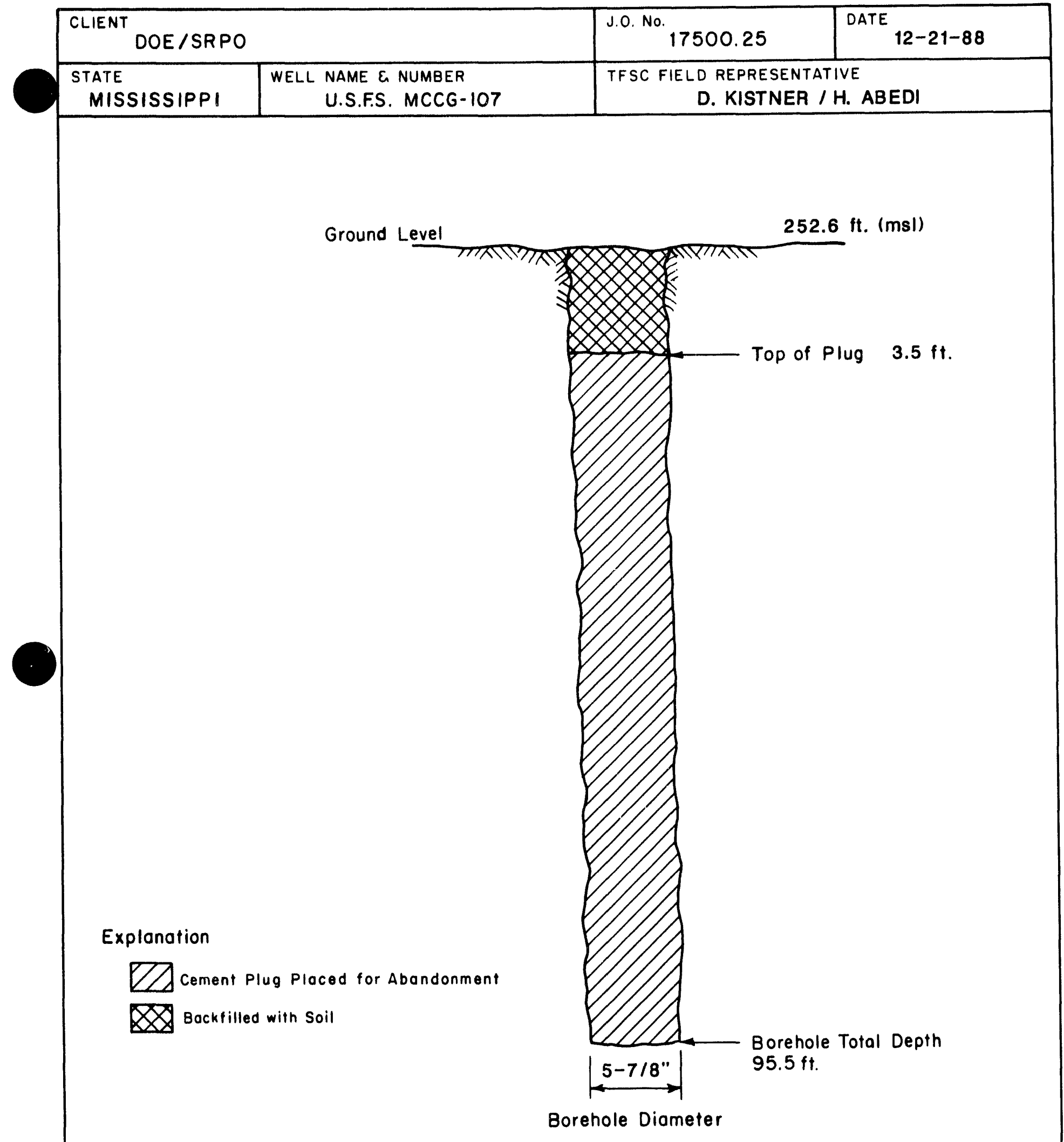

Note:

Not to scole.

All depths measured from ground level elevotion $252.6 \mathrm{ft}$. 
BOREHOLE COMPLETION SKETCH

\begin{tabular}{|l|l|l|l|}
\hline $\begin{array}{l}\text { CLIENT DOE / SRPO } \\
\text { STATE }\end{array}$ & $\begin{array}{l}\text { J.0. No. } \\
\text { MISSISSIPPI }\end{array}$ & $\begin{array}{c}\text { WELL NAME E. NUMBER } \\
\text { U.S.F.S. MCCG - } 110\end{array}$ & $\begin{array}{r}\text { TFSC FIELD REPRESENTATIVE } \\
\text { D. KISTNER / H. ABEDI }\end{array}$ \\
\hline
\end{tabular}

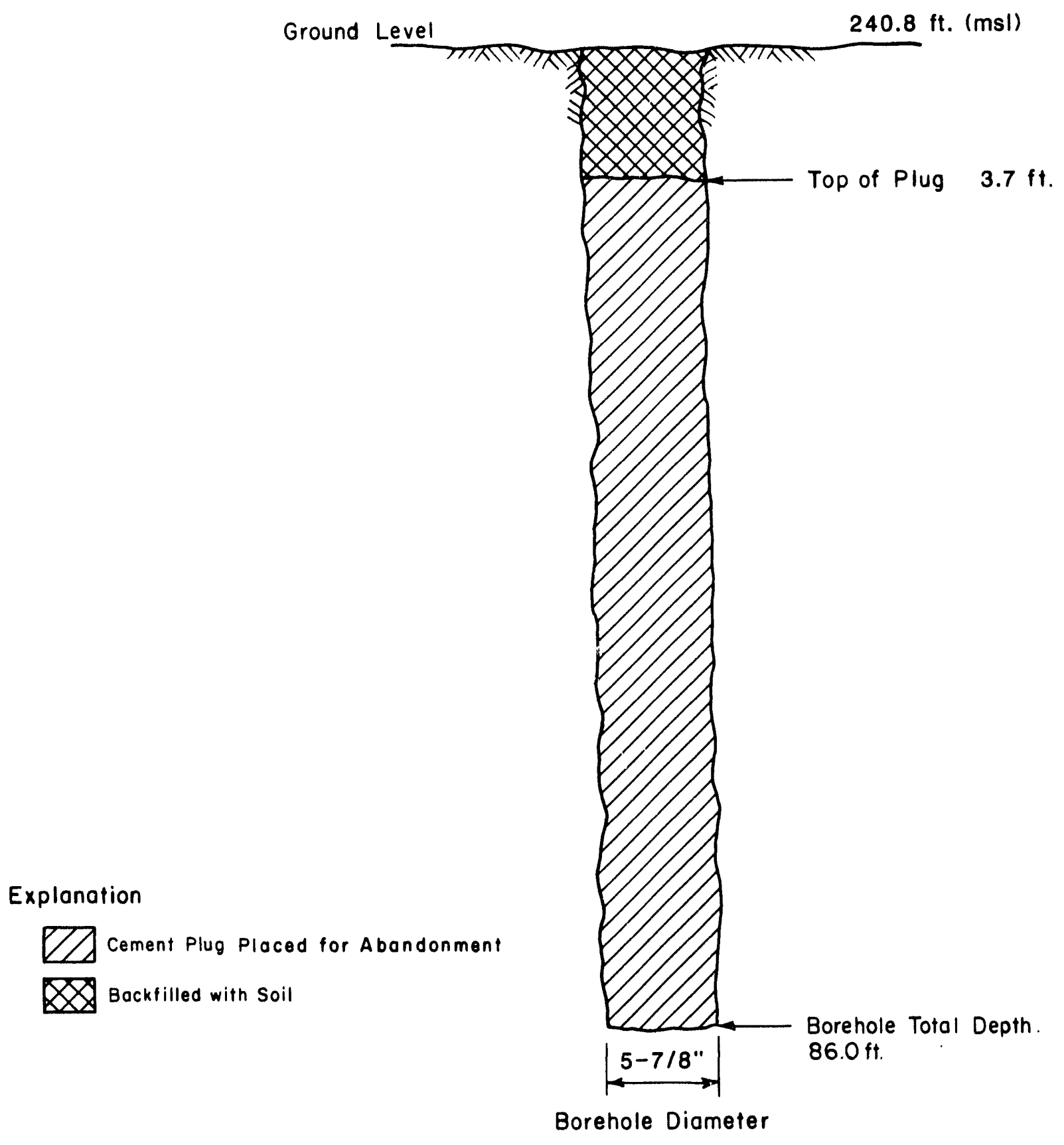

Note:

Not to scale.

All depths measured from ground level elevation $240.8 \mathrm{ft}$. 
BOREHOLE COMPLETION SKETCH

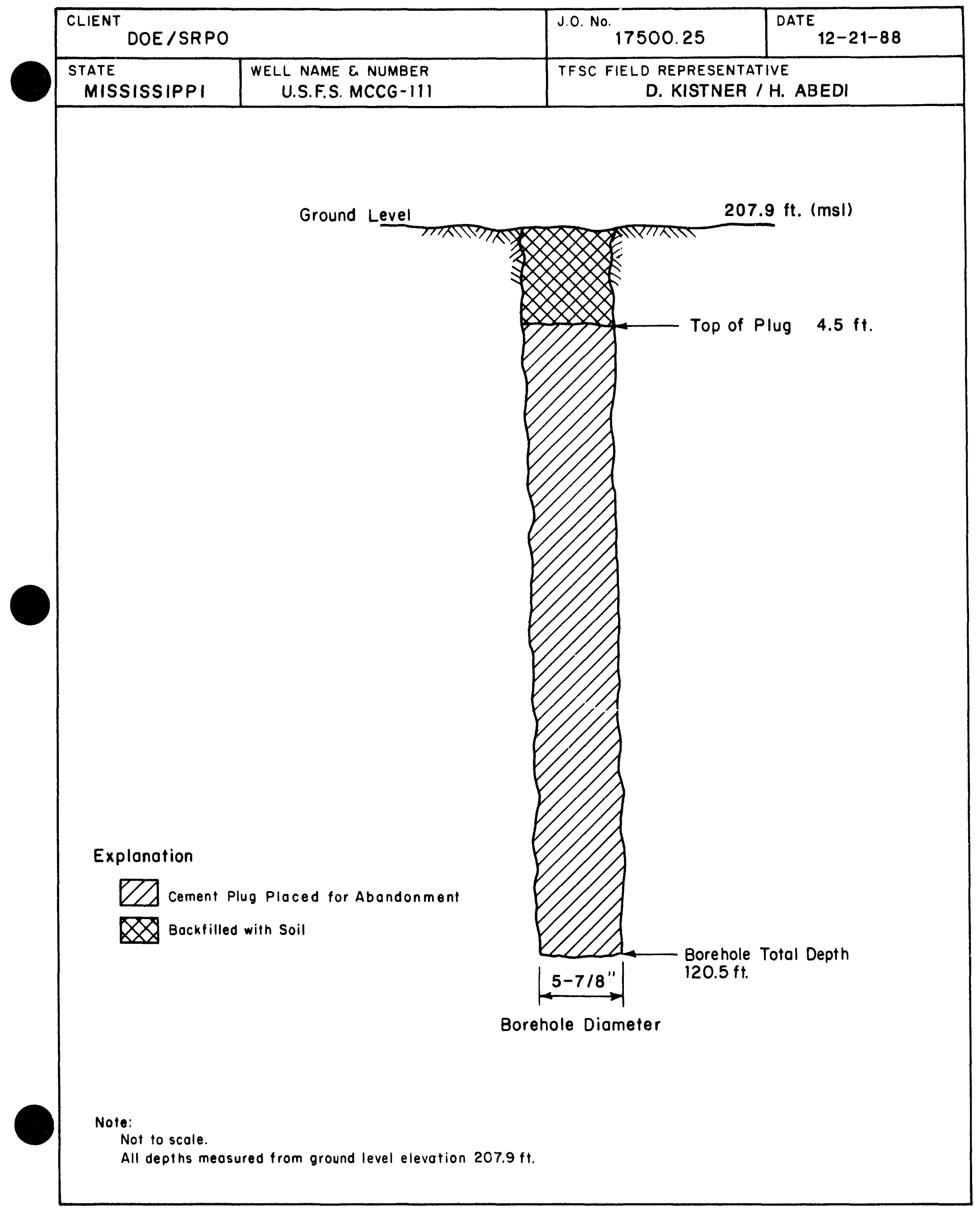


BOREHOLE COMPLETION SKETCH

\begin{tabular}{|l|c|c|c|}
\hline $\begin{array}{l}\text { CLIENT DOE / SRPO } \\
\text { D. No. }\end{array}$ & $\begin{array}{l}\text { J. No } \\
\text { STATE } \\
\text { MISSISSIPPI }\end{array}$ & WELL NAME E NUMBER & TFSC FIELD REPRESENTATIVE \\
U.S.F.S. MCCG-112 & T. REGAN \\
\hline
\end{tabular}

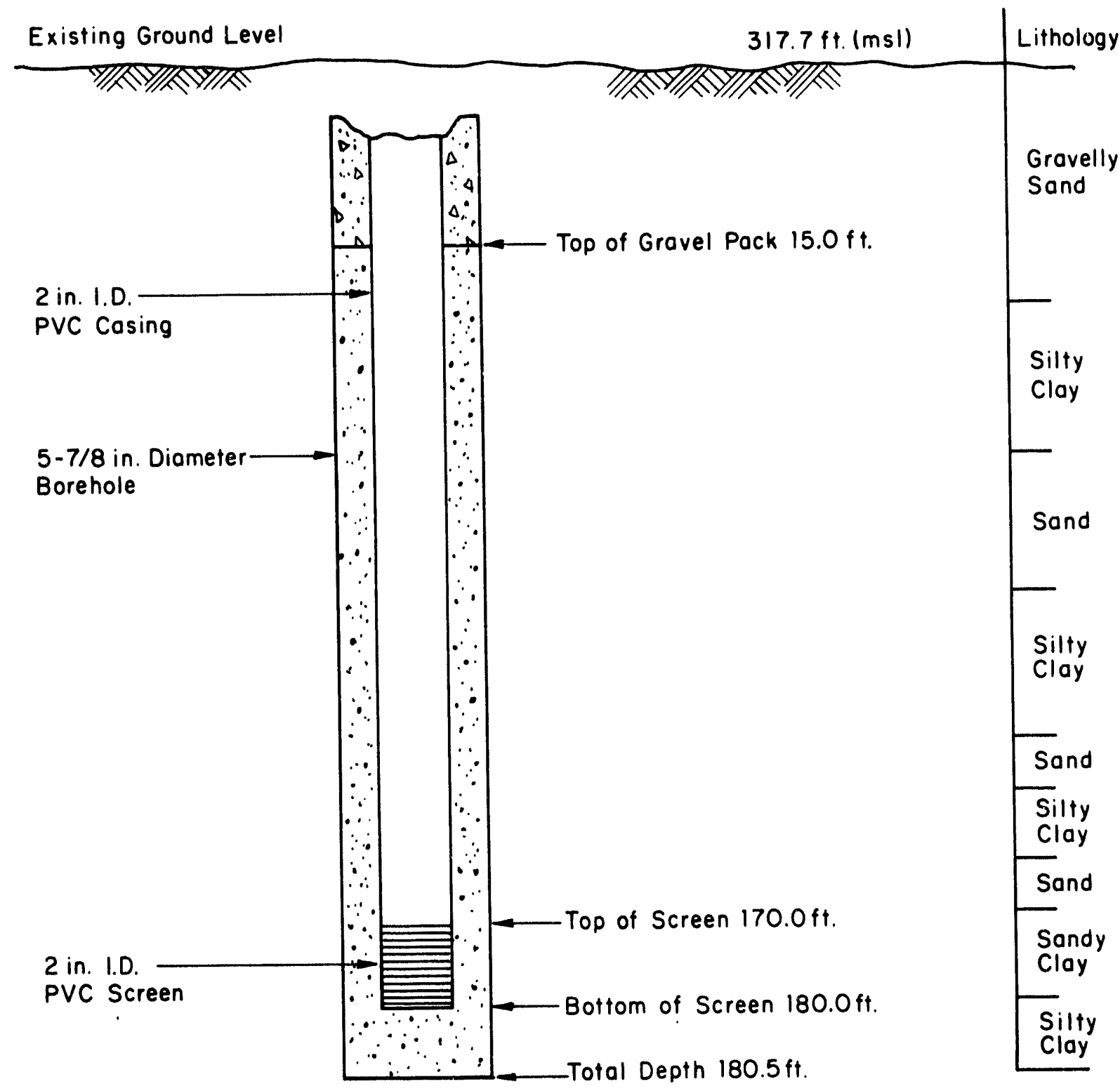

\section{Explanation}

$\therefore$ Cement Grout

Z7 Bentonite Seal

$\because \because$ Gravel Pack

Notes:

1. All Depths Measured From Ground Level

2. Not to Scale

3. Well Not Found During Abandonment Operations December 1988 
BOREHOLE COMPLETION SKETCH

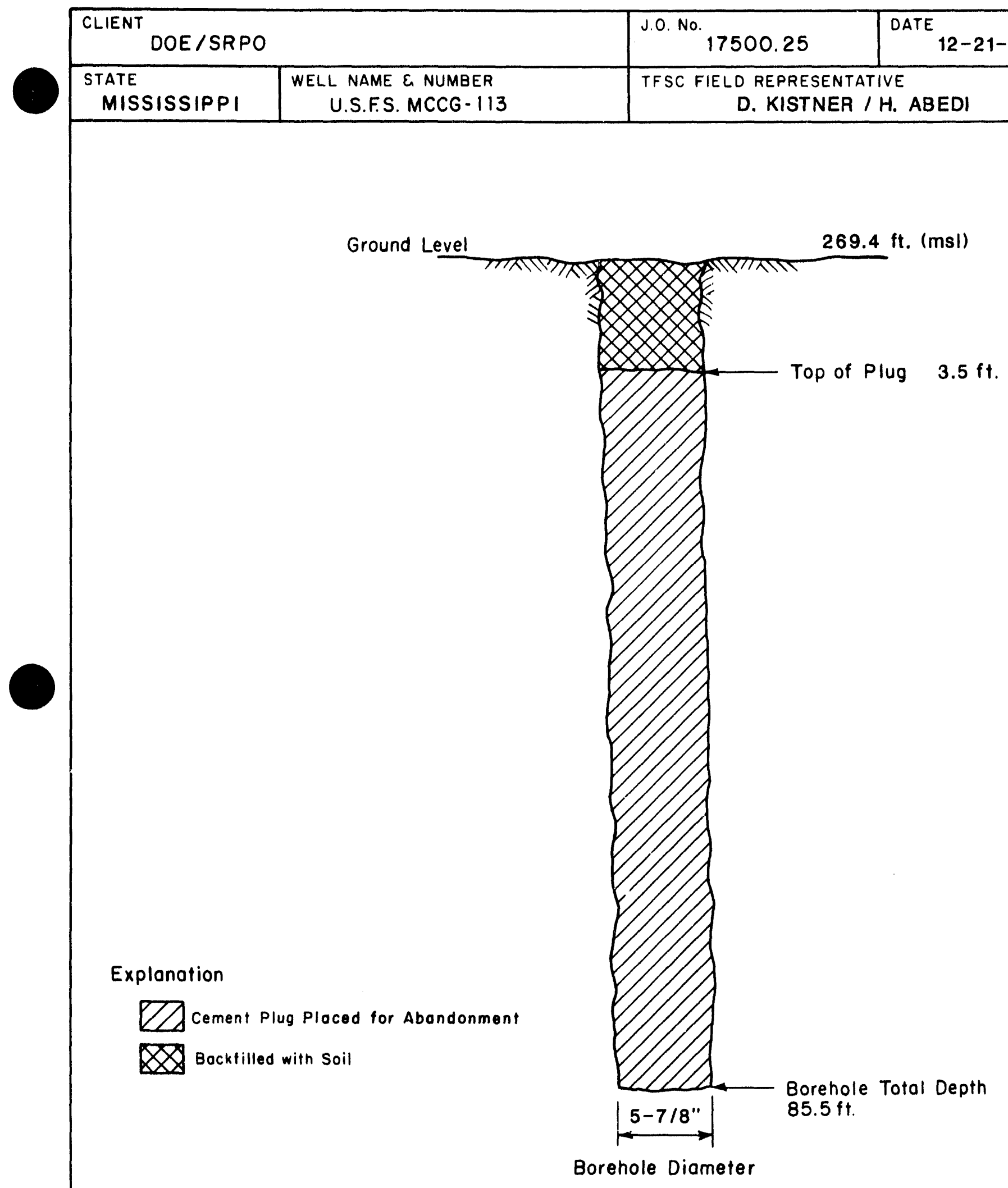

Note:

Not to scale.

All depths measured from ground level elevation $269.4 \mathrm{ft}$. 


\section{BOREHOLE COMPLETION SKETCH}

\begin{tabular}{|l|l|l|l|}
\hline $\begin{array}{l}\text { CLIENT DOE /SRPO } \\
\text { STATE }\end{array}$ & $\begin{array}{l}\text { J.0. No. } 17500.25 \\
\text { MATE } \\
\text { MISSISSIPPI }\end{array}$ & $\begin{array}{c}\text { WELL NAME E NUMBER } \\
\text { U.S.F.S. MCCG- } 114\end{array}$ & $\begin{array}{r}\text { TFS FIELD REPRESENTATIVE } \\
\text { D. KISTNER / H. ABEDI }\end{array}$ \\
\hline
\end{tabular}

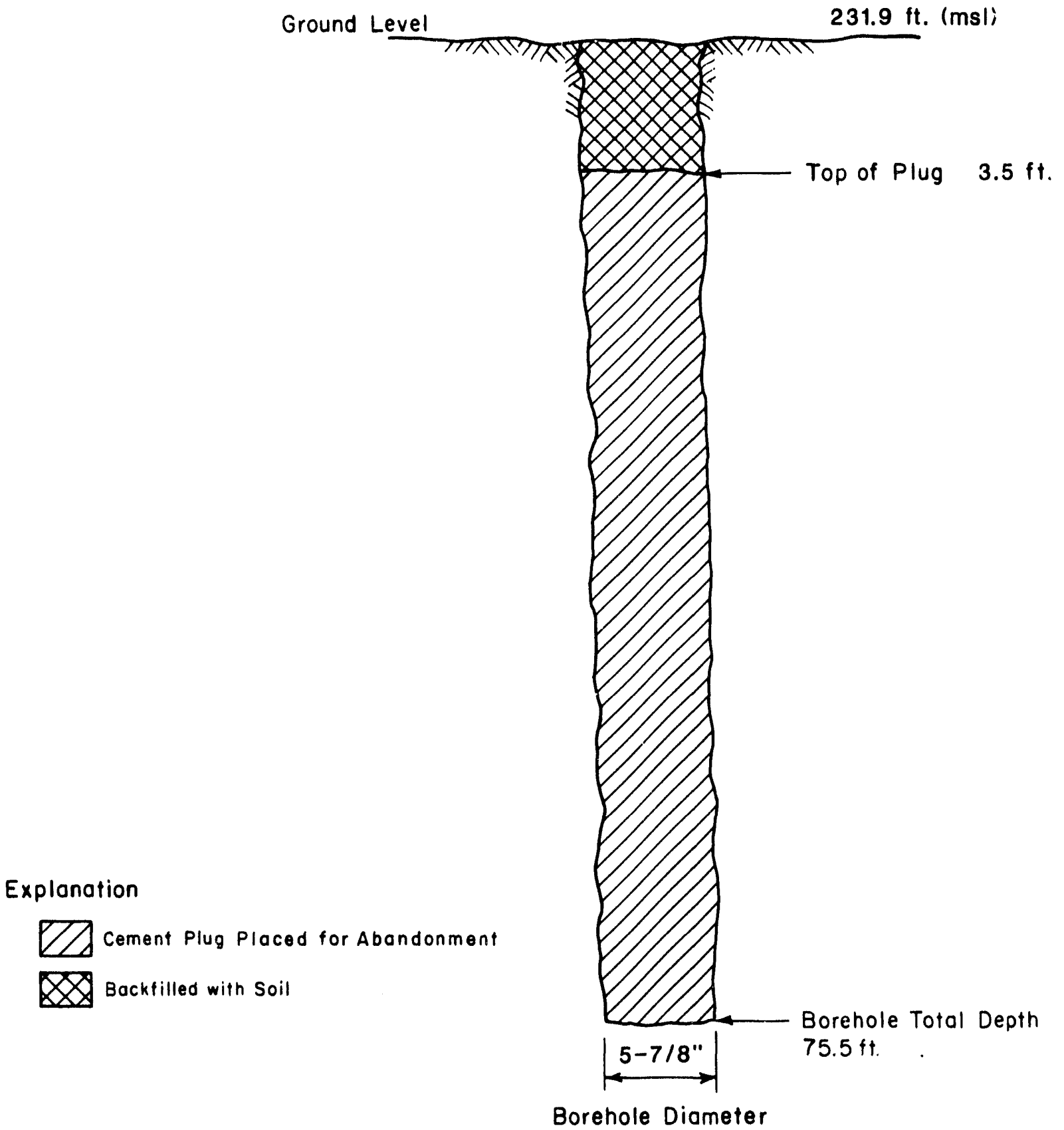

Note:

Not to scale.

All depths measured from ground level elevation $231.9 \mathrm{ff}$. 
BOREHOLE COMPLETION SKETCH

\begin{tabular}{|l|c|c|c|}
\hline $\begin{array}{l}\text { CL.IENT DOE/SRPO } \\
\text { STATE } \\
\text { MISSISSIPPI }\end{array}$ & $\begin{array}{l}\text { J.0. No. } 17500.25 \\
\text { WELL NAME \& NUMBER } \\
\text { U.S.F.S. MCCG -115 }\end{array}$ & $\begin{array}{c}\text { TFSC FIELD REPRESENTATIVE } \\
\text { D. KISTNER / H. ABEDI }\end{array}$ \\
\hline
\end{tabular}

Ground Level

$189.7 \mathrm{ft} .(\mathrm{ms} /)$

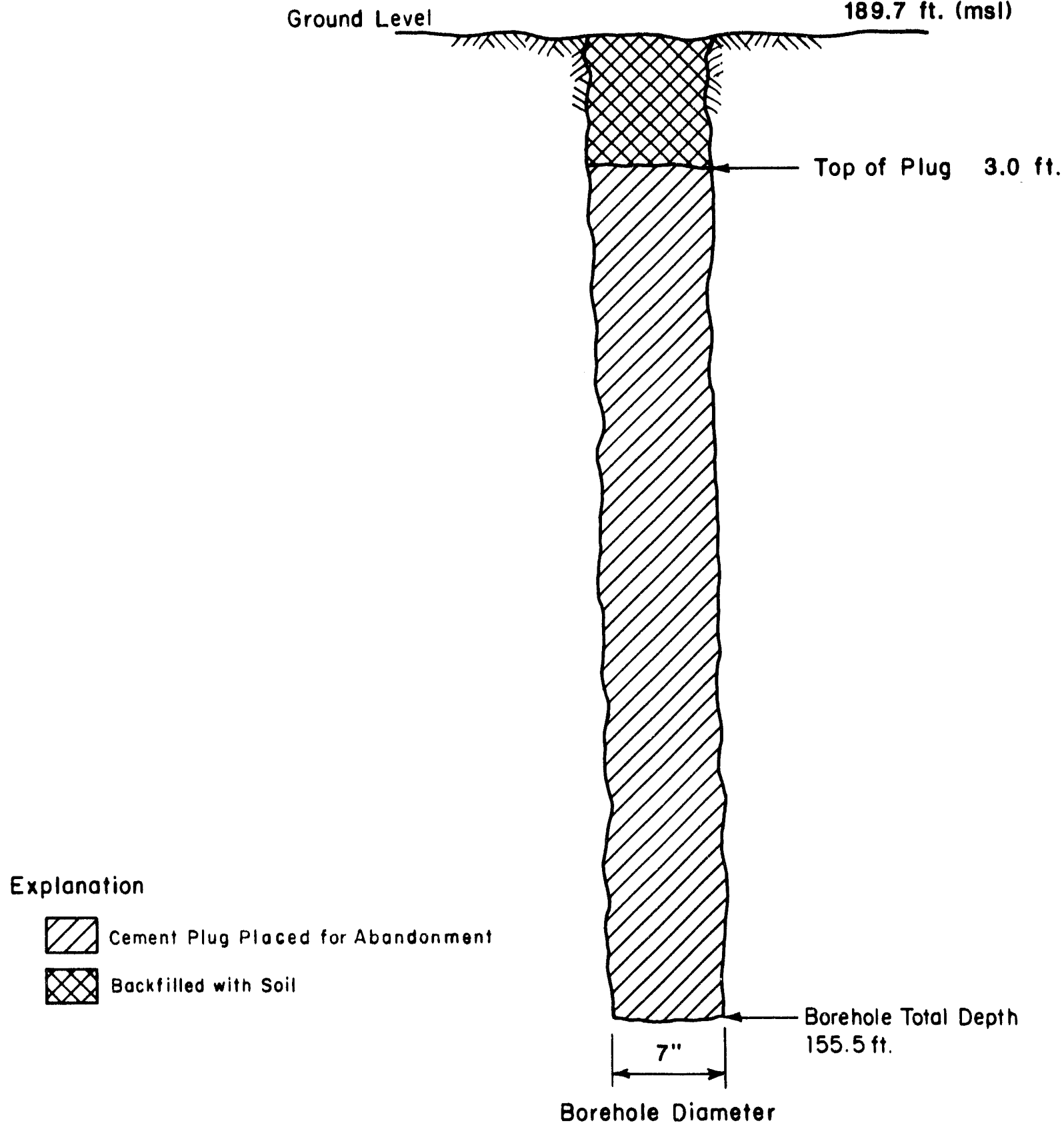

Note:

Not to scale.

All depths measured from ground level elevation $189.7 \mathrm{ft}$. 
BOREHOLE COMPLETION SKETCH

\begin{tabular}{|c|c|c|c|}
\hline \multicolumn{2}{|c|}{$\begin{array}{c}\text { CLIENT } \\
\text { DOE / SRPO }\end{array}$} & J.0. No. 17500.25 & ${ }_{12-21-88}$ \\
\hline $\begin{array}{l}\text { STATE } \\
\text { MISSISSIPPI }\end{array}$ & $\begin{array}{l}\text { WELL NAME \& NUMBER } \\
\text { U.S.F.S. MCCG }-117\end{array}$ & $\begin{array}{r}\text { TFSC FIELD REPRES } \\
\text { D. KISTN }\end{array}$ & $\begin{array}{l}\text { VE } \\
\text { I. ABEDI }\end{array}$ \\
\hline
\end{tabular}

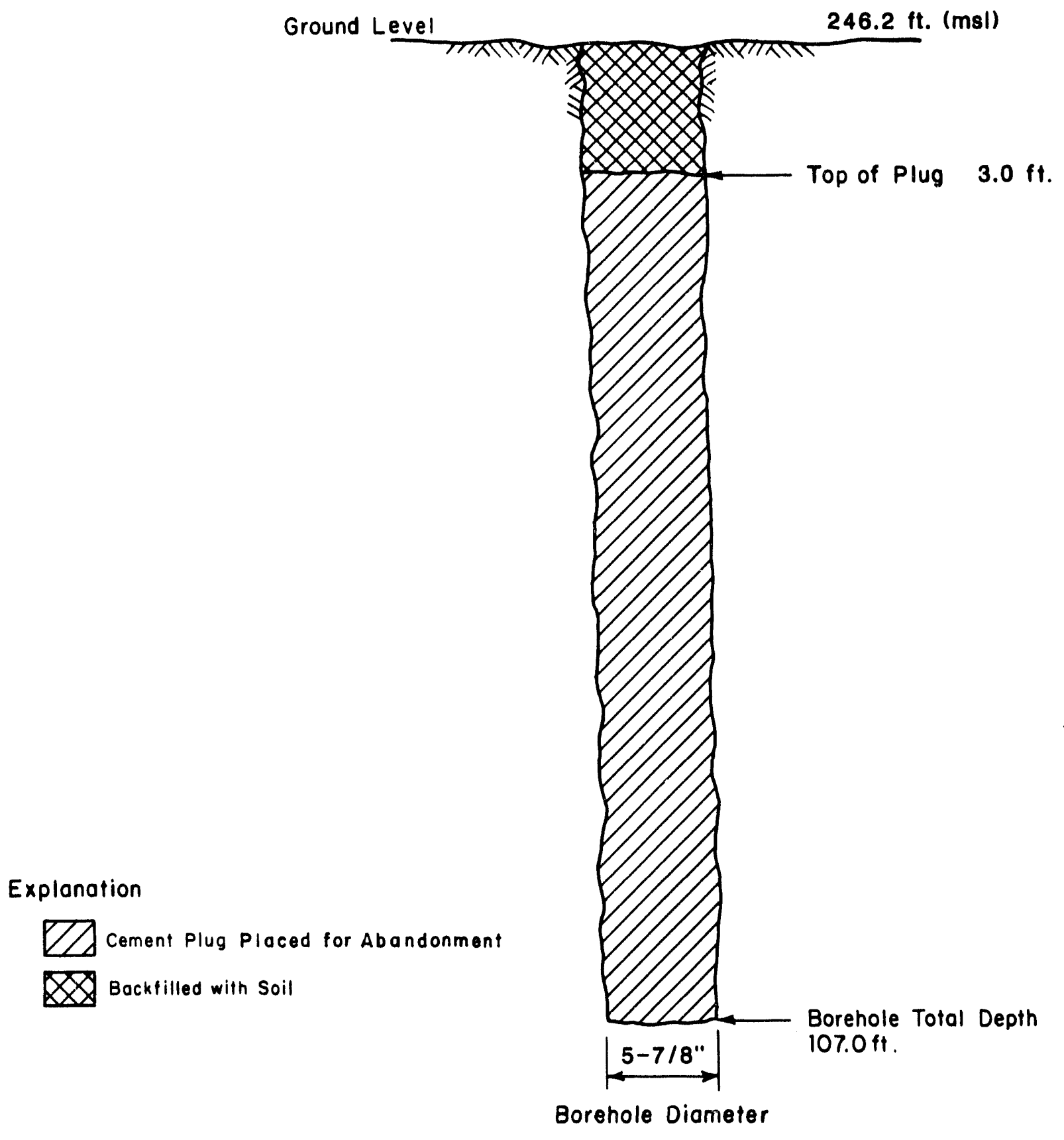

Note:

Not to scale.

All depths measured from ground level elevation $246.2 \mathrm{ft}$. 
BOREHOLE COMPLETION SKETCH

\begin{tabular}{|l|c|c|c|}
\hline $\begin{array}{l}\text { CLIENT DOE / SRPO } \\
\text { STATE }\end{array}$ & $\begin{array}{l}\text { J.0. No. } 17500.25 \\
\text { DATE } \\
\text { MISSISSIPPI }\end{array}$ & $\begin{array}{c}\text { TFSC FIELD REPRESENTATIVE } \\
\text { T. REGANE E NUMBER }\end{array}$ \\
\hline
\end{tabular}

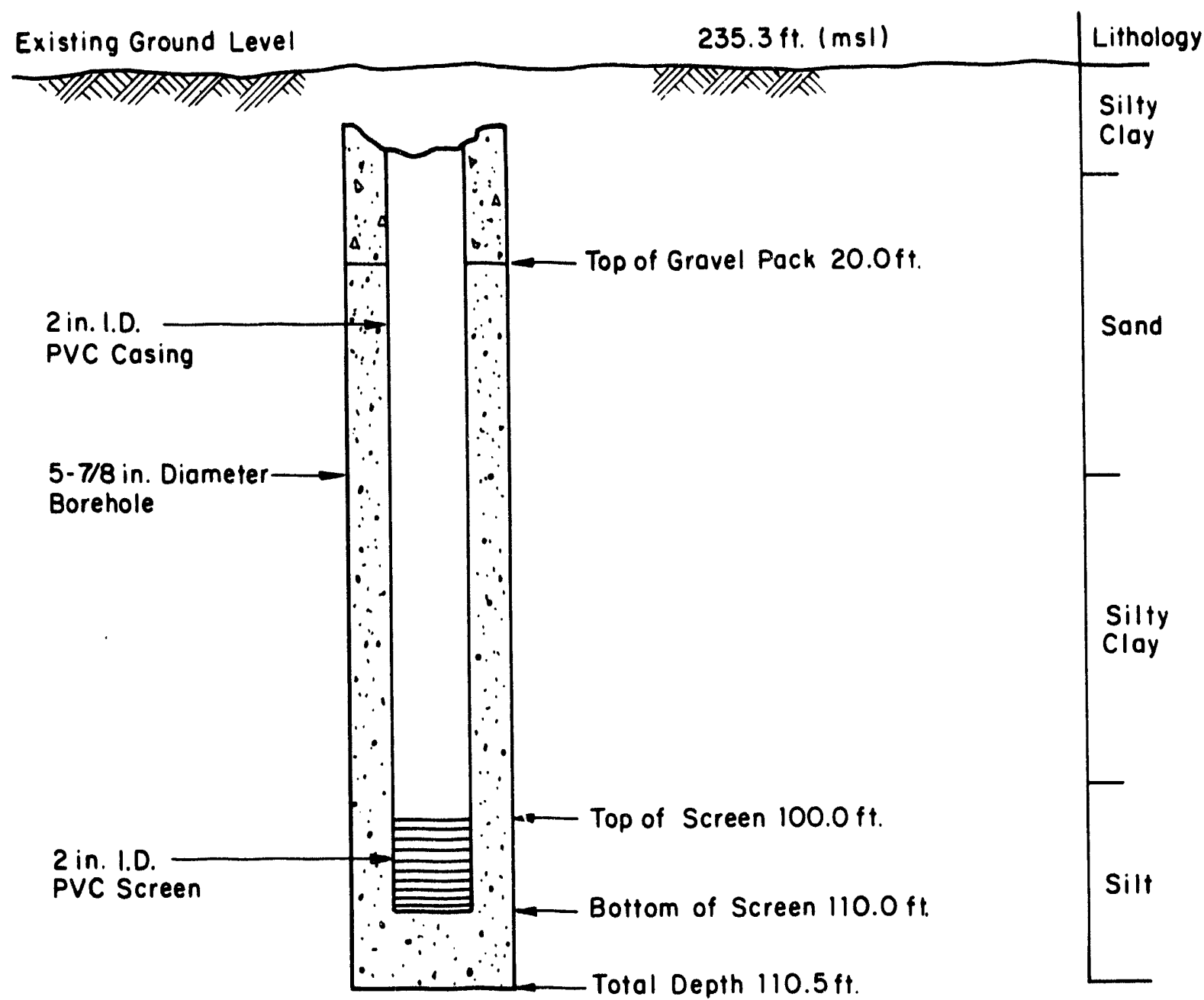

Explanation

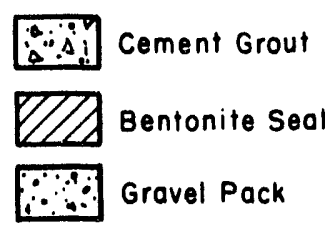

Notes:

1. All Depths Measured From Ground Level

2. Not to Scale

3. Well Not Found During Abandonment Operations December 1988 
BOREHOLE COMPLETION SKETCH

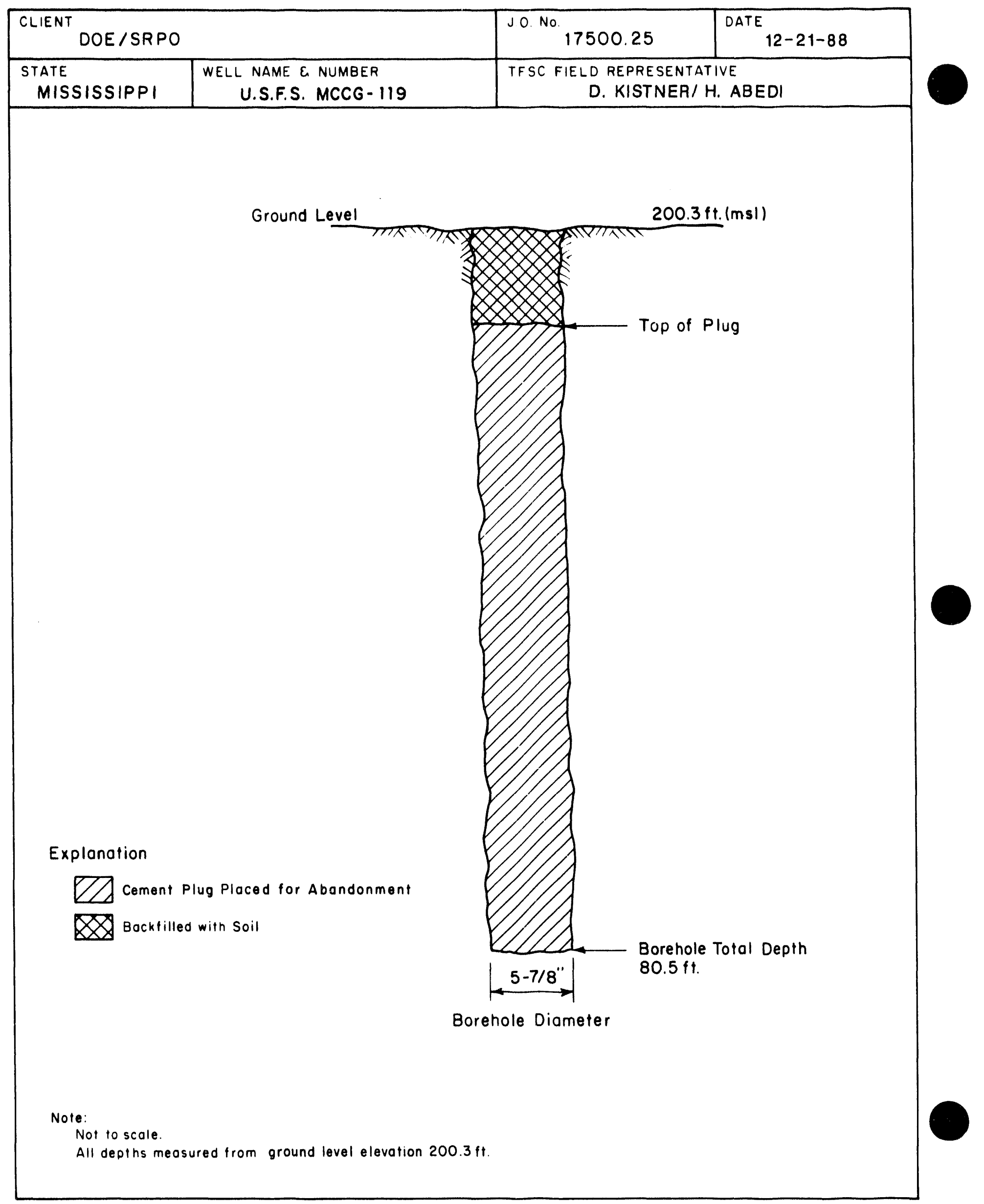


BOREHOLE COMPLETION SKETCH

\begin{tabular}{|l|l|l|l|}
\hline \multicolumn{2}{|c|}{ CLIENT DOE / SRPO } & $\begin{array}{l}\text { J. No. } 17500.25 \\
\text { DATE }\end{array}$ & $12-21-88$ \\
\hline $\begin{array}{l}\text { STATE } \\
\text { MISSISSIPPI }\end{array}$ & $\begin{array}{c}\text { WELL NAME E NUMBER } \\
\text { L.R.F.P. MRIG - 2OI }\end{array}$ & $\begin{array}{r}\text { TFSC FIELD REPRESENTATIVE } \\
\text { D. KISTNER/ H. ABEDI }\end{array}$ \\
\hline
\end{tabular}

\section{Explanation}

$\triangle$ cement Plug Ploced for Abandonment

Backfilled with Soil

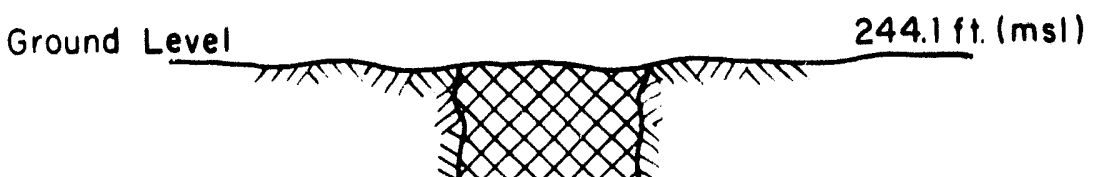

Top of Plug

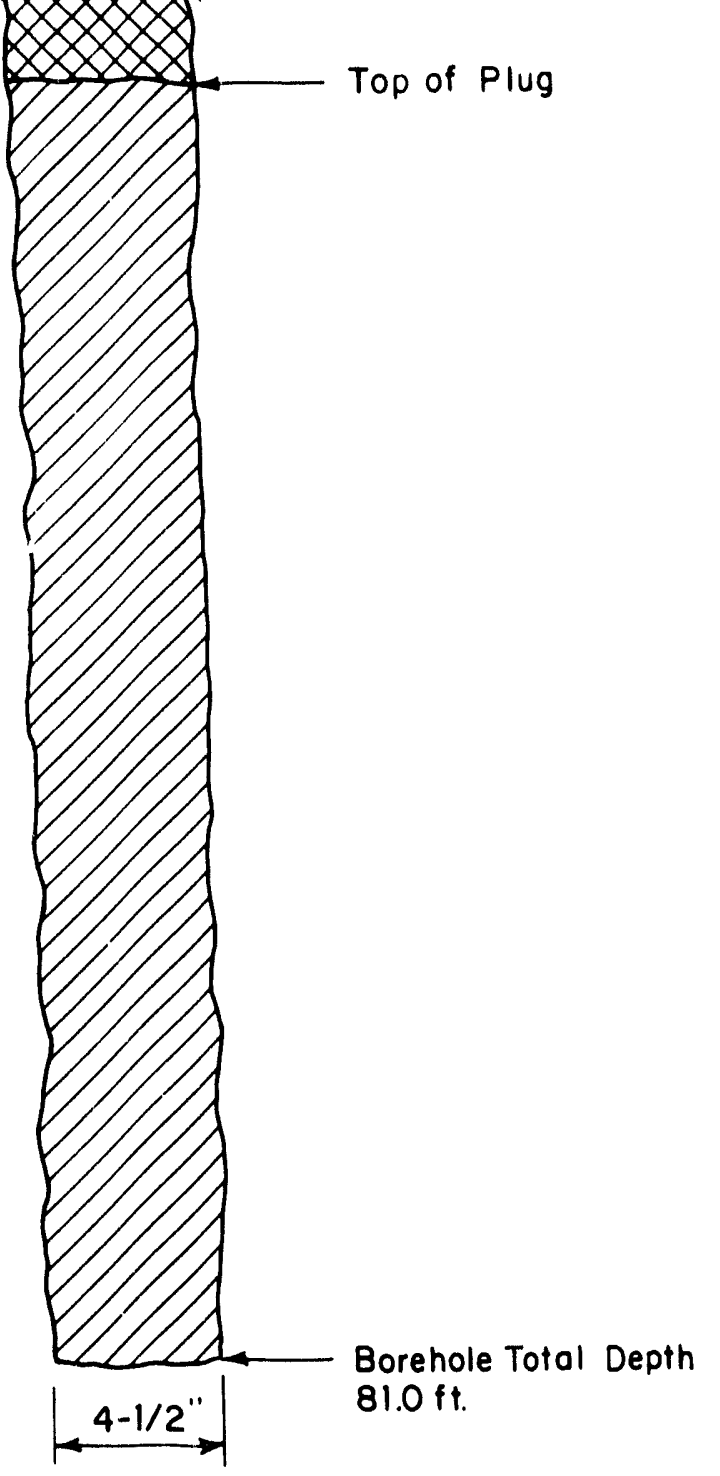

Borehole Diameter

Note:

Not to scale.

All depths measured from ground level elevation $244.1 \mathrm{ft}$. 
BOREHOLE COMPLETION SKETCH

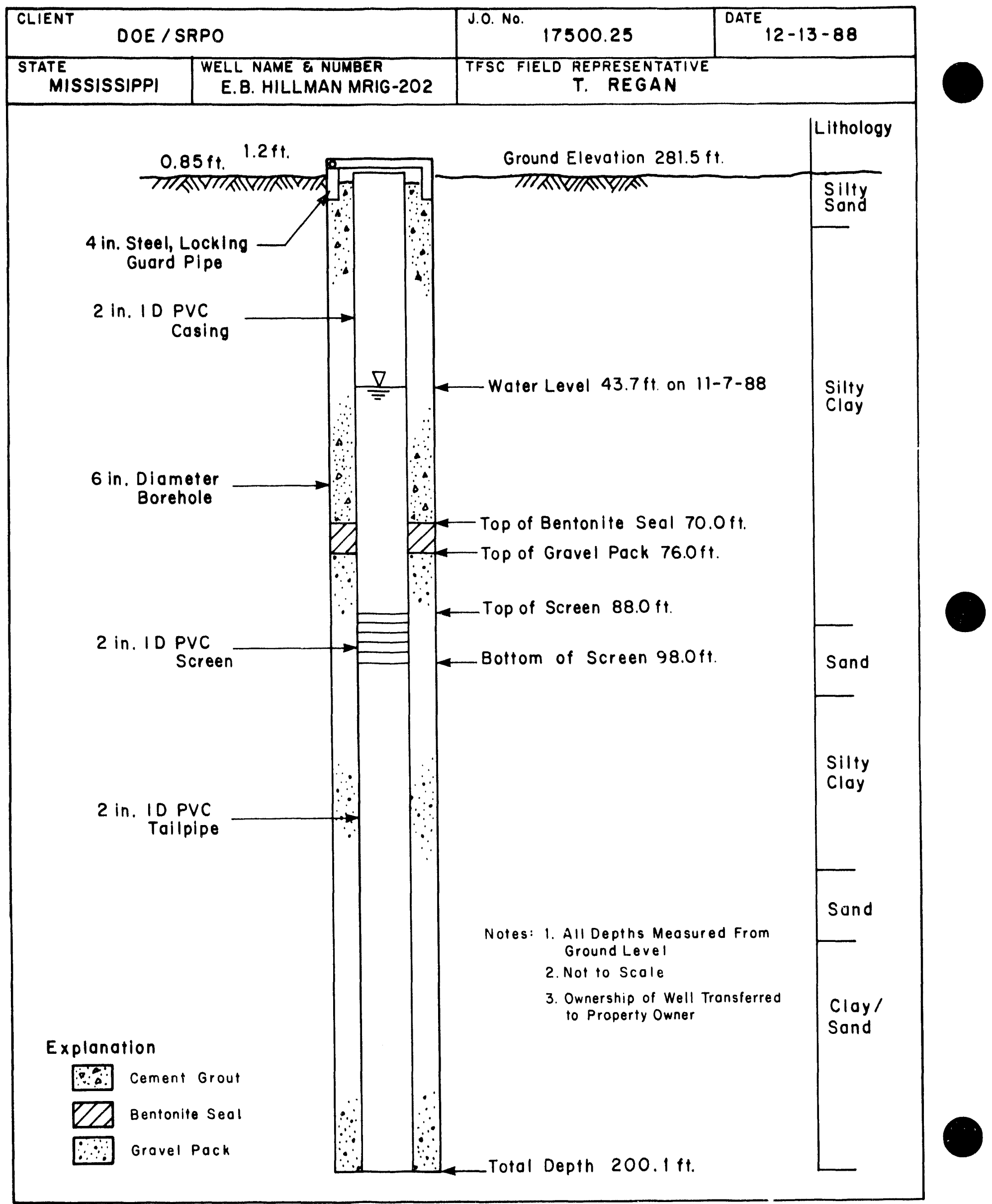


BOREHOLE COMPLETION SKETCH

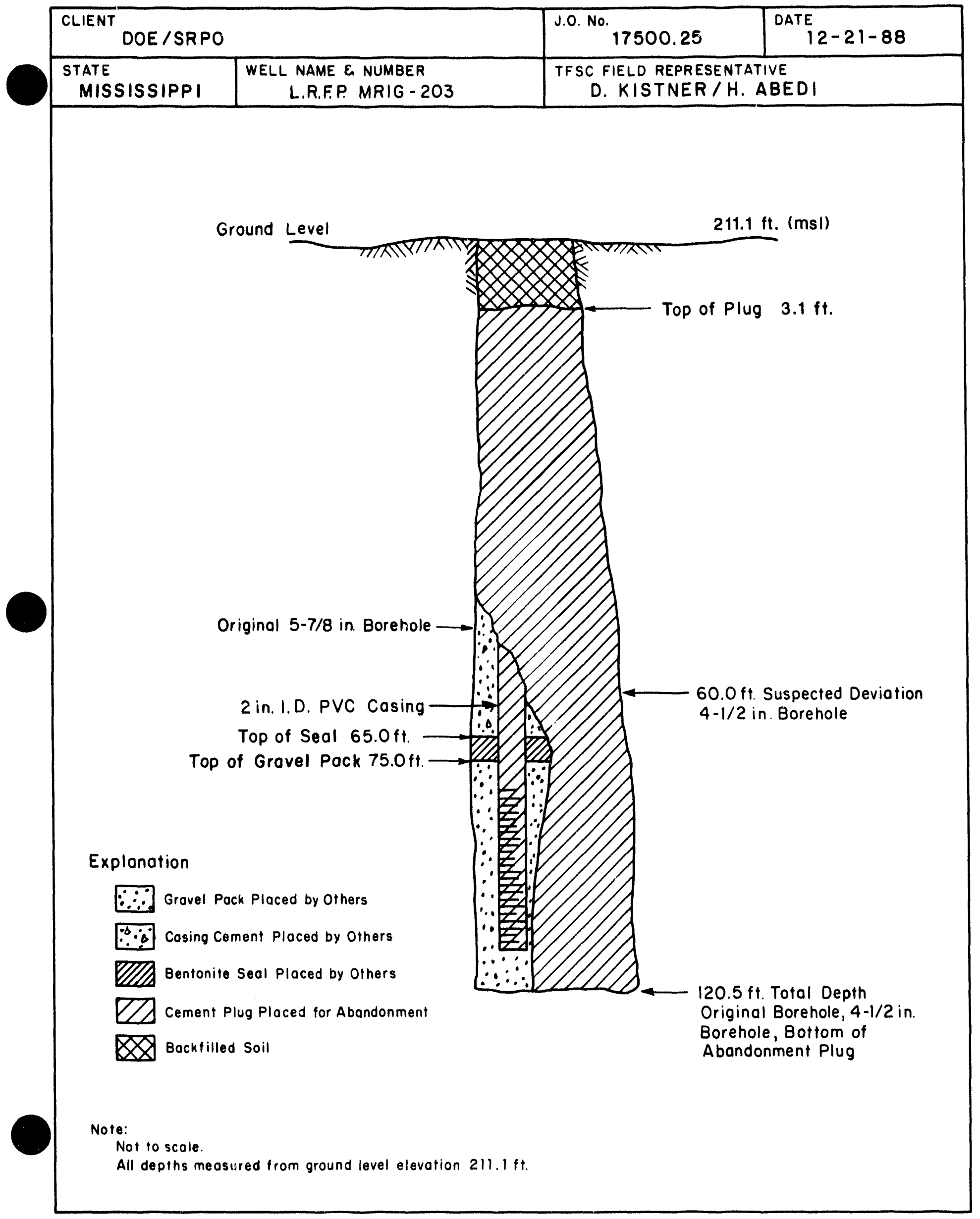


BOREHOLE COMPLETION: : KETCH

\begin{tabular}{|c|c|c|c|}
\hline \multicolumn{2}{|l|}{ CLIENT } & J.0. No. 17500.25 & ${ }^{\text {DATE }} 12-21-88$ \\
\hline $\begin{array}{l}\text { STATE } \\
\text { MISSISSIPPI }\end{array}$ & $\begin{array}{l}\text { WELL NAME \& NUMBER } \\
\text { L.R.F.P. MRIG-204 }\end{array}$ & $\begin{array}{r}\text { TFSC FIELD REPRES } \\
\text { D. KISTN }\end{array}$ & $\begin{array}{l}\text { IVE } \\
\text { †. ABEDI }\end{array}$ \\
\hline
\end{tabular}

Ground Level

Explanation

Da cement plug Ploced for Abandonment

$\triangle$ Backfilled with Soil

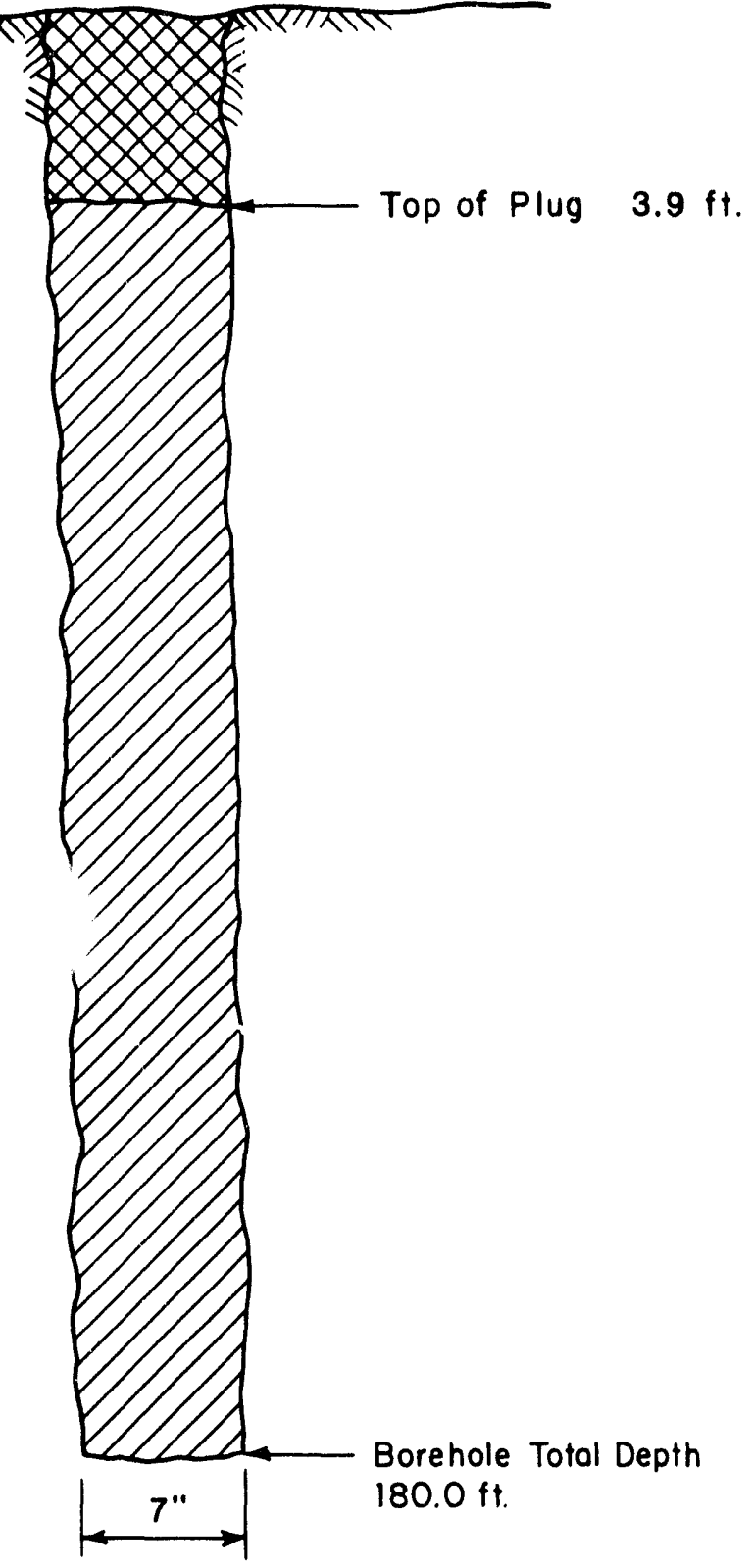

Borehole Diameter

Note:

Not to scale.

All depths measured from ground level elevation $275.1 \mathrm{ft}$. 
BOREHOLE COMPLETION SKETCH

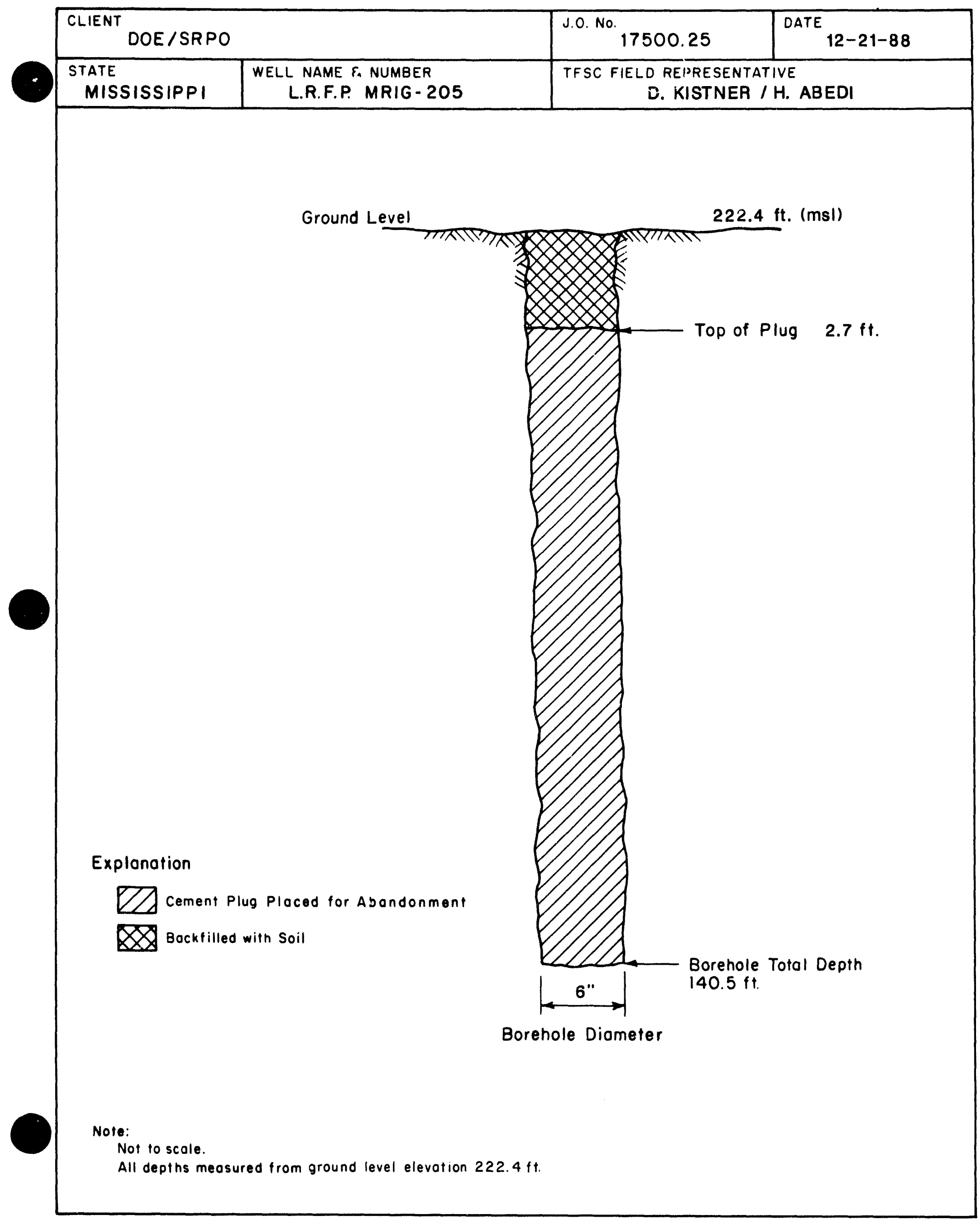


BOREHOLE COMPLETION SKETCH

\begin{tabular}{|l|c|c|c|}
\hline $\begin{array}{l}\text { CLIENT } \\
\text { DOE/SRPO }\end{array}$ & $\begin{array}{l}\text { J.0. No. } 17500.25 \\
\text { DATE }\end{array}$ & \multicolumn{1}{c|}{$12-88$} \\
\hline $\begin{array}{c}\text { STATE } \\
\text { MISSISSIPPI }\end{array}$ & $\begin{array}{c}\text { WELL NAME E. NUMBER } \\
\text { L.R.F.P. MRIG-209 }\end{array}$ & $\begin{array}{c}\text { TFSC FIELD REPRESENTATIVE } \\
\text { D. KISTNER / H. ABEDI }\end{array}$ \\
\hline
\end{tabular}

Ground Leyel

$240.6 \mathrm{ft}$. (msl)

\section{Explanation}

D cement Plug Placed for Abondonment

$\bigotimes$ Backfilled with Soil

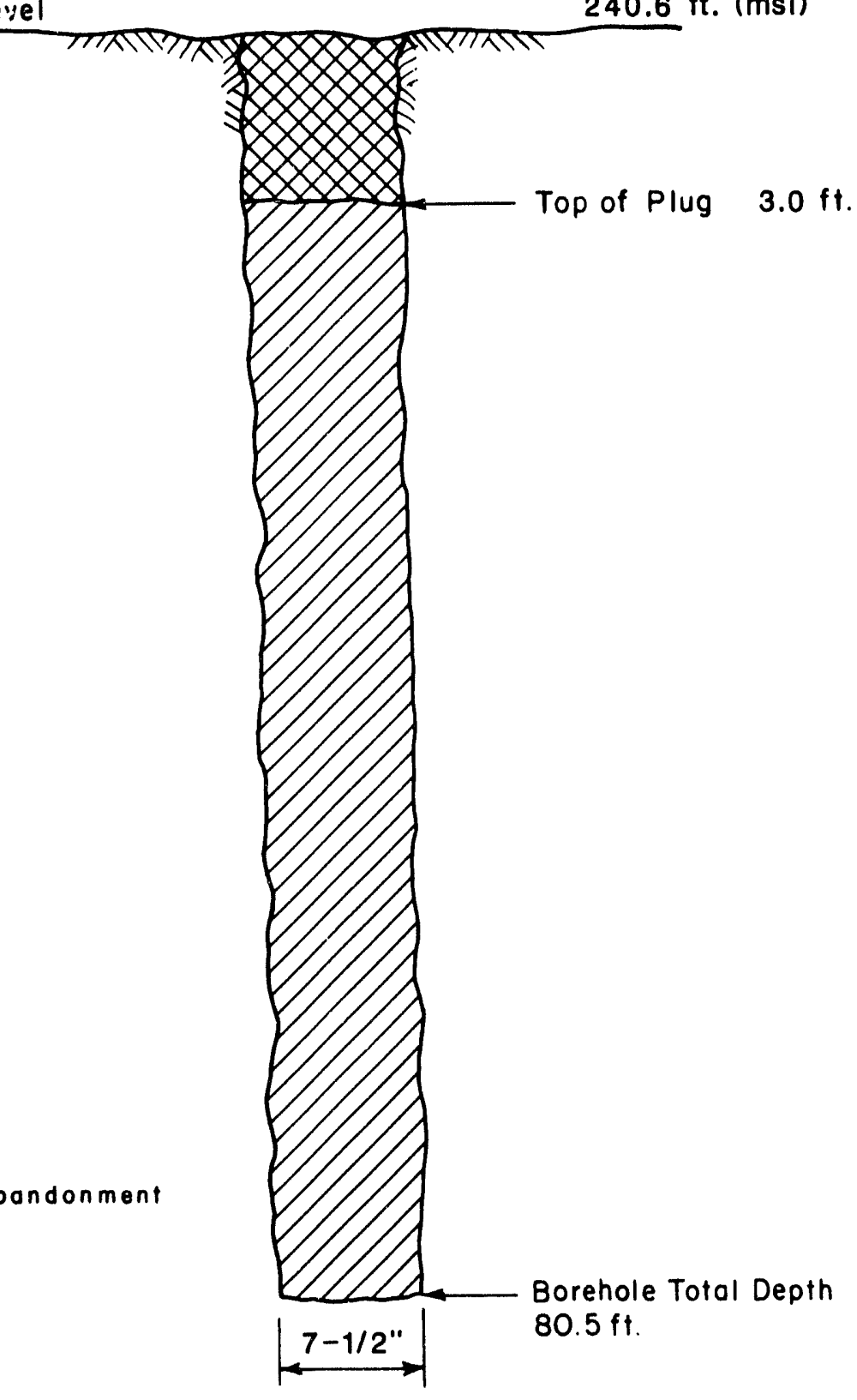

Borehole Diameter

Note:

Not to scale.

All depths measured from ground level elevation $240.6 \mathrm{ft}$. 
BOREHOLE COMPLETION SKETCH

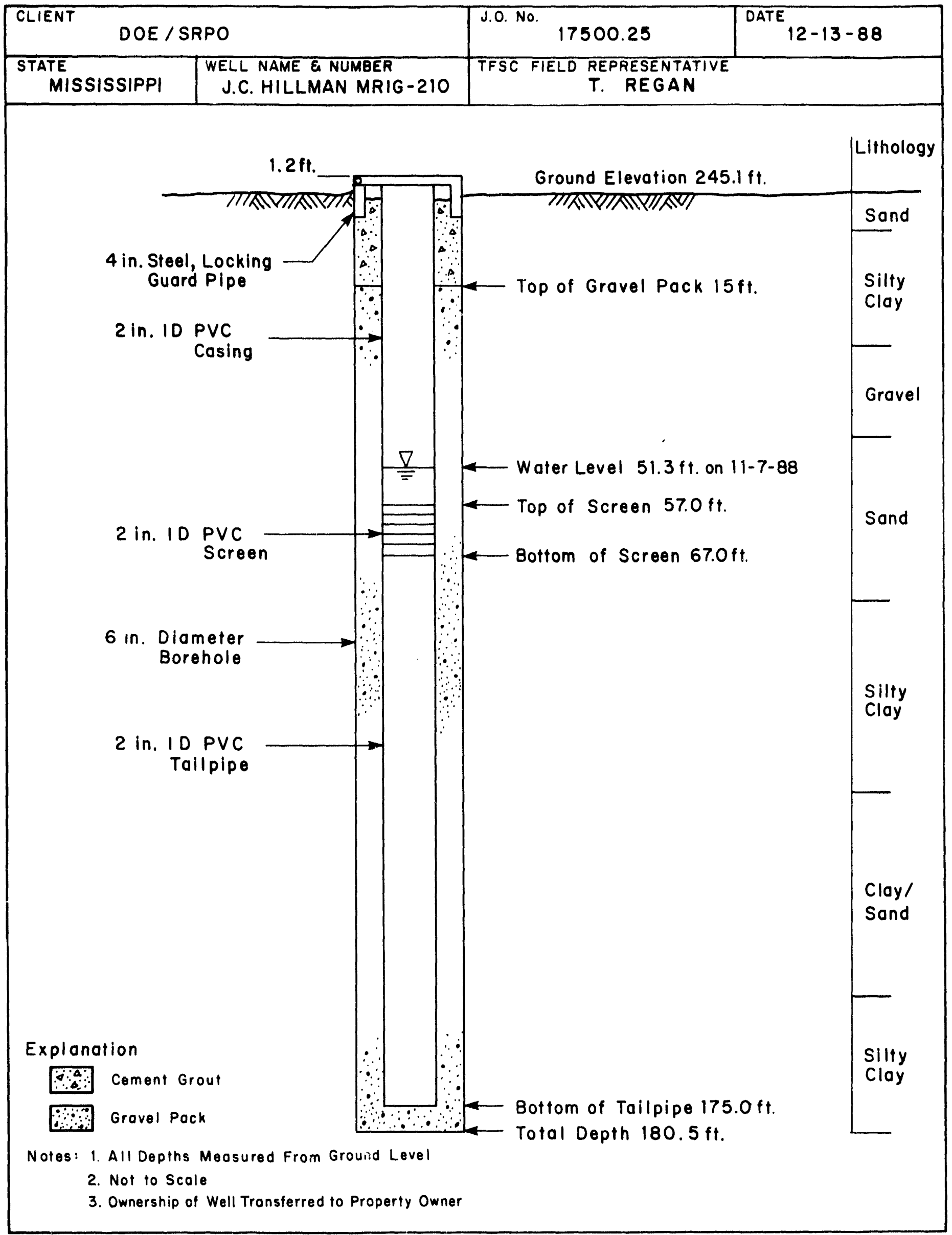


BOREHOLE COMPLETION SKETCH

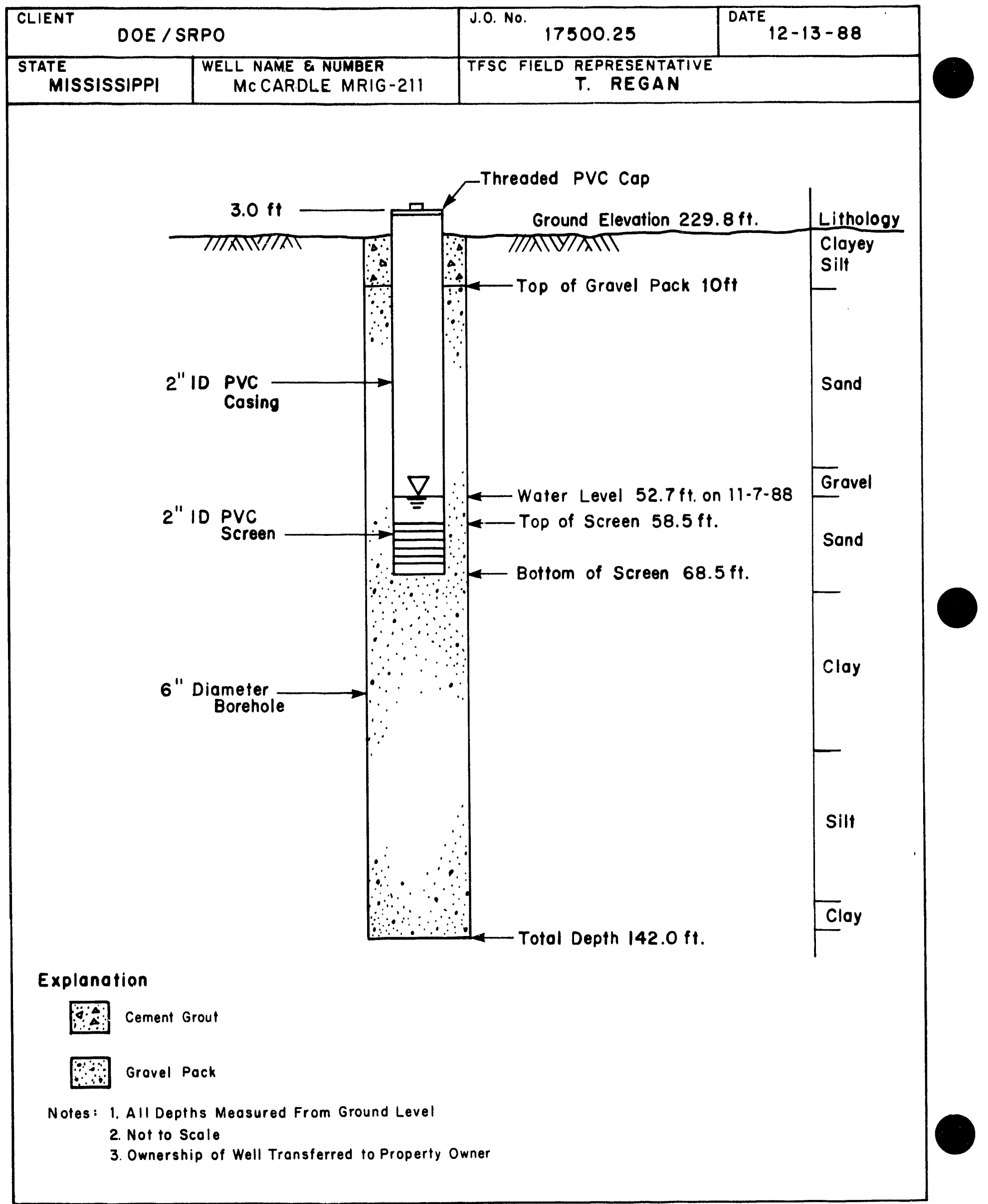


BOREHOLE COMPLETION SKETCH

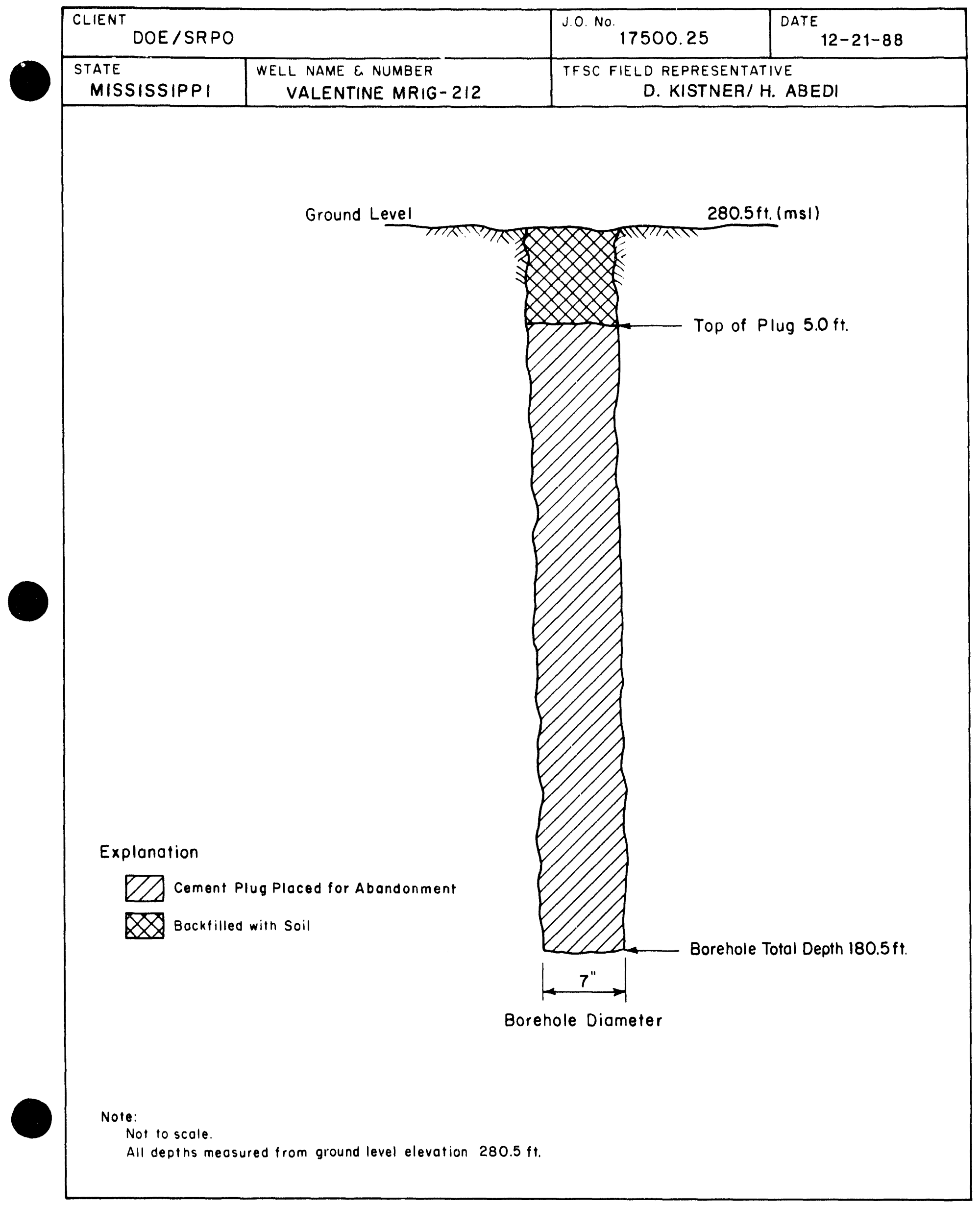




\begin{tabular}{|l|l|l|l|}
\hline $\begin{array}{l}\text { CLIENT DOE / SRPO } \\
\text { STATE }\end{array}$ & $\begin{array}{l}\text { J.0. No. } 17500.25 \\
\text { MISSISSIPPI }\end{array}$ & $\begin{array}{c}\text { WELL NAME \& NUMBER } \\
\text { MCLENDON MRIG-213 }\end{array}$ & $\begin{array}{c}\text { TFSC FIELD REPRESENTATIVE } \\
\text { D. KISTNER / H. ABEDI }\end{array}$ \\
\hline
\end{tabular}

Ground Level IITाभा $178.3 \mathrm{ft}$ (msl)

Explanation

D cement plug placed for Abandonment

$\triangle$ Bockfilled with Soil

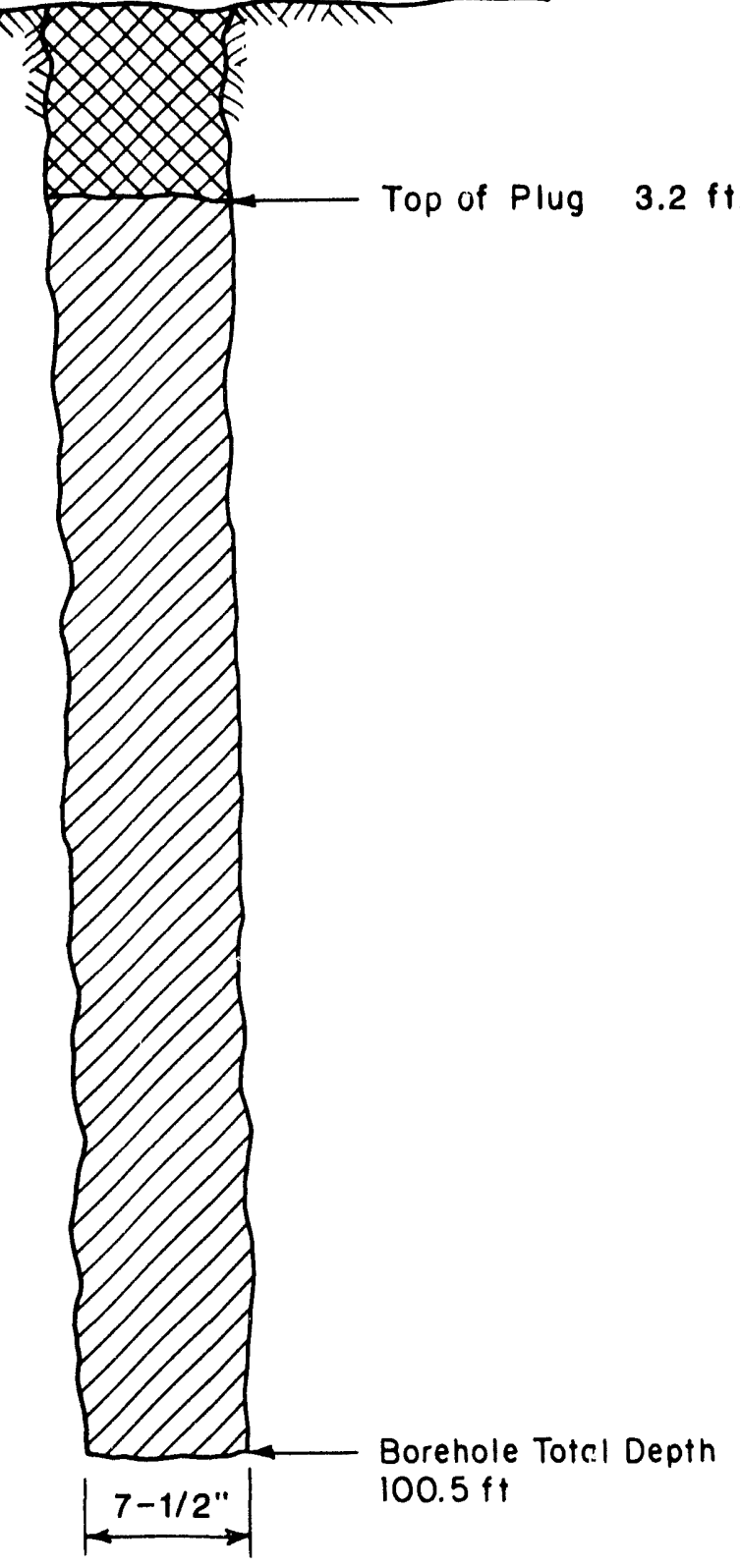

Borehole Diameter

Note:

Not to scale.

All depths measured from ground level elevation $178.3 \mathrm{ft}$. 
BOREHOLE COMPLETION SKETCH

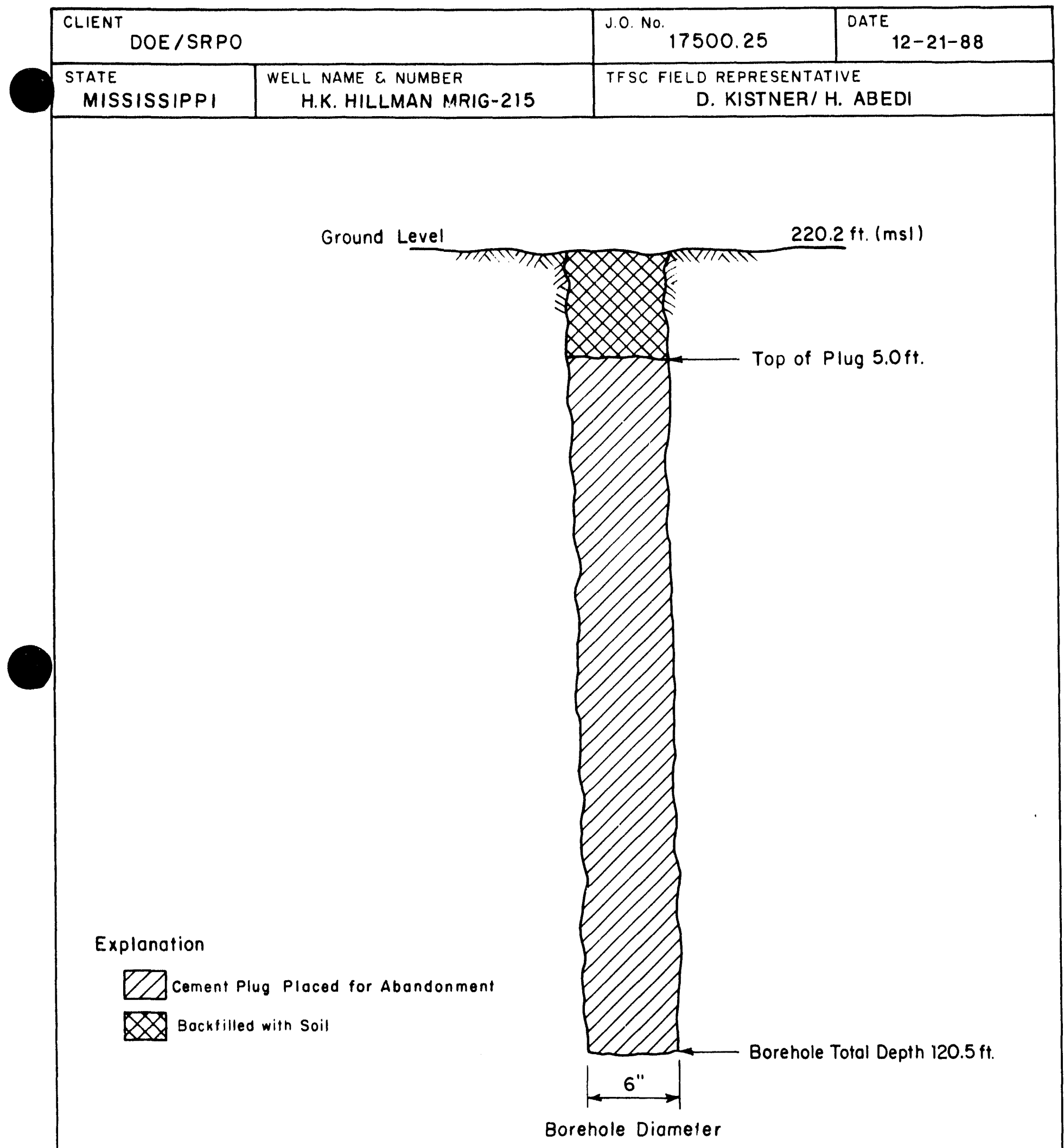

Note:

Not to scole.

All depths meosured from ground level elevation $220.2 \mathrm{ft}$ 
BOREHOLE COMPLETION SKETCH

\begin{tabular}{|l|l|l|l|}
\hline $\begin{array}{l}\text { CLIENT DOE / SRPO } \\
\text { STATE } \\
\text { MISSISSIPPI }\end{array}$ & $\begin{array}{l}\text { J.0. No. } \\
17500.25\end{array}$ & $\begin{array}{c}\text { DATE } \\
12-21-88\end{array}$ \\
\hline
\end{tabular}

Ground Level

218.5 ft. (msl)

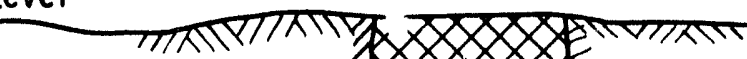

Top of Plug $2.9 \mathrm{ft}$.

2 in. I.D. PVC Casing

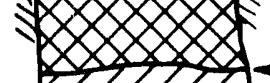

Top of Plug 2.9 ft.

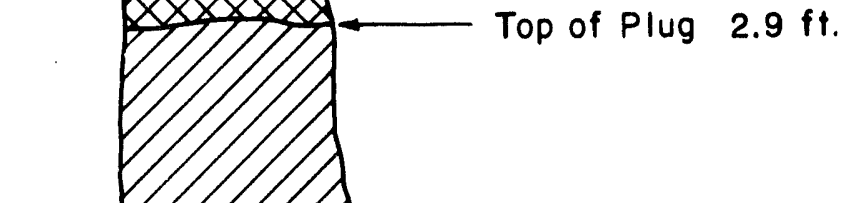

Explanation

$\therefore$ Gravel Pack Placed by Others

$\because \therefore$ Casing Cement Placed by Others

Q7) Bentonite Seal Placed by Others

20 cement Plug Placed for Abandonment

$40.0 \mathrm{ft}$. Suspected
$7-1 / 2$ in. Borehole

Top of Seal $79.0 \mathrm{ft}$.

Top of Gravel Pack $85.0 \mathrm{ft}$

Backfilled Soil

$120.5 \mathrm{ft}$. Total Depth

Origional Borehole, 7-1/2 in. Deviated Borehole, Bottom of Abandonment Plug

Nore:

Not 10 scale.

All depths measured from ground level elevation $218.5 \mathrm{ft}$. 
BOREHOLE COMPLETION SKETCH

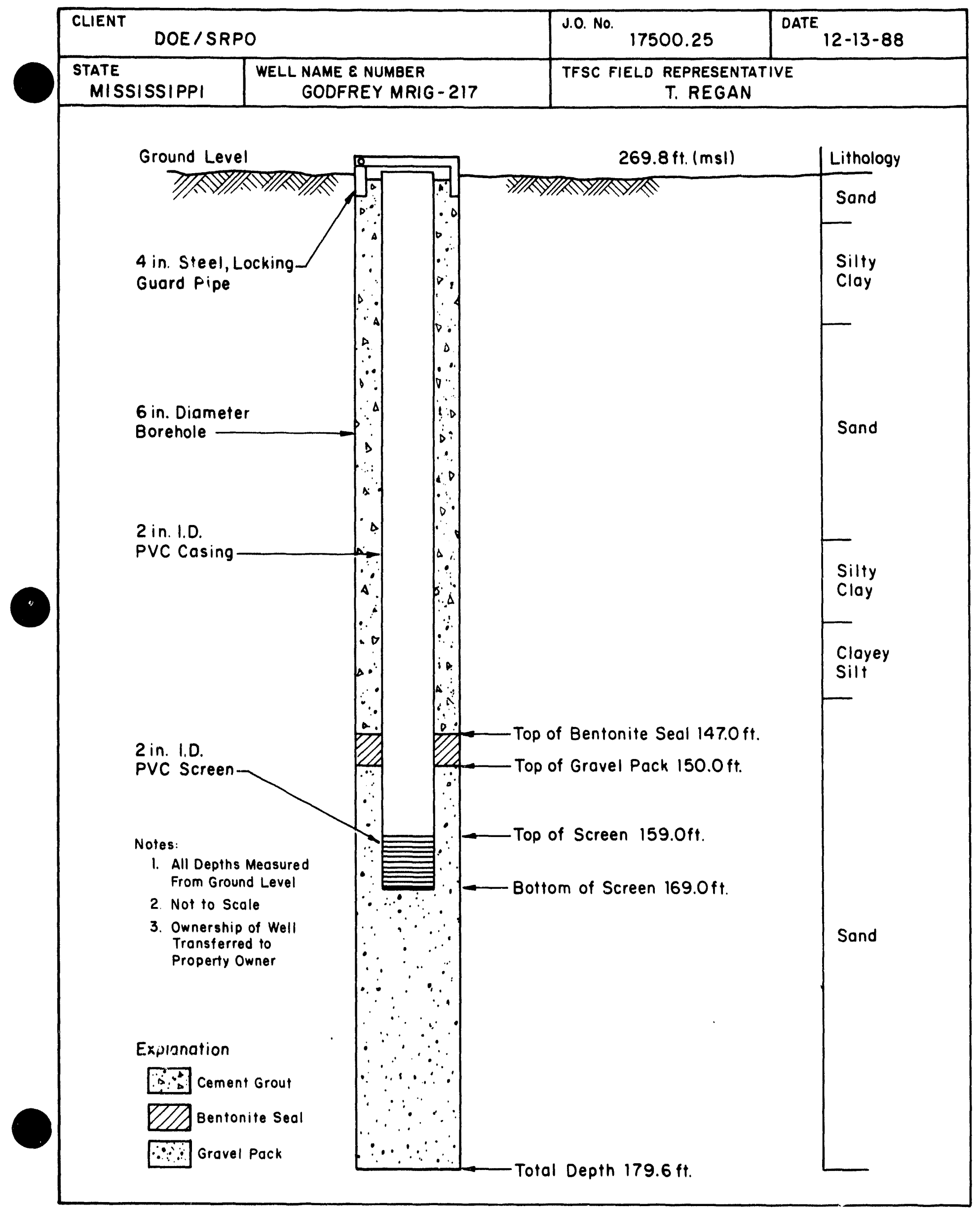


BOREHOLE COMPLETION SKETCH

\begin{tabular}{|l|c|c|c|}
\hline $\begin{array}{l}\text { CLIENT DOE / SRPO } \\
\text { STATE } \\
\text { MISSISSIPPI }\end{array}$ & $\begin{array}{c}\text { J.0. No. } \\
17500.25\end{array}$ & $\begin{array}{c}\text { DATE NAME E NUMBER } \\
12-21-88\end{array}$ \\
\hline
\end{tabular}

Ground Level

$239.7 \mathrm{ft} .(\mathrm{msl})$

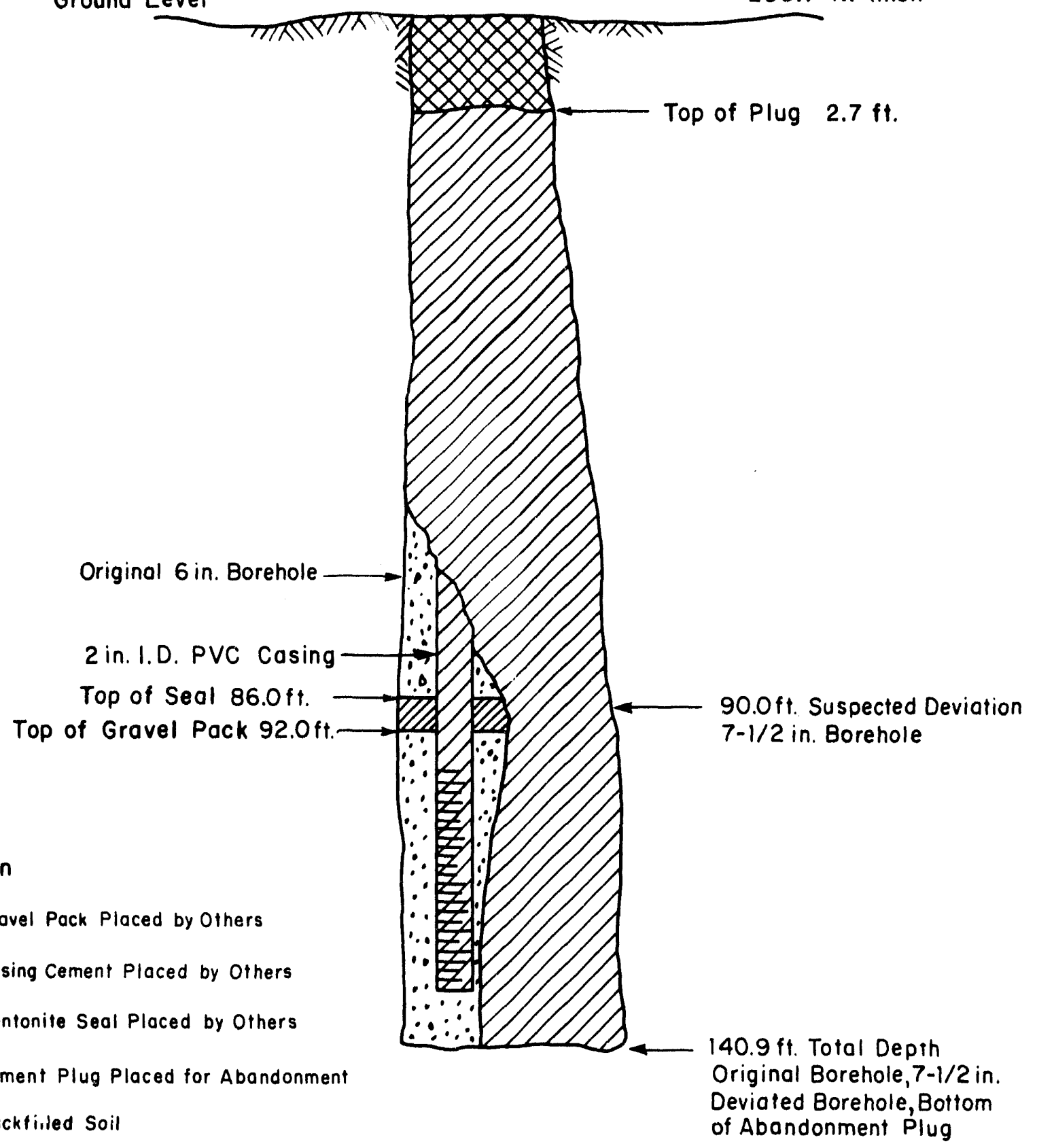

Explanation

$\therefore \therefore$ Grovel Pack Placed by Others

$\because$ id Cosing Cement Placed by Others

Q7en Bentonite Seal Placed by Others

21 cement Plug Placed for Abandonment

Original Borehole, $7-1 / 2$ in.

$\$$ Backfilied Soil

of Abandonment Plug

Note:

Not to scale.

All depths measured from ground level elevation $239.7 \mathrm{ff}$. 
BOREHOLE COMPLETION SKETCH

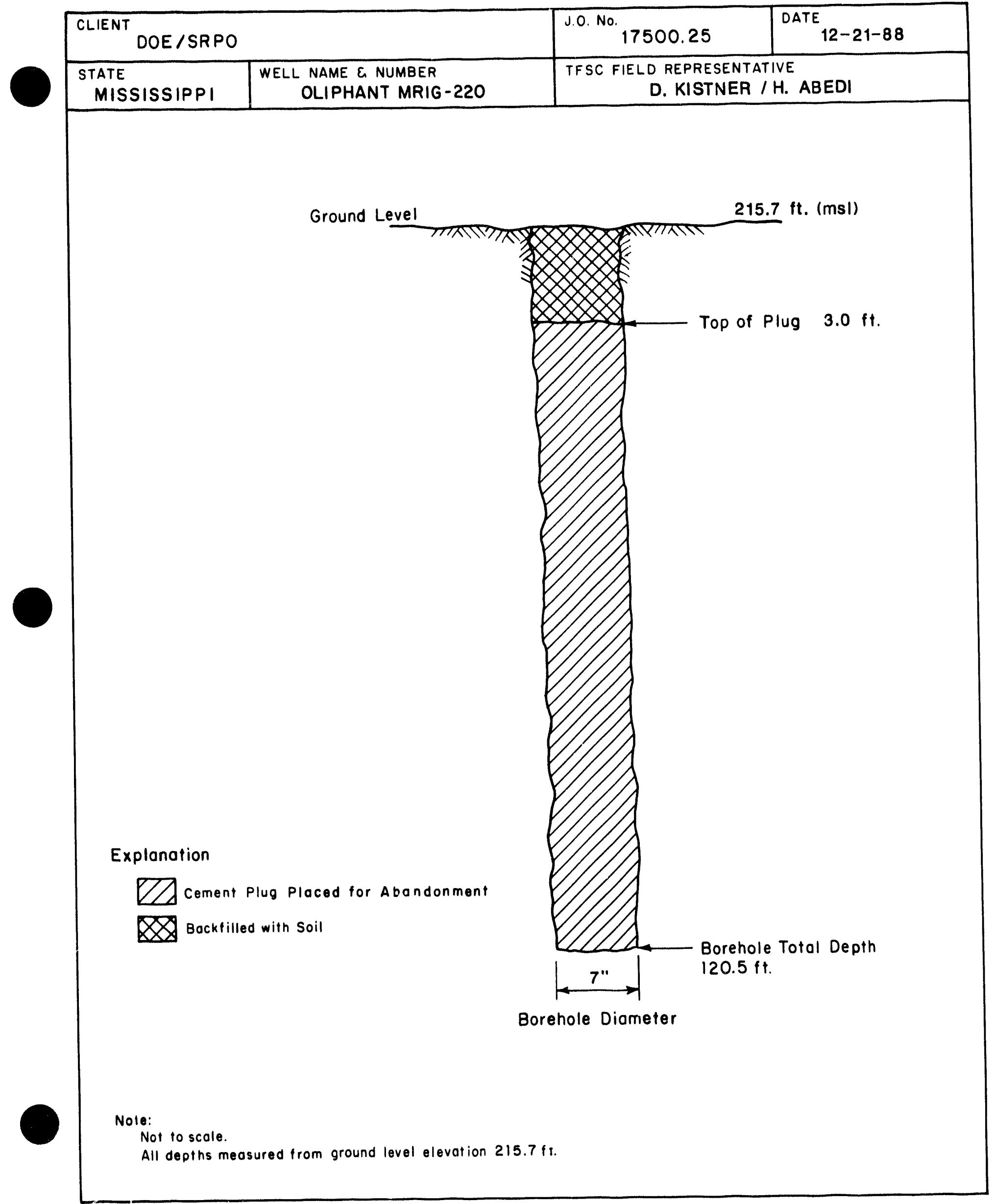




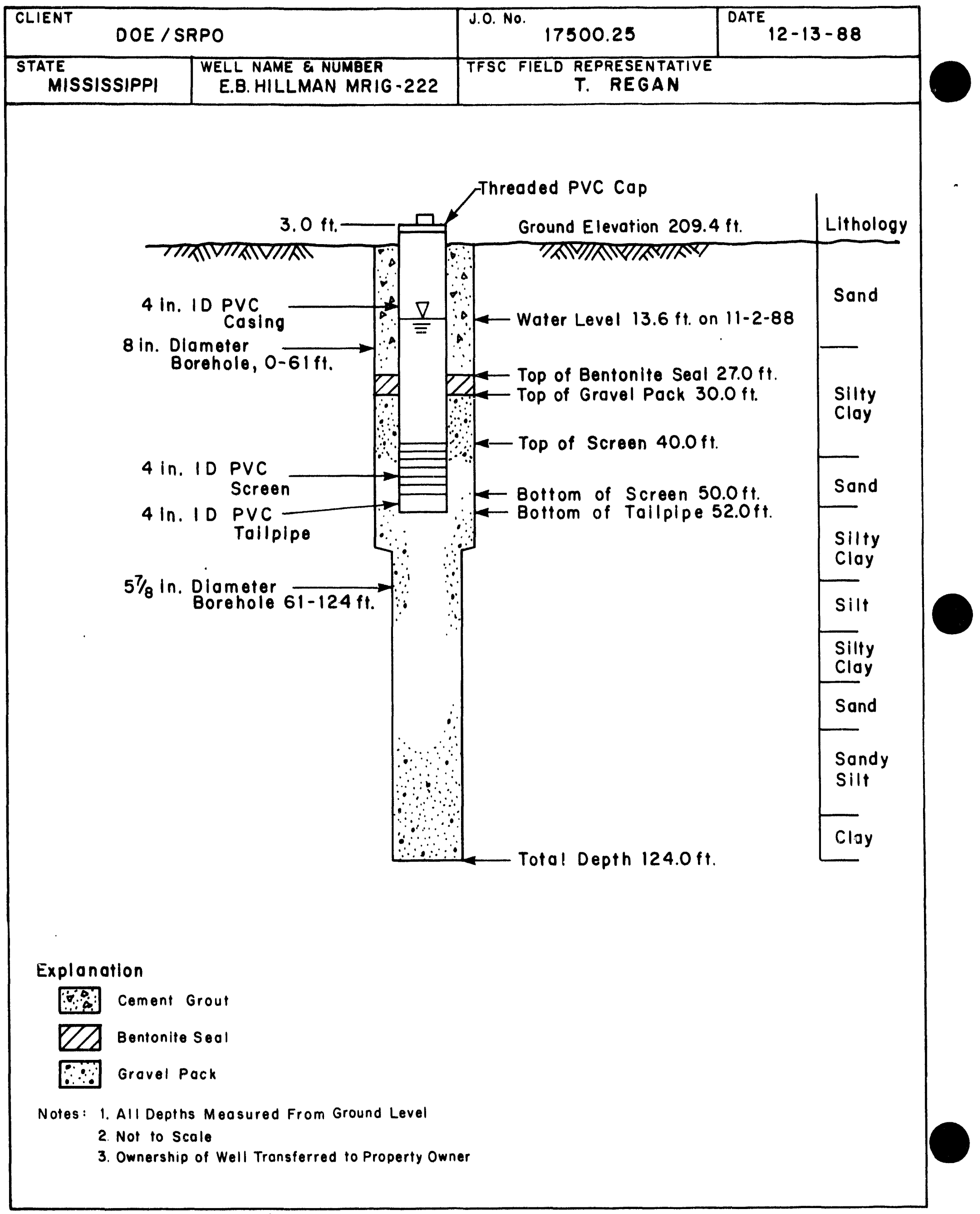


BOREHOLE COMPLETION SKETCH

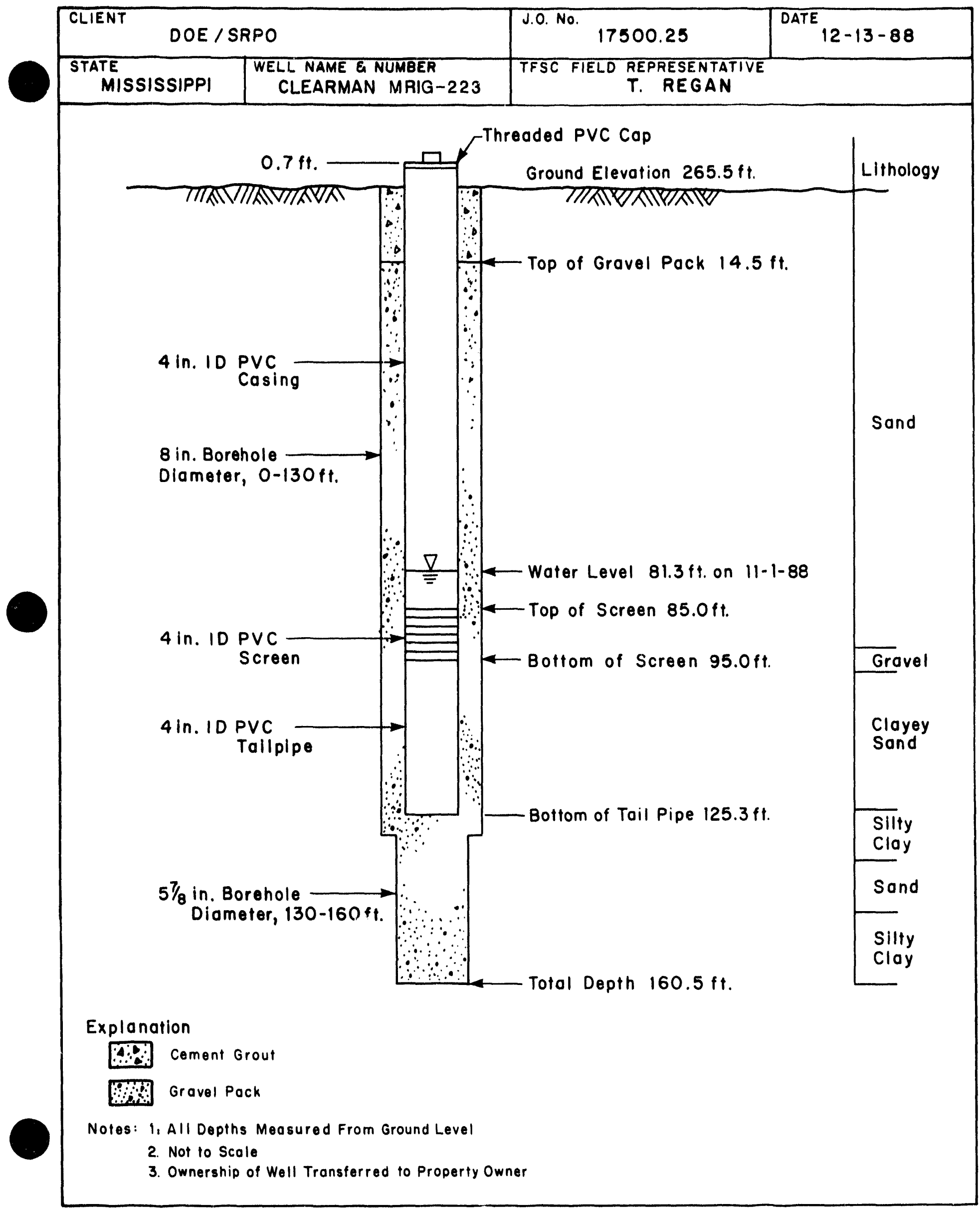




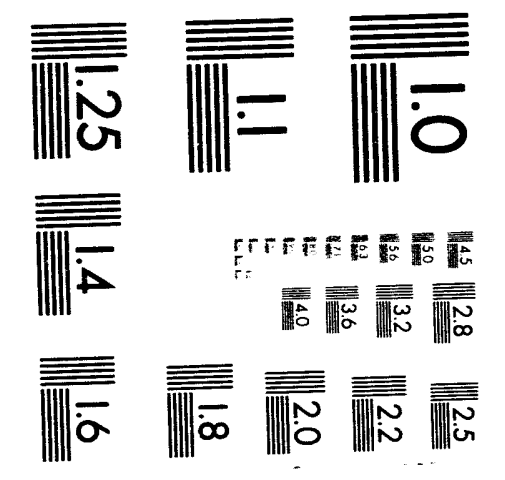



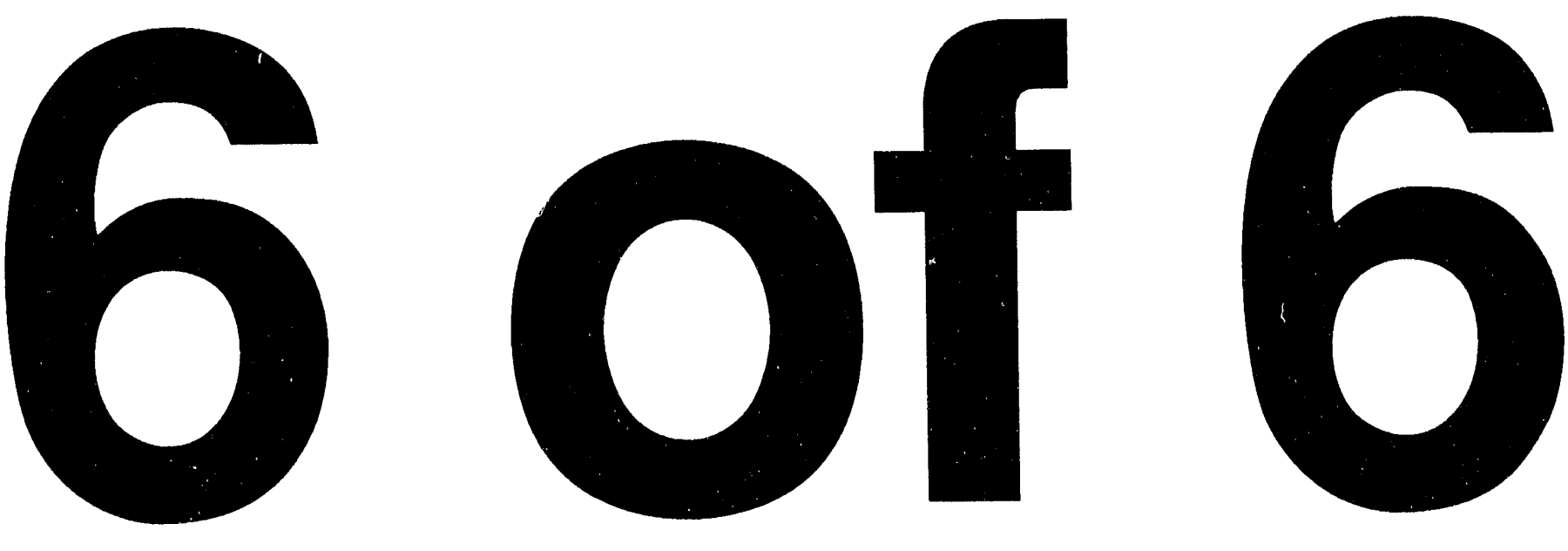
BOREHOLE COMPLETION SKETCH

\begin{tabular}{|l|l|l|l|}
\hline $\begin{array}{l}\text { CLIENT DOE / SRPO } \\
\text { STATE }\end{array}$ & $\begin{array}{l}\text { J.0. No. } \\
\text { MISSISSIPPI }\end{array}$ & $\begin{array}{c}\text { WELL NAME E NUMBER } \\
\text { PHILLIPS MRIG-224 }\end{array}$ & $\begin{array}{r}\text { TFSC FIELD REPRESENTATIVE } \\
\text { D. KISTNER / H. ABEDI }\end{array}$ \\
\hline
\end{tabular}

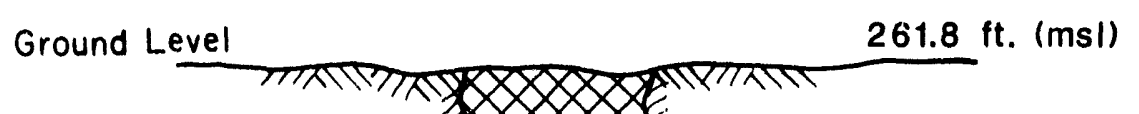

Explanation

$P A$ cement plug placed for Abandonment

Bockfilled with Soil

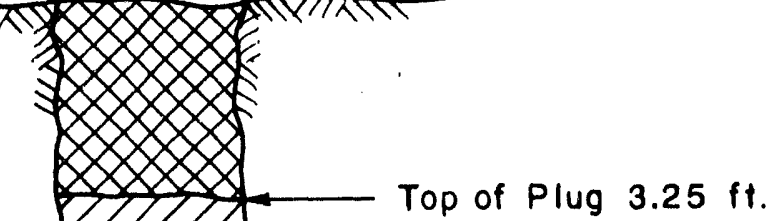

Borehole Total Depth

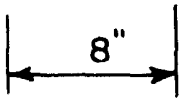

$130.0 \mathrm{ft}$.

Borehole Diometer

Note:

Not to scale.

All depths rieasured from ground level elevation $261.8 \mathrm{ft}$. 
BOREHOLE COMPLETION SKETCH

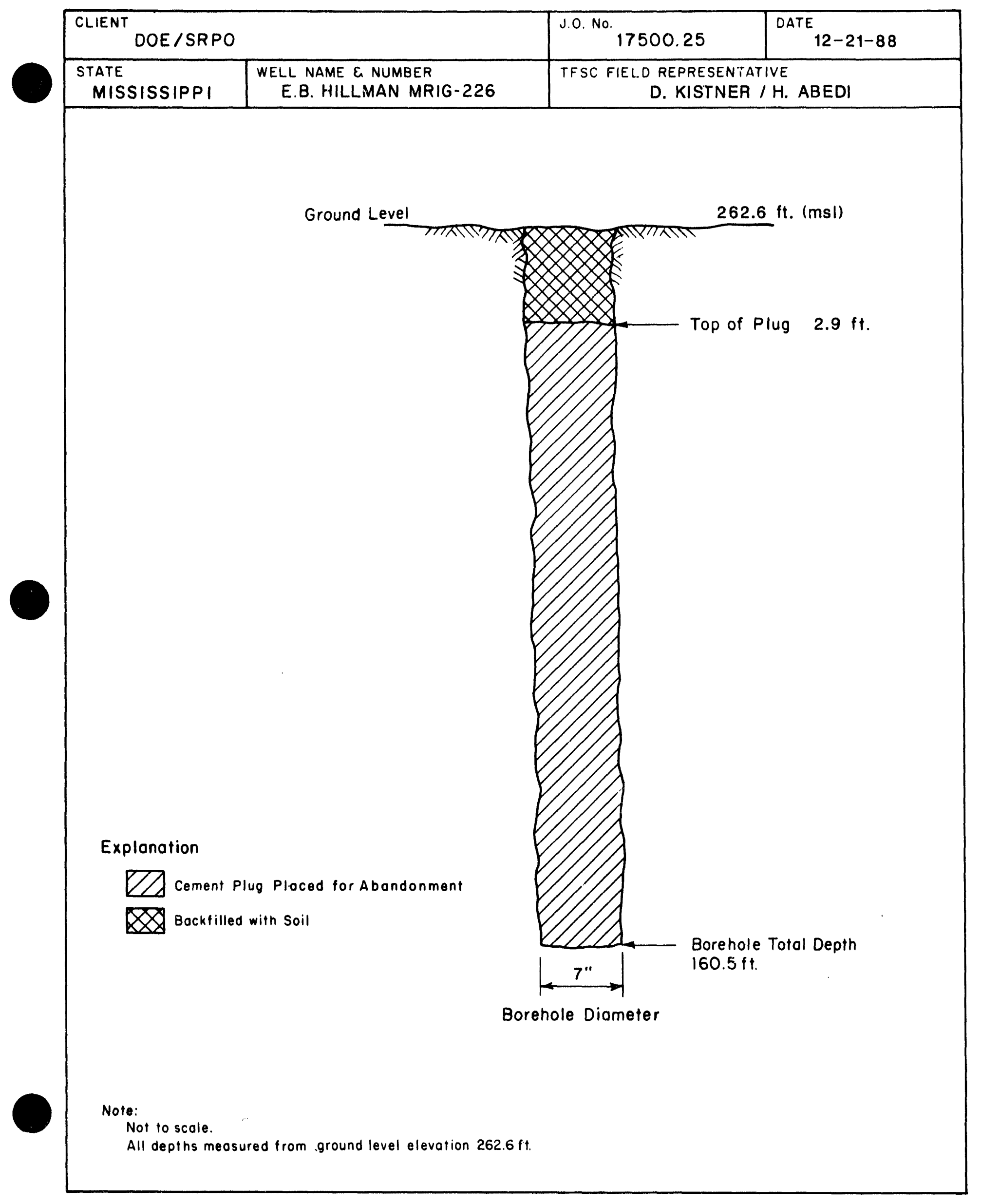


BOREHOLE COMPLETION SKETCH

\begin{tabular}{|l|l|l|l|}
\hline $\begin{array}{l}\text { CLIENT DOE / SFPO } \\
\text { STATE }\end{array}$ & $\begin{array}{l}\text { J.0. No. } 17500.25 \\
\text { MISSISSIPPI }\end{array}$ & $\begin{array}{r}\text { WELL NAME \& NUMBER } \\
\text { E. B. HILLMAN MRIG-227 }\end{array}$ & $\begin{array}{r}\text { TFSC FIELD REPRESENTATIVE } \\
\text { D. KISTNER / H. ABEDI }\end{array}$ \\
\hline
\end{tabular}

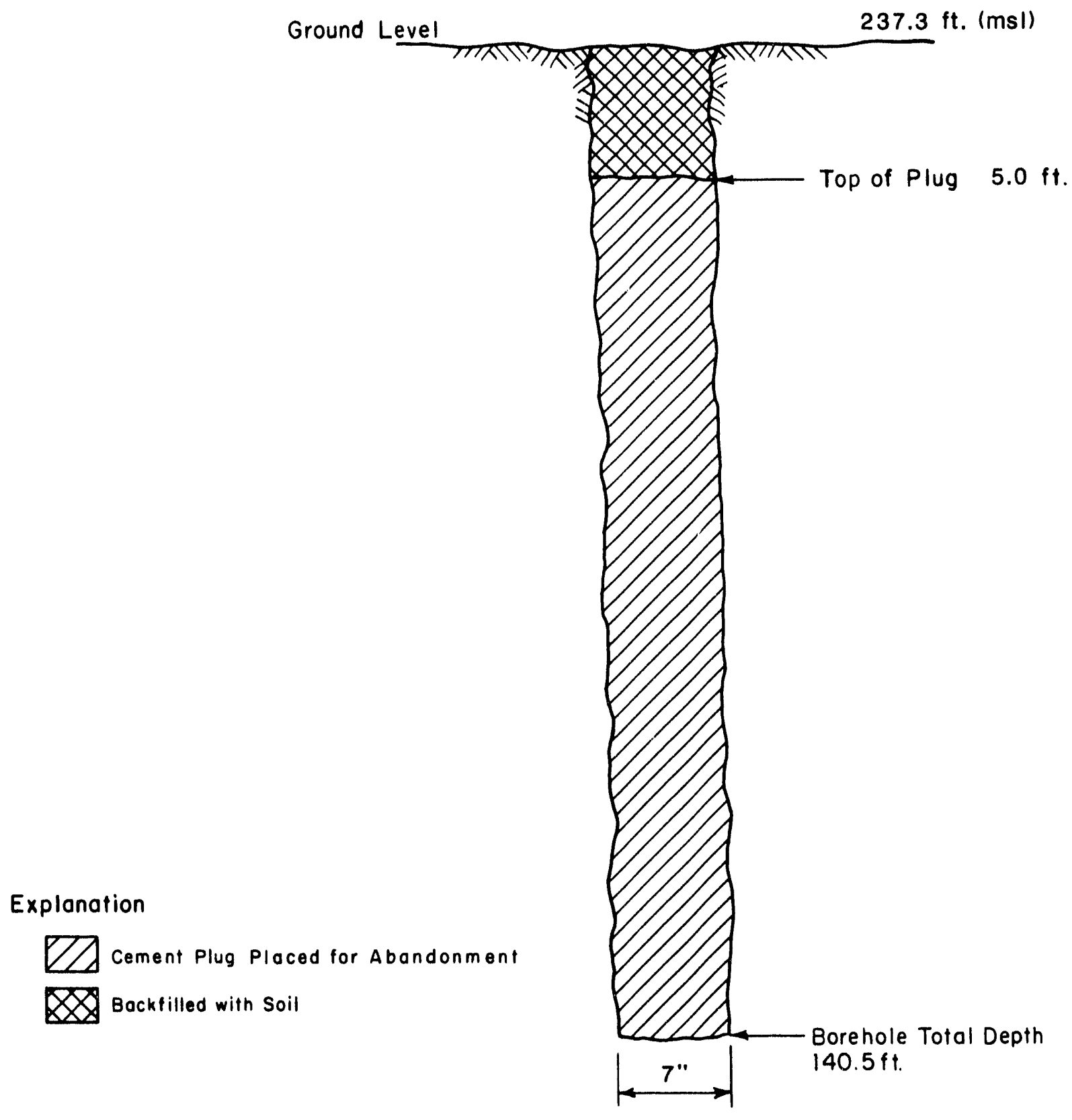

Borehole Diameter

Note:

Not to scale.

All depths meosured from ground level elevation $237.3 \mathrm{ft}$. 
BOREHOLE COMPLETION SKETCH

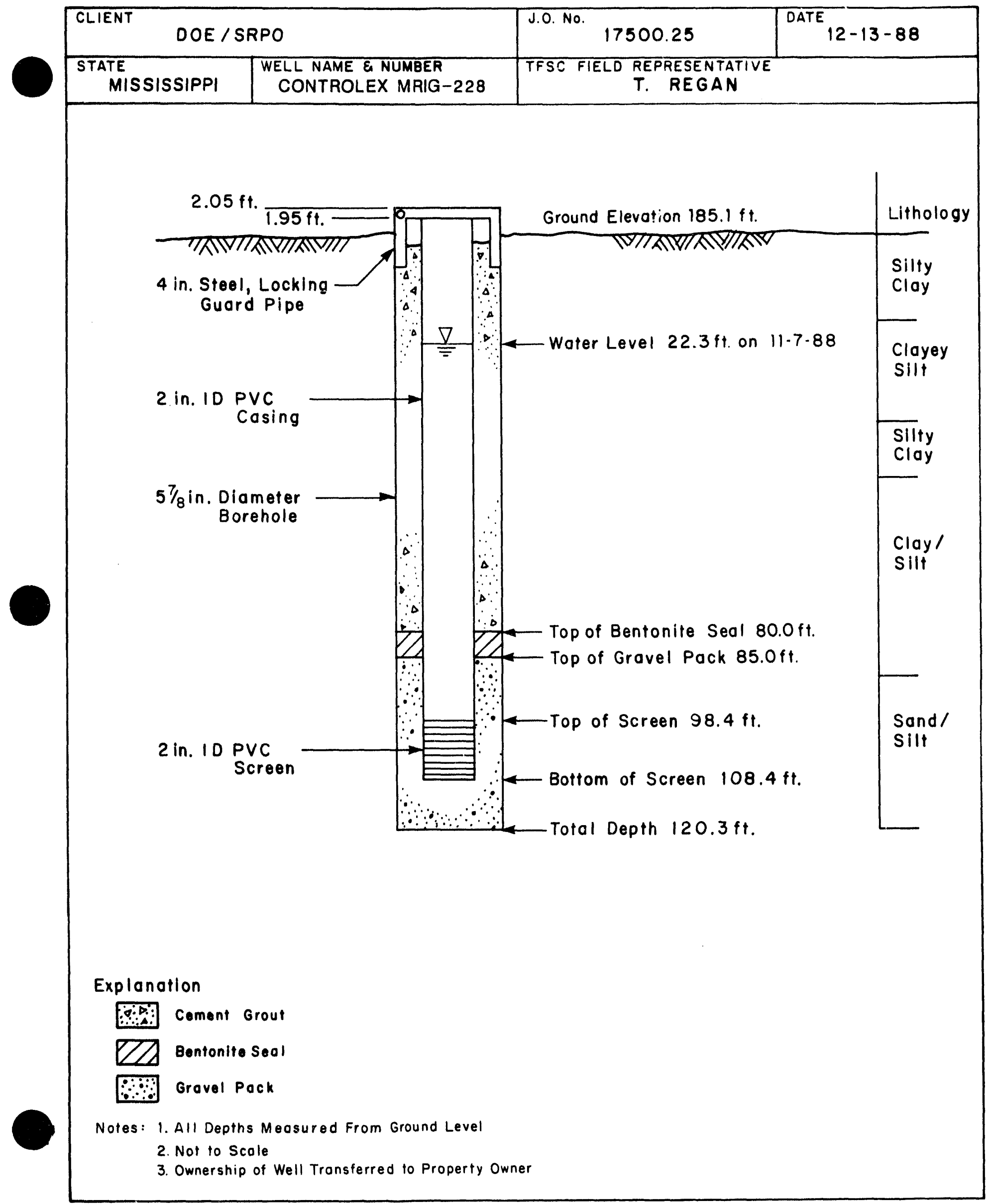


BOREHOLE COMPLETION SKETCH

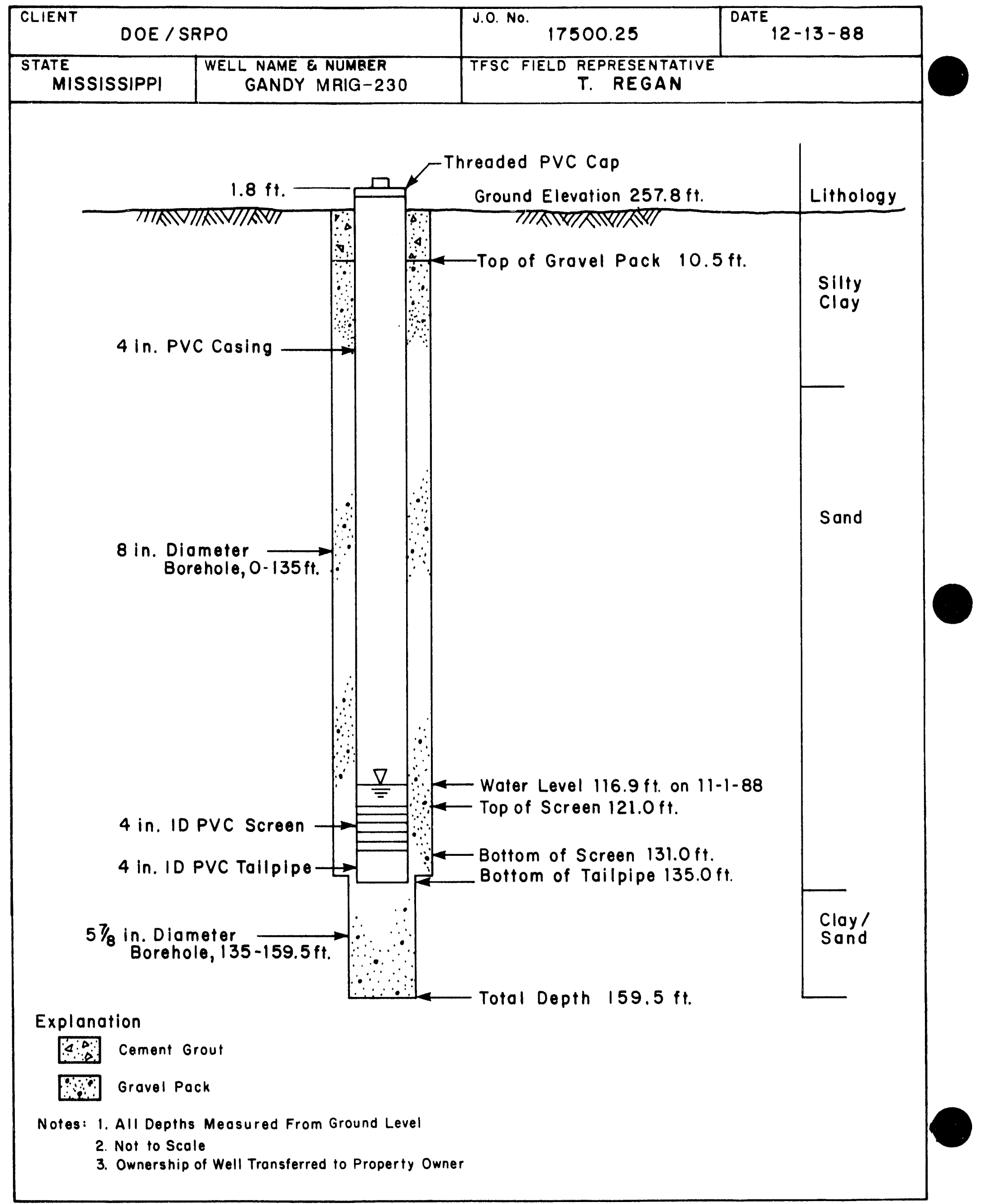




\section{BOREHOLE COMPLETION SKETCH}

\begin{tabular}{|l|l|l|l|}
\hline $\begin{array}{l}\text { CLIENT DOE/SRPO } \\
\text { STATE }\end{array}$ & $\begin{array}{l}\text { J.0. No. } 17500.25 \\
\text { DISSISSIPPI }\end{array}$ & $\begin{array}{c}\text { WELL NAME \& NUMBER } \\
\text { L.R.F.P. MRIG-232 }\end{array}$ & $\begin{array}{c}\text { TFSC FIELD REPRESENTATIVE } \\
\text { D. KISTNER / H. ABEDI }\end{array}$ \\
\hline
\end{tabular}

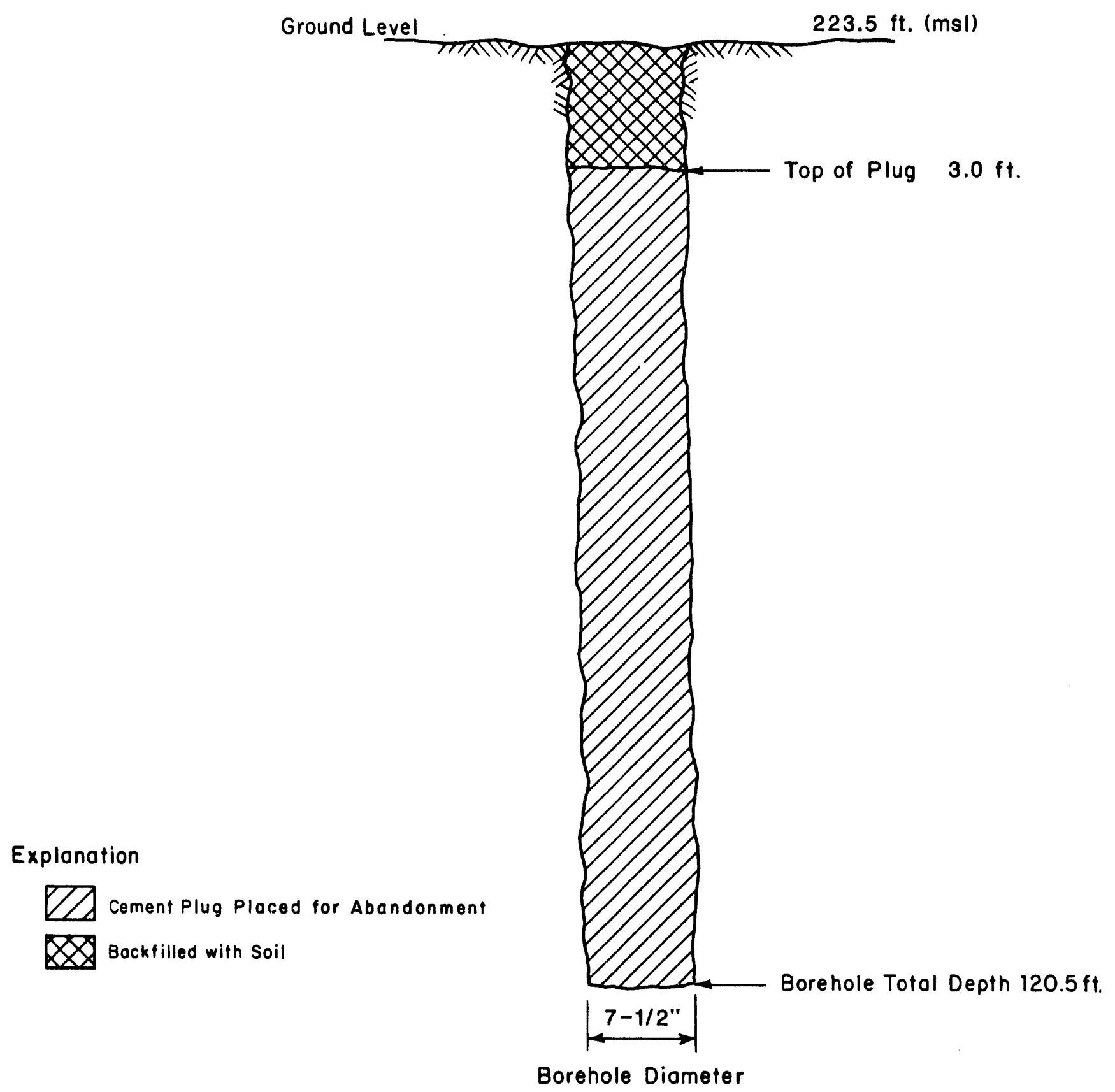

Note:

Not to scale.

All depths meosured from ground level elevation $223.5 \mathrm{ft}$. 
APPENDIX I

Damage Releases and Transfer Agreements 


\title{
U.S. Department of Energy \\ Salt Repository Project Office
}

\author{
Amarillo Petroleum Building \\ Suite 205 \\ 203 W. 8th Street \\ P.O. Box 9334 \\ Amarillo. Texas $79105-9334$ \\ Apri1 24, 1989
}

Mr. Joe Clayton

Forest Service, United States

Department of Agriculture

Suite 1141

100 West Capitol Street

Jackson, MS 39269

SUBJECT: USFS TEST SITES - CYPRESS CREEK SALT DOME PERRY COUNTY, MISSISSIPPI

Dear Mr. Elayton:

Regarding surface use for geologic and other studies and oll-sites provided under terms of agreement by and between the United States Department of Energy and the United States Department of Agriculture, Forest Service, dated February 21, 1979, please be advised, as follows:

Stone \& Webster Engineering Corporation (SWEC) acting as subcontractor for the United States Department of Energy has conducted decommissioning activities, plugging and abandoning of wells, removal of equipment and material, and restoration of sites under the terms of said agreement. The wells decommissioned by SWEC were for hydrologic monitoring studies and related activities on or in the vicinity of lands in the Desoto National Forest within Section 33, T3N-R1OW, Sections 4, 5, 8 thrcugh 11, 14, 15, 17, 20 through 23, 27, 28, and 33, T2N-R1OW, Perry County, Mississippi and Section 4, TIN-R13W, Forrest County, Mississippi, and are listed on Attachment 1 to this letter.

With reference to Paragraph B-10 of said agreement, the studies provided under the terms of the agreement have been completed, the wells have been plugged and abandoned in accordance with rules and Regulations of the Mississippi

Department of Natural Resources and the Mississippi 011 \& Gas Board. The we11sites have been restored to as near original condition as possible and the United States Department of Energy has no further use for the sites.

Accordingly, this letter is written notice under Paragraph $C-1$ of said agreement that the United States Department of Energy does hereby terminate 
said agreement. At your earliest convenience, it would be appreciated if you acknowledge receipt of this letter, acceptance of site restoration and termination of the agreement.

Sincerely,

UNITED STATES DEPARTMENT OF ENERGY

By:

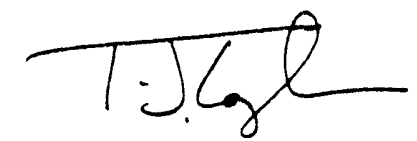

Theodore J. Taylor

Project Manager

The undersigned does hereby acknowledge receipt of this letter, acceptance of site restoration, and termination of said agreement.

UNITED STATES DEPARTMENT OF AGRICULTURE, FOREST SERVICE
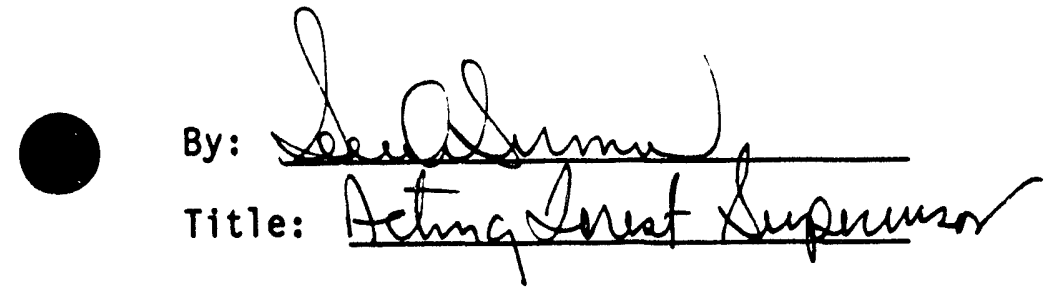


\section{ATTACHRENT 1}

WELL

NATE \& NUMBER

U.S.F.S. MCCG-1

U.S.F.S. MCCG-IWS

U.S.E.S. MCCG-2

U.S.E.S. MCCG-2WS

U.S.E.S. MCCH-3A

U.S.F.S. $\mathrm{MCCH}-3 \mathrm{~B}$

U.S.E.S. $\mathrm{MCCH}-3 \mathrm{C}$

U.S.F.S. MCCH-3D

U.S.E.S. MCCH-3WS

U.S.F.S. MA-5A

U.S.E.S. MH-5B

U.S.F.S. MH-5C

U.S.E.S. M-5D

U.S.F.S. W-5WS

U.S.E.S. MCCG-102

U.S.F.S. ACCG-102

U.S.F.S. MCCG-103

U.S.F.S. ACCG-104

U.S.F.S. HCCG-105

U.S.F.S. MCCG-106

U.S.F.S. MCCG-107

U.S.F.S. MCCG-110

U.S.E.S. ICCG-111

U.S.F.S. $\operatorname{accG-112}$
LOCATION

TOWNSHIP AND RANGE

S9, T2N, R1OW, 2702 FT. EWL, 2528 FT. NSL

S9, T2N, R1OW, 78 ET. N45W of MCCG-1

S33. T3N, RIOW, 1119 FT. SNL, 1961 ET. WEL

S33, T3N, R1OW, 94 FT. S47W of MCCG-2

S33, T2N, RIOW, 1084 FT. WEL, 681 FT. NSL

S33, T2N, R1OW, 1030 FT. WEL, 546 FT NSL

S33, T2N, R1OW, 984 FT. WEL, 641 FT NSL

S33, I2N, R1OW, 1129 FT. WEL, 696 FT NSL

S33, T2N, R1OW, 110 FT. DUE WEST OF MCCH-3A

S4, T1N, R13W, 2059 FT. WEL, 2194 ET. NSL

S4, T1N, RI3W, 1984 FT. WEL, 2284 FT. NSL

S4, T1N, R13W, 1914 FT. WEL, 2174 FT. NSL

S4, TIN, R13W, 1984 FT. WEL, 2119 FT. NSL

S4, TIN, RI3W, 101 FT. S77W of MAH-5A

S4, T2N, RIOW, 1029 ET. SIL, 2170 FT. WEL

S28, T2N, R1OW, 2029.3 FT. SNL, 2110.2 FT. WEL

S28, T2N, RIOH, 2394.7 FT. SNT, 1496.2 FT. EWL

S5, T2N, R1OW, 700.1 ET. SKR, 1959.2 FT. EKL

S5, T2N, RIOW, S15.2 ET. NSL, 466.2 FT. EWL

S4, T2N, R1OW, 1002.7 FT. MSI, 1336.4 TT. WEL

S8, T2N, R1OW, 3162.1 FT. SRT, 899.0 TT. EWL

S10, T2N, R1ON, 1390.1 FT. SIT, 2663.7 FT. WEL

S8, T2N, R1OW, 3130.9 FT. SRL, 3027.6 FT. EWL

S11, T2A, R1OW, 88.4 TT. ASL, 1016.5 TT. EWL 


\section{ATTACHAEYT l (Cont.)}

WELL

NAME \& NUMBER

LOCATION

TOWNSHIP AND RANGE

U.S.E.S. YCCG-113

S17, T2N, R10W, 914.4 FT. SNL, 710.0 FT. EWI

U.S.F.S. MCCG-114

S15, T2N, RIOW, 296.7 FT. NSL, 1200.2 FT. EWL

U.S.F.S. MCCG-115

S20, T2N, RIOW, 2620.8 FT. SNL, 1918.0 FT, WEL

U.S.F.S. MCCG-117

S28, T2N, R1OW, 458.6 FT. SNL, 142.3 FT. WEL

U.S.F.S. MCCG -118

S22, T2N, R1OW, 2269.1 FT. SNL, 1314.7 FT. WEL

U.S.F.S. HCCG-119

S33, T2N, R1OW, 2046.8 FT. SNL, 1281.4 FT. EWL 
The underalgned, for and on belialf of the underalgned and the repreacntativea, lielra and asolgna of the underalgned, hereby acknowledges recelpt from stone \& Weboter Englncering Corporation (SWEC) of the aum of Ten and 00/100 Dollars $(\$ 10.00)$

and otlier valuable consideration, the recelpt and oufflelency of whlch Is hereby acknowledged, In full and complete settlement and at afaction of any and all claims, demando, dameger, loose, actlona, and cause of action of every kind and character, which the underalgned or tlictr representat Ives, helro and asolgns have or miglit have by reason of ownership of the property or real property righte in the property, described below on which the U.S. Government, and Ito agento, oervonto, employees, ouccesoro, osigne and contractors have conducted drilling, hydrologle monltorlng otudieo, well plugging. ite restoration, and other operations. The herelnafter deacribed property 10 aloo described in certaln agreements executed by the undigralgned whlch granted right-of-entry and the right to perform hydrologle monitoring otudies and other operations on the real property of the undersigned.

The owner has declded to accept the above payment in lles of SWEC completing the following ite reatoration activitleot

Site clean up and restoration of lands used in the activities and operations set out above on lands hereinafter described.

In conolderation of the obove payment, the underalgned hereby forever discharges and releases the U.S. Government, and Its ogents, Bervanta, employee, succesors, osigne, and contractors from any and all clalme, demando, damages, losere, ections and causen of oction whlch the underalgned or thelr repreaentativea, helro, or abigno, have or might have arlolng out of, accruing to or revulting from the performance of hydrologle monltoring otudies and related activities on or in the vicinity of the property descrlbed below.

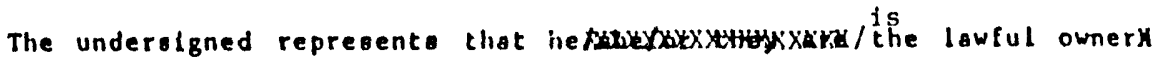
of the property deccibed below and to the proper party to recelve payment hereunder.

Description of Property situated in the fRritibxiak County of Perry State of Mississippi 1

SWlanEl Section 3, Township 3 North, Range 10 West.

Nㅐ-4

IN WITNESS WIEREOF, we have liereunto set our hands and beals this the $22 \pm \underline{2}$ day of Anril 1989.

WITNESS,
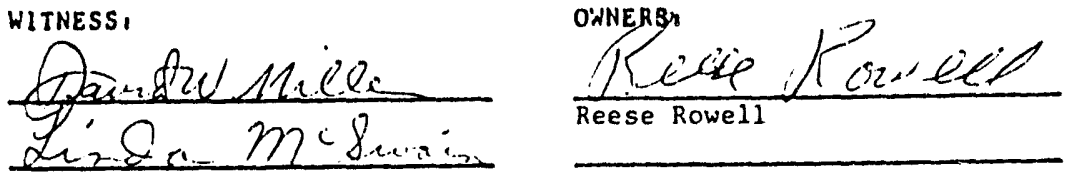

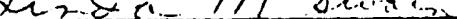

SRuxIKkXd County of Perry

STATE OF Mississippi

Apr 11 - 1989, before me personally appeared

On this day of Reese Rowel1 1989, before the personally'

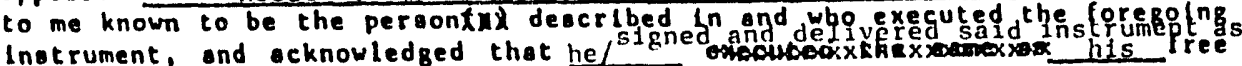
act and deed.

Given under my hand and offlclal seal, this the day of Apr11, 1989.

(SEAL)

Notary Publle In and for LRyYLuKXax Perry Countyx, Mississippi (state)

Hy Commisesion Explresi 
STATE OF MISSISSIPPI

COUNTY OF HINDS

PERSONALLY APPEARED before we the undergigned authority in and for said County and State, the within named David W. Hiller one of the subscribing witnesses to the within and foregoing Instrument, who being first duly oworn, deposeth and saith that he saw the within named Reese Rowell

whose name is subscribed thereto, $81 \mathrm{gn}$ and dellver the above and foregoing Damage Release, that he, this afflant, subscribed his name as a witness thei'eto in the presence of the said Reese Rowell

and that he saw the other subscribing witness sign the same in the presence of the sald Reese Rowe11

and that the witnesses signed in the presence of each other, on the day and year there in named.

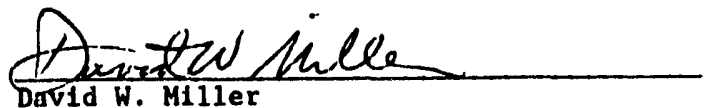

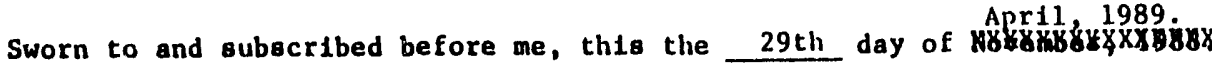

(AffIX Seal)

My commission expires:

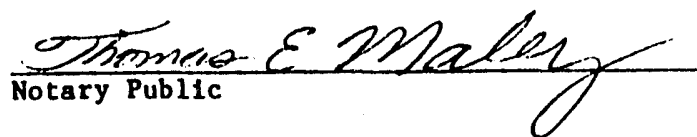

$3-28-90$

STATE OF MISSISSIPPI

COUNTY OF HINDS

PERSONALLY APPREARED before me the undersigned authority in and for said County and State, the within named David W.Miller one of the subscribing witnesses to the within and foregoing instrument, who being first duly sworn, deposeth and salth that he saw the within named

whose name subscribed thereto, sign and deliver the above and foregoing Damage Release, that he, this afflant, subscribed his name as a witness thereto in the presence of the said and that he saw the other subscribing witness sign the same in the presence of the sald

and that the witnesses signed in the presence of each other, on the day and year therein named.

David W. MIIler

Sworn to and subscribed before me, this the day of November, 1988.

(Aff1x Seal)

My commission explres:

Notary Pubilc

Address of Grantor:

Reese Rowe11

Route 1

New Augusta, MS 39462
Address of Grantee:

U. S. Government et al

Stone Webster Engincering Corporation

245 Summer Street

P. O. Box 2325

Boston, Mass. 02017

ATTN: Mr. C. A. Foster 
The underalgned, for and on belialf of the undersigned and the representat lves, helrs nnd anolpill of the undernlgned, hereby acknowledpen recelpt from stone Webster Euglnecrlng Corporation (SWEC) of the sum of Ten dollars and $00 / 100(\$ 1 C .00)$

and other valuable consideration, the recelpt and sufficlency of wilch Is liereby scknowledged, In full and complete nettlement and nat lafaction of any and all clalms, demnnda, Inmages, losere, actlona, and causeg of nction of every kind aild clianncter, which the underalgned or their representativen, lielra and aosignn linve or mlght have by reason of ownerahip of the property or real property righte in the property, descrlbed below on which the U.S. Government, and tto agents, gervanta, employees, nuccessors, asnlgns and contractors have conducted drlillng, hydrologlc monttoring atudies, well plugginy. olte restoration, and other operations. The lierelnafter described property is alno described in certaln ogreements executed by the undersigned whish granted right-of-entry and the right to perform hydrologle monltoring otudien and other operations on the real property of the underalgned.

The Owner lias declded to nncept the above payment in lleu of SWEC completing the following bite restoration activitles,

Site clean up and restorat: on.

In consideration of the above nayment, the undersigned hereby forever discliarges and releases the U.S. Government, and 1 ts agents, nervanta, employees, auccessors, asolgna, and contractors from any and all clalma, demandn, Jamapen, Iosen, act long and caunen of nctlon whlch the undernipinod or thelr representatives, helra, or asalgna, have or mlght have arlalng out of accruing to or regulting from the performance of hydrologic monltoring tudies and related ativities on or in the vicinity of the property described below.

The undersigned reprenents liat he/she/or they are the lawful owners of the property deacribed below and to the proper party to recelve payment liereunder.

Deacription of Property sltunted In the (Pariah or County) of Marion

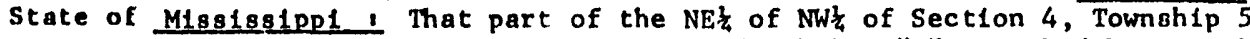
North, Range 17 West, shown on Drawing titled Exhibit " $A$ " attached hereto, being a parcel of land 400 feet by 250 seet, together with road easement 20 feet wide by 550 feet long, as shown on Exh:b1t "A", and leading to sald parcel.

IN WITNESS WIIEREOF, we havi. hereunto net our hands and seals this the 2nd day of December 1988.
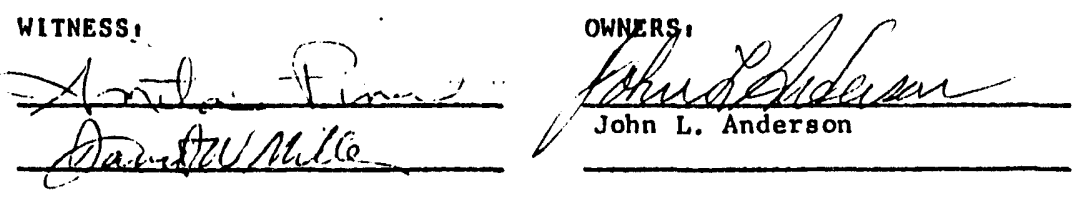

STATE OF

(Parloh or County) of anpeared

On thls day of - 1988, before me personally to me known to be clie person(o) described In and who executed the foregotnp; Inntrument, and acknowledged that __ executed the same as__free act and deed.

(SEAL)

Notary Publle In and for (Parloh or County). (State) 
PERSONALI,Y APPEARED before me the undreralgned nuthority in nind ror satd County and State, the withln named Davill W. Mllier one of the subscrihlus wltnesses to the within and foregolug Instrument, who being irst duly sworn, deposeth nnd salth that he snw the withli nnmed John L. Anderso:

whose name is subseribed thereto, slgn and deliver the ibove nud roregoing Damage Release, that he, this affiant, subserlbed his name as a witness thereto in the presence of the sald ___.John L. Anderson

and that he saw the other subscribing, withess sign the same til the presence of the" sald _ John_L. Anderson

and that the witnesses signed til the iresence of ench other, on the day and year there In named.
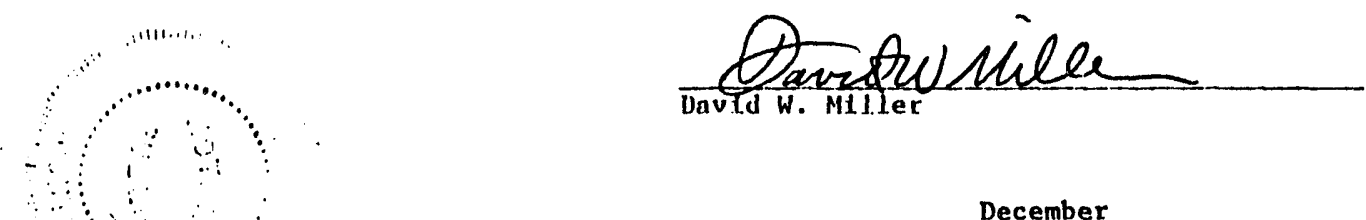

Swornoto and subscribed before me, this the 5th day of December 1983. (Afriseai)

My commlsston exptres:

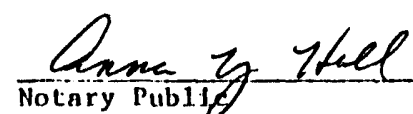

$2-5-90$

\section{STATE OF MISSISSIPPI}

COUN''Y OF IILNDS

PERSONALISY APPREARED before me the undersigned authority in and for snid County and State, the within named Dav1d W.Miller one of the subscribing witnesses to the within and foregoing instrument, who being irst duly sworn, deposeth and salth that he saw the within named

whose name subscribed thereto. sign and deliver the alove and foregolng

Damage Release, that he, thls afflant, subscribed his name as a witness thereto in the presence of the satd

and that he saw the other subscribins: witness sign the same fin the presence of the said

and that the witnesses stgned in the presence of each other, on the day and year there in named.

\section{David W. Miller}

Sworn to and subscribed before me, tl.ts the day of November, 1988.

(Afflx Seal)

My commission expires : 


$$
\text { ar } \$ 1015 \therefore 3 ? \%
$$

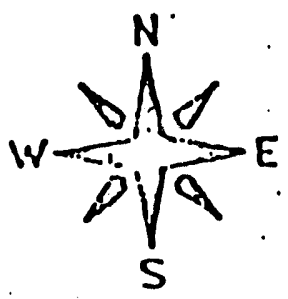

$$
\begin{aligned}
& \text { DATE: FEB. } 22,1979 \\
& \text { STALE: }
\end{aligned}
$$

$$
\text { Exhibit "A". }
$$

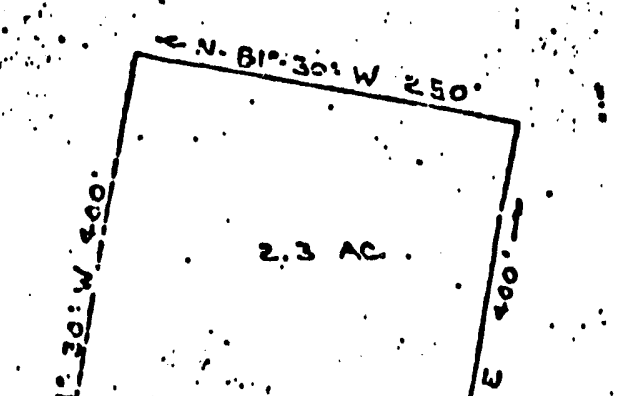

$$
\begin{aligned}
& \text { MU } \\
& \text { NH } 6 A, 6 B, 6 C, 6 D, 6 \text { W }
\end{aligned}
$$

1787

STATE OF MISSISSIPPI MARION COUNTY

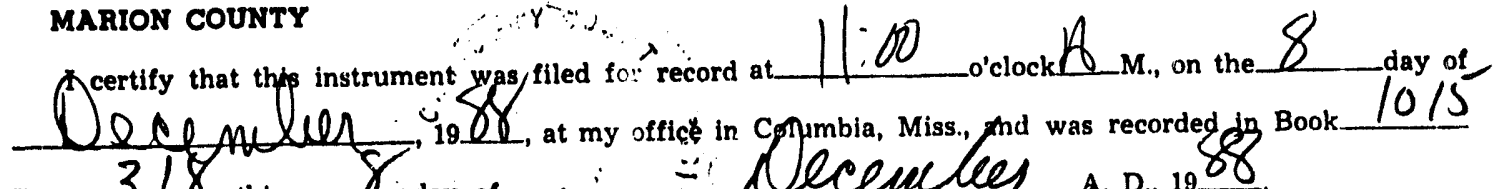

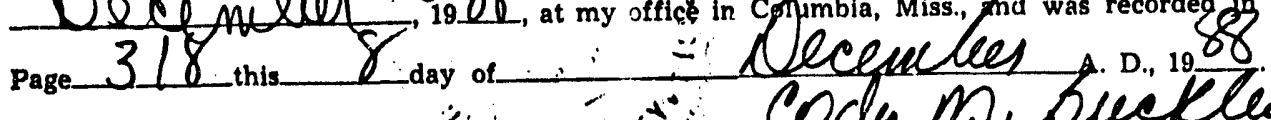
(SEAL) "अ

Cody D. Buckley, cry. clerk 
The U.S. Government, elther directly or through contrncts wlith othern lina drllled certaln hydrologic and geologle Borlugn (tlie "Borling") on the property of John L, Anderson_llie "Landowiler").

Deacription of the Property oltuated li (liarloli or County of) Marion

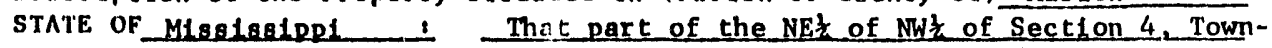
shlp 5 North. Range 17 West. shown on Drawing $t$ tited Exhiblt "A" attached here to, betng a parcel of land 400 feet by 250 feet. together whth road easement 20 feet wide by 550 feet lone. as shown on Exhtb1t "A" and leading to said parcel: Mll 6WS

WHEREAS, the Landowner may whol, to use the borling as a water well, and

WIIEREAS, the U.S. Government dit not drill the borlng a a water well;

TIIEREFORE, In conslderation of the U.S. Government leaving the Borling In Its present conditlon to be used by the Landowner as the landowner desireg, the undersigned Landowner, does hereby forever discharge and release the U.S. Government and 1 th agents, mervanta, emplóyees, succensorn, assigis, and contractoro whlch are or may be in any manner what ooever responstble for the Borlng from any and all clalms, demands, damagen, losses, actiong and causes of nitton which the underalgned Landowner or their representatives. lielro, or naslgno han apnlunt any of the nbove, lincluding speclfleally, but not limlted to, any clalin whlch la related to the sultabllity of the Borlng as a water well.

In execut Ing and delluering the release, the underalgned Landowner relled wholly upon hin Judgment, knowlodge and bellef nn to the nature, extent aind duration of the damages which he may autaln in the future. The underalgned further repreants anis warrants that he lias not been influenced by any representationn, otatements or warrantiea made by any person, $f(\mathrm{rm}$, association, partnership or corpuration or agency of the U:S. Government liereby released, or by any ngenti. employee or of licer reprenenting them, or any of them, concernlug the na ure, extent and duration of any potentlal damages, losses or legal llabllity.

The underolgned represents that he/gho/or they are the lawful owner of the property descrlbed above ani are the proper party to execute this release/agreement.

In whtness whereof, we have herounto net our hands and aealo thla the 2nd day of December - 1988.
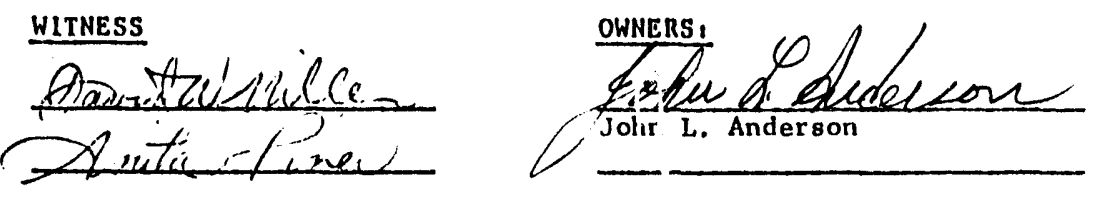
STATE OF MISSISSIPPI

$$
\text { f. } 1015
$$

COUNTY OF HINDS

PERSONALIY APPEARFD before me the undersigned authorlty in and for sald County and State, the within named David W. Miller one of the subscribing witnesses to the within and foregoing instrument, who being first duly sworn, deposeth and sath that he saw the within named John L. Anderson

whose name is subgcrlbed therete, sign and dellver the above and foregoing Release, that he, this affant, subscribed his name as a whtness thereto in the presence of the sald Join L. Anderson

and that he saw the other subscribirg witness sign the same in the presence of the said John L. Anderson

and that the witnesses signed in thi presence of each otlier, on the day and year therein named.

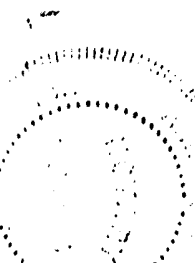

Sworn.to and subscribed before me, this the 5th day of December 1988.

(Aff $1 \times$ Sea 1 )

My commisston exptres:

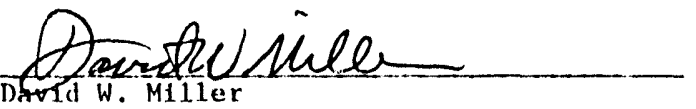

$2-5-90$

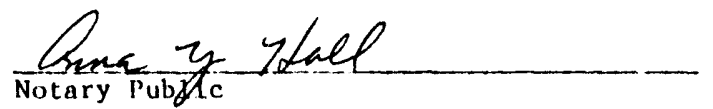

STATE OF MISSISSIPPI

COUNTY OF HINDS

PERSONALLY APPREARED before me the indersigned authorlty in and for sald County and State, the within named David W.Mllle: one of the subscribing witnesses to the within and foregoing instrument, who belng first duly sworn, deposeth and sat th that ho saw the within named

whose name subscribed theret:, sign and delfver the above and foregoing

Damage Release, that he, this affla.t, subscribed his name as a wltness thereto in the presence of the said

and that he saw the other subscrtbitg witness sign the same in the presence of the said

and that the witnesses signed in the presence of each other, on the day and year therein named.

David W. Miller

Sworn to and subscribed before me, his the day of November, 1988.

$($ Af $\mathrm{f} 1 \times$ Sea 1$)$

My commission explres: 


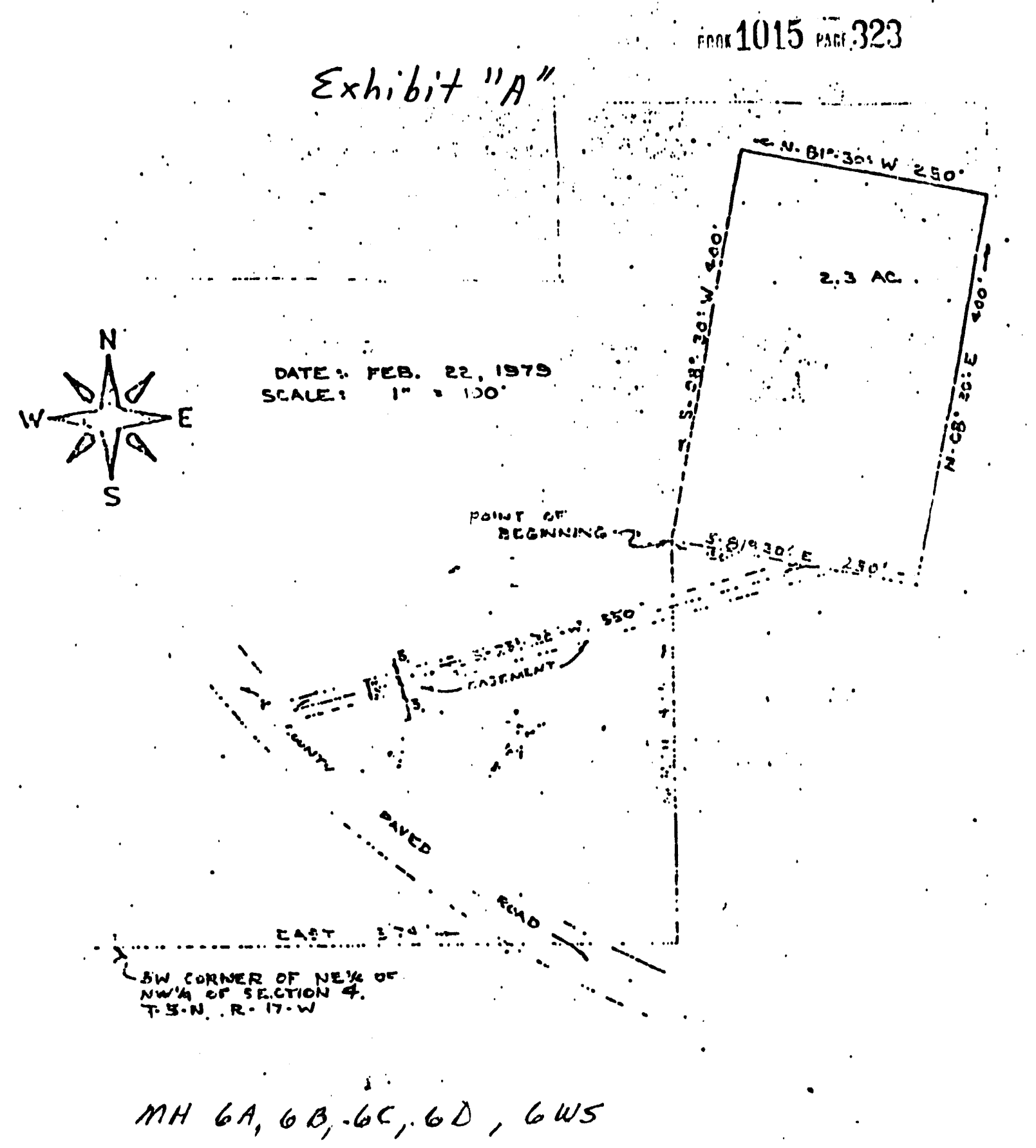

STATE OF MISSISSIPPI

7288 MARION COUNTY

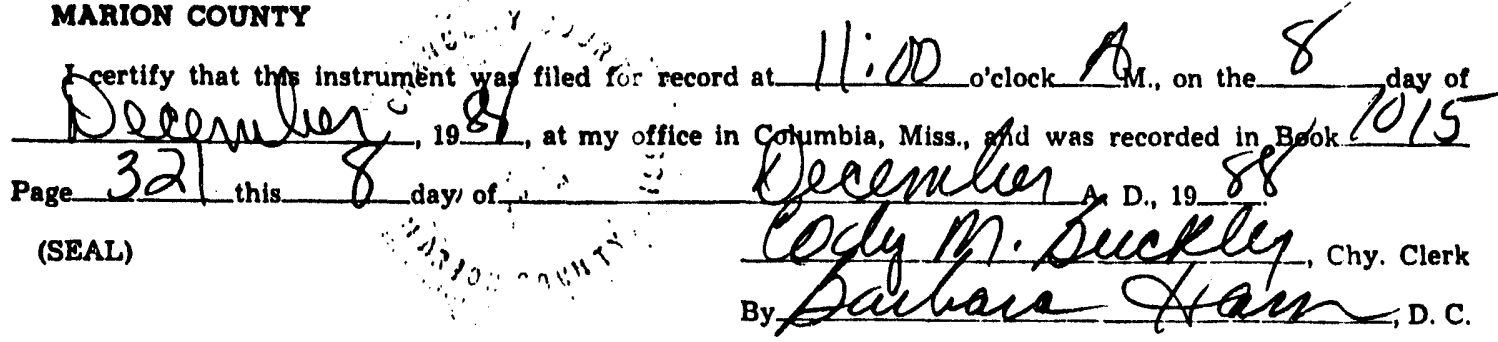


The underolgned, for and on belinif of the undernigned and the representat iven, lielre and anolunn of the undernigned, linraby acknowledpen recelpt from Stone t Webter Englneering Corporation (SWEC) of the num of One Dollar and other valuable conslderation, the recelpt and oufficlency of which Io hereby acknowledged, In full and complete settlement and cat lofaction of any and all clatmo, demands, damages, losaes, act lonn. and caunea of action of every kind and character, which the undersigned or thelr representativeo, helrs and asolgns liave or might have by reason of ouneralip of the property or real property righte in the property, dencribed below on whlch the U.S. Government, and lta apenta, nervanth. employees, successora, asalgne and contractora have conducted drilling, hydrologic monltorlng otudles, well plugglng, nite restoration, and other operations. The lierelnafter degcrlbed property lo nlao deucribed in certall agreements (the "Agreements") execuled by the underolgned whlch granted right-of-entry and the right to perform hydrologle monitorling otudies and other operations on the real property of the underelgned.

SWEC has completed well plugging and/or olte reatoration work nccording to the requirements of the Apreementa. In constderntion of the above pnyment, the undersigned liereby corever dincliarpes and relenses the U.S.

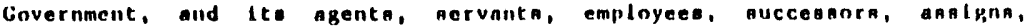
and contractors from any and all clalmg, demanda, damages, loanen, act lonn and causes of action which the underilgned or thelr representatives, helrn, or donigne, liave or miglit have arlinilig out of, eccrulng to or renult lilg from the performance of hydrologlc monltorinp atind es and related activities on or lil the vicintty of the property deacribed below.

The undersigned represente that lie/slie/or they are the lawful owners of the property described below and 18 the proper party to execute this Release Agreement.

Description of Property situnted in the of Furrest - STATE or Hississippi county

For description see Exhiblt " $A$ " attached hereto.

in HITHESS WIIEREOF, we liave liereunto ant our liando and beala this the 10til day of November $198 \mathrm{~B}$.

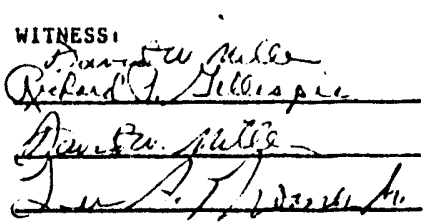

OWNERS ,

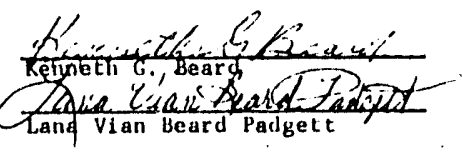

STATE OF

- (Parlall or comuty) of

On this day of - 1988, before me personally appeared

to me known to be the person(o) described In and who executed the foregoing Instrument, and ackiowledged that lree act and deed. executed the ame an

(SEAL)

Notary Public In end for (Parlsh

My Comalesion Explree,

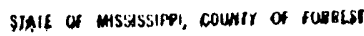

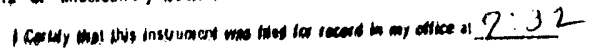

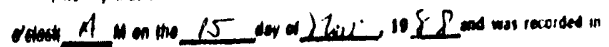

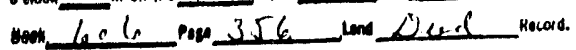

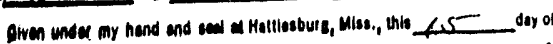

Sins. 1088 JMMYC.HAVAKE 
STATE OF MISSISSIPPI

COUNrY oF IIINUS

PERSONALLY APPEARED before me the undersigned authorlty in and for sald County and State, the Within named David W. Miller

one of the subscribing witnesses to the within and foregolning lnstrument, who

beling first duly sworn, deposeth and salth that he saw the within named

Kennetli G. Heard

subscribed thereto, sign and deliver the same to the sald stonc \& Wehster

Engineering Corporation that he, this afflant, subscribed his name as

It ness thereto in the presence of the sald Kenneth G. Beard

witness thereto In the presence or tho ald and that he saw the other subscriblng

witness sign the same in the presence of the sald Kennetli c. Beard

and that the witnesses signed In

the presence of each other, on the day and year therein named.
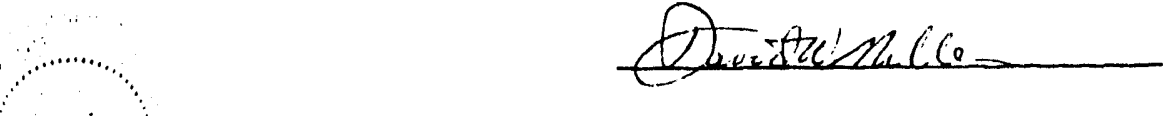

Sworn to and subscribed before me, thls the 11th day of Novenber . $19 \underline{88}$.

(Arfix.Sis)

My commission expl res:

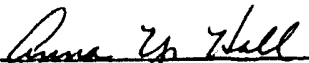

tehmays, ceso

Notary Pubilc

STATE OF MISSISSIPPI

COUNTY OF

HiNDS

PERSONALLY APPEARED before me the underalgned authorlty in and for bald County and State, tho within named David W. Miller

one of the subsortblng witnesses to the within and foregoing Instrument, who

belng first duly sworn, deposeth and sath that he oaw the within named

Lang Vian

those name Is subscribed thereto, $81 \mathrm{gn}$ and deliver the same to the sald Stolle \& Webster

Enoineerlne Corporation that he, this afflant, subscribed his name as itness thereto in the presence of the sald

witnd that he saw the other subscribling vitness sign the same in the presence of the sald Lana Vlan Beard Padget

and thet the witnesses slgned in

the presence of each other, on the day and year therein named.

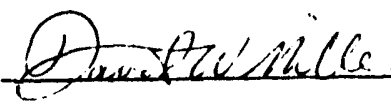

andint

Syorn to and subseribed before me, this the 11th day of November 1988.

(Affix Seal)

My. commisstion explresi

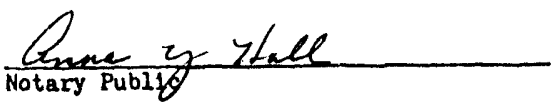

2.tuming, 1990

SIAIE OF MISTSFIPPI , COUNIY OF, FORREST

if Jimmy C. Havard. Chancery Cletk of Forrest connly, ow haioby callity liat the foregoing is a live and correct cons of

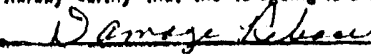

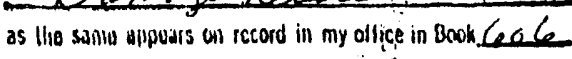

Parn 356

Givou under hiy halld and ollicial seal on this the $15-5 x$

day of Jlaxandere $1988^{\circ} \because \cdots$

IIMAYY a HAVARD. Chancery chak ini"

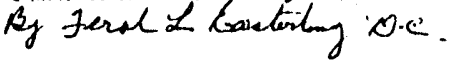


Township 5 North, Range 13 West, Forrest County, Mississippi.

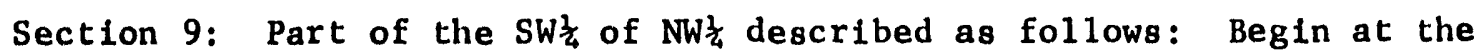
Southwest corner of the SW $\frac{1}{4}$ of NWh, Section 9, run East 180 yards, thence North 161 yards to the public road, thence Northwesterly along public road to the Section 1ine, thence South 282 yards to the point of beginning, all in Section 9, containing 8.44 acres, more or less; and

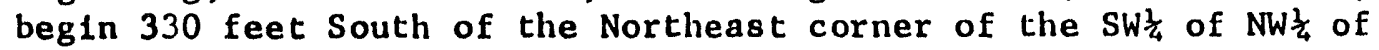
Section 9, thence run West 570 feet, thence run South 210 feet, thence run West 210 feet, thence run South 780 feet, thence run East 120 feet, thence run North 660 feet, thence run East 660 feet, thence run North 330 feet to the point of beginning, less part to highway.

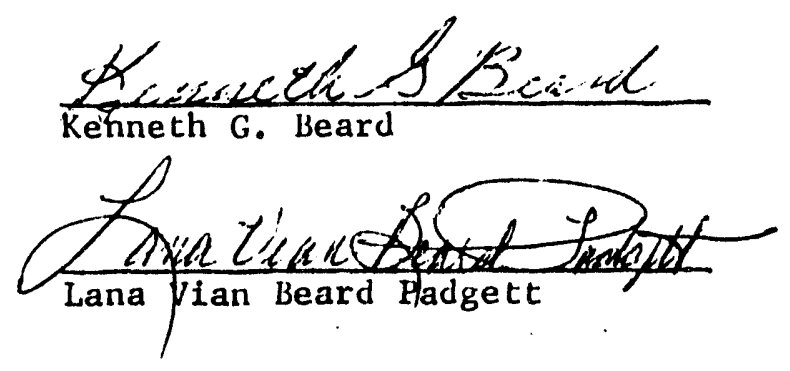


The U.s. Covernment, eltlier directly or tlirough contrncto with othern linn drilled certall liydrologle and geologle Boring" (tlie "Borling") on the property of Kenneth C. Beard and Lana Vlan Beard Padeet

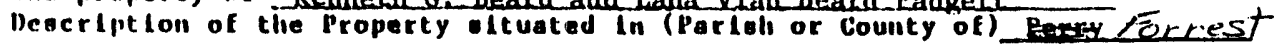
STATE OF_Mlssissipp L i For descriotion see Exhidb it "A" attached hereto.

WIIEREAS, the Landowner may wiols to use the borlng as a water well, and

WIIEREAS, the U.s. Government dld not drlil the borlng as ater well,

TILEREFRE, In conelderation of the U.s. Government leaving the Borlng In Its present condition to be used by the Landowner as the Landowner desires, the undersigned Landowner, does liereby forever discharge and relesse the U.S. Government and its agente, iervants, emplöyees, successors, asolgils, and contractors whlch are or may be in any manner whatsoever responsible for the Borlng from any and all clalms, demands, Jamagen, losses, actlons and caunes of action which the underulgned Landowner or their representatives. helra, or onolgne has apalnot any of the above. linclinding opeclfleally, but not limited to, any clalin which lo related to the oultablity of the Borling an water well.

In execut ling and dellverling the releave, the underalgned landowner relled wholly upon hio Judgment, knowledge and bellef an to the nature, extent and duration of the damages whlch he may oustall in the future. The undersigned curtlier reprenents and warrants that he has not been Influenced by ony representationn. otatemento or warrantieo made by any person. $11 \mathrm{rm}$, asooclation, partnerghip or corporation or agency of the U.S. Government hereby released, or by ally agento, employee or offlcer representing them, or any of them, concernlug the nature, extent and duration of any potentlal damagen, looses or legal llabllity.

The underalgied represents that he/olio/or they are the lawful owner of the property described above and are the proper party to execute this release/agreement.

In vitues whereof, we have liereunto net our hand and seals this the $2 Z^{t h}$ day of January $2898 x 1989$.
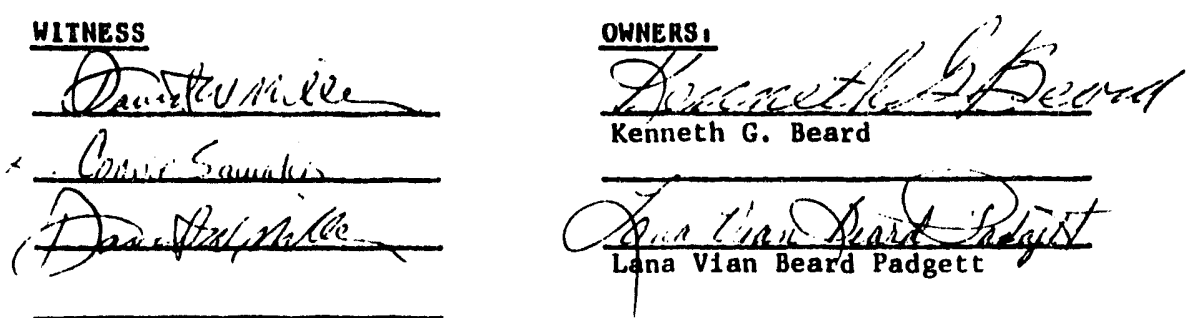
STATE OF MISSISSIPPI

COUNTY OF HINDS

PERSONALLY APPEARED before me the undersigned authority in and for said County and State, the within named David W. Mller one of the subscribing witnesses to the within and foregoing Instrument, who being firat duly sworn, deposeth and saith that he saw the within named Keuneth G. Beard

whose name is subscribed thereto, sign and dellver the above and foregoing Damage Release, that he, this afflant, subscribed his name as a witness thereto in the presence of the said kicnneth G. Beard

and that he saw the other subscribing witness olgn the same in the presence of the sa1d Kenneth G. Beard

and that the witnesses signed in the presence of each other, on the day and year therein named.

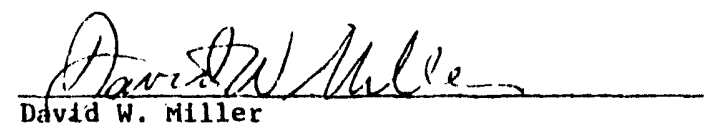

Sworn to and subscribed before me, this the 30 th day of lanuary, 1989.

(Af I IX Seal)

My comnission explres:

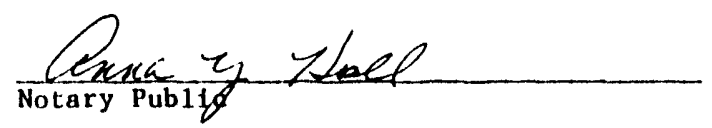

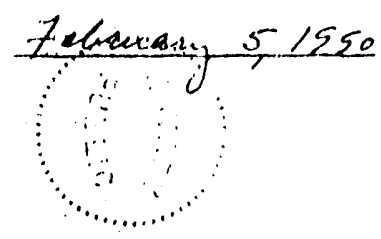

STATE OF MISSISSIPPI

COUNTY OF HINDS

PERSONALLY APPREARED before me the undersigned authority in and for said County and State, the within named David W.Mllier the subscribing witness to the within and foregoing instrument, who being first duly sworn, deposeth and salth that he saw the within named Lana Vian Beard Padsett

whose name is subscribed thereto, sign and deliver the above and foregoing

Damage Release, that he, this afflant, subscribed his name as a wituess thereto in the presence of the sald Lana Vlan Beard padgett

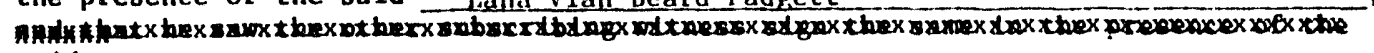
andedx

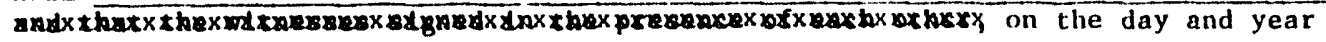
therein named.

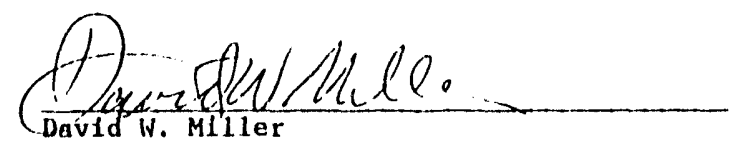

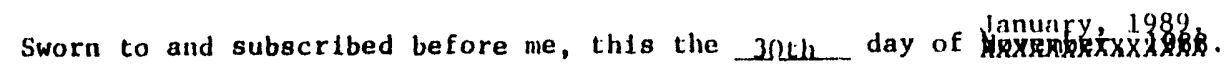

( $\Lambda f f 1 x$ Seal)

My commission expires:
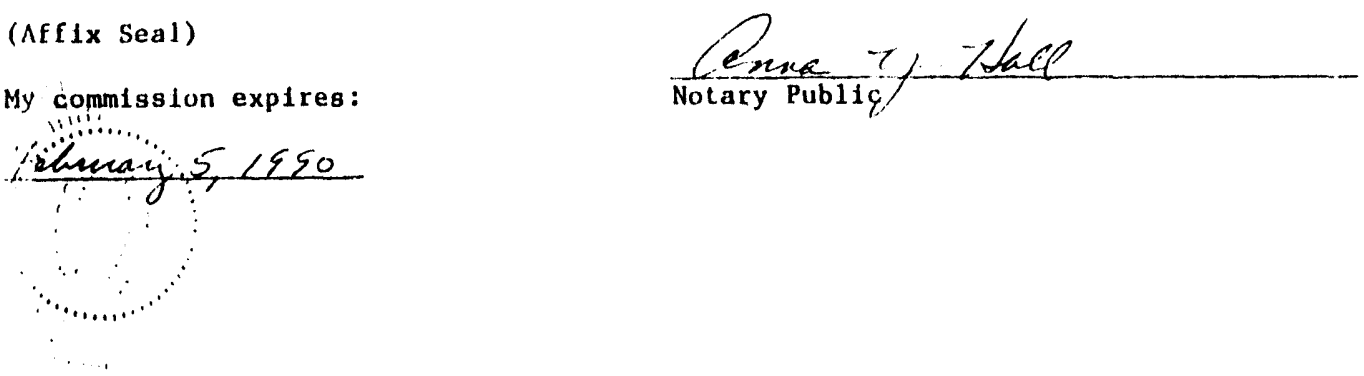
Township 5 North, Range 13 West, Forrest County, Mississippl.

Section 9: Part of the SWt. of NWt. described a follows: Begin at the -Soutliwedt corner of the swit of NWt , Section 9, run Eagt 180 yards, thence North 161 yards to the public road, thence Northwesterly along public road to the Section IIne, thence South 282 yarde to the point of beginnilig, all in Section 9, contalning 8.44 acres, more or less; and begIn 330 feet South of the Northeant corner of the SWt of NWt $\frac{1}{4}$ of Section 9, thence run West 570 feet, thence run South 210 feet, thence run West 210 feet, thence run South 780 feet, thence run East 120 feet, thence run North 660 feet, thence run Eayt 660 feet, thence run North 330 feet to the point of beginning, 1988 part to highway.

STATE OF MISSISSIPPI, Perry Counly

Filed for record on the ב

Job) $\quad 1989$ a18:45 cinck A.M. and recorded this

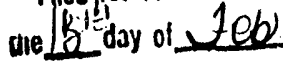
1:89. aleed lik

Aiflif Q LOII, Cliancery Clerk Pobin Dartin o.c 
The inderolgned, for and on belialf of the undernlgned and the reprenentat lves, lielra nind noslynn of the underntgued, liernby acknowledpren recelfic (rom stone Webater linglueering Cocporntion (SWEC) of the anun of Onr Dollar and other valuable conglderation, the recelpt and sufficlency of whlch is liereby ackinowledged, In full and complete aetelement and

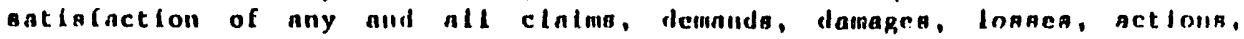
nind causen of action of every klud nuil clinencter, whlch the underalyined or llielr repregententiven, heira and naslgina linve or inlglit liave by reanou of ownersilf of the property or real property rights in the property, descrllied below on which the U.S. Covernment, and Its apenta, servants, employeen, successors, asalgus and contractors have conducted drilling, hydrologlc inonltorlng studies, well plupgling, slte restoratlon, and other operations. The lineliniter deseribed property lo nloo denertbed in certaln ngrenements (the "Agreomente") executed by the underalpined which biration riphtol-entry and the right to perform hydrologle monttoring studies and other operations on the real property of the undersigned.

SWEC has completed well plugping and/or olte reatoration work accordlinp to the requirements of the Agremente. In constderation of the alove paymint, the inderalghed liereby forever Jlacharges and relentes the U.S. lovernment, and teo npenta, nervanta, cumployees, auccennora, oaslpin, and contractorg from any and nll clalma, Jemands, damagea, losnes, actlons and causes of action whlch the underslgied or thelr representatives, heirs, or asnigns, liave or miphit have arialing out of, accrulng to or resulting from the performnice of liydrolople monltorlinp ntudles and related activitien on or lin the vielintey of the property dencribed below.

The undergigned represents that he/she/or they are the lawful owners of the property descrlbed below and ia the proper party to execute this Release Agreement.

Deacription of Property Situated In the (1)arlali or (iounly) of Narien - STATE UF Mississippi

For description see Exhibit "A" attached hereto. MH 8

IN WITNESS WillikeOF, we have liereunto nat our handa and aeala thla the 2loth day of Hebruary , $1988.19 \%$,
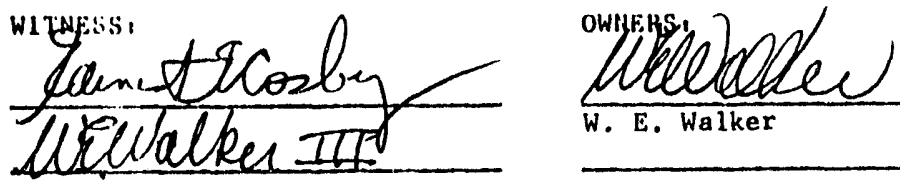

W. E. Walker

STATE OF MISSISSIPPI

COUNTY (II HJNDS

'TIIIS DAY persunally appeared before me, the undersigno! atulhority in and for said County and State, the within named W. li. Walker who acknowledged that he signed and delivered the within and foregoing inntrume'nt on the day and year therein mentioned.

(iIVEN under my hand and seal of office, this 20 th day of Defireary, $1988.17 \%$
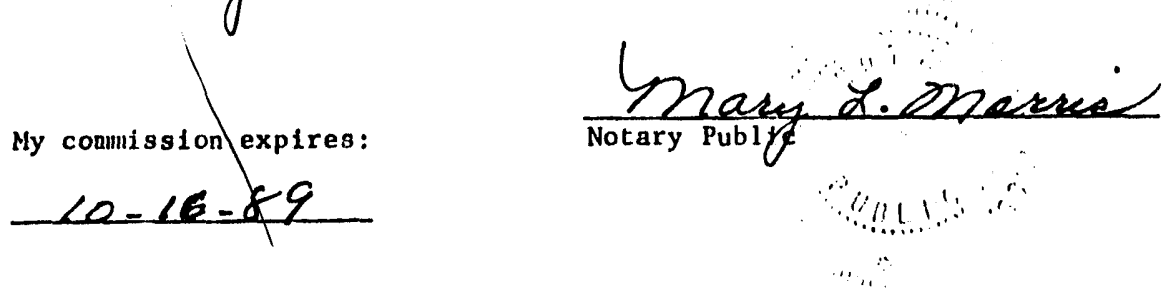
A parcel of land In the bast llalf of the Northeast quarter (i: $1 / 2$ of NE $1 / 4$ ) of Section 10 , 'lownshlp 2 North, Range 17 West. Marion County, Maslssippt, and more particularly described as commencling at the Soulliwest Corner of the Northeast Quarter of the Northeast Quarter (NE 1/4 of NE 1/4) of the above sald Section 10; thence North 209.15 feet; thence bist 238.01 loet to the Polnt of Beglmndng; thence Sunth $13^{\circ}$ 15' East 600 feet; thence North $76^{\circ}{ }^{\circ} 5^{\prime}$ liast 250 feet; thence North $13^{\circ} 15^{\prime}$ West 60 ') feet; thence South $76^{\circ} 45^{\prime}$ West 250 feet to the lolut of Begloning. Also all that land $1 \%$ ing between the above described tract and the asphalt paved road and befing In the East llalf of the Northeast Quarter (E $1 / 2$ of $\mathrm{NE} 1 / 4)$ of sald Section, contalning 4.27 acres, more or less, plat attached hereto.

SIGNED FOR IDEN'IFICATION :

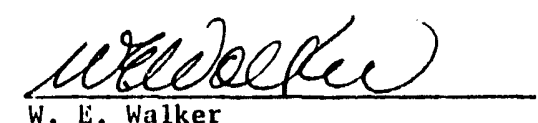




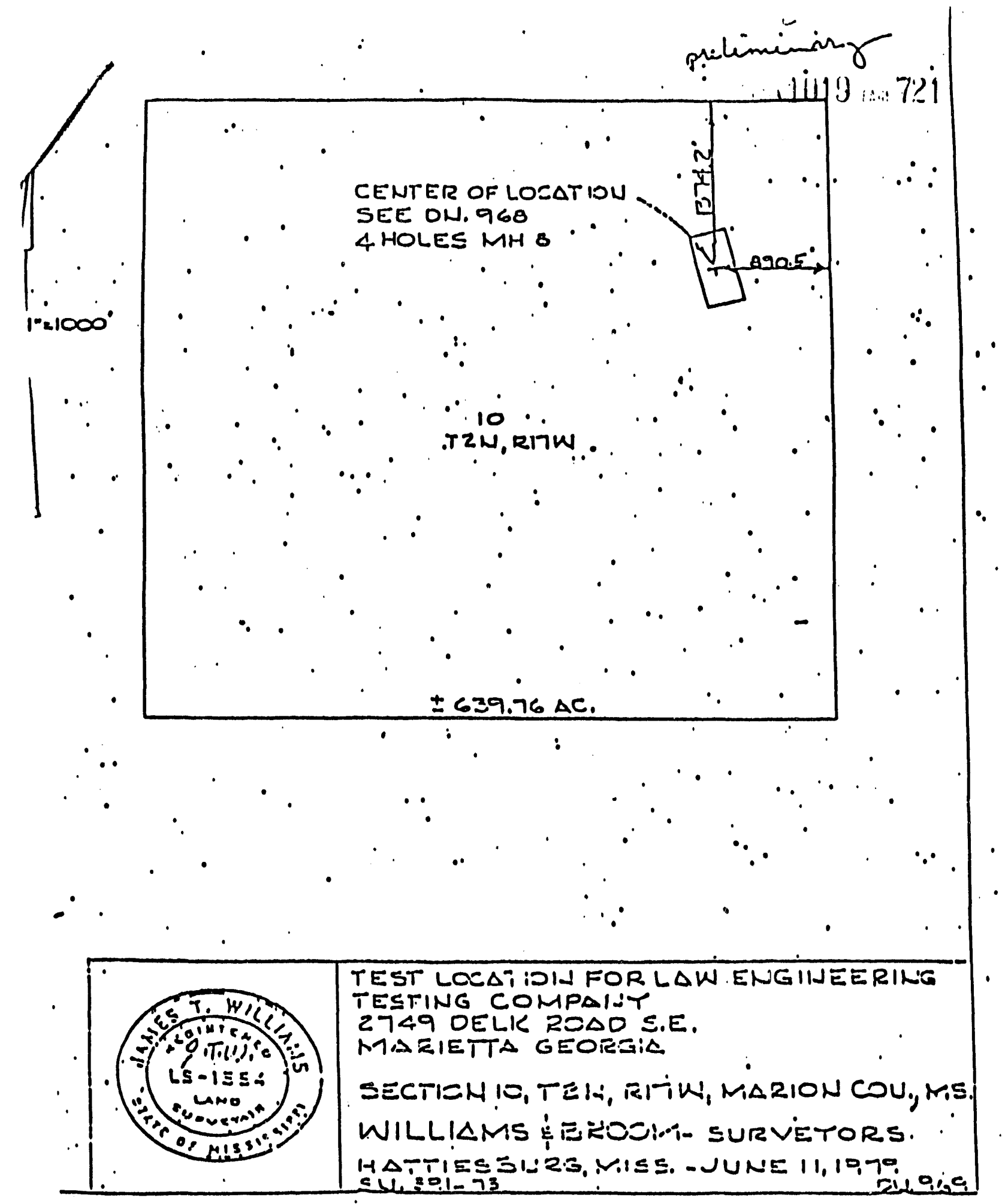




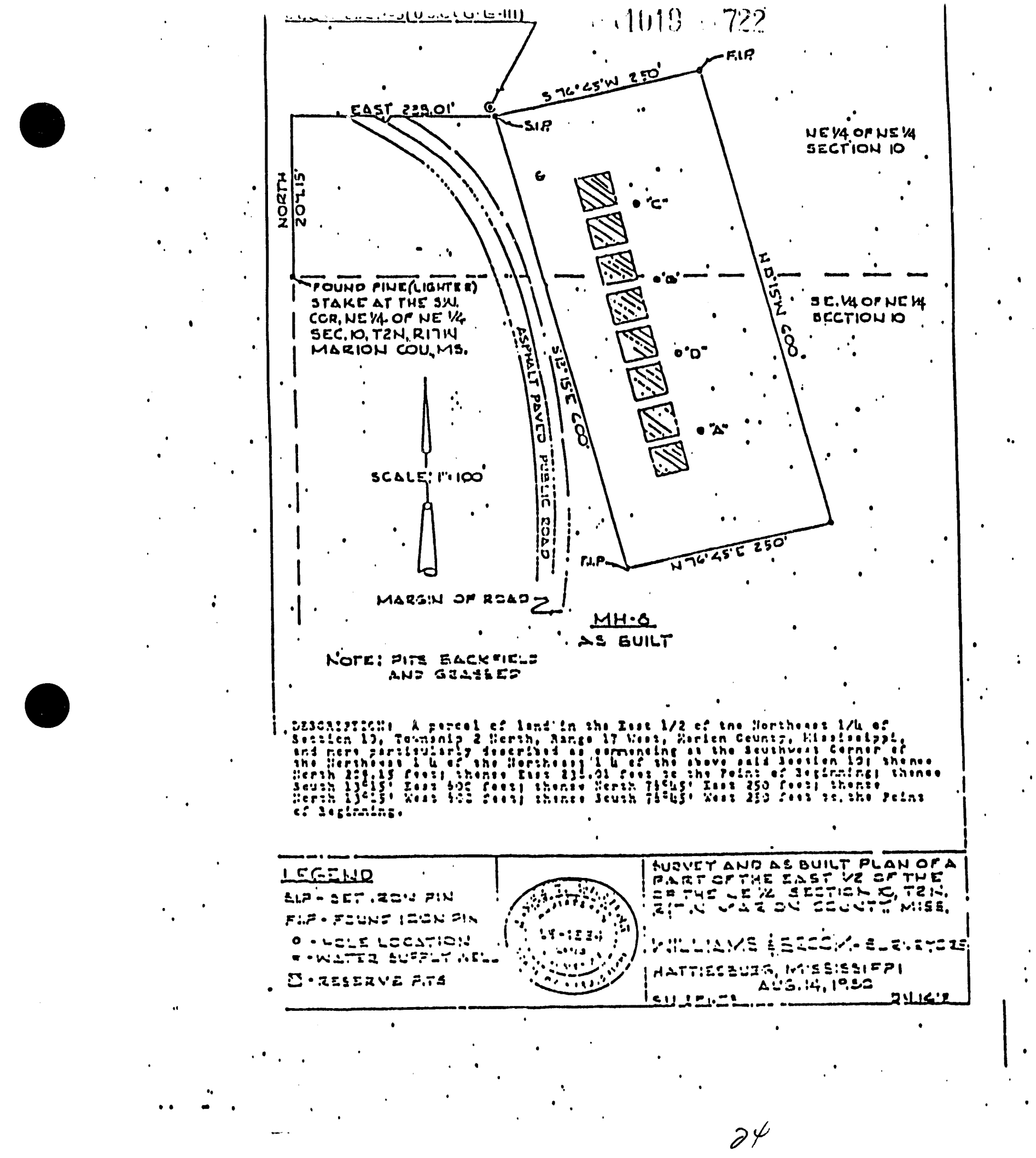

STATE OF MISSISSIPPI

MARION COUNTY

I certify that this instrument was filed for record at. 89 . (SEAL) 
The underalpned, for sind on belialf of the underalgned and the

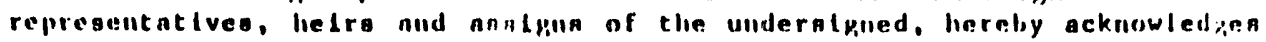
recelpt from stone o Webater Englneerink Corporation (SWEC) of the allm of One Dollar and other valunile consideration, the recelpt and oufficlency of whlch to liereby acknowledged, In full and complete aettlement and

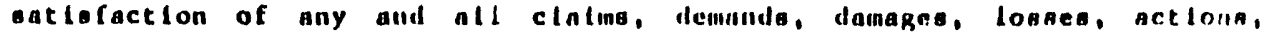
and causes of action of every kind nnd chnrncter, which the undernipned or their representativen, helra and anslgna liave or mlght have by rengon of ownerahip of the property or real property righte in the property, described below on whicl the U.S. Government, and Its agents, pervanta, employees, auccessors, asnigis and contractors liave conducted drilling, hydrologle monltorling otudies, well plughing, olte restoration, and other operations. The herelnafter descrlbed property to aloo described in certaln agreemente (the "Agreements") executed by the undersigned which granted right-of-entry and the right to perform hydrologle monitoring otudies and other operations on the real property of the underalgned.

SWEC has completed well plughling and/or alte restoration work according to the requirements of the Apreements. In consideration of the atove payment, the undernigned lieruby forever dlincliarpes and releases the U.s. Government, and tes ngentr, nervanta, employees, aucceanora, analpina, and contractora from any nind all clalma, desnanda, damagea, losaes, artiona and causes of action which the underalgned or thelr representatives, heirs, or assigns, have or mlght have arialng out of, accruing to or regulting from the performnice of hydrologlc monltorinp atudiea and related activities on or In the vicinlty of the property deacribed below. "

The underglgned represents that he/glie/or they are the lawful owliers of the property described bilow and to the proper party to execute thils Release Agraement.

Descilpitiun of foplerty sltunted in the (Parloh or County)
of Periy

For description see Exhibit " $A$ " attached hereto.

IN WITNESS WIIEREOF, we have hereunto art our hands and beals thla the 21st_ day of Novinber . 1988.

\section{WITNESS ।}

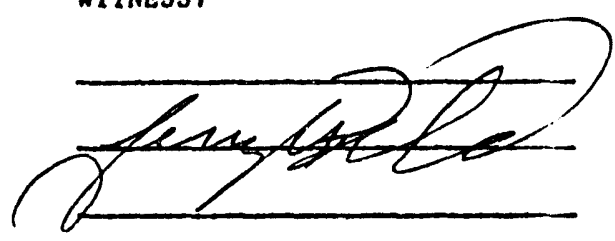

OWNERS,

LEAF RIVER FOREST PRODUCTS, INC.

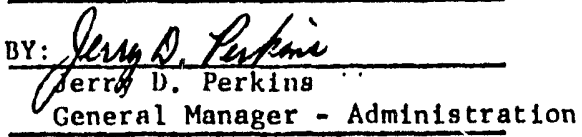

STATE OF MISSISSIPPI

COUNTY OF PERKY

Personally appeared before me, the undersigned authority in and for sald County and State, Jerr" D. Perkins, General Manager - Admintetration of Leaf River Forest Products, Inc., who acknowledged that he signed and delivered the above and for'going instrument on the day and year there in mentioned as the act and decd of sald corporation, being duly authorized so to do.

Given under my hand, and offictal seal on this $2 /{ }^{5}$ day of

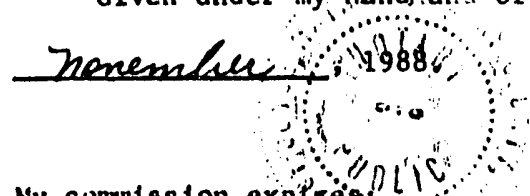

My countssion expités! ?

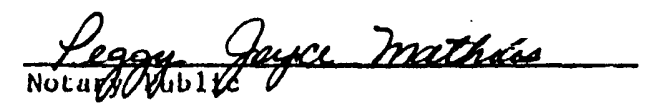

My Commission Explres Jan, 22, 196 


\section{PARCEL I: MRIG 9}

A parcel of land In the Northeast qunrter of the Northwest quarter Section 26, Township 5 Not th, Range 10 West, Perry County, MLssisslipl, and more particulary described an commencing at the Southeast Corner of the Northeast Quarter of the Northwest Quarter of sald Section 26; thence North 164.1 feet; thence Weat 499.25 feet to the point of besinnlng, thence South 69 degrees $28^{\prime}$ West 200 feet; thence North 20 degrees 32 ' West 250 feet; thence North 69 degrees $28^{\prime}$ East to the edge of public road; thence south along sald edge of public road to a point East of point of beginnin:; thence West to point of beginning.

\section{PARCEL II: MRIG 10}

$\Lambda$ parcel of land in the Northeast Quarter of the Northwest Quarter, Section 12, Township 5 No:th, Range 10 West, Perry County, Mississippl, and more particularly descrlbed as comencing at the Southwest Cornar of the Northeast Quarter of the Northwest Quarter of the above sald Section 12; thence North 759.8 feet; thence East 425.1 feet to the point of beginning; thence South 36 degrees $53^{\prime} 30^{\prime \prime}$ East 200 feet; thence North 57 degrees $21^{\prime} 30^{\prime \prime}$ East 104.9 feet; thence North 48 degrees $53^{\prime}$ East $145.8^{\circ}$ fect; thence North 36 degrees $53^{\prime} 30^{\prime \prime}$ West 197 feet; thence South 53 degrees 06' 30" West 250 feet to the polnt of beginning.

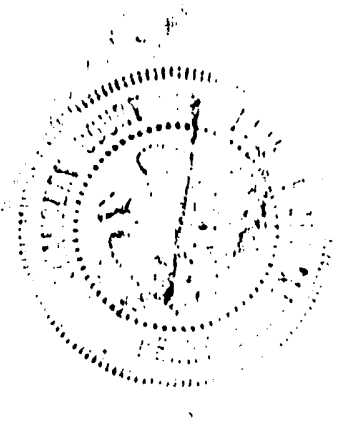

gTATE of mississippl, Perry County

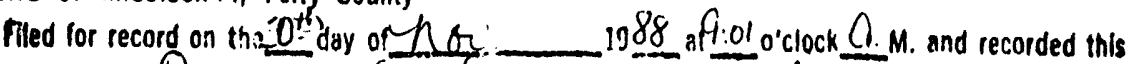
tho lettray of Dec

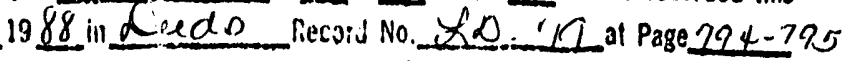

ALH liiu LOTT, Chancery Clerk

By Rebe Mratei D. C 


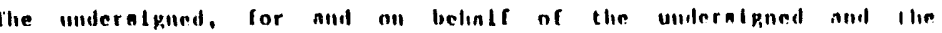

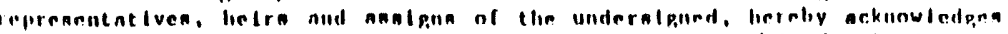

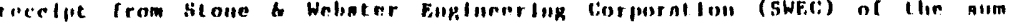
"l len dol lare and $00 / 100=-\ldots(\$ 10.00)$

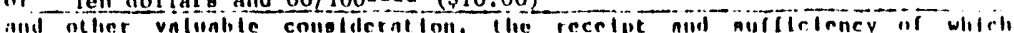
Is liereby ackilowledged, In lull anil complete antllement and natlufactinn

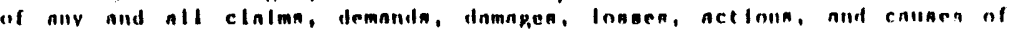

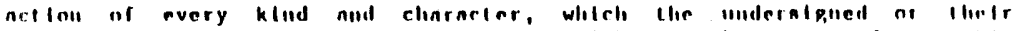

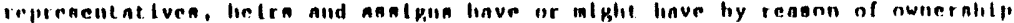

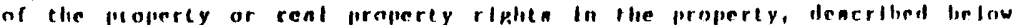

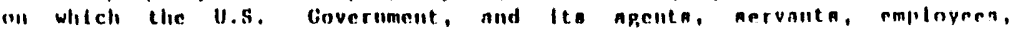
ancecanorn, nasipns and contenctora linve condlucted drllllop. hydralapic

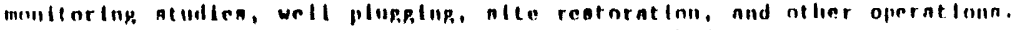

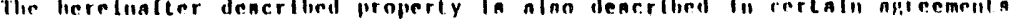
excented by the indernigned which pranted ripht-of-entry and the right to perform hydrolopic montoring ntudteo and otler operationn on lie reat property of the undersigned.

The owiser ling decided to accent tlie nbove inyment in liell of swic complecting the followling olte rentoration activitles, Site clean up and restoration,

III conslderation of the aloove pnyment, the inderalpened linreby forever diaclingers and relenaes the U.S. Coverument, and lth apenta, nervallta,

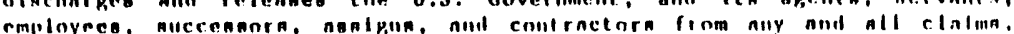

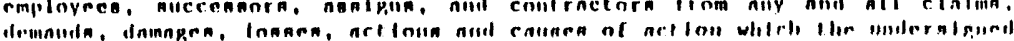

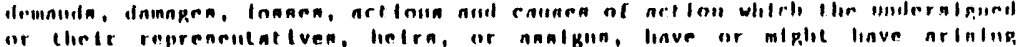

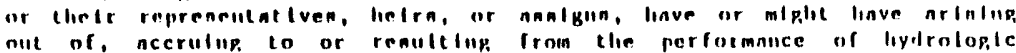
immltorlup stulles and related activitles on or lil the vicinlty of llie property dencribed below.

Tlic inderalpund reprenanta lint he/alie/or they nre the lawlinl ownera of the property deocribed below and is the proper party to recelve mayment hereunder.

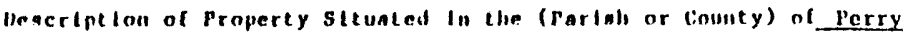
state of Mlssiagippi i South 20 acres of the SEh of NEל and the NEt of SE3 of Section 9, Township 4 North, Range 10 West, Perry County, Misstssippl; MRIH 11A, 11B, 11C, 11D and IIWS:

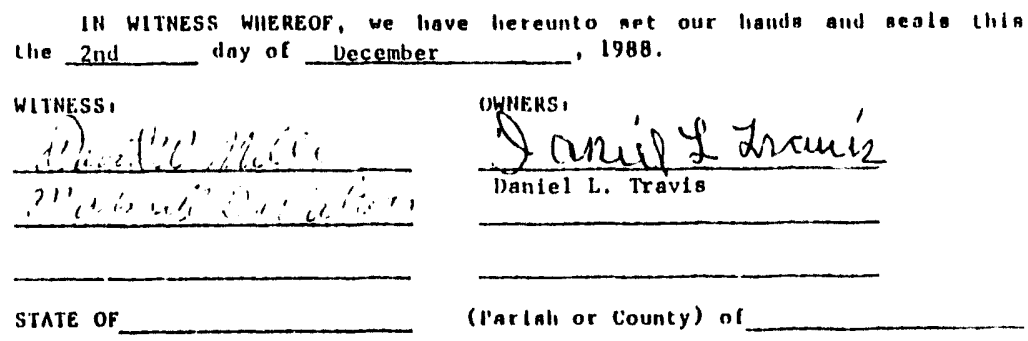

On this

day of

1988, before me persoutly nppenred

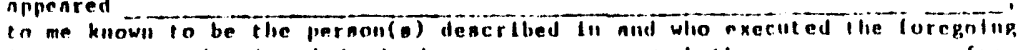
lint rument, aild ncknowledged that ___ exceuted the name an__fre nct and deed.

(SEAL)

inenry Pubtic In and inr (inilati or Cominy), (stale)

Hy Commiaglon Explrea 
STAtli al MLsistsithl

COUNTY (II) IIINDS:

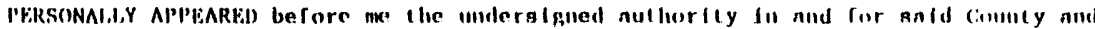

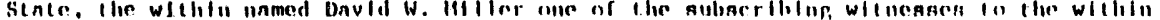

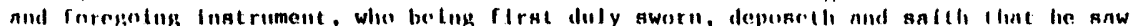
lilie wllhlil lianed ..... paniel l. Trayis.

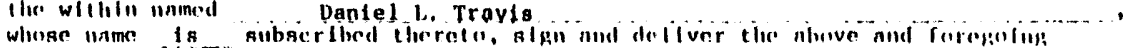
a

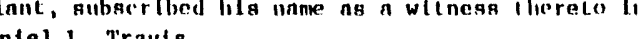

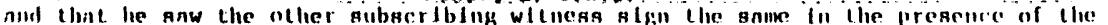
anld Daniel L. Travis

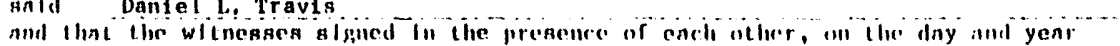
mid lhat the with
lliureln unined.
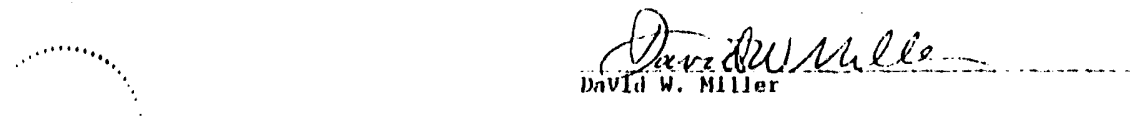

Swoprn to and subscribed before me, this the 5 th... day of necemberser, 1988.

(ArIIX Sen1)

My commission explres:

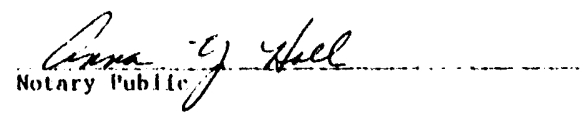

$2-5-90$

STATE OF MISSISSIPPI

COUNTY OF HINISS

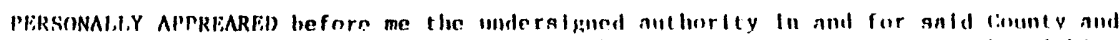

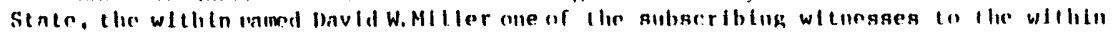

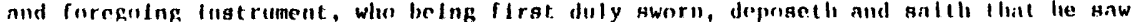
llie witlill named

whose

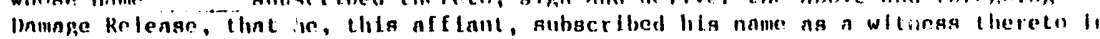
the presence of the suld

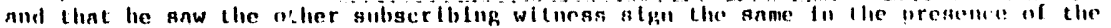
A:ild

and that the witnesses signed lil the presence of ench ofther, on the day and yenr therell inned.

invidi is. iillier

Sworn to nnd subscribed before ine, this the day of November, $198 \%$.

\section{(Alflx seal)}

My commiaslon explres:

Notary pubilic
- 1.t. ine ' $\because$ nday of UrC.

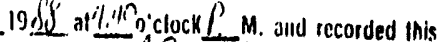

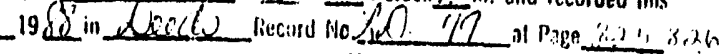




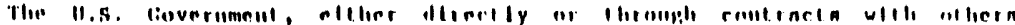

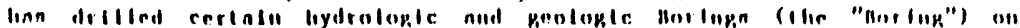

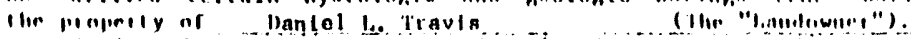

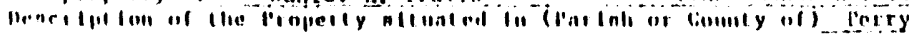

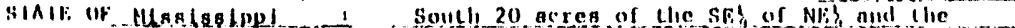

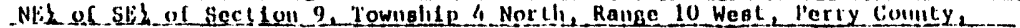

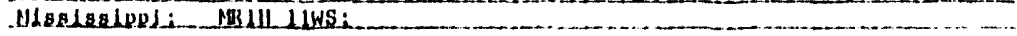

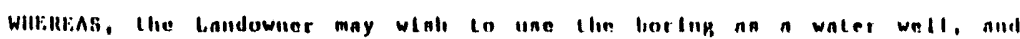

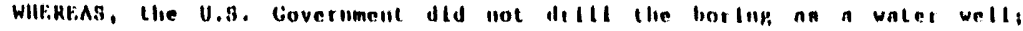

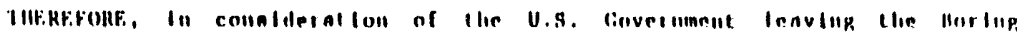

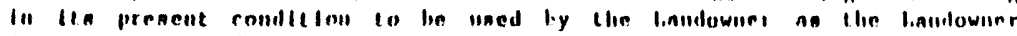

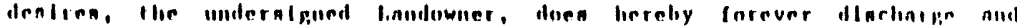

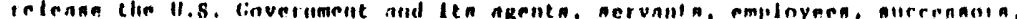

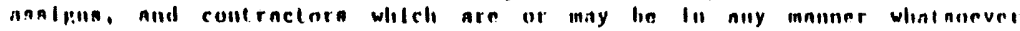

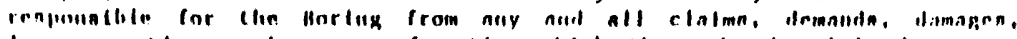

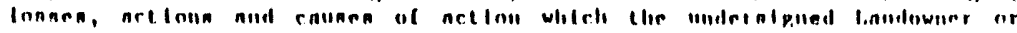

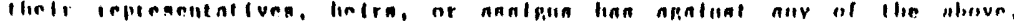

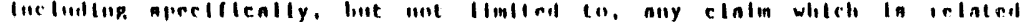
Io the sultoblllty of the llorling ne anter well.

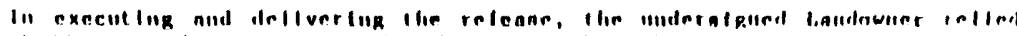

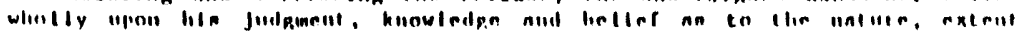

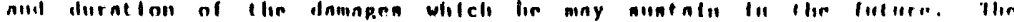

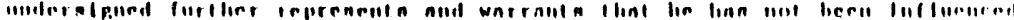

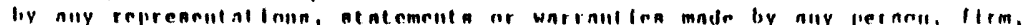

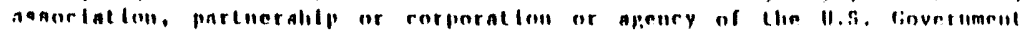

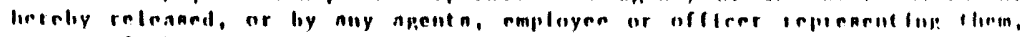

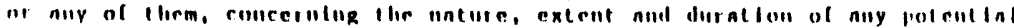
damnjere, lo ien ar legnl IInhlilty.

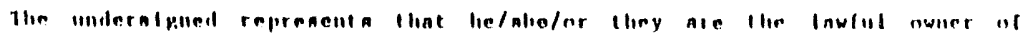

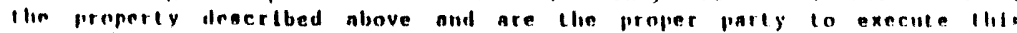
intrage/abirrmeilt.

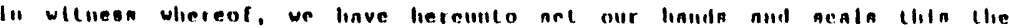
2nd Iny of Vecember , 19H11.

State of

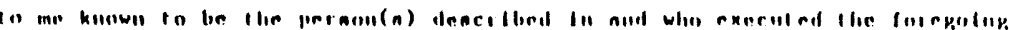

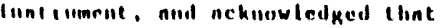

lien net and deed. exectilend tlie nome ne

(SE:AL)

Ily Commiasion Explrens

Notniy Publle In nond for (Pat lali on (Eiminty) of

state of 


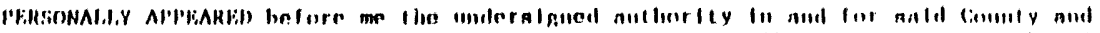

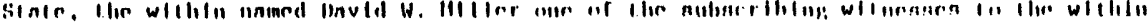

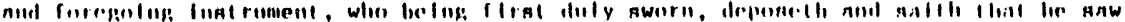
llin wlllill uned bantol l. Travis

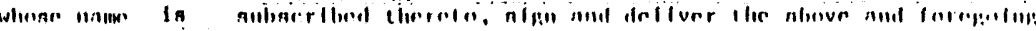

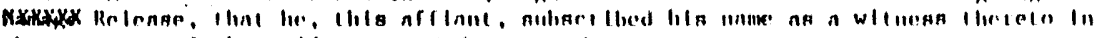
llie preacher of llie anla Dantel te. Irenuta

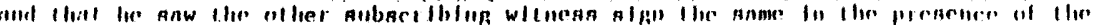

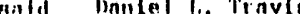

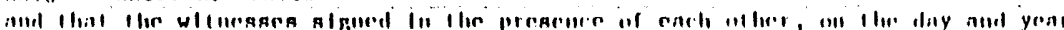

llieretil llamed.

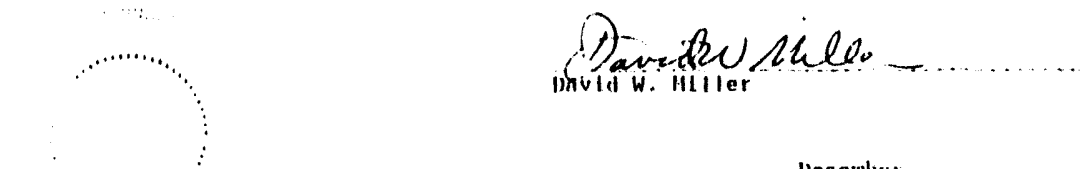

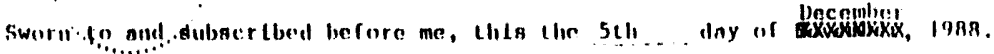

$(A|1| x \operatorname{sen} 1)$

My cimmlsalinl explres:

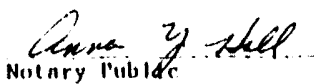

$\alpha \cdot 5 \cdot 2, \ldots$

Notnry inible

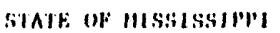

COUNTY OF IIINIIS

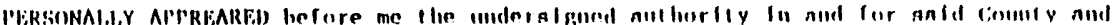

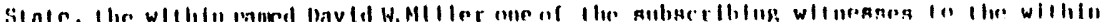

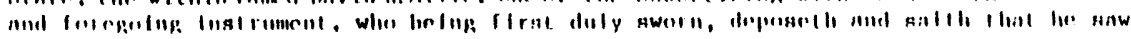
Ilin willilin linmed

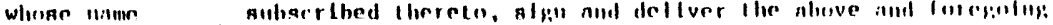

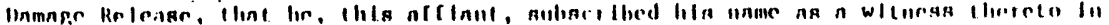

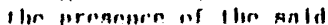

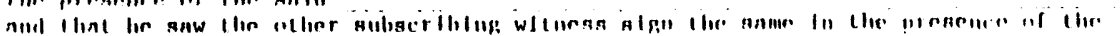
M:ili

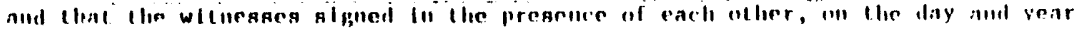
therelı nnumed.

invild ij. iilitier

Sworn In nuld subertlied before me, thla the day "I November, 1984.

(Alfix senl)

My commigaton explrea:

Piotenry Public

State of mississipH, l'irry Counly

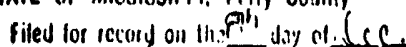

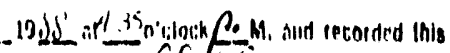

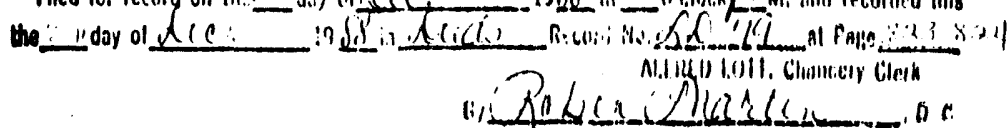


The underalgned, for and on belialf of the underalgnod and the representat I vee, lielrs nild analpun of the undernlgned, liereby acknowledyen recelpt from stone Webater Englneerink Corporation (SWEC) of the allm of One Dollar and other valunble consideration, the recelpt and oufflelency of whlch to hereby acknowledged, In full and complete settement and oatlofaction of any and all clatmo, demninds, damages, losen, act lonk, and causes of action of every kind and clinracter, which the underalgined or their representativen, he:ro and naglung have or mlght have by renton of ownership of the property or real property righto in the property, deecrlbed below on whlch the U.S. Government, and Its agents, aervants, employees, successors, assigis and contractors liave conducted drilllig. hydrologic monttoring atudien, well plugglng, olte restoration, and otiter operations. The herelnafter sescrlbed property 10 aloo described in certiln agreements (the "Agreements") executed by the underolgned which Branted right-of-entry and the right to perform hydrologic monitoring otudies and other operatione on the real property of the underalgned.

SWEC hag completed well plugging and/or olte restoration work according to the requirements of the Agreements. In consideration of the above payment, the underalgned lieruby forever dlacharpes and releases the U.S.

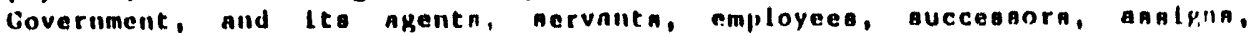
and contractors from any and all clatma, demanda, damages, loses, actions and causes of action whlch the underslgned or thelr representatives, helra, or assigne, have or mlght hive arising out of, accruing to or result ing from the performance of hydroiogle monttorinp atidien and related activitles on or in the vicinlty of the property deacribed below.

The undersigned repregents that he/ghe/or they are the lawful owners of the property descrlbed below and 1 a the proper party to execute this Release Agreement.

Deacription of Property stuated in the (Parloh or County)
of Perry

For description see Exhibit "A" attached hereto.

IN WITNESS WIIEREOF, we liave hereunto art our hande and acals thla the 218t day of November 1988.

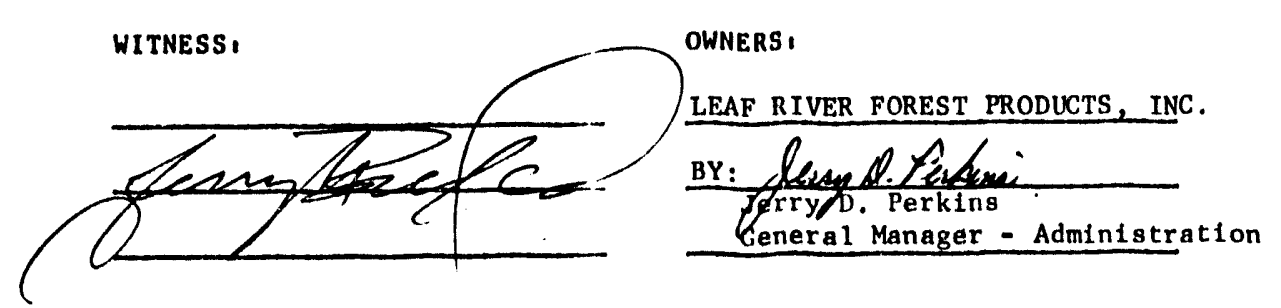

STATE OF MISSISSIPPI

COUNTY OF PERRY

Personally appeared before me, the undersigned authority in and for sald County and State, Jerry D. Perkins, General Manager - Administration of Leaf River Forest Products, Inc., who acknowledged that he signed and delivered the above and fore; oing instrument on the day and year thereir. mentioned as the act and deed of said corporation, being duly authorizec: so to do.

Given under my hariat. and offictal seal on this $2 \mathbb{L}^{5^{*}}$ day of

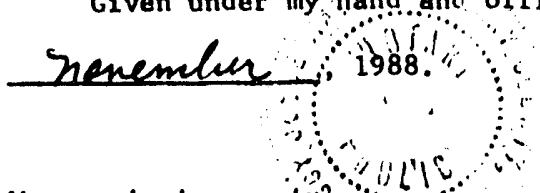

My commission exptras;

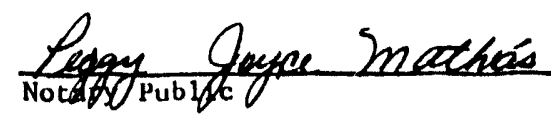

Commbston Explros Jan. 22, 1990 


\section{EXHIBIT "A"}

- NWt $\frac{1}{4}$ of SW $\frac{1}{4}$ of Section 4, Townsh1p 4 North, Range 9 West - MRIG 232

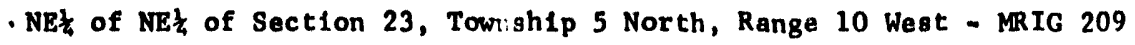

- NWt of NWt of Section 22, Townsh1p 5 North, Range 10 West - MRIG 205

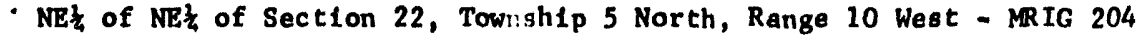

- NEt of SWt of Section 23, Towrship 5 North, Range 10 West - MRIG 203

- SE $\frac{1}{4}$ of SWl of Section 22, Townsh1p 5 North, Range 10 West - MRIG 201

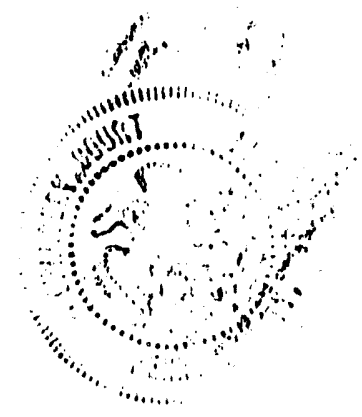




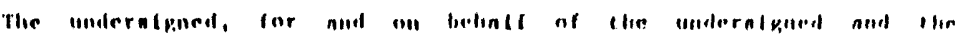

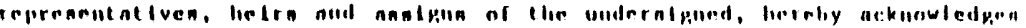

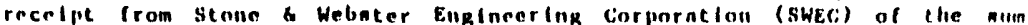
of One lollar and otlier valuable connideration, the recelpt and nuflictency of whicli in hereby acknowledped, In riull nind romplete notelenente nind

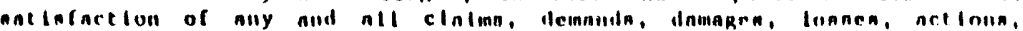

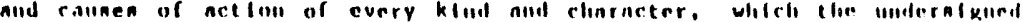

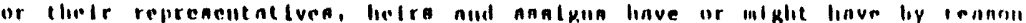

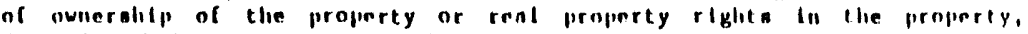
dencrthed below on whicli tlie U.S. Coverument, anil ito apenta, aervanta,

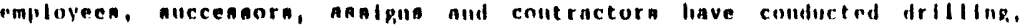

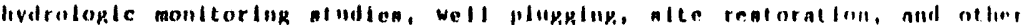

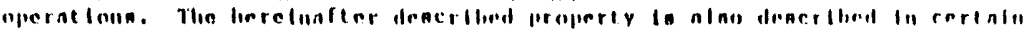

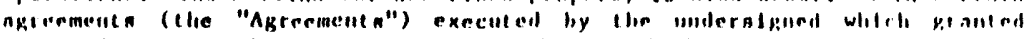
riphi-nf-entry and the riglit to perform liydrologle monleorling atudies and other operations on the real property of the underatgued.

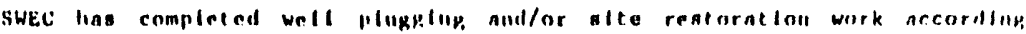

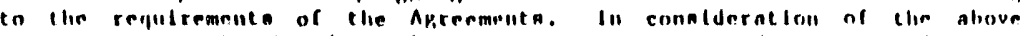

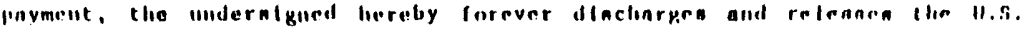

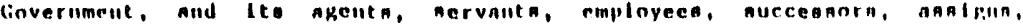

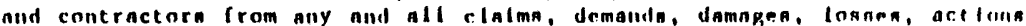
and causes of action which the undernlphed or their reprenentativen, lirira, or agalgne, have or mipht linve arialilk out of, accrulilg to or reaulelip. from the pertormnice of hydrolokle monlterlup ntinllen alld related nctlvitiea oll or lil the vicintey of the property dencribed below.

The undersigned repreannta that he/ahe/or they are the lawful owners of the property described below and in the proper party to execute this Release Agreement.

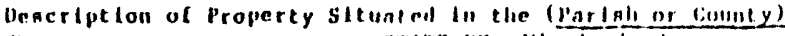
l'erry - STATE of Miselasiout

SEt of Sw\} of Section 26, Jowliship 5 North, Range IU! Weat. MRIf: 202

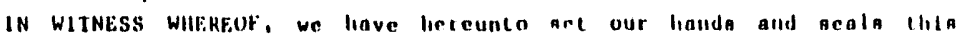
the loll day of Novemier. 1988.

WITHESS ,

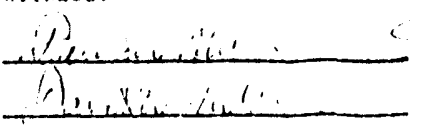

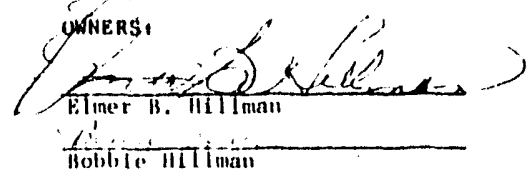

STATE OF - (1'aclali or county) of

On thls day of

- 1988, before me peraonally Appeared

to me known to be the peranin(a) deacribed In and who executed the foregotur, lint rument, and acknowledged that free act and deed. executed the same as

(SEAL)

Hy Comuntost on Explrea,

Wotnry Minitic in and lor (Baninti
or coumly).


STA'LE OF MTSSISSIPPI

COINTY OF IIIHDS

PERSONAII,Y APPEARR before me the underalgned authorlty in and for sald County and state, the witlin named bavid W. Miller, the subscrlifng

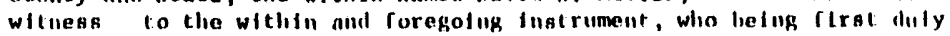

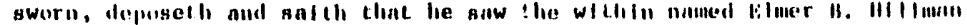
and wife. Hobble 1111 Iman, whose names are gnbsershed thereto, sI gil and deliver Che alsove ancl foregoing Damage Release, that he, Lhis affiant, subacribed lils name as a withess thereto lil the presence of the aald Nimer H. Ililiman and wife, Hobbte lilliman.

\section{Amerterylle havid W. Milier}

Sworn.to and suhacribed before me, this the 5 th day of December, 1988. $\therefore$ ?

(Affix Seal)

$\because, \ldots . . . .{ }^{\circ}$

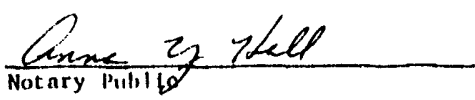

My commission expires:

$2-5.50$

itrate of MassissippI, Perry Counly

filed lor recerd on the $\delta^{\wedge}$ day of $\mathcal{L}_{\mathrm{S}} \mathrm{s}$.

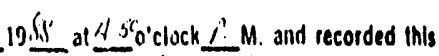
the 2 -2 iday of dece.

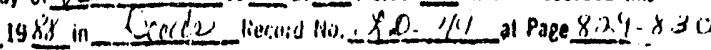

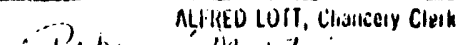

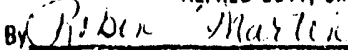


The undersigned, for and on behalf of the underalgned and the representatives, helro and aoshng of the undernigned, hereby acknowledgen recelpt from stone Weboter Englneerlng Corporation (SWEC) of the num of One Dollar and other valuable consideration, the recelpt and aufficlency of which is hereby ackriowledged, In full and complete settlement and oat lofaction of any and all clatms, demands, damages, loones, ections, and caunes of actlon of every $k$ ind and cliaracter, which the undersigned or their representatives, heiro and asolgne have or inlght have by reason of owreralip of the property or real property righte in the property, describet beloy on which the U.S. Covernment, and te agents, oervants, employees, uccessors, osigns and contractors have conducted drilling, hydrologle monltorlng otudieo, well plugging, olte restoration, and other operations. The lierelnafter described property is aloo deocribed in certaln agreements (the "Agreemente") executed by the underigned which granted right-of-entry and the right to perform hydrologlc monitoring atudies and other operations on the real property of the undersigned.

SWEC has completed well plugging and/or itte regtoration work according to the requirements of the Agreements. In conolderation of the above payment, the underalgined hereby forever dlocliarges and releagco the U.S. Goverument, and lto agenta, servaitn, employees, successorn, anignn, and contractors from any and all clalms, demands, damages, losees, actions and causes of action which the undersigned or thelr representatives, heirs, or asolgng, have or mlght have eriging out of, accrulng to or resulting from the performance of hydrologle monltoring atudles and related activities on or In the vicinity of the property descrlbed below.

The undersigned represents that he/olie/or they are the lawful owners of the property descrlbed below and is the proper party to execute this Release Agreement.

Description of Property situated In the (Parloli or County) of , STATE bF Mis818:1ppt

For description see Exhibit "A" attached hereto. MRIG 210

IN WITNESS WIIEREOF, we have liereunto not our hand and gealo thlo the $28 \mathrm{th}$ day of January - 1989.

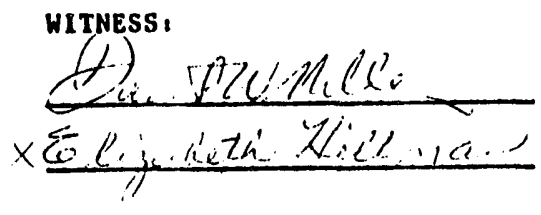

OWNERS :

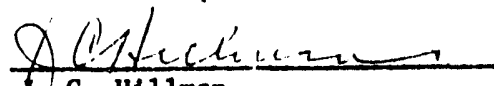
H. C. Hiliman 
The U.S. Covernment, elther directly or through contracte with othern lian drilled certain hydrologlc and geologle Boring" (the "Borling") on the property ol J. C. IIIl liman (The "landowner"). Dencription of the Property ituated In (Parlah or county of) Perry STATE OF Mississippi For description see Exhibit " $\Lambda$ " attached hereto.

WHEREAS, the Landowiler may wigh to use the boring as a water well, and

WILERAS, the U.S. Government did not drILI the borling as a water well;

TILERFORE, In conalderation of the U.S. Government leaving the Boring In the present condition to be used by the Landowner as the Landowner destrea, the undersigned Landowner, does hereby forever discharge and release the U.S. Government and tte agente, ervante, employees, ouccessors, asigns, and contractore which are or may be In any manner whatooever respoisible for the Boring from any and all clatme, demande, damages, losses, actions and causes of action.whlch the undersigned Landowner or their representativea, helrs, or asolgne has against any of the above. Including specifically, but not limited to, any claim which lo related to the sultabllity of the Horling a a water well.

In executing and dellverlng the release, the underalgned Landowner relled wholly upon his Judgment, knowledre and bellef an to tlie nature, extent and duration of the damanes "wilch he may oustill in the future. The undersigned further represents and warrants that he has not been Influenced by any representations, otatements or warranties made by any person, firm, association, partnership or corporation or agency of the U.S. Government hereby released, or by any agento, employee or offlcer representing them, or any of them, concerning the nature, extent and duration of any potential damages, losees or legal liablitty.

The undersigned represents that lie/olie/or they are the lawful owner of the property described above and are the proper party to execute this release/agreement.

In witness wherepf, we have hereunto act our hands and oealo this the 15 th day of November . 1988.

WITNESS

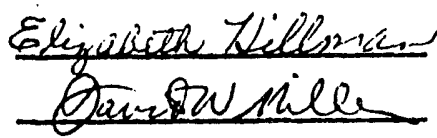

OWNERS:

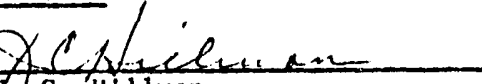

J. C. IIIIman

State of (ParLili or County) of

On this - 1988, before me personally appeared

to me known to be the peroon(a) descrlbed In and who executed the foregoing Inetrument, and acknowledged that executed the oame as

free act and deed.

(SEAL)

My Coamlaton Explreos

Notary Public In and for (Parlah or (ioninty) of

state os 
EXHIBIT "A"

MRIG 210

Township 5 North, Range 10 West

Section 36: Beginning at the Northeast corner of the SEt of NWt of said Section 36, and run thence West along the North boundary of said forty acres to the Northwest corner of said SE⿺ of NWh, thence run South 535 feet, thence run in a Southeasterly direction along the North boundary of the old Hattiesburg-Ricliton Public road a distance of 1075 feet, thence North 650 feet, thence East 446 feet, thence North 417 feet back to the point of beginning; Less and except a parcel of land described as beginning at the Northeast corner of the SEt of NWt of sald Section 36, and running thence South. 417 feet, thence run west $446 \mathrm{feet}$, thence run South 650 feet to the point of beginning of "the land herein described and excepted, from the point of beginning run North 522 feet, thence run West 417 feet, thence run South 295 feet to the North boundary of the 01d Public Road, thence run Southeasterly along the old road 500 feet to the point of beginning.

SIGNED FOR IDENTIFICATION:

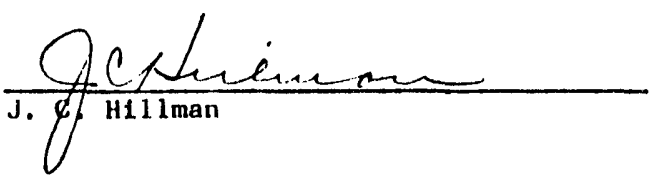


STATE OF MLSSISSIPPI

COUN'T OF IIINDS

(1)

PERSONALLY APPIARED before me the undersigned authority 1 in and for said county and State, the within named David W. Mller one of the subscribing witnesses to the within and foregoing lingtrument, who being first duly sworn, deposeth nind saith that he saw the withti immed ....... H. H11man whose name to subscribed thereto, sion and deliver the above and foregiili.......' Damage Release, that he, this afflant, subscribed his name as a witness thereto in the presence of the sald J. C. Hiliman

and that he saw the other subscribing witness sign the saine in the presence of the said J. C. H1llman

and that the witnesses signed in the presence of each other, on the day and year choriflin ninned.

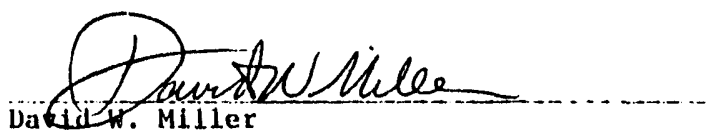

Sworn to and subscribed before me, this the 9 th day of January, 1989 .

(Arfy'seal)

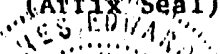

rinyidomatioston explres:

o

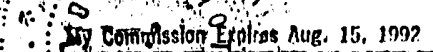

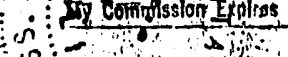

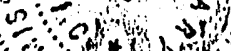

ifing

"rolingosis

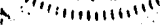

STATE OF MISSISSLIPI

COUNTY OF IIINDS

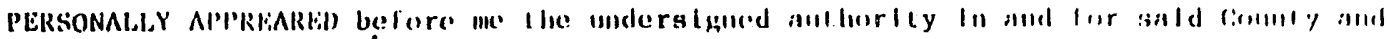
State, the within naured Dav1d W.Mlller one of the subscribing witnesses to the withll and foregoing instrument, who being first duly sworn, deposeth and saith that he saw the within named

whose name subscribed thereto, sign and deliver the above and foregoins

Damage Release, that he, this afflant, subscribed his name as a witness thereto ill the presence of the said

and that he saw the other subscribing witness sign the same in thic iresence if the said

and that the witnesses signed in the presence of each other, on the day and year

therein named.

David w. Miller

Sworn to and subscrtbed before me, this the___ day of November, 1988.

(Affix Seal)

My comnission expires:

Notary Public

sitate of MISSISSIPPI, Perry County

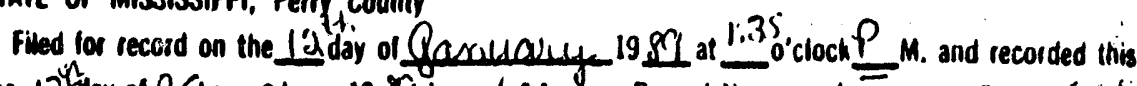

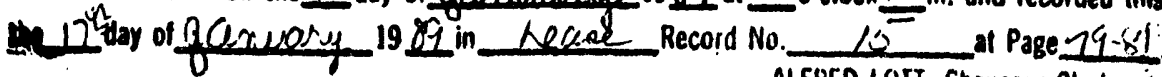


The underolgned, for and on behalf of the undernigned and the representat lves, lielrs nind anlgnn of the undernlgned, lieroby acknowledgen recelpt from stone Webnter Englneertng Corporntion (SWEC) of the num of One Dollar and otlier valuable consideration, the recelpt and oufficlency of wilch io hereby acknowledged, In full and complete settlement and oatefaction of nny and all clalima, lemando, damagen, Ionnes, actinnn, and causes of action of every klnd nunl clinrncter, whlch the undernigned or their represententiven, helro nild naglans have or might have by renann of ownership of the property or real property rights in the property, descrlbed below on whlch the U.S. Government, and to agents, servanto, employeca, ouccessors, asolgns and contractors have conducted drilling. hydrologlc monltorlng atudies, well plugging, nite restoration, and otlier operatlons. The liercinnfter descrlbed property is also descrlbed in certaln agreaments (the "Agreements") executed by the undersigned whlch granted right-of-entry and the right to perform hydrologlc monttoring otudies and other operatione on the real property of the underaigned.

SWEC lias completed well plugglng and/or site restoration work according to the requirements of the ARreementa. In conolderation of the above nayment, the undernlgned liereby forever dlocliargen and relenace the U.S. Govermment, nind tts ngentn, nervnutn, employees, nuccesnorn, annlgnn, and contractors from any and all clalms, demands, damages, loses, actions and caunco of action which the underalgned or thelr reprenentatives, helra, or asalgis, have or might liave ariolng out of, accruing to or resulting from the performance of hydrologic monttorling atidies and related activities on or in the vicinity of the property deacribed below.

The underolgned repreoents that lie/glie/or they are the lawful ownras of the property deacribed below and lo the proper party to execute thls Release Agreement.

Description of Property sltuated In the (Parlali or County)

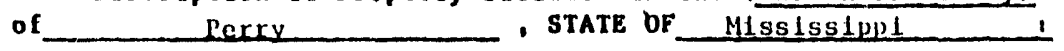

$\mathrm{SE}_{4}$ of $S W^{\mathrm{t}} \mathrm{f}_{4}$ of Section 25 , Township 5 North, Range 10 West.

IN WITNESS WIIEREOF, we have hereunto not our hando and gealo this the 27 th day of 1989.

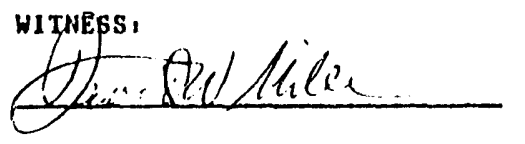

OWNERS ,
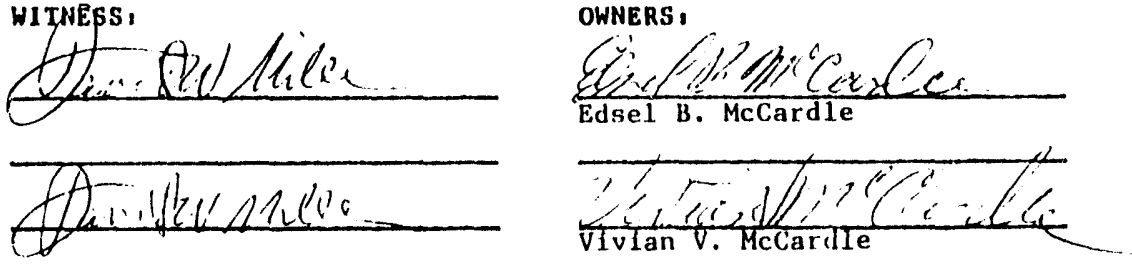
The U.S. Government, elther directly or through contracte with othera han drllled certaln hydrologic and geologle Borlngo (the "Borlng") on the property of Edsel B. McCardle and wife, Vivian V. McCardle (The "Landowner"). Description of the Property ituated In (Parlali or county of) perry

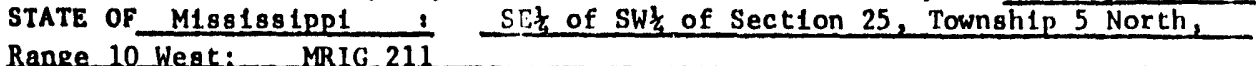

MIEREAS, the Landowner may wish to une the boring as a water well, and

WHEREAS, the U.S. Government did not drill the boring as a water well;

THEREFORE, In consideration of the U.S. Government leaving the Borlng In 1ta present condition to be used by the Landowner a the Landowner desires, the underalgned Landowner, does hereby forever diocharge and release the U.S. Government and Lt agents, servants, employees, ouccesoors, assigno, and contractors whlch are or may be In any manner whatsoever responsible for the Borlng from any and all clalma, demands, damages, losses, actions and causes of action. Whlch the underalgned landowner or thelr representatives, helra, or asolgne has agalnnt any of the above, Including apeclfically, but not limited to, any clalm which io related to the sultabllity of the Borlny, as ater well.

In execut Ing and dellvering the release, the underalgned Landowner relled wholly upon hlo judgment, knowledge and bellef an to the nature, extent and duration of the damagea whlch he may ausaln in the future. The underaigned further reprenents and warrants that he has not been Influenced by any representationa, tatements or warrantles made by any person, $f(\mathrm{rm}$, association, partnershlp or corporation or agency of the. U.S. Government hereby released, or by any agents, employee or officer representing them. or any of them, concerning the nature, extent and duration of any potential damages, losses or legal llabllity.

The undersigned represents thit he/sho/or they are the lawful owner of the property described above and are the proper party to execute this release/agreement.

In witneso whereof, wo have hereunto nnt our handa and seals thls the 15 th day of November 1988 .
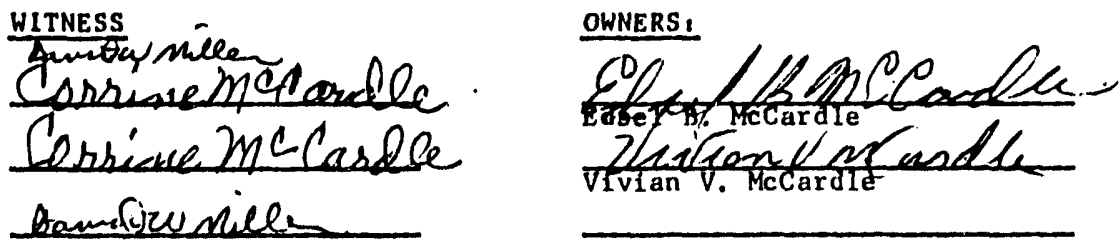

State of (Parlsh or County) of

On this appeared day of 1988, before me personally

to me known to be the person(s) described in and who executed the foregoing Inat rument, and acknowledged that executed the ane as

free act and deed.

(SEAL)

Hy Comeniesion Explresi 
PERSONALLY APPEARED before me the undersigned authority in and for said Count: and state, the within named David W. Hiller one of the subscribing witnesses to the within and foregoing instrument, who beln; first duly sworn, deposeth and salth that he saw the within named Edsel B. McCard ic and wife, Vivian V. McCardle

whose names are subscribed thercto, sign and deliver the above and foregotn:; makxyx Release, that he, this affint, subscribed his name as a witness therelo in the presence of the sald Edse1 B. McCardle and wife, Vivian V. McCardle

and that he saw the other subscribing witness sign the same in the presence ot the sa1d Edsel B. McCardle and wife, Vivian V. McCardle

and that the witnesses signed in tie presence of each other, on the day and ycar there in named.

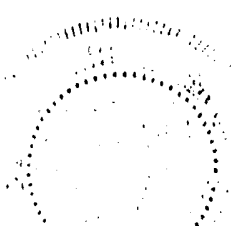

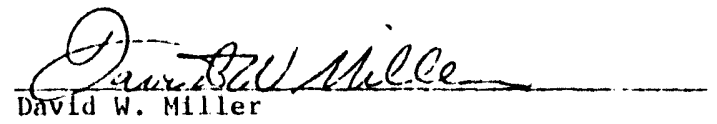

Swarn to and subscribed before me, this the $21 \mathrm{st}$ day of November, 1988.

(Af $f x \cdot$ Sea 1$)$

My commission explres:

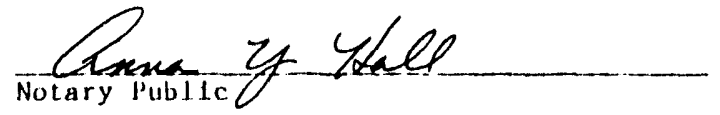

$2-5-1990$

STATE OF MISSISSIPPI

COUNTY OF HINDS

PERSONALLY APPREARED before me the indersigned authority in and for sald Count $v$ and State, the within named David W.MILlor one of the subscribing witnesses to the within and foregoing instrument, who beins: first duly sworn, deposeth and saith that he saw the within named whose name subscribed thereto, sign and deltver the above and foregoing axoux Release, that he, this afflilt, subscribed his name as a witness theret" in the presence of the satd

and that he saw the other subscribi: said

and that the witnesses algned in the presence of each other, on the day and yeir there in named. day of November, 1988 .

(Aff $1 x$ Seal)

My commission expires:

STATE OF MISSISSIPPI, Perry County

Filed for record on the 23 day of unos the $28^{\text {th }}=$ day of $n$ ore. 1388 in ind

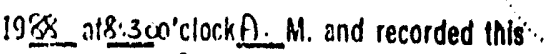

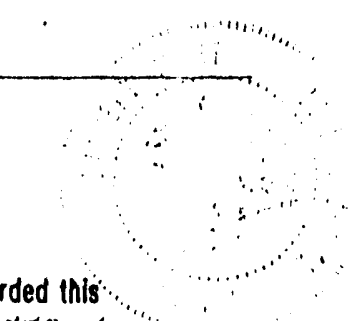
hiculi No. $50-79$ at Page $153-54$ ALFRED LOTT, Chancery Clerk 
RGillespie

Project File M-9

WPC $245 / 12$

(6428-1750026-B4T)

\section{CAFoster}

Mr. Lloyd Valentine and Mrs. Mary Katherine Valentine Route 3, Box 158

Richton, Mississippi 39476

DECOMMISSIONING OF MRIG-212 WELL AND RESTORATION OF LANDS IN NE $\frac{1}{4}$ NE $\frac{1}{4}$ SECTION 35 , T5N-R1OW PERRY COUNTY, MISSISSIPPI
April 14, 1989

J.0. No. 17500

Dear Mr. \& Mrs. Valentine:

The subject well was turned over to you by Law Engineering Testing Company and accepted by you under terms of Release dated May 6, 1981. Stone \& Webster checked the well for you to determine the feasibility of your using it as a water well and found casing in the well had parted or broken and the well was not suitable for use as a water well. You were informed of this on November 9 and 10, 1988 and directed SWEC's representatives to plug and abandon the well.

On November 10 and 11 Stone \& Webster Engineering Corporation (SWEC) plugged and abandoned the subject well in accordance with the Rules and Regulations of the Mississippi Department of Natural Resources.

You were contacted in December, 1988, and January and February, 1989, regarding site restoration and you said the site restoration was satisfactory. You told Stone \& Webster representative David W. Miller you were satisfied with the restoration work and that the restoration was acceptable.

Stone \& Webster has plugged and abandoned the subject well and restored the surface of lands therearound in accordance with the terms and conditions of Extension of Right of Entry and Use Agreement by and between yourselves and The Earth Technology Corporation dated July 20, 1987, recorded in the official Records of Perry County, Mississippi in Lease Book 14, Page 43. Therefore, SWEC has no further obligation with respect to the lands covered thereby or the well thereon. The agreement will expire by its own terms. 
Unless Stone \& Webster hears to the contrary from you within ten (10) days from the date hereof, Stone \& Webster will consider all obligations fulfilled and the matter closed.

Yours very truly,

STONE \& WEBSTER ENGINEERING CORPORATION

By: $\frac{(A / 4)-C s t a d}{\text { C. A. Foster }}$

$\mathrm{CAF}: \mathrm{jr}$ 
The inderntgned, for and on belinlf of the underalgned and the

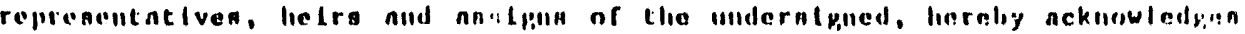
recelpt from stone \& Webater Einplineerlnk Corporation (SWEC) of the nilm of One Dollar and other valualile conglderation, the recelpt and anficlency of whlch is hereby acknowledged, in full and complete nettlement and oatlsfaction of any and all clating, demands, dainages, losnes, actions, and causes of action of every $k$ ind and character, which the undersigued or their representativea, lietra and anolkina have or inght have by renson of ownership of the property or real property riglita in the property, descrtbed below on whIch the U.S. Government, and It agents, servants, employees, successors, asalgns and contractorg have conducted drllllin, hydrologlc monltorlng atudles, well plugplng, alte regtoratlon, and other operntions. The lierelnafter deacribed property la nlan deacribed In certoln agreements (the "Agreementa") executed by the undernigned which pranted right-of-entry and the right to perforin hydrologic monltoring atudiea and other operations on the real property of the undersigned.

SWEC lias completed well plupiplng and/or alte reatorntion work accordlup to the requirements of the Aprenments. In consideration of the abeve pnyment, the underolgned lierrby forever dlacliarges and relenges the U.S. Government, and 1 to agent, gervanta, employees, ouccesaora, analpin, and contractors from any and all clatma, demanla, damages, logses, act and causes of action which th. undersigned or thelr representatives, helrs, or assigna, have or mlght hilve arlaing out of, accrulng to or regulting from the performance of liydrologle monltoring, atudlea and related act lvitiea on or lin the viclnity of the property descrlbed below.

The undersigned represents that he/ghe/or they are the lawful owners of the property deacrlbed be low and is the proper party to execute this Release Agreement.

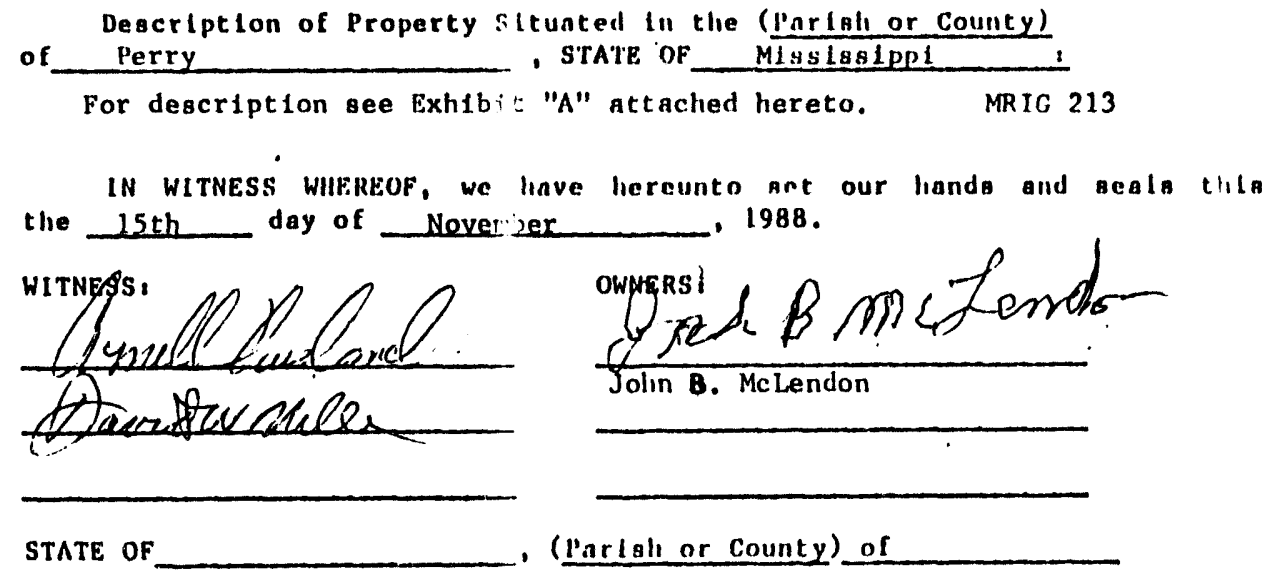

On thls

day of

, 1988, before me personally appeared

to me known to be the peraon(a) deacrlbed in and who executed the foregoing Instrument, and acknowledged that free act and deed. executed the oame an

(SEAL)

Notary Public ln and for (Parlah
or (Sounty).

My Commlssion Explreo 
STATE, OF MISSISSIPPI

COUNTY OF IIINDS

PERSONALLY APPEARED before me the indersigned authority in and for said County and State, the within named David W. Hiler one of the subscribing witnesses to the within and foregoing instrument, who beln! f Irst duly sworn, deposeth and saith that he saw the within named John B. McLendon.

whose name is subscribed thereto, sign and deliver the above and foregolng

Damage Release, that he, this affiant, subscribed his name as a witness thereto in the presence of the said John B. McLendon

and that he saw the other subscribing witness sign the same in the presence of the said John B. McLendon

and that the witnesses signed in the presence of each other, on the day and year there in nnmed.
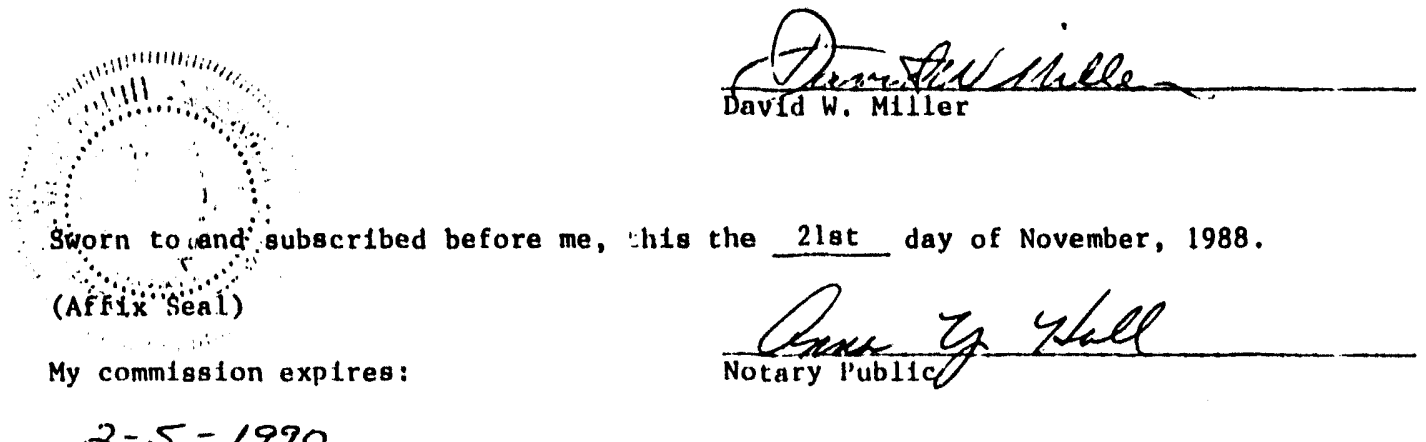

$2-5-1990$

\section{STATE OF MISSISSIPPI}

COUNTY OF HINDS

PERSONALLY APPREARED before me the undersigned authority in and for sald County and State, the within named David W.Mille one of the subscribing witnesses to the within and foregoing instrument, who being f1rst duly sworn, deposeth and saith that he saw the within named

whose name subscribed theret, , $1 \mathrm{gn}$ and deliver the above and foregoing Damage Release, that he, this affiati, subscribed his name as a witness thereto in the presence of the said

and that he saw the other subscribing witness sign the same in the presence of the sald

and that the witnesses signed in the presence of each other, on the day and yer $r$ therein named.

(Affix Seal)

My commission expires: 


\section{Township 5 North, Range 10 We: $t$}

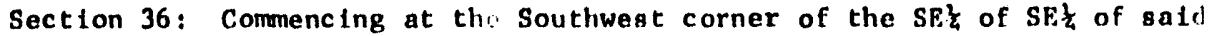
Section 36, thence run East 61'6 feet for a point of beginning, thence run North 104 feet, thence run Enst 52 feet, thence run South 104 feet, thence run West 52 feet to the potnt of beginning; and Beginning at the Southwest

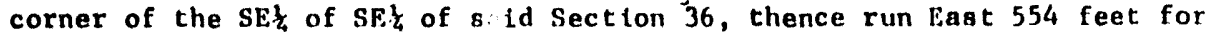
a point of beginning, thence run North 104 feet, thence run East 52 feet, thence run South 104 feet, thrince run West 52 feet to the point of beginning.

MRIG 213

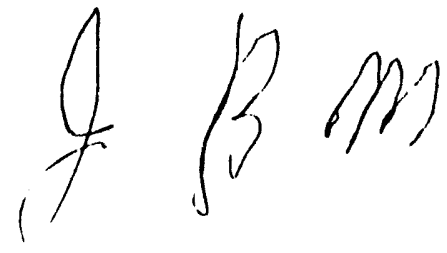

STATE OF MISSISSIPPI, Perry Counly

Flied for record on the 23 day a hou ${ }^{19} 88$ at 8:50'clock A.M. and recorded this curs day of tou. 19,8 in Spee Record No. \%ैo.79 at Page759.61 ALFRED LOTT, Chancery Clerk

By Dudsers bote D. C 


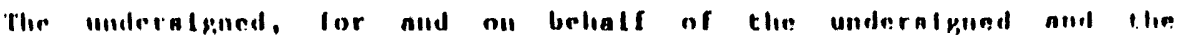

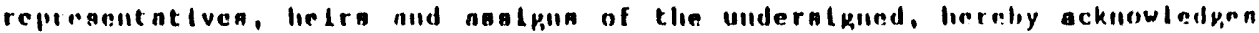
recelpt from stowe o Webater Enplinecrlnk Corporation (SWEC) of the anm of One llollar nud other valuable conslderation, the recelpt and nufficlency of whicli Is lieroby ackiowledged, II liil and complete nettlement nind

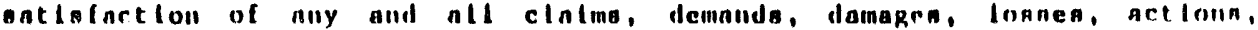
and cannes of nctlon of every klul and clinencter, which tie underalpured

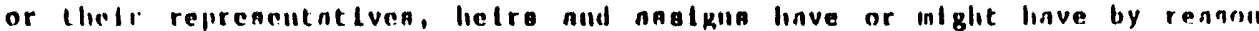
of owlieralilp of the property or renl property righte lin the property, descrlhed below on whlch the U.s. Government, and Ita anenta, pervanta, employeca. aucceasors, analgas and contractors have conducted drilling, liydrologic monltorling atulles, well plugping. site restoration, and otlier operations. The lincelinaftior described property le alao deacribed in certalin agreements (the "Agreenente") executed by the underalgned which granted right-nf-entry and the right to perform liydrologle montoring atudies and other operations on the real property of the undersigied.

SWEC hag completed wal plugplng and/or elte reatoration work accordling to the requiremente of the Apreementa. In conalderation of the ahove

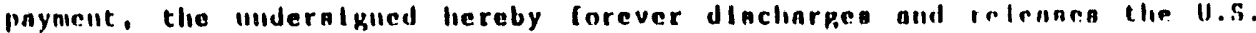

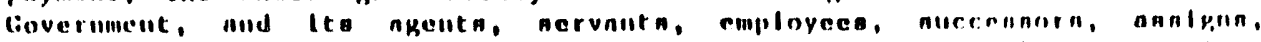

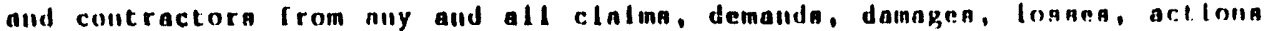
and causes of action whicli the undersigned or thelr repreacutativea, helra,

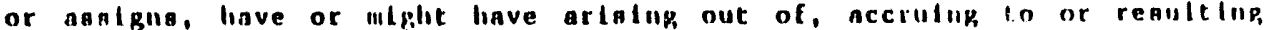
from the performince of liyilrologle montentup ntilian and related activitiea oil or lil the viclilty ol the property deacribed below.

The underalgned represente that he/alie/or they nre the lawful ownira of the property deacribed below and la the proper party to exccute this Release Agrecment.

Description of Property situnted lu the (1'arlah or County)
of perry

E. of NWt of NW? of Section 1, Township 4 North, Range 10 West. MR16 215

IN Withess WIllilliur, we liave hereunto not our hallda and aenla thin the 9 th day of January XX4kx 1989

WITNESS

OWNEHS ,

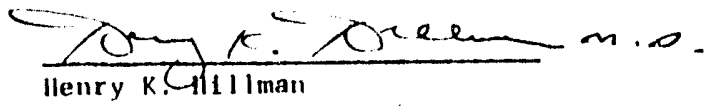

STATE OF MISSISS1UU1

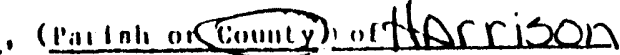

THI:; DAY personally appeared before me, the undersigned nuthority in and for sald county and state, the within named HFNRY K. IIIlIMAMN who acknowlriged that he afgned and delivered the withil and foregoing ingtrument on the day and year therein mentioned. 1989

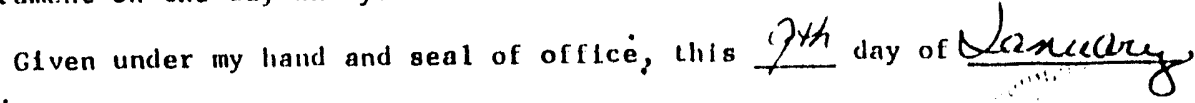

$$
\text { (Affta } \therefore \text { eaij) }
$$

M: cqumiasion explres:

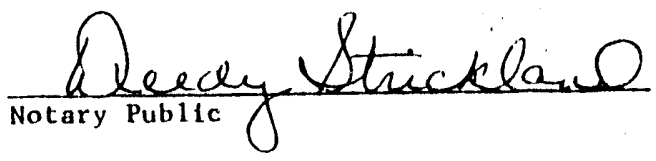

Myctmmission Expires August 31.199 ;

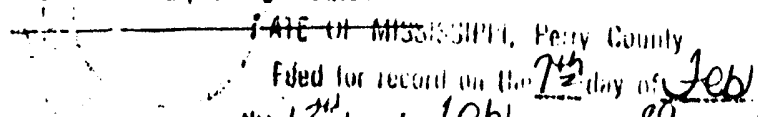

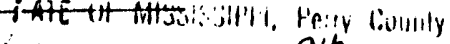

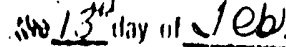
1: 89 i" aleed 889,8750

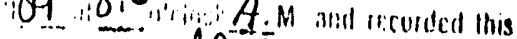

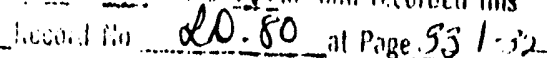
NLI liEI LOH, Chancery Clerk 
The underolgned, for and on belintf of the undernigned and the representativen, helrs nind nnilpinn of the undernipned, liernby ucknouledsen recelpt from Stone Webater Englneering Corporation (SWEC) of the fium of One Dollar and other valualile constderation, the recelpt and aufficlency of which in hereby acknowlidged, In full and complete nettlement and

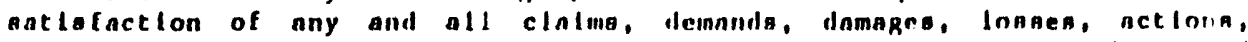
and caunes of action of every klud nind chnencter, whlch the underalyined or their repreaentatives, heirs and ansigna linve or inglit liave by renson of ownership of the property or real property riglite in the property, descrtbed below on whlch the U.S. Government, and Its agenta, aervants, employees, successors, asalp,is and contracturs have conducted drilling, hydrologlc monltoring atudles, well plupglng, atte restoration, and other operatlons. The lierelnafter debcrlbed property lo nlao deacrlbed in certaln agreements (the "Agreements") executed by the undernigned which granted right-of-entry and the right. to perform hydrologlc monltoring otudies and other operations on the real property of the undersigned.

SWEC has completed well plusphing and/or alte reatoration work according, to the requitements of the Apreementa. In consideration of the above payment, the underslgned lieriby forever dlacharges and releases the U.S. Government, and tts agenta, gervanta, employees, successors, ansipin, and contractors from ally and all clalms, demaride, damages, losen, actlona and causes of action which thr undersigned or thelr representative, heirs, or asalgna, have or mlght hi:ve arlaling out of, accrulng to or resulting from the performance of hydrologic monltoring atudien and related activitica on or in the vicinity of the property described below.

The underatgied represents thint he/she/or they are the lawful ownirs of the property described be low and is the proper party to execute thils Release Agreement.

Description of Property Situated in the (Parloh or County)

of Perry , STATE of Mississippi

NWt of SW $\frac{1}{4}$ of Section 1, Tounship 4 North, Range 10 West. MRIG 216.

IN WITNESS WIIEREOF, ve have hereunto aet our hands and seala thlo the 15 th day of Nov mber . 1988 .
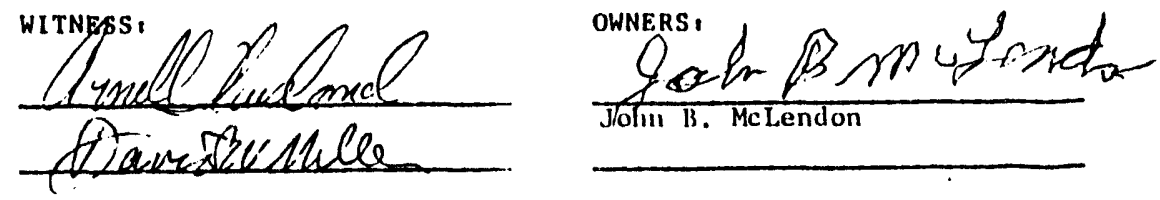

STATE OF - (Parlall or County) of

On this day of 1988, before me personally appeared

to me known to be the peraon(";) descrlbed In and who executed the foregoing inst rument, and acknowledged that free act and deed. executed the same as

(SEAL)

My Commlasion Explrea
Notary Public In and for (Parlsh or County). (State) 
PERSONALLY APPEARED before me the undersigned authority in and for said County and State, the within named David W. Miller one of the subscribing witnesses to the within and foregoing instrument, who being first duly aworn, deposeth and saith that he saw the within named John B. McLendoin

whose name is subscribed theretu, sign and deliver the above and foregoing

Damage Release, that he, this affint, subscribed his name as a witness thereto in the presence of the said John B. YcLendon

and that he saw the other subscribilig witness sign the same in the presence of the sald John B. McLendon

and that the witnesses signed in thi presence of each other, on the day and year therein named.

(1)

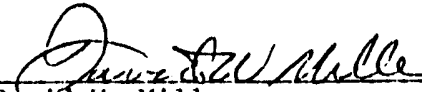

and

David W. Miller

Sworn to and subscribed before me, ints the 21st day of November, 1988.

(A) :

(Afftix Sea1)

My commission expires:

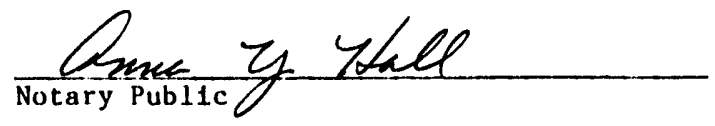

$2-5-1990$

STATE OF MISSISSIPPI

COUNTY OF HINDS

PERSONALLY APPREARED before me the inderstgned authority in and for sald County and State, the within named David W.Mille: one of the subscribing witnesses to the within and foregoing instrument, who being first duly sworn, deposeth and salth that he saw the within named

subscribed thereto, sign and deliver the above and foregoing

Damage Release, that he, this afflant, subscribed his name as a witness thereto in the presence of the sald

and that he saw the other subscribing witness sign the same in the presence of the said

and that the witnesses signed in the presence of each other, on the day and yenr therein named.

David W. Miller

Sworn to and subscribed before me, shis the day of November, 1988.

(Affix Seal)

My commission expires:

STATE OF MISSISSIPPI, Perry County

Filed for record on the 23 day of ring day of unow. 1988 in 5320 1988 al 8.300'clock D.M. and recorded this at Page? $762 \cdot 10=$ Record No. $220-29$ at Page 762.63

$$
\text { ALFRED LOTT, Clancery Clerk }
$$

By Oustert Loel 
RE IEASE

WHEREAS, L.AW Engireering Jesting Company (the "Company"). esther directly or inrough contracts with others. has driljed certain hydrologic and oeslogic borings (the "Boring") on the property of L. W. and Ervis D. Codfrey

(the " Landowner"): and

WHERFAS, the Landownet may wish to use the Boring as a waterweld, and

WHEREAS, the Compary did not drijl the Borjag as a waterwell:

THEREFORE, in the consideration of the company leaving the Boring in its preserit condition to be used iy the landowner as the Landowner desjres. the uncersigred Landowner. does hereby release. acqujt and forever discharge the Corpany. its successors. employees. represeritatives, drilling contractcre and ary and all other persoris. firms. partmerships, associations or corporations wijich are or may be in any manner whatsoever responsjtje for the soring fron anj and al claims, demands, acte, damages, causes of action, costs. experses. and any and al? other liabjljtjes whatsoever, both in egujty or ctherwise, whether known or uriknowr. which the undersigned Landowiner has against any of the aboze. including specifjcally any clajm which might have been asserted against the company due to itie sujtabjlits of the Boring as a walerwe!l.

It is unierstooc aric asteed that tis release is executed for and in cersiciration of the company leeving the boring in its piesent concition fCr use by the Landowse:. and the company in no way represents that the soring is suilable for use as a vaterwedl.

In execuling anc delivering the release, the undersigned Lanoowne: relied wholly upor his judgnent, knowleoge and belief as to the nature, extent and duration of the damages whict, he may sustain in the future. The undersigned further represents and wargante that he has not been in!luences by any refresertatione, statements cr wargansier made iy any pessor. firm, association. Fartnesshif or corporasic:. rezeby released, $c$ : by any ogents, empioyee o: offlces representing them, s: any of them. concerning ire neture. extent and duraticn of any potential damages. losses or legal Jiabilaty.

This release contains tre enilge agreement between the partues hereto and the terss of this relezse are contractuel in nature.

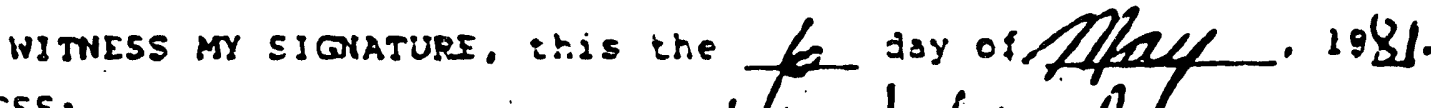
h:-isess:
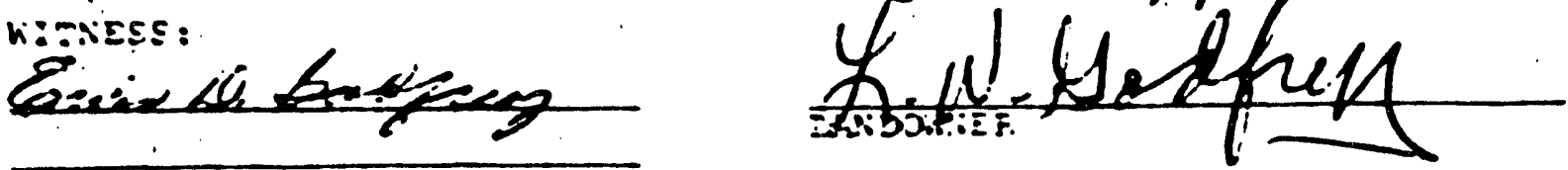

$$
\text { W: R:S- }
$$


The undersigned, for and on behalf of the undersigned and the representatives, helrs nind asigna of the undernlgned, hereby acknowledges recelpt from stone \& Weboter Englneering corporation (SWEC) of the sum of One Dollar and other valuable consideration, the recelpt and oufficiency of which la hereby acknowledged, In full and complete oettement and satiofaction of any and all clatme, deminda, damager, Losen, actionn, and causes of action of every klnil and chingeter, which the underolgined or their representatives, helro and assigna linve or inight have by reason of ownership of the property or real property rights in the property, described below on which the U.S. Governicent, and Its agents, nervanta. employees, successors, asolgns and contractors have conducted drillinb, hydrologlc monltoring etudies, well plugglig, olte restoration, and other operations. The herelnafter descrlbed property lo nloo deacribed in certaln agreements (the "Agreementa") executed by the undersigned whlch granted right-of-entry and the right to perform hydrologic monitoring otudies and other operations on the real property of the undersigned.

SWEC has completed well plughling and/or hite reatoration work nccorilng to the requirements of the Agreementa. In consideration of the above payment, the undersigned hereby forever discharges and relenses the U.S. Government, and 1 to agents, servant, cinployees, ouccessors, assigns, and contractore from any and all clatima, dimands, damages, losea, actions and causes of action which the underalgined ir thelr representatives, helra, ir assigns, have or might liave arising out of, accruing to or resulting, from the performance of liydrologlc monttorlin:, atidien and related activitiea oll or in the vicinity of the property deacribed below.

The undersigned represents that he/she/or they are the lawful owners of the property described below and is tlit proper party to execute this Release Agrecment.

Description of Property situnted in the
perry

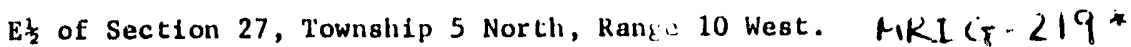

IN WITNESS. WHiHEOF, we have hereunte ant our hande and beala this Llie $10 \mathrm{th}$ day of Noyeulber . 1988.

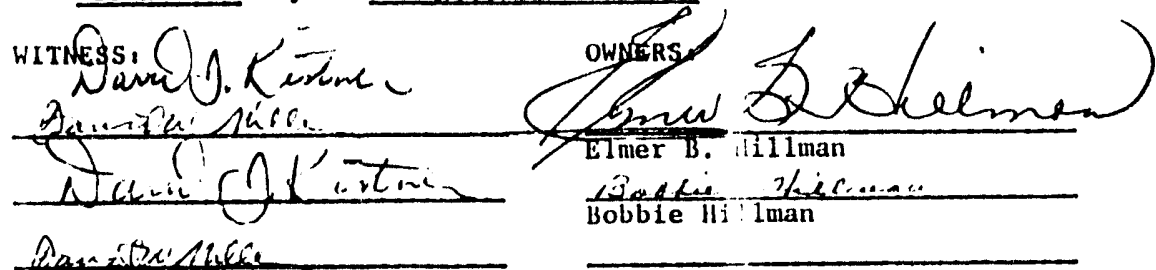

STATE OF (liarlah or county) of

On this day of 1988, before me personally appeasid lo me known to be the person(a) descrlbed lil and who executed the forcgoing instrunent, and acknowledged that ree act and deed. exicuted the saine as

(SEAL)

Notary Puilic in and lor $\frac{(\text { parlsh }}{\text { (ptate) }}$
or County),

My Commlesion Explres:

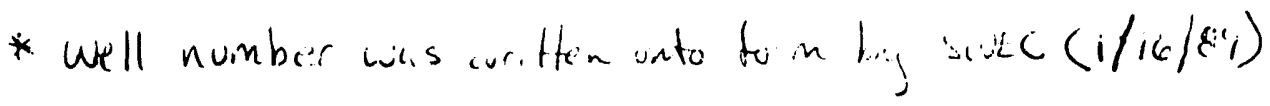


STATE OF MISSISSIPPI

COUNCY OF HINDS

PERSONALLY APPEARED before me the undersigned a thorlty in and for sald County and Stato, the within named David W. Miller

one of the subscribling witnesses to the within and foregolning instrument, who

being flrot duly oworn, deposeth and salth that he oaw the within named Elmur B. Hillman and wife, Bobble lliliman the same to the sald Stone \& Webster Engineering Corporation - that he, this afflant, subscribed his name as a witness thereto in the presence of the sald :1mer 1 . lliliman and wife Bobbie Hiliman , anc that he saw the other subscribing witness sign the same in the presence of the said Eluer B. Hliliman and wife. Boblie lil1man and that the witnesses signed in the presence of each other, on the day and year therein named.

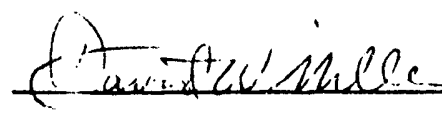

Sworn taind subscribed before me, this the 11tin day of November $19 \underline{88}$. (Affix $\operatorname{seq}_{0}$

My commiesion explres: Iebuing 458

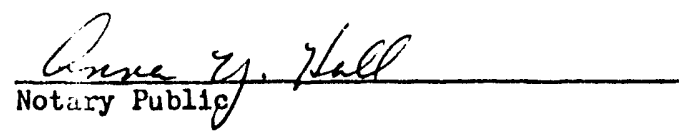

STATE OF MISSISSIPPI

COUNTY OF

PERSONALLY APPEARED before me the undersigned atithority in and for sald County and State, the within named

one of the subscrlbing witnesses to the within end foregolng instrument, who belne first duly sworn, deposeth and salth that re saw the within named

subscribed thereto, sign and deliver the same to the sald

whose name

- that he, this aiflant, subscribed his name as a witness thereto In the presence of the sald witness sign the same in the presence of the sal:!

- and that he saw the other subscribing wind that the witnesses signed in the presence of each other, on the day and year 'herein named.

Sworn to and subscribed before me, this the day of . 19

(Aff1x Seal)

My comission explres:

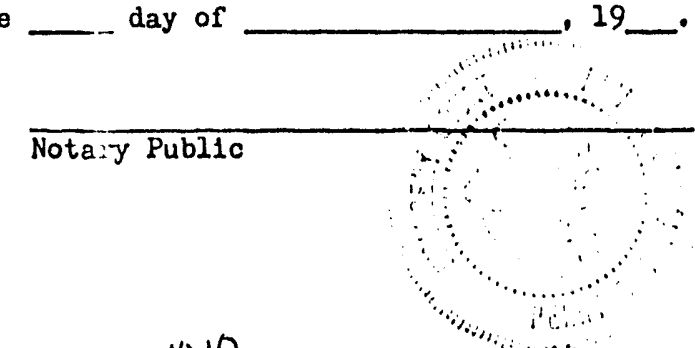

itaTE Of MUSSISSIPPI, Perry County

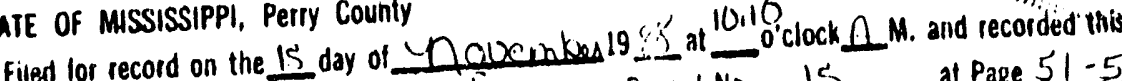
the 2 2day of - Dove in bu 19.88 in Lease Ricord No 15 at Page. $51-52$ 
The undersigned, for nild on belinlf of the underalgned and the representatives, lieirs nind nnilgun of the undernigned, hereby acknowledpea recelpt from Stone Weboter Englneerling Corporation (SWEC) of the aum of One Dollar and other valuable consideration, the recelpt and sufficlency of which is hereby acknowledged, In full and complete settlement and satiofaction of any and all clalma, demnnda, damagen, lonaes, actions, and causes of action of every kInd and clinracter, which the underntpined or their representativea, hetrs and anglgna have or mlght have by renaon of ownership of the property or real property rights in the property, described below on whlch the U.S. Government, and Its agents, gervants, employees, successors, asolgris and contractors have conducted drilling, hydrologic monitoring studies, well plugging. site restoration, and other operations. The herelnafter deacribed property 1 a also deacribed in certaln agreements (the "Agreements") executed by the underalgned whlch granted right-of-entry and the right to perform hydrologle monitoring otudies and other operations on the real property of the undersigned.

SWEC has completed well plupging and/or alte regtorntlon work nccording to the requirements of the Apreementa. In conalderation of the above payment, the underalgned lieriby lorever dinclinges and relenases the U.S. Government, and its agents, aervalls, employees, auccessors, asslgna, and contractors from any and all clalms, demande, damages, loses, actions and causes of action which the undersigned or thelr representatives, helrs, or assigns, have or might hive arising out of, accruing to or resulting from the performance of hydrolingle monltoring, atudiea and related activities on or in the vicinity of the property described below.

The undersigned represents that he/she/or they are the lawful owners of the property deocribed be low and is the proper parti to execute thls Release Agreement.

Description of Property Situated in the (Parlsh or County) of Perry - STATE 'OF Mississippl

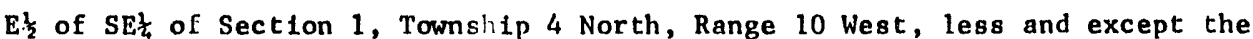

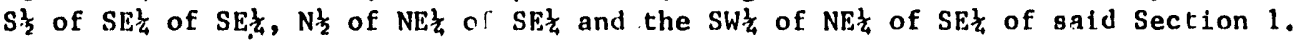

IN WITNESS WIIFREOF, we have hereunto ant our hande and seala thla

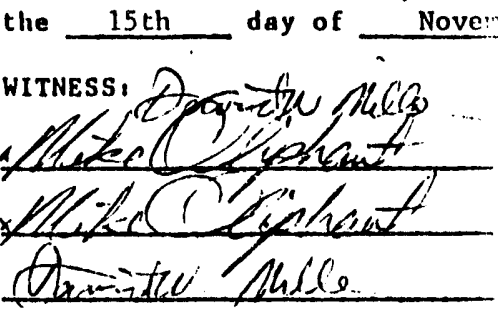
, 1988.

STATE OF

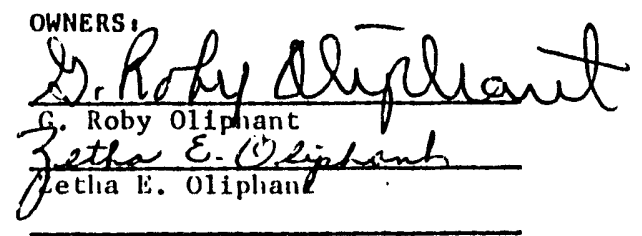

On this (Parlali or County) of

appeared

to me known to be the peraon( $n$ ) deacribed In and who executed the foregoing Instrument, and acknowledged that free act and deed. executed the ome as

(SEAL)

Notary Public In and for $\frac{(\text { Parlsli }}{(\text { State })}$
or County).

My Comulasion Explres: 
PERSONALLY APPEARED before me the undersigned authority in and for said County and State, the within named David $W$. Miler one of the subscribing witnesses to the within and foregoing Instrument, who belng: first duly sworn, deposeth and salth that he snw the within named G. Roby 0liphant and wife, Zetha E. 0liphant

whose names are subscribed thereto, sign and deliver the above and foregoing Damage Release, that he, this afflat, subscribed his name as a witness thereto in the presence of the said G. Roby J11phant and wife, Zetha E. Oliphant and that he saw the other subscribing witness sign the same in the presence of the sald G. Roby Oliphant and wife, Zetha E. Oliphant

and that the witnesses signed in the presence of each other, on the day and yeir there in named.

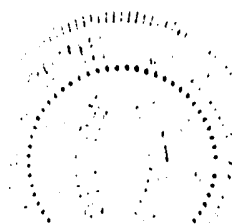

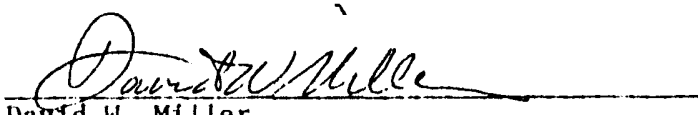

Swoprintand subscribed before me, this the $21 \mathrm{st}$ day of November, 1988.

\section{(Af $\mathrm{f} x$ Sëa 1 )}

My commission expires:

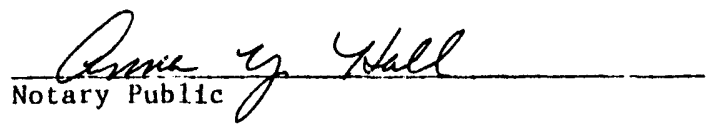

$2-5=1930$

\section{STATE OF MISSISSIPPI}

COUNTY OF UINDS

PERSONALLY APPREARED before me the indersigned authority in and for sald County and State, the within named David W.M1lle" one of the subscribing witnesses to the within and foregoing instrument, who being first duly sworn, deposeth and salth that he saw the within named

whose name subscribed theret, sign and dellver the above and foregolng Damage Release, that he, this afflat, subscribed his name as a witness thereto in the presence of the said

and that he saw the other subscribi.ug witness sign the same in the presence of the said

and that the witnesses signed in the presence of each other, on the day and year therein named.

\section{David W. Miller}

Sworn to and subscribed before me, his the day of November, 1988.

(Affix Sea1)

My commission explres:

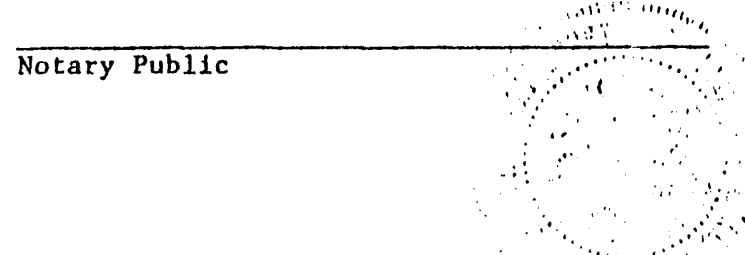

STATE OF MISSISSIPPI, Perry County Filed for record on the 23 day of enct 20 day of nou. 1988 in Deed
1988 at 8.38'clock $\cap . M$. and recorded this. reccrd No.8D-79 at Page $764=765$

ALFRED LOTT, Chancery Clerk

By Audxey shore 


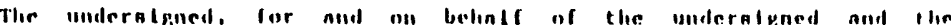

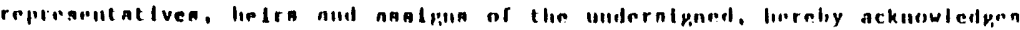
recelfte Crom stone o Webater Euglueerlng Corporntion (sWEC) of llie num of One Dollar and nther valuable consideration, the recelpt and suffiriency of whlch to liereby acknowledged, In litl and complete nettlement anil

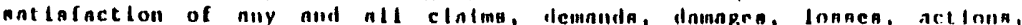

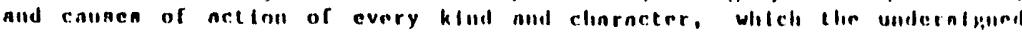

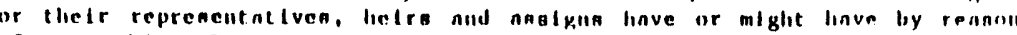
of owlierelilp of the property or real property riglite in llie property. dencrlbed belus on whlch the U.S. Covernnent, and Ita npenta, nervanta, employeca, nuccensors, anglgng and contenctorn lave conducted drillinp. hydrologle monttorling atudien, well pluppling, alte reatoration, and otlier

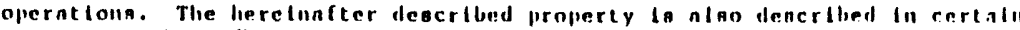
aprements (the "Agrectuente") executed by the underalgned whlch prantme riplit-of-entry and the riglit to perlorm liydrolngle monltoring atudies and other operations on the real property of the underalgiled.

SWEC las completed well plugpling alil/or olte reatorntion work accorlling to the requiremente of the Aprepmenta. In conslderntion of the aboue pnyment, the underalgued hereby forever dlacliarpea nuil releasen the U.s.

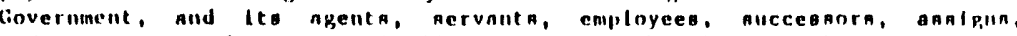

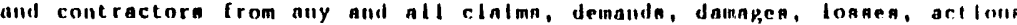
and causen of action whlch the underalgued or thelr reprenelltatives, lir lra, or asalgin, liave or mipht linve arlaling nut of, accrulng to or resultiug from tlie performnice of liyilrolopic monltarlap ntidlea and related activition on or III the vicintly of the property degeribed below.

The underolgned represente that lie/glie/or they are the Inwful owners of the property described below and ta the proper party to execute this Release Agreement.

Dencrlption of Property sttunted lin the (Pailali or count y) of - STATE UF Mississiup

SEt of St? of Section 35, Townshlp 5 North, Range 10 West. Nitli 222

IN WITNESS WHFREOF, we linve liereunto nat our lianda alli geala thita the 1011 day of November
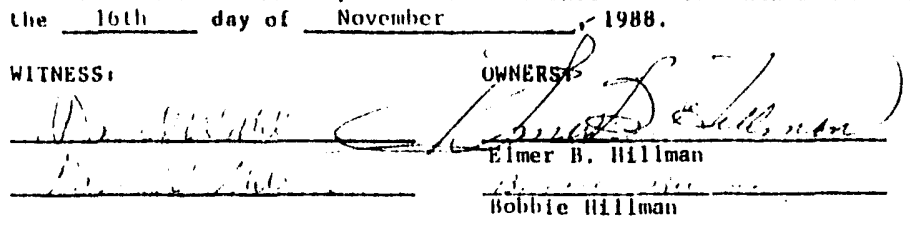

SIATE O (1), Int of (onuly) of

on this day or - 1988, before me peraonally appenred

to me known to be the peraon(a) deacribed in nind whin exceiled the coregotup linstrunent, and ncknowledged that exccuted the same a

tree act and deed.

(GEAL)

Hy Conmiasion Explres: inenry Publle lin ond lor (larlali II (10u11(y)). 


\section{STATE OF MISSISSIPPI}

\section{COUNTY OF HINDS}

PISRONAII.Y APPEAREI) liefore me the undersfgned nuthority in and for sald

county and sinte, the within named bavid w. Mtller. Lilir suliseriling

wilness to the withlu and foregolng instrument, who heing first duly sworn, deposeth and and th that he snw the within naned liliner B. IItllunn null wife, Hoblife Illliman, whose names are subscrthed thereto, sign and deliver the ahove and foregning bamnge Relense, that he, lihs afflant, sulsacrilied his llane as n wilness therelo in the presence of the snld bimer B. IIIl iman and wile, Hobble IIIIImall.
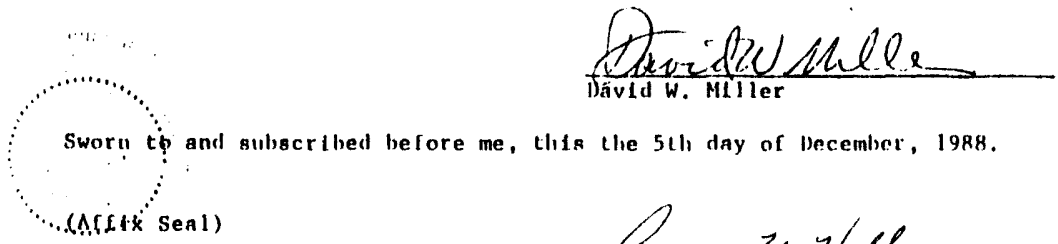
Dávid W. Miller

\section{$($ (Afflix Sea 1$)$}

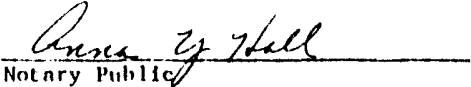

My conmisalon expltres:

$\therefore-5>0$

STATE OF MISSISSIPPI. Perry County

filed for record on the $x^{\prime \prime \prime}$ day ol גere Filed tar record on the

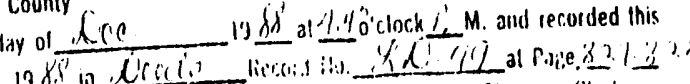
fiujulu Liji, Chancery Cleak

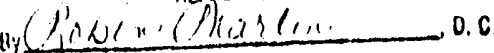


The underalpned, for and on behalf of the underalpingd and the

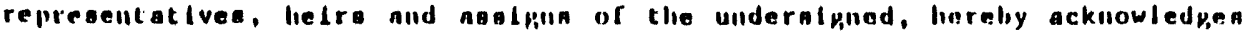
recelpt from stone Weboter Englneerling Corporation (SWEC) of the num of One Dollar and other valuable consideration, the recelpt and uufficlency of which to liereby acknowledged, In full and complete settlement and oatlofaction of any and all claims, demands, damages, losnes, actions, and causes of action of every kind and character, whlch the underalgned or their representatives, lielra and assigno have or mlght have by reacoll of ownerahip of the property or real property right in lie property, described below on whlch the U.S. Government, and its ngents, servants, employeen, auccessors, asigns and contractors liave conducted drilling, hydrologic monltorlng studles, well plugging, site restoration, and other operations. The lierelnafter deocribed property lo also deacribed in certaln agreements (the "Agreements") executed by the underaipned which pranted right-of-entry and the right to perform liydrologlc monltoring atudies and other operations on the real property of the underaignad.

SWEC lias completed well plugpling and/or olte reatoration work nccordlng to the requlrements of the Agreementa. In considerntion of the above payment, the underalgned lieruby lorever diacharpes and relenaen the U.s. Government, and t to agenta, nervalita, employees, aucceonors, eaglgna, and contractora from any and all clatima, demanda, damages, lonaes, act lons and causes of action which the undersigned or thelr tepresentatives, helrs, or anigue, liave or mlght have arloling out of, accruing to or resulting from the porformance of hydrologlc molitering atudlea and related activitlea on or lil the vicintty of the property deacribed below.

The undersigned represents that he/she/or they are the lawful owners of the property described below and is the proper party to execute this Releage Agreement.

Description of Property situnted I" the (Parlah or County) of Perry . STATE UF Mississipol

For description see Exhibit " $A$ " attached hereto. MRIG 223

IN WITNESS HIIEREOF, we linve hereunto art our handa alld aealo thia the 16 th day of 1988.

WITNESS,

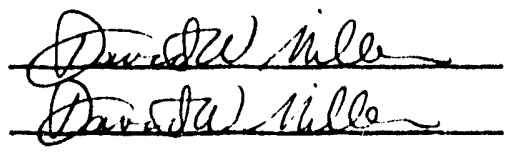

\section{OWNERS :}

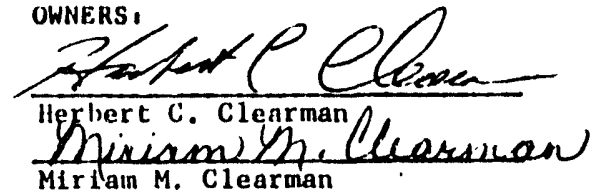

STATE OF - (larlali or Counly) of

On this day of 1988, before me peroonally appeared

to me known to be the person(a) described in and who executed the foregoing linstrument, and acknowledged that free act and deed. executed the oame as

\begin{abstract}
cree act and deed,
\end{abstract}
(SEAL)

Notnry Publle In and for $\frac{\text { (Parligh }}{\text { (State) }}$
or County).

My Commisaton Explres: 
Townsh1p 4 North, Range 10 West

Section 2: NWt of NWh, 1 ess and except the $W \frac{1}{2}$ of $W \frac{1}{2}$ of NWt of NWt, and 1 ess and except all that part of the NWt of NWt lying South of the paved road, containing 23.10 acres, more or 1 ess; and

Beginning at the intersection of the West line of the NEt of NWt with the centerline of a paved public road, and run North along ald West IIne for 749 feet to a fence that 18 running in a southeasterly direction, thence run South $76^{\circ}$ East along a fence and the projection of said fence for 823 feet to the intersection of the centerline of the aforesaid public road, said point being 978 feet northeasterly along said public road from the point of beginning, thence run southwesterly along the centerline of sald public road for 978 feet to the point of beginning, containing 6.9 acres, more or less.

MRIG 223

SIGNED FOR IDENTIFICATION:

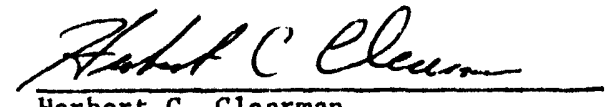

Herbert C. Clearman

Diviam th. Dlemenas Miriam M. Clearman 
54

STATE OF MISSISSIPPI

COUNTY OF HINDS

PERSONALLY APPEARED before me the undersigned authority in and for said County and State, the within named David W. Miller, one of the subscribing witnesses to the within and foregoing instrument, who being first duly sworn, deposeth and saith that he saw the within named Herbert C. Clearman and wife, Miriam M. Clearman, whose names are subscribed thereto, sign and deliver the above and foregoing Damage Release, that he, this affiant, subscribed his name as a witness thereto in the presence of the said Herbert C. Clearman and wife, Miriam M. Clearman.

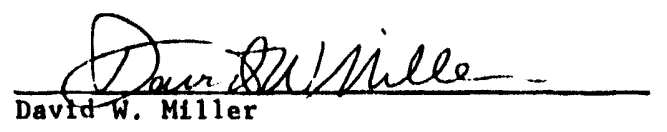

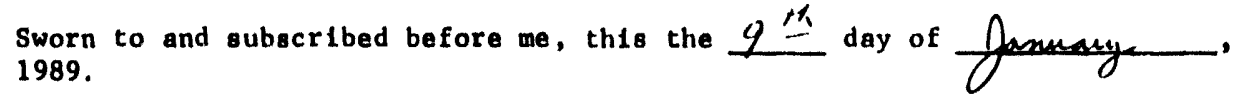

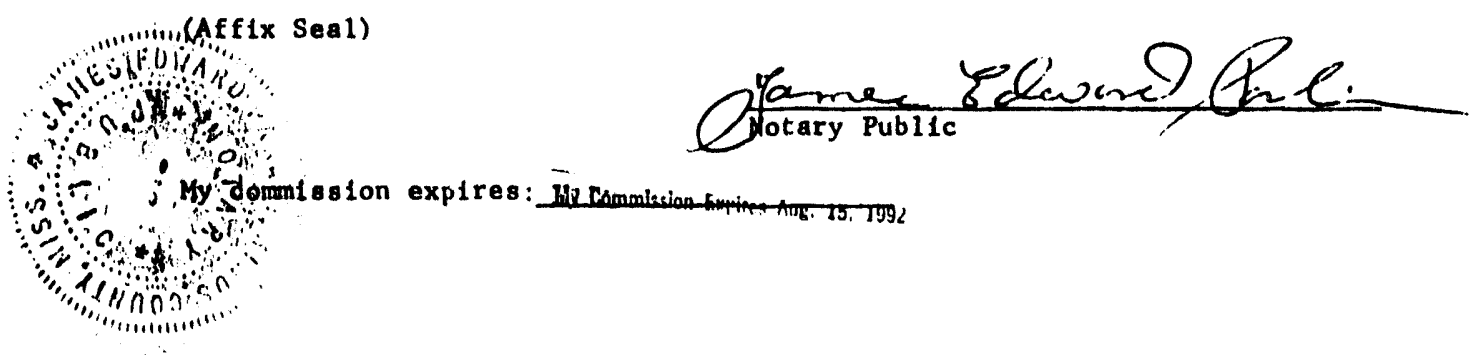

STATE OF MISSISSIPPI, Perry County

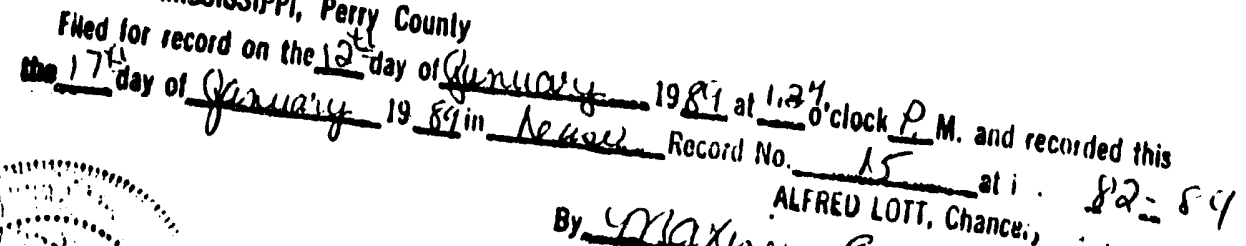
By Lelayeace BL RE LOTT, Chancei, . 
DAMAGE RELEASE

The undersigned, for aud on belinlf of the undernlgyed and the representatives, hel ra and anilpinn of the undernlgned, hereby acknowledges receipt from stone Webster Enplupering corporation (SWEC) of the nim of One Dollar and other valuable consideration, the receipt and sufficiency of which is hereby acknowledged, In lull and complete nettlement and

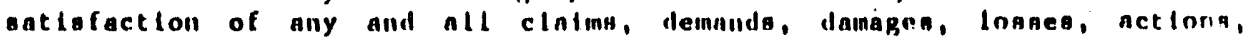
aid causes of action of ever: kind and chinencter, which the underalponel or their representatives, hel ra mind nasibila line or ing lit have by reason of owneralitp of the property or rent property riglitg In the property, described below on which the U.S, Government, and its agents, servants, employees, successors, asolgis and contractors have conducted. drilling, hydrologic monitoring studies, well plupsilig. site restoration, and other operations. The lierelnafter drocrlbed property $l a$ also described in certain agreements (chic "Agreements"; executed li the undersigned which granted riglit-of-entry and the right to perform hydrologic monitoring studies and other operations on the real property of the undersigned.

SWEC has completed well plopping and/or ate restoration work according to the requirements of the Agreements. In consideration of the above payment, the underalgued hereby forever dinclinrpes and relines the U.S. Government, and t ta agents, servants, employees, nuccenarn, analpin, and contractors from any and all claims, demands, damages, losses, actions and causes of action which the undersigned or their representatives, hel rs, or assigns, have or might halve earthling out of, accruing to or resulting from the performance of liydrologle monltorlup, atwillan and related activities on or li l the vicinity of the property described below.

The undersigned represents that he/she/or they are the lawful owners of the property described be low and to the proper party to execute this Release Agreement.

Description of Property Situated In the (Parish or County)

of Perry - STATE OF Mississippi

For description see Exhibit "A" attached hereto.

MIG 224

IN WITNESS WIIFReOF, we have hereunto art our lands and seals thea the 15 th day of Nover:ber , 1988.

WITNESSES :

Dorothy Phillips

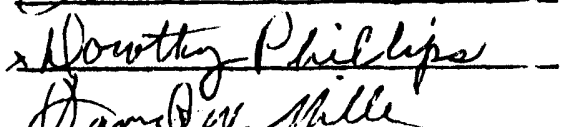
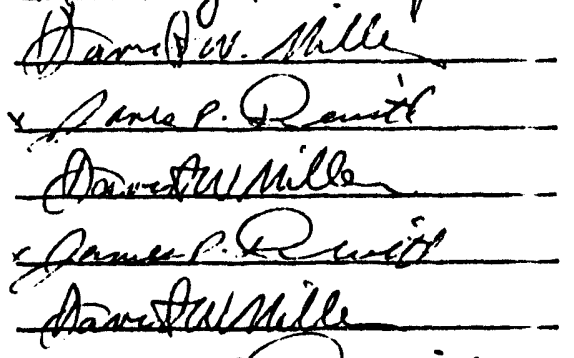

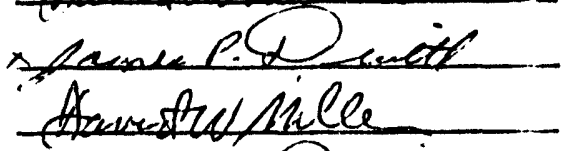

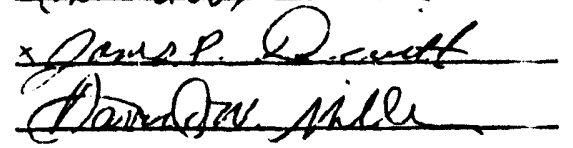

OWNERS:

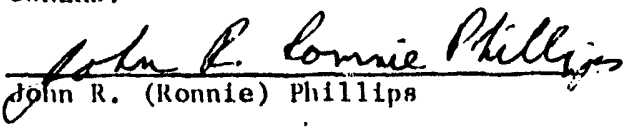

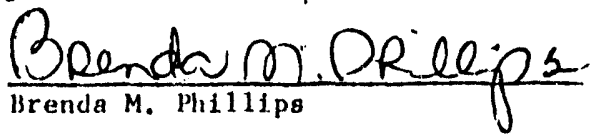

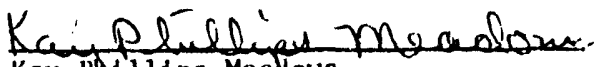

Kay Pi111ps Meadows

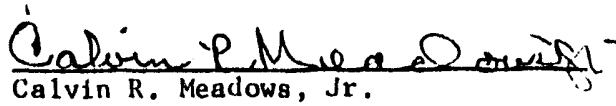

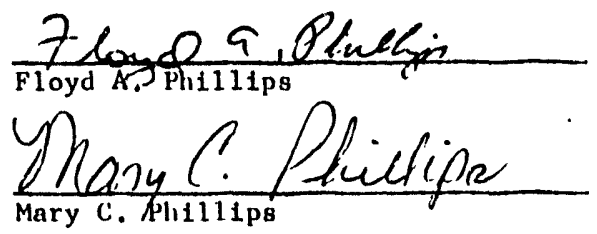


PERSONALLY APPEARED before me the undersigned authority in and for sald County and State, the within named David W. M1!ler one of the subscribing witnesses to the within and foregoing instrument, who belng first duly sworn, deposeth and saith that he saw the within named John_R. (Ronnte) Fi1111ps and wife. Brenda M. Ph1111pg whose names are subscribed thereti, sign and deliver the above and foregoing Damage Release, that he, this afflalt, subscribed his name as a witness thereto in the presence of the sald John R. (Ronnie) Phtllitps and wife. Brenda M. Phillips and that he saw the other subscribing witness sign the same in the presence of the sald John R. (Ronnte) Phil11ps and wife. Brenda M. Ph1111ps

and that the witnesses signed in the presence of each other, on the day and year therein named.

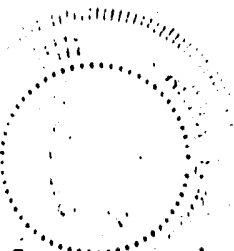

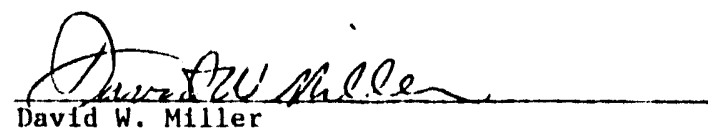

Swori'to' and subscribed before me, this the $21 \mathrm{st}$ day of November, 1988.

(Af $\mathrm{ix}$ Seal)

My commission explres:

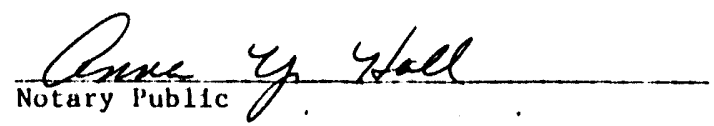

$$
a^{2}-5-1990
$$

STATE OF MISSISSIPPI

COUNTY OF HINDS

PERSONALI,Y APPREARED before me the undersigned authority in and for sald County and State, the within named David W.Miller one of the subscribing witnesses to the within and foregoing instrument, who being first duly sworn, deposeth and salth that he saw the within named Kay Phillipg Meadows and husband, Calvin R. Meadows, Jr. whose names are subscribed theret, sign and deliver the above and foregolng Damage Release, that he, this afflant, subscribed his name as a witness thereto in the presence of the sald Kay Ph111: ps Meadows and husband, Calvin R. Meadows, Ir, , and that he saw the other subscribli:; witness sign the same in the presence of "he sald Kay PhI111ps Meadows and husba: d, Calvin_R. Meadows, Ir.

and that the witnesses signed in the presence of each other, on the day and year there in named.

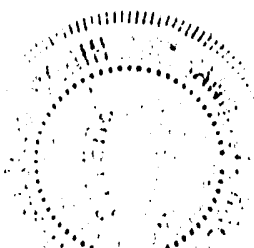

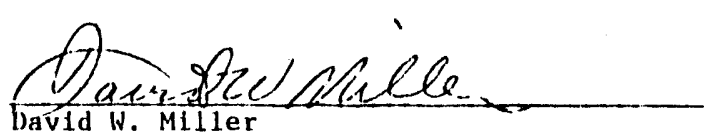

Sworti "to and subscribed before me, tils the $218 t$ day of November, 1988.

(Aff1x Seal)

My commission explres:

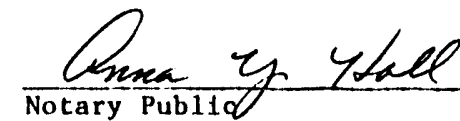


STATE OF MISSISSIPPI

COUNTY OF HINDS

PERSONALLY APPEARED before me the undersigned authority in and for said County and State, the within named David W. Miller one of the subscribing witnesses to the within and foregoing ingtrument, who beins: first duly sworn, deposeth and saith that he saw the within named Floyd A. PhIlligs and wife, Mary Ce Ph1111ps

whose names are subscribed there:0, sign and deliver the above and foregoing:

Damage Release, that he, this affint, subscribed h1s name as a witness thereto in the presence of the sald Floyd A. Phlllips and wife. Mary C. Phillips

and that he saw the other subscribing witness sign the same in the presence of the sald Floyd A. Phillips and wife, Mrry C. Pht1lips

and that the witnesses signed in the presence of each other, on the day and year therein named.<smiles>C1=CC=Cc2ccccc2C=C1</smiles>

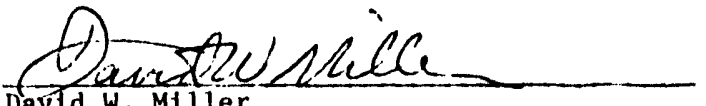

Sworn to and subscribed before me, this the $218 \mathrm{st}$ day of November, 1988 .

(Affix Sea1)

My commission expires:

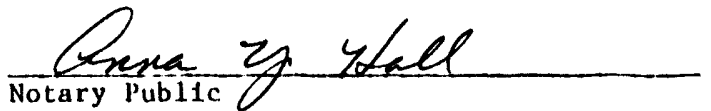

$x^{2}-5-1990$

STATE OF MISSISSIPPI

COUNTY OF HINDS

PERSONALLY APPREARED before me the indersigned authority in and for said County and State, the within named David W.M1ller one of the subscribing witnesses to the within and foregoing instrument, who being first duly sworn, deposeth and saith that he saw the within named

whose name subscribed thereto, sign and deliver the above and foregoing

Damage Release, that he, this affiat, subscribed his name as a witness thereto in the presence of the said

and that he saw the other subscribing witness sign the same in the presence of the sald

and that the witnesses signed in thre presence of each other, on the day and year therein named.

David W. Miller

Sworn to and subscribed before me, this the day of November, 1988.

(Aff1x Sea1)

My commission expires: 
LXHIBIT "A"

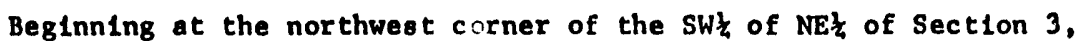
Township 4 North, Range 10 Wrot, Perry County, M1881881pp1, thence run South 974 feet, thence run East 210 feet, thence run South 200 feet to the centeriline of a gravel road, thence run Northeasterly along the centerline of 8 ald road to the centerline of a blacktop public road, thence run Southeasterly along the centerline of sald blacktop road to the east boundary of sald forty $(40)$, thence run North 762 feet, thence run West 1,350 feet to the point of beginning, containing 25 acres, more or less.
\end{abstract}

STATE OF MISSISSIPPI, Perry County

Filed for record on the 23 day of now. 1988 at8.3. $30^{\prime}$ clock $\theta . M$. and recorded this

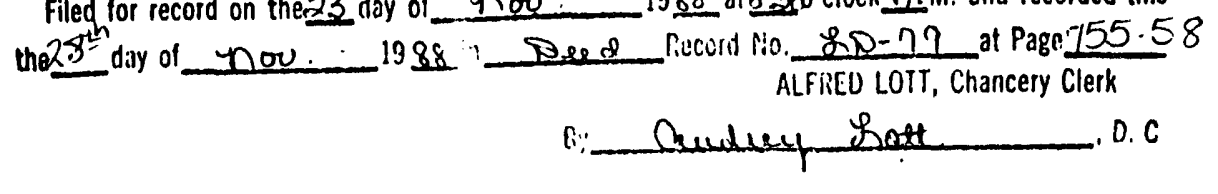


The underalgned, for and on behalf of the underalgned and the representativea, helrs and asipins of the underalgned, liereby acknowledgen rccelpt from stone \& Weboter Englneering Corporation (SWEC) of the sum of One Dollar and other valuable consideration, the recelpt and sufficlency of which to hereby acknowledged, In full and complete oettlement and ontiofaction of any and all clalmo, demnids, danages, Iosees, actionn, and causes of action of every klnd and clinencter, whlch the underalgied or their representatives, helra alld anglgni have or inglit liave by reason of ownerahlp of the property or real property righta in the property, deacrlbed below on whlch the U.S. Government, ind its agents, eervants, employees, successors, asaigns and contractors have conducted drilling. hydrologic monitoring tudies, well pluggini, olte restoration, and other operations. The hereinafter descrlbed propercy is also described in certaln agreements (the "Agreements") executed by the undersigned which granted right-of-entry and the right to perform hydrologlc monltoring otudies and other operations on the real property of the underolgned.

SWEC has completed well plugging and/or al te reatoration work according to the requirements of the Agreements. in consideration of the above payment, the underalgned hereby forever Jincharges and relenses the U.S. Government, and lts agents, servanta, employees, successors, asplpna, and contractora from any and all clalma, desunda, danages, loses, actiona and causes of action wilch the undersigned or thele representativea, helrs, or asolgna, have or mlglit have arialng out of, accrulng to or resulting from the performance of liydrologlc montoring atudlea and related activities oil or in the vicinity of the property deocribed below.

The undersigned represente that he/she/or they are the lawful owners of the property described below and is the proper party to execute this Release Agreement.

Description of Property Sltuated in the
of Perry

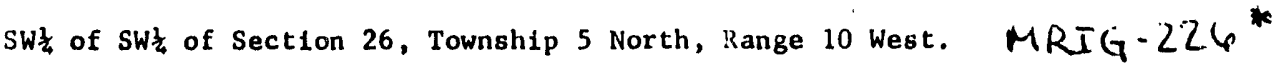

IN WITNESS WIIEREOF, we have hereunto net our hande and geals thla the loth day of November

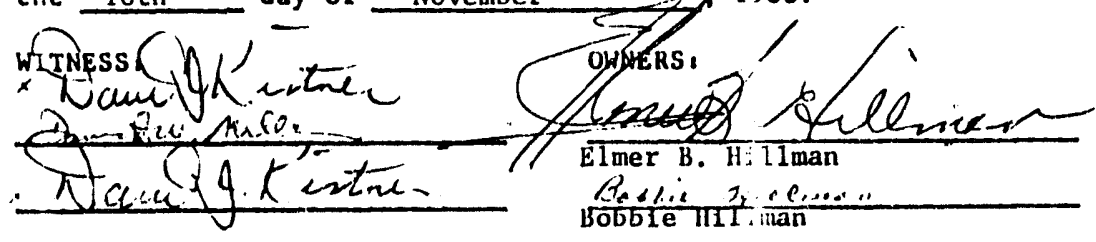

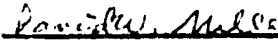

STATE OF - (Parlali or County) of

On this day of - 1988, before me personally appeared

to me known to be the person(s) descrlbed In and who executed the foregoing instrument, and acknowledged that free act and deed. executed the same as

(SEAL) Notary Pub Ic In and for (Parlsh or County).

(State)

My Commisaton Explres:

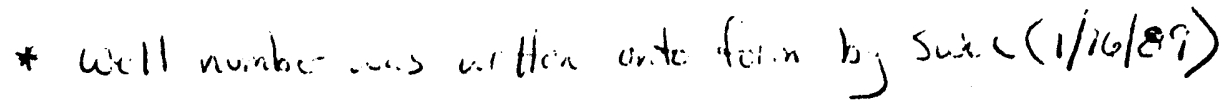


STATE OF MISSISSIPPI

COUNTY OF HINDS

PERSONALLY APPEARED before me the undersigned authorlty in and for sald County and State, the within named David W. Miller

one of the subscribing witnesses to the within and foregoining instrument, who

belng first duly sworn, deposeth and salth that he saw the within named

Elmer B. Hillman and wife, Bobble Hillman

whose names are

subscribed thereto, sign and deliver the same to the sald stone \& Webster

Engineering Corporation on that he, this afflant, subscribed his name as a

witness thereto in the presence of the sald Elmer B. Hillman and wife, Bobbie Hillman a: that he saw the other subscribing witness sign the same In the presence of the sild Elmer B. Hillman and wife.

Bobbie Hillman and that the witnesses signed in the presence of each other, on the day and year therein named.

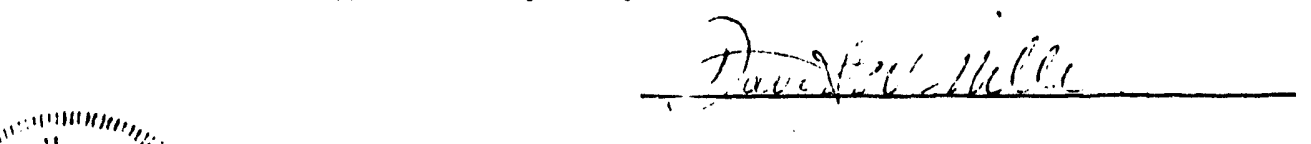

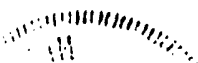

Sworn.to" and subieribed before me, this the $11: \mathrm{h}$ day of November $19 \underline{88}$.

(Afix

My cominisstion expires:

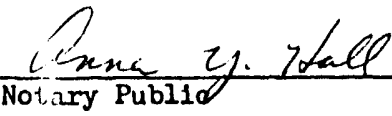

Zebricän

\section{STATE OF MISSISSIPPI}

COUNTY OF

PERSONALLY APPEARED before me the undersigned a:dthority in and for said County and State, the within named

one of the subscribing witnesses to the within and foregolng Instrument, who

beling flrst duly sworn, deposeth and salth that he saw the within named

subscribed thereto, sign and deliver the same to the sald

- whose name

witness thereto in the presence of the sald

, ari that he saw the other subscribing

witness sign the same In the presence of the said

and that the witnesses signed in the presence of each other, on the day and year therein named.

Sworn to and subscribed before me, this the day of 19

(Afrix Seal)

My commission explres!

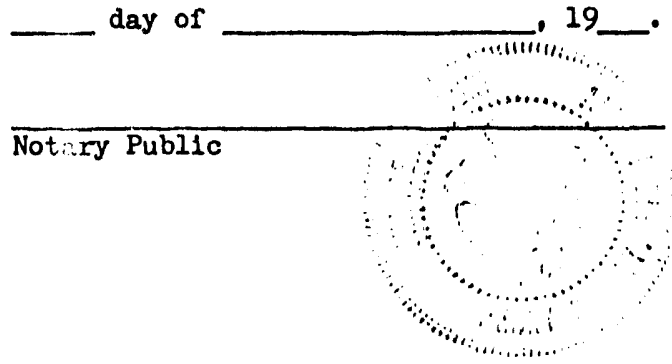

staTE OF MASSISSIPPI, Perry Counfy

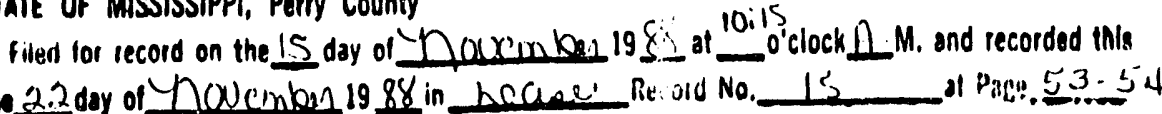

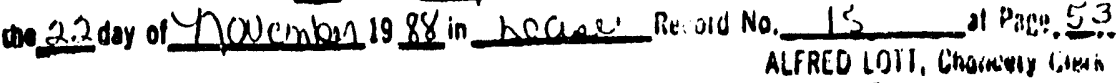

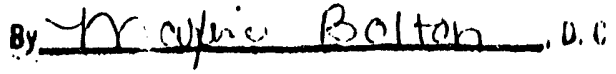


The underalgned, for and on belialf of the underalgned and the representatives, lieirs nind nosibnn of the undernigned, hereby acknowledpen recelpt from stone Webater Englneerln: Corporation (SWEC) of the num of One Dollar and other valuable consideration, the recelpt and sufficlency of which 1s hereby acknowledged, In full and complete oettlement and satiofaction of any and all clatims, detinnds, damages, losees, actions, and causes of action of every $k$ ind and character, which the undersigned or thelr representatives, helrs and assifiso have or might have by reason of owmershlp of the property or real property rights in the property, described below on whlch the U.S. Government, and its agents, servants, employees, successors, assigns and contrictors have conducted drilling, liydrologic monitoring studies, well plugising, olte restoration, and other operations. The hereinafter descrlbed propierty to nlso described in certaln agreements (the "Agreenents") executed by the undersigned which granted right-of-entry and the right to perforia hydrologle monitoring atudies and other operations on the real property $c f$ the undergigned.

SWEC has completed well plugging and/or alte regtoration work according to the requirements of the Agreements. In consideration of the above payment, the underalgned liereby forever Ilscliarges and releases the U.S. Government, and its agents, ocrvants, cmployees, ouccessors, asigna, and contractors from any and all clatms, iemands, damages, loses, actions and causes of action whicli the undersigned or their representatives, heirs, or asolgns, have or might have arloling nut of, accruing to or resulting from the performance of hydrologic monltoring, atudles and related activitlea oll or in the vicinity of tlic property desc: lbed below.

The undersigned represents that lie/slie/or they are the lawful owners of the property described below and 18 the proper party to execute this Release Agreement.

Description of Property sltuated In tlie
Perry

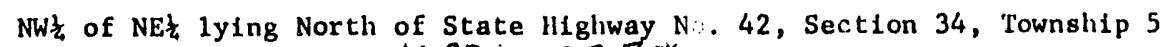
North, Range 10 West. MRIG $-227 *$

IN WITNESS WIIEREOF, we have hereunio get our hands and seals thlo

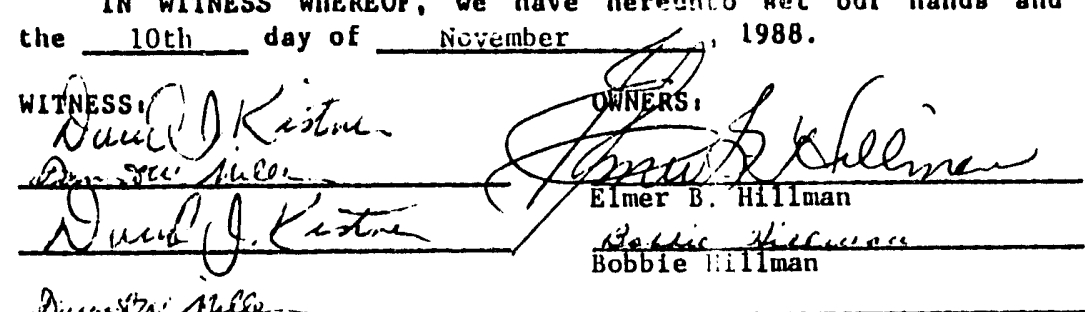

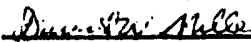

STATE OF - (partall or County) of

On this day of 1988, before me perionally appeared to me known to be the person(g) described In and who executed the foregoing instrument, and acknowledged that

[ree act and deed.

(SEAL) Notary lublic in and for (Parlsh or Count $y$ ).

My Commission Explresi

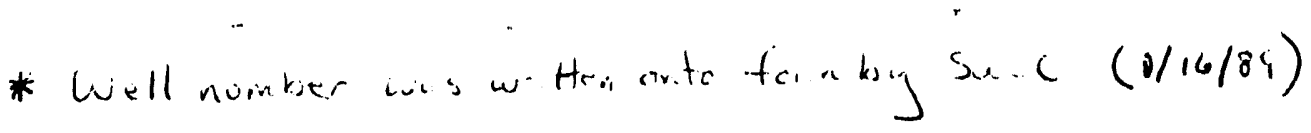


STATE OF MISSISSIPPI

COUNTY OF HINDS

PERSONALLY APPEARED before me the undersigned a thorlty in and for sald County and State, the within named David W. Miller

one of the subscrlbing witnesses to the within ind foregoining instrument, who being first duly sworn, deposeth and salth that he saw the within named Elmer B. Hillman and wife, Bobbie ll11lman

, whose names are subscribed thereto, s1gn and deliver the same to the sald Stone \& Webster Engineering Corporation on that he, this afflant, subscribed his name as a witnoss thereto in the presence of the sald Blmer B. Hillman and wife, Bobble H1l iman , and that he saw the other subscribing witness sign the same in the presence of the sald Elmer B. HLIlman and wife,

Bobhie Hillman and that the witnesses signed in the presence of each other, on the day and year therein named.

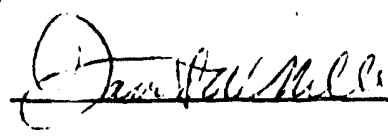

$$
\text { . }
$$

$111:$ day of November $19 \underline{88}$.

Sworn to and subscribed before me, this the

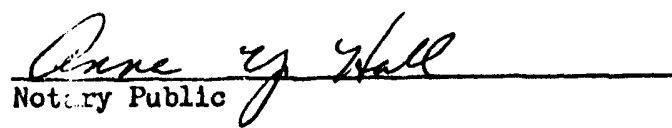

My commission explres:

Feliman 5 1550

STATE OF MISSISSIPPI

COUNTY OF

PERSCNALLY APPEARED before me the underslgned alithority in and for sald County and State, the within named

one of the subscribling witnesses to the within and foregolng instrument, who

belne flrst duly sworn, deposeth and soith that he saw the within named

subscribed thereto, sign and deliver the same tc the sald
witness thereto in the presence of the sald

anc? that he saw the other subscribing Witness sign the same In the presence of the said

and that the witnesses signed in the presence of each other, on the day and year therein named.

Sworn to and subscribed before me, this the day of

(Aff1x Seal)

My connission explresi

Notary Public

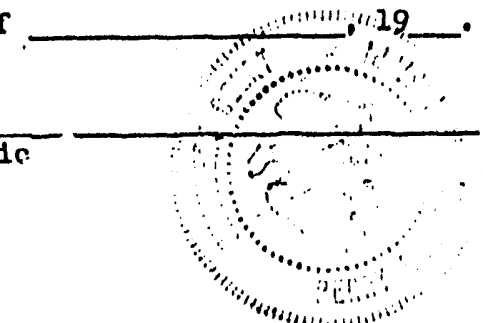

STATE of MISSISSIPPI, Perry County

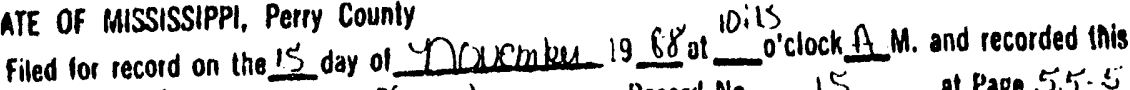

By Y: vollerey Boltoh_o.6 
The II.S. Government, elther directly or tlirough contracts with others lias drilled certaln liydrologic and geologle Borliga (tlie "Horlng") on the property of Controlex Enterprises, lne. (The "landowner"). Description of the property ituated in (Parioli or County of) Perry

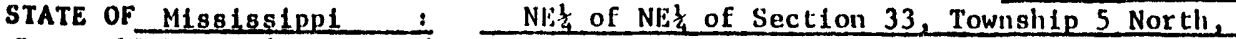
Range 10 West, lying South of lligliway No. 42 as presently lald out;

WIIEREAS, the Landowner may wish to use the borling as a water well, and

WiEREAS, lise U.S. Goveriment did not drIll the boring as a water well;

THEREFORE, In conelderation of the U.S. Government leaving the Boring In Ito present condition to be used by the Landowner as the handowner desires, the undersigied Landowner, does hereby forever discliarge and release the U.S. Government and tto agents, oervanta, employees, ouccessors, assigns, and contractora which are or may be in any mnner whatioever responsible for the Borling from any and all clalma, demanda, damages, losses, actions and causen of action whlch the underslgned Landowner or their representativen, helra, or asolgno has againat any of tlie above, Including opecifically, but not limited to, any clalu whlch lo related to the oultablilty of the Borling as a water well.

In executing and delivering the releare, the underalgned Landowner relled wholly upon his Judgment, knowledre nnd bellef an to the nature, extent and duration of the damages whlch lie may oustalin In the future. The undersigned further repreaents and warrante that he has not bcen Influenced by any representationa, statements or warrantles made by any peraon, firm, association, partnership or corporation or agency of the U.S. Government liereby released, or by any agent"l, employee or of ficer representing them, or any of them, concernlin the nature, extent and duration of any potential damages. Losses or legal llabllity.

The undersigned representa that he/she/or they are the lawrü owner of the property described above and are the proper party to execute this release/agreement.

In withess whereof, we have icreunto net our hands and oealo this the $15 \mathrm{th}$ day of November 1988 .

WITNESS

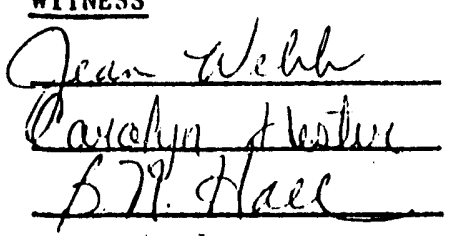

OWNERS:

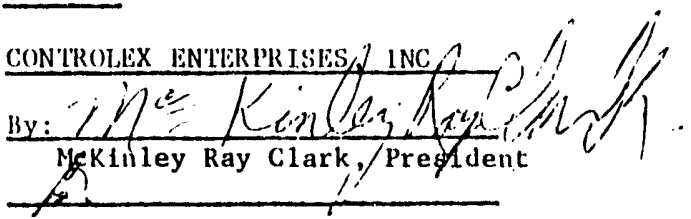

\section{STATE OF MISSISSIPPI}

COUNTY UF JACKSON

BEFORE ME, the undersigned officer in and for the county and state aforesald, this day personally appeared the within named Mckinley Ray clark who acknowledged that as President on behalf of and by authority of Controlex Enterprises, Inc., a Corporation, he signed, sealed and delivered the foregoing conveyance on the day and year therein named, as the free and voluntary act of the said Corporation after being first authorized so to do.

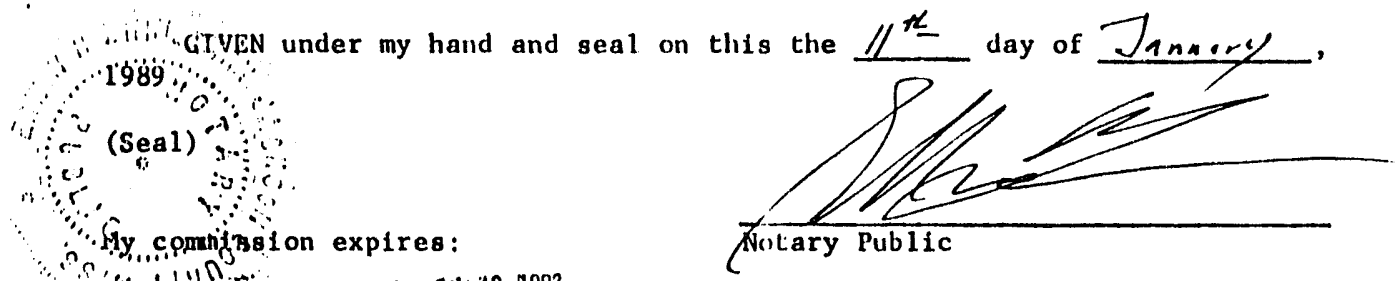


The underalgned, for and on belialf of the undernigned and the representatives, helrs and aospan of the undernlgned, hereby acknowledgen recelpt from stone Webater EngIneerlnp corporation (SWEC) of the num of One Dollar and other valuable conglderation, the recelpt and sufficlency of whlch 18 hereby acknowledped, In full and complete settlement and at lofaction of any and all clalme, demanda, damages, Insmes, actionn, and cnuses of actlon of every klnd and clinracter, whlch the underalgned or thelr representatives, helrg nnd nasigns have or mlght liave by rengon of owneralitp of the property or renl property rights In the property, described below on whlch the U.S. Government, and Ito ogente, gervants, employees, successors, asalgns and contractors have conducted drilllng, hydrologlc monltorling atudies, well plugglng, site reatoration, and other operatlons. The herelnafter descrlbed property lo aiso dencrlbed in certaln agreements (the "Agreemento") executed by the underalgned whlch granted right-of-entry and the right to perform hydrologle monltoring otudies and otlier operations on the real property of the underoigned.

SWEC has completed well plugglng and/or olte rentoration work according to the requltements of clie Apreementin. In considerntion of the above paynent, the undernigned liereby forever dincliarpes and relennen the I.s. Government, and Itn agenta, nervnnta, employeen, aucceonorn, onflpinn, and contractorg from any and all claims, demands, damages, lospen, act lonn and cnuses of action whlch the underalgned or thelr reprenentatlves, helrs, or analgna, have or might have arlalng out of, accrulng to or regulting, from the performance of liydrologic monttoring, atidles and related activities on or In the vicinlty of the property deocrlbed below.

The underglgneil represents that he/glie/or they are the lawful owners of the property denirlbed below and is the proper party to execute this Releabe Agreeinent.

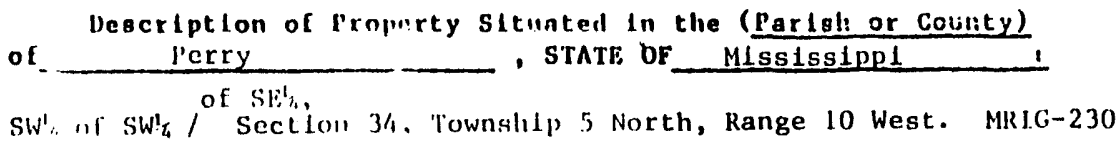

IN WITNESS WIIFHi:OF, we have hereunto nat our hands and ocela thlo thir 27th day ol January . Kseax 1989.

WI THI:S3,

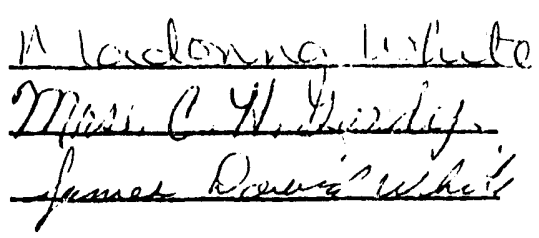

OWNERS
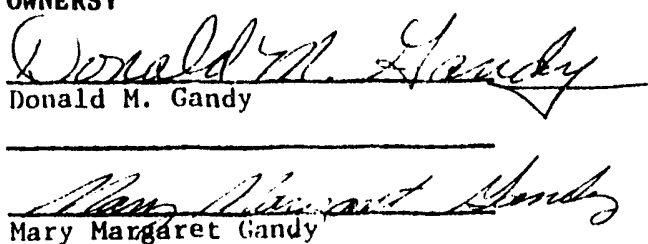

STATE OF MISSISSIPPI

COUN'TY OF

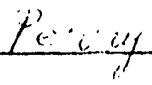

TIIIS DAY personilly appeared hefore me, the undersigned authority in and for sald County and State, the within named Donald $M$. Gandy and wife, Mary Margaret Gandy who acknowledged that they stgucd and delivered the within and foregoing instrument on the day and year thercln mentioned. 1989.

'GIVEN under my ha:ad and seal of office, this $6^{\text {th }}$ day of steruary,'
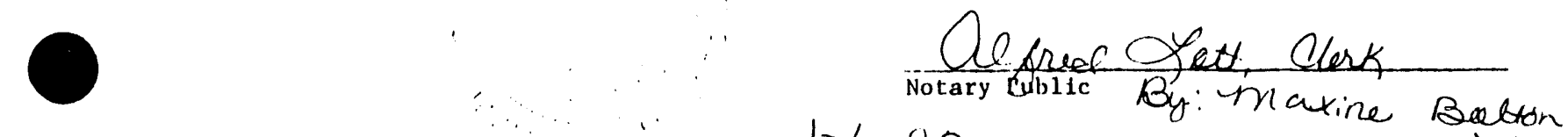

My commissión expires:

$1-6-93$

$\Delta \cdot C$. 
The U.S. Government, either directly or tlirough contencte with othern linn drilled certaln liydrologlc and geologle Borlnan (the "Morlug") on the moperty of Dona1d M. Gandy et ux, Mary Margaret Gandy (Llie "Landowner"), Dencription of the Property oltuated I" (Parloli or County of) Perry

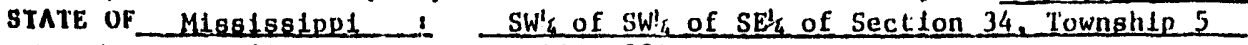
North, Range 10 West MRIG -230

HILAEAS, the Landowier may wioli to une the borlng as a water well, and

WIEREAS, the U.S. Government did not Irill the boring as a water well,

TIEREFORF, In congldeintion of the U.s. Government leaving the Boring In Itn present condition to be uned by the Landowner as the handowier desiren, the underalgind Landowner, loes hereby forever dlocharge and release the U.S. Coverunint nind tta agenti, ervanto, emplóyeer, auccensors, asolpun, aild contractoin which arc or may be In any manner whataoever respountble for the Boilin, from nily nind all clalmo, demanda, damages, loseen, nctlong and cannce of actlon which the underalgned Landowner or thelr represententiven, lintra, or naglpine has aralnat any of the nbove,

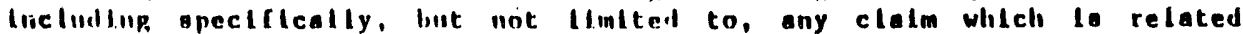
to the nultablilty of the Dorlug as n waler well.

In ex"ait: Ing nind delluerling the releanr, the underolgned Landowner relled wholly "low hlo Julgment, knowledpe nud bellef an to the nature, extent. and duintion of the dnmagea whicli he may ountali in the future. The undernlfund further repreaenta and warrante that he has not been Influnnced by any representat lonf, otntement, or warrantiea made by any person, firm, aosoclation, partnerghip or corporation or agency of tie U.s. Government hereby releaved, or by nily ngents, employee or offlcer representing them, or any of them, concernlii): the nature, extent and duration of any potential damager, losees or legal lisebility.

The underalgited reprenente that he/olio/or they are the lawful owner of the property deocribed above and are the proper party to execute this release/agreement.

In withese whereof, we liave liereunto net our hands and seals this the

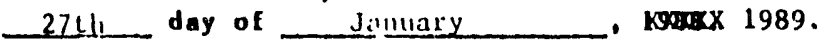
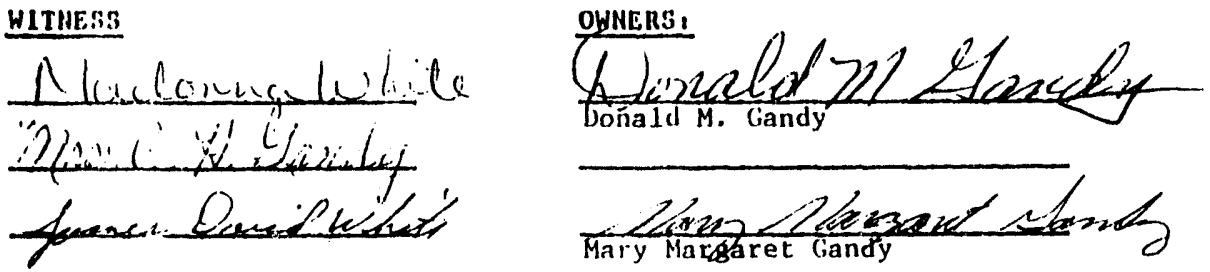

STATE OF MISSISSIPP I

COUNTY OF DRRY

TIIIS DAY personally appeared before me, the underslgned authorlty in and for sald County and Stale, the within named Donald M. Gandy and wife, Mary Margaret Gandy who acknowledged that they signed and delivered the within and foregoing instrument on the day and year therein mentloned. 1989.

GIVEN under my hand and scal of of fice, this $6^{\text {chs }}$ day of tebruary.

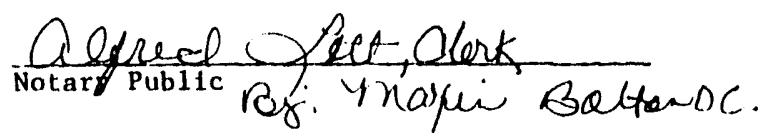

My commission expites: $1 \cdot 6-92$ 

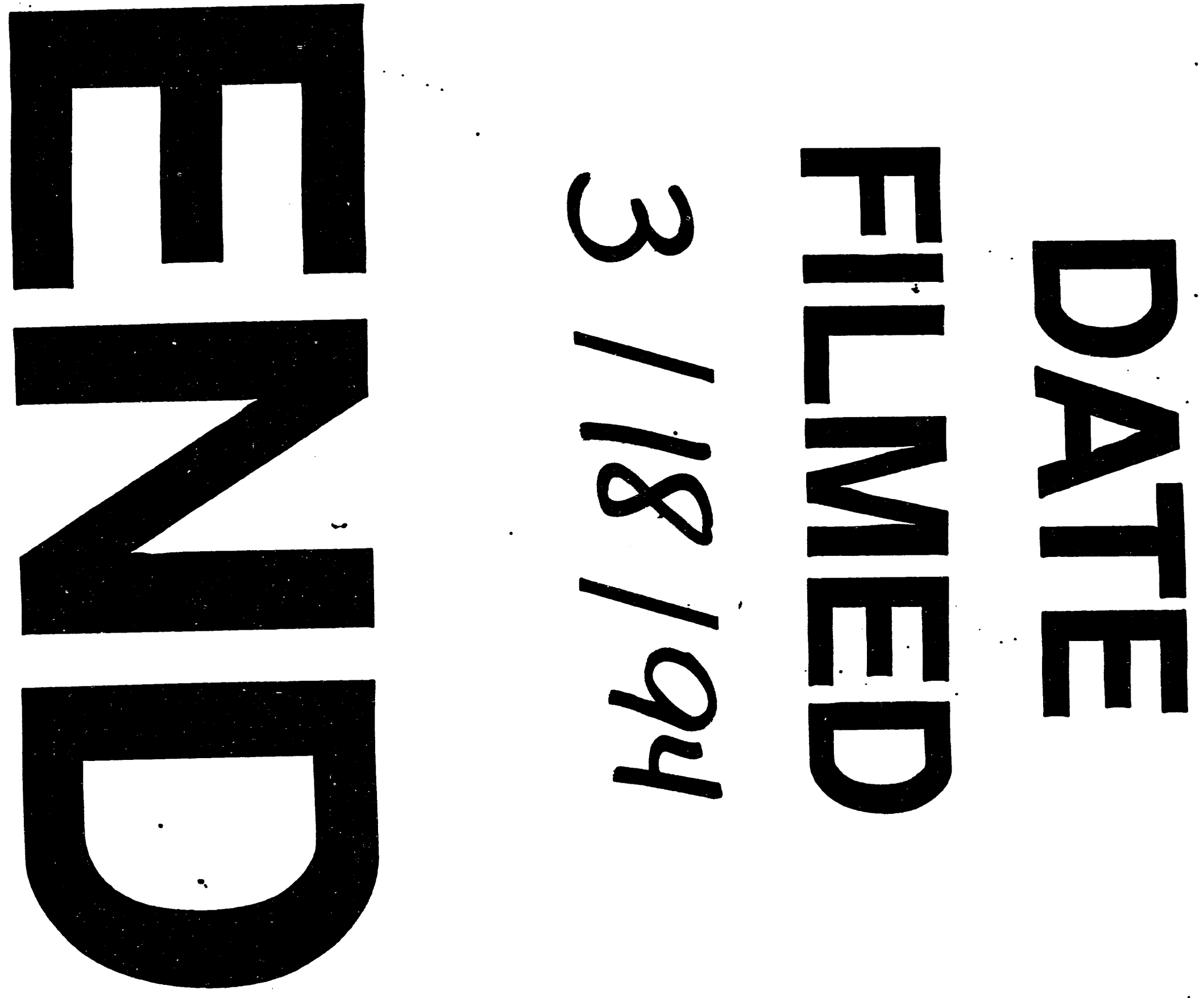
